DOE/OR/01-1136

Annual Report on the Background Soil Characterization Project on the Oak Ridge Reservation, Oak Ridge, Tennessee

Results of Phase I Investigation

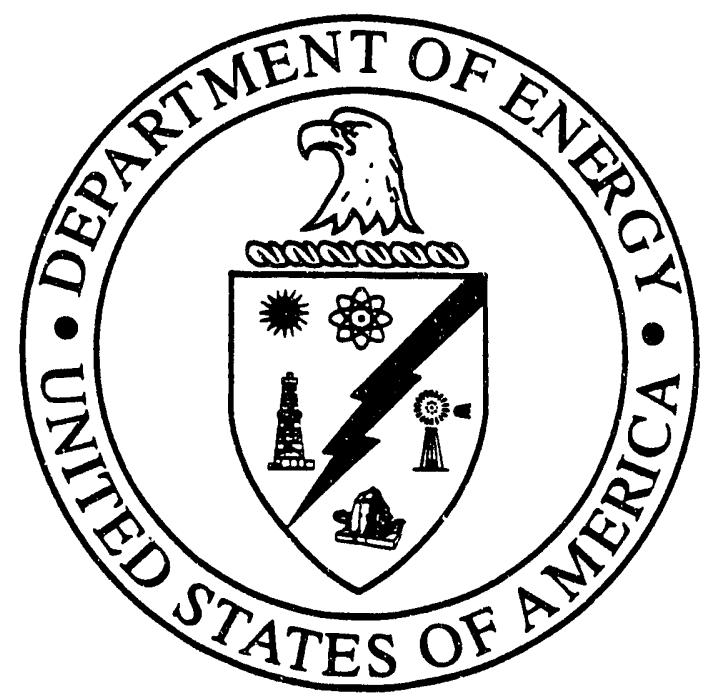




\section{NOTICE ABOUT UNUSABLE DATA}

The europium-155 data contained in this report have been determined to be unusable, because of possible interference with the gamma spectra of other naturally occurring radionuclides in soils. Although these data were used throughout the analyses discussed in this report, including the evaluation of risk, the effects of eliminating the europium-155 data from risk considerations are minimal, because the risks posed to human health from ingestion of and external exposure to uncontaminated soil on the Oak Ridge Reservation (ORR) containing background levels of europium-155 are relatively small $\left(5.97 \times 10^{-11}\right.$ and $1.49 \times 10^{-7}$, respectively). Furthermore, individual risks are negligible when compared with the total pathway risk from ingestion of and external exposure to background levels of all radionuclides on the ORR (pathway risks are $7.39 \times 10^{-6}$ and $6.38 \times 10^{-4}$, respectively). Hence, elimination of the europium-155 data from this data set would not significantly change the risk values, and similar statements are also true for the europium-155 background risks for Anderson and Roane counties. Revised Phase I results will be presented in the project final report.

This report has been reproduced directly from the best avallable copy.

Available to DOE and DOE contractors from the Office of Scientific and Technical Information, P.O. Box 62, Oak Ridge, TN 37831; prices available from $615-576-8401$.

Available to the public from the National Technical Information Service, U.S. Department of Commerce, 5285 Port Royal Rd., Springfield, VA 22161. 
DOE/OR/01-1136

ES/ER/TM-43

\title{
Annual Report on the Background Soil Characterization Project on the Oak Ridge Reservation, Oak Ridge, Tennessee \\ Results of Phase I Investigation
}

\author{
Environmental Restoration Division \\ P.O. Box 2003 \\ Oak Ridge, Tennessee 37831-7298
}

Date Issued-May 1993

\author{
Prepared by \\ Environmental Sciences Division \\ Oak Ridge National Laboratory \\ ESD Publication 3944 \\ Prepared for
}

U.S. Department of Energy

Office of Environmental Restoration and Waste Management under budget and reporting code EW 20

MARTIN MARIETTA ENERGY SYSTEMS, INC.

Oak Ridge K-25 Site

Oak Ridge Y-12 Plant

Oak Ridge National Laboratory

under contract DE-AC05-84OR21400

managing the

for the
U.S. DEPARTMENT OF ENERGY

Paducah Gaseous Diffusion Plant Portsmouth Gaseous Diffusion Plant under contract DE-AC05-76OR00001 
Authors

D. R. Watkins

J. T. Ammons

J. L. Branson

B. B. Burgoa

P. L. Goddard

T. L. Hatmaker

L. A. Hook

B. L. Jackson

C. W. Kimbrough

S. Y. Lee

D. A. Lietzke

C. W. McGinn

B. D. Nourse

R. L. Schmoyer

R. A. Shaw

S. E. Stinnette

J. Switek

J. C. Wright

\section{Author Affiliations}

D. R. Watkins (Project Manager), L. A. Hook, S. Y. Lee, and J. Switek are members of the Environmental Sciences Division; B. L. Jackson is a member of the Computing Applications Division; P. L. Goddard is with the K-25 Site Program Office; T. L. Hatmaker and J. C. Wright are with the Measurement Applications and Development Group; C. W. McGinn, B. D. Nourse, R. A. Shaw, and S. E. Stinnette are members of the Health and Safety Research Division; and R. L. Schmoyer is a member of the Engineering Physics and Mathematics Division, all part of the Oak Ridge National Laboratory. C. W. Kimbrough is manager of the Analytical Projects Office. All of these organizations are managed by Martin Marietta Energy Systems, Inc. J. T. Ammons, J. L. Branson, and B. B. Burgoa are with the Department of Plant and Soil Science at The University of Tennessee in Knoxville. D. A. Lietzke is a consultant. 


\title{
Annual Report on the Background Soil Characterization Project on the Oak Ridge Reservation, Oak Ridge, Tennessee
}

\author{
Results of Phase I Investigation
}

(DOE/OR/01-1136)

\author{
APPROVALS
}

S. P. Ridtte, Chief

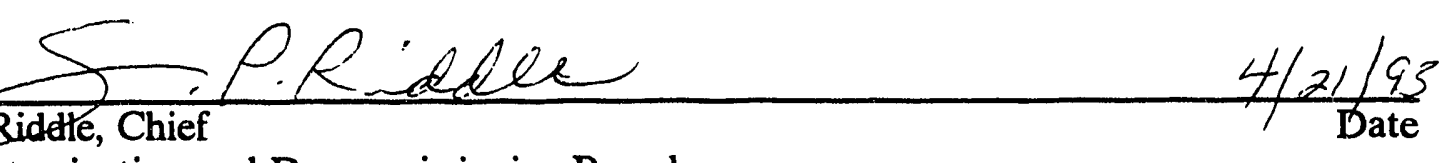

Decontamination and Decommissioning Branch

DOE Oak Ridge Field Office

D. M. Carden

$\frac{4 / 21 / 93}{\text { Date }}$

DOE Program Manager

DOE Oak Ridge Field Office

D. T. Bell

David T. Bell

ER Program Manager

Martin Marietta Energy Systems, Inc.

D. R. Watkins

MUlatemi

BSCP Manager

$4 / 21 / 93$

Martin Marietta Energy Systems, Inc. 


\section{CONTENTS}

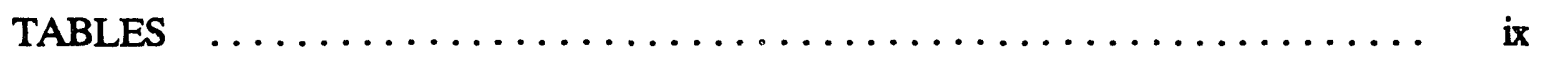

FIGURES $\ldots \ldots \ldots \ldots \ldots \ldots \ldots \ldots \ldots \ldots \ldots \ldots \ldots \ldots \ldots \ldots \ldots \ldots \ldots$

ABBREVIATIONS $\ldots \ldots \ldots \ldots \ldots \ldots \ldots \ldots \ldots \ldots \ldots \ldots \ldots \ldots \ldots \ldots$

EXECUTTVE SUMMARY $\ldots \ldots \ldots \ldots \ldots \ldots \ldots \ldots \ldots \ldots \ldots \ldots \ldots \ldots$

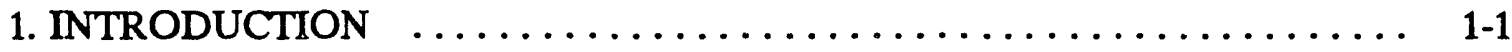

1.1 PROJECT OBJECTIVES AND APPROACH $\ldots \ldots \ldots \ldots \ldots \ldots \ldots \ldots$ 1-1

1.2 REPORT ORGANIZATION $\ldots \ldots \ldots \ldots \ldots \ldots \ldots \ldots \ldots \ldots \ldots \ldots \ldots$ 1-1

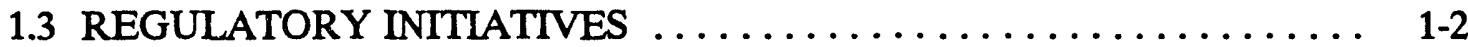

1.4 DATA QUALITY OBJECTIVES $\ldots \ldots \ldots \ldots \ldots \ldots \ldots \ldots \ldots \ldots \ldots \ldots$

2. BACKGROUND AND DATA USER INFORMATION $\ldots \ldots \ldots \ldots \ldots \ldots \ldots$ 2-1

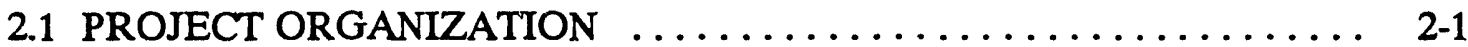

2.2 DATA MANAGEMENT AND VERIFICATION .............. 2-1

2.2.1 Responsibilities for Data Management and Verification ........ 2-1

2.2.2 Data Storage and Records Management ............... 2-1

2.3 DATA USER GUIDELINES $\ldots \ldots \ldots \ldots \ldots \ldots \ldots \ldots \ldots \ldots \ldots . \ldots \ldots$

2.3.1 How To Use Phase I Data-A Field Perspective ........... . 2-7

2.3.2 How To Use Phase I Data-An Analytical Perspective .......... . 2-8

2.3.3 Statistical Guidelines for Users of Background Soil Data . . . . . . . . 2-9

2.3.4 Data User Guidelines for Risk Assessments .............. 2-11

2.3.5 Data Access Considerations ................... 2-13

3. FIELD INVESTIGATION AND GAMMA SCREENING ANALYSES $\ldots . . .3$ 3-1

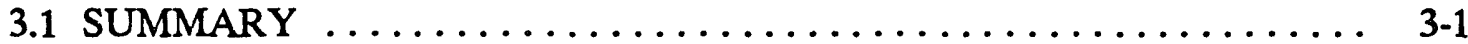

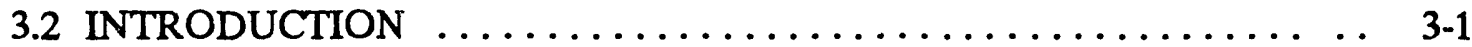

3.3 SAMPLING SITE SELECTION $\ldots \ldots \ldots \ldots \ldots \ldots \ldots \ldots \ldots \ldots \ldots$ 3-3

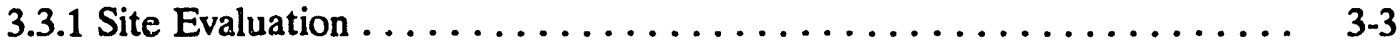

3.3.2 Selected Sites . . . . . . . . . . .

3.3.3 Composited Sample Sites $\ldots \ldots \ldots \ldots \ldots \ldots \ldots \ldots \ldots \ldots$ 3.7

3.3.4 Selection and Initial Evaluation of Off-Site Locations . . . . . . . . . 3. 3-7

3.4 SITE AND SOIL DESCRIPTIONS . . . . . . . . . . . . . . . 3-8

3.5 SAMPLING PROCEDURES $\ldots \ldots \ldots \ldots \ldots \ldots \ldots \ldots \ldots \ldots \ldots . \ldots \ldots$

3.6 SOIL SAMPLING AND SAMPLE PREPARATION $\ldots \ldots \ldots \ldots \ldots \ldots$ 3-8

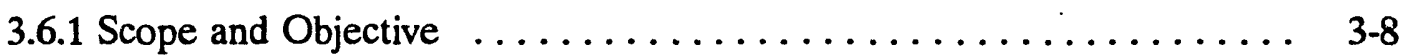

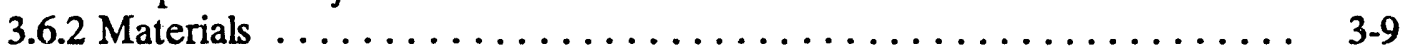

3.6.3 Field Activities $\ldots \ldots \ldots \ldots \ldots \ldots \ldots \ldots \ldots \ldots \ldots \ldots$ 3-9.

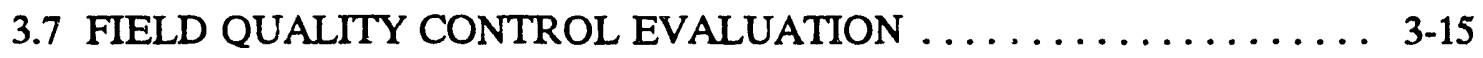

\begin{tabular}{l}
3.8 QUALITATIVE RESULTS OF GAMMA SPECTROSCOPY \\
SCREENING $\ldots \ldots \ldots \ldots \ldots \ldots \ldots \ldots \ldots \ldots \ldots \ldots \ldots \ldots \ldots \ldots \ldots$ \\
\hline
\end{tabular} 
4. ANALYTICAL LABORATORY ANALYSES

AND DATA VALIDATION $\ldots \ldots \ldots \ldots \ldots \ldots \ldots \ldots \ldots \ldots \ldots, 4,1$

4.1 SUMMARY OF DATA VALIDATION $\ldots \ldots \ldots \ldots \ldots \ldots \ldots \ldots, 4,1$

4.2 INTRODUCTION $\ldots \ldots \ldots \ldots \ldots \ldots \ldots \ldots \ldots \ldots \ldots \ldots \ldots, \quad 4,2$

4.3 SELECTION OF LABORATORIES $\ldots \ldots \ldots \ldots \ldots \ldots \ldots \ldots \ldots, 4,3$

4.4 QUALITY ASSURANCE/QUALITY CONTROL $\ldots \ldots \ldots \ldots \ldots \ldots, 4.3$

4.4.1 Organic Data Qualifiers ....................... 4-4

4.4.2 Inorganic Data Qualifiers ..................... 4.5

4.5 DATA VALIDATION $\ldots \ldots \ldots \ldots \ldots \ldots \ldots \ldots \ldots \ldots, 4.5$

4.5.1 Organic Data Validation Results $\ldots \ldots \ldots \ldots \ldots \ldots \ldots \ldots, 4,6$

4.5.2 Inorganic Data Validation Results ................. 4-16

4.5.3 Radiochemical Validation Results ................. 4-21

4.6 SCREENING ANALYSES FOR VOLATILE ORGANIC COMPOUNDS $\ldots \ldots \ldots \ldots \ldots \ldots \ldots \ldots \ldots \ldots \ldots \ldots, 4-28$

4.7 ORGANIC COMPOUND ANALYSES $\ldots \ldots \ldots \ldots \ldots \ldots \ldots \ldots \ldots, 4-28$

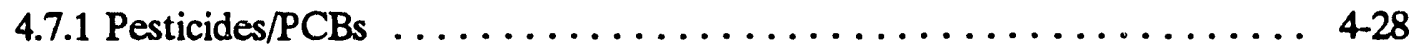

4.7.2 Polynuclear Aromatic Hydrocarbons $\ldots \ldots \ldots \ldots \ldots \ldots \ldots \ldots \ldots$ 4-28

4.7.3 Herbicides ............................ 4.30

4.8 INORGANIC COMPOUND ANALYSES $\ldots \ldots \ldots \ldots \ldots \ldots \ldots \ldots, 4,30$

4.8.1 Metals $\ldots \ldots \ldots \ldots \ldots \ldots \ldots \ldots \ldots \ldots \ldots \ldots \ldots \ldots \ldots, 4,30$

4.8.2 Cyanide and Sulfate $\ldots \ldots \ldots \ldots \ldots \ldots \ldots \ldots \ldots \ldots \ldots, 4,31$

4.9 RADIONUCLIDE ANALYSES $\ldots \ldots \ldots \ldots \ldots \ldots \ldots \ldots \ldots \ldots \ldots \ldots \ldots \ldots \ldots, 41$

5. STATISTICAL ANALYSIS $\ldots \ldots \ldots \ldots \ldots \ldots \ldots \ldots \ldots \ldots \ldots \ldots \ldots$ 5-1

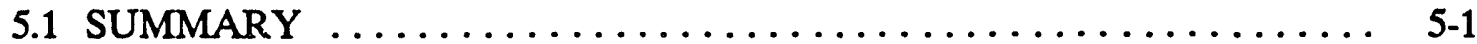

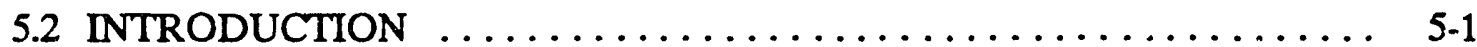

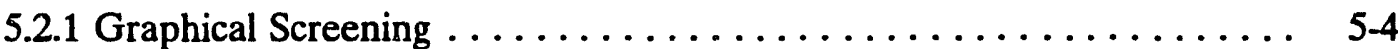

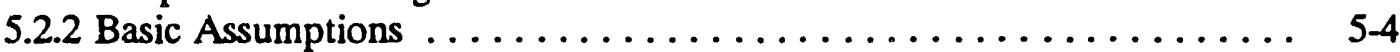

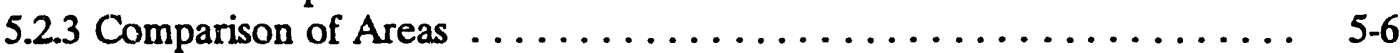

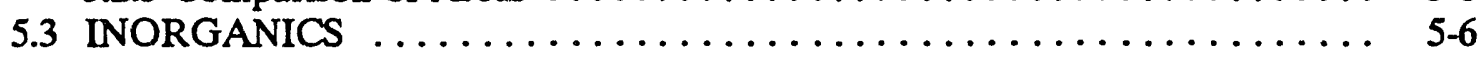

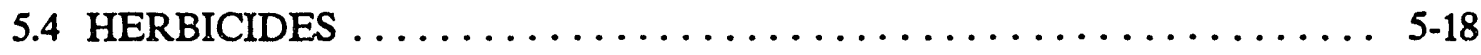

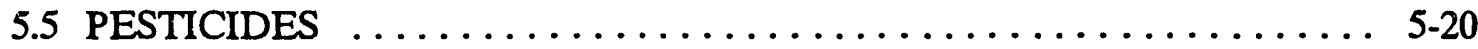

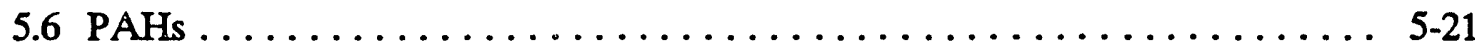

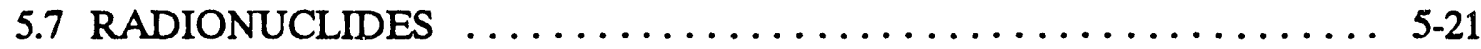

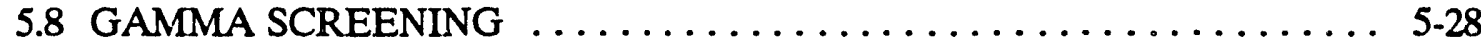

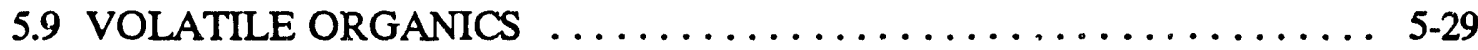

5.10 VARIANCE COMPONENTS $\ldots \ldots \ldots \ldots \ldots \ldots \ldots \ldots \ldots \ldots .5,29$

5.11 ADDITIONAL REMARKS $\ldots \ldots \ldots \ldots \ldots \ldots \ldots \ldots \ldots \ldots \ldots, 5.36$

6. DATA INTERPRETATION $\ldots \ldots \ldots \ldots \ldots \ldots \ldots \ldots \ldots \ldots \ldots \ldots \ldots \ldots$ 6-1

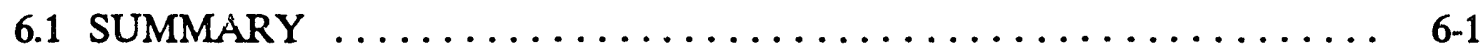

6.2 BASIC IDEAS AND CONCEPTS OF INTERPRETING

SOILS DATA $\ldots \ldots \ldots \ldots \ldots \ldots \ldots \ldots \ldots \ldots \ldots \ldots \ldots \ldots \ldots, 6 \ldots \ldots, 2$

6.3 VALID DATA COMPARISONS $\ldots \ldots \ldots \ldots \ldots \ldots \ldots \ldots \ldots \ldots \ldots, 6.4$

6.4 INTERPRETATION OF PHASE I DATA $\ldots \ldots \ldots \ldots \ldots \ldots \ldots \ldots, 6.5$

6.4.1 Organic Compounds ..................... 6.6

6.4.2 Inorganic Compounds and Metals $\ldots \ldots \ldots \ldots \ldots \ldots \ldots \ldots \ldots \ldots, 6.6$

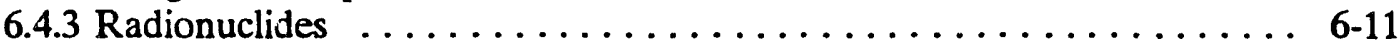


7. BACKGROUND RISK EVALUATION $\ldots \ldots \ldots \ldots \ldots \ldots \ldots \ldots \ldots \ldots$. $7-1$

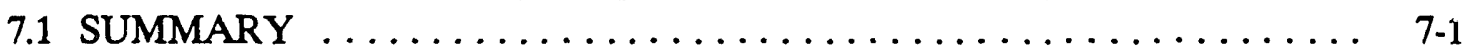

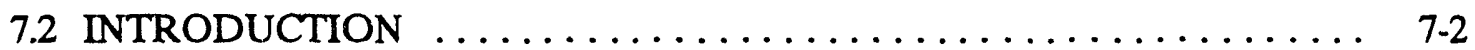

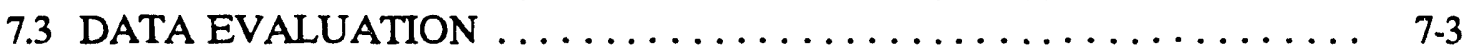

7.3.1 Data Usability ............................ 7.3

7.3.2 General Site-Specific Data Collection Considerations . . . . . . . . 7 7-3

7.3.3 General Site-Specific Data Evaluation Considerations $\ldots \ldots \ldots \ldots$ 7-3

7.3.4 Identification of Constituents Included

in the Background Risk Evaluation . . . . . . . . . . . . . . 7-4

7.4 EXPOSURE ASSESSMENT . . . . . . . . . . . . . $\ldots \ldots \ldots \ldots$.

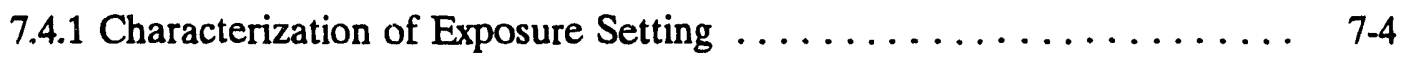

7.4.2 Identification of Exposure Pathways . . . . . . . . . . . . .

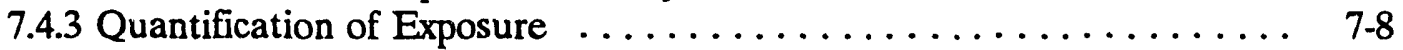

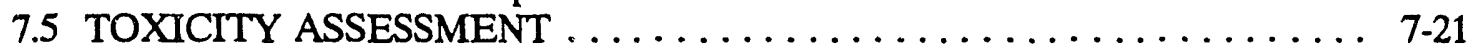

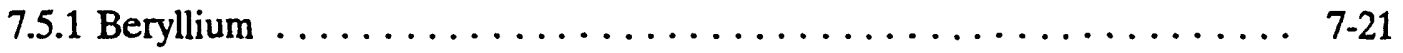

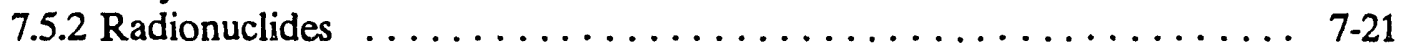

7.6 RISK CHARACTERIZATION . . . . . . . . $\ldots \ldots \ldots \ldots \ldots \ldots$

7.6.1 EPA Guidance-Carcinogens ................... 7-29

7.6.2 EPA Guidance-Noncarcinogens ................ 7-30

7.6.3 Background Risk and Hazard Index Comparisons

Between the ORR and Anderson and Roane Counties ... . . . . . 7-30

7.6.4 Background Risk Characterization for the ORR .......... 7-43

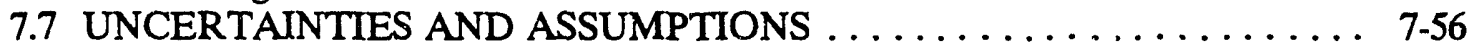

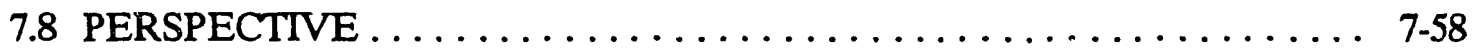

8. ASSESSMENT OF OVERALL DATA QUALITY OBJECTIVES $\ldots \ldots \ldots .8$

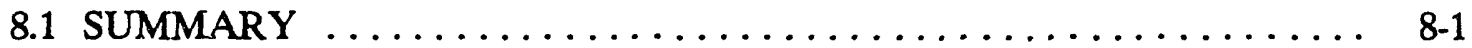

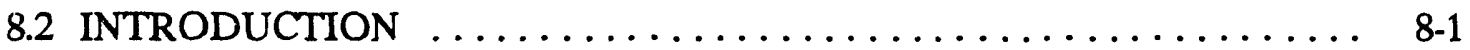

8.3 DATA QUALITY OBJECTIVES FOR

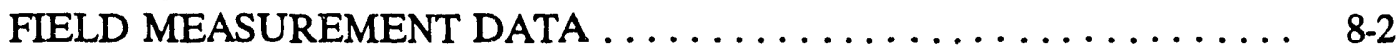

8.4 DATA QUALITY OBJECTIVES FOR

LABORATORY MEASUREMENT DATA ............. 8-2

8.5 ASSESSMENT OF COMPLIANCE

WITH DATA QUALITY OBJECTIVES . . . . . . . . . . . . 8 8 3

8.5 .1 Audits and Surveillances $\ldots \ldots \ldots \ldots \ldots \ldots \ldots \ldots \ldots \ldots . \ldots \ldots$

8.5.2 Data Quality Indicators for Field Measurement Data ......... 8-3

8.5.3 Data Quality Indicators for Analytical Laboratory Measurement and Soil Preparation Laboratory Data . . . . . . . . . . . . . . . 8-4

8.5.4 Training of Field and Soil Preparation Laboratory Personnel . . . . . 8 8-5

8.5.5 Field Data and Records Management ................ 8-5

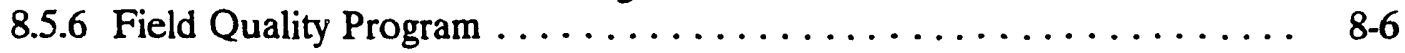

8.5 .7 Field Data Validation $\ldots \ldots \ldots \ldots \ldots \ldots \ldots \ldots \ldots \ldots \ldots . \ldots \ldots$

8.5.8 Field Quality Control Assessment . . . . . . . . . . . . . 8-8

8.5.9 Analytical Data Quality Assessment ... . . . . . . . . . 8 8-12

8.5.10 Data Gaps .......................... 8.16

9. PLANNED PHASE II ACTIVITIES $\ldots \ldots \ldots \ldots \ldots \ldots \ldots \ldots \ldots \ldots \ldots$ 9-1

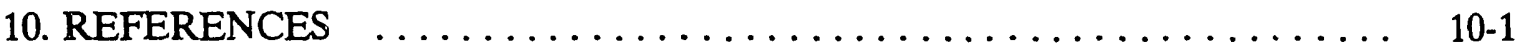


APPENDIX A-SITE DESCRIPTIONS, SOIL PROFILE DESCRIPTIONS,

AND GENERAL ANALYSIS OF SITES $\ldots \ldots \ldots \ldots \ldots \ldots$ A-1

A.1 OAK RIDGE RESERVATION SITE DESCRIPTIONS $\ldots \ldots \ldots \ldots \ldots \ldots$ A-3

A.2 ROANE COUNTY SITE DESCRIPTIONS $\ldots \ldots \ldots \ldots \ldots \ldots \ldots \ldots$ A-9

A.3 ANDERSON COUNTY SITE DESCRIPTIONS $\ldots \ldots \ldots \ldots \ldots \ldots \ldots$ A-12

A.4 OAK RIDGE RESERVATION SOIL PROFILES $\ldots \ldots \ldots \ldots \ldots \ldots \ldots$ A-15

A.5 ROANE COUNTY SOIL PROFILES $\ldots \ldots \ldots \ldots \ldots \ldots \ldots \ldots \ldots \ldots$ A-39

A.6 ANDERSON COUNTY SOIL PROFILES .................. A-51

A.7 ANALYSIS OF OAK RIDGE RESERVATION SITES $\ldots \ldots \ldots \ldots \ldots \ldots$ A-63

A.8 ANALYSIS OF ROANE COUNTY SITES $\ldots \ldots \ldots \ldots \ldots \ldots \ldots \ldots$ A 6.68

A.9 ANALYSIS OF ANDERSON COUNTY SITES $\ldots \ldots \ldots \ldots \ldots \ldots \ldots$ A-70

A.10 ANALYSIS OF DATA FROM COMPOSITED SAMPLES $\ldots \ldots \ldots \ldots$ A-72

APPENDIX B-SCREENING ANALYSIS DATA $\ldots \ldots \ldots \ldots \ldots \ldots \ldots$ B-1

APPENDIX C-ORGANIC ANALYSIS DATA $\ldots \ldots \ldots \ldots \ldots \ldots \ldots$ C-1

APPENDIX D-INORGANIC ANALYSIS DATA $\ldots \ldots \ldots \ldots \ldots \ldots \ldots$ D-1

APPENDIX E-RADIONUCLIDE ANALYSIS DATA $\ldots \ldots \ldots \ldots \ldots \ldots$ E-1

APPENDIX F-RELATION OF SAMPLE NUMBERS TO

LABORATORY SAMPLE DELIVERY GROUPS (SDGs) $\ldots \quad$ F-1

APPENDIX G-DESCRIPTIONS OF SAMPLES FOR PHASE I

ARRANGED NUMBERICALLY

BY SAMPLE IDENTIFICATION NUMBER .......... G-1

APPENDIX H-OCCURRENCES OF REJECTED DATA $\ldots \ldots \ldots \ldots \ldots$ H-1 


\section{TABLES}

2.1 Soil horizons and corresponding sample designations for Phase I . . . . 2-4

4.1 Definition of data validation qualifiers $\ldots \ldots \ldots \ldots \ldots \ldots \ldots \ldots$

4.2 Summary distribution of pesticide/PCB data validation results . . . . . . 4-10

4.3 Summary distribution of chlorinated herbicide data validation results . . . 4 4-12

4.4 Summary distribution of polynuclear aromatic hydrocarbon

data validation results $\ldots \ldots \ldots \ldots \ldots \ldots \ldots \ldots \ldots \ldots \ldots \ldots \ldots$ 4-17

4.5 Summary distribution of inorganic data validation results $\ldots \ldots \ldots \ldots .4-21$

4.6 Summary distribution of radiochemical data validation results . . . . . . 4-29

$5.1 \quad$ Summary statistics for inorganics $(\mathrm{mg} / \mathrm{kg}) \quad \ldots \ldots \ldots \ldots \ldots \ldots \ldots$

5.2 Additional summary statistics for inorganics:
for mostly undetected $95 \%$ UCBs for detection probability $\ldots \ldots \ldots \ldots \ldots$ 5-17

5.3 Herbicides $95 \%$ UCBs for detection probabilities $\ldots \ldots \ldots \ldots \ldots \ldots$ 5-18

$5.4 \quad$ Pesticides $95 \%$ UCBs for detection probabilities $\ldots \ldots \ldots \ldots \ldots \ldots$ 5-20

5.5 PAHs 95\% UCBs for detection probability for analytes

with fewer than three detects $\ldots \ldots \ldots \ldots \ldots \ldots \ldots \ldots \ldots \ldots \ldots$ 5-22

$5.6 \quad$ Additional summary statistics for PAHs with several detects $\ldots \ldots \ldots \ldots$ 5-22

5.7 Radionuclide $95 \%$ UCBs for detection probability

$5.8 \quad$ Additional radionuclides summary statistics $(\mathrm{pCi} / \mathrm{g}) \ldots \ldots \ldots \ldots \ldots \ldots$.

5.9 Overall results for cesium-137 (in $\left.\mathrm{pCi} / \mathrm{cm}^{2}\right) \ldots \ldots \ldots \ldots \ldots \ldots \ldots$

5.10A Standard deviation estimates for inorganics $\ldots \ldots \ldots \ldots \ldots \ldots \ldots \ldots$

5.10B Standard deviation estimates for PAHs $\ldots \ldots \ldots \ldots \ldots \ldots \ldots \ldots \ldots$

$5.10 \mathrm{C}$ Standard deviation estimates for radionuclides $\ldots \ldots \ldots \ldots \ldots \ldots$. $\ldots .35$

7.1 Oak Ridge Reservation background soil analytes evaluated quantitatively $\ldots \ldots \ldots \ldots \ldots \ldots \ldots \ldots \ldots \ldots \ldots \ldots \ldots \ldots \ldots \ldots \ldots$ 
7.2 Oak Ridge Reservation background soil analytes evaluated

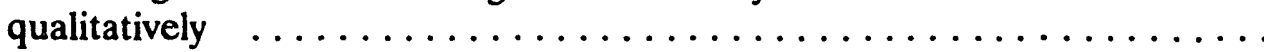

7.3 On-site resident exposure scenario $\ldots \ldots \ldots \ldots \ldots \ldots \ldots \ldots \ldots \ldots$

7.4A Chronic daily intake of ORR background soil by the on-site resident-Dismal Gap ...................... 7-13

7.4B Chronic daily intake of ORR background soil by the on-site resident-Dismal Gap ...................... 7-14

7.4C Chronic daily intake of ORR background soil by the on-site

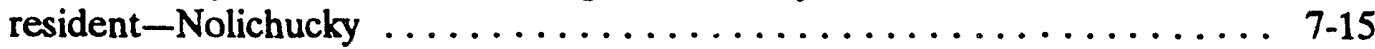

7.4D Chronic daily intake of ORR background soil by the on-site resident-Nolichucky

7.4E Chronic daily intake of ORR background soil by the on-site resident

7.5 Toxicity information for carcinogenic potential ORR analytes of concern-ingestion pathway $\ldots \ldots \ldots \ldots \ldots \ldots \ldots \ldots \ldots \ldots \ldots .22$

7.6 Toxicity information for noncarcinogenic potential analytes of concern on the Oak Ridge Reservation $\ldots \ldots \ldots \ldots \ldots \ldots \ldots \ldots . \ldots . \ldots .23$

7.7 Toxicity information for external exposure to radionuclides on the Oak Ridge Reservation

7.8 Comparative background cancer risk estimates from exposure to soil constituents from the Oak Ridge Reservation, Anderson County, and Roane County-Dismal Gap lithology

7.9 Comparative background hazard index estimates from exposure to soil constituents from the Oak Ridge Reservation, Anderson County, and Roane County-Dismal Gap lithology

7.10 Comparative background risk estimates from exposure to soil constituents from the Oak Ridge Reservation, Anderson County, and Roan County-Dismal Gap

7.11 Comparative hazard index estimates from exposure to background soil constituents from the Oak Ridge Reservation, Anderson County, and Roane County-Dismal Gap

7.12a Background cancer risk estimates from exposure to soil constituents on the Oak Ridge Reservation 
7.12b Background cancer risk estimates from exposure to Oak Ridge

Reservation soil constituents .................. 7-45

7.12c Background cancer risk estimates from exposure to Oak Ridge

Reservation soil constituents .................... . 7-47

7.13a Background hazard index estimates for residents exposed to Oak Ridge

Reservation soil constituents-Dismal Gap . . . . . . . . . . . 7 7-50

7.13b Background hazard index estimates for residents exposed to Oak Ridge

Reservation soil constituents-Dismal Gap ............... 7-51

7.13c Background hazard index estimates for residents exposed to Oak Ridge

Reservation soil constituents-Nolichucky $\ldots \ldots \ldots \ldots \ldots \ldots \ldots$ 7-52

7.13d Background hazard index estimates for residents exposed to Oak Ridge

Reservation soil constituents-Dismal Gap . . . . . . . . . . . 7-53

7.13e Background hazard index estimates for residents exposed to Oak Ridge

Reservation soil constituents-Dismal Gap . . . . . . . . . . . . . 7-54

7.13f Background hazard index estimates for residents exposed to Oak Ridge

Reservation soil constituents-Nolichucky .............. 7-55

$7.14 \quad$ Site-specific uncertainty factors $\ldots \ldots \ldots \ldots \ldots \ldots \ldots \ldots \ldots \ldots \ldots$

8.1 Comparison of rinse water and source water for metals on the ORR . . 8-10

8.2 Comparison of source water and rinse water

for Anderson and Roane counties . . . . . . . $\ldots \ldots \ldots \ldots \ldots$ 8-11

8.3 Distribution of data usability $\ldots \ldots \ldots \ldots \ldots \ldots \ldots \ldots \ldots \ldots$

B.1 Volatile organic analysis results for soil samples $\ldots \ldots \ldots \ldots \ldots \ldots$ B-5

B.2 Weighted gamma screening results for soil samples $\ldots \ldots \ldots \ldots \ldots \ldots$ B-35

B.3 Unweighted gamma screening results for soil samples $\ldots \ldots \ldots \ldots \ldots \ldots$ B-43

C.1 Organic analysis results for soil samples $\ldots \ldots \ldots \ldots \ldots \ldots \ldots \ldots \ldots$

D.1 Inorganic analysis results for composite soil samples $\ldots \ldots \ldots \ldots \ldots \ldots$ D-5

E.1 Radionuclides analysis results for composite soil samples $\ldots \ldots \ldots \ldots$ E-5

F.1 Relation of sample numbers to laboratory

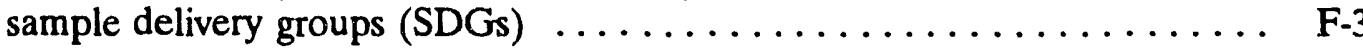


G.1 Descriptions of samples for Phase I arranged numerically by sample identification number $\ldots \ldots \ldots \ldots \ldots \ldots \ldots \ldots \ldots, \mathrm{G}-3$

H.1 Occurrences of $R$ validation prefix - Phase $I \ldots \ldots \ldots \ldots \ldots \ldots, H$ 


\section{FIGURES}

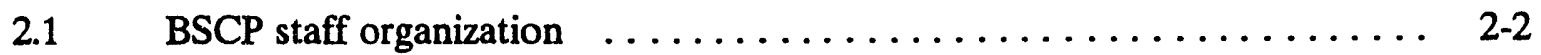

3.1 Approximate locations of BSCP sampling areas $\ldots \ldots \ldots \ldots \ldots \ldots \ldots$

3.2 Sampling site locations for the ORR $\ldots \ldots \ldots \ldots \ldots \ldots \ldots \ldots \ldots$

3.3 Sampling sites in Roane County $\ldots \ldots \ldots \ldots \ldots \ldots \ldots \ldots \ldots \ldots \ldots$

3.4 Sampling sites in Anderson County $\ldots \ldots \ldots \ldots \ldots \ldots \ldots \ldots \ldots \ldots$

5.1 Log of aluminum concentrations for horizon A-an example of graphics used to check for outliers . . . . . . . . . . . . . . . . . . .

5.2 Log of aluminum concentrations for horizon B-illustrating significant differences between sampling areas ............ 5-19

5.3 Cesium-137 results (square root of $\mathrm{pCi} / \mathrm{cm}^{2}$ ) by area from gamma scan $\ldots \quad$ 5-30

7.1 Comparison of total background cancer risks calculated from soil samples from the Dismal Gap Formation in Anderson County, Dismal Gap in Roane County, Dismal Gap on the ORR, and the Nolichucky Formation on the ORR

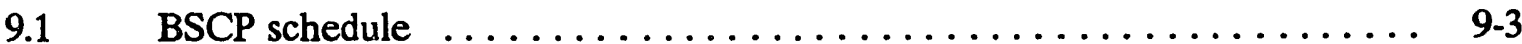




\section{ABBREVIATIONS}

\begin{tabular}{|c|c|}
\hline AA & atomic absorption \\
\hline AESG & Analytical Environmental Support Group at the Oak Ridge K-25 Site \\
\hline AND & Anderson County \\
\hline APO & Analytical Projects Office \\
\hline BSCP & Background Soil Characterization Project \\
\hline CCB & continuing calibration blank \\
\hline CCV & continuing calibration verification \\
\hline CDI & chronic daily intake \\
\hline CERCLA & Comprehensive Environmental Response, Compensation, and Liability \\
\hline CLP & $\begin{array}{l}\text { Act }(1980) \\
\text { Contract Laboratory Program }\end{array}$ \\
\hline $\mathrm{COC}$ & chain of custody \\
\hline CRDL & contract required detection limit \\
\hline CVAA & cold vapor atomic absorption \\
\hline DOE & U.S. Department of Energy \\
\hline DOE-OR & DOE Oak Ridge Field Office \\
\hline $\mathrm{DQ}$ & data quality \\
\hline DQO & Data Quality Objective \\
\hline EPA & U.S. Environmental Protection Agency \\
\hline ER & environmental restoration \\
\hline ESD & Environmental Sciences Division of ORNL \\
\hline FD & field duplicate \\
\hline FLAA & flame atomic absorption \\
\hline FWHM & full-width half-maximum \\
\hline GC & gas chromatography \\
\hline GFAA & graphite furnace atomic absorption \\
\hline GI & gastrointestinal \\
\hline HEAST & Health Effects Assessment Summary Tables \\
\hline HI & hazard index \\
\hline HPLC & high performance liquid chromatography \\
\hline HSWA & Hazardous and Solid Waste Amendments to RCRA (1984) \\
\hline ICB & initial calibration blank \\
\hline ICP & inductively coupled plasma \\
\hline ICRP & International Commission on Radiological Protection \\
\hline ICS & interference check sample \\
\hline ICV & initial calibration verification \\
\hline ID & identification number \\
\hline IDL & instrument detection limit \\
\hline IRIS & Integrated Risk Information System \\
\hline LCS & laboratory control sample \\
\hline LET & linear energy transfer \\
\hline LLWDDD & Low-Level Waste Disposal Development Demonstration \\
\hline LTB & lower tolerance bound \\
\hline MAD & Measurement, Applications, and Development Group \\
\hline MDA & minimum detectable activity \\
\hline
\end{tabular}




$\begin{array}{ll}\text { MDL } & \text { method detection limit } \\ \text { MS } & \text { mass spectroscopy } \\ \text { MSA } & \text { Method of Standard Additions } \\ \text { MSD } & \text { matrix spike duplicate } \\ \text { MS/MSD } & \text { matrix spike/matrix spike duplicate } \\ \text { NAA } & \text { neutron activation analysis } \\ \text { ND } & \text { no data } \\ \text { NEPA } & \text { National Environmental Policy Act (1968) } \\ \text { NIST } & \text { National Institute for Standards and Testing } \\ \text { NPL } & \text { National Priorities List } \\ \text { OREIS } & \text { Oak Ridge Environmental Information System } \\ \text { ORNL } & \text { Oak Ridge National Laboratory } \\ \text { ORR } & \text { Oak Ridge Reservation } \\ \text { OSWER } & \text { Office of Solid Waste and Emergency Response } \\ \text { OU } & \text { operable unit } \\ \text { PAH } & \text { polynuclear aromatic hydrocarbon } \\ \text { PC } & \text { personal computer } \\ \text { PCB } & \text { polychlorinated biphenyl } \\ \text { PE } & \text { performance evaluation } \\ \text { PEM } & \text { performance evaluation mixture } \\ \text { PQL } & \text { practical quantitation limit } \\ \text { PSD } & \text { percent standard deviation } \\ \text { QA/QC } & \text { quality assurance/quality control } \\ \text { RAGS } & \text { Risk Assessment Guidance for Superfund } \\ \text { RCRA } & \text { Resource Conservation and Recovery Act (1976) } \\ \text { RfD } & \text { reference dose } \\ \text { ROA } & \text { Roane County } \\ \text { ROW } & \text { right of way } \\ \text { RPD } & \text { relative percent difference } \\ \text { RSD } & \text { percent standard deviation } \\ \text { SARA } & \text { Superfund Amendments and Reauthorization Act (1986) } \\ \text { SDG } & \text { Sample Delivery Group } \\ \text { SF } & \text { slope factor } \\ \text { SOP } & \text { standard operating procedure } \\ \text { SOW } & \text { statement of work } \\ \text { SPL } & \text { Soil Preparation Laboratory } \\ \text { SVOC } & \text { semi-volatile organic compound } \\ \text { SWMU } & \text { solid waste management unit } \\ \text { TIC } & \text { tentatively identified compound } \\ \text { TSD } & \text { treatment, storage, and disposal } \\ \text { TVA } & \text { Tennessee Valley Authority } \\ \text { UCB } & \text { upper confidence bound } \\ \text { UT } & \text { University of Tennessee-Knoxville } \\ \text { USDA } & \text { U.S. Department of Agriculture } \\ \text { VOA } & \text { volatile organic analysis } \\ \text { VOC } & \text { volatile organic compound } \\ \text { WAG } & \text { waste area grouping } \\ \text { WM } & \text { waste management } \\ & \text { Tha } \\ \end{array}$


certain operating criteria to safeguard the environment (RCRA 1976). These TSD facilities are referred to as solid waste management units (SWMUs) which are defined as any "discernible waste management unit at a RCRA facility from which hazardous waste or hazardous constituents might migrate, irrespective of whether or not the unit was intended for the management of solid or hazardous waste." Such units include any area at a facility at which hazardous wastes or hazardous constituents have been routinely and systematically released (EPA 1989a).

- Hazardous and Solid Waste Amendments (HSWA). These amendments to RCRA were enacted in 1984 and provided EPA with the authority to enforce corrective actions by broadening the scope of the RCRA Corrective Action Program. In addition to evaluating and correcting releases to the uppermost aquifer from regulated RCRA units, HSWA promotes the cleanup of continuing releases to any environmental media resulting from waste management units and practices at RCRA facilities (HSWA 1984). Among the most significant provisions of HSWA are the following:

1. Section 3004(u), Corrective Action for Continuing Releases. Sution 3004(u) states that for permits issued after November 8, 1984, corrective action is required for releases of hazardous waste or constituents from any SWMU at any TSD facility seeking a permit for permanent operation, regardless of when waste was placed in the unit. Thus, corrective actions apply to current as well as past releases.

2. Section 3004(v), Corrective Action Beyond the Facility Boundary. Section 3004(v) authorizes EPA to require that corrective action be taken by the facility owner or operator for releases that have migrated off-site beyond the facility boundary. Such action should be taken where necessary in order to protect human health and the environment unless the owner/operator demonstrates to the satisfaction of the administrator that permission to undertake such action was denied.

- Comprehensive Environmental Response, Compensation, and Liability Act (CERCLA), also referred to as Superfund. Created in 1980, it established a program to identify sites (operable units) from which environmental releases of hazardous substances might occur or have occurred. At such sites, Superfund promotes the evaluation of damage to natural resources, ensures cleanup by the responsible party or the government, and creates a claims procedure for parties involved in site cleanup and natural resource reclamation. Sites identified by CERCLA are evaluated and then placed on the National Priorities List (NPL), if appropriate. The Oak Ridge Reservation was listed on the NPL in December 1989 in the Federal Register (54 FR 48184) (EPA 1989b).

- Superfund Amendments and Reauthorization Act (SARA). Created in 1986 as a 5-year extension of the Superfund/CERCLA program to clean up hazardous releases at uncontrolled or abandoned hazardous waste sites.

- National Environmental Policy Act (NEPA). Created in 1968, it directs public officials to consider the impacts of their actions (e.g., construction, remediation) on the human environment as a part of all decision-making processes.

When the ORR was placed on the National Priorities List, CERCLA became the primary regulatory driver for environmental studies and clean-up actions. Part of the 
requirements of CERCLA are that remedial actions be based on nine criteria. Those criteria are (1) overall protection of human health and the environment; (2) compliance with applicable, or relevant and appropriate requirements; (3) long-term effectiveness and permanence; (4) reduction of toxicity, mobility or volume through treatment; (5) short-term effectiveness; (6) implementability; (7) cost; (8) state acceptance; and (9) community acceptance. To determine whether or not proposed remedial activities for contaminated sites can meet these criteria, the concentration of suspected contaminants must be compared to the conientrations of those same constituents in natural environments. The purpose of the BSCP is to determine the concentrations of all key organics, inorganics (metals), and radionuclides in background soils in the Oak Ridge area, so that they could be used for comparing the concentrations found at contaminated sites undergoing remedial investigation under CERCLA. Key constituents are those that are of interest to ongoing, as well as anticipated, remedial actions and investigations.

\subsection{DATA QUALTYY OBJECTTVES}

Determination of naturally occurring concentrations in soils in the Oak Ridge area necessitates a systematic investigation because there are several different underlying formations from which soils are derived, and because of the natural variability within the different soil types. To evaluate the ranges of concentrations of organics, metals, and radionuclides with high confidence levels, the project participants followed the steps described in this section for project planning found in the report Characterizing Heterogeneous Wastes: Methods and Recommendations (Rupp and Jones 1991). This section outlines the approach taken to establish DQOs for this project.

\section{State the Problem To Be Resolved}

The problem to be resolved by conducting the Background Soil Characterization Project is to determine the range in concentration of naturally occurring organics, metals, and radionuclides in soils. Ranges of concentrations for these constituents are required because of the variability found in any naturally occurring substance and because of the varying soil types resulting from different underlying geologic formations in the Oak Ridge area. The sample collection program was designed to account for some of this variability (Sect. 5.2, Energy Systems 1992) through the collection of field duplicates.

\section{Identify the Decision To Be Made}

Decisions will be made with respect to characterization of background concentrations of organics, inorganics (metals), and radionuclides found in nature. Standards for cleanup of potentially contaminated soils in the ORR will be based on the concentrations above those established as background in this project for typical constituents. IF data from this project can be used to determine that levels of organics, metals, and radionuclides at a suspected contaminated site are no greater than those found in nature, THEN those constituents will not be considered contaminants of concern for that particular site. However, IF the concentrations of these constituents are significantly greater than those found in nature, THEN appropriate remedial activities will be evaluated in site specific cleanup projects to reduce the elevated concentrations to those of naturally occurring levels or to technically feasible levels. 


\section{Identify Inputs to the Decision}

The approach taken to provide needed quantitative data on background concentration levels is based on collecting and analyzing samples from representative soil sections. The determination that sample collection locations are representative was made by assimilating information from relevant disciplines. Those disciplines included site history, geology, soil science, statistics, and analytical chemistry. To ascertain that samples would reflect accurate background concentrations, the history of the sample collection site was determined to be unaffected by process and research operations of the Oak Ridge Reservation, and the site was determined to have the same underlying geologic units and soils as those underlying suspected and contaminated sites. To determine the probable ranges of background concentrations, a statistically based sample collection and analysis program was designed. To provide defensible laboratory analyses upon which to base statistical analysis and the resulting conclusions, analytical chemists determined that EPA Analytical Level IV QC and documentation would be required.

\section{Narrow the Boundaries of the Study}

Upon defining the problem to be resolved and the decisions to be made from project data, the boundaries of the study were narrowed in three ways: (1) appropriate locations for sample collection were determined, (2) analytical parameters were agreed upon, and (3) statistical analytical procedures designed. From these decisions, the appropriate levels of quality assurance documentation required from field sampling and laboratory activities were established. The process for selecting sample collection sites is described in the Project Plan for the Background Soil Characterization Project on the Oak Ridge Reservation, Oak Ridge, Tennessee (Energy Systems 1992). Therein the process is discussed in detail, as are the analytical parameters of interest, for both the field and laboratory activities, the associated quality assurance documentation requirements for each, and the statistical analysis techniques.

\section{Develop a Decision Rule}

Upon completion of sample collection and analysis according to the requirements discussed above, the results are statistically analyzed, compiled, and reported including the ranges of concentrations for each constituent. This information will be used to address the following statement: IF concentrations of contaminants of concern at potentially contaminated sites are above those established as background, THEN appropriate remedial measures will be evaluated for application at that site.

\section{Develop Uncertainty Constraints}

The uncertainty of all results from this project must be as low as reasonably achievable or, fut in other words, the confidence level must be high, because the information developed in this study will be used as a basis upon which to make decisions in remedial projects that are estimated to cost millions of dollars and require several years to implement. It is important that resources be directed at sites that are truly contaminated. To achieve the lowest uncertainty in the statistical analysis conducted as a part of this project, high quality data, as well as adequate sample sizes, are required. The project team decided that analytical data used in the analysis should require EPA Level IV quality control and documentation to ensure high quality data. Preliminary screening analyses were assigned EPA Analytical 
Level II quality control documentation. In order to ensure that sample collection and field observations were done in such a way as to ensure technically complete and accurate and legally defensible information, these activities were conducted according to procedures that had been reviewed and approved by technical experts, knowledgeable managers, and regulators, subject to appropriate quality assurance oversight.

\section{Optimize Design for Obtaining Data}

The data collection design for this project is described in the Project Plan (Sect. 5.3, Energy Systems 1992). This design was optimized to account for variability within soil types by compositing soil samples. Additional optimization was achieved by conducting field screening analyses on soils to ensure that the site was not contaminated by unrecorded disposals. The field screening analyses were supplemented by laboratory analysis for manmade contaminants that would be unacceptable in a site used for determining natural background concentrations.

The sampling plan was further optimized by repeating the sample collection and statistical analyses obtained on the ORR at two separate remote areas in adjacent counties. These areas were selected to ensure the same underlying geologic formations, and consequently, the same soil types. This repeat analysis technique is designed to ver fy the results of the analysis conducted on those samples collected within the boundaries of the ORR.

\section{Development of Uncertainty Constraints}

It is difficult, at best, to assign a simple uncertainty constraint on this or any environmental investigation. These types of investigations differ from other experiments where uncertainty constraints are commonly used, in that little is known about the sample population (background concentration) before the experiment. In many uses of uncertainty constraints, there is some knowledge of the sample population (such as the length of a manufactured item or a combination of poker hands) before the experiment. Furthermore, while uncertainty constraints can be calculated for the end result of the data acquisition effort (the analytical results), there are several controlling aspects of an environmental investigation that do not lend themselves to quantifiable uncertainties. Among those factors that do not lend themselves to quantifiable uncertainties are the following:

1. The certainty that the sample was collected within the geologic unit for which it was intended.

2. The certainty that the sample was collected within the soil horizon for which it was intended.

3. The certainty that the sample collection locations accurately reflect the actual constituent concentrations of areally distributed soil types.

4. The certainty that sample analyses accurately reflect the actual concentrations in the sample.

Each of the above controlling factors is based on the best professional judgement of highly qualified individuals, but even then a numerical value on these factors would be difficult to calculate objectively. It was for those reasons that the uncertainty descriptors, such as high, medium, and low, are recommended for the DQO process. 
The uncertainty constraints that can be calculated for the BSCP have been described in Appendix D of the BSCP Plan. These include probability calculations on the laboratory analyses. The analyses upon which these calculations are based were the basis for the sampling program. This program was in turn based on a cursory examination of data available. However, the available data came from an experiment that was much different from the BSCP. Those data were collected upgradient of a known contaminant source in the RCRA investigation of the K-1070-A Contaminated Burial Ground, which is in the Knox Formation. BSCP data were collected from strata that included representative soil groups but were removed from any known contaminant sources.

The quantifiable uncertainty constraints that can be made in this experiment are based on two scenarios or combinations of them. The two scenarios are (1) that concentrations will be above the detection limits of laboratory instrumentation, and (2) that concentrations will be below the detection limits of laboratory instruments.

In the first scenario, where many or all analytical results are above the detection limit of the laboratory instrument, the distribution, standard deviation, mean, and median will be computed. Upper confidence bounds of any percentile can be computed from this information, and for this experiment, the 95th percentile will be reported. However, it is important to understand that the range of the 95th percentile will be based upon the results of the data and cannot be stated until all data have been received and validated. The range of the 95th percentile will vary according to the range of the analytical results. If the analytical results for a certain constituent vary only slightly, the spread between the median and the corresponding 95th percentile will be small. On the other hand, if the analytical results for any constituent vary considerably, then the spread between the median value and the 95th will be large.

In the second scenario, where all analytical results are below the detection limit of the laboratory instrument, confidence bounds for detection probabilities will be reported. As discussed in Appendix D of the Project Plan for the $\mathrm{JSCP}$, when the sample size is 4, as is the case in this experiment where four composited soil samples are analyzed, the $90 \%$ lower confidence bound for the probability that another composited sample would also be less than the detection limit is 0.56 . If the composited samples from different geologic units and/or horizons were to be combined, and all have concentrations less than detection limits, thereby increasing the sample size to 12 , for example, then the $90 \%$ confidence bound would be 0.83 . However, combining the sample populations to increase sample size will need to be evaluated for technical defensibility before statements on the probabilities of doing so can be made.

A detailed explanation of the statistical implications of the BSCP can be found in Appendix D of the BSCP Plan (Energy Systems 1992). 


\section{BACKGROUND AND DATA USER INFORMATION}

\section{PROJECT ORGANIZATION}

The management of the Background Soil Characterization Project (BSCP) is under the DOE and Martin Marietta Energy Systems management structure for the Environmental Restoration (ER) Program at Oak Ridge. The BSCP staff organization is summarized in Fig. 2.1. Functional responsibilities for individual participants in project activities are described in the BSCP Plan (Energy Systems 1992).

\section{DATA MANAGEMENT AND VERIFICATION}

\subsection{Responsibilities for Data Management and Verification}

Records of data collection and analysis of samples for the BSCP are generated by field and laboratory personnel. The BSCP data base, using SAS software, has been established on a mainframe computer system at ORNL to store the data. The purpose of the data base is to provide retrievability, integrity, security, and organization of the data, according to the Data Management Plan (Sect. 7) in the BSCP Plan (Energy Systems 1992). All project data have been verified to be correct and representative of the background soil sampling sites, validated against project requirements, and assessed for compliance with project data quality objectives. All validated project data packages from the contract laboratories were verified by data management personnel to be correct as input into the project data base and cross-checked with field records to corroborate the one-to-one correspondence of laboratory results with field sampling sites from where soil samples were originally obtained.

Field data were verified in two ways. First, field activities were subject to surveillance (JS-BSCP-92-01) and were found to be satisfactory in regards to in-force standard operating procedures (SOPs). However, the SOPs were found to be in need of refining to ensure that all items specified in the Project Plan were accounted for. Second, all field records for Phase I sampling were reviewed site-by-site and checked for completeness against the ESP-500 procedures, as called for in the Project Plan. These records were found to be complete but were in need of an index or user's guide (see Sect. 2.3). Validation of analytical laboratory data is discussed fully in Sect. 4.5.

Data summaries, statistical analysis, risk analysis, and availability of data are discussed briefly in this section. Programs have been developed to provide working data reports to the technical coordinator, analytical coordinator, field operations personnel, and in-house laboratory personnel. These working reports are available throughout the project and facilitate accurate record keeping and status reporting of progress.

\section{Data Storage and Records Management}

The BSCP data base is cataloged and resides on a disk pool volume on the IBM 3090 computer system. A partitioned data set of source programs is cataloged and resides on the disk pool volume. Read, write, execute, and delete access to these data sets are restricted. Daily and weekly backups are performed. Working data sets may be accessed on PC diskette, 


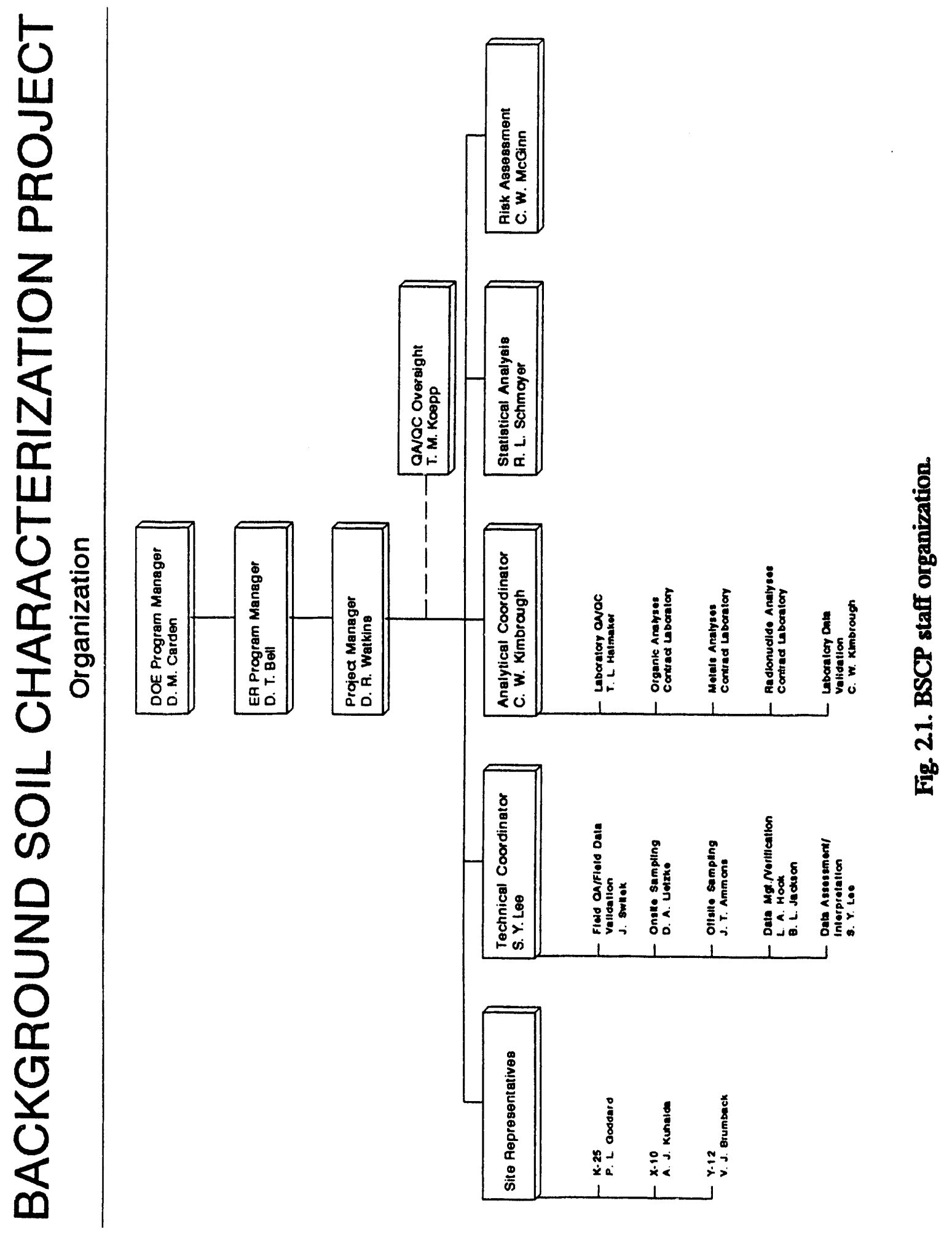


PC fixed disk, the STC10 VAX, or UNIX workstation. However, all data appear in final form in the SAS data base on the IBM 3090.

The following field data records and laboratory analysis records have been entered or transferred to the SAS data base:

- field sample tracking information entered from ORNL Environmental Sciences Division (ESD) and University of Tennessee sampling crew field sample logbooks and from sample compositing/sample processing laboratory logbooks;

- gamma sample laboratory parameter information, activity measurements, and concentration summaries transferred from diskettes provided by the ESD/Radioanalytical Laboratory;

- volatile organic analysis screening results provided by the Y-12 Plant analytical laboratory, which are transferred and included in the SAS data base;

- organic (pesticides, PCBs, herbicides, and PAHs) sample laboratory information and concentration levels entered from analysis data sheets provided by Lockheed Analytical Services;

- inorganic sample laboratory information and concentrations entered from analysis data sheets provided by Lockheed Analytical Services; and

- radionuclide sample laboratory parameter information, concentrations, and detection limits entered from analysis data sheets provided by Ecotek LSI.

Data sets of analytical laboratory results were provided to the statistical coordinator for conducting statistical analysis, generating data summaries, and performing data reduction. The statistical coordinator in turn provides data summaries to the risk analysis coordinator. Baseline risk to human health is calculated for later use in comparison with risks associated with contaminated sites.

Validated and verified analytical data and field data will be transferred to the Oak Ridge Environmental Information System (OREIS) with the approval of the project manager. Other ER Division projects needing background soil concentration data may access needed data from OREIS.

An example of a typical summary printout showing types of analyses (except gamma screening data) is provided in Table 2.1 . 


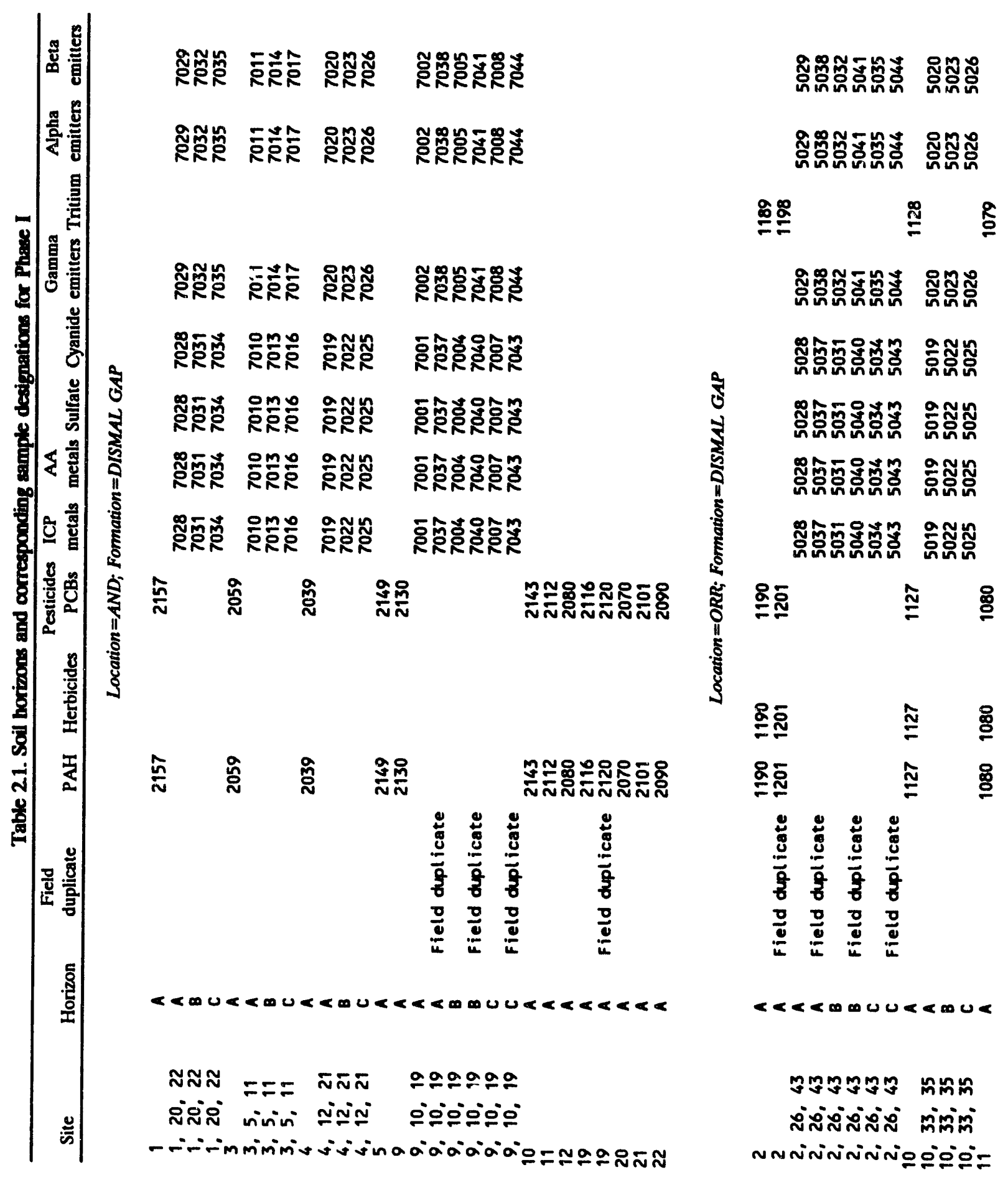




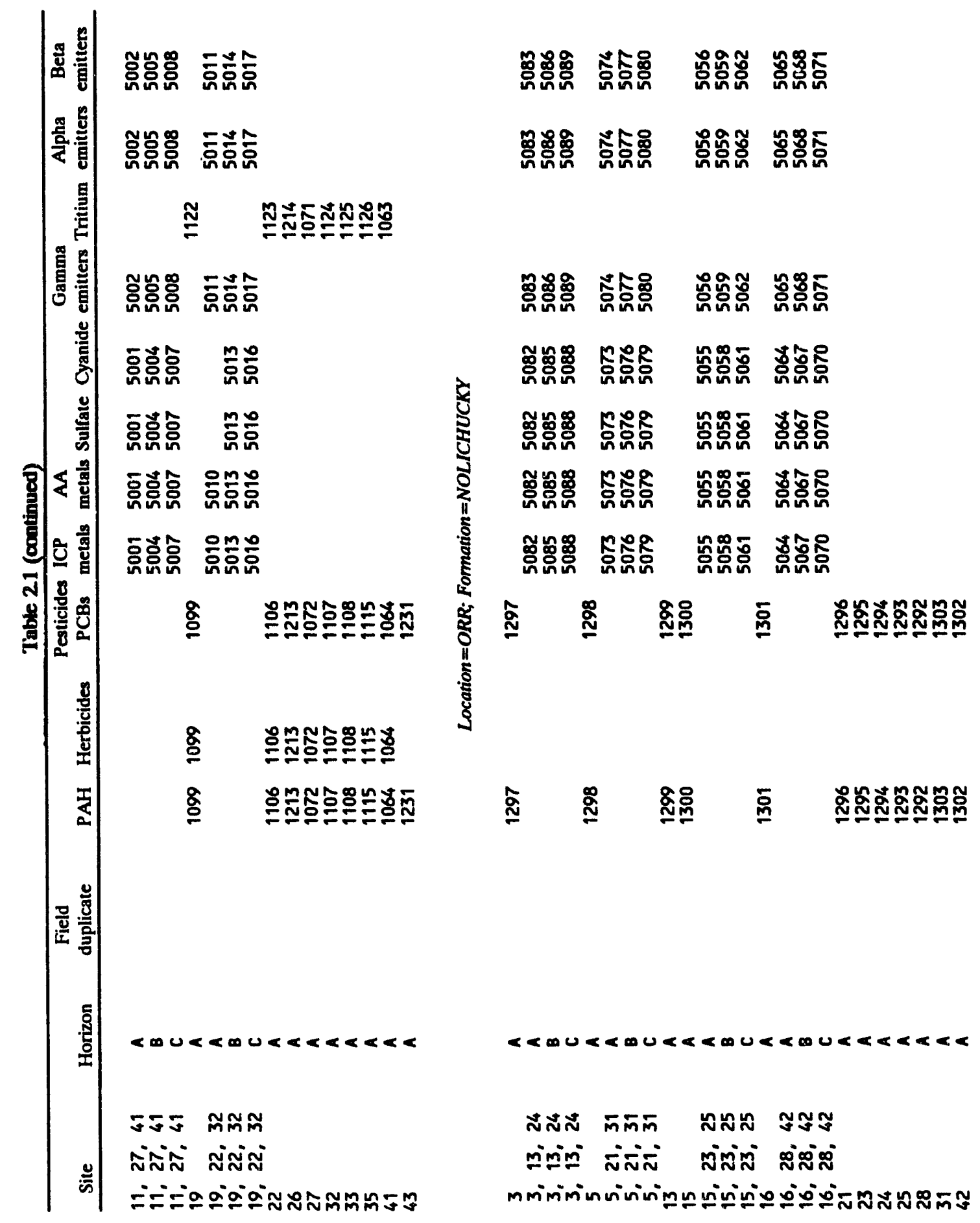




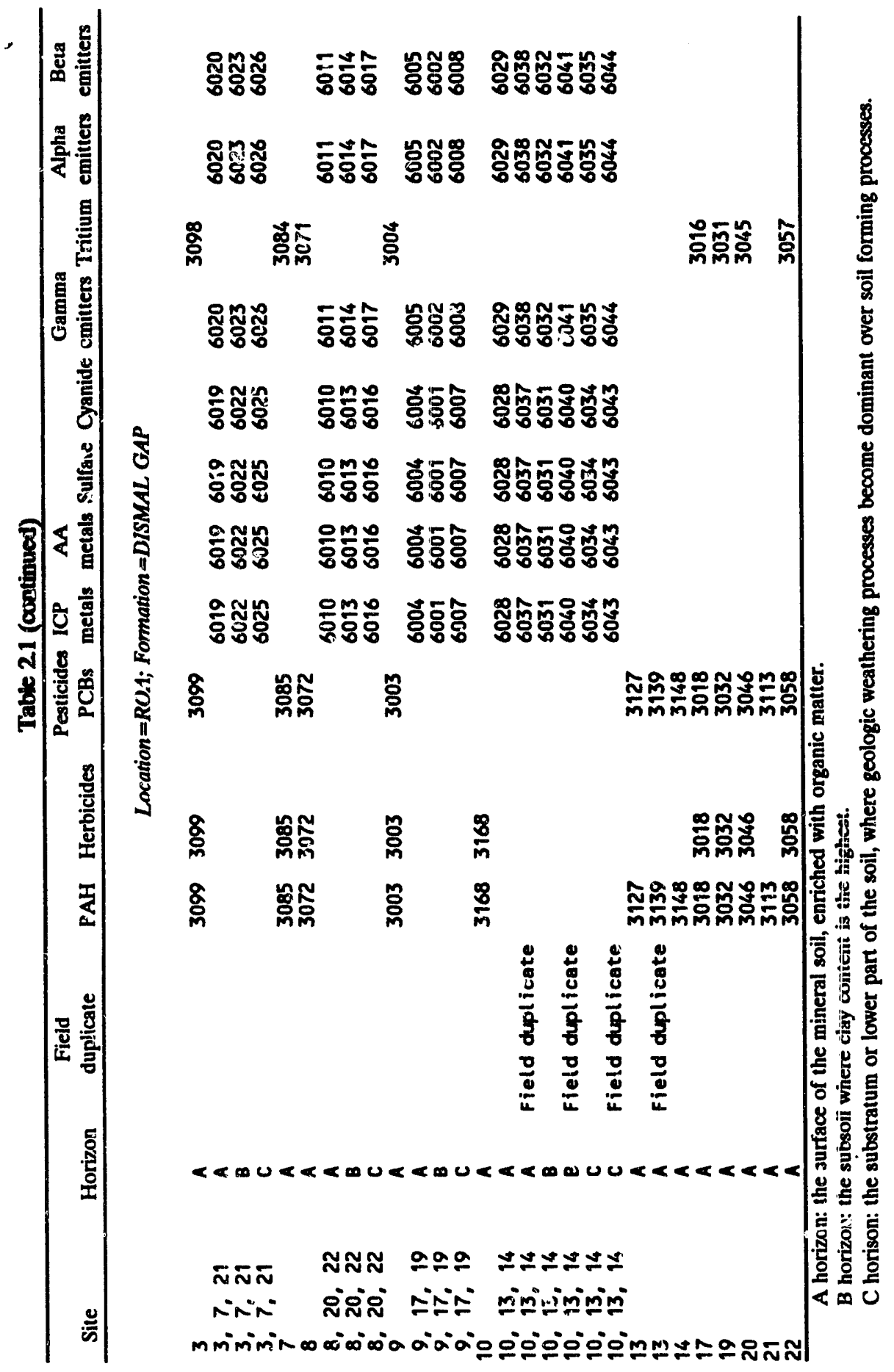




\section{DATA USER GUIDELINES}

\subsection{How To Use Phase I Data-A Field Perspective}

The purpose of this section is to advise data users how to use the BSCP data base. The BSCP Plan (Energy Systems 1992) discussed the approach for site selection and sampling requirements. Reading the plan will help in understanding objectives and the scope of activities. If your intended use of background soils data is beyond our scope, you must develop scientific rationale to justify such use. Users are advised to read the entire text $o$ this report instead of just the data summaries appearing in Sect. 5 and Appendixes B throu $\mathrm{h}$. E. The following checklist of pertinent questions is provided to guide the prospective data user.

1. Do you know your site geological formations and soil characteristics? Have you read the BSCP sampling protocols? Will you be using a qualified soil scientist for collecting samples?

2. Did you compare your analytical methods with those contained in the BSCP Plan (Energy Systems 1992)? Were the samples analyzed according to the EPA methods and procedures referenced in BSCP Plan? Extraction and dissolution methods for metals, organics, and some radionuclides should be the same if one would like to compare contaminated site characterization results with background soil concentration values. The use of neutron activation analysis data to complement analytical data in this report will be discussed in the project final report.

3. What geologic formation is beneath your soil sampling site? This question is important when contaminants, such as metals and radionuclides, occur naturally in soils and bedrock. The following qualifications may change after full statistical analyses of both Phase I and Phase II results.

a. Rome Formation: Naturally occurring metal and radionuclide BSCP data may not be applicable.

b. Conasauga Group: For Pumpkin Valley, Rutledge (Friendship), and Maynardville formations, BSCP data may not be applicable, but for Dismal Gap (Maryville) and Nolichucky formations, BSCP Phase I data may be applicable.

c. Knox Group: For Copper Ridge and Chepultepec formations, BSCP Phase II data may be applicable. For Longview, Kingsport, and Mascot formations, BSCP Phase II dat may or may not be applicable, depending on correlative factors.

d. Chickamauga Group: For Bethel Valley area, Phase II Bethel Valley section BSCP data may be applicable and for the K-25 area, Phase II K-25 section BSCP data may be applicable.

4. Was your sample collected from a ridge top or upper side slope and from a residual soil? If your sample came from a floodplain or from a concave-shaped landform with alluvial-colluvial soils, then the data will probably vary, but the Phase I data will probably be on the conservative side.

5. Was your sample collected from a forested mineral soil surface layer (A horizon or $A+E$ horizons) or from an Ap horizon in a grassland field? You can use the mean values from the A horizon from the geologic formation that you checked above. 
6. Was your sample collected from the surface of a site that has been disturbed or stripped of topsoil in the past $\mathbf{4 5}$ years? If so, then the B horizon data from the particular geologic formation may be used for comparison.

7. Was your sample collected from a depth of 3 feet or more below the surface? You can compare your data with the mean values for the $\mathrm{C}$ horizon for the geologic formation that you checked above.

8. Was your sample collected from fill materials or cover above waste trenches? Can you identify the geologic formation source of those soil materials? Then you can compare your data with the $C$ horizon data. If the geologic source of the cover or fill material cannot be identified according to its geologic origin, or if it was imported, do not compare your data with Phase I BSCP data! If the fill came from Chestnut Ridge or from Melton Hill on the Oak Ridge Reservation (ORR), wait until Phase II BSCP data are available.

9. Are your results equal to or lower than the mean value (transformed?) plus two sigma deviation units? If so, your sample is probably not contaminated. If your results are significantly higher than the mean plus two sigma units, then your sample may be contaminated. Note: The data user should keep in mind that some properties of natural soils are extremely variable and complex and that the BSCP data represent only a very small subset of soils on the ORR.

10. With respect to man-made organic compounds and radionuclides, these represent a separate issue and are not connected to geology. We do not want to limit the application of BSCP data because of these artificial soil constituents. What we do want to do is to base the analytic thresholds on instrument detection limits or on detection limits associated with method dilution factors.

\section{How To Use Phase I Data-An Analytical Perspective}

The data reported in this document have been collected, analyzed, and validated according to the guidelines and requirements detailed in the BSCP Plan (Energy Systems 1992). The data were analyzed according to methods detailed in Sect. 4.7 of this report, and the data were validated according to the criteria described in Sect. 4.4. For these data to be properly used by future users, the user must use similar data analysis methods as described in this report. In addition, the user must ensure that any deviation in protocols be considered during the planning stage.

To use these data properly, the user must understand the purpose of the data validation and the validation qualifiers used. The purpose of validation was to assess quality of the data against EPA's nationally applicable criteria. The criteria followed for most of the chemical data were the EPA Contract Laboratory Program (CLP) Data Validation Criteria. The criteria used for the non-CLP chemical and radiological data were prepared according to the requirements provided in the BSCP Plan and the EPA CLP Data Validation Criteria. The validated data were given validation qualifiers that explain the overall judgment of the data validator as to the worthiness of the data points. Two types of qualifiers are provided in the data tables: laboratory qualifiers and validation qualifiers. The definitions of the contract 
laboratory qualifiers are found in Sects. 4.4.1 and 4.4.2 of this report. The data validation qualifiers used in this project are listed in Table 4.1.

Data with validation qualifiers J, UJ, UNN, UN, and NJ in Table 4.1 can be used, but the data user must be aware that the data must be used with the limitations that the qualifier defines. An example would be that a project could use the data qualified as J, but it must be understood that they are using a data value that represents an approximate concentration of the analyte and not a true concentration.

The following questions are presented to provide additional guidance.

1. Did you compare your analytical methods with those contained in the BSCP Plan (Energy Systems 1992)?

2. Were the samples analyzed according to the EPA methods and procedures contained in the BSCP Plan (Energy Systems 1992)?

3. Did you follow the same sample preparation methods and requirements as those stated in the BSCP Plan (Energy Systems 1992)?

4. Did you use total dissolution methods for radiological analyses?

5. Did you incorporate any deviations or modifications in the methods as described in the BSCP Plan (Energy Systems 1992) or in this report?

6. Is your data based on wet weight or dry weight?

7. Are the units associated with your data the same as those presented in this report?

8. Did you compare your detection limits with those contained in the BSCP Plan (Energy Systems 1992)? Are you using instrument detection limits, method detection limits, practical quantitation limits, or contract required detection limits? For explanation of terminology on detection limits, refer to EPA/SW-846 (2nd ed.) and to the EPA/CLP statement of work for organic and inorganic analyses.

9. Did you refer to the data validation qualifiers (list of data validation qualifier definitions can be found in Sect. 4.4) for data in this report when evaluating your data?

\subsubsection{Statistical Guidelines for Users of Background Soil Data}

The scope of possible applications of the BSCP data is so broad that it is not feasible to elaborate on statistical methods appropriate for each possible application. The following is presented as a starting point.

Is your goal

- to design a soil sampling program for which the BSCP is to be a reference? Refer to the BSCP Plan (Energy Systems 1992) and to Sect. 5 (particularly Sect. 5.10) of this report 
for discussions of laboratory and spatial variance and compositing. See Sect. 5.2 (particularly Sect. 5.2.3) for analytes of interest.

- to determine target values for remediation? See Sect. 5.2 for general discussion on the computation of confidence bounds, and Sects. 5.3-5.9 for particular analytes of interest.

- to obtain a target value that is within the normal background range? Use a lower tolerance bound for an upper percentile (e.g., the 95th).

- to obtain a target value that is near the mean (or median) of normal background levels? Use a confidence bound for the mean. If you want to be confident that a target is no higher than the mean, use a lower confidence bound. (Use an upper confidence bound for the mean only if you want to be confident that the target is above the mean.)

- to determine if the detection of a PAH, pesticide, herbicide, or other normally absent substance is inconsistent with a practical definition of background (i.e., one for which some limited anthropogenic effects are admitted)? Refer to upper confidence bounds for detection probabilities, discussed in Sect. 5 (particularly Sect. 5.2), but note that some of these confidence bounds are not useful because overall sample sizes for Phase I are small.

- to determine if detected concentrations are within normal background levels? Refer to appropriate upper percentile estimates and lower tolerance bounds in Sect. 5 and discussion in Sects. 5.2 and 5.10.

\section{Exclusions}

Certain applications will be sufficiently sensitive to warrant a close look at the background data and statistical methods of analysis. How well the lognormal and alternate models apply for the particular analytes of concern should be considered. Data already collected may not be automatically compared to BSCP data without further scrutiny and analysis-for example, if samples are not composited or composited at significantly different levels other than three, or if they are biased, perhaps through the use of a nonrandomized sampling site selection process resulting in the selection of hot spots. Alternatives for composites of other than three are discussed in Sect. 5.10. The statistical variability of new observations, which may be expressed in means or percentiles from replicates, should be considered.

Confidence bounds and other statistics are intended to reasonably delineate states of knowledge. For some purposes, some of the BSCP data statistics may seem unreasonably high or low. In most cases the problem is not in the statistics but is rather in the actual uncertainty in the state of knowledge. If a statistic is questionable, the costs of getting additional information, for example, by additional sampling, should be weighed against the losses due to relying on values that may be too high or low. Practical considerations should go beyond statistical confidence and significance. For example, in light of risks, some background levels may represent low remediation targets. 


\subsection{Data User Guidelines for Risk Assessments}

The following questions are intended to focus attention on aspects of using BSCP data for risk assessments.

\section{What is risk assessment as it pertains to the BSCP?}

Risk assessment is used to evaluate potential risks to human health from exposure to constituents in background soils (from the ORR, Anderson County, and Roane County). There are two types of risk, carcinogenic risk and noncarcinogenic (systemic) risk. For carcinogens, risks are estimated as the incremental probability of an individual developing cancer over a lifetime as a result of exposure to the carcinogen. Cancer risk from the exposure to contamination is expressed as excess cancer risk; that is, cancer incurred in addition to normally expected rates of cancer development. An excess cancer risk of $1.0 \times$ $10^{-6}$ indicates one person in one million is predicted to incur cancer from exposure to this contamination level.

Noncarcinogenic effects are systemic toxic effects-that is, they are toxic effects to an organ or system which occur when a threshold dose is reached. Unlike carcinogenic risk, which is represented by a probability of incurring cancer over a lifetime, systemic risk is posed only if a threshold is exceeded.

\section{What are the primary goals of this risk assessment?}

The primary objectives of this BSCP risk assessment are to (1) evaluate the Phase I background data in terms of potential adverse effects to human health (carcinogenic and systemic); (2) produce a comprehensive database for naturally occurring constituent concentrations in soils on the ORR; (3) provide the context for discussion of risks associated with ORR site related contamination (which includes identifying contaminants of concern); and (4) provide a comparison, based on risk, between soils collected from the three sampling areas (ORR, Anderson County, and Roane County).

\section{How are risks and hazard indices determined?}

To evaluate potential risk to human health from background constituents, EPA-approved dose/response information must be available-that is, slope factors (for carcinogenic risk analysis) and reference doses (for analysis of noncarcinogenic/systemic effects).

Carcinogenic effects are expressed is terms of risk. The risk is calculated by multiplying the daily intake of a constituent by the EPA-approved slope factor. There are three regions of concern according to EPA guidelines for contaminated sites: risk $<1.0 \times 10^{-6}$, no concern; risk between $1.0 \times 10^{-6}$ and $1.0 \times 10^{-4}$, range of concern; and risk $>1.0 \times 10^{-4}$, unacceptable. Risks due to background soil concentrations are reported in this manner, but the results are only for comparison with site-related risk; the results do not pertain to remediation goals.

Systemic risks are expressed in terms of a hazard index. The hazard index is calculated by determining the ratio of the daily intake of a constituent to the EPA-approved reference dose. If this ratio is less than 1.0 , no adverse effects from exposure to this chemical are expected; if the hazard index is greater than 1.0 , adverse systemic effects may possibly occur. 


\section{How are the calculated risk values to be used?}

The most important aspect of the background soil data for risk assessment is in application to the selection of potential contaminants of concern. These background values can be used to attain an accurate assessment of the risk to human health posed by contaminants found at higher concentrations [two orders of magnitude above background concentrations (EPA 1990)] than naturally occurring background concentrations on the ORR. The total soil background risk reported in this document can be used to discuss site-related risk in the context of background risk.

Although background risk numbers are presented for Anderson and Roane counties in addition to the ORR, risk assessments conducted on the reservation are to employ the background risk numbers calculated for the ORR, as these data best represent background levels at an ORR site. The background risk numbers presented in Sect. 7 should be used in a baseline risk assessment or in a feasibility study for screening of alternatives on the ORR. In some cases (refer to Sect. 7), the background risk is unacceptable for an analyte in terms of EPA guidance $\left(>1 \times 10^{-4}\right)$. This should also be reported in the site-specific risk assessment. Cleanup goals should not be below the reported background level.

The risk assessment in this report is subject to uncertainty pertaining to sampling and analysis, exposure estimation, and toxicological data. Several sources of uncertainty exist that are associated with site risk assessments. The following are examples of factors that may contribute to uncertainty in the risk assessment (Sect. 7).

- Assuming that risk doses within an exposure route are additive does not account for synergism or antagonism, which may overestimate or underestimate risks.

- Not all toxicity values represent the same degree of certainty. These values are subject to change as new evidence becomes available.

- Assuming exposures to be constant does not account for environmental fate, transport, or transfer that may alter concentrations.

In addition, land use for this risk assessment was assumed to be residential. Although the assumption of residential land use is generally recommended when determining risk at a site (EPA 1989), risk numbers that result are at the conservative end of the scale, when in fact residential use may not be the most likely future land use for the ORR. This assumption contributes to the uncertainty by possibly overestimating risks. Identifying these, and other, key site-related variables and assumptions that contribute to uncertainty will enable the risk estimates to be placed in proper perspective (EPA 1989).

What are the uncertainties associated with the risk and hazard index numbers?

Risk assessment as a scientific activity is subject to uncertainty. Although the methodology used in this risk assessment follows EPA guidelines, uncertainties pertaining to sampling and analysis, exposure estimation, and toxicological data still exist.

The major assumptions used in risk assessment are that (1) contaminant concentrations detected and reported by the analytical laboratory are representative of the analyte 
concentrations in the soil, (2) the intake rates and exposure parameters representative of actual potentially exposed populations, and (3) all contaminant exposuise and intakes are from the site-related exposure media.

Given these assumptions, there are other areas which can result in uncertainty. The toxicological data (slope factors and RfDs) are often updated and revised, which could alter risk values. Furthermore, these values are often extrapolations from animals to humans, which also induces uncertainties in toxicity values. In addition, not all of the detected background chemicals reported in this study currently have toxicity values; hence, this can underestimate total risk because quantitative assessment of such chemicals is currently not obtainable.

\subsection{Data Access Considerations}

BSCP analytical results are available from OREIS. Users wishing to access the data should refer to ER/C-P2702, Rev. 0, "Obtaining Access to Data in OREIS," and the "Oak Ridge Environmental Information System (OREIS) User Interface Manual for General Users, Version 1.0."

All data definitions are consistent within OREIS and are described in the OREIS documentation. Based upon user responses to the previous and the following guideline questions, the various fields can be queried to extract specific information.

Additional considerations follow.

1. Does the user want to distinguish between data collected for screening purposes and those for higher quality analytical results? Attention must be given to qualifiers which indicate the original purpose for which the data were collected and then determine the appropriate use of the data.

2. Does the user want to distinguish among results for the same analyte but determined by different analytical methods? Users are cautioned to separate the results by method before calculating summary statistics.

3. Does the user want to reproduce the risk calculations using alternate risk factors or exposure scenarios? The mean and upper $95 \%$ confidence bounds were calculated using a maximum likelihood estimation technique to appropriately account for values reported at their detection limit. 


\section{FIELD INVESTIGATION AND GAMMA SCREENING ANALYSES}

\subsection{SUMMARY}

This section discusses pertinent aspects of obtaining soil samples for analysis in Phase I according to project objectives. To this end, the section covers sampling site selection, sample preparation procedures, field quality control, and results of site screening activities. To meet sampling requirements, field operations were planned and executed as follows:

- In the first half of Phase I, the Dismal Gap Formation was sampled at 24 locations, both on-site (12 on the ORR) and off-site (12 in Roane County).

- In the second half of Phase I, 24 more sites were sampled (12 on the ORR in the Nolichucky Shale and 12 in the Dismal Gap in Anderson County) for a total of 48 sites in Phase I. These operations were conducted during FY 1992.

\subsection{INTRODUCTION}

The Oak Ridge Reservation (ORR) lies in an area characterized by elongated ridges and broad to narrow valleys which run northeast to southwest. Geologically, the area is characterized by four principal rock groups (the Rome, Conasauga, Knox, and Chickamauga). There are two major categories of soils: residual soils developed from in-place weathered residuum of the geologic groups and soils developed in partially sorted colluvial and alluvial soil materials. Within the first of these residual soil groups, only certain formations of the Conasauga Group are considered in this Phase I report because this group represents the dominant soils at waste area groupings and operable units in imminent remedial action projects on the ORR. Soils formed in the Knox and Chickamauga groups will be sampled and analyzed in Phase II of this project. Soils from the Rome Formation do not appear with regularity at contaminated sites on the ORR and for that reason are not addressed in this project. The hydrologic system on the ORR, including both surface water and groundwater, is controlled regionally by the Clinch River. The climate of the area is generally temperate with warm, humid summers and cool winters, and the average annual rainfall in the Oak Ridge area is approximately $136 \mathrm{~cm}$.

Soil sampling for Phase I was restricted to residual soils of the two most representative Conasauga Group geologic formations out of six: the Dismal Gap Formation (formerly Maryville Limestone) and Nolichucky Shale within the Bear Creek Valley section. Three areas within this geologic section were chosen. The ORR area extended from the Clinch River on the west to the west end of the Y-12 Plant burial grounds on the east. Two off-site areas in the same geologic strike zone were located to the southwest in Roane County and to the northeast in Anderson County (Fig. 3.1). Only residual soils of the Dismal Gap Formation were sampled in both on-site ORR and off-site locations in Phase I of the project. The selection of which parent materials to sample in each sampling area reflected the availability of limited ressurces and the intention to maximize project effectiveness, in addition to 


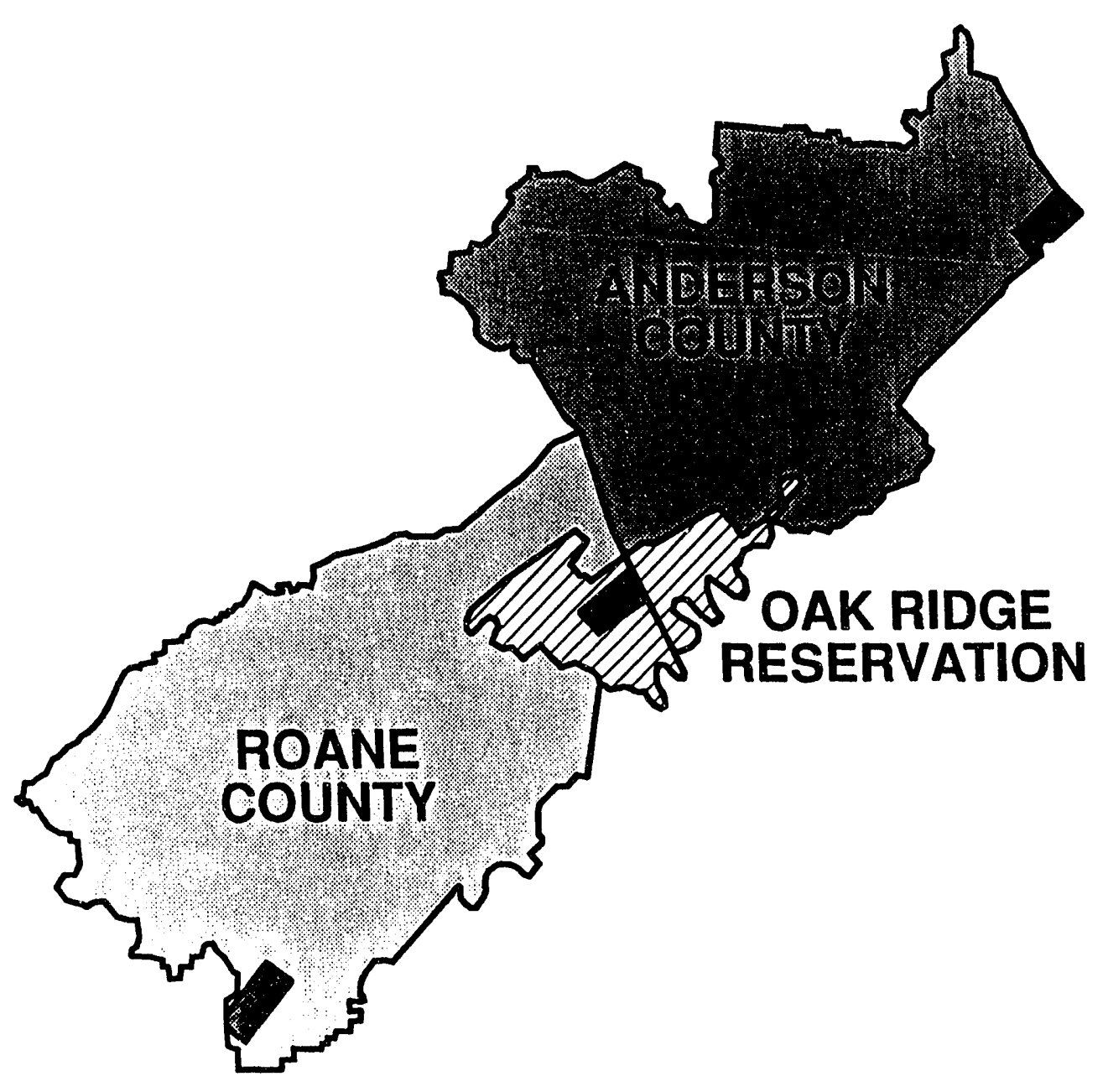

Fig. 3.1. Approximate locations of BSCP sampling areas. 
considering technical factors, such as site accessibility and the availability of suitable sampling sites that fit the selection criteria discussed in Sect. 3.2. It is not known whether all of the data from the Bear Creek section of the Conasauga can be used in the Melton Valley section of the Conasauga, since these two sections were separated by a considerable lateral extent during deposition, even though faulting has now brought both sections quite close together. The primary concerns here are those metals and radionuclides that are inherited from geologic materials.

\subsection{SAMPLING SITE SEIECTION}

Sampling sites on the ORR were mostly confined to the Roane County portion, but some ORR Phase II sites were located in Anderson County (Fig. 3.2). Recent digitized soil maps (available from the Oak Ridge Environmental Information System), where residual soils had been related to the underlying geologic formations, provided the base map for generating potential sites. A statistical program was utilized to randomly select grid coordinates that fell on predetermined soil map units. No two sites were to be less than 250 feet apart. This methodology resulted in the generation of a base map with 44 potential sampling locations for the Dismal Gap and Nolichucky soils. Each potential sampling site was assigned a unique number from 1 to 44 . In addition, the statistical program determined primary and secondary sampling sites. Secondary sites are alternate site locations in case the primary sites are unacceptable in terms of the selection criteria discussed below. In several cases on the ORR, both primary and secondary sites were not acceptable, resulting in the soil scientist looking nearby for potential sites that would meet the criteria. Potential sites in southwestern Roane (Fig. 3.3) and northeastern Anderson (Fig. 3.4) counties were selected differently because of ownership, vegetation (Figs. 3.1-3.4), and disturbance constraints. Anderson County and Roane County sites are located within the shaded remote site areas, as shown in Fig. 3.1. In these off-site locations more than 24 potential sites were located in the field. Those sites eventually chosen were located along the entire distance of the evaluated area and had to meet the vegetation and disturbance requirements discussed below.

\subsubsection{Site Evaluation}

Individual site evaluation used the following criteria:

- Vegetation and disturbance. The site had to be in forest that had not been disturbed for at least the past $\mathbf{4 0} \pm 5$ years. Forest was either hardwoods, mixed old-field successional pines-cedars and hardwoods, or planted loblolly pine plantations. Recently replanted pine plantations were rejected because of too recent surface disturbance. Each site was located by relating the map grid point to the actual soil map unit delineation and then to the actual landform in the woods. If the vegetation parameter was met, then the next evaluation parameter was considered.

- Initial soil evaluation. Several soil evaluations were made in an area surrounding the potential 3- by 3-meter sampling site to determine if the soil there was entirely of residual origin and not colluvium, or of a thin capping of colluvium over residuum, which was considered to be an acceptable site. The center of the actual sampling site was then located, and plastic ribbon was tied around one or more trees. The closest route in from 


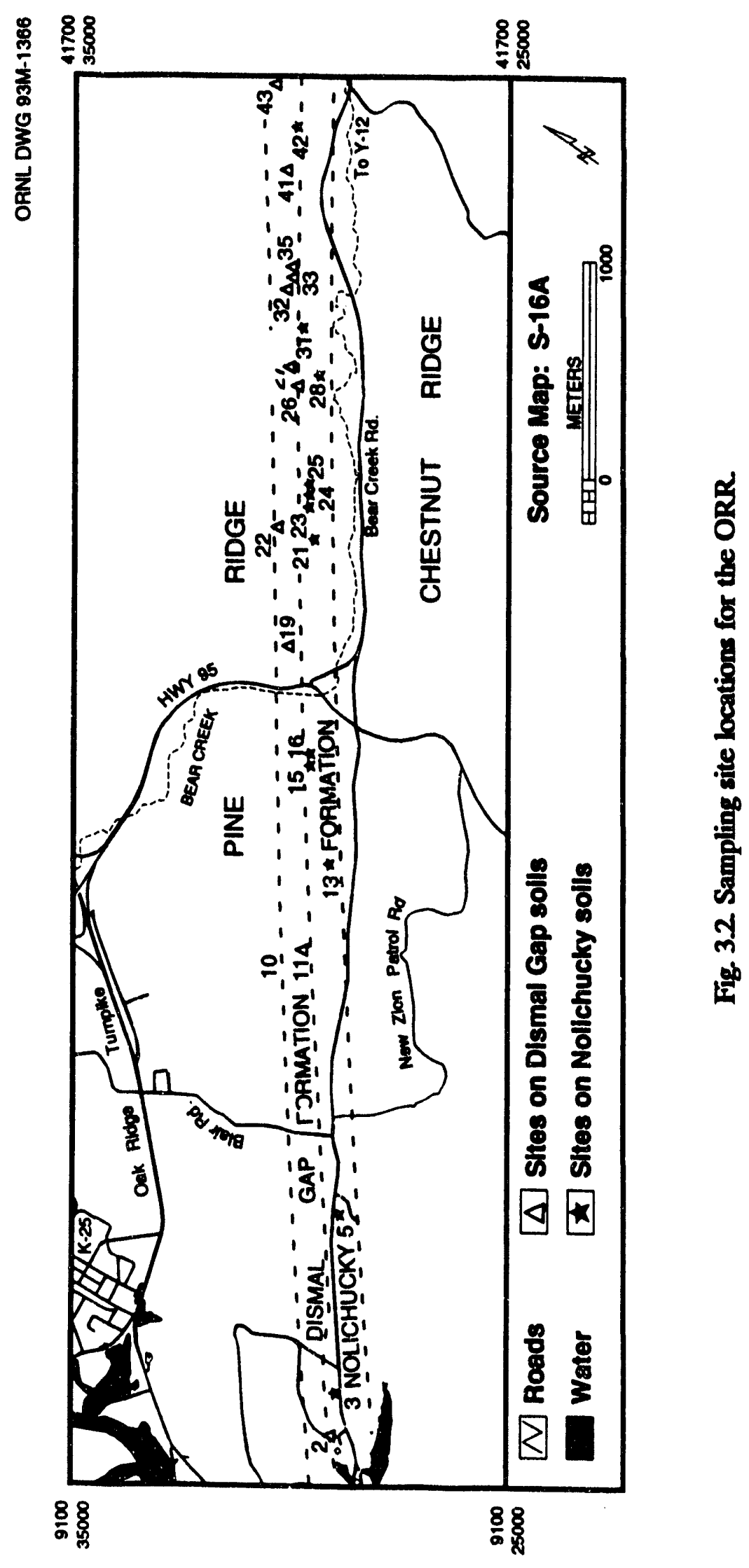


ORNL DWG 93M-1364

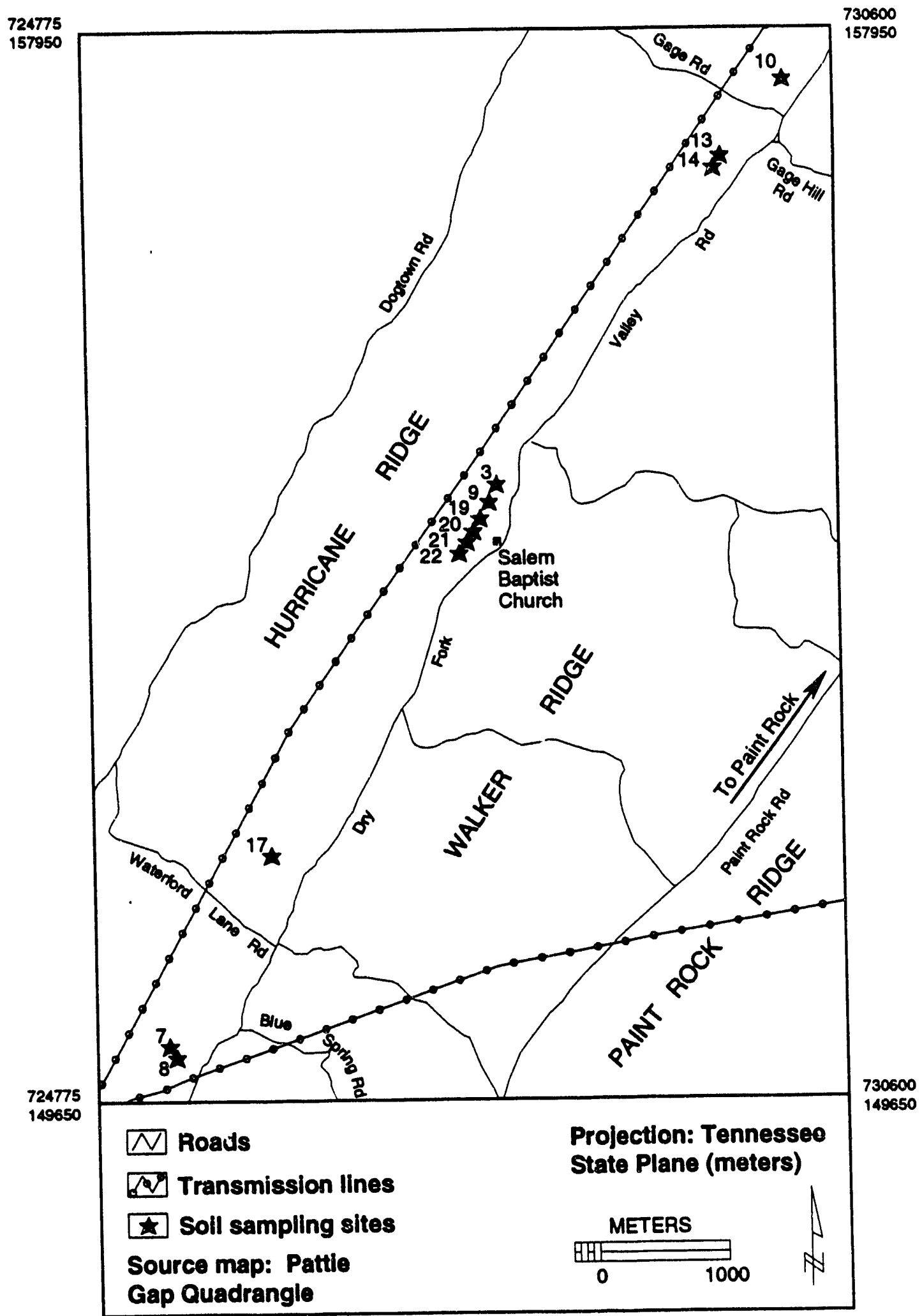

Fig. 3.3. Sampling sites in Roane County. 


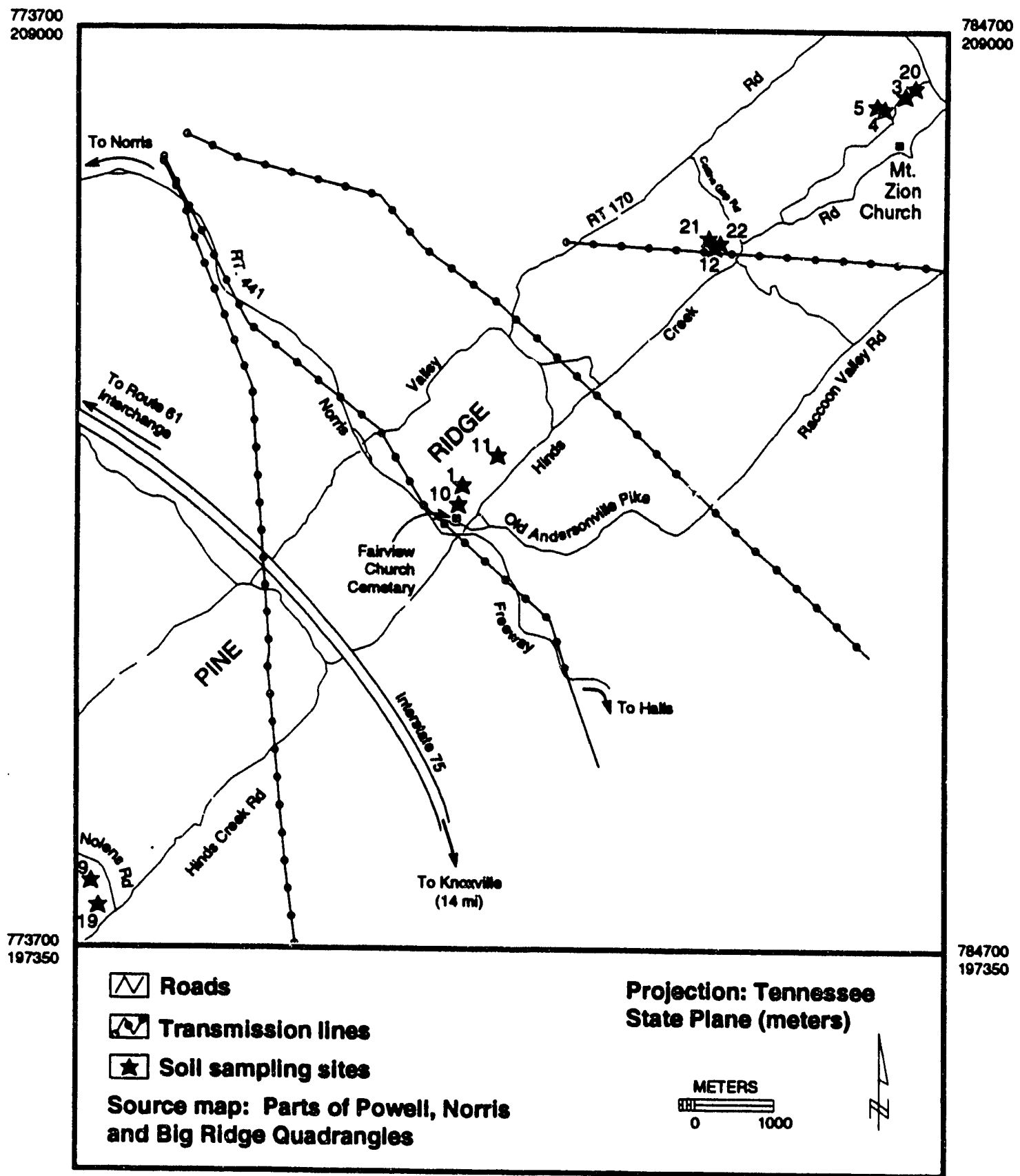

Fig. 3.4. Sampling sites in Anderson County. 
the road was also flagged so that the site could be located again some time after the initial evaluation and after trees had leafed.

\subsubsection{Selected Sites}

After the initial vegetation and soil screenings were finisithed for all of the potential Dismal Gap sites, the following ORR Dismal Gap sites were found to be suitable:

- Dismal Gap/primary: $\quad 11,22,26,32,33$, and 41 ;

- Dismal Gap/secondary: $1,2,4,10,19,27,35$, and 43 .

All primary sites were used. Secondary sites were selectedl so that there would be no major gaps in coverage. Because of this, secondary sites 1 and 4 were rejected.

After the initial vegetation and suil screenings were done for all of the potential Nolichucky sites, the following sites were found to be suitable:

- Nolichucky Shale/primary: $\quad 15,23,24,25$, and 31 ;

- Nolichucky Shale;secondary: $3,5,13,16,21,28$, and 42 .

The 12 ORR Nolichucky sites were located as required, although it was more difficult to accomplish because of more recent replanting of pines on less sloping Nolichucky landforms. Some sites were moved to a new locations in order to obtain sufficient soil samples.

\subsubsection{Composited Sample Sites}

After 12 sites were chosen for each formation, a randomizing process was used to determine the grouping of threes for the compositing procedure specified in the sampling plan in Sect. 5.3 of the BSCP Plan (Energy Systems 1992).

Following are the groupings for Dismal Gap and Nolichucky sites:

- Dismal Gap: $\quad\left[\begin{array}{lll}27 & 41 & 11\end{array}\right]\left[\begin{array}{lll}22 & 19 & 32\end{array}\right]\left[\begin{array}{lll}33 & 10 & 35\end{array}\right]\left[\begin{array}{llll}2 & 43 & 26\end{array}\right]$,

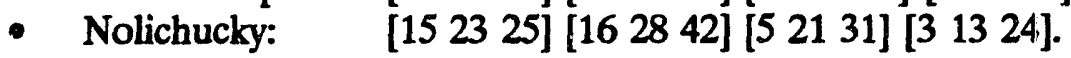

The exact sequence of sampling a site within any particular sampling group was not important. This concluded the preliminary site selection and initial evaluation process for the ORR sites.

\subsubsection{Selection and Initial Evaluation of Off-Site Locations}

Conventional U.S. Geulogical Survey topographic maps were utilized in locating potential sampling areas in southwest Roane County and in northeast Anderson County, so that these potential areas were in the same strike belt Conasauga Group section as the ORR Dismal Gap and Nolichucky sites. The University of Tennessee samplirig crew made the potential site selection by using he same vegetation and soil parameters clescrihed above. Independent confirmation was obtained that the Roane County sites were in the Dismal Gap Formation. Because of both present and past land uses off-site, the potential number of sampling areas 
was severely limited, but no two adjacent sampling sites could be closer than 250 feet. Twelve sampling sites that met the vegetation, soils, and past land use criterir were selected in Roane County and 12 in Anderson County. A radiation scan was not per 'ormed for any off-site sampling location.

After the 12 Roane and 12 Anderson sites were selected, a random drawing process was used to generate combinations of sites for purposes of compositing. The following are the combinations that were generated:

- Roane County: [ [9 17 19] [ [ $\left.\begin{array}{llll}3 & 7 & 21\end{array}\right]\left[\begin{array}{lll}8 & 20 & 22\end{array}\right]\left[\begin{array}{llll}10 & 13 & 14\end{array}\right]$,

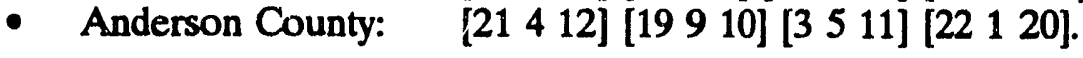

\subsection{SITE AND SOIL DESCRIPTIONS}

The site and soil narrative descriptions are presented in Appendix A for on-site ORR locations and off-site locations in Roane and Anderson counties. Each site in Phase $I$ is described in numerical order. In the appendix ORR sampling sites are described first, followed by descriptions of the Roane County and Anderson County sites.

\subsection{SAMPLING PROCEDURES}

Field operations and sample handling are governed by the following procedures developed specifically for this project:

- Background Soil Characterization Project, Procedure BSCP-SOP-01, Rev. 1, May 23, 1992; and

- Background Soil Characterization Project, Procedure BSCP-SOP-02, Rev. 0, August 6, 1992.

These procedures were developed based on the following references: EPA $(1980,1987 \mathrm{a}$, 1987b, and 1991a); ANSI/ASTM (1980); and Kimbrough et al. (1988).

A performance-based training plan was initiated for all personnel involved with soil sampling activities. The technical coordinator tested the team sampling leader in all aspects of sampling and sample management in which he/she will be involved. Only those actually doing the soil sampling and signing chain-of-custody forms received performance-based training and testing. Technicians received on-the-job training for those activities in which they were involved and were supervised in these activities either by the technical coordinator or by the sampling team leader.

\subsection{SOIL SAMPLING AND SAMPLE PREPARATION}

\subsubsection{Scope and Objective}

Procedure BSCP-SOP-01, Rev. 1 describes the siting of soil sampling locations and soil sampling methodology. The objectives of the procedure are to (1) select representative 
sampling sites and (2) obtain representative soil samples for characterization. This procedure was prepared to meet the project quality assurance/quality control and health and safety objectives (BSCP Plan, Energy Systems 1992).

\subsection{Materials}

Required equipment for field sampling operations is described in Procedure BSCP-SOP-01, Rev. 1.

\subsubsection{Field Activities}

\subsubsection{Locating sampling areas}

The soil scientist and technician located potential sampling sites based on location of grid nodes on site location maps (Figs. 3.2 through 3.4). At selected sites, the following stepwise assessment was made prior to sampling based on the following criteria:

1. Field evidence must substantiate that the present forest vegetation had not been disturbed for the last $\mathbf{4 0} \pm 5$ years. Young pine plantations were not considered. Only old hardwood forest, old field forest regrowth, and old pine plantations areas were considered as potential sampling sites. If a primary site was not suitable because of recent surface disturbance, it was rejected with an explanatory note in the soil scientist's logbook, and the secondary site was observed for its potential suitability.

2. If a site, based on vegetation cover, was deemed to have potential, the first soil observation near the grid point that qualifies for the soil to be sampled marked one corner of the proposed sample site. This was one way of reducing soil scientist bias. After one corner of the sample site had been located, additional soil observations were made within a 4-meter radius of the located grid point to determine if the proposed site was uniform enough for sampling or for additional sampling in the future. Proposed sampling areas were located on the most stable part of the landform with the intent that there would have been minimal overland runoff and removal of surface soil materials over the past $\mathbf{4 0}$ or so years. The purpose of the additional soil observations was to determine that most of the site was composed of residual soils, not of thick colluvium or a thin (50 to $100 \mathrm{~cm}$ ) colluvial capping. However, because of several constraints of locating enough suitable sites, soils with a thin colluvial or alluvial capping less than $50 \mathrm{~cm}$ thick were considered suitable for sampling.

3. If the soils and vegetation cover were suitable, then an approximately 3-by 3-meter area was selected and located by flagging around nearby trees. Soil observations were made at the four corners of this square area, and brief soil evaluations were made. Disturbance within the square was kept to a minimum. Soil from these limited observations was not placed within the 3- by 3-meter area. The site number was painted on at least one marker stake. This stake was driven into the ground at one corner of the sampling square. Other stakes were placed at the other three corners. These stakes remained in place until all sampling had been completed. Care was taken so that there was minimal surface disturbance of the sampling area when digging pits. On a sloping site the sampling pit was always located at the lowest point and the upslope face, if suitable, was sampled. Often in a forested area, stump holes that have filled in are exposed in digging 
the pit and another pit face had to be selected. In situations where there was highly variable depth to rock, a pit face other than the upslope face had to be sampled. Soil removed from the pit was placed outside the 3-meter site. After all soil sampling had been completed, the pits were filled.

4. The most feasible route from the sampling site to the road was flagged so that the site could be easily relocated.

5. All ORR sites were scanned prior to any sampling using a hand-held radiation detector. An air reading and a ground level reading were obtained. If the ground level radiation reading was higher than $100 \mathrm{cpm}$, then the site was considered contaminated. Where ground level readings were above $80 \mathrm{cpm}$, a reading was taken in the top of the auger hole to determine if there was a higher level of radioactivity in the upper mineral soil. Off-reservation sites in Roane and Anderson counties were not scanned with the detector. Volatile organic compound (VOC) emissions from the selected sites were monitored by an industrial hygienist during sampling (only $25 \%$ of ORR sites).

6. After all sampling had been completed, a permanent steel marker fencepost, suitably labeled, was placed at the center of each site (only ORR sites) so that the site could be relocated.

\subsubsection{Sampling methods}

After arriving at or near the sampling site, all laboratory precleaned, rinsed, and aluminum foil-wrapped sampling equipment to be used for sampling was thoroughly rinsed with deionized water and then rewrapped with aluminum foil. A small pit was dug in a topographically lower part of the sampling square so that the area above the pit was not disturbed. Soil horizons were evaluated in this small pit. If the soil exposure was suitable, the pit width was enlarged so that enough soil was exposed to acquire the volume of needed sample. Initial pit excavation was done with a steel shovel or spade. The soil profile was described from the pit face to be sampled before collecting Environmental Sciences Division (ESD) composite samples of $\mathrm{A}, \mathrm{B}$, and $\mathrm{C}$ horizons. The newly exposed pit face was cut back a minimum (about 1-2 cm) with stainless steel soil sampling equipment to expose a fresh face. The forest litter layer was removed down to the mineral surface. If a pit had been opened previously for other sampling, the old pit face was cut back a minimum of $18 \mathrm{~cm}$ to expose a fresh face to obtain undisturbed samples. A fresh, precleaned, and field-rinsed stainless steel sampling tool was used for sampling each soil horizon.

Surface horizon sampling. There are at least two possible conditions that will be encountered in sampling the surface layer. First, the site will be located in an area that has never been plowed. The horizonation will usually be an $\mathrm{O}$ horizon followed by an $\mathrm{A}$ horizon. This A horizon is usually thin, less than $10 \mathrm{~cm}$ thick and underlain by an $\mathrm{E}$ horizon. Second, the site will be located in an old field with naturally regenerated forest or in a pine plantation with trees at least 40 years of age. Here the soil will have an $O$ horizon of forest litter followed by an A horizon that is 4 to about 8 or $10 \mathrm{~cm}$ thick. Beneath this horizon is an Ap horizon that typically extends to a depth of 15 to $18 \mathrm{~cm}$ and may not be recognized as an old Ap horizon but as an $E$ horizon. In the event of past landuse disturbance, the upper $A$ horizon has reformed since the last disturbance. The upper organic enriched horizons, $A, A p$, and $E$ horizon, will be sampled and labeled "A horizon." Sampling will usually require the 
removal of tree roots. It is likely that many tree roots will have to be cut and removed. Poison ivy grows nearly everywhere. Care must be taken by samplers to protect against poison ivy. A small stainless steel shovel or spatula will be used to push soil into the mouth of the sample jar. If soil goes past the mouth of the jar and comes into contact with the sampler's hand, it is discarded. All sampling is done in this manner where the soil that is collected comes into contact only with the stainless steel sampling tool. The only exception is for gamma screening samples, where because of the geometry of the sampling container, the soil must be packed by use of the fingers or a small freshly cut stick.

Three different soil samples were collected from the surface A horizon soil. Noncomposited A horizon samples were collected for (1) VOC analysis in a 250-mL amber glass bottle, (2) tritium analysis in a $1000-\mathrm{mL}$ clear glass bottle, and (3) organic compound (such as PAHs, pesticides, and herbicides) analyses in a 1000-mL amber bottle. Bottles were capped, labeled, and sealed with a custody seal. One additional A horizon sample was collected in a 2.liter bottle and labeled "ESD A Horizon Composite." All A horizon samples were placed in a chilled ice chest in the field and then placed into a refrigerator maintained at $4^{\circ} \pm 4^{\circ} \mathrm{C}$.

Each soil sample had an attached label to uniquely identify that sample. If an $A$ horizon field duplicate sample was obtained for VOC, organics, or tritium analysis, it was identified by the letter "FD" after the sample identification number. The choice of site from which to obtain an A horizon duplicate was at the discretion of the sampling crew. Any used gloves were discarded into a trash bag.

Subsoil (B horizon) sampling. The subsoil, either a Bt horizon or a Bw horizon, was sampled at all sites but only for compositing purposes. Only horizons $8 \mathrm{~cm}$ or thicker were sampled individually. Thin subsoil horizons were grouped so that a minimum $15-\mathrm{cm}$ thickness was sampled. The surface of the subsoil horizon was exposed by removing any soil horizons above. Final removal of overlying soil was done using stainless steel equipment. At least $1.5 \mathrm{~kg}$ of the subsoil samples were collected at a designated depth determined from field description using stainless steel sampling equipment and placed into a suitably labeled glass 2-liter container or three or four 1-liter jars if field splits were to be generated in the soil preparation laboratory (SPL). If the $\mathrm{Bt}$ or Bw horizon was less than $15 \mathrm{~cm}$ thick, its entire thickness was sampled. Otherwise, only the upper $15 \mathrm{~cm}$ was sampled. Samplers wore suitable gloves as needed for the hand work, and the presence of poison ivy roots necessitated protection at some sites. B horizon samples were all labeled "ESD B Horizon Composite."

C horizon or substratum sampling. Soils having a shallow depth to the $\mathrm{C}$ or $\mathrm{Cr}$ horizon were sampled with hand digging equipment. This included soils in the Dismal Gap and Nolichucky formations and some soils in the Chickamauga. Soils in the Copper Ridge and Chepultepec formations may require augering equipment to penetrate deep enough to reach such soil materials. The $C$ horizon or substratum is defined as that depth in the soil where there is minimal evidence of translocated clay and where there is minimal expression of pedogenic soil structure. Some soils will be underlain by saprolite, other soils will be underlain by saprolitic materials, and still others will have clayey materials lying directly on bedrock. Depth to the soil layer to be sampled was established by the project soil scientist at each site as sampling was done. However, earlier observations assisted in determining the approximate depth of sampling. At least $1.5 \mathrm{~kg}$ of $\mathrm{C}$ horizon soil samples were collected from depths 
predetermined from field description using clean stainless steel equipment (hand auger or power-driven auger), placed in glass jars, and labeled "ESD C Horizon Composite."

Composited samples and composited SPL splits. Soil samples from A, B, and C horizons were collected from at least one set of sites per geologic formation on the ORR, Roane, and Anderson losalities for purposes of compositing. The practice for collecting field duplicates for compositing purposes in Phase I and Phase II required that a set of $\mathrm{A}, \mathrm{B}$, and C horizon samples be collected from one face of the soil pit. Then, the field duplicate set was obtained from a side face of the same soil pit to expedite field operations rather than digging another soil pit. Field duplicates for compositing purposes were identified by the letters "FD" after the sample number.

"Field" splits will be generated in Phase II in the following manner. After the compositing is done for one horizon from the three sites to be composited, enough sample will be weighed out from each horizon, thoroughly mixed, and then placed into precleaned sample jars. One composited sample is designated, for example, "metals, A horizon." Another jar, filled with the same soil, will have the same designation but a different number and will be listed as a composited split in the laboratory notebook. Both field duplicates and sample splits will be obtained in Phase II of the BSCP.

Gamma screening samples. Six 5-cm depth increment samples were collected from a 10 by $10-\mathrm{cm}$ area in special plastic containers for cesium-137 determination by gamma spectroscopy. Detailed steps for collecting ESD gamma soil samples follow.

1. After a site had been located and preliminary observations made including a rad-scan, a pit was dug to a depth of 50 to $60 \mathrm{~cm}$ at one corner of the 3- by 3-meter site.

2. Surface litter and organic matter layers were removed to expose the mineral soil surface in an area larger than that to be sampled (about $500 \mathrm{~cm}^{2}$ ).

3. A $10 \times 10 \times 5 \mathrm{~cm}$ stainless steel frame was laid on the soil surface and carefully hammered into the soil to its 5-cm depth.

4. Soil from three sides of the frame was removed, and a knife or a spatula was used to sever roots and soil from beneath the frame. The soil-filled fraine was removed and placed onto aluminum foil.

5. The soil inside the frame was packed into a 500-mL marinelli beaker. The label was filled out after packing and cross-checked with the field book entry. Large roots $(>1 \mathrm{~cm}$ diameter) were not put into the container. When samples had a considerable number of coarse fragments-for example, soils in the Knox Group-fine earth was packed into the container first and the coarse fragments were added on top. The container lid was placed, taped, and custody sealed.

6. The sampling frame and equipment were wiped clean of soil by using paper towels and a brass wire brush before collecting the next $5-\mathrm{cm}$ increment.

7. The soil from the sampling area was removed down to the top of the next depth in an area larger than that to be sampled. The clean stainless steel frame was placed on the 
soil and driven into its full $5-\mathrm{cm}$ depth. The soil was removed and packed following the above procedure.

8. This procedure was repeated at $5-\mathrm{cm}$ increments to a depth of $30 \mathrm{~cm}$.

\subsubsection{Preparation of composited soil samples in the SPL}

The following steps are employed in preparing soil samples for analysis.

1. Composite samples (to be composited) of $\mathrm{A}, \mathrm{B}$, and $\mathrm{C}$ horizons brought from sampling sites were refrigerated until soil sampling of all three sites in the predetermined group was completed.

2. Individual composite samples were placed on clean blotting paper to partially dry prior to sieving. All of the samples were passed through a $4.75-\mathrm{mm}$ stainless steel sieve in the laboratory. The coarse fragments $(>4.75 \mathrm{~mm})$ were discarded after determination of the weight contribution to the whole soil sample. An equal amount (about $1 \mathrm{~kg}$ or more) of three equivalent horizon samples (passed through the $4.75-\mathrm{mm}$ sieve) was composited and mixed in a stainless steel container. Mixing involved pouring the sample from one stainless steel container into another several times while the pouring container rotated. If a sample splitter was used, it produced a mixed composited sample sooner, but care had to be taken to not raise dust. One-third of each composited sample was stored in a precleaned glass jar for metal analyses, one-third in a polypropylene bottle for radionuclide analyses, and the remaining one-third (labeled "extra") in a glass bottle for use in measurement of soil properties, such as $\mathrm{pH}$, and in neutron activation analysis (NAA). Additional samples and jars were required if composited splits were generated. The compositing procedure resulted in the destruction of the original field $A, B$, and $C$ horizon soil samples. New sample numbers were assigned to all SPL composited soil samples, and a new chain-of-custody form was completed. The sampling time (and date) for composited samples corresponded to the constituent sample with the earliest sampling date.

3. The composited soil samples and noncomposited A horizon soil samples were preserved in the SPL refrigerator until packed for shipment. Samples were shipped to the designated contract laboratories through the Analytical Projects Office according to Procedure BSCP-SOP-02, Rev. 0.

Additionally, note that

- Soil profile descriptions were recorded in the field sampling notebook. Soil profile descriptions were not made until the soil pit was dug to the depth required for sampling $\mathrm{B}$ and $\mathrm{C}$ horizons. Any horizons that were field grouped for sampling due to thinness were noted in the field book.

- A variance form was used where field conditions necessitated a change in sampling procedure (none were needed in Phase I). It was intended that the sampling procedure be the same for all sites underlain by a particular geologic formation(s). 


\subsubsection{NAA samples}

Composited samples of all A, B, and C horizons that had been labeled "extra" and preserved in a refrigerator were subsampled for NAA. A 40-mL precleaned glass sample jar with a teflon seal was filled with soil from a large clear glass "extra" jar. A small sampling device was used to obtain a vertical cross-section sample from the large glass jar. Sampling was done in this manner until the $40-\mathrm{mL}$ jar was filled. The small sample jar was given the same "extra" composited sample number but designated "NAA." A laboratory chain-of-custody form was filled out and the samples transferred to the Analytical Chemistry Division at ORNL. After the samples had been returned to the SPL, the moisture content of each was determined. The moisture content will be used to convert all NAA values to an oven-dry-soil basis.

\subsubsection{Cleaning sample containers and sampling tooks}

Precleaned glass jar sample containers used by field sampling teams were obtained from a commercial supplier. Analytical results of the last rinse water for the lot were provided by the supplier. Sampling devices were cleaned by field sampling teams in the SPL using Method ESP-900 (Environmental Surveillance Procedures, Kimbrough et al. 1988). Soil-contaminated tools were brought into the soils laboratory. They were first washed in tap water and a detrgent, then thoroughly rinsed with warm tap water. The tools were then carefully rinsed with SPL distilled water for a total of five rinses. The tools were given another five rinses with deionized distilled water and then wrapped while wet in one or more thicknesses of aluminum foil and placed in a cardboard box ready for transport to the field. An acid rinse and a solvent rinse called for in the above ESP-900 procedure was not applied to stainless steel field and laboratory equipment. A final deionized water rinse of the sampling devices was performed in the field prior to sampling. The effectiveness of the equipment cleaning and the potential contamination during sampling trips were monitored by submitting rinse water samples for analysis (five times by on-site and off-site sampling teams). The quality of the deionized and organic-free water used was monitored by collecting samples (once from on-site and off-site water sources) in standard precleaned sample containers and submitting them to the analytical laboratory for analysis.

\subsubsection{Maintenance and calibration of SPL balances, oven, refrigerator, and other equipment used in soil preparation activities}

The SPL balance was used to weigh soil for compositing, to obtain the weight of coarse fragments, and to determine moisture contents of soil samples. The electronic balance is recalibrated every 6 months. In use, the balance is zeroed before anything is placed on the pan. The weight is recorded after the balance stabilizes and an "OK" appears in the display window. The accuracy of the balance is verified by using a standard weight. In addition, a set of brass weights ranging from 1 to $2000 \mathrm{~g}$ is used to determine both accuracy and precision. This information was recorded in the BSCP laboratory notebook.

Temperature monitoring was done periodically of both the refrigerator and the ice chests used to cool soil samples in the field and for transfer to analytical laboratories. Temperature measurements made with a max/min thermometer indicated that a temperature range of $4^{\circ}$ $\pm 4^{\circ} \mathrm{C}$ can be maintained most of the time. The addition of several warm samples can, for a short period of time, raise the temperature above $8^{\circ} \mathrm{C}$. There were a few instances where 
a VOC trip blank was taken to the field with too much ice, resulting in partial freezing of the trip blank before soil samples were added to the ice chest. Temperature data were recorded in the laboratory notebook.

The oven in the SPL was monitored periodically to ensure that the drying temperature was maintained between 100 and $104^{\circ} \mathrm{C}$. These monitoring data are recorded in the laboratory notebook.

The deionized water used for sampling equipment rinsing was monitored periodically for conductivity. This information was put in the laboratory notebook.

\subsubsection{Maintenance and transfer of records}

Original records are maintained in the SPL (Building 1505, Room 375 at ORNL) for all BSCP ORR sampling activities. For University of Tennessee sampling activities, some original documents are kept there and copies in Room 375. Records are kept in a file cabinet with a list of contents. After each phase of the project has been completed and the data verified, copies were made of each document and the originals transferred to archived storage. Transfer was accomplished by a chain-of-custody procedure where the original documents to be transferred are listed individually. Copies remain in the SPL for reference and review.

\subsubsection{Management of noncontaminated waste in the SPL}

Waste generated in the SPL consists of emptied glass jars, excess soil over and above what was needed for compositing purposes, soil in gamma scan containers, soil in VOC. sample bottles returned from the Y-12 Plant VOC analytical laboratory, and blotting paper. Since none of the above waste materials contained any hazardous metals, organics, or radionuclides, disposal was done in the following manner. The plastic lids and teflon seals were removed from the glass jars and placed into a suitable trash container at the rear of Building 1505. The glass jars were placed in the glass dumpster at the rear of Building 1505. Blotting paper was placed into the waste container in Room 375 for removal by cleaning personnel. Excess soil was returned to the woods close to its origin and spread on the forest floor in a thin layer.

Should the SPL have any contaminated samples, they would be disposed of under laboratory standard operating procedures.

\subsection{FIELD QUALITY CONTROL EVALUATION}

There were three major objectives for achieving field quality control:

1. selection of representative sampling sites that were not disturbed by recent activities, including ORR facility activities or off-site activities such as farming operations or recreational uses that resulted in surface soil disturbance;

2. collection of representative samples and their transfer to analytical laboratories; and 
3. prevention of cross contamination at any site and cross contamination between sites, which included maintaining a complete chain of custody and detailed records of all field and laboratory compositing activities.

Any sign of recent (in the past 40 to 50 years) land disturbance or the presence of man-made organic compounds or radionuclides above global fallout levels would immediately result in a site being rejected. Potential sites were initially chosen on the basis of the lack of any recent land disturbance which, for most sites, was the presence of old-field successional forest. Nearly all of the sites had been cultivated and severely eroded prior to being abandoned or planted in pine trees on the ORR or allowed to revert back to forest on private lands.

Site screening included the following on ORR sites:

1. Sites were scanned for radiation. Any ground level reading above 100 counts per minute resulted in that site being rejected.

2. Selected sites were monitored for organics by an industrial hygienist while the pit was opened either for the first time or when the pit was reopened to collect additional samples.

3. Samples of each A horizon were collected for VOC analysis at all sites. Site screening at Roane County and Anderson County sites consisted of collecting VOA samples from all $A$ horizons. The BSCP Plan states that VOC analyses will be done according to EPA analytical Level II. Analytical laboratory data in the BSCP adhere to EPA Level IV methods, procedures, and documentation requirements. The Y-12 Laboratory utilized Level IV methodology and procedures in determining VOC levels, but since the results were to be used only for screening purposes to reject unacceptable sampling sites (by pre-activity), these results were required to be reported and documented to only Level II because more rigorous requirements were unnecessary.

Field quality levels ranged from data quality (DQ) Level II to DQ Level IV. However, in practice, DQ Level IV was adhered to throughout all field sampling activities, including screening samples for VOCs where samples were placed into precleaned glass containers. Field quality control procedures are listed in Sects. 6.6.1.3 to 6.6.1.9 of the BSCP Plan (Energy Systems 1992). The following discussion covers the procedures that were followed in collecting samples.

Prior to going to the field, all stainless steel sampling equipment was thoroughly washed with soap and water followed by a prescribed number of distilled water rinses. After the final rinse the equipment was wrapped with aluminum foil. The sampling equipment was taken to the field in the back of a pickup truck. At or near the site, the sampling equipment was unwrapped and given a field rinse, then immediately rewrapped until it was used. Some sites were located a considerable distance from the closest point of access. Here the rinsing was done at the truck, the equipment wrapped in aluminum foil, placed into a backpack, and carried to the site. A small pit was dug with a steel shovel deep enough to place the sample jar below the soil horizon that was to be sampled. A sampling tool was unwrapped and used to remove soil from the pit face directly into the jar. At no time did fingers touch a soil sample placed into a precleaned glass sample container. Soil that was pushed by the sampling 
tool beyond the mouth of the jar was discarded. Placing soil into the ESD gamma poly containers was the only exception to this rule. Placing the entire volume of soil into the gamma poly container required that the soil be packed into the lower restricted space with either the fingers or a freshly cut stick of a convenient diameter. After each soil horizon was sampled, a new sample tool was used to collect samples from the next soil horizon. All used stainless steel sampling tools were returned to the laboratory for standard cleaning, rinsing, and aluminum foil wrapping. Shovels that were used to open and fill pits were thoroughly cleaned between sites to prevent any cross contamination. In addition, soil removed from pits was placed outside the 3- by 3-meter sample area.

Each sample was given its own identification number in the field. This number and the description of each sample were first recorded in the field logbook. From the field logbook, sample container labels were filled out and placed on glass jars after the jar was filled. Each sample that was logged into the field logbook was then transcribed onto a field chain-ofcustody form, which was signed by all personnel involved in the sampling operation.

ESD laboratory operations for Phase I consisted of placing soil samples in a refrigerator, preparing laboratory chain-of-custody forms, packing samples into ice chests, and taking them to laboratory shipping. In the latter half of Phase I activities, preparation of laboratory chainof-custody forms and new container labels, packing, and shipping were done by Analytical Project Office personnel, according to Procedure BSCP-SOP-02, Rev. 0.

Compositing samples resulted in the destruction of the individual site A horizon, B horizon, and $C$ horizon samples and the creation of new composited samples. All of these activities were recorded in the ESD soils laboratory logbook. New sample numbers were first recorded in the laboratory logbook, then transcribed onto container labels and the appropriate chain-of-custody form.

The field variance system (Sect. 6.6.1.9 of the BSCP Plan, Energy Systems 1992) was not utilized in any Phase I activities. The necessity to use the field variance process (Sect. 6.6.1.9 of the BSCP Plan) did not arise during Phase I activities in this project.

\subsection{QUALITATIVE RESULTS OF GAMMA SPECTROSCOPY SCREENING}

The objective of the gamma screening was to determine if any of the Phase I sites had been affected by ORR facility activities in the past. Gamma spectroscopy indicates activities of several radionuclides in soils. Except for cesium-137 and other fallout radionuclides, several important natural radionuclides such as potassium-40, thorium, and radon-226 also occur in soils. Therefore, total cesium-137 activities in the upper $30 \mathrm{~cm}$ of soil profiles at each site were used as a screening parameter. If the potential site had a cesium-137 radioactivity level due to local sources much higher than regional background fallout level, it could be rejected as a sampling site if there was no obvious explanation from the site description. The average background level of Cs-137 for the southeastern United States is about $10 \mathrm{pCi} / \mathrm{cm}^{2}$ (discussed in Energy Systems 1992). However, soils located on a sediment depositional landform or from a soil with a thin colluvial or alluvial capping could have up to $14 \mathrm{pCi} / \mathrm{cm}^{2}$, and soils from erosional landforms could have much lower values. Soils located on a stable landform would be ideal for the BSCP. However, it was necessary to use some sites that were less desirable than the ideal, but which, in fact, represent the real world better as there are no ideal sites. 
In Phase I activities there were 12 ORR Dismal Gap sites, 12 ORR Nolichucky sites, 12 Roane County Dismal Gap sites, and 12 Anderson County Dismal Gap sites. Six gamma samples at 5-cm depth increments were collected from each site to a total depth of $30 \mathrm{~cm}$. The surface area sampled was $100 \mathrm{~cm}^{2}$ for each depth increment.

The gamma screening samples were counted on a high-resolution, solid state, coaxial, intrinsic, germanium detector coupled to an ND9900 multichannel analyzer with 4096 channels. The gamma system had previously been calibrated with a laboratory control sample (National Bureau of Standards SRM 4353 Rocky Flats Soil) in the geometry used to contain the soil samples. The documentation of analytical results was prepared at DQ Level II, but the analytical procedure used for the soil samples was DQ Level IV. For example, the laboratory control sample, laboratory blank, and duplicate counts were performed within a batch of $\mathbf{2 0}$ or fewer samples and documented. In addition, weekly and daily sources were counted to verify that the detectors remained in calibration.

Cesium-137 values were summed for the upper $30 \mathrm{~cm}$ of the soil profile (see Appendix B). Statistical analysis indicates that there are significant differences between ORR, Anderson, and Roane sites. Roane County sites have lower mean values, but this can be accounted for by present and recent past land use practices causing localized erosion in Roane County. One Roane site, No. 13 , had a total cesium- 137 value of $1.98 \mathrm{pCi} / \mathrm{cm}^{2}$. From looking at the soil profile description (Appendix A), there is a strong indication that this severely eroded site has only very recently beccusc stabilized with a forest litter layer, thus reducing surface erosion. Two Roane sites had high cesium-137 values compared to the expected average background level of approximately $10 \mathrm{pCi} / \mathrm{cm}^{2}$. Both sites had a surface capping of either colluvium or alluvium, a situation where there is lateral water and sediment movement and localized transport from higher areas. It should be pointed out that the source of cesium-137 is atmospheric deposition generally over the entire region in which the ORR and Roane and Anderson counties are located. In addition, the ORR has superimposed on it the contribution of cesium from sources within the ORR. There is no transport or movement of cesium or another constituent postulated or implied from these results between on-site and off-site sampling areas. One Anderson County site had the highest value, 14.424 $\mathrm{pCi} / \mathrm{cm}^{2}$. This site, AND-19, located on the lower part of a convex slope, has an overthickened surface horizon with about $13 \mathrm{~cm}$ of modern sediment overwash that contains considerable cesium. The ORR Dismal Gap data are slightly higher than the Anderson County and Roane County Dismal Gap data due to higher minimum values, which indicates a longer period of minimal disturbance for the ORR sites. The Nolichucky data have the highest mean values and also the highest minimum values. This is most likely due to the more gentle slope gradients that resulted in less lateral transport of particles downslope. The ORR sites are significantly different from the Roane and Anderson sites. Variations in the cesium-137 gamma screening data could be accounted for by past land use and by landform variability. 


\section{ANALYTICAL LABORATORY ANALYSES AND DATA VALIDATION}

\subsection{SUMMMARY OF DATA VALIDATION}

The data generated in Phase I of the Background Soil Characterization Project (BSCP) were validated according to project-specific validation guidelines. These guidelines were prepared according to the U.S. Environmental Protection Agency (EPA) Contract Laboratory Program (CLP) Validation Guidelines and the BSCP Project Plan (Energy Systems 1992). There were a total of 43 data packages received for Phase I ( 34 chemical and 9 radiological). The laboratories reported 6556 results with only a total of 395 results (6.0\%) being rejected by data validation (occurrences of rejected data appear in Appendix $\mathrm{H}$ ). The quality control (QC) problems observed in the chemical data validation consisted of calibration problems; blank spike, matrix spike (MS), and surrogate recoveries outside QC limits; and coelution problems. The major concern in the chemical data centered on the analysis of polynuclear aromatic hydrocarbons (PAHs). The analytical laboratory had problems related to the method, with only $74 \%$ of the data being usable. There were minor problems with herbicides and metals; $50 \%$ of the dalapon results and $71 \%$ of the osmium results were rejected. The problems encountered in the radiological data ranged from calibration problems to blank spike and MS recoveries outside of QC limits. Usability was lowest on two isotopes, technetium-99 and neptunium-237, for which virtually all data were rejected. The technetium-99 data were rejected because of the possibility of technetium loss during the muffle furnace step of the analytical procedure. The neptunium-237 results were rejected because of calibration errors and calculation errors in matrix spike/matrix spike duplicate (MS/MSD) and blank spike recoveries that upon correction yielded recoveries that were outside QC limits. The most prominent data gaps are being addressed through further evaluation in the case of neptunium-237 and through resampling with reanalysis to obtain replacement technetium-99 data. Lists of sample numbers belonging to each sample delivery group (SDG) are presented in Appendix F. Information on numbers of samples involved in these summary percentages is provided in Tables 4.2 through 4.6.

Lessons learned during the course of Phase I can be of benefit to future ER projects. The initial planning process focused on sampling, with a general idea of what analyses were required. Upon review of $\mathrm{QC}$ requirements and analytical methods required, the project had to re-evaluate the schedule and budget to address analytical needs. In addition, the BSCP was the first ER project to utilize fully the new Analytical Projects Office. The laboratories performing the work - the first large project they had received from Energy Systems-required a period of adjustment to Energy Systems requirements and needs. Many of the concerns that surfaced during the validation of Phase I data may be attributed to this learning period; however, there were some problems that Energy Systems might have been able to avert. A project-specific preaudit [with reference to the BSCP Project Plan (Energy Systems 1992) and the APO Statement of Work] of the laboratories, including review of the laboratories' procedures and quality assurance (QA) review process, would have been helpful. In addition, sending performance evaluation samples to the laboratory for each of the methods requested would have indicated what type of data packages each laboratory provides and demonstrated the laboratory's ability to perform sufficiently the requested analyses. For example, during the 
validation of the technetium-99 data, we requested a copy of the laboratory's procedure for analyzing technetium and discovered that the laboratory furnaced the samples at $500^{\circ} \mathrm{C}$. This temperature caused the rejection of the technetium data. A preaudit would have revealed the furnacing step of the procedure before the samples were shipped. Because no preaudit was performed, the project must now study the effects of muffle furnacing at high temperatures on the volatility of technetium in order to determine the acceptability and usefulness of the data.

\section{INTRODUCTION}

The overall objectives of this project include determining the background concentrations of selected organics, metals, and radionuclides in natural soil samples and providing validated data for use in remedial investigations and risk assessments. Specific objectives relative to analytical laboratory work are detailed in Sect. 8.3. These considerations directed the selection of analytical parameters for laboratory analyses.

The assumptions used to select the analytical parameters follow:

- Background concentrations of naturally occurring inorganic, organic, and radiological analytes of interest are those normally found in soils and sediments of natural origin. Contamination is indicated when these analytes are found in concentrations above the natural background. Analytes of interest include heavy metals, organic compounds, and radionuclides that are used in or generated by industrial, agricultural, and research activities associated with the Oak Ridge Reservation.

- Analytes not occurring naturally were assumed to have an a priori concentration equivalent to zero background, which would be below the analytical detection limits. Some of these analytes include man-made compounds, such as volatile organics and some semivolatile organics. Radionuclides were an exception because of nuclear activation and fission products that may have been added to the natural background by environmental transport processes, such as atmospheric deposition.

The analytical methodologies used for this project were consistent with EPA analytical Level IV. The EPA CLP procedures were used where appropriate, and EPA SW-846 (EPA 1991b) methods were used for the non-CLP parameters. Because the CLP contract-required detection limits were too high for this project, the laboratories adapted EPA SW-846 detection limits to their procedures.

Data validation is an important step in the overall data quality process. It is during the data validation process that the precision, accuracy, and completeness of the data received are assessed. Data validation provides the end users of the data with an overall quality assessment of the data. This assessment indicates whether there were any quality concerns, such as whether the laboratory was in control of its analytical process. The validators of the data qualify the data with respect to the criteria established by the project and give qualifiers to the data in relation to how the data meet the established criteria. The qualifiers given to the data indicate the potential usability of the data to the end user, but do not necessarily constitute the final conclusion on the overall usability of the data. The end users of the data need to consider the representativeness and comparability of the data and the ultimate use. 


\subsection{SELECTION OF LABORATORIES}

The laboratories selected to perform the analyses were

- selected and approved by the Analytical Projects Office through a rigorous, multi-point, competitive elimination process,

- capable of performing the requested analyses as stated in the project work plan, and

- low in cost.

The laboratories selected for the BSCP were Lockheed Analytical Services in Las Vegas, Nevada, for chemical analyses and EcoTek LSI in Atlanta, Georgia, for radiological analyses. Four laboratories responded to the statement of work that was issued (Project Sampling and Analysis Flan, Sect. 5.3, Energy Systems 1992), and these two were chosen based on their responses. Not all of the laboratories submitted prices for each analyte required for this project, so common analytes were selected, and a price comparison was performed. Of the laboratories submitting prices for the chemical portion of the project, only Lockheed provided pricing and availability for all requested parameters. Lockheed also had the lowest overall cost of the respor ling laboratories.

Only two laboratories submitted responses to the statement of work (SOW) for the radiological analyses. A comparison of the responses indicated that EcoTek was capable of performing the analyses at low cost.

\subsection{QUALITY ASSURANCE/QUALITY CONTROL}

Analytical QA and QC were conducted according to the requirements of the EPA CLP. The required analytical level defined by the EPA Data Quality Objectives (DQOs) document is Level IV (EPA 1987b). This level is characterized by rigorous QA/QC protocols and documentation. The pesticide/polychlorinated biphenyl (PCB) analyses were performed according to the EPA CLP Organics SOW, March 1990 (EPA 1990a). The metals analyses (except osmium) were performed according to the EPA CLP Inorganics SOW, March 1990 (EPA 1990b). All other analyses were performed under "CLP-like" procedures, with the minimum QC outlined in the Project Plan (Energy Systems 1992). Holding time requirements for these methods can be found in Table 6.4 of the BSCP Plan (Energy Systems 1992).

During this project there we:e some modifications to the analytical program specified in the BSCP Plan (Energy Systems 1992). The modifications and their effects on the project follow.

- The method for the volatile organic analysis was changed from EPA Method 8240 to EPA Method 8260 because the Y-12 Plant analytical laboratory uses a gas chromatographic system with a capillary column for separation instead of a column packed with graphitized carbon coated with carbowax (as required by Method 8240). This change did not affect the detection limits specified by the Project Plan.

- The analysis of nitrate was removed fro $\lrcorner$ the analy ical program because of the 24-h holding time. Compositing the samples de ayed shipment of the samples for a minimum 
of two days after collection, which meant that the nitrate holding time was already exceeded. Therefore, analyzing for nitrate would not have been valid.

- The Project Plan indicates that EPA 200.7 CLP-M be used for the preparation and analysis of silicon; however, in this project, silicon analysis is prepared according to EPA Method 3050 and analyzed according to EPA 200.7 CLP-M. This change does affect the recovery of silicon since ine preferred method is hydrogen fluoride digestion. The impact of this change will be lower recoveries of silicon, but the technical coordinator requested that all samples be prepared by nitric/hydrochloric acid digestion.

\subsubsection{Organic Data Qualifiers}

The following organic laboratory data qualifiers are used in this project.

\begin{tabular}{|c|l|}
\hline Qualifier & Explanation \\
\hline U & Indicates compound was analyzed for but not detected. \\
J & Indicates an estimated value. \\
N & $\begin{array}{l}\text { Indicates presumptive evidence of a compound [used only for tentatively } \\
\text { identified compounds (TICs)]. }\end{array}$ \\
P & $\begin{array}{l}\text { Used for pesticide/aroclor target analytes when there is greater than 25\% } \\
\text { difference for detected concentrations between the two gas chromatograph (GC) } \\
\text { columns. The lower of the two is reported and flagged. }\end{array}$ \\
C & $\begin{array}{l}\text { Use sr pesticide results where the identification has been confirmed by } \\
\text { GC/mass spectrograph. }\end{array}$ \\
B & $\begin{array}{l}\text { Used when the analyte is found in the associated blank as well as in the sample. } \\
\text { Identifies all compounds indicated a: a secondary dilution factor. }\end{array}$ \\
A & Indicates that a TIC is a suspected aldol-condensation product. \\
X & $\begin{array}{l}\text { Other specific flags may be used to properly define the results. If they are used } \\
\text { they must be fully described and attached to the Sample Data Package. }\end{array}$ \\
\hline
\end{tabular}




\subsection{Inorganic Data Qualifiers}

The following inorganic laboratory data qualifiers are used in this project.

\begin{tabular}{|c|c|}
\hline Qualifier & Explanation \\
\hline $\mathrm{U}$ & Indicates compound was analyzed for but not detected. \\
\hline $\mathbf{J}$ & Indicates an estimated value. \\
\hline $\mathbf{N}$ & Spiked sample recovery not within control limits. \\
\hline $\mathbf{E}$ & Reported value estimated because of the presence of interference. \\
\hline $\mathbf{M}$ & Duplicate injection precision not met. \\
\hline B & $\begin{array}{l}\text { Reported value was obtained from a reading that was less than the contract } \\
\text { required detection limit, but greater than or equal to the instrument detection } \\
\text { limit (IDL). }\end{array}$ \\
\hline $\mathbf{S}$ & Reported value was determined by the method of standard additions (MSA). \\
\hline $\mathbf{W}$ & $\begin{array}{l}\text { Post-digestion spike for furnace atomic absorption is out of control limits, while } \\
\text { sample absorbance is less that } 50 \% \text { of spike absorbance. }\end{array}$ \\
\hline * & Duplicate analysis not within control limits. \\
\hline+ & Correlation coefficient for the MSA is less than 0.995 . \\
\hline
\end{tabular}

\subsection{DATA VALIDATION}

The data validation for Phase I of this project was conducted by the K-25 Analytical Environmental Support Group (AESG), with assistance from the ORNL Measurement, Applications, and Development (MAD) Group. All sample data were delivered to the analytical coordinator from ORNLMAD, who had ultimate responsibility for the data throughout the validation process. ORNLMAD screened the data packages to ensure that project deliverables were provided, and K-25 AESG performed the technical review of the data.

The criteria for the data validation are outlined in the BSCP Plan (ES/ER/TM-26/R1). However, the project plan did not provide detailed requirements; therefore, project specific criteria had to be developed by the K-25 AESG (and presented in AESG's standard operating procedures SOPs). They were prepared according to the EPA CLP Validation Guidelines, as well as the validation guidelines outlined in the BSCP Plan.

The quality of the data validation process was ensured by a defined and documented process. Initially, the data package underwent a contract compliance check, during which it was screened for completeness of project deliverables. The validated data were given validation qualifiers that reflected the overall judgment of the data validator about the worthiness of each data point. The validation qualifiers used in this project are listed in Table 4.1. Second, the data were reviewed and evaluated against the project-specific data validation criteria. This second step was then assessed through a peer review, which examined the qualified data, checked the rationale of the professional judgments, and evaluated the 
reasonableness of the findings in light of the DQOs. The peer-reviewed data package was then reviewed by a third party who concentrated on the rationale and reasonableness of the qualifications. This extensive review and oversight process was designed to ensure that consistency was maintained throughout the project. Upon completion of the validation, a report was issued. A summary of the findings is presented below.

\subsection{Organic Data Validation Results}

\subsubsection{Pesticide/PCB validation results}

The analysis of pesticide/PCB samples was performed according to the USEPA Contract Laboratory Program Statement of Work for Organic Analysis, Multi-Media, Multi-Concentration, March 1990. There were 60 samples analyzed for the pesticide/PCB compounds listed in the SOW. However, only 59 samples were analyzed for the following compounds: gamma-chlordane, aldrin, all aroclor compounds, endosulfan sulfate, endosulfan II, endrin, endrin aldehyde, endrin ketone, methoxychlor, toxaphene, 4,4'-DDE, and 4,4'-DDT. There were only 58 sample results reported for aroclor-1242. The reason for not reporting a result for all compounds is being actively investigated. The results of this investigation will be provided in the project final report.

Holding times. Both the extraction and analysis holding times were met for all samples, except samples in SDGs 0514260 and 0727260 . Samples in SDG 0514260 were re-extracted outside of the extraction holding time, thus qualifying the data as estimated (J). The extraction holding time for samples in SDG 0727260 was exceeded by one day, so the data were qualified as estimated (J). (SDGs are related to actual sample numbers in Appendix F.)

$G C / E C D$ instrument performance. The frequency and sequence of the resolution check mixture and the performance evaluation mixtures (PEMs) were evaluated.

1. A resolution check mixture was analyzed at the beginning of every initial calibration sequence on each GC column and instrument used for analysis.

2. The depth of the valleys between two adjacent compounds (dieldrin and DDE) in the resolution check mixture could not be verified as being $\geq 60 \%$ of the height of the shorter peak Dieldrin and DDE were qualified as estimated (J) in SDGs 0523260, 0508260, 0511260, 042260, 0424260, 0430260, 0514260, 0519260, .

3. A PEM was analyzed at the beginning and end of each initial calibration sequence and at the beginning of every other 12-hour analytical sequence.

4. Adjacent peaks in the PEM were reviewed and appeared to be $100 \%$ resolved for all compounds except beta-BHC and gamma-BHC on one column. Retention times were within the specified retention time windows. Beta-BHC and gamma-BHC were qualified as estimated (J) for SDG 0523260, 0508260, 0511260, 0430260, 0514260, 0519260.

5. The relative percent difference (RPD) between the calculated amount and the true amount for each of the single component pesticides and surrogates in the PEMs were $\leq 25 \%$ for all target compounds except

- 4, 4'-DDT, qualified as estimated (J) in SDG 0523260;

- beta-BHC, qualified as estimated (J) in SDG 0508260;

- beta-BHC and methoxychlor in sample 3072 of SDG 0511260 , qualified as estimated (J); 
Table 4.1. Definition of data validation qualifiers

\begin{tabular}{|c|c|}
\hline Qualifier & Definition \\
\hline $\mathbf{U}$ & $\begin{array}{l}\text { The analyte was analyzed for but was not detected above the } \\
\text { reported sample quantitiation limit. }\end{array}$ \\
\hline $\mathbf{J}$ & $\begin{array}{l}\text { The analyte was positively identified; the associated numerical } \\
\text { value is the approximate concentration of the analyte in the } \\
\text { sample. }\end{array}$ \\
\hline $\mathbf{N}$ & $\begin{array}{l}\text { The analysis indicates the presence of an analyte for which } \\
\text { there is presumptive evidence to make a tentative } \\
\text { identification. }\end{array}$ \\
\hline $\mathbf{N}$ & $\begin{array}{l}\text { The analysis indicates the presence of an analyte that has } \\
\text { been tentatively identified, and the associated numerical } \\
\text { value represents its approximate concentration. }\end{array}$ \\
\hline UJ & $\begin{array}{l}\text { The analyte was not detected above the reported sample } \\
\text { quantitation limit. However, the reported quantitation limit } \\
\text { is approximate and may or may not represent the actual limit } \\
\text { of quantitation necessary to accurately and precisely measure } \\
\text { the analyte in the sample. }\end{array}$ \\
\hline $\mathbf{R}$ & $\begin{array}{l}\text { The sample results are rejected because of serious } \\
\text { deficiencies in the ability to analyze the sample and meet } \\
\text { quality control criteria. The presence or absence of the } \\
\text { analyte cannot be verified. }\end{array}$ \\
\hline UN & $\begin{array}{l}\text { The laboratory did not register this compound, but there was } \\
\text { presumptive evidence of a compound that was within the } \\
\text { retention time window but was not reported. No other } \\
\text { qualification of the data was made. }\end{array}$ \\
\hline UNN & $\begin{array}{l}\text { The laboratory did not report the compound, but there was } \\
\text { presumptive evidence of a compound that was within the } \\
\text { retention time window but was not reported. The data were } \\
\text { qualified as estimated, J, because of other discrepancies with } \\
\text { the data. }\end{array}$ \\
\hline $\mathbf{R N}$ & $\begin{array}{l}\text { The laboratory did not report the compound, but there was } \\
\text { evidence of a compound that was within the retention time } \\
\text { window but was not reported. The data were qualified as } \\
\text { unusable, } R \text {, because of other discrepancies with the data. }\end{array}$ \\
\hline
\end{tabular}


- alpha-BHC, qualified as estimated (J) in samples $1064,1072,1080$, and 3003 of SDG 042260;

- beta-BHC and methoxychlor, qualified as estimated (J) in sample 3018 of SDG 042260;

- alpha-BHC, qualified as estimated (J) in samples 1099 and 1106 of SDG 0424260;

- beta-BHC and methoxychlor, qualified as estimated (J) in samples 1107, 1108, and 1115 of SDG 0424260 ;

- beta-BHC and methoxychlor, qualified as estimated (J) in samples 1127 and 3032 of SDG 0430260;

- 4, 4'-DDT, qualified as estimated (J) for SDG 0722260;

- beta-BHC, qualified as estimated (J) in SDG 0727260; and

- beta-BHC, qualified as estimated (J) in SDG 0803260.

Initial and verification calibration. Results on initial calibration and calibration verification forms were examined to ensure that reported results met required QC criteria.

1. Individual standard mixtures $A$ and $B$ contained all of the single component compounds and surrogates and were analyzed at low, midpoint, and high concentrations during the initial calibration on each GC column and instrument used for analysis.

2. Adjacent peaks in the individual standard mixtures were reviewed and appeared to be at least $90 \%$ resolved for all target compounds.

3. Retention times reviewed were within the specified retention time windows. Endosulfan I and alpha-BHC had almost the same retention time window, which qualified the data as estimated (J) in SDG 0508260.

4. All percent relative standard deviation (\%RSD) results for the calibration factors met the QC criterion of $520 \%$ for target compounds, with the exception of

- alpha-BHC, qualified as estimated (J) in SDG 0803260 and 0727260;

- 4, 4'-DDT, qualified as estimated (J) in SDG 0722260;

- alpha-BHC, delta-BHC, 4, 4'-DDD and 4, 4'-DDE, qualified as estimated (J) in SDGs 0519260 and 0508260 ;

- alpha-BHC, 4, 4'-DDD and 4, 4'-DDE, qualified as estimated (J) in SDG 0430260 and sample 3072 of SDG 0511260;

- alpha-BHC and endrin aldehyde, qualified as estimated (J) in samples 1099 and 1106 of SDG 0424260 and samples 1064, 1072, 1080, and 3003 of SDG 042260;

- alpha-BHC, 4, 4'DDE, 4, 4'-DDD, and 4, 4'-DDT, qualified as estimated (J) in samples 1107, 1108, and 1115 of SDG 0424260 and sample 3018 of SDG 042260;

- alpha-BHC, delta-BHC, gamma-BHC, 4, 4'-DDD and 4, 4'-DDE, qualified as estimated (J) in samples 3058, 3099, and 3085 of SDG 0511260; and

- alpha-BHC, gamma-BHC, 4, 4'-DDD, 4, 4'-DDT, and endrin aldehyde, qualified as estimated (J) in SDG 0523260.

5. Surrogates met the criterion of $\leq 30 \%$ RSD.

6. A single concentration calibration standard was analyzed for multicomponent compounds.

7. All RPDs between calculated and nominal amounts for each target compound and surrogate in the midpoint continuing calibration concentrations met the QC criterion of $\leq 25 \%$, with the exception of

- aldrin, which was qualified as estimated (J) in SDG 0430260 and 
- delta-BHC, heptachlor, and 4, 4'-DDD, which were qualified as estimated (J) in SDG 0523260.

Laboratory blanks. Samples were extracted with a method blank, and an instrument blank was run immediately prior to analysis of either a PEM or an individual continuing calibration midpoint standard mixture. The was no significant contamination found in the blanks, with the exception of PBBLK02 of SDG 0514260. PBBLK02 was found to contain aroclor-1242, which was also identified in two of the samples. Therefore, samples 3046 and 3148 were qualified as nondetected $(U)$ since the concentration of the samples was less than five times the concentration found in the associated blanks.

Surrogates. All surrogates were within the 60-150\% QC limits, with the following exceptions:

- sample 3058 of SDG 0511260, all target compounds in this sample were qualified as estimated (J);

- sample 3018 of SDG 042260, no qualification was necessary because all surrogates were outside the limits on the high side and no target compounds were detected;

- sample 3113 of SDG 0514260, all target compounds in this sample were qualified as estimated (J);

- some surrogates for SDG 0722260 were outside the QC limits. Sample 2130 showed a TCMX recovery of $175 \%$. Samples 2090 and 2143 showed one recovery of DCB below the minimum QC criterion of $60 \%$ and sample 2149 showed DCB recoveries less than the QC criterion of $60 \%$ on both columns; therefore, late eluters (those eluting within 10 min of the DCB surrogate) were qualified as estimated (J) in sample 2149;

- samples 2179 and 1462 of SDG 0727260 showed recoveries of DCB of less than the QC criterion of $60 \%$ on both columns; therefore, late eluters (those eluting within $10 \mathrm{~min}$ of the DCB surrogate) were qualified as estimated (J) in samples 1462 and 2179;

- SDG 0727260 showed recovery of DCB less than the QC criterion of $60 \%$ on both columns; therefore, late eluters (those eluting within 10 min of the DCB surrogate) were qualified as estimated (J) in this SDG.

Matrix spike/matrix spike duplicates. Results were checked to ensure that they met the required QC criteria. MS/MSD data are not used to qualify data alone. All MS/MSD recoveries were within $\mathrm{QC}$ limits, with the exception of the following:

- some MS/MSD recoveries exceeded the QC limit of $150 \%$. However, there was no qualification of the data because no target compounds were found in the samples. This was found in SDGs 0727260 and 0803260 ;

- Endrin failed to be recovered in the MS of SDG 0523260 and was poorly recovered in the matrix spike duplicate (MSD). However, since there were no problems with recovery and breakdown of endrin in the standards and PEMs, there was no qualification of the data.

Overall assessment. The laboratory did not always adhere to CLP protocol. In particular,

- Extract volumes were condensed to $4 \mathrm{~mL}$ instead of $10 \mathrm{~mL}$.

- Only $1 \mathrm{~mL}$ of MS/MSD solutions was added to samples instead of the required $2 \mathrm{~mL}$. 
- Chromatograms for standards were noncompliant (less than $10 \%$ full scale for single component compounds and less than $25 \%$ full scale for multicomponent compounds).

- The florisil cartridge check and cleanup were not performed as required.

- Target compounds were detected on both columns above the detection limit, but below the contract required quantitation limit; however, they were not reported on Form $1 \mathrm{s.}$

- $\quad$ All pesticide/PCB data were determined to be usable.

- A summary of the pesticide/PCB data validation results is presented in Table 4.2.

Table 4.2. Summary distribution of pesticide/PCB data validation results

\begin{tabular}{|c|c|c|c|c|c|c|}
\hline Compound & $\mathbf{U}$ & UJ & $\mathbf{P}$ & $\mathbf{J}$ & Sum & $\begin{array}{c}\text { Percent } \\
\text { usable }\end{array}$ \\
\hline $\begin{array}{l}\text { Alpha-BHC } \\
\text { Beta-BHC } \\
\text { Delta-BHC } \\
\text { Gamma-BHC (Lindane) }\end{array}$ & $\begin{array}{l}15 \\
19 \\
28 \\
10\end{array}$ & $\begin{array}{l}45 \\
41 \\
32 \\
50\end{array}$ & & & $\begin{array}{l}60 \\
60 \\
60 \\
60\end{array}$ & $\begin{array}{l}100 \% \\
100 \% \\
100 \% \\
100 \%\end{array}$ \\
\hline $\begin{array}{l}\text { Gamma-chlordane } \\
\text { Aldrin } \\
\text { Aroclor } 1016 \\
\text { Aroclor } 1221\end{array}$ & $\begin{array}{l}52 \\
34 \\
52 \\
52\end{array}$ & $\begin{array}{c}8 \\
26 \\
8 \\
8\end{array}$ & & & $\begin{array}{l}60 \\
60 \\
60 \\
60\end{array}$ & $\begin{array}{l}100 \% \\
100 \% \\
100 \% \\
100 \%\end{array}$ \\
\hline $\begin{array}{l}\text { Aroclor } 1232 \\
\text { Aroclor } 1242 \\
\text { Aroclor } 1248 \\
\text { Aroclor } 1254\end{array}$ & $\begin{array}{l}52 \\
49 \\
52 \\
52\end{array}$ & $\begin{array}{l}8 \\
8 \\
8 \\
8\end{array}$ & 1 & & $\begin{array}{l}60 \\
58 \\
60 \\
60\end{array}$ & $\begin{array}{l}100 \% \\
100 \% \\
100 \% \\
100 \%\end{array}$ \\
\hline $\begin{array}{l}\text { Aroclor } 1260 \\
\text { Dieldrin } \\
\text { Endonsulfan I } \\
\text { Endonsulfan II }\end{array}$ & $\begin{array}{c}52 \\
2 \\
47 \\
52\end{array}$ & $\begin{array}{c}8 \\
58 \\
13 \\
8\end{array}$ & & & $\begin{array}{l}60 \\
60 \\
60 \\
60\end{array}$ & $\begin{array}{l}100 \% \\
100 \% \\
100 \% \\
100 \%\end{array}$ \\
\hline $\begin{array}{l}\text { Endonsulfan sulfate } \\
\text { Endrin } \\
\text { Endrin aldehyde } \\
\text { Endrin ketone }\end{array}$ & $\begin{array}{l}48 \\
52 \\
42 \\
49\end{array}$ & $\begin{array}{c}11 \\
8 \\
17 \\
11\end{array}$ & & & $\begin{array}{l}59 \\
60 \\
59 \\
60\end{array}$ & $\begin{array}{l}100 \% \\
100 \% \\
100 \% \\
100 \%\end{array}$ \\
\hline $\begin{array}{l}\text { Heptachlor } \\
\text { Heptachlor epoxide } \\
\text { Methoxyclor } \\
\text { Toxaphene }\end{array}$ & $\begin{array}{l}12 \\
52 \\
42 \\
52\end{array}$ & $\begin{array}{c}48 \\
8 \\
18 \\
8\end{array}$ & & & $\begin{array}{l}60 \\
60 \\
60 \\
60\end{array}$ & $\begin{array}{l}100 \% \\
100 \% \\
100 \% \\
100 \%\end{array}$ \\
\hline $\begin{array}{l}\text { Alpha-chlordane } \\
4,4^{\prime}-\text { DDD } \\
4,4^{\prime}-\text { DDE } \\
4,4^{\prime}-\text { DDT }\end{array}$ & $\begin{array}{l}47 \\
23 \\
17 \\
20\end{array}$ & $\begin{array}{l}13 \\
37 \\
43 \\
39\end{array}$ & & 1 & $\begin{array}{l}60 \\
60 \\
60 \\
60\end{array}$ & $\begin{array}{l}100 \% \\
100 \% \\
100 \% \\
100 \%\end{array}$ \\
\hline
\end{tabular}




\subsubsection{Chlorinated herbicide validation results}

The analysis of chlorinated herbicide samples was performed according to the USEPA $S W-846$ Method 8150, Second Edition, with the QC performed in a "CLP-like" manner. There were 24 samples analyzed for the chlorinated herbicide compounds.

Holding times. All holding times were met for both extraction and analysis.

Initial and verification calibration. Some of the chlorinated herbicides were found to be outside the QC limits $\left(r^{2} \geq 0.990\right)$. The data were qualified by reviewing the exceedance of the QC limits in regard to other problems encountered during the validation.

- In SDGs 0508260 and 0511260 , the data were qualified as nondetected (U) because dalapon, dichloroprop, dinoseb, and the surrogate 2, 4 dichlorophenylmethylacetate were outside QC limits. However, there were no compounds detected in the samples, and the second column values were within QC limits, with the exception of dalapon. Since dalapon failed the QC criteria on both columns, this compound was qualified as estimated nondetected (UJ) for all samples except 1213.

- In SDGs 0803260 and $0727260 / 0728260 / 0729260$, the data were qualified as nondetected $(U)$ because 2, 4-DB was outside the $Q C$ limits on one column, while dinoseb and the surrogate 2, 4 dichlorophenylmethylacetate were outside the limits on the second column. Since no compounds were detected in the samples and since the compounds met the QC criteria on at least one column, the data were qualified nondetected.

- The data in SDG 0430260 were qualified because dalapon and MCPA, 2, 4-dichlorophenyl-methylacetate were outside QC limits on both columns and 2, 4-DB was outside on one column and dichloroprop was outside on the other column. Another initial calibration should have been run because of the failure of the surrogate on both columns. Therefore, all data are qualified estimated nondetected (UJ) because the surrogate value was not within the QC limits. Dalapon was rejected (R) because of its gross failure of the QC criteria.

- Dalapon in SDG 0424260 was rejected because it was found to be significantly outside the QC limits.

- All calibration verifications were run under the initial calibration with the exceptions of SDGs 042260 and 0424260 . Dalapon was rejected (R) in SDG 042260 because it failed the QC limit (\%D $\leq 15 \%$ ), while dichloroprop, dinoseb, and 2, 4-DB were qualified estimated nondetected (UJ).

- Dicamba, MCPP and 2, 4-D were qualified estimated nondetected (UJ) because they were found outside the QC limits (\%D $\leq 15 \%)$.

Laboratory blanks. There were no significant contamination problems found except for SDG 0430260 . In this SDG, the laboratory experienced a contamination problem and diluted all the samples and QC samples by a factor of $1: 10$.

Surrogates. All surrogate recoveries were found within the QC criteria of 50-150\%, with the exception of some samples within SDGs 0430260 (1064, 1080, 1127, and 3032), 0424260 (1099, 1106, 1107, and 1115), 0511260 (3046 and 3072), and 0508260 (1201-FD). Samples that had surrogate recoveries outside the QC limits on both columns and no detects reported were qualified as estimated nondetected (UJ). However, if surrogate recoveries were less than $10 \%$ on both columns, the data were rejected (R). 
Laboratory control samples. All samples met requirements for laboratory control sample (LCS) recoveries except for the following:

- Silvex and 2, 4, 5-T had LCS recoveries that were slightly outside the QC limits; therefore, data for SDGs 0508260 and 0511260 were qualified as estimated (J).

- All samples of SDG 0424260 were qualified estimated nondetected (UJ) because the LCS recoveries were outside QC limits.

- All data in SDGs 0803260 and $0727260 / 0728260 / 0729260$ were qualified estimated nondetected (UJ) because no LCS was provided. The reason for this omission is under investigation, and an explanation for the omission will be provided in the project final report.

Overall assessment. The overall performance of the laboratory was acceptable, but the following problems were noted:

- Initial calibration information was not provided for SDG 0424260;

- There were contamination problems with some of the SDGs, and the laboratory had to dilute some samples by a factor of $1: 20$;

- An improper amount of soil was used. The proper amount was $50 \mathrm{~g}$, but the laboratory used $25 \mathrm{~g}$ in some of the SDGs;

- Verification of practical quantitation limits (PQLs) was not possible because the information was not provided;

- All chlorinated herbicide data were determined to be usable with the exception of dalapon (50\% usable); and

- A summary of the chlorinated herbicide data validation results is presented in Table 4.3.

Table 4.3. Summary distribution of chlorinated herbicide data validation results

\begin{tabular}{|c|c|c|c|c|c|c|c|c|c|c|c|}
\hline Compound & $\mathbf{U}$ & UJ & $\mathbf{J}$ & JN & $\mathbf{R}$ & $\mathbf{R N}$ & UN & UJN & $\mathbf{P}$ & Sum & $\begin{array}{c}\text { Percen } \\
\text { usable }\end{array}$ \\
\hline $\begin{array}{l}\text { Dalapon } \\
\text { Dicamba } \\
\text { Dichloroprop } \\
\text { Dinoseb } \\
\text { MCPA }\end{array}$ & $\begin{array}{l}5 \\
5 \\
5 \\
5\end{array}$ & $\begin{array}{l}12 \\
19 \\
19 \\
19 \\
19\end{array}$ & & & 12 & & & & & $\begin{array}{l}24 \\
24 \\
24 \\
24 \\
24\end{array}$ & $\begin{array}{l}50 \\
100 \\
100 \\
100 \\
100\end{array}$ \\
\hline $\begin{array}{l}\text { MCPP } \\
\text { Silvex } \\
2,4-D \\
2,4-D B \\
2,4,5-T\end{array}$ & $\begin{array}{l}5 \\
5 \\
5\end{array}$ & $\begin{array}{l}19 \\
24 \\
19 \\
19 \\
24\end{array}$ & & & & & & & & $\begin{array}{l}24 \\
24 \\
24 \\
24 \\
24\end{array}$ & $\begin{array}{l}100 \\
100 \\
100 \\
100 \\
100\end{array}$ \\
\hline
\end{tabular}

\subsubsection{Polynuclear aromatic hydrocarbons}

The analysis of PAH samples was performed according to the USEPA SW-846 Method 8310, Second Edition, with the QC performed in a "CLP-like" manner. There were 65 samples analyzed for the PAH compounds, with the following exceptions: 64 samples for 
anthracene, 63 samples for benzo[b]fluoranthene, and 66 samples for phenanthrene. These exceptions are being investigated, and the results will be contained in the project final report.

Holding times. All samples met established holding times except for those associated with SDG 0722260. These samples were re-extracted 14 days outside of the extraction holding times; therefore, all detected results were estimated $(J)$, and nondetected results were qualified estimated nondetected (UJ).

Initial and verification calibration. The initial calibration is assessed by the review of the data against the correlation coefficient. The QC limit for the correlation coefficient is $r^{2} \geq$ 0.990 .

- Benzo[a]anthracene and chrysene for SDGs 0422260, 0424260, 0430260, 0508260, $0511260,0514260,0519260,0722260$, and $0722260 / 0723260$ were found to coelute and were qualified as unusable (R) for all positive hits because it was impossible to distinguish one from the other and as nondetected $(U)$ for results less than reporting limits.

- Anthracene and acenaphthene for SDGs 0422260 and 0424260 exceeded the initial calibration QC limits and were qualified estimated $(J)$ for positive hits and estimated nondetected (UJ) for nondetects.

- Pyrene and decafluorobiphenyl (the surrogate) for SDGs 0508260 and 0511260 exceeded the initial calibration QC limits so all pyrene data were flagged as estimated (J) for detects and estimated nondetected (UJ) for nondetects. All other data must be estimated (J) because of the coelution of the surrogate with a target compound.

- The surrogate, decafluorobiphenyl, and fluroanthene coelute; therefore, detected fluoranthene results in SDG 0511260 were qualified as unusable (R). All other data must be estimated $(J)$ because of the coelution of the surrogate with a target compound.

- Positive hits for fluoranthene were qualified unusable (R) because decafluorobiphenyl and fluroanthene coelute. All other data must be estimated (J) because of the coelution of the surrogate with a target compound.

- Decafluorbiphenyl and benzo[a]anthracene/chrysene for SDG 0523260 exceeded the initial calibration QC limits. All data were estimated (J) for detected compounds and estimated nondetected for nondetected compounds.

- Benzo[g,h,i]perylene coelutes with dibenzo[ $a, h]$ anthracene; therefore, results for SDGs 0727260,0727260 , and 0803260 for these two compounds must be qualified as unusable (R) because the laboratory could not quantify the MS/MSDs and LCS recoveries for dibenzo $[a, h]$ anthracene.

- Anthracene, benzo[k]fluoranthene, benzo[ $a]$ pyrene, and benzo $[g, h, i]$ perylene/ dibenzo $[a, h]$ anthracene for SDG 0727260 exceeded initial calibration QC limits, so detected results for anthracene and benzo $[k]$ fluoranthene were qualified as estimated $(J)$ and estimated nondetects for nondetected results of these compounds. Because benzo $[a]$ pyrene is only slightly below criteria (0.9891), it was not qualified.

- Anthracene, benzo[k]fluoranthene, benzo[a]pyrene, and benzo[g,hi]perylene/ dibenzo[a,h]anthracene for samples 1458 and 1464 of SDG 0803260 exceeded initial calibration QC limits, so detected results for anthracene and benzo[ $k]$ fluoranthene were qualified as estimated $(J)$ and estimated nondetects for nondetected results of these compounds. Because benzo[a]pyrene is only slightly below criteria (0.9303), it was not qualified. 
The verification of the calibration was assessed by determining the percent difference of the verification calibration result sample to the initial calibration result. All verification analyses were within the QC criteria (\%D $\leq 15 \%$ ) except the following:

- Benzo[k]fluroanthene, indeno-1,2,3(c,d)-pyrene, benzo[a]pyrene, and benzo[g, $h, i]$ perylene for samples 1099, 1106, 1107, 1108, 1115, and 3018 of SDG 0422260 exceeded QC limits, and detected compounds were qualified as estimated (J), and nondetected compounds were qualified as estimated nondetect (UJ).

- Naphthalene, acenaphthalene, fluorene, phenanthrene, fluoranthene, indeno-1,2,3(c,d)-pyrene, benzo[k]fluroanthene, and benzo[g,h,i]perylene for SDGs 0430260, 0508260, and 0511260 exceeded QC limits, and detected compounds were qualified as estimated $(\mathrm{J})$, and nondetected compounds were qualified as estimated nondetect (UJ).

- Naphthalene, acenaphthalene, fluorene, phenanthrene, fluoranthene, indeno-1,2,3(c,d)-pyrene, benzo $k]$ fluroanthene, anthracene, pyrene, benzo[gh, $i]$ perylene, and benzo[a]pyrene for SDGs 0514260 and 0519260 exceeded QC limits, and detected compounds were qualified as estimated (J), and nondetected compounds were qualified as estimated nondetect (UJ).

- Naphthalene, acenaphthalene, fluorene, phenanthrene, fluoranthene, indeno-1,2,3(c,d)-pyrene, benzo[k]fluroanthene, anthracene, pyrene, benzo[ $a]$ anthracene/chrysene, and benzo $[a]$ pyrene for SDG 0523260 exceeded QC limits, and detected compounds were qualified as estimated $(J)$, and nondetected compounds were qualified as estimated nondetect (UJ).

- Benzo[k]fluoranthene for SDG 0722260 exceeded QC limits, and detected compounds were qualified as estimated (J), and nondetected compounds were qualified as estimated nondetect (UJ).

- Benzo[k]fluoranthene, dibenzo[a,h]anthracene, and benzo[g,h,i]perylene for SDGs 0727260 and 0803260 were qualified as estimated $(J)$, and nondetected compounds were qualified as estimated nondetect (UJ).

Laboratory blanks. There were no extensive contamination problems found. However, in SDGs 0422260 and 0424260 , the laboratory experienced a contamination problem and diluted all the samples and QC samples by a factor of $1: 100$ and $1: 10$, respectively. Because of this problem, all samples were estimated (J) for detected compounds and estimated nondetect for nondetected compounds.

Surrogates. All surrogate recoveries were found within the QC criteria of 50-150\%, with the exception of the following:

- Surrogate recoveries were below $10 \%$ for SDG 0422260 ; therefore, all positive results were qualified as estimated $(\mathrm{J})$, and all nondetected compounds were qualified as estimated nondetect (UJ).

- Surrogate recoveries were reported outside the QC limits for all samples except sample 1099 of SDG 0424260. All results except for sample 1099 were qualified as JN for detected compounds and UJN for nondetected compounds. The $\mathrm{N}$ qualification was added because of the laboratory's inability to properly integrate the surrogate peak.

- Decafluorobiphenyl had a $0 \%$ recovery for sample 1213 in SDG 0508260 , so all nondetects were rejected (R), and all positive results were estimated (J). 
- Decafluorobiphenyl had extremely high values for samples 1190 and 1201 of SDG 0508260 , so all positive results were estimated $(\mathrm{J})$, and all nondetects were estimated nondetects (UJ).

- All results for SDG 0511260 were qualified as J (detects) and UJ (nondetects), and nondetect results for sample 3099 were qualified unusable $(R)$ because a surrogate recovery of $0 \%$ was reported.

- Surrogate recoveries were outside of QC limits for SDG 0514260, so all positive results were estimated (J), and all nondetects were estimated nondetects (UJ).

- Surrogate recoveries were outside of QC limits for samples 3148 and 3168 of SDG 0519260 , so all positive results were estimated $(J)$, and all nondetects were estimated nondetects (UJ).

- Surrogate recoveries were outside of QC limits for all samples of SDG 0523260 except samples 1293, 1295, 1300, and 1301, so all positive results of the samples outside of QC limits were estimated (J), and all nondetects were estimated nondetects (UJ).

- Surrogate recoveries were outside of QC limits for samples 2039, 2143, 2130, and 2059 of SDG 0722260 so all positive results were estimated (J), and all nondetects were estimated nondetects (UJ).

- All samples in SDG 0722260/0723260 exceeded the surrogate QC limits; therefore, all positive results of the samples outside of QC limits were estimated (J) and all nondetects were estimated nondeterts (UJ). Sample 2080 had a surrogate recovery below $10 \%$, so all positive results were estimated (J), and nondetects were rejected (R).

Matrix spike/matrix spike duplicates. Reported results were checked to ensure that they met the required QC criteria. MS/MSD data are not used to qualify data alone. All MS/MSD recoveries were within QC limits, with the exception of the following:

- SDGs 0422260 and 0424260 had MS recoveries for naphthalene and acenaphthylene of $0 \%$.

- SDGs 0508260, 0511260 had MS recoveries for naphthalene, acenaphthylene, phenanthrene, fluorene, and acenaphthene of $0 \%$.

- SDG 0514260 had MS recoveries for fluorene of $0 \%$. Naphthalene was reported at twice the amount spiked.

- SDG 0523260 had MS recoveries for naphthalene, acenaphthylene, fluorene, and acenaphthene MSD of $0 \%$.

- All results for dibenzo[a,h]anthracene and benzo[g,h,i]perylene of SDG 0722260 were rejected $(R)$ because these two compounds coelute.

- SDGs 0727260 and 0803260 had MS recoveries for anthracene and dibenzo $[a, h]$ anthracene MS/MSD of $0 \%$.

Laboratory control samples. All samples met requirements for LCS recoveries except for the following:

- An LCS was not provided in SDG 0422260; therefore, all the data were qualified as estimated (detects) and UJ (nondetects).

- The LCS for SDG 0424260 was diluted 1:10, indicating a problem. Because of this problem, samples in this SDG were estimated J (detects) or UJ (nondetects).

- Fluorene results for SDG 0511260 were estimated J (detects) or UJ (nondetects) because LCS recoveries for fluorene were outside QC limits (D-142\%). 
- Acenaphthylene results for SDG 0523260 were estimated J (detects) or rejected R (nondetects) because a $0 \%$ LCS recovery was reported.

Overall assessment. There were three major problems identified with the PAHs: coelution, compound identification, and reporting of diluted and undiluted samples. The conditions used by the laboratory for Method 8310 resulted in coelution problems. Initially, the laboratory was using decafluorobiphenyl as a surrogate, which coeluted with fluoranthene. The laboratory also experienced coelution problems with benzo[a]anthracene and chrysene because a change of conditions took place after June 1,1992, including a change of surrogates to 2-fluorobiphenyl. Coelution problems were resolved for the surrogate and fluoranthene and for benzo[a]anthracene and chrysene; however, this led to a coelution problem between benzo[g,h,i]perylene and dibenzo[ $a, h]$ anthracene.

There were several identification problems with the PAHs. The laboratory did not consistently report compounds. The laboratory's method of determining retention time windows and their criteria for determining whether a compound is inside or outside the retention time window is not consistent. For samples experiencing this problem, the compounds were qualified as $\mathrm{N}$, because there was presumptive evidence of the compound. The laboratory was contacted to address this issue.

The laboratory does not consistently perform dilutions when a compound exceeds the initial calibration linear range. When dilutions are performed, the laboratory reports both the diluted and undiluted samples on the same Form 1s. The laboratory was found to report sample results at the PQL even though a positive hit was found in the undiluted sample, while it was not found in the diluted sample. The laboratory did not provide rationale for these practices, and the project is actively pursuing a resolution to these reporting discrepancies. The $\mathbf{N}$ qualifier was again used in these cases because the validator felt that there was presumptive evidence of a compound. A summary of the PAH data validation results is presented in Table 4.4.

\section{5 .2 Inorganic data validation results}

The analysis of inorganic species was performed according to the USEPA Contract Laboratory Program Statement of Work for Inorganic Analysis, Multi-Media, Multi-Concentration, July 1987. The analytes that are not governed under this SOW are osmium and sulfate, which were analyzed using a "CLP-like" SW-846 method with a QC protocol similar to CLP. There were 63 samples analyzed for all analytes listed in the Project Plan. There were 62 samples analyzed for boron, cyanide, lithium, osmium, strontium, and sulfate. The reason for not reporting a result for all 63 samples is under active investigation. The results of the investigation will be reported in the project final report. Sodium results were not received for all samples because the laboratory inadvertently reported these results in some SDGs, even though sodium analysis was not requested in the analytical SOW. 
Table 4.4. Summary distribution of polynuclear aromatic hydrocarbon data validation results

\begin{tabular}{lccccccccccc}
\hline \multicolumn{1}{c}{ Compound } & U & UJ & J & JN & R & RN & UN & UJN & P & Sum & $\begin{array}{c}\text { Percent } \\
\text { usable }\end{array}$ \\
\hline Acenaphthene & 6 & 22 & 16 & 5 & 5 & & & 11 & 65 & 92 \\
Acenaphthylene & 2 & 31 & 5 & 0 & 13 & 2 & & 12 & 65 & 77 \\
Anthracene & 1 & 35 & 4 & 1 & 11 & 2 & & 11 & 65 & 80 \\
Benzo[a]anthracene & 5 & 12 & 6 & 5 & 27 & & & 10 & 65 & 58 \\
Benzo[a]pyrene & 2 & 24 & 15 & 6 & 4 & & & 14 & 65 & 94 \\
Benzo[b]fluoranthene & 3 & 26 & 13 & 6 & 6 & & & 11 & 65 & 90 \\
Benzo[g,h,i]perylene & & 24 & 2 & & 26 & 1 & & 12 & 65 & 58 \\
Benzo[k]fluoranthene & & 30 & 5 & & 13 & 1 & 1 & 15 & 65 & 78 \\
Chrysene & 5 & 20 & & & 29 & 1 & & 10 & 65 & 54 \\
Dibenzo[a,h]anthracene & 2 & 24 & 1 & & 28 & & & 10 & 65 & 57 \\
Fluoranthene & 2 & 8 & 5 & & 46 & 1 & & 3 & 65 & 28 \\
Fluorene & 2 & 28 & 4 & 4 & 14 & 2 & & 11 & 65 & 75 \\
Indeno-1,2,3(c,d)-pyrene & 3 & 34 & 1 & & 15 & 2 & & 10 & 65 & 74 \\
Naphthalene & 2 & 33 & 8 & 16 & 10 & 1 & & 11 & 65 & 83 \\
Phenanthrene & 2 & 23 & 12 & 11 & 4 & & & 13 & 65 & 94 \\
Pyrene & 3 & 20 & 13 & 1 & 8 & & & 20 & 65 & 88 \\
\hline
\end{tabular}

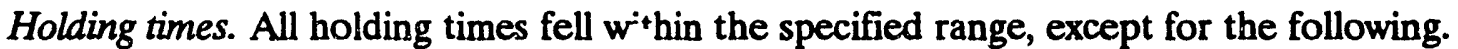
Mercury and sulfate were analyzed outside of their specified holding times for samples 5001, 5004, and 5007. Sample 5010 also had the holding time exceeded for sulfate. In addition, sample 3144 (water sample) had a pH of 5 upon receipt at the laboratory. The required pH under CLP is 2.

Initial calibration and calibration verification. Either the calibrations for the SDGs for graphite furnace atomic absorption (GFAA) met all the requirements, or the deviations did not warrant any action by the validator. The calibration for inductively coupled plasma analyses of SDG numbers $042260,0430260,0508260,0511260,0514260$, and 05192 ' did not comply with the CLP criteria or the manufacturer's criteria. In addition, there were three SDGs where the calibration did not comply for the inductively coupled plasma (ICP) analytes of boron, lithium, osmium, and silicon. These three SDGs are $0.22260,0723260$, and 0803260 . In each caso, the laboratory used the update function of the instrument instead of the calibration called for in the CLP SOW. In addition, an update slope function was used in conjunction with the update function. The update slope determines percent correction factors to be used by the instrument to "recalibrate." This, too, is a deviation from CLP; however, the laboratory did not use the proper manufacturer's guiciance in applying this correction. The laboratory allowed percent corrections to exceed the manufacturer's criteria for recalibration withou. performing a recalibration. The technical judgment was to not qualify the data as estimated (J) because of acceptable ICV and continuing calibration verifications (CCVs), but it may be necessary to consider the added uncertainty for certain uses of the data, as well as regulatory and defensibility concerns. This issue is still under investigation and will be further clarified in the project final report. 
The cyanide results were qualified estimated $(J)$ or estimated nondetect because there was no evidence that the middle standard or ICV was distilled as specified by CLP.

The osmium CCV samples (CCV-3, $-4,-5$, and -6 ) for SDGs $0722260 / 0723260$ were outside the criteria at $110.9,113.0,112.1$, and 111.4 , respectively. This would qualify the osmium data as estimated (J), but the MS recovery finding supersedes this qualification because it qualifies the data as unusable (R).

Laboratory blank results. The analysis of laboratory blanks provides a means of assessing the existence of contamination in the analytical method. Blanks did not show evidence of significant contamination except for the analytes discussed below.

- For SDG 0422260, the level of selenium in the preparation blank was comparable to that found in some of the samples, so those samples were qualified as nondetect $(U)$.

- Sample 6004 of SDG 0430260 was qualified as nondetect for lithium because the sample result was less than five times the value of the associated CCV.

- The lithium result for sample 6010 (SDG 0511260) was also qualified as nondetect because the resuit was less than five times the associated CCV. In addition, calcium and selenium were qualified as nondetects because the results of the preparation blanks were comparable to the sample results.

- The preparation blanks for SDG 0514260 contained levels of calcium and thallium comparable to those found in the samples; therefore, these samples were qualified as nondetect.

- Thallium results for SDG 0519260 were qualified nondetect because the preparation blank results were comparable to those found in the samples.

- Boron and silicon results for SDG 0727260/0728260/0729260 were qualified as estimated nondetects (UJ) because the continuing calibration blank (CCB) before or between which they were determined had values approaching the negative reporting limit and well beyond the negative IDL. Calcium results were qualified nondetect when the calcium sample results were less than five times the concentrations in the preparation blank.

- Antimony data for SDG $0722260 / 0723260$ were qualified nondetect when sample results were less than five times the concentration found in the preparation blanks.

- The boron and silicon results in SDG 0803260 were qualified as estimated nondetected (UJ) and estimated (J), respectively. The boron result was qualified estimated nondetected because the CCBs between which it was determined had values approaching the negative reporting limit and well beyond the negative IDL. Silicon results were qualified estimated (J) because the CCBs between which the sample was analyzed had values exceeding the negative reporting limit.

- Overall, the laboratory did not comply with the sample analysis order for CCBs and CCVs. The laboratory analyzed the CCB before the CCV, which is against the specifications of the CLP SOW. In addition, in some cases the laboratory analyzed a rinse blank before the CCB. By doing so, the evaluation of the CCBs does not provide information regarding carryover contamination.

Interference check sample. The a alysis of an interference check sample (ICS) was to verify the interelement and background correction factors. All ICS results were acceptable, except for the results of vanadium in SDGs 0514260 and 0519260 . The vanadium was outside the criteria on both the initial and final ICS; therefore, all vanadium data were qualified estimated (J). 
Matrix spikes. The spiking levels and analytes did not agree with CLP requirements, so it was difficult to apply CLP criteria. In addition, post-digestion spikes were not performed as specified by CLP. The data were qualified because MS samples were outside criteria.

- The magnesium and potassium results for SDG 0422260 were qualified estimated (J). Osmium results were qualified as estimated nondetects because the predigestion spike was outside criteria.

- The results for SDG $0422260,0430260 / 0508260 / 0511260$ for silicon were qualified as estimated $(\mathrm{J})$ because the spike recovery was below the lower limit.

- The osmium results for SDGs $0430260,0508260,0511260$ were qualified as estimated nondetect (UJ) because the predigestion spike was outside criteria.

- Silver results for SDG 0727260/0728260/0729260 were qualified as estimated nondetect (UJ) because of low predigestion spike recoveries. Silicon was qualified as estimated (J) because of low recoveries, while osmium results were rejected $(R)$ because of very low recoveries.

- Antimony and silver results for SDG 0722260/0723260 were qualified as estimated nondetected (UJ) because spike recovery was low. Magnesium and potassium results were qualified estimated $(J)$ because the predigestion spike results were outside criteria, greater than $125 \%$. All osmium results were rejected $(R)$ because the spike recovery (2.2\%) was out of range (criteria $75-125 \%$ ).

- Silicon and cadmium results for SDG 0803260 were qualified estimated (J) because of low spike resoveries. Sulfate was qualified as estimated $(J)$ because the postdigestion spike recovery was very low.

Duplicates. All laboratory duplicates were within the QC limits except for the following. Copper, iron, boron, and sulfate results for SDGs 0422260, 0430260, 0508260, and 0511260 were qualified as estimated (J) because the duplicate results exceeded criteria. Chromium results for SDG 0803260 were qualified estimated $(J)$ because the duplicate results exceeded criteria.

Laboratory control samples. An aqueous LCS was used. The CLP SOW specifies the use of a solid LCS when analyzing solid samples. The results from the aqueous LCS may not be indicative of analyte recovery, making the evaluation difficult. Osmium results in SDGs $042260,0430260,0508260$, and 0511260 were qualified estimated $(J)$ or estimated nondetected (UJ) because LCS recoveries were outside of criteria. Osmium results for SDGs 0514260 , 0519260 , and 0803260 were rejected $(R)$ because of very poor recovery.

Method of standard additions. The MSA was performed on the following samples. MSAs were performed on lead samples 5031, 5034, 5040,6028, 6034, 6040, 5079, 5088, 1468, and $1468 \mathrm{D}$, with no problems except that the spiking levels used in sample 5079 and 5088 were not adapted well to the concentration of the samples. MSAs were performed on chromium samples 7034, 7037-FD, 7040-FD, 7043-FD, and 1468, with no problems.

Serial dilutions. The serial dilution results for SDG 0422260/0430260/0508260/0511260 for silicon exceeded the acceptance criteria; therefore, all silicon data were qualified as estimated (J). Silicon results for SDG 0727260/0728260/0729260 were qualified estimated (J) because the serial dilution exceeded acceptance criteria. Silicon and zinc serial dilution results exceeded acceptance criteria for SDG 0803260. 
Other laboratory $Q C$. Accompanying the soil samples were equipment water rinsates. These equipment rinsates were taken after Phase I soil sampling was completed, whereas potential contamination of samples is normally identified from rinsates taken during the sample collection process. Association of rinsates with particular samples was not identified, so specific qualification of data could not be performed. Rinsate samples were never associated with any sample numbers.

- SDG 0422260: Antimony analytical spike recoveries were below limits, so those results were qualified as estimated (J).

- SDG 0430260: Antimony GFAA analytical spike recovery is based on a spike concentration of $20 \mathrm{mg} / \mathrm{L}$. The laboratory qualified sample 6001 with a "W"; all antimony results should have been similarly qualified since the values of the analytical spike ranged from $73-80 \%$. On this basis, all antimony results were qualified as estimated nondetects.

- SDG 0508260: Antimony GFAA results for sample 5019, 5022, and 5010 were qualified as estimated nondetected because the analytical spike recovery was low.

- SDG 0519260: Antimony GFAA results were qualified as estimated nondetected (UJ) because of low analytical spike recoveries.

- SDG 0727260/0728260/0729260: The arsenic results for sample 5070 were qualified estimated nondetected (UJ) because the analytical spike results exceeded limits.

- SDG 0722260/0723260: Antimony GFAA results were qualified as estimated nondetected (UJ) because of low recoveries for the analytical spike.

Overall assessment. There were numerous deviations from CLP protocol that could affect data comparability and create increased uncertainty in the quality of the data. Some of the deviations follow:

- Calibration of the Leeman ICP did not follow CLP or manufacturers' instructions.

- Spiking levels for MSs, post-digestion spikes, and GFAA analytical spikes were inconsistent with CLP, and the analytes in the MS were not in agreement with the CLP.

- Preparation volumes were noncompliant.

- The lab analyzed post-digestion spikes when they were not called for.

- MSs for GFAA were analyzed with an analytical spike added, which is not called for in CLP.

- Reanalysis was performed when the blank exceeded the absolute value of the contract-required detection limit or reporting limit was not performed.

- Aqueous rather than solid LCS samples were analyzed with soil samples.

These deviations are being discussed with the laboratory and will be addressed for resolution by the APO. All inorganic data were determined to be usable with the exception of osmium (29\%). A summary of the inorganic data validation results is presented in Table 4.5. The $\mathrm{CN}^{-}$results were qualified estimated (J) or estimated nondect (UJ) because there was no evidence that a middle standard or ICV was distilled as specified CLP. Sample results for sulfate were qualified as estimated because of either missed holding times or duplicate RPDs outside QC limits. 
Table 4.5. Summary distribution of inorganic data validation results

\begin{tabular}{lcccccccc}
\hline Compound & No qualifier & U & J & UJ & B & R & Sum & $\begin{array}{c}\text { Percent } \\
\text { usable }\end{array}$ \\
\hline Aluminum & 58 & 5 & & & & 63 & 100 \\
Antimony & & 20 & 3 & 31 & 9 & 63 & 100 \\
Arsenic & 30 & 8 & 1 & 2 & 22 & 63 & 100 \\
Barium & 58 & 5 & & & & 63 & 100 \\
Beryllium & 13 & 5 & & & 45 & 63 & 100 \\
Boron & 11 & 23 & 13 & 15 & & 63 & 100 \\
Cadmium & & 61 & 1 & 1 & & 63 & 100 \\
Calcium & 22 & 24 & & & 17 & 63 & 100 \\
Chromium & 52 & 6 & 5 & & & 63 & 100 \\
Cobalt & 36 & 5 & & & 22 & 63 & 100 \\
Copper & 43 & 5 & 15 & & & 63 & 100 \\
Cyanide & 4 & 1 & 12 & 45 & & 62 & 100 \\
Iron & 43 & 4 & 15 & & 1 & 63 & 100 \\
Lead & 41 & 6 & & 1 & 15 & 63 & 100 \\
Lithium & 46 & 7 & & & 9 & 62 & 100 \\
Miagnesium & 39 & 5 & 18 & & 1 & 63 & 100 \\
Manganese & 58 & 4 & & & 1 & 63 & 100 \\
Mercury & 15 & 44 & 3 & & 1 & 63 & 100 \\
Molybdenum & & 55 & & & 8 & 63 & 100 \\
Nickel & 58 & 5 & & & & 63 & 100 \\
Osmium & & 2 & & 16 & & 44 & 62 & 29 \\
Potassium & 39 & 5 & 18 & & 1 & 63 & 100 \\
Selenium & 1 & 36 & 4 & 1 & 21 & 63 & 100 \\
Silicon & 16 & 2 & 43 & 2 & & 63 & 100 \\
Silver & & 36 & & 27 & & 63 & 100 \\
Sodium & 41 & 3 & & & & 44 & 100 \\
Strontium & 57 & 5 & & & & 62 & 100 \\
Sulfate & 45 & & 15 & & 2 & 62 & 100 \\
Thallium & & 49 & & 1 & 13 & 63 & 100 \\
Vanadium & 42 & 5 & 15 & & 1 & 63 & 100 \\
Zinc & 57 & 3 & 1 & & 2 & 63 & 100 \\
\hline & & & & & & & \\
\hline
\end{tabular}

\subsection{Radiochemical Validation Results}

\subsubsection{Thorium isotopes}

Fifty-eight samples were analyzed for isotopic thorium by the alpha spectrometry technique.

Holding times. The holding times for isotopic thorium were met. 
Calibration. All calibration criteria for SDG 2684 were met. The laboratory included the daily full-width half-maximum information, centroid information, and efficiency information. Background information pertaining to these samples was acceptable, and all monthly calibration information was acceptable and corrected by the daily instrument performance check.

Incorrect calibration information was provided for SDGs 2658, 2423, and 2419 by the laboratory. Without the correct information, the data must be qualified estimated $(J)$ for results greater than minimum detectable activity (MDA) and UJ for results less than MDA.

The laboratory did not provide daily calibration information for SDGs 2638 and 2633, so it is impossible to determine the behavior of the instrument on the day of the analysis. All results greater than MDA were qualified as $\mathrm{J}$, and all results less than MDA were qualified as UJ.

Laboratory blank results. All laboratory blank results were less than MDA. The blank results obtained for SDG 2423 were invalid because there was no daily calibration information to support this blank result.

Tracer results. Thorium-229 was used as the tracer for this analysis. All tracer recoveries were within the QC limits (15-125\%) except for SDGs 2419, 2423, and 2633; therefore, all data must be qualified estimated (J) for results greater than MDA and unusable (R) for results less than MDA. The tracer results for SDG 2684 were within QC limits, but an outdated tracer solution was used; therefore, all results greater than MDA were estimated $(J)$, and all results less than MDA were rejected (R).

Matrix spike/matrix spike duplicates. Thorium-230 was the spike used in the MS/MSD. All MS/MSD results were within the QC limits (75-125\%) except for SDGs 2419 and 2423. RPDs between the MS/MSDs were all within the QC limits ( $550 \%$ maximum).

Duplicates. The RPD acceptance criterion was $\pm 35 \%$ for samples with values greater than or equal to 5 times the MDA. All duplicate results met this criterion.

Blank spike. The spike was thorium-230. All the blank spike results were within QC limits (75-125\%).

Chemical separation specificity. No energy or library matches were provided to check the chemical separation specificity of the isotope. All results were qualified as estimated (J) for results greater than MDA and $\mathrm{UJ}$ for results less than MDA.

Overall assessment. The data in SDG 2684 were estimated (J) because an outdated tracer was used. The data in SDG 2638 were estimated (J) because of the failure to run a daily calibration. The data in SDGs 2633, 2658 were estimated (J) because energy spectra and library matches were unavailable to assess the chemical separation specificity. The data in SDGs 2419 and 2423 were estimated (J) for results greater than MDA and rejected (R) for results less than MDA because the tracer recovery in the blank spike was outside of limits. 


\subsubsection{Uranium isotopes}

Fifty-eight samples were analyzed for isotopic uranium by alpha spectrometry.

Holding times. All technical holding times were met.

Calibration. All samples passed the initial and verification calibration criteria except for sample 6038 of SDG 2423. Results for sample 6038 were qualified estimated (J) for results greater than MDA and UJ for results less than MDA.

Laboratory blank results. There were no detected activities found above the MDA except for SDGs 2391 and 2658. Uranium-238 was found in the laboratory blank of SDG 2391 above the MDA, but it was less than $10 \%$ of the sample activity. Uranium-234, -235 , and -238 were found in the laboratory blank of SDG 2658 above the MDA. All the samples had positive results greater than the MDA, but less than five times the blank value. Therefore, all results less than five times the blank were qualified $U$.

Tracer results. All tracer recoveries were within the QC limits (15-125\%) except for sample 6038 of SDG 2423 which had tracer recoveries below the QC limits. Results above the MDA were qualified J, and results below the MDA were rejected (R).

Matrix spike/matrix spike duplicate. All MS/MSD recoveries were within the QC limits (75-125\%) with the exception of uranium-238 for SDGs 2419 and 2423 . In addition, there was no radioactive source report, calculation or logsheet for uranium-238 + natural spike. Therefore, all results above the MDA were qualified J, and all results below the MDA were qualified as UJ.

Duplicates. Duplicates were assessed by determining the RPD criterion of $\pm 35 \%$ for samples with results greater than or equal to 5 times the MDA. All duplicate RPDs were within the QC limits except for uranium-235 of SDG 2684. All data associated with this SDG were qualified $\mathrm{J}$ for results greater than the MDA.

Blank spike results. All blank spike recoveries were within the QC limits (75-125\%).

Chemical separation specificity. All results greater than the MDA were qualified estimated (J) and UJ for results less than the MDA. This qualification was made because no energy spectra or library matches were received so that chemical separation could be assessed.

Overall assessment. Data from SDGs 2684, 2633, 2391, 2419, 2638, and 2658 were qualified estimated $(J)$ because there was no energy spectra or library matches to assess chemical separation. Samples in SDG 2423 were qualified estimated (J) because of the failure of the MS to meet acceptance criteria.

\subsubsection{Neptunium-237}

Twenty-six samples were analyzed for isotopic neptunium by alpha spectrometry.

Holding times. All technical holding times were met. 
Calibration. The tracer (neptunium-239) for this analysis was run by gas proportional counter, and the determination of neptunium-237 was done by alpha spectrometry; therefore, calibration information was needed for each instrument. For SDGs 2423, 2638, 2633, 2419, and 2391, no calibration information was provided for the gas proportional counter, and no daily QC data were provided for this instrument. Calibration information was provided for the alpha spectrometer. Because no calibration information was provided for the gas proportional counter, all data for this SDG were rejected (R). For SDGs 2684 and 2658, the laboratory used the incorrect activity value in the calibration calculations, so all results above the MDA were qualified as estimated (J).

Laboratory blank results. There were no detected activities found above the MDA. The laboratory did not provide laboratory blank data for SDG 2684, so all data above the MDA were estimated $(\mathrm{J})$.

Tracer results. All tracer recoveries were within the QC limits (15-125\%).

Matrix spike/matrix spike duplicate. MS/MSD recoveries were within the QC limits (75-125\%) for SDG 2391, but all other SDGs were outside QC limits. The laboratory used an incorrect activity value, which changed their MS/MSD results. The data were qualified estimated $(J)$ for results greater than the MDA because of the failure to meet QC criteria.

Duplicates. Duplicates were assessed by determining the RPD criterion of $\pm 35 \%$ for samples with results greater than or equal to 5 times the MDA. All duplicate RPDs were within the QC limits.

Blank spike results. All blank spike recoveries were outside the QC limits (75-125\%). All results greater than the MDA were qualified estimated (J) while all results less than MDA were rejected $(R)$.

Chemical separation specificity. No alpha spectrometry energy spectra and library matches were given to verify that there were no interferences. The preparation notes mention the presence of an iron hydroxide precipitate at the time of plating, suggesting the presence of uranium, which would interfere with the plutonium spectrum. The data must be qualified as estimated (J) for results above MDA.

Overall assessment. The data for SDGs 2684 and 2658 were rejected (R) because of calibration errors and MS/MSD and blank spike errors that, when corrected, caused the data to fail criteria requirements. The data for SDGs 2419 and 2391 were rejected (R), because there was no self-absorption information to assess calibration. The data for SDGs 2638, 2633 and 2423 were rejected (R), because MS and blank spike recoveries were outside of limits and there were no data for the calibration of the gas proportional counter. If self-absorption curves are provided, the data qualification may be changed to "estimated," which may render the majority of the ${ }^{237} \mathrm{~Np}$ data usable to the project. These results will appear in the project final report. 


\subsubsection{Plutonium isotopes}

Twenty-six samples were analyzed for plutonium-238, 5 samples for plutonium-239, 21 samples for plutonium-239/240, and 5 samples for plutonium-240 by alpha spectrometry.

Holding times. All technical holding times were met.

Calibration. All calibration criteria were met for all samples except for sample 5029 of SDG 2419 and SDG 2633. Sample 5029 was qualified estimated (J) for results greater than MDA and UJ for results less than MDA because no background information was provided. The data in SDG 2633 were qualified estimated (J) for results greater than MDA and UJ for results less than MDA because daily calibration information was not provided, so it was impossible to determine instrument behavior at the time of analysis.

Laboratory blank results. There were no detected activities found above the MDA.

Tracer results. All tracer recoveries were within the QC limits (15-125\%). However, data for SDGs 2684 and 2391 had to be qualified estimated (J) for results above the MDA and unusable ( $R$ ) for results below the MDA because of the use of an outdated tracer solution.

Matrix spike/matrix spike duplicate. All MS/MSD recoveries were within the QC limits $(75-125 \%)$.

Duplicates. Duplicates were assessed by determining the RPD criterion of $\pm 35 \%$ for samples with results greater than or equal to five times the MDA. All duplicate RPDs were within the QC limits.

Blank spike results. All blank spike recoveries were within the QC limits (75-125\%).

Chemical separation specificity. No alpha spectrometry energy spectra and library matches were given to verify that there were no interferences. The preparation notes mention the presence of an iron hydroxide precipitate at the time of plating, suggesting the presence of uranium, which would interfere with the plutonium spectrum. The data must be qualified as estimated (J) for results above MDA and $\mathrm{UJ}$ for results below the MDA.

Overall assessment. The data for SDGs 2684 and 2391 were rejected (R) because of the use of an outdated tracer solution. The data for SDG 2633 were qualified as estimated (J) for results greater than the MDA and UJ for results less than the MDA because there was no daily instrument performance check information. The data for SDGs $2638,2658,2419$, and 2423 were estimated $(J)$ for results greater than the MDA and UJ for results less than the MDA because there were no energy spectra or library matches to assess chemical separation specificity.

\subsubsection{Strontium-89/90}

Twenty-six samples were analyzed for strontium-89/90 by gas fow proportional counting.

Holding times. All technical holding times were met. 
Calibration. All calibration criteria were met for all samples except for the following:

- unable to determine when the calibration was performed for SDGs 2633,2638 , and 2658 , so the data were rejected $(R)$ and

- no self-absorption data were provided for SDG 2684 , so the data were rejected (R).

Laboratory blank results. There were no detected activities found above the MDA.

Matrix spike/matrix spike duplicate. All MS/MSD recoveries were within the QC limits (75-125\%), with the exception of SDGs 2633, 2638, and 2658, which had MS recoveries below the QC limits.

Duplicates. Duplicates were assessed by determining the RPD criterion of $\pm 35 \%$ for samples with results greater than or equal to five times the MDA. All duplicate RPDs were within the QC limits.

Blank spike results. All blank spike recoveries were within the QC limits (75-125\%).

Overall assessment. The data for SDGs 2684, 2658, 2638, and 2633 were rejected (R) because self-absorption information was not provided. The data for SDG 2423 were qualified as estimated (J) for results greater than the MDA and UJ for results less than the MDA, because the calibration and yttrium-ingrowth information was not provided.

\subsubsection{Gamma spectrometry}

Sixty-one samples were analyzed by gamma spectrometry.

Holding times. All technical holding times were met.

Calibration. All calibration criteria were met for all samples and were within the upper and lower ranges.

Laboratory blank results. No analytical laboratory blank samples were analyzed.

Duplicates. Duplicates were assessed by determining the RPD criterion of $\pm 35 \%$ for samples with results greater than or equal to five times the MDA. All duplicate RPDs were within the QC limits, except for SDGs 2419 and 2423, which had RPD results outside the QC limits.

Overall assessment. The data were qualified estimated (J) for results above the MDA and $\mathrm{UJ}$ for results below the MDA, because the laboratory's performance or method accuracy could not be adequately assessed.

\subsubsection{Total uranium}

Twenty-six samples were analyzed for total uranium by pulsed laser phosphorimetry.

Holding times. All technical holding times were met. 
Calibration. All calibration criteria were met for all samples except that the correlation coefficients for the high and low standards did not meet criteria, so these data were qualified $J$ for detects and $U J$ for non-detects.

Laboratory blank results. There were no detected activities found above the detection limit except for SDGs 2633 and 2638, which had blank results above the detection limit, but the sample results were greater than five times the blank results.

Matrix spike/matrix spike duplicate. All MS/MSD recoveries were within the QC limits (75-125\%) for SDG 2658. MS/MSD recoveries for the other SDGs were outside the QC limits. The percent RPD for all SDGs were within the QC criteria.

Duplicates. Duplicates were assessed by determining the RPD criterion of $\pm 35 \%$ for samples with results greater than or equal to five times the MDA. All duplicate RPDs were within the QC limits.

Blank spike results. All blank spike recoveries were within the QC limits (75-125\%).

Overall assessment. The data were qualified estimated (J) for results above the MDA and $\mathrm{UJ}$ for results below the MDA because the MS recovery was below the lower QC limit.

\subsubsection{Technetium-99}

Twenty-six samples were analyzed for technetium-99 by liquid scintillation.

Overall assessment. All nondetect values were qualified unusable (R) based on the possibility that most or all of the technetium-99 was lost as a result of ashing in a muffle furnace at $500^{\circ} \mathrm{C}$. Resampling and reanalysis with appropriate changes in methodology are being actively pursued, and these results will be reported in the final project report.

\subsubsection{Tritium}

Twenty-two samples were analyzed for tritium by liquid scintillation.

Holding times. All technical holding times were met.

Calibration. The liquid scintillation counter was calibrated with National Institute of Standards and Technology traceable quench standards; however, this information was not included in the data package. The quench curve was established and the quench measured for each sample. limit.

Laboratory blank results. There were no detected activities found above the detection

Matrix spike/matrix spike duplicate. All MS/MSD recoveries were within the QC limits (75-125\%) for SDG 2399. MS/MSD recoveries for SDGs 2369 and 2391 were outside the QC limits, thus qualifying the data as estimated (J) for results above the MDA and UJ for results below the MDA. The percent RPD for all SDGs were within the QC criteria. 
Duplicates. Duplicates were assessed by determining the RPD criterion of $\pm 35 \%$ for samples with results greater than or equal to five times the MDA. All duplicate RPDs were within the QC limits for SDG 2399, but outside the criterion for SDG 2391. This qualifies the data for SDG 2391 as estimated (J) for results above the MDA and UJ for results below the MDA. The percent RPD for all SDGs were within the QC criteria.

Blank spike results. All blank spike recoveries were within the QC limits (75-125\%).

Overall assessment. The data for SDGs 2369 and 2391 were qualified estimated (J) for results above the MDA and UJ for results below the MDA because the MS recovery was outside the QC limits. The data for SDG 2391 were qualified UJ because of the inability to assess activity, error, and MDA values. A summary of the radiochemical data validation results is presented in Table 4.6.

\subsection{SCREENING ANALYSES FOR VOLATILE ORGANIC COMPOUNDS}

Analyses for volatile organic compounds were performed on all noncomposited surface soil samples. These analyses were conducted as a screen to determine whether there was any disposal of wastes at the site or evidence of contamination of ground water plumes under the site. Since the analysis was being performed as a screen, the analytical level was set at data quality level II, which provided quantitative data with less rigorous QAVQC and documentation compared to EPA Level IV.

The results of most volatile organic screens were negative. Data are presented in Appendix B. Some samples were found with detectable quantities of compounds that typically appear as laboratory contaminants (acetone and 2-butanone). Twenty eight samples showed acetone as a contaminant and 6 samples showed both acetone and 2-butanone as contaminants. In addition, there were two samples that showed a compound other than these two contaminants. One sample showed detectable quantities of trichlorofluoromethane, and the other sample showed detectable quantities of chloroform. Each of these compounds was found in low concentration and could conceivably be associated with the laboratory performing the analysis.

\subsection{ORGANIC COMPOUND ANALYSES}

\subsubsection{Pesticides/PCBs}

The method used for this project is the CLP method for pesticides/PCBs based on the SOW of March 1990 (EPA 1990a). The method involves the extraction of samples with methylene chloride. The concentrated extract is then readied for cleanup by diluting it with acetone. The cleanup is performed with a florisil cartridge, and after cleanup, the extract solution volume is adjusted with hexane to its final volume. The sample is then ready for analysis by $\mathrm{GC}$ techniques.

\subsubsection{Polynuclear Aromatic Hydrocarbons}

The method used for this project is EPA Method 8310. This method is used to determine the concentration of certain PAHs. The method provides high performance liquid chromatography (HPLC) conditions for the detection of levels of certain PAHs in the parts 
Table 4.6. Summary distribution of radiochemical data validation results

\begin{tabular}{|c|c|c|c|c|c|c|}
\hline Analyte & $\begin{array}{c}\text { No } \\
\text { qualifier }\end{array}$ & $\mathbf{J}$ & UJ & $\mathbf{R}$ & Sum & $\begin{array}{c}\text { Percent } \\
\text { usable }\end{array}$ \\
\hline $\begin{array}{l}\text { Americium-241 } \\
\text { Barium-133 } \\
\text { Cesium-137 } \\
\text { Chromium-51 } \\
\text { Cobalt-57 }\end{array}$ & $\begin{array}{l}4 \\
4 \\
4 \\
4 \\
4\end{array}$ & $\begin{array}{c}2 \\
1 \\
41 \\
1 \\
1\end{array}$ & $\begin{array}{l}55 \\
56 \\
16 \\
56 \\
56\end{array}$ & & $\begin{array}{l}61 \\
61 \\
61 \\
61 \\
61\end{array}$ & $\begin{array}{l}100 \% \\
100 \% \\
100 \% \\
100 \% \\
100 \%\end{array}$ \\
\hline $\begin{array}{l}\text { Cobalt-60 } \\
\text { Curium-243 } \\
\text { Curium-244 } \\
\text { Curium-245 } \\
\text { Curium-247 }\end{array}$ & 4 & 2 & $\begin{array}{c}56 \\
13 \\
2 \\
13 \\
11\end{array}$ & & $\begin{array}{c}61 \\
13 \\
2 \\
13 \\
13\end{array}$ & $\begin{array}{l}100 \% \\
100 \% \\
100 \% \\
100 \% \\
100 \%\end{array}$ \\
\hline $\begin{array}{l}\text { Europium-152 } \\
\text { Europium-154 } \\
\text { Europium-155 } \\
\text { Hafnium-181 } \\
\text { Iridium-192 }\end{array}$ & $\begin{array}{l}4 \\
4 \\
4 \\
4 \\
4\end{array}$ & $\begin{array}{l}1 \\
53 \\
1\end{array}$ & $\begin{array}{l}56 \\
57 \\
4 \\
45 \\
44\end{array}$ & & $\begin{array}{l}61 \\
61 \\
61 \\
49 \\
49\end{array}$ & $\begin{array}{l}100 \% \\
100 \% \\
100 \% \\
100 \% \\
100 \%\end{array}$ \\
\hline $\begin{array}{l}\text { Neptunium-237 } \\
\text { Niobium } \\
\text { Plutonium-238 } \\
\text { Plutonium-239 } \\
\text { Plutonium-239/240 }\end{array}$ & & $\begin{array}{l}8 \\
1 \\
3\end{array}$ & $\begin{array}{c}1 \\
43 \\
17 \\
3 \\
18\end{array}$ & $\begin{array}{l}25 \\
1 \\
1\end{array}$ & $\begin{array}{c}26 \\
43 \\
26 \\
5 \\
21\end{array}$ & $\begin{array}{c}4 \% \\
100 \% \\
96 \% \\
80 \% \\
100 \%\end{array}$ \\
\hline $\begin{array}{l}\text { Plutonium-240 } \\
\text { Potassium-40 } \\
\text { Radium-226 } \\
\text { Ruthenium-103 } \\
\text { Strontium-90 }\end{array}$ & $\begin{array}{c}4 \\
4 \\
13\end{array}$ & $\begin{array}{c}55 \\
57 \\
1 \\
1\end{array}$ & $\begin{array}{c}4 \\
2 \\
1 \\
56 \\
2\end{array}$ & 10 & $\begin{array}{l}5 \\
61 \\
58 \\
61 \\
26\end{array}$ & $\begin{array}{c}80 \% \\
100 \% \\
100 \% \\
100 \% \\
62 \%\end{array}$ \\
\hline $\begin{array}{l}\text { Technetium-99 } \\
\text { Thorium-228 } \\
\text { Thorium-230 } \\
\text { Thorium-232 } \\
\text { Thorium-234 }\end{array}$ & 55 & $\begin{array}{c}57 \\
58 \\
58 \\
3\end{array}$ & 1 & 26 & $\begin{array}{l}26 \\
58 \\
58 \\
58 \\
58\end{array}$ & $\begin{array}{l}0 \% \\
100 \% \\
100 \% \\
100 \% \\
100 \%\end{array}$ \\
\hline $\begin{array}{l}\text { Total uranium } \\
\text { Tritium } \\
\text { Uranium-233/234 } \\
\text { Uranium-235 } \\
\text { Uranium-236 }\end{array}$ & & $\begin{array}{c}25 \\
5 \\
46 \\
25 \\
4\end{array}$ & $\begin{array}{c}1 \\
14 \\
12 \\
33 \\
54\end{array}$ & 3 & $\begin{array}{l}26 \\
22 \\
58 \\
58 \\
58\end{array}$ & $\begin{array}{l}100 \% \\
86 \% \\
100 \% \\
100 \% \\
100 \%\end{array}$ \\
\hline $\begin{array}{l}\text { Uranium-238 } \\
\text { Zinc-65 } \\
\text { Zirconium-95 }\end{array}$ & $\begin{array}{l}4 \\
4\end{array}$ & 46 & $\begin{array}{l}12 \\
57 \\
57\end{array}$ & & $\begin{array}{l}58 \\
61 \\
61\end{array}$ & $\begin{array}{l}100 \% \\
100 \% \\
100 \%\end{array}$ \\
\hline
\end{tabular}


per billion (ppb) range. Prior to the use of this method, a sample extraction technique must be used. The extraction techniques called for in the Project Plan are EPA Method 3510 (water) and Method 3550 (soil). Method 3510 takes one liter of the sample which is extracted with methylene chloride using a separatory funnel. This extract is then dried, concentrated, and exchanged into acetonitrile to be compatible with this analysis method. Method 3550 takes a $\mathbf{3 0}$ gram sample that is mixed with anhydrous sodium sulfate to form a free flowing powder. This powder is extracted three times with a 1:1 mixture of methylene chloride:acetone using sonication. Then the extract is separated from the sample by vacuum filtration and exchanged into acetonitrile for analysis. A 5- to 25-mL aliquot of the extract is injected into the HPLC, and the compounds in the effluent are detected by ultraviolet and fluorescence detectors.

\subsubsection{Herbicides}

The method being used for this project is EPA Method 8150. This method is a GC method for determining certain chlorinated herbicides. This method provides procedures for extraction, esterification, and gas chromatographic conditions. The extraction method for soil samples requires $50 \mathrm{~g}$ of the sample. This is extracted three times using acetone, and acetone/diethyl ether. The esterification is performed using a Diazald kit to prepare diazomethane. The diazomethane is added to the extracted solution and then concentrated at room temperature. The residue is dissolved in hexane and analyzed by GC.

\subsection{NNORGANIC COMPOUND ANALYSES}

\subsubsection{Metals}

The analysis of metals for this project is performed using ICP, GFAA, flame atomic absorption (FLAA), cold vapor atomic absorption (CVAA), neutron activation analysis (NAA), inductively coupled plasma/mass spectroscopy (ICP/MS) analytical techniques. The data reported in this report detail the data obtained for ICP, GFAA, FLAA, and CVAA. Data for the ICP/MS and NAA are being generated and will appear in the project final report.

The ICP, GFAA, FLAA, and CVAA methods are being performed according to the EPA Contract Laboratory Program Statement of Work for Inonganics, March 1990 (EPA 1990b) (except for osmium which is being done by EPA Method 7550). This method addresses preparation and analysis of the samples. Soil samples are prepared by digesting $1 \mathrm{~g}$ of the sample in nitric acid and hydrogen peroxide. The digestate is then refluxed with nitric acid or hydrochloric acid. Hydrochloric acid is used as the final reflux acid for the GFAA analysis of antimony, and FLAA analysis or ICP analysis of aluminum, antimony, barium, beryllium, calcium, cadmium, chromium, cobalt, copper, iron, lead, magnesium, manganese, nickel, potassium, silver, sodium, thallium, vanadium, and zinc. Nitric acid is employed as the final reflux acid for the GFAA analysis of arsenic, cadmium, chromium, lead, selenium, and thallium. The final analysis volume is brought to $100 \mathrm{~mL}$.

The preparation method summarized above is the same as the method called out in Method 7550. The preparation method for CVAA is detailed in the CLP SOW (EPA 1990b). It involves the reduction of mercury to its elemental state and then analyzing. 
The analysis of samples by ICP is based on the measurement of atomic emissions by an optical spectroscopic technique. Samples nebulized into an aerosol state are transported to the plasma torch where the excitation occurs. Characteristic atomic line emission spectra are produced by a radio irequency inductively coupled plasma. The spectra are dispersed by a grating spectrometer and intensities of the lines are processed.

Background correction is performed in order to compensate for the variable background contribution. The analysis of samples by GFAA and FLAA is based on the atomization of the sample. In these techniques the samples are atomized by a furnace or a flame. In FLAA, a light beam is directed through the flame into a monochromator and onto a detector that measures the amount of absorbed light. The absorption depends upon the presence of free unexcited ground-state atoms in the flame. The wavelength of the light beam is characteristic of only the metal being determined, so the energy absorbed by the flame is a measure of the concentration of the metal in the sample. In GFAA, the principle is essentially the same except that a furnace is being used to atomize the sample.

The analysis of mercury involves the aeration of mercury from solution in a closed system. The mercury vapor passes through a cell positioned in the path of the light from the AA spectrometer. The absorbance is measured as a function of mercury concentration.

Osmium is being analyzed by FLAA (refer to the section on GFAA and FLAA).

\subsection{Cyanide and Sulfate}

Cyanide and sulfate are analyzed in this project. The cyanide analysis is performed according to EPA CLP SOW for Inorganics, March 1990 (EPA 1990b). This method involves the colorimetric measurement of cyanide converted to cyanogen chloride. Cyanogen chloride is formed by reacting chloramine- $\mathrm{T}$ at a $\mathrm{pH}$ less than $\mathbf{8}$ without hydrolyzing to the cyanate. After the reaction is complete, color is formed on the addition of pyridine-pyrazolone or pyridine-barbituric acid reagent. The absorbance is read at $620 \mathrm{~nm}$ when using pyridine-pyrazolone and $578 \mathrm{~nm}$ when using pyridine-barbituric acid.

The sulfate ion is being determined by EPA Method 9038 . This method is being used at this time to provide consistency with future analyses. The analysis for sulfate ion is performed by converting the sulfate ion to a barium sulfate suspension. The resulting turbidity is determined by a nephelometer and compared with a curve prepared from a standard sulfate solution.

It should be pointed out that soil samples were to be analyzed for nitrates in the BSCP at the outset. However, consultation with the analytical laboratories revealed that EPA's very short holding time requirement of $\mathbf{4 8}$ hours would be exceeded consistently in practice and result in total rejection of the data. For this reason, further efforts to obtain nitrate data in the project were suspended at that time.

\subsection{RADIONUCLIDE ANALYSES}

The methods employed to analyze for radionuclides include gamma spectrometry, alpha spectrometry, gas proportional counting, and liquid scintillation. Results appear in Appendix E. 


\section{STATISTICAL ANALYSIS}

\subsection{SUMMARY}

This section contains summary statistics for the Phase I Background Soil Characterization Project (BSCP) data, including detection frequencies, median estimates (as measures of central tendency), upper 0.95 quantile estimates as measures of the upper ends of the normal background ranges, and confidence bounds for these estimates. The purpose of this section is to provide a rational and consistent basis for data analysis and interpretation, as well as risk analysis, not only for this study, but also for use as a guide to future studies in this area. Methods of statistical analysis are described. For data that are primarily nondetects, detection probability confidence bounds are given. These bounds are based only on simple properties of the binomial distribution. For data for which there are detects, the method of statistical analysis assumes lognormal distributions with possibly different means, but equal variances in different areas. The statistical methods incorporate nondetect information exactly, without resorting to approximations, such as setting nondetect values to detection limits.

All data were first examined graphically to assess these assumptions and to check for outliers. The data generally appear reasonable, although there are a few anomalous observations. Further consideration in light of Phase II data are likely to resolve the anomalies. Comparisons across areas are made. There are some statistically significant differences, including differences between the Anderson, Roane, and the Oak Ridge Reservation (ORR) Dismal Gap formations, but the practical importance of the differences is not considered in this section. Laboratory and spatial variances are estimated and compared. On the basis of these estimates and the relative costs of laboratory and field sampling, an advantage of using composited soil samples is demonstrated.

\subsection{INTRODUCTION}

Statistical analysis of the Phase I data was performed

- to assess the data graphically - that is, to screen for statistical outliers and to make a preliminary decision about the statistical distributions of the soil constituents;

- to compute summary statistics, the means and confidence bounds for soil constituent levels, tolerance bounds, and estimates of and confidence bounds for detection probabilities;

- to resolve and estimate laboratory and field components of variance; and

- to compare, to a limited extent, the four different sampling areas: the background sampling areas in the Dismal Gap Formation in Anderson and Roane counties and on the ORR and the background sampling area in the Nolichucky Formation on the ORR.

The purpose of this section is to provide a statistical overview. Naturally, results for different analytes behave differently. They tend to have different statistical distributions, variance properties, patterns of detection, or patterns of missing or rejected data. For many 
of the analytes, the statistical analysis assumes that the data are lognormally distributed with equal variances but possibly different means in the different areas. How appropriate these assumptions are may vary with the analyte. Time and budget constraints preclude tailoring statistical analyses individually to each analyte, but such special attention may be warranted in certain cases, especially for analytes whose background levels are near levels of risk concern. In such cases, the following discussion may provide useful guidance, but the user of the background data should perform his own analysis.

Many of the background results are nondetects (see Table 4.1 for a description of the validation codes), and the results given in the background data sets are then detection limits. In these cases, we assume only that the actual analyte concentration was between zero and the detection limit. The method of analysis we use to handle nondetects (the method of maximum likelihood) makes full use of these nondetects, without "imputing" them or resorting to other compromises, such as setting nondetects to zero or the corresponding detection limits. It would be useful to know the detection limits for analyses that were detects, but those limits were not provided.

Data designated with the validation code " $R$ " (rejected) are not used in the following analyses, but the remainder were, including data designated "J" (estimated). Some data were rejected for most of the analyses. We assume that the decision to reject data was not based on the level of the reported result. To the extent that this assumption is violated, statistics presented here are likely to be biased upward. This is particularly true for detects and nondetects that are subject to the same data quality problems, but are differentially assigned "R" and "J" qualifiers.

All results were plotted to check for outliers and other anomalies. For soil constituents with levels that were for the most part detected (i.e., primarily the inorganics and radionuctides and gamma screening data), the same plots were used to decide whether a parametric statistical distribution (e.g., normal or lognormal) is appropriate for modelling the data, and whether the statistical scatter in the data is similar over the different areas. On the basis of this visual assessment, we decided that the lognormal distribution and homogeneityof-variance assumptions were adequate for the preliminary data analyses considered here. Graphical data assessment is discussed further in Sect. 5.2.1.

For the mostly detected constituents, an array of means, standard errors, and confidence bounds was computed using the SAS Lifereg procedure and the method of maximum likelihood with lognormal errors and homogeneity of variance (SAS 1990). This procedure is described further in this section. Maximum likelihood estimation for log-normal (possibly censored) data is discussed in Lawless (1982, Sect. 5.2). For these mostly detected constituents, separate means are estimated for the lognormal analyte distributions for each area and horizon, but results for all areas contribute to a single variance estimate. In this way the data are pooled over areas, thus reducing the statistical noise in the estimates and making confidence limits tighter. Results cannot, in the same way, be pooled across horizons, because of the statistical dependence of results at different horizons but at the same location.

When the vast majority of the results are nondetects, as with herbicides, pesticides, and polynuclear aromatic hydrocarbons (PAHs), the usual statistics cannot be computed, and only detection probabilities are estimated. Then, a parametric statistical distribution is not needed. But, for the detection probability estimates and confidence bounds to be useful, samples sizes 
must be fairly large (e.g., >50). In our case, this necessitates combining several data groups (e.g., over areas).

Summary statistics are given in Sects. 5.3-5.9 for inorganics, herbicides, pesticides, PAHs, radionuclides, volatile organics, and gamma screening data.

Means and confidence bounds for means are computed as standard procedure. However, focusing exclusively on means skirts the issue of data scatter and the question of how large a constituent level has to be before it can reasonably be assumed to exceed background. To address this question, we use tolerance bounds: If a background distribution percentile is known exactly, it would be logical to assume that a particular sample exceeds background if it exceeds some particular upper percentile of the background distribution that is selected as a reasonable bound on the usual background range. For example, if the sample exceeds the 95th percentile, then either (1) contamination is present or (2) it is an unusual (1-in-20) background sample. As the percentile level increases, (1) becomes ever more and (2) ever less tenable. Of course background percentiles are not known exactly, but they can be estimated with background data. Tolerance bounds, which are just confidence bounds for percentiles, account for estimation error. Of particular interest are lower tolerance bounds for upper percentiles; if a sample value is below such a lower tolerance bound, then we can be confident that it does not exceed the corresponding percentile. If the percentile level is not too high, then we can be confident that the sample level is within the usual background range. For the same reason, a lower tolerance bound for an upper percentile is a reasonable candidate for a remediation target.

For analyses with a sufficient number of detects, tolerance bounds along with their corresponding percentile estimates, and the mean estimates and upper confidence bounds (UCBs) provide a good assessment of the statistical accuracy of the results. For analyses with zero detécts, and sometimes, for numerical reasons, these statistics cannot be computed. In cases with almost all nondetects, UCBs for detection probabilities can be used instead. UCBs for detection probabilities (binomial proportions) are discussed in Owens (1962, p. 273).

UCBs for detection probabilities can be used as follows. If we are confident that the true detection probability is less than the UCB, then, if that UCB is small enough and if the detection limits do not change much in the future, any future detect suggests contamination. For example, if the detection probability is less than 0.05 , a detect indicates either a 1-in-20chance background event or contamination. To be useful, the detection probability UCBs should be around 0.05 or less. Note that these results are for composites of three. Of course, the detection probabilities depend on the detection limits, which can change in future surveys.

For noncomposites or composites of other than three, variance components (i.e., field and laboratory) must be estimated. This is discussed in Sect. 5.10, where the advantage of compositing is also demonstrated for the background data.

Analysis of lognormal data is generally accomplished by computing means, standard errors, etc. of the logs. A problem arises when the results are transformed back to the original scale, because the mean of the logs is not the same as the log of the mean. However, the median (50th percentile) of the logs is the log of the median. Other percentiles transform in the same way and so do conficience bounds for them. For this reason, we shall, at this stage, restrict attention to medians and other percentiles, instead of means. Medians are usually 
considered to be more appropriate measures of central tendency for skewed distributions, such as the lognormal.

Finally, brief comparisons of results for different areas are discussed in Sects. 5.2.3 and 5.3-5.9. Comparisons of medians are of interest because of their implications on (1) combining areas for data analysis (e.g., to increase degrees of freedom for error estimates) and (2) extrapolations to other areas (e.g., formations). Differences in background values among areas for which there are data may rule out extrapolating to other areas.

\subsection{Graphical Screening}

All results, whether detects or nondetects, were plotted to check for outliers, homogeneity of variance (approximately equal scatter), and deviations from lognormality, which, for these data with so few observations for each area, amounts to checking for outliers. The volume of plots precludes presenting them all here; however, Fig. 5.1 shows that the ORR Dismal Gap duplicated original is an outlier. Major outliers and anomalous results are noted in Sects. 5.3-5.9.

Other types of data screening are possible. Data location coordinates are not available in the background data sets, but in theory the data could be interpreted in terms of location (e.g., with respect to spatial trends). Thus, a different outlier assessment could be made.

\subsubsection{Basic Assumptions}

Soil maps for the ORR and surrounding regions are complex patchworks; any particular formation is represented as the union of numerous small disjoint regions. For that formation, a subset of that union, suitable for background sampling and within particular boundaries, defines a targeted area for the BSCP (e.g., ORR Dismal Gap). As described in the project plan, to the extent feasible, targeted areas were sampled randomly. Triplicates were randomly selected for compositing. Therefore, to the extent that areas are sampled randomly, the data, both composites and noncomposites, are simple random samples. A close approximation to random sampling of areas was achieved for ORR areas. Access limitations are more severe off-site, and so the approximation is not as good there. Nevertheless, on the basis of graphical inspection, on- and off-site data seem to have similar distributions, and so we assume that the goal of simple random sampling was met for all areas.

For those analytes that were mostly undetected, distribution assumptions other than simple random sampling play no role in the analysis. However, for many of the inorganics and radionuclides, there are detects. For these analyses, on the basis of the plots, the ad hoc decision was made to model the data as lognormal with equal variances (but possibly different means) within areas. Separate analyses are made for each horizon. By using the same statistical model for all of the detected analytes, the analysis is greatly simplified, which is consistent with the goal of providing a statistical overview. Furthermore, more formal assessment of the model assumptions (e.g., by means of goodness-of-fit tests) is difficult (e.g., there are few observations in each area or nondetects) and fraught with logical problems (e.g., failing to reject a model may be due only to weakness of the goodness-of-fit test, which is itself very complicated to assess). Nevertheless, the lognormal and equal variance assumptions may be less appropriate for some analytes than others, and closer scrutiny may be warranted in applications different from this. 


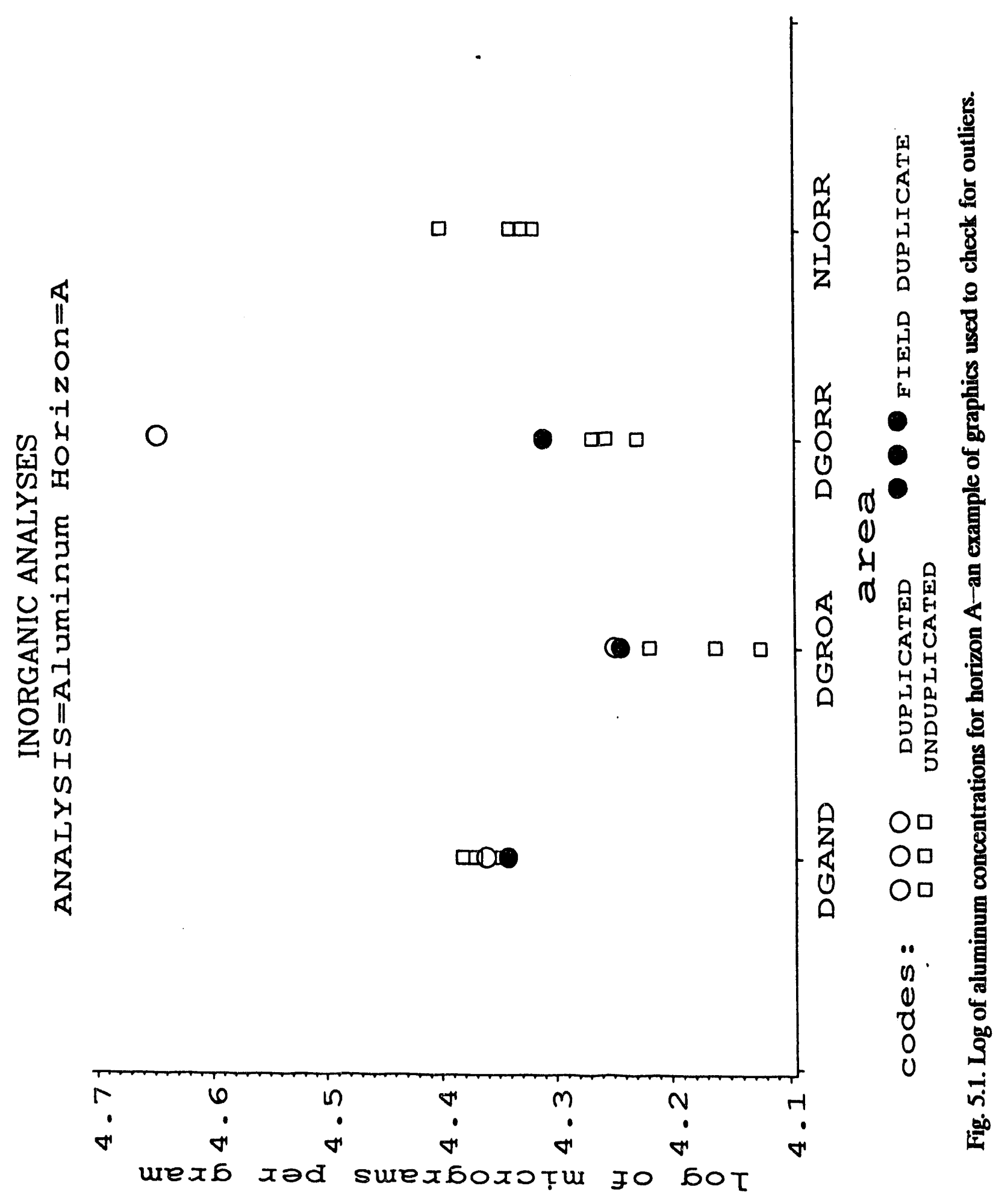




\subsection{Comparison of Areas}

Comparisons of areas can be made using chi-square likelihood ratio tests with the SAS Proc Lifereg and the lognormal equal variance model, even when there are nondetects. This is essentially a one-way analysis of variance, but nondetects are admitted into the analysis. When there are no nondetects, areas can also be compared using F-tests or t-tests, for example, with SAS Proc GLM (SAS 1990). This is the usual one-way analysis of variance, which is a standard statistical procedure.

When there are no nondetects, the chi-square and F-test significance levels are the same asymptotically (i.e., in theory for large sample sizes). In practice, with sample sizes such as this study's, the chi-square significance levels are generally smaller. [The likelihood ratio and Ftests actually coincide in this case (see Wilks 1962, Chapter 13). The approximation incurred in the likelihood ratio test is only through using the chi-square to approximate the F-distribution.]

To be consistent for the cases of nondetects and all-detects, the chi-square test was used to make all comparisons, but since the corresponding significance levels tend to be smaller and because many comparisons are made (i.e., for many analytes), the 0.01 significance cut-off was used, rather than the usual 0.05 , to declare areas significantly different. In only a very few of the nondetect cases was a chi-square test significant with $\mathrm{p}<0.01$ and the corresponding F-test not significant with $p>0.05$. These cases are noted.

When the statistical areas differ significantly, the question becomes how they differ (comparison of means). Unlike the SAS Proc GLM, the software in Proc Lifereg has not been developed to answer this question easily. Under the present cost and time constraints, pursuing that question fully when there are nondetects is not feasible. A step made in that direction is to test for differences among the three Dismal Gap areas, which illustrates an approach. When there are no nondetects, the question can be addressed by testing particular comparisons using Proc GLM.

Formal comparisons can also be made of detection frequencies (using a different chisquare test). Here, frequencies are the focus only when there are few or no detects. Then, frequency comparisons are negative.

\subsection{INORGANICS}

For many inorganics, which include metals, cyanide, and sulfates, some or all values are nondetects, but most results are detects. In addition, the following paragraphs (despite best efforts) still contain incomplete results for some analytes in the inorganic data sets of this project. Available project resources do not permit resampling to obtain complete data.

Data screening reveals that many of the ORR A horizon compusite results for sites 2 , 26, 43 are much higher than the other values for the ORR A horizon, including the field duplicate, which also happens to be from site grouping $2,26,43$. Figure 5.1 illustrates this for aluminum. It is also true for magnesium, nickel, vanadium, and zinc, and to a slightly lesser extent for barium, cobalt, copper, iron, lithium, potassium, and strontium. The duplicates are 
consistently high, suggesting the possibility of laboratory error. The apparently large laboratory error adversely affects estimates and confidence bounds.

For arsenic, chromium, and lead in horizons A and B, one of the Nolichucky values is a low outlier and a nondetect. Much of the data for sodium are missing, and most of the osmium results are validation rejects. Copper and vanadium values from ORR B horizon site groupings 11, 27, 41 are outliers. There are no entries for ORR A horizon site groupings 19, 22,32 for boron, cyanide, lithium, osmium, strontium, or sulfate. Many of the calcium values are nondetects. For cyanide, occasional results came out negative. Obviously, the negative results would be better set to zero. But this still causes difficulty in the cyanide analysis, because the zero implies that the cyanide detection limit is zero.

Summary statistics for inorganics that have a sufficient number of detects are given in Table 5.1. They include estimates of the medians, made under the assumption that the data are lognormal with equal variances across areas. The estimates are based on all of the data, whether detects or not. A 95\% UCB for the median is also given, along with an estimate of the 95th percentile (X95) of the distribution and LTB9595, the 95\% lower tolerance bound for the 95 th percentile. $N$, the number of samples, and the number of detects is also given. For this table, results for field duplicates and originals were averaged. The percentile estimate and lower tolerance bounds are for composites of three. The estimates and confidence bounds are computed using the Lifereg procedure in SAS, which gives standard errors of percentile estimates in addition to the estimates themselves.

Table 5.1. Summary statistics for inorganics (mg/kg)

\begin{tabular}{cccccccc}
\hline \multicolumn{7}{c}{ ANALYSIS=Aluminum } \\
\hline Horizon & Areas & N & Detect & Median & UCB95 & X95 & LTB9595 \\
\hline A & DG AND & 4 & 4 & 23100 & 26100 & 29400 & 25600 \\
A & DG ROA & 4 & 4 & 15400 & 17400 & 19600 & 17000 \\
A & DG ORR & 4 & 4 & 20700 & 23300 & 26300 & 22900 \\
A & NOL ORR & 4 & 4 & 22200 & 25000 & 28300 & 24600 \\
B & DG AlND & 4 & 4 & 35500 & 40200 & 45400 & 39400 \\
B & DG ROA & 4 & 4 & 23700 & 26800 & 30300 & 26200 \\
B & DG ORR & 4 & 4 & 31100 & 35200 & 39800 & 34500 \\
B & NOL ORR & 4 & 4 & 34800 & 39300 & 44500 & 38600 \\
C & DG AND & 4 & 4 & 38900 & 41700 & 44700 & 41200 \\
C & DG ROA & 4 & 4 & 25100 & 27000 & 28900 & 26700 \\
C & DG ORR & 4 & 4 & 39000 & 41900 & 44900 & 41400 \\
C & NOL ORR & 4 & 4 & 37900 & 40700 & 43600 & 40200 \\
\hline
\end{tabular}


Table 5.1 (continued)

\begin{tabular}{|c|c|c|c|c|c|c|c|}
\hline \multicolumn{8}{|c|}{ ANALYSIS=Antimony } \\
\hline Horizon & Areas & $\mathbf{N}$ & Detect & Median & UCB95 & X95 & LTB9595 \\
\hline $\mathbf{A}$ & DG AND & 4 & 1 & 0.885 & 0.929 & 0.936 & 0.882 \\
\hline $\mathbf{A}$ & DG ROA & 4 & 0 & . & . & . & 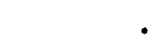 \\
\hline $\mathbf{A}$ & DG ORR & 4 & 0 & & . & & . \\
\hline A & NOL ORR & 4 & 1 & 0.463 & 0.485 & 0.490 & 0.470 \\
\hline B & DG AND & 4 & 1 & 0.663 & 1.000 & 1.200 & 0.780 \\
\hline B & DG ROA & 4 & 0 & - & . & . & \\
\hline B & DG ORR & 4 & 0 & . & . & . & 0.838 \\
\hline B & NOL ORR & 4 & 4 & 0.717 & 0.965 & 1.300 & 0.836 \\
\hline C & DG AND & 4 & 1 & 0.693 & 1.110 & 1.350 & - \\
\hline C & DG ROA & 4 & 0 & - & • & . & • \\
\hline C & DG ORR & 4 & 0 & - & - & & 0.803 \\
\hline C & NOL ORR & 4 & 4 & 0.673 & 0.938 & 1.310 & \\
\hline \multicolumn{8}{|c|}{ ANALYSIS=Arsenic } \\
\hline Horizon & Areas & $\mathbf{N}$ & Detect & Median & UCB95 & X95 & LTB9595 \\
\hline $\mathbf{A}$ & DG AND & 4 & 4 & 4.35 & 7.22 & 12.00 & 6.60 \\
\hline A & DG ROA & 4 & 4 & 5.86 & 9.72 & 16.10 & 8.89 \\
\hline $\mathbf{A}$ & DG ORR & 4 & 4 & 6.24 & 10.30 & 17.20 & 9.47 \\
\hline A & NOL ORR & 4 & 3 & 3.09 & 5.14 & 8.49 & 4.72 \\
\hline B & DG AND & 4 & 3 & 3.75 & 7.10 & 12.90 & 6.22 \\
\hline B & DG ROA & 4 & 4 & 7.03 & 13.00 & 24.20 & 11.60 \\
\hline B & DG ORR & 4 & 4 & 7.77 & 14.40 & 26.70 & 12.80 \\
\hline B & NOL ORR & 4 & 3 & 3.17 & 5.93 & 10.90 & 5.29 \\
\hline C & DG AND & 4 & 3 & 3.88 & 5.26 & 7.03 & 4.91 \\
\hline C & DG ROA & 4 & 4 & 7.43 & 10.00 & 13.50 & 9.46 \\
\hline C & DG ORR & 4 & 4 & 12.60 & 17.00 & 22.90 & 16.10 \\
\hline C & NOL ORR & 4 & 3 & 6.79 & 9.23 & 12.30 & 8.67 \\
\hline \multicolumn{8}{|c|}{ ANALYSIS=Barium } \\
\hline Horizon & Areas & $\mathbf{N}$ & Detect & Median & UCB95 & X95 & LTB9595 \\
\hline $\mathbf{A}$ & DG AND & 4 & 4 & 80.7 & 102.0 & 128.0 & 97.9 \\
\hline $\mathbf{A}$ & DG ROA & 4 & 4 & 87.9 & 111.0 & 139.0 & 107.0 \\
\hline A & DG ORR & 4 & 4 & 99.1 & 125.0 & 157.0 & 120.0 \\
\hline A & NOL ORR & 4 & 4 & 75.4 & 94.9 & 119.0 & 91.5 \\
\hline B & DG AND & 4 & 4 & 76.0 & 84.4 & 93.9 & 83.1 \\
\hline B & DG ROA & 4 & 4 & 69.4 & 77.2 & 85.8 & 75.9 \\
\hline B & DG ORR & 4 & 4 & 96.7 & 108.0 & 120.0 & 106.0 \\
\hline B & NOL ORR & 4 & 4 & 86.2 & 95.8 & 107.0 & 94.3 \\
\hline C & DG AND & 4 & 4 & 83.2 & 95.0 & 109.0 & 93.1 \\
\hline C & DG ROA & 4 & 4 & 73.0 & 83.3 & 95.2 & 81.6 \\
\hline C & DG ORR & 4 & 4 & 109.0 & 124.0 & 142.0 & 122.0 \\
\hline C & NOL ORR & 4 & 4 & 80.9 & 92.3 & 105.0 & 90.4 \\
\hline
\end{tabular}


Table 5.1 (continued)

\begin{tabular}{|c|c|c|c|c|c|c|c|}
\hline \multicolumn{8}{|c|}{ ANALYSIS=Beryllium } \\
\hline Horizon & Areas & $\mathbf{N}$ & Detect & Median & UCB95 & X95 & LTB9595 \\
\hline $\mathbf{A}$ & DG AND & 4 & 4 & 0.833 & 1.070 & 1.36 & 1.030 \\
\hline $\mathbf{A}$ & DG ROA & 4 & 4 & 0.647 & 0.828 & 1.06 & 0.796 \\
\hline A & DG ORR & 4 & 4 & 0.781 & 0.999 & 1.28 & 0.961 \\
\hline A & NOL ORR & 4 & 4 & 0.786 & 1.010 & 1.29 & 0.968 \\
\hline B & DG AND & 4 & 4 & 0.962 & 1.280 & 1.71 & 1.220 \\
\hline B & DG ROA & 4 & 4 & 0.628 & 0.836 & 1.11 & 0.800 \\
\hline B & DG ORR & 4 & 4 & 0.728 & 0.969 & 1.29 & 0.927 \\
\hline B & NOL ORR & 4 & 4 & 1.000 & 1.330 & 1.78 & 1.280 \\
\hline C & DG AND & 4 & 4 & 1.170 & 1.480 & 1.87 & 1.430 \\
\hline C & DG ROA & 4 & 4 & 0.825 & 1.040 & 1.31 & 1.000 \\
\hline C & DG ORR & 4 & 4 & 1.020 & 1.280 & 1.62 & 1.240 \\
\hline $\mathrm{C}$ & NOL ORR & 4 & 4 & 1.170 & 1.470 & 1.86 & 1.420 \\
\hline \multicolumn{8}{|c|}{ ANALYSIS=Boron } \\
\hline Horizon & Areas & $\mathbf{N}$ & Detect & Median & UCB95 & X95 & LTB9595 \\
\hline $\mathbf{A}$ & DG AND & 4 & 0 & & & & \\
\hline A & DG ROA & 4 & 3 & 25.9 & 39.3 & 59.4 & 33.6 \\
\hline A & DG ORR & 3 & 1 & 13.5 & 23.3 & 31.0 & 16.6 \\
\hline A & NOL ORR & 4 & 0 & • & $\cdot$ & $\cdot$ & • \\
\hline B & DG AND & 4 & 0 & & & $\cdot$ & \\
\hline B & I,G ROA & 4 & 3 & 15.2 & 25.7 & 41.9 & 21.5 \\
\hline B & DG ORR & 4 & 3 & 21.4 & 35.7 & 59.0 & 29.7 \\
\hline B & NOL ORR & 4 & 0 & . & $\cdot$ & $\cdot$ & . \\
\hline C & DG AND & 4 & 0 & . & . & & . \\
\hline C & DG ROA & 4 & 4 & 23.4 & 31.4 & 42.1 & 28.8 \\
\hline C & DG ORR & 4 & 4 & 27.4 & 36.7 & 49.2 & 33.7 \\
\hline $\mathrm{C}$ & NOL ORR & 4 & 0 & $\cdot$ & $\cdot$ & $\cdot$ & $\cdot$ \\
\hline \multicolumn{8}{|c|}{$\begin{array}{c}\text { ANALYSIS=Cadmium } \\
\text { (No detects) }\end{array}$} \\
\hline \multicolumn{8}{|c|}{ ANALYSIS=Calcium } \\
\hline Horizon & Areas & $\mathbf{N}$ & Detect & Median & UCB95 & $\mathrm{X} 95$ & LTB9595 \\
\hline A & DG AND & 4 & 4 & 1350 & 1930 & 2760 & 1770 \\
\hline A & DG ROA & 4 & 2 & 798 & 1180 & 1630 & 1060 \\
\hline A & DG ORR & 4 & 2 & 907 & 1320 & 1850 & 1190 \\
\hline A & NOL ORR & 4 & 2 & 490 & 734 & 1000 & 645 \\
\hline B & DG AND & 4 & 4 & 764 & 1050 & 1460 & 969 \\
\hline B & DG ROA & 4 & 1 & 420 & 644 & 801 & 526 \\
\hline B & DG ORR & 4 & 2 & 706 & 1030 & 1350 & 898 \\
\hline B & NOL ORR & 4 & 3 & 663 & 928 & 1270 & 849 \\
\hline C & DG AND & 4 & 4 & 383 & 605 & 955 & 540 \\
\hline C & DG ROA & 4 & 1 & 277 & 513 & 691 & 377 \\
\hline C & DG ORR & 4 & 3 & 824 & 1310 & 2050 & 1160 \\
\hline $\mathrm{C}$ & NOL ORR & 4 & 2 & 591 & 969 & 1470 & 852 \\
\hline
\end{tabular}


Table 5.1 (continued)

\begin{tabular}{|c|c|c|c|c|c|c|c|}
\hline \multicolumn{8}{|c|}{ ANALYSIS=Chromium } \\
\hline Horizon & Areas & $\mathbf{N}$ & Detect & Median & UC'B95 & X95 & LTB9595 \\
\hline $\mathbf{A}$ & DG AND & 4 & 4 & 28.10 & 61.9 & 136.0 & 53.9 \\
\hline A & DG ROA & 4 & 4 & 27.30 & 60.1 & 132.0 & 52.3 \\
\hline A & DG ORR & 4 & 4 & 24.70 & 54.3 & 120.0 & 47.3 \\
\hline A & NOL ORR & 4 & 3 & 9.38 & 20.8 & 45.5 & 18.2 \\
\hline B & DG AND & 4 & 4 & 35.00 & 81.0 & 187.0 & 70.0 \\
\hline $\bar{B}$ & DG ROA & 4 & 4 & 38.10 & 88.1 & 204.0 & 76.1 \\
\hline B & DG ORR & 4 & 4 & 37.40 & 86.5 & 200.0 & 74.7 \\
\hline $\bar{B}$ & NOL ORR & 4 & 3 & 11.70 & 27.2 & 62.4 & 23.6 \\
\hline $\bar{C}$ & DG AND & 4 & 4 & 37.70 & 45.5 & 55.0 & 44.2 \\
\hline $\mathrm{C}$ & DG ROA & 4 & 4 & 38.70 & 46.8 & 56.5 & 45.4 \\
\hline C & DG ORR & 4 & 4 & 46.00 & $\$ 5.6$ & 67.1 & 54.0 \\
\hline C & NOL ORR & 4 & 4 & 54.40 & 65.7 & 79.3 & 63.7 \\
\hline \multicolumn{8}{|c|}{ ANALYSIS=Cobalt } \\
\hline Horizon & Areas & $\mathrm{N}$ & Detect & Median & UCB395 & X95 & LTB9595 \\
\hline $\mathbf{A}$ & DG AND & 4 & 4 & 12.40 & 16.5 & 22.1 & 15.8 \\
\hline A & DG ROA & 4 & 4 & 21.40 & 28.5 & 38.1 & 27.3 \\
\hline $\mathbf{A}$ & DG ORR & 4 & 4 & 14.50 & 19.4 & 25.8 & 18.5 \\
\hline $\mathbf{A}$ & NOL ORR & 4 & 4 & 14.40 & 19.3 & 25.7 & 18.4 \\
\hline B & DG AND & 4 & 4 & 12.20 & 19.2 & 30.2 & 17.9 \\
\hline B & DG ROA & 4 & 4 & 9.80 & 15.4 & 24.3 & 14.4 \\
\hline B & DG ORR & 4 & 4 & 8.92 & 14.1 & 22.1 & 13.1 \\
\hline & NOL ORR & 4 & 4 & 13.40 & 21.1 & 33.3 & 19.7 \\
\hline C & DG AND & 4 & 4 & 14.60 & 21.3 & 31.1 & 20.1 \\
\hline C & DG ROA & 4 & 4 & 10.10 & 14.8 & 21.6 & 13.9 \\
\hline C & DG ORR & 4 & 4 & 12.00 & 17.5 & 25.6 & 16.5 \\
\hline C & NOL ORR & 4 & 4 & 14.50 & 21.2 & 31.0 & 20.0 \\
\hline \multicolumn{8}{|c|}{ ANALYSIS=Copper } \\
\hline Horizon & Areas & $\mathrm{N}$ & Detect & Median & UCB95 & X95 & LTB9595 \\
\hline $\mathbf{A}$ & DG AND & 4 & 4 & 14.9 & 16.9 & 19.2 & 16.6 \\
\hline A & DG ROA & 4 & 4 & 11.0 & 12.5 & 14.3 & 12.3 \\
\hline A & DG ORR & 4 & 4 & 16.1 & 18.3 & 20.8 & 18.0 \\
\hline A & NOL ORR & 4 & 4 & 11.7 & 13.3 & 15.1 & 13.0 \\
\hline B & DG AND & 4 & 4 & 19.0 & 28.3 & 42.2 & 26.6 \\
\hline B & DG ROA & 4 & 4 & 13.7 & 20.4 & 30.4 & 19.2 \\
\hline B & DG ORR & 4 & 4 & 12.5 & 18.6 & 27.7 & 17.5 \\
\hline B & NOL ORR & 4 & 4 & 19.3 & 28.7 & 42.8 & 27.0 \\
\hline C & DG AND & 4 & 4 & 27.3 & 33.7 & 41.6 & 32.6 \\
\hline C & DG ROA & 4 & 4 & 23.8 & 29.4 & 36.3 & 28.5 \\
\hline C & DG ORR & 4 & 4 & 28.7 & 35.4 & 43.7 & 34.3 \\
\hline C & NOL ORR & 4 & 4 & 24.9 & 30.7 & 37.9 & 29.7 \\
\hline
\end{tabular}


Table 5.1 (continued)

\begin{tabular}{|c|c|c|c|c|c|c|c|}
\hline \multicolumn{8}{|c|}{ ANALYSIS=Cyanide } \\
\hline Horizon & Areas & $\mathbf{N}$ & Detect & Median & UCB95 & $\mathrm{X} 95$ & LTB9595 \\
\hline A & DG AND & 4 & 1 & 0.1340 & 0.253 & 0.410 & 0.195 \\
\hline A & DG ROA & 4 & 2 & 0.3190 & 0.583 & 0.979 & 0.447 \\
\hline $\mathbf{A}$ & DG ORR & 3 & 1 & 0.1300 & 0.281 & 0.398 & 0.177 \\
\hline $\mathbf{A}$ & NOL ORR & 4 & 0 & & & & . \\
\hline B & DG AND & 4 & 1 & 0.0688 & 0.210 & 0.291 & 0.102 \\
\hline B & DG ROA & 4 & 0 & & & & . \\
\hline B & DG ORR & 3 & 2 & 0.2460 & 0.594 & 1.040 & 0.292 \\
\hline B & NOL ORR & 4 & 0 & . & • & . & . \\
\hline C & DG AND & 4 & 0 & . & - & . & - \\
\hline C & DG ROA & 4 & 0 & - & - & - & \\
\hline C & DG ORR & 3 & 2 & 0.2660 & 0.760 & 1.450 & 0.278 \\
\hline C & NOL ORR & 4 & 0 &. & $\dot{ }$ & $\cdot$ & $\dot{ }$ \\
\hline \multicolumn{8}{|c|}{ ANALYSIS=Iron } \\
\hline Horizon & Areas & $\mathbf{N}$ & Detect & Median & UCB95 & X95 & LTB9595 \\
\hline $\mathbf{A}$ & DG AND & 4 & 4 & 25600 & 28600 & 32000 & 28100 \\
\hline $\mathbf{A}$ & DG ROA & 4 & 4 & 25400 & 28500 & 31800 & 28000 \\
\hline $\mathbf{A}$ & DG ORR & 4 & 4 & 29400 & 32900 & 36900 & 32400 \\
\hline $\mathbf{A}$ & NOL ORR & 4 & 4 & 27900 & 31200 & 34900 & 30700 \\
\hline B & DG AND & 4 & 4 & 39400 & 44400 & 50000 & 43500 \\
\hline B & DG ROA & 4 & 4 & 32600 & 36700 & 41400 & 36100 \\
\hline B & DG ORR & 4 & 4 & 37300 & 42100 & 47400 & 41300 \\
\hline B & NOL ORR & 4 & 4 & 42400 & 47800 & 53900 & 46900 \\
\hline C & DG AND & 4 & 4 & 42700 & 45500 & 48500 & 45000 \\
\hline C & DG ROA & 4 & 4 & 38800 & 41400 & 44100 & 41000 \\
\hline C & DG ORR & 4 & 4 & 43000 & 45800 & 48800 & 45400 \\
\hline $\mathrm{C}$ & NOL ORR & 4 & 4 & 41700 & 44400 & 47400 & 44000 \\
\hline \multicolumn{8}{|c|}{ ANALYSIS=Lead } \\
\hline Horizon & Areas & $\mathbf{N}$ & Detect & Median & UCB95 & X95 & LTB9595 \\
\hline A & DG AND & 4 & 4 & 28.60 & 67.70 & 160.0 & 58.20 \\
\hline A & DG ROA & 4 & 4 & 23.60 & 55.80 & 132.0 & 48.00 \\
\hline A & DG ORR & 4 & 4 & 20.30 & 48.00 & 113.0 & 41.30 \\
\hline A & NOL ORR & 4 & 3 & 5.51 & 13.10 & 30.8 & 11.40 \\
\hline B & DG AND & 4 & 4 & 18.80 & 40.50 & 87.2 & 35.40 \\
\hline B & DG ROA & 4 & 4 & 12.80 & 27.60 & 59.3 & 24.10 \\
\hline B & DG ORR & 4 & 4 & 11.80 & 25.40 & 54.6 & 22.20 \\
\hline B & NOL ORR & 4 & 3 & 4.19 & 9.08 & 19.4 & 7.98 \\
\hline C & DG AND & 3 & 3 & 21.30 & 34.50 & 49.2 & 28.50 \\
\hline C & DG ROA & 4 & 4 & 15.70 & 23.90 & 36.3 & 22.30 \\
\hline C & DG ORR & 4 & 4 & 14.70 & 22.30 & 34.0 & 20.80 \\
\hline $\mathrm{C}$ & NOL ORR & 4 & 4 & 23.70 & 36.10 & 54.8 & 33.70 \\
\hline
\end{tabular}


Table 5.1 (continned)

\begin{tabular}{|c|c|c|c|c|c|c|c|}
\hline \multicolumn{8}{|c|}{ ANALXSIS=Lithium } \\
\hline Horizon & Areas & $\mathbf{N}$ & Detect & Median & UCB95 & X95 & LTB9595 \\
\hline $\mathbf{A}$ & DG AND & 4 & 4 & 10.4 & 11.8 & 13.4 & 11.5 \\
\hline $\mathbf{A}$ & DG ROA & 4 & 2 & 11.2 & 13.2 & 14.5 & 12.2 \\
\hline $\mathbf{A}$ & DG ORR & 3 & 3 & 16.2 & 18.8 & 20.9 & 17.6 \\
\hline $\mathbf{A}$ & NOL ORR & 4 & 4 & 10.9 & 12.4 & 14.1 & 12.1 \\
\hline $\mathbf{B}$ & DG AND & 4 & 4 & 19.2 & 22.9 & 27.3 & 22.3 \\
\hline B & DG ROA & 4 & 4 & 19.2 & 22.9 & 27.3 & 22.2 \\
\hline B & DG ORR & 4 & 4 & 22.1 & 26.4 & 31.4 & 25.6 \\
\hline B & NOL ORR & 4 & 4 & 23.8 & 28.4 & 33.9 & 27.6 \\
\hline C & DG AND & 4 & 4 & 20.7 & 24.3 & 28.6 & 23.7 \\
\hline C & DG ROA & 4 & 4 & 24.6 & 28.8 & 33.8 & 28.1 \\
\hline C & DG ORR & 4 & 4 & 27.6 & 32.4 & 38.0 & 31.6 \\
\hline C & NOL ORR & 4 & 4 & 23.4 & 27.5 & 32.3 & 26.8 \\
\hline \multicolumn{8}{|c|}{ ANALYSIS=Magnesium } \\
\hline Horizon & Areas & $\mathbf{N}$ & Detect & Median & UCB95 & X95 & LTB9595 \\
\hline $\mathbf{A}$ & DG AND & 4 & 4 & 2690 & 3310 & 4080 & 3200 \\
\hline $\mathbf{A}$ & DG ROA & 4 & 4 & 1580 & 1950 & 2400 & 1890 \\
\hline $\mathbf{A}$ & DG ORR & 4 & 4 & 2850 & 3510 & 4320 & 3390 \\
\hline $\mathbf{A}$ & NOL ORR & 4 & 4 & 2010 & 2470 & 3050 & 2390 \\
\hline B & DG AND & 4 & 4 & 2890 & 3260 & 3680 & 3200 \\
\hline B & DG ROA & 4 & 4 & 1980 & 2240 & 2530 & 2200 \\
\hline B & DG ORR & 4 & 4 & 3280 & 3700 & 4180 & 3630 \\
\hline B - & NOL ORR & 4 & 4 & 2720 & 3080 & 3480 & 3020 \\
\hline C & DG AND & 4 & 4 & 3560 & 4100 & 4730 & 4010 \\
\hline C & DG ROA & 4 & 4 & 3010 & 3460 & 3990 & 3390 \\
\hline C & DG ORR & 4 & 4 & 4370 & 5030 & 5790 & 4920 \\
\hline C & NOL ORR & 4 & 4 & 3380 & 3890 & 4480 & 3810 \\
\hline \multicolumn{8}{|c|}{ ANALYSIS=Manganese } \\
\hline Horizon & Areas & $\mathbf{N}$ & Detect & Median & UCB95 & X95 & LTB9595 \\
\hline $\mathbf{A}$ & DG AND & 4 & 4 & 708 & 957 & 1290 & 913 \\
\hline $\mathbf{A}$ & DG ROA & 4 & 4 & 1720 & 2330 & 3140 & 2220 \\
\hline $\mathbf{A}$ & DG ORR & 4 & 4 & 997 & 1350 & 1820 & 1290 \\
\hline $\mathbf{A}$ & NOL ORR & 4 & 4 & 653 & 883 & 1190 & 842 \\
\hline B & DG AND & 4 & 4 & 279 & 469 & 789 & 432 \\
\hline B & DG ROA & 4 & 4 & 341 & 574 & 966 & 529 \\
\hline B & DG ORR & 4 & 4 & 279 & 469 & 789 & 432 \\
\hline B & NOL ORR & 4 & 4 & 265 & 445 & 749 & 410 \\
\hline $\mathrm{C}$ & DG AND & 4 & 4 & 535 & 989 & 1830 & 898 \\
\hline C & DG ROA & 4 & 4 & 265 & 491 & 907 & 446 \\
\hline C & DG ORR & 4 & 4 & 344 & 635 & 1170 & 577 \\
\hline C & NOL ORR & 4 & 4 & 321 & 594 & 1100 & 540 \\
\hline
\end{tabular}




\section{$5-13$}

Table 5.1 (continued)

\begin{tabular}{|c|c|c|c|c|c|c|c|}
\hline \multicolumn{8}{|c|}{ ANALYSIS=Mercury } \\
\hline Horizon & Areas & $\mathbf{N}$ & Detect & Median & UCB95 & X95 & LTB9595 \\
\hline $\mathbf{A}$ & DG AND & 4 & 1 & 0.104 & 0.1160 & 0.1220 & 0.1090 \\
\hline A & DG ROA & 4 & 2 & 0.161 & 0.1760 & 0.1890 & 0.1720 \\
\hline A & DG ORR & 4 & 4 & 0.316 & 0.3420 & 0.3700 & 0.3360 \\
\hline $\mathbf{A}$ & NOL ORR & 4 & 4 & 0.185 & 0.2000 & 0.2170 & 0.1960 \\
\hline B & DG AND & 4 & 0 & & & & \\
\hline B & DG ROA & 4 & 1 & 0.142 & 0.1690 & 0.1790 & 0.1520 \\
\hline B & DG ORR & 4 & 2 & 0.154 & 0.1780 & 0.1950 & 0.1620 \\
\hline B & NOL ORR & 4 & 0 & . & & . & . \\
\hline $\mathrm{C}$ & DG AND & 4 & 0 & . & & & \\
\hline $\mathrm{C}$ & DG ROA & 4 & 1 & 0.141 & 0.1570 & 0.1610 & 0.1450 \\
\hline C & DG ORR & 4 & 1 & 0.060 & 0.0685 & 0.0685 & 0.0571 \\
\hline C & NOL ORR & 4 & 0 & . & . & . & $\therefore$ \\
\hline \multicolumn{8}{|c|}{ ANALYSIS=Molybdenum } \\
\hline Horizon & Areas & $\mathbf{N}$ & Detect & Median & UCB95 & X95 & LTB9595 \\
\hline A & DG AND & 4 & 0 & . & . & . & . \\
\hline A & DG ROA & 4 & 0 & . & . & . & • \\
\hline A & DG ORR & 4 & 0 & . & . & . & . \\
\hline A & NOL ORR & 4 & 0 & . & & . & . \\
\hline B & DG AND & 4 & 1 & 1.50 & 1.85 & 1.95 & 1.58 \\
\hline B & DG ROA & 4 & 0 & . & . & . & . \\
\hline B & DG ORR & 4 & 0 & & . & . & . \\
\hline B & NOL ORR & 4 & 1 & 1.57 & 1.93 & 2.04 & 1.68 \\
\hline $\mathrm{C}$ & DG AND & 4 & 2 & 1.72 & 1.87 & 1.97 & 1.75 \\
\hline C & DG ROA & 4 & 0 & . & . & - & . \\
\hline C & DG ORR & 4 & 0 & . & . & . & . \\
\hline $\mathrm{C}$ & NOL ORR & 4 & 0 & . & $\cdot$ & . & - \\
\hline \multicolumn{8}{|c|}{ ANALYSIS=Nickel } \\
\hline Horizon & Areas & $\mathbf{N}$ & Detect & Median & UCB95 & X95 & LTB9595 \\
\hline $\mathbf{A}$ & DG AND & 4 & 4 & 20.8 & 24.8 & 29.6 & 24.1 \\
\hline A & DG ROA & 4 & 4 & 16.7 & 20.0 & 23.8 & 19.4 \\
\hline $\mathbf{A}$ & DG ORR & 4 & 4 & 23.5 & 28.0 & 33.4 & 27.2 \\
\hline $\mathbf{A}$ & NOL ORR & 4 & 4 & 17.3 & 20.6 & 24.6 & 20.0 \\
\hline B & DG AND & 4 & 4 & 24.3 & 27.1 & 30.3 & 26.7 \\
\hline B & DG ROA & 4 & 4 & 17.9 & 20.0 & 22.4 & 19.7 \\
\hline B & DG ORR & 4 & 4 & 22.9 & 25.6 & 28.6 & 25.1 \\
\hline B & NOL ORR & 4 & 4 & 20.8 & 23.3 & 26.0 & 22.9 \\
\hline C & DG AND & 4 & 4 & 29.2 & 33.5 & 38.5 & 32.8 \\
\hline C & DG ROA & 4 & 4 & 26.9 & 30.9 & 35.4 & 30.2 \\
\hline C & DG ORR & 4 & 4 & 28.8 & 33.1 & 38.0 & 32.4 \\
\hline $\mathrm{C}$ & NOL ORR & 4 & 4 & 24.3 & 27.9 & 32.0 & 27.3 \\
\hline
\end{tabular}


Table 5.1 (continued)

\begin{tabular}{|c|c|c|c|c|c|c|c|}
\hline \multicolumn{8}{|c|}{ ANALYSIS=Potassium } \\
\hline Horizon & Areas & $\mathbf{N}$ & Detect & Median & UCB95 & $\mathrm{X95}$ & LTB9595 \\
\hline $\mathbf{A}$ & DG AND & 4 & 4 & 3890 & 4760 & 5830 & 4610 \\
\hline $\mathbf{A}$ & DG ROA & 4 & 4 & 1300 & 1600 & 1950 & 1550 \\
\hline A & DG ORR & 4 & 4 & 2300 & 2820 & 3450 & 2730 \\
\hline A & NOL ORR & 4 & 4 & 2950 & 3610 & 4420 & 3500 \\
\hline B & DG AND & 4 & 4 & 3850 & 4520 & 5310 & 4410 \\
\hline B & DG ROA & 4 & 4 & 1730 & 2040 & 2390 & 1980 \\
\hline B & DG ORR & 4 & 4 & 2590 & 3040 & 3560 & 2960 \\
\hline B & NOL ORR & 4 & 4 & 3690 & 4340 & 5090 & 4230 \\
\hline $\mathrm{C}$ & DG AND & 4 & 4 & 4460 & 5130 & 5910 & 5020 \\
\hline $\mathrm{C}$ & DG ROA & 4 & 4 & 2490 & 2870 & 3300 & 2800 \\
\hline C & DG ORR & 4 & 4 & 3130 & 3600 & 4150 & 3520 \\
\hline $\mathrm{C}$ & NOL ORR & 4 & 4 & 5020 & 5780 & 6650 & 5650 \\
\hline \multicolumn{8}{|c|}{ ANALYSIS=Selenium } \\
\hline Horizon & Areas & $\mathbf{N}$ & Detect & Median & UCB95 & X95 & LTB9595 \\
\hline $\mathbf{A}$ & DG AND & 4 & 4 & 0.746 & 0.933 & 1.170 & 0.869 \\
\hline $\mathbf{A}$ & DG ROA & 4 & 1 & 0.728 & 0.998 & 1.140 & 0.821 \\
\hline $\mathbf{A}$ & DG ORR & 4 & 0 & & & & \\
\hline $\mathbf{A}$ & NOL ORR & 4 & 3 & 0.566 & 0.713 & 0.885 & 0.666 \\
\hline B & DG AND & 4 & 4 & 0.676 & 0.811 & 0.973 & 0.768 \\
\hline B & DG ROA & 4 & 1 & 0.429 & 0.598 & 0.617 & 0.434 \\
\hline B & DG ORR & 4 & 0 & & & & \\
\hline B & NOL ORR & 4 & 3 & 0.649 & 0.782 & 0.934 & 0.742 \\
\hline C & DG AND & 4 & 3 & 0.494 & 0.643 & 0.821 & 0.581 \\
\hline C & DG ROA & 4 & 0 & . & . & - & . \\
\hline C & DG ORR & 4 & 0 & & & & \\
\hline C & NOL ORR & 4 & 3 & 0.816 & 1.060 & 1.350 & 0.966 \\
\hline \multicolumn{8}{|c|}{ ANALYSIS=Silicon } \\
\hline Horizon & Areas & $\mathbf{N}$ & Detect & Median & UCB95 & X95 & LTB9595 \\
\hline $\mathbf{A}$ & DG AND & 4 & 4 & 221 & 246 & 274 & 242 \\
\hline A & DG ROA & 4 & 4 & 484 & 539 & 600 & 530 \\
\hline A & DG ORR & 4 & 4 & 506 & 563 & 627 & 554 \\
\hline A & NOL ORR & 4 & 4 & 245 & 272 & 303 & 268 \\
\hline B & DG AND & 4 & 4 & 239 & 276 & 317 & 270 \\
\hline B & DG ROA & 4 & 4 & 519 & 597 & 687 & 584 \\
\hline B & DG ORR & 4 & 4 & 491 & 565 & 650 & 553 \\
\hline B & NOL ORR & 4 & 4 & 248 & 285 & 328 & 279 \\
\hline C & DG AND & 4 & 4 & 214 & 250 & 292 & 244 \\
\hline $\mathrm{C}$ & DG ROA & 4 & 4 & 423 & 494 & 577 & 482 \\
\hline C & DG ORR & 4 & 4 & 514 & 600 & 701 & 586 \\
\hline $\mathrm{C}$ & NOL ORR & 4 & 4 & $28 r_{3}$ & 328 & 383 & 320 \\
\hline \multicolumn{8}{|c|}{$\begin{array}{c}\text { ANALYSIS=Silver } \\
\text { (No detects) }\end{array}$} \\
\hline
\end{tabular}


Table 5.1 (continued)

\begin{tabular}{|c|c|c|c|c|c|c|c|}
\hline \multicolumn{8}{|c|}{$\begin{array}{c}\text { ANALYSIS=Sodium } \\
\text { (No detects) }\end{array}$} \\
\hline \multicolumn{8}{|c|}{ ANALYSIS=Strontium } \\
\hline Horizon & Areas & $\mathbf{N}$ & Detect & Median & UCB95 & X95 & LTB9595 \\
\hline $\mathbf{A}$ & DG AND & 4 & 4 & 6.18 & 7.70 & 9.58 & 7.42 \\
\hline A & DG ROA & 4 & 4 & 4.97 & 6.18 & 7.70 & 5.96 \\
\hline A & DG ORR & 3 & 3 & 7.93 & 10.20 & 12.30 & 9.25 \\
\hline $\mathbf{A}$ & NOL ORR & 4 & 4 & 4.55 & 5.67 & 7.05 & 5.46 \\
\hline B & DG AND & 4 & 4 & 4.32 & 5.19 & 6.24 & 5.04 \\
\hline B & DG ROA & 4 & 4 & 4.63 & 5.57 & 6.70 & 5.41 \\
\hline B & DG ORR & 4 & 4 & 7.52 & 9.04 & 10.90 & 8.79 \\
\hline B & NOL ORR & 4 & 4 & 5.52 & 6.63 & 7.97 & 6.44 \\
\hline $\mathrm{C}$ & DG AND & 4 & 4 & 3.76 & 4.85 & 6.26 & 4.66 \\
\hline C & DG ROA & 4 & 4 & 4.17 & 5.38 & 6.95 & 5.17 \\
\hline C & DG ORR & 4 & 4 & 8.97 & 11.60 & 15.00 & 11.10 \\
\hline C & NOL ORR & 4 & 4 & 5.19 & 6.70 & 8.66 & 6.44 \\
\hline \multicolumn{8}{|c|}{ ANALYSIS=Sulfate } \\
\hline Horizon & Areas & $\mathbf{N}$ & Detect & Median & UCB95 & X95 & LTB9595 \\
\hline $\mathbf{A}$ & DG AND & 4 & 4 & 14.1 & 17.4 & 21.5 & 16.8 \\
\hline A & DG ROA & 4 & 4 & 69.9 & 86.4 & 107.0 & 83.4 \\
\hline $\mathbf{A}$ & DG ORR & 3 & 3 & 86.7 & 111.0 & 133.0 & 101.0 \\
\hline A & NOL ORR & 4 & 4 & 18.7 & 23.1 & 28.6 & 22.3 \\
\hline B & DG AND & 4 & 4 & 41.8 & 60.6 & 88.0 & 57.2 \\
\hline B & DG ROA & 4 & 4 & 134.0 & 195.0 & 283.0 & 184.0 \\
\hline B & DG ORR & 4 & 4 & 103.0 & 149.0 & 217.0 & 141.0 \\
\hline B & NOL ORR & 4 & 4 & 79.0 & 115.0 & 166.0 & 108.0 \\
\hline $\mathrm{C}$ & DG AND & 4 & 4 & 16.0 & 20.0 & 24.8 & 19.3 \\
\hline C & DG ROA & 4 & 4 & 47.2 & 58.6 & 72.9 & 56.7 \\
\hline $\mathrm{C}$ & DG ORR & 4 & 4 & 129.0 & 161.0 & 200.0 & 155.0 \\
\hline $\mathrm{C}$ & NOL ORR & 4 & 4 & 38.3 & 47.6 & 59.2 & 46.0 \\
\hline \multicolumn{8}{|c|}{ ANALYSIS = Thallium } \\
\hline Horizon & Areas & $\mathbf{N}$ & Detect & Median & UCB95 & X95 & LTB9595 \\
\hline $\mathbf{A}$ & DG AND & 4 & 0 & & & & \\
\hline A & DG ROA & 4 & 1 & 0.104 & 0.423 & 0.527 & 0.145 \\
\hline A & DG ORR & 4 & 1 & 0.164 & 0.608 & 0.825 & 0.240 \\
\hline A & NOL ORR & 4 & 0 & . & . & . & . \\
\hline B & DG AND & 4 & 0 & & & . & \\
\hline B & DG ROA & 4 & 1 & 0.218 & 0.424 & 0.527 & 0.275 \\
\hline B & DG ORR & 4 & 2 & 0.315 & 0.523 & 0.761 & 0.403 \\
\hline B & NOL ORR & 4 & 1 & 0.320 & 0.623 & 0.773 & 0.410 \\
\hline C & DG AND & 4 & 0 & & & & \\
\hline C & DG ROA & 4 & 1 & 0.257 & 0.404 & 0.511 & 0.327 \\
\hline $\mathrm{C}$ & DG ORR & 4 & 2 & 0.336 & 0.499 & 0.667 & 0.427 \\
\hline $\mathrm{C}$ & NOL ORR & 4 & 4 & 0.576 & 0.813 & 1.150 & 0.714 \\
\hline
\end{tabular}


Table 5.1 (continued)

\begin{tabular}{cccccccr}
\hline \multicolumn{7}{c}{ ANALYSIS=Vanadium } & \\
\hline Horizon & Areas & N & Detect & Median & UCB95 & X95 & LTB9595 \\
\hline A & DG AND & 4 & 4 & 30.3 & 32.7 & 35.3 & 32.4 \\
A & DG ROA & 4 & 4 & 32.2 & 34.8 & 37.5 & 34.4 \\
A & DG ORR & 4 & 4 & 34.2 & 36.8 & 39.8 & 36.4 \\
A & NOL ORR & 4 & 4 & 32.4 & 35.0 & 37.7 & 34.5 \\
B & DG AND & 4 & 4 & 44.8 & 65.8 & 96.6 & 61.9 \\
B & DG ROA & 4 & 4 & 39.1 & 57.4 & 84.3 & 54.0 \\
B & DG ORR & 4 & 4 & 23.4 & 34.4 & 50.5 & 32.4 \\
B & NOL ORR & 4 & 4 & 45.9 & 67.4 & 98.9 & 63.4 \\
C & DG AND & 4 & 4 & 42.6 & 45.8 & 49.2 & 45.3 \\
C & DG ROA & 4 & 4 & 35.0 & 37.6 & 40.4 & 37.2 \\
C & DG ORR & 4 & 4 & 46.6 & 50.1 & 53.8 & 49.5 \\
C & NOL ORR & 4 & 4 & 41.4 & 44.5 & 47.8 & 44.0 \\
\hline & & & ANALYSIS=Zinc & & & \\
\hline Horizon & Areas & N & Detect & Median & UCB95 & X95 & LTB9595 \\
\hline A & DG AND & 4 & 4 & 49.7 & 57.0 & 65.4 & 55.8 \\
A & DG ROA & 4 & 4 & 40.7 & 46.7 & 53.6 & 45.7 \\
A & DG ORR & 4 & 4 & 50.6 & 58.1 & 66.7 & 56.9 \\
A & NOL ORR & 4 & 4 & 37.9 & 43.5 & 49.9 & 42.6 \\
B & DG AND & 4 & 4 & 51.0 & 55.9 & 61.2 & 55.1 \\
B & DG ROA & 4 & 4 & 41.1 & 45.1 & 49.4 & 44.4 \\
B & DG ORR & 4 & 4 & 51.5 & 56.4 & 61.8 & 55.6 \\
B & NOL ORR & 4 & 4 & 44.5 & 48.7 & 53.4 & 48.0 \\
C & DG AND & 4 & 4 & 59.5 & 65.9 & 73.0 & 64.9 \\
C & DG ROA & 4 & 4 & 51.1 & 56.5 & 62.6 & 55.6 \\
C & DG ORR & 4 & 4 & 61.5 & 68.1 & 75.4 & 67.0 \\
C & NOL ORR & 4 & 4 & 44.6 & 49.4 & 54.7 & 48.6 \\
\hline
\end{tabular}

A sense of the statistical accuracy of the results can be obtained by comparing the estimates to their corresponding confidence bounds: the median to the UCB95 and the X95 to the LTB9595, and by comparing the two confidence bounds. Consider, for example, the beryllium, A horizon, ORR row in Table 5.1. The median and 95th percentile estimates are 0.78 and $1.28 \mathrm{mg} / \mathrm{kg}$ per gram. But, as indicated, we can be $95 \%$ confident only that the median is less than 1.00, and $95 \%$ confident that the 95th percentile (for composites of three) exceeds $0.96 \mathrm{mg} / \mathrm{kg}$. On the basis of these data and statistical arguments, one could not rule out beryllium contamination at a new test location, unless the level there was less than about 0.96. Since we are $95 \%$ certain only that the median is less than 1.00 , we cannot be sure that we will not receive samples in the future which are not contaminated, but for which contamination cannot be ruled out on the basis of these data. This is an unavoidable consequence of the study's small sample sizes. Of course, in practice, on the basis of risk analysis, EPA guidelines, etc., levels much higher than this might be needed to trigger an alarm. Nevertheless, on a purely statistical basis, the results are inadequate. To increase 
statistical precision, further combining of data may be necessary [e.g., over additional areas (from Phase II data)], or it may simply be necessary to collect more data.

On the other hand, the overlap between LTB9595 (0.96) and UCB95 (1.00) is slight. With additional Phase II data, the resolution will be better. In Sect. 5.6 it is shown that for some of the PAHs (e.g., acenaphthene in Anderson County), it already is.

The usual summary statistics are not meaningful when all of the observations are nondetects. For these inorganics, Table 5.2 is an alternative. Table 5.2 contains $95 \%$ UCBs for the detection probability for those analytes having fewer than three detects in all four areas. Field duplicates were dropped. (So there may be a few discrepancies between values of the variable "Detect" in Tables 5.1 and 5.2.) The maximum detection limit is also given. The maximum detection limits are computed only from nondetects. (Detection limits for the detects were not provided.)

Results in Table 5.2 have been combined over areas to increase the sample sizes. Still, the UCBs are considerably above 0.05 . To be useful they will have to be recomputed using more data or more combinations of areas, assuming the proportion of detects in the additional data is about the same as in the original data. Probabilities of detecting higher amounts in the more numerous original samples (before compositing) can also be estimated.

Table 5.2. Additional summary statistics for inorganics: for mostly undetected $95 \%$ UCBs for detection probability (Data combined over sampling areas)

\begin{tabular}{lccccc}
\hline & & & & $\begin{array}{c}\text { Maximum } \\
\text { detection }\end{array}$ & \\
Analysis & Horizon & $\mathrm{N}$ & Detect & limit $(\mathrm{mg} / \mathrm{kg})$ & UCB \\
\hline Antimony & $\mathrm{A}$ & 16 & 2 & 1.40 & 0.34 \\
Cadmium & $\mathrm{A}$ & 16 & 0 & 0.25 & 0.17 \\
Cadmium & $\mathrm{B}$ & 16 & 0 & 0.24 & 0.17 \\
Cadmium & $\mathrm{C}$ & 16 & 0 & 0.31 & 0.17 \\
Cyanide & $\mathrm{C}$ & 16 & 2 & 0.44 & 0.34 \\
Mercury & $\mathrm{C}$ & 16 & 2 & 0.21 & 0.34 \\
Molybdenum & $\mathrm{A}$ & 16 & 1 & 9.80 & 0.26 \\
Molybdenum & $\mathrm{B}$ & 16 & 2 & 9.80 & 0.34 \\
Molybdenum & $\mathrm{C}$ & 16 & 2 & 12.80 & 0.34 \\
Osmium & $\mathrm{A}$ & 4 & 0 & 14.80 & 0.53 \\
Osmium & $\mathrm{B}$ & 5 & 0 & 15.20 & 0.45 \\
Osmium & $\mathrm{C}$ & 5 & 0 & 19.90 & 0.45 \\
Silver & $\mathrm{A}$ & 16 & 0 & 2.10 & 0.17 \\
Silver & $\mathrm{B}$ & 16 & 0 & 2.20 & 0.17 \\
Silver & $\mathrm{C}$ & 16 & 0 & 2.80 & 0.17 \\
Sodium & $\mathrm{A}$ & 1 & 0 & 301.00 & 0.95 \\
Sodium & $\mathrm{B}$ & 1 & 0 & 332.00 & 0.95 \\
Sodium & $\mathrm{C}$ & 1 & 0 & 305.00 & 0.95 \\
Thallium & $\mathrm{A}$ & 16 & 2 & 0.51 & 0.34 \\
\hline
\end{tabular}


Using the criteria "significance level $<0.01$," the following analyte-horizon combinations show significant differences among areas: aluminum $A, B, C$; antimony $A$; arsenic $C$; barium $B(p=0.06$ for F-test), $C$; copper $A$; lithium $A$; magnesium $A, B$; manganese $A$; mercury $A$, C; nickel $B(p=0.08$ for F-test); potassium $A, B, C$; silicon $A, B, C$; strontium $B, C$; sulfate $A, B, C$; vanadium $C$; and zinc $B(p=0.08$ for $F$-test), $C$. Of these, all but zinc $C$ show significant differences among the three Dismal Gap areas.

To further explore the differences of antimony, arsenic, lithium, mercury, and sulfate A, which had some nondetects, see Table 5.1. For arsenic, for example, it is clear from the table that levels are higher for the ORR Dismal Gap Formation than for the other areas. The analytes with no nondetects can be examined in the same way, or using the usual F-test (Proc GLM) approach. Consider, for example, aluminum. The horizon $A$ data have the outlier mentioned above, so consider horizon B. Here there is a significant $(p=0.006)$ difference between the Dismal Gap Formation in Anderson County and the Dismal Gap Formation in Roane County, but not between the Nolichucky and Dismal Gap formations on the ORR, or for the comparison of the Dismal Gap Formation on- and off-reservation, or for the comparison of on- and off-reservation in general. This is illustrated in Fig. 5.2. Other comparisons can also be made.

\subsection{HERBICIDES}

All results for herbicides are from the Dismal Gap Formation A horizon, and all are nondetects. There are data for only 11 sites from the ORR and 8 from Roane County. There are no statistical outliers. Graphical examination reveals that the field duplicate and original are generally in extremely close agreement. This suggests that perhaps the designation " $U$ " for nondetect has been applied too conservatively. Of course, these data are nevertheless handled here as nondetects. The summary Table 5.3 parallels Table 5.2 for the inorganics. For a fixed $N$ (number of samples), as long as the number of detects is fixed (e.g., at 0 ), the UCB is the same.

Table 5.3. Herbicides 95\% UCBs for detection probabilities (Data combined over sampling areas)

\begin{tabular}{lrcrr}
\hline Analysis & N & Detect & $\begin{array}{c}\text { Maximum } \\
\text { detection } \\
\text { limit }(\mu \mathrm{g} / \mathrm{kg})\end{array}$ & UCB \\
\hline 2,4,5-T & 19 & 0 & 316 & 0.15 \\
2,4-D & 19 & 0 & 1894 & 0.15 \\
2,4-DB & 19 & 0 & 1421 & 0.15 \\
Dalapon & 7 & 0 & 937 & 0.35 \\
Dicamba & 19 & 0 & 421 & 0.15 \\
Dichlorprop & 19 & 0 & 1052 & 0.15 \\
Dinoseb & 19 & 0 & 221 & 0.15 \\
MCPA & 19 & 0 & 394685 & 0.15 \\
MCPP & 19 & 0 & 299961 & 0.15 \\
Silvex & 19 & 0 & 263 & 0.15 \\
\hline
\end{tabular}




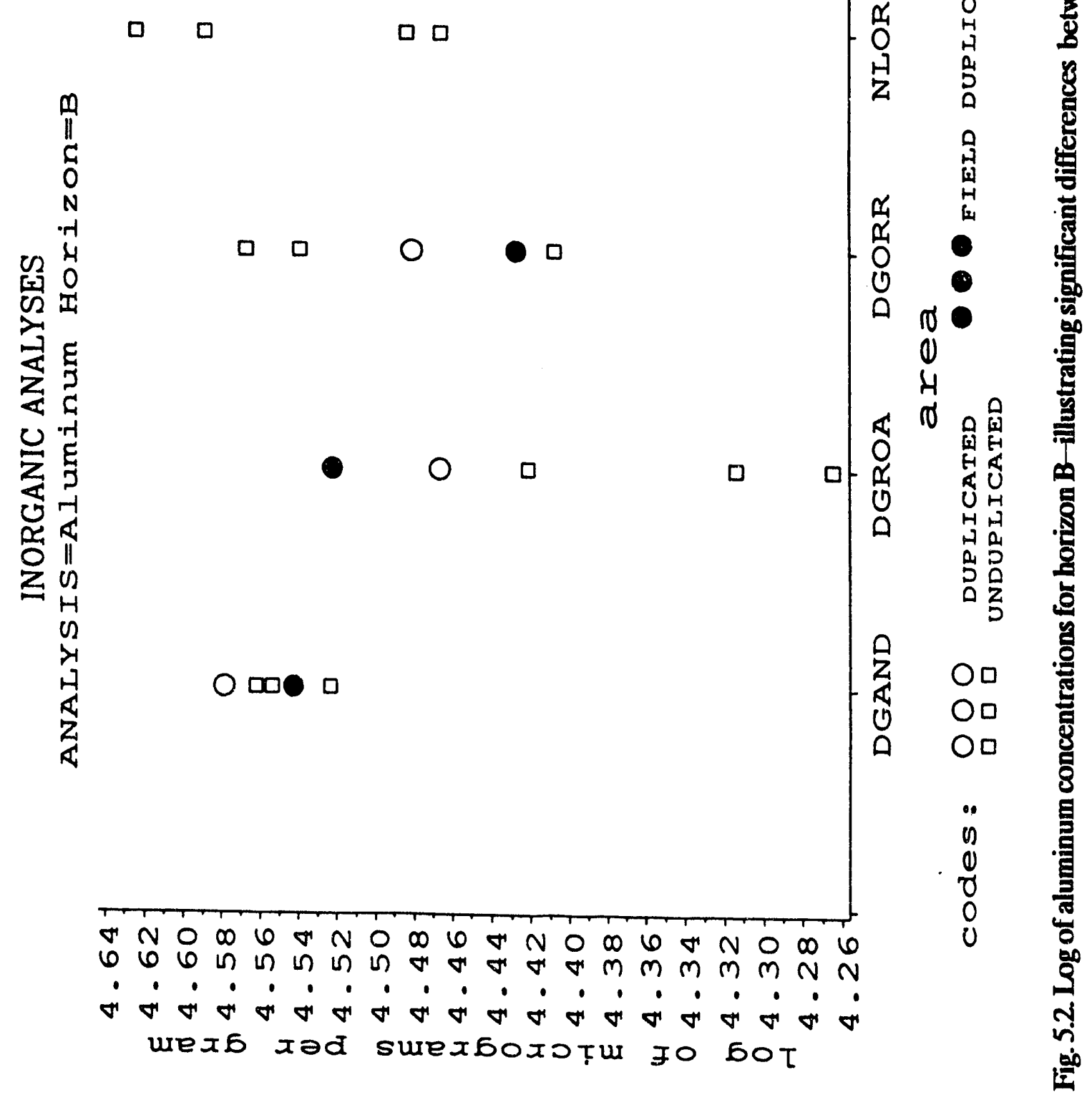


As with the inorganics, these UCBs are currently too large to be of much practical value. By combining results with future (Phase II) results, the number of samples will be increased. Assuming that future data contain very few nondetects, these UCBs will come down much closer to the 0.05 range. Then, a detect will reflect, statistically, a departure from background.

\subsection{PESTICIDES}

All pesticide results are for the $A$ horizon. There are no statistical outliers and no validation rejects. As with the herbicides, field duplicates and original results are all very close. Table 5.4 for pesticides is analogous to Table 5.2 for inorganics. To compute the table, field duplicates were deleted. Where duplicate analyses were performed, originals were also deleted. There are only two detects.

Table 5.4. Pesticides 95\% UCBs for detection probabilities (Data combined over sampling areas)

\begin{tabular}{lcccc}
\hline & \multicolumn{3}{c}{$\begin{array}{c}\text { Maximum } \\
\text { detection }\end{array}$} \\
Analysis & $\mathrm{N}$ & Detect & limit $(\mu \mathrm{g} / \mathrm{kg})$ & UCB \\
\hline 4,4'-DDD & 48 & 0 & 13.0 & 0.06 \\
$4,4^{\prime}$-DDE & 48 & 0 & 13.0 & 0.06 \\
4,4'-DDT & 48 & 1 & 13.0 & 0.10 \\
Aldrin & 48 & 0 & 6.3 & 0.06 \\
Aroclor-1016 & 48 & 0 & 130.0 & 0.06 \\
Aroclor-1221 & 48 & 0 & 254.0 & 0.06 \\
Aroclor-1232 & 48 & 0 & 130.0 & 0.06 \\
Aroclor-1242 & 48 & 1 & 130.0 & 0.10 \\
Aroclor-1248 & 48 & 0 & 130.0 & 0.06 \\
Aroclor-1254 & 48 & 0 & 130.0 & 0.06 \\
Aroclor-1260 & 48 & 0 & 130.0 & 0.06 \\
Dieldrin & 48 & 0 & 13.0 & 0.06 \\
Endosulfan I & 48 & 0 & 6.3 & 0.06 \\
Endosulfan II & 48 & 0 & 13.0 & 0.06 \\
Endosulfan sulfate & 48 & 0 & 13.0 & 0.06 \\
Endrin & 48 & 0 & 13.0 & 0.06 \\
Endrin aldehyde & 48 & 0 & 13.0 & 0.06 \\
Endrin ketone & 48 & 0 & 13.0 & 0.06 \\
Heptachlor & 48 & 0 & 6.3 & 0.06 \\
Heptachlor epoxide & 48 & 0 & 6.3 & 0.06 \\
Methoxychlor & 48 & 0 & 63.0 & 0.06 \\
Toxaphene & 48 & 0 & 630.0 & 0.06 \\
alpha-BHC & 48 & 0 & 6.3 & 0.06 \\
alpha-Chlordane & 48 & 0 & 6.3 & 0.06 \\
beta-BHC & 48 & 0 & 6.3 & 0.06 \\
delta-BHC & 48 & 0 & 6.3 & 0.06 \\
gamma-BHC (Lindane) & 48 & 0 & 6.3 & 0.06 \\
gamma-Chlordane & 48 & 0 & & \\
\hline & & & 0.06 \\
\hline
\end{tabular}




\subsection{PAHS}

All PAH results are for the A horizon. Most, but not all, of the results are nondetects. Many results have the validation designation "R," and are thus not used in the statistical analysis. (All of the originals in the original-reanalysis pairs are so designated). There are no statistical outliers.

Again, the field duplicates and originals are nearly identical for all of the PAHs and are exactly equal for most. There are large deviations between the detection limits for some analyses; in the case of acenaphthene, the deviations are several factors of 10.

Table 5.5 shows the pattern of detects and UCBs for the detection probability. Table 5.6 gives summary statistics for those few PAHs having one or more detects. Tables 5.5 and 5.6 parallel Tables 5.2 and 5.1 for the inorganics.

Of the PAHs, only pyrene showed a significant $(p<0.01)$ difference across areas, based on the Lifereg analysis. Pyrene also shows significant differences within the Dismal Gap areas. Notice that for a few of these analytes and areas, in contrast to the inorganics, LTB9595 does exceed UCB95 (e.g., for acenaphthene or benzopyrene in Anderson County).

\subsection{RADIONUCLIDES}

Many (74) of the radionuclide soil results are validation rejects, and in general, the data structures for radionuclides vary considerably with analyte. Only a few of the anomalies are listed here: There is almost no curium-244 data or neptunium-237 data. There are no Nolichucky ORR results for many analytes (e.g., hafnium-181 and iridium-192). There are fairly large discrepancies between many of the field duplicates and originals (e.g., for americium-241 Roane County horizon A, or cobalt-60 Roane County horizon C). One of the niobium-95 detection limits $(82,000 \mathrm{pCi} / \mathrm{g})$ is clearly an outlier. All of the technetium- 99 data are validation rejects.

Results for the mostly undetected analytes are summarized in Table 5.7. Results for the detects are summarized in Table 5.8.

On the basis of the chi-square tests in Proc Lifereg, the following radionuclides-horizons show significant differences $(p<0.01$ ) over areas: plutonium-238 A; radium-226 A, B, C; thorium-228 A; thorium-232 A; thorium-234 A, C. The all-detect chi-square tests tracked the usual (F-test) results quite closely, though the significance levels are all a little lower. All of these showed significant differences within the Dismal Gap areas, except for thorium-228 A and thorium-232 A.

To further explore the nature of the differences, see Table 5.8 or the data itself. For data with all detects, formal comparisons can also be made using Proc GLM. For example, on the ORR, horizon A thorium-232 levels in the Nolichucky Formation are significantly higher than levels in the Dismal Gap Formation ( $p=0.0007$ ).

Duplications of these results and related factors are discussed in Sect. 5.11 and in Sects. 6 and 8 of this report. 
Table 5.5. PAHs 95\% UCBs for detection probability for analytes with fewer than three detects

(Data combined over sampling areas)

\begin{tabular}{lcccc}
\hline Analysis & N & Detect & $\begin{array}{c}\text { Maximum } \\
\text { detection limit } \\
(\mu \mathrm{g} / \mathrm{kg})\end{array}$ & UCB \\
\hline Acenaphthylene & 42 & 2 & 15130 & 0.14 \\
Anthracene & 42 & 0 & 4342 & 0.07 \\
Benzo[ghi]perylene & 35 & 1 & 500 & 0.13 \\
Benzo[k]fluoranthene & 42 & 1 & 112 & 0.11 \\
Chrysene & 30 & 0 & 987 & 0.10 \\
Dibenzo[ah]anthracene & 34 & 0 & 197 & 0.08 \\
Fluoranthene & 12 & 2 & 355 & 0.44 \\
Indeno123cdpyrene & 42 & 0 & 283 & 0.07 \\
Naphthalene & 43 & 2 & 11841 & 0.14 \\
\hline
\end{tabular}

Table 5.6. Additional summary statistics for PAHis with several detects

\begin{tabular}{lccrrrrr}
\hline Analysis & Areas & N & Detect & Median & UCB95 & X95 & LTB9595 \\
\hline Acenaphthene & DG AND & 10 & 8 & 3.400 & 6.02 & 19.60 & 8.44 \\
Acenaphthene & NOL ORR & 12 & 1 & 35.800 & 118.00 & 206.00 & 73.90 \\
Acenaphthylene & DG AND & 8 & 1 & 7.870 & 53.30 & 127.00 & 23.70 \\
Benzo[a]anthracene & DG AND & 11 & 10 & 1.750 & 2.39 & 4.88 & 3.01 \\
Benzo[a]pyrene & DG AND & 11 & 7 & 2.420 & 3.08 & 4.98 & 3.64 \\
Benzo[a]pyrene & DG ROA & 11 & 1 & 0.959 & 1.84 & 1.97 & 1.01 \\
Benzo[a]pyrene & NOL ORR & 12 & 1 & 0.942 & 1.77 & 1.94 & 1.03 \\
Benzo[b]fluoranthene & DG ROA & 11 & 2 & 1.250 & 1.63 & 1.94 & 1.48 \\
Benzo[b]fluoranthene & NOL ORR & 12 & 4 & 1.160 & 1.42 & 1.79 & 1.42 \\
Fluoranthene & DG AND & 8 & 2 & 1.950 & 20.30 & 50.00 & 6.37 \\
Fluoiene & DG AND & 8 & 3 & 3.370 & 14.30 & 71.80 & 14.10 \\
Fluorene & DG ROA & 11 & 1 & 5.650 & 50.60 & 121.00 & 20.70 \\
& & & 1 & 10.000 & 93.60 & 214.00 & 33.90 \\
Fluorene & DG ORR & 11 & 1 & 171.000 & 342.000 & 495.00 & 233.00 \\
Naphthalene & DG ROA & 12 & 2 & 11.00 & 37.80 \\
Phenanthrene & DG AND & 11 & 10 & 14.800 & 25.30 & 86.00 & 37.00 \\
Phenanthrene & DG ROA & 11 & 1 & 36.400 & 112.00 & 211.00 & 73.20 \\
Pyrene & NOL ORR & 12 & 2 & 10.500 & 11.10 & 11.30 & 10.40 \\
\hline
\end{tabular}


Table 5.7. Radionuclide $95 \%$ UCBe for detection probubility with fewer than two detects (Data combinod over zampling areas)

\begin{tabular}{|c|c|c|c|c|c|c|}
\hline Analysis & Type & Horizon & $\mathbf{N}$ & Detect & $\begin{array}{l}\text { Maximum detection } \\
\text { limit }(\mathrm{pCi} / \mathrm{g})\end{array}$ & $\begin{array}{c}\text { UCB } \\
(\mathrm{pCi} / \mathrm{g})\end{array}$ \\
\hline Americiurn-241 & gamma & A & 16 & 0 & 0.06 & 0.17 \\
\hline Americium-241 & gamma & B & 15 & 0 & 0.05 & 0.18 \\
\hline Americium-241 & gamma & C & 15 & $\mathbf{0}$ & 0.06 & 0.18 \\
\hline Barium-133 & gamma & $\mathbf{A}$ & 16 & 0 & 0.01 & 0.17 \\
\hline Barium-133 & gamma & $\mathbf{B}$ & 15 & 0 & 0.01 & 0.18 \\
\hline Barium-133 & gamma & C & 15 & 0 & 0.01 & 0.18 \\
\hline Chromium-51 & gamma & $\mathbf{A}$ & 16 & $\mathbf{0}$ & 0.10 & 0.17 \\
\hline Chromium-51 & gamma & $\mathbf{B}$ & 15 & 0 & 0.09 & 0.18 \\
\hline Chromium-51 & gamma & C & 15 & $\mathbf{0}$ & 0.09 & 0.18 \\
\hline Cobalt-57 & gamma & $\mathbf{A}$ & 16 & 0 & 0.01 & 0.17 \\
\hline Cobalt-57 & gamma & B & 15 & 0 & 0.01 & 0.18 \\
\hline Cobalt -57 & gamma & C & 15 & 0 & 0.01 & 0.18 \\
\hline Cobalt -60 & gamma & $\mathbf{A}$ & 16 & 0 & 0.01 & 0.17 \\
\hline Cobalt -60 & gamma & B & 15 & 0 & 0.01 & 0.18 \\
\hline Cobalt -60 & gamma & C & 15 & 0 & 0.01 & 0.18 \\
\hline Curium-243 & gamma & A & 12 & 0 & 0.19 & 0.22 \\
\hline Curium-243 & gamma & B & 3 & 0 & 0.06 & 0.63 \\
\hline Curium-243 & gamma & C & 3 & 0 & 0.07 & 0.63 \\
\hline Curium-244 & alpha & $\mathbf{A}$ & 7 & $\mathbf{0}$ & 3.50 & 0.35 \\
\hline Curium-244 & alpha & B & 1 & 0 & 0.83 & 0.95 \\
\hline Curium-245 & gamma & $\mathbf{A}$ & 12 & 0 & 0.11 & 0.22 \\
\hline Curium-245 & gamma & B & 3 & 0 & 0.11 & 0.63 \\
\hline Curium-245 & gamma & C & 3 & 0 & 0.11 & 0.63 \\
\hline Curium-247 & gamma & B & 3 & $\mathbf{0}$ & 0.01 & 0.63 \\
\hline Curium-247 & gamma & $\mathrm{C}$ & 3 & $\mathbf{0}$ & 0.01 & 0.63 \\
\hline Europium-152 & gamma & $\mathbf{A}$ & 16 & $\mathbf{0}$ & 0.07 & 0.17 \\
\hline Europium-152 & gamma & B & 15 & 0 & 0.06 & 0.18 \\
\hline Europium-152 & gamma & C & 15 & 0 & 0.06 & 0.18 \\
\hline Europium-154 & gamma & $\mathbf{A}$ & 16 & 0 & 0.02 & 0.17 \\
\hline Europium-154 & gamma & B & 15 & 0 & 0.02 & 0.18 \\
\hline Europium-154 & gamma & C & 15 & $\mathbf{0}$ & 0.02 & 0.18 \\
\hline Hafnium-181 & beta & $\mathbf{A}$ & 12 & 0 & 0.01 & 0.22 \\
\hline Hafnium-181 & beta & B & 11 & 0 & 0.01 & 0.24 \\
\hline Hafnium-181 & beta & C & 11 & 0 & 0.01 & 0.24 \\
\hline Iridium-192 & beta & A & 11 & 0 & 0.01 & 0.24 \\
\hline Iridium-192 & beta & B & 11 & 0 & 0.01 & 0.24 \\
\hline Iridium-192 & beta & C & 11 & 0 & 0.01 & 0.24 \\
\hline Neptunium-237 & alpha & $\mathbf{A}$ & 1 & 0 & 0.03 & 0.95 \\
\hline Niobium-95 & beta & $\mathbf{A}$ & 12 & 0 & 0.01 & 0.22 \\
\hline Niobium-95 & beta & B & 11 & 0 & $82000.00^{\circ}$ & 0.24 \\
\hline Niobium-95 & beta & C & 10 & 0 & 0.03 & 0.26 \\
\hline Plutonium-238 & alpha & C & 3 & 0 & 0.10 & 0.63 \\
\hline Plutonium-239/240 & alpha & $\mathbf{A}$ & 12 & 1 & 0.07 & 0.34 \\
\hline Plutonium-240 & alpha & $\mathbf{A}$ & 4 & $\mathbf{0}$ & 0.06 & 0.53 \\
\hline Ruthenium-103 & beta & A & 16 & 0 & 0.11 & 0.17 \\
\hline Ruthenium-103 & beta & B & 15 & 0 & 0.01 & 0.18 \\
\hline Ruthenium-103 & beta & C & 15 & 1 & 0.01 & 0.28 \\
\hline Uranium-236 & alpha & A & 16 & 1 & 0.08 & 0.26 \\
\hline Uranium-236 & alpha & B & 15 & 1 & 0.06 & 0.28 \\
\hline Uranium-236 & alpha & C & 15 & 0 & 0.11 & 0.18 \\
\hline Zinc-65 & gamma & $\mathbf{A}$ & 16 & 0 & 0.03 & 0.17 \\
\hline Zinc-65 & gamma & B & 15 & 0 & 0.03 & 0.18 \\
\hline Zinc-65 & gamma & $\mathrm{C}$ & 15 & $\mathbf{0}$ & 0.03 & 0.18 \\
\hline Zirconium-95 & beta & A & 16 & 0 & 0.02 & 0.17 \\
\hline Zirconium-95 & beta & B & 15 & 0 & 0.02 & 0.18 \\
\hline Zirconium-95 & beta & C & 15 & 0 & 0.02 & 0.18 \\
\hline
\end{tabular}

- Statistical outlier 
Table 5.8. Additional radionuclides summary statistics (pci/g)

\begin{tabular}{|c|c|c|c|c|c|c|c|}
\hline \multicolumn{8}{|c|}{ ANALYSIS=CESIUM-137 TYPE=GAMMA } \\
\hline Horizon & Areas & $\mathbf{N}$ & Detect & Median & UCB95 & X95 & LTB9595 \\
\hline $\mathbf{A}$ & DG AND & 4 & 3 & 0.11500 & 0.4880 & 1.9600 & 0.3710 \\
\hline $\mathbf{A}$ & DG ROA & 4 & 3 & 0.27000 & 1.1400 & 4.6100 & 0.8730 \\
\hline $\mathbf{A}$ & DG ORR & 4 & 4 & 0.59800 & 2.4700 & 10.2000 & 1.8800 \\
\hline $\mathbf{A}$ & NOL ORR & 4 & 4 & 0.52700 & 2.1800 & 8.9800 & 1.6500 \\
\hline B & DG AND & 4 & 4 & 0.06190 & 0.2100 & 0.7100 & 0.1640 \\
\hline B & DG ROA & 4 & 4 & 0.00935 & 0.0317 & 0.1070 & 0.0248 \\
\hline B & DG ORR & 3 & 2 & 0.03950 & 0.1700 & 0.4530 & 0.0908 \\
\hline B & NOL ORR & 4 & 3 & 0.00798 & 0.0286 & 0.0915 & 0.0214 \\
\hline C & DG AND & 4 & 3 & 0.03180 & 0.2550 & 1.7200 & 0.1020 \\
\hline $\mathrm{C}$ & DG ORR & 3 & 2 & 0.03490 & 0.4040 & 1.8900 & 0.0912 \\
\hline \multicolumn{8}{|c|}{ ANALYSIS=CURIUM-247 TYPE=GAMMA } \\
\hline Horizon & Areas & $\mathbf{N}$ & Detect & Median & UCB95 & X95 & LTB9595 \\
\hline $\mathbf{A}$ & NOL ORR & 4 & 2 & 0.00552 & 0.00649 & 0.00716 & 0.00578 \\
\hline \multicolumn{8}{|c|}{ ANALYSIS=EUROPIUM-155 TYPE=GAMMA } \\
\hline Horizon & Areas & $\mathbf{N}$ & Detect & Median & UCB95 & $\mathrm{X} 95$ & LTB9595 \\
\hline $\mathbf{A}$ & DG AND & 4 & 4 & 0.0806 & 0.0895 & 0.0994 & 0.0880 \\
\hline $\mathbf{A}$ & DG ROA & 4 & 4 & 0.0790 & 0.0877 & 0.0975 & 0.0863 \\
\hline $\mathbf{A}$ & DG ORR & 4 & 4 & 0.0948 & 0.1050 & 0.1170 & 0.1040 \\
\hline $\mathbf{A}$ & NOL ORR & 4 & 4 & 0.0896 & 0.0995 & 0.1100 & 0.0978 \\
\hline B & DG AND & 4 & 4 & 0.0741 & 0.1210 & 0.1960 & 0.1090 \\
\hline B & DG ROA & 3 & 3 & 0.0838 & 0.1470 & 0.2220 & 0.1160 \\
\hline B & DG ORR & 3 & 2 & 0.0435 & 0.0771 & 0.1150 & 0.0609 \\
\hline B & NOL ORR & 4 & 4 & 0.0908 & 0.1480 & 0.2400 & 0.1340 \\
\hline C & DG AND & 4 & 4 & 0.0750 & 0.1140 & 0.1740 & 0.1070 \\
\hline C & DG ROA & 4 & 4 & 0.0582 & 0.0888 & 0.1350 & 0.0827 \\
\hline C & DG ORR & 3 & 3 & 0.0911 & 0.1480 & 0.2120 & 0.1220 \\
\hline C & NOL ORR & 4 & 4 & 0.1070 & 0.1640 & 0.2500 & 0.1530 \\
\hline \multicolumn{8}{|c|}{ ANALYSIS=PLUTONIUM-238 TYPE=ALPHA } \\
\hline Horizon & Areas & $\mathbf{N}$ & Detect & Median & UCB95 & X95 & LTB9595 \\
\hline A & DG ROA & 4 & 2 & 0.1060 & 0.1570 & 0.221 & 0.1340 \\
\hline B & DG ROA & 1 & 1 & 0.0980 & 0.1160 & 0.116 & 0.0946 \\
\hline B & DG ORR & 2 & 2 & 0.0853 & 0.0964 & 0.101 & 0.0857 \\
\hline \multicolumn{8}{|c|}{ ANALYSIS=PLUTONIUM-239 TYPE=ALPHA } \\
\hline Horizon & Areas & $\mathbf{N}$ & Detect & Median & UCB95 & X95 & LTB9595 \\
\hline $\mathbf{A}$ & DG AND & 4 & 1 & 0.00914 & 0.0473 & 0.0513 & 0.0106 \\
\hline
\end{tabular}


Table 5.8 (continued)

\begin{tabular}{|c|c|c|c|c|c|c|c|}
\hline \multicolumn{8}{|c|}{ ANALYSIS=POTASSIUM-40 TYPE=GAMMA } \\
\hline Horizon & Areas & $\mathbf{N}$ & Detect & Median & UCB95 & X95 & LTB9595 \\
\hline A & DG AND & 4 & 4 & 19.3 & 24.0 & 29.9 & 23.20 \\
\hline A & DG ROA & 4 & 4 & 11.1 & 13.8 & 17.2 & 13.30 \\
\hline $\mathbf{A}$ & DG ORR & 4 & 4 & 16.3 & 20.3 & 25.3 & 19.70 \\
\hline A & NOL ORR & 4 & 4 & 15.2 & 18.9 & 23.5 & 18.30 \\
\hline B & DG AND & 4 & 4 & 26.2 & 63.0 & 151.0 & 53.50 \\
\hline B & DG ROA & 4 & 4 & 18.2 & 43.8 & 105.0 & 37.20 \\
\hline B & DG ORR & 3 & 2 & 3.8 & 10.6 & 21.9 & 7.01 \\
\hline B & NOL ORR & 4 & 4 & 16.6 & 40.0 & 96.1 & 33.90 \\
\hline C & DG AND & 4 & 4 & 22.3 & 26.8 & 32.1 & 26.00 \\
\hline C & DG ROA & 4 & 4 & 23.4 & 28.0 & 33.6 & 27.20 \\
\hline $\mathrm{C}$ & DG ORR & 3 & 3 & 19.5 & 24.0 & 28.0 & 22.10 \\
\hline $\mathrm{C}$ & NOL ORR & 4 & 4 & 25.2 & 30.2 & 36.1 & 29.30 \\
\hline \multicolumn{8}{|c|}{ ANALYSIS=RADIUM-226 TYPE $=$ ALPHA } \\
\hline Horizon & Areas & $\mathrm{N}$ & Detect & Median & UCB95 & X95 & LTB9595 \\
\hline $\mathbf{A}$ & DG AND & 4 & 4 & 1.820 & 2.220 & 2.710 & 2.150 \\
\hline A & DG ROA & 4 & 4 & 0.833 & 1.020 & 1.240 & 0.985 \\
\hline A & DG ORR & 4 & 4 & 0.786 & 0.961 & 1.170 & 0.931 \\
\hline $\mathbf{A}$ & NOL ORR & 4 & 4 & 0.740 & 0.904 & 1.100 & 0.876 \\
\hline B & DG AND & 4 & 4 & 1.710 & 2.180 & 2.780 & 2.090 \\
\hline B & DG ROA & 4 & 4 & 0.865 & 1.100 & 1.410 & 1.060 \\
\hline B & DG ORR & 4 & 3 & 0.753 & 0.985 & 1.220 & 0.909 \\
\hline B & NOL ORR & 4 & 4 & 0.880 & 1.120 & 1.430 & 1.080 \\
\hline C & DG AND & 4 & 4 & 1.670 & 1.840 & 2.030 & 1.810 \\
\hline $\mathrm{C}$ & DG ROA & 4 & 4 & 0.786 & 0.866 & 0.954 & 0.853 \\
\hline $\mathrm{C}$ & DG ORR & 4 & 4 & 0.763 & 0.840 & 0.926 & 0.828 \\
\hline $\mathrm{C}$ & NOL ORR & 4 & 4 & 0.970 & 1.070 & 1.180 & 1.050 \\
\hline \multicolumn{8}{|c|}{ ANALYSIS=THORIUM-228 TYPE=ALPHA } \\
\hline Horizon & Areas & $\mathbf{N}$ & Detect & Median & UCB95 & X95 & LTB9595 \\
\hline A & DG AND & 4 & 4 & 1.200 & 1.450 & 1.75 & 1.410 \\
\hline A & DG ROA & 4 & 4 & 0.988 & 1.190 & 1.44 & 1.160 \\
\hline A & DG ORR & 4 & 4 & 0.713 & 0.862 & 1.04 & 0.837 \\
\hline A & NOL ORR & 4 & 4 & 1.510 & 1.820 & 2.20 & 1.770 \\
\hline $\mathrm{B}$ & DG AND & 4 & 4 & 1.010 & 1.620 & 2.61 & 1.510 \\
\hline B & DG ROA & 4 & 4 & 0.733 & 1.180 & 1.90 & 1.090 \\
\hline B & DG ORR & 4 & 4 & 1.030 & 1.650 & 2.66 & 1.540 \\
\hline B & NOL ORR & 4 & 4 & 1.590 & 2.570 & 4.13 & 2.380 \\
\hline $\mathrm{C}$ & DG AND & 4 & 4 & 1.090 & 1.970 & 3.57 & 1.780 \\
\hline C & DG ROA & 4 & 4 & 0.712 & 1.290 & 2.34 & 1.160 \\
\hline $\mathrm{C}$ & DG ORR & 4 & 3 & 0.612 & 1.110 & 2.01 & 1.010 \\
\hline $\mathrm{C}$ & NOL ORR & 4 & 4 & 1.570 & 2.840 & 5.15 & 2.560 \\
\hline
\end{tabular}


Table 5.8 (continued)

\begin{tabular}{|c|c|c|c|c|c|c|c|}
\hline \multicolumn{8}{|c|}{ ANALYSIS=THORIUM-230 TYPE=ALPHA } \\
\hline Horizon & Areas & $\mathbf{N}$ & Detect & Median & UCB95 & X95 & LTB9595 \\
\hline $\mathbf{A}$ & DG AND & 4 & 4 & 0.912 & 1.160 & 1.47 & 1.120 \\
\hline A & DG ROA & 4 & 4 & 0.746 & 0.947 & 1.20 & 0.912 \\
\hline A & DG ORR & 4 & 4 & 0.565 & 0.717 & 0.91 & 0.691 \\
\hline $\mathbf{A}$ & NOL ORR & 4 & 4 & 0.966 & 1.230 & 1.56 & 1.180 \\
\hline B & DG AND & 4 & 4 & 0.958 & 1.330 & 1.84 & 1.260 \\
\hline B & DG ROA & 4 & 4 & 0.868 & 1.200 & 1.67 & 1.140 \\
\hline B & DG ORR & 4 & 4 & 0.727 & 1.010 & 1.40 & 0.957 \\
\hline B & NOL ORR & 4 & 4 & 1.000 & 1.390 & 1.93 & 1.320 \\
\hline C & DG AND & 4 & 4 & 0.833 & 1.180 & 1.67 & 1.120 \\
\hline C & DG ROA & 4 & 4 & 0.508 & 0.719 & 1.02 & 0.681 \\
\hline C & DG ORR & 4 & 4 & 0.571 & 0.809 & 1.14 & 0.766 \\
\hline $\mathrm{C}$ & NOL ORR & 4 & 4 & 0.877 & 1.240 & 1.76 & 1.180 \\
\hline \multicolumn{8}{|c|}{ ANALYSIS=THORIUM-232 TYPE=ALPHA } \\
\hline Horizon & Areas & $\mathbf{N}$ & Detect & Median & UCB95 & X95 & LTB9595 \\
\hline $\mathbf{A}$ & DG AND & 4 & 4 & 1.060 & 1.260 & 1.500 & 1.230 \\
\hline $\mathbf{A}$ & DG ROA & 4 & 4 & 0.945 & 1.120 & 1.340 & 1.090 \\
\hline A & DG ORR & 4 & 4 & 0.683 & 0.813 & 0.968 & 0.791 \\
\hline A & NOL ORR & 4 & 4 & 1.490 & 1.780 & 2.120 & 1.730 \\
\hline B & DG AND & 4 & 4 & 1.100 & 1.500 & 2.040 & 1.430 \\
\hline B & DG ROA & 4 & 4 & 1.280 & 1.740 & 2.370 & 1.660 \\
\hline B & DG ORR & 4 & 4 & 1.020 & 1.390 & 1.890 & 1.320 \\
\hline B & NOL ORR & 4 & 4 & 1.500 & 2.030 & 2.770 & 1.940 \\
\hline C & DG AND & 4 & 4 & 1.070 & 1.670 & 2.600 & 1.560 \\
\hline C & DG ROA & 4 & 4 & 0.680 & 1.060 & 1.650 & 0.988 \\
\hline C & DG ORR & 4 & 4 & 0.841 & 1.310 & 2.040 & 1.220 \\
\hline $\mathrm{C}$ & NOL ORR & 4 & 4 & 1.370 & 2.130 & 3.320 & 1.990 \\
\hline \multicolumn{8}{|c|}{ ANALYSIS=THORIUM-234 TYPE=BETA } \\
\hline Horizon & Areas & $\mathbf{N}$ & Detect & Median & UCB95 & X95 & LTB9595 \\
\hline A & DG AND & 4 & 4 & 1.06 & 1.15 & 1.25 & 1.14 \\
\hline A & DG ROA & 3 & 3 & 1.43 & 1.56 & 1.67 & 1.51 \\
\hline $\mathbf{A}$ & DG ORR & 4 & 4 & 1.63 & 1.77 & 1.92 & 1.75 \\
\hline A & NOL ORR & 4 & 4 & 1.42 & 1.54 & 1.67 & 1.52 \\
\hline B & DG AND & 4 & 4 & 1.05 & 1.21 & 1.41 & 1.18 \\
\hline B & DG ROA & 3 & 3 & 1.29 & 1.53 & 1.73 & 1.43 \\
\hline B & DG ORR & 3 & 3 & 1.18 & 1.40 & 1.59 & 1.31 \\
\hline B & NOL ORR & 4 & 4 & 1.10 & 1.28 & 1.48 & 1.24 \\
\hline $\mathrm{C}$ & DG AND & 4 & 4 & 1.02 & 1.09 & 1.17 & 1.08 \\
\hline C & DG ROA & 3 & 3 & 1.35 & 1.45 & 1.54 & 1.41 \\
\hline C & DG ORR & 4 & 4 & 1.16 & 1.24 & 1.33 & 1.23 \\
\hline C & NOL OR:R & 4 & 4 & 1.07 & 1.14 & 1.22 & 1.13 \\
\hline
\end{tabular}


Table 5.8 (continued)

\begin{tabular}{|c|c|c|c|c|c|c|c|}
\hline \multicolumn{8}{|c|}{ ANALYSIS=TOTAL URANIUM TYPE=ALPHA } \\
\hline Horizon & Areas & $\mathrm{N}$ & Detect & Median & UCB95 & X95 & LTB9595 \\
\hline $\mathbf{A}$ & DG AND & 4 & 4 & 0.999 & 2.190 & 4.79 & 1.910 \\
\hline $\mathbf{A}$ & DG ROA & 4 & 3 & 0.646 & 1.430 & 3.09 & 1.250 \\
\hline A & DG ORR & 4 & 4 & 1.310 & 2.870 & 6.29 & 2.510 \\
\hline A & NOL ORR & 4 & 4 & 1.150 & 2.510 & 5.50 & 2.190 \\
\hline B & DG ROA & 1 & 1 & 0.450 & 1.650 & 1.65 & 0.345 \\
\hline B & DG ORR & 2 & 2 & 0.316 & 0.791 & 1.16 & 0.326 \\
\hline $\mathrm{C}$ & DG ROA & 1 & 1 & 1.300 & 9.340 & 9.34 & 0.869 \\
\hline $\mathrm{C}$ & DG ORR & 2 & 2 & 0.299 & 1.210 & 2.15 & 0.315 \\
\hline \multicolumn{8}{|c|}{ ANALYSIS $=$ TRITIUM TYPE $=$ TRITIUM } \\
\hline Horizon & Areas & $\mathbf{N}$ & Detect & Median & UCB95 & X95 & LTB9595 \\
\hline A & DG ORR & 9 & 5 & 0.0304 & 0.0445 & 0.0808 & 0.0466 \\
\hline \multicolumn{8}{|c|}{ ANALYSIS=URANIUM-233/234 TYPE=ALPHA } \\
\hline Horizon & Areas & $\mathbf{N}$ & Detect & Median & UCB95 & X95 & LTB9595 \\
\hline $\mathbf{A}$ & DG AND & 4 & 4 & 0.925 & 1.080 & 1.25 & 1.040 \\
\hline A & DG ROA & 4 & 4 & 0.934 & 1.090 & 1.26 & 1.050 \\
\hline A & DG ORR & 4 & 4 & 0.937 & 1.090 & 1.27 & 1.060 \\
\hline B & DG AND & 4 & 4 & 0.916 & 1.310 & 1.87 & 1.220 \\
\hline B & DG ROA & 4 & 4 & 0.766 & 1.090 & 1.56 & 1.020 \\
\hline B & DG ORR & 4 & 4 & 1.110 & 1.590 & 2.27 & 1.480 \\
\hline $\mathrm{C}$ & DG AND & 4 & 4 & 0.871 & 1.110 & 1.42 & 1.060 \\
\hline C & DG ROA & 4 & 4 & 0.671 & 0.856 & 1.09 & 0.815 \\
\hline $\mathrm{C}$ & DG ORR & 4 & 4 & 0.663 & 0.846 & 1.08 & 0.805 \\
\hline \multicolumn{8}{|c|}{ ANALYSIS=URANIUM-235 TYPE=ALPHA } \\
\hline Horizon & Areas & $\mathbf{N}$ & Detect & Median & UCB95 & $\mathrm{X} 95$ & LTB9595 \\
\hline A & DG ROA & 4 & 3 & 0.0546 & 0.0660 & 0.0766 & 0.0603 \\
\hline A & DG ORR & 4 & 2 & 0.0563 & 0.0673 & 0.0790 & 0.0629 \\
\hline B & DG AND & 4 & 2 & 0.0380 & 0.0676 & 0.1140 & 0.0573 \\
\hline B & DG ROA & 4 & 2 & 0.0323 & 0.0583 & 0.0967 & 0.0480 \\
\hline B & DG ORR & 4 & 2 & 0.0666 & 0.1200 & 0.2000 & 0.0995 \\
\hline C & DG AND & 4 & 2 & 0.0384 & 0.0521 & 0.0669 & 0.0457 \\
\hline $\mathrm{C}$ & DG ORR & 4 & 2 & 0.0366 & 0.0496 & 0.0639 & 0.0432 \\
\hline
\end{tabular}


Table 5.8 (continued)

\begin{tabular}{|c|c|c|c|c|c|c|c|}
\hline \multicolumn{8}{|c|}{ ANALYSIS=URANIUM-235 TYPE=GAMMA } \\
\hline Horizon & Areas & $\mathbf{N}$ & Detect & Median & UCB95 & X95 & LTB9595 \\
\hline A & DG AND & 4 & 4 & 0.0606 & 0.0727 & 0.0872 & 0.0706 \\
\hline $\mathbf{A}$ & DG ROA & 4 & 4 & 0.0768 & 0.0922 & 0.1110 & 0.0896 \\
\hline $\mathbf{A}$ & DG ORR & 4 & 4 & 0.0792 & 0.0950 & 0.1140 & 0.0923 \\
\hline $\mathbf{A}$ & NOL ORR & 4 & 4 & 0.0713 & 0.0855 & 0.1030 & 0.0831 \\
\hline B & DG AND & 4 & 4 & 0.0537 & 0.0733 & 0.1000 & 0.0696 \\
\hline B & DG ROA & 4 & 4 & 0.0639 & 0.0873 & 0.1190 & 0.0829 \\
\hline B & DG ORR & 3 & 3 & 0.0700 & 0.1000 & 0.1310 & 0.0870 \\
\hline B & NOL ORR & 4 & 4 & 0.0412 & 0.0563 & 0.0769 & 0.0534 \\
\hline C & DG AND & 4 & 4 & 0.0345 & 0.0444 & 0.0571 & 0.0427 \\
\hline C & DG ROA & 4 & 4 & 0.0626 & 0.0806 & 0.1040 & 0.0774 \\
\hline $\mathrm{C}$ & DG ORR & 4 & 4 & 0.0433 & 0.0557 & 0.0717 & 0.0536 \\
\hline C & NOL ORR & 4 & 4 & 0.0473 & 0.0608 & 0.0782 & 0.0584 \\
\hline \multicolumn{8}{|c|}{ ANALYSIS=URANIUM-236 TYPE=ALPHA } \\
\hline Horizon & Areas & $\mathbf{N}$ & Detect & Median & UCB95 & X95 & LTB9595 \\
\hline B & DG ORR & 3 & 1 & 0.0263 & 0.298 & 0.354 & 0.0243 \\
\hline \multicolumn{8}{|c|}{ ANALYSIS=URANIUM-238 TYPE=ALPHA } \\
\hline Horizon & Areas & $\mathbf{N}$ & Detect & Median & UCB95 & X95 & LTB9595 \\
\hline A & DG AND & 4 & 4 & 0.890 & 1.000 & 1.13 & 0.979 \\
\hline $\mathbf{A}$ & DG ROA & 4 & 4 & 0.992 & 1.120 & 1.26 & 1.090 \\
\hline A & DG ORR & 4 & 4 & 1.020 & 1.150 & 1.30 & 1.130 \\
\hline B & DG AND & 4 & 4 & 0.966 & 1.310 & 1.78 & 1.230 \\
\hline B & DG ROA & 4 & 4 & 0.825 & 1.120 & 1.52 & 1.050 \\
\hline B & DG ORR & 4 & 4 & 1.120 & 1.520 & 2.05 & 1.420 \\
\hline C & DG AND & 4 & 4 & 0.871 & 1.090 & 1.36 & 1.040 \\
\hline C & DG ROA & 4 & 4 & 0.743 & 0.928 & 1.16 & 0.887 \\
\hline C & DG ORR & 4 & 4 & 0.666 & 0.833 & 1.04 & 0.796 \\
\hline
\end{tabular}

\subsection{GAMMA SCREENING}

The purpose of the gamma screening is to affirm that background cesium-137 levels are not higher than normal for the southeastern United States (about $10 \mathrm{pCi} / \mathrm{cm}^{2}$ ). So far, a few results seem low ( $<8 \mathrm{pCi} / \mathrm{cm}^{2}$ ), and one has exceeded 13 (Table 5.9).

Table 5.9. Overall results for cesium-137 (in pCi/ $\mathrm{cm}^{2}$ )

\begin{tabular}{lcccccc}
\hline Formation & Location & N & Mean & Std dev & Min & Max \\
\hline Dismal Gap & AND & 12 & 7.870 & 3.007 & 3.775 & 14.424 \\
Dismal Gap & ROA & 13 & 6.071 & 3.322 & 0.160 & 11.937 \\
Dismal Gap & ORR & 12 & 8.541 & 1.525 & 6.024 & 11.053 \\
Nolichucky & ORR & 12 & 9.504 & 1.642 & 6.760 & 11.962 \\
\hline
\end{tabular}


The data are also illustrated in Fig. 5.3. The very low results are from severely eroded sites; the very high result is from a sediment deposition site.

All of the data are detects, and so they were analyzed using the usual F-tests (Proc GLM). Probably because they arise as counts, the data seem to be better modelled using the square-root rather than the log transformation. Both transformations and no transformation were investigated, and the following conclusions about differences across areas are not materially affected by the choice. (The gamma analysis results depart from the general lognormal approach. The data have been available longer, so a slightly more careful analysis was performed.)

There are significant differences between areas ( $p=0.0001)$, mainly because the two onreservation areas exhibit significantly higher levels than the two off-reservation areas $(p=$ 0.005 ). Some conclusions depend on whether the deposition and erosion sites are included or excluded [e.g., the Anderson and Roane Dismal Gap areas differ significantly $(p=0.05)$ when all of the data are used, but do not $(p=0.69)$ when the sediment and deposition sites are excluded]. These $p$-values were computed using the square-root transformation.

\subsection{VOLATILE ORGANICS}

No statistical analyses were performed on the volatile organics data. The purpose of these analyses was to screen for volatile organics-ideally to affirm that, since sampling is from background areas, volatile organics are absent. Although there are a few exceptions, this is generally true. The exceptions are discussed in Sect. 6.

\subsection{VARIANCE COMPONENTS}

For radionuclides (except tritium) and inorganics, the variable LTB9595 is a tolerance bound for composites of three. Tolerance bounds for noncomposites or composites of otherthan-three can also be computed from the background data, but to do this, estimates of laboratory and spatial (field) standard deviations are needed. V, the variance of a composite of $\mathbf{k}$ satisfies

$$
\mathrm{V}=\mathrm{L}+\mathrm{S} / \mathrm{k}
$$

[Equation 5.1]

where $\mathrm{L}$ is the variance of laboratory error; $\mathrm{S}$ is the spatial variance of single (noncomposited) samples. ( $\mathrm{S}$ is the hypothetical variance of single samples measured without error.)

Strictly, Equation 5.1 applies to untransformed data, but it holds approximately for logtransformed data, and we will assume it for the lognormal model. For the lognormal model, computing a tolerance bound for a composite of $\mathrm{k}$ requires an estimate of $\mathrm{V}$.

For composites of three, the quantity $\mathrm{V}$ can be estimated simply by computing the maximum likelihood estimate of the pooled standard deviation of individual (composited) observations using Proc Lifereg. $\mathrm{L}$ can be estimated from field duplicate differences, using data from all horizons. S can then be estimated using Equation 5.1. 


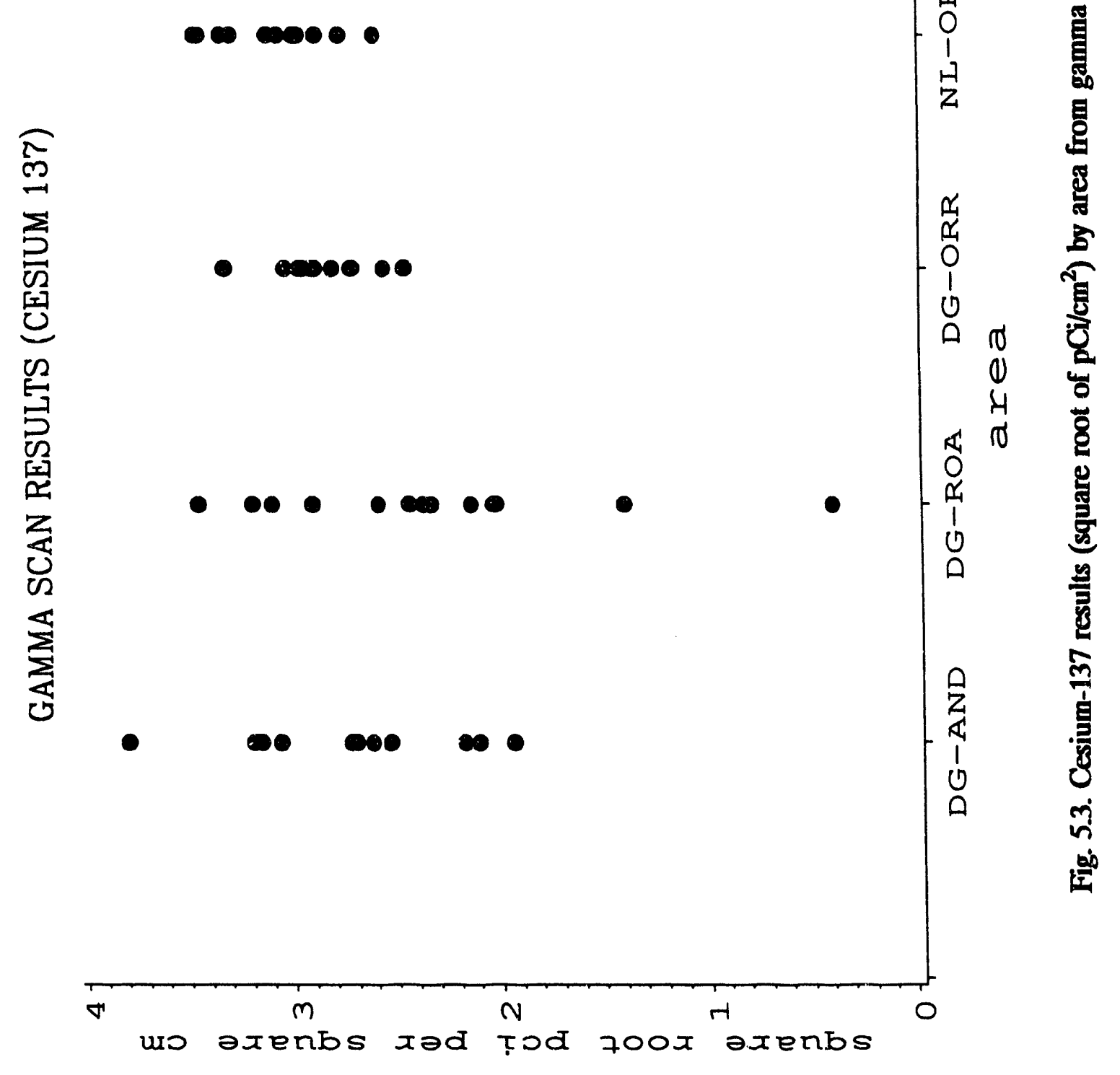


Tables 5.10a, 5.10b, and 5.10c contain standard deviation estimates for log concentrations of inorganics, PAHs, and radionuclides. (Each standard deviation is the square root of the corresponding variance, $\mathrm{V}, \mathrm{L}$, or S, of the log-transformed observations. A degree-of-freedom adjustment has been made so that the standard deviations coincide with the usual unbiased standard deviations when there are no nondetects.) These standard deviation estimates can be used to compute tolerance bounds for composites of other than three, and, as discussed below, to assess the advantage of compositing.

For a number of horizon areas, the spatial standard deviation is missing (.), which indicates that the best estimate is actually zero. In particular, this happens in groups having relatively small overall standard deviation (of composites) and relatively large laboratory standard deviation. It can be a consequence of the noise inherent in these small-sample-size standard deviations or of anomalous discrepancies between field duplicates, as in Fig. 5.1. However, it is also due in part to the fact that, unfortunately, the laboratory and spatial standard deviations in Table 5.10 are biased: The laboratory standard deviations are computed from field duplicate differences, but the field duplicates differ not just by laboratory error, but by the small-scale spatial error as well (field duplicate samples were taken about 3 feet apart). Since the field standard deviations are computed from the standard deviation of composites (or overall standard deviation) using Equation 5.1, the spatial standard deviations tend to be biased down and the laboratory standard deviations are biased up.

There are presently a few (i.e., one or two) standard samples-too few to be of much value-but with additional standards data (Phase II), we would be able to improve the standard deviation estimates in Table 5.10.

Means of untransformed observations do not depend on the degree of compositing, and the same holds approximately for their logs. Thus, estimates of and confidence bounds for the mean of BSCP composites of three are estimates and confidence bounds for other background observations, whatever the degree of compositing. For composites of other than three, $V$ can be approximated using Equation 5.1 and Table 5.10. With mean and V estimates so obtained, approximate tolerance bounds for composites of other than three can be computed.

If there are sufficient data (i.e., sufficiently many degrees of freedom for error estimation), variability of $\mathrm{V}$ estimates can be ignored, and tolerance bounds can be computed from confidence bounds for means. After Phase II, when data from ten areas are available, there will be sufficient data to do this. (There will be 30 degrees of freedom for error for the data with four composited samples per area.)

At present there are only four areas (and thus 12 degrees of freedom for error), so variability of $\mathrm{V}$ estimates cannot be ignored. Computing tolerance bounds for composites of other than three when variance estimates are themselves variable requires approximation. How best to do this is an open question, which will not be pursued here. (Difficulties are due to statistical dependence between variance component estimates and, when there are nondetects, between the mean and overall variance estimates.) 
Table 5.10a Standard deviation estimates for inorganics

\begin{tabular}{|c|c|c|c|c|c|c|c|}
\hline Analysis & Horizon & $\begin{array}{l}\text { Number } \\
\text { of areas }\end{array}$ & $\mathrm{N}$ & Detect & $\begin{array}{c}\text { Std dev for } \\
\text { composites } \\
\mathrm{mg} / \mathrm{kg}\end{array}$ & $\begin{array}{c}\text { Std dev for } \\
\text { laboratory } \\
\mathrm{mg} / \mathrm{kg}\end{array}$ & $\begin{array}{c}\text { Spatial } \\
\text { std dev } \\
\mathrm{mg} / \mathrm{kg}\end{array}$ \\
\hline Aluminum & A & 4 & 16 & 16 & 0.07371 & 0.08340 & . \\
\hline Aluminum & B & 4 & 16 & 16 & 0.07510 & 0.08340 & . \\
\hline Aluminum & C & 4 & 16 & 16 & 0.04292 & 0.08340 & \\
\hline Antimony & A & 4 & 16 & 2 & 0.01716 & 0.00021 & 0.02972 \\
\hline Antimony & B & 4 & 16 & 5 & 0.18080 & 0.00021 & 0.31315 \\
\hline Antimony & C & 4 & 16 & 5 & 0.20313 & 0.00021 & 0.35183 \\
\hline Arsenic & A & 4 & 16 & 15 & 0.30859 & 0.12668 & 0.48739 \\
\hline Arsenic & B & 4 & 16 & 14 & 0.37651 & 0.12668 & 0.61411 \\
\hline Arsenic & C & 4 & 16 & 14 & 0.18120 & 0.12668 & 0.22441 \\
\hline Barium & A & 4 & 16 & 16 & 0.14032 & 0.08984 & 0.18669 \\
\hline Barium & B & 4 & 16 & 16 & 0.06459 & 0.08984 & . \\
\hline Barium & C & 4 & 16 & 16 & 0.08079 & 0.08984 & . \\
\hline Beryllium & A & 4 & 16 & 16 & 0.15022 & 0.07614 & 0.22430 \\
\hline Beryllium & B & 4 & 16 & 16 & 0.17462 & 0.07614 & 0.27219 \\
\hline Beryllium & C & 4 & 16 & 16 & 0.14157 & 0.07614 & 0.20672 \\
\hline Boron & A & 4 & 15 & 6 & 0.25206 & 0.18610 & 0.29447 \\
\hline Boron & B & 4 & 16 & 7 & 0.24898 & 0.18610 & 0.28649 \\
\hline Boron & C & 4 & 16 & 8 & 0.17858 & 0.18610 & \\
\hline Calcium & A & 4 & 16 & 11 & 0.22739 & 0.10830 & 0.34631 \\
\hline Calcium & B & 4 & 16 & 10 & 0.19691 & 0.10830 & 0.28484 \\
\hline Calcium & C & 4 & 16 & 11 & 0.28515 & 0.10830 & 0.45689 \\
\hline Chromium & A & 4 & 16 & 15 & 0.48154 & 0.09505 & 0.81764 \\
\hline Chromium & B & 4 & 16 & 15 & 0.51104 & 0.09505 & 0.86970 \\
\hline Chromium & C & 4 & 16 & 16 & 0.11510 & 0.09505 & 0.11244 \\
\hline Cobalt & A & 4 & 16 & 16 & 0.17544 & 0.19448 & \\
\hline Cobalt & B & 4 & 16 & 16 & 0.27732 & 0.19448 & 0.34244 \\
\hline Cobalt & C. & 4 & 16 & 16 & 0.23125 & 0.19448 & 0.21673 \\
\hline Copper & A & 4 & 16 & 16 & 0.07799 & 0.12343 & \\
\hline Copper & B & 4 & 16 & 16 & 0.24288 & 0.12343 & 0.36230 \\
\hline Copper & C & 4 & 16 & 16 & 0.12808 & 0.12343 & 0.05920 \\
\hline Cyanide & A & 4 & 15 & 6 & 0.40933 & 0.65423 & . \\
\hline Cyanide & B & 4 & 16 & 3 & 0.43961 & 0.65423 & . \\
\hline Cyanide & C & 4 & 16 & 2 & 0.51676 & 0.65423 & . \\
\hline Iron & A & 4 & 16 & 16 & 0.06865 & 0.07635 & . \\
\hline Iron & B & 4 & 16 & 16 & 0.07311 & 0.07635 & . \\
\hline Iron & C & 4 & 16 & 16 & 0.03892 & 0.07635 & . \\
\hline Lead & A & 4 & 16 & 15 & 0.52447 & 0.08267 & 0.89706 \\
\hline Lead & B & 4 & 16 & 15 & 0.46754 & 0.08267 & 0.79705 \\
\hline Lead & C & 4 & 15 & 15 & 0.25815 & 0.08267 & 0.42358 \\
\hline Lithium & A & 4 & 15 & 13 & 0.07931 & 0.08759 & . \\
\hline Lithium & B & 4 & 16 & 16 & 0.10748 & 0.08759 & 0.10788 \\
\hline Lithium & C & 4 & 16 & 16 & 0.09763 & 0.08759 & 0.07468 \\
\hline Magnesium & A & 4 & 16 & 16 & 0.12717 & 0.08648 & 0.16148 \\
\hline Magnesium & B & 4 & 16 & 16 & 0.07447 & 0.08648 & . \\
\hline Magnesium & C & 4 & 16 & 16 & 0.08606 & 0.08648 & . \\
\hline Manganese & A & 4 & 16 & 16 & 0.18364 & 0.22096 & \\
\hline Manganese & B & 4 & 16 & 16 & 0.31729 & 0.22096 & 0.39440 \\
\hline Manganese & C & 4 & 16 & 16 & 0.37468 & 0.22096 & 0.52411 \\
\hline
\end{tabular}




\section{5-33}

Table 5.10a (continued)

\begin{tabular}{|c|c|c|c|c|c|c|c|}
\hline Analysis & Horizon & $\begin{array}{l}\text { Number } \\
\text { of areas } \\
\end{array}$ & $\mathbf{N}$ & Detect & $\begin{array}{c}\text { Std dev for } \\
\text { composites } \\
\mathrm{mg} / \mathrm{kg}\end{array}$ & $\begin{array}{c}\text { Std dev for } \\
\text { laboratory } \\
\mathrm{mg} / \mathrm{kg}\end{array}$ & $\begin{array}{c}\text { Spatial } \\
\text { std dev } \\
\mathrm{mg} / \mathrm{kg}\end{array}$ \\
\hline Mercury & A & 4 & 16 & 11 & 0.04857 & 0.12164 & - \\
\hline Mercury & B & 4 & 16 & 3 & 0.07096 & 0.12164 & . \\
\hline Mercury & $\mathrm{C}$ & 4 & 16 & 2 & 0.04032 & 0.12164 & . \\
\hline Molybdenum & A & 4 & 16 & 1 & 0.00077 & 0.09063 & . \\
\hline Molybdenum & B & 4 & 16 & 3 & 0.08452 & 0.09063 & . \\
\hline Molybdenum & $\mathrm{C}$ & 4 & 16 & 2 & 0.04168 & 0.09063 & . \\
\hline Nickel & $A$ & 4 & 16 & 16 & 0.10725 & 0.08665 & 0.10947 \\
\hline Nickel & B & 4 & 16 & 16 & 0.06789 & 0.08665 & . \\
\hline Nickel & C & 4 & 16 & 16 & 0.08447 & 0.08665 & . \\
\hline Potassium & A & 4 & 16 & 16 & 0.12317 & 0.09948 & 0.12578 \\
\hline Potassium & B & 4 & 16 & 16 & 0.09787 & 0.09948 & . \\
\hline Potassium & C & 4 & 16 & 16 & 0.08604 & 0.09948 & - \\
\hline Selenium & A & 4 & 16 & 8 & 0.13627 & 0.03428 & 0.22843 \\
\hline Selenium & B & 4 & 16 & 8 & 0.11093 & 0.03428 & 0.18273 \\
\hline Selenium & C & 4 & 16 & 7 & 0.15584 & 0.03428 & 0.26331 \\
\hline Silicon & A & 4 & 16 & 16 & 0.06522 & 0.07328 & . \\
\hline Silicon & B & 4 & 16 & 16 & 0.08560 & 0.07328 & 0.07662 \\
\hline Silicon & $\mathrm{C}$ & 4 & 16 & 16 & 0.09479 & 0.07328 & 0.10413 \\
\hline Strontium & A & 4 & 15 & 15 & 0.13506 & 0.11303 & 0.12804 \\
\hline Strontium & B & 4 & 16 & 16 & 0.11233 & 0.11303 & . \\
\hline Strontium & C & 4 & 16 & 16 & 0.15581 & 0.11303 & 0.18576 \\
\hline Sulfate & A & 4 & 15 & 15 & 0.13133 & 0.22660 & . \\
\hline Sulfate & A & 4 & 15 & 15 & 0.13133 & 0.11303 & 0.18576 \\
\hline Sulfate & B & 4 & 16 & 16 & 0.22732 & 0.22660 & 0.03112 \\
\hline Sulfate & C & 4 & 16 & 16 & 0.13291 & 0.22660 & . \\
\hline Thallium & A & 4 & 16 & 2 & 0.49324 & 0.00230 & 0.85431 \\
\hline Thallium & B & 4 & 16 & 4 & 0.26926 & 0.00230 & 0.46635 \\
\hline Thallium & $C$ & 4 & 16 & 7 & 0.20932 & 0.00230 & 0.36253 \\
\hline Vanadium & A & 4 & 16 & 16 & 0.04627 & 0.07229 & . \\
\hline Vanadium & B & 4 & 16 & 16 & 0.23423 & 0.07229 & 0.38589 \\
\hline Vanadium & $C$ & 4 & 16 & 16 & 0.04365 & 0.07229 & . \\
\hline Zinc & A & 4 & 16 & 16 & 0.08398 & 0.07695 & 0.05824 \\
\hline Zinc & B & 4 & 16 & 16 & 0.05562 & 0.07695 & . \\
\hline Zinc & C & 4 & 16 & 16 & 0.06218 & 0.07695 & . \\
\hline
\end{tabular}




\section{5-34}

Table 5.10b. Standard deviation estimates for PAHs

\begin{tabular}{|c|c|c|c|c|c|c|c|}
\hline Analysis & Horizon & $\begin{array}{c}\text { No. } \\
\text { of } \\
\text { areas }\end{array}$ & $\mathbf{N}$ & Detect & $\begin{array}{c}\text { Overall } \\
\text { std dev } \\
\mu \mathrm{g} / \mathrm{kg}\end{array}$ & $\begin{array}{c}\text { Std dev for } \\
\text { laboratory } \\
\mu \mathrm{g} / \mathrm{kg}\end{array}$ & $\begin{array}{c}\text { Spatial } \\
\text { std dev } \\
\mu \mathrm{g} / \mathrm{kg}\end{array}$ \\
\hline Acenaphthene & $\mathbf{A}$ & 4 & 44 & 9 & 0.48495 & 0.12120 & 0.46956 \\
\hline Acenaphthylene & $\mathbf{A}$ & 4 & 42 & 1 & 0.74977 & 0.00250 & 0.74977 \\
\hline Benzo[a]anthracene & A & 4 & 34 & 10 & 0.28882 & 0.06289 & 0.28189 \\
\hline Benzo[a]pyrene & A & 4 & 45 & 9 & 0.19959 & 0.07481 & 0.18504 \\
\hline Benzo[b]fluoranthene & $\mathbf{A}$ & 4 & 42 & 6 & 0.12126 & 0.00304 & 0.12122 \\
\hline Benzo[ghi]perylene & A & 4 & 35 & 1 & 0.00022 & 0.00538 & . \\
\hline Benzo[k]fluoranthene & A & 4 & 42 & 1 & 0.00147 & 0.00443 & . \\
\hline Fluoranthene & $\mathbf{A}$ & 3 & 12 & 2 & 0.98880 & 0.00000 & 0.98880 \\
\hline Fluorene & A & 4 & 42 & 5 & 0.84931 & 0.04101 & 0.84832 \\
\hline Naphthalene & $\mathbf{A}$ & 4 & 43 & 2 & 0.29493 & 0.00174 & 0.29493 \\
\hline Phenanthrene & A & 4 & 45 & 11 & 0.48603 & 0.04292 & 0.48413 \\
\hline Pyrene & $\mathbf{A}$ & 4 & 43 & 3 & 0.01773 & 0.00244 & 0.01757 \\
\hline
\end{tabular}


Table 5.10c. Standard deviation estimates for radionuctides

\begin{tabular}{|c|c|c|c|c|c|c|c|}
\hline Analysis & Horizon & $\begin{array}{c}\text { No. of } \\
\text { areas }\end{array}$ & $\mathbf{N}$ & Detect & $\begin{array}{l}\text { Std dev. for } \\
\text { composites } \\
\text { pCi/kg }\end{array}$ & $\begin{array}{c}\text { Std dev for } \\
\text { laboratory } \\
\text { pCi/kg }\end{array}$ & $\begin{array}{l}\text { Spatial } \\
\text { std dev } \\
\text { pCi/kg }\end{array}$ \\
\hline Cesium-137 & A & 4 & 16 & 14 & 0.86473 & 0.75538 & 0.72901 \\
\hline Cesium-137 & B & 4 & 15 & 13 & 0.75205 & 0.75538 & . \\
\hline Cesium-137 & $\mathrm{C}$ & 4 & 15 & 5 & 1.23108 & 0.75538 & 1.68371 \\
\hline Curium-247 & A & 4 & 10 & 2 & 0.08841 & 0.00234 & 0.15307 \\
\hline Europium-155 & A & 4 & 16 & 16 & 0.06406 & 0.10821 & . \\
\hline Europium-155 & B & 4 & 14 & 13 & 0.30421 & 0.10821 & 0.49245 \\
\hline Europium-155 & $\mathrm{C}$ & 4 & 15 & 15 & 0.26000 & 0.10821 & 0.40949 \\
\hline Plutonium-238 & A & 4 & 16 & 3 & 0.22482 & 0.16557 & 0.26342 \\
\hline Plutonium-238 & B & 2 & 3 & 3 & 0.07899 & 0.16557 & . \\
\hline Plutonium-239 & A & 1 & 4 & 1 & 0.52589 & . & . \\
\hline Plutonium-239/240 & A & 4 & 13 & 1 & 0.03148 & 0.00738 & 0.05301 \\
\hline Plutonium-239/240 & B & 2 & 3 & 1 & 0.00331 & 0.00738 & . \\
\hline Plutonium-239/240 & $\mathrm{C}$ & 2 & 3 & 1 & 0.00424 & 0.00738 & . \\
\hline Potassium -40 & A & 4 & 16 & 16 & 0.13353 & 0.08258 & 0.18174 \\
\hline Potassium-40 & $\mathrm{B}$ & 4 & 15 & 14 & 0.54074 & 0.08258 & 0.92561 \\
\hline Potassium-40 & $\mathrm{C}$ & 4 & 15 & 15 & 0.11142 & 0.08258 & 0.12957 \\
\hline Radium-226 & A & 4 & 16 & 16 & 0.12216 & 0.03761 & 0.20131 \\
\hline Radium-226 & B & 4 & 16 & 15 & 0.14817 & 0.03761 & 0.24823 \\
\hline Radium-226 & $\mathrm{C}$ & 4 & 16 & 16 & 0.05899 & 0.03761 & 0.07871 \\
\hline Strontium-90 & A & 2 & 2 & 1 & 0.00000 & 0.00212 & . \\
\hline Thorium-228 & A & 4 & 16 & 16 & 0.11554 & 0.09349 & 0.11760 \\
\hline Thorium-228 & B & 4 & 16 & 16 & 0.29020 & 0.09349 & 0.47584 \\
\hline Thorium-228 & $\mathrm{C}$ & 4 & 16 & 15 & 0.36248 & 0.09349 & 0.60660 \\
\hline Thorium-230 & A & 4 & 16 & 16 & 0.14532 & 0.16105 & . \\
\hline Thorium-230 & B & 4 & 16 & 16 & 0.19924 & 0.16105 & 0.20318 \\
\hline Thorium-230 & C & 4 & 16 & 16 & 0.21185 & 0.16105 & 0.23838 \\
\hline Thorium-232 & $\mathbf{A}$ & 4 & 16 & 16 & 0.10641 & 0.09421 & 0.08571 \\
\hline Thorium-232 & B & 4 & 16 & 16 & 0.18747 & 0.09421 & 0.28074 \\
\hline Thorium-232 & C & 4 & 16 & 16 & 0.27056 & 0.09421 & 0.43929 \\
\hline Thorium-234 & A & 4 & 15 & 15 & 0.04938 & 0.12634 & . \\
\hline Thorium-234 & B & 4 & 14 & 14 & 0.09249 & 0.12634 & . \\
\hline Thorium-234 & $\mathrm{C}$ & 4 & 15 & 15 & 0.04145 & 0.12634 & . \\
\hline Total uranium & A & 4 & 16 & 15 & 0.47783 & 0.21374 & 0.74021 \\
\hline Total uranium & B & 2 & 3 & 3 & 0.59390 & 0.21374 & 0.95973 \\
\hline Total uranium & $\mathrm{C}$ & 2 & 3 & 3 & 0.90163 & 0.21374 & 1.51715 \\
\hline Tritium & A & 2 & 16 & 5 & 0.27557 & 0.01087 & 0.27536 \\
\hline Uranium-233/234 & A & 4 & 16 & 12 & 0.09143 & 0.07204 & 0.09753 \\
\hline Uranium-233/234 & B & 4 & 16 & 12 & 0.21695 & 0.07204 & 0.35444 \\
\hline Uranium-233/234 & $\mathrm{C}$ & 4 & 16 & 12 & 0.14909 & 0.07204 & 0.22608 \\
\hline Uranium-235(alpha) & $\mathbf{A}$ & 4 & 16 & 7 & 0.08668 & 0.24853 & - \\
\hline Uranium-235(alpha) & B & 4 & 16 & 6 & 0.32833 & 0.24853 & 0.37161 \\
\hline Uranium-235(alpha) & $\mathrm{C}$ & 4 & 16 & 6 & 0.12257 & 0.24853 & . \\
\hline Uranium-235(gamma) & $\mathbf{A}$ & 4 & 16 & 16 & 0.11092 & 0.10610 & 0.05605 \\
\hline Uranium-235(gamma) & B & 4 & 15 & 15 & 0.19241 & 0.10610 & 0.27803 \\
\hline Uranium-235(gamma) & $\mathrm{C}$ & 4 & 16 & 16 & 0.15365 & 0.10610 & 0.19251 \\
\hline Uranium-236 & A & 4 & 16 & 1 & 0.00187 & 0.01397 & . \\
\hline Uranium-236 & B & 4 & 15 & 1 & 0.80136 & 0.01397 & 1.38778 \\
\hline Uranium-238 & A & 4 & 16 & 12 & 0.07291 & 0.07382 & . \\
\hline Uranium-238 & B & 4 & 16 & 12 & 0.18569 & 0.07382 & 0.29512 \\
\hline Uranium-238 & $\mathrm{C}$ & 4 & 16 & 12 & 0.13604 & 0.07382 & 0.19793 \\
\hline
\end{tabular}


Because the laboratory standard deviations are too large and the spatial standard deviations are too small, the ratio, $R$, of spatial-to-laboratory standard deviations is biased down. This ratio is of interest because it can be shown that although compositing provides less detail for field interpretation, the degree of compositing $k$ that minimizes the overall variance for a fixed total cost is given by $R(A / F)^{1 / 2}$, where $F$ is the cost per field sample and $A$ is the cost per sample sent to the laboratory. [Using Lagrange multipliers, minimize $(\mathrm{V}+\mathrm{S} / \mathrm{k}) \mathbf{N}$ subject to $\mathrm{NA}+\mathrm{NkF}=\mathrm{C}$, where $\mathrm{N}$ is the number of laboratory samples and $\mathrm{C}$ is the fixed total cost.] Note that $A$ includes the costs of data entry, verification, and validation.

In 1992, costs to the BSCP for laboratory analysis, data entry, validation, etc. were about 4.5 times the cost of field sampling. Because field samples were composited, this implies that $\mathrm{A} / \mathrm{F}>4.5$, and thus $(\mathrm{A} / \mathrm{F})^{1 / 2}>2.1$. From Tables 5.10a through 5.10c, values of $R$ (which are biased down) are in the vicinity of 4 for arsenic, 3 for beryllium, 10 for lead, and 1.5-11 for potassium-40 (depending on horizon). In these cases, the optimal compositing levels (k) vary from (at least) 3 up to (at least) 20 and support the use of composited samples in the background study. Although it is difficult to quantify the cost of statistical variability, compositing translates to direct savings to the project.

\subsection{ADDITIONAL REMARKS}

Many of the results need further consideration, particularly the field duplicate and original results for some of the inorganics because of the large discrepancy between them. In contrast, the duplicate and original results are very close for the organics. Perhaps the designation " $U$ " for nondetect has been applied too conservatively for the organics (e.g., the detection limits are programmatic or contract limits rather that the true laboratory detection limits). In this background study, the use of high detection limits may not be conservative, because it tends to obscure how low background values actually may be.

The procedure for making soil preparation laboratory (SPL) duplicate composited samples from primary composites should be reconsidered. SPL composited sample splits will provide for better assessment of laboratory error-as well as a method of validation that depends only on simple statistical comparisons of results (rather than expensive and timeconsuming reviews of paperwork).

Statistics presented in this section may be biased upwards (too high), because of the assignment of validation codes on the basis of detect-nondetect status. Because this is a background study, the goal of which is to determine reference values as opposed to a study designed to characterize contamination levels, upward bias will likely be regarded as nonconservative.

Because many analytes do differ significantly with area, many that do not differ based on current data probably would if sample sizes were larger or statistical variability was smaller. The practical importance of differences is not considered in this section. In cases where no significant difference was found, confidence limits for the true differences, or minimum detectable differences, should be considered. In many cases the minimum detectable differences may themselves be of practical importance. This would indicate a need for further sampling (or more powerful statistical methods). 
Some of the analyses are inherently noisy. This is seen in the wide departure of the confidence bounds from their corresponding median or percentile estimates. In certain cases risk arguments may demonstrate that the results are adequate (or more than adequate) despite the noise. In other cases the noise may be remedied by some method of combining data that is more complicated than the lognormal model used here (e.g., a multivariate analysis using vectors of measurements over horizons). It is likely, however, that in many cases the only good way to remedy the noise - an unavoidable consequence of the survey's limited sample sizes-is to get more data. 


\section{DATA INTERPRETATION}

\subsection{SUMMARY}

This section fulfills project objectives for technical evaluation of project data that will maximize its usefulness to other Environmental Restoration projects and field investigations.

Chemical compounds, minerals, elements, and radionuclides in soils can have several sources. Extraction procedures remove differing amounts of various soil constituents, and the location of the soil in the landscape can affect the data. Interpretation of this type of data, then, must be done very carefully.

Screening of sites on the Oak Ridge Reservation (ORR) by a hand-held radiation detector, plus gamma screening and analysis of volatile organic compounds (VOCs) for all Phase I sites did not reveal any gross contamination, although one or more of the Roane County and Anderson County sites had some detects for organic contaminants. Both VOC and organic analytical techniques can be used elsewhere in the ORR without restriction as to site properties or soil conditions. The Phase I data indicate that any VOC or organic detects in suspected contaminated sites should be taken as a sign of contamination.

Inorganic compounds in soils present a much more difficult situation regarding interpretation. Some inorganics are definitely inherited from the underlying geologic formations, while others have an anthropogenic source from either global fallout or from local and regional sources. For example, lead and arsenic can have two sources, while nearly all mercury can be considered a surface contaminant. Several metal elements including cadmium, osmium, and silver were not detected in any soil samples. The presence of any of these would be an indicator of possible contamination.

Radionuclides also have two primary sources, the underlying geology and global and regional anthropogenic sources. However, a third possible source of radionuclides on the ORR cannot be ignored, and their presence can be interpreted as a sign of local contamination. Cesium-137 and strontium-90 also can have a local source as well as a global fallout source. Some important radionuclides, such as thorium and potassium- 40 , have a total geologic source; some other radionuclides, such as curium-247, tritium, and technetium-99, have a mostly local source. Concentrations of these radionuclides above background levels should be taken as indication of potential contamination from local sources.

In summary, none of the ORR Phase I sites exhibited any indication of disturbance in the past 50 years approximately. For this reason, Phase I data can be considered "background" and used as a basis of comparison with similar areas on the ORR where contamination is known or suspected provided the underlying geology and soil classifications are the same. Individual site-based interpretations are presented 'a Appendix A (in Sects. A.7, A.8, and A.9) for the ORR, Roane County, and Anderson County sampling areas. Analysis of data from composited soil samples is discussed in Sect. A.10 of Appendix A. 


\subsection{BASIC IDEAS AND CONCEPTS OF INTERPRETING SOUL DATA}

The intent of Background Soil Characterization Project (BSCP) data interpretation is to partition soil chemical analysis data according to three sources: (1) the inherited geologic source, (2) the world-wide or regional atmospheric depositional source, and (3) the source introduced by local surface or subsurface deposition. Some portion of all of these sources can be considered to be hazardous, but the component of greatest concern to be screened out and eliminated from this study is hazardous materials resulting from manufacturing and processing activities.

Soil extraction factors that can affect the measured chemical content of soils: The interpretation of analytical results of data from a soil environment can often be an exercise in both frustration and uncertainty. The chemical extraction of inorganic soil components is also fraught with great uncertainties. The $\mathrm{pH}$ of unbuffered soil extractants can change from sample to sample, resulting in the extraction of differing amounts of what is to be measured. Differing extracting methods and procedures result in differing amounts of what is measured. The scientific literature is full of differing methods for extracting soil components.

The soil system is dynamic in both time and space. Included is a very dynamic biotic component. For example, some inorganic ions are quite immobile, but if transformed into organic compounds, they can be come very mobile and potentially hazardous. Methyl mercury is a prime example. Biotic compounds of arsenic and lead behave similarly. Therefore, the interpretation of results must be based on a knowledge of what goes on at various depths in a soil system and how the whole soil system reacts and interacts. In this BSCP activity, samples were collected from specific soil horizons rather than from prescribed depths. The only exceptions were the gamma screening samples. Gamma screening was done primarily to determine the atmospheric input of cesium-137, so the upper $30 \mathrm{~cm}$ of the soil profile was sampled in $5-\mathrm{cm}$ increments.

Landscape factors that may affect the chemical content of soils: Several soil-landscape variables can affect what is measured. Some variables can act independently, whereas other variables interact in unpredictable ways. One major variable that can affect results and interpretation of those results is the location of the soil in the landscape. A soil can be affected by the adjacent soils, especially those soils at higher elevations. Rainfall can infiltrate or run off from higher soils. Rainfall that has infiltrated soil at higher elevations can then move laterally below the surface to affect soils down slope. The primary objective of BSCP was to sample soils that were (1) geomorphically stable, (2) located in the highest part of the landform so that there would be minimal effects from the immediate adjacent soils, (3) not disturbed in the past 50 years or more and had a hardwood forest, (4) not eroded, and (5) formed in residuum. However, reality dictated that some chosen sites were on side slopes, some had a thin capping of either old colluvium or old alluvium, and some were located in older loblolly pine plantations or in old-field successional mixed pine and hardwood forests.

If future sites are sampled on recently eroded side slopes, in recently established pine plantations, in depositional, concave toeslope-footslope areas, or in floodplains, the results generated can be higher or lower compared with data from the Phase I soils that meet the sampling objectives described in the preceding paragraph. In other words, the Phase I BSCP data represent only a certain portion of the landscape that is underlain by Dismal Gap and Nolichucky residual soils. Many of the solid waste storage sites and other potentially 
contaminated areas on the ORR are located on lower landscape positions. The background levels of contaminants in colluvial or floodplain soils can either be higher or lower than the background levels measured in this project.

Factors that can affect the chemical contents of $A, B$, and $C$ soil horizons: Samples were obtained from (1) the A horizon of the soil, (2) the B horizon of the soil, and (3) the " $\mathrm{C}$ " horizon (including either the lower $\mathrm{B}$ horizon, a transitional $\mathrm{BC}$ or $\mathrm{C} \cdot \mathrm{B}$ horizon, or the $\mathrm{C}$ horizon.) The $A$ horizon contains the most organic carbon and also the laighest biotic activity. Here, soil fauna can decompose or transform one compound into another or inorganic compounds can be transformed into more mobile organic compounds. Both aerobic and anaerobic respiration can occur in this surface mineral horizon of the soil.

The B horizor of most soils, commonly known as the upper subsoil, is the soil zone in which there is a net accumulation of soil clay minerals and iron oxides. Here, soil fauna tend to degrade organic compounds that bave been translocated from the $A$ horizon above, releasing metal ions from an organic form to an inorganic form. Respiration in this part of the soil tends to be aerobic on ped surfaces and along root channels and anaerobic within peds. Dissolved organic carbon moves into and through this soil horizon. Whether it is degraded and releases any metal ions depends on its rate of movement and time of residence. Saturated flow will tend to move dissolved organic carbon compounds and other ions rapidly through larger flow zones so that the soil fauna never come into contact with it. Another process that often occurs in this upper subscil zone results in the destruction of clay minerals and the release of both silica and alunina ions and their lateral or downward translocation.

The $C$ horizon occurs at a highly variable depth in the soil. It begins at the upper zone where saprolite or saprolitic material with its geologic strike and dip can be recognized or where pedogenic soil structure becomes minor. Here, soil processes are minimal, Lut there is often some biotic component, especially the soil fauna associated vith roots and dissolved organic carbon that move downward along ped surfaces and along fracture and joint suriaces. Soil moisture remains nearly constant, and most soil fauna respiration is anaerobic, except along cracks and pores open to the surface. Where $C$ horizons occur close to the surface (less than $50 \mathrm{~cm}$ to about $100 \mathrm{~cm}$ ) as in most Dismal Gap and Nolichucky soils, there is a much higher organic component than in the " $\mathrm{C}$ " horizon of Copper Ridge soils that were sampled below a depth of 140 to $160 \mathrm{~cm}$. The $\mathrm{C}$ horizon zone of the soil tends to be the location where there is deposition of ions translocated through the horizons above. Here, manganese and other ions with similar chemistry are often found in higher concentrations. In this part of the soil, water movement becomes increasingly channelized into well-defined flow zones. Flow zones in this part of the soil usually have a rather intense reduction potential because the oxygen partial pressure is very low. Here, some ions that are generally quite immobile are transformed into more mobile forms. For example, manganese oxides are reduced, resulting in greater mobility. The same happens with iron oxides that are transformed from ferric to ferrous forms and acquire a layer of oriented hydration water or hydroxyl groups. Other ions having similar geochemical properties can also tecome mobile in this zone.

Factors that must be considered in the inicial comparison of results: When making a comparison with a new site, the best interpretation of results involves having a set of data from the $A, B$, and $C$ horizons of a particular site under similar vegetation to observe trends of those ions in question. For example, contamination via surface deposition on a grassy slope should be confined to the surface if ions are immobile because of the shallow rooting of 
grasses. In a forest, contamination via surface deposition is a different situation. Here, stem flow can deliver contaminants deeply into the soil through root flow zones and rapidly into shallow water tables. Tree drip can produce zones where the level of contamination may be higher. A high degree of spatial variability is normal in a forested soil. The data from an A horizon at one site should not be compared with the $\mathrm{B}$ or $\mathrm{C}$ horizon data from another site. Inorganic and natural radionuclide data from Dismal Gap and Nolichucky soils may not be directly compared with data from Pumpkin Valley, Rutledge, or Maynardville soils because soils from different geologic formations can have different levels of inherited metals and natural radionuclides. Inorganic data from residual soils may not be compat od with alluvial or colluvial soils. However, such restriction of data use will be reevaluated after Phase II investigation.

\subsection{VALID DATA COMPARISONS}

Gamma Scanning: Gamma scanning via the methodology in ES/ER/TM-26/R1 can be used anywhere and for all conditions for sample/site screening purposes, based on cesium-137 activity levels. This procedure requires that a standard cross-sectional area be sampled. This is accomplished by using a rigid form that has sides $10 \mathrm{~cm}$ long and $5 \mathrm{~cm}$ deep. All soil must be put into the special scanning container. The sampling form must to be thoroughly cleaned of soil to minimize cross-contamination. Obtaining a series of samples at 5-cm-thick increments ensures that all cesium- 137 has been found for upland residual soils. However, cesium levels for alluvial and colluvial soils can be much different. Colluvial soils usually have higher levels of cesium- 137 because of surface and subsurface transport from soils higher in the landscape. In floodplain and low-terrace landscapes, it will often be necessary to sample deeper, where modern deposition of sediments has occurred, because the products of airborne deposition can be buried below a depth of $30 \mathrm{~cm}$. The other possibility is that the cesium-137 and other associated contaminants may have been removed from the site by surface erosion.

The gamma-scanning error term must be considered in attaching significance to any data. The error term for some elements is very low, less than $10 \%$, whereas the error terms for other elements, namely uranium-235 and uranium-238 tend to be large ( $>50 \%$ ). Uranium screening data were reported but should not be used because the contract laboratory will use more sensitive analytical methods.

Volatile Organic Compounds: VOC analysis can be done at any site on the ORR. Some precautions must be considered in interpreting results. Certain organic compounds, such as acetone, butanone, and other laboratory-induced compounds, commonly show up in the results. These are mostly the result of contamination of the analytical apparatus. The interpretation of other results must be based on the life of such volatile compounds in an aerobic surface soil environment.

Pesticides, Herbicides, and PCBs: The analysis for these compounds is done on field moist soil samples. The surface leaf litter in a forest soil is removed, and a sample is immediately collected. For bare or grass-covered soil, a sample is collected from the upper 5 to $10 \mathrm{~cm}$ of the soil. Additional samples can be collected at depth to determine the extent of downward migration. If an upwelling plume is suspected, a sample or samples can be collected at depth to confirm or reject the hypothesis. The interpretation of results must be based on the life of such compounds in a soil environment. Some compounds have a very long 
half-life, whereas others are readily decomposed by the indigenous soil fauna. Most of the compounds that were analyzed for BSCP have a very long half-life or the daughter products still have undesirable biochemical properties. Therefore, it is helpful in interpretation if the time when the suspected contamination occurred is known.

Inorganics: Inorganics occur as cations and anions, as well as in the mineral fraction. Some cations are relatively mobile, whereas others are not. Most anions are mobile because there are very few anion retention sites in soils, the notable exceptions being the organic carbon component of the surface soil layer (A horizon) and oxides that coat ped surfaces in the subsoil (B horizon) or fracture faces in the $\mathrm{C}$ horizon.

Many cations of metals, such as aluminum and iron, are dominant components of all mineral soils and are not diagnostic of any contamination. Some metals are inherited from the underlying geology. If the distribution of these metals remains the same throughout the various sampling depths or increases with depth, they usually have a geologic origin, especially if they are not mobile in a soil environment. Results must be interpreted carefully so that anthropogenic contamination can be distinguished from geologically inherited inorganics. Some metals can be introduced by the use of sampling equipment. A comparison of ORR source water (No. 1465) with the inorganics field rinse water (No. 1459) showed that the rinse water contained higher amounts of aluminum, iron, manganese, silica, strontium, and copper. Geologic inherited inorganics must be determined from the particular geologic formation because different geologic formations have differing levels of rare earths and heavy metals. One must also be aware that sedimentation conditions vary within any geologic formation. Another complicating factor in interpreting results is the past land use of a site. Past fertilizer and lime applications can result in increased amounts of heavy metals and rare earths in the surface soil, especially if rock phosphate was used. The widespread use of certain fungicides and pesticides, such as copper sulfate or lead arsenate, can also affect interpretations. Comparisons of inorganic results should be confined to the same geologic formation or section of the formation. Without some baseline data from the Melton Valley section of the Conasauga Group, comparison of Bear Creek Conasauga section data with Melton Valley section data is tenuous, even from the same formation and from soils of similar morphology.

Radionuclides: The interpretation of radionuclides can be difficult. The presence of certain nuclides, such as cesium-137, is nearly always the result of airborne deposition, whereas other nuclides are all inherited from the underlying rock. Uranium isotopes (uranium-235 and uranium-238) present special problems in interpretation because some are inherited from the underlying rock and some are the result of airborne dust deposition. Therefore, comprehensive assessment will be done after Phase II investigation.

\subsection{INTERPRETATION OF PHASE I DATA}

Detailed analytical results of the soil chemical analyses for organics, inorganics, and radionuclides are given in Appendixes $\mathrm{C}, \mathrm{D}$, and $\mathrm{E}$, respectively. The analytical procedures are referenced to the EPA Contract Laboratory Program Statement of Work (EPA 1990a and $b$ ). 


\subsubsection{Organic Compounds}

Screening analysis for VOCs were negative except for the following sites. Site ROA-8 in Roane County contained 1,1,1-trichloroethane. Site ORR-31 on the reservation had trichlorofluoromethane, but the field duplicate for this site did not contain any contaminants. The presence of detectable VOAs for any potentially contaminated ORR site samples can be taken as a sign of probable contamination.

The analyses of organochlorine pesticides, polychlorinated biphenyls (PCBs), herbicides, and polynuclear aromatic hydrocarbons (PAHs) were performed only on surface soil samples. The results indicate that no herbicides were detected in the A horizon of any Dismal Gap or Nolichucky site. Pesticides were identified at only two sites, ROA-8 (4,4'-DDT) and ROA-14 (Aroclor 1242). Both of these sites are currently in old-field successional woods.

PAHs were detected at 7 of 12 Roane County sites, 7 of 24 sites on the ORR, and all Anderson County sites. ORR sites 13, 16, 19, 23, 24, 31, and 42 showed evidence of PAHs. The ORR-2 primary sample showed evidence of PAHs, but the field duplicate sample did not. The ORR-2 VOC sample also showed chloroform. This site is located immediately grid east of the K-25 Site water treatment plant. Of interest is that the ORR sites closest to a major highway did not show any indication of PAHs. Those sites that did indicate the presence of PAHs occur in a random pattern. ORR-13 was located in an old farm yard and also close to a recently planted pine plantation; thus there was some potential for contamination with acenaphtene. ORR site 16 is located close to the intersection of a high-pressure gas pipeline and an electrical transmission right-of-way. This site showed evidence of benzo[a]pyrene, benzo[b]fluoranthene, and benzo[k]fluoranthene. Can these PAHs come from diesel exhaust smoke or have another source? ORR-23 was located fairly close to a well cluster where three wells had been drilled approximately 5 to 6 years ago. ORR-31 was located near the edge of an area where mature pines had been harvested. It is possible that the presence of benzo[b]fluoranthene might be related to this past activity. The ORR-31 primary sample also showed evidence of a VOC, trichlorofluoromethane, but the field duplicate sample did not. The ORR -42 site, located some distance upslope into the woods, but close to the west end of the Y-12 burial grounds, contained estimated " $B$ " detects of both benzo[b]fluoranthene and phenanthrene. However, ORR-43, located between ORR-42 and the Y-12 burial grounds, does not contain any PAHs above detection limits.

The presence of organic compounds above minimum detection levels can be taken as a sign of probable contamination on the ORR. However,.some of organic compounds are detected more often off-site than on-site, suggesting that the presence of the organic compounds in the ORR is not related to DOE activity.

\subsubsection{Inorganic Compounds and Metals}

Inorganics and metals were analyzed using five analytical techniques. The acid extraction method, however, causes considerable laboratory variability, and some elements discussed here are more susceptible to extraction by acid than others depending on the natural soil $\mathrm{pH}$ and the nature of the compound. Acid extraction data will not be comparable to NAA data, to total analysis data, or to cation-anion exchangeable data. Some of the data distribution by soil horizon reflect the translocation of certain constituents, while other data indicate the surface addition of contaminants (e.g., lead and mercury), and still other data indicate a geologic 
origin. Several metals were usually below detection limits, including cadmium, osmium, and silver. The following discussion uses median values determined from statistical analysis.

The primary sources of the following information are $\mathrm{K}$. Rankama and T. G. Sahama, Geochemistry, The University of Chicago Press, 1950; and A. L. Page, R. H. Miller and D. R. Keeney (Eds.), Methods of Soil Analysis, Part 2-Chemical and Microbiological Properties, Agronomy Monograph No. 9, Part 2, 2nd ed., American Society of Agronomy, Soil Science Society of America, Madison, Wisconsin, 1982.

Aluminum: Aluminum is a natural constituent of all inorganic soils. Acid extraction removes large quantities of aluminum from soils. Most of the aluminum comes from clay lattices. The data indicate that Roane County Dismal Gap soils have significantly lower acidextractable median aluminum levels than do ORR and Anderson County Dismal Gap soils. Acid-extractable aluminum levels are always lower in the A horizon of soils because the clay mineral content is lower and is higher in clay-enriched subsoil B horizons and in C horizons of Dismal Gap and Nolichucky soils.

Antimony: This element was extracted with consistency only from the ORR Nolichucky sites. It reaches a maximum in the $\mathrm{B}$ and $\mathrm{C}$ horizons. Its most likely source is the underlying rock.

Arsenic: Arsenic can be inherited from the underlying rock and also can be added to the soil as a surface contaminant from coal exhaust gases and from spraying arsenic-containing pesticides on fruit trees. Acid-extractable arsenic contents of A, B, and C horizons were the highest for ORR Dismal Gap sites. The remainder of the Phase I data does not exhibit any trends or relationships indicating either a geologic origin or contamination from regional coalfired power plants. Arsenic can occur in the soil in the following valance states: $-3,0,+3$, and +5 in compounds that have varying solubility and dissociation constants. These compounds can be translocated within the soil on fine clay particles. Biologic processes can transform inorganic forms to volatile organic forms that are readily taken up by plants. Levels of arsenic that are higher in the A horizon than in the subjacent B and C horizons should be taken as a sign of potential contamination.

Barium: Acid-extractable barium occurs rather uniformly in all soil horizons and at all Phase I locations. Barium occurs in slightly higher levels in the ORR Dismal Gap soils than in off-site Dismal Gap soils. The source of most barium in soils is from geologic origins.

Beryllium: Beryllium was detected or estimated to be in all samples from all Phase I sites but in very small amounts. Median beryllium levels were lowest in surface $A$ horizons and higher in B and C horizons. Because beryllium levels are so low and exhibit very high variability, more samples would be needed to establish a reasonable background level. Beryllium is closely associated with aluminum, where it can be substituted for aluminum in the lattice structures of clay minerals and is probably correlated with the acid-extractable aluminum levels reported in this study. Data did not show systematic differences between onsite and off-site locations. Roane County sites have lower concentrations than other sites.

Boron: Boron was not detected in any of the ORR Nolichucky sites nor in any of the Anderson County Dismal Gap sites. Boron does occur in all horizons of the ORR and Roane County Dismal Gap sites. The presence and distribution of boron are from geologic origins. 
However, some boron, from volcanic eruptions, is deposited on the soil surface and then subjected to biologic uptake or to downward translocation. The results suggest a possible analytical problem on this data set and need to be reevaluated with Phase II results.

Cadmium: There was no detectable cadmium at any Phase I site because natural occurrence in soils is typically low. Therefore, any detectable cadmium can probably be interpreted as local contamination.

Calcium: One must keep in mind that an acid extraction for determination of calcium is of questionable validity. Total calcium from NAA will yield a much more valid result for actual levels of soil calcium. However, interpretation of these data follows. Higher levels of calcium in A horizons than in $\mathrm{B}$ or $\mathrm{C}$ horizons usually indicate that there have been past additions of agricultural "limestone". Where there is a small increase of calcium in the $A$ horizon over the level in the $B$ and $C$ horizons, it can also be due to biologic translocation. The low median calcium levels in Roane County Dismal Gap soils in conjunction with other data, including potassium-40 data, indicate that the Roane County soils are more highly weathered and probably had lower levels of calcium in the underlying parent rock. The ORR Dismal Gap rocks were intermediate in calcium levels, and the Anderson County Dismal Gap rocks contained the highest calcium levels.

Chromium: Chromium is present in about the same median amounts in all Dismal Gap sites. The Nolichucky data have problems. Composited data from ORR 16-28-42 (Samples 5064 and 5067) have nondetectable " $U$ " levels of chromium in the A and B horizons, but there is a detectable level in the $C$ horizon beneath (sample 5070). In contrast, the other ORR Nolichucky sites have chromium distributed throughout all three soil horizons. This results in median values being low in the A and B horizons of Nolichucky soils. The high median chromium levels in the $\mathrm{C}$ horizons of the ORR Nolichucky soils are due to high levels in only two of the four sites, highly unlikely given the random grouping of sites for compositing. These data may not be correct either. In conclusion, chromium data for ORR Nolichucky soils are highly suspect, but the data seem to be reasonable for all of the Dismal Gap sites. There are two common sources of chromium in soils, a small geologically inherited component and as a flyash contaminant from coal-powered electric generating plants.

Cobalt: Median levels of cobalt appear to be distributed throughout all Phase I sites. Roane County and ORR Dismal Gap sites have higher median levels of cobalt in A horizons than in B horizons. There are probably two sources, that inherited from the underlying geology and that occurring as a contaminant from the flyash of coal-powered electric generating plants.

Copper: Median levels of copper seem to be fairly evenly distributed throughout all Phase I soils. Median copper levels of $\mathrm{C}$ horizons are higher than in $\mathrm{A}$ and $\mathrm{B}$ horizons. Copper occurs in soils in $\mathrm{Cu}^{+2}$ and $\mathrm{Cu}^{+3}$ compounds with varying solubilities and dissociation constants. Copper can be biologically translocated in the soil as well as being translocated downward attached to clay minerals. Some of the copper reported in this study may also come from the stainless steel sampling equipment. A comparison of the ORR rinse water with the source water indicates that rinsate copper levels are higher than in the source water. This trend was not seen in the Anderson County source and rinse water comparison. 
Cyanide: Cyanide levels are either below detection limits or just above detection limits as " $\mathrm{B}$ " estimates. The presence of higher cyanide levels in any soil would be a sign of potential contamination.

Iron: Iron is an important component of most well-drained upland soils, and large amounts are readily extracted by an acid extraction procedure. Extractable iron levels are usually associated with the clay content. Iron levels are always lower in the A horizons of soils because of various soil processes that result in the translocation of iron compounds from $A$ and $\mathrm{E}$ soil horizons to subsoil $\mathrm{B}$ horizons. Reduced iron $\left(\mathrm{Fe}^{+2}\right)$ is quite soluble, moving both laterally and downward with soil water. Acid-extractable iron cannot be considered diagnostic of any soil contamination.

Lead: Acid-extractable lead is the highest in all surface A horizons, indicating that a substantial source is from airborne deposition. The Anderson County sites have higher levels, perhaps a reflection of being downwind from a major metropolitan area (Knoxville) and also downwind from the Bull Run Steam Plant, where flyash and automobile exhaust deposition would contribute to higher levels of lead. Some lead in soils is inherited from the underlying rock.

Some of the lead data from the ORR Nolichucky Formation appears to have a laboratory problem. Samples 5064 and 5067 (A and B horizons) have nondetected lead levels, while sample 5070 from the $C$ horizon has a value above detection and is not estimated. The lack of data for the $\mathrm{A}$ and $\mathrm{B}$ horizon results in median values being much lower. With the exception of these two samples, the rest of the Phase I data have detectable or estimated lead in all samples.

Lithium: Acid-extractable lithium occurs in all samples, but the highest levels were in C horizons. High levels of lithium in the soil surface over levels found in lower B and C horizons would indicate a possibility of surface contamination.

Magnesium: Acid-extractable magnesium is a common constituent of most soils and sometimes occurs in higher amounts than calcium. Magnesium levels are lower in Roane County Dismal Gap soils than in Anderson County and ORR Dismal Gap soils. These data indicate that the Roane County Dismal Gap soils are different from the ORR and Anderson County Dismal Gap soils. Magnesium levels are higher in $C$ horizons than in A and B horizons. This distribution results from the usually net downward movement of this element. Some surface A horizons can have slightly higher levels of both magnesium and calcium, a result of biologic uptake. Past additions of agricultural lime can also result in higher levels of surface magnesium. The ORR Nolichucky soils have less magnesium than the ORR Dismal Gap soils, a reflection of the lower carbonate content of the Nolichucky Formation.

Manganese: Acid-extractable manganese has a varied soil distribution. Median contents are the highest in surface $\mathrm{A}$ horizons and the lowest in $\mathrm{C}$ horizons. Manganese in soils has several valance states, with some compounds having quite high solubility and others being quite insoluble. Most soil manganese compounds have a valance of $+2,+3$, and +4 . Most soil manganese occurs in the oxide form, which has a very low solubility. Manganese along with iron compounds in the soil is involved with oxidation-reduction processes as either an electron donor or acceptor. Many soils are deficient in manganese. Manganese is of interest in soils because of its association with other trace and potentially toxic metals. 
Mercury: Mercury occurs in all surface A horizons of ORR Dismal Gap and ORR Nolichucky soils, but only one Anderson County and only two Roane County A horizons from composited sets contained mercury. Only ORR composited sets contained detectable amounts of mercury in $\mathrm{B}$ and $\mathrm{C}$ horizons. The presence of mercury in soils can be taken as an indicator of airborne deposition, and probably none is inherited from the underlying geology.

Molybdenum: Molybdenum levels were below the detection level for all Phase I sites, but there were a few " $B$ " estimated detects. The presence of molybdenum above detection can probably be taken as a sign that there is probably surface contamination.

Nickel: Acid-extractable nickel occurs in all Phase I soils. Both Anderson County and ORR Dismal Gap soils have higher amounts of nickel throughout than the Roane County Dismal Gap soils and the ORR Nolichucky soils. Most of the nickel has a geologic origin, but some nickel may come from automobile exhaust or from oil-burning power plants. However, the level of nickel is the lowest in the soil surface and highest in the $\mathrm{C}$ horizon, indicating a dominant geologic source.

Osmium: No osmium was detected in any Phase I soils. Its presence could be taken as a sign of contamination.

Potassium: Potassium is an important element in all soils. Its natural occurrence in soils is of geologic origin. Potassium, being an important plant nutrient that is nearly always limiting, is added to soils in fertilizer. That potassium fertilizer was added to some soils in this Phase I study cannot be ruled out, but distributions by soil horizon and by location indicate that very little potassium fertilizer was ever applied to the sampled sites. Roane County Dismal Gap soils have the lowest potassium levels, another indication that these soils are different from the ORR and Anderson County Dismal Gap soils. Anderson County Dismal Gap soils have the highest potassium levels in all three horizons when compared to the ORR Dismal Gap median levels. ORR Nolichucky soils, having a higher clay mineral content and also a higher mica content, have quite high potassium levels, especially in the $\mathrm{C}$ horizon. Potassium levels in soils cannot be taken as an indicator of any contamination.

Selenium: Selenium, as estimated "B" detects, occurs in all surface A and B horizons of Anderson County Dismal Gap soils and only trace amounts in Roane County Dismal Gap soils. No selenium was detected in any ORR Dismal Gap soils. Three of four ORR Nolichucky composited site groups contained selenium but only as estimated " $B$ " detects. The presence of selenium above detection can probably be taken as an indication of probable contamination.

Silicon: Silica is a dominant component of all inorganic soils. Acid-extractable silica levels were the lowest in all three soil horizons of ORR Nolichucky soils and the highest for ORR and Roane County Dismal Gap soils. However, since the acid-extractable silica does not represent total silica content in the soil, it may not reflect the actual amount of silica in the soil.

Silver: Silver was not detected in any Phase I soil. Its presence above detection can be considered a probable sign of potential contamination. 
Sodium: Sodium was not analyzed. Because sodium ions are so mobile, the presence of sodium, unless in very high amounts from road salt contamination, cannot be used as a sign of probable contamination.

Strontium: Acid-extractable strontium occurs in all Phase I soils, but there is no evident pattern with respect to either location or soil horizon. Strontium-90 was not detected in any of the radionuclide analyses. If the $\mathrm{A}, \mathrm{B}$, and $\mathrm{C}$ soil horizons contain roughly equal amounts of strontium, the most likely source is inherited from the underlying geology, mostly from feldspars. If the soil surface contains higher levels of strontium, the most likely source is from coal flyash deposition. If strontium-90 occurs in the soil, it is a sign of contamination.

Sulfate: The most likely source of sulfate anions found in the surface horizons of soils is coal- and oil-fired electric power plants. Some sulfate can be inherited from the underlying geology where pyritic compounds weather. The Dismal Gap Formation, at least on the ORR, is known to contain pyritic materials.

Thallium: All thallium detects are " $B$ " estimated detects. The presence of thallium above detection would be an indication of probable contamination.

Vanadium: Acid-extractable vanadium occurs in all Phase I soils, and there are no readily apparent trends with respect to location or to soil horizon.

Zinc: Acid-extractable zinc occurs in all Phase I soils, with $\mathrm{A}$ horizons containing the least and $\mathrm{C}$ horizons containing the most. The most likely source of the reported zinc is from the underlying geology.

\subsubsection{Radionuclides}

Radionuclides in soils originate from three major sources: (1) naturally occurring in soils and bedrock; (2) global fallout after atmospheric bomb tests and nuclear reactor accidents in the former Soviet Union; and (3) local sources originating from uranium enrichment, isotope production, and reprocessing activities. This project tried to include all radionuclides that have been or could be detected in any known contaminated areas of the ORR.

Radionuclides from global fallout and local sources are expected to be associated mainly with surface soils (A horizon), and radionuclides from natural sources are expected to be present in both surface and subsurface soils (B and $C$ horizons). Therefore, except for gamma- producing radionuclides, global fallout and local sources of radionuclides, such as plutonium isotopes, neptunium-237, strontium- 90 , technetium- 99 , and tritium, were analyzed only in A horizon soil samples and were not analyzed in B and C horizon soils.

A total of 34 isotopes was analyzed. In most cases, the majority of radionuclides were not detected above the reported sample quantitation limit (qualifier UJ), or the analytical results were rejected because of serious deficiencies in the ability to analyze the sample and meet quality control criteria (qualifier R). The quantitation limit is approximate and may or may not represent the actual limit of quantification necessary to accurately and precisely measure the analyte in the samples, but it could be used as an upper bound of background concentration. On the other hand, the analytical results having data validation qualifier " $R$ " 
are reported because the presence or absence of the analyte cannot be verified, although the analytical deficiencies were caused mainly by the absence of the analyte.

Radionuclides commonly detected are cesium-137; europium-155; potassium-40; radium-226; curium-247; plutonium-238, and -238/240; thorium-228, $-230,-232$, and -234 ; and uranium-233, $-234,-235$, and -238 (Table 5.8).

Cesium-137: The major source of this radionuclide is global fallout. Analytical results showed that both Dismal Gap and Nolichucky Formation A horizon soils on the ORR have a higher concentration than Dismal Gap formation soils at Anderson and Roane counties. The amount of the radionuclide decreased drastically in $B$ and $C$ horizons. A difference was also noticed from gamma screening results (see Sect. 6.2). The observation could suggest that the past operation of Oak Ridge facilities may be a contributing factor for the higher level of cesium-137 concentration. A more reasoned explanation is that the ORR sites have been much less disturbed for the past 50 years than the sites off the reservation. However, the statistical difference is significant but the actual quantity is very small. Background risk assessment of ORR should, therefore, use ORR values rather than the overall average value, and contaminated site assessment (cleanup reference value) should use off-site values.

Curium-247: Curium-247 is produced at ORNL as a part of isotope production activities. Therefore, some of sediments/soils and wastes contain curium isotopes. The analyte was positively identified in two soil samples from Nolichucky ORR. However, the concentrations are very close to the detection limit.

Europium-155: Europium-155 is a fission product having a relatively short half-life (5 years). The main source of this radionuclide is global fallout. The results show that the amount in the soil is very small and that there is no significant difference among horizons or among sites. The distribution results indicated that local sources are not a contributing factor, although some of the ORR contaminated sites have europium-155 in soil and waste.

Plutonium-238 and -239/240: Plutonium isotopes are global fallout radionuclides and were positively identified in only a few samples from $\mathbf{A}$ horizon of Dismal Gap Roane and Anderson counties and in B horizon of Dismal Gap Roane County and Dismal Gap ORR. The presence of the radionuclides in both on-site and off-site locations suggests that there is no contribution to the concentration by local sources. However, the plutonium-238 to plutonium-239/240 activity ratio does not match the known ratio (0.045) in global fallout. The observed values are at least ten times higher than the known ratio. This problem will be discussed in the project final report after Phase II data evaluation.

Potassium-40: Potassium-40 is the most abundant naturally occurring isotope in soils. In most cases, variability of potassium -40 is related to amounts of micaceous minerals and organic matter in the soils.

Radium-226: Radium-226 is a naturally occurring radionuclide in soils and one of the uranium-238 decay products. The analytical results show that soils from Dismal Gap Anderson County have significantly higher amounts of radium-226 than other soils. The Dismal Gap Roane County soils have a higher amount of radium than the Dismal Gap ORR soils but may not be significant. No noticeable trends or differences in the distribution of the radionuclide 
were observed between horizons. Therefore, environmental risk and contaminated site assessments should use on-site concentration values.

Technetium-99: Technetium-99 is one of the fission products that is introduced to the environment by reprocessing of spent fuel or from global fallout. Technetium- 99 data are still being evaluated at the time of this report, because of uncertainty in the representativeness of the analytical method used by the laboratory. All technetium results in this report were rejected (as discussed in Sect. 4.5.3.8). However, further evaluation of the analysis method and laboratory procedures may produce usable results, which will be incorporated in the project final report. In addition, resampling and reanalysis is being considered to ensure the availability of technetium data.

Thorium-228, $-230,-232$, and -234 : Thorium isotopes occur naturally in soils and are important for health risk assessment if the concentration in soils is elevated. Thorium-232 is a stable isotope, and the others are products of uranium or thorium decay. In the A and B soil horizons, the thorium-232 concentration of Dismal Gap ORR sites is lower than others, but that of Nolichucky ORR sites is higher than others. This observation suggests that the thorium-232 distribution is related to geologic formation rather than local input. Thorium-228 was distributed in the same pattern as thorium-232. Thorium-234 distribution in the soils did not show a consistent pattern.

Tritium: Although tritium forms naturally in the atmosphere, this source is not usually a significant one. Tritium has been used in many different projects at ORNL, resulting in a considerable amount of discharge to waste steams. Tritium was analyzed in most A horizon samples from the Dismal Gap Formation. Ten ORR sites and eight sites in Roane County were analyzed. Tritium was detected in five ORR sites with concentrations under $0.062 \mathrm{pCi} / \mathrm{g}$. The estimated quantitation limit was $0.04 \mathrm{pCi} / \mathrm{g}$. No tritium was detected in any of the Roane County samples.

Uranium-233/234, -235, and -238: Uranium was quantified by two different methods, alpha spectroscopy for the isotopic series and gamma spectroscopy for uranium-235 and uranium-238. Uranium isotope series occur naturally in soils, but the ORR soils were expected to have additional inputs from local sources, such as Oak Ridge K-25 Site and Oak Ridge Y-12 Plant operations. However, the analytical results of background soils do not confirm such speculation. Uranium-233/234 data show that $B$ horizon soils from Dismal Gap ORR had a higher concentration than other sites but that $C$ horizon soils from Dismal Gap ORR contained less than other $C$ horizon soils, except Nolichucky ORR soils. Uranium analysis data for the Nolichucky ORR are so low that they should be reexamined. The $A$ and B horizon soils of the Dismal Gap ORR site have somewhat higher concentrations than C horizon, but the off-site soils have the same trends. The uranium-235 data determined by the alpha method showed the same trend with uranium-233/234 data, although the number of detects for uranium-235 was lower. The uranium-235 results obtained from gamma spectrum are higher than the alpha analysis results. In these results, the Nolichucky ORR data are more reasonably close to the results from the other sites. Again, the results do not show a difference in uranium concentration between on-site and off-site soil samples. The uranium-238 results by alpha analyses show the same trend as the results of uranium-233/234. In the final project report, the results of neutron activation analysis will be incorporated. 


\section{BACKGROUND RISK EVALUATION}

\subsection{SUMMARY}

The Phase I background soil data, collected from the Dismal Gap and Nolichucky formations (horizon A) on the Oak Ridge Reservation (ORR) and from Anderson and Roane counties, were evaluated in terms of potential adverse effects to human health. This background risk evaluation provides a context for the discussion and comparison of risks associated with site-related contamination and for determining contaminants of concern for that site.

Three primary pathways of exposure were evaluated for inorganic analytes and radionuclides and include (1) direct ingestion of soil, (2) dermal contact with soil, and (3) external exposure to radionuclides in the soil. Background risks for individual analytes and total pathway risk estimates (i.e., the sum of the background risks of all analytes within a pathway) were determined.

The constituents detected in the uncontaminated background soil samples were evaluated within the context of EPA-approved guidelines for contaminated soils in which there are three regions of concern for carcinogenic risk $\left(<1.0 \times 10^{-6}\right.$ no concern; $1.0 \times 10^{-6}$ through $1.0 \times 10^{-4}$, range of concern; and $>1.0 \times 10^{-4}$, unacceptable) and two areas of concern in terms of systemic risk (hazard index $<1.0$, no concern; and $>1.0$, concern). The background risks are reported in this manner, but the results are only for comparison with risks determined for contaminated sites; the results do not pertain to remediation decisions.

In summary, with a few exceptions, the carcinogenic-risk and noncarcinogenic-hazard indices determined for individual analytes (found in horizon A of the Dismal Gap Formation) were very similar for the three sampling areas (ORR and Anderson and Roane counties). The total pathway background risks for ingestion of soil are $7.39 \times 10^{-6}, 8.07 \times 10^{-6}$, and $6.14 \times 10^{-6}$; for dermal exposure to soil are $1.13 \times 10^{-5}, 1.20 \times 10^{-5}$, and $9.33 \times 10^{-6}$; and for external exposure to radionuclides are $6.38 \times 10^{-4}, 8.51 \times 10^{-4}$, and $5.43 \times 10^{-4}$ for the ORR, Anderson County, and Roane County, respectively. The main contributor to the risk for the ingestion and dermal exposure pathways is beryllium. Cesium-137, potassium-40, radium-226, and thorium-228 are the main contritutors to risk for the external exposure pathway.

The total pathway hazard indices for ingestion of soil are $0.875,0.685$, and 0.973 ; and for

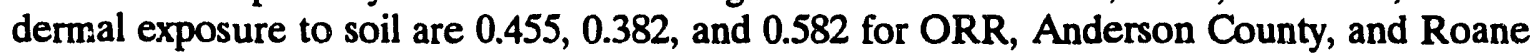
County, respectively. The total hazard indices for both pathways (i.e., ingestion of inorganics plus dermal exposure to inorganics) in the Dismal Gap Formation are 1.33, 1.07, and 1.55 for ORR, Anderson County, and Roane County, respectively. Arsenic and manganese are the major contributors to the $\mathrm{HI}$ for the ingestion pathway; and the main contributors to the $\mathrm{HI}$ for the dermal exposure pathway are manganese and vanadium. These background risk levels are discussed further and assessed in Sect. 7.6.

These background risk estimates should be considered only in the context of comparison with site-related risk. The EPA action level of $1.0 \times 10^{-4}$, refers to risks related to hazardous waste sites. The background risk results are not indicative of concerns or actions that would 
be identified with similar potential risks from a contaminated site, and care should be taken not to misinterpret these results to pertain to remediation decisions.

\subsection{INTRODUCTION}

A primary goal of producing a comprehensive data base for naturally occurring concentrations of soil constituents on the ORR is to support the need (for risk assessment) to differentiate contamination from naturally occurring constituents. The overall objective of this section is to evaluate the Phase I background data relative to risk. The risk assessment methodology in this study is based on the Risk Assessment Guidance for Superfund (RAGS) (EPA 1989c) so that risk results for exposure to background soil constituents will be comparable to site-related risk. Quantitative analysis of the inorganic (metals) and radionuclide analytes found in undisturbed soil (no organic analytes were detected) will identify the potential risks to human health associated with exposure to these naturally occurring constituents.

Specific objectives of this section are to (1) evaluate the potential risks from exposure to constituents in background soils on the ORR in order to provide a context for the discussion of risks associated with site-related contamination in future risk assessments and (2) provide a comparison based on background risk between the soils collected at the three sampling locations. Because remedial investigation (RI) activities will use soil data specific to the reservation to meet background needs, this evaluation focuses on background risks associated with ORR soils. Accordingly, the results of each step of the background risk evaluation are presented in full for the ORR soils. The same background risk evaluation process has been applied to the Dismal Gap Formation soil data from Anderson and Roane counties; however, only the total exposure risk and hazard results are presented for these locations as part of the comparison of background risks associated with the three sampling locales.

The organization of risk evaluation of background soils for Phase I from the Dismal Gap and Nolichucky formations is presented in this report. The first step involves evaluating the data from a risk assessment perspective and identifying the soil constituents that will be considered in the assessment. This process would parallel the selection of potential contaminants of concern at a contaminated site. Next is an assessment of the exposure potential and the identification of exposure pathways. Subsequently, exposure is estimated quantitatively, and the toxicity of the soil constituents is appraised. The results of the exposure and toxicity assessments are brought together in the background risk characterization section, which includes a comparison of background risks between the three locations and a more detailed description of the ORR soils background risk evaluation. The following sections describe the methodology used in evaluating site analytical data, physical characteristics, potential pathways, and receptors in quantifying the potential risk to human health from background soil constituents. 


\subsection{DATA EVAI,UATION}

\subsubsection{Data Usability}

In future site-specific investigations of the risk to human health posed by soil contamination at the ORR, the background risk associated with each analyte in this section of the Background Soil Characterization Project (BSCP) can be compared with the risk determinations for the analytes found in contaminated soils on the ORR. In addition, the $t \mathrm{t}-1$ soil background risk reported here can be used to discuss site-related risk in the context of background risk. However, the most important aspect of these data for risk assessment is in their application to the selection of potential contaminants of concern. The background values can be used to attain an accurate assessment of the risk to human health posed by contaminants found at higher concentrations than naturally occurring background concentrations (two orders of magnitude above background concentrations, according to EPA guidance; EPA/540/G-90/008, October 1990).

The risk evaluation of background soils on the ORR is to provide a context for the discussion of risks associated with site-related contamination in future risk assessments. To meet this objective, the background constituents detected in uncontaminated soils were evaluated by the same methods typically used to assess the potential risks resulting from exposure to contaminated soil. Similarly, the results of the background evaluation have been discussed within the context of the Comprehensive Environmental Response, Compensation, and Liability Act (CERCLA) framework, which uses the potential risks estimated from site-related contamination to determine if remedial action is necessary at a waste site. Although reported in this manner, the background risk results are not indicative of concerns or actions that would be identified with similar potential risks from a contaminated site. Care should be taken not to misinterpret these results to pertain to remediation decisions.

\subsubsection{General Site-Specific Data Collection Considerations}

General guidance for collecting soil samples is given in the Project Plan for the BSCP on the ORR in Oak Ridge, Tennessee (Energy Systems 1992). Guidance for soil sampling is also included in the EPA publication Preparation of Soil Sampling Protocol, Techniques and Strategies (EPA 1983). Standard procedures were also followed for the collection of samples (Kimbrough et al. 1988) and the Engineering Support Branch Standard Operation' Procedures and Quality Assurance Manual (EPA 1991a). Sample site selection and data collection are discussed in Sects. 3.2 and 3.4 of this report.

\subsection{General Site-Specific Data Evaluation Considerations}

The validated data included in this study consist of organic, inorganic, and radionuclide analyses of soils from two formations (Dismal Gap and Nolichucky), three horizons (A, B, and C), and three sampling areas (Roane County, Anderson County, and the ORR). Because organics are not naturally occurring background constituents, they were not evaluated in this risk assessment. For complete statistical analysis of the data, refer to Sect. 5. In this background risk evaluation section, both carcinogenic (cancer risk) and noncarcinogenic [hazard index (HI)] effects posed to a child and an adult in a hypothetical on-site residential scenario will be determined for the ORR soil samples taken from horizon A only. The analytes detected in horizon $A$ for the ORR sampling areas will be divided into two data sets 
(i.e., Dismal Gap horizon A and Nolichucky horizon A). Also included in this section is a comparison of total risk (child + adult) and total HI (child + adult) for the three sampling areas (Roane and Anderson counties and the ORR) for the Dismal Gap Formation.

In this phase of the BSCP study, background soil samples taken from undisturbed locations on the ORR (from horizon $A$ of the Dismal Gap and Nolichucky formations) best represent the background constituents found or the reservation and, therefore, best represent the background risk associated with these analytes.

\subsubsection{Identification of Constituents Included in the Background Risk Evaluation}

The identification of specific soil constituents included in the assessment of background soil risk is based on methodology from Sect. 5 of RAGS (EPA 1989c). The number of constituents that can be quantitatively evaluated in the risk evaluation is limited by the availability of chemical-specific EPA-approved dose/response information. The constituents considered in the quantitative assessment of risk and noncarcinogenic effects from the background soil are listed in Table 7.1. Note, beryllium is the only inorganic analyte found in the backgrrand soil samples for which an EPA-approved slope factor is available.

The risk from exposure to some constituents detected in soil cannot be quantivied because there are no current EPA-approved slope factors (SF) or reference doses (RfDs) available. Therefore, exposure to these constituents can only be evaluated qualitativaly (Table 7.2). A quantitative assessment of these soil constituents is not performed as part of this evaluation.

\subsection{EXPOSURE ASSESSMENT}

An exposure assessment combines information about site characteristics and constituent data with the exposure assumptions used by the risk assessor. The objectives of the exposure assessment are to determine or estimate the magnitude, frequency, and duration of present and future pathways of potential human exposure to site-contaminants by:

- characterizing the exposure setting,

- identifying exposure pathways, and

- quantifying exposures.

\subsubsection{Characterization of Exposure Setting}

Characterization of the exposure setting involves identifying the general physical characteristics of the site (e.g., climate and vegetation) and the characteristics of the populations on $\mathrm{cr}$ near the site. This characterization ensures that all potential constituent migration pathways and potential receptors are evaluated in the risk assessment. Details of the physical and environmental characteristics of the ORR and Anderson and Roane counties have already been discussed in Sect. 4 of the Project Plan for the BSCP (Energy Systems 1992).

To estimate human health risk tor background soil (i.e., uncontaminated soil), the soil sampling areas were selected from areas with minimal soil erosion and deposition, minimal 


\section{7-5}

Table 7.1. Oak Ridge Reservation background soil analytes evaluated quantitatively

\begin{tabular}{|c|c|c|c|c|c|}
\hline Analyte & $\begin{array}{c}\text { Frequency } \\
\text { of } \\
\text { detection }\end{array}$ & $\begin{array}{c}\text { Minimum } \\
\text { detection } \\
\text { concentration }\end{array}$ & $\begin{array}{c}\text { Maximum } \\
\text { detected } \\
\text { concentration }\end{array}$ & $\begin{array}{c}\text { Median } \\
\text { concentration }\end{array}$ & $\begin{array}{l}\text { Upper } 95 \% \\
\text { confidence } \\
\text { bound on the } \\
\text { median }\end{array}$ \\
\hline \multicolumn{6}{|c|}{ Dismal Gap } \\
\hline \multicolumn{6}{|l|}{ Inorganics (mg/kg) } \\
\hline Arsenic & $4 / 4$ & 5.300 & 7.300 & 6.23910 & 10.3490 \\
\hline Barium & $4 / 4$ & 77.20 & 212.0 & 99.0600 & 124.690 \\
\hline Beryllium & $4 / 4$ & 0.550 & 2.200 & 0.78063 & 0.99871 \\
\hline Boron & $1 / 3$ & 16.40 & 21.10 & 13.5410 & 23.3130 \\
\hline Chromium VI & $4 / 4$ & 19.40 & 32.10 & 24.6530 & 54.3050 \\
\hline Cyanide & $1 / 3$ & 0.440 & 0.440 & 0.13003 & 0.28146 \\
\hline Manganese & $4 / 4$ & 768.0 & 2220 & 997.070 & 1347.50 \\
\hline Mercury & $4 / 4$ & 0.230 & 0.400 & 0.31591 & 0.34211 \\
\hline Mercury (salts) & $4 / 4$ & 0.230 & 0.400 & 0.31591 & 0.34211 \\
\hline Nickel & $4 / 4$ & 19.50 & 56.70 & 23.4810 & 27.9970 \\
\hline Nickel (salts) & $4 / 4$ & 19.50 & 56.70 & 23.4810 & 27.9970 \\
\hline Strontium & $3 / 3$ & 6.100 & 16.80 & 7.93480 & 10.2180 \\
\hline Vanadium & $4 / 4$ & 27.90 & 54.00 & 34.1560 & 36.8490 \\
\hline Zinc & $4 / 4$ & 42.30 & 108.0 & 50.6480 & 58.1260 \\
\hline \multicolumn{6}{|l|}{ Radionuclides (pCi/g) } \\
\hline Cesium-137 & $4 / 4$ & 0.0210 & 0.900 & 0.59825 & 2.47050 \\
\hline Europium-155 & $4 / 4$ & 0.0840 & 0.110 & 0.09480 & 0.10530 \\
\hline Potassium-40 & $4 / 4$ & 14.000 & 22.00 & 16.3465 & 20.3483 \\
\hline Radium-226 & $4 / 4$ & 0.7000 & 0.860 & 0.78646 & 0.96091 \\
\hline Thorium-228 & $4 / 4$ & 0.5000 & 0.940 & 0.71322 & 0.86202 \\
\hline Thorium-230 & $4 / 4$ & 0.3100 & 0.830 & 0.56516 & 0.71725 \\
\hline Thorium-232 & $4 / 4$ & 0.4100 & 0.970 & 0.68310 & 0.81335 \\
\hline Thorium-234 & $4 / 4$ & 1.5000 & 1.900 & 1.63470 & 1.77100 \\
\hline Uranium-233/234 & $4 / 4$ & 0.6100 & 1.400 & 0.93725 & 1.08890 \\
\hline Uranium-235 & $4 / 4$ & 0.0569 & 0.120 & 0.07919 & 0.09499 \\
\hline Uranium-238 & $4 / 4$ & 0.7500 & 1.700 & 1.02470 & 1.15490 \\
\hline
\end{tabular}


Table 7.1 (continued)

\begin{tabular}{|c|c|c|c|c|c|}
\hline Analyte & $\begin{array}{l}\text { Frequency } \\
\text { of } \\
\text { detection }\end{array}$ & $\begin{array}{l}\text { Minimum } \\
\text { detection } \\
\text { concentration }\end{array}$ & $\begin{array}{c}\text { Maximum } \\
\text { detected } \\
\text { concentration }\end{array}$ & $\begin{array}{c}\text { Median } \\
\text { concentration }\end{array}$ & $\begin{array}{l}\text { Upper } 95 \% \\
\text { confidence } \\
\text { bound on the } \\
\text { median }\end{array}$ \\
\hline \multicolumn{6}{|c|}{ Nolichucky } \\
\hline \multicolumn{6}{|l|}{ Inorganics $(\mathrm{mg} / \mathrm{kg})$} \\
\hline Antimony & $1 / 4$ & 0.490 & 0.490 & 0.46320 & 0.48482 \\
\hline Arsenic & $3 / 4$ & 5.800 & 6.400 & 3.08630 & 5.14460 \\
\hline Barium & $4 / 4$ & 59.70 & 106.0 & 75.3620 & 94.8610 \\
\hline Beryllium & $4 / 4$ & 0.730 & 0.850 & 0.78616 & 1.00580 \\
\hline Chromium VI & $3 / 4$ & 26.40 & 29.90 & 9.37800 & 20.8120 \\
\hline Manganese & $4 / 4$ & 405.0 & 935.0 & 653.480 & 883.140 \\
\hline Mercury & $4 / 4$ & 0.180 & 0.190 & 0.18493 & 0.20027 \\
\hline Mercury (salts) & $4 / 4$ & 0.180 & 0.190 & 0.18493 & 0.20027 \\
\hline Nickel & $4 / 4$ & 15.20 & 20.00 & 17.2860 & 20.6100 \\
\hline Nickel (salts) & $4 / 4$ & 15.20 & 20.00 & 17.2860 & 20.6100 \\
\hline Selenium & $3 / 4$ & 0.560 & 0.740 & 0.56611 & 0.71345 \\
\hline Strontium & $4 / 4$ & 3.200 & 6.100 & 4.55220 & 5.66690 \\
\hline Vanadium & $4 / 4$ & 29.40 & 35.20 & 32.4070 & 34.9620 \\
\hline Zinc & $4 / 4$ & 33.90 & 40.70 & 37.8920 & 43.4870 \\
\hline \multicolumn{6}{|l|}{ Radionuclides (pCi/g) } \\
\hline Cesium-137 & $4 / 4$ & 0.38000 & 0.7100 & 0.52689 & 2.17570 \\
\hline Curium-247 & $2 / 4$ & 0.00530 & 0.0070 & 0.00552 & 0.00649 \\
\hline Europium-155 & $4 / 4$ & 0.08400 & 0.0980 & 0.08955 & 0.09947 \\
\hline Potassium-40 & $4 / 4$ & 14.0000 & 17.000 & 15.1952 & 18.9151 \\
\hline Radium-226 & $4 / 4$ & 0.39000 & 1.4000 & 0.74007 & 0.90422 \\
\hline Thorium-228 & $4 / 4$ & 1.20000 & 2.2000 & 1.50820 & 1.82290 \\
\hline Thorium-230 & $4 / 4$ & 0.85000 & 1.2000 & 0.96648 & 1.22660 \\
\hline Thorium-232 & $4 / 4$ & 1.20000 & 2.0000 & 1.49480 & 1.77980 \\
\hline Thorium-234 & $4 / 4$ & 1.30000 & 1.5000 & 1.42250 & 1.54120 \\
\hline Uranium-235 & $4 / 4$ & 0.04320 & 0.0969 & 0.07127 & 0.08549 \\
\hline
\end{tabular}


Table 7.2 Oak Ridge Reservation background soil analytes evaluated qualitatively

\begin{tabular}{|c|c|c|c|c|c|}
\hline Analyte & $\begin{array}{l}\text { Frequency } \\
\text { of detection }\end{array}$ & $\begin{array}{l}\text { Minimum } \\
\text { detected } \\
\text { concentration }\end{array}$ & $\begin{array}{c}\text { Maximum } \\
\text { detected } \\
\text { concentration }\end{array}$ & $\begin{array}{c}\text { Median } \\
\text { concentration }\end{array}$ & $\begin{array}{l}\text { Upper } 95 \% \\
\text { confidence } \\
\text { bound on the } \\
\text { median }\end{array}$ \\
\hline
\end{tabular}

\section{Dismal Gap}

Inorganics (mg/kg)

$\begin{array}{lrrrrr}\text { Aluminum } & 4 / 4 & 16900 & 44300 & 20656 & 23311 \\ \text { Calcium } & 2 / 4 & 991 & 1860 & 907.04 & 1320.6 \\ \text { Chromium } & 4 / 4 & 19.4 & 32.10 & 24.653 & 54.305 \\ \text { Cobalt } & 4 / 4 & 11.3 & 36.7 & 14.523 & 19.363 \\ \text { Copper } & 4 / 4 & 12.4 & 30.1 & 16.137 & 18.339 \\ \text { Iron } & 4 / 4 & 23800 & 49000 & 29438 & 32946 \\ \text { Lead } & 4 / 4 & 14.6 & 35.4 & 20.306 & 47.993 \\ \text { Lithium } & 3 / 3 & 12.7 & 27.0 & 16.165 & 18.753 \\ \text { Magnesium } & 4 / 4 & 2090 & 7430 & 2845.9 & 3505.9 \\ \text { Potassium } & 4 / 4 & 1890 & 5390 & 2300.3 & 2815.1 \\ \text { Silicon } & 4 / 4 & 461 & 697 & 505.90 & 563.01 \\ \text { Sulfate } & 3 / 3 & 28.0 & 163 & 86.736 & 110.92 \\ \text { Thallium } & 1 / 4 & 0.790 & 0.790 & 0.16355 & 0.60786\end{array}$

\section{$\underline{\text { Nolichucky }}$}

Inorganics (mg/kg)

\begin{tabular}{lrrrrr} 
Aluminum & $4 / 4$ & 20800 & 25100 & 22189 & 25041 \\
Calcium & $2 / 4$ & 498 & 952 & 490.00 & 734.28 \\
Chromium & $3 / 4$ & 26.40 & 29.90 & 9.3780 & 20.812 \\
Cobalt & $4 / 4$ & 11.1 & 17.5 & 14.446 & 19.262 \\
Copper & $4 / 4$ & 11.0 & 12.7 & 11.701 & 13.298 \\
Iron & $4 / 4$ & 23000 & 32100 & 27901 & 31226 \\
Lead & $3 / 4$ & 15.3 & 20.4 & 5.5086 & 13.131 \\
Lithium & $4 / 4$ & 7.60 & 15.5 & 10.926 & 12.426 \\
Magnesium & $4 / 4$ & 1730 & 2410 & 2008.0 & 2473.7 \\
Potassium & $4 / 4$ & 2640 & 3230 & 2949.8 & 3610.1 \\
Silicon & $4 / 4$ & 185 & 328 & 244.69 & 272.31 \\
Sulfate & $4 / 4$ & 14.1 & 25.4 & 18.657 & 23.085 \\
\hline
\end{tabular}


groundwater discharge, and minimal influence of past and present DOE activities (on-site) and agricultural practices (off-site). A hypothetical on-site resident scenario will be used to determine human health risk associated with background soils; this scenario uses conservatively based calculations, is an accepted default scenario by EPA, and is unlikely to underestimate the exposure to background constituents for individuals residing on or in the vicinity of the ORR.

\subsection{Identification of Exposure Pathways}

The identification of exposure pathways of concern is determined by evaluating all of the components (source, transport medium, exposure point, potential receptors, and routes of exposure) necessary to complete the potential exposure pathway. For an exposure pathway to be considered complete, each of these components must be identified and linked to each of the other components. Routes of exposure (ingestion, inhalation, dermal absorption, and external exposure to radiation) and potential receptors are crucial in identifying the validity of an exposure pathway. For example, an exposure scenario that includes dermal absorption of subsurface soil contaminants would not be valid for general personnel (industrial) receptors. However, for excavation workers, dermal absorption of subsurface soil contaminants could be possible, and such a scenario would be valid.

In this assessment, potential health effects from background soils are considered for horizon A surface soil in Roane and Anderson counties and from the ORR. Because soil samples taken on the ORR are the most representative of the background concentrations on the reservation, a detailed background risk analysis will use only the ORR soil data, and general comparisons will be made with the background risk determinations and results for Anderson and Roane counties. The following discussion evaluates the potential pathways related to the on-site resident scenario resulting from exposure to constituents in the soil.

A hypothetical residential exposure scenario is used to assess the risk from soil because it is protective of human health and is typically employed in the evaluation of risk from the exposure to contamination on the ORR. If we assume that concentration levels in the soil are constant, the potential pathways affecting the on-site resident would include direct exposure to soil as well as exposure to constituents in the soil transferred to the air. The direct exposure to soil would involve the ingestion and dermal contact routes of intake and exposure to radionuclides. Because of the uncertainty of modeling the air pathway, only direct exposure pathways (ingestion, dermal, and exposure to radionuclides) are addressed here.

\subsubsection{Quantification of Exposure}

Exposure, in the context of human health risk, is defined as the direct contact of a person with a chemical or physical agent. To quantify exposure, one must determine exposure concentrations and calculate chemical intakes for the various exposure pathways identified for the site. The potential exposure pathways at background soil sampling areas are considered quantitatively in the following section.

This section follows the procedure involved in developing the chronic daily intake (CDI) of a constituent (also termed "intake" or "dose" for external exposure to radionuclides). The $\mathrm{CDI}$ is the amount of a constituent an individual takes into one's body per day via ingestion or dermal contact. The first consideration in deriving the CDI is the methodology employed 
in the development of an exposure concentration, which is the amount of each constituent in the various media to which receptors are exposed. To calculate the CDI, one evaluates the exposure concentration in the context of the scenario, exposure pathway, and constituent-specific exposure variables, such as duration of exposure and intake rate. The quantification of exposure and calculation of the CDI for the resident are discussed in Sects. 7.4.3.1 and 7.4.3.2.

\subsubsection{Derivation of representative exposure concentrations}

This section and Sect. 7.4.3.2 address methods used in calculating the exposure concentrations for the hypothetical on-site residential exposure scenario and pathways evaluated in this background risk assessment. EPA guidance requires evaluation using the onsite residential scenario, which is the most conservative. This typically requires determination of risks associated with adult residents, as well as young children (especially with respect to dermal contact and ingestion of soil). As a result of the statistical data evaluation process described in Sect. 5, the set of background soil concentration data used in this background risk assessment was compiled. The results are summarized in Tables 7.1 and 7.2 and include the frequency of detection, the minimum and maximum detected concentrations of each analyte, and the analyte median concentration as well as the upper $95 \%$ confidence bound (UCB95) on the median. The UCB95 is assumed to be representative of the analyte concentration and is used in the calculations of the CDI and dose. This upper confidence bound is used to ensure that the exposure concentrations are not underestimated. Refer to Sect. 5 for a complete statistical evaluation of the data and the list of analytes reported as nondetects. The nondetected analytes are not evaluated in this risk assessment.

\subsubsection{Exposure to residents}

The potential exposure pathways associated with the on-site residential land use scenario are direct ingestion of soil, dermal contact with soil, and external exposure to the radionuclides in the soil. The representative concentrations (UCB95) of constituents in sampling area soils in Table 7.1 are the concentrations used to quantify exposures via soil-related pathways.

Table 7.3 lists the exposure variables associated with each exposure route considered for the on-site resident. The variables used in each exposure equation have been derived from standard intake rates, skin surface areas, and adherence factors (EPA 1991e). It was assumed that the resident would be exposed to soil constituents for $350 \mathrm{~d} /$ year for 30 years. All pathways were divided into two parts. First, a 6-year exposure duration was evaluated for young children, which accounts for receptors with high intake rates relative to body weight. Second, a 24-year exposure duration was assumed for adults. For example, for the soil ingestion pathway, a child ingestion rate $(200 \mathrm{mg} /$ day $)$ and body weight $(15 \mathrm{~kg})$ was assumed for 6 years, while an adult ingestion rate $(100 \mathrm{mg} /$ day $)$ and body weight $(70 \mathrm{~kg})$ was assumed for 24 years. CDIs for ingestion and dermal contact, and dose for radionuclide external exposure, for the background soil samples are listed in Table 7.4. Table 7.4 is separated by formation (Dismal Gap and Nolichucky) and according to whether a background risk or HI could be calculated (i.e., if an SF or RfD was available). In the cases where toxicity information (SF or RfD) is not available, CDIs are given so that when values become available in the future, a background risk or HI can be calculated for the constituents present in this BSCP. 
Table 7.3. On-site resident exposure scenario

\begin{tabular}{|c|c|c|c|c|}
\hline \multicolumn{2}{|c|}{ Variable } & & Value used & Explanation/source \\
\hline \multicolumn{5}{|c|}{ Residential ingestion scenario } \\
\hline \multicolumn{5}{|c|}{ Chronic daily intake $(\mathrm{mg} / \mathrm{kg}$-day $)=\mathrm{CS} \times \mathrm{IR} \times \mathrm{FI} \times \mathrm{EF} \times \mathrm{ED}$} \\
\hline \multicolumn{5}{|c|}{$\mathbf{B W} \times \mathbf{A T}$} \\
\hline \multicolumn{5}{|c|}{ Intake $(\mathrm{pCi})=\mathrm{CS} \times \mathrm{CF} \times \mathrm{IR} \times \mathrm{EF} \times \mathrm{ED}$} \\
\hline CS & $=$ & $\begin{array}{l}\text { Concentration in } \\
\text { soil }\end{array}$ & $\begin{array}{l}\text { Chemical-specific (mg/kg; } \\
\text { pCi/g) }\end{array}$ & $\begin{array}{l}\text { Concentration is obtained } \\
\text { from the data in Table } 7.1\end{array}$ \\
\hline \multirow[t]{2}{*}{ IR } & $=$ & Ingestion rate & $0.0002 \mathrm{~kg} / \mathrm{day}$ & $\begin{array}{l}\text { Child rate (Section 6, } \\
\text { RAGS, EPA 1989c) }\end{array}$ \\
\hline & & & $0.0001 \mathrm{~kg} / \mathrm{day}$ & $\begin{array}{l}\text { Adult rate (Section 6, } \\
\text { RAGS, EPA 1989c) }\end{array}$ \\
\hline $\mathrm{CF}$ & $=$ & Conversion factor & $10^{3} \mathrm{~g} / \mathrm{kg}$ & $\begin{array}{l}\text { Conversion factor necessary } \\
\text { to convert to appropriate } \\
\text { units. }\end{array}$ \\
\hline FI & $=$ & Fraction ingested & 1 (unitless) & $\begin{array}{l}\text { Maximum value used; } \\
\text { equivalent to } 100 \%\end{array}$ \\
\hline EF & $=$ & Exposure frequency & 350 d/year & $\begin{array}{l}\text { OSWER Directive 9285.6- } \\
03 \text { (EPA 1991e) }\end{array}$ \\
\hline ED & $=$ & Exposure duration & 24 years & $\begin{array}{l}\text { Two-part (childhood and } \\
\text { adult) residential exposure } \\
\text { for a 30-year duration } \\
\text { (OSWER Directive, EPA } \\
\text { 1991e) }\end{array}$ \\
\hline \multirow[t]{2}{*}{ BW } & $=$ & Body weight & $15 \mathrm{~kg}$ & $\begin{array}{l}\text { Child (OSWER Directive, } \\
\text { EPA 1991e) }\end{array}$ \\
\hline & & & $70 \mathrm{~kg}$ & $\begin{array}{l}\text { Adult (Section 6, RAGS, } \\
\text { EPA 1989c) }\end{array}$ \\
\hline \multirow[t]{2}{*}{ AT } & $=$ & Averaging time & $365 \mathrm{~d} \times \mathrm{ED}$ & $\begin{array}{l}\text { Averaging time } \\
\text { for noncarcinogens }\end{array}$ \\
\hline & & & 365 d/year $\times 70$ years & $\begin{array}{l}\text { Averaging time } \\
\text { for carcinogens }\end{array}$ \\
\hline
\end{tabular}


Table 7.3 (continued)

\begin{tabular}{|c|c|c|c|c|}
\hline \multicolumn{3}{|c|}{ Variable } & Value used & Explanation/source \\
\hline \multicolumn{5}{|c|}{ Residential dermal contact scenario } \\
\hline \multicolumn{5}{|c|}{ Chronic daily intake (mg/kg-day) $=$ CS $\times$ CF $\times$ SA $\times$ AF $\times$ ABS $\times$ EF $\times$ ED } \\
\hline & & & \multicolumn{2}{|c|}{$\mathrm{BW} \times \mathrm{AT}$} \\
\hline $\operatorname{CS}=$ & $=$ & $\begin{array}{l}\text { Concentration in } \\
\text { soil }\end{array}$ & Chemical-specific (mg/kg) & $\begin{array}{l}\text { Concentration is contained } \\
\text { in Table } 7.1\end{array}$ \\
\hline$C F=$ & $=$ & Conversion factors & $10^{-6} \mathrm{~kg} / \mathrm{mg}$ and $10^{4} \mathrm{~cm}^{2} / \mathrm{m}^{2}$ & $\begin{array}{l}\text { Necessary to convert } \\
\text { to appropriate units }\end{array}$ \\
\hline \multirow[t]{2}{*}{ SA } & $=$ & $\begin{array}{l}\text { Available surface } \\
\text { area }\end{array}$ & $0.728 \mathrm{~m}^{2} / \mathrm{d}$ & $\begin{array}{l}\text { 50th percentile total body } \\
\text { surface area }\end{array}$ \\
\hline & & & $1.94 \mathrm{~m}^{2} / \mathrm{d}$ & $\begin{array}{l}\text { for children and adults, } \\
\text { respectively (Section 6, } \\
\text { RAGS, EPA 1989c) }\end{array}$ \\
\hline $\mathrm{AF}=$ & $=$ & Adherence factor & $1.00 \mathrm{mg} / \mathrm{cm}^{2}$ & $\begin{array}{l}\text { Adherence factor for soil, } \\
\text { (EPA Region IV Interim } \\
\text { Guidance) }\end{array}$ \\
\hline ABS & $=$ & Absorption factor & 0.001 (unitless) & $\begin{array}{l}\text { Equivalent to } 0.1 \% \\
\text { for inorganics (EPA New } \\
\text { Interim, Region IV, } \\
\text { Guidance } 2 / 11 / 92 \text { ) }\end{array}$ \\
\hline $\mathrm{EF}$ & $=$ & Exposure frequency & $350 \mathrm{~d} /$ year & $\begin{array}{l}\text { OSWER Directive (EPA } \\
\text { 1991e) }\end{array}$ \\
\hline ED : & $=$ & Exposure duration & 24 years & $\begin{array}{l}\text { Two-part (childhood and } \\
\text { adult) residential exposure } \\
\text { for a 30-year duration } \\
\text { (OSWER Directive, EPA } \\
\text { 1991e) }\end{array}$ \\
\hline \multirow[t]{2}{*}{$\mathrm{BW}$} & $=$ & Body weight & $15 \mathrm{~kg}$ & $\begin{array}{l}\text { Child (OSWER Directive, } \\
\text { EPA 1991e) }\end{array}$ \\
\hline & & & $70 \mathrm{~kg}$ & $\begin{array}{l}\text { Adult (Section 6, RAGS, } \\
\text { EPA 1989c) }\end{array}$ \\
\hline \multirow[t]{2}{*}{ AT } & $=$ & Averaging time & 365 d/year $\times \mathrm{ED}$ & $\begin{array}{l}\text { Averaging time } \\
\text { for noncarcinogens }\end{array}$ \\
\hline & & & 365 d/year $\times 70$ years & $\begin{array}{l}\text { Averaging time } \\
\text { for carcinogens }\end{array}$ \\
\hline
\end{tabular}


Table 7.3 (continued)

\begin{tabular}{|c|c|c|c|c|}
\hline \multicolumn{3}{|c|}{ Variable } & Value used & \multirow[t]{2}{*}{ Explanation/source } \\
\hline \multicolumn{4}{|c|}{ Residential external exposure scenario } & \\
\hline \multicolumn{5}{|c|}{ Dose $(\mathrm{pCi}-\mathrm{yr} / \mathrm{g})=\mathrm{CS} \times \mathrm{ED} \times(1-\mathrm{Se}) \times \mathrm{Te}$} \\
\hline $\operatorname{cs}$ & $=$ & $\begin{array}{l}\text { Concentration } \\
\text { in soil }\end{array}$ & Chemical-specific (pCi/g) & $\begin{array}{l}\text { Concentration is obtained } \\
\text { from the data in Table } 7.1\end{array}$ \\
\hline ED & $=$ & Exposure duration & $\begin{array}{l}6 \text { years } \\
24 \text { years }\end{array}$ & $\begin{array}{l}\text { Two-part (childhood and } \\
\text { adult) residential exposure } \\
\text { for a 30-year duration } \\
\text { (OSWER Directive, EPA } \\
\text { 1991e) }\end{array}$ \\
\hline $\mathrm{Se}$ & $=$ & $\begin{array}{l}\text { Gamma shielding } \\
\text { factor (unitless) }\end{array}$ & 0.2 & $\begin{array}{l}\text { RAGS-part B, EPA 1991; } \\
\text { sect. } 4.1 .2 \text { (default value) }\end{array}$ \\
\hline $\mathrm{Te}$ & $=$ & $\begin{array}{l}\text { Gamma exposure } \\
\text { time factor (unitless) }\end{array}$ & 1.0 & $\begin{array}{l}\text { RAGS-part B, EPA 1991; } \\
\text { sect. 4.1.2 (default value, } \\
24 \mathrm{~h} / 24 \mathrm{~h} \text { ) }\end{array}$ \\
\hline
\end{tabular}




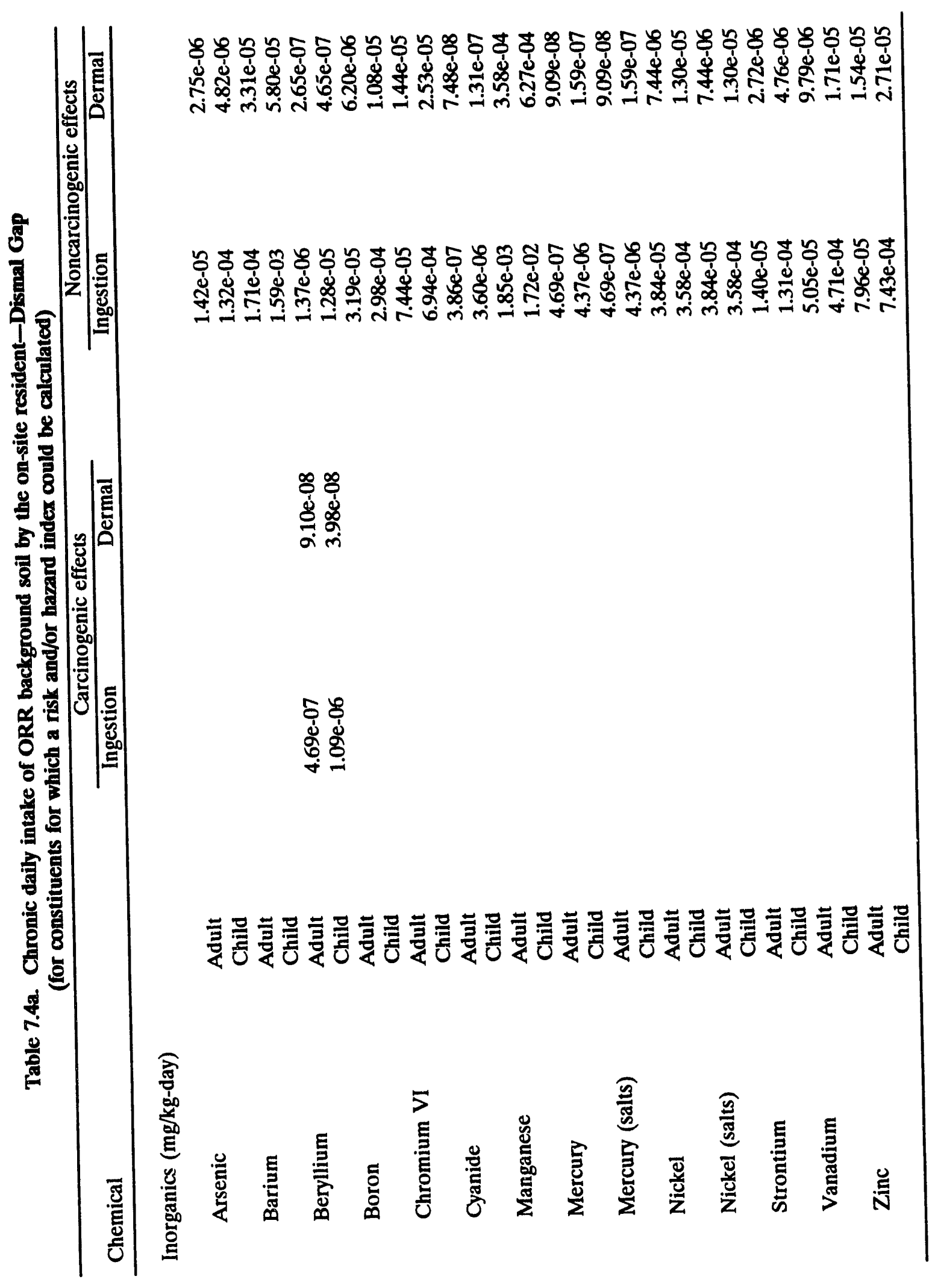




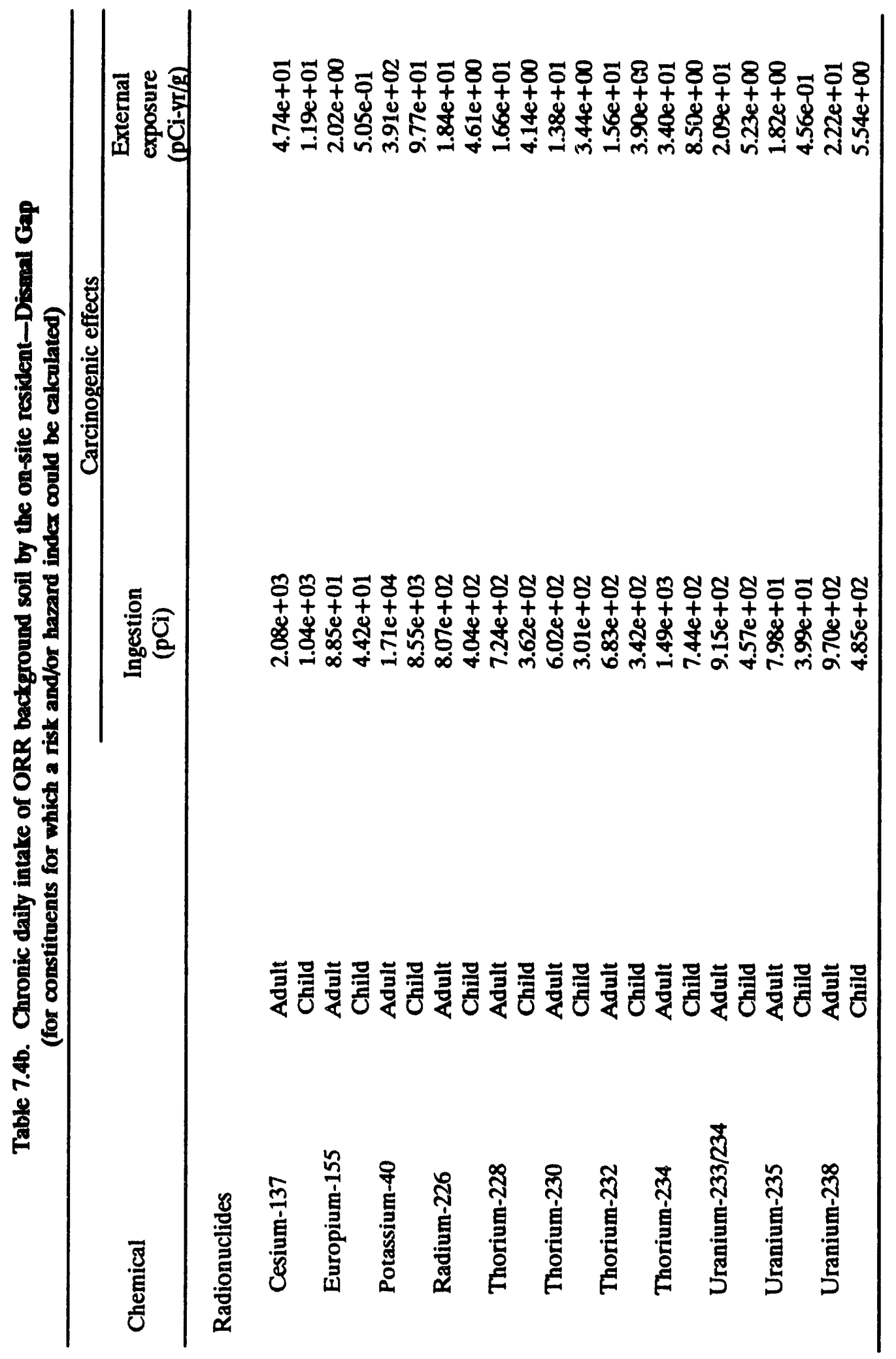




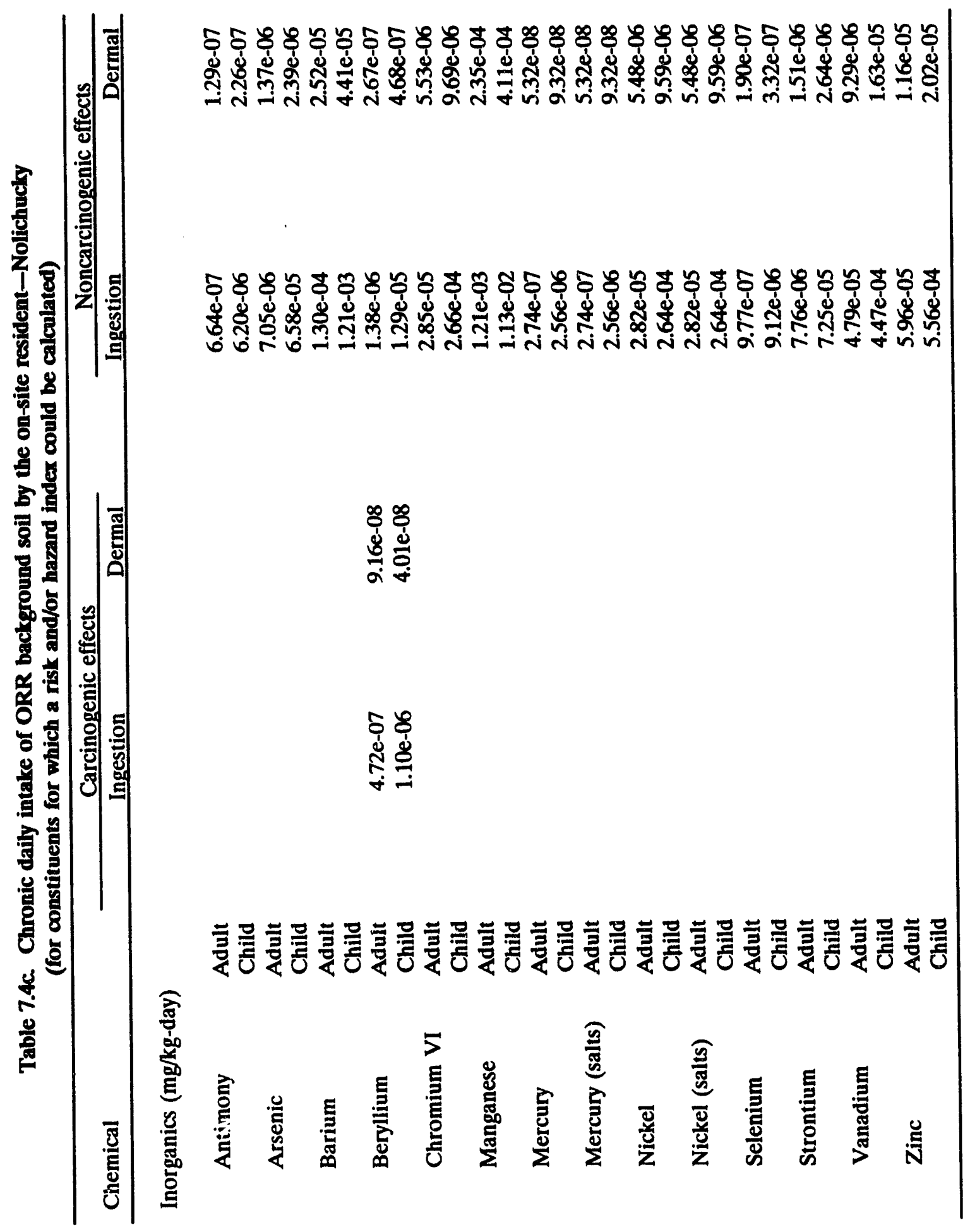




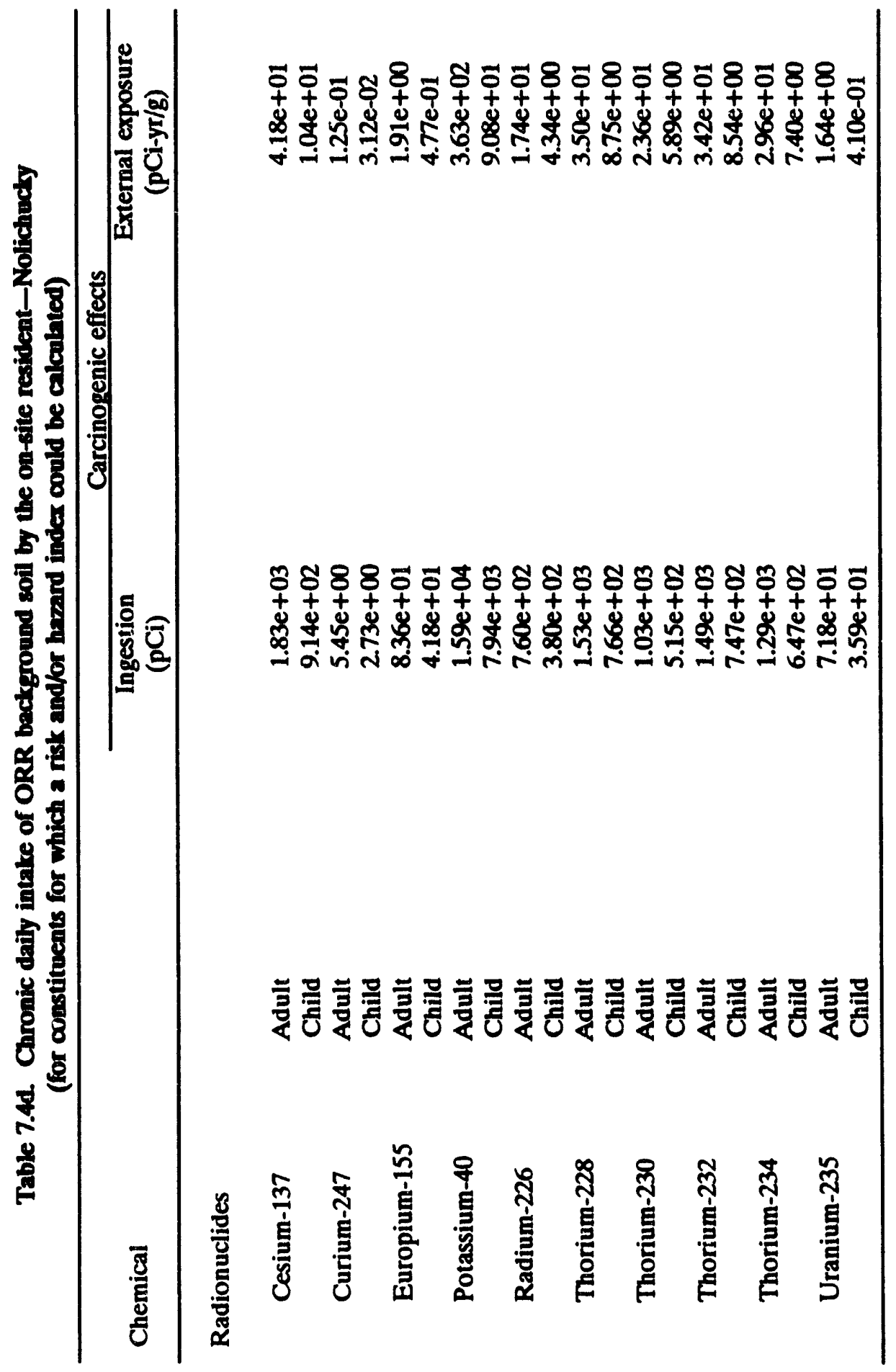




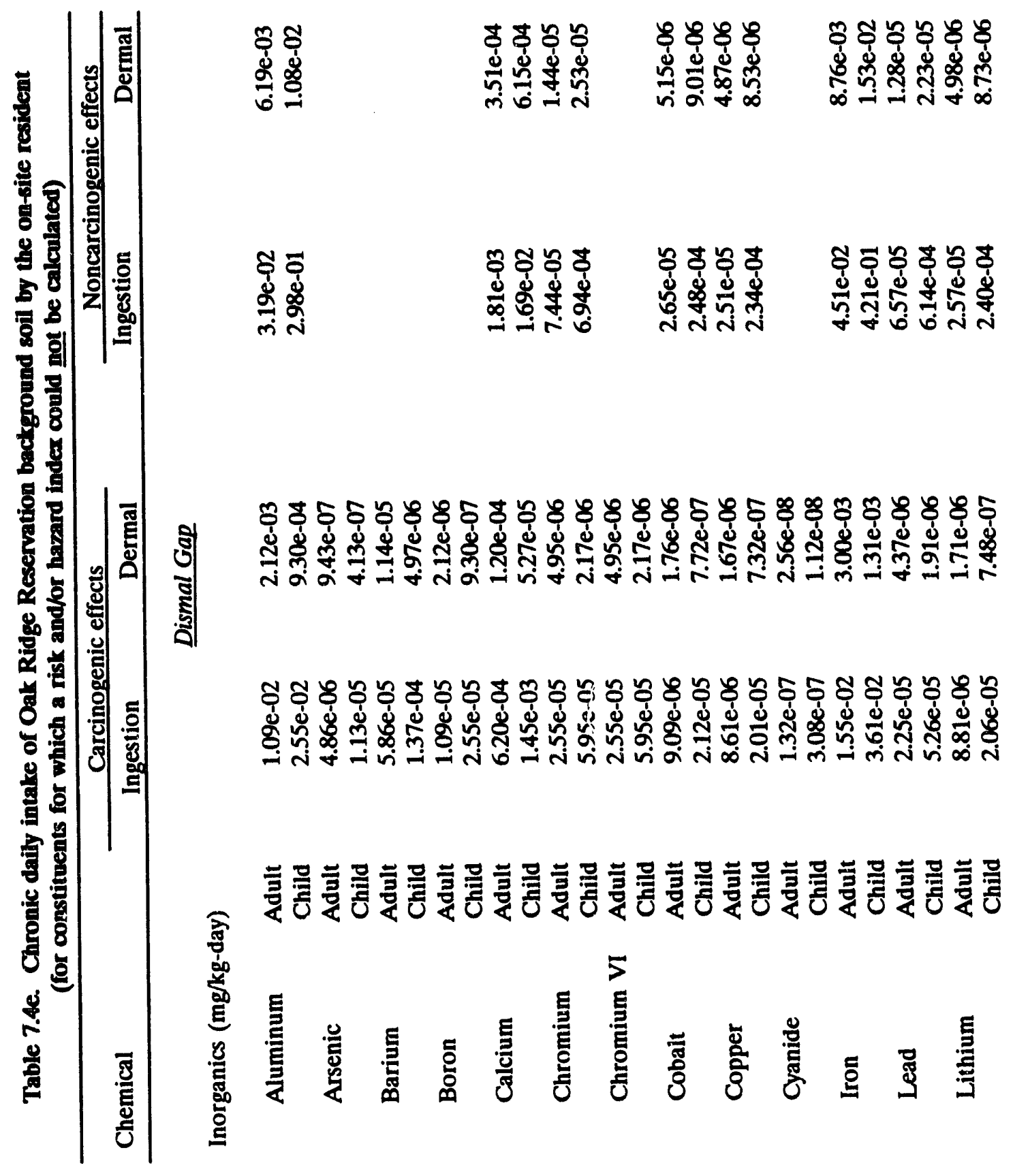




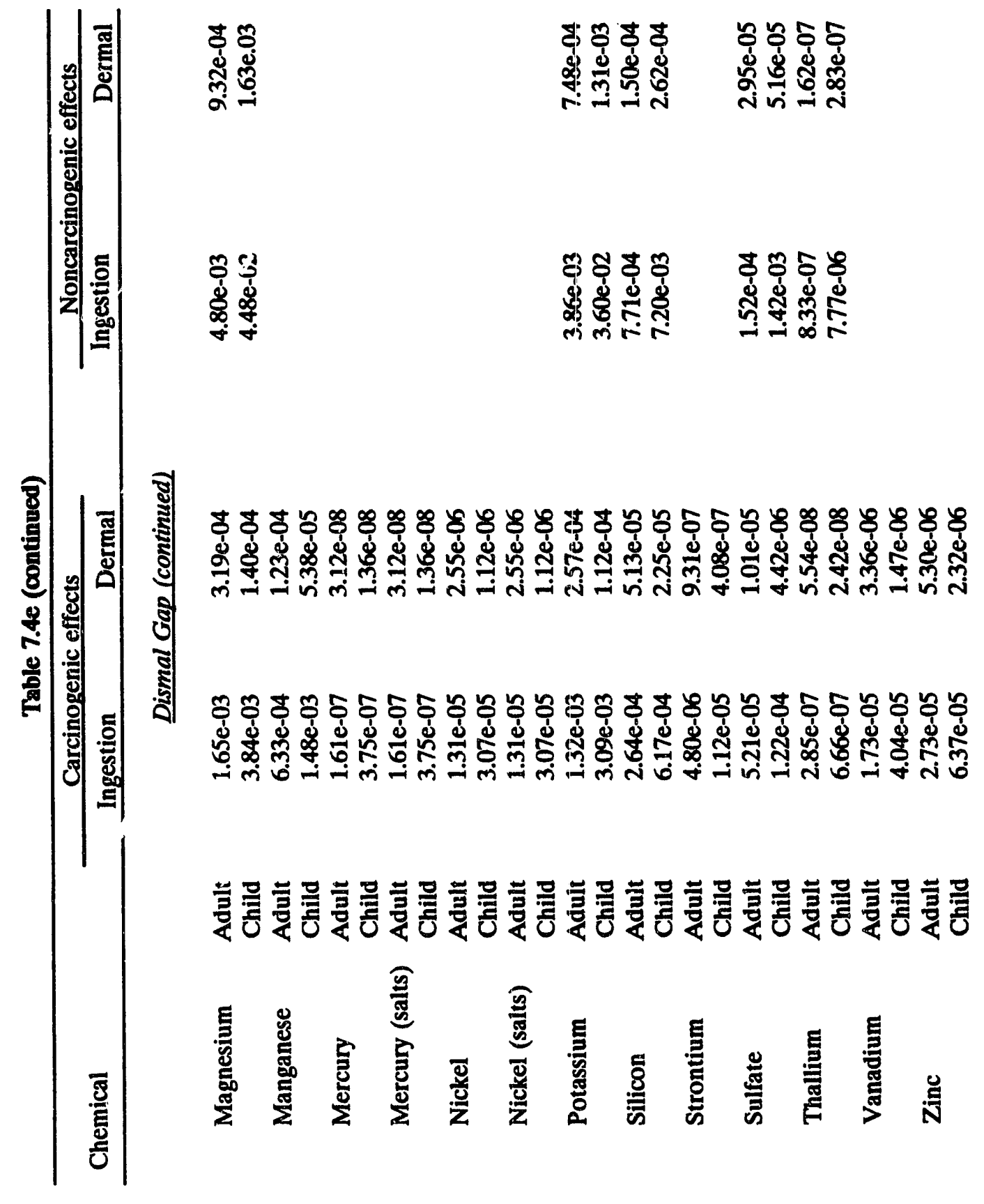




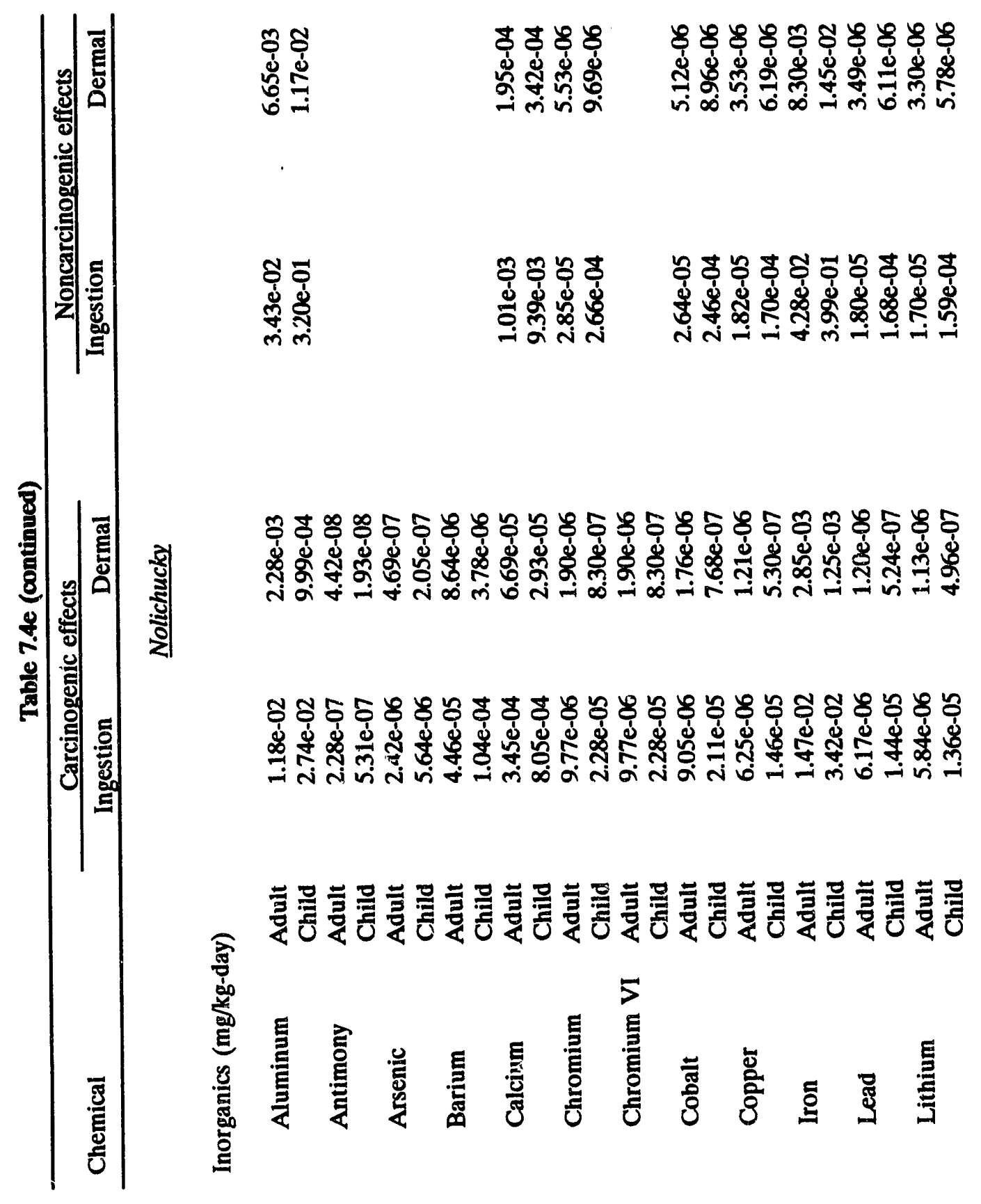




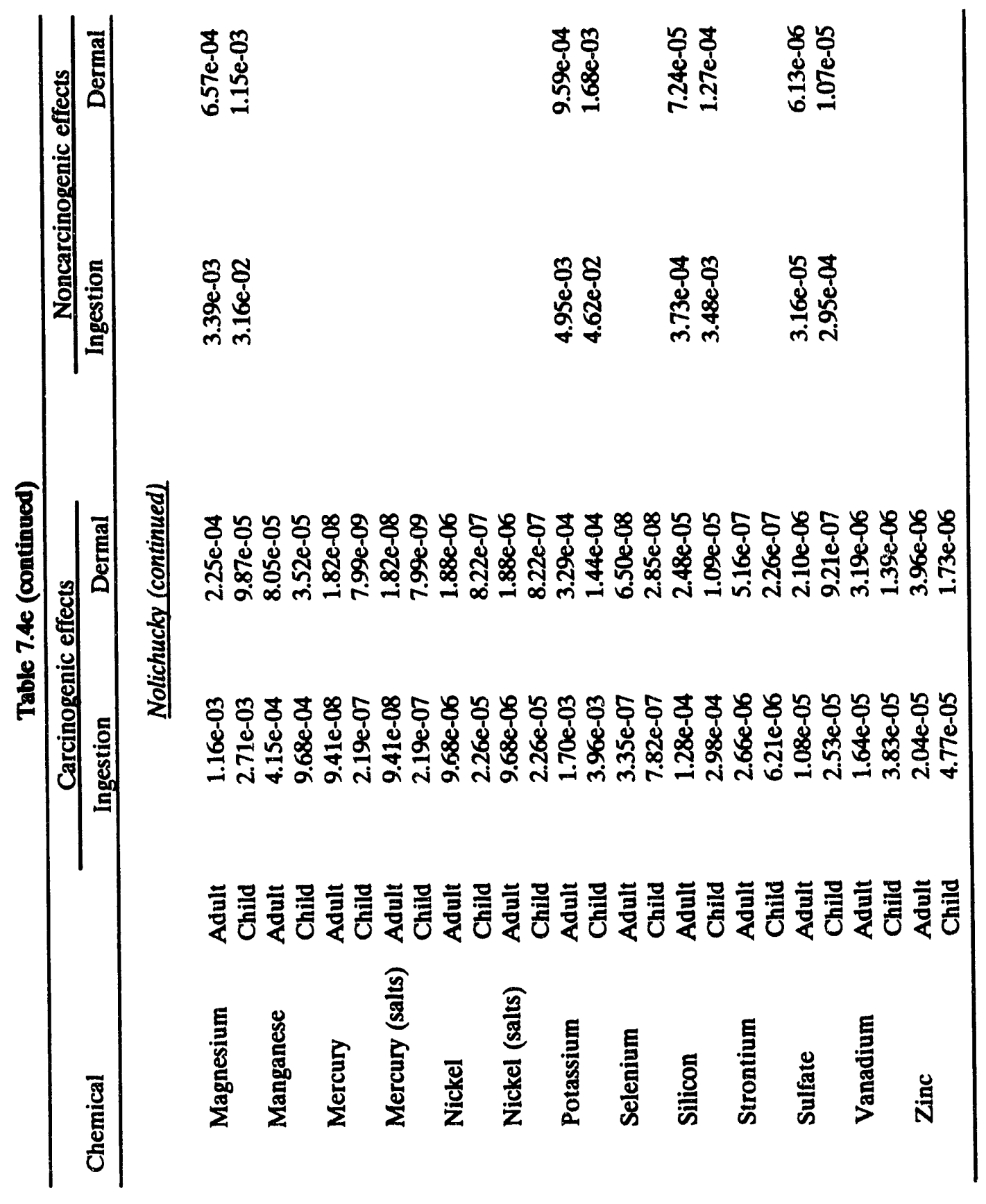




\subsection{TOXICITY ASSESSMENT}

The purpose of any toxicity assessment is to evaluate the potential for constituents to cause adverse health effects in exposed individuals. This usually consists of an evaluation of the relationship between the extent of exposure to a particular constituent and the increased likelihood or severity of adverse health effects as a result of that exposure relative to a baseline. The toxicity assessment generally involves two steps. The first step comprises determining whether exposure to an agent can cause an increase in the incidence of a particular health effect and whether that health effect will occur in humans. The second step involves characterizing the relationship between the received dose of the constituent and the incidence of adverse health effects in exposed populations.

The constituent-specific information in Sects. 7.5.1 and 7.5.2 provides general information as well as constituent-specific discussion about health effects related to those constituents of concern evaluated in the risk assessment for the background soil. Carcinogenic and noncarcinogenic health effects are considered. Data used in this section are from human and laboratory animal research and from occupational studies to characterize likely health effects resulting from exposure to the constituents of potential concern. Tables 7.5 through 7.7 summarize toxicity information for the constituents. The health effects described in this section are conservative and may not necessarily represent the actual health effects incurred by exposure to constituent levels presented in this background soil evaluation.

\subsubsection{Beryllium}

Pure beryllium is a hard, grayish metal that is present in the earth's crust. It can be found in emissions from coal combustion; in surface water and soil; and in house dust, food, drinking water, and cigarette smoke. Industry employs beryllium in several ways, including in brake systems for airplanes, for neutron monochromatization, as window material for $\mathrm{x}$-ray tubes, and in radiation detectors. Additionally, beryllium compounds are used in manufacturing ceramics and refractories, chemical reagents, and gas mantle hardeners. The highest risk for exposure to beryllium occurs among workers employed in beryllium manufacturing, fabricating, or reclaiming industries. However, people who live near these industries and who are sensitive to extremely low concentrations of beryllium in the air are also at risk. In addition, smokers inhale unusually high concentrations of beryllium, depending on the source of tobacco.

A limited amount of data indicates that the oral toxicity of beryllium is low; however, the inhaled toxicity of beryllium is well documented. Humans inhaling massive doses of beryllium compounds may develop acute berylliosis. Additionally, beryllium and its compounds are presumed to have cancer-causing potential in the human lung when inhaled. The cancer-causing ability has been investigated in workers exposed to beryllium. The degree of harm depends on the amount and duration of exposure. Short-term exposure to beryllium may cause noncarcinogenic health effects, such as acute pneumonitis berylliosis, while long-term exposure may cause lung cancer (ATSDR 1988a).

\subsubsection{Radionuclides}

Radionuclides are unstable atoms of chemical elements that will emit charged particles to achieve a more stable state. These charged particles are termed "alpha and beta radiation" 
Table 7.5. Toxicity information for carcinogenic potential ORR analytes of concern-ingestion pathwey

\begin{tabular}{|c|c|c|c|c|}
\hline Chemical & $\begin{array}{l}\text { Oral slope } \\
\text { factor }\end{array}$ & $\begin{array}{l}\text { EPA and } \\
\text { lung class }\end{array}$ & $\begin{array}{l}\text { Type of } \\
\text { cancer }\end{array}$ & $\begin{array}{l}\text { Slope factor basis/ } \\
\text { slope factor source }\end{array}$ \\
\hline \multicolumn{5}{|c|}{ Inorganics (mg/kg-day) ${ }^{-1}$} \\
\hline Antimony & ND & ND & ND & IRIS/HEAST \\
\hline Arsenic & ND & $\mathbf{A}^{\mathbf{c}}$ & Skin & Water/IRIS/HEAST \\
\hline Barium & ND & ND & ND & IRIS/HEAST \\
\hline Beryllium & $4.30 \mathrm{e}+00^{\circ}$ & $\mathbf{B} 2^{\mathbf{c}}$ & ND & $\begin{array}{l}\text { Intratracheal } \\
\text { Instillation/IRIS/ } \\
\text { HEAST }\end{array}$ \\
\hline Boron & ND & ND & ND & IRIS/HEAST \\
\hline Chromium & ND & $A^{c}$ & ND & IRIS/HEAST \\
\hline Chromium (VI) & ND & $A^{c}$ & tumors ${ }^{a}$ & IRIS/HEAST \\
\hline Cyanide & ND & $D^{c}$ & ND & IRIS/HEAST \\
\hline Manganese & ND & $D^{c}$ & ND & IRIS/HEAST \\
\hline Mercury & ND & $D^{c}$ & ND & IRIS/HEAST \\
\hline Mercury (salts) & ND & ND & ND & IRIS/HEAST \\
\hline Nickel & ND & ND & ND & IRIS/HEAST \\
\hline Nickel (salts) & ND & ND & ND & IRIS/HEAST \\
\hline Selenium & ND & $D^{c}$ & ND & IRIS/HEAST \\
\hline Strontium & ND & ND & ND & IRIS/HEAST \\
\hline Vanadium & ND & ND & ND & IRIS/HEAST \\
\hline Zinc & ND & $D^{c}$ & ND & IRIS/HEAST \\
\hline
\end{tabular}

Radionuclides ( $\mathrm{pCi})^{-1}$

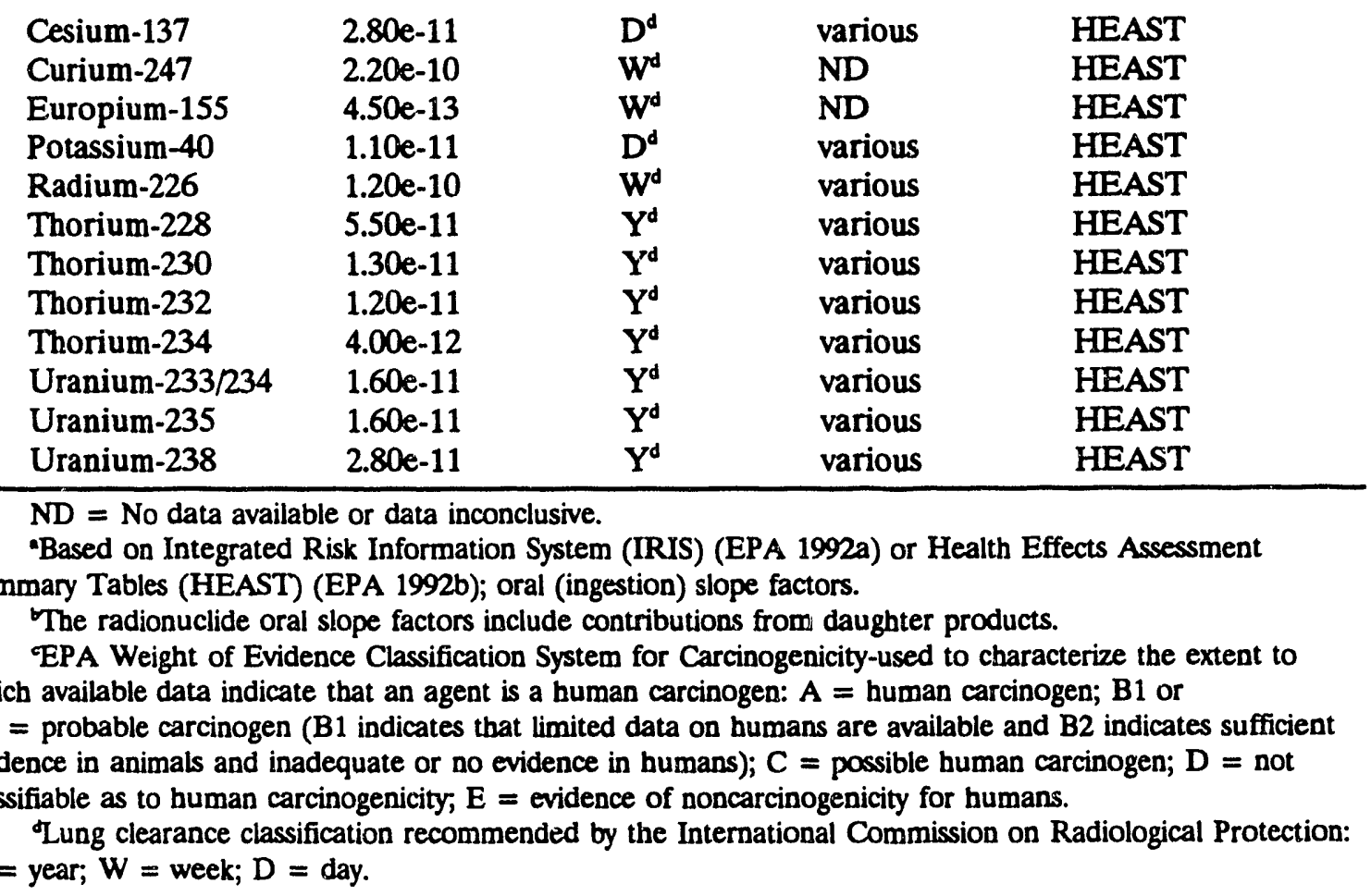




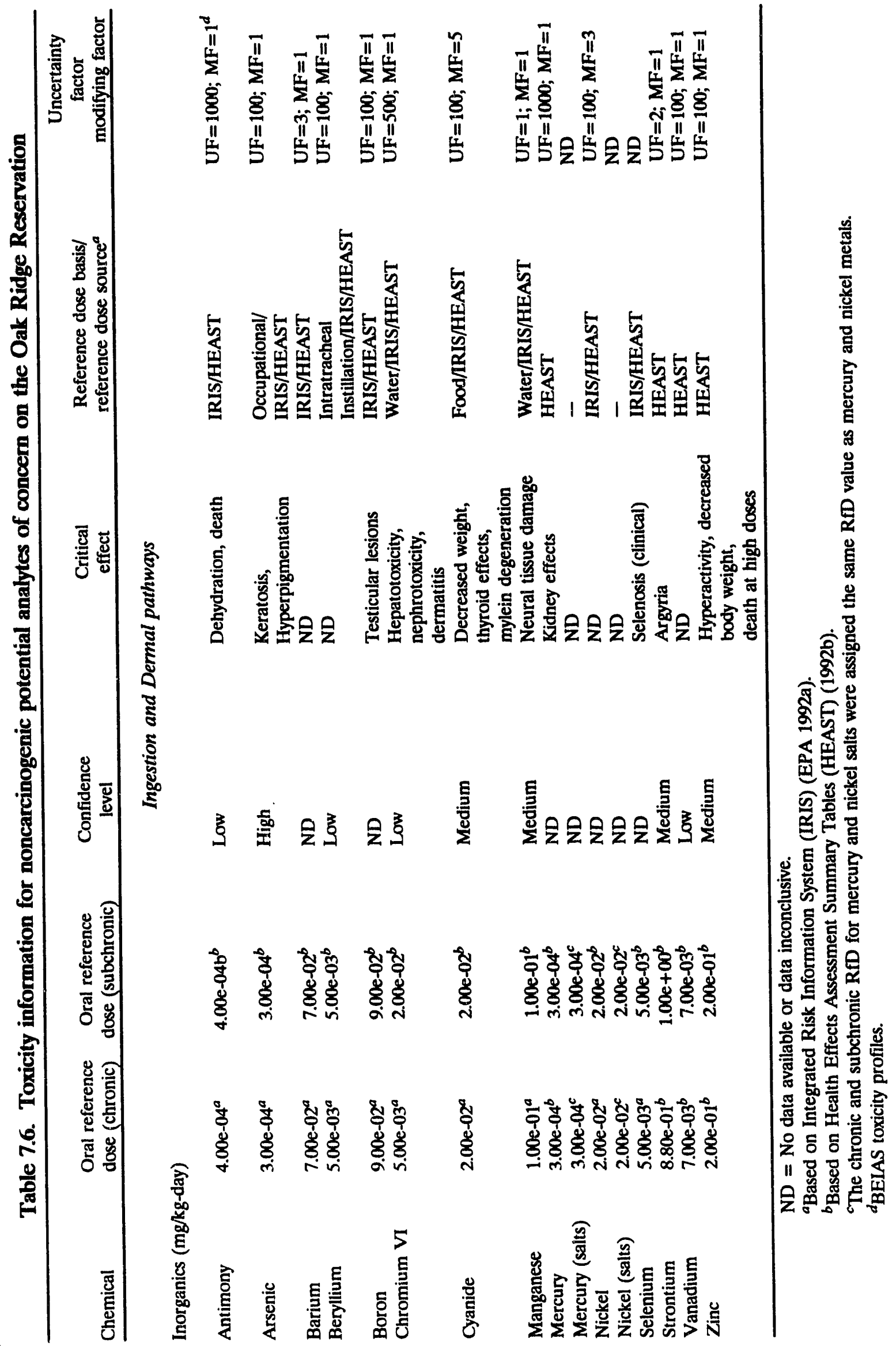


Table 7.7. Toxicity information for external exposure to radionuclides on the Oak Ridge Reservation

\begin{tabular}{llcl}
\hline Chemical & $\begin{array}{c}\text { External } \\
\text { exposure slope } \\
\text { factor }^{\text {, }}\end{array}$ & $\begin{array}{c}\text { ICRP } \\
\text { lung } \\
\text { class }^{c}\end{array}$ & $\begin{array}{c}\text { Type } \\
\text { of } \\
\text { cancer }\end{array}$ \\
\hline $\begin{array}{l}\text { Radionuclides }(\mathrm{g} / \mathrm{pCi}-\mathrm{yr}) \\
\text { Cesium-137 }\end{array}$ & $2.00 \mathrm{e}-06$ & $\mathrm{D}$ & Various \\
Curium-247 & $9.20 \mathrm{e}-07$ & $\mathrm{~W}$ & $\mathrm{ND}$ \\
Europium-155 & $5.90 \mathrm{e}-08$ & $\mathrm{~W}$ & ND \\
Potassium-40 & $5.40 \mathrm{e}-07$ & $\mathrm{D}$ & Various \\
Radium-226 & $6.00 \mathrm{e}-06$ & $\mathrm{~W}$ & Various \\
Thorium-228 & $5.60 \mathrm{e}-06$ & $\mathrm{Y}$ & Various \\
Thorium-230 & $5.40 \mathrm{e}-11$ & $\mathrm{Y}$ & Various \\
Thorium-232 & $2.60 \mathrm{e}-11$ & $\mathrm{Y}$ & Various \\
Thorium-234 & $3.50 \mathrm{e}-09$ & $\mathrm{Y}$ & Various \\
Uranium-233/234 & $4.20 \mathrm{e}-11$ & $\mathrm{Y}$ & Various \\
Uranium-235 & $2.40 \mathrm{e}-07$ & $\mathrm{Y}$ & Various \\
Uranium-238 & $3.60 \mathrm{e}-08$ & $\mathrm{Y}$ & Various \\
\hline
\end{tabular}

ND = No data available or data inconclusive.

${ }^{a}$ Based on Integrated Risk Information System (IRIS) (EPA 1992a).

${ }^{b}$ The radionuclide external exposure slope factors include contributions from daughter products.

'Lung clearance classification recommended by the International Commission on Radiological Protection (ICRP). $Y=$ year; $W=$ week; $D=$ day. 
and "neutral gamma rays." Interaction of these charged particles (and gamma rays) with matter will produce ionization events, or radiation, which may cause living cell tissue damage. Because the deposition of energy by ionizing radiation is a random process, sufficient energy may be deposited (in a critical volume) within a cell and result in cell modification or death (ICRP 1991). In addition, ionizing radiation has sufficient energy that interactions with matter will produce an ejected electron and a positively charged ion (known as free radicals) that are highly reactive and may combine with other elements, or compounds within a cell, to produce toxins or otherwise disrupt the overall chemical balance of the cell (EPA 1991b). These free radicals can also react with deoxyribonucleic acid (DNA), causing genetic damage, cancer induction, or even cell death.

Radionuclides are characterized by the type and energy level of the radiation emitted. Radiation emissions fall into two major categories: particulate (electrons, alpha particles, beta particles, and protons) or electromagnetic radiation (gamma and x-rays) (ASTDR 1989d). Therefore, all radionuclides are classified by the EPA as Group A carcinogens based on their property of emitting ionizing radiation and on the extensive weight of evidence provided by epidemiological studies of humans with cancers induced by high doses of radiation. Alpha particles are emitted at a characteristic energy level for differing radionuclides. The alpha particle has a charge of +2 and a comparably large size. Alpha particles have the ability to react (and/or ionize) with other molecules, but they have very little penetrating power and lack the ability to pass through a piece of paper or human skin. However, alpha-emitting radionuclides are of concern when there is a potential for inhalation or ingestion of the radionuclide. Alpha particles are directly ionizing and deposit their energy in dense concentrations [termed high linear energy transfer (high LET)], resulting in short paths of highly localized ionization reactions. The probability of cell damage increases as a result of the increase in ionization events occurring in smaller areas; this may also be the reason for increased cancer incidence caused by inhalation of radon gas. In addition, the cancer incidence in smokers may be directly attributed to the naturally occurring alpha emitter, polonium-210, in common tobacco products (Hammonds and Hoffman 1992).

Beta emissions generally refer to beta negative particle emissions. Radionuclides with an excess of neutrons achieve stability by beta decay. Beta radiation, like alpha radiation, is directly ionizing but, unlike alpha activity, beta particles deposit their energy along a longer track length (low-LET), resulting in more space between ionization events (Hammonds and Hoffman 1992). Beta-emitting radionuclides can cause injury to the skin and superficial body tissue but are most destructive when inhaled or ingested. Many beta emitters are similar chemically to naturally occurring essential nutrients and will therefore tend to accumulate in certain specific tissues. For example, strontium- 90 is chemically similar to calcium and, as a result, accumulates in the bones, where it causes continuous exposure. The health effects of beta particle emissions depend upon the target organ. Those seeking the bones would cause a prolonged exposure to the bone marrow and affect blood cell formation, possibly resulting in leukemia, other blood disorders, or bone cancers. Those seeking the liver would result in liver diseases or cancer, while those seeking the thyroid would cause thyroid and metabolic disorders. In addition, beta radiation may lead to damage of genetic material (DNA), causing hereditary defects.

Gamma emissions are simply the energy that has been released from transformations of the atomic nucleus. Gamma emitters and x-rays behave similarly but differ in their origin: gamma emissions originate in nuclear transformations, and $x$-rays result from changes in the 
orbiting electron structure. Radionuclides that emit gamma radiation can induce internal and external effects. Gamma rays have high penetrating ability in living tissue and are capable of reaching all internal body organs. Without such sufficient shielding as lead, concrete, or steel, gamma radiation can penetrate the body from the outside and does not require ingestion or inhalation to penetrate sensitive organs. Gamma rays are characterized as low-LET radiation, as is beta radiation; however, the behavior of beta radiation differs from that of gamma radiation in that beta particles deposit most of their energy in the medium through which they pass, while gamma rays often escape the medium because of higher energies, thereby creating difficulties in determining actual internal exposure. For this reason, direct whole-body measurements are necessary to detect gamma radiation, while urine/fecal analyses are usually effective in detecting beta radiation (Hammonds and Hoffman 1992).

People receive gamma radiation continuously from naturally occurring radioactive decay processes going on in the earth's surface, from radiation naturally occurring inside their bodies, from the atmosphere as fallout from nuclear testing or explosions, and from space or cosmic sources. Cesium-137 (from nuclear fallout) decays to barium-137, the highest contributor to fallout-induced gamma radiation (NCRP 1977). Beta radiation from the soil is a less penetrating form of radiation but has many contributing sources. Potassium-40, cesium-137, lead-214, and bismuth-214 are among the most common environmental beta emitters. Tritium is also a beta emitter but contributes little to the soil beta radiation because of the low energy of its emission and its low concentration in the atmosphere (NCRP 1977). Alpha radiation is also emitted by the soil but is not measurable more than a few centimeters from the ground surface. The majority of alpha emissions are attributable to radon-222 and radon-220 and their decay products (NCRP 1977). This contributes to what is called background exposure to radiation (ATSDR 1989d).

The general health effects of radiation can be divided into stochastic (related to dose) and nonstochastic (not related to dose) effects. The risk of development of cancer from exposure to radiation is a stochastic effect. Examples of nonstochastic effects include acute radiation syndrome and cataract formation, which occur only at high levels of exposures (Killough and Eckerman 1983).

Radiation can damage cells in different ways. It can cause damage to DNA within the cell, and the cell either may not be able to recover from this type of damage or may survive but function abnormally. If an abnormally functioning cell divides and reproduces, a tumor or mutation in the tissue may develop. The rapidly dividing cells that line the intestines and stomach and the blood cells in bone marrow are extremely sensitive to this damage. Organ damage results from the damage caused to the individual cells. This type of damage has been reported with doses of 10 to 500 rads ( 0.1 to 5.0 gray, in SI units). Acute radiation sickness is seen only after doses of $>50$ rads ( 0.5 gray) which is a dose rate usually achieved only in a nuclear accident (ATSDR 1989d).

When the radiation-damaged cells are reproductive cells, genetic damage can occur in the offspring of the person exposed. The developing fetus is especially sensitive to radiation. The type of malformation that may occur is related to the stage of fetal development and the cells that are differentiating at the time of exposure. Radiation damage to children exposed in the womb is related to the dose the pregnant mother receives. Mental retardation is a possible effect of fetal radiation exposure (ATSDR 1989d). 
The most widely studied population that has had known exposure to radiation is the atomic bomb survivors of Hiroshima and Nagasaki, Japan. Data indicate an increase in the rate of leukemia and cancers in this population. However, the rate at which cancer incidence is significantly affected by low radiation exposures, such as results of exposure to natural background and industrially contaminated sites, is still undergoing study and is uncertain (Hammonds and Hoffman 1992). In studies conducted to determine the rate of cancer and leukemia increase, as well as genetic defects, several radionuclides must be considered. A brief physical description, an industrial profile, and radiation emission information pertaining to the primary radionuclides, which are major contributors to background risk (see Sect. 7.6), are given in Sects. 7.5.2.1 through 7.5.2.4.

\subsubsection{Cesium-137}

Cesium occurs in nature as cesium-133 in the aluminosilicates, pollucite (a hydrated silicate of aluminum and cesium) and lepidolite; in the borate, rhodizite; and in other sources (Budavari et al. 1989, Klaassen et al. 1986). Cesium-137 is one of the artificial isotopes of cesium and is one of the principal radionuclides present in reactor effluents under normal operations. Cesium-137 may also be produced in nuclear and thermonuclear explosions, through which it would be a primary contributor to human exposure through fallout radiation, assimilation through the food chain, or beta dose to the skin (Budavari et al. 1989, Klaasen et al. 1986). In addition, cesium-137, along with strontium-90, is one of the most important fission products that was widely distributed in near-surface soils because of historical weapons testing. Measurable concentrations still exist in the soil today, almost exclusively in the upper $15 \mathrm{~cm}$ of soil; these concentrations decrease roughly exponentially with depth.

Cesium-137 may also have important roles in medical treatments (a teletherapy source or intercavitary or interstitial radiation source in treatment of malignancies) and as an encapsulated energy source (Budavari et al. 1989, Casarett 1968). Cesium-137 decays to and reaches radioactive equilibrium with its daughter product, barium-137m (Budavari et al. 1989, Casarett 1968). Barium-137m is a very short-lived gamma emitter that can contribute to external gamma exposure (Budavari et al. 1989).

\subsubsection{Potassium -40}

Potassium is a silvery white, light, very soft, chemically reactive member of the alkali metal family. Potassium is used in manufacturing certain types of soap and glass, and potassium nitrate (saltpeter) is used in matches and explosives. Potassium- 40 is a naturally occurring radioisotope of potassium and is found in the earth's crust in measurable quantities. It is a major constituent of both igneous and sedimentary rocks, especially granite $(>30 \mathrm{pCi} / \mathrm{g})$ and shale $(22 \mathrm{pCi} / \mathrm{g})$, respectively. Potassium- 40 has a half-life of 1.3 billion years and is used in radioactive dating of rocks. In addition, potassium- 40 is one of 17 naturally occurring radioisotopes that decay to stable isotopes.

Potassium- 40 is always present in the body; it decays with emission of beta particles and a gamma ray, but the rate of decay is so relatively slow that it requires a whole body count to detect. The rate is considered slow, but potassium- 40 expels more than a million beta particles per minute in the average adult. Although potassium- 40 is present in the body, it is not found in fatty tissues. Therefore, a measurement of the total quantity of potassium- 40 
(along with other data) can be used to determine the relative proportions of lean and fatty tissue in the body (Glasstone 1967). The lifetime total cancer risk SF is greater when potassium-40 is ingested than when it is inhaled. The external exposure is only half as great as the internal risk of ingestion.

\subsubsection{Radium-226}

Radium is a naturally occurring radioactive element that exists in several isotopic forms. The radium isotopes are formed from the decay of uranium and thorium. Radium-226 is formed from uranium-238 and uranium-234, and radium-226 has the longest half-life of the radium isotopes (radium-228, radium-224, and radium-223). In general, the activity concentration of radium-226 measured in most soils and rocks is comparable to those of uranium-238 and uranium-234, suggesting that radium does not tend to migrate from either of its uranium precursors under stable conditions. Radium-226 is primarily an alpha and gamma emitter.

Radium has been used as a component of luminous paints for clock and instrument dials. It has also been used in the treatment of cancer, in radiography, and in research. Radium is released into the environment in coal fly ash and in uranium mining and processing wastes. The background level of radium in industrial regions in soil is about $8.1 \mathrm{pCi} / \mathrm{g}$. Clays and soil components generally retard the movement of radium in the environment, but acidic processing wastes can enhance its movement. Radium may bioaccumulate in plants and animals, and exposure through the food chain is possible.

Many environmental problems can be directly attributed to the decay products or daughters of radium. The primary daughters are isotopes of radon-a colorless, odorless, radioactive gas. Radon gas can infiltrate basements and water systems, resulting in significant exposure via inhalation pathways.

\subsubsection{Thorium-228}

Thorium is a naturally occurring radioactive element commonly found in the earth's crust. It is also produced from monazite, a by-product of mineral sand mined for titanium and zirconium. Much of the thorium mined in the United States is exported. Thorium is used for fuel for nuclear reactors, mantles for camping lanterns, welding electrodes, aerospace alloys, high temperature materials, special lighting fixtures, and nuclear weapons. Thorium is also introduced into the environment from the use of phosphate fertilizers.

Natural thorium is primarily thorium-232, which has a slow decay process. The decay series for thorium-232 proceeds through radium-228 to thorium-228, ending in lead-208, a stable isotope. Thorium-228, as do all thorium isotopes, emits alpha, beta, and/or gamma radiation on decay. However, the major radiation energies of concern from thorium-228 are alpha and gamma emissions.

\subsection{RISK CHARACTERIZATION}

The purpose of the risk characterization is to integrate and summarize the information presented in the toxicity and exposure assessments. Potential carcinogenic effects are 
characterized by estimating the probability that an individual will develop cancer over a lifetime from projected intakes (and exposure) and chemical-specific dose-response data (i.e., SFs). Potential noncarcinogenic (systemic) effects are characterized by comparing projected intakes of contaminants to toxicity values (i.e., RfDs). The results of this background risk analysis for the hypothetical on-site residential exposure scenario (discussed in Sect. 7.4) are summarized in this section.

Note that the inorganic analytes listed in the tables include chromium VI, mercury salts, and nickel salts. The analytical laboratory reported detected concentrations for the total chromium, mercury, and nickel found. Because (1) the concentrations were reported in this form (i.e., no distinction between valences and speciation); (2) the percent gastrointestinal (\%GI) absorption toxisity values are known for chromium VI, mercury salts, and nickel salts; and (3) the RfDs are known for mercury and nickel, it was necessary to assess all types of these analytes, which included the most toxic form of the metals. The RfDs for mercury and nickel salts were assumed to be the same as those listed for metallic mercury and nickel. The total pathway hazard indices include only one $\mathrm{HI}$ value for each pair (i.e., chromium and chromium VI, mercury and mercury salts, and nickel and nickel salts); the most conservative HI values were included in the total pathway HI (i.e., HI for chromium VI, mercury salts, and nickel salts were included in the pathway totals) to ensure that exposure is not underestimated.

Also note that the CDIs, background risks, and background hazard indices are shown with three significant figures. In most cases, SFs and/or intake parameters are given with only one significant figure; therefore, only one significant figure should be reported for the calculated risks and HI values. However, for clarity and for comparison (of the calculated values) in this section of the BSCP, three significant figures will be given.

\subsubsection{EPA Guidance-Carcinogens}

For carcinogens, risks are estimated as the incremental probability of an individual's developing cancer over a lifetime as a result of exposure to the carcinogen (i.e., the term "incremental" refers to excess individual lifetime cancer risk). Cancer risk from exposure to contamination is expressed as excess cancer risk, that is, cancer incurred in addition to normally expected rates of cancer development. An excess cancer risk of $1.0 \times 10^{-6}$ indicates one person in one million is predicted to incur cancer from exposure to this contamination level. Excess cancer risks falling between $1.0 \times 10^{-6}$ and $1.0 \times 10^{-4}$ are within the range of concern, and cancer risks above $1.0 \times 10^{-4}$ are considered unacceptable by the EPA (1989c). The excess cancer risk is determined by the application of an SF, which is a chemical-specific value based on carcinogenic dose-response data. Because the SF are the upper 95\% confidence limit on the probability of a carcinogenic response, the carcinogenic risk estimate represents an upper confidence bound estimate. Therefore, there is only a $5 \%$ probability that the actual risk will be higher than the estimate presented, and the actual risk may well be less than the estimate.

Slope factors used in the evaluation of risk from exposure to constituents in background soils are listed in Tables 7.5 and 7.7. Slope factors are not currently available for all background constituents, and several constituents are not indicated by epidemiological studies to be carcinogenic; consequently, these do not have SFs. Furthermore, SFs are not available for several background constituents because their carcinogenicity has not been determined. 
These constituents may contribute to carcinogenic effects from exposure to the soil, but their effect cannot be quantified at the present time.

\subsubsection{EPA Guidance-Noncarcinogens}

Noncarcinogenic effects are evaluated by comparing an exposure experienced over a specified time period (e.g., 30 years) with an $\mathrm{RfD}$ derived for a similar exposure period. Some RfDs available for the constituents present in the background soil are given in Table 7.6. To evaluate the noncarcinogenic effects of exposure to soil, the hazard quotient (the ratio of the exposure dose to the RfD) is calculated for each constituent. This noncarcinogen hazard quotient (also referred to as $\mathrm{HI}$ ) assumes that, below a given level of exposure (i.e., the $\mathrm{RfD}$ ), even sensitive populations are unlikely to experience adverse health effects. If the exposure level (intake) exceeds this threshold [i.e., if intake/RfD exceeds one (1.0)], there may be concern for potential systemic health effects; the level of concern does not necessarily increase linearly as the hazard quotient approaches or exceeds unity. In other words, the $\mathrm{HI}$ is not a percentage or probability.

\subsubsection{Background Risk and Hazard Index Comparisons Between the ORR and Anderson and Roane Counties}

Background soil samples were collected from soils of the Dismal Gap Formation in Anderson and Roane counties and on the ORR. For detected analytes for which an SF or an RfD were available, background risk or an $\mathrm{HI}$ were calculated for each analyte for each of the three sampling areas. The results of these calculations are summarized in Tables 7.8 and 7.9. A comparison can be made between the calculated human health risk (or HI) for each analyte at the three sampling areas. This comparison can be used to quantitatively and qualitatively assess the similarities and differences in carcinogenic risk and systemic effects posed by analytes found in background soil on and in the vicinity of ORR. For the purpose of this comparison only, the total excess cancer risk and $\mathrm{HI}$ are used [the risk to a child + the risk to an adult (Table 7.8), and the chronic HI for an adult + the subchronic $\mathrm{HI}$ for a child (Table 7.9)]. Adult- or child-specific risks would vary in a similar manner.

\subsubsection{Background risk comparisons between the ORR and Anderson and Roane counties}

The following discussion describes similarities and differences between calculated background risk values for Roane and Anderson counties and the ORR. First consider the background risk estimates for the residential ingestion of soil pathway (Table 7.8). The human health cancer risk estimates for each analyte are approximately the same for the three sampling areas (ORR, Anderson County, and Roane County), with the exception of thorium-228 and cesium-137. The background risk posed by thorium-228 in Anderson County is approximately twice the magnitude found on the ORR and is approximately 1.5 times larger when compared with Roane County; background risk posed by thorium-228 is approximately the same for ORR and Roane County. The background risk posed by cesium-137 on the ORR is approximately twice that found in Roane County and five times that calculated for Anderson County. The background risk posed by cesium-137 in Roane County is approximately twice that shown for Anderson County. The thorium-228 and cesium-137 horizon A data have been shown statistically to vary significantly over sampling locations (refer to the statistical analysis, Sects. 5.7 and 5.8). 
Table 7.8. Comparative background risk estimates from exposure to soil constituents from the Oak Ridge Reservation, Anderson County, and Roane County--Dismal Gap lithology"

\begin{tabular}{lccc}
\hline Analyte & Oak Ridge & Anderson & Roane \\
Reservation & County & County \\
\hline
\end{tabular}

Exposure pathway: residential ingestion of soil

$\begin{array}{llll}\text { Beryllium } & 6.72 \mathrm{e}-06 & 7.18 \mathrm{e}-06 & 5.57 \mathrm{e}-06 \\ \text { Cesium-137 } & 8.72 \mathrm{e}-08 & 1.72 \mathrm{e}-08 & 4.04 \mathrm{e}-08 \\ \text { Europium-155 } & 5.97 \mathrm{e}-11 & 5.07 \mathrm{e}-11 & 4.98 \mathrm{e}-11 \\ \text { Potassium-40 } & 2.82 \mathrm{e}-07 & 3.33 \mathrm{e}-07 & 1.91 \mathrm{e}-07 \\ \text { Radium-226 } & 1.45 \mathrm{e}-07 & 3.35 \mathrm{e}-07 & 1.54 \mathrm{e}-07 \\ \text { Thorium-228 } & 5.97 \mathrm{e}-08 & 1.00 \mathrm{e}-07 & 8.28 \mathrm{e}-08 \\ \text { Thorium-230 } & 1.17 \mathrm{e}-08 & 1.90 \mathrm{e}-08 & 1.55 \mathrm{e}-08 \\ \text { Thorium-232 } & 1.23 \mathrm{e}-08 & 1.91 \mathrm{e}-08 & 1.70 \mathrm{e}-08 \\ \text { Thorium-234 } & 8.93 \mathrm{e}-09 & 5.81 \mathrm{e}-09 & 7.89 \mathrm{e}-09 \\ \text { Uranium-233/234 } & 2.20 \mathrm{e}-08 & 2.17 \mathrm{e}-08 & 2.19 \mathrm{e}-08 \\ \text { Uranium-235 } & 1.91 \mathrm{e}-09 & 1.47 \mathrm{e}-09 & 1.86 \mathrm{e}-09 \\ \text { Uranium-238 } & 4.07 \mathrm{e}-08 & 3.54 \mathrm{e}-08 & 3.94 \mathrm{e}-08 \\ & & & \\ \text { Total pathway risk } & 7.39 \mathrm{e}-06 & 8.07 \mathrm{e}-06 & 6.14 \mathrm{e}-06\end{array}$

Exposure pathway: residential dermal exposure to soil

Beryllium

Total pathway risk
$1.13 e-05$

$1.20 \mathrm{e}-05$

$1.13 \mathrm{e}-05$

$1.20 e-05$

9.33e-06

Exposure pathway: residential external exposure to radiation

\footnotetext{
Cesium-137

Europium-155

Potassium-40

Radium-226

Thorium-228

Thorium-230

Thorium-232

Thorium-234

Uranium-233/234

Uranium-235

Uranium-238
}

Total pathway risk

\begin{abstract}
1.19e-04
$1.49 \mathrm{e}-07$

2.64e-04

$1.38 \mathrm{e}-04$

$1.16 \mathrm{e}-04$

9.30e-10

5.08e-10

$1.49 \mathrm{e}-07$

$1.10 \mathrm{e}-09$

5.47e-07

9.98e-07
\end{abstract}

$6.38 \mathrm{e}-04$

$6.57 \mathrm{e}-04$
$2.34 \mathrm{e}-05$

1.27e-07

3.12e-04

3.19e-04

$1.95 \mathrm{e}-04$

$1.50 \mathrm{e}-09$

7.88e-10

$9.69 \mathrm{e}-08$

$1.08 \mathrm{e}-09$

4.19e-07

8.67e-07

8.51e-04

8.71e-04 5.49e-05

1.24e-07

$1.79 \mathrm{e}-04$

$1.46 \mathrm{e}-04$

$1.61 \mathrm{e}-04$

$1.23 \mathrm{e}-09$

7.02e-10

1.31e-07

1.09e-09

5.31e-07

9.66e-07

5.43e-04

$5.58 \mathrm{e}-04$

a Total cancer risk (risk to an adult plus risk to a child).

${ }^{b}$ The sum of the risk from all analytes for all pathways. 
Table 7.9. Comparative background harard inder estimates from exposure to soil constituents from the Oak Ridge Reservation, Anderson County, and Roane County-Dismal Gap lithology"

\begin{tabular}{|c|c|c|c|}
\hline Analyte & $\begin{array}{c}\text { Oak Ridge } \\
\text { Reservation }\end{array}$ & $\begin{array}{c}\text { Anderson } \\
\text { County }\end{array}$ & $\begin{array}{l}\text { Roane } \\
\text { Covnty }\end{array}$ \\
\hline \multicolumn{4}{|c|}{ Exposure pathway: residential ingestion of soil } \\
\hline Antimony & - & $3.29 \mathrm{e}-02$ & - \\
\hline Arsenic & $4.88 \mathrm{e}-01$ & $3.40 \mathrm{e}-01$ & $4.59 \mathrm{e}-01$ \\
\hline Barium & $2.52 e-02$ & $2.05 e-02$ & $2.24 \mathrm{e}-02$ \\
\hline Beryllium & $2.83 e-03$ & $3.02 e-03$ & $2.34 \mathrm{e}-03$ \\
\hline Boron & $3.67 e-03$ & - & $6.18 \mathrm{e}-03$ \\
\hline Chromium VI & $4.96 \mathrm{e}-02$ & $5.66 \mathrm{e}-02$ & $5.48 \mathrm{e}-02$ \\
\hline Cyanide & $1.99 e-04$ & $1.79 e-04$ & $4.13 e-04$ \\
\hline Manganese & $1.91 e-01$ & $1.36 \mathrm{e}-01$ & $3.29 e-01$ \\
\hline Mercury & $1.61 \mathrm{e}-02$ & $5.48 e-03$ & $8.29 \mathrm{e}-03$ \\
\hline Mercury (salts) & $1.61 e-02$ & $5.48 e-03$ & $8.29 e-03$ \\
\hline Nickel & $1.98 e-0 i$ & $1.76 e-02$ & $1.41 e-02$ \\
\hline Nickel (salts) & $1.98 \mathrm{e}-02$ & $1.76 e-02$ & $1.41 \mathrm{e}-02$ \\
\hline Selenium & - & $2.64 e-03$ & $2.83 e-03$ \\
\hline Strontium & $1.47 e-04$ & $1.10 \mathrm{e}-04$ & $8.87 e-05$ \\
\hline Vanadium & $7.45 e-02$ & $6.62 e-02$ & $7.03 e-02$ \\
\hline Zinc & $4.11 e-03$ & $4.03 e-03$ & $3.30 \mathrm{e}-03$ \\
\hline Total pathway hazard index $x^{b}$ & $8.75 e-01$ & $6.85 \mathrm{e}-01$ & $9.73 e-01$ \\
\hline
\end{tabular}

Exposure pathway: residential dermal exposure to soil

\begin{tabular}{lccc} 
Antimony & - & $1.13 \mathrm{e}-02$ & - \\
Arsenic & $2.52 \mathrm{e}-02$ & $1.76 \mathrm{e}-02$ & $2.37 \mathrm{e}-02$ \\
Barium & $1.30 \mathrm{e}-02$ & $1.06 \mathrm{e}-02$ & $1.16 \mathrm{e}-02$ \\
Beryllium & $2.92 \mathrm{e}-03$ & 3.12 .03 & $2.42 \mathrm{e}-03$ \\
Boron & $1.89 \mathrm{e}-04$ & - & $3.19 \mathrm{e}-04$ \\
Chromium VI & $3.99 \mathrm{e}-02$ & $4.55 \mathrm{e}-02$ & $4.41 \mathrm{e}-02$ \\
Cyanide & $2.57 \mathrm{e}-05$ & $2.31 \mathrm{e}-05$ & $5.33 \mathrm{e}-05$ \\
Manganese & $1.97 \mathrm{e}-01$ & $1.40 \mathrm{e}-01$ & $3.40 \mathrm{e}-01$ \\
Mercury & $8.34 \mathrm{e}-04$ & $2.83 \mathrm{e}-04$ & $4.28 \mathrm{e}-04$ \\
Mercury (salts) & $5.56 \mathrm{e}-03$ & $1.89 \mathrm{e}-03$ & $2.85 \mathrm{e}-03$ \\
Nickel & $1.02 \mathrm{e}-03$ & $9.07 \mathrm{e}-04$ & $7.73 \mathrm{e}-04$ \\
Nickel (salts) & $2.05 \mathrm{e}-02$ & $1.81 \mathrm{e}-02$ & $1.46 \mathrm{e}-02$ \\
Selenium & $-\mathrm{C}$ & $2.27 \mathrm{e}-04$ & $2.43 \mathrm{e}-04$ \\
Strontium & $7.84 \mathrm{e}-06$ & $5.91 \mathrm{e}-06$ & $4.75 \mathrm{e}-06$ \\
Vanadicin & $1.50 \mathrm{e}-01$ & $1.33 \mathrm{e}-01$ & $1.41 \mathrm{e}-01$ \\
Zinc & $4.25 \mathrm{e}-04$ & $4.17 \mathrm{e}-04$ & $3.41 \mathrm{e}-04$ \\
& & & \\
Total pathway hazard index ${ }^{b}$ & $4.55 \mathrm{e}-01$ & $3.82 \mathrm{e}-01$ & $5.82 \mathrm{e}-01$ \\
\hline
\end{tabular}

Total hazard index (HI) (HI chronic for adult plus HI subchronic for child).

${ }^{b}$ The total pathway HI does not include HI values for mercury and nickel metals. 
The calculated background risk of $5.97 \times 10^{-8}$ for thorium-228 from the background concentrations reported for thorium-228 in horizon A on the ORR is a conservative estimate and will be used in this section. Also note that, for both cesium-137 and thorium-228, all three sampling areas have background risk estimates far below the EPA range of concern (i.e., $<1.0 \times 10^{-6}$ ). The total pathway risks for ingestion of soil (i.e., the sum of the background risk for each analyte in that pathway) are very similar between the three sampling areas (7.39 $\times 10^{-6}$ for ORR, $8.07 \times 10^{-6}$ for Anderson County and $6.14 \times 10^{-6}$ for Roane County) and are all $>1.0 \times 10^{-6}$, which is within the EPA's range of concern for contaminated soils (refer to Sect. 7.6.1 for a general discussion on EPA guidance). The pathway risks for the ingestion of soil that contains radionuclides only are $6.70 \times 10^{-7}$ (i.e., $7.39 \times 10^{-6}$ minus $6.72 \times 10^{-6}$ ), $8.90 \times 10^{-7}$, and $5.70 \times 10^{-7}$, respectively, for ORR, Anderson County, and Roane County, and these background risk estimations are not within the EPA range of concern. The potential risks resulting from the ingestion of soil containing only beryllium are $6.72 \times 10^{-6}$ on the ORR, $7.18 \times 10^{-6}$ for Anderson County soils, and $5.57 \times 10^{-6}$ for Roane County (refer to Table 7.8). Therefore, background risk posed by ingestion of beryllium is driving the total pathway risk for the ingestion of soil into the EPA range of concern (i.e., between $1.0 \times 10^{-6}$ and $1.0 \times 10^{-4}$ ).

Second, background risk estimates (Table 7.8) for beryllium for the residential dermal exposure to soil pathway are quite similar between the three sampling areas and are also in the EPA range of concern for contaminated soils but fall below the unacceptable level of $1.0 \times 10^{-4}$ (see Sect. 7.6.1 for EPA guidance summary). Upon comparison of the background risks posed by the third exposure pathway, external exposure to radionuclides in soil (Table 7.8), it is found that cesium-137 is the only analyte that shows a significant difference between the three sampling areas; this observation is addressed statistically in Sects. 5.7 and 5.8 and qualitatively in Sects. 6.3 and 6.4.3. Cesium-137 results from man-made activities (see Sect. 6.4.3 and Sect. 7.5.2.1). The estimated background risk for cesium-137 on the ORR is approximately five times that found in Anderson County and twice that found in Roane County. The calculated background risk from external exposure to radiation posed by cesium-137 in Roane and Anderson counties is within the EPA range of concern for contaminated sites; the cesium-137 background risk on the ORR is slightly $>1.0 \times 10^{-4}$. The total pathway risks for external exposure to radionuclides (the sum of the background risk for each radionuclide, in this case) are very similar between the three sampling areas (Table 7.8).

The total background risks (i.e., cumulative background risk from all analytes for all pathways) for soils from the Dismal Gap Formation for the ORR, Anderson County, and Roane County are $6.57 \times 10^{-4}, 8.71 \times 10^{-4}$, and $5.58 \times 10^{-4}$, respectively (Table 7.8). This information is illustrated graphically in Fig. 7.1 (these risk values were determined using the UCB95 analyte concentrations and are represented as the top line of each box). The total background risk for the Nolichucky Formation on the ORR is $7.45 \times 10^{-4}$ and is also shown in Fig. 7.1.

These background risk estimates should be considered only in the context of comparison with site-related risk. The EPA action level of $1.0 \times 10^{-4}$ refers to risks related to hazardous waste sites. Therefore, the background risk results are not indicative of concerns or actions that would be identified with similar potential risks from a contaminated site.

Because of the variation between the cesium-137 levels (concentrations) on the ORR and those in Anderson and Roane counties, some uncertainty exists concerning the 


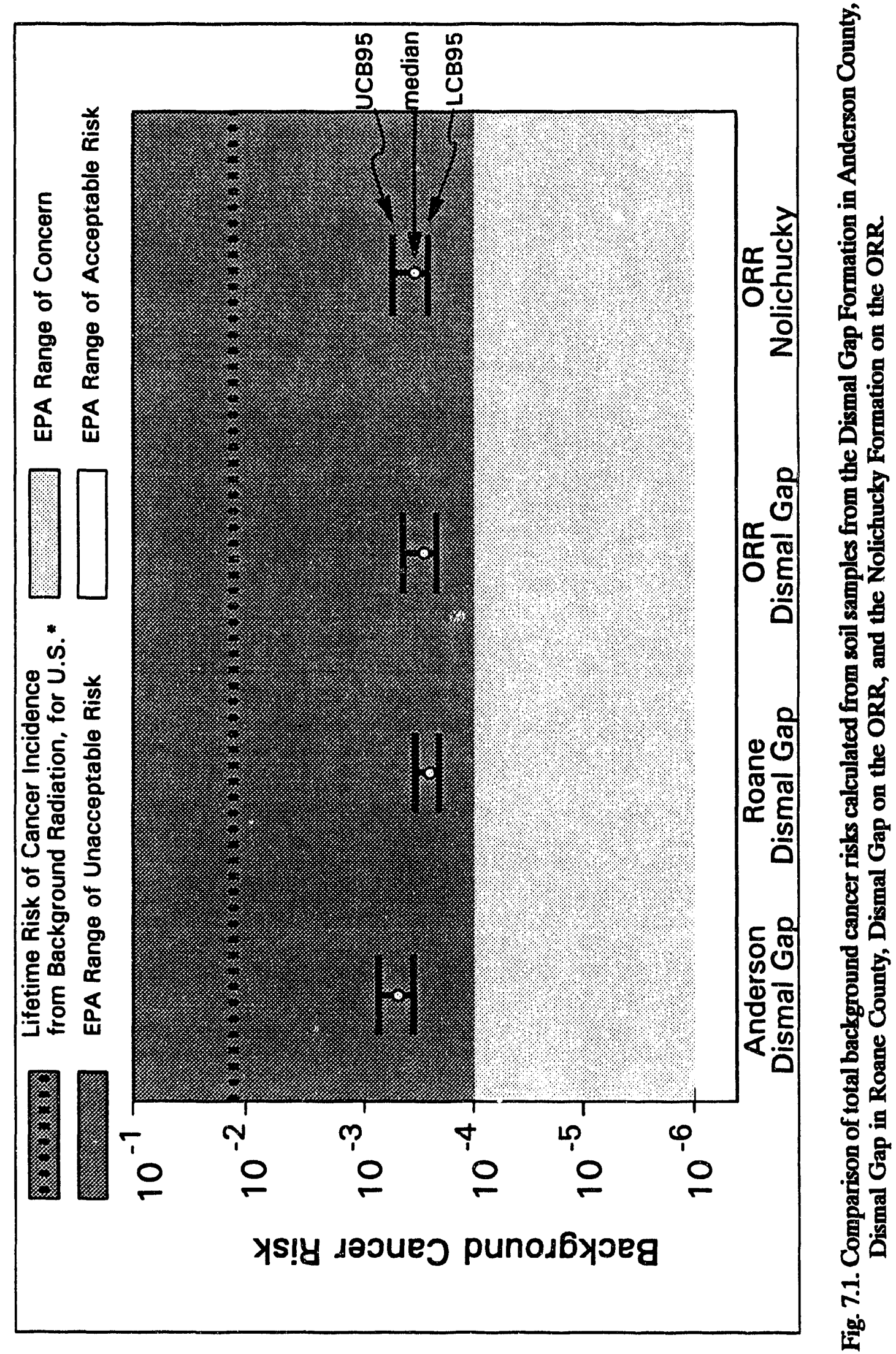


background risk results for the cesium-137 data in the risk analysis discussed previously. The gamma scan method was used in this project to screen cesium-137 levels to find appropriate locations for soil sampling sites; data resulting from this method are not commonly used for analysis of radionuclides or for evaluating risk. However, because of the concern stated previously, the cesium-137 gamma screening data (0 to $5 \mathrm{~cm}$ depth) were evaluated in terms of background risk and will be discussed here; these screening data were addressed statistically in Sect. 5.8 and qualitatively in Sect. 6.3. The residential ingestion of soil pathway for the Dismal Gap Formation on the ORR and in Anderson and Roane counties shows background risks (from the cesium-137 gamma scan) of $6.18 \times 10^{-8}, 4.75 \times 10^{-8}$, and $3.91 \times 10^{-8}$, respectively; these risks are below the EPA range of concern. The background risks from the residential external exposure pathway for the ORR, Anderson County, and Roane County are $8.41 \times 10^{-5}, 6.46 \times 10^{-5}$, and $5.32 \times 10^{-5}$, respectively; these risks are within the EPA range of concern (i.e., $1.0 \times 10^{-6}$ through $1.0 \times 10^{-4}$ ). For both exposure pathways, the risks from cesium-137 (based on gamma scan data) on the ORR are approximately 1.5 times greater than those for Anderson and Roane counties. If these cesium-137 risk numbers were used in the calculation of total cumulative excess cancer risks, the excess cancer risks for the ORR, Anderson County, and Roane County would be $6.22 \times 10^{-4}, 9.12 \times 10^{-4}$, and $5.56 \times 10^{-4}$, respectively.

\subsubsection{Background hazard index comparisons between the ORR and Anderson and Roane counties}

Table 7.9 contains the total HI (chronic HI for an adult + subchronic $\mathrm{HI}$ for a child) estimates determined for background analytes (where $\mathrm{RfD}$ values were available) found in soil in the three sampling areas. As indicated in Table 7.9, the metals detected vary between the three locations; antimony and selenium are not found in soil from the ORR, antimony is also not found in Roane County soil, and boron is not found in Anderson County. Upon evaluation of the ingestion of soil pathway, when analytes were found in two or all three of the sampling areas, the calculated background $H I$ values were very similar. ORR mercury (and mercury salts) HI values, however, were found to be approximately twice those determined for Roane County and approximately three times those found in Anderson County. The ORR cyanide HI value is approximately one-half that found in Roane County and the ORR strontium HI value is approximately 1.5 times higher than that found in Roane County. The statistical analysis (Sect. 5.3) for mercury concentrations shows significant differences in concentration for the horizon A data for the three sampling areas. No statistical differences between sampling areas for the cyanide and strontium data (horizon A) were reported in Sect. 5.3.

No single analyte (Table 7.9) was found to have a background $\mathrm{HI}$ greater than 1.0. Exposure via ingestion, for all soil locations, has a cumulative total pathway HI (the sum of the background $\mathrm{HI}$ for each analyte in that pathway) of $<1.0$. Therefore, systemic effects resulting from ingestion of soil are not a concern for the background analyte concentrations.

The evaluation of dermal exposure to soil (Table 7.9) results in most analytes having similar background $H I$ values across the three sampling areas. The $H I$ for nickel in the ORR soil differs only slightly from that found in Anderson County; the nickel $\mathrm{HI}$ is approximately 1.5 times that found in Roane County. The HI for ORR mercury (and mercury salts) is approximately twice that found in Roane County and three times that found in Anderson County. The ORR background HI for exposure to cyanide is approximately half that found 
in Roane County. Furthermore, the total pathway HI determinations for the three sampling areas are very similar and are all $<1.0$. Therefore, even sensitive populations are unlikely to experience adverse systemic health effects when exposed to soil constituents at background concentrations.

In Sects. 7.6.3 through 7.6.3.2, comparisons were made between the calculated risks or HI values from background constituents in soils from the Dismal Gap Formation from three sampling areas (ORR, Roane County, and Anderson County). In summary, with the few exceptions noted, the background risk and hazard indices (Tables 7.8 and 7.9) determined for individual analytes are very similar for the three sampling areas. In Sect. 7.6.4, carcinogenic and systemic effects will be evaluated quantitatively for soil samples that best represent the background analytes found on the ORR (i.e., the horizon A soil samples taken from the ORR in the Dismal Gap and Nolichucky formations).

\subsubsection{Background risk and HI comparisons using the LCB95, median, and UCB95 analyte concentrations}

The following discussion of the range in risk associated with the concentration variability for background soil constituents applies only to this background soil data set. The results associated with the background data set do not necessarily represent the actual variability of constituent concentrations in soils of the Dismal Gap and Nolichucky formations. Tables 7.10 and 7.11 assess the variability in the risk and $\mathrm{HI}$ estimates for the three sampling areas with respect to the analyte concentrations used in this background evaluation. These tables include risk (Table 7.10) and hazard indices (Table 7.11) for three analyte concentrations [i.e., the lower $95 \%$ confidence bound on the median concentration (LCB95), the median concentration, and the UCB95]. All other tables in Sect. 7 (excluding Tables 7.10 and 7.11) use the UCB95 as the representative analyte concentrations.

The differences between the UCB95 and LCB95 total risk estimates for the ORR, Anderson County, and Roane County are $2.89 \times 10^{-6}, 3.09 \times 10^{-6}$, and $2.37 \times 10^{-6}$, respectively. This information is also illustrated graphically in Fig. 7.1. The cumulative background risks determined using the UCB95 analyte concentrations are represented by the top line; the risk determined using the median analyte concentrations is shown as the open circle, and when the LCB95 concentration was used to calculate risk, this information is represented by the bottom line. Note, that the variability between the three risk numbers (within each sampling area) is relatively small and, therefore, the overall total background risk to human health does not significantly change by varying the analyte concentration in this manner.

The results tabulated in Table 7.11 show the variability in the hazard indices for each sampling area using the three analyte concentrations. Again, the differences in the HI values are very small. The results of these comparisons (risk and HI) illustrate high confidence in the quantitative validity of this background soil ciata set (see Sect. 5). However, it should be understood that these conclusions may not necessarily apply to the actual variability of constituent concentrations in soils. Depending on the application of the background data either the UCB95 or the LCB95 is more appropriate in terms of human health (refer to Sect. 2.3.4, Data User Guidelines). 


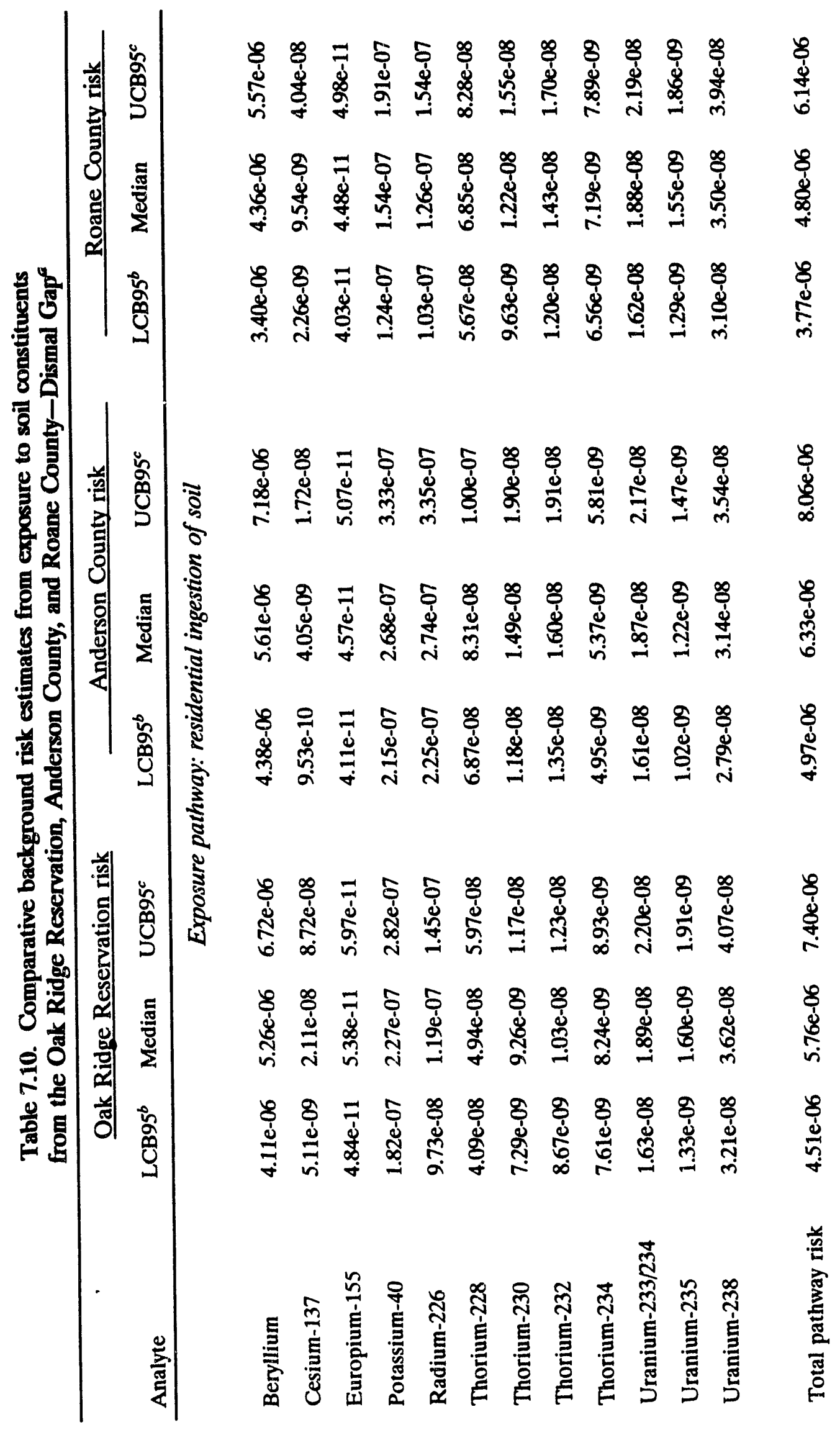




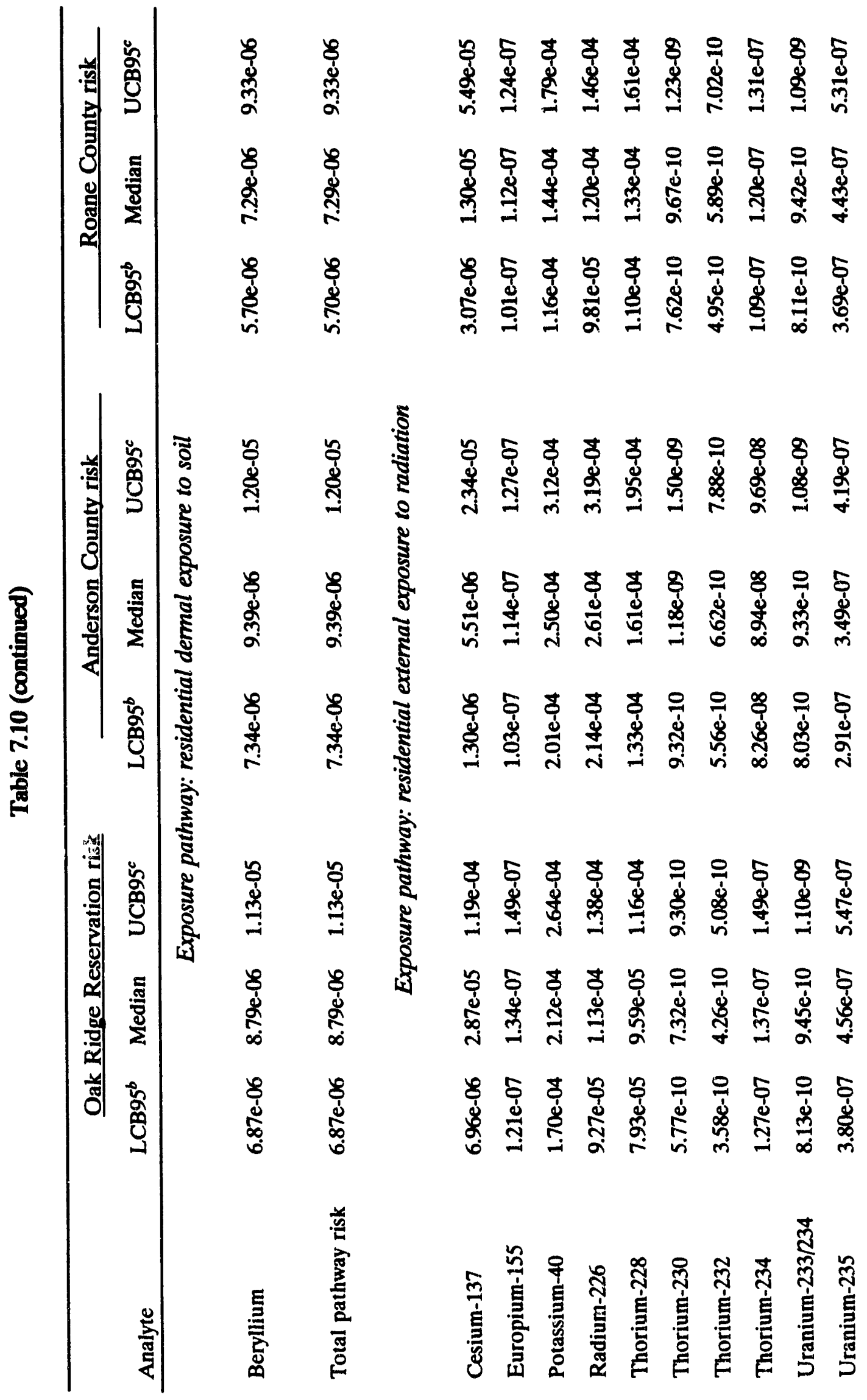




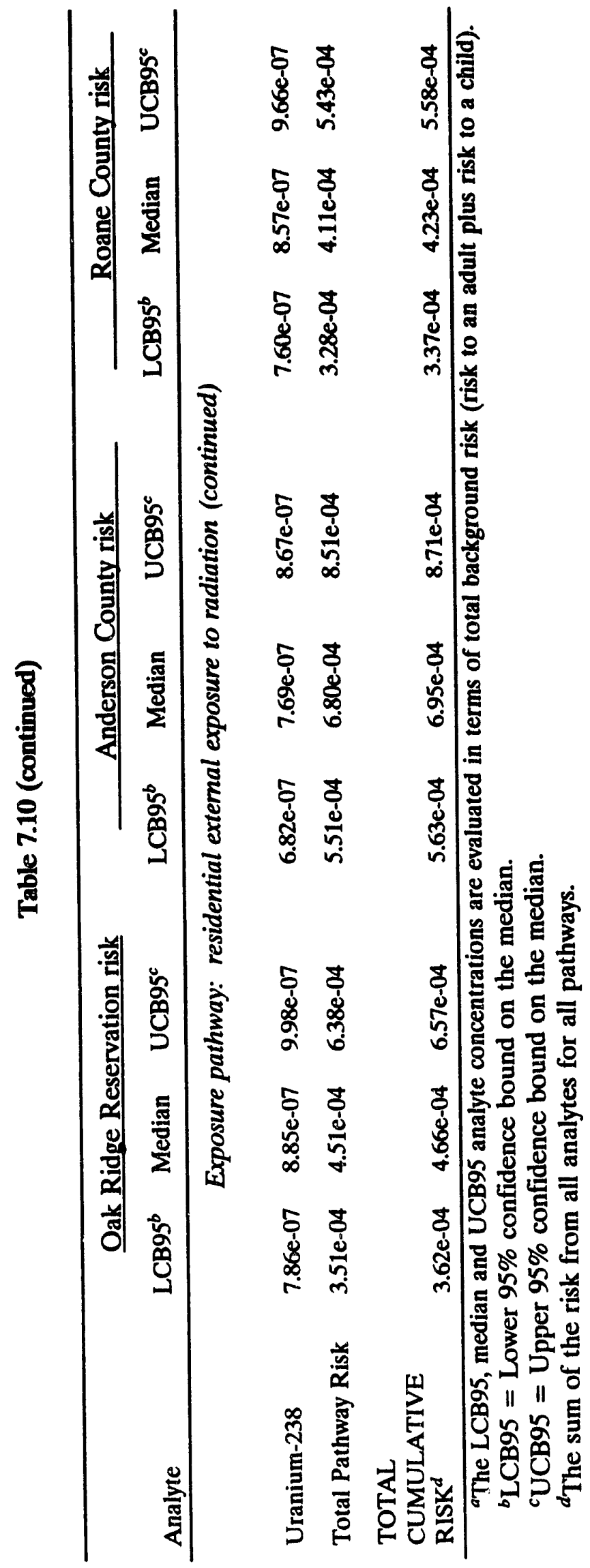




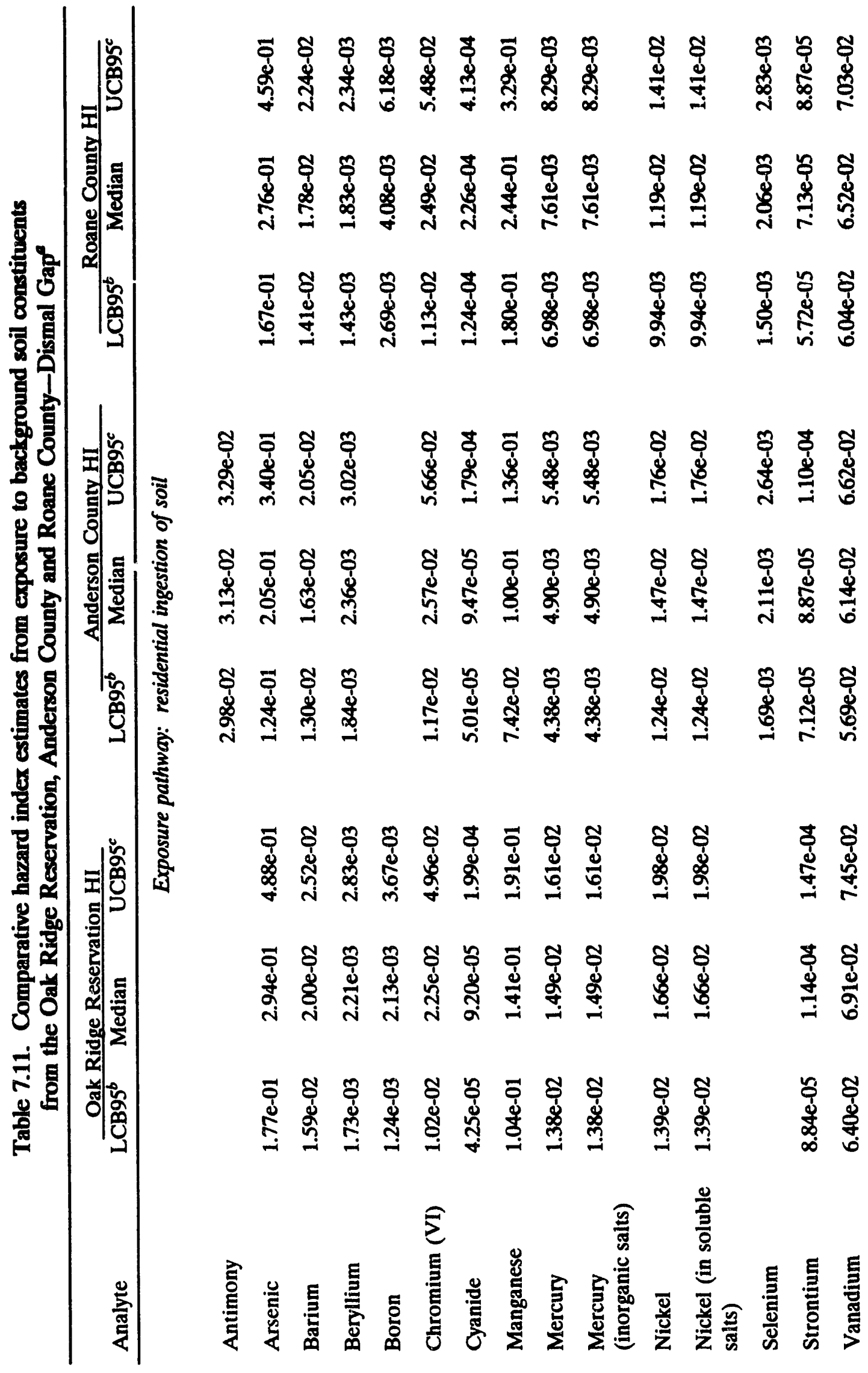




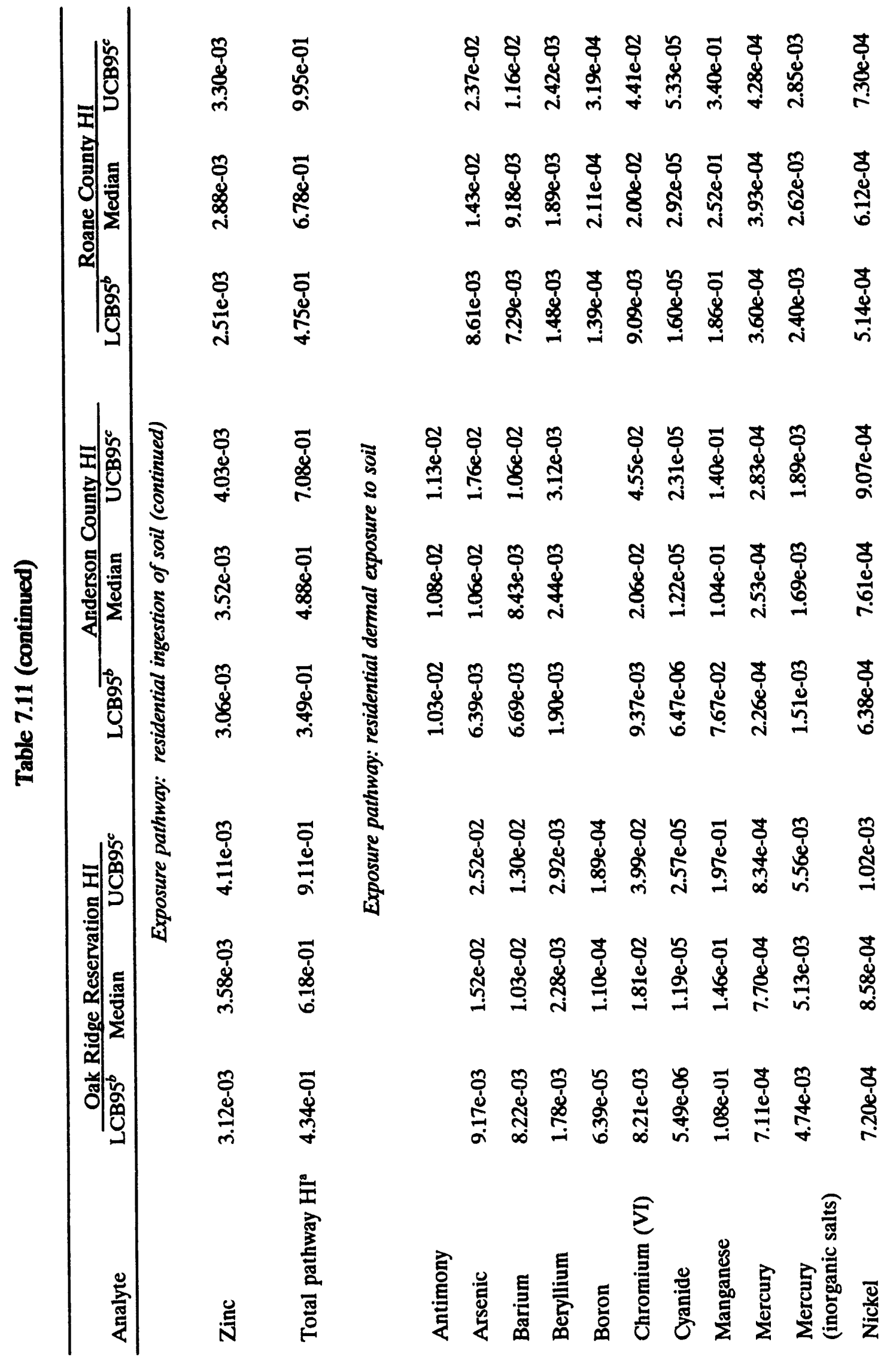




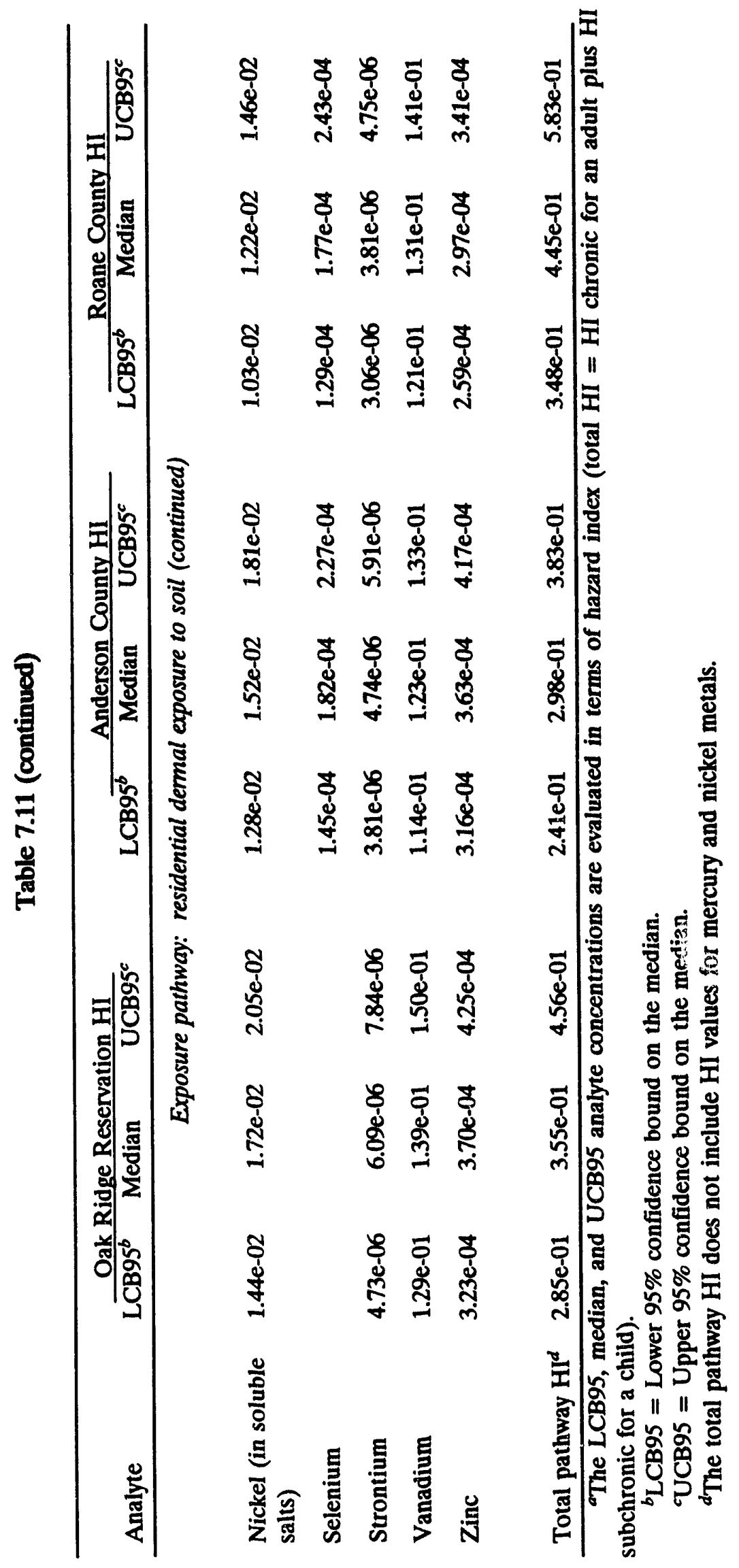




\subsubsection{Background Risk Characterization for the ORR}

The carcinogenic and systemic health effects are evaluated for elements that have both an SF and an RfD. Other soil constituents, however, have only one (or neither) of the two toxicity values. Each constituent detected in the ORR background soil has been included in the risk or HI calculation, provided the constituent has at least one of the toxicity values. If neither of the toxicity values is available for a constituent, a quantitative CDI can be calculated (Table 7.4), but the carcinogenic and systemic effect can only be evaluated qualitatively (Table 7.2). Such constituents may contribute to carcinogenic and noncarcinogenic effects from exposure to the soil, but their effect cannot be quantified at present. For constituents that have both an oral SF and an oral RfD, both their carcinogenic and systemic health effects are quantified.

\subsubsection{Carcinogenic background risk characterization for the ORR}

As discussed in Sect. 7.4, an on-site resident would be exposed to background soil constituents via ingestion of soil, dermal contact with soil, and external exposure to radionuclides in the soil. Table 7.12a-c lists the chemical-specific toxicity information, the calculated CDIs (and dose), the calculated background cancer risk for an adult and a child, the total background cancer risk (adult + child), and the total pathway risk for the analytes found in the ORR background soils for which oral SFs were available. In general, for beryllium (Table 7.12a), the background cancer risk for a child exposed to beryllium is slightly higher than that of an adult for the ingestion pathway, and the child's risk is slightly lower than that of an adult for dermal exposure. Beryllium is the only inorganic analyte found on the ORR, horizon $A$, for which an oral SF is available. Exposure to beryllium via soil ingestion or dermal contact in both the Dismal Gap and Nolichucky formations results in a total (adult + child) background cancer risk between $1.0 \times 10^{-6}$ and $1.0 \times 10^{-4}$ (the EPA range of concern for contaminated soils). The total pathway background cancer risks from exposure to beryllium across these two pathways combined (cumulative risk) are $1.80 \times 10^{-5}$ and $1.81 \times 10^{-5}$ for the Dismal Gap and Nolichucky formations, respectively. These total background cancer risks are also within the EPA range of concern for contaminated soils.

Also included in Table 7.12b are the adult and child background cancer risk estimates for ingestion of soil containing radionuclides. In general, the adult resident has a higher cancer risk from ingestion of the soil than does the child resident. The total (adult + child) pathway background cancer risks for ingestion of soil containing radionuclides are $6.72 \times 10^{-7}$ and $6.60 \times 10^{-7}$ for the Dismal Gap and Nolichucky formations, respectively. These risk values are less than the lower limit of the EPA range of concern, which is considered acceptable for exposure to site contaminants.

The adult and child background cancer risk estimates for external exposure to ORR soils containing radionuclides are summarized in Table 7.12c. Again, in general, the adult resident has a greater background cancer risk than the child resident. The total pathway background cancer risks (adult + child) for Dismal Gap and Nolichucky lithologies are $6.38 \times 10^{-4}$ and $7.26 \times 10^{-4}$, respectively. These total background soil constituents risk estimates fall in the EPA region of unacceptable risk $\left(>1.0 \times 10^{-4}\right)$ for exposure to contaminated sites.

The total (adult + child) exposure (ingestion of beryllium and radionuclides, dermal contact with beryllium, and external exposure to radionuclides) background cancer risks are $6.57 \times 10^{-4}$ and $7.45 \times 10^{-4}$ for soils from the Dismal Gap and Nolichucky formations, 


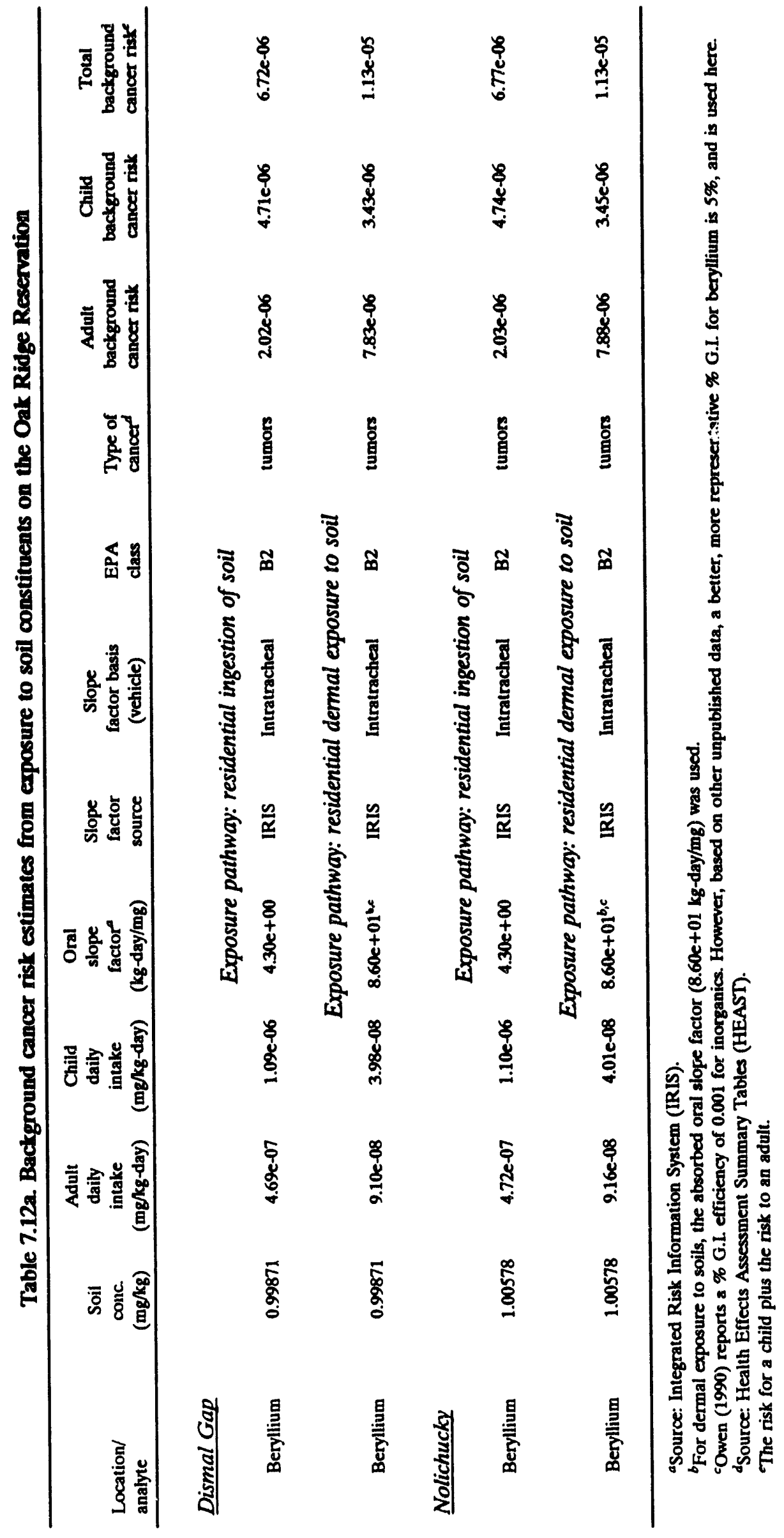




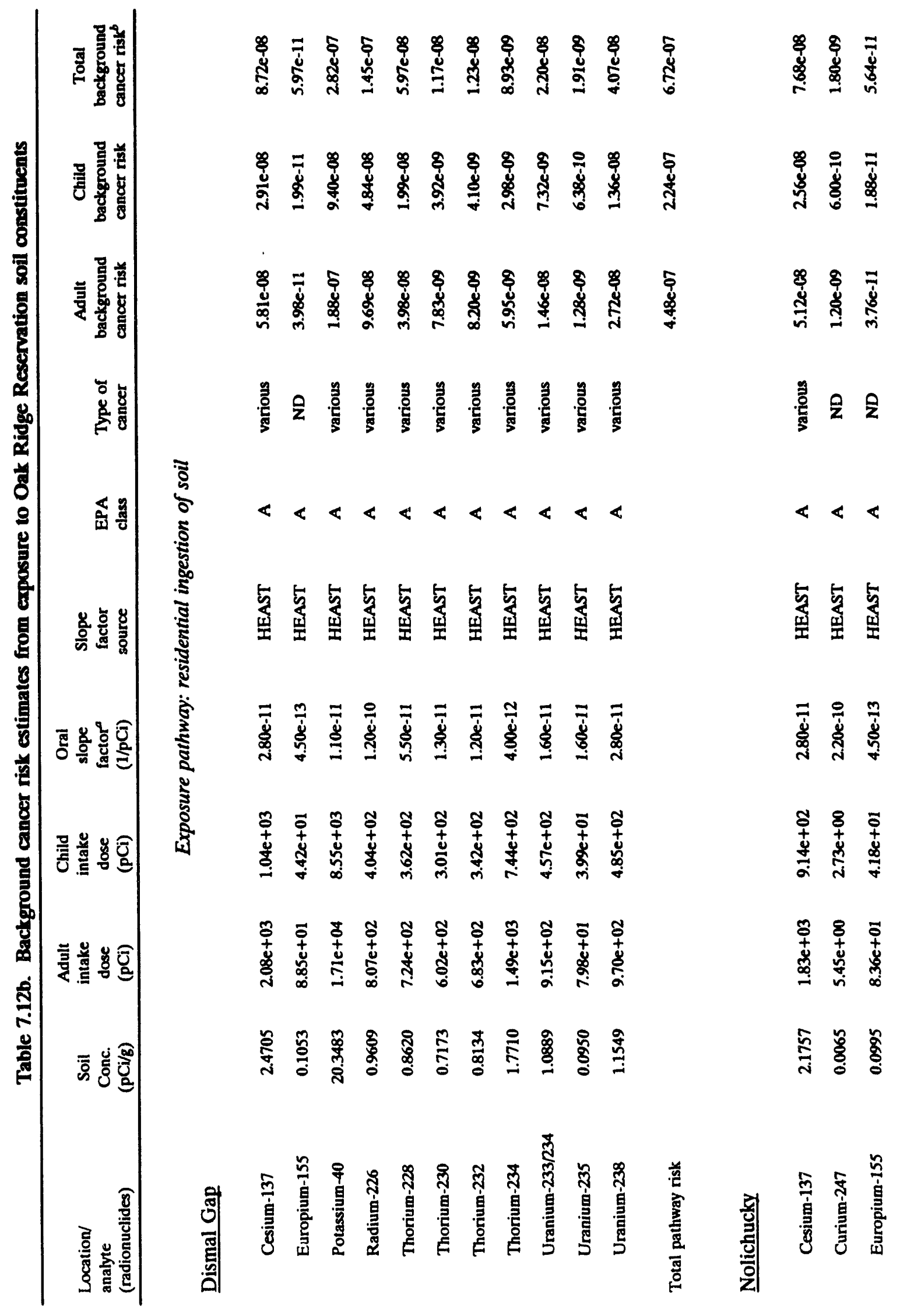




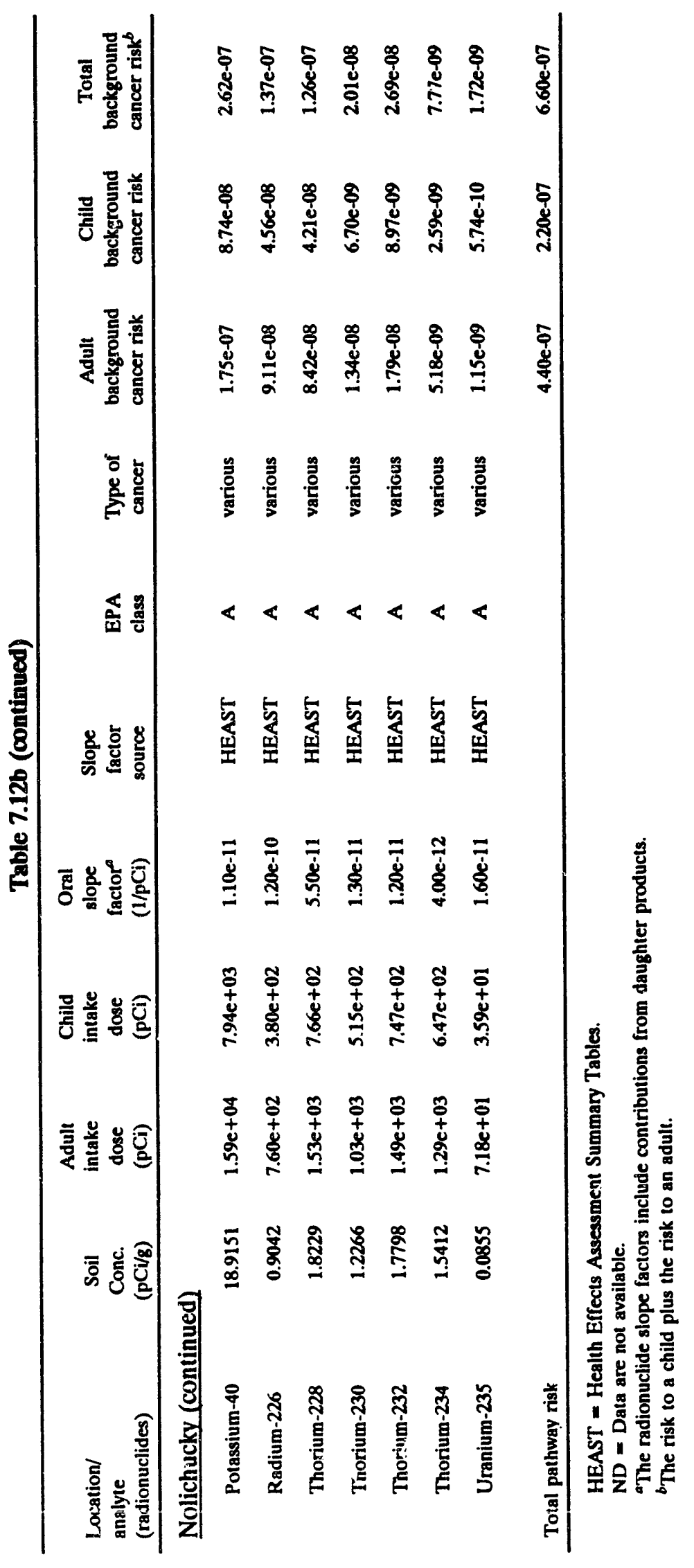




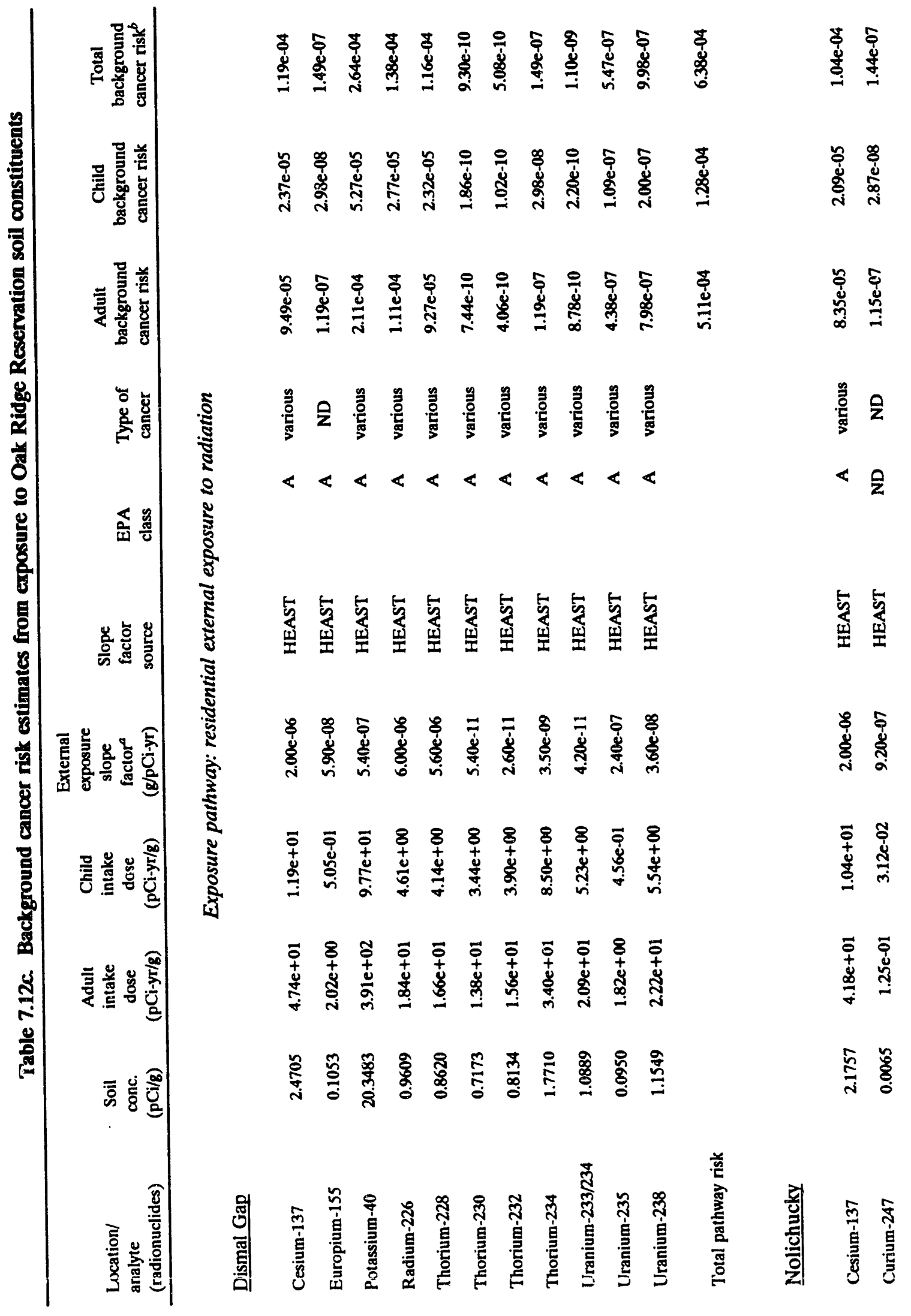




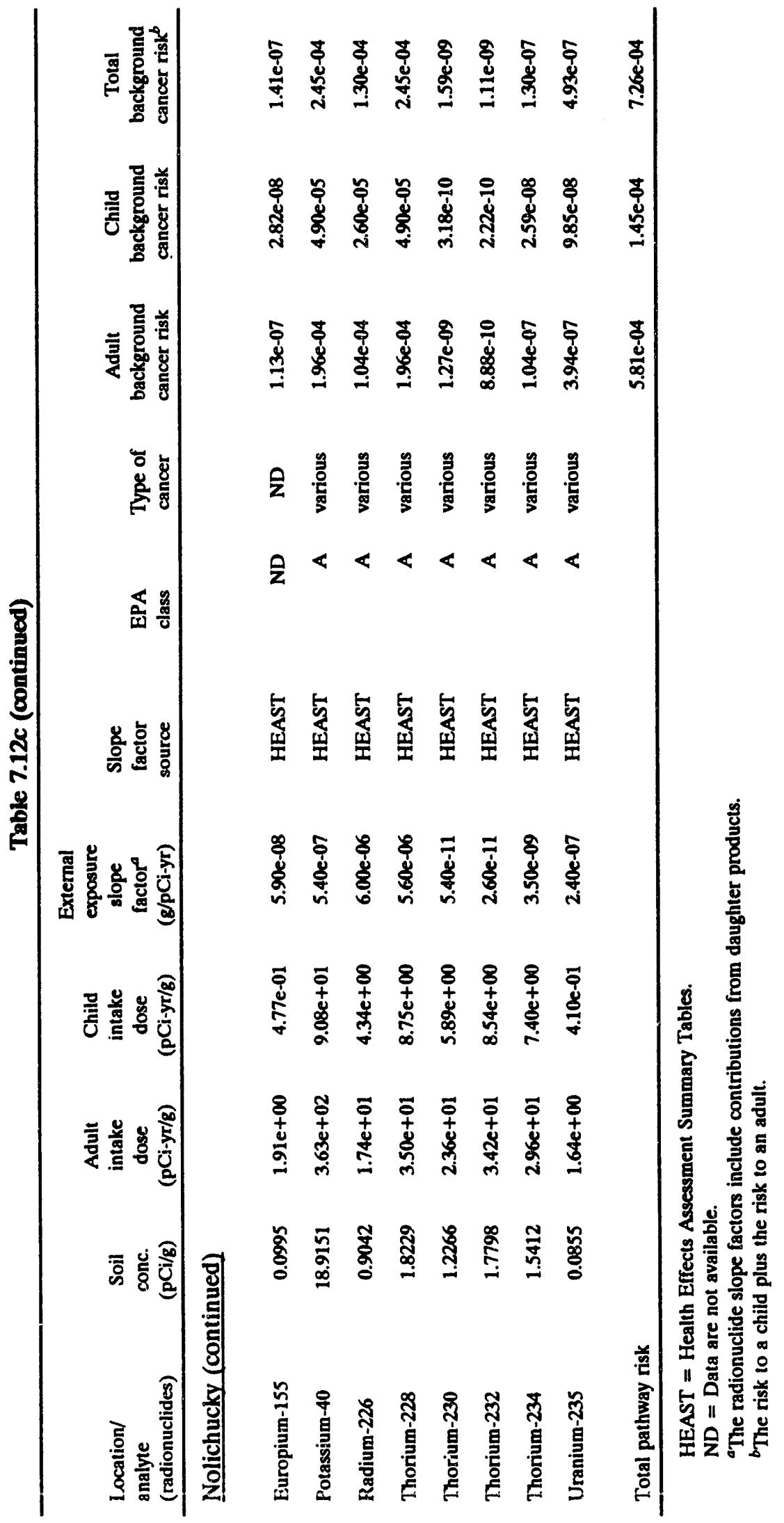


respectively (i.e., the sum of total pathway risks found in Tables 7.12a through 7.12c). These relatively high total exposure risk results are predominantly from the background risks associated with the external expcsure to radiation.

\subsubsection{Noncarcinogenic background risk characterization for the ORR}

The results of the assessment of systemic toxicity indicate that neither the ingestion of inorganics nor the dermal absorption of inorganics pathway is a concern. Table 7.13a-f lists the RfDs, CDIs for a child and an adult, background hazard indices for a child and an adult, and the total (adult + child) background hazard indices for systemic toxicants.

In general, the child HI (subchronic) is greater than the adult HI (chronic) for both the ingestion of soil and the dermal contact pathways. The total ingestion pathway HIs (adult + child) for the Dismal Gap (Table 7.13b) and Nolichucky (Table 7.13c) formations are $8.75 \times 10^{-1}$ and $5.25 \times 10^{-1}$, respectively. Furthermore, the total dermal pathway HI values (adult + child) are $4.55 \times 10^{-1}$ (Table 7.13e) and $3.77 \times 10^{-1}$ (Table 7.13f) for Dismal Gap and Nolichucky, respectively. The total (adult + child) pathway (ingestion and dermal) background His for Dismal Gap and Nolichucky are 1.33 and 0.862 , respectively. The Dismal Gap soil HIs are above the EPA threshold of 1.0. Hence, there is a concern for human health from systemic effects from these natural background constituents, according to EPA guidance for site contamination.

\subsubsection{Summary of the background risk and hazard index characterization for the ORR}

In summary, the total pathway risk estimates for the carcinogens found in background soil samples taken on the ORR are (1) between $1.0 \times 10^{-6}$ and $1.0 \times 10^{-4}$ for ingestion and dermal contact with beryllium; (2) less than $1.0 \times 10^{-6}$ for ingestion of radionuclides; and (3) greater than $1.0 \times 10^{-4}$ for external exposure to radionuclides. The main contributor to the risk for the ingestion and dermal exposure pathways is beryllium. Cesium-137, potassium-40, radium-226, and thorium-228 are the main contributors to risk for the external exposure pathway. Note, because the technetium-99 data did not pass validation, the results (risk) from exposure to this radionuclide are not included in this risk evaluation. However, an EPAapproved slope factor for technetium- 99 is available. Therefore, if technetium- 99 were included in future risk evaluations, the background risk associated with radionuclides may be greater than reported here.

The hazard indices estimated for ingestion of inorganics in background soil and for dermal contact with the background soil are below the EPA guideline of 1.0; therefore, these pathways pose no expected adverse effects to human health. The total pathway HI (ingestion + dermal) for Dismal Gap Formation samples is slightly above this threshold. Arsenic and manganese are the major contributors to the $\mathrm{HI}$ for the ingestion pathway; and the main contributors to the $\mathrm{HI}$ for the dermal exposure pathway are manganese and vanadium. Note that the risks associated with background soils on the ORR were estimated to provide a frame of reference for interpreting the magnitude and relative importance of risks evaluated at hazardous waste sites on the ORR. Therefore, risks from uncontaminated background soil samples found to be within or above the EPA region of concern do not indicate concerns or actions that would be identified with similar potential risks from a contaminated site. 


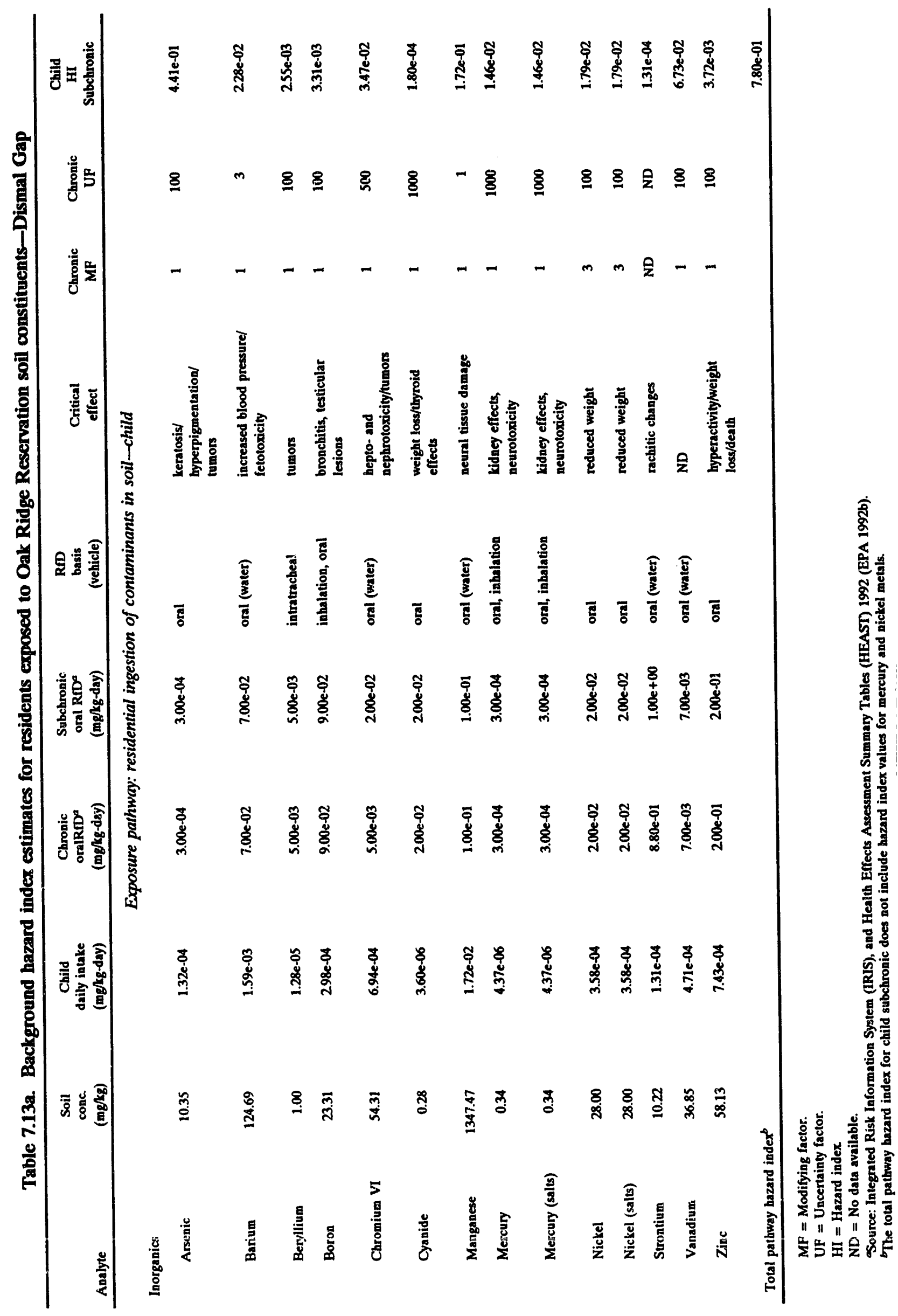


$7-51$

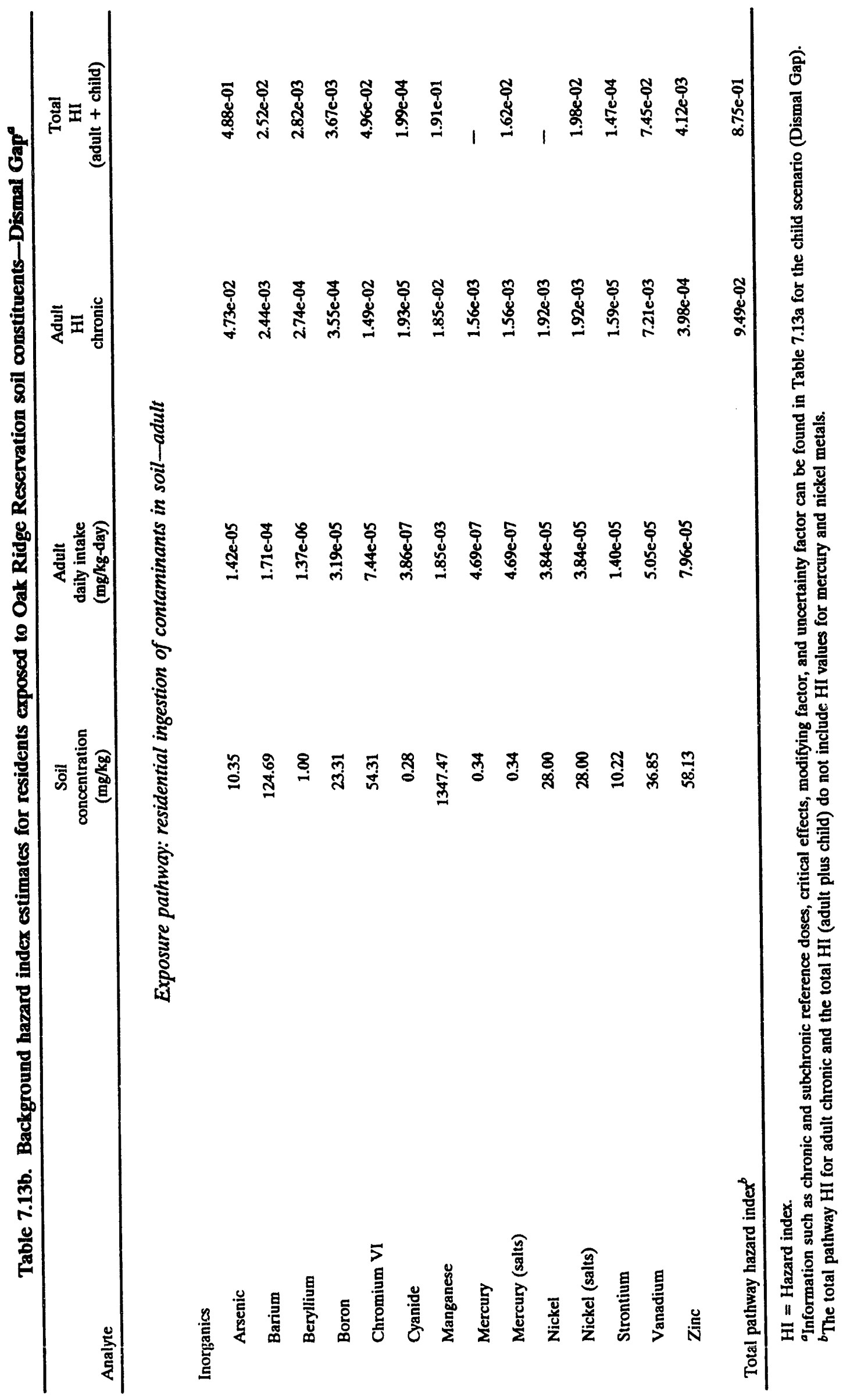




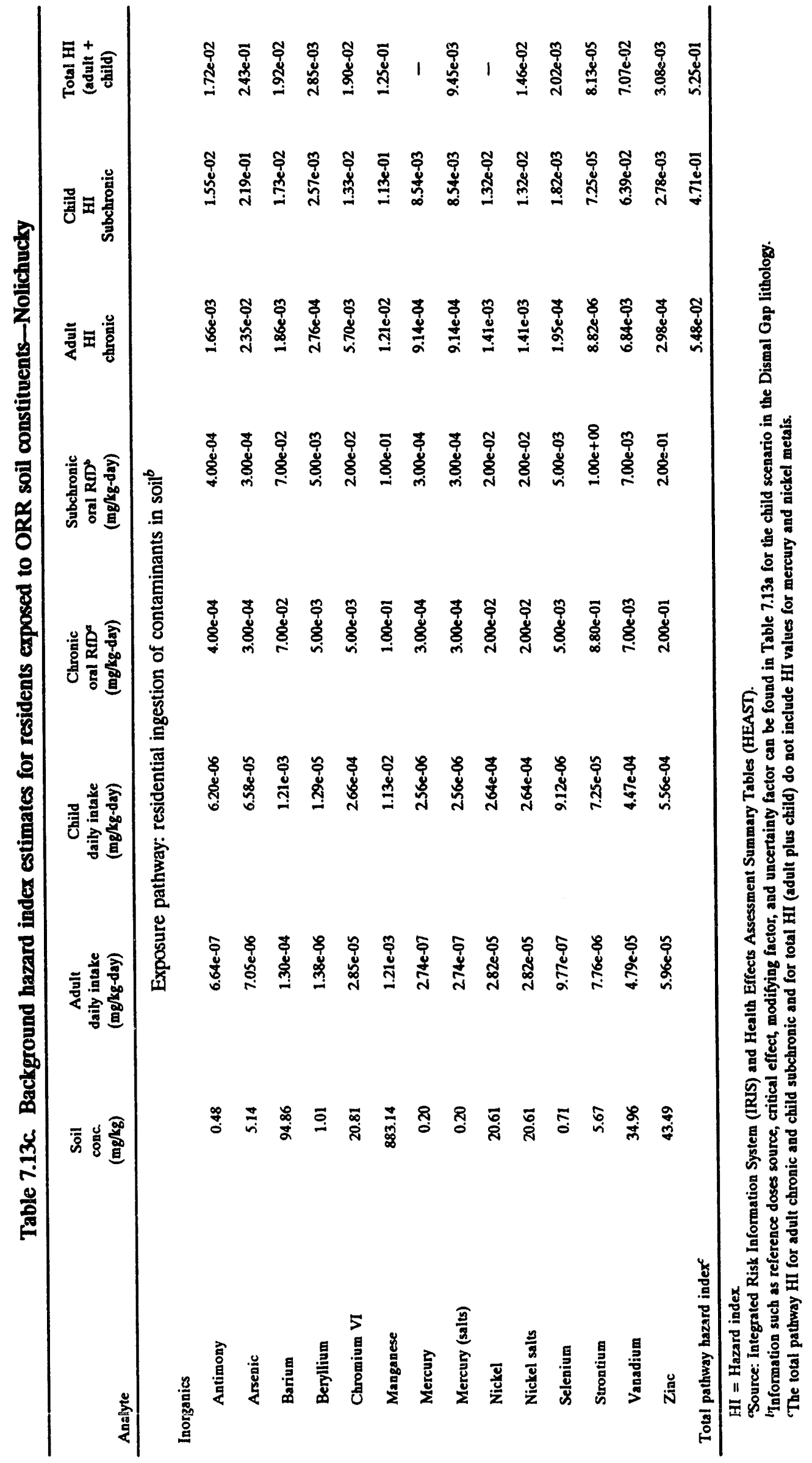




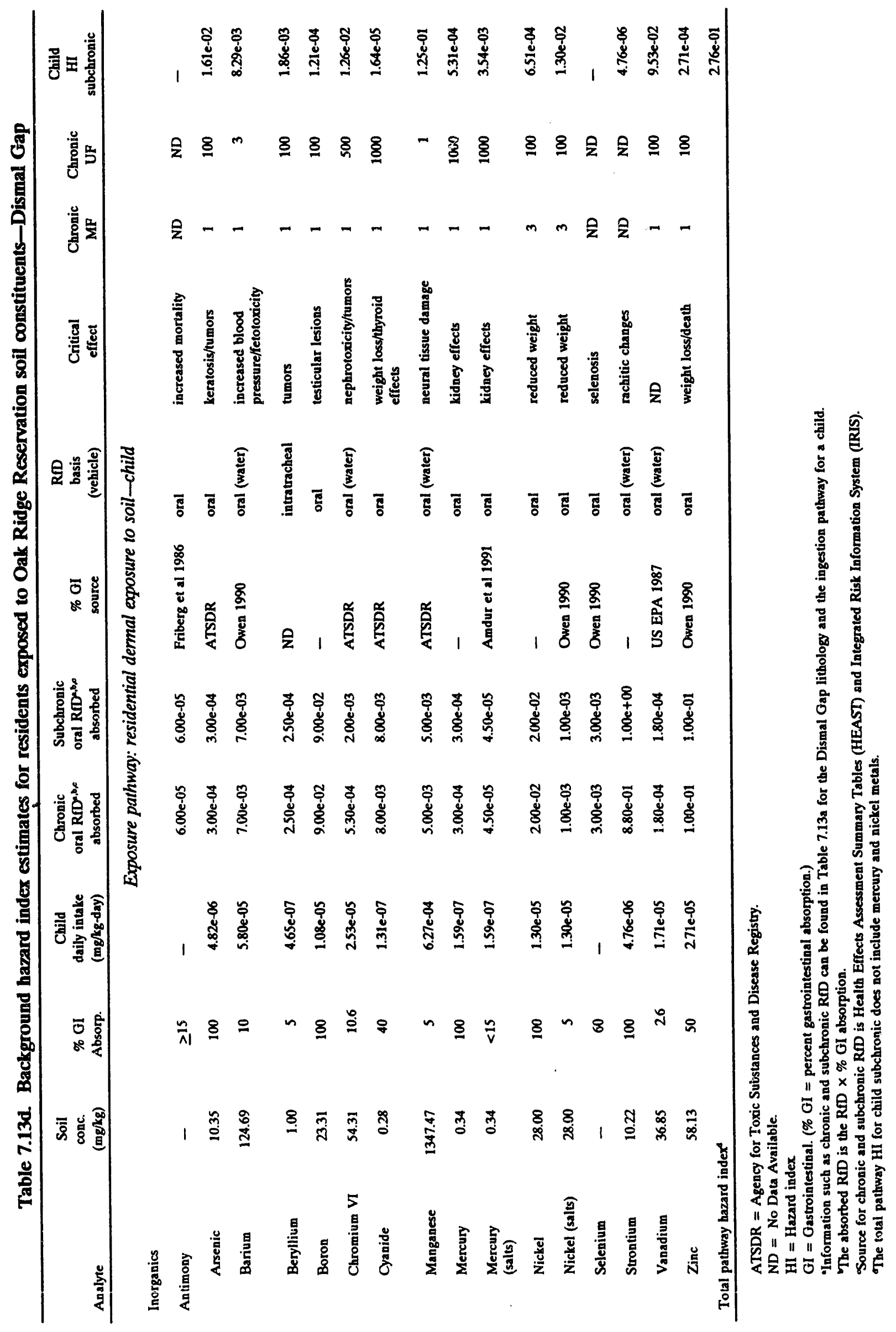




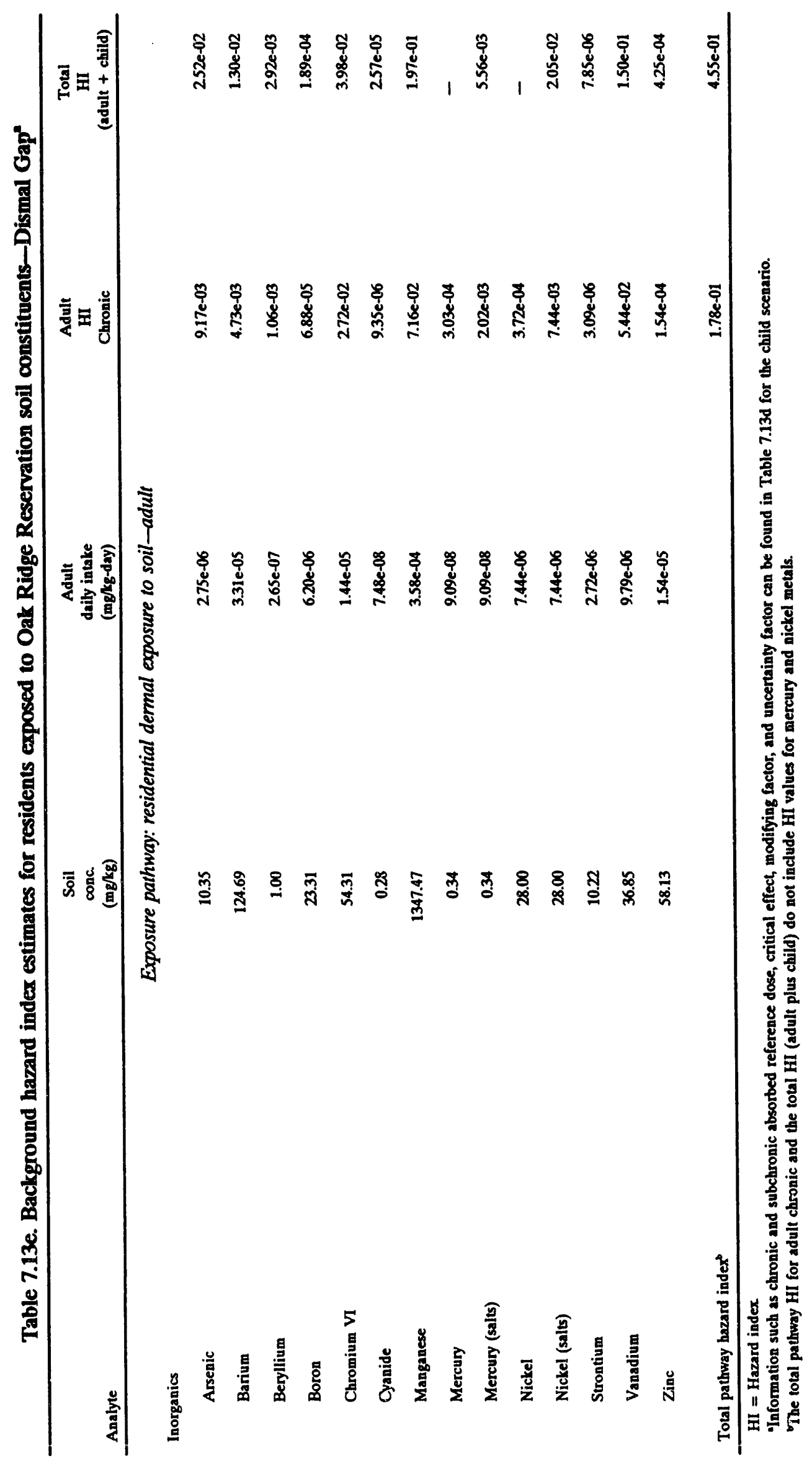




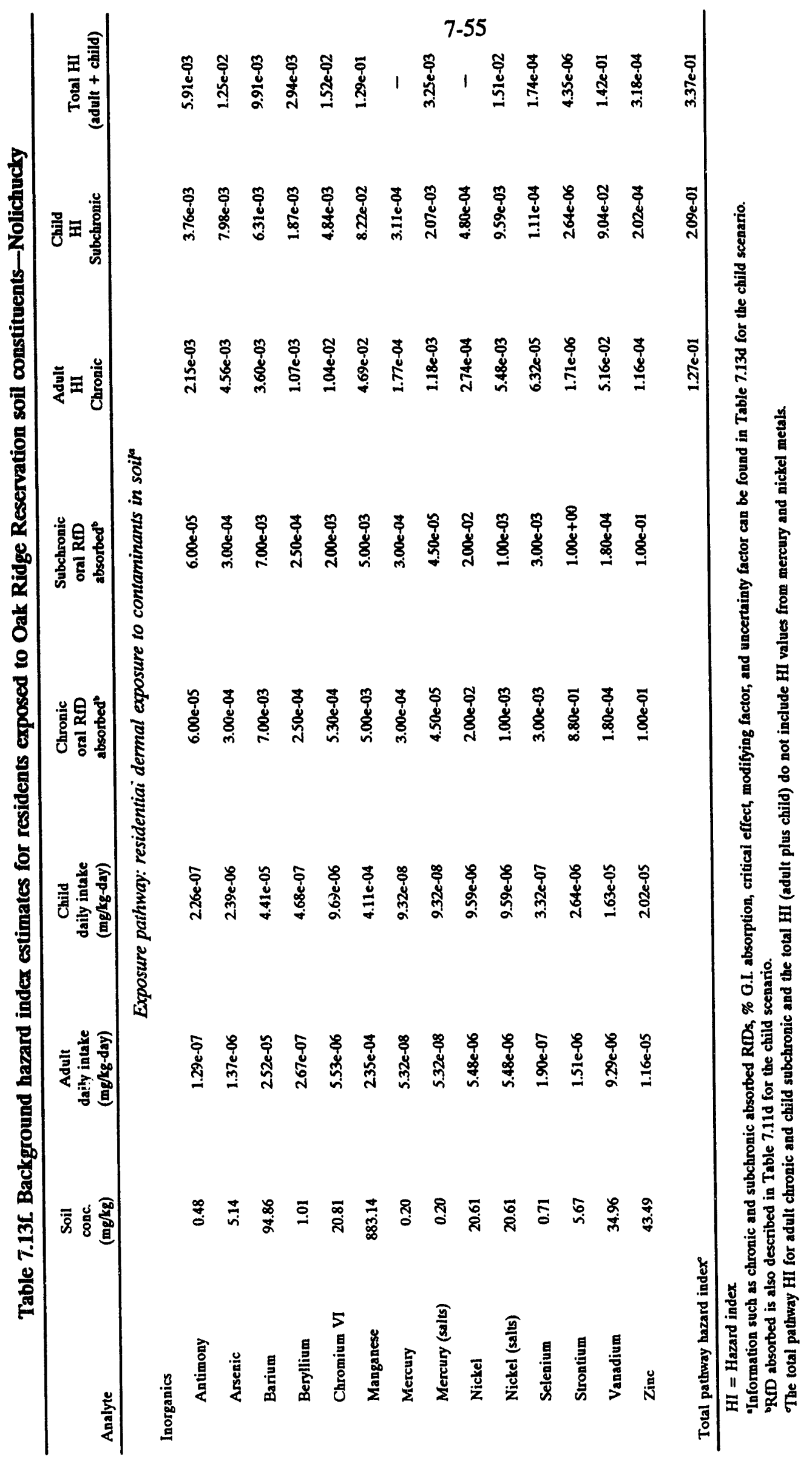




\subsection{UNCERTAINTIES AND ASSUMPTIONS}

Risk assessment as a scientific activity is subject to uncertainty (Table 7.14). The methodology used in this background risk evaluation follows EPA guidelines. The risk evaluation in this report is subject to uncertainty pertaining to sampling and analysis, exposure estimation, and toxicological data.

The major assumptions used in risk assessment are (1) that contaminant concentrations detected and reported by the analytical laboratory are representative of true analyte concentrations in soils (i.e., the analyte concentration remains constant over the sampling and analysis time period); (2) that the intake rates and exposure parameters are representative of actual potentially exposed populations; and (3) that all contaminant exposure and intake are from the site-related exposure media (i.e., no other sources contribute to the receptor's health risk). Even if these assumptions are true, other areas of uncertainty may apply. The toxicological data (SFs and RfDs) are frequently updated and revised, which can lead to overor underestimation of risks. These values are often extrapolations from animals to humans, which also induces uncertainties in toxicity values. In addition, as mentioned earlier, in the analytical analyses for metals (total metal only) risks may be overestimated because the metals that are present are conservatively assumed to be in their most toxic forms. Furthermore, not all of the background chemicals reported in Table 7.2 currently have toxicity values; this can lead to an underestimation of total risk because quantitative analysis of such chemicals is currently not possible.

In addition, current analytical methods are limited in their ability to achieve detection limits that are appropriate for use in risk assessment. Therefore, risks may be overestimated as a result of analyte concentrations being reported at the method detection limit (MDL) which may be greater than the concentration at which adverse health effects would occur. The risk of increased incidence of cancer from exposure to low-level radiation is estimated by application of a risk factor to either the radiation dose or the radionuclide intake. Regardless of the type of risk factor used, the same basic uncertainties remain. These uncertainties are related to the model used for determining the health effects of radiation exposure. The model most frequently used for determining risk of radiation exposure is the linear nonthreshold model which assumes there is some increased risk for any increment of radiation exposure with no threshold below which effects are not seen. This is the most conservative model for evaluating radiation risk; it uses data from high-dose radiation exposures (such as from the survivors of the atomic bomb) and extrapolates risk from these high exposures to the lowlevel environment or occupational dose range. The current EPA-recommended radiation risk factors are based on the 1980 National Academy of Sciences Biological Effects of Ionizing Radiation Committee (BEIR III) report. The BEIR III recommendations were increased slightly by EPA to reflect recent information on the health effects of exposure to ionizing radiation. In early 1990, the National Academy of Sciences published the results of the most recent studies of the health effects of ionizing radiation, the BEIR V report, which increases the estimates of cancer risk by a factor of 3 to 5 over the BEIR III report. These increases are based primarily on a re-evaluation of the doses received by the atomic bomb victims. 


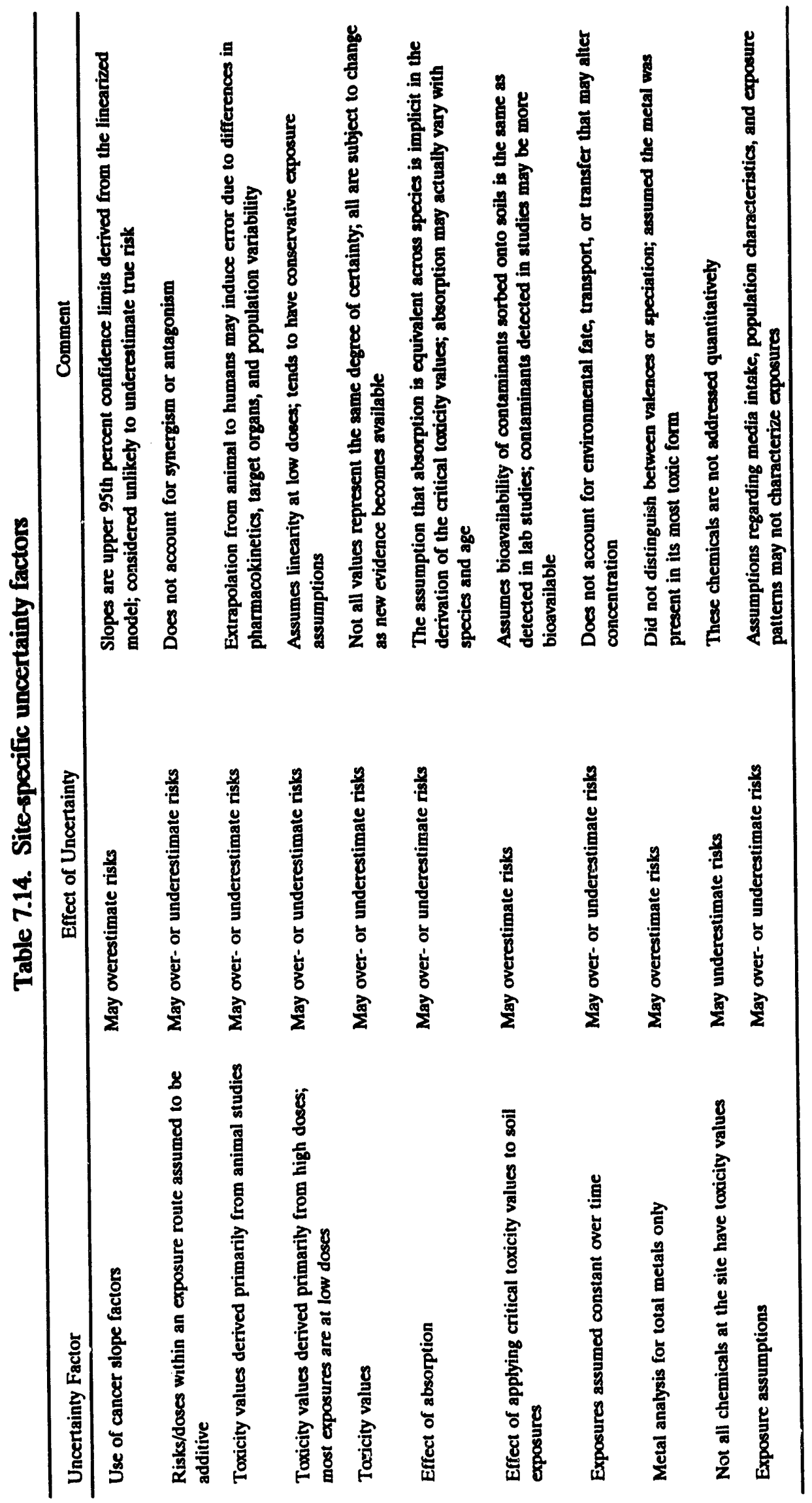




\subsection{PERSPECTIVE}

In order to put the results from the BSCP risk evaluation into perspective, one should consider the probability of an individual's developing cancer from unavoidable exposure to naturally occurring background radiation in general. In the Background Information Document for the Environmental Impact Statement for NESHAPS Radionuclides (EPA 1989t), EPA evaluated risks from exposure to average nationwide levels of background radiation. The risk of fatal cancer for the U.S. population exposed to low-LET radiation over a lifetime (70.7 years) was estimated to be $2.4 \times 10^{-3}$, which accounts for approximately $1.5 \%$ of U.S. cancer deaths. The average lifetime cancer risk for high-LET radiation exposure is estimated to be $6.5 \times 10^{-3}$ and accounts for approximately $4 \%$ of all U.S. cancer deaths. The total risk of fatal cancer because of background radiation was approximately $8.9 \times 10^{-3}$. From EPA's risk factors for low-LET radiation, the ratio of cancer incidence to fatal cancers was determined to be 1.6. Therefore, the lifetime risk of cancer incidence in the general population is approximately $1.4 \times 10^{-2}$ (see Fig. 7.1), which is approximately 100 times greater than the upper bound $\left(1.0 \times 10^{-4}\right)$ of EPA's range of concern and above the levels registered in the vicinity of the ORR in this study.

To understand the background risk information presented in this report, it is important to discern between adverse health effects resulting from unavoidable versus avoidable exposure. The risk of cancer presented in the previous discussion, approximately $1.4 \times 10^{-2}$, is the result of the unavoidable exposure to natural radiation sources; that is, a risk that we are all subject to because we live on the surface of the planet Earth. The majority of the risks modeled from the exposure to background soil constituents discussed in this section are a subset of the unavoidable risk associated with exposure to natural radiation sources. The EPA has determined that risk from exposure to hazardous waste sites are avoidable sources of exposure. The risk resulting from exposure to such sources is referred to as incremental or excess cancer risk because it is a cancer risk in addition to that which is unavoidable. Therefore, to be protective of human health, the $1 \times 10^{-4}$ threshold for excess cancer risk was selected to aid risk managers in the evaluation of preventable risks associated with CERCLA sites.

It should be clear that an essential objective for all RIs is to differentiate between risks that are unavoidable (background) and avoidable risks associated with site contamination. To clarify, if unavoidable background risks from exposure to soil on the ORR $\left(6 \times 10^{-4}\right)$ are not separated from risks resulting from exposure to site contamination, the risk will always be in the EPA's unacceptable range. The information presented in this document should be used to make this differentiation and ensure that risk management decisions are based on excess cancer risk. 


\section{ASSESSMENT OF OVERALL DATA QUALTTY OBJECTIVES}

\subsection{SUMMARY}

Phase I activities established both field and laboratory data quality objectives at the project planning stage. The project plan (Energy Systems 1992) discusses training, audits and surveillances, and data management, as well as the establishment of PARCC (precision, accuracy, representativeness, completeness, and comparability) parameters for evaluating the field and analytical data.

Field sampling training was able to reduce possible variability related to personnel changes. The sampling procedure was designed to effectirely reduce the possibility of cross-contamination throughout sampling activities. Audits and surveillances contributed to improving procedures and practices. Data management activities ensured the organization, consistency, traceability, integrity, and security of the data sets generated.

Representative sampling sites were selected by evaluating soil morphology and vegetation and by testing volatile organic compounds (VOCs) and fallout activity. Overall quality of site selection is satisfactory, but a few off-site (AND and ROA) locations had excess loss of surface soils due to erosion. Soil erosion is one of the contributing factors to lower average cesium-137 values of off-site locations in comparison to ORR sites. Except for trip blanks, laboratory source waters were used only for washing sampling equipment. Therefore, quality of deionized water was a minor issue.

Analytical data quality was determined by analyzing (1) laboratory blanks to assess contamination levels in the analytical process; (2) laboratory control samples to assess analytical method bias, precision, and comparability; (3) matrix spikes to assess bias of the method for the matrix, as well as precision of the method when performed in duplicate; and (4) duplicates to assess precision of the sampling process and/or the analytical methods.

During the laboratory review process and the independent validation process, the data were evaluated and qualified as discussed in Sects. 4.3 and 4.4, respectively. The majority of the data were usable. Among the organics, PAH was only $74 \%$ usable. Among the radionuclides, $4 \%$ of neptunium-237, $62 \%$ of strontium- 90 , and $0 \%$ of technetium- 99 were usable. The reasons of rejection are discussed in Sect. 8.5.9.2. Lists of sample numbers belonging to each sample delivery group and sample numbers related to sites, horizons, formations, and analyses are presented in Appendixes F and G, respectively.

\subsection{INTRODUCTION}

The purpose of this section is to present and assess the results of field sampling and analytical laboratory quality assurance (QA) and quality control (QC) activities for Phase I of the BSCP. These QAVQC results are presented to illustrate that the data collected during Phase I are of sufficient quality to meet project objectives. The quality assurance program was designed to meet the requirements of QAMS-005/80 (EPA 1980a), ASME NQA-1 (ASME 1989), and with the Environmental Restoraticn Division Quality Assurance Program Plan 
(ES/ER/TM-4/R1). The quality assurance objectives were defined in the Project Plan (ES/ER/TM-26/R1).

\subsection{DATA QUALITY OBJECTIVES FOR FIELD MEASUREIMENT DATA}

The field QAVQC objectives for BSCP Phase I data follow.

1. Data generated would withstand scientific scrutiny.

2. Data would be gathered using appropriate procedures for site selection, field sampling, chain of custody, laboratory analyses, and data reporting.

3. Data could be used elsewhere on the Oak Ridge Reservation (ORR) for comparison of similar residuum soils, or fill from soils from the same geologic formation.

The specific QA objective for all data collected during Phase I was, therefore, to obtain precise and accurate measurements consistent with the intended use of the data and within the limitations of the very few number of samples plus errors introduced or inherent in the sampling and analytical procedures used.

These objectives were met through the development and implementation of (1) a QA oversight program of audits and surveillances, (2) standard operating procedures accompanied by a training program, (3) field sampling QC requirements, and (4) data and records management systems.

\subsection{DATA QUALTY OBJECTIVES FOR LABORATORY MEASUREMENT DATA}

The laboratory QA/QC objectives for BSCP Phase I data follow.

1. Laboratory data generated would withstand scientific scrutiny and be subject to data validation procedures.

2. Data would be generated using appropriate procedures for chain of custody, laboratory analyses, and data reporting.

3. Data would be complete and of known precision and accuracy and will be technically defensible and legally admissible.

These objectives were met through the development of a detailed Analytical Statement of Work to ensure that the laboratories involved understood the requirements of the analytical QC program. Also, the laboratories were to follow approved EPA procedures for their chemical analyses and HASL-300 (AEC 1972) for radiochemical analyses to ensure that the data generated were from widely accepted methods. Finally, these objectives were met through an extensive data validation process which evaluated the CLP data packages for their technical and legal integrity. 


\subsection{ASSESSMENT OF COMPLIANCE WITH DATA QUALTTY OBJECTIVES}

\subsubsection{Audits and Surveillances}

Audits and surveillances were performed by DOE Oak Ridge Field Office, Martin Marietta Energy Systems, Inc., and others who reviewed and evaluated the adequacy of field and laboratory performance and ascertained whether the QA/AC Plan was adequately and uniformly implemented. Results of the audits and surveillances were documented and reported to management.

The following field surveillances were conducted for Phase I field quality control:

June 12, 1992: $\quad$ Energy Systems Surveillance Report JS-BSCP-92-01

February 25, 1993: $\quad$ DOE Oak Ridge EQA-92-12-10

February 25, 1993: $\quad$ Energy Systems QA Report JS-BSCP-93-01, Phase I Field Data Validation

Corrective actions were taken after the reports were received.

The following analytical laboratory surveillances were conducted for Phase I analytical quality control:

September 2, 1992: $\quad$ Environmental Restoration Surveillance Report 92ERTI-9, Data Validation Methods

October 22-23, 1992: $\quad$ Environmental Restoration Surveillance Report 92ERTI-10, Data Validation Status

October 27, 1992: $\quad$ Environmental Restoration Surveillance Report 92ERTI-11, Surveillance of Lockheed Analytical Laboratory in Las Vegas, Nevada

Written responses and corrective actions were taken after the reports were received.

\subsubsection{Data Quality Indicators for Field Measurement Data}

Both qualitative and quantitative criteria are used as indicators of the quality of the field data. In determining whether the data are usable, especially in the decision process, the integrity and authenticity of the data must be evaluated, and the analytical uncertainty must be known. Field indicators generally used to qualitatively assess the data quality are representativeness, comparability, completeness, sensitivity, and whether the data are reasonable in terms of soil morphology, conceptual models of soil genesis, general soil forming processes, and site location criteria specific for each site.

Analysis of field duplicates provides an assessment of the small-scale natural variability of soil samples. Soil preparation laboratory (SPL) duplicates of composited samples provide for some assessment of analytical laboratory variability, the variability introduced by the SPL compositing method, and also natural soil variability. Other quantitative measures of field quality control include proper sample preservation, use of field and source water blanks, equipment rinsates, and suitable precleaned containers. 


\subsubsection{Data Quality Indicators for Analytical Laboratory Measurement and Soil Preparation Laboratory Data}

Five qualitative and quantitative parameters are used as data quality indicators. The review of data according to these parameters and the validation of the field and analytical program are used to determine the usability of the data generated. The data quality indicators to be used are precision, atcuracy, representativeness, comparability, and completeness. Precision and accuracy are quantitative characteristics, whereas representativeness, comparability, and completeness are qualitative characteristics for evaluating the field and analytical performance.

\subsection{Precision}

Precision is the measure of the reproducibility of measurements under a given set of conditions. It is a quantitative measure of the variability of a group of measurements compared to their average values. Precision is usually stated in terms of standard deviation (s) and relative percent difference (RPD). The overall precision of measurement data is a mixture of field sampling and laboratory analytical factors. Analytical precision is much easier to control and quantify than sampling precision. The historical data available to assess method performance are dependent upon the samples received in the laboratory, while sampling precision is unique to each site. Sampling precision in Phase I was determined by collecting and analyzing field duplicate samples. The results from these measurements provide data on the overall measurement. Analytical precision was determined by the measurement of laboratory replicates. The measurement of the sampling precision is determined by subtracting the analytical precision from the overall measurement precision.

\subsection{Accuracy}

Accuracy is a measure of the bias in a measurement system. It is difficult to measure for the entire data collection activity. Sources of error are the sampling process, field contamination, preservation, handling, sample matrix, sample preparation, and analysis techniques. The sampling accuracy can be assessed by evaluating the results of field blanks, while the analytical accuracy can be assessed through the use of matrix spike and laboratory control samples.

\subsubsection{Representativeness}

Representativeness is a qualitative parameter that is most concerned with the proper design of the sampling program. It is an expression of how accurately and precisely the data represent a characteristic of a population, the parameter variability at a sampling point, or an environmental condition. Representativeness can be assessed by the use of collocated samples. Collocated samples are by definition samples collected such that the samples are equally representative of a given point in space and time. This will provide both precision and representativeness information.

\subsubsection{Completeness}

Completeness is defined as the percentage of measurements made which are judged to be valid measurements. The completeness goal of a project is satisfied if a sufficient amount 
of valid data is generated for its intended use. The completeness of the project is assessed by determining the number of measurements judged to be valid from the data validation and evaluation process.

\subsubsection{Comparability}

Comparability is a qualitative parameter expressing the confidence with which one data set can be compared against another data set. The sample data should be comparable with other measurement data for similar samples and sample conditions. Comparability is assessed by determining whether the standard techniques (field and analytical) stated in the plan are used and that the analytical results are reported in the appropriate units. Data sets can only be compared with confidence when the precision and accuracy are known.

\subsection{Training of Field and Soil Preparation Laboratory Personnel}

The BSCP training program includes actual training in BSCP procedures. Training is completed as required in the appropriate standard operating procedure (SOP), and training records are maintained by the appropriate coordinator for the BSCP. Generally, the extent of field/laboratory training is commensurate with the scope, complexity, and nature of the activity along with the educational experience and proficiency of the person being trained.

QC measures for field locations, including selection of sampling locations, field data recording, and sample collection, were implemented to meet the Phase I project objectives. Sampling sites on the ORR were the responsibility of the ORR sampling team leader, while sites in Anderson and Roane counties were the responsibility of the University of Tennessee sampling team leader. Discussion of each site is presented in Sect. 3 and Appendix A. Methods for field activities, including record keeping, sample identification, maintenance of sample custody, and laboratory instrument calibration were specified in SOPs.

\subsubsection{Field Data and Records Management}

Field data management activities ensured the organization, consistency, traceability, integrity, and security of the data sets generated during Phase I to enable the project to meet its objectives. A unique identification code was assigned to each sample to ensure internal consistency and compatibility. Sufficient information was recorded at each sampling site to ensure that data were traceable to the sampling task: the location, sample identification, sample depth, and sampling date. The chain-of-custody form listed the laboratory destination.

Records generated by the program that are required to (1) provide a complete and accurate history of sample collection, analysis, and data reporting; (2) document conduct of project business; and (3) support any future legal or administrative actions that may be taken are retained in the project files. Similarly, records that furnish documentation or evidence of. quality (e.g, project plans and results of QA oversight activities) were designated QA records and added to the project files.

Records identified for Phase I sampling and analysis activities included project plans and approvals, field and laboratory notebooks, chain-of-custody and request-for-analysis forms, and instrument listings for gamma screening spectroscopy. 
All field activities followed standard record keeping and chain-of-custody procedures. These included recording site-specific information in bound notebooks, with routine reviews of the notebooks. Notebooks for ORR activities were divided into field notebooks, in which all field activities were recorded, and lab notebooks where all laboratory activities were recorded. Sample custody was established by the sampling team upon collection, through the use of standard chain-of-custody forms, and maintained throughout sample processing and delivery to the shipper for transport to the analytical service laboratories. Phase I field QAVC procedures included field duplicates, equipment-cleaning rinse samples, and VOC trip water blanks. Specific field QC activities are discussed in Sect. 3.

\subsubsection{Field Quality Program}

\subsubsection{Selection of sampling sites}

Representative sampling sites were selected that were not disturbed by recent activities, including ORR facility activities since 1942 and off-site activities, such as farming operations or recreational uses, that resulted in surface soil disturbance.

A brief description of each site is presented in Appendix A. All sites met the minimum qualifications specified in Sect. 3. Anderson County and Roane County sites had a more varied land use history for the past 50 years than the ORR sites. Some of the off-reservation sites were still being used for cattle pasture. This shows up in the gamma scanning results, which have a much wider range than for the ORR sites, which were, for the most part, abandoned 50 years ago, although there had been some logging on some of the ORR sites.

Any sign of recent (in the past 40 to 50 years) land disturbance, the presence of man-made organic compounds, or the presence of radionuclides above global fallout levels would immediately result in a site being rejected. Potential sites were initially chosen on the basis of the lack of any recent land disturbance which, for most sites, was the presence of old-field successional forest. Nearly all of the sites had been cultivated and severely eroded prior to being abandoned or planted in pines on the ORR or allowed to revert back to forest on private lands.

\subsubsection{Collection of samples}

Representative samples were collected and transferred to temporary refrigerator storage in the SPL (Room 375), to the Y-12 Plant analytical laboratory, or to the ORNL shipping department for transfer to off-site analytical laboratories.

All VOC, organic, and tritium soil samples from A horizons were immediately preserved on ice or within 15 minutes after being sarupled. Samples that were collected for compositing were usually not preserved until after they had been partially dried, sieved, mixed, and put into suitable bottles. From that time, composited samples were preserved at $4^{\circ} \mathrm{C} \pm 4^{\circ}$. Observations that were made during Phast: II activities indicated that an ice chest and the refrigerator could generally maintain a temperature within 4 degrees of $4^{\circ} \mathrm{C}$. If a large number of warm samples was placed into the ice chest or in a refrigerator, the temperature might exceed $8^{\circ} \mathrm{C}$ for a short period of time. Temperatures were checked with a $\mathrm{max} / \mathrm{min}$ thermometer. Great efforts were made to ensure that representative samples were collected. On the ORR, the designated sampling team leader sampled all of the sites except for one 
absence between April 20 and 23. All off-ORR sites were sampled by or activities were monitored by the University of Tennessee sampling team leader.

\subsubsection{Handling of samples}

Efforts were made to prevent cross-contamination at any site and between sites and to maintain a complete chain of custody and detailed records of all field and laboratory compositing activities.

All pit digging equipment was thoroughly cleaned before going from one site to another. All of the SPL cleaned stainless steel sampling equipment that was to be used at one site was given a field rinse at the truck, rewrapped in aluminum foil, and carried to the site. One sampling piece was used for each soil horizon and then placed into a container for used equipment. All dirty equipment was cleaned, rinsed, and wrapped in aluminum foil in the SPL after the day's sampling had been done. The field logbook was where site and sample descriptions were first recorded and where a unique sample number was assigned. From the field logbook, all container labels were filled out and then placed on the sample jar. Field chain-of-custody forms were also filled out from the field logbook. Laboratory chain-of-custody forms were filled out in the SPL for samples to be sent to analytical laboratories. Two field logbooks were used for Phase I field activities. All activities that were done in the SPL were recorded in the BSCP Phase I laboratory notebook.

Field and SPL quality levels ranged from DQ Level II to DQ Level IV. However, in practice, DQ Level IV was adhered to throughout all field sampling activities, including screening samples for VOCs where samples were placed into precleaned glass containers. SPL work with ESD composite soil samples was done under DQ Level IV.

Field quality control procedures are listed in Sects. 6.6.1.3 to 6.6.1.9 in ES/ER/TM-26/R1 (Energy Systems 1992). The following discussion covers the procedures that were followed in collecting samples. Prior to going to the field, all stainless steel sampling equipment was thoroughly washed in the SPL with soap and water followed by a prescribed number of distilled water rinses. After the final rinse the wet equipment was immediately wrapped with one or more thicknesses of aluminum foil. The sampling equipment was taken to the field in the back of a pickup truck. At or near the site, the sampling equipment was unwrapped and given a field rinse, then immediately rewrapped until it was used. Some sites were located a considerable distance from the closest point of access. Here the rinsing was done at the truck, the field-rinsed equipment wrapped in aluminum foil, placed into a backpack, and carried to the site. A small pit was dug with a steel shovel deep enough to place the sample jar below the soil horizon that was to be sampled. A sampling tool was unwrapped and used to remove soil from the pit face directly into the jar. At no time were fingers used to place a soil sample into a precleaned glass sample container. Soil that was pushed by the sampling tool beyond the mouth of the jar was discarded. Placing soil into the ESD gamma poly containers was the only exception to this rule. Placing the entire volume of soil into the gamma poly container required that the soil be packed into the lower restricted space with either the fingers or a freshly cut stick of a convenient diameter. After each soil horizon was sampled, a new sample tool was used to collect samples from the next soil horizon. All used stainless steel sampling tools were returned to the laboratory for standard cleaning, rinsing, and aluminum foil wrapping. Stainless steel sampling equipment was not given an acid rinse because of potential pitting and etching problems, nor was it given a solvent rinse as it would have been necessary 
to do this in a radiation-contaminated hood. Shovels that were used to open and fill pits were thoroughly cleaned between sites to prevent any cross-contamination. In addition, soil removed from pits was placed outside the 3 - by 3 -meter sample area.

Each sample was given its own identification number in the field. This number and the description of each sample were first recorded in the field logbook. From the field logbook, sample container labels were filled out and placed on glass jars after the jar was filled. Each sample that was logged into the field logbook was then transcribed onto a field chain-of-custody form, which was signed by all personnel involved in the sampling operation.

ESD SPL operations for Phase I consisted of placing soil samples in a refrigerator, compositing operations, preparing laboratory chain-of-custody forms, packing samples into ice chests, and taking them to shipping. In the latter part of Phase I activities, preparation of laboratory chain-of-custody forms, new container labels, packing, and shipping were done by APO personnel, according to Procedure BSCP-SOP-02, Rev. 0.

Compositing samples resulted in the destruction of individual samples obtained within a given horizon and the creation of new composited samples. All of these activities were recorded in the ESD soils laboratory logbook. New sample numbers were first recorded in the laboratory logbook, then transcribed onto container labels and the appropriate chain-ofcustody form.

No field variances (Sect. 6.6.1.9 of the BSCP Plan, Energy Systems 1992) were needed and none were made in any Phase I activities.

\subsubsection{Field Data Validation}

As part of the QA/QC effort to satisfy the data quality objectives of the project, validation of the project field data is vital to ensure that the field data set is complete with respect to procedure ESP-500, ESH/Sub/87-21706/1, as specified in the project plan. A validation worksheet listing the ESP-500 elements was prepared for each site sampled in Phase I of the project, and the elements were checked off as they were found. The results of the validation effort revealed that project field records are essentially complete but were distributed among several sources, so that there was need for a general index of records and their contents. This activity identified a lack of complete records on sample preservation and a lack of land owner contact information for sites off the ORR. These areas were addressed by project staff.

\subsubsection{Field Quality Control Assessment}

Samples and data collected to evaluate QC for the Phase I field and laboratory activities were outlined in ES/ER/TM-26/R1 (Energy Systems 1992). The frequency and type of QC samples collected were predetermined in the sampling plan based primarily on cost limitations. The specific QA objectives for all data were to obtain reproducible, precise, and accurate measurements consistent with the intended use of the data and within the limitations of the number of samples, sampling methodology, and analytical procedures used. For this report, field QC includes actions ranging from site selection to sample receipt by the shipper. This includes but is not limited to sample collection, custody, processing, preservation, 
cross-contamination, and field record keeping. Each of these actions is discussed herein as appropriate.

Site and sample representativeness is an assessment of how well environmental conditions are represented by the sites sampled and whether contamination of samples occurred between collection and analysis. Representativeness is evaluated relative to field activities through review of site selection rationale, frequency of sampling individual sites, and selection of analytical parameters to be characterized.

Comparability for field activities is the confidence with which data collected at different times from the same site may be compared. Objectives for comparability between samples are met by (1) narrowly defined sampling methodologies, (2) site surveillance and use of standard sampling devices and monitoring devices, (3) training of personnel, and (4) documentation of sampling locations.

Cross-contamination is a possible problem during field sampling and composite sample preparation. To minimize such a possibility, this project practiced the following procedures: (1) each sample container was precleaned and had a certified rinsate water analysis;

(2) sampling equipment was used only once and rinsed on site before sampling; (3) contact with distilled rinse water and stainless steel was a possible source of contamination, but the possible influence to data quality was negligible (see water analysis results); (4) the soil sampling procedure was designed to effectively reduce possible cross-contamination among samples from different horizons within a soil profile; and (5) laboratory analytical procedures were developed to ensure no cross-contamination among the soil samples.

\subsubsection{Soil}

All samples were collected in accordance with ES/ER/TM-26/R1 (Energy Systems 1992) regarding sample collection procedures, sampling devices, sample container compatibility, preservation, custody, and preanalytical SPL processing. Soil variability was evaluated in Phase I through the collection of field duplicates.

The major purpose for obtaining field duplicates was to assess small-scale soil variability. Field duplicate samples for VOA and organic analysis were sampled in two corners of the sampling square, or about 3 meters apart. If the primary sample, for example, contained a VOC and the field duplicate did not, then the primary sample with the VOC was rejected. Field duplicate samples for compositing purposes were collected from different faces of the soil pit, or from opposite ends of the primary sampling face, or a distance between 100 to $120 \mathrm{~cm}$. Data from composited samples allowed for an initial look at SPL variability in compositing and whether or not the analytical laboratory made an error. At least one notable laboratory error was found when comparing primary and duplicate data for the A horizon (ORR 5028 and ORR 5037) where the problem occurred and the B and C horizons (ORR 5031, 5034, 5040, 5043) which had very comparable values. The Phase I field duplicates were not intended to entirely assess analytical laboratory variability. A laboratory reference soil was intended to assess analytical laboratory variability, but not enough reference soil samples were sent. 


\subsubsection{Water}

Field QC samples were treated identically to primary samples in terms of sample identification, custody, request for analytical services, and data processing. Results from QC samples were not used to adjust the results obtained for primary or duplicate soil samples. QC samples included trip water blanks, rinse water collected from field sampling equipment, and source water used to rinse sampling equipment in the laboratory and in the field.

Trip blank A sealed container of organic-free water was used to identify contamination contributed to soil samples during transport from the field to the $Y-12$ anaiytical lab. Trip blanks were transported to and from the field in the same manner and preserved in the same manner as primary samples. Information from trip blanks is relevant to the interpretation of VOCs in soil samples and in VOC field rinsates.

Rinse water. Field rinse water is obtained by the rinsing of sample collection tools after arriving as close as possible to the sampling site. Analysis and comparison of the rinsate with the source water determine if the cleaning procedures are adequate to avoid carry-over of contamination from one site to another.

Comparison results from all rinse water samples from field and laboratory equipment cleaning operations with source water are presented in Tables 8.1 and 8.2. Sample identification numbers and analytes for which there were no differences are not presented.

Table 8.1. Comparison of rinse water and source water for metals on the ORR

(Units are micrograms per liter.)

\begin{tabular}{lcc}
\hline Element & $\begin{array}{c}\text { Source water, } \\
\text { sample 1465 }\end{array}$ & $\begin{array}{c}\text { Rinse water, } \\
\text { sample 1459 }\end{array}$ \\
\hline $\mathrm{Al}$ & $18.0 \mathrm{U}$ & $54.6 \mathrm{~B}$ \\
$\mathrm{Fe}$ & $5.0 \mathrm{U}$ & $65.5 \mathrm{~B}$ \\
$\mathrm{Mn}$ & $1.0 \mathrm{U}$ & $3.7 \mathrm{~B}$ \\
$\mathrm{Zn}$ & $11.6 \mathrm{~B}$ & $6.1 \mathrm{~B}$ \\
$\mathrm{Si}$ & $25.0 \mathrm{U}$ & $58.4 \mathrm{~B}$ \\
$\mathrm{Sr}$ & $1.4 \mathrm{~B}$ & 5.1 \\
$\mathrm{Cu}$ & $3.7 \mathrm{~B}$ & $7.9 \mathrm{~B}$ \\
\hline
\end{tabular}

$\mathrm{B}=$ estimated between IDL and CRDL;

$\mathrm{U}=$ nondetect. 
Table 8.2 Comparison of source water and rinse water for Anderson and Roane counties

(Units are micrograms per liter.)

\begin{tabular}{lllll}
\hline \multirow{2}{*}{ Element } & \multicolumn{2}{c}{ Anderson County } & \multicolumn{2}{c}{ Roane County } \\
\cline { 2 - 5 } & $\begin{array}{l}\text { Source water, } \\
\text { sample 2177 }\end{array}$ & $\begin{array}{l}\text { Rinse water, } \\
\text { sample } 2174\end{array}$ & $\begin{array}{l}\text { Source water, } \\
\text { sample } 2177\end{array}$ & $\begin{array}{l}\text { Rinse water, } \\
\text { sample 3144 }\end{array}$ \\
\hline Antimony & & & $2.0 \mathrm{U}$ & $1.0 \mathrm{U}$ \\
Arsenic & & $2.0 \mathrm{U}$ & $10.0 \mathrm{U}$ \\
Chromium & & $2.0 \mathrm{U}$ & $2.8 \mathrm{~B}$ \\
Lead & & $1.8 \mathrm{U}$ & $1.0 \mathrm{U}$ \\
Manganese & & $8.0 \mathrm{U}$ & $7.0 \mathrm{U}$ \\
Mercury & & $0.2 \mathrm{U}$ & $0.08 \mathrm{~B}$ \\
Selenium & & & $2.0 \mathrm{U}$ & $5.0 \mathrm{U}$ \\
Silicon & 419.0 & 398.0 & 419.0 & 95.5 \\
Zinc & $6.0 \mathrm{~B}$ & $5.4 \mathrm{~B}$ & $6.0 \mathrm{U}$ & $5.0 \mathrm{U}$ \\
Boron & 209.0 & 170.0 & $170.0 \mathrm{U}$ & $42.0 \mathrm{U}$ \\
Lithium & & & $24.0 \mathrm{U}$ & $9.0 \mathrm{U}$ \\
Osmium & & $32.0 \mathrm{U}$ & $64.0 \mathrm{U}$ \\
Sulfate & & & $\mathrm{NA}$ & 1230.0 \\
Cyanide & & & NA & $4.0 \mathrm{U}$ \\
\hline & & & & \\
\hline
\end{tabular}

$\mathrm{B}=$ estimated; $\mathrm{U}=$ nondetect; no qualifier letter is a detect.

All of these values, except for strontium rinse water, are either below detection limits (U) or are estimates (B). The rest of the data are the same for both samples. The data indicate (1) the variability in laboratory detection limits from day to day and (2) the ORR field rinse water was removing ions from the stainless steel field sampling equipment. The comparison of the ORR source and metals field rinses indicates that the rinse water has increased amounts of iron, manganese, and aluminum. The increase in silicon is probably from water storage in 1-gallon glass jugs carried to the field.

No tritium was detected in any of the field rinses or source water samples. Europium-155 and potassium- 40 were detected in the radionuclides ORR rinse water but were not detected in the ORR source water. No organics were detected in the ORR field rinse water samples. Trip blanks and field rinse water samples for the ORR VOC analysis were estimated to contain (J qualifier) acetone and 2-butanone. Comparisons of rinse water with source water 
do not indicate any sampling contamination problems; rather, most of the listed differences are the result of laboratory contamination or instrument calibration lower limits of detection.

Most of these values are either below detection limits or are estimates. The rest of the data are the same for both samples and are not listed. Of interest is that some numbers are higher in the source than in the rinse water. This is most likely due to instrument variability and sensitivity from day to day.

Trip blanks and field rinse water samples for Anderson County VOC analysis showed the following estimates $\mathrm{J}$ or detects:

\begin{tabular}{|c|c|}
\hline Sample & Compound \\
\hline 2175 & acetone J Rinse water \\
\hline $\begin{array}{l}2178 \\
2037\end{array}$ & $\begin{array}{l}\text { acetone J Source water } \\
\text { acetone J Rinse water }\end{array}$ \\
\hline
\end{tabular}

Chloroform was detected $(14 \mu \mathrm{g} / \mathrm{L})$ in sample 2024. This is a trip blank. This is most likely laboratory contamination since no associated soil samples contained this compound.

The source water for Anderson County organic analyses did not contain any detects. The field rinse water did not contain any organic detects.

Some of the Roane County trip blanks and rinse water samples for VOC analysis contained estimated $\mathrm{J}$ detects of acetone and 2-butanone. These are considered to be due to instrument contamination. Of note was the presence of carbon disulfide and 1,2-dichloropropane in the following VOC trip blanks: ROA [3042, 3043], [3068, 3069], and $[3095,3096]$. Each of these is a pair. The accompanying VOC soil samples for each trip blank pair were all resampled because of contamination from sealing the sample lid with a particular brand of masking tape used by the University of Tennessee sampling crews. This practice was promptly discontinued upon discovery of the problem.

The Anderson County rinse water for radionuclide analysis contained potassium-40, but Roane County rinse water for radionuclides did not contain any detects for tritium. Neither the Anderson nor Roane source water sample contained any rad detects.

\subsection{Analytical Data Quality Assessment}

The laboratory QC program was designed to ensure that all data generated and reported are scientifically valid, consistent with accepted methods, and of known accuracy. All inorganics and organics were analyzed by Lockheed Analytical Laboratory (Las Vegas, Nevada), while all radiological samples were analyzed by EcoTek, LSI (Atlanta, Georgia). The laboratories analyzed the following QC samples:

- laboratory blanks to assess the contamination levels in the analytical process;

- laboratory control samples to assess method bias, precision, and comparability;

- matrix spikes to assess the bias of the method for the matrix, as well as the precision of the method when performed in duplicate; and

- field duplicates to assess precision of the sampling process and/or the analytical methods. 


\subsubsection{Analytical data validation}

Section 4.4 details the data validation program followed for the BSCP. The criteria for the BSCP were prepared specific to the methods defined for this project. The results of the data validation with respect to the methods used for this project are provided in Sect. 4.4. This section details the results of data validation with respect to formation characteristics and provides an overall assessment of the data. Two sets of data qualifiers were used for this project. During the laboratory review process, the data were qualified by the laboratory generating the data. These qualifiers are defined in Sect. 4.3. The data were also qualified during the data validation process. These qualifiers are defined in Sect. 4.4.

\subsubsection{Qualification of the data}

A total of 43 data packages were provided by the laboratories: 9 inorganic, 10 pesticide/PCB, 5 chlorinated herbicide, 10 polynuclear aromatic hydrocarbon, and 9 radiological. This section provides an overall summary of the $\mathrm{QC}$ problems found during the data validation process. The distribution of usable data by method is presented in Table 8.3.

Table 8.3. Distribution of data usability

\begin{tabular}{llc}
\hline \multicolumn{1}{c}{ Analysis type } & \multicolumn{1}{c}{ Method } & $\begin{array}{c}\text { Percent } \\
\text { Usable }\end{array}$ \\
\hline Organics & Pesticide/PCB & 100 \\
& Chlorinated herbicides & 95 \\
& Polynuclear aromatic hydrocarbons & 74 \\
Inorganics & Metals & 98 \\
& Cyanide & 100 \\
& Sulfate & 100 \\
& Gamma emitters & 100 \\
& Thorium isotopes & 100 \\
& Uranium isotopes & 100 \\
& Total uranium & 100 \\
& Neptunium-237 & 4 \\
& Plutonium isotopes & 95 \\
& Strontium-90 & 62 \\
& Technetium-99 & 0 \\
& Tritium & 86 \\
\hline
\end{tabular}

Includes estimated values. 
Pesticide/PCB. Ten data packages were provided for pesticide/PCB analysis. The sample delivery groups provided by the laboratory are listed here by number.
042260
0424260
0430260
0508260
0511260
0514260
0519260
0523260
0722260
0803260

The data were found to be $100 \%$ usable. The $\mathrm{QC}$ problems found during the data validation that qualified the data $\mathrm{J}$ or $\mathrm{UJ}$ follow:

- Two SDGs were extracted outside of holding times.

- Problems were observed in the GC/ECD instrument performance.

- There were calibration concerns, and surrogate recoveries were outside QC limits.

Chlorinated herbicides. Five data packages were provided by the laboratory for chlorinated herbicide analysis. The sample delivery groups issued by the laboratory are listed here by number.
042260
0424260
0430260
0508260
0511260

The data were found to be $95 \%$ usable. There were 12 samples analyzed for dalapon which were rejected because surrogate recoveries were below $10 \%$. Some data were qualified J or $\mathrm{UJ}$ because of calibration problems and surrogate recoveries that were outside of QC limits.

Polynuclear aromatic hydrocarbons. Ten data packages were provided by the laboratory for polynuclear aromatic hydrocarbon analysis. The sample delivery groups issued by the laboratory are listed here by number.

042260

0424260

0430260

0508260

0511260

0514260

0519260

0523260

0722260 
$072260 / 0723260$

The data were found to be $74 \%$ usable. The QC problems found during the data validation process that rejected the data were

- very poor surrogate recoveries, $<10 \%$;

- LCS and MS/MSD recoveries outside QC limits; and

- surrogate coelution problems.

In addition to the rejected data, the data were also qualified J/UJ and JN/UJN/RN. The data were qualified J/UJ because of missed holding times (1 SDG), coelution problems, calibration problems, laboratory blank contamination (2SDGs), surrogate recoveries and LCS recoveries outside QC limits. The data were qualified JN/UJN/RN because of problems with the laboratory's method of identifying peaks within the retention time windows.

Inorganics. Nine data packages were provided by the laboratory for the analysis of inorganic analytes. The sample delivery groups issued by the laboratory are listed here by number.

\author{
042260 \\ 0422260/0430260/0508260/0511260 for silicon only \\ 0430260 \\ 0508260 \\ 0511260 \\ 0514260 \\ 0519260 \\ 0727260/0728260/0729260 \\ $072260 / 0723260$
}

The data were found to be $98 \%$ usable for the metals and $100 \%$ usable for cyanide and sulfate. The rejected metals data were for osmium due to predigestion recoveries being outside QC limits. The data also had some analytes qualified as J or UJ. The reasons for this qualification were

- calibration problems,

- the cyanide middle standard or ICV was not properly distilled,

- laboratory blank contamination, and

- $\quad$ MS and analytical spike recoveries were outside QC limits.

Radiochemical analyses. The usability of radiochemical data generated in this project were broken down by method and/or isotope. The data for gamma, isotopic thorium, isotopic uranium, and total uranium were found to be $100 \%$ usable. The remaining isotopes had percent usability values ranging from 0 to $95 \%$. The data generated for technetium- 99 were not usable in this project because of the possibility of technetium loss during the muffle furnace step of the procedure. This problem is being actively investigated and will be discussed in the project final report. Isotopic neptunium was found to be only $4 \%$ usable due to calibration errors and blank spike and matrix spike calculation errors, which when corrected yielded recoveries outside QC limits. The isotopic plutonium data were found to have $5 \%$ of the data unusable due to the laboratory's use of an outdated tracer solution. 
Strontium-90 data were found to have $38 \%$ of the data qualified unusable because the laboratory did not provide self-absorption curves, which would provide information about how much activity was being absorbed by the sample relative to the activity reaching the detector. Also, $14 \%$ of the tritium data were found to be unusable because of very poor matrix spike recoveries. Specific actions taken with the laboratory and results will be discussed in the project final report.

Other isotopes may have been qualified as $\mathrm{J}$ or $\mathrm{UJ}$ for various reasons. Some of the reasons for this qualification were

- $\quad$ blank spike and matrix spike recoveries were outside QC limits;

- unable to determine chemical separation specificity;

- no daily instrument performance check;

- calibration problems;

- laboratory performance or method accuracy could not be determined; and

- unable to assess activity, error, and/or minimum detectable activities in samples.

\subsection{Data Gaps}

The occurrences of rejected analytical data for organics, inorganics, and radionuclides are tabulated in Appendix H. Most rejects are in PAHs and radionuclides. The rejected PAHs include a variety of compounds (as discussed in Sect. 4), whereas only one inorganic (osmium) was affected. Rejection of radionuclides can be ascribed to two isotopes: neptunium-237 and technetium-99. Specifics are discussed in Sects. 4.5.2 and 4.5.3.

These gaps in intended Phase I data availability are being addressed with continued evaluation and resampling/reanalysis following Phase II validation. Specifics are discussed in Sects. 4.1 and 9. 


\section{PLANNED PHASE II ACTIVITIES}

Remaining project activities for FY 1993 with respect to the approved, revised project schedule (Rev. 3) appearing in Fig. 9.1 include the following:

- The first half of Phase II field samples were obtained during FY 1992. The Copper Ridge Dolomite Formation was sampled both on-site and off-site at 36 locations (12 on the ORR, 12 in Roane County, and 12 in Anderson County).

- The second half of Phase II field operations was completed early in FY 1993 by obtaining soil samples at an additional 36 sites on the ORR (12 Chepultepec Dolomite sites in the Knox Group and 24 limestone sites in the Chickamauga Group) for a total of 72 sampling sites in Phase II. The 48 sampling sites from Phase I plus the 72 from Phase II make a total of 120 sites, as called for in Sects. 5.2 and 5.3 of the Project Plan (Energy Systems 1992), thereby completing the sampling of the three major soil groups (the Chickamauga, the Knox, and the Conasauga) in and around the ORR. These results will be reported in the Phase II data report and in the project final report.

- All ORR sampling sites will be accurately located with Global Positioning System equipment.

- Chemical and radiological analyses of Phase II soil samples will be completed, followed by validation of Phase II analytical data packages.

- After validation, processed data will be placed in required formats, checked for consistency, and input verified during data management activities.

- Project data will be transferred to and incorporated into the Oak Ridge Environmental Information System (OREIS) within 30 days after completion of data processing and verification activities.

- Neutron activation analysis (NAA) samples from Phase I were processed in a single batch with Phase II soil samples in ORNL's Analytical Chemistry Division laboratory to conserve equipment set-up time and sample preparation and to expedite logistics. NAA results for Phase I will be available at the same time as Phase II results and will be validated and presented in the Phase II data report.

- Inductively coupled plasma/mass spectroscopy data are also being provided by the commercial analytical laboratory concurrently with Phase II data. These data will also be validated and presented in the Phase II data report.

- Further evaluation to render more neptunium-237 data usable and resampling and reanalysis to obtain replacement technetium-99 data will be completed to dovetail with analysis, interpretation, and reporting activities for the project final report.

- Statistical analysis and interpretation of the data obtained in Phase II will be performed after validation and verification. Phase II data will be compiled and presented in the 
Phase II data report, due June 30, 1993. These data will be provided to the risk assessment group for study and analysis.

- Overall, integrated statistical analysis and data interpretation of all analytical results from both Phase I and Phase II will be completed, followed by integrated risk assessment study and production of the project final report. Project completion is scheduled for October 29, 1993. Specific schedule elements and corresponding dates appear in Fig. 9.1.

The above sequence of events refers to the project schedule originally presented in the Project Plan (Energy Systems 1992) that appears here as a revision (Rev. 3) in Fig. 9.1. The Phase I data report (referred to in the schedule) and the BSCP Annual Report deliverable are one and the same document. 


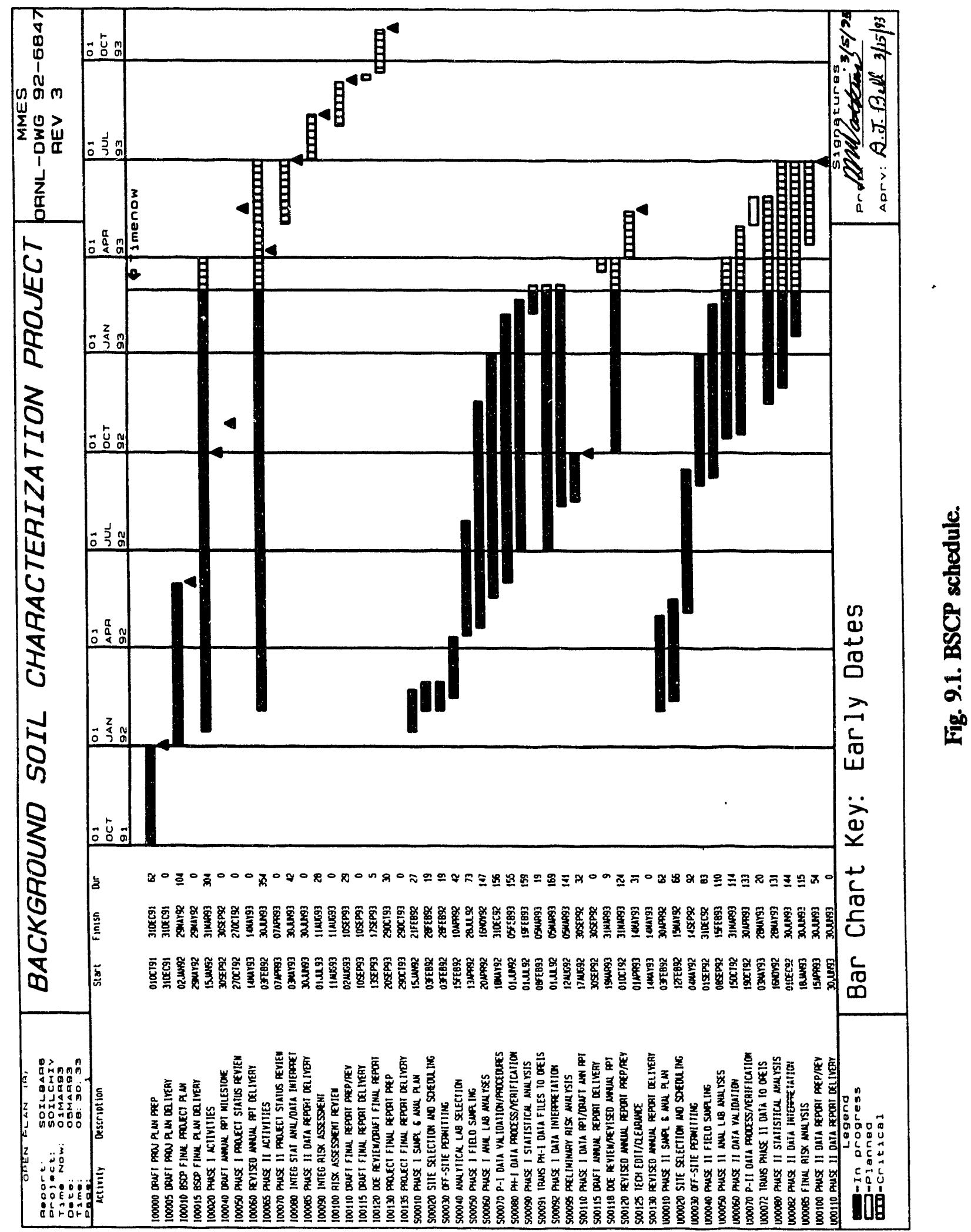




\section{REFERENCES}

AEC (U.S. Atomic Energy Commission). 1972. Health and Sajety Laboratory Procedures Manual. HASL-300. New York, NY.

ANSI/ASTM (American Society for Testing and Materials). 1980. Soil Investigation and Sampling by Auger Borings. ANSI ASTM D 1452-80. Philadelphia, PA.

ASME (American Society of Mechanical Engineers). 1989. Quality Assurance Program Requirements for Nuclear Facilities. ANSI/ASME NQA-1. New York, NY.

ATSDR (Agency for Toxic Substances and Disease Registry). 1988. Toxicological Profile for Chromium. U.S. Department of Health and Human Services, Public Health Service. Atlanta, GA.

ATSDR (Agency for Toxic Substances and Disease Registry). 1989. Draft Toxicological Profile for Plutonium. U.S. Department of Health and Human Services, Public Health Service. Atlanta, GA.

Budavari, S., M. J. O'Neill, A. Smith, P. E. Heckelman, ed. 1989. The Merck Index: An Encyclopedia of Chemicals, Drugs, and Biologicals. 11th 3e. Merck and Co., Inc. Rahway, NJ.

Casarett, A. P. 1968. Radiation Biology. Prentice-Hall, Inc. Englewood Cliffs, NJ.

Eckerman K. E. and M. W. Young. 1980. A Method for Calculating Residual Radioactivity Levels Following Decommissioning. INTEGRATED-0707. U. S. Nuclear Regulatory Commission. Office of Standards Development. Washington, D. C.

Energy Systems (Martin Marietta Energy Systems, Inc.). 1992. Project Plan for the Background Soil Characterization Project on the Oak Ridge Reservation, Oak Ridge, Tennessee. ES/ER/TM-26/R1. Environmental Restoration Division. Oak Ridge, Tennessee.

EPA (U. S. Environmental Protection Agency). 1980. Samplers and Sampling Procedures for Hazardous Waste Streams. EPA/600/2-80/018. Washington, D.C.

EPA (U. S. Environmental Protection Agency). 1980a. Interim Guidelines and Specifications for Preparing Quality Assurance Project Plans. QAMS-005/80. Office of Monitoring Systems and Quality Assurance, Office of Research and Development.

EPA (U. S. Environmental Protection Agency). 1983. Preparation of Soil Sampling Protocol, Techniques, and Strategies. PB83-206979. University of Nevada-Las Vegas. Las Vegas, Nevada.

EPA (U. S. Environmental Protection Agency). 1987a. A Compendium of Superfund Field Operations Methods. EPA/540/P-87/001. Washington, D.C. 
EPA (U. S. Environmental Protection Agency). 1987b. Data Quality Objectives for Remedial Response Activities. EPA/540/G-87/003. Washington, D. C.

EPA (U. S. Environmental Protection Agency). 1989a. RCRA Facility Investigation (RFI) Guidance. PB89-200299, (EPA 530/SW-89-031). Waste Management Division, Office of Solid Waste. Washington, D. C.

EPA (U. S. Environmental Protection Agency). 1989b. Federal Register (54 FR 48184). Washington, D. C.

EPA (U. S. Environmental Protection Agency). 1989c. Risk Assessment Guidance for Superfund Volume I: Human Health Evaluation Manual (Part A). EPA/540/1-89/002. Washington, D. C.

EPA (U. S. Environmental Protection Agency). 1990. Guidance for Data Useability in Risk Assessment. Interim Final, EPA/540/G-90/008, Directive 9285.7-05, Office of Emergency and Remedial Response.

EPA (U. S. Environmental Protection Agency). 1990a. Contract Laboratory Program Statement of Work for Organics. Las Vegas, Nevada.

EPA (U. S. Environmental Protection Agency). 1990b. Contract Laboratory Program Statement of Work for Inorganics. Las Vegas, Nevada.

EPA (U. S. Environmental Protection Agency). 1991a. Engineering Support Branch Standard Operating Procedures and Quality Assurance Manual. Region IV. Athens, GA.

EPA (U. S. Environmental Protection Agency). 1991b. Test Methods for Evaluating Solid Waste. SW-846, 3rd Edition. Washington, D. C.

EPA (U. S. Environmental Protection Agency). 1991c. Laboratory Data Validation Functional Guidelines for Evaluating Organic Analyses. Hazardous Site Control Division. Latest Edition. Alexandria, Virginia.

EPA (U. S. Environmental Protection Agency). 1991d. Laboratory Data Validation Functional Guidelines for Evaluating Inorganic Analyses. Hazardous Site Control Division. Latest Edition. Alexandria, Virginia.

EPA (U. S. Environmental Protection Agency). 1991e. Human Health Evaluation Manuah Supplement Guidance. "Standard Default Exposure Factors." OSWER Directive 9285.6-03. Office of Solid Waste and Emergency Response. Washington, D. C.

EPA (U. S. Environmental Protection Agency). 1992a. Integrated Risk Information System Database. Office of Research and Development. Washington, D.C.

EPA (U. S. Environmental Protection Agency). 1992b. Health Effects Assessment Summary Tables. Office of Research and Development and Office of Emergency and Remedial Response. OERR 9200.6-3303 (92-8). Washington, D.C. 
Glasstone, S. 1967. Sourcebook on Atomic Energy. Division of Technical Information, U.S. Atomic Energy Commission. D. Van Nostrand Co., Inc. NJ.

Hammonds, J. A, and F. O. Hoffman. 1992. Toxicity PRofiles for Radionuclides. SENES Oak Ridge Inc., Center for Risk Analysis. Oak Ridge, TN.

ICRP (International Commission on Radiological Protection). 1991. 1990 Recommendations of the International Commission on Radiological Protection. ICRP Publication No. 60. Pergamon Press, Inc. New York, NY.

Killough, G. G., and K F. Eckerman. 1983. "Internal Dosimetry." In Radiological Assessment, NUREG/CR-3332, ORNL-5966, pp. 7-1-7-98. Eds. J. E. Till and H. R. Meyer. United States Nuclear Regulatory Commission. Washington, D.C.

Kimbrough, C. W., L. W. Long, and L. W. McMahon (eds.). 1988. Environmental Surveillance Procedures Quality Control Program. ESH/Sub/87-21706/1. Martin Marietta Energy Systems, Inc., Oak Ridge, Tennessee.

Klaassen, C. D., M. O. Amdur, J. Doull, eds. 1986. Casarett and Doull's Toxicology: The Basic Science of Poisons. 3rd ed. Macmillan Publishing Co. New York, NY.

Lawless, J. F. 1982. Statistical Models and Methods for Lifetime Data. John Wiley \& Sons, New York, NY.

MMES (Martin Marietta Energy Systems, Inc.). 1993. Obiaining Access to Data in OREIS. ER Division Procedure ER/C-P2702, Rev. 0. Environmcntal Restoration Division. Oak Ridge, Tennessee.

NCRP (National Council on Radiation Protection). 1977. Environmental Radiation Measurements. NCRP Report No. 50. Washington, DC.

Owens, D. B. 1962. Handbook of Statistical Tables. Wesley Publishing Co.. Reading, Massachusetts.

Page, A. L., R. H. Miller and D. R. Keeney (eds). 1982. Methods of Soil Analysis, Part 2 -Chemical and Microbiological Properties. Agronomy Monograph No. 9, Part 2, 2nd ed. American Society of Agronomy, Soil Science Society of America. Madison, Wisconsin.

Rankama, K. and T. G. Sahama. 1950. Geochemistry. The University of Chicago Press.

Rupp, G. L. and R. R. Jones. 1991. Characterizing Heterogeneous Wastes: Methods and Recommendations. EPA 800/R-92/033. Harry Reid Center for Environmental Studies. University of Nevada-Las Vegas. Las Vegas, Nevada.

SAS. 1990. SAS/STAT Users Guide, Volume 2, GLM-Varcomp. SAS Institute, Inc. Cary, N.C. 
Sax, N. I. and R. J. Lewis, Sr. 1987. Hazardous Chemicals Desk Reference. Van Nostrand Reinhold Co., Inc. New York, NY.

Sehmel, G. A. 1984. Deposition and Resuspension in Atmospheric Science and Power Production. DOE/TIC-27601. Edited by D. R. Anderson. Technical Information Center Office of Scientific and Technical Information. U. S. Department of Energy. Oak Ridge, Tennessee.

Seiler, H. G., H. Sigel, and A. Sigel. 1988. Handbook on Toxicity of Inonganic Compounds. Marcel Dekker, Inc. New York, NY.

Wilks, S. S. 1962. Mathematical Statistics. John Wiley \& Sons. New York, NY. 
Appendix A

SITE DESCRIPTIONS, SOIL PROFILE DESCRIPTIONS, AND GENERAL ANALYSIS OF SITES 


\section{A.1 OAR RIDGE RESERVATION SITE DESCRIPTIONS}

ORR Site No. 2. This site is located off Bear Creek Road close to the Clinch River, grid location N29300, E10300. The actual site is located northeast of the K-25 water treatment plant. Follow the road to where the power line right of way (ROW) crosses it. Then turn right, go up hill for about $200 \mathrm{ft}$, then left into the woods about $50 \mathrm{ft}$. The site is located in a stand of large oak, red maple, sweet gum and white pine that was probably cleared and pastured at one time but evidently never plowed. The underlying geology is the Dismal Gap Formation. The soils are typical of the soils of largest extent that were mapped on this formation. The soils are very heterogeneous. The geologic dip in the $\mathrm{C}$ horizon varies from about 30 degrees to nearly vertical. Differential weathering of thin strata gives rise to very abrupt and irregular degrees of weathering with some strata being very highly weathered and other strata still hard and dense.

ORR Site No. 3. This site is located across Bear Creek Road from the Scientific Ecology Group plant, grid N 29300, E11200. The actual site is located about $50 \mathrm{ft}$ north of the cut bank on the north side of the road. The vegetation on this site consists of old-field successional forest that has not been disturbed since the field was abandoned. Most of the old pines have died and fallen over. The present regrowth consists of young pine,oak, hickory, sassafras, and cedar with a sparse ground cover of honeysuckle and poison ivy. The underlying geology is the Nolichucky Shale. Because this site is on a stable landform, the soils were not badly eroded before abandonment.

ORR Site No. 5. This site is located on the south side of Bear Creek Road about $300 \mathrm{ft}$ west of the main entrance to the Central Training Facility and about $25 \mathrm{ft}$ south of the security fence, grid N29300, E15300. The site is located in an old field that was severely eroded before or after abandonment. Shallow gullies are on either side of this site. Vegetation is old-field successional forest. Most of the old pines have died and fallen over. Present vegetation consists of a dense stand of young pines along with cedar, oak, dogwood, and sweet gum along with a generous stand of honeysuckle and greenbriar. This site is the only ORR site that has a thick organic layer consisting primarily of mosses. The soils on this site are very similar to those of ORR Site No. 3, except that they are more eroded with the plow layer resting directly on the clayey subsoil beneath. The underlying geology is the Nolichucky Shale.

ORR Site No. 10. This site is located about $300 \mathrm{ft}$ north of the Tennessee Valley Authority (TVA) power line ROW that parallels Bear Creek Road on the north side of the road, grid N30200, E21460. This site is located in an old field that had been severely eroded some time before abandonment. Vegetation is old-field successional forest that is now mostly hardwoods consisting of white oak, hickory cedar, and ash. The pine have disappeared. This site, being fairly open, has a dense ground cover of honeysuckle, Virginia creeper and poison ivy. The soils on this site are more typical of soils on steeper slopes. In fact, this site is the steepest of the ORR Phase 1 sites. The underlying geology is the lower Dismal Gap Formation, with strata in the $\mathrm{C}$ and $\mathrm{Cr}$ horizons nearly vertical.

ORR Site No. 11. This site is located about $50 \mathrm{ft}$ north of the TVA power line ROW that parallels Bear Creek Road on the north side of the road, ORR Site No. 10 is located about $250 \mathrm{ft}$ upslope. This site is located in an old field that had been severely eroded prior to or after abandonment. Vegetation is old-field successional forest. Many pines have died and 
fallen over. There is considerable young brush and sprouts of dogwood, sassafras, cherry, red maple, and sweet gum along with an abundant ground cover of poison ivy and honeysuckle. The soils are typical of stable uplands underlain by the upper Dismal Gap Formation.

ORR Site No. 13. This site is located south of the TVA power line ROW that parallels Bear Creek Road on the north side. The actual site, grid N29480, E23220, is located about $100 \mathrm{ft}$ south of the ROW in forest. The site is located in an old farmyard. The remains of the chimney are located about $200 \mathrm{ft}$ east. Present vegetation consists of sugar maple and tulip poplar. The surface soil should have a higher $\mathrm{pH}$ and higher fertility. There is much evidence of worm activity on this site The only ground cover is a thick stand of poison ivy. The actual soil pit is situated within a brecciated zone of the Nolichucky Shale. Because of the past tectonic brecciation the soil is deeply weathered.

ORR Site No. 15. This site is located close to the intersection of the natural gas line ROW and the TVA power line ROW, grid N29700, E25720. The route into this site and ORR Site No. 16 is through the first gate west of the Bear Creek and Route 95 intersection on the north side of the road. This field road is followed until the intersection of the TVA power line and the gas line ROW is crossed, then about $25 \mathrm{ft}$ north of the gas line ROW. The site is located in a severely eroded old field which was planted to loblolly pines. The trees are about 40 to $50 \mathrm{y}$ old and nearly ready for harvesting for poles. There is a hardwood understory consisting of oak, red maple, dogwood and a sparse ground cover of honeysuckle and grasses. The underlying geology is the lower Nolichucky Shale, which in this area, is fairly permeable as the saprolite fragments are mostly coated with red iron oxides.

ORR Site No. 16. This site is located close to the intersection of the gas line ROW and the TVA power line ROW, grid N29700, E25928. The route into this site and ORR Site No. 16 is through the first gate west of the Bear Creek and Route 95 intersection on the north side of the road. This field road is followed until the intersection of the TVA power line and the gas line ROW is crossed, then along the power line ROW. The site is located on a sideslope of a severely eroded old field which was planted to loblolly pines. The trees are about 40 to $50 \mathrm{y}$ old and nearly ready for harvesting for poles. There is a hardwood understory consisting of oak, red maple, dogwood and a sparse ground cover of honeysuckle and grasses. The soils are typical that are underlain by the middle to lower Nolichucky Shale, which in this area, is less permeable than at ORR Site No. 15, as the saprolite fragments are mostly coated with black manganese oxides and clay plugged.

ORR Site No. 19. This site is located east of the Bear Creek and Route No. 95 intersection within the area known as the Bear Creek Low-level Waste Disposal Development and Demonstration (LLWDDD) Site. The route to this site starts at the intersection of Bear Creek Road and Gum Hollow Road. Follow Gum Hollow Road to the intersection with the TVA power line ROW. Turn left and follow this road to the intersection with the telegraph ROW. Turn right onto the telegraph ROW road for about $400 \mathrm{ft}$. The site is about $60 \mathrm{ft}$ east into the woods. The site is located in an old field. Vegetation is old-field successional forest. Most of the old pines $h$ ive died and fallen over. Present vegetation is mostly young hardwoods of cherry, sweet gum, cedar, poplar, dogwood, and Virginia pine. The ground surface is leaf covered with only scattered honeysuckle and poison ivy. The soils are typical of stable land surfaces that are underlain by the steeply dipping lower Dismal Gap Formation. This site has thin very fine grained sandstone strata in the $C$ horizon. The original location of this site was in a stand of young recently replanted pines and was moved about $300 \mathrm{ft}$ to the present location. 
ORR Site No. 21. This site is located in the area known as the Bear Creek LLWDDD. The route to this site starts at the intersection of Bear Creek Road and Gum Hollow Road. Follow Gum Hollow Road to the intersection with the TVA power line ROW. Turn left and follow this road for about $1,600 \mathrm{ft}$. The site is in the woods on the south side of the ROW, grid N29700, E30940. The site is located in an old field that had been severely eroded before abandonment. Vegetation is old-field successional foresi and some planted loblolly pines. Some pines have been harvested or have died and fallen over. Present vegetation consists of cedar, poplar, dense sourwood, oak, and red maple. Some grape vines and some prairie flowering plants are on the site. In addition to leaves, mosses and lichens cover part of the ground surface. The soils are typical for moderate slopes that are underlain by the Nolichucky Shale. Note that this site was moved a considerable distance from its original location. The original location was underlain by a deeply weathered section of the lower Maynardville Limestone which interbeds with the uppermost Nolichucky. When soil mapping was done in the Bear Creek LLWDDD site, this particular area was thought to be part of the Nolichucky, since the Maynardville was supposed to be high-grade limestone which gives rise to much different soils. Subsequent mapping and study finally put this shale-limestone interbedded area into the Maynardville which necessitated moving the site onto about the middle of the Nolichucky.

ORR Site No. 22. This site is located within the area known as the Bear Creek LLWDDD. The route to this site starts at the intersection of Bear Creek Road and Gum Hollow Road. Follow Gum Hollow Road to the intersection with the TVA power line ROW. Turn left and follow this road for about $800 \mathrm{ft}$. There is a cluster of wells on the north side of the ROW. Turn right onto a rough track and go to the top of the ridge then turn left and go down to where a large windthrow blocks the track. The site is about $100 \mathrm{ft}$ beyond the windthrow then about $50 \mathrm{ft}$ south into the woods, grid N30520, E31250. Vegetation at this site is old-field successional forest of Virginia pine, shortleaf pine, dogwood, and cedar. The surface is leaf covered and there is a sparse stand of honeysuckle. This site is located in the transition zone between the Dismal Gap Formation and the Rogersville Shale. The saprolite in the sampling pit was mostly Rogersville with a very steep dip.

ORR Site No. 23. This site is located within the area known as the Bear Creek LLWDDD. The route to this site starts $a^{\prime}$ the intersection of Bear Creek Road and Gum Hollow Road. Follow Gum Hollow Road to the intersection with the TVA power line ROW. Turn left and follow this road for about $800 \mathrm{ft}$. There is a cluster of wells on the north side of the ROW. Turn right onto a rough track for about $50 \mathrm{ft}$. The site is in the woods about $20 \mathrm{ft}$ to the east, grid N29800, E31830. Vegetation at this site is old-field successional forest. Most of the pines have died and fallen over. Present vegetation is red maple, sourwood and dogwoods, plus a ground cover of honeysuckle and poison ivy. The soils are typical examples of the Dismal Gap Formation. The $\mathrm{C}$ horizon at this site contained glauconitic strata that were sampled.

ORR Site No. 24. This site is located within the area known as the Bear Creek LLWDDD. The route to this site starts at the intersection of Bear Creek Road and Gum Hollow Road. Follow Gum Hollow Road to the intersection with the TVA power line ROW. Turn left and follow this road for about $600 \mathrm{ft}$. The site is on the south side of the ROW then into the woods about $100 \mathrm{ft}$, grid, $\mathrm{N} 29900, \mathrm{E} 31980$. The vegetation at this site is old-field 
successional forest with some planted loblolly pine about $30 \mathrm{y}$ old. Present vegetation is a dense stand of poplar, dogwood, cedar, pine, and red maple with a sparse ground cover of poison ivy and honeysuckle. The soils are typical examples of the Nolichucky Shale. Of interest were the thick manganese oxide coatings on fragment surfaces in the $\mathrm{C} \& \mathrm{Cr}$ horizons that were covered by translocated clay.

ORR Site No. 25. This site is located within the area known as the Bear Creek LLWDDD. The route to this site starts at the intersection of Bear Creek Road and Gum Hollow Road. Follow Gum Hollow Road to the intersection with the TVA power line ROW. Turn left and follow this road for about $400 \mathrm{ft}$. The site is on the south side of the ROW then into the woods about $100 \mathrm{ft}$, grid, N29800, E32290. Present vegetation on this site is planted loblhlly pines that are about $\mathbf{4 0}$ to $50 \mathrm{y}$ old. There is an understory of red mapie, dogwoud, and svieet gum. The ground surface is covered by a thick layer of pine needles and there is a moderate gro'nd cover of poison ivy and honeysuckle. The soils are typical examples of the Nolichucky Shale.

ORR Site No. 26. This site is located east of the ORR area known as the Bear Creek LLWDDD. The route to this site starts at the intersection of Bear Creek Road and Gum Hollow Branch Road. Turn off Bear Creek onto Gum Hollow. Go to where the TVA power line ROW crosses. Turn right down the ROW track for about $1400 \mathrm{ft}$. The site is about 75 tc $80 \mathrm{ft}$ north of the ROW in the woods, grid N30000, E34370. The site is in a severely eroded old field that was abandoned well before 1942. Vegetation is old-field successional forest where hardwoods have supplanted the early pine stage. Present forest vegetation consists of red maple, oak, hickory, and a few cedar. The forest canopy is sufficiently dense that therc is very little vegetative ground cover. The ground surface is covered with leaves. The soils are typical examples of the Dismal Gap Formatior

ORR Site No. 27. This site is located east of the ORR area known as the Bear Creek LLWDDD. The route to this site starts at the intersection of Bear Creek Road and Gum Hollow Branch Road. Turn off Bear Creek onto Gum Hollow. Go to where the TVA power line ROW crosses. Tumn right down the ROW track for about $1800 \mathrm{ft}$. The site is about $100 \mathrm{ft}$ north cf the ROW in the woods, grid N30100, E34990. ORR Site 27 is separated from ORR Site 26 by a drainageway. The site is in an eroded old field that was abandoned well before 1942. Vegetation is old-field successional forest where hardwoods have supplanted the early pine stage. Present forest vegetation consists of red and black oak, white oak, a few Virginia pine and some dead cedars. The understory consists of beech, red maple, dogwood. Scattered ferns occur under the dense forest canopy, othervise the ground surface is covered by leaves. This site and ORR Site No. 2 have the oldest forest vegetation for any of the ORR Phase 1 sites. The soils on this site are typical examples of the Dismal Gap Formation.

ORR Site No. 28. This site occurs along the new haul road that was constructed a few years ago to transport cover materials from the Chestnut Ridge borrow area to the Y-12 burial grounds. The rouse to this site starts at the intersection of Bear Creek Road and the new haul road. Turn orto the new haul road. The site is about $300 \mathrm{ft}$ beyond the gate on Bear Creek into the woods about $20 \mathrm{ft}$ from the edge of the TVA north-south power line ROW, grid N29500, E34580. The site is in a very severely eroded old field when it was abandoned. Vegetation is old-field successional forest. Most of the older pines have either been harvested or died and fallen over. Present vegetation consists of a dense stand of young Virginia pine along with cedar and poplar. There is a sparse ground cover of honeysuckle, mosses, and ferns. The soils are typical examples of the Nolichucky Shale. 
ORR Site No. 31. This site occurs along the new haul road that was constructed a few years ago to transport cover materials from the Chestnut Ridge borrow area to the Y-12 burial grounds. The route to this site starts at the intersection of Bear Creek Road and the new haul road. Turn onto the new haul road. The site is about $3400 \mathrm{ft}$ from the intersection. The site is on the south side of the haul road and approximately $200 \mathrm{ft}$ into cutover woods, grid N29900, E35820. The site is in a very severely eroded old field when it was abandoned. Vegetation is old-field successional forest. The pines were harvested 10 to 15 y ago. Present vegetation consists of a dense stand of sweet gum, dogwood, cedar, hickory, poplar, and ash. There is a sparse ground cover of honeysuckle and poison ivy. Even though there hal been some tree harvesting, the actual site showed little, if any, disturbance from the harve. $t$. This site occurs low on a long sideslope. Initial soil investigations indicated that there might be some alluvial or colluvial capping. However, when the soil pit was opened the soil morphology indicated that the upper $61 \mathrm{~cm}$ of the soil formed in late Pleistocene Age or early Holocene Age slope wash was derived from the Nolichucky Shale. The clay enriched subsoil has a clayey texture, but the substratum beneath did not contain any relic rock structure.

ORR Site No. 32. This site occurs along the new haul road that was constructed a few years ago to transport cover materials from the Chestnut Ridge borrow area to the Y-12 burial grounds. The route to this site starts at the intersection of Bear Creek Road and the new haul road. Turn onto the new haul road. The site is about $5400 \mathrm{ft}$ from the intersection and about $100 \mathrm{ft}$ grid north into the woods from the edge of the TVA power line ROW, grid N30200, E36760. Vegetation is old-field successional forest. Most of the pine trees were harvested or have died and fallen over. Present vegetation consists of a dense stand of hardwood underbrush plus abundant honeysuckle and some greenbriar. The soils on this site are typical examples of the upper Dismal Gap Formation.

ORR Site No. 33. This site occurs along the new haul road that was constructed a few years ago to transport cover materials from the Chestnut Ridge borrow area to the Y-12 burial grounds. The route to this site starts at the intersection of Bear Creek Road and the new haul road. Turn onto the new haul road. The site is about $5700 \mathrm{ft}$ from the intersection and about 75 to $100 \mathrm{ft}$ grid north into the woods from the edge of the TVA power line ROW, grid N30000, E36970. This site is about $250-300 \mathrm{ft}$ east of site 32 . Vegetation is old-field successional forest. Most of the pine trees were harvested or have died and fallen over. Present vegetation consists of a dense stand of hardwood underbrush plus abundant honeysuckle and some greenbriar. The soils on this site are typical examples of the middle Dismal Gap Formation.

ORR Site No. 35. This site occurs along the new haul road that was constructed a few years ago to transport cover materials from the Chestnut Ridge borrow area to the Y-12 burial grounds. The route to this site starts at the intersection of Bear Creek Road and the new haul road. Turn onto the new haul road. The site is about $6000 \mathrm{ft}$ from the intersection and about $150 \mathrm{ft}$ grid north into the woods from the edge of the IVA power line ROW, grid $\mathrm{N} 30000, \mathrm{E} 37280$. This site is about $250-300 \mathrm{ft}$ east of site 33 . Vegetation is old-field successional forest. Most of the pine trees were harvested or have died and fallen over. Present vegetation consists of a dense stand of sweet gum, dogwood, haw, sourwood and some poplar. About $90 \%$ of the ground surface is leaf covered with the remainder covered by honeysuckle, mosses and other forbes (weeds). The soils on this site are typical examples of the lower Dismal Gap Formation. 
ORR Site No. 41. This site occurs along the new haul road that was constructed a few years ago to transport cover materials from the Chestnut Ridge borrow area to the Y-12 burial grounds. The route to this site starts at the intersection of Bear Creek Road and the new haul road. Turn onto the new haul road. The site is about $7400 \mathrm{ft}$ from the intersection and about $400 \mathrm{ft}$ grid north into the woods from the edge of the TVA power line ROW, grid $\mathrm{N} 30200, \mathrm{E} 39570$. This site occurs in an old field that was severely eroded prior to or after abandonment. Vegetation is old-field successional forest. Most older pines have died and fallen over. The present vegetation is a fairly dense regrowth of pine, red maple, beech, and poplar. The ground surface is covered with leaves. The soils are typical examples of the Middle Dismal Gap Formation.

ORR Site No. 42. This site occurs on the new haul road that was constructed a few years ago to transport cover materials from the Chestnut Ridge borrow area to the Y-12 burial grounds. The route to this site starts at the intersection of Bear Creek Road and the new haul road. Turn onto the new haul road. The site is about $8100 \mathrm{ft}$ from the intersection and about $30 \mathrm{ft}$ grid north into the woods from the edge of the TVA power line ROW, grid $\mathrm{N} 30000, \mathrm{E} 40500$. This site is in an old field that was severely eroded prior to or after abandonment. Vegetation is old-field successional forest. Most of the old pine trees have died and fallen over or were harvested. Present vegetation is a dense regrowth of poplar, sweet gum, cedar, oak, dogwood, and beech. There is a sparse ground cover of honeysuckle and poison ivy. The soils on this site are fairly typical examples of the middle to upper Nolichucky Shale.

ORR Site No. 43. This site is the easternmost site on the ORR. It is fairly close to west end of the Y-12 burial grounds. This site occurs on the new haul road that was constructed a few years ago to transport cover materials from the Chestnut Ridge borrow area to the Y-12 burial grounds. The route to this site starts at the intersection of Bear Creek Road and the new haul road. Turn onto the new haul road. The site is about $9100 \mathrm{ft}$ from the intersection and about $200 \mathrm{ft}$ grid north into the woods from the edge of the TVA power line ROW, grid N30400, E41460. This site occurs in an old field that was severely eroded prior to or after abandonment. Vegetation is old-field successional forest. Present vegetation is red maple, poplar, abundant dogwood along with a dense ground cover of honeysuckle and poison ivy. The soils on this site are typical of stable upland positions that are underlain by the lower Dismal Gap Formation. This site is very close to the Rogersville Shale boundary. 


\section{A.2 ROANE COUNTY SITE DESCRIPTIONS}

ROANE Site No. 3. This site is on property belonging to Bowater or Kenneth Sutton. On the Pattie Gap quadrangle, go south on Dry Fork Valley Road. This property lies northwest from Salem Baptist Church. The site is in the hardwood forest $550 \mathrm{ft}$ northwest of the church, $350 \mathrm{ft}$ west of the creek, and $200 \mathrm{ft}$ southeast of the power lines. The present vegetation is oak, sweetgum, red maple, dogwood, and poison ivy. The underlying geology is the Dismal Gap Formation. The site lies between several rills and gullies in a convex toeslope position.

ROANE Site No. 7. On the Pattie Gap quadrangle, go south on Dry Fork Valley Road. After passing Blue Spring Road, the power lines will cross the road. The next driveway on the right will lead to Mr. and Mrs. John Walker's home. There is an old house in the field to the right. Go through the cattle gate and follow the field road to the tobacco patch. The site is in the hardwood thicket to the right. It is approximately $300 \mathrm{ft}$ northeast of Mr. Walker's present house and $50 \mathrm{ft}$ south of the fence dividing Mr. Walker's property from Kenneth Sutton's property. This site has been cleared at one time. The present vegetation is tulip poplar, dogwood, red maple, and some Virginia pine. Age of the deciduous trees indicates they have been established at least $40 \mathrm{y}$. There are many spots where rills are beginning to develop. The underlying geology is the Dismal Gap Formation. There is evidence of colluvium on this site. Oddly, in several places, sandstone fragments can be seen on the surface. Presently, this area is occupied by cattle for pasture.

ROANE Site No. 8. On the Pattie Gap quadrangle, go south on Dry Fork Valley Road. After passing Blue Spring Road, the power lines will cross the road. The next driveway on the right will lead to Mr. and Mrs. John Walker's home. There is an old house in the field to the right. Go through the cattle gate and follow the field road to the tobacco patch. The site is in the hardwood thicket to the right. It is approximately $250 \mathrm{ft}$ northeast of Mr. Walker's present house and $70 \mathrm{ft}$ south of the fence dividing Mr. Walker's property from Kenneth Sutton's property. This site was cleared at one time. The present vegetation is tulip poplar, dogwood, red maple, and some Virginia pine. Age of the deciduous trees indicates they have been established at least $40 \mathrm{y}$. There are many spots where rills are beginning to develop. The underlying geology is the Dismal Gap Formation. There is evidence of colluvium on this site. Oddly, in several places sandstone fragments can be seen on the surface. Presently, this area is occupied by cattle for pasture.

ROANE Site No. 9. This site is on property belonging to Bowater or Kenneth Sutton. On the Pattie Gap quadrangle, go south on Dry Fork Valley Road. This property lies northwest from Salem Baptist Church. The site is in the hardwood forest $500 \mathrm{ft}$ northwest of the church, $500 \mathrm{ft}$ west of the creek, and $200 \mathrm{ft}$ southeast of the power lines. The present vegetation is oak hardwoods mixed with Virginia pine. The underlying geology is the Dismal Gap Formation, but there is an alluvial or colluvial capping about $30 \mathrm{~cm}$ thick. The site lies between several rills and gullies in a convex toeslope position.

ROANE Site No. 10. This site is on property belonging to Don Manning. On the Pattie Gap quadrangle, go south on Dry Fork Valley Road. Turn onto Gage Road. There is an outbuilding of white blocks at the first drive to the right. Go through the gate and immediately turn left. Cross the small drain and go up to the knob above. The site is in the thicket north of the main creek. It is approximately $350 \mathrm{ft}$ northwest of Dry Fork Valley Road and $300 \mathrm{ft}$ east from Gage Road. This site was cleared at one time. The present vegetation 
is shortleaf pine, $r \in$ maple, and oak. Age of the deciduous trees indicates they have been established at least $40 \mathrm{y}$. The underlying geology is the Dismal Gap Formation. There is evidence of alluvium on this site. Several large rounded rocks were on site and in the profile. The area north of this site was a sagegrass field with multiple gullies and rills. Presently, this area is occupied by cattle for pasture.

ROANE Site No. 13. This site is on property belonging to Don Manning. On the Pattie Gap quadrangle, go south on Dry Fork Valley Road. Go past Gage Road. The next driveway on the right leads to a white frame house belonging to Ms. Bea Wise. Behind the house is a red barn. To the east of the barn a fence divides Ms. Wise's property from Mr. Manning's. The site is in the forest $300 \mathrm{ft}$ east of the peak of the barn roof. The present vegetation is Virginia pine, red maple, dogwood, poison ivy, and chestnut oak. Age of the deciduous trees indicates they have been established at least $40 \mathrm{y}$. The underlying geology is the Dismal Gap Formation. The site is at the bottom of a long slope in a bench position. There is evidence of alluvium in the area, but none was seen in this profile.

ROANE Site No. 14. This site is on property belonging to Don Manning. On the Pattie Gap quadrangle, go south on Dry Fork Valley Road. Go past Gage Road. The next driveway on the right leads to a white frame house belonging to Ms. Bea Wise. Behind the house is a red barn. To the east of the barn a fence divides Ms. Wise's property from Mr. Manning's. The site is in the forest $100 \mathrm{ft}$ east of the peak of the barn roof. The present vegetation is tulip poplar, red maple, dogwood, poison ivy, and chestnut oak. The understory vegetation is thick. Age of the deciduous trees indicates they have been established at least $40 \mathrm{y}$. The underlying geology is the Dismal Gap Formation. However, this formation is very thin and in digging the soil pit the Rogersville Formation was also exposed. The site is in a midslope position. The slope rapidly drops into a drain below the site.

ROANE Site No. 17. This site is on property belonging to Robert and Mattie Sue Viar. On the Pattie Gap quadrangle, go south on Dry Fork Valley Road. The Viar's live in the last house on the right before reaching Waterford Lane. The site is in the pine forest $500 \mathrm{ft}$ west of the Viar's home. The present vegetation is Virginia pine, eastern red cedar, and sweetgum. The owners indicated this land has not been cleared in the last $40 \mathrm{y}$. The underlying geology is the Dismal Gap Formation. The site lies between the convergence of two drains on a convex slope.

ROANE Site No. 19. This site is on property belonging to Bowater or Kenneth Sutton. On the Pattie Gap quadrangle, go south on Dry Fork Valley Road. This property lies northwest from Salem Baptist Church. The site is in the hardwood forest $750 \mathrm{ft}$ northwest of the church, $750 \mathrm{ft}$ west of the creek, and $200 \mathrm{ft}$ southeast of the power lines. The present vegetation is oak, red maple, hickory, eastern red cedar, and dogwood. The underlying geology is the Dismal Gap Formation. The site lies between several rills and gullies on a convex toeslope.

ROANE Site No. 20. This site is on property belonging to Bowater or Kenneth Sutton. On the Pattie Gap quadrangle, go south on Dry Fork Valley Road. This property lies northwest from Salem Baptist Church. The site is in the hardwood forest $950 \mathrm{ft}$ northwest of the church, $1000 \mathrm{ft}$ west of the creek, and $200 \mathrm{ft}$ southeast of the power lines. The present 
vegetation is chestnut oak, sweetgum, and dogwood. The underlying geology is the Dismal Gap Formation. The site lies between several rills and gullies in a toeslope position. Highly eroded.

ROANE Site No. 21. This site is on property belonging to Bowater or Kenneth Sutton. The property line could not be clearly defined. On the Pattie Gap quadrangle, go south on Dry Fork Valley Road. This property lies northwest from Salem Baptist Church. The site is in the hardwood forest $1100 \mathrm{ft}$ northwest of the church, $1200 \mathrm{ft}$ west of the creek, and $200 \mathrm{ft}$ southeast of the power lines. The present vegetation is oak, red maple, tulip poplar, sweetgum, and club mosses. There was no evidence of a plow layer. The underlying geology is the Dismal Gap Formation. The site lies between several rills and gullies in a midslope position.

ROANE Site No. 22. This site is on property belonging to Bowater or Kenneth Sutton. The property line could not be clearly defined. On the Pattie Gap quadrangle, go south on Dry Fork Valley Road. This property lies northwest from Salem Baptist Church. The site is in the hardwood forest $1400 \mathrm{ft}$ northwest of the church, $1500 \mathrm{ft}$ west of the creek, and $200 \mathrm{ft}$ southeast of the power lines. The present vegetation is oak, Virginia pine, tulip poplar, eastern red cedar, and club mosses. The underlying geology is the Dismal Gap Formation. The site lies between several rills and gullies in a midslope position on a bench. 


\section{A.3 ANDERSON COUNTY SITE DESCRIPTIONS}

ANDERSON Site No. 1. Take Highway 441 north. Turn right on to Hinds Creek Road. Go $200 \mathrm{ft}$ and turn left on to Old Andersonville Pike. Turn into the parking lot at Fairview Church. The sites are in a hardwood thicket adjacent to the northeast end of the cemetery. Located in the Norris Quadrangle, this site is located on property belonging to Marguriet Atkins. It is approximately $200 \mathrm{ft}$ northeast of Fairview cemetery and $500 \mathrm{ft}$ west from Hinds Creek Road. This site had been cleared at one time. The present vegetation is tulip poplar, dogwood, red maple, and some Virginia pine. Age of the deciduous trees indicates they have been established at least $50 \mathrm{y}$. The underlying geology is the Dismal Gap Formation. There was a water seep at approximately $40 \mathrm{~cm}$. The slope dips strongly $(30 \%)$ approximately $50 \mathrm{ft}$ from the site. Presently, this area is occupied by cattle for pasture.

ANDERSON Site No. 3. Take Highway 441 north. Turn right on to Hinds Creek Road. After several miles, the road forks. Take the road on the left, which is Judson Road. The site is in a hardwood forest $100 \mathrm{ft}$ northwest of Judson Road and $\mathbf{0 . 4}$ miles from the intersection of Judson Road and Moore Valley Road (Cooper's Gap). Located on the Big Ridge Park quadrangle, this site is located on property belonging to John Irwin. The present vegetation is moss, poison ivy, dogwood, red maple, and Virginia pine. Age of the deciduous trees indicates they have been established at least $50 \mathrm{y}$. The underlying geology is the Dismal Gap Formation. The site is on a wide slope. The surface drops to a drainage way approximately 150 to the northwest. There are no houses near this site, although there are indications of an old home site approximately $300 \mathrm{ft}$ southwest of the site near Judson Road.

ANDERSON Site No. 4. Take Highway 441 north. Turn right on to Hinds Creek Road. After several miles, the road forks. Take the road on the left, which is Judson Road. The site is in a hardwood forest $300 \mathrm{ft}$ northwest of Judson Road and $\mathbf{0 . 6}$ miles from the intersection of Judson Road and Moore Valley Road (Cooper's Gap). Located on the Big Ridge Park quadrangle, this site is located on property belonging to John Irwin. The present vegetation is poison ivy, dogwood, elm, cherry, sourwood, red maple, and Virginia pine. Age of the deciduous trees indicates they have been established at least $50 \mathrm{y}$. The underlying geology is the Dismal Gap Formation. The site is in the middle of a steep slope. It was difficult to find a good level position in which to dig.

ANDERSON Site No. 5. Take Highway 441 north. Turn right on to Hinds Creek Road. After several miles, the road forks. Take the road on the left, which is Judson Road. The site is in a hardwood forest $300 \mathrm{ft}$ northwest of Judson Road and 0.6 miles from the intersection of Judson Road and Moore Valley Road (Cooper's Gap). This site is $300 \mathrm{ft}$ south from Anderson Site No. 4, but slightly higher on the slope. Located on the Big Ridge Park quadrangle, this site is located on property belonging to John Irwin. The present vegetation is poison ivy, dogwood, oak, and Virginia pine. Age of the deciduous trees indicates they have been established at least $50 \mathrm{y}$. The underlying geology is the Dismal Gap Formation. The site is in the middle of a steep slope. It was difficult to find a good level position in which to dig.

ANDERSON Site No. 9. Take Highway 441 north. Turn left onto East Wolf Valley Road. After passing Nolan Lane, go approximately $400 \mathrm{ft}$ and turn right into a driveway angling off the road and up a steep grade. This site is on property belonging to Bill Lee on the Powell quadrangle. After passing Mr. Lee's house, follow the old logging road until reaching property owned by Billy Hughes. The site is in a hardwood forest on the right side of the logging road 
downslope toward East Wolf Valley Road. It is approximately $1300 \mathrm{ft}$ north of East Wolf Valley Road and 0.25 miles west from Nolan Lane. The surrounding forest shows evidence of having been logged in the last $40 \mathrm{y}$. The site is in a midislope position on a convex slope. The trees on this site are older and were probably not logged as recently as those closer to the logging road. The present vegetation is tulip poplar, dogwood, red maple, and some Virginia pine. The underlying geology is the Dismal Gap Formation. This site is $300 \mathrm{ft}$ north of Anderson Site No. 19.

ANDERSON Site No. 10. Take Highway 441 north. Turn right on to Hinds Creek Road. Go $200 \mathrm{ft}$ and turn left on to Old Andersonville Pike. Turn into the parking lot at Fairview Church. The sites are in a hardwood thicket adjacent to the northeast end of the cemetery. Located in the Norris Quadrangle, this site is located on property belonging to Marguriet Atkins. It is approximately $50 \mathrm{ft}$ northeast of Fairview cemetery and $450 \mathrm{ft}$ west from Hinds Creek Road. This site had been cleared at one time. The present vegetation is tulip poplar, dogwood, red maple, and some Virginia pine. Age of the deciduous trees indicates they have been established at least $50 \mathrm{y}$. The underlying geology is the Dismal Gap Formation. The site is in a level upland position. Presently, this area is occupied by cattle for pasture.

ANDERSON Site No. 11. Take Highway 441 north. Turn right on to Hind's Creek Road. Drive several miles until two signs saying Hidden Drive are passed. We never did figure out where Hidden Drive was located. There is a sawmill on the left and an open field. A 400-ft concrete driveway lead up to Allen Johnson's home. A large knob sits to the left (west) of Mr. Johnson's house. A road runs around the edge of this knob. This site is located $600 \mathrm{ft}$ northwest from Hind's Creek Road and $200 \mathrm{ft}$ west of Mr. Johnson's house. The present forest vegetation is tulip poplar, may apple, beech, red maple, and hickory. The understory vegetation is very thick. This site has probably been cleared at some time. Age of the deciduous trees indicates they have been established at least $50 \mathrm{y}$. The underlying geology is the Dismal Gap Formation.

ANDERSON Site No. 12. Take Highway 441 north. Turn right on to Hind's Creek Road. Drive several miles until Hill Road enters on the right. Property is directly across from Hill Road. Located on the Big Ridge Park quadrangle, this site is located on property belonging to Swan Kidwell. Mr. Kidwell lives in the gray house with an equipment shed on the left side of Hind's Creek Road just prior to reaching Hill Road. Go up the hill behind Mr. Kidwell's house. Cross under the power lines to the wooded area on the northwest side. This site is located $500 \mathrm{ft}$ northwest from Hind's Creek Road and $100 \mathrm{ft}$ west of the power lines. It is $200 \mathrm{ft}$ west from Anderson Site No. 22 on a wide slope. A drain lies between Sites 12 and 22 . The present forest vegetation is dogwood, sweetgum, hickory, and Virginia pine. A thick carpet of grass underlies the trees. This site has probably been cleared at some time. Age of the deciduous trees indicates they have been established at least $50 \mathrm{y}$. The underlying geology is the Dismal Gap Formation. The site presently is used as pasture for cattle.

ANDERSON Site No. 19. Take Highway 441 north. Turn left onto East Wolf Valley Road. After passing Nolan Lane, go approximately $400 \mathrm{ft}$ and turn right into a driveway angling off the road and up a steep grade. This site is on property belonging to Bill Lee. After passing Mr. Lee's house follow the old logging road until reaching property owned by Billy Hughes. The site is in a hardwood forest on the right side of the logging road downslope toward East Wolf Valley Road. It is approximately $1000 \mathrm{ft}$ north of East Wolf Valley Road and 0.25 miles west from Nolan Lane. This site is on the Powell quadrangle. The surrounding 
forest shows evidence of having been logged in the last $40 \mathrm{y}$. The site is in a midslope position on a convex slope. The trees on this site are older and were probably not logged as recently as those closer to the logging road. The present vegetation is tulip poplar, sassafras, red maple, moss, and some Virginia pine. The underlying geology is the Dismal Gap Formation. This site is $300 \mathrm{ft}$ south of Anderson Site No. 9.

ANDERSON Site No. 20. Take Highway 441 north. Turn right on to Hinds Creek Road. After several miles, the road forks. Take the road on the left, which is Judson Road. The site is in a hardwood forest $100 \mathrm{ft}$ northwest of Judson Road and 0.3 miles from the intersection of Judson Road and Moore Valley Road (Cooper's Gap). Located on the Big Ridge Park quadrangle, this site is located on property belonging to John Irwin. The present vegetation is moss, poison ivy, dogwood, red maple, and Virginia pine. Age of the deciduous trees indicates they have been established at least $50 \mathrm{y}$. The underlying geology is the Dismal Gap Formation. The site is on a wide slope. There are several areas in which rills have developed and erosion is evident. There are no houses near this site, although there are indications of an old home site approximately $500 \mathrm{ft}$ southwest of the site near Judson Road.

ANDERSON Site No. 21. Take Highway 441 north. Turn right on to Hind's Creek Road. Drive several miles until Hill Road enters on the right. Property is directly across from Hill Road. Located on the Big Ridge Park quadrangle, this site is located on property belonging to Swan Kidwell. Mr. Kidwell lives in the gray house with an equipment shed on the left side of Hind's Creek Road just prior to reaching Hill Road. Go up the hill behind Mr. Kidwell's house. Cross under the power lines to the wooded area on the northwest side. This site is located $700 \mathrm{ft}$ northwest from Hind's Creek Road and $150 \mathrm{ft}$ west of the power lines. It is $200 \mathrm{ft}$ northeast from Anderson Site No. 12. The site is located in a saddle between two slopes at the head of a drain. The present forest vegetation is dogwood,sweetgum, hickory, and Virginia pine. A thick carpet of grass underlies the trees. This site has probably been cleared at some time. Age of the deciduous trees indicates they have bsen established at least $50 \mathrm{y}$. The underlying geology is the Dismal Gap Formation. The site presently is used for pasture for cattle.

ANDERSON Site No. 22. Take Highway 441 north. Turn right on to Hind's Creek Road. Drive several miles until Hill Road enters on the right. Property is directly across from Hill Road. Located on the Big Ridge Park quadrangle, this site is located on property belonging to Swan Kidwell. Mr. Kidwell lives in the gray house with an equipment shed on the left side of Hind's Creek Road just prior to reaching Hill Road. Go up the hill behind Mr. Kidwell's house. Cross under the power lines to the wooded area on the northwest side. This site is located $500 \mathrm{ft}$ northwest from Hind's Creek Road and $300 \mathrm{ft}$ west of the power lines. It is $200 \mathrm{ft}$ east from Anderson Site No. 12 on a convex slope. A drain lies between sites 12 and 22. The present forest vegetation is dogwood,tulip poplar, eastern red cedar, and Virginia pine. A thick carpet of grass underlies the trees. This site has probably been cleared at some time. Age of the deciduous trees indicates they have been established at least $50 \mathrm{y}$. The underlying geology is the Dismal Gap Formation. The site presently is used as pasture for cattle. 


\section{A.4 OAK RIDGE RESERVATION SOIL PROFILES}

\section{SITE NUMBER}

ORR-2

See pages $20,66,67,70,71,91$ in the phase 1 field book No. 1 .

Location: Oak Ridge Reservation. Grid N29300, E10300.

Classification: Ruptic Ultic Dystrochrepts; clayey $\mathrm{Bt}$, loamy-skeletal $\mathrm{Bw}, \mathrm{C}$, and $\mathrm{Cr}$, mixed, thermic.

Geomorphic Position: Middle of convex sideslope.

Slope and Aspect: 16\% West Northwest.

Parent Material(s): Middle Dismal Gap Formation.

Vegetation: Oaks, few red maple, sweet gum, and white pine. This site was probably cleared and probably pastured but never plowed.

Described By. D. A. Lietzke.

Sampling Crew: Lietzke, Wilson.

Rad. Scan: Air 25; Ground level 45.

Weather Yesterday: Partly cloudy, cool, scattered showers.

Present Weather: Partly cloudy, cool.

Date: May 6, 1992.

\section{Soil Description}

Oi $\quad 4$ to $0 \mathrm{~cm}$; leaf litter, $100 \%$ coverage, few tree sprouts.

A $\quad 0$ to $5 \mathrm{~cm}$; dark brown (10YR 3/3) silt loam; strong fine granular structure; very friable; abrupt wavy boundary.

E 5 to $14 \mathrm{~cm}$; yellowish brown (10YR 5/4) silt loam; weak fine subangular blocky structure; very friable; clear wavy boundary.

BE 14 to $20 \mathrm{~cm}$; yellowish brown (10YR 5/6) shaly silty clay loam; weak fine subangular blocky structure; friable; clear wavy boundary.

$\mathrm{Bt} 20$ to $32 \mathrm{~cm}$; yellowish red (5YR 5/6) shaly clay; strong medium subangular blocky structure; firm; all peds coated with yellowish brown (10YR 5/6) materials; abrupt irregular boundary.

$\mathrm{BC} \& \mathrm{Cr} 32$ to $74 \mathrm{~cm}$; strong brown (7.5YR 5/6) fragment interiors in BC materials and light yellowish brown (2.5Y 6/4) fragment interiors in $\mathrm{C}$ and $\mathrm{Cr}$ materials. Fine earth and clay flows are strong brown (7.5YR 4/6); nearly all rock controlled structure; firm; Much glauconite in BC materials; abrupt irregular boundary.

Cr $\quad 74$ to $80 \mathrm{~cm}$; Not described.

NOTES: The ESD composite samples were collected from a pit face oriented with strike. There was less variability than the field duplicate samples that were collected from a pit face oriented across the strike. See pages 66 and 67 in the field log book for sketches. The field duplicate samples were collected from the appropriate inclined soil horizon as shown in the sketch on page 67.

Depths of sampling are: $\mathrm{A}, 0$ to $14 \mathrm{~cm} ; \mathrm{B}, 20$ to $32 \mathrm{~cm}$;, 32 to $50 \mathrm{~cm}$. 


\section{SITE NUMBER}

ORR-3

See pages 94, 109 and 128 in phase 1 field book No. 1 and pages 4 and 5 in phase 1, field book No. 2 .

Location: Oak Ridge Reservation. Grid N29300, E11200. This site is within $50 \mathrm{ft}$ of the top edge of Bear Creek road embankment.

Classification: Typic Hapludults; clayey, mixed, thermic.

Geomorphic Position: Broad slightly convex sideslope.

Slope and Aspect: 5\% South.

Parent Matcrial(s): Middle Nolichucky Shale.

Vegetation: Old field successional forest. Most old pines have died and fallen over. Present regrowth is V. pine, W. pine, oak, hickory, sassafras. cedar, with a sparse poison ivy ground cover.

Described By: D. A. Lietzke.

Sampling Crew: Lietzke, Burgoa.

Rad Scan: Air 50 ; Ground level 40.

Weather Yesterday: Clear, very warm, but with an evening shower.

Present Weather: Partly cloudy, very warm and humid.

Date: July 22, 1992.

\section{Soil Description}

$\mathrm{Oi} \quad 3$ to $0 \mathrm{~cm}$; leaf litter and pine needles.

A 0 to $5 \mathrm{~cm}$; dark brown (10YR $3 / 3$ ) silt loam; strong fine granular structure; very friable; abrupt wavy boundary.

Ap 5 to $15 \mathrm{~cm}$; yellowish brown (10YR 5/4) silt loam; moderate medium granular structure; friable; abrupt wavy boundary.

B\&E 15 to $18 \mathrm{~cm}$; dark yellowish brown (10YR 4/6) B part, and yellowish brown (10YR 5/6) E part; silty clay loam; weak medium subangular blocky structure; friable; abrupt wavy boundary.

Bt 18 to $40 \mathrm{~cm}$; strong brown (7.5YR 4/6) clay; moderate medium subangular blocky structure; firm; strong brown (7.5YR 4/4) ped coatings; abrupt irregular boundary.

C $\quad 40$ to $51 \mathrm{~cm}$; light olive brown (2.5Y 5/4) fragment interiors; plugged with strong brown (7.5YR 5/4) clay; all rock structure; abrupt irregular boundary.

$\mathrm{Cr} \quad 51$ to $60 \mathrm{~cm}$; brown (7.5YR 5/2) fragment interiors with most surfaces coated with dark red (2.5YR 3/6) iron-clay complexes.

NOTES: Sampling depths for ESD composites are: A, 0 to $15 \mathrm{~cm} ; \mathrm{B}, 18$ to $40 \mathrm{~cm} ; \mathrm{C}, 40$ to $51 \mathrm{~cm}$. 


\section{SITE NUMBER}

ORR-5

See pages 95, 109, 133 in Phase 1, field book No. 1, and pages 1, 2 in phase 1, field book No. 2.

Location: Oak Ridge Reservation. Grid: N29300, E15300. About $300 \mathrm{ft}$ west of Central Training facility main entrance and $25 \mathrm{ft}$ south of the boundary fence.

Classification: Typic Hapludults; clayey, mixed, thermic.

Geomorphic Position: Broad bench landform on a convex spur.

Slope and Aspect: $1 \%$

Parent Material(s): Middle Nolichucky Shale with several carbonate strata.

Vegetation: Old field successional forest. Most old pines have died and fallen over. Present regrowth is a dense stand of young pines, cedar, oak, dogwood, and sweet gum along with a generous stand of honeysuckle and greenbriar.

Described By: D. A. Lietzke.

Sampling Crew: Lietzke, Burgoa.

Rad. Scan: Air 40; Ground level 50.

Weather Yesterday: Clear and very warm with showers during the past evening.

Present Weather: Cloudy and cooler.

Date: July 22, 1992.

\section{Soil Description}

Oi $\quad 5$ to $3 \mathrm{~cm}$; mosses and pine needles.

Oe $\quad 3$ to $0 \mathrm{~cm}$; hemic materials, sapric materials and a dense root mat.

Ap 0 to $10 \mathrm{~cm}$; yellowish brown (10YR 5/4) silt loam; moderate fine granular structure; very friable; abrupt wavy boundary.

Bt 10 to $40 \mathrm{~cm}$; strong brown (7.5YR $4 / 6$ ) clay or silty clay; moderate medium subangular blocky structure; firm; strong brown (7.5YR 4/4) ped coatings; abrupt irregular boundary.

$\mathrm{C} \& \mathrm{Cr} \quad 40$ to $60 \mathrm{~cm}$; olive brown $(2.5 \mathrm{Y} 4 / 3)$ fragment interiors with red and black surface coatings; strong brown (7.5YR 5/4) clay coatings on some surfaces and also as plugged zones.

NOTES: This is the only ORR phase 1 site that has a thick moss layer.

Sampling depths are: A, 0 to $10 \mathrm{~cm} ; \mathrm{B}, 10$ to $40 \mathrm{~cm}$;, 40 to $55 \mathrm{~cm}$. 


\section{SITE NUMBER \\ ORR-10}

See pages $21,39,54,55,91$ in the phase 1 field book No. 1 .

Location: Oak Ridge Reservation. Grid N30200, E21460.

Classification: Typic Dystrochrepts; loamy-skeletal, mixed, thermic.

Geomorphic Position: Upper convex sideslope just below ridge crest.

Slope and Aspect: 20\% South Southwest.

Parent Material(s): Lower Dismal Gap Formation.

Vegetation: Hardwood forest that re-generated from older severely eroded old-field forest succession. Present trees are white oak, hickory, cedar, ash. Ground cover of leaves and thick stand of poison ivy, five-leaf ivy and honeysuckle.

Described By. D. A. Lietzke.

Sampling Crew. Lietzke, Wilson, Farmer

Rad. Scan: Air 20; Ground level 20.

Weather Yesterday: Cool, widely scattered showers in AM with clearing and warmer in the PM as the front went through.

Present Weather. Clear and warm.

Date: May 1, 1992.

Oi $\quad 4$ to $0 \mathrm{~cm}$; leaf litter.

\section{Soil Description}

Oa $\quad 0$ to $1 \mathrm{~cm}$; sapric and hemic materials with a root mat.

A 1 to $12 \mathrm{~cm}$; brown (10YR 4/3) shaly silt loam; strong fine granular structure; very friable; clear wavy boundary.

Bw 12 to $24 \mathrm{~cm}$; yellowish brown (10YR 5/6) shaly or very shaly silt loam or silty clay loam; moderate medium subangular blocky structure; very friable; some peds coated with darker materials; abrupt irregular boundary. (An intermittent Bt horizon, formed in a carbonate stratum was dug out before sampling for ESD composites started.)

C 24 to $46 \mathrm{~cm}$; light olive brown (2.5Y 5/6) fragment interiors. Most fragment surfaces thickly coated with yellowish brown (10YR 5/4) silty materials. There are thin seams of red (2.5YR 4/8) clay originally derived from carbonate seams in the rock. 60 to $90 \%$ geologic structure. interbedded with harder $\mathrm{Cr}$ materials at lower depths.

$\mathrm{Cr} \quad 46$ to $80 \mathrm{~cm}$; light olive brown (2.5Y 5/6) fragment interiors; surface coatings of dark red (2.5YR 3/6) and yellowish brown (10YR 5/4).

NOTES: Three sides of the pit were Typic Dystrochrepts. The fourth side had a Bt horizon but it was confined to a thin carbonate stratum and it had disappeared when the pit was enlarged to sample for ESD composites. The Oa horizon was not sampled. Sample depths are: A, 1 to $12 \mathrm{~cm}$ : B, 12 to $24 \mathrm{~cm} ; \mathrm{C}, 24$ to $46 \mathrm{~cm}$. 


\section{STTE NUMBER \\ ORR-11}

See pages $16,17,32,91$ in the phase 1 field book No. 1 .

Location: Oak Ridge Reservation. About $50 \mathrm{ft}$ grid north of TVA power line ROW. Grid N29800, E21460

Cassification: Typic Hapludults; clayey, mixed, thermic.

Geomorphic Position: Lower slightly convex sideslope. Severely eroded prior to or after abandonment.

Slope and Aspect: 13\% Southwest.

Parent Material(s): Upper Dismal Gap Formation and transition zone to lower Nolichucky.

Vegetation: Old field regrowth. Most pines have died and have fallen over. Young brush and sprouts of dogwood, sassafras, cherry, red maple and sweet gum, with an abundant ground cover of poison ivy and honeysuckle.

Described By. D. A. Lietzke.

Sampling Crew: Lietzke, Wilson, Phillips, Farmer

Rad Scan: Air 15; Ground level 70.

Weather Yesterday. Partly cloudy and cool.

Present Weather: Clear and warmer.

Date: March 17, 1992.

\section{Soil Description}

$\mathrm{Oi} \quad 4$ to $0 \mathrm{~cm}$; leaves and pine needles.

Oa $\quad 0$ to $1 \mathrm{~cm}$; black (10YR 2/1) organic matter and root mat.

Ap 1 to $18 \mathrm{~cm}$; brown (10YR 4/3) silty clay loam with fresh shale fragments; moderate fine granular structure; friable; common fine roots; abrupt wavy boundary.

$\mathrm{Bt} \quad 18$ to $36 \mathrm{~cm}$; yellowish red (5YR 5/6) silty clay or clay; moderate medium subangular blocky structure; friable; few fine roots and few medium roots; strong brown (7.5YR $4 / 6$ ) clay coats on all ped faces; gradual wavy boundary.

BC 36 to $48 \mathrm{~cm}$; strong brown (7.5YR 4/6) shaly silty clay or shaly clay; moderate coarse prismatic parting to moderate medium to coarse subangular blocky structure; firm; many fragments are oriented with respect to the geologic strike and dip. yellowish and reddish parent materials saprolitic colors are also present.

C 48 to $85 \mathrm{~cm}$; red (2.5YR 4/8) and yellowish red (5YR 5/8 fragment interiors; material easily crushes to shaly or very shaly silty clay loam; 70 to $90 \%$ rock structure; yellowish red (5YR 4/6) coatings on many fragment faces.

$\mathrm{Cr} \quad 85$ to $132 \mathrm{~cm}$; fragment interiors range from red to olive; some fragment faces are coated with dark red (10R 3/6) while other fragment surfaces are coated with yellowish brown (10YR 5/4) clay; some fragments have light olive gray (5Y 6/2) fringes indicating wetness conditions. Light brownish gray $(2.5 Y 6 / 2)$ flow zones also occur in this horizon. Fine roots are in flow zones.

NOTES: Sampling depths are: A, 1 to $18 \mathrm{~cm} ; \mathrm{B}, 23$ to $36 \mathrm{~cm}$;, 51 to $62 \mathrm{~cm}$. 


\section{SITE NUMBER}

ORIR-13

See nages 95, 109, 135 in phase 1 field book No. 1, and pages 12 and 13 of phase 1, field book No. 2 .

Location: Oak Ridge Reservation. Grid: N294/80, E23220.

Ciasification: 'Typic Hapludults; clayey, mixed, thermic.

Geomorphic Position: Broad convex ridgetop.

Slope and Aspact: 3\% Northwest.

Parent Material(s): Middle Nolichucky Shale.

Vegetation: Old house site. Probably a yard at one time. Present vegetation is maple and poplar with a dense ground cover of poison ivy.

Described By: D. A. Lietzke.

S anpling Crew: Lietzke, Burgoa.

Rad. Scan: Air 50; Ground level 60.

Weather Yesterday: Partly cloudy, very warm.

Present Weather: Cloudy, very humid.

Date: July 22, 1992.

\section{Soil Description}

Oi 2 to $0 \mathrm{~cm}$; leaf litter.

Ap 0 to $23 \mathrm{~cm}$; brown (10YR 4/3) silt loam or shaly silt loam; strong fine granular structure; very friable; abrupt wavy boundary.

Bt 23 to $46 \mathrm{~cm}$; strong brown (7.5YR 4/4) clay or shaly clay; moderate medium subangular blocky structure; friable; brown (10YR 4/4) ped coatings; clear wavy boundary.

C 46 to $69 \mathrm{~cm}$; brecciated zone with many disoriented small fragments with olive yellow (2.5Y 6/6) interiors; rock controlled structure; friable; crushes easily to a shaly or very shaly silty clay loam or silty clay.

NOTES: This site is in a brecciated zone that is deeply weathered. Sampling depths for ESD Composites are: A, 0 to $23 \mathrm{~cm} ; \mathrm{B}, 23$ to $46 \mathrm{~cm}$, C, 46 to $56 \mathrm{~cm}$. 


\section{SITE NUMBER}

\section{ORR-15}

See pages $95,111,116,117$ in phase 1 field book No. 1 .

Location: Oak Ridge Reservation. Grid: N29700, E25720. About $25 \mathrm{ft}$ north of gas line ROW.

Classification: Typic Hapludults; clayey, mixed, thermic.

Geomorphic Position: Broad, slightly convex, ridgetop.

Slope and Aspect: 2-3\% South Southwest.

Parent Material(s): Lower Nolichucky Shale.

Vegetation: Stand of 40 to 50 year old loblolly pines, with an understory of oaks, red maple, dogwood, and a ground cover of honeysuckle and grasses.

Described By: D. A. Lietzke.

Sampling Crew: Lietzke, Wilson.

Rad Scan: Air 30; Ground level 40.

Weather Yesterday: Cloudy with scattered showers, cool.

Present Weather: Partly cloudy and cool.

Date: May 27, 1992.

\section{Soil Description}

Oi $\quad 2$ to $0 \mathrm{~cm}$; pine needles and a few leaves.

A $\quad 0$ to $2 \mathrm{~cm}$; brown (10YR 4/3) silt loam; moderate fine granular structure; very friable; abrupt wavy boundary.

Ap 2 to $16 \mathrm{~cm}$; yellowish brown (10YR 5/6) silt loam or silty clay loam; weak medium granular structure; friable; abrupt wavy boundary.

Bt 16 to $36 \mathrm{~cm}$; yellowish red (5YR 5/6) clay; moderate medium subangular blocky structure; firm; strong brown (7.5YR 4/6) clay films on all ped surfaces; few parent material reds and yellows in some ped interiors in the lower part; abrupt irregular boundary.

$\mathrm{C} / \mathrm{Cr} \quad 36$ to $60 \mathrm{~cm}$; olive yellow (2.5Y 6/8) fragment interiors and thin strong brown (7.5YR 4/6) clay seams. Yellowish red (5YR 5/6) clay flows coating C horizon material; most $\mathrm{Cr}$ horizon fragments coated with red iron oxides; all rock controlled structure; $\mathrm{C}$ and $\mathrm{Cr}$ materials occur in thin strata that had weathered differentially with respect to each other.

Cr $\quad 60$ to $70 \mathrm{~cm}$; brown (7.5YR 4/4) fragment interiors of less weathered strata and yellowish red (5YR 4/6) fragment interiors of more highly weathered strata; dark red (2.5YR 3/6-3/4) iron oxides on many fragment surfaces.

NOTES: Sampling depths are: A, 0 to $16 \mathrm{~cm} ; \mathrm{B}, 16$ to $36 \mathrm{~cm} ; \mathrm{C}, 36$ to $60 \mathrm{~cm}$. 


\section{SITE NUMBER \\ ORR-16}

See pages $98,11,113$ in phase 1 field book No. 1 .

Location: Oak Ridge Reservation. Grid: N29700; E25928.

Classification: Typic Hapludults; clayey, mixed, thermic.

Geomorphic Position: Sideslope, slight convexity.

Slope and Aspect: 10\% Southeast. Severely eroded prior to abandonment.

Parent Material(s): Middle Nolichucky Shale.

Vegetation: Loblolly Pine plantation with approximately 40 year old trees. Understory of dogwoods, oaks, sweetgum. Sparse ground cover of honeysuckle and poison ivy.

Described By: D. A. Lietzke.

Sampling Crew: Lietzke, Burgoa.

Rad Scan: Air 45; Ground level 60.

Weather Yesterday: Clear and warm.

Present Weather: Clear and warm.

Date: July 20, 1992.

\section{Soil Description}

Oi $\quad 3$ to $0 \mathrm{~cm}$; pine needles and some leaves.

A $\quad 0$ to $7 \mathrm{~cm}$; dark yellowish brown (10YR 4/4) shaly silt loam; moderate fine granular structure; very friable; abrupt wavy boundary.

Ap $\quad 7$ to $20 \mathrm{~cm}$; yellowish brown (10YR 5/4) shaly silty clay loam; moderate medium granular structure; very friable; clear wavy boundary.

Bt 20 to $33 \mathrm{~cm}$; strong brown (7.5YR 4/6) clay or shaly clay; moderate medium subangular blocky structure; friable; all peds coated; clear wavy boundary.

BC $\quad 33$ to $43 \mathrm{~cm}$; strong brown (7.5YR 5/4) very shaly silty clay loam; weak medium subangular blocky structure and areas of rock controlled structure; firm; strong brown (7.5YR 5/4) clay coatings on most fragment surfaces, with the clay covering a thick layer of black manganese oxides; abrupt irregular boundary.

C 43 to $88 \mathrm{~cm}$; yellowish brown (10YR 5/6) fragment interiors; crushes to shaly silty clay or very shaly silty clay loam; $90 \%$ rock controlled structure; clay plugged; some black coatings; firm; abrupt irregular boundary.

NOTES: Sampling depths for ESD composites are: A, 0 to $20 \mathrm{~cm} ; \mathrm{B}, 20$ to $33 \mathrm{~cm}$;, 43 to $63 \mathrm{~cm}$. 


\section{SITE NUMBER \\ ORR-19}

See pages $21,33,35,37,42,43,91$ in phase 1 field book No. 1 .

Location: Oak Ridge Reservation. $60 \mathrm{ft}$ east of telegraph ROW within the Bear Creek LLWDDD. Grid N33000, E28530.

Classification: Typic Hapludults; clayey, mixed, thermic.

Geomorphic Position: Top of broad spur ridge.

Slope and Aspect: 4\% West.

Parent Material(s): Lower Dismal Gap Formation.

Vegetation: Old-field forest. Most pines have fallen. Young hardwoods of cherry, sweet gum, cedar, poplar, dogwood and virginia pine. Sparse ground cover of honeysuckle. Ground surface $100 \%$ leaf covered.

Described By: D. A. Lietzke.

Sampling Crew: Lietzke, Wilson, Farmer.

Rad Scan: Air 30; _Ground level 40.

Weather Yesterday: Cool, cloudy, light showers.

Present Weather: Cool, cloudy with cold front moving through.

Date: April 27, 1992.

\section{Soil Description}

Oi $\quad 5$ to $0 \mathrm{~cm}$; leaf litter.

Oa $\quad 0$ to $1 \mathrm{~cm}$; sapric and hemic materials and dense root mat.

A 1 to $6 \mathrm{~cm}$; dark brown (10YR 3/3) silt loam; moderate fine granular structure; very friable; clear wavy boundary.

Ap 6 to $16 \mathrm{~cm}$; yellowish brown (10YR 5/4) shaly silt loam; moderate fine granular structure; very friable; abrupt wavy boundary.

BE $\quad 16$ to $25 \mathrm{~cm}$; strong brown (7.5YR 5/6) shaly silty clay loam; weak medium subangular blocky structure; friable; clear wavy boundary.

Bt 25 to $51 \mathrm{~cm}$; yellowish red (5YR 4/6) silty clay or clay; moderate medium subangular blocky structure; friable; strong brown (7.5YR 5/6) clay on all ped faces; clear wavy boundary.

BC $\quad 51$ to $54 \mathrm{~cm}$; transition to $\mathrm{C}$ horizon with loss of soil structure and start of geologic strike and dip controlled rock structure.

C 54 to $84 \mathrm{~cm}$; alternating strata of brown (7.5YR 4/3) very fine grained sandstone saprolite and shale-siltstone saprolite. Saprolite is plugged with yellowish red (5YR $4 / 6)$ clay.

Cr $\quad 84$ to $90 \mathrm{~cm}$; Harder shale strata. Fragment interiors are olive brown $(2.5 \mathrm{Y} 4 / 4)$. Most surface are coated with black manganese oxides and some yellowish red (5YR 4/6) clay.

NOTES: The Bt horizon is continuous around the pit. The $\mathrm{Cr}$ horizon in shale starts at $60 \mathrm{~cm}$ depth while the $\mathrm{Cr}$ in the sandstone strata occurs at a depth of 80 to $84 \mathrm{~cm}$ below the surface. Sampling depths are: A, 0 to $16 \mathrm{~cm} ; \mathrm{B}, 25$ to $51 \mathrm{~cm}$; C, 54 to $74 \mathrm{~cm}$. This site was moved from its original location, which was in a young stand of planted pines, to a location in an old field successional forest. 


\section{SITE NUMBER}

ORR-21

See pages $99,107,139,158,159$ in phase 1 field book No. 1.

Location: Oak Ridge Reservation. Grid: N29700, E30940. Site in old field grown up to pines. Classification: Ruptic Ultic Dystrochrepts; clayey Bt, loamy-skeletal Bw, C and Cr, mixed, thermic.

Geomorphic Position: Nearly level bench-like area on a convex sideslope.

Slope and Aspect: 9\% South. Severely eroded.

Parent Material(s): Middle Nolichucky Shale.

Vegetation: Old field successional forest with a few tall pines remaining along with cedar, poplar, dense stands of sourwood, oak and red maple. Some grape vines and some mosses and lichen groundcover.

Described By: D. A. Lietzke.

Sampling Crew: Lietzke, Burgoa.

Rad. Scan: Air 35 ; Ground level 80.

Weather Yesterday: Clear and warm.

Present Weather: Partly cloudy and very warm.

Date: July 21, 1992.

\section{Soil Description}

$\mathrm{Oi} \quad 2$ to $0 \mathrm{~cm}$; leaf litter.

Ap $\quad 0$ to $10 \mathrm{~cm}$; dark yellowish brown (10YR 4/6) shaly silty clay loam; moderate medium granular structure; friable, abrupt wavy boundary.

Bt 10 to $26 \mathrm{~cm}$; yellowish red (5YR 5/6) shaly clay; strong medium subangular blocky structure; firm; strong brown (7.5YR 4/4) ped coatings; abrupt irregular boundary.

$\mathrm{Cr} 26$ to $48 \mathrm{~cm}$; inclined shaly strata with brown (7.5YR 4/2) fragment interiors; red (10YR 4/6) surface coatings of iron-clay complex; abrupt irregular boundary.

2C 48 to $63 \mathrm{~cm}$; yellowish brown and yellowish red saprolitic materials that crushes to silty clay loam; this stratum impregnated with red iron compounds and black manganese oxides.

$3 \mathrm{Cr} \quad 63$ to $65 \mathrm{~cm}$; harder shale saprolite.

NOTES: This site was moved from its original location. The original location was on the Maynardville Formation. See the Phase 1 map for the original and moved locations.

Sampling depths are: A, 0 to $10 \mathrm{~cm} ; \mathrm{B}, 10$ to $26 \mathrm{~cm}$;, 26 to $48 \mathrm{~cm}$. 


\section{SITE NUMBER}

ORR-22

See pages $24,35,37,46,47,91$ in phase 1 field book No. 1 .

Location: Oak Ridge Reservation. Within Bear Creek LLWDDD site. Grid N30520, E31250.

Classification: Ruptic Ultic Dystrochrepts; clayey Bt, loamy-skeletal C and Cr horizons; mixed, thermic.

Geomorphic Position: Broad convex ridge crest.

Slope and Aspect: 3\% Southwest.

Parent Material(s): Lower Dismal Gap Formation and transition to Rogersville shale. The pit was in mostly Rogersville saprolite.

Vegetation: Old-field woods of virginia pine, shortleaf pine, dogwoods, cedars. Surface $100 \%$ leaf covered and with a sparse groundcover of honeysuckle.

Described By: D. A. Lietzke.

Sampling Crew: Lietzke, Wilson, Farmer

Rad Scan: Air 40; Ground level 40.

Weather Yesterday: Cloudy with scattered showers.

Present Weather: Cloudy, cool, with scattered showers.

Date: April 28, 1992.

\section{Soil Description}

Oi\&Oa 2 to $0 \mathrm{~cm}$; leaf litter, litter fragments and root mat.

A 0 to $5 \mathrm{~cm}$; dark grayish brown (10YR 4/2) silt loam; moderate fine granular structure; very friable; clear wavy boundary.

Ap 5 to $15 \mathrm{~cm}$; brown (10YR $5 / 3$ ) shaly silt loam; weak medium granular structure; friable; abrupt wavy boundary.

Bt 15 to $27 \mathrm{~cm}$; yellowish red (5YR 4/6) clay; moderate medium subangular blocky structure; firm; brown (7.5YR 4/4) clay on all ped faces; abrupt irregular boundary.

$\mathrm{C} \& \mathrm{Cr} 127$ to $46 \mathrm{~cm}$; Alternating thin strata of soft and hard shale-siltstone saprolite with light olive brown (2.5Y 5/4) fragment interiors; plugged with brown (7.5YR 4/4) clay.

$\mathrm{Cr} 2 \quad 46$ to $54 \mathrm{~cm}$; harder shale saprolite with most fragment surfaces coated with dark red (2.5YR 3/4-3/6) iron oxides.

NOTES: Sampling depths are: A, 0 to $15 \mathrm{~cm} ; \mathrm{B}, 15$ to $27 \mathrm{~cm} ; \mathrm{C}, 27$ to $46 \mathrm{~cm}$. 


\section{SITE NUMBER \\ ORR-23}

See pages 101, 107, 120, 121 in phase 1 field book No. 1.

Location: Oak Ridge Reservation. Grid: N 29800, E 31830 . About $50 \mathrm{ft}$ north of TVA power line ROW and about $20 \mathrm{ft}$ east of road that goes to ORR-22.

Classification: Ruptic Ultic Dystrochrepts; clayey $\mathrm{Bt}$, loamy-skeletal $\mathrm{Bw}, \mathrm{C}$, and $\mathrm{Cr}$, mixed, thermic.

Geomorphic Position: Interfluve between two drainageways.

Slope and Aspect: 2 to 3\%, South.

Parent Material(s): Lower Nolichucky Shale.

Vegetation: Old-field pines. Most have died and fallen over. Present canopy vegetation is red maple, sourwood and dogwoods. Groundcover of honeysuckle and poison ivy.

Described By: D. A Lietzke.

Sampling Crew: Lietzke, Wilson, Burgoa.

Rad. Scan: Air 30; Ground level 70.

Weather Yesterday: Partly cloudy and cool.

Present Weather. Cloudy with showers

Date: May 28, 1992.

\section{Soil Description}

Oi $\quad 4$ to $2 \mathrm{~cm}$; pine needles and leaves.

Oa 2 to $0 \mathrm{~cm}$; hemic materials, sapric organic materials, many fine roots, and a root mat at the mineral soil surface. (The $\mathrm{Oa}$ has variable thickness ranging from $0 \mathrm{~cm}$ to about $2 \mathrm{~cm}$ in the area that was cleared of organic materials for sampling the mineral soil.)

A 0 to $1 \mathrm{~cm}$; dark yellowish brown (10YR 4/4) shaly silt loam; moderate fine granular structure; very friable; abrupt wavy boundary.

Ap 1 to $12 \mathrm{~cm}$; dark yellowish brown (10YR 4/6) shaly silty clay loam; weak medium granular structure; friable; abrupt wavy boundary.

Bt 12 to $30 \mathrm{~cm}$; yellowish red (5YR 4/6) shaly clay; moderate fine subangular blocky structure; firm; strong brown (7.5YR 4/4) coatings on all ped surfaces; abrupt irregular boundary.

$\mathrm{C} \& \mathrm{Cr} 30$ to $50 \mathrm{~cm}$; strong brown (7.5YR 5/8) glauconitic fragment interiors and reddish brown (5YR 4/4) clay flows; rock oriented strike and dip structure.

$\mathrm{Cr} \& \mathrm{C} \quad 50$ to $60 \mathrm{~cm}$; Thin strata of both $\mathrm{C}$ and $\mathrm{Cr}$ siltstone saprolite materials. In addition to the siltstone stratum, there is a red (10R 4/6) glauconitic stratum and a confined flow zone stratum dominated by light brownish gray (10YR 6/2) wetness mottles; some fragments in the $C$ horizon are coated with reddish brown (5YR 4/4) clay. Siltstone fragment interiors are yellowish brown (10YR 5/6).

NOTES: This pit was dug and sampled for ESD composites and gamma scan samples in a light rain. The profile was not described until June 1, 1992. Sampling depths are: $A, 0$ to $12 \mathrm{~cm} ; \mathrm{B}, 12$ to $30 \mathrm{~cm} ; \mathrm{C}, 30$ to $50 \mathrm{~cm}$. 


\section{SITE NUMBER}

ORR-24

See pages 101, 107 and 108 in phase 1 field book No. 1, and pages 8 and 9 in Phase 1 field Book No. 2.

Location: Oak Ridge Reservation. Grid: N29900, E31980.

Classification: Ruptic Ultic Dystrochrepts; clayey $\mathrm{Bt}$, loamy-skeletal, $\mathrm{Bw}, \mathrm{C}$ and $\mathrm{Cr}$, mixed, thermic.

Geomorphic Position: Convex sideslope.

Slope and Aspect: 8\% North.

Parent Material(s): Middle Nolichucky Shale.

Vegetation: Old field successional forest with some planted loblolly pine about $30 \mathrm{y}$ old. Dense undergrowth of poplar, dogwood, cedar, pine, red maple. Sparse ground cover of poison ivy and honeysuckle.

Described By: D. A. Lietzke.

Sampling Crew: Lietzke, Burgoa.

Rad. Scan: Air 50 ; Ground level 70.

Weather Yesterday: Clear to partly cloudy, very warm with a shower in the evening.

Present Weather: Partly cloudy, very warm, very humid.

Date: July 22, 1992.

\section{Soil Description}

Oi $\quad 4$ to $1 \mathrm{~cm}$; pine needles and some leaves.

Oa\&Oe 1 to $0 \mathrm{~cm}$; hemic materials, sapric materials and a root mat.

A $\quad 0$ to $5 \mathrm{~cm}$; dark brown (10YR 3/3) silt loam; strong fine granular structure; very friable; abrupt wavy boundary.

Ap 5 to $19 \mathrm{~cm}$; dark yellowish brown (10YR 4/4) shaly silt loam; moderate fine granular structure; very friable; abrupt wavy boundary.

Bw\&Bt 19 to $26 \mathrm{~cm}$; dark yellowish brown (10YR 4/4) shaly or very shaly silty clay loam; weak medium subangular blocky structure; friable; abrupt wavy boundary.

$\mathrm{C} \& \mathrm{Cr} 26$ to $49 \mathrm{~cm}$; light olive brown $(2.5 \mathrm{Y} 5 / 3)$ fragment interiors; rock controlled structure; yellowish brown (10YR 5/4) coatings on most fragment surfaces. These coatings cover thick black manganese oxide coatings.

$\mathrm{Cr} \& \mathrm{C} \quad 49$ to $60 \mathrm{~cm}$; highly weathered shale and siltstone saprolite.

NOTES: Sampling depths for ESD composites are: A, 0 to $19 \mathrm{~cm} ; \mathrm{B}, 19$ to $26 \mathrm{~cm}$;, 26 to $49 \mathrm{~cm}$. 


\section{SITE NUMBER \\ ORR-25}

See pages 95, 107, 124, 125 in phase 1 field book No. 1.

Location: Oak Ridge Reservation. Grid: N 29800, E 32290 . Site about $50 \mathrm{ft}$ south of TVA electric power line ROW.

Classification: Ruptic Ultic Dystrochrepts; clayey Bt, loamy-skeletal Bw, C and $\mathrm{Cr}$, mixed, thermic.

Geomorphic Position: Upland summit, slightly convex.

Slope and Aspect: 2 to 3\%, Southeast.

Parent Material(s): Middle Nolichucky Shale.

Vegetation: Loblolly pine plantation, 40 to 50 y old. Lower story of red maple, dogwood, and sweet gum. Thick layer of pine needles on the ground surface along with moderate poison ivy and honeysuckle.

Described By: D. A. Lietzke.

Sampling Crew: Lietzke, Wilson, Burgoa.

Rad. Scan: Air 45; Ground level 60.

Weather Yesterday: Partly cloudy and cool.

Present Weather: Overcast with showers.

Date: May 28, 1992.

\section{Soil Description}

Oi $\quad 4$ to $2 \mathrm{~cm}$; pine needles and leaves.

Oe\&Oa 2 to $0 \mathrm{~cm}$; sapric materials, hemic materials and root mat.

A $\quad 0$ to $1 \mathrm{~cm}$; yellowish brown (10YR 5/4) shaly silt loam; moderate fine granular structure; very friable; abrupt wavy boundary.

Ap 1 to $17 \mathrm{~cm}$; yellowish brown (10YR 5/4) shaly silty clay loam; weak medium granular structure; friable; abrupt wavy boundary.

Bt\&Bw 17 to $34 \mathrm{~cm}$; brown (7.5YR 4/4) shaly clay; about $50 \%$ moderate medium subangular blocky structure in $\mathrm{Bt}$ strata and rock controlled strike and dip structure in Bw; Fragment interiors are light yellowish brown (2.5Y 6/4); firm; abrupt irregular boundary.

$\mathrm{C} \& \mathrm{Cr} 34$ to $50 \mathrm{~cm}$; strong brown (7.5YR 5/4) very shaly clay; strike and dip controlled orientation of shale fragments; light olive brown shale fragment interiors; abrupt irregular boundary.

$\mathrm{Cr} \quad 50$ to $60 \mathrm{~cm}$; light olive brown (2.5Y 5/4) fragment interiors; red and black coatings on fragment surfaces; thin carbonate seams are red (2.5YR 4/8) clay; larger cracks contain strong brown (7.5YR 5/4) translocated clay.

NOTES: ESD composite samples were collected in a light rain. The ESD gamma scan samples were collected and the profile described on the 1st of June, 1992 when the weather was partly cloudy. Sampling depths are: A, 0 to $17 \mathrm{~cm}$; B, 17 to $34 \mathrm{~cm} ; \mathrm{C}, 34$ to $50 \mathrm{~cm}$. 


\section{SITE NUMBER \\ ORR-26}

See pages $24,74,75,78,79,91$ in phase 1 field book No. 1 .

Location: Oak Ridge Reservation. Grid N 30000, E34370. The site is 75 to $80 \mathrm{ft}$ north of the TVA power line ROW.

Classification: Ruptic Ultic Dystrochrepts; clayey Bt, loamy-skeletal Bw, C, Cr, mixed, thermic. Geomorphic Position: Lower convex sideslope.

Slope and Aspect: 14\% South Southeast.

Parent Material(s): Middle Dismal Gap Formation.

Vegetation: This site has been plowed but was abandoned before the Atomic Energy Commission (AEC) takeover in 1942. Present forest is hardwoods of red maple, oaks, hickories, and a few cedars.

Described By: D. A. Lietzke.

Sampling Crew: Lietzke, Wilson.

Rad. Scan: Air 15; Ground level 25.

Weather Yesterday: Partly cloudy in the morning with afternoon scattered showers.

Present Weather. Cloudy, scattered showers, cool. Got rained out before completing sampling and soil description.

Date: May 11, 1992.

\section{Soil Description}

Oi 4 to $0 \mathrm{~cm}$; leaf litter and root mat.

A $\quad 0$ to $6 \mathrm{~cm}$; very dark grayish brown (10YR 3/2) shaly silt loam; strong fine granular structure; very friable; abrupt wavy boundary.

Ap 6 to $15 \mathrm{~cm}$; dark yellowish brown (10YR 4/4) shaly silt loam; moderate fine granular structure; very friable; abrupt wavy boundary.

Bt 15 to $30 \mathrm{~cm}$; red $(2.5 \mathrm{YR} 4 / 6)$ shaly clay or shaly silty clay; moderate medium subangular blocky structure; friable; Strong brown (7.5YR) coatings on all ped faces; abrupt irregular boundary.

BC\&C 30 to $50 \mathrm{~cm}$; light olive brown (2.5Y $5 / 4)$ fragment interiors; soil matrix is yellowish red (5YR 4/6) very shaly clay. BC has some weak soil structure. the $C$ has all rock structure; abrupt irregular boundary.

$\mathrm{C} \& \mathrm{Cr} \quad 50$ to $80 \mathrm{~cm}$; olive brown (2.5Y 4/4) fragment interiors; $\mathrm{Cr}$ fragments coated with black manganese oxides; C horizon fragments coated with yellowish red (5YR 4/6) and strong brown (7.5YR 5/6) materials.

NOTES: The BC\&C and C\&Cr horizons occur in thin strata that have geologic strike and dip. It was not feasible to describe each separately, nor could they be individually sampled.

Sampling depths: A, 0 to $15 \mathrm{~cm} ; \mathrm{B}, 15$ to $30 \mathrm{~cm} ; C, 30$ to $60 \mathrm{~cm}$. The field duplicate samples, because they were sampled from another side of the pit have different depths as follows: A, 0 to $15 \mathrm{~cm} ; \mathrm{B}, 18$ to $36 \mathrm{~cm}$; and C, 20 to $40 \mathrm{~cm}$. 


\section{SITE NUMBER \\ ORR-27}

See pages $8,9,25,32,91$ in phase 1 field book No. 1 .

Location: Oak Ridge Reservation, Bear Creek area. $100 \mathrm{ft}$ north of TVA power line right-of-way. Grid N30100, E34990.

Classification: Ruptic Ultic Dystrochrepts; clayey $\mathrm{Bt}$ and loamy-skeletal $\mathrm{Bw}, \mathrm{C}$, and $\mathrm{Cr}$ horizons; mixed, thermic.

Geomorphic Position: Mid side slope with slight convexity

Slope and Aspect: 7\%, Southwest.

Parent Material(s): Upper Dismal Gap Formation.

Vegetation: Old-field regrowth of red and black oak, chestnut oak, white oak, a few virginia pine, some dead cedars. Understory of beech, oak, red maple, dogwood, and a few green herbs. Ground covered $100 \%$ by leaves.

Described By: D. A. Lietzke.

Sampling Crew: Lietzke, Wilson, Phillips, Farmer.

Rad Scan: Air 10; Ground level 15.

Previous Weather: Rain and snow showers on March 14th and 15th.

Weather Today: Clear, 18 to 20 degrees in morning.

Date: March 16, 1992.

\section{Soil Description}

$\mathrm{Oi} \quad 4$ to $0 \mathrm{~cm}$; leaf litter.

A1 0 to $5 \mathrm{~cm}$; very dark grayish brown (10YR 3/2) silt loam; strong fine granular structure; very friable; many fine roots; abrupt wavy boundary.

Ap 5 to $10 \mathrm{~cm}$; dark brown (10YR 3/3) silt loam; moderate fine granular structure; very friable; common fine roots; abrupt wavy boundary.

AB 10 to $23 \mathrm{~cm}$; yellowish brown (10YR 5/4) silt loam; weak fine subangular blocky structure; few medium roots; clear wavy boundary.

Bt 23 to $34 \mathrm{~cm}$; strong brown (7.5YR 4/6) silty clay loam; moderate medium subangular blocky structure; few roots; abrupt irregular boundary.

C $\quad 34$ to $74 \mathrm{~cm}$; olive brown (2.5Y $4 / 4-5 / 4)$ fragment interiors; material crushes to a shaly or very shaly silty clay loam; yellowish red (5YR 4/6) clay coatings on some fragment faces while other fragment faces coated with black manganese oxides in the lower part of the horizon; more than $70 \%$ rock controlled structure; abrupt irregular boundary.

$\mathrm{Cr} \quad 74$ to $80 \mathrm{~cm}$; olive brown (2.5Y 4/4) fragment interiors; dark red (2.5YR 3/6) and black coatings on most fragment faces.

Notes: Depth to the $\mathrm{Cr}$ varied from 45 to $74 \mathrm{~cm}$ across the face that was sampled. Only the Argillic part of the soil was described and sampled. One side of the pit had $\mathrm{Cr}$ materials coming to within $20 \mathrm{~cm}$ of the surface. This old-field site was abandoned some time before the AEC takeover and had already reverted to hardwoods.

Depths of sampling for ESD composites are: A, 0 to $10 \mathrm{~cm} ; \mathrm{B}, 23$ to $34 \mathrm{~cm} ; \mathrm{C}, 34$ to $53 \mathrm{~cm}$. 


\section{SITE NUMBER \\ ORR-28}

See pages 103, 107, 129 in phase 1 field book No. 1, and pages 152 and 153 in phase 1 field book No. 2.

Location: Oak Ridge Reservation. Grid: N29500, E34580. About $20 \mathrm{ft}$ grid west of edge of power line ROW.

Classification: Ruptic Ultic Dystrochrepts; clayey $\mathrm{Bt}$, loamy-skeletal $\mathrm{Bw}, \mathrm{C}$ and $\mathrm{Cr}$ horizons; mixed, thermic.

Geomorphic Position: Mid sideslope with convexity. Severely eroded before abandonment.

Slope and Aspect: $5 \%$ Southeast.

Parent Material(s): Middle Nolichucky Shale.

Vegetation: Old field successional forest where older pines have been harvested. Present vegetation of young pines, cedars and poplar. Sparse ground cover of honeysuckle, mosses and ferns.

Described By. D. A. Lietzke.

Sampling Crew: Lietzke, Burgoa.

Rad Scan: Air 45; Ground level 60.

Weather Yesterday: Clear and warm.

Present Weather: Clear and warm.

Date: July 21, 1992.

\section{Soil Description}

Oi $\quad 2$ to $0 \mathrm{~cm}$; mosses, pine needles, leaves and root mat.

A $\quad 0$ to $2 \mathrm{~cm}$; brown (10YR 4/3) silt loam or shaly silt loam; strong fine granular structure; very friable; abrupt wavy boundary.

Ap 2 to $16 \mathrm{~cm}$; yellowish brown (10YR $5 / 4$ ) silt loam or shaly silt loam; moderate fine granular structure; very friable; abrupt wavy boundary.

Bt 16 to $25 \mathrm{~cm}$; yellowish brown (10YR 5/8) shaly clay; moderate medium subangular blocky structure; friable; strong brown (7.5YR 5/6) ped coatings; abrupt irregular boundary.

C 25 to $35 \mathrm{~cm}$; light olive brown (2.5Y 5/4) fragment interiors; red (2.5YR 4/6) iron-clay coatings on some fragment surfaces; other areas plugged with yellowish red (5YR 5/4) clay; abrupt irregular boundary.

$\mathrm{Cr} \quad 35$ to $52 \mathrm{~cm}$; brown (10YR 4/3) fragment interiors; most surface coated with red and yellowish red clay and iron oxides.

NOTES: If the Bt horizon is continuous around all four sides of the pit the soil is classified as a Typic Hapludult. If the Bt horizon is interrupted by a Bw, C or $\mathrm{Cr}$ horizon then the soil is classified as a Ruptic Ultic Dystrochrept.

Sampling depths are: A, 0 to $16 \mathrm{~cm}$; B, 16 to $25 \mathrm{~cm}$ C, 26 to $35 \mathrm{~cm}$.

Depths of sampling for ESD composites are: A, 0 to $10 \mathrm{~cm} ; \mathrm{B}, 23$ to $34 \mathrm{~cm}$; , 34 to $53 \mathrm{~cm}$. 


\section{SITE NUMBER}

ORR-31

See pages $103,113,114,156,157$ in phase 1 field book No. 1.

Location: Oak Ridge Reservation. Grid: N29900, E35820.

Classification: Typic Hapludults; clayey, mixed. thermic.

Geomorphic Position: Lower part of long slightly convex sideslope.

Slope and Aspect: 3\% Southeast.

Parent Material(s): Upper Nolichucky Shale.

Vegetation: Old field successional forest. The older pines have been harvested in the past 10 to $15 \mathrm{y}$. Dense regrowth of sweet gum, dogwood, cedar, hickory, poplar, and ash. Sparse ground cover of honeysuckle and poison ivy.

Described By: D. A. Lietzke.

Sampling Crewr. Lietzke, Burgoa.

Rad Scan: Air 35 ; Ground level 60 .

Weather Yesterday: Clear and warm.

Present Weather. Partly cloudy, very warm and humid.

Date: July 21, 1992.

\section{Soil Description}

Oi $\quad 2$ to $0 \mathrm{~cm}$; leaf litter.

A $\quad 0$ to $8 \mathrm{~cm}$; brown (10YR 4/3) silt loam; strong fine granular structure; very friable; abrupt wavy boundary.

Ap 8 to $19 \mathrm{~cm}$; yellowish brown (10YR 5/4) silt loam; moderate fine granular structure; friable; abrupt wavy boundary.

Bt 19 to $40 \mathrm{~cm}$; dark yellowish brown (10YR 4/6) silty clay; moderate medium subangular blocky structure; friable; dark yellowish brown (10YR 4/4) ped coatings; clear wavy boundary.

C 40 to $61 \mathrm{~cm}$; yellowish brown (10YR 5/4) silt loam or silty clay loam; weak coarse subangular blocky structure; firm; light brownish gray (10YR 6/2) wetness mottles; strong brown (7.5YR 4/4) ped zones, but most peds are not coated; abrupt irregular boundary.

$2 \mathrm{Cr} \quad 61$ to $70 \mathrm{~cm}$; dark reddish gray (5YR 4/2) fragment interiors, with surfaces coated with black manganese oxides.

NOTES: The upper $61 \mathrm{~cm}$ appears to be colluvium of entirely Nolichucky origins and of early Holocene age.

Sampling depths are: A, 0 to $19 \mathrm{~cm}$ B, 19 to $40 \mathrm{~cm}$;, 40 to $55 \mathrm{~cm}$. 


\section{SITE NUMBER}

\section{ORR-32}

See pages $25,33,35,37,50,51,91$ in phase 1 field book No. 1 .

Location: Oak Ridge Reservation. About $100 \mathrm{ft}$ grid north of TVA power line ROW. Grid N30200, E36760.

Classification: Ruptic Ultic Dystrochrepts; clayey $\mathrm{Bt}$ and loamy-skeletal, $\mathrm{Bw}, \mathrm{C}$ and $\mathrm{Cr}$ horizons; mixed, thermic.

Geomorphic Position: Mid point of long convex sideslope.

Slope and Aspect: 12\% South.

Parent Material(s): Upper Dismal Gap Formation.

Vegetation: Old-field. Pines have almost all died and fallen over. Present vegetation is thick hardwood underbrush and abundant honeysuckle.

Described By: D. A. Lietzke.

Sampling Crew: Lietzke, Wilson, Farmer

Rad Scan: Air 15; Ground level 40.

Weather Yesterday: Cloudy, cool, widely scattered showers.

Present Weather: Cloudy, cooler with clearing in the afternoon.

Date: April 28, 1992.

\section{Soil Description}

Oi 4 to $0 \mathrm{~cm}$; leaf litter and root mat.

A 0 to $5 \mathrm{~cm}$; dark brown (10YR 3/3) silt loam; strong fine granular structure; very friable; abrupt wavy boundary.

Ap 5 to $20 \mathrm{~cm}$; dark yellowish brown (1/,YR 4/4) silt loam or shaly silt loam; moderate fine granular structure; very friable; abrupt wavy boundary.

Bt 20 to $32 \mathrm{~cm}$; yellowish red (5YR 4/6) silty clay or clay; moderate medium subangular blocky structure; strong brown (7.5YR 5/4) clay coatings on all ped surfaces; clear irregular boundary.

C $\quad 32$ to $53 \mathrm{~cm}$; light olive brown (2.5Y 5/6) fragment intericis coated with brown (7.5YR 4/4) clay; 60 to $80 \%$ rock controlled structure; clay plugged; abrupt irregular boundary.

Cr 53 to $64 \mathrm{~cm}$; light olive brown (2.5Y 5/6) fragment interiors; many fragment faces coated with black manganese oxides; some fragment faces coated with red (2.5YR $4 / 6$ ) iron-clay materials; some strong brown (7.5YR 4/6) clay in thin flow zone seams.

NOTES: Sampling depths: A, 0 to $20 \mathrm{~cm} ; \mathrm{B}, 20$ to $32 \mathrm{~cm} ; \mathrm{C}, 32$ to $53 \mathrm{~cm}$. 


\section{STIE NUMBER}

ORR-33

See pages $25,35,37,62,63,91$ in phase 1 field book No. 1 .

Location: Oak Ridge Reservation. Grid N30000, E36970.

Classification: Ruptic Ultic Dystrochrepts; Clayey Bt, loamy-skeletal Bw, C, and Cr horizons, mixed, thermic.

Geomorphic Position: Middie of slightly convex lower sideslope.

Slope and Aspect: 13\% South Southwest.

Parent Material(s): Middle Dismal Gap Formation.

Vegetation: Original old-field successional forest of pines has died and fallen over. Present vegetation consists of dogwood, beech, sweet gum, poplar, red maple, pine, with a thick ground cover of honeysuckle.

Describea By. D. A. Lietzke.

Sampling Crew: Lietzke, Wilson.

Rad Scan: Air 25; Ground level 40.

Weather Yesterday: Early morning showers then clearing and very windy.

Present Weather: Clear, sunny afternoon with a light breeze.

Date: May 4, 1992.

\section{Soil Description}

Oi $\quad 7$ to $0 \mathrm{~cm}$; leaf litter, twigs and small branches, and a dense root mat.

A $\quad 0$ to $5 \mathrm{~cm}$; dark yellowish brown (10YR 4/4) silt loam; strong fine granular structure; very friable; abrupt wavy boundary.

Ap 5 to $12 \mathrm{~cm}$; yellowish brown (10YR 5/6) shaly silt loam; moderate fine granular structure; friable; abrupt wavy boundary.

Bt 12 to $27 \mathrm{~cm}$; yellowish red (5YR 5/6) clay; moderate medium subangular blocky structure; firm; strong brown (7.5YR 5/6) clay films on all ped faces; clear wavy boundary.

BC 27 to 53 cra; strong brown (7.5YR 5/6) shaly or very shaly silty clay loam; weak coarse subangular blocky structure; firm; 10 to $50 \%$ rock structure in thin harder strata; abrupt irregular boundary.

C 53 to $63 \mathrm{~cm}$; olive brown (2.5Y 4/4) fragment interiors; thick strong brown (7.5YR $5 / 6)$ contings on most fragment surfaces and plugging cracks and fractures; abrupt irregular boundary.

$\mathrm{Cr} \quad 63$ to $80 \mathrm{~cm}$; light olive brown (2.5Y 5/6) fragment interiors; surfaces coated with hlack manganese oxides or yellowish brown (10YR 5/8) iron hydroxides.

NOTES: Sampling depths are: A, 0 to $12 \mathrm{~cm} ; \mathrm{B}, 12$ to $27 \mathrm{~cm} ; \mathrm{C}, 53$ to $63 \mathrm{~cm}$. 
SITE NUMBER

ORR-35

See pages 28, 37, 39, 58, 59, 91 in phase 1 field book No. 1.

Location: Oak Ridge Reservation. Grid: N30000, E37280. Site is located about $150 \mathrm{ft}$ north of cleared edge of ROYY.

Classification: Typic Hapludults; clayey, mixed, thermic.

Geomorphic Position: Convex area just below rest of ridge.

Slope and Aspect: $13 \%$ South Southwest.

Parent Material(s): Lower Dismal Gap Formation.

Vegetation: Old field successional forest where most pines have died and fallen or were harvested. Present vegetation is stveet gum, dogwood, haw, sourwood with some poplar. About $90 \%$ of ground surface is leaf covered the remainder is covered by honeysuckle, mosses and other forbes.

Describ- : By: D. A. Lietzke.

Sampling Crew: Lietzke, Wilson, Farmer.

Rad. Scan: Air 15; Ground level 35.

Weather Yesterday: Cloudy with early morning showers, then clearing and windy in the afternoon.

Present Weather. Clear and windy.

Date: May 4, 1992.

\section{Soil Description}

$\mathrm{Oi} \quad 2$ to $0 \mathrm{~cm}$; leaf litter.

A $\quad 0$ to $8 \mathrm{~cm}$; dark brown (10YR 3/3) silt loam; strong fine granular structure; very friable; abrupt wavy boundary.

Ap 8 to $19 \mathrm{~cm}$; brown (10YR 5/3) silt loam; weak fine granular structure; very friable; abrupt wavy boundary.

E\&B 19 to $31 \mathrm{~cm}$; yellowish brown (10YR 5/4) silt loam, E-part, and strong brown (7.5YR 5/6) silty clay loam, B-part; weak medium subangular blocky structure; very friable; abrupt irregular boundary.

Bt $\quad 31$ to $52 \mathrm{~cm}$; strong brown (7.5YR 4/6) clay; moderate medium subangular blocky structure; friable; peds coated with yellowish red (5YR 4/6) clay; abrupt irregular boundary.

BC 52 to $63 \mathrm{~cm}$; strong brown (7.5YR 4/6) shaly clay; weak medium subangular blocky structure; with about 10 to $25 \%$ rock structure remaining; highly clay plugged. Abrupt irregular boundary.

$\mathrm{C} \& \mathrm{Cr} 63$ to $80 \mathrm{~cm}$; dark yellowish brown (10YR 4/6) fragment interiors; some fragment surface coated with yellowish red (5YR 4/6) clay and others coated with red (2.5YR $4 / 6)$ iron-clay materials.

NOTES: Sampling depths are: A, 0 to $19 \mathrm{~cm} ; \mathrm{B}, 31$ to $52 \mathrm{~cm} ; \mathrm{C}, 63$ to $80 \mathrm{~cm}$. 


\section{STTE NUMBER ORR-41}

See pages 12, 13, 28, 29, 91 in phase 1 field book No. 1.

Location: Oak Ridge Reservation. About $400 \mathrm{ft}$ north of the TVA ROW. Grid N30200, E39570.

Classification: Typic Hapludults; clayey, mixed, thermic.

Geomorphic Position: Convex summit of small ridge. Soils were severely eroded prior to or after abandonment.

Slope and Aspect: 2-3\%.

Parent Material(s): Middle Dismal Gap Formation.

Vegetation: Old-field successional forest. Most older pines have died and fallen over. Present regrowth of pines red maple, beech and poplar. Ground surface covered with leaves.

Described By: D. A. Lietzke.

Sampling Crew: Lietzke, Wilson, Phillips, Farmer

Rad Scan: Air 10; Ground level 50.

Weather Yesterday: Partly cloudy.

Present Weather. Clear and warmer.

Date: March 17, 1992.

\section{Soil Description}

Oi $\quad 4$ to $0 \mathrm{~cm}$; leaf litter.

A $\quad 0$ to $5 \mathrm{~cm}$; very dark grayish brown (10YR 3/2) silt loam; moderate fine granular structure; very friable; many fine roots; abrupt wavy boundary.

Ap 5 to $12 \mathrm{~cm}$; dark yellowish brown (10YR 4/4) heavy silt loam; weak fine granular structure; very friable; common fine roots; abrupt wavy boundary.

Bt 12 to $28 \mathrm{~cm}$; strong brown (7.5YR 4/6) silty clay loam or silty clay; moderate medium subangular blocky structure; friable; common medium roots and common fine roots; strong brown (7.5YR 5/6) clay flows on ped faces; clear wayy boundary.

BC 28 to $40 \mathrm{~cm}$; strong brown (7.5YR $5 / 6)$ shaly silty clay or shaly clay; weak coarse subangular blocky structure; some areas without soil structure; firm; few fine roots; clay plugged with abrupt irregular boundary.

C 40 to $64 \mathrm{~cm}$; strong brown (7.5YR 4/6) and yellowish brown (10YR 5/8) fragment interiors; Material crushes easily to a shaly or very shaly silty clay loam; 60 to $80 \%$ rock controlled structure; friable; few fine roots; considerable strong brown (7.5YR 4/6) clay on fragment surfaces; abrupt irregular boundary.

$\mathrm{Cr} 164$ to $80 \mathrm{~cm}$; harder fragment interiors of strong brown (7.5YR 4/6) and yellowish brown (10YR 5/8); most fragments coated with dark red (10R 3/6) iron oxides.

$\mathrm{Cr} 280$ to $90 \mathrm{~cm}$; harder fragment interiors of light olive gray $(5 \mathrm{Y} 6 / 2)$ and yellowish brown (10YR 5/8) with a few red coatings; most fragments are wet and this horizon is at the top of a perched water zone.

NOTES: Sampling depths for ESD composites are: A, 0 to $12 \mathrm{~cm} ; \mathrm{B}, 14$ to $28 \mathrm{~cm} ; \mathrm{C}, 50$ to $60 \mathrm{~cm}$. 


\section{SITE NUMBER}

ORR-42

See pages 105, 111, 143, 154, 155 in phase 1 field book No. 1.

Location: Oak Ridge Reservation. Grid: N30000, E40500. Site in woods about $30 \mathrm{ft}$ from edge of power line ROW.

Classification: Ruptic Ultic Dystrochrepts; clayey Bt, loamy-skeletal Bw, C, Cr horizon; mixed, thermic.

Geomorphic Position: Long, gentle, convex sideslope.

Slope and Aspect: 3\% South.

Parent Material(s): Middle to upper Nolichucky Shale.

Vegetation: Old field successional where most older pines have died and fallen over or were harvested. Dense regrowth of poplar, sweet gum, cedar, oak, dogwood, and beech. Sparse honey suckle and poison ivy ground cover.

Described By: D. A. Lietzke.

Sampling Crew: Lietzke, Burgoa.

Rad. Scan: Air 30; Ground level 90.

Weather Yesterday: Clear and warm.

Present Weather: Clear and warm.

Date: July 21, 1992.

\section{Soil Description}

Oi $\quad 2$ to $0 \mathrm{~cm}$; leaves.

Oa $\quad 0$ to $1 \mathrm{~cm}$; hemic materials, sapric materials and root mat.

Ap 1 to $15 \mathrm{~cm}$; yellowish brown (10YR 5/4) silt loam or shaly silt loam; moderate fine granular structure; very friable; abrupt wavy boundary.

Bt 15 to $33 \mathrm{~cm}$; dark yellowish brown (10YR 4/4) shaly clay; moderate medium subangular blocky structure; firm; all peds coated with brown (10YR 5/3) material; abrupt irregular boundary.

C $\quad 33$ to $47 \mathrm{~cm}$; mottled dark yellowish brown (10YR 4/4), brown (10YR 5/3) and grayish brown (10YR 5/2) wetness streaks; extremely shaly clay; all rock controlled structure; abrupt irregular boundary.

Cr 47 to $56 \mathrm{~cm}$; brown (10YR 4/3) fragment interiors; red (10R 4/6) and black coatings on most fragment surfaces; some grayish brown (10YR $5 / 2)$ in flow zones.

NOTES: The Bw and Bt were sampled together. The Bw had similar morphology but higher fragment content.

This site has limestone seams 2 to $5 \mathrm{~cm}$ thick that have weathered out to a yellowish brown clay. Some of this clayey material was included in the very shaly $\mathrm{C}$ horizon sample.

Sampling depths are: A, 1 to $15 \mathrm{~cm}$; VB, 15 to $33 \mathrm{~cm}$;, 33 to $47 \mathrm{~cm}$. 


\section{SITE NUMBER}

ORR-43

See pages $28,82,83,86,87,91$ in phase 1 field book No. 1 .

Location: Oak Ridge Reservation. Grid N30400, E41460. About $200 \mathrm{ft}$ grid north of edge of ROW.

Classification: Typic Hapludults; clayey, mixed, thermic.

Geomorphic Position: Broad, slightly convex sideslope.

Slope and Aspect: 2-3\% South Southwest.

Parent Material(s): Lower Dismal Gap Formation within transition zone to Rogersville Shale. Vegetation: Old field hardwoods of red maple, poplar, abundant dogwood, beech, with thick honeysuckle, and poison ivy groundcover.

Described By: D. A. Lietzke.

Sampling Crew: Lietzke, Wilson.

Rad. Scan: Air 20; Ground level 55.

Weather Yesterday: Clear, warmer. Last rain on May 9th.

Present Weather: Clear and warm.

Date: May 11, 1992.

\section{Soil Description}

Oi $\quad 4$ to $0 \mathrm{~cm}$; leaf litter.

A 0 to $6 \mathrm{~cm}$; dark brown (10YR 3/3) silt loam; strong fine granular structure; very friable; abrupt wavy boundary.

Ap 6 to $15 \mathrm{~cm}$; brown (10YR 5/3) silt loam or shaly silt loam; moderate fine granular structure; very friable; abrupt wavy boundary.

Bt1 15 to $37 \mathrm{~cm}$; strong brown (7.5YR 4/6) clay or silty clay; moderate medium subangular blocky structure; firm; clear wavy boundary.

Bt2 37 to $51 \mathrm{~cm}$; strong brown (7.5 YR 4/6) shaly silty clay; moderate medium and coarse subangular blocky structure; firm; Manganese oxide plugged at base of horizon; abrupt irregular boundary.

C 51 to $70 \mathrm{~cm}$; light olive brown fragment interiors; very soft saprolite with yellowish red (2.5 YR 4/6) clay streaks and yellowish red (5YR 4/6) soil material coating some fragment surfaces and filling spaces.

NOTES: Sampling depths: A, 0 to $15 \mathrm{~cm} ; \mathrm{B}, 15$ to $37 \mathrm{~cm}$;, 51 to $70 \mathrm{~cm}$.

Sampling depths for ORR-43 field duplicates are: A, 0 to $15 \mathrm{~cm} ; \mathrm{B}, 15$ to $40 \mathrm{~cm}$;, 53 to $70 \mathrm{~cm}$. The reason for the different depths is that strike and dip oriented subsoil and substratum horizons vary around the sides of a pit. 


\section{AS ROANE COUNTY SOIL PROFILES}

\section{STTE NUMBER}

ROANE No. 3

Location: Pattie Gap Quadrangle, Bowater property, $550 \mathrm{ft}$ northwest from Salem Baptist Church, $200 \mathrm{ft}$ southeast from power line, $350 \mathrm{ft}$ west of creek.

Classification: Typic Hapludults; fine-loamy, mixed, thermic.

Control Section: 31 to $81 \mathrm{~cm}$ of the argillic horizon.

Geomorphic Position: Convex toeslope position.

Slope and Aspect: 11\% Southeast.

Parent Material(s): Thin colluvial mantle over Dismal Gap Formation.

Vegetation: Oak, red maple, sweetgum, dogwood, poison ivy.

Described By. J. L. Branson.

Sampling Crew: Branson, Timpson, Morris.

Time Since Last Rain: 0 days.

Present Weather: $75^{\circ} \mathrm{F}$ raining during sampling.

Date: May 7, 1992.

\section{Soil Description}

Oi $\quad 4$ to $0 \mathrm{~cm}$; leaves and pine needles.

Ap 0 to $15 \mathrm{~cm}$; dark yellowish brown (10YR 4/4) loam; moderate medium granular structure; friable; clear wavy boundary.

BE 15 to $31 \mathrm{~cm}$; strong brown (7.5YR 5/6) and dark yellowish brown (10YR 4/4) silt loam; moderate medium subangular blocky structure; friable; clear wavy boundary.

Bt1 31 to $53 \mathrm{~cm}$; yellowish brown (10YR 5/6) clay loam; weak coarse subangular blocky structure; firm; gradual wavy boundary.

$\mathrm{Bt} 253$ to $85 \mathrm{~cm}$; mottled yellowish brown (10YR 5/6), red (2.5YR 4/6), and light yellowish brown ( $2.5 \mathrm{Y} 6 / 4)$ clay loam; moderate medium subangular blocky structure; firm; gradual smooth boundary.

C $85+\mathrm{cm}$; mottled yellowish brown (10YR 5/6), dark red (2.5YR 3/6), and light brownish gray (2.5Y 6/2) loam; rock controlled structure; firm.

NOTES: This site is on a footslope in an area that is highly dissected. The area around the site has numerous rills and gullies. This area shows no evidence of having been cleared or farmed within the last $50 \mathrm{y}$. The area $300 \mathrm{ft}$ east of this site has been clearcut by Bowater within the last 60 days. 


\section{SITE NUMBER \\ ROANE No. 7}

Location: Pattie Gap Quadrangle, John and Imogene Walker property, $400 \mathrm{ft}$ west from Dry Fork Valley Road, $50 \mathrm{ft}$ south from the property line between the Walker's and Kenneth Sutton's tobacco patch, 300 northeast from the Walker's house.

Classification: Typic Hapludults; fine-loamy, mixed, thermic.

Control Section: 16 to $56 \mathrm{~cm}$ of the argillic horizon.

Geomorphic Position: Upland.

Slope and Aspect: 4\% South.

Parent Material(s): Colluvium over Dismal Gap Formation.

Vegetation: Virginia pine, red maple, dogwood, tulip poplar.

Described By: J. L. Branson.

Sampling Crew: Branson, Livingston.

Time Since Last Rain: 1 day.

Present Weather: $65^{\circ} \mathrm{F}$ cloudy, sprinkled during sampling.

Date: May 6, 1992.

\section{Soil Description}

A 0 to $6 \mathrm{~cm}$; very dark grayish brown (10YR 3/2) silt loam; moderate fine granular structure; friable; abrupt smooth boundary.

Ap 6 to $16 \mathrm{~cm}$; yellowish brown (10YR 5/4) silt loam; weak fine subangular blocky structure; friable; clear smooth boundary.

Bt1 16 to $44 \mathrm{~cm}$; yellowish brown (10YR 5/6) silty clay loam; moderate medium subangular blocky structure; friable; clear smooth boundary.

2Bt2 44 to $56 \mathrm{~cm}$; mottled red (2.5YR 4/6), strong brown (7.5YR 4/6), and light brownish gray (2.5Y 6/2) clay; weak medium subangular blocky structure; firm; gradual smooth boundary.

2C 56 to $93 \mathrm{~cm}$; mottled: red (2.5YR 4/6), strong brown (7.5YR 5/6), and light brownish gray (2.5Y 6/2) clay; rock controlled structure; firm; clear wavy boundary.

$2 \mathrm{Cr} \quad 93+\mathrm{cm}$; soft red shale fragments with some olive and yellow shale

NOTES: This site is in a group of deciduous trees surrounded by cattle pasture. Indications are that area that had been cleared at one time and plowed. The owners have assured me this area has not been cleared or cropped in the last $50 \mathrm{y}$. There is evidence of erosion with small rills beginning to form and shallow surface horizons. Presently the land is open to cattle. 


\section{SITE NUMBER \\ ROANE No. 8}

Location: Pattie Gap Quadrangle, John and Imogene Walker property, $550 \mathrm{ft}$ west from Dry Fork Valley Road, $70 \mathrm{ft}$ south from the property line between the Walker's and Kenneth Sutton's tobacco patch, 250 northeast from the Walker's house.

Classification: Typic Hapludults; fine-loamy, mixed, thermic.

Control Section: 17 to $67 \mathrm{~cm}$ of the argillic horizon.

Geomorphic Position: Upland.

Slope and Aspect: 6\% South.

Parent Material(s): Colluvium over Dismal Gap Formation.

Vegetation: Virginia pine, red maple, dogwood, sweetgum.

Described By: J. L. Branson.

Sampling Crew: Branson, Livingston.

Time Since Last Rain: 1 day.

Present Weather: $50^{\circ} \mathrm{F}$ cloudy, overcast.

Date: May 6, 1992.

\section{Soil Description}

A $\quad 0$ to $5 \mathrm{~cm}$; very dark grayish brown (10YR $3 / 2)$ silt loam; strong fine granular structure; very friable; abrupt smooth boundary.

Ap 5 to $17 \mathrm{~cm}$; yellowish brown (10YR 5/6) silt loam; moderate fine granular structure; very friable; clear smooth boundary.

Bt1 17 to $44 \mathrm{~cm}$; strong brown (7.5YR 5/6) silty clay loam; moderate medium subangular blocky structure; friable; gradual smooth boundary.

2Bt2 44 to $80 \mathrm{~cm}$; strong brown (7.5YR 5/6) clay; moderate medium angular blocky structure; firm; gradual smooth boundary.

2C 80 to $95 \mathrm{~cm}$; mottled red (2.5YR 4/6), yellowish brown (5YR 4/6), and light reddish brown (2.5YR 6/4) clay; rock controlled structure; very firm consistency.

NOTES: This site is in a group of deciduous trees surrounded by cattle pasture. Indications are that area that had been cleared at one time and plowed. The owners have assured me this area has not been cleared or cropped in the last $50 \mathrm{y}$. There is evidence of erosion with small rills beginning to form and shallow surface horizons. Presently the land is open to cattle. 


\section{A-42 \\ SITE NUMBER \\ ROANE No. 9}

Location: Pattie Gap Quadrangle, Bowater property, $500 \mathrm{ft}$ northwest from Salem Baptist Church, $200 \mathrm{ft}$ southeast frum power line, $500 \mathrm{ft}$ west of creek.

Classification: Ochreptic Hapludults; fine-loamy, mixed, thermic, shallow.

Control Section: 30 to $52 \mathrm{~cm}$ of the argillic horizon.

Geomorphic Position: Colluvial footslope position.

Slope and Aspect: 12\% Southeast.

Parent Material(8): Dismal Gap Formation.

Vegetation: Oak hardwoods mixed with Virginia pine.

Described By. J. L. Branson.

Sampling Crew. Branson, Timpson.

Time Since Last Rain: 3 days.

Present Weather: $76^{\circ} \mathrm{F}$ sunny.

Date: April 14, 1992.

\section{Soil Description}

$\mathrm{Oi} \quad 2$ to $0 \mathrm{~cm}$; leaves and twigs.

A 0 to $9 \mathrm{~cm}$; dark grayish brown (10YR 4/2) silt loam or loam; moderate medium subangular blocky structure; very friable; clear wavy boundary.

E 9 to $16 \mathrm{~cm}$; brown (10YR 5/3) silt loam or loam; moderate medium subangular blocky structure; friable; clear wavy boundary.

BE 16 to $30 \mathrm{~cm}$; yellowish brown (10YR 5/6) silt loam; weak medium subangular blocky structure; friable; clear wavy boundary.

Bt $\quad 30$ to $52 \mathrm{~cm}$; yellowish red (5YR 5/6) silty clay loam; weak medium subangular blocky structure; friable consistency; clear wavy boundary.

$\mathrm{Cr} \quad 52+\mathrm{cm}$; soft red shale with some olive and yellowish brown strata.

NOTES: This site is on a footslope in an area that is highly dissected. The area around the site has numerous rills and gullies. This area shows no evidence of having been cleared or farmed within the last $50 \mathrm{y}$. The area $300 \mathrm{ft}$ east of this site has been clearcut by Bowater within the last 60 days. 


\section{SITE NUMBER}

ROANE No. 10

Location: Pattie Gap Quadrangle, Don Manning property, $350 \mathrm{ft}$ northwest from Dry Fork Valley Road, $300 \mathrm{ft}$ east from Gage Road

Classification: Ochreptic Hapludults; fine-loamy, mixed, thermic, shallow.

Control Section: 18 to $28 \mathrm{~cm}$ of the argillic horizon.

Geomorphic Position: Upland bench.

Slope and Aspect: 3\% Southeast.

Parent Material(s): Thin alluvial capping over Dismal Gap Formation.

Vegetation: shortleaf pine, red maple, oak.

Described By: J. L. Branson.

Sampling Crew: Branson, Gallagher, Morris.

Time Since Last Rain: 2 days.

Present Weather: $85^{\circ} \mathrm{F}$ sunny.

Date: May 15, 1992.

\section{Soil Description}

$\mathrm{Oi} \quad 7$ to $5 \mathrm{~cm}$; leaf litter.

Oa $\quad 5$ to $0 \mathrm{~cm}$; decomposed plant material.

Ap 0 to $18 \mathrm{~cm}$; dark yellowish brown (10YR 4/4) silt loam; weak medium granular structure; friable; clear irregular boundary.

2Bt 18 to $28 \mathrm{~cm}$; yellowish brown (10YR 5/4) shaly clay loam; moderate fine subangular blocky structure; firm; clear smooth boundary.

2BC 28 to $40 \mathrm{~cm}$; yellowish brown (10YR 5/4) shaly clay; moderate medium angular blocky structure; firm; clear smooth boundary.

2CB 40 to $62 \mathrm{~cm}$; strong brown (7.5YR 4/6) very shaly clay; moderate medium angular blocky structure; firm; clear smooth boundary.

$2 \mathrm{Cr} 1 \quad 62$ to $83 \mathrm{~cm}$; soft red shale with yellow and olive shale fragments

$2 \mathrm{Cr} 283$ to $108+\mathrm{cm}$; soft green shale with a thick band of manganese stained material and concretions.

NOTES: This site is in a group of deciduous trees surrounded by cattle pasture. Indications were the area had been cleared at one time and plowed. The owners have assured me this area has not been cleared or cropped in the last $50 \mathrm{y}$. The site area is at the bottom of a long slope. This looks to be a terrace remanent. There were rounded rock fragments in the profile and a large rock approximately $20 \mathrm{~cm}$ in diameter. There is evidence of erosion with small rills and gullies forming in the sagegrass field to the north of the site. Presently the land is open to cattle. 


\section{SITE NUMBER}

\section{ROANE No. 13}

Location: Pattie Gap Quadrangle, Don Manning property, $350 \mathrm{ft}$ northwest from Dry Fork Valley Road, $300 \mathrm{ft}$ west from the property line between Bea Wise and Don Manning, $350 \mathrm{ft}$ directly west from Ms. Wise's red barn.

Classification: Typic Hapludults; fine-loamy, mixed, thermic.

Control Section: 13 to $63 \mathrm{~cm}$ of the argillic horizon.

Geomorphic Position: Upland bench.

Slope and Aspect: 3\% East.

Parent Material(s): Dismal Gap Formation.

Vegetation: Virginia pine, red maple, chestnut oak, dogwood, poison ivy.

Described By: J. L. Branson.

Sampling Crew: Branson, Gallagher, Timpson.

Time Since Last Rain: 1 day.

Present Weather: $75^{\circ} \mathrm{F}$ sunny.

Date: May 14, 1992.

\section{Soil Description}

A $\quad 0$ to $2.5 \mathrm{~cm}$; very dark brown (10YR 2/2) loam; strong fine granular structure; friable; abrupt smooth boundary.

Ap $\quad 2.5$ to $13 \mathrm{~cm}$; yellowish brown (10YR 5/4) and brown (10YR 5/3) silt loam; weak fine and medium granular structure; friable; clear wavy boundary.

Bt1 13 to $50 \mathrm{~cm}$; strong brown (7.5YR 4/6) on clay skins and strong brown ped interiors (7.5YR 5/6); clay; moderate fine and medium subangular blocky structure; firm; clear wavy boundary.

Bt2 50 to $78 \mathrm{~cm}$; strong brown (7.5YR 4/6) clay; weak medium and fine subangular blocky structure; firm; clear wavy boundary.

C 78 to $93+\mathrm{cm}$; strong brown (7.5YR 4/6) clay; rock controlled structure; firm.

NOTES: This site is in a wooded area. The site area is at the bottom of a long slope. Because it is in a low position, the possibility of this being an area of terrace smears is likely. However, there were no indications in this profile to substantiate this point. 


\section{SITE NUMBER}

ROANE No. 14

Location: Pattie Gap Quadrangle, Don Manning property. $350 \mathrm{ft}$ northwest from Dry Fork Valley Road, $50 \mathrm{ft}$ west from the property line between Bea Wise and Don Manning, $100 \mathrm{ft}$ directly west from Ms. Wise's red barn.

Classification: Typic Hapludults; fine-loamy, mixed, thermic.

Control Section: 19 to $58 \mathrm{~cm}$ of the argillic horizon.

Geomorphic Position: Upland on a convex sideslope.

Slope and Aspect: 13\% East.

Parent Material(s): Dismal Gap Formation and Rogersville Formation transition zone.

Vegetation: red maple, oak, tulip poplar, dogwood, poison ivy.

Described By: J. L. Branson.

Sampling Crew: Branson, Gallagher, Timpson.

Time Since Last Rain: 1 day.

Present Weather: $80^{\circ} \mathrm{F}$ sunny.

Date: May 14, 1992.

\section{Soil Description}

A $\quad 0$ to $8 \mathrm{~cm}$; very dark brown (10YR 2/2) silt; moderate, medium granular structure; very friable; clear smooth boundary.

Ap 8 to $19 \mathrm{~cm}$; brown (10YR 4/3) loam; moderate medium granular structure; friable; clear wavy boundary.

Bt 19 to $41 \mathrm{~cm}$; strong brown (7.5YR 4/6) silty clay loam; weak medium subangular blocky structure; firm; clear wavy boundary.

Bt\&C 41 to $58 \mathrm{~cm}$; mottled red (2.5YR 4/6), grayish brown (10YR 5/2), and olive brown ( $2.5 \mathrm{Y} 4 / 4)$; channery silty clay; moderate medium subangular blocky structure; firm; gradual wavy boundary.

$\mathrm{Cr} \quad 58$ to $104+\mathrm{cm}$; primarily dark red and olive shale

NOTES: This site is in a wooded area. The site area is in the middle of a short slope. The Dismal Gap is very thin and fluctuates in this area. The site showed some indications of having Dismal Gap residuum, but when the pit was opened there was more Rogersville shale than Dismal Gap. This area has a great deal of groundcover, primarily vines and poison ivy. The area from the site drops immediately into a drainageway. 


\section{SITE NUMBER}

\section{ROANE No. 17}

Location: Pattie Gap Quadrangle, Robert and Mattie Sue Viar property, $500 \mathrm{ft}$ west from Dry Fork Valley Road, $1000 \mathrm{ft}$ north from Waterford Lane

Claseification: Typic Hapludults; clayey, kaolinitic, thermic.

Control Section: 28 to $78 \mathrm{~cm}$ of the argillic horizon.

Geomorphic Position: Upland on the crown of a convex slope.

Slope and Aspect: 2\% East.

Parent Material(8): Dismal Gap Formation.

Vegetation: Virginia pine, eastern red cedar, sweetgum.

Described By: J. L. Branson.

Sampling Crew: Branson, Timpson.

Time Since Laet Rain: 1 day.

Present Weather. $65^{\circ} \mathrm{F}$ cloudy, light rain.

Date: April 20, 1992.

\section{Soil Description}

Oi $\quad 2$ to $0 \mathrm{~cm}$; pine needles, leaves, and twigs

A $\quad 0$ to $15 \mathrm{~cm}$; yellowish brown (10YR 5/4) silt loam; weak fine granular structure; friable; clear smooth boundary.

$\mathrm{AB} \quad 15$ to $28 \mathrm{~cm}$; dark yellowish brown (10YR 4/6) silt loam; moderate fine subangular blocky structure; friable; clear smooth boundary.

Bt1 28 to $60 \mathrm{~cm}$; yellowish red (5YR 4/6) clay; moderate medium subangular blocky structure; friable; gradual smooth boundary.

Bt2 60 to $80 \mathrm{~cm}$; yellowish red (5YR 4/6) clay; moderate medium subangular blocky structure; friable; clear smooth boundary.

C 80 to $104+\mathrm{cm}$; mottled yellowish red (5YR 4/6), strong brown (7.5YR 4/6), and light olive brown (2.5Y 5/4) clay loam; rock controlled structure; friable.

NOTES: This site lies between the convergence of two drains, both of which continually carry at least some flowing water. The site is on a convex slope in an area that had been cleared at one time. The owners have assured me this area has not been cleared or cropped in the last $50 \mathrm{y}$. Presently the land is open to cattle. 


\section{SITE NUMBER}

ROANE No. 19

Location: Pattie Gap Quadrangle, Kenneth Sutton/Bowater property, $750 \mathrm{ft}$ northwest from Salem Baptist Church, $200 \mathrm{ft}$ southeast from power line, $750 \mathrm{ft}$ west of creek.

Classification: Typic Hapludults; fine-loamy, mixed, thermic.

Control Section: 18 to $47 \mathrm{~cm}$ of the argillic horizon.

Geomorphic Position: Convex toeslope position.

Slope and Aspect: $15 \%$ Southeast.

Parent Material(s): Dismal Gap Formation.

Vegetation: Oak, red manle, hickory, dogwood, red cedar.

Described By: J. L. Branson.

Sampling Crew: Branson, Gallagher.

Time Since Last Rain: 2 days.

Present Weather: $80^{\circ} \mathrm{F}$ sunny.

Date: April 23, 1992.

\section{Soil Description}

Oe $\quad 2$ to $0 \mathrm{~cm}$; decomposing leaves

A 0 to $6 \mathrm{~cm}$; very dark grayish brown (10YR 3/2) silt loam; strong fine granular structure; very friable; abrupt smooth boundary.

Ap 6 to $18 \mathrm{~cm}$; dark yellowish brown (10YR 4/4) silt loam; weak medium granular structure; very friable; clear smooth boundary.

Bt 18 to $47 \mathrm{~cm}$; strong brown (7.5YR 4/6) clay loam; moderate medium subangular blocky structure; friable; clear wavy boundary.

$\mathrm{Cr} \quad 47$ to $98+\mathrm{cm}$; soft red shale with yellow and green shale fragments.

NOTES: This site is on a toeslope in an area that is highly dissected. The area around the site has numerous rills and gullies. This area shows no evidence of having been cleared or farmed within the last $50 \mathrm{y}$. We were unsure as to where the boundary between Kenneth Sutton and Bowater was located, but had permission to sample on both properties. 


\section{SITE NUMBER \\ ROANE No. 20}

Location: Pattie Gap Quadrangle, Kenneth Sutton or Bowater property. Site is located $800 \mathrm{ft}$ from Salem Baptist Church, $200 \mathrm{ft}$ southeast of power line and $900 \mathrm{ft}$ west of the creek. Classification: Ochreptic Hapludults; fine-loamy, mixed, thermic, shallow.

Geomorphic Position: Convex toeslope position.

Slope and Aspect: 12\% Southeast.

Parent Material(s): Dismal Gap Formation.

Vegetation: Oak, sweetgum, dogwood, poison ivy.

Described By: J. L. Branson.

Sampling Crew: Branson, Gallagher.

Time Since Last Rain: 1 day.

Present Weather: 60 degrees, partly cloudy.

Date: May 5, 1992.

\section{Soil Description}

Oi $\quad 2$ to $0 \mathrm{~cm}$; leaves ar: ${ }^{2}$,ine needles.

Ap 0 to $12 \mathrm{~cm}$; yellowish brown (10YR 5/4) silt loam; weak medium granular structure; very friable; clear smooth boundary.

Bt 12 tc $35 \mathrm{~cm}$; yellowish red (5YR 4/6) clay; strong medium subangular blocky structure; firm; g-adual smooth boundary.

C 35 to $52 \mathrm{~cm}$; dark red (2.5YR $3 / 6)$ saprolite that crushes easily to clay; rock controlled struc ure; abrupt irregular boundary.

Cr $\quad 52$ to $72 \mathrm{~cm}$; weathered reddish and olive shale. 


\section{SITE NUMBER}

ROANE No. 21

Location: Pattie Gap Quadrangle, Kenneth Sutton/Bowater property, $1100 \mathrm{ft}$ northwest from Salem Baptist Church, $200 \mathrm{ft}$ southeast from power line, $1200 \mathrm{ft}$ west of creek. Classification: Typic Hapludults; fine-loamy, mixed, thermic.

Control Section: 18 to $64 \mathrm{~cm}$ of the argillic horizon.

Geomorphic Position: Midslope.

Slope and Aspect: $13 \%$ Southeast.

Parent Material(s): Dismal Gap Formation.

Vegetation: Oak, tulip poplar, Virginia pine, sweetgum, red maple, club mosses.

Described By: J. L. Branson.

Sampling Crew: Branson, Morris.

Time Since Last Rain: 2 days.

Present Weather: $80^{\circ} \mathrm{F}$ sunny.

Date: May 11, 1992.

\section{Soil Description}

Oi $\quad 3$ to $0 \mathrm{~cm}$; leaves, moss, and pine needles

A $\quad 0$ to $5 \mathrm{~cm}$; brown (10YR 4/3) shaly silty clay loam; moderate, medium granular structure; very friable; clear smooth boundary.

Ap 5 to $18 \mathrm{~cm}$; dark grayish brown (10YR 4/2) silty clay loam; weak medium subangular blocky structure; friable; clear wavy boundary.

Bt1 18 to $36 \mathrm{~cm}$; strong brown (7.5YR 4/6) clay loam; moderate medium subangular blocky structure; firm; clear smooth boundary.

Bt2 36 to $64 \mathrm{~cm}$; strong brown (7.5YR 5/6) clay loam; moderate coarse subangular blocky structure; firm; clear smooth boundary.

BC 64 to $74 \mathrm{~cm}$; brown (7.5YR 5/4) silty clay loam; weak medium subangular blocky structure; friable; gradual smooth boundary.

$\mathrm{Cr} \quad 74+\mathrm{cm}$; soft red shale with some olive and yellowish brown thin strata.

NOTES: This site is on a convex slope in an area that is highly dissected. The area around the site has numerous rills and gullies. This area shows no evidence of having been cleared or farmed within the last $50 \mathrm{y}$. Surfaces nearby show evidence of extensive erosion and shallow depths. We were unsure as to where the boundary between Kenneth Sutton and Bowater was located, but had permission to sample on both properties. 


\section{SITE NUMBER \\ ROANE No. 22}

Location: Pattie Gap Quadrangle, Kenneth Sutton/Bowater property, $1400 \mathrm{ft}$ northwest from Salem Baptist Church, $200 \mathrm{ft}$ southeast from power line, $1500 \mathrm{ft}$ west of creek.

Classification: Typic Hapludults; fine-loamy, mixed, thermic.

Control Section: 19 to $56 \mathrm{~cm}$ of the argillic horizon.

Geomorphic Position: Midslope bench.

Slope and Aspect: 13\% Southeast.

Parent Material(s): Dismal Gap Formation.

Vegetation: Oak, tulip poplar, Virginia pine, red cedar, club mosses.

Described By: J. L. Branson.

Sampling Crew: Branson, Gallagher.

Time Since Last Rain: 1 day.

Present Weather: $60^{\circ} \mathrm{F}$ overcast, sprinkled lightly during sampling.

Date: May 5, 1992.

\section{Soil Description}

Oi $\quad 3$ to $0 \mathrm{~cm}$; leaves and pine needles.

Ap 0 to $19 \mathrm{~cm}$; yellowish brown (10YR 5/6) silt loam; moderate medium subangular blocky structure; friable; clear, smooth boundary.

Bt1 19 to $29 \mathrm{~cm}$; strong brown (7.5YR 5/8) clay loam; moderate medium subangular blocky structure; firm; clear smooth boundary.

Bt2 29 to $45 \mathrm{~cm}$; strong brown (7.5YR 4/6) clay loam; moderate medium subangular blocky structure; firm; clear smooth boundary.

Bt\&C 45 to $56 \mathrm{~cm}$; red (2.5YR 4/6) and light olive brown (2.5Y 5/4) clay; weak coarse subangular blocky structure; firm; clear smooth boundary.

C56 to $100+\mathrm{cm}$; mottled red (2.5YR 4/6), strong brown (7.5YR 5/8), and light yellowish brown (2.5Y 6/4) clay loam; rock controlled structure.

NOTES: This site is on a slight bench in an area that is highly dissected. The area around the site has numerous rills and gullies. This area shows no evidence of having been cleared or farmed within the last 50 y. Surfaces nearby show evidence of extensive erosion and shallow depths. We were unsure as to where the boundary between Kenneth Sutton and Bowater was located, but had permission to sample on both properties. 


\section{A.6 ANDERSON COUNTY SOIL PROFILES}

\section{SITE NUMBER \\ ANDERSON No. 1}

Location: Norris Quadrangle, Marguriet Atkins property, 200 north of Fairview cemetery, $500 \mathrm{ft}$ west from Hinds Creek Road.

Classification: Ochreptic Hapludults; clayey, kaolinitic, thermic, shallow.

Control Section: 28 to $44 \mathrm{~cm}$ argillic horizon.

Geomorphic Position: Midslope.

Slope and Aspect: 6\% North.

Parent Material(s): Dismal Gap Formation.

Vegetation: Virginia pine, tulip poplar, red maple, dogwood.

Described By. J. L. Branson.

Sampling Crew. Branson, Livingston, Gallagher.

Time Since Last Rain: 0 day.

Present Weather. $85^{\circ} \mathrm{F}$ partly cloudy, heavy rain during sampling.

Date: July 17, 1992.

\section{Soil Description}

A $\quad 0$ to $10 \mathrm{~cm}$; dark brown (10YR 3/3) silt loam; weak medium granular structure; very friable; clear smooth boundary.

Ap 10 to $28 \mathrm{~cm}$; yellowish brown (10YR 5/6) loam; weak medium granular structure; friable ; clear wavy boundary.

Bt 28 to $44 \mathrm{~cm}$; yellowish red (5YR 5/6) channery clay; moderate medium subangular blocky structure; firm; clear wavy boundary.

Cr $\quad 44$ to $104 \mathrm{~cm}$; soft olive, red, and yellow shale.

NOTES: This site had been cleared at one time. Age of the deciduous trees indicated they had been established at least $50 \mathrm{y}$. No evidence of a plow layer. Slope dips strongly (30\%) approximately 50 from the site. Presently, this area is occupied by cattle for pasture. 


\section{SITE NUMBER \\ ANDERSON No. 3}

Location: Big Ridge Park Quadrangle, John Irwin property, $100 \mathrm{ft}$ northwest from Judson Road, 0.4 miles south from Moore Valley Road (also known as Cooper Gap).

Classification: Typic Hapludults; fine-loamy, mixed, thermic.

Control Section: 16 to $53 \mathrm{~cm}$ entire argillic horizon.

Geomorphic Position: Upland, convex slope.

Slope and Aspect: 7\% southeast.

Parent Material(s): Dismal Gap Formation.

Vegetation: Virginia Pine, dogwood, red maple, mosses, poison ivy, sumac, red cedar.

Described By: J. L. Branson.

Sampling Crew: Branson, Timpson, Livingston, Gallagher, Entrekin.

Time Since Last Rain: 0 days.

Present Weather: $85^{\circ} \mathrm{F}$ Heavy rain during sampling.

Date: July 15, 1992.

\section{Soil Description}

$\mathrm{Oi} \quad 2$ to $0 \mathrm{~cm}$; moss and pine needles

A 0 to $4 \mathrm{~cm}$; dark brown (10YR $4 / 3$ ) loam; moderate medium granular structure; friable; abrupt smooth boundary.

Ap 4 to $16 \mathrm{~cm}$; yellowish brown (10YR 5/4) loam; moderate fine granular structure; friable; clear smooth boundary.

Bt1 16 to $40 \mathrm{~cm}$; yellowish red (5YR 4/6) clay; moderate medium angular blocky structure; firm; clear wavy boundary.

Bt2 40 to $53 \mathrm{~cm}$; yellowish red (5YR 4/6) clay loam; moderate medium angular blocky structure; firm; clear wavy boundary.

Cr 53 to $90 \mathrm{~cm}$; soft olive, red, and yellow shale 


\section{SITE NUMBER \\ ANDERSON No. 4}

Location: Big Ridge Park Quadrangle, John Irwin property, $300 \mathrm{ft}$ northwest from Judson Road, 0.6 miles south from Moore Valley Road (also known as Cooper Gap).

Classification: Typic Hapludults; fine-loamy, mixed, thermic.

Control Section: 24 to $70 \mathrm{~cm}$ argillic horizon.

Geomorphic Position: Midslope, convex shape.

Slope and Aspect: 24\% Southeast.

Parent Material(s): Dismal Gap Formation.

Vegetation: Virginia pine, dogwood, red maple, elm, poison ivy, cherry, sourwood.

Described By: J. L. Branson.

Sampling Crew: Branson, Timpson, Livingston, Gallagher, Entrekin.

Time Since Last Rain: 4 days.

Present Weather. $85^{\circ} \mathrm{F}$ Cloudy.

Date: July 15, 1992.

\section{Soil Description}

$\mathrm{Oi} \quad 3$ to $0 \mathrm{~cm}$; leaf litter, small roots, and pine needles.

A $\quad 0$ to $5 \mathrm{~cm}$; yellowish brown (10YR 5/4) loam; weak medium granular structure; very friable; abrupt smooth boundary.

Ap 5 to $24 \mathrm{~cm}$; yellowish brown (10YR 5/6) silt loam; weak fine granular structure; friable; abrupt smooth boundary.

Bt1 24 to $37 \mathrm{~cm}$; strong brown (7.5YR 5/6) silty clay loam; moderate fine subangular blocky structure; friable; clear smooth boundary.

Bt2 37 to $53 \mathrm{~cm}$; yellowish red (5YR 4/6) clay loam; moderate medium subangular blocky structure; friable; clear wavy boundary.

$\mathrm{Bt \& Cr} 53$ to $70 \mathrm{~cm}$; Bt: yellowish red (5YR 5/6) clay loam; moderate medium subangular block structure; friable; Cr: soft olive, red, and yellow shale; abrupt wavy boundary.

$\mathrm{Cr} \quad 70$ to $100 \mathrm{~cm}$; soft olive, red, and yellow shale. 


\section{SITE NUMBER}

ANDERSON No. 5

Location: Big Ridge Park Quadrangle, John Irwin property. $300 \mathrm{ft}$ northwest from Judson Road, 0.6 miles south from Moore Valley Road (also known as Cooper. Gap), $300 \mathrm{ft}$ south of Anderson No. 4.

Classification: Typic Hapludults; fine-loamy, mixed, thermic.

Control Section: 6 to $56 \mathrm{~cm}$ of the argillic horizon.

Geomorphic Position: Midslope, convex shape.

Slope and Aspect: 25\% Southeast.

Parent Material(8): Dismal Gap Formation.

Vegetation: Virginia pine, dogwood, oak, poison ivy.

Described By: J. L. Branson.

Sampling Crew: Branson, Timpson, Livingston, Gallagher, Entrekin.

Time Since Last Rain: 4 days.

Present Weather: $85^{\circ} \mathrm{F}$ Cloudy.

Date: July 15, 1992.

\section{Soil Description}

Oi 2 to $0 \mathrm{~cm}$; leaf litter, small roots, and pine needles.

A $\quad 0$ to $6 \mathrm{~cm}$; dark yellowish brown (10YR 4/4) loam; weak medium granular structure; very friable; clear smooth boundary.

Ap 6 to $15 \mathrm{~cm}$; dark yellowish brown (10YR 4/6) loam; weak fine granular structure; friable; clear smooth boundary.

Bt1 15 to $45 \mathrm{~cm}$; yellowish brown (10YR 5/6) clay loam; moderate medium subangular blocky structure; friable; clear smooth boundary.

Bt2 45 to $70 \mathrm{~cm}$; strong brown (7.5YR 5/8) clay loam; moderate medium subangular blocky structure; friable; clear smooth boundary.

$\mathrm{Cr} \quad 70$ to $122 \mathrm{~cm}$; soft olive, red, and yellow shale. 


\section{SITE NUMBER \\ ANDERSON No. 9}

Location: Powell Quadrangle, Bill Lee property, $1300 \mathrm{ft}$ north from Hinds Creek Road, 0.25 miles west from Nolan Road, $300 \mathrm{ft}$ north from Anderson No. 19.

Classification: Ochreptic Hapludults; clayey, kaolinitic, thermic, shallow.

Control Section: 12 to $35 \mathrm{~cm}$ of the argillic horizon.

Geomorphic Position: Middle portion of a convex slope.

Slope and Aspect: 15\% Southeast.

Parent Material(s): Dismal Gap Formation.

Vegetation: Virginia pine, red maple, tulip poplar, dogwood.

Described By. J. L. Branson.

Sampling Crew: Branson, Timpson, Livingston, Gallagher, Entrekin.

Time Since Last Rain: 2 days.

Present Weather: $75^{\circ} \mathrm{F}$ cloudy.

Date: July 17, 1992.

\section{Soil Description}

Oi $\quad 2$ to $0 \mathrm{~cm}$; leaves and pine needles.

A 0 to $5 \mathrm{~cm}$; dark brown (10YR 3/3) loam; moderate fine granular structure; very friable; clear, smooth boundary.

Ap 5 to $12 \mathrm{~cm}$; yellowish brown (10YR 5/4) loam; weak fine and medium granular structure; friable; clear smooth boundary.

Bt 12 to $35 \mathrm{~cm}$; strong brown (7.5YR 5/6) clay; moderate medium and coarse subangular blocky structure; firm; clear wavy boundary.

BC 35 to $50 \mathrm{~cm}$; strong brown (7.5YR 4/6) clay; 60\% of structure is rock controlled; firm; clear wavy boundary.

CB $\quad 50$ to $62 \mathrm{~cm}$; strong brown (7.5YR 5/6) clay loam; $80 \%$ structure is rock controlled; firm; clear, wavy boundary.

Cr $\quad 62$ to $105 \mathrm{~cm}$; soft olive, red, and yellow shale. 


\section{SITE NUMBER \\ ANDERSON No. 10}

Location: Norris Quadrangle, Marguriet Atkins property, $50 \mathrm{ft}$ north of Fairview cemetery, $450 \mathrm{ft}$ west of Hinds Creek Road

Classification: Typic Hapludults; fine-loamy, mixed, thermic.

Control Section:: 40 to $77 \mathrm{~cm}$ argillic horizon.

Geomorphic Position: Upland slightly convex summit.

Slope and Aspect: $1 \%$ northeast.

Parent Material(s): Dismal Gap Formation.

Vegetation: Virginia pine, tulip poplar, red maple, dogwood.

Described By: J. L. Branson.

Sampling Crew: Branson, Livingston, Gallagher.

Time Since Last Rain: 0 day.

Present Weather: $85^{\circ} \mathrm{F}$ partly cloudy, heavy rain during sampling.

Date: July 17, 1992.

\section{Soil Description}

Oi $\quad 2$ to $0 \mathrm{~cm}$; roots and leaf litter.

A $\quad 0$ to $10 \mathrm{~cm}$; very dark grayish brown (10YR 3/2) silt loam; weak medium granular structure; friable; clear wavy boundary.

E 10 to $21 \mathrm{~cm}$; brown (10YR 5/3) silt loam; weak medium granular structure; friable; clear smooth boundary.

BE 21 to $40 \mathrm{~cm}$; yellowish brown (10YR 5/4) and strong brown (7.5YR 5/6) clay loam; weak medium subangular blocky structure; friable; clear smooth boundary.

Bt $\quad 40$ to $77 \mathrm{~cm}$; yellowish red (5YR 5/6) clay loam; weak medium subangular blocky structure; friable; clear smooth boundary.

$\mathrm{Cr} \quad 77$ to $136 \mathrm{~cm}$; soft olive, red, and yellow shale. 


\section{SITE NUMBER \\ ANDERSON No. 11}

Location: Norris Quadrangle, Allen Johnson property, $600 \mathrm{ft}$ northwest from Hinds Creek Road, $200 \mathrm{ft}$ west of Mr. Johnson's house

Classification: Typic Hapludults; fine-loamy, mixed, thermic.

Control Section: 18 to $56 \mathrm{~cm}$ of the argillic horizon.

Geomorphic Position: Upland position.

Slope and Aspect: 12\% Southeast.

Parent Material(s): Dismal Gap Formation.

Vegetation: tulip poplar, may apple, beech, red maple, hickory.

Described By. J. L. Branson.

Sampling Crew. Branson, Timpson, Livingston, Gallagher, Entrekin.

Time Since Last Rain: 2 days.

Present Weather: $65^{\circ} \mathrm{F}$ partly cloudy.

Date: May 27, 1992 sampled for gamma samples and description. July 16, 1992 sampled for zero contamination samples.

\section{Soil Description}

$\mathrm{Oa} \quad 2$ to $0 \mathrm{~cm}$; crumbly organic matter.

A 0 to $6 \mathrm{~cm}$; brown (10YR 4/3) silt loam; moderate fine granular structure; very friable; clear wavy boundary.

Ap 6 to $12 \mathrm{~cm}$; yellowish brown (10YR 5/6) silt loam or loam; weak fine granular structure; friable; clear wavy boundary.

BE 12 to $18 \mathrm{~cm}$; strong brown (7.5YR 5/6) and brown (7.5YR 5/4) loam; moderate fine subangular blocky structure; friable; clear wavy boundary.

Bt1 18 to $30 \mathrm{~cm}$; brown (7.5YR 5/4) clay; weak medium subangular blocky parting to moderate fine subangular blocky structure; firm; clear wavy boundary; yellowish red (5YR 5/6) on ped faces.

Bt2 30 to $56 \mathrm{~cm}$; strong brown (7.5YR 5/6) clay loam; weak medium subangular blocky parting to moderate fine subangular blocky structure; friable; yellowish red (5YR 5/6) on clay skins; clear irregular boundary.

CB 56 to $92 \mathrm{~cm}$; brown (7.5YR 5/4); clay loam; about 50\% fine medium subangular blocky structure, remainder is rock controlled; friable to firm; gradual wavy boundary.

Cr $\quad 92$ to $140 \mathrm{~cm}$; soft olive, red, and yellowish brown shale. 


\section{SITE NUMBER \\ ANDERSON No. 12}

Location: Big Ridge Park Quadrangle, Swan Kidwell property, $500 \mathrm{ft}$ northwest of Hinds Creek Road, $450 \mathrm{ft}$ from Mr. Kidwell's house, $100 \mathrm{ft}$ west of the power lines.

Classification: Typic Hapludults; fine-loamy, mixed, thermic.

Control Section: 16 to $56 \mathrm{~cm}$ argillic horizon.

Geomorphic Position: Midslope.

Slope and Aspect: $15 \%$ Southeast.

Parent Material(s): Dismal Gap Formation.

Vegetation: Virginia pine, dogwood, sweetgum, hickory.

Described By: J. L. Branson.

Sampling Crew: Branson, Timpson, Livingston, Gallagher, Entrekin.

Time Since Last Rain: 1 day.

Present Weather: $80^{\circ} \mathrm{F}$ partly cloudy.

Date: July 16, 1992.

\section{Soil Description}

Oi $\quad 2$ to $0 \mathrm{~cm}$; leaf litter and pine needles.

A $\quad 0$ to $7 \mathrm{~cm}$; dark brown (10YR 3/3) silt loam; moderate fine granular structure; very friable; abrupt smooth boundary.

Ap 7 to $16 \mathrm{~cm}$; yellowish brown (10YR 5/4) loam; weak medium granular structure; friable; clear, wavy boundary.

Bt1 16 to $41 \mathrm{~cm}$; strong brown (7.5YR 4/6) clay; moderate coarse subangular blocky structure; firm; clear, wavy boundary.

Bt2 41 to $56 \mathrm{~cm}$; yellowish red (5YR 5/6) clay; moderate coarse subangular blocky structure; firm; clear wavy boundary.

$\mathrm{Cr} 156$ to $90 \mathrm{~cm}$; soft olive, red, and yellow shale.

Cr2 90 to $122 \mathrm{~cm}$; soft shale primarily red with some olive and yellowish brown colors.

NOTES: This site had been cleared at one time. Age of the deciduous trees indicated they had been established at least $50 \mathrm{y}$. No evidence of a plow layer. Slope dips strongly (30\%) off to the side. Presently, this area is occupied by cattle for pasture. 


\section{SITE NUMBER}

ANDERSON No. 19

Location: Powell Quadrangle, Bill Lee property, $100 \mathrm{ft}$ north from Hinds Creek Road, 0.25 miles west from Nolan Road, $300 \mathrm{ft}$ south from Anderson No. 9.

Classification: Typic Hapludults; clayey, kaolinitic, thermic.

Control Section: 23 to $72 \mathrm{~cm}$ of the argillic horizon.

Geomorphic Position: Lower portion of a convex slope.

Slope and Aspect: 6\% Southeast.

Parent Material(s): Dismal Gap Formation.

Vegetation: Virginia pine, red maple, tulip poplar, sassafras, mosses.

Described By: J. L. Branson.

Sampling Crew: Branson, Timpson, Livingston, Gallagher, Entrekin.

Time Since Last Rain: 2 days.

Present Weather. $75^{\circ} \mathrm{F}$ Cloudy.

Date: July 17, 1992.

\section{Soil Description}

Oi $\quad 4$ to $0 \mathrm{~cm}$; moss, small roots, and pine needles.

A $\quad 0$ to $13 \mathrm{~cm}$; very dark grayish brown (10YR 3/2) silt loam; moderate medium granular structure; very friable; abrupt smooth boundary.

Ap 13 to $23 \mathrm{~cm}$; yellowish brown (10YR 5/6) loam; weak fine and medium granular structure; friable; abrupt smooth boundary.

Bt1 23 to $39 \mathrm{~cm}$; yellowish red (5YR 5/6) clay; moderate medium subangular blocky structure; firm; clear smooth boundary.

Bt2 39 to $72 \mathrm{~cm}$; yellowish red (5YR 5/8) clay; moderate medium subangular blocky structure; firm; clear wavy boundary.

BC $\quad 72$ to $95 \mathrm{~cm}$; yellowish red (5YR 5/8) clay loam; weak medium subangular blocky structure; friable; gradual wavy boundary.

$\mathrm{Cr} \quad 95$ to $135 \mathrm{~cm}$; soft olive, red, and yellow shale. 


\section{A-60 \\ SITE NUMBER \\ ANDERSON No. 20}

Location: Big Ridge Park Quadrangle, John Irwin property, $100 \mathrm{ft}$ northwest from Judson Road, 0.3 miles south from Moore Valley Road (also known as Cooper Gap), $300 \mathrm{ft}$ north of Anderson No. 3.

Classification: Typic Hapludults; clayey, kaolinitic, thermic.

Control Section: 13 to $63 \mathrm{~cm}$ of the argillic horizon.

Geomorphic Position: Upland, convex slope, area is highly eroded.

Slope and Aspect: $12 \%$ Southwest.

Parent Material(8): Dismall Gap Formation.

Vegetation: Virginia pine, dogwood, tulip poplar, poison ivy, mosses.

Described By: J. L. Branson.

Sampling Crew: Branson, Timpson, Livingston, Gallegher, Entrekin.

Time Since Last Rain: 0 days.

Present Weather: $85^{\circ} \mathrm{F}$ Heavy rain during sampling.

Date: July 15, 1992.

\section{Soil Description}

Oi $\quad 2$ to $0 \mathrm{~cm}$; moss and leaf litter.

A $\quad 0$ to $5 \mathrm{~cm}$; dark brown (7.5YR 4/4) silt loam; moderate medium granular structure; friable; clear smooth boundary.

Ap 5 to $13 \mathrm{~cm}$; yellowish brown (10YR 5/6) silt loam; weak fine granular structure; friable; gradual smooth boundary.

Bt1 13 to $21 \mathrm{~cm}$; strong brown (7.5YR $5 / 6$ ) silty clay loam; moderate medium subangular blocky structure; friable; abrupt smooth boundary.

Bt2 21 to $42 \mathrm{~cm}$; yellowish red (5YR 5/6) clay; moderate medium subangular blocky structure; firm; clear wavy boundary.

Bt3 42 to $84 \mathrm{~cm}$; red (2.5YR 4.8) clay; strong, medium subangular blocky structure; friable; clear, wavy boundary.

$\mathrm{Cr} \quad 84$ to $90 \mathrm{~cm}$; soft light olive, red and yellowish brown shale. 


\section{SITE NUMBER}

ANDERSON No. 21

Location: Big Ridge Park Quadrangle, Swan Kidwell property, $700 \mathrm{ft}$ northwest from Hinds Creek Road, $650 \mathrm{ft}$ northwest from Mr. Kidwell's house, $150 \mathrm{ft}$ west from power lines, $200 \mathrm{ft}$ northwest from Anderson No. 12.

Classification: Ochreptic Hapludults; clayey, kaolinitic, thermic, shallow.

Control Section: 12 to $28 \mathrm{~cm}$ argillic horizon.

Geomorphic Position: Upland in the curve connecting two parallel slopes at the top of a small ridge.

Slope and Aspect: $11 \%$ Southeast.

Parent Material(s): Dismal Gap Formation.

Vegetation: Virginia pine, dogwood, sweetgum, hickory.

Described By: J. L. Branson.

Sampling Crew:Branson, Timpson, Livingston, Gallagher, Entrekin

Time Since Last Rain: 1 day.

Present Weather: $80^{\circ} \mathrm{F}$ partly cloudy to sunny.

Date: July 16, 1992.

\section{Soil Description}

$\mathrm{Oi} \quad 2$ to $0 \mathrm{~cm}$; leaf litter and pine needles.

A $\quad 0$ to $6 \mathrm{~cm}$; dark brown (10YR 3/3) silt loam; moderate medium granular structure; friable; abrupt smooth boundary.

Ap 6 to $12 \mathrm{~cm}$; yellowish brown (10YR 5/4) loam; moderate medium granular structure; friable; clear smooth boundary.

Bt $\quad 12$ to $28 \mathrm{~cm}$; yellowish red (5YR 4/6) clay; strong medium subangular blocky structure; firm; clear wavy boundary.

$\mathrm{Cr} \quad 28$ to $87 \mathrm{~cm}$; soft olive, red, and yellow shale.

NOTES: This site had been cleared at one time. Age of the deciduous trees indicated they had been established at least $50 \mathrm{y}$. No evidence of a plow layer. Slope dips strongly (30\%) approximately $\mathbf{5 0}$ from the site. Presently, this area is occupied by cattle for pasture. 


\section{SITE NUMBER}

ANDERSON No. 22

Location: Big Ridge Park Quadrangle, John Irwin property, $100 \mathrm{ft}$ northwest from Judson Road, 0.3 miles south from Moore Valley Road (also known as Cooper Gap).

Classification: Ochreptic Hapludults; fine-loamy, mixed, thermic, shallow.

Control Section: 4 to $28 \mathrm{~cm}$ entire argillic horizon.

Geomorphic Position: Upland.

Slope and Aspect: 18\% Southeast.

Parent Material(s): Dismal Gap Formation.

Vegetation: Virginia Pine, dogwood, cedar, poison ivy, red cedar.

Described By. J. L. Branson.

Sampling Crew: Branson, Livingston.

Time Since Last Rain: 0 days.

Present Weather: $85^{\circ} \mathrm{F}$ Heavy rain during sampling.

Date: July 15, 1992-zero contamination sampling. July 16, 1992-gamma sampling.

\section{Soil Description}

Oi $\quad 2$ to $0 \mathrm{~cm}$; roots, leaf litter, and pine needles.

A $\quad 0$ to $4 \mathrm{~cm}$; dark brown (10YR 4/3) loam; weak fine granular structure; friable; abrupt smooth boundary.

Ap 4 to $14 \mathrm{~cm}$; yellowish brown (10YR 5/4) silt loam; weak medium granular structure; friable; clear wavy boundary.

Bt 14 to $28 \mathrm{~cm}$; yellowish red (5YR 5/6) clay; moderate medium subangular blocky structure; firm; clear irregular boundary.

$\mathrm{Cr} \quad 28$ to $98 \mathrm{~cm}$; soft olive, red, and yellow shale

Note: A water seep occurred at approximately $50 \mathrm{~cm}$. 


\section{A.7 ANALYSIS OF OAK RIDGE RESERVATION SITES}

ORR-2. This site, based on both geologic information and soil survey data, is situated within the Dismal Gap Formation. The soils are typical of ORR Dismal Gap soils in that they possess a very high degree of spatial variability. There was no visual field evidence of any recent surface disturbance, nor had the site ever been plowed. The A horizon sample consisted of $A$ and $E$ horizon soil material, the $B$ horizon sample consisted of the entire argillic horizon and the $C$ horizon consisted of transition horizons between the argillic horizon and the $\mathrm{Cr}$ horizon. The VOA's sample (1257) contained chloroform. This is considered to be due to instrument contamination. The related water trip blank did not contain chloroform. Two sets of $A$ horizon samples were collected for tritium analysis $(1189,1198)$. No tritium was detected in either sample but the detection limits reported were different. Two sets of A horizon samples were collected for organics analysis $(1190,1201)$. Most of the reported numbers, below detection limits, are similar, but statistical analysis would be needed to determine if any of the reported results are different. The ESD gamma scan analysis for cesium-137 gave a median value of $8.33 \mathrm{pCi} / \mathrm{cm}^{2}$, a typical value for cesium in the upper $30 \mathrm{~cm}$ of the soil on a sloping site.

ORR-3. This site is located on the Nolichucky Formation and within $50 \mathrm{ft}$ of the north edge of the cutslope above Bear Creek Road. This site is in old-field successional woods and there was no field evidence of recent surface disturbance. The A horizon soil sample consisted of a thin A horizon and the old Ap horizon beneath. The B horizon sample consisted entirely of argillic horizon soil material and the $C$ horizon sample was collected entirely of $\mathrm{C}$ horizon material between the $\mathrm{B}$ horizon above and the $\mathrm{Cr}$ horizon beneath. Acetone from instrument contamination was detected in a VOA sample (1271), but the reported value is lower than for other samples that have a " $U$ " qualifier. The organics analysis indicated no detects. The ESD gamma scanning results gave a median cesium-137 value of $8.47 \mathrm{pCi} / \mathrm{cm}^{2}$ in the upper $30 \mathrm{~cm}$ of the soil profile.

ORR-5. This site is located in the Nolichucky Formation. This site is located behind the security fence of the Central Training Facility and about $50 \mathrm{ft}$ south of Bear Creek Road. The vegetation is old-field successional forest that was dominated by pines. This site had a layer of pine needles and mosses $5 \mathrm{~cm}$ thick, and differed from many other sites in this respect. The A horizon sample consisted of an old Ap horizon. The B horizon sample cronsisted of the entire argillic horizon, and the $\mathrm{C}$ horizon consisted of a mixture of $\mathrm{C}$ and $\mathrm{Cr}$ horizon materials due to the steeply dipping strata. VOA sample (1272) contained acetone (from instrument contamination). All organics were below detection limits. ESD gamma scanning results gave a median cesium-137 value of $9.03 \mathrm{pCi} / \mathrm{cm}^{2}$ in the upper $30 \mathrm{~cm}$ of the soil profile, a typical value of a stable site where there has been no recent erosion.

ORR-10. This site is situated in the transition zone between the Dismal Gap Formation and the Rogersville Formation. Vegetation includes hardwoods indicating that this site had reverted from agricultural activities well before other sites due to very severe erosion before abandonment. The $\mathrm{A}$ horizon sample consisted entirely of $\mathrm{A}$ horizon materials, the $\mathrm{B}$ horizon consisted of cambic materials, and the $\mathrm{C}$ horizon of $\mathrm{C}$ and $\mathrm{Cr}$ materials, an example of a fairly typical Dismal Gap soil. VOA sample (1258) contained acetone (instrument contamination). The tritium result was rejected. All organic results were below detection limits. ESD gamma scanning results gave a median cesium-137 value of $10.97 \mathrm{pCi} / \mathrm{cm}^{2}$ in the upper $30 \mathrm{~cm}$ of the soil profile, an indication that this site was stable even though the slope was about $20 \%$, and 
evidence indicates that the site had been severely eroded at one time before global fallout started.

ORR-11. This site is located about $400 \mathrm{ft}$ down slope from ORR-10. This site is located in the Dismal Gap Formation. Vegetation consisted of old-field successional forest indicating that this site was open when abandoned in 1942-43. The A horizon sample consisted of the old Ap horizon, the B horizon sample consisted of the entire thickness of the argillic horizon, and the $C$ horizon sample consisted mostly of $C$ materials. Since ORR-10 and ORR-11 are close together, the results should be closely comparable, except for the differences in past land use, surface stability, and present vegetation. VOA analysis (sample number 1259) shows nothing above detection limits, except for acetone (instrument contamination). No tritium was detected. All organic results were below detection limits. ESD gamma scanning results gave a median cesium-137 value of $7.26 \mathrm{pCi} / \mathrm{cm}^{2}$ in the upper $30 \mathrm{~cm}$ of the soil profile, considerably lower than for ORR-10 indicating that there has been some soil erosion since global fallout started.

$O R R-13$. This site is located in an abandoned farm yard. The soil had a thick dark surface layer indicating that it had formed beneath grass vegetation. This site, in the Nolichucky Formation, is underlain by a brecciated zone having higher porosity than is typical. VOA sample (1273) had acetone as an instrument contaminant. Organics results indicated the estimated " $\mathrm{J}$ " presence of a PAH, benzo[b]fluoranthene. All other data were below detection limits. Recent pine harvesting and replanting activity close to this site might have caused this PAH to be in the soil. ESD cesium-137 gamma scanning results gave a median value of $8.94 \mathrm{pCi} / \mathrm{cm}^{2}$ in the upper $30 \mathrm{~cm}$ of the soil, indicating that this site has been stable.

$O R R-15$ and ORR-16. These sites are located about $250 \mathrm{ft}$ apart on the Nolichucky Formation. They have similar vegetation of 40 - to 50 -year old planted loblolly pines. The major difference is that one site, ORR-15, is located on a nearly level landform, and ORR-16 is located on a sideslope with $10 \%$ slope gradient and was severely eroded prior to abandonment. With the exception of acetone due to instrument contamination, there were no VOAs above detection limits. The organic results were also very similar with all results below detection limits except for "J" estimates of benzo[ $a]$ pyrene, benzo[b]fluoranthene and benzo $[b]$ fluorasthene at very low levels at ORR-16. ESD gamma scanning results from ORR-15 gave a median value of $8.08 \mathrm{pCi} / \mathrm{cm}^{2}$ in the upper $30 \mathrm{~cm}$ of the soil, indicating that this site has been stable. ESD cesium-137 gamma results for ORR-16 gave a median value of $9.93 \mathrm{pCi} / \mathrm{cm}^{2}$ in the upper $30 \mathrm{~cm}$ of the soil profile, indicating that this site has also been stable since global fallout began.

ORR-19. This site is located on the Dismal Gap Formation. It is in an old field with old-field successional forest dominated by pines. All samples were collected from appropriate soil horizons. With the exception of acetone resulting from instrument contamination, there were no VOAs above detection limits. Tritium was not found at this site, but the results were rejected due to analytical laboratory problems. The organic results were all below detection limits, except for fluorene which has a "J" qualifier. ESD cesium-137 gamma scanning results from ORR-19 gave a median value of $9.01 \mathrm{pCi} / \mathrm{cm}^{2}$ in the upper $30 \mathrm{~cm}$ of the soil, indicating that this site has been stable.

$O R R-21$. This site underlain by the Nolichucky Formation. Present vegetation is old-field successional forest once dominated by pines. This site is situated on a bench landform below 
an upper convex slope and had been severely eroded prior to abandonment. All VOA results were below detection limits with the exception of acetone due to instrument contamination. All organics results were below detection limits. ESD cesium-137 gamma scanning results from ORR-21 gave a median value of $11.46 \mathrm{pCi} / \mathrm{cm}^{2}$ in the upper $30 \mathrm{~cm}$ of the soil indicating that this site has probably received some soil deposition from higher areas since global fallout began.

ORR-22. This site is in the transition zone between the Dismal Gap Formation and the Rogersville Formation. It is situated on a high point in the landscape. The site is in an old field. The present forest vegetation is old field successional dominated by pines. The A horizon sample consists of a recently formed A horizon and the old Ap horizon beneath. The $B$ horizon consists of the entire thickness of the argillic horizon and the $\mathrm{C}$ horizon sample consists of a mixture of the $\mathrm{C}$ and $\mathrm{Cr}$ soil materials. The soil is very typical of the geology and landform location. The VOA data show no detects except for acetone, which is the result of instrument contamination. Tritium was detected at this site (sample number 1123). All organics were below detection limits. ESD gamma scanning results for cesium 137 gave a mean value of $9.63 \mathrm{pCi} / \mathrm{cm}^{2}$, which indicates that this site has been stable since global fallout commenced and that little cesium had been removed by erosion.

$O R R-23, O R R-24$, and $O R R-25$. These three sites are closely related in terms of their geology, landscape position, vegetation, and past land use. They are all underlain by the Nolichucky Formation, and all are in forest dominated by old-field successional pines. All three sites have similar soil morphology with a superficial layer of organic materials. The A horizon samples consisted of a thin A horizon and the old Ap horizon beneath. The $B$ horizon samples consisted of a mixture of the argillic and cambic horizons, and the $\mathrm{C}$ horizon samples were a mixture of $\mathrm{C}$ and $\mathrm{Cr}$ soil materials. Duplicate samples were collected for VOA analysis from two of the three sites. The results were all below detection except for acetone, which is due to instrument contamination. The organic results were also all very similar for all three sites, except for "J" estimates of acenapthene in ORR-23 and pyrene in ORR-24. The results for benzoanthrene, chrysene and fluoranthene were all rejected. ESD cesium-137 gamma scanning results are similar for ORR-23 and ORR-24, 9.17 and $10.49 \mathrm{pCi} / \mathrm{cm}^{2}$ respectively, indicating site stability. ORR-25 had a result of $7.69 \mathrm{pCi} / \mathrm{cm}^{2}$ indications that some erosion had occurred since global fallout started.

$O R R-26$ and $O R R-27$. These sites are also close together. Both are underlain by the Dismal Gap Formation, have similar forest vegetation and past landuse, and are separated by a quite deeply incised drainageway. Both sites are in old fields that were abandoned well before 1942-43. The early successional pines on both sites had all been replaced by hardwoods. The A horizon samples consisted of a recently formed A horizon and the older Ap horizon beneath. The B horizon samples consisted of the entire thickness of the argillic horizon, and the $\mathrm{C}$ horizons consisted of a mixture of $\mathrm{C}$ and $\mathrm{Cr}$ horizon materials. VOA results were all non-detects with the exception of Acetone and 2-butanone which are due to instrument contamination. ORR-26 contained "J" estimated tritium while ORR-27 did not. The organic results for both sites were below detection limits. ESD gamma scanning data for cesium-137 indicate low median results of 8.59 for ORR-26 and $6.35 \mathrm{pCi} / \mathrm{cm}^{2}$ for ORR-27. ORR-26 is on a steeper slope gradient than ORR-27, but appears to be more stable. One cannot rule out a forest fire on ORR-27 that could have led to some soil erosion. 
ORR-28. This site is located a short distance south of ORR-26 and ORR-27. ORR-28 is underlain by the Nolichucky Formation and is in a dense stand of young pines with some scattered hardwoods. The old-field successional pines had already been harvested from this site, or had died and fallen over. The soil profile is typical of Nolichucky soils. The A horizon sample consisted of a thin recently formed A horizon and the older Ap horizon beneath. The $B$ horizon sample consisted entirely of the argillic horizon and the $C$ horizon sample consisted of mostly $\mathrm{C}$ horizon materials. Two samples were sent for VOA analysis. There were no detects is the VOA results with the exception of acetone " $\mathrm{J}$ " value due to instrument contamination. All organics were below detection limits, with two PAHs rejected. ESD gamma scan results for cesium-137 showed a median value of $9.69 \mathrm{pCi} / \mathrm{cm}^{2}$ indicating that this site had not been subjected to erosion since global fallout started, even though this site had been severely eroded prior to abandonment.

ORR-31. This site is underlain by the Nolichucky Formation, but the upper $61 \mathrm{~cm}$ consisted of colluvium. Most of the old-field successional pines had been harvested in the past 10 to 15 years, and there was evidence that the larger area around this site had been disturbed, but that the actual site did not show any evidence of disturbance. This site was considered marginal in terms of site quality during the site selection process, but with the difficulty of locating suitable Nolichucky sites, it was sampled. Two samples were collected for VOA analysis. One result showed the presence of trichlorofluoromethane but the other sample did not. Both samples had acetone and one had 2-butanone, which are considered to be due to instrument contamination. The organic results had a "J" estimate for benzo[b]fluoranthene and the result for fluoranthene was rejected. All other organics were below detection limits. ESD gamma scanning data showed a median value of $11.14 \mathrm{pCi} / \mathrm{cm}^{2}$ for cesium-137, a value slightly higher than predicted. This site is on the lower part of a long sideslope and had received some deposition of soil from higher on the slope. Specific data for this site do not indicate that it should be rejected.

$O R R-32, O R R-33$, and $O R R-35$. These sites are all located adjacent to each other and are separated by about 250 to $300 \mathrm{ft}$. All three sites are underlain by the Dismal Gap Formation and have had similar old-field successional forest that was dominated by pines. Most of the pines have recently died and fallen over releasing a dense understory of brush, small pines, and small hardwoods. All three sites had similar soil morphology. The A horizon sample consisted of a thin recently formed A horizon and the older Ap horizon beneath. The $B$ horizon sample consisted of the entire thickness of the argillic horizon, and the $C$ horizon sample consisted of a mixture of $\mathrm{C}$ and $\mathrm{Cr}$ materials. Acetone was found in all VOA samples but is considered to be due to instrument contamination. All three sites have detectable tritium. All organics were below detection limits. Data from ESD cesium-137 gamma scanning had some spread, indicating that one site (ORR-35) was more subject to erosion than the other two sites. ORR-32 had a median value of 7.88, ORR-33 had a value of 9.38 , and ORR-35 had a value of $5.87 \mathrm{pCi} / \mathrm{cm}^{2}$. These differences cannot be explained in terms of soil morphology, slope gradient, vegetation, or landscape position.

$O R R-41, O R R-42$, and $O R R-43$. These sites are located in close proximity and near the west end of the Y-12 burial grounds. ORR-41 and ORR-43 are underlain by the Dismal Gap Formation, while ORR -42 is underlain by the Nolichucky Formation. All sites had typical soils of their geologic formation and had similar morphology. With the exception of acetone all A horizon VOA analytes were below detection limits. Tritium was not detected in ORR-41, the other sites were not analyzed. No organics were above detection limits in ORR-41 and 
ORR-43. Benzo[b]fluoranthene and pyrene were estimated at very low levels in ORR-42. The results of some PAHs for these sites were rejected. ESD cesium-137 gamma scan data for two of the three sites indicated that there had been minimal surface instability since global fallout started. ORR-41 had a median value of $10.89 \mathrm{pCi} / \mathrm{cm}^{2}$ in the upper $30 \mathrm{~cm}$ of soil, ORR-42 had a value of 6.75 , indicating erosion, and ORR -43 had a value of 8.48 . Soil morphology for ORR -42 indicates that this site had been somewhat less stable than the sites on either side. 


\section{A.8 ANALYSIS OF ROANE COUNTY SITES}

ROA-3, ROA-9, ROA-19, ROA-20, ROA-21, and ROA-22. These sites are located close to each other in the central part of the sampling transect. All of these sites had old-field successional forest of pines and hardwoods.

ROA-3. This site is located in a toeslope position. The entire soil profile consists of colluvium/alluvium derived from soils of Conasauga Group rocks rather than residuum from the Dismal Gap Formation. Acetone and 2-Butanone were "J" estimates in the VOA analysis, but they are due to instrument contamination. There were no other VOA analytes above detection limits. No tritium was detected in the A horizon sample from this site. In the organics analysis, only Naphthalene was estimated to be present. All other organics were below detection limits. ESD cesium-137 gamma scan analysis for this site showed a median value of $5.63 \mathrm{pCi} / \mathrm{cm}^{2}$, a low value indicating that this site has experienced erosion since the start of global fallout.

ROA-7 and ROA-8. These sites are in close proximity. ROA-7 and ROA-8 are on a lower sideslope. The upper $44 \mathrm{~cm}$ of the ROA-7 and the ROA-8 soil profiles are formed in colluvium, and the soil beneath is residuum of the Dismal Gap Formation. Present forest is old-field successional dominated by pines. This site is in a group of trees surrounded by cattle pasture and the site is open to cattle grazing. With the exception of acetone, there were no VOAs detected and no tritium detect. Benzo $[b]$ anthracene was an estimated " $\mathrm{J}$ " detect but there were no other organics detected. ESD cesium-137 gamma scan results gave a value of $6.64 \mathrm{pCi} / \mathrm{cm}^{2}$ for ROA-7, indicating that this site has been eroding since global fallout started. The corresponding value for ROA-8 is $11.93 \mathrm{pCi} / \mathrm{cm}^{2}$, indicating that there has been some deposition on this site.

$R O A-9$. This site is located in a toeslope position. The upper $52 \mathrm{~cm}$ of the soil profile is in colluvium. The $A$ horizon and the $B$ horizon samples are colluvial materials, while the $\mathrm{C}$ horizon sample consists of $\mathrm{Cr}$ materials from the Dismal Gap Formation. There were no VOAs detected, no tritium detected, and no organics detected. ESD cesium-137 gamma scan showed a typical uneroded site median value of $10.15 \mathrm{pCi} / \mathrm{cm}^{2}$ for the upper $30 \mathrm{~cm}$ of the soil profile.

ROA-10. This site is located at the north end of the Roane County transect. This site is surrounded by an open field and cattle have access to this site. The upper $18 \mathrm{~cm}$ of the soil formed in alluvium, but the lower part formed in residuum of the Dismal Gap Formation. Present forest is old-field successional with both pines and hardwoods. With the exception of acetone there were no VOAs or organics detected. ESD cesium-137 gamma scan results gave a value of $8.56 \mathrm{pCi} / \mathrm{cm}^{2}$, indicating that this site has been relatively stable.

ROA-13 and ROA-14. These sites are in close proximity. The soil at ROA-13 formed in residuum of the Dismal Gap Formation. Present forest is old-field successional dominated by pines. The site is at the base of a long slope. With the exception of acetone and 2-butanone, there were no VOAs detected. Benzo[b]fluoranthene was an estimated " $\mathrm{J}$ " detect. There were no other organics detected. ESD cesium-137 gamma scan results gave a value of $1.98 \mathrm{pCi} / \mathrm{cm}^{2}$, a very low value indicating that this site has been eroding. ROA-14 occurs on a convex sideslope. The upper $41 \mathrm{~cm}$ of the soil profile formed in colluvium. Below $41 \mathrm{~cm}$ the soil formed in the transition zone between the Dismal Gap and Rogersville formations. 
Present forest is old-field successional dominated by hardwoods. With the exception of acetone, there were no VOAs detected. Benzo[a]pyrene was an estimated " $\mathrm{J}$ " detect, but there were no other organics detected. ESD gamma scan results gave a value of $8.20 \mathrm{pCi} / \mathrm{cm}^{2}$ for this site, an indication of relative stability.

ROA-17. This site is isolated. The soil on this site is residuum of the Dismal Gap Formation. Present forest vegetation is old-field successional dominated by pines. The site is open to cattle. With the exception of acetone and 2-butanone resulting from instrument contamination, there were no other VOAs detected, no tritium detected, and no organics detected. ESD cesium-137 gamma scan results gave a value of $9.61 \mathrm{pCi} / \mathrm{cm}^{2}$, indicating that this site has not been eroding.

ROA-19. This site is located in a toeslope position. The upper $47 \mathrm{~cm}$ of the soil profile is formed in colluvium. The A horizon and B horizon samples came from this soil material. The $C$ horizon sample came from residuum of the Dismal Gap Formation. There were no VOAs detected, no tritium detected, and no organics detected in the A horizon sample. ESD cesium-137 gamma scan results showed a median value of $4.16 \mathrm{pCi} / \mathrm{cm}^{2}$, an indication that this site has been eroding since the start of global fallout.

ROA-20. This site is located in a toeslope position. The soil is derived from residuum of the Dismal Gap Formation. There were no VOAs detected, no tritium detect, but fluorene was a "J" estimated detect in the A horizon. There were no other detects for organics. ESD cesium-137 gamma scan results gave a value of $6.11 \mathrm{pCi} / \mathrm{cm}^{2}$, indicating that there has been some soil erosion since global fallout started.

$R O A-21$. This site is located in a midslope position. The upper $74 \mathrm{~cm}$ of the soil profile formed in colluvium from the Dismal Gap Formation. The $2 \mathrm{Cr}$ horizon beneath is residuum of the Dismal Gap. Present forest is old-field successional dominated by pines. There were no VOAs detected and no organics detected. The ESD cesium-137 gamma scan data results show a value of $5.40 \mathrm{pCi} / \mathrm{cm}^{2}$, an indication that this site has been eroding since global fallout started.

$R O A-22$. Is located on a bench landform. The upper $45 \mathrm{~cm}$ of the soil profile is colluvium. Below is residuum of the Dismal Gap Formation. Present forest is old-field successional but now dominated by hardwonds. There were no VOAs detected, no tr:ium detected, but there was an estimated "J" detect for naphthalene in A horizon samples. ESD cesium-137 gamma scan results gave a value of $4.16 \mathrm{pCi} / \mathrm{cm}^{2}$ to a depth of $30 \mathrm{~cm}$ for this site, indicating that there has been erosion since global fallout started. 


\section{A.9 ANALYSIS OF ANDERSON COUNTY SITES}

AND-1, AND-10, and AND-11. These sites are located in proximity to each other. AND-1 formed in Dismal Gap residuum and is located in a woodlot that is also used for cattle pasture. The A horizon sample consisted of an old Ap horizon, the B horizon sample consisted of the entire thickness of the argillic horizon, and the $C$ horizon samples of $\mathrm{Cr}$ horizon materials. This site is also on a $30 \%$ slope and subject to accelerated soil erosion. No VOAs occurred above detection limits, but several organics were detected. The results from ESD cesium-137 gamma scanning gave a value of $6.58 \mathrm{pCi} / \mathrm{cm}^{2}$ in the upper $30 \mathrm{~cm}$ of soil. This value indicates that this site has 'een and perhaps is still eroding, although at a very slow rate. AND-10 occurs in an old field with old-field successional forest dominated by pines. This site is on a nearly level ridgetop. The A horizon sample consisted of an A horizon, the B horizon sample consisted of the entire thickness of the argillic horizon, and the $\mathrm{C}$ horizon sample consisted of $\mathrm{Cr}$ materials. With the exception of acetone, all VOA analytes were below detection limits, but there were several organics estimated. Most were PAHs. In addition, there were several organic rejects. ESD cesium-137 gamma scanning results gave a median value of $9.39 \mathrm{pCi} / \mathrm{cm}^{2}$, which agrees with the soil morphology indication of surface stability. AND-11 occurs in a stand of hardwoods but was once an old field. The soil morphology is typical of a more strongly weathered and developed soil from the Dismal Gap Formation than what is generally typical. With the exception of acetone, there were no VOA analytes above detection limits. There were several "J" estimated organics. The ESD cesium-137 gamma scanning results gave a median value of $10.27 \mathrm{pCi} / \mathrm{cm}^{2}$ for the upper $30 \mathrm{~cm}$ of the soil profile. This value indicates that this site has not been eroding.

$A N D-3, A N D-4, A N D-5, A N D-20$, and $A N D-22$. These five sites are clustered close together. They are all under the same ownership and have a similar old-field successional forest that is dominated by pines. The underlying geology is the Dismal Gap Formation. AND-3 formed wholly in residuum. AND-4 formed in $53 \mathrm{~cm}$ of colluvium and the underlying residuum. AND-5 formed in $70 \mathrm{~cm}$ of colluvium and the underlying residuum. AND-20 formed in $21 \mathrm{~cm}$ of colluvium and the underlying residuum. AND-22 formed wholly in residuum. With the exception of acetone resulting from instrument contamination, there were no VOA analytes above detection limits. All sites showed estimated " $\mathrm{J}$ " amounts of several PAHs. ESD cesium-137 gamma scan results indicated that AND-3 with a value of $4.73 \mathrm{pCi} / \mathrm{cm}^{2}$ and AND-22 with a value of $3.80 \mathrm{pCi} / \mathrm{cm}^{2}$ had been quite eroded. AND-20 with a value of $7.03 \mathrm{pCi} / \mathrm{cm}^{2}$ had been eroded to some extent, but AND-4 with a value of $9.97 \mathrm{pCi} / \mathrm{cm}^{2}$ had not experienced any erosion.

AND-9 and AND-19. These sites are located close to each other, separated by about $300 \mathrm{ft}$. Both sites have typical soils that formed in Disma! Gap residuum. AND-9 occurs on a convex sideslope while AND-19 occurs on the lower part of a sideslope. With the exception of acetone there were no VOA analytes above detection limits for either site. However, there were several " $J$ " estimated organics, mostly PAHs for both sites. ESD cesium-137 gamma scan results for AND-9 show a median value of $8.95 \mathrm{pCi} / \mathrm{cm}^{2}$ in the upper $30 \mathrm{~cm}$ of the soil profile, while AND-19 shows a median value of 14.42 . The soil profile description indicates that there has been some soil deposition at this site.

AND-12 and AND-21. These sites are about $200 \mathrm{ft}$ apart. Both sites are underlain by the Dismal Gap Formation, and both soils are typical of Dismal Gap residual soils. Both have similar old-field successional forest dominated by pines but have slightly differing landscape 
positions. Cattle are allowed to graze on both sites. With the exception of acetone, there were no VOA analytes above detection limits. Both sites contain estimated "J" PAHs. AND-12 also contains Aroclor 1242 above detection limits. ESD cesium-137 gamma scan data shows a median value of $7.31 \mathrm{pCi} / \mathrm{cm}^{2}$, a lower than normal value, indicating that there has been some soil erosion from this site. The value for AND-21 is 6.35, also a lower than normal value indicating that there has been soil erosion from this site. 


\section{A.10 ANALYSIS OF DATA FROM COMPOSITED SAMPLES}

All of the composited sample data seem to be reasonable in light of geostatistical concepts with the exception of the following samples (ordered by sample number).

5004 Copper is very low compared in this B horizon sample compared to the A and C horizons.

5014 Cesium-137 and potassium-40 values were not detected. However, ESD gamma scan data show these to be present. No Europium-155 was in this sample, but was found in samples from above and below this horizon.

5028 Much of the data is 2 times higher than the field duplicate. It is very difficult to explain this other than laboratory dilution error.

5031 Cobalt in this B horizon sample is very low in comparison to the horizons above and below.

5068 No cesium-137 is reported, yet the ESD gamma scan data show that some is present.

5070 Chromium is very high compared to levels below detection limits in the $A$ and $B$ horizons above. Lead has the same problem when the $A$ and $B$ horizon samples are below detection limits.

5079 Both chromium and lead have very high values when compared to $A$ and $B$ horizon samples above.

7020 No cesium-137 was found in this A horizon sample even though the ESD gamma scan shows its presence.

7029 Potassium -40 has a very high value compared to the $B$ and $C$ horizon samples giving a reverse distribution. ESD gamma scan data always show an increase of K-40 in the B horizon.

6002 The cesium-137 value is higher in the B horizon than in the $\mathrm{A}$ horizon. This is not substantiated by ESD gamma scan data.

6020 There is no cesium-137 detect for this A horizon sample though the ESD gamma scan data indicate its presence. 
Appendix B

SCREENING ANALYSIS DATA 
B-2

NOTE: When available, the validation qualifiers are used in this appendix. When validation qualifiers are not available, the corresponding laboratory data qualifiers are used. 


\section{VALIDATOR DATA QUALIFIER DEFINITIONS}

The following definitions provide brief explanations of the qualifiers assigned to the data in this appendix.

$\mathbf{U}$

J

$\mathbf{N}$

$\mathbf{N}$

UJ

$\mathbf{R}$ The sample results are rejected because of serious deficiencies in the ability to analyze the sample and meet quality control criteria. The presence or absence of the analyte cannot be verified.

UN The laboratory did not register this compound, but there was presumptive evidence of a compound that was within the retention time window but was not reported. No other qualification of the data was made.

UJN The laboratory did not report the compound, but there was presumptive evidence of a compound that was within the retention time window but was not reported. The data were qualified as estimated, $\mathrm{J}$, because of other discrepancies with the data.

RN The laboratory did not report the compound, but there was evidence of a compound that was within the retention time window but was not reported. The data were qualified as unusable, $R$, because of other discrepancies with the data. 


\section{LABORATORY DATA QUALIFIER DEFINITIONS}

The following contract laboratory data qualifiers are used in this project.

\section{Organic}

\begin{tabular}{|c|c|}
\hline Qualifier & Explanation \\
\hline $\mathrm{U}$ & Indicates compound was analyzed for but not detected. \\
\hline $\mathrm{J}$ & Indicates an estimated value. \\
\hline $\mathbf{N}$ & $\begin{array}{l}\text { Indicates presumptive evidence of a compound [used only for tentatively } \\
\text { identified compounds (TTCS)]. }\end{array}$ \\
\hline $\mathbf{P}$ & $\begin{array}{l}\text { Used for pesticide/aroclor target analytes when there is greater than } 25 \% \\
\text { difference for detected concentrations between the two gas chromatograph (GC) } \\
\text { columns. The lower of the two is reported and flagged. }\end{array}$ \\
\hline C & $\begin{array}{l}\text { Used for pesticide results where the identification has been confirmed by } \\
\text { GC/mass spectrograph (MS). }\end{array}$ \\
\hline B & Used when the analyte is found in the associated blank as well as in the sample. \\
\hline D & Identifies all compounds in an analyte at a secondary dilution factor. \\
\hline $\mathbf{A}$ & Indicates that a TIC is a suspected aldol-condensation product. \\
\hline $\mathbf{x}$ & $\begin{array}{l}\text { Other specific flags may be used to properly define the results. If they are used } \\
\text { they must be fully described and attached to the Sample Data Package. }\end{array}$ \\
\hline
\end{tabular}

Inorganic

\begin{tabular}{|c|c|}
\hline Qualifier & Explanation \\
\hline $\mathrm{U}$ & Indicates compound was analyzed for but not detected. \\
\hline $\mathbf{J}$ & Indicates an estimated value. \\
\hline $\mathbf{N}$ & Spiked sample recovery not within control limits. \\
\hline $\mathrm{E}$ & Reported value estimated because of the presence of interference. \\
\hline $\mathbf{M}$ & Duplicate injection precision not met. \\
\hline B & $\begin{array}{l}\text { Reported value was obtained from a reading that was less than the CRDL, but } \\
\text { greater than or equal to the instrument detection limit (IDL). }\end{array}$ \\
\hline $\mathbf{S}$ & Reported value was determined by the method of standard additions (MSA). \\
\hline W & $\begin{array}{l}\text { Post-digestion spike for furnace atomic absorption is out of control limits, while } \\
\text { sample absorbance is less that } 50 \% \text { of spike absorbance. }\end{array}$ \\
\hline * & Duplicate analysis not within control limits. \\
\hline+ & Correlation coefficient for the MSA is less than 0.995 . \\
\hline
\end{tabular}


Table B.1. Volatile organic analysis results for soil samples

\begin{tabular}{|c|c|c|c|}
\hline Anatysis & Units & A horizon & Qual \\
\hline \multicolumn{4}{|c|}{ Location $=A N D ;$ Formation $=$ DISMAL GAF } \\
\hline $\begin{array}{l}\text { Acetone } \\
\text { Benzene } \\
\text { Bromodichloromethane } \\
\text { Bromoform } \\
\text { Bromomethane } \\
\text { Carbon Disulfide } \\
\text { Carbon Tetrachloride } \\
\text { Chlorobenzene } \\
\text { Chloroethane } \\
\text { Chloroform } \\
\text { Chloromethane } \\
\text { Cis-1,2-Dichloroethene } \\
\text { Cis-1,3-Dichloropropene } \\
\text { Dibromochloromethane } \\
\text { Ethyl Benzene } \\
\text { Methylene Chloride } \\
\text { Ortho Xylene } \\
\text { Styrene } \\
\text { Tetrachloroethene } \\
\text { Toluene } \\
\text { Trans-1,2-Dichloroethene } \\
\text { Trans-1,3-Dichloropropene } \\
\text { Trichloroethene } \\
\text { Vinyl Chloride } \\
\text { Xylene (Meta + Para) } \\
1,1-D \text { ichloroethane } \\
1,1-D \text { ichloroethene } \\
1,1,1-\text { Trichloroethane } \\
1,1,2-\text { Trichloroethane } \\
1,1,2,2-\text { Tetrachloroethane } \\
1,2-0 \text { ichloroethane } \\
1,2-0 \text { ichloropropane } \\
2-\text { Butanone } \\
2-\text { Hexanone } \\
4-\text { Methyl-2-Pentanone }\end{array}$ & $\begin{array}{l}\mu g / \mathbf{k g} \\
\mu g / \mathbf{k g} \\
\mu g / \mathbf{k g} \\
\mu g / \mathbf{k g} \\
\mu g / \mathbf{k g} \\
\mu g / \mathbf{k g} \\
\mu g / \mathbf{k g} \\
\mu g / \mathbf{k g} \\
\mu g / \mathbf{k g} \\
\mu g / \mathbf{k g} \\
\mu g / \mathbf{k g} \\
\mu g / \mathbf{k g} \\
\mu g / \mathbf{k g} \\
\mu g / \mathbf{k g} \\
\mu g / \mathbf{k g} \\
\mu g / \mathbf{k g} \\
\mu g / \mathbf{k g} \\
\mu g / \mathbf{k g} \\
\mu g / \mathbf{k g} \\
\mu g / \mathbf{k g} \\
\mu g / \mathbf{k g} \\
\mu g / \mathbf{k g} \\
\mu g / \mathbf{k g} \\
\mu g / \mathbf{k g} \\
\mu g / \mathbf{k g} \\
\mu g / \mathbf{k g} \\
\mu g / \mathbf{k g} \\
\mu g / \mathbf{k g} \\
\mu g / \mathbf{k g} \\
\mu g / \mathbf{k g} \\
\mu g / \mathbf{k g} \\
\mu g / \mathbf{k g} \\
\mu g / \mathbf{k g} \\
\mu g / \mathbf{k g} \\
\mu g / \mathbf{k g}\end{array}$ & $\begin{array}{r}100 \\
5 \\
5 \\
5 \\
10 \\
5 \\
5 \\
5 \\
10 \\
5 \\
10 \\
5 \\
5 \\
5 \\
5 \\
5 \\
5 \\
5 \\
5 \\
5 \\
5 \\
5 \\
5 \\
10 \\
5 \\
5 \\
5 \\
5 \\
5 \\
5 \\
5 \\
5\end{array}$ & 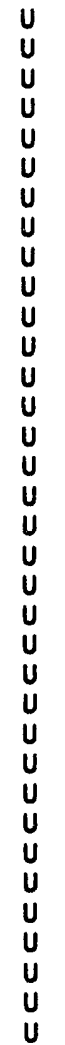 \\
\hline
\end{tabular}

$$
\text { Location }=A N D ; \text { Formation }=\text { DISMAL GAP; Site }=3
$$

Acetone
Benzene
Bromodichloromethane
Bromoform
Bromomethane
Carbon Disulfide
Carbon Tetrachloride
Chlorobenzene
Chloroethane
Chloroform
Chloromethane
Cis-1,2-Dichloroethene
Cis-1,3-Dichloropropene
Dibromochloromethane
Ethyl Benzene
Methylene Chloride
Ortho Xylene
Styrene
Tetrachloroethene
Toluene
Trans-1,2-Dichloroethene
Trans-1,3-Dichloropropene
Trichloroethene
Vinyl Chloride
Xylene (Meta + Para)
$1,1-D$ ichloroethane
$1,1-D i c h l o r o e t h e n e$

$\begin{array}{lrl}\mu \mathrm{g} / \mathbf{k g} & 5 & \mathrm{~J} \\ \mu \mathrm{g} / \mathbf{k g} & 5 & U \\ \mu \mathrm{g} / \mathbf{k g} & 5 & U \\ \mu \mathrm{g} / \mathbf{k g} & 5 & U \\ \mu \mathrm{g} / \mathbf{k g} & 10 & U \\ \mu \mathrm{g} / \mathbf{k g} & 5 & U \\ \mu \mathrm{g} / \mathbf{k g} & 5 & U \\ \mu \mathrm{g} / \mathbf{k g} & 5 & U \\ \mu \mathrm{g} / \mathbf{k g} & 10 & U \\ \mu \mathrm{g} / \mathbf{k g} & 5 & U \\ \mu \mathrm{g} / \mathbf{k g} & 10 & U \\ \mu \mathrm{g} / \mathbf{k g} & 5 & U \\ \mu \mathrm{g} / \mathbf{k g} & 5 & U \\ \mu \mathrm{g} / \mathbf{k g} & 5 & U \\ \mu \mathrm{g} / \mathbf{k g} & 5 & U \\ \mu \mathrm{g} / \mathbf{k g} & 5 & U \\ \mu \mathrm{g} / \mathbf{k g} & 5 & U \\ \mu \mathrm{g} / \mathbf{k g} & 5 & U \\ \mu \mathrm{g} / \mathbf{k g} & 5 & U \\ \mu \mathrm{g} / \mathbf{k g} & 5 & U \\ \mu \mathrm{g} / \mathbf{k g} & 5 & U \\ \mu \mathrm{g} / \mathbf{k g} & 5 & U \\ \mu \mathrm{g} / \mathbf{k g} & 5 & U \\ \mu \mathrm{g} / \mathbf{k g} & 10 & U \\ \mu \mathrm{g} / \mathbf{k g} & 5 & U \\ \mu \mathrm{g} / \mathbf{k g} & 5 & U \\ \mu \mathrm{g} / \mathbf{k g} & 5 & U\end{array}$


Table B.1 (continued)

\begin{tabular}{|c|c|c|c|c|c|}
\hline Analysis & Units & A horizon & Qual. & $\begin{array}{l}\text { A borizon } \\
\text { field dup }\end{array}$ & Qual. \\
\hline $\begin{array}{l}\text { 1,1,1-Trichloroethane } \\
1,1,2-T r \text { ichloroethane } \\
1,1,2,2-\text { Tetrachloroethane } \\
\text { 1,2-Dichloroethane } \\
\text { 1,2-Dichloropropane } \\
\text { 2-Butanone } \\
\text { 2-Hexanone } \\
\text { 4-Methyl-2-Pentanone }\end{array}$ & $\begin{array}{l}\mu g / k g \\
\mu g / k g \\
\mu g / k g \\
\mu g / k g \\
\mu g / k g \\
\mu g / k g \\
\mu g / k g \\
\mu g / k g\end{array}$ & $\begin{array}{r}5 \\
5 \\
5 \\
5 \\
5 \\
100 \\
50 \\
50\end{array}$ & $\begin{array}{l}\mathbf{U} \\
\mathbf{U} \\
\mathbf{U} \\
\mathbf{U} \\
\mathbf{U} \\
\mathbf{U} \\
\mathbf{U} \\
\mathbf{U}\end{array}$ & & \\
\hline
\end{tabular}

$$
\text { Location }=A N D ; \text { Formation }=\text { DISMAL GAP; Site }=4
$$

Acetone
Benzene
Bromodichloromethane
Bromoform
Bromomethane
Carbon Disulfide
Carbon Tetrachloride
Chlorobenzene
Chloroethane
Chloroform
Chloromethane
Cis-1,2-Dichloroethene
Cis-1,3-Dichloropropene
Dibromochloromethane
Ethyl Benzene
Methylene Chloride
Ortho Xylene
Styrene
Tetrachloroethene
Toluene
Trans-1,2-Dichloroethene
Trans-1,3-Dichloropropene
Trichloroethene
Vinyl Chloride
Xylene (Meta + Para)
$1,1-0$ ichloroethane
$1,1-D i c h l o r o e t h e n e$
$1,1,1-$ Trichloroethane
$1,1,2-$ Trichloroethane
$1,1,2,2-$ Tetrachloroethane
$1,2-D i c h l o r o e t h a n e$
$1,2-D i c h l o r o p r o p a n e$
$2-8 u t a n o n e$
$2-$ Hexanone
$4-$ Methyl-2-Pentanone

$\begin{array}{lrl}\mu g / k g & 13 & J \\ \mu g / k g & 5 & U \\ \mu g / k g & 5 & U \\ \mu g / k g & 5 & U \\ \mu g / k g & 10 & U \\ \mu g / k g & 5 & U \\ \mu g / k g & 5 & U \\ \mu g / k g & 5 & U \\ \mu g / k g & 10 & U \\ \mu g / k g & 5 & U \\ \mu g / k g & 10 & U \\ \mu g / k g & 5 & U \\ \mu g / k g & 5 & U \\ \mu g / k g & 5 & U \\ \mu g / k g & 5 & U \\ \mu g / k g & 5 & U \\ \mu g / k g & 5 & U \\ \mu g / k g & 5 & U \\ \mu g / k g & 5 & U \\ \mu g / k g & 5 & U \\ \mu g / k g & 5 & U \\ \mu g / k g & 5 & U \\ \mu g / k g & 5 & U \\ \mu g / k g & 10 & U \\ \mu g / k g & 5 & U \\ \mu g / k g & 5 & U \\ \mu g / k g & 5 & U \\ \mu g / k g & 5 & U \\ \mu g / k g & 5 & U \\ \mu g / k g & 5 & U \\ \mu g / k g & 5 & U \\ \mu g / k g & 5 & U \\ \mu g / k g & 100 & U \\ \mu g / k g & 50 & U \\ \mu g / k g & 50 & U \\ & & 5\end{array}$

$$
\text { Location }=A N D ; \text { Formation }=\text { DISMAL GAP; Site }=5
$$

$\begin{array}{ll}\text { Acetone } & \mu \mathrm{g} / \mathrm{kg} \\ \text { Benzene } & \mu \mathrm{g} / \mathrm{kg} \\ \text { Bromodichloromethane } & \mu \mathrm{g} / \mathrm{kg} \\ \text { Bromoform } & \mu \mathrm{g} / \mathrm{kg} \\ \text { Bromomethane } & \mu \mathrm{g} / \mathrm{kg} \\ \text { Carbon Disulfide } & \mu \mathrm{g} / \mathrm{kg} \\ \text { Carbon Tetrachloride } & \mu \mathrm{g} / \mathrm{kg} \\ \text { Chlorobenzene } & \mu \mathrm{g} / \mathrm{kg} \\ \text { Chloroethane } & \mu \mathrm{g} / \mathrm{kg} \\ \text { Chloroform } & \mu \mathrm{g} / \mathrm{kg} \\ \text { Chloromethane } & \mu \mathrm{g} / \mathrm{kg} \\ \text { Cis-1,2-Dichloroethene } & \mu \mathrm{g} / \mathrm{kg} \\ \text { Cis-1,3-Dichloropropene } & \mu \mathrm{g} / \mathrm{kg} \\ \text { Dibromochloromethane } & \mu \mathrm{g} / \mathrm{kg} \\ \text { Ethyl Benzene } & \mu \mathrm{g} / \mathrm{kg} \\ \text { Methylene Chloride } & \mu \mathrm{g} / \mathrm{kg} \\ \text { Ortho Xylene } & \mu \mathrm{g} / \mathrm{kg}\end{array}$

$\begin{array}{rr}8 & J \\ 5 & U \\ 5 & U \\ 5 & U \\ 10 & U \\ 5 & U \\ 5 & U \\ 5 & U \\ 10 & U \\ 5 & U \\ 10 & U \\ 5 & U \\ 5 & U \\ 5 & U \\ 5 & U \\ 5 & U \\ 5 & U\end{array}$


B-7

Table B.1 (continued)

\begin{tabular}{|c|c|c|c|c|c|}
\hline Analysis & Units & A horizon & Qual. & $\begin{array}{l}\text { A horizon } \\
\text { field dup }\end{array}$ & Qual. \\
\hline $\begin{array}{l}\text { Styrene } \\
\text { Tetrachloroethene } \\
\text { Toluene } \\
\text { Trans-1,2-Dichloroethene } \\
\text { Trans-1,3-Dichloropropene } \\
\text { Trichloroethene } \\
\text { Vinyl Chloride } \\
\text { Xylene (Meta + Para) } \\
1,1-D i c h l \text { oroethane } \\
1,1-D \text { ichloroethene } \\
1,1,1-\text { Trichloroethane } \\
1,1,2-\text { Trichloroethane } \\
1,1,2,2-\text { Tetrachloroethane } \\
1,2-D \text { ichloroethane } \\
1,2-D \text { ichloropropane } \\
2-\text { - utanone } \\
\text { 2-Hexanone } \\
\text { 4-Methyl-2-Pentanone }\end{array}$ & $\begin{array}{l}\mu g / \mathbf{k g} \\
\mu g / \mathbf{k g} \\
\mu g / \mathbf{k g} \\
\mu g / \mathbf{k g} \\
\mu g / \mathbf{k g} \\
\mu g / \mathbf{k g} \\
\mu g / \mathbf{k g} \\
\mu g / \mathbf{k g} \\
\mu g / \mathbf{k g} \\
\mu g / \mathbf{k g} \\
\mu g / \mathbf{k g} \\
\mu g / k g \\
\mu g / k g \\
\mu g / k g \\
\mu g / \mathbf{k g} \\
\mu g / k g \\
\mu g / \mathbf{k g} \\
\mu g / \mathbf{k g}\end{array}$ & $\begin{array}{r}5 \\
5 \\
5 \\
5 \\
5 \\
5 \\
10 \\
5 \\
5 \\
5 \\
5 \\
5 \\
5 \\
5 \\
5 \\
100 \\
50 \\
50\end{array}$ & $\begin{array}{l}U \\
U \\
U \\
U \\
U \\
U \\
U \\
U \\
U \\
U \\
U \\
U \\
U \\
U \\
U \\
U \\
U \\
U \\
U\end{array}$ & & \\
\hline
\end{tabular}

Acetone
Benzene
Bromodichloromethane
Bromoform
Bromomethane
Carbon Disulfide
Carbon Tetrachloride
Chlorobenzene
Chloroethane
Chloroform
Chloromethane
Cis-1,2-Dichloroethene
Cis-1,3-D ichloroplomane
Dibromochloromethane
Ethyl Benzene
Methylene Chloride
Ortho Xylene
Styrene
Tetrachloroethene
Toluene
Trans-1,2-Dichloroethene
Trans-1,3-Dichloropropene
Trichloroethene
Vinyl Chloride
Xylene (Meta + Para)
$1,1-D$ ichloroethane
$1,1-D$ ichloroethene
$1,1,1-$ Trichloroethane
$1,1,2-$ Trichloroethane
$1,1,2,2-$ Tetrachloroethane
$1,2-D i c h l o r o e t h a n e$
$1,2-D$ ichloropropane
$2-$ Butanone
$2-$ Hexanone
$4-$ Methyl-2-Pentanone

$$
\text { Location }=A N D ; \text { Formation }=\text { DISMAL GAP; Site }=10
$$

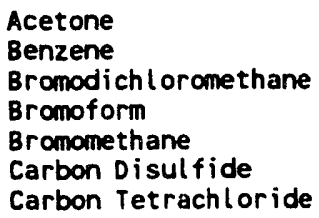

\begin{tabular}{|c|c|}
\hline 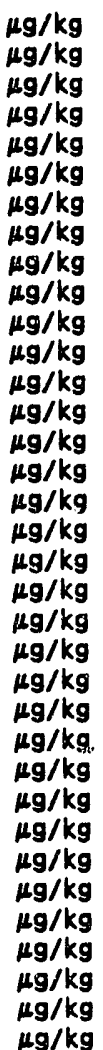 & $\begin{array}{r}8 \\
5 \\
5 \\
5 \\
10 \\
5 \\
5 \\
5 \\
10 \\
5 \\
10 \\
5 \\
5 \\
5 \\
5 \\
5 \\
5 \\
5 \\
5 \\
5 \\
5 \\
5 \\
5 \\
10 \\
5 \\
5 \\
5 \\
5 \\
5 \\
5 \\
5 \\
5 \\
100 \\
50 \\
50\end{array}$ \\
\hline
\end{tabular}


Table B.1 (continued)

\begin{tabular}{|c|c|c|c|c|c|}
\hline Analysis & Units & A horizon & Qual. & $\begin{array}{l}\text { A borizon } \\
\text { field dup }\end{array}$ & Qual. \\
\hline $\begin{array}{l}\text { Chlorobenzene } \\
\text { Chloroethane } \\
\text { Chloroform } \\
\text { Chloromethane } \\
\text { Cis-1,2-Dichloroethene } \\
\text { Cis-1,3-Dichloropropene } \\
\text { Dibromochloromethane } \\
\text { Ethyl Benzene } \\
\text { Methylene Chloride } \\
\text { Ortho Xylene } \\
\text { styrene } \\
\text { Tetrachloroethene } \\
\text { Toluene } \\
\text { Trans-1,2-Dichloroethene } \\
\text { Trans-1,3-Dichloropropene } \\
\text { Trichloroethene } \\
\text { Vinyl Chloride } \\
\text { Xylene (Meta + Para) } \\
1,1-D \text { ichloroethane } \\
1,1-D i c h l o r o e t h e n e \\
1,1,1-1 r i c h l o r o e t h a n e \\
1,1,2-T r i c h l o r o e t h a n e \\
1,1,2,2-\text { Tetrachloroethane } \\
1,2-D \text { ichloroethane } \\
1,2-D \text { ichloropropane } \\
2-\text { - Butanone } \\
2-\text { Hexanone } \\
4-\text { Methyl-2-Pentanone }\end{array}$ & $\begin{array}{l}\mu g / \mathbf{k g} \\
\mu g / \mathbf{k g} \\
\mu g / \mathbf{k g} \\
\mu g / \mathbf{k g} \\
\mu g / \mathbf{k g} \\
\mu g / \mathbf{k g} \\
\mu g / \mathbf{k g} \\
\mu g / \mathbf{k g} \\
\mu g / \mathbf{k g} \\
\mu g / \mathbf{k g} \\
\mu g / \mathbf{k g} \\
\mu g / \mathbf{k g} \\
\mu g / \mathbf{k g} \\
\mu g / \mathbf{k g} \\
\mu g / \mathbf{k g} \\
\mu g / \mathbf{k g} \\
\mu g / \mathbf{k g} \\
\mu g / \mathbf{k g} \\
\mu g / \mathbf{k g} \\
\mu g / \mathbf{k g} \\
\mu g / \mathbf{k g} \\
\mu g / \mathbf{k g} \\
\mu g / \mathbf{k g} \\
\mu g / \mathbf{k g} \\
\mu g / \mathbf{k g} \\
\mu g / \mathbf{k g} \\
\mu g / \mathbf{k g} \\
\mu g / \mathbf{k g}\end{array}$ & $\begin{array}{r}5 \\
10 \\
5 \\
10 \\
5 \\
5 \\
5 \\
5 \\
5 \\
5 \\
5 \\
5 \\
5 \\
5 \\
5 \\
5 \\
10 \\
5 \\
5 \\
5 \\
5 \\
5 \\
5 \\
5 \\
5 \\
100 \\
50 \\
50\end{array}$ & $\begin{array}{l}U \\
U \\
U \\
U \\
U \\
U \\
U \\
U \\
U \\
U \\
U \\
U \\
U \\
U \\
U \\
U \\
U \\
U \\
U \\
U \\
U \\
U \\
U \\
U \\
U \\
U \\
U \\
U\end{array}$ & & \\
\hline
\end{tabular}

$$
\text { Location }=A N D ; \text { Formation }=\text { DISMAL GAP; Site }=11
$$

Acetone
Benzene
Bromodichloromethane
Bromoform
Bromomethane
Carbon Disulfide
Carbon Tetrachloride
Chlorobenzene
Chloroethane
Chloroform
Chloromethane
Cis-1,2-Dichloroethene
Cis-1,3-Dichloropropene
Dibromochloromethane
Ethyl Benzene
Methylene Chloride
Ortho Xylene
Styrene
Tetrachloroethene
Toluene
Trans-1,2-Dichloroethene
Trans-1,3-Dichloropropene
Trichloroethene
Vinyl Chloride
Xylene (Meta + Para)
$1,1-D$ ichloroethane
$1,1-D i c h l o r o e t h e n e$
$1,1,1-$ Trichloroethane
$1,1,2-$ Trichloroethane
$1,1,2,2-$ Tetrachloroethane
$1,2-D$ ichloroethane
$1,2-D i c h l o r o p r o p a n e$
$2-$ Butanone
$2-$ Hexanone
$4-$ Methyl-2-Pentanone

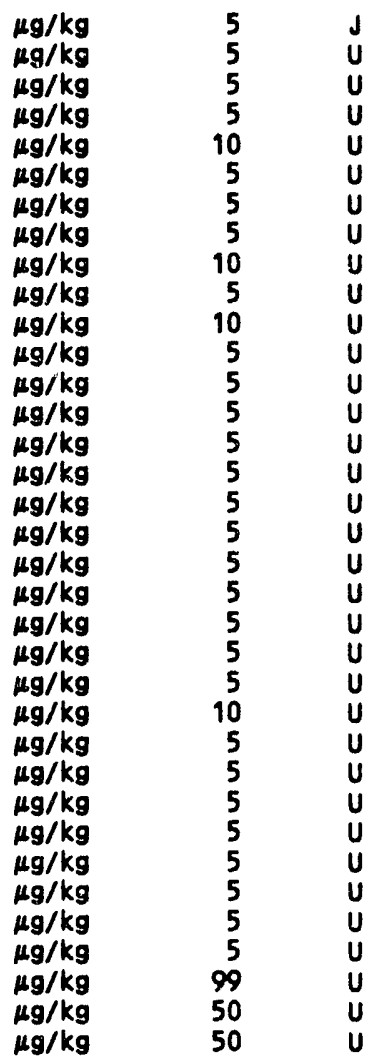


Table B.1 (continued)

\begin{tabular}{lll}
\hline & A horizon \\
Anatysis & Units A horizon Qual. field dup Qual. \\
\hline
\end{tabular}

$$
\text { Location }=A N D ; \text { Formation }=\text { DISMAL GAP; Site }=12
$$

Acetone
Benzene
Bronodichloromethane
Bromoform
Bromomethane
Carbon Disulfide
Carbon Tetrachloride
Chlorobenzene
Chloroethane
Chloroform
Chloromethane
Cis-1,2-Dichloroethene
Cis-1,3-Dichloropropene
Dibromochloromethane
Ethyl Benzene
Methylene Chloride
Ortho Xylene
Styrene
Tetrachloroethene
Toluene
Trans-1,2-Dichloroethene
Trans-1,3-Dichloropropene
Trichloroethene
Vinyl Chloride
Xylene (Meta + Para)
$1,1-D i c h l o r o e t h a n e$
$1,1-D i c h l o r o e t h e n e$
$1,1,1-$ Trichloroethane
$1,1,2-$ Trichloroethane
$1,1,2,2-$ Tetrachloroethane
$1,2-D$ ichloroethane
$1,2-D i c h l o r o p r o p a n e$
$2-$ Butanone
$2-$ Hexanone
$4-$ Methyl-2-Pentanone

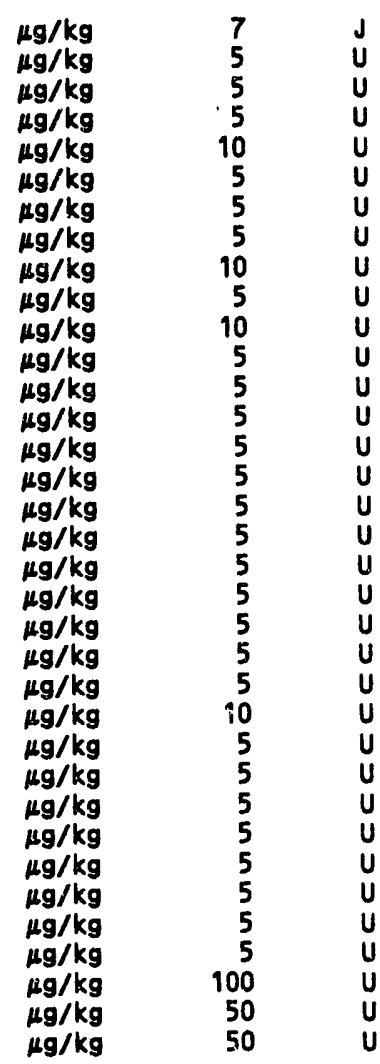

Location $=A N D ;$ Formation $=$ DISMAL GAP; Site $=19$

\begin{tabular}{|c|c|}
\hline $\begin{array}{l}\text { Acetone } \\
\text { Benzene } \\
\text { Bromodichloromethane } \\
\text { Bromoform } \\
\text { Bromomethane } \\
\text { Carbon Disulfide } \\
\text { Carbon Tetrachloride } \\
\text { Chlorobenzene } \\
\text { Chloroethane } \\
\text { Chloroform } \\
\text { Chloromethane } \\
\text { Cis-1,2-Dichloroethene } \\
\text { Cis-1,3-Dichloropropene } \\
\text { Dibromochloromethane } \\
\text { Ethyl Benzene } \\
\text { Methylene Chloride } \\
\text { Ortho Xylene } \\
\text { Styrene } \\
\text { Tetrachloroethene } \\
\text { Toluene } \\
\text { Trans-1,2-Dichloroethene } \\
\text { Trans-1,3-Dichloropropene } \\
\text { Trichloroethene } \\
\text { Vinyl Chloride } \\
\text { Xylene (Meta + Para) } \\
1,1-D \text { ichloroethane }\end{array}$ & $\begin{array}{l}\mu g / \mathrm{kg} \\
\mu g / \mathrm{kg} \\
\mu g / \mathrm{kg} \\
\mu \mathrm{g} / \mathrm{kg} \\
\mu \mathrm{g} / \mathrm{kg} \\
\mu \mathrm{g} / \mathrm{kg} \\
\mu \mathrm{g} / \mathrm{kg} \\
\mu \mathrm{g} / \mathrm{kg} \\
\mu \mathrm{g} / \mathrm{kg} \\
\mu \mathrm{g} / \mathrm{kg} \\
\mu g / \mathrm{kg} \\
\mu \mathrm{g} / \mathrm{kg} \\
\mu \mathrm{g} / \mathrm{kg} \\
\mu \mathrm{g} / \mathrm{kg} \\
\mu \mathrm{g} / \mathrm{kg} \\
\mu \mathrm{g} / \mathrm{kg} \\
\mu \mathrm{g} / \mathrm{kg}\end{array}$ \\
\hline
\end{tabular}

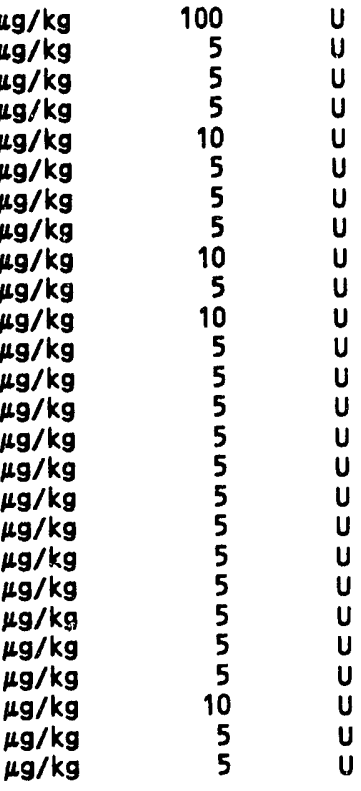


Table B.1 (continued)

\begin{tabular}{|c|c|c|c|c|c|}
\hline Analysis & Units & A borizon & Qual. & $\begin{array}{l}\text { A horizon } \\
\text { field dup }\end{array}$ & Qual. \\
\hline $\begin{array}{l}\text { 1,1-Dichloroethene } \\
1,1,1-\text { Trichloroethane } \\
\text { 1,1,2-Trichloroethane } \\
\text { 1,1,2,2-Tetrachloroethane } \\
\text { 1,2-D ichloroethane } \\
\text { 1,2-Dichloropropane } \\
\text { 2-Butanone } \\
\text { 2-Hexanone } \\
\text { 4- Methyl-2-Pentanone }\end{array}$ & $\begin{array}{l}\mu g / \mathbf{k g} \\
\mu g / \mathbf{k g} \\
\mu g / \mathbf{k g} \\
\mu g / \mathbf{k g} \\
\mu \mathrm{g} / \mathbf{k g} \\
\mu \mathrm{g} / \mathbf{k g} \\
\mu \mathrm{g} / \mathbf{k g} \\
\mu \mathrm{g} / \mathbf{k g} \\
\mu \mathrm{g} / \mathbf{k g}\end{array}$ & $\begin{array}{r}5 \\
5 \\
5 \\
5 \\
5 \\
5 \\
100 \\
50 \\
50\end{array}$ & $\begin{array}{l}\mathbf{U} \\
\mathbf{U} \\
\mathbf{U} \\
\mathbf{U} \\
\mathbf{U} \\
\mathbf{U} \\
\mathbf{U} \\
\mathbf{U} \\
\mathbf{U}\end{array}$ & & \\
\hline
\end{tabular}

$$
\text { Location }=A N D ; \text { Formation }=\text { DISMAL GAP; Site }=20
$$

Acetone
Benzene
Bromodichloromethane
Bromoform
Bromomethane
Carbon D isulfide
Carbon Tetrachloride
Chlorobenzene
Chloroethane
Chloroform
Chloromethane
Cis-1,2-Dichloroethene
Cis-1,3-Dichloropropene
Dibromochloromethane
Ethyl Benzene
Methylene Chloride
Ortho Xylene
Styrene
Tetrachloroethene
Toluene
Trans-1,2-Dichloroethene
Trans-1,3-Dichloropropene
Trichloroethene
Vinyl Chloride
Xylene (Meta + Para)
$1,1-D$ ichloroethane
$1,1-0$ ichloroethene
$1,1,1-T r$ ichloroethane
$1,1,2-$ Trichloroethane
$1,1,2,2-$ Tetrachloroethane
$1,2-D$ ichloroethane
$1,2-D$ ichloropropane
$2-$ Butanone
$2-$ Hexanone
$4-$ Methyl-2-Pentanone

$\mu g / k g$
$\mu g / k g$
$\mu g / k g$
$\mu g / k g$
$\mu g / k g$
$\mu g / k g$
$\mu g / k g$
$\mu g / k g$
$\mu g / k g$
$\mu g / k g$
$\mu g / k g$
$\mu g / k g$
$\mu g / k g$
$\mu g / k g$
$\mu g / k g$
$\mu g / k g$
$\mu g / k g$
$\mu g / k g$
$\mu g / k g$
$\mu g / k g$
$\mu g / k g$
$\mu g / k g$
$\mu g / k g$
$\mu g / k g$
$\mu g / k g$
$\mu g / k g$
$\mu g / k g$
$\mu g / k g$
$\mu g / k g$
$\mu g / k g$
$\mu g / k g$
$\mu g / k g$
$\mu g / k g$
$\mu g / k g$
$\mu g / k g$

$\begin{array}{rr}5 & U \\ 5 & U \\ 5 & U \\ 5 & U \\ 10 & U \\ 5 & U \\ 5 & U \\ 5 & U \\ 10 & U \\ 5 & U \\ 10 & U \\ 5 & U \\ 5 & U \\ 5 & U \\ 5 & U \\ 5 & U \\ 5 & U \\ 5 & U \\ 5 & U \\ 5 & U \\ 5 & U \\ 5 & U \\ 5 & U \\ 10 & U \\ 5 & U \\ 5 & U \\ 5 & U \\ 5 & U \\ 5 & U \\ 5 & U \\ 5 & U \\ 5 & U \\ 50 & U \\ 50 & U\end{array}$

Location $=A N D ;$ Formation $=$ DISMAL GAP; Site $=21$

\begin{tabular}{|c|c|}
\hline $\begin{array}{l}\text { Acetone } \\
\text { Benzene } \\
\text { Bromodichloromethane } \\
\text { Bromoform } \\
\text { Bromomethane } \\
\text { Carbon D isulfide } \\
\text { Carbon Tetrachloride } \\
\text { Chlorobenzene } \\
\text { Chloroethane } \\
\text { Chloroform } \\
\text { Chloromethane } \\
\text { Cis-1,2-Dichloroethene } \\
\text { Cis- } 1,3-\text { Dichloropropene } \\
\text { Dibromochloromethane } \\
\text { Ethyl Benzene }\end{array}$ & $\begin{array}{l}\mu g / \mathbf{k g} \\
\mu g / \mathbf{k g} \\
\mu g / \mathbf{k g} \\
\mu g / \mathbf{k g} \\
\mu g / \mathbf{k g} \\
\mu g / \mathbf{k g} \\
\mu g / \mathbf{k g} \\
\mu g / \mathbf{k g} \\
\mu g / k g \\
\mu g / \mathbf{k g} \\
\mu g / \mathbf{k g} \\
\mu g / \mathbf{k g} \\
\mu g / \mathbf{k g} \\
\mu g / \mathbf{k g} \\
\mu g / \mathbf{k g}\end{array}$ \\
\hline
\end{tabular}

Methylene Chloride $\quad \mathbf{H g} / \mathrm{kg}$

$\begin{array}{rr}6 & J \\ 5 & U \\ 5 & U \\ 5 & U \\ 10 & U \\ 5 & U \\ 5 & U \\ 5 & U \\ 10 & U \\ 5 & U \\ 10 & U \\ 5 & U \\ 5 & U \\ 5 & U \\ 5 & U \\ 5 & U\end{array}$


Table B.1 (continued)

\begin{tabular}{|c|c|c|c|c|c|}
\hline Analysis & Units & A horizon & Qual. & $\begin{array}{l}\text { A horizon } \\
\text { field dup }\end{array}$ & Qual. \\
\hline $\begin{array}{l}\text { Ortho Xylene } \\
\text { Styrene } \\
\text { Tetrachloroethene } \\
\text { Toluene } \\
\text { Trans-1,2-Dichloroethene } \\
\text { Trans- } 1,3-D \text { ichloropropene } \\
\text { Trichloroethene } \\
\text { Vinyl Chloride } \\
\text { Xylene (Meta + Para) } \\
1,1-D \text { ichloroethane } \\
1,1-D \text { ichloroethene } \\
1,1,1-\text { Trichloroethane } \\
1,1,2-\text { Trichloroethane } \\
1,1,2,2-\text { Tetrachloroethane } \\
1,2-D \text { ichloroethane } \\
1,2-D \text { ichloropropane } \\
2-\text {-Butanone } \\
\text { 2-Hexanone } \\
\text { 4-Methyl-2-Pentanone }\end{array}$ & $\begin{array}{l}\mu g / \mathbf{k g} \\
\mu g / \mathbf{k g} \\
\mu g / \mathbf{k g} \\
\mu g / \mathbf{k g} \\
\mu g / \mathbf{k g} \\
\mu g / \mathbf{k g} \\
\mu g / \mathbf{k g} \\
\mu g / \mathbf{k g} \\
\mu g / \mathbf{k g} \\
\mu g / \mathbf{k g} \\
\mu g / \mathbf{k g} \\
\mu g / \mathbf{k g} \\
\mu g / \mathbf{k g} \\
\mu g / \mathbf{k g} \\
\mu g / \mathbf{k g} \\
\mu g / \mathbf{k g} \\
\mu g / \mathbf{k g} \\
\mu g / \mathbf{k g} \\
\mu g / \mathbf{k g}\end{array}$ & $\begin{array}{r}5 \\
5 \\
5 \\
5 \\
5 \\
5 \\
5 \\
10 \\
5 \\
5 \\
5 \\
5 \\
5 \\
5 \\
5 \\
5 \\
100 \\
50 \\
50\end{array}$ & $\begin{array}{l}U \\
U \\
U \\
U \\
U \\
U \\
U \\
U \\
U \\
U \\
U \\
U \\
U \\
U \\
U \\
U \\
U \\
U \\
U\end{array}$ & & \\
\hline
\end{tabular}

\begin{tabular}{|c|c|c|}
\hline $\begin{array}{l}\text { Acetone } \\
\text { Benzene } \\
\text { Bromodichloromethane } \\
\text { Bromoform } \\
\text { Bromomethane } \\
\text { Carbon Disulfide } \\
\text { Carbon Tetrachloride } \\
\text { Chlorobenzene } \\
\text { Chloroethane } \\
\text { Chloroform } \\
\text { Chloromethane } \\
\text { Cis-1,2-Dichloroethene } \\
\text { Cis-1,3-Dichloropropene } \\
\text { Dibromochloromethane } \\
\text { Ethyl Benzene } \\
\text { Methylene Chloride } \\
\text { Ortho Xylene } \\
\text { Styrene } \\
\text { Tetrachloroethene } \\
\text { Toluene } \\
\text { Trans-1,2-Dichloroethene } \\
\text { Trans-1,3-Dichloropropene } \\
\text { Trichloroethene } \\
\text { Vinyl Chloride } \\
\text { Xylene (Meta + Para) } \\
1,1-D \text { ichloroethane } \\
1,1-D \text { ichloroethene } \\
1,1,1-\text { Trichloroethane } \\
1,1,2-\text { Trichloroethane } \\
1,1,2,2-\text { Tetrachloroethane } \\
1,2-0 \text { ichloroethane } \\
1,2-D \text { ichloropropane } \\
2-\text { Butanone } \\
2-\text { Hexanone } \\
4-\text { Methyl-2-Pentanone }\end{array}$ & 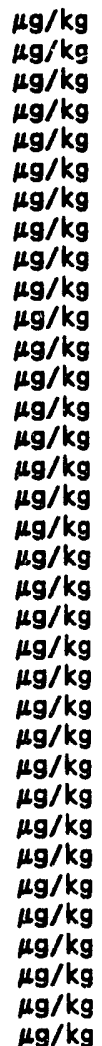 & $\begin{array}{r}10 \\
5 \\
5 \\
5 \\
10 \\
5 \\
5 \\
5 \\
10 \\
5 \\
10 \\
5 \\
5 \\
5 \\
5 \\
5 \\
5 \\
5 \\
5 \\
5 \\
5 \\
5 \\
5 \\
10 \\
5 \\
5 \\
5 \\
5 \\
5 \\
5 \\
5 \\
50 \\
50\end{array}$ \\
\hline
\end{tabular}

\begin{tabular}{|c|c|c|c|}
\hline $\begin{array}{l}\text { Acetone } \\
\text { Benzene } \\
\text { Bromodichloromethane } \\
\text { Bromoform } \\
\text { Bromomethane } \\
\text { Carbon Disulfide } \\
\text { Carbon Tetrachloride } \\
\text { Chlorobenzene }\end{array}$ & $\begin{array}{l}\mu g / \mathrm{kg} \\
\mu g / \mathrm{kg} \\
\mu g / \mathrm{kg} \\
\mu g / \mathrm{kg} \\
\mu g / \mathrm{kg} \\
\mu g / \mathrm{kg} \\
\mu g / \mathrm{kg} \\
\mu g / \mathrm{kg}\end{array}$ & $\begin{array}{r}26 \\
5 \\
5 \\
5 \\
10 \\
5 \\
5 \\
5\end{array}$ & $\begin{array}{l}B J \\
U \\
U \\
U \\
U \\
U \\
U \\
U\end{array}$ \\
\hline
\end{tabular}


Table B.1 (continued)

\begin{tabular}{|c|c|c|c|c|c|}
\hline Analysis & Units & A borizon & Qual. & $\begin{array}{l}\text { A horizon } \\
\text { field dup }\end{array}$ & Qual. \\
\hline $\begin{array}{l}\text { Chloroethane } \\
\text { Chloroform } \\
\text { Chloromethane } \\
\text { Cis-1,2-Dichloroethene } \\
\text { Cis-1,3-Dichloropropene } \\
\text { Dibromochloromethane } \\
\text { Ethyl Benzene } \\
\text { Freon-113 } \\
\text { Methylene Chloride } \\
\text { Ortho Xylene } \\
\text { Styrene } \\
\text { Tetrachloroethene } \\
\text { Toluene } \\
\text { Trans-1,2-Dichloroethene } \\
\text { Trans-1,3-Dichloropropene } \\
\text { Trichloroethene } \\
\text { Trichlorofluoromethane } \\
\text { Vinyl Acetate } \\
\text { Vinyl Chloride } \\
\text { Xylene (Meta + Para) } \\
\text { 1,1-Dichloroethane } \\
1,1-D \text { ichloroethene } \\
1,1,1-\text { Trichloroethane } \\
1,1,2-\text { Trichloroethane } \\
1,1,2,2-T e t r a c h l o r o e t h a n e \\
1,2-D i c h l o r o e t h a n e \\
1,2-D i c h l o r o p r o p a n e \\
1,4-D i c h l o r o b e n z e n e \\
2-\text { Butanone } \\
2-C h l o r o e t h y l \text { Vinyl Ether } \\
2-\text { Hexanone } \\
4-\text { Methyl-2-Pentanone }\end{array}$ & $\begin{array}{l}\mu g / \mathbf{k g} \\
\mu g / \mathbf{k g} \\
\mu g / \mathbf{k g} \\
\mu g / \mathbf{k g} \\
\mu g / \mathbf{k g} \\
\mu g / \mathbf{k g} \\
\mu g / \mathbf{k g} \\
\mu g / \mathbf{k g} \\
\mu g / \mathbf{k g} \\
\mu g / \mathbf{k g} \\
\mu g / \mathbf{k g} \\
\mu g / \mathbf{k g} \\
\mu g / \mathbf{k g} \\
\mu g / \mathbf{k g} \\
\mu g / \mathbf{k g} \\
\mu g / \mathbf{k g} \\
\mu g / \mathbf{k g} \\
\mu g / \mathbf{k g} \\
\mu g / \mathbf{k g} \\
\mu g / \mathbf{k g} \\
\mu g / \mathbf{k g} \\
\mu g / \mathbf{k g} \\
\mu g / \mathbf{k g} \\
\mu g / \mathbf{k g} \\
\mu g / \mathbf{k g} \\
\mu g / \mathbf{k g} \\
\mu g / \mathbf{k g} \\
\mu g / \mathbf{k g} \\
\mu g / \mathbf{k g} \\
\mu g / \mathbf{k g} \\
\mu g / \mathbf{k g} \\
\mu g / \mathbf{k g}\end{array}$ & $\begin{array}{r}10 \\
8 \\
10 \\
5 \\
5 \\
5 \\
5 \\
5 \\
5 \\
5 \\
5 \\
5 \\
5 \\
5 \\
5 \\
5 \\
5 \\
5 \\
10 \\
5 \\
5 \\
5 \\
5 \\
5 \\
5 \\
5 \\
5 \\
5 \\
99 \\
5 \\
50 \\
50\end{array}$ & $\begin{array}{l}U \\
U \\
U \\
U \\
U \\
U \\
U \\
U \\
U \\
U \\
U \\
U \\
U \\
U \\
U \\
U \\
U \\
U \\
U \\
U \\
U \\
U \\
U \\
U \\
U \\
U \\
U \\
U \\
U \\
U \\
U \\
U\end{array}$ & & \\
\hline
\end{tabular}

Location $=$ ORR; Formation $=$ DISMAL GAP; Site $=10$

\begin{tabular}{|c|c|c|}
\hline 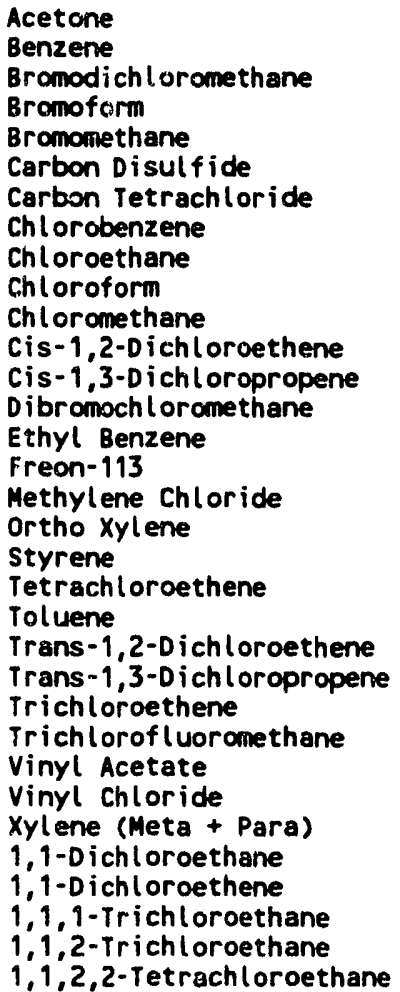 & $\begin{array}{l}\mu g / k g \\
\mu g / k g \\
\mu g / k g \\
\mu g / k g \\
\mu g / k g \\
\mu g / k g \\
\mu g / k g \\
\mu g / k g \\
\mu g / k g \\
\mu g / k g \\
\mu g / k g \\
\mu g / k g \\
\mu g / k g \\
\mu g / k g \\
\mu g / k g \\
\mu g / k g \\
\mu g / k g \\
\mu g / k g \\
\mu g / k g \\
\mu g / k g \\
\mu g / k g \\
\mu g / k g \\
\mu g / k g \\
\mu g / k g \\
\mu g / k g \\
\mu g / k g \\
\mu g / k g \\
\mu g / k g \\
\mu g / k g \\
\mu g / k g \\
\mu g / k g \\
\mu g / k g \\
\mu g / k g\end{array}$ & $\begin{array}{r}14 \\
5 \\
5 \\
5 \\
10 \\
5 \\
5 \\
5 \\
10 \\
5 \\
10 \\
5 \\
5 \\
5 \\
5 \\
10 \\
5 \\
5 \\
5 \\
5 \\
5 \\
5 \\
5 \\
5 \\
10 \\
10 \\
10 \\
5 \\
5\end{array}$ \\
\hline
\end{tabular}


Table B.1 (continued)

\begin{tabular}{|c|c|c|c|c|c|}
\hline Analysis & Units & A horizon & Qual. & $\begin{array}{l}\text { A horizon } \\
\text { field dup }\end{array}$ & Qual. \\
\hline $\begin{array}{l}\text { 1,2-D ichlorobenzene } \\
\text { 1,2-D ichloroethane } \\
\text { 1,2-D ichloropropane } \\
\text { 1,3-D ichlorobenzene } \\
\text { 1,4-D ichlorobenzene } \\
\text { 2-Butanone } \\
\text { 2-Chloroethyl Vinyl Ether } \\
\text { 2-Hexanone } \\
\text { 4-Methyl-2-Pentanone }\end{array}$ & $\begin{array}{l}\mu g / \mathbf{k g} \\
\mu g / k g \\
\mu g / k g \\
\mu g / k g \\
\mu g / k g \\
\mu g / k g \\
\mu g / k g \\
\mu g / k g \\
\mu g / k g\end{array}$ & $\begin{array}{r}5 \\
5 \\
5 \\
5 \\
5 \\
98 \\
5 \\
49 \\
49\end{array}$ & $\begin{array}{l}U \\
U \\
U \\
U \\
U \\
U \\
U \\
U \\
U\end{array}$ & & \\
\hline
\end{tabular}

\begin{tabular}{|c|c|c|}
\hline 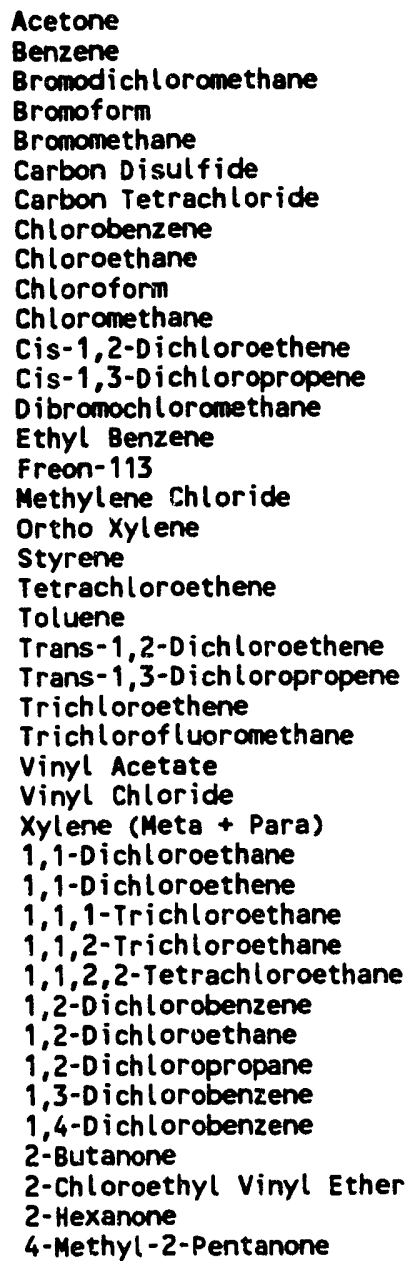 & 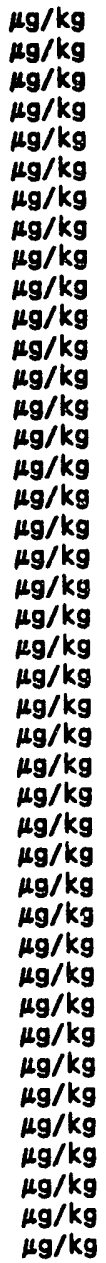 & $\begin{array}{r}52 \\
5 \\
5 \\
5 \\
10 \\
5 \\
5 \\
5 \\
10 \\
5 \\
10 \\
5 \\
5 \\
5 \\
5 \\
10 \\
5 \\
5 \\
5 \\
5 \\
5 \\
5 \\
5 \\
5 \\
10 \\
10 \\
10 \\
5 \\
5 \\
5 \\
5 \\
5 \\
5 \\
5 \\
5 \\
100 \\
5\end{array}$ \\
\hline
\end{tabular}

$$
\text { Location }=\text { ORR; Formation }=\text { DISMAL GAP; Site }=19
$$

$\begin{array}{lrrr}\text { Acetone } & \mu \mathrm{g} / \mathrm{kg} & 41 & \mathrm{BJ} \\ \text { Benzene } & \mu \mathrm{g} / \mathrm{kg} & 5 & U \\ \text { Bromodichloromethane } & \mu \mathrm{g} / \mathrm{kg} & 5 & U \\ \text { Bromoform } & \mu \mathrm{g} / \mathrm{kg} & 5 & U \\ \text { Bromomethane } & \mu \mathrm{g} / \mathrm{kg} & 10 & U \\ \text { Carbon Disulfide } & \mu \mathrm{g} / \mathrm{kg} & 5 & U \\ \text { Carbon Tetrachloride } & \mu \mathrm{g} / \mathrm{kg} & 5 & \mathrm{U} \\ \text { Chlorobenzene } & \mu \mathrm{g} / \mathrm{kg} & 5 & \mathrm{U} \\ \text { Chloroethane } & \mu \mathrm{g} / \mathrm{kg} & 10 & U\end{array}$


Table B.1 (continued)

\begin{tabular}{|c|c|c|c|c|c|}
\hline Analysis & Units & A horizon & Qual. & $\begin{array}{l}\text { A borizon } \\
\text { field dup }\end{array}$ & Qual. \\
\hline $\begin{array}{l}\text { Chloroform } \\
\text { Chloronethane } \\
\text { Cis-1,2-Dichloroethene } \\
\text { Cis-1,3-Dichloropropene } \\
\text { Dibromochloromethane } \\
\text { Ethyl Benzene } \\
\text { Freon-113 } \\
\text { Methylene Chloride } \\
\text { Ortho Xylene } \\
\text { styrene } \\
\text { Tetrachloroethene } \\
\text { Toluene } \\
\text { Trans-1,2-Dichloroethene } \\
\text { Trans-1,3-Dichloropropene } \\
\text { Trichloroethene } \\
\text { Trichlorofluoromethane } \\
\text { Vinyl Acetate } \\
\text { Vinyl Chloride } \\
\text { Xylene (Meta + Para) } \\
1,1-D \text { ichloroethane } \\
1,1-D i c h l o r o e t h e n e \\
1,1,1-\text { Trichloroethane } \\
1,1,2-\text { Trichloroethane } \\
1,1,2,2-\text { Tetrachloroethane } \\
1,2-D \text { ichloroethane } \\
1,2-D \text { ichloropropane } \\
1,4-D \text { ichlorobenzene } \\
2-\text { - utanone } \\
2-\text { Chloroethyl Vinyl Ether } \\
2-\text { Hexanone } \\
4-\text { Methyl-2-Pentanone }\end{array}$ & $\begin{array}{l}\mu g / k g \\
\mu g / k g \\
\mu g / k g \\
\mu g / k g \\
\mu g / k g \\
\mu g / k g \\
\mu g / k g \\
\mu g / k g \\
\mu g / k g \\
\mu g / k g \\
\mu g / k g \\
\mu g / k g \\
\mu g / k g \\
\mu g / k g \\
\mu g / k g \\
\mu g / k g \\
\mu g / k g \\
\mu g / k g \\
\mu g / k g \\
\mu g / k g \\
\mu g / k g \\
\mu g / k g \\
\mu g / k g \\
\mu g / k g \\
\mu g / k g \\
\mu g / k g \\
\mu g / k g \\
\mu g / k g \\
\mu g / k g \\
\mu g / k g \\
\mu g / k g\end{array}$ & $\begin{array}{r}5 \\
10 \\
5 \\
5 \\
5 \\
5 \\
5 \\
5 \\
5 \\
5 \\
5 \\
5 \\
5 \\
5 \\
5 \\
5 \\
5 \\
10 \\
5 \\
5 \\
5 \\
5 \\
5 \\
5 \\
5 \\
5 \\
5 \\
100 \\
5 \\
50 \\
50\end{array}$ & $\begin{array}{l}U \\
U \\
U \\
U \\
U \\
U \\
U U \\
U \\
U \\
U \\
U \\
U \\
U \\
U \\
U \\
U \\
U \\
U \\
U \\
U \\
U \\
U \\
U \\
U \\
U \\
U \\
U \\
U \\
U \\
U \\
U\end{array}$ & & \\
\hline
\end{tabular}

Acetone
Benzene
Bromodichloromethane
Bromoform
Bromomethane
Carbon Disulfide
Carbon Tetrachloride
Chlorobenzene
Chloroethane
Chloroform
Chloromethane
Cis-1,2-Dichloroethene
Cis-1,3-Dichloropropene
Dibromochloromethane
Ethyl Benzene
Freon-113
Methylene Chloride
Ortho Xylene
Styrene
Tetrachloroethene
Toluene
Trans-1,2-0ichloroethene
Trans-1,3-Dichloropropene
Trichloroethene
Trichlorofluoromethane
Vinyl Acetate
Vinyl Chloride
Xylene (Meta + Para)
$1,1-0$ ichloroethane
$1,1-D$ ichloroethene
$1,1,1-$ Trichloroethane
$1,1,2-$ Trichloroethane
$1,1,2,2-T e t r a c h l o r o e t h a n e$
$1,2-0$ ichloroethane

\begin{tabular}{|c|c|}
\hline $\begin{array}{l}\mu g / k g \\
\mu g / k g \\
\mu g / k g \\
\mu g / k g \\
\mu g / k g \\
\mu g / k g \\
\mu g / k g \\
\mu g / k g \\
\mu g / k g \\
\mu g / k g \\
\mu g / k g \\
\mu g / k g \\
\mu g / k g \\
\mu g / k g \\
\mu g / k g \\
\mu g / k g \\
\mu g / k g \\
\mu g / k g \\
\mu g / k g \\
\mu g / k g \\
\mu g / k g \\
\mu g / k g \\
\mu g / k g \\
\mu g / k g \\
\mu g / k g \\
\mu g / k g \\
\mu g / k g \\
\mu g / k g \\
\mu g / k g \\
\mu g / k g \\
\mu g / k g \\
\mu g / k g \\
\mu g / k g \\
\mu g / k g\end{array}$ & $\begin{array}{r}26 \\
5 \\
5 \\
5 \\
10 \\
5 \\
5 \\
5 \\
10 \\
5 \\
10 \\
5 \\
5 \\
5 \\
5 \\
5 \\
5 \\
5 \\
5 \\
5 \\
5 \\
5 \\
5 \\
5 \\
5 \\
5 \\
10 \\
5 \\
5 \\
5 \\
5 \\
5\end{array}$ \\
\hline
\end{tabular}




\section{B-15}

Table B.1 (continued)

\begin{tabular}{|c|c|c|c|c|c|}
\hline Analysis & Units & A horizon & Qual. & $\begin{array}{l}\text { A horizon } \\
\text { field dup }\end{array}$ & Qual. \\
\hline $\begin{array}{l}\text { 1,2-Dichloropropane } \\
\text { 1,4-Dichlorobenzene } \\
\text { 2-Butanone } \\
\text { 2-Chloroethyl Vinyl Ether } \\
\text { 2-Hexanone } \\
\text { 4-Methyl-2-Pentanone }\end{array}$ & $\begin{array}{l}\mu g / \mathbf{k g} \\
\mu g / \mathbf{k g} \\
\mu g / \mathbf{k g} \\
\mu g / \mathbf{k g} \\
\mu g / \mathbf{k g} \\
\mu \mathrm{g} / \mathbf{k g}\end{array}$ & $\begin{array}{r}5 \\
5 \\
100 \\
5 \\
51 \\
51\end{array}$ & $\begin{array}{l}U \\
U \\
U \\
U \\
U \\
U\end{array}$ & & \\
\hline
\end{tabular}

\begin{tabular}{|c|c|c|}
\hline 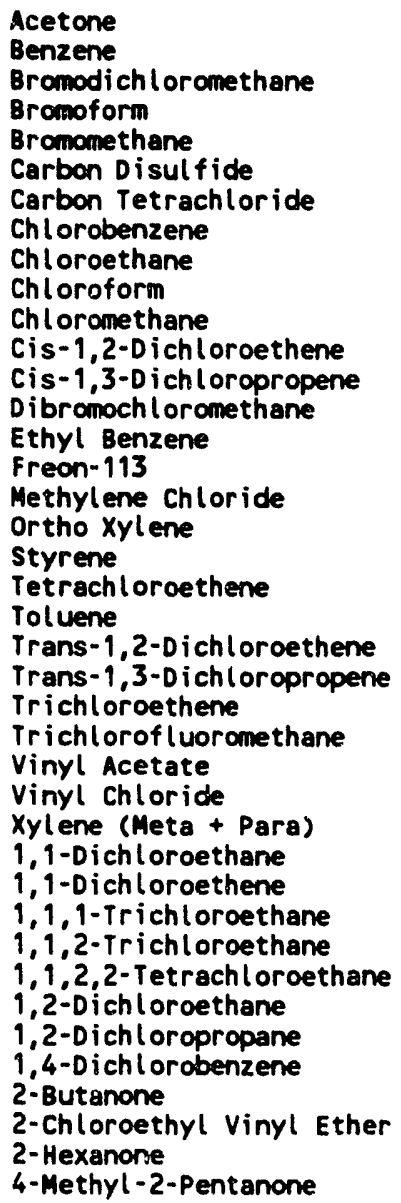 & 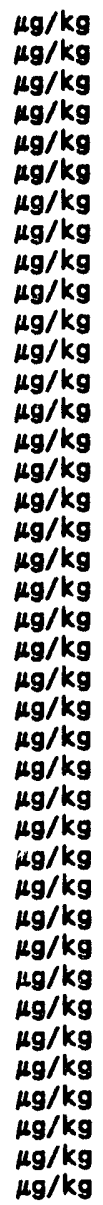 & $\begin{array}{r}64 \\
5 \\
5 \\
5 \\
10 \\
5 \\
5 \\
5 \\
10 \\
5 \\
10 \\
5 \\
5 \\
5 \\
5 \\
5 \\
5 \\
5 \\
5 \\
5 \\
5 \\
5 \\
5 \\
5 \\
5 \\
5 \\
10 \\
5 \\
5 \\
5 \\
5 \\
5 \\
50 \\
50\end{array}$ \\
\hline
\end{tabular}

$$
\text { Location }=\text { ORR; Formation }=\text { DISMAL GAP; Site }=27
$$

\begin{tabular}{|c|c|c|}
\hline $\begin{array}{l}\text { Acetone } \\
\text { Benzene } \\
\text { Bromodichloromethane } \\
\text { Bromoform } \\
\text { Bromomethane } \\
\text { Carbon D isulf ide } \\
\text { Carbon Tetrachloride } \\
\text { Chlorobenzene } \\
\text { Chloroethane } \\
\text { Chloroform } \\
\text { Chloromethane } \\
\text { Cis-1,2-Dichloroethene } \\
\text { Cis-1,3-D ich loropropene } \\
\text { Dibromochloromethane }\end{array}$ & $\begin{array}{l}\mu g / k g \\
\mu g / k g \\
\mu g / k g \\
\mu g / k g \\
\mu g / k g \\
\mu g / k g \\
\mu g / k g \\
\mu g / k g \\
\mu g / k g \\
\mu g / k g \\
\mu g / k g \\
\mu g / k g \\
\mu g / k g \\
\mu g / k g\end{array}$ & $\begin{array}{r}53 \\
5 \\
5 \\
5 \\
10 \\
5 \\
5\end{array}$ \\
\hline
\end{tabular}


Table B.1 (continued)

\begin{tabular}{|c|c|c|c|c|c|}
\hline Analysis & Units & A horizon & Qual. & $\begin{array}{l}\text { A horizon } \\
\text { field dup }\end{array}$ & Qual. \\
\hline 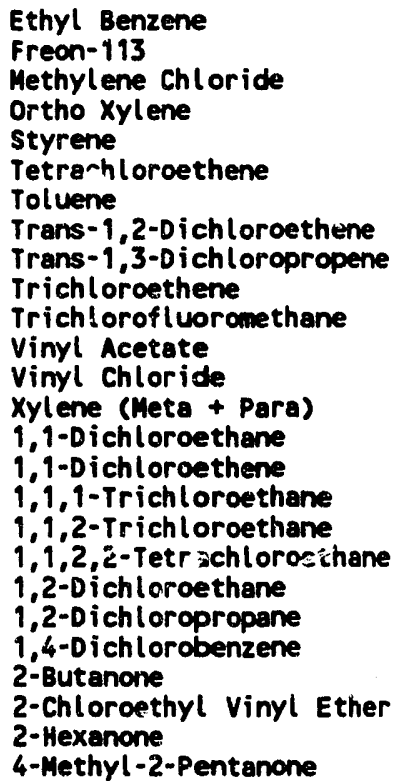 & 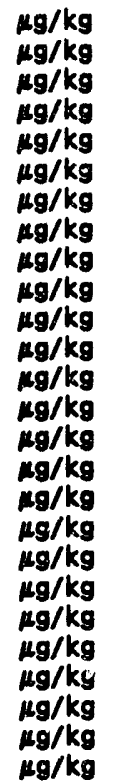 & $\begin{array}{r}5 \\
5 \\
5 \\
5 \\
5 \\
5 \\
5 \\
5 \\
5 \\
5 \\
5 \\
5 \\
10 \\
5 \\
5 \\
5 \\
5 \\
5 \\
5 \\
5 \\
5 \\
5 \\
8 \\
5 \\
49 \\
49\end{array}$ & 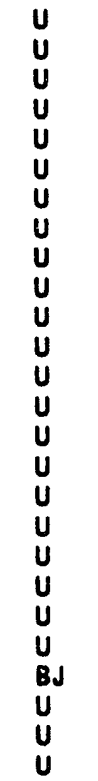 & & \\
\hline
\end{tabular}

Acetone
Benzene
Bromodichloronethane
Bromoform
Bromomethane
Carbon Disulfide
Carbon Tetrachloride
Chlorobenzene
Chloroethane
Chloroform
Chloromethane
Cis-1,2-Dichloroethene
Cis-1,3-Dichloropropene
Dibromochloromethane
Ethyl Benzene
Froon-113
Methylene Chloride
Ortho Xylene
Styrene
Tetrachloroethene
Toluene
Trans-1,2-Dichloroethen:
Trans-1,3-Dichloropropene
Trichloroethene
Trichlorofluoromethane
Vinyl Acetate
Vinyl Chloride
Xylene (Meta + Para)
$1,1-D i c h l o r o e t h a n e$
$1,1-D i c h l o r o e t h e n e$
$1,1,1-$ Trichloroethane
$1,1,2-$ Trichloroetthane
$1,1,2,2-$ Tetrachloroethane
$1,2-D$ ichloroethane
$1,2-D i c h l o r o p r o p a n e$
$1,4-D$ ichlorobenzene
$2-$ Butanone
$2-$ Chloroethyl Vinyl Ether
$2-$ Hexanone
$4-$ Methyl-2-Pentanone

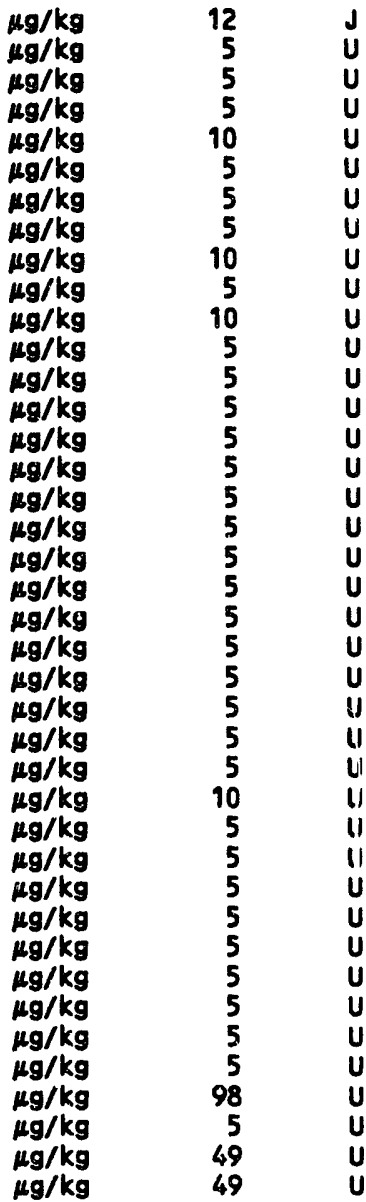


Table B.1 (continued)

\begin{tabular}{ll}
\hline & \\
Analysis & Anorizon \\
& Units A horizon Qual. field dup Qual. \\
\hline
\end{tabular}

Location $=$ ORR; Formation $=$ DISMAL GAP; Site $=33$

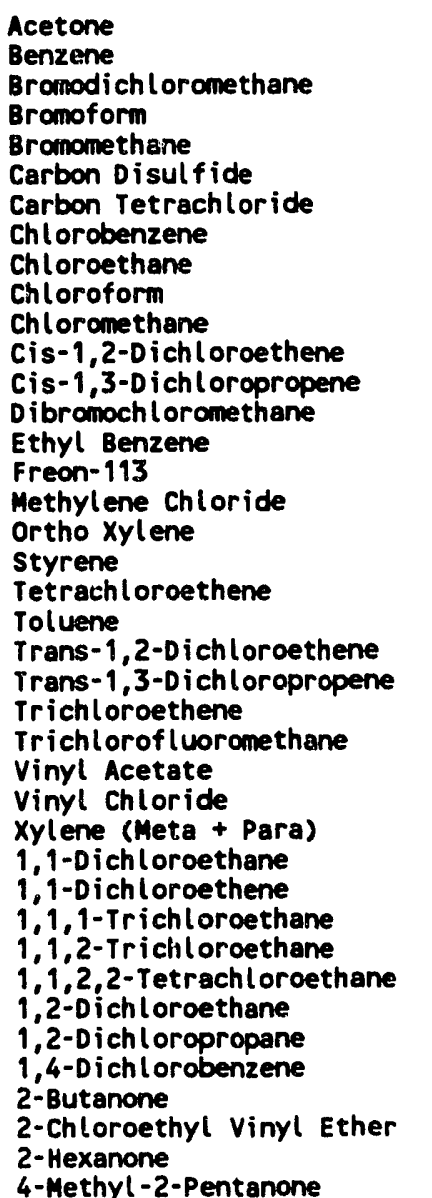

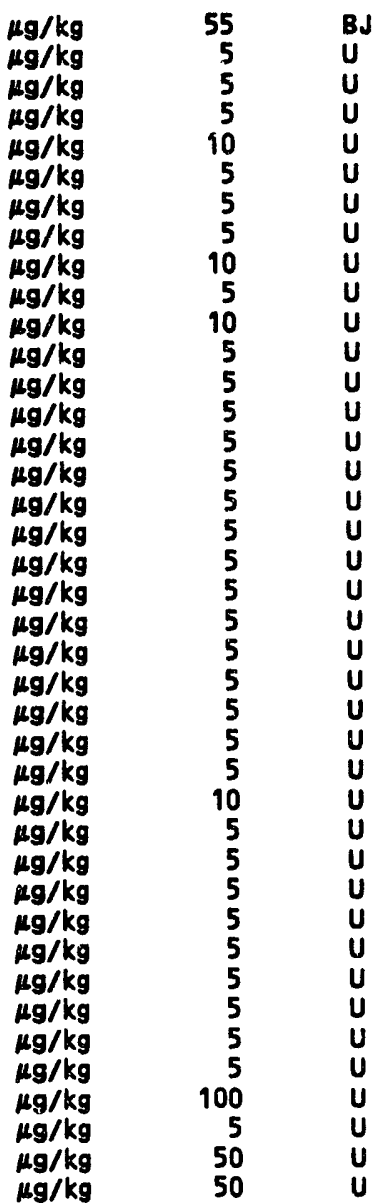

$$
\text { Location }=\text { ORR; Formation }=\text { DISMAL GAP; Site }=35
$$

\begin{tabular}{|c|c|}
\hline $\begin{array}{l}\text { Acetone } \\
\text { Benzene } \\
\text { Bromodichloromethane } \\
\text { Bromoform } \\
\text { Bromomethane } \\
\text { Carbon Disul fide } \\
\text { Carbon Tetrachloride } \\
\text { Chlorobenzene } \\
\text { Chloroethane } \\
\text { Chloroform } \\
\text { Chloromethane } \\
\text { Cis-1,2-Dichloroethene } \\
\text { Cis-1,3-Dichloropropene } \\
\text { Dibromochloromethane } \\
\text { Ethyl Benzene } \\
\text { Freon-113 } \\
\text { Methylene Chloride } \\
\text { Ortho Xylene } \\
\text { Styrene } \\
\text { Tetrachloroethene } \\
\text { Toluene } \\
\text { Trans-1,2-Dichloroethene } \\
\text { Trans-1,3-Dichloropropene }\end{array}$ & $\begin{array}{l}\mu g / k g \\
\mu g / k g \\
\mu g / k g \\
\mu g / k g \\
\mu g / k g \\
\mu g / k g \\
\mu g / k g \\
\mu g / k g \\
\mu g / k g \\
\mu g / k g \\
\mu g / k g \\
\mu g / k g \\
\mu g / k g \\
\mu g / k g \\
\mu g / k g \\
\mu g / k g \\
\mu g / k g \\
\mu g / k g \\
\mu g / k g\end{array}$ \\
\hline
\end{tabular}

$\begin{array}{rr}7 & \text { BJ } \\ 5 & U \\ 5 & U \\ 5 & U \\ 10 & U \\ 5 & U \\ 5 & U \\ 5 & U \\ 10 & U \\ 5 & U \\ 10 & U \\ 5 & U \\ 5 & U \\ 5 & U \\ 5 & U \\ 5 & U \\ 5 & U \\ 5 & U \\ 5 & U \\ 5 & U \\ 5 & U \\ 5 & U \\ 5 & U\end{array}$


Table B.1 (continued)

\begin{tabular}{|c|c|c|c|c|c|}
\hline Analysis & Units & A horizon & Qual. & $\begin{array}{l}\text { A horizon } \\
\text { field dup }\end{array}$ & Qual. \\
\hline 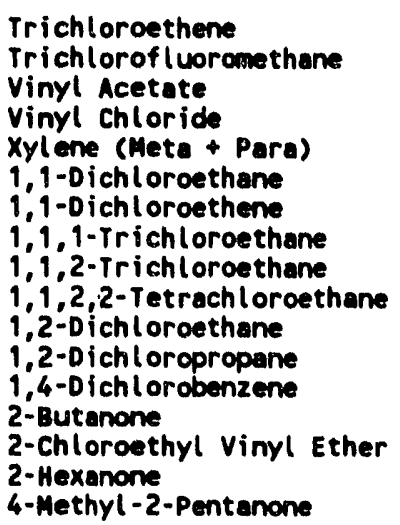 & $\begin{array}{l}\mu g / k g \\
\mu g / k g \\
\mu g / k g \\
\mu g / k g \\
\mu g / k g \\
\mu g / k g \\
\mu g / k g \\
\mu g / k g \\
\mu g / k g \\
\mu g / k g \\
\mu g / k g \\
\mu g / k g \\
\mu g / k g \\
\mu g / k g \\
\mu g / k g \\
\mu g / k g \\
\mu g / k g\end{array}$ & $\begin{array}{r}5 \\
5 \\
5 \\
10 \\
5 \\
5 \\
5 \\
5 \\
5 \\
5 \\
5 \\
5 \\
5 \\
6 \\
5 \\
50 \\
50\end{array}$ & $\begin{array}{l}U \\
U \\
U \\
U \\
U \\
U \\
U \\
U \\
U \\
U \\
U \\
U \\
U \\
B J \\
U \\
U \\
U\end{array}$ & & \\
\hline
\end{tabular}

\begin{tabular}{|c|c|}
\hline $\begin{array}{l}\text { ne } \\
\text { ne } \\
\text { dichloromethane } \\
\text { form } \\
\text { methane } \\
n \text { Disulfide } \\
n \text { Tetrachloride } \\
\text { obenzene } \\
\text { oethane } \\
\text { oform } \\
\text { omethane } \\
\text {-2-Dichloroethene } \\
\text { 3-Dichloropropene } \\
\text { mochloromethane } \\
\text { Benzene } \\
\text {-113 } \\
\text { lene Chloride } \\
\text { Xylene } \\
\text { ne } \\
\text { chloroethene } \\
\text { ne } \\
\text {-1,2-Dichloroethene } \\
\text { - I,3-Dichloropropene } \\
\text { loroethene } \\
\text { lorofluoromethane } \\
\text { Acetate } \\
\text { Chloride } \\
\text { e (Meta + Para) } \\
\text { ichloroethane } \\
\text { ichloroethene } \\
\text { - Trichloroethane } \\
\text { - Trichloroethane } \\
\text { 2-Tetrachloroethane } \\
\text { ichloroethane } \\
\text { ichloropropane } \\
\text { ichlorobenzene } \\
\text { anone } \\
\text { oroethyl vinyl Ether } \\
\text { anone } \\
\text { hyl-2-Pentanone }\end{array}$ & $\begin{array}{l}\mu g / \mathrm{kg} \\
\mu g / \mathrm{kg} \\
\mu g / \mathrm{kg} \\
\mu g / \mathrm{kg} \\
\mu g / \mathrm{kg} \\
\mu g / \mathrm{kg} \\
\mu g / \mathrm{kg} \\
\mu g / \mathrm{kg} \\
\mu g / \mathrm{kg} \\
\mu g / \mathrm{kg} \\
\mu g / \mathrm{kg} \\
\mu g / \mathrm{kg} \\
\mathrm{Mg} / \mathrm{kg} \\
\mu g / \mathrm{kg} \\
\mu g / \mathrm{kg} \\
\mu g / \mathrm{kg} \\
\mu g / \mathrm{kg} \\
\mu g / \mathrm{kg} \\
\mu g / \mathrm{kg} \\
\mu g / \mathrm{kg} \\
\mu g / \mathrm{kg} \\
\mu g / \mathrm{kg} \\
\mu g / \mathrm{kg} \\
\mu g / \mathrm{kg} \\
\mu g / \mathrm{kg} \\
\mu g / \mathrm{kg} \\
\mu g / \mathrm{kg} \\
\mu g / \mathrm{kg} \\
\mu g / \mathrm{kg}\end{array}$ \\
\hline
\end{tabular}

Location $=$ ORR; Formation $=$ DISMAL GAP; Site $=43$

$\begin{array}{lrrr}\text { Acetone } & \mu g / k g & 100 & U \\ \text { Benzene } & \mu g / k g & 5 & U \\ \text { Bromodichloromethane } & \mu g / k g & 5 & U \\ \text { Bromoform } & \mu g / k g & 5 & U \\ \text { Bromomethane } & \mu g / k g & 10 & U\end{array}$


Table B.1 (continued)

\begin{tabular}{|c|c|c|c|c|c|}
\hline Analysis & Units & A horizon & Qual. & $\begin{array}{l}\text { A horizon } \\
\text { field dup }\end{array}$ & Qual. \\
\hline $\begin{array}{l}\text { Carbon Disulfide } \\
\text { Carbon Tetrachloride } \\
\text { Chlorobenzene } \\
\text { Chloroethane } \\
\text { Chloroform } \\
\text { Chloromethane } \\
\text { Cis-1,2-Dichloroethene } \\
\text { Cis-1,3-D ichloropropene } \\
\text { Dibromochloromethane } \\
\text { Ethyl Benzene } \\
\text { Freon-113 } \\
\text { Methylene Chloride } \\
\text { Ortho Xylene } \\
\text { styrene } \\
\text { Tetrachloroethene } \\
\text { Toluene } \\
\text { Trans-1,2-Dichloroethene } \\
\text { Trans-1,3-Dichloropropene } \\
\text { Trichloroethene } \\
\text { Trichlorofluoromethane } \\
\text { Vinyl Acetate } \\
\text { Vinyl Chloride } \\
\text { xylene (Meta + Para) } \\
1,1-D i c h l o r o e t h a n e \\
1,1-D i c h l o r o e t h e n e \\
1,1,1-T r i c h l o r o e t h a n e \\
1,1,2-\text { Trichloroethane } \\
1,1,2,2-\text { Tetrachloroethane } \\
1,2-\text { Dichloroethane } \\
1,2-D i c h l o r o p r o p a n e \\
1,4-D \text { ichlorobenzene } \\
2-\text { Butanone } \\
2-\text { Chloroethyl Vinyl Ether } \\
2-\text { Hexanone } \\
4-\text { Methyl-2-Pentanone }\end{array}$ & $\begin{array}{l}\mu g / \mathbf{k g} \\
\mu g / \mathbf{k g} \\
\mu g / \mathbf{k g} \\
\mu g / \mathbf{k g} \\
\mu g / \mathbf{k g} \\
\mu g / \mathbf{k g} \\
\mu g / \mathbf{k g} \\
\mu g / \mathbf{k g} \\
\mu g / \mathbf{k g} \\
\mu g / \mathbf{k g} \\
\mu g / \mathbf{k g} \\
\mu g / \mathbf{k g} \\
\mu g / \mathbf{k g} \\
\mu g / \mathbf{k g} \\
\mu g / \mathbf{k g} \\
\mu g / \mathbf{k g} \\
\mu g / \mathbf{k g} \\
\mu g / \mathbf{k g} \\
\mu g / \mathbf{k g} \\
\mu g / \mathbf{k g} \\
\mu g / \mathbf{k g} \\
\mu g / \mathbf{k g} \\
\mu g / \mathbf{k g} \\
\mu g / \mathbf{k g} \\
\mu g / \mathbf{k g} \\
\mu g / \mathbf{k g} \\
\mu g / \mathbf{k g} \\
\mu g / \mathbf{k g} \\
\mu g / \mathbf{k g} \\
\mu g / \mathbf{k g} \\
\mu g / \mathbf{k g} \\
\mu g / \mathbf{k g} \\
\mu g / \mathbf{k g} \\
\mu g / \mathbf{k g} \\
\mu g / \mathbf{k g}\end{array}$ & $\begin{array}{r}5 \\
5 \\
5 \\
10 \\
5 \\
10 \\
5 \\
5 \\
5 \\
5 \\
5 \\
5 \\
5 \\
5 \\
5 \\
5 \\
5 \\
5 \\
5 \\
5 \\
5 \\
10 \\
5 \\
5 \\
5 \\
5 \\
5 \\
5 \\
5 \\
5 \\
5 \\
100 \\
5 \\
50 \\
50\end{array}$ & 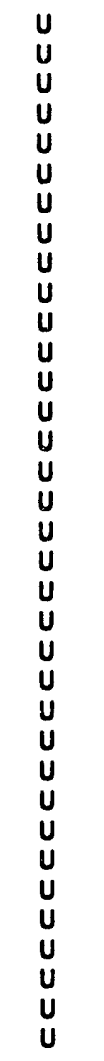 & & \\
\hline \multicolumn{6}{|c|}{ Location $=$ ORR; Formation $=$ NOLICHUCKY; Site $=3$} \\
\hline $\begin{array}{l}\text { Acetone } \\
\text { Benzene } \\
\text { Bromodichloromethane } \\
\text { Bromoform } \\
\text { Bromomethane } \\
\text { Carbon Disulfide } \\
\text { Carbon Tetrachloride } \\
\text { Chlorobenzene } \\
\text { Chloroethane } \\
\text { Chloroform } \\
\text { Chloromethane } \\
\text { Cis-1,2-Dichloroethene } \\
\text { Cis-1,3-Dichloropropene } \\
\text { Dibromochloromethane } \\
\text { Ethyl Benzene } \\
\text { Freon- } 113 \\
\text { Methylene Chloride } \\
\text { Ortho Xylene } \\
\text { styrene } \\
\text { Tetrachloroethene } \\
\text { Toluene } \\
\text { Trans-1,2-Dichloroethene } \\
\text { Irans-1,3-Dichloropropene } \\
\text { Trichloroethene } \\
\text { Trichlorofluoromethane } \\
\text { Vinyl Acetate } \\
\text { Vinyl Chloride } \\
\text { Xylene (Meta + Para) } \\
1,1-D i c h l o r o e t h a n e \\
1,1-D \text { ichloroethene } \\
1,1,1-\text { Trichloroethane } \\
1,1,2-\text { Trichloroethane }\end{array}$ & 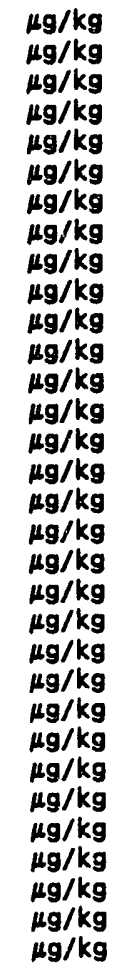 & $\begin{array}{r}12 \\
5 \\
5 \\
5 \\
10 \\
5 \\
5 \\
5 \\
10 \\
5 \\
10 \\
5 \\
5 \\
5 \\
5 \\
5 \\
5 \\
5 \\
5 \\
5 \\
5 \\
5 \\
5 \\
5 \\
5 \\
5 \\
10 \\
5 \\
5 \\
5 \\
5 \\
5\end{array}$ & $\begin{array}{l}J \\
U \\
U \\
U \\
U \\
U \\
U \\
U \\
U \\
U \\
U \\
U \\
U \\
U \\
U \\
U \\
U \\
U \\
U \\
U \\
U \\
U \\
U \\
U \\
U \\
U \\
U \\
U \\
U \\
U \\
U \\
U \\
U \\
U\end{array}$ & & \\
\hline
\end{tabular}


Table B.1 (continued)

\begin{tabular}{|c|c|c|c|c|c|}
\hline Analysis & Units & A horizon & Qual. & $\begin{array}{l}\text { A horizon } \\
\text { field dup }\end{array}$ & Qual. \\
\hline $\begin{array}{l}\text { 1,1,2,2-Tetrachloroethane } \\
1,2-D \text { ichloroethane } \\
\text { 1,2-Dichloropropane } \\
\text { 1,4-Dichlorobenzene } \\
\text { 2--Butanone } \\
\text { 2-Chloroethyl Vinyl Ether } \\
\text { 2-Hexanone } \\
\text { 4-Methyl-2-Pentanone }\end{array}$ & $\begin{array}{l}\mu g / \mathrm{kg} \\
\mu g / \mathrm{kg} \\
\mu g / \mathrm{kg} \\
\mu g / \mathrm{kg} \\
\mu g / \mathrm{kg} \\
\mu g / \mathrm{kg} \\
\mu g / \mathrm{kg} \\
\mu g / \mathrm{kg}\end{array}$ & $\begin{array}{r}5 \\
5 \\
5 \\
5 \\
98 \\
5 \\
49 \\
49\end{array}$ & $\begin{array}{l}U \\
U \\
U \\
U \\
U \\
U \\
U \\
U\end{array}$ & & \\
\hline
\end{tabular}

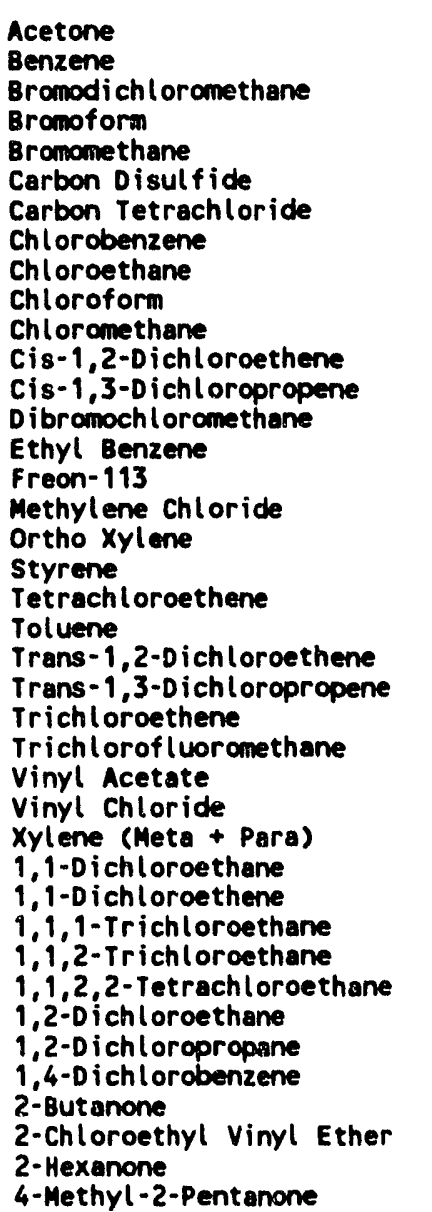

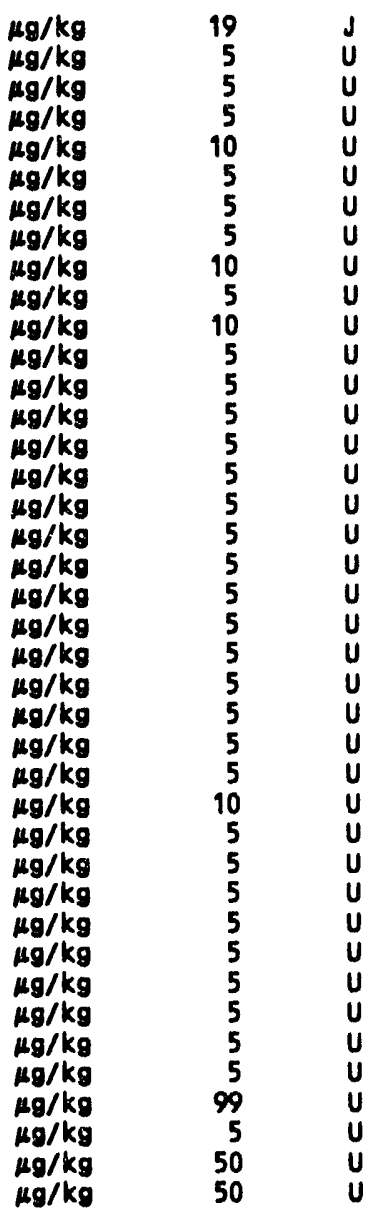

$$
\text { Location }=\text { ORR; Formation }=\text { NOLICHUCKY; Site }=13
$$

$\begin{array}{ll}\text { Acetone } & \mu g / k g \\ \text { Benzene } & \mu g / k g \\ \text { Bromodichloromethane } & \mu g / k g \\ \text { Bromoform } & \mu g / k g \\ \text { Bromomethane } & \mu g / k g \\ \text { Carbon Disulfide } & \mu g / k g \\ \text { Carbon Tetrachloride } & \mu g / k g \\ \text { Chlorobenzene } & \mu g / k g \\ \text { Chloroethane } & \mu g / k g \\ \text { Chloroform } & \mu g / k g \\ \text { Chloromethane } & \mu g / k g \\ \text { Cis-1,2-Dichloroethene } & \mu g / k g \\ \text { Cis-1,3-Dichloropropene } & \mu g / k g \\ \text { Dibromochloromethane } & \mu g / k g\end{array}$

$\begin{array}{lrl}\mu g / \mathbf{k g} & 10 & J \\ \mu g / \mathbf{k g} & 5 & U \\ \mu g / \mathbf{k g} & 5 & U \\ \mu \mathrm{g} / \mathbf{k g} & 5 & U \\ \mu g / \mathbf{k g} & 10 & U \\ \mu g / \mathbf{k g} & 5 & U \\ \mu g / \mathbf{k g} & 5 & U \\ \mu \mathrm{g} / \mathbf{k g} & 5 & U \\ \mu g / \mathbf{k g} & 10 & U \\ \mu g / \mathbf{k g} & 5 & U \\ \mu g / \mathbf{k g} & 10 & U \\ \mu g / \mathbf{g g} & 5 & U \\ \mu g / \mathbf{k g} & 5 & U \\ \mu g / \mathbf{k g} & 5 & U\end{array}$


Table B.1 (continued)

\begin{tabular}{|c|c|c|c|c|c|}
\hline Analysis & Units & A horizon & Qual. & $\begin{array}{l}\text { A horizon } \\
\text { field dup }\end{array}$ & Qual. \\
\hline $\begin{array}{l}\text { Ethyl Benzene } \\
\text { Freon-113 } \\
\text { Methylene Chloride } \\
\text { Ortho Xylene } \\
\text { Styrene } \\
\text { Tetrachloroethene } \\
\text { Toluene } \\
\text { Trans-1,2-Dichloroethene } \\
\text { Trans-1,3-Dichloropropene } \\
\text { Trichloroethene } \\
\text { Trichlorofluoromethane } \\
\text { Vinyl Acetate } \\
\text { Vinyl Chloride } \\
\text { xylene (Meta + Para) } \\
1,1-D \text { ichloroethane } \\
1,1-D i c h l o r o e t h e n e \\
1,1,1-\text { Trichloroethane } \\
1,1,2-\text { Trichloroethane } \\
1,1,2,2-\text { Tetrachloroethane } \\
1,2-0 \text { ichloroethane } \\
1,2-0 \text { ichloropropare } \\
1,4-D i c h l o r o b e n z e n e \\
2-\text { Butanone } \\
2-\text { Chloroethyl Vinyl Ether } \\
2-\text { Hexanone } \\
4-\text { Methyl-2-Pentanone }\end{array}$ & $\begin{array}{l}\mu g / \mathbf{k g} \\
\mu g / \mathbf{k g} \\
\mu g / \mathbf{k g} \\
\mu g / \mathbf{k g} \\
\mu g / \mathbf{k g} \\
\mu g / \mathbf{k g} \\
\mu g / \mathbf{k g} \\
\mu g / \mathbf{k g} \\
\mu g / \mathbf{k g} \\
\mu g / \mathbf{k g} \\
\mu g / \mathbf{k g} \\
\mu g / \mathbf{k g} \\
\mu g / \mathbf{k g} \\
\mu g / \mathbf{k g} \\
\mu g / \mathbf{k g} \\
\mu g / \mathbf{k g} \\
\mu g / \mathbf{k g} \\
\mu g / \mathbf{k g} \\
\mu g / \mathbf{k g} \\
\mu g / \mathbf{k g} \\
\mu g / \mathbf{k g} \\
\mu g / \mathbf{k g} \\
\mu g / \mathbf{k g} \\
\mu g / \mathbf{k g} \\
\mu g / \mathbf{k g} \\
\mu g / \mathbf{k g}\end{array}$ & $\begin{array}{r}5 \\
5 \\
5 \\
5 \\
5 \\
5 \\
5 \\
5 \\
5 \\
5 \\
5 \\
5 \\
10 \\
5 \\
5 \\
5 \\
5 \\
5 \\
5 \\
5 \\
5 \\
5 \\
99 \\
5 \\
50 \\
50\end{array}$ & $\begin{array}{l}U \\
U \\
U \\
U \\
U \\
U \\
U \\
U \\
U \\
U \\
U \\
U \\
U \\
U \\
U \\
U \\
U \\
U \\
U \\
U \\
U \\
U \\
U \\
U \\
U \\
U \\
U\end{array}$ & & \\
\hline \multicolumn{6}{|c|}{ Location $=$ ORR; Formation $=$ NOLICHUCKY; Site $=15$} \\
\hline 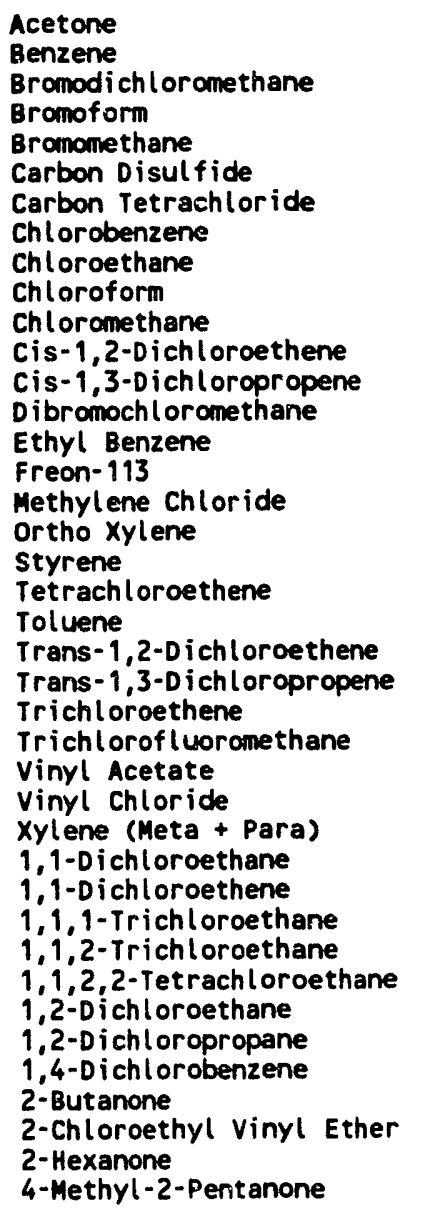 & $\begin{array}{l}\mu g / \mathbf{k g} \\
\mu g / \mathbf{k g} \\
\mu g / \mathbf{k g} \\
\mu g / \mathbf{k g} \\
\mu g / \mathbf{k g} \\
\mu g / \mathbf{k g} \\
\mu g / \mathbf{k g} \\
\mu g / \mathbf{k g} \\
\mu g / \mathbf{k g} \\
\mu g / \mathbf{k g} \\
\mu g / \mathbf{k g} \\
\mu g / \mathbf{k g} \\
\mu g / \mathbf{k g} \\
\mu g / \mathbf{k g} \\
\mu g / \mathbf{k g} \\
\mu g / \mathbf{k g} \\
\mu g / \mathbf{k g} \\
\mu g / \mathbf{k g} \\
\mu g / \mathbf{k g} \\
\mu g / \mathbf{k g} \\
\mu g / \mathbf{k g} \\
\mu g / \mathbf{k g} \\
\mu g / \mathbf{k g} \\
\mu g / \mathbf{k g} \\
\mu g / \mathbf{k g} \\
\mu g / \mathbf{k g} \\
\mu g / \mathbf{k g} \\
\mu g / \mathbf{k g} \\
\mu g / \mathbf{k g} \\
\mu g / \mathbf{k g} \\
\mu g / \mathbf{k g} \\
\mu g / \mathbf{k g} \\
\mu g / \mathbf{k g} \\
\mu g / \mathbf{k g} \\
\mu g / \mathbf{k g} \\
\mu g / \mathbf{k g} \\
\mu g / \mathbf{k g} \\
\mu g / \mathbf{k g} \\
\mu g / \mathbf{k g} \\
\mu g / \mathbf{k g}\end{array}$ & $\begin{array}{r}18 \\
5 \\
5 \\
5 \\
10 \\
5 \\
5 \\
5 \\
10 \\
5 \\
10 \\
5 \\
5 \\
5 \\
5 \\
5 \\
5 \\
5 \\
5 \\
5 \\
5 \\
5 \\
5 \\
5 \\
5 \\
5 \\
10 \\
5 \\
5 \\
5 \\
5 \\
5 \\
5 \\
5 \\
5 \\
5 \\
98 \\
5 \\
49 \\
49\end{array}$ & 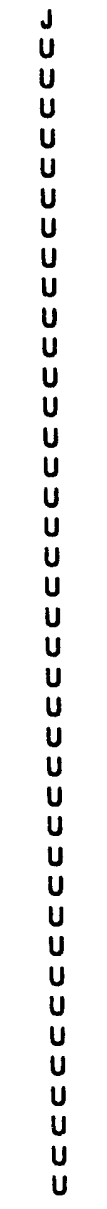 & & \\
\hline
\end{tabular}


Table B.1 (continued)

\begin{tabular}{llc} 
& & A horizon \\
Analysis & Units A horizon Qual. field dup Qual. \\
\hline
\end{tabular}

Location $=$ ORR; Formation $=$ NOLICHUCKY; Site $=16$

Acetone
Benzene
Bromodichloromethane
Bronoform
Bronomethane
Carbon Disulfide
Carbon Tetrachloride
Chlorobenzene
Chloroethane
Chloroform
Chloromethane
Cis-1,2-Dichloroethene
Cis-1,3-Dichloropropene
Dibromochloromethane
Ethyl Benzene
Freon-113
Methylene Chloride
Ortho Xylene
Styrene
Tetrachloroethene
Toluene
Trans-1,2-Dichloroethene
Trans-1,3-Dichloropropene
Trichloroethene
Trichlorofluoromethane
Vinyl Acetate
Vinyl Chloride
Xylene (Meta + Para)
$1,1-0$ ichloroethane
$1,1-0$ ichloroethene
$1,1,1-$ Trichloroethane
$1,1,2-T r$ ichloroethane
$1,1,2,2-$ Tetrachloroethane
$1,2-0$ ichloroethane
$1,2-0$ ichloropropane
$1,4-0$ ichlorobenzene
$2-$ Butanone
$2-$ Chloroethyl Vinyl Ether
$2-$ Hexanone
$4-$ Methyl-2-Pentanone

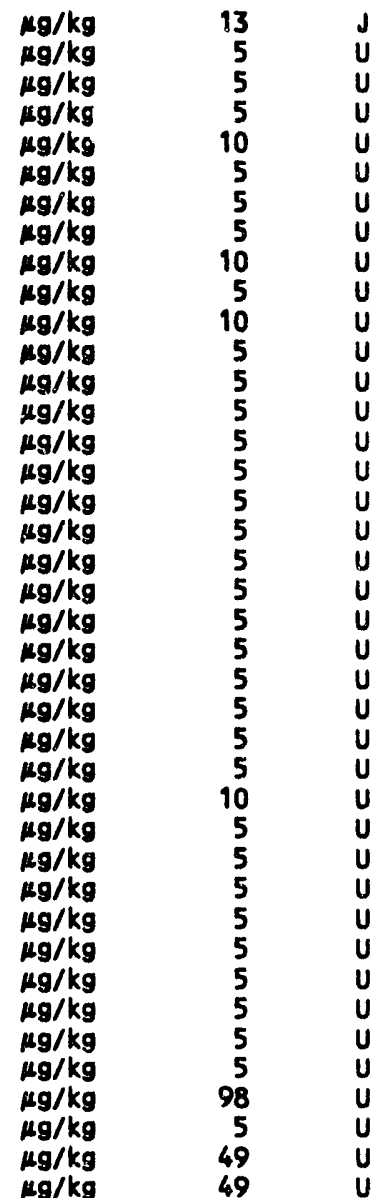

Location $=$ ORR; Formation $=$ NOLJCHUCKY; Site $=21$

Acetone
Benzene
Bromodichloromethane
Bromoform
Bromonethane
Carbon Disulfide
Carbon Tetrachloride
Chlorobenzene
Chloroethane
Chloroform
Chloromethane
Cis-1,2-Dichloroethene
Cis-1,3-Dichloropropene
Dibromochloromethane
Ethyl Benzene
Freon-113
Methylene Chloride
Ortho Xylene
Styrene
Tetrachloroethene
Toluene
Trans-1,2-Dichloroethene
Trans-1,3-Dichloropropene


Table B.1 (continued)

\begin{tabular}{|c|c|c|c|c|c|}
\hline Analysis & Units & A horizon & Qual. & $\begin{array}{l}\text { A horizon } \\
\text { field dup }\end{array}$ & Qual. \\
\hline $\begin{array}{l}\text { Trichloroethene } \\
\text { Trichlorofluoromethane } \\
\text { Vinyl Acetate } \\
\text { Vinyl Chloride } \\
\text { Xylene (Meta + Para) } \\
1,1-\text { ichloroethane } \\
1,1-\text { Dichloroethene } \\
1,1,1-\text { Trichloroethane } \\
1,1,2-\text { Trichloroethane } \\
1,1,2,2-\text { Tetrachloroethane } \\
1,2-0 \text { ichloroethane } \\
1,2-\text { ichloropropane } \\
1,4-\text { Dichlorobenzene } \\
\text { 2--Butanone } \\
\text { 2-Chloroethyl Vinyl Ether } \\
\text { 2-Hexanone } \\
\text { 4-Methyl-2-Pentanone }\end{array}$ & $\begin{array}{l}\mu g / \mathbf{k g} \\
\mu g / \mathbf{k g} \\
\mu g / \mathbf{k g} \\
\mu g / \mathbf{k g} \\
\mu g / \mathbf{k g} \\
\mu g / \mathbf{k g} \\
\mu g / \mathbf{k g} \\
\mu g / \mathbf{k g} \\
\mu g / \mathbf{k g} \\
\mu g / \mathbf{k g} \\
\mu g / \mathbf{k g} \\
\mu g / \mathbf{k g} \\
\mu g / \mathbf{k g} \\
\mu g / \mathbf{k g} \\
\mu g / \mathbf{k g} \\
\mu g / \mathbf{k g} \\
\mu g / \mathbf{k g}\end{array}$ & $\begin{array}{r}5 \\
5 \\
5 \\
10 \\
5 \\
5 \\
5 \\
5 \\
5 \\
5 \\
5 \\
5 \\
5 \\
98 \\
5 \\
49 \\
49\end{array}$ & $\begin{array}{l}U \\
U \\
U \\
U \\
U \\
U \\
U \\
U \\
U \\
U \\
U \\
U \\
U \\
U \\
U \\
U \\
U\end{array}$ & $\begin{array}{r}5 \\
5 \\
5 \\
10 \\
5 \\
5 \\
5 \\
5 \\
5 \\
5 \\
5 \\
5 \\
5 \\
100 \\
5 \\
50 \\
50\end{array}$ & $\begin{array}{l}U \\
U \\
U \\
U \\
U \\
U \\
U \\
U \\
U \\
U \\
U \\
U \\
U \\
U \\
U \\
U \\
U \\
U\end{array}$ \\
\hline
\end{tabular}

Location $=$ ORR; Formation $=$ NOLICHUCKY; Site $=23$

\begin{tabular}{|c|c|c|c|c|}
\hline $\begin{array}{l}\text { Acetone } \\
\text { Benzene } \\
\text { Bromodichloromethane } \\
\text { Bromoform } \\
\text { Bromomethane } \\
\text { Carbon Disulfide } \\
\text { Carbon Tetrachloride } \\
\text { Chlorobenzene } \\
\text { Chloroethane } \\
\text { Chloroform } \\
\text { Chloromethane } \\
\text { Cis-1,2-Dichloroethene } \\
\text { Cis-1,3-Dichloropropene } \\
\text { Dibromochloromethane } \\
\text { Ethyl Benzene } \\
\text { Freon- } 13 \\
\text { Methylene Chloride } \\
\text { Ortho Xylene } \\
\text { Styrene } \\
\text { Tetrachloroethene } \\
\text { Toluene } \\
\text { Trans-1,2-Dichloroethene } \\
\text { Trans-1,3-Dichloropropene } \\
\text { Trichloroethene } \\
\text { Trichlorofluoromethane } \\
\text { Vinyl Acetate } \\
\text { Vinyl Chloride } \\
\text { Xylene (Meta + Para) } \\
1,1-D \text { ichloroethane } \\
1,1-D \text { ichloroethene } \\
1,1,1-\text { Trichloroethane } \\
1,1,2-T r i c h l o r o e t h a n e \\
1,1,2,2-\text { Tetrachloroethane } \\
1,2-D \text { ichloroethane } \\
1,2-D \text { ichloropropane } \\
1,4-D \text { ichlorobenzene } \\
2-\text { Butanone } \\
2-\text { Chloroethyl Vinyl Ether } \\
2-\text { Hexanone } \\
4-\text { Methyl-2-Pentanone }\end{array}$ & $\begin{array}{l}\mu g / k g \\
\mu g / k g \\
\mu g / k g \\
\mu g / k g \\
\mu g / k g \\
\mu g / k g \\
\mu g / k g \\
\mu g / k g \\
\mu g / k g \\
\mu g / k g \\
\mu g / k g \\
\mu g / k g \\
\mu g / k g \\
\mu g / k g \\
\mu g / k g \\
\mu g / k g \\
\mu g / k g \\
\mu g / k g \\
\mu g / k g \\
\mu g / k g \\
\mu g / k g \\
\mu g / k g \\
\mu g / k g \\
\mu g / k g \\
\mu g / k g \\
\mu g / k g \\
\mu g / k g \\
\mu g / k g \\
\mu g / k g \\
\mu g / k g \\
\mu g / k g \\
\mu g / k g \\
\mu g / k g \\
\mu g / k g \\
\mu g / k g \\
\mu g / k g \\
\mu g / k g \\
\mu g / k g \\
\mu g / k g \\
\mu g / k g\end{array}$ & $\begin{array}{r}14 \\
5 \\
5 \\
5 \\
10 \\
5 \\
5 \\
5 \\
10 \\
5 \\
10 \\
5 \\
5 \\
5 \\
5 \\
5 \\
5 \\
5 \\
5 \\
5 \\
5 \\
5 \\
5 \\
5 \\
5 \\
5 \\
10 \\
5 \\
5 \\
5 \\
5 \\
5 \\
5 \\
5 \\
5 \\
5 \\
5 \\
50 \\
50\end{array}$ & 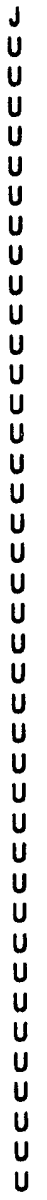 & $\begin{array}{r}18 \\
5 \\
5 \\
5 \\
10 \\
5 \\
5 \\
5 \\
10 \\
5 \\
10 \\
5 \\
5 \\
5 \\
5 \\
5 \\
5 \\
5 \\
5 \\
5 \\
5 \\
5 \\
5 \\
5 \\
5 \\
5 \\
10 \\
5 \\
5 \\
5 \\
5 \\
5 \\
100 \\
5 \\
50 \\
50\end{array}$ \\
\hline
\end{tabular}

$$
\text { Location }=\text { ORR; Formation }=\text { NOLICHUCKY; Site }=24
$$

Acetone

Benzene

Bromodichloromethane

Bromoform

Bromomethane

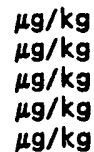

$\mu \mathrm{g} / \mathrm{kg}$

$\mu \mathrm{g} / \mathrm{kg}$

$\mu \mathrm{g} / \mathrm{kg}$ 
Table B.1 (continued)

\begin{tabular}{|c|c|c|c|c|c|}
\hline Analysis & Units & A horizon & Qual. & $\begin{array}{l}\text { A borizon } \\
\text { field dup }\end{array}$ & Qual. \\
\hline 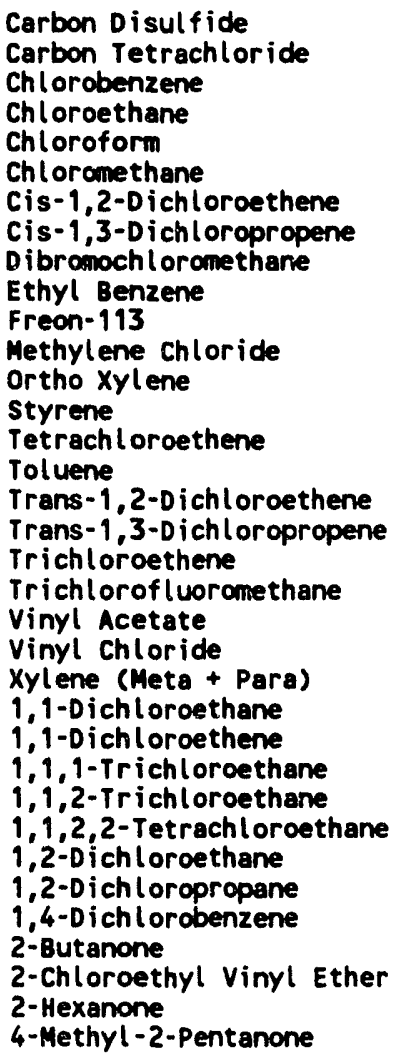 & $\begin{array}{l}\mu g / k g \\
\mu g / \mathbf{k g} \\
\mu g / \mathbf{k g} \\
\mu g / \mathbf{k g} \\
\mu g / \mathbf{k g} \\
\mu g / \mathbf{k g} \\
\mu g / \mathbf{k g} \\
\mu g / \mathbf{k g} \\
\mu g / \mathbf{k g} \\
\mu g / \mathbf{k g} \\
\mu g / \mathbf{k g} \\
\mu g / \mathbf{k g} \\
\mu g / \mathbf{k g} \\
\mu g / \mathbf{k g} \\
\mu g / \mathbf{k g} \\
\mu g / \mathbf{k g} \\
\mu g / \mathbf{k g} \\
\mu g / \mathbf{k g} \\
\mu g / \mathbf{k g} \\
\mu g / \mathbf{k g} \\
\mu g / \mathbf{k g} \\
\mu g / \mathbf{k g} \\
\mu g / \mathbf{k g} \\
\mu g / \mathbf{k g} \\
\mu g / \mathbf{k g} \\
\mu g / \mathbf{k g} \\
\mu g / \mathbf{k g} \\
\mu g / \mathbf{k g} \\
\mu g / \mathbf{k g} \\
\mu g / \mathbf{k g} \\
\mu g / \mathbf{k g} \\
\mu g / \mathbf{k g} \\
\mu g / \mathbf{k g} \\
\mu g / \mathbf{k g} \\
\mu g / \mathbf{k g}\end{array}$ & $\begin{array}{r}5 \\
5 \\
5 \\
10 \\
5 \\
10 \\
5 \\
5 \\
5 \\
5 \\
5 \\
5 \\
5 \\
5 \\
5 \\
5 \\
5 \\
5 \\
5 \\
5 \\
5 \\
10 \\
5 \\
5 \\
5 \\
5 \\
5 \\
5 \\
5 \\
5 \\
5 \\
100 \\
5 \\
50 \\
50\end{array}$ & 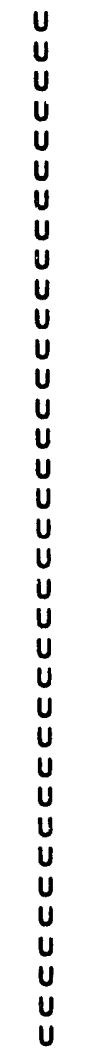 & $\begin{array}{r}5 \\
5 \\
5 \\
10 \\
5 \\
10 \\
5 \\
5 \\
5 \\
5 \\
5 \\
5 \\
5 \\
5 \\
5 \\
5 \\
5 \\
5 \\
5 \\
5 \\
5 \\
10 \\
5 \\
5 \\
5 \\
5 \\
5 \\
5 \\
5 \\
5 \\
5 \\
100 \\
5 \\
50 \\
50\end{array}$ & 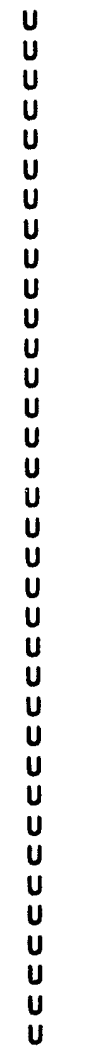 \\
\hline
\end{tabular}

$$
\text { Location }=\text { ORR; Formation }=\text { NOLICHUCKY } ; \text { Site }=25
$$

Acetone
Benzene
Bromodichloromethane
Bromoform
Bromomethane
Carbon D isulfide
Carbon Tetrachloride
Chlorobenzene
Chloroethane
Chloroform
Chloromethane
Cis-1,2-Dichloroethene
Cis-1,3-Dichloropropene
Dibromochloromethane
Ethyl Benzene
Freon-113
Methylene Chloride
Ortho Xylene
Styrene
Tetrachloroethene
Toluene
Trans-1,2-Dichloroethene
Trans-1,3-Dichloropropene
Trichloroethene
Trichlorofluoromethane
Vinyl Acetate
Vinyl Chloride
Xylene (Meta + Para)
$1,1-D i c h l o r o e t h a n e$
$1,1-D i c h l o r o e t h e n e$
$1,1,1-T r i c h l o r o e t h a n e$
$1,1,2-T r i c h l o r o e t h a n e$

$\begin{array}{lrl}\mu g / k g & 24 & J \\ \mu g / k g & 5 & U \\ \mu g / k g & 5 & U \\ \mu g / k g & 5 & U \\ \mu g / k g & 10 & U \\ \mu g / k g & 5 & U \\ \mu g / k g & 5 & U \\ \mu g / k g & 5 & U \\ \mu g / k g & 10 & U \\ \mu g / k g & 5 & U \\ \mu g / k g & 10 & U \\ \mu g / k g & 5 & U \\ \mu g / k g & 5 & U \\ \mu g / k g & 5 & U \\ \mu g / k g & 5 & U \\ \mu g / k g & 5 & U \\ \mu g / k g & 5 & U \\ \mu g / k g & 5 & U \\ \mu g / k g & 5 & U \\ \mu g / k g & 5 & U \\ \mu g / k g & 5 & U \\ \mu g / k g & 5 & U \\ \mu g / k g & 5 & U \\ \mu g / k g & 5 & U \\ \mu g / k g & 5 & U \\ \mu g / k g & 5 & U \\ \mu g / k g & 10 & U \\ \mu g / k g & 5 & U \\ \mu g / k g & 5 & U \\ \mu g / k g & 5 & U \\ \mu g / k g & 5 & U \\ \mu g / k g & 5 & U\end{array}$


Table B.1 (continued)

\begin{tabular}{|c|c|c|c|c|c|}
\hline Analysis & Units & A horizon & Qual. & $\begin{array}{l}\text { A horizon } \\
\text { field dup }\end{array}$ & Qual. \\
\hline $\begin{array}{l}\text { 1,1,2,2-Tetrachloroethane } \\
\text { 1,2-Dichloroethane } \\
\text { 1,2-Dichloropropane } \\
\text { 1,4-Dichlorobenzene } \\
\text { 2-Butanone } \\
\text { 2-Chloroethyl Vinyl Ether } \\
\text { 2-Hexanone } \\
\text { 4-Methyl-2-Pentanone }\end{array}$ & $\begin{array}{l}\mu \mathrm{g} / \mathbf{k g} \\
\boldsymbol{\mu g} / \mathbf{k g} \\
\mu \mathrm{g} / \mathbf{k g} \\
\mu \mathrm{g} / \mathbf{k g} \\
\mu \mathrm{g} / \mathbf{k g} \\
\mu \mathrm{g} / \mathbf{k g} \\
\mu \mathrm{g} / \mathbf{k g} \\
\mu \mathrm{g} / \mathbf{k g}\end{array}$ & $\begin{array}{r}5 \\
5 \\
5 \\
5 \\
98 \\
5 \\
49 \\
49\end{array}$ & $\begin{array}{l}\mathbf{U} \\
\mathbf{U} \\
\mathbf{U} \\
\mathbf{U} \\
\mathbf{U} \\
\mathbf{U} \\
\mathbf{U} \\
\mathbf{U}\end{array}$ & & \\
\hline
\end{tabular}

Location $=$ ORR; Formation $=$ NOLICHUCKY $;$ Site $=28$

\begin{tabular}{|c|c|c|c|c|}
\hline 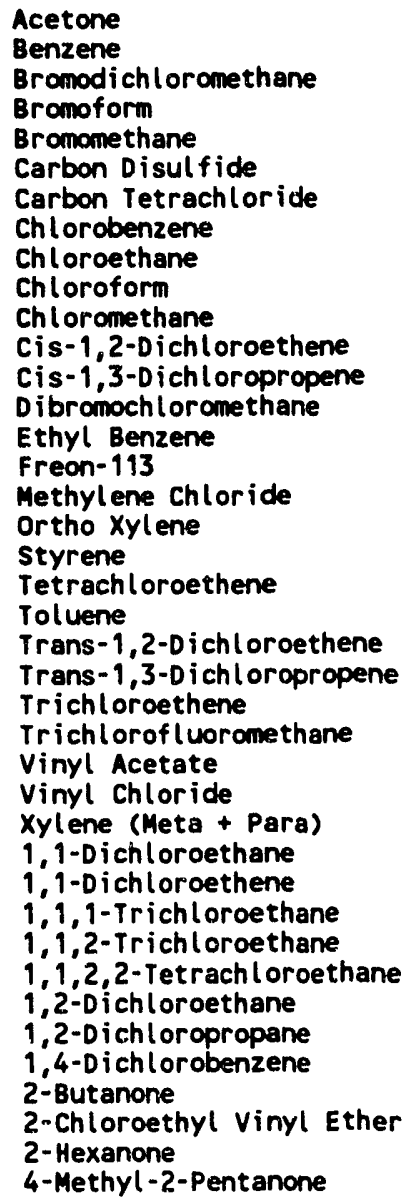 & $\begin{array}{l}\mu g / \mathbf{k g} \\
\mu g / k g \\
\mu g / k g \\
\mu g / k g \\
\mu g / k g \\
\mu g / k g \\
\mu g / k g \\
\mu g / k g \\
\mu g / k g \\
\mu g / k g \\
\mu g / k g \\
\mu g / k g \\
\mu g / k g \\
\mu g / k g \\
\mu g / k g \\
\mu g / k g \\
\mu g / k g \\
\mu g / k g \\
\mu g / k g \\
\mu g / k g \\
\mu g / k g \\
\mu g / k g \\
\mu g / k g \\
\mu g / k g \\
\mu g / k g \\
\mu g / k g \\
\mu g / k g\end{array}$ & $\begin{array}{r}22 \\
5 \\
5 \\
5 \\
10 \\
5 \\
5 \\
5 \\
10 \\
5 \\
10 \\
5 \\
5 \\
5 \\
5 \\
5 \\
5 \\
5 \\
5 \\
5 \\
5 \\
5 \\
5 \\
5 \\
5 \\
5 \\
10 \\
5 \\
5 \\
5 \\
5 \\
5 \\
5 \\
5 \\
5 \\
5 \\
5 \\
49\end{array}$ & $\begin{array}{l}J \\
U \\
U \\
U \\
U \\
U \\
U \\
U \\
U \\
U \\
U \\
U \\
U \\
U \\
U \\
U \\
U \\
U \\
U \\
U \\
U \\
U \\
U \\
U \\
U \\
U \\
U \\
U \\
U \\
U \\
U \\
U \\
U \\
U \\
U \\
U \\
U \\
U \\
U \\
U\end{array}$ & $\begin{array}{r}14 \\
5 \\
5 \\
5 \\
10 \\
5 \\
5 \\
5 \\
10 \\
5 \\
10 \\
5 \\
5 \\
5 \\
5 \\
5 \\
5 \\
5 \\
5 \\
5 \\
5 \\
5 \\
5 \\
5 \\
5 \\
5 \\
10 \\
5 \\
5 \\
5 \\
5 \\
5 \\
5 \\
5 \\
50 \\
5 \\
5 \\
5 \\
5 \\
5 \\
5 \\
5 \\
5 \\
5 \\
5 \\
5 \\
5 \\
5 \\
5 \\
5 \\
5 \\
5 \\
5 \\
5\end{array}$ \\
\hline
\end{tabular}

$$
\text { Location }=\text { ORR; Formation }=\text { NOLICHUCKY; Site }=31
$$

$\begin{array}{ll}\text { Acetone } & \mu \mathrm{g} / \mathrm{kg} \\ \text { Benzene } & \mu \mathrm{g} / \mathrm{kg} \\ \text { Bromodichloromethane } & \mu \mathrm{g} / \mathrm{kg} \\ \text { Bromoform } & \mu \mathrm{g} / \mathrm{kg} \\ \text { Bromomethane } & \mu \mathrm{g} / \mathrm{kg} \\ \text { Carbon Disulfide } & \mu \mathrm{g} / \mathrm{kg} \\ \text { Carbon Tetrachloride } & \mu \mathrm{g} / \mathrm{kg} \\ \text { Chlorobenzene } & \mu \mathrm{g} / \mathrm{kg} \\ \text { Chloroethane } & \mu \mathrm{g} / \mathrm{kg} \\ \text { Chloroform } & \mu \mathrm{g} / \mathrm{kg} \\ \text { Chloromethane } & \mu \mathrm{g} / \mathrm{kg} \\ \text { Cis-1,2-Dichloroethene } & \mu \mathrm{g} / \mathrm{kg} \\ \text { Cis-1,3-Dichloropropene } & \mu \mathrm{g} / \mathrm{kg} \\ \text { Dibromochloromethane } & \mu \mathrm{g} / \mathrm{kg}\end{array}$

$\begin{array}{rrrr}27 & J & 78 & J \\ 5 & U & 5 & U \\ 5 & U & 5 & U \\ 5 & U & 5 & U \\ 10 & U & 10 & U \\ 5 & U & 5 & U \\ 5 & U & 5 & U \\ 5 & U & 5 & U \\ 10 & U & 10 & U \\ 5 & U & 5 & U \\ 10 & U & 10 & U \\ 5 & U & 5 & U \\ 5 & U & 5 & U \\ 5 & U & 5 & U\end{array}$


Table B.1 (continued)

\begin{tabular}{|c|c|c|c|c|c|}
\hline Analysis & Units & A horizon & Qual. & $\begin{array}{l}\text { A horizon } \\
\text { field dup }\end{array}$ & Qual. \\
\hline 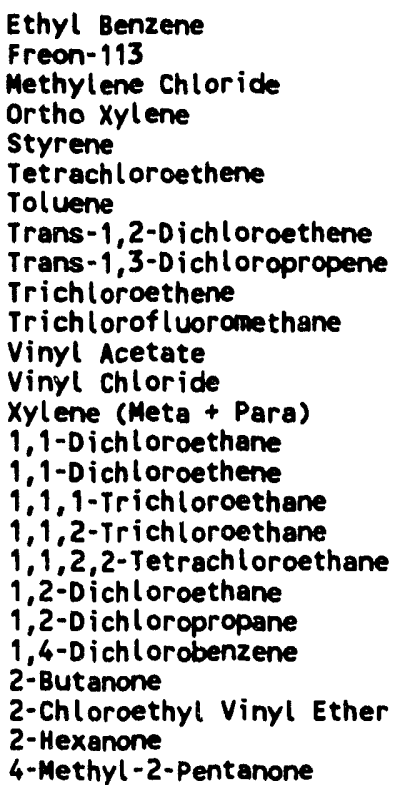 & $\begin{array}{l}\mu g / k g \\
\mu g / k g \\
\mu g / k g \\
\mu g / k g \\
\mu g / k g \\
\mu g / k g \\
\mu g / k g \\
\mu g / k g \\
\mu g / k g \\
\mu g / k g \\
\mu g / k g \\
\mu g / k g \\
\mu g / k g \\
\mu g / k g \\
\mu g / k g \\
\mu g / k g \\
\mu g / k g \\
\mu g / k g \\
\mu g / k g \\
\mu g / k g \\
\mu g / k g \\
\mu g / k g \\
\mu g / k g \\
\mu g / k g \\
\mu g / k g \\
\mu g / k g\end{array}$ & $\begin{array}{r}5 \\
5 \\
5 \\
5 \\
5 \\
5 \\
5 \\
5 \\
5 \\
5 \\
24 \\
5 \\
10 \\
5 \\
5 \\
5 \\
5 \\
5 \\
5 \\
5 \\
5 \\
5 \\
100 \\
5 \\
50 \\
50\end{array}$ & $\begin{array}{l}U \\
U \\
U \\
U \\
U \\
U \\
U \\
U \\
U \\
U \\
\mathbf{U} \\
\mathbf{U} \\
\mathbf{U} \\
\mathbf{U} \\
\mathbf{U} \\
\mathbf{U} \\
\mathbf{U} \\
\mathbf{U} \\
\mathbf{U} \\
\mathbf{U} \\
\mathbf{U} \\
\mathbf{U} \\
\mathbf{U} \\
\mathbf{U} \\
\mathbf{U}\end{array}$ & $\begin{array}{r}5 \\
5 \\
5 \\
5 \\
5 \\
5 \\
5 \\
5 \\
5 \\
5 \\
5 \\
5 \\
10 \\
5 \\
5 \\
5 \\
5 \\
5 \\
5 \\
5 \\
5 \\
5 \\
7 \\
5 \\
50 \\
50\end{array}$ & $\begin{array}{l}U \\
U \\
U \\
U \\
U \\
U \\
U \\
U \\
U \\
U \\
U \\
U \\
U \\
U \\
U \\
U \\
U \\
U \\
U \\
U \\
U \\
U \\
U \\
U \\
U \\
B J \\
U \\
U \\
U \\
U\end{array}$ \\
\hline
\end{tabular}

$$
\text { Location }=\text { ORR; Formation }=\text { NOLICHUCKY } ; \text { Site }=42
$$

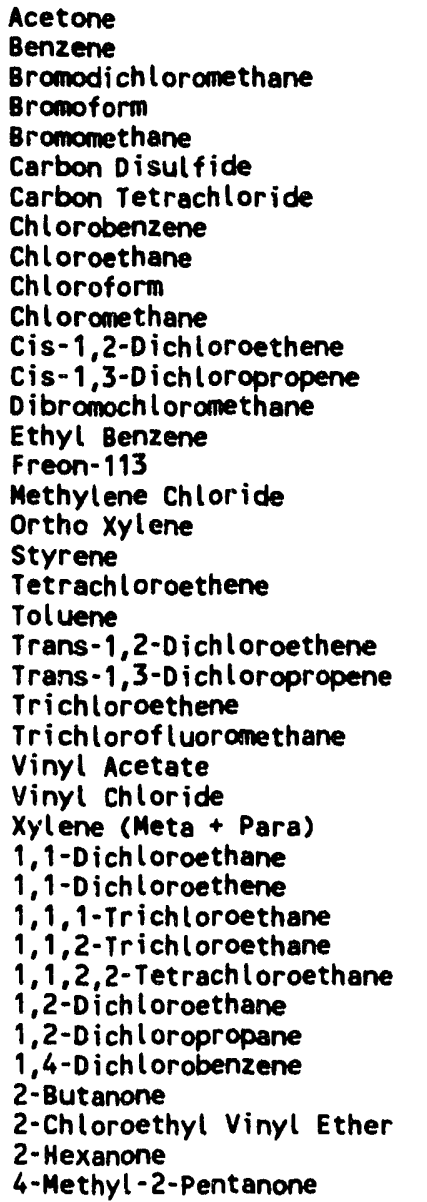

$\begin{array}{lr}\mu g / k g & 18 \\ \mu g / k g & 5 \\ \mu g / k g & 5 \\ \mu g / k g & 5 \\ \mu g / k g & 10 \\ \mu g / k g & 5 \\ \mu g / k g & 5 \\ \mu g / k g & 5 \\ \mu g / k g & 10 \\ \mu g / k g & 5 \\ \mu g / k g & 10 \\ \mu g / k g & 5 \\ \mu g / k g & 5 \\ \mu g / k g & 5 \\ \mu g / k g & 5 \\ \mu g / k g & 5 \\ \mu g / k g & 5 \\ \mu g / k g & 5 \\ \mu g / k g & 5 \\ \mu g / k g & 5 \\ \mu g / k g & 5 \\ \mu g / k g & 5 \\ \mu g / k g & 5 \\ \mu g / k g & 5 \\ \mu g / k g & 5 \\ \mu g / k g & 5 \\ \mu g / k g & 5 \\ \mu g / k g & 5 \\ \mu g / k g & 5 \\ \mu g / k g & 5 \\ \mu g / k g & 5 \\ \mu g / k g & 5 \\ \mu g / k g & 5 \\ \mu g / k g & 5 / k g \\ \mu g / k g & 5 \\ \mu g & 5 \\ \mu g & 5 \\ \mu g\end{array}$

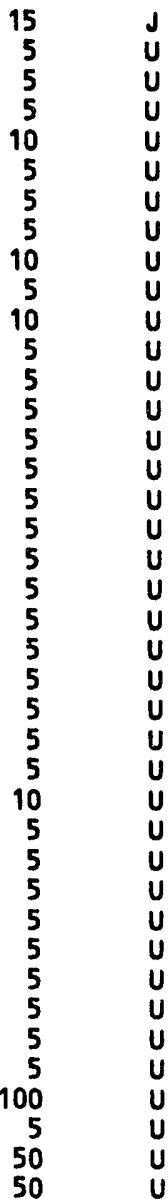


Table B.1 (continued)

\begin{tabular}{lll}
\hline & A horizon \\
Anatysis & Units A horizon Qual. field dup Qual. \\
\hline
\end{tabular}

Location $=$ ROA; Formation $=$ DISMAL GAP; Site $=3$

\begin{tabular}{|c|c|c|}
\hline $\begin{array}{l}\text { Acetone } \\
\text { Benzene } \\
\text { Bromodichloromethane } \\
\text { Bromoform } \\
\text { Bromomethane } \\
\text { Carbon D isulfide } \\
\text { Carbon Tetrachloride } \\
\text { Chlorobenzene } \\
\text { Chloroethane } \\
\text { Chloroform } \\
\text { Chloromethane } \\
\text { Cis-1,2-Dichloroethene } \\
\text { Cis-1,3-Dichloropropene } \\
\text { Dibromochloromethane } \\
\text { Ethyl Benzene } \\
\text { Methylene Chloride } \\
\text { Ortho Xylene } \\
\text { Styrene } \\
\text { Tetrachloroethene } \\
\text { Toluene } \\
\text { Trans-1,2-Dichloroethene } \\
\text { Trans-1,3-Dichloropropene } \\
\text { Trichloroethene } \\
\text { Vimyl Chloride } \\
\text { Xylene (Meta + Para) } \\
1,1-D \text { ichloroethane } \\
1,1-D i c h l o r o e t h e n e \\
1,1,1-T r i c h l o r o e t h a n e \\
1,1,2-T r i c h l o r o e t h a n e \\
1,1,2,2-\text { Tetrachloroethane } \\
1,2-0 \text { ichloroethane } \\
1,2-D \text { ichloropropane } \\
2-\text { Butanone } \\
2-\text { Hexanone } \\
4-\text { Methyl-2-Pentanone }\end{array}$ & 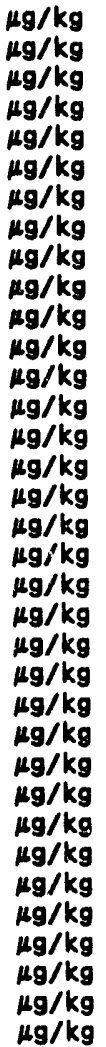 & $\begin{array}{r}\mathbf{5} \\
\mathbf{5} \\
\mathbf{5} \\
\mathbf{5} \\
10 \\
\mathbf{5} \\
\mathbf{5} \\
\mathbf{5} \\
10 \\
\mathbf{5} \\
10 \\
\mathbf{5} \\
\mathbf{5} \\
5 \\
5 \\
\mathbf{5} \\
\mathbf{5} \\
\mathbf{5} \\
\mathbf{5} \\
\mathbf{5} \\
5 \\
\mathbf{5} \\
\mathbf{5} \\
10 \\
\mathbf{5} \\
\mathbf{5} \\
\mathbf{5} \\
\mathbf{5} \\
\mathbf{5} \\
\mathbf{5} \\
\mathbf{5} \\
\mathbf{5} \\
\mathbf{5}\end{array}$ \\
\hline
\end{tabular}

$$
\text { Location }=\text { ROA; Formation }=\text { DISMAL GAP; Site }=7
$$

Acetone
Benzene
Bromodichloromethane
Bromoform
Bromontethane
Carbon Disulfide
Carbon Tetrachloride
Chlorobenzene
Chloroethane
Chloroform
Chloromethane
Cis-1,2-Dichloroethene
Cis-1,3-Dichloropropene
Dibromochloromethane
Ethyl Benzene
Freon-113
Methylene Chloride
Ortho Xylene
Styrene
Tetrachloroethene
Toluene
Trans-1,2-Dichloroethene
Trans-1,3-Dichloropropene
Trichloroethene
Trichlorofluoromethane
Vinyl Acetate
Vinyl Chloride
Xylene (Meta + Para)

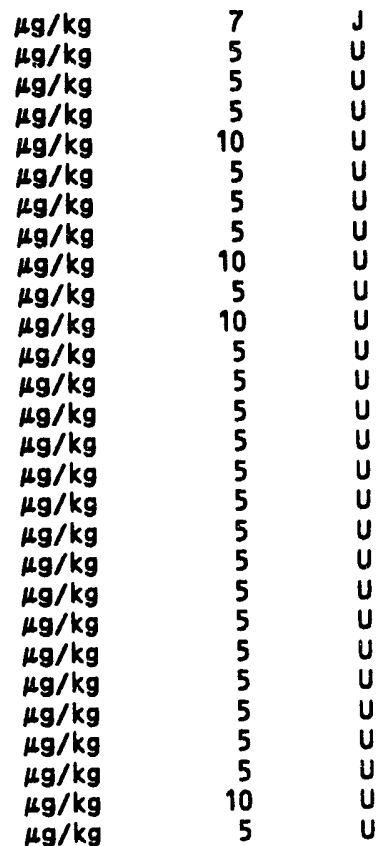


Table B.1 (continued)

\begin{tabular}{|c|c|c|c|c|c|}
\hline Analysis & Units & A horizon & Qual. & $\begin{array}{l}\text { A horizon } \\
\text { field dup }\end{array}$ & Qual. \\
\hline $\begin{array}{l}\text { 1,1-Dichloroethane } \\
1,1-\text { Dichloroethene } \\
1,1,1-\text { Trichloroethane } \\
1,1,2-\text { Trichloroethane } \\
\text { 1,1,2,2-Tetrachloroethane } \\
1,2-\text { Dichloroethane } \\
\text { 1,2-Dichloropropane } \\
\text { 1,4-Dichlorobenzene } \\
\text { 2-Butanone } \\
\text { 2-Chloroethyl Vinyl Ether } \\
\text { 2-Hexanone } \\
\text { 4-Methyl-2-Pentanone }\end{array}$ & $\begin{array}{l}\mu g / k g \\
\mu g / k g \\
\mu g / k g \\
\mu g / k g \\
\mu g / k g \\
\mu g / k g \\
\mu g / k g \\
\mu g / k g \\
\mu g / k g \\
\mu g / k g \\
\mu g / k g \\
\mu g / k g\end{array}$ & $\begin{array}{r}5 \\
5 \\
5 \\
5 \\
5 \\
5 \\
5 \\
5 \\
100 \\
5 \\
50 \\
50\end{array}$ & $\begin{array}{l}U \\
U \\
U \\
U \\
U \\
U \\
U \\
U \\
U \\
U \\
U \\
U\end{array}$ & & \\
\hline
\end{tabular}

Acetone

Bromodichloromethane

$\mu \mathrm{g} / \mathrm{kg}$

$\mu \mathrm{g} / \mathrm{kg}$

Bromoform

Bromomethane

Carbon Disulfide

$\mathrm{Mg} / \mathrm{kg}$

$\mu \mathrm{g} / \mathrm{kg}$

Carbon Tetrachloride $\quad \mu g / k g$

Chlorobenzene $\quad \mu g / k g$

Chloroethane

chloroform

Chloromethane

$\mathrm{Mg} / \mathrm{kg}$

$\mu \mathrm{g} / \mathrm{kg}$

$\mu g / k g$

Cis-1,3-Dichloropropene $\quad \mu \mathrm{g} / \mathrm{kg}$

Dibromochloromethane $\quad \mu g / k g$

Ethyl Benzene

Freon-113

Methylene Chloride

ortho Xylene

Styrene

Tetrachloroethene

Toluene

Trans-1,2-Dichloroethene

Trans-1,3-Dichloropropene

Trichloroethene

Trichlor of luoromethane

Vinyl Acetate

Vinyl Chloride

Xylene (Meta + Para)

1,1-Dichloroethane

1,1-Dichloroethene

1,1,1-Trichloroethane

1,1,2-Trichloroethane

1,1,2,2-Tetrachloroethane

1,2-D ichloroethane

1,2-Dichloropropane

1,4-Dichlorobenzene

2-Butanone

2-Chloroethyl Vinyl Ether

2-Hexanone

4-Methyl-2-Pentanone

$\mu g / k g$

$\mathrm{\mu g} / \mathrm{kg}$

$\mu g / k g$

$\mu \mathrm{g} / \mathrm{kg}$

$\mu g / k g$

$\mu \mathrm{g} / \mathrm{kg}$

Mg/kg

$\mu \mathrm{g} / \mathrm{kg}$

$\mathrm{mg} / \mathrm{kg}$

$\mathrm{\mu g} / \mathrm{kg}$

Mo/kg

$\mu \mathrm{g} / \mathrm{kg}$

$\mu \mathrm{g} / \mathrm{kg}$

$\mathrm{Hg} / \mathrm{kg}$

$\mu \mathrm{g} / \mathrm{kg}$

$\mu g / k g$

$\mathrm{kg} / \mathrm{kg}$

$\mu \mathrm{g} / \mathrm{kg}$

$\mu \mathrm{g} / \mathrm{kg}$

$\mathrm{Hg} / \mathrm{kg}$

$\mathrm{\mu g} / \mathrm{kg}$

$\mu \mathrm{g} / \mathrm{kg}$

$\mu \mathrm{g} / \mathrm{kg}$

$\mu \mathrm{g} / \mathrm{kg}$

$\mu \mathrm{g} / \mathrm{kg}$

$\mu g / \mathrm{kg}$

$\begin{array}{rr}7 & J \\ 5 & U \\ 5 & U \\ 5 & U \\ 10 & U \\ 5 & U \\ 5 & U \\ 5 & U \\ 10 & U \\ 5 & U \\ 10 & U \\ 5 & U \\ 5 & U \\ 5 & U \\ 5 & U \\ 5 & U \\ 5 & U \\ 5 & U \\ 5 & U \\ 5 & U \\ 5 & U \\ 5 & U \\ 5 & U \\ 5 & U \\ 5 & U \\ 5 & U \\ 10 & U \\ 5 & U \\ 5 & U \\ 5 & U \\ 8 & \\ 5 & U \\ 5 & U \\ 5 & U \\ 5 & U \\ 5 & U \\ 100 & U \\ 5 & U \\ 50 & U \\ 50 & U \\ & \end{array}$

$$
\text { Location }=\text { ROA; Formation }=\text { DISMAL GAP; Site }=9
$$

$\begin{array}{ll}\text { Acetone } & \boldsymbol{\mu g} / \mathbf{k g} \\ \text { Benzene } & \boldsymbol{\mu g} / \mathbf{k g} \\ \text { Bromodichloromethane } & \boldsymbol{\mu g} / \mathrm{kg} \\ \text { Bromoform } & \boldsymbol{\mu g} / \mathbf{k g} \\ \text { Bromomethane } & \boldsymbol{\mu g} / \mathbf{k g} \\ \text { Carbon Disulfide } & \boldsymbol{\mu g} / \mathbf{k g} \\ \text { Carbon Tetrachloride } & \boldsymbol{\mu g} / \mathbf{k g} \\ \text { Chlorobenzene } & \boldsymbol{\mu g} / \mathbf{k g} \\ \text { Chloroethane } & \boldsymbol{\mu g} / \mathbf{k g} \\ \text { Chloroform } & \boldsymbol{\mu g} / \mathbf{k g}\end{array}$

$\begin{array}{rr}100 & U \\ 5 & U \\ 5 & U \\ 5 & U \\ 10 & U \\ 5 & U \\ 5 & U \\ 5 & U \\ 10 & U \\ 5 & U\end{array}$


Table B.1 (continued)

\begin{tabular}{|c|c|c|c|c|c|}
\hline Analysis & Units & A horizon & Qual. & $\begin{array}{l}\text { A borizon } \\
\text { field dup }\end{array}$ & Qual. \\
\hline $\begin{array}{l}\text { Chloromethane } \\
\text { Cis-1,2-Dichloroethene } \\
\text { Cis-1,3-Dichloropropene } \\
\text { Dibromochloromethane } \\
\text { Ethyl Benzene } \\
\text { Methylene Chloride } \\
\text { Ortho Xylene } \\
\text { Styrene } \\
\text { Tetrachloroethene } \\
\text { Toluene } \\
\text { Trans-1,2-Dichloroethene } \\
\text { Trans-1,3-Dichloropropene } \\
\text { Trichloroethene } \\
\text { Vinyl Chloride } \\
\text { Xylene (Meta + Para) } \\
1,1-D \text { ichloroethane } \\
1,1-D \text { ichloroethene } \\
1,1,1-\text { Trichloroethane } \\
1,1,2-\text { Trichloroethane } \\
1,1,2,2-\text { Tetrachloroethane } \\
1,2-D \text { ichloroethane } \\
1,2-D \text { ichloropropane } \\
2-\text { Butanone } \\
2-\text { Hexanone } \\
4-\text { Methyl-2-Pentanone }\end{array}$ & 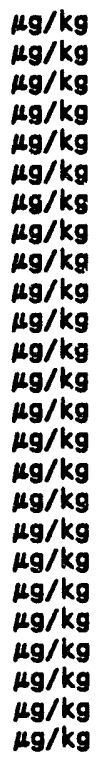 & $\begin{array}{r}10 \\
5 \\
5 \\
5 \\
5 \\
5 \\
5 \\
5 \\
5 \\
5 \\
5 \\
5 \\
5 \\
10 \\
5 \\
5 \\
\mathbf{5} \\
\mathbf{5} \\
\mathbf{5} \\
\mathbf{5} \\
\mathbf{5} \\
\mathbf{5} \\
100 \\
\mathbf{5 0} \\
\mathbf{5 0}\end{array}$ & $\begin{array}{l}\mathbf{U} \\
\mathbf{U} \\
\mathbf{U} \\
\mathbf{U} \\
\mathbf{U} \\
\mathbf{U} \\
\mathbf{U} \\
\mathbf{U} \\
\mathbf{U} \\
\mathbf{U} \\
\mathbf{U} \\
\mathbf{U} \\
\mathbf{U} \\
\mathbf{U} \\
\mathbf{U} \\
\mathbf{U} \\
\mathbf{U} \\
\mathbf{U} \\
\mathbf{U} \\
\mathbf{U} \\
\mathbf{U} \\
\mathbf{U} \\
\mathbf{U} \\
\mathbf{U} \\
\mathbf{U}\end{array}$ & & \\
\hline
\end{tabular}

$$
\text { Location }=\text { ROA; Formation }=\text { DISMAL GAP; Site }=10
$$

\begin{tabular}{|c|c|c|}
\hline $\begin{array}{l}\text { Acetone } \\
\text { Benzene } \\
\text { Bromodichloromethane } \\
\text { Bromoform } \\
\text { Bromonethane } \\
\text { Carbon Disulfide } \\
\text { Carbon Tetrachloride } \\
\text { Chlorobenzene } \\
\text { Chloroethane } \\
\text { Chloroform } \\
\text { Chloromethane } \\
\text { Cis-1,2-Dichloroethene } \\
\text { Cis-1,3-Dichloropropene } \\
\text { Dibromochloromethane } \\
\text { Ethyl Benzene } \\
\text { Freon-113 } \\
\text { Methylene Chloride } \\
\text { Ortho Xylene } \\
\text { Styrene } \\
\text { Tetrachloroethene } \\
\text { Toluene } \\
\text { Trans-1,2-Dichloroethene } \\
\text { Trans-1,3-Dichloropropene } \\
\text { Trichloroethene } \\
\text { Trichlorofluoromethane } \\
\text { Vinyl Acetate } \\
\text { Vinyl Chloride } \\
\text { Xylene (Meta + Para) } \\
1,1-D \text { ichloroethane } \\
1,1-D \text { ichloroethene } \\
1,1,1-\text { Trichloroethane } \\
1,1,2-\text { Trichloroethane } \\
1,1,2,2-\text { Tetrachloroethane } \\
1,2-D \text { ichloroethane } \\
1,2-D \text { ichloropropane } \\
1,4-D \text { ichlorobenzene } \\
2-\text { Butanone } \\
2-\text { Chloroethyl Vinyl Ether } \\
2-\text { Hexanone } \\
4-\text { Methyl-2-Pentanone }\end{array}$ & $\begin{array}{l}\mu g / k g \\
\mu g / k g \\
\mu g / k g \\
\mu g / k g \\
\mu g / k g \\
\mu g / k g \\
\mu g / k g \\
\mu g / k g \\
\mu g / k g \\
\mu g / k g \\
\mu g / k g \\
\mu g / k g \\
\mu g / k g \\
\mu g / k g \\
\mu g / k g \\
\mu g / k g \\
\mu g \\
\mu g \\
\mu g \\
\mu g \\
\mu g \\
\mu g\end{array}$ & $\begin{array}{r}8 \\
5 \\
5 \\
5 \\
10 \\
5 \\
5 \\
5 \\
10 \\
5 \\
10 \\
5 \\
5 \\
5 \\
5 \\
5 \\
5 \\
5 \\
5 \\
5 \\
5 \\
5 \\
5 \\
5 \\
5 \\
5 \\
5 \\
5 \\
5 \\
5 \\
5 \\
5 \\
5 \\
5 \\
5 \\
5 \\
5\end{array}$ \\
\hline
\end{tabular}


Table B.1 (continued)

Anahysis Units A horizon Qual. field dup Qual.

Location $=$ ROA; Fomation $=$ DISMAL GAP; Site $=13$

\begin{tabular}{|c|c|}
\hline $\begin{array}{l}\text { chloroethene } \\
\text { chloropropene } \\
\text { lene } \\
\text { oromethane } \\
\text { de } \\
\text { + Para) } \\
\text { ethane } \\
\text { ethene } \\
\text { oroethone } \\
\text { oroethane } \\
\text { achloroethane } \\
\text { ethane } \\
\text { proparie } \\
\text { benzene } \\
\text { l vinyl Ether } \\
\text { entanone }\end{array}$ & $\begin{array}{l}\mu g / \mathrm{kg} \\
\mu g / \mathrm{kg} \\
\mu g / \mathrm{kg} \\
\mu g / \mathrm{kg} \\
\mu g / \mathrm{kg} \\
\mu g / \mathrm{kg} \\
\mu g / \mathrm{kg} \\
\mu g / \mathrm{kg} \\
\mu g / \mathrm{kg} \\
\mu g / \mathrm{kg} \\
\mu g / \mathrm{kg} \\
\mu g / \mathrm{kg} \\
\mu g / \mathrm{kg} \\
\mu g / \mathrm{kg} \\
\mu g / \mathrm{kg} \\
\mu g / \mathrm{kg} \\
\mu g / \mathrm{kg} \\
\mu g / \mathrm{kg} \\
\mu g / \mathrm{kg} \\
\mu g / \mathrm{kg} \\
\mu g / \mathrm{kg} \\
\mu g / \mathrm{kg} \\
\mu g / \mathrm{kg} \\
\mu g / \mathrm{kg} \\
\mu g / \mathrm{kg} \\
\mu g / \mathrm{kg} \\
\mu g / \mathrm{kg} \\
\mu g / \mathrm{kg} \\
\mu g / \mathrm{kg} \\
\mu g / \mathrm{kg} \\
\mu g / \mathrm{kg} \\
\mu g / \mathrm{kg} \\
\mu g / \mathrm{kg}\end{array}$ \\
\hline
\end{tabular}

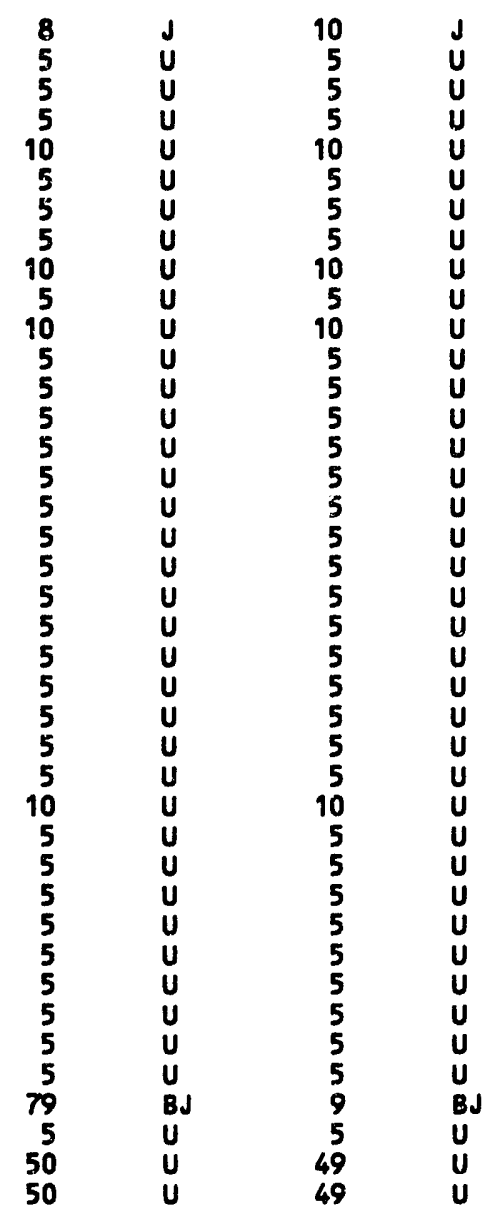

$$
\text { Location }=\text { ROA; Formation }=\text { DISMAL GAP; Sire }=14
$$

Acetone
Benzene
Bronodichloromethane
Bronoform
Bromomethane
Carbon Disulfide
Carbon Tetrachloride
Chlurobenzene
Chloroethane
Chloroform
Chtoromethane
Cis-1,2-Dichloroethene
Cis-1,3-Dichloropropene
Dibromochloromethane
Ethyl Benzene
Freon-113
Methylene Chloride
Ortho Xylene
Styrene
Tetrachloroethene
Toluene
Trans-1,2-Dichloroethene
Trans-1,3-Dichloropropene

$\mu g / \mathbf{k g}$
$\mu g / k g$
$\mu g / k g$
$\mu g / k g$
$\mu g / k g$
$\mu g / k g$
$\mu g / k g$
$\mu g / k g$
$\mu g / k g$
$\mu g / k g$
$\mu g / k g$
$\mu g / k g$
$\mu g / k g$
$\mu g / k g$
$\mu \gamma / \mathbf{k g}$
$\mu g / k g$
$\mu g / k g$
$\mu g / k g$
$\mu g / k g$
$\mu g / k g$
$\mu g / k g$
$\mu g / k g$
$\mu g / k g$

$\begin{array}{rr}8 & J \\ 5 & U \\ 5 & U \\ 5 & U \\ 10 & U \\ 5 & U \\ 5 & U \\ 5 & U \\ 10 & U \\ 5 & U \\ 10 & U \\ 5 & U \\ 5 & U \\ 5 & U \\ 5 & U \\ 5 & U \\ 5 & U \\ 5 & U \\ 5 & U \\ 5 & U \\ 5 & U \\ 5 & U \\ 5 & U\end{array}$


Table B.1 (continued)

\begin{tabular}{|c|c|c|c|c|c|}
\hline Analysis & Units & A horizon & Qual. & $\begin{array}{l}\text { A liorizon } \\
\text { field dup }\end{array}$ & Qual. \\
\hline $\begin{array}{l}\text { Trichloroethene } \\
\text { Trichloroflworonethane } \\
\text { Vinyl Acetate } \\
\text { Vinyl Chloride } \\
\text { Xylene (Meta + Para) } \\
\text { 1,1-Dichloroethane } \\
1,1-0 \text { ichloroethene } \\
1,1,1-\text { Trichloroethane } \\
1,1,2-\text { Trichloroethane } \\
1,1,2,2-\text { Tetrachloroethane } \\
1,2-\text { ichloroethane } \\
1,2-\text { ichloropropane } \\
1,4-\text { Dichlorobenzene } \\
\text { 2--Butanone } \\
\text { 2-Chloroethyl Vinyl Ether } \\
\text { 2-Hexanone } \\
\text { 4-Methyl-2-Pentanone }\end{array}$ & $\begin{array}{l}\mu g / k g \\
\mu g / k g \\
\mu g / k g \\
\mu g / k g \\
\mu g / k g \\
\mu g / k g \\
\mu g / k g \\
\mu g / k g \\
\mu g / k g \\
\mu g / k g \\
\mu g / k g \\
\mu g / k g \\
\mu g / k g \\
\mu g / k g \\
\mu g / k g \\
\mu g / k g \\
\mu g / k g\end{array}$ & $\begin{array}{r}5 \\
5 \\
5 \\
10 \\
5 \\
5 \\
5 \\
5 \\
5 \\
5 \\
5 \\
5 \\
5 \\
35 \\
5 \\
50 \\
50\end{array}$ & $\begin{array}{l}\mathbf{U} \\
\mathbf{U} \\
\mathbf{U} \\
\mathbf{U} \\
\mathbf{U} \\
\mathbf{U} \\
\mathbf{U} \\
\mathbf{U} \\
\mathbf{U} \\
\mathbf{U} \\
\mathbf{U} \\
\mathbf{U} \\
\mathbf{B} \mathbf{J} \\
\mathbf{U} \\
\mathbf{U} \\
\mathbf{U}\end{array}$ & & \\
\hline \multicolumn{6}{|c|}{ Location $=$ ROA; Fomation $=$ DISMAL GAP; Site $=17$} \\
\hline 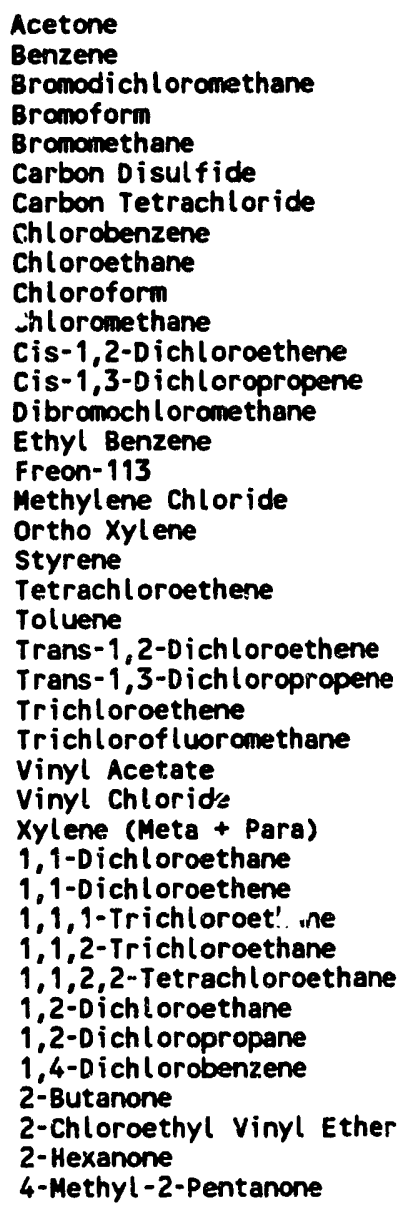 & $\begin{array}{l}\mu g / \mathbf{k g} \\
\mu g / \mathbf{k g} \\
\mu g / k g \\
\mu g / k g \\
\mu g / k g \\
\mu g / k g \\
\mu g / k g \\
\mu g / k g \\
\mu g / k g \\
\mu g / k g \\
\mu g / k g \\
\mu g / k g \\
\mu g / k g \\
\mu g / k g \\
\mu g / k g \\
\mu g / k g \\
\mu g / k g \\
\mu g / k g \\
\mu g / k g \\
\mu g / k g \\
\mu g / k g \\
\mu g / k g \\
\mu g / k g \\
\mu g / k g \\
\mu g / k g \\
\mu g / k g \\
\mu g / k g \\
\mu g / k g \\
\mu g / k g \\
\mu g / k g \\
\mu g / k g \\
\mu g / k g \\
\mu g / k g \\
\mu g / k g \\
\mu g / k g \\
\mu g / k g \\
\mu g / k g \\
\mu g / k g \\
\mu g / k g \\
\mu g / k g\end{array}$ & 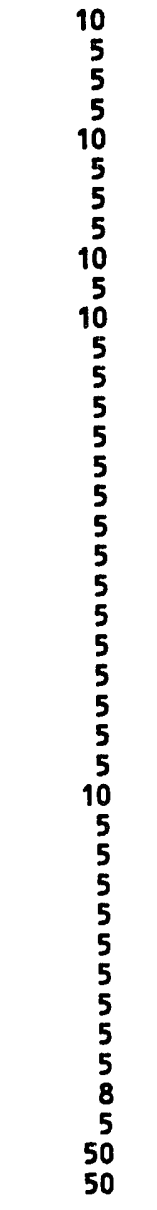 & 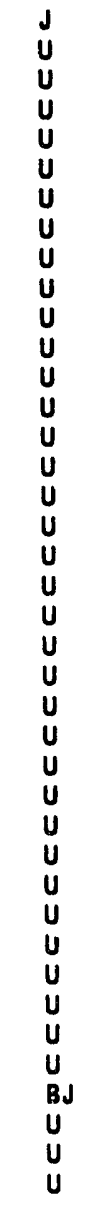 & & \\
\hline \multicolumn{6}{|c|}{ Location $=$ ROA; Fommation $=$ DISMAL GAP; Site $=19$} \\
\hline $\begin{array}{l}\text { Acetone } \\
\text { Benzene } \\
\text { Bromodichloromethane } \\
\text { Bromoform } \\
\text { Bromomethane }\end{array}$ & $\begin{array}{l}\mu g / \mathbf{k g} \\
\mu g / k g \\
\mu g / k g \\
\mu g / k g \\
\mu g / k g\end{array}$ & $\begin{array}{r}100 \\
5 \\
5 \\
5 \\
10\end{array}$ & $\begin{array}{l}U \\
U \\
U \\
U \\
U\end{array}$ & & \\
\hline
\end{tabular}


Table B.1 (continued)

\begin{tabular}{|c|c|c|c|c|c|}
\hline Analysis & Units & A horizon & Qual. & $\begin{array}{l}\text { A borizon } \\
\text { field dup }\end{array}$ & Qual. \\
\hline 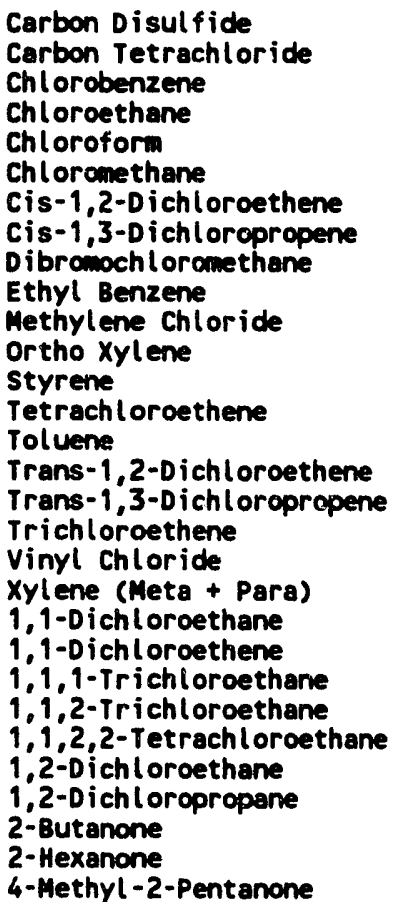 & 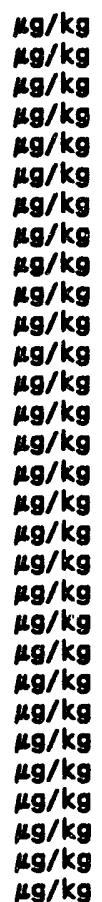 & $\begin{array}{r}5 \\
5 \\
5 \\
10 \\
5 \\
10 \\
5 \\
5 \\
5 \\
5 \\
5 \\
5 \\
5 \\
5 \\
5 \\
5 \\
5 \\
5 \\
10 \\
5 \\
5 \\
5 \\
5 \\
5 \\
5 \\
5 \\
5 \\
100 \\
50 \\
50\end{array}$ & $\begin{array}{l}\mathbf{U} \\
\mathbf{U} \\
\mathbf{U} \\
\mathbf{U} \\
\mathbf{U} \\
\mathbf{U} \\
\mathbf{U} \\
\mathbf{U} \\
\mathbf{U} \\
\mathbf{U} \\
\mathbf{U} \\
\mathbf{U} \\
\mathbf{U} \\
\mathbf{U} \\
\mathbf{U} \\
\mathbf{U} \\
\mathbf{U} \\
\mathbf{U} \\
\mathbf{U} \\
\mathbf{U} \\
\mathbf{U} \\
\mathbf{U} \\
\mathbf{U} \\
\mathbf{U} \\
\mathbf{U} \\
\mathbf{U} \\
\mathbf{U} \\
\mathbf{U} \\
\mathbf{U} \\
\mathbf{U}\end{array}$ & & \\
\hline
\end{tabular}

Location $=$ ROA; Formation $=$ DISMAL GAP; Site $=20$

\begin{tabular}{|c|c|c|}
\hline 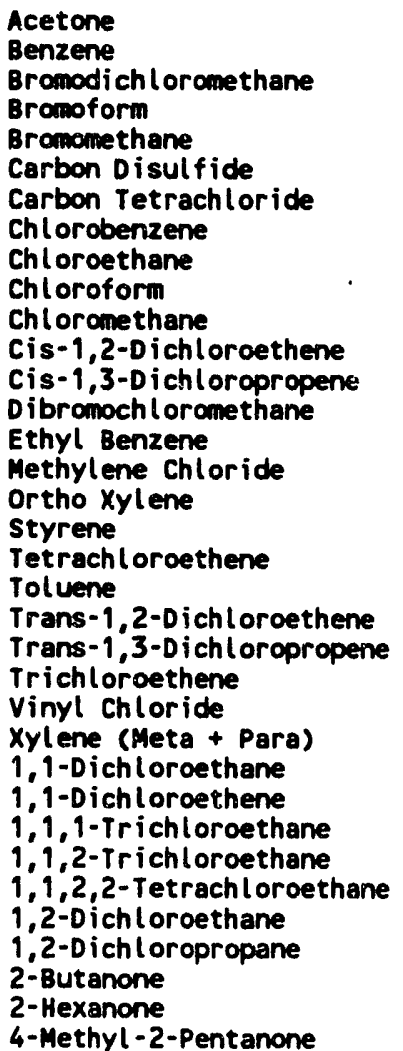 & 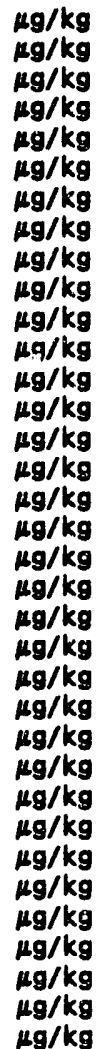 & $\begin{array}{r}100 \\
5 \\
5 \\
5 \\
10 \\
5 \\
5 \\
5 \\
10 \\
5 \\
10 \\
5 \\
5 \\
5 \\
5 \\
5 \\
5 \\
5 \\
5 \\
5 \\
5 \\
5 \\
5 \\
10 \\
5 \\
5 \\
5 \\
5 \\
5 \\
5 \\
5 \\
5 \\
100 \\
50 \\
50\end{array}$ \\
\hline
\end{tabular}




\section{B-33}

Table B.1 (continued)

\begin{tabular}{|c|c|c|c|}
\hline Analysis & Units & A horizon & 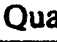 \\
\hline \multicolumn{4}{|c|}{ Location $=$ ROA; Formation $=$ DISMAL GAF } \\
\hline $\begin{array}{l}\text { Acetone } \\
\text { Benzene } \\
\text { Bromodichloromethane } \\
\text { Bromoform } \\
\text { Bromomethane } \\
\text { Carbon D isulfide } \\
\text { Carbon Tetrachloride } \\
\text { Chlorobenzene } \\
\text { Chloroethane } \\
\text { Chloroform } \\
\text { Chloromethane } \\
\text { Cis-1,2-Dichloroethene } \\
\text { Cis-1,3-Dichloropropene } \\
\text { Dibromochloromethane } \\
\text { Ethyl Benzene } \\
\text { Methylene Chloride } \\
\text { Ortho Xylene } \\
\text { styrene } \\
\text { Tetrachloroethene } \\
\text { Toluene } \\
\text { Trans-1,2-Dichloroethene } \\
\text { Trans-1,3-Dichloropropene } \\
\text { Trichloroethene } \\
\text { Vinyl Chloride } \\
\text { Xylene (Meta + Para) } \\
1,1-D i c h l o r o e t h a n e \\
1,1-D i c h l o r o e t h e n e \\
1,1,1-\text { Trichloroethane } \\
1,1,2-\text { Trichloroethane } \\
1,1,2,2-\text { Tetrachloroethane } \\
1,2-0 \text { ichloroethane } \\
1,2-D \text { ichloropropane } \\
2-\text { Butanone } \\
2-\text { Hexanone } \\
4-\text { Methyl-2-Pentanone }\end{array}$ & $\begin{array}{l}\mu g / \mathbf{k g} \\
\mu g / \mathbf{k g} \\
\mu g / \mathbf{k g} \\
\mu g / \mathbf{k g} \\
\mu g / \mathbf{k g} \\
\mu g / \mathbf{k g} \\
\mu g / \mathbf{k g} \\
\mu g / \mathbf{k g} \\
\mu g / \mathbf{k g} \\
\mu g / \mathbf{k g} \\
\mu g / \mathbf{k g} \\
\mu g / \mathbf{k g} \\
\mu g / \mathbf{k g} \\
\mu g / \mathbf{k g} \\
\mu g / \mathbf{k g} \\
\mu g / \mathbf{k g} \\
\mu g / \mathbf{k g} \\
\mu g / \mathbf{k g} \\
\mu g / \mathbf{k g} \\
\mu g / \mathbf{k g} \\
\mu g / \mathbf{k g} \\
\mu g / \mathbf{k g} \\
\mu g / \mathbf{k g} \\
\mu g / \mathbf{k g} \\
\mu g / \mathbf{k g} \\
\mu g / \mathbf{k g} \\
\mu g / \mathbf{k g} \\
\mu g / \mathbf{k g} \\
\mu g / \mathbf{k g} \\
\mu g / \mathbf{k g} \\
\mu g / \mathbf{k g} \\
\mu g / \mathbf{k g} \\
\mu g / \mathbf{k g} \\
\mu g / \mathbf{k g} \\
\mu g / \mathbf{k g}\end{array}$ & $\begin{array}{r}100 \\
5 \\
5 \\
5 \\
10 \\
5 \\
5 \\
5 \\
10 \\
5 \\
10 \\
5 \\
5 \\
5 \\
5 \\
5 \\
5 \\
5 \\
5 \\
5 \\
5 \\
5 \\
5 \\
10 \\
5 \\
5 \\
5 \\
5 \\
5 \\
5 \\
5 \\
5 \\
100 \\
50 \\
50\end{array}$ & $\begin{array}{l}\mathbf{U} \\
\mathbf{U} \\
\mathbf{U} \\
\mathbf{U}\end{array}$ \\
\hline
\end{tabular}

$$
\text { Location }=\text { ROA; Formation }=\text { DISMAL GAP; Site }=22
$$

\begin{tabular}{|c|c|c|}
\hline 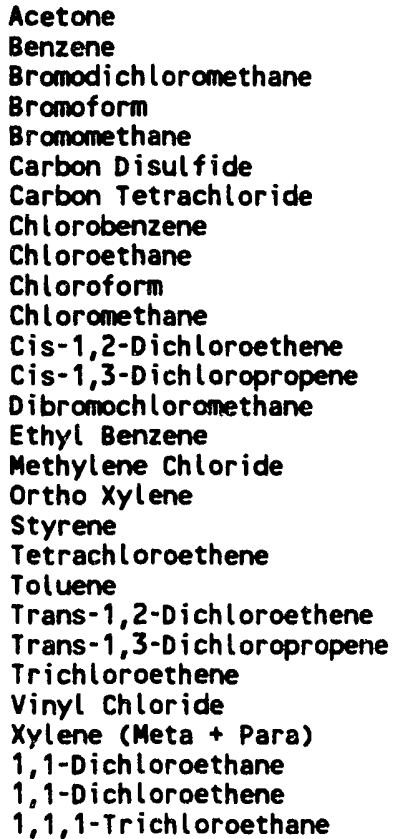 & $\begin{array}{l}\mu g / \mathbf{k g} \\
\mu g / k g \\
\mu g / \mathbf{k g} \\
\mu g / k g \\
\mu g / k g \\
\mu g / k g \\
\mu g / k g \\
\mu g / k g \\
\mu g / k g \\
\mu g / k g \\
\mu g / k g \\
\mu g / k g \\
\mu g / k g \\
\mu g / k g \\
\mu g / k g \\
\mu g / k g \\
\mu g / k g \\
\mu g / k g \\
\mu g / k g \\
\mu g / k g \\
\mu g / k g \\
\mu g / k g \\
\mu g / k g \\
\mu g / k g \\
\mu g / k g \\
\mu g / k g \\
\mu g / k g \\
\mu g / k g\end{array}$ & $\begin{array}{r}98 \\
5 \\
5 \\
5 \\
10 \\
5 \\
5 \\
5 \\
10 \\
5 \\
10 \\
5 \\
5 \\
5 \\
5 \\
5 \\
5 \\
5 \\
5 \\
5 \\
5 \\
5 \\
5 \\
10 \\
5 \\
5\end{array}$ \\
\hline
\end{tabular}

A horizon

field dup Qual. 
B-34

Table B.1 (continued)

\begin{tabular}{|c|c|c|c|c|c|}
\hline Analysis & Units & A horizon & Qual. & $\begin{array}{l}\text { A horizon } \\
\text { field dup }\end{array}$ & Qual. \\
\hline $\begin{array}{l}\text { 1,1,2-Trichloroethane } \\
1,1,2,2-\text { Tetrachloroethane } \\
\text { 1,2-Dichloroethane } \\
\text { 1,2-Dichloropropane } \\
\text { 2-Butanone } \\
\text { 2-Hexanone } \\
\text { 4-Methyl-2-Pentanone }\end{array}$ & $\begin{array}{l}\mu g / \mathrm{kg} \\
\mu g / \mathrm{kg} \\
\mu g / \mathrm{kg} \\
\mu g / \mathrm{kg} \\
\mu g / \mathrm{kg} \\
\mu g / \mathrm{kg} \\
\mu g / \mathrm{kg}\end{array}$ & $\begin{array}{r}5 \\
5 \\
5 \\
5 \\
98 \\
49 \\
49\end{array}$ & $\begin{array}{l}u \\
U \\
U \\
U \\
U \\
U \\
U\end{array}$ & & \\
\hline
\end{tabular}


Table B.2. Weighted gamma screening results for soil samples

\begin{tabular}{|c|c|c|c|c|c|c|c|}
\hline \multirow[b]{2}{*}{ Analysis } & \multirow[b]{2}{*}{ Units } & \multicolumn{6}{|c|}{ Depth (cm) } \\
\hline & & $00-05$ & $05-10$ & $10-15$ & $15-20$ & $20-25$ & $25-30$ \\
\hline \multicolumn{8}{|c|}{ Location $=A N D ;$ Formation $=$ DISMAL GAP; Site $=1$} \\
\hline Cs-137 & $\mathrm{PCi} / \mathrm{g}$ & 1.0405 & 0.1665 & 0.0405 & $\mathbf{N}^{*}$ & N & 0.0024 \\
\hline $\begin{array}{l}X-40 \\
U-238 \\
R a+226 \\
T h-232 \\
U-235\end{array}$ & $\begin{array}{l}\text { pCi/g } \\
\text { pCi/g } \\
\text { pCi/g } \\
\text { pCi/g } \\
\text { pCi/g }\end{array}$ & $\begin{array}{c}15.3184 \\
0.5906 \\
0.8824 \\
N\end{array}$ & $\begin{array}{l}17.2832 \\
1.5935 \\
0.6374 \\
0.9491 \\
\end{array}$ & $\begin{array}{c}24.2441 \\
0.6665 \\
1.1162 \\
N\end{array}$ & $\begin{array}{l}28.9573 \\
3.2284 \\
0.5316 \\
1.1710 \\
N\end{array}$ & $\begin{array}{c}27.5187 \\
N \\
0.5379 \\
1.1096 \\
H\end{array}$ & $\begin{array}{r}26.7698 \\
1.0655 \\
0.5178 \\
1.1050 \\
0.0548\end{array}$ \\
\hline
\end{tabular}

Location $=A N D ;$ Formation $=$ DISMAL GAP; Site $=3$

$\begin{array}{llcccccr}\text { Cs }-137 & \text { PCi/g } & 0.5910 & 0.1505 & 0.0473 & 0.0247 & 0.0148 & N \\ K-40 & \text { PCi/g } & 22.0278 & 20.0736 & 20.3150 & 22.0944 & 23.9437 & 22.9413 \\ U-238 & \text { PCi/g } & N & 2.1177 & N & 1.6575 & N & 4.4991 \\ R a-226 & \text { PCi/g } & 0.5822 & 0.6817 & 0.5955 & 0.5933 & 0.6653 & 0.5522 \\ T h-232 & \text { PCi/g } & 1.1588 & 1.1267 & 1.1139 & 1.2530 & 1.3566 & 1.3722 \\ U-235 & \text { PCi/g } & 0.1369 & N & 0.1372 & 0.0526 & N & N\end{array}$

Location $=A N D ;$ Formation $=$ DISMAL GAP; Site $=4$

$\begin{array}{llcccccc}\text { Cs-137 } & \text { PCi } / 9 & 1.4287 & 0.1510 & 0.0498 & 0.0627 & 0.0692 & 0.0263 \\ K-40 & \text { PCi/g } & 38.6418 & 35.0762 & 34.7543 & 38.6450 & 39.4480 & 36.5679 \\ U-238 & \text { PCi/g } & N & N & N & N & N \\ R a-226 & \text { PCi/g } & 0.6041 & 0.5853 & 0.5594 & 0.5266 & 0.5971 & 0.5300 \\ T h-232 & \text { PCi/g } & 1.0622 & 0.9718 & 0.9949 & 1.1156 & 1.2681 & 1.1060 \\ U-235 & \text { PCi/g } & N & N & N & N & N & N\end{array}$

Location $=A N D ;$ Formation $=$ DISMAL GAP; Site $=5$

$\begin{array}{llcccccr}\text { Cs }-137 & \text { PCi/g } & 0.7101 & 0.0924 & 0.0095 & N & N & N \\ K-40 & \text { PCi } / g & 42.2530 & 42.8831 & 42.2073 & 42.2013 & 39.4710 & 36.9445 \\ U-238 & \text { PCi/g } & N & N & 3.4697 & 3.5550 & N & 1.1683 \\ R a-226 & \text { PCi } / 9 & 0.5661 & 0.5771 & 0.5308 & 0.5410 & 0.5666 & 0.6190 \\ T h-232 & \text { PCi } / 9 & 1.1098 & 1.1491 & 1.1711 & 1.1772 & 1.1599 & 1.1139 \\ U-235 & \text { PCi/g } & N & 0.2555 & N & N & 0.2688 & 0.0803\end{array}$

Location $=A N D ;$ Formation $=$ DISMAL GAP; Site $=9$

$\begin{array}{llcccrrr}\text { Cs }-137 & \text { PCi /g } & 1.2969 & 0.2461 & 0.0855 & 0.0120 & 0.0403 & 0.0043 \\ K-40 & \text { pCi/g } & 17.1819 & 18.9354 & 20.4686 & 22.2786 & 22.6142 & 20.6699 \\ U-238 & \text { pCi/g } & 1.1784 & N & N & 5.1406 & 4.5186 & 2.2496 \\ \text { Ra-226 } & \text { pCi/g } & 0.5175 & 0.7025 & 0.7756 & 0.8913 & 0.7526 & 0.7397 \\ \text { Th-232 } & \text { pCi/g } & 0.8142 & 1.2333 & 1.4551 & 1.4779 & 1.5131 & 1.3998 \\ U-235 & \text { pCi/g } & N & 0.4942 & N & 0.2734 & 0.2443 & 0.0979\end{array}$

Location = AND; Formation $=$ DISMAL GAP; Site $=10$

$\begin{array}{llrrrrrr}\text { Cs }-137 & \text { PCi/g } & 1.7352 & 0.4507 & 0.0784 & 0.1257 & 0.0380 & 0.0010 \\ K-40 & \text { PCi/g } & 11.8015 & 11.8693 & 12.2725 & 12.5808 & 16.4545 & 18.3984 \\ U-238 & \text { PCi } / g & 4.0791 & 2.6709 & 1.0747 & 0.6061 & 1.2632 & 1.0706 \\ R a-226 & \text { PCi/g } & 0.7584 & 0.6983 & 0.7517 & 0.7760 & 0.7012 & 0.7227 \\ T h-232 & \text { PCi/g } & 0.9708 & 0.8848 & 0.9176 & 0.9236 & 1.0609 & 1.0965 \\ U-235 & \text { PCi } / g & 0.2239 & 0.1365 & 0.1481 & 0.1374 & 0.1045 & 0.0549\end{array}$


Table B.2 (continued)

\begin{tabular}{|c|c|c|c|c|c|c|c|}
\hline \multirow[b]{2}{*}{ Analysis } & \multirow[b]{2}{*}{ Units } & \multicolumn{6}{|c|}{ Depth (cm) } \\
\hline & & $00-05$ & $05-10$ & $10-15$ & $15-20$ & $20-25$ & $25-30$ \\
\hline \multicolumn{8}{|c|}{ Location $=A N D ;$ Formation $=$ DISMAL GAP; Site $=11$} \\
\hline $\begin{array}{l}\text { Cs-137 } \\
K-40 \\
U-238 \\
R a-226 \\
T h-232 \\
U-235\end{array}$ & 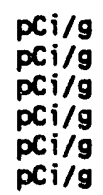 & $\begin{array}{r}1.3817 \\
27.4654 \\
2.9494 \\
0.5614 \\
0.8008 \\
0.3359\end{array}$ & $\begin{array}{r}0.3935 \\
26.5695 \\
2.7268 \\
0.5051 \\
0.8003 \\
0.1403\end{array}$ & $\begin{array}{r}0.1478 \\
27.4711 \\
2.5674 \\
0.4774 \\
0.8681 \\
0.1355\end{array}$ & $\begin{array}{r}0.0640 \\
30.7996 \\
1.3655 \\
0.5233 \\
0.9982 \\
0.0286\end{array}$ & $\begin{array}{r}0.0289 \\
34.7116 \\
3.0768 \\
0.5704 \\
1.1924 \\
0.1508\end{array}$ & $\begin{array}{r}0.0109 \\
35.2470 \\
1.9468 \\
0.6033 \\
1.2761 \\
0.0515\end{array}$ \\
\hline
\end{tabular}

Location $=A N D ;$ Formation $=$ DISMAL GAP; Site $=12$

$\begin{array}{llcccccr}C s-137 & P C i / g & 9.0709 & 0.1372 & 0.0255 & 0.0175 & 0.0314 & 0.0386 \\ K-40 & P C i / g & 24.4291 & 26.2972 & 30.1365 & 32.0915 & 34.8006 & 33.3333 \\ U-238 & P C i / g & N & N & N & 4.7411 & 3.5144 & 4.4903 \\ R a-226 & P C i / g & 0.5542 & 0.6450 & 0.6896 & 0.7044 & 0.7390 & 0.6715 \\ T h-232 & P C i / g & 0.7771 & 0.8903 & 0.9694 & 1.2797 & 1.3595 & 1.3283 \\ U-235 & P C i / 9 & 0.1039 & N & 0.1844 & 0.2166 & 0.1761 & 0.2364\end{array}$

Location = AND; Formation $=$ DISMAL GAP; Site $=19$

$\begin{array}{llrrrrrr}C s-137 & \text { PCi/g } & 2.1956 & 0.3944 & 0.0641 & 0.0341 & 0.0048 & 0.0092 \\ K-40 & \text { PCi/g } & 10.8236 & 13.7935 & 14.9526 & 19.0090 & 21.5009 & 21.5915 \\ U-238 & \text { PCi/g } & 0.9936 & 0.8918 & 2.4189 & 2.7871 & 2.3963 & 1.3233 \\ R a-226 & \text { PCi/g } & 0.5843 & 0.7096 & 0.6770 & 0.6492 & 0.6780 & 0.6273 \\ T h-232 & \text { PCi/g } & 0.8043 & 1.0076 & 1.0909 & 1.2560 & 1.3327 & 1.2870 \\ U-235 & \text { PCi/g } & 0.1024 & 0.1452 & 0.1432 & 0.1029 & 0.1650 & 0.0666\end{array}$

Location $=A N D ;$ Formation $=$ DISMAL GAP; Site $=20$

$\begin{array}{llcccccc}C s-137 & P C i / g & 1.1134 & 0.0549 & 0.0363 & N & 0.0403 & N \\ K-40 & P C i / 9 & 17.3193 & 16.7671 & 18.7084 & 21.6877 & 20.6868 & 19.2249 \\ U-238 & P C i / g & N & N & N & N & 4.0640 & N \\ R a-226 & P C i / g & 0.7066 & 0.6747 & 0.6899 & 0.7344 & 0.7655 & 0.5821 \\ T h-232 & P C i / g & 1.0379 & 0.9686 & 1.1993 & 1.4205 & 1.4101 & 1.2856 \\ U-235 & P C i / g & N & N & N & N & 0.2252 & 0.0902\end{array}$

Location $=A N D ;$ Formation $=$ DISMAL GAP; Site $=2 I$

$\begin{array}{llccccrr}C s-137 & \text { PCi/g } & 0.8445 & 0.1873 & 0.0844 & 0.0787 & 0.0172 & 0.0154 \\ K-40 & \text { PCi/g } & 21.1842 & 21.1774 & 29.5182 & 28.1374 & 26.8110 & 28.8829 \\ U-238 & \text { PCi/g } & N & N & 5.6762 & 3.7402 & 4.0914 & 4.3602 \\ R a-226 & \text { PCi/g } & 0.4616 & 0.5328 & 0.5716 & 0.4946 & 0.5707 & 0.5243 \\ T h-232 & \text { PCi/g } & 0.8694 & 0.8480 & 1.1365 & 1.1462 & 1.1399 & 1.2445 \\ U-235 & \text { PCi/g } & N & N & N & 0.1963 & 0.2800 & 0.2204\end{array}$

Lacation $=A N D ;$ Formation $=$ DISMAL GAP; Site $=22$

$\begin{array}{llrrrrrr}\text { Cs-137 } & \text { PCi/g } & 0.5353 & 0.0534 & 0.0255 & 0.0269 & 0.0248 & 0.0075 \\ K-40 & \text { PCi/g } & 29.6099 & 26.6361 & 27.9045 & 25.3770 & 28.1517 & 29.2053 \\ U-238 & \text { PCi/g } & 3.1628 & 2.6898 & 3.2686 & 3.3346 & 2.9950 & 1.2268 \\ R a-226 & \text { PCi/g } & 0.6262 & 0.6201 & 0.6371 & 0.6038 & 0.6204 & 0.5593 \\ T h-232 & \text { PCi/g } & 1.0610 & 1.0666 & 1.2126 & 1.2661 & 1.1991 & 1.1141 \\ U-235 & \text { PCi/g } & 0.1576 & 0.1492 & 0.1560 & 0.1608 & 0.1619 & 0.0565\end{array}$


Table B.2 (continued)

\begin{tabular}{|c|c|c|c|c|c|c|c|}
\hline \multirow[b]{2}{*}{ Analysis } & \multirow[b]{2}{*}{ Units } & \multicolumn{6}{|c|}{ Depth (cm) } \\
\hline & & $00-05$ & $05-10$ & $10-15$ & $15-20$ & $20-25$ & $25-30$ \\
\hline \multicolumn{8}{|c|}{ Location $=$ ORR; Formation $=$ DISMAL GAP; Site $=2$} \\
\hline $\begin{array}{l}\text { Cs }-137 \\
K-40 \\
U-238 \\
R a-226 \\
T h-232 \\
U-235\end{array}$ & $\begin{array}{l}\text { pCi/g } \\
p_{C i} i / g \\
p C i / g \\
p C i / g \\
p C i / g \\
p C i / g\end{array}$ & $\begin{array}{r}1.7189 \\
15.1972 \\
3.2767 \\
0.9044 \\
1.0133 \\
0.0967\end{array}$ & $\begin{array}{r}0.2115 \\
16.3894 \\
2.3829 \\
0.7510 \\
0.9939 \\
0.1449\end{array}$ & $\begin{array}{r}0.0747 \\
19.4542 \\
2.3630 \\
0.6634 \\
1.0647 \\
0.1463\end{array}$ & $\begin{array}{r}0.0422 \\
21.4761 \\
2.0251 \\
0.6080 \\
1.0588 \\
0.1388\end{array}$ & $\begin{array}{r}0.0284 \\
23.1861 \\
2.4461 \\
0.7755 \\
1.1916 \\
0.1908\end{array}$ & $\begin{array}{r}0.0131 \\
22.8751 \\
2.7837 \\
0.5888 \\
1.3749 \\
0.1606\end{array}$ \\
\hline
\end{tabular}

Location $=$ ORR; Formation $=$ DISMAL GAP; Site $=10$

$\begin{array}{llrrrrrr}\text { Cs }-137 & \text { PCi/g } & 1.3656 & 0.8561 & 0.2386 & 0.1730 & 0.0296 & 0.0148 \\ K-40 & \text { PCi/g } & 22.0029 & 23.1574 & 16.1954 & 19.3380 & 17.1920 & 17.0671 \\ U-238 & \text { PCi/g } & 3.5354 & 3.6073 & 2.3943 & 2.8313 & 2.4951 & 0.4048 \\ R a-226 & \text { PCi/g } & 0.7174 & 0.6503 & 0.4937 & 0.5869 & 0.8397 & 0.5044 \\ T h-232 & \text { PCi/g } & 1.1676 & 1.2337 & 0.9389 & 1.1499 & 1.1452 & 1.0976 \\ U-235 & \text { PCi/g } & 0.1695 & 0.1837 & 0.0671 & 0.1521 & 0.1485 & 0.0242\end{array}$

Location $=$ ORR; Formation $=$ DISMAL GAP; Site $=11$

$\begin{array}{llrrrrrr}C s-137 & P C i / g & 1.3536 & 0.4110 & 0.1274 & 0.0915 & 0.0516 & 0.0220 \\ K-40 & P C i / g & 27.3335 & 26.2783 & 24.7049 & 22.4150 & 28.9250 & 30.8557 \\ R a-226 & P C i / g & 0.8693 & 0.7244 & 0.6513 & 0.6776 & 0.7523 & 0.8685 \\ T h-232 & \text { PCi/g } & 1.4545 & 1.3252 & 1.3623 & 1.3867 & 1.5733 & 1.6720 \\ U-235 & \text { PCi/g } & 0.1348 & 0.1714 & 0.1145 & 0.1162 & 0.1227 & 0.1354\end{array}$

Location $=$ ORR; Formation $=$ DISMAL GAP; Site $=19$

$\begin{array}{lllrrrrr}C s-137 & \text { pCi/g } & 1.15976 & 0.0860 & 0.0184 & 0.0049 & 0.0072 & 0.0044 \\ K-40 & \text { PCi/g } & 9.78573 & 12.2284 & 13.9657 & 15.6700 & 17.5225 & 19.3992 \\ U-238 & \text { pCi/g } & 2.35735 & 2.1892 & 0.6857 & 1.4209 & 0.5366 & 1.7466 \\ R a-226 & \text { pCi/g } & 0.55824 & 0.6651 & 0.6638 & 0.7204 & 0.7024 & 0.7533 \\ T h-232 & \text { pCi/g } & 0.69456 & 0.9246 & 0.9387 & 1.0822 & 1.1208 & 1.2394 \\ U-235 & \text { pCi/g } & 0.13685 & 0.1363 & 0.0849 & 0.0545 & 0.0412 & 0.1009\end{array}$

Location $=$ ORR; Formation $=$ DISMAL GAP; Site $=22$

$\begin{array}{llrrrrrr}\text { Cs }-137 & \text { PCi/g } & 1.5205 & 0.3829 & 0.0907 & 0.0285 & 0.0265 & 0.0101 \\ K-40 & \text { pCi/g } & 13.5478 & 15.9145 & 18.1063 & 26.8466 & 28.8003 & 27.9817 \\ U-238 & \text { pCi/g } & 3.2313 & 2.3585 & 2.4392 & 2.9163 & 2.8677 & 1.3261 \\ R a-226 & \text { pCi/g } & 0.6656 & 0.6936 & 0.7541 & 0.7897 & 0.6973 & 0.5653 \\ T h-232 & \text { PCi/g } & 0.9694 & 1.0370 & 1.1640 & 1.4235 & 1.4542 & 1.4183 \\ U-235 & \text { pCi/g } & 0.1789 & 0.1117 & N & 0.1689 & N & 0.0530\end{array}$

Location $=$ ORR; Formation $=$ DISMAL GAP; Site $=26$

$\begin{array}{llrrrrrr}\text { Cs }-137 & \text { PCi } / g & 1.3703 & 0.2945 & 0.0957 & 0.0251 & 0.0116 & 0.0129 \\ K-40 & \text { pCi/g } & 18.3172 & 17.9569 & 20.0332 & 20.9431 & 21.5800 & 26.3039 \\ U-238 & \text { PCi/g } & 3.3415 & 2.8359 & 2.7844 & 2.9593 & 2.5301 & 1.6265 \\ \text { Ra-226 } & \text { PCi/g } & 0.8390 & 0.5569 & 0.6158 & 0.6438 & 0.6505 & 0.6739 \\ \text { Th-232 } & \text { PCi/g } & 1.0653 & 0.9838 & 1.0935 & 1.1993 & 1.3079 & 1.4047 \\ U-235 & \text { pCi/g } & 0.1761 & 0.1001 & 0.1578 & 0.1472 & 0.1718 & 0.0495\end{array}$

Location $=$ ORR; Formation $=$ DISMAL GAP; Site $=27$

$\begin{array}{llrrrrrr}\text { Cs }-137 & \text { PCi/g } & 1.2572 & 0.3332 & 0.1850 & 0.0111 & 0.0082 & 0.0013 \\ K-40 & \text { PCi/g } & 12.6756 & 14.8813 & 14.8364 & 15.4542 & 16.6053 & 16.9683 \\ R a-226 & \text { PCi/g } & 0.7552 & 0.8678 & 0.6976 & 0.7580 & 0.7483 & 0.6641 \\ \text { Th-232 } & \text { PCi/g } & 0.9894 & 1.1002 & 1.0336 & 1.0406 & 1.0213 & 0.9938 \\ U-235 & \text { PCi/g } & 0.1628 & 0.1161 & 0.1443 & 0.1126 & 0.1126 & 0.0949\end{array}$


Table B.2 (continced)

\begin{tabular}{|c|c|c|c|c|c|c|c|}
\hline \multirow[b]{2}{*}{ Analysis } & \multirow[b]{2}{*}{ Units } & \multicolumn{6}{|c|}{ Depth (cm) } \\
\hline & & $00-05$ & $05-10$ & $10-15$ & $15-20$ & $20-25$ & $25-30$ \\
\hline \multicolumn{8}{|c|}{ Location $=$ ORR; Formation $=$ DISMAL GAP; Site $=32$} \\
\hline $\begin{array}{l}\text { Cs-137 } \\
K-40 \\
U-238 \\
R a-226 \\
T h-232 \\
U-235\end{array}$ & $\begin{array}{r}p C i / g \\
p C i / g \\
p C i / g \\
p C i / g \\
p C i / g \\
p C i / g \\
I\end{array}$ & $\begin{array}{r}1.0464 \\
15.4072 \\
1.0328 \\
0.6552 \\
1.0246 \\
0.0761 \\
\text { ation }=0\end{array}$ & $\begin{array}{r}0.2435 \\
15.6139 \\
1.2187 \\
0.5872 \\
0.9444 \\
0.0511 \\
R ; \text { Format }\end{array}$ & $\begin{array}{r}0.1281 \\
15.8296 \\
2.0979 \\
0.6975 \\
0.9937 \\
0.1186 \\
=D I S M\end{array}$ & $\begin{array}{c}0.0150 \\
16.9094 \\
1.2029 \\
0.6931 \\
1.0446 \\
0.0571 \\
\text { GAP; Sit }\end{array}$ & $\begin{array}{r}0.0001 \\
20.7083 \\
0.8208 \\
0.6028 \\
1.1923 \\
0.0617 \\
=33\end{array}$ & $\begin{array}{r}0.0005 \\
21.1364 \\
0.7408 \\
0.5331 \\
1.0643 \\
0.0455\end{array}$ \\
\hline $\begin{array}{l}\text { Cs }-137 \\
K-40 \\
U-238 \\
R a-226 \\
T h-232 \\
U-235\end{array}$ & $\begin{array}{l}p C i / g \\
p C i / g \\
p C i / g \\
p C i / g \\
p C i / g \\
p C i / g\end{array}$ & $\begin{array}{r}2.6559 \\
19.1068 \\
7.8303 \\
1.2161 \\
1.3637 \\
0.1426\end{array}$ & $\begin{array}{r}0.3720 \\
17.4362 \\
3.4469 \\
0.7522 \\
1.0643 \\
0.1776\end{array}$ & $\begin{array}{r}0.0489 \\
19.8696 \\
3.7913 \\
0.6108 \\
1.1503 \\
0.1254\end{array}$ & $\begin{array}{r}0.0306 \\
18.0485 \\
3.1028 \\
0.6012 \\
1.1354 \\
0.1659\end{array}$ & $\begin{array}{r}0.0283 \\
16.6901 \\
2.1457 \\
0.6634 \\
1.1780 \\
N\end{array}$ & $\begin{array}{r}0.0200 \\
19.5761 \\
2.4243 \\
0.7477 \\
1.1920 \\
0.1333\end{array}$ \\
\hline
\end{tabular}

Location $=O R R ;$ Fommation $=$ DISMAL,$G A P ;$ Site $=35$

$\begin{array}{lllllllr}C s-137 & \text { PCi } / g & 0.87748 & 0.09969 & 0.02041 & 0.01806 & 0.00300 & 0.0191 \\ K-40 & \text { PCi } / 9 & 6.20873 & 5.68209 & 5.81069 & 6.62107 & 7.71612 & 10.6939 \\ U-238 & \text { PCi/g } & 3.08327 & 2.05896 & 0.75256 & 3.83585 & 1.26806 & 2.4462 \\ R a-226 & \text { PCi/g } & 0.72322 & 0.81582 & 0.88972 & 0.75208 & 0.71532 & 0.8264 \\ T h-232 & \text { PCi/g } & 0.80594 & 0.94075 & 0.90856 & 0.89607 & 0.89224 & 1.0554 \\ U-235 & \text { PCi/g } & 0.11851 & 0.10982 & 0.13253 & 0.12612 & 0.02210 & 0.1065\end{array}$

Location $=$ ORR; Formation $=$ DISMAL GAP; Site $=41$

$\begin{array}{llrlrrrr}\text { Cs-137 } & \text { PCi/g } & 2.0686 & 0.60128 & 0.3249 & 0.0779 & 0.0207 & 0.0216 \\ \text { K-40 } & \text { PCi/g } & 10.7427 & 9.92154 & 11.0152 & 18.5631 & 18.6508 & 20.0673 \\ R 8-226 & \text { PCi/g } & 0.7361 & 0.72228 & 0.6847 & 0.6112 & 0.5917 & 0.7496 \\ \text { Th-232 } & \text { PCi/g } & 1.0634 & 1.00867 & 1.0485 & 1.4132 & 1.5553 & 1.6006 \\ U-235 & \text { PCi/g } & 0.1896 & 0.13170 & 0.1131 & 0.1402 & 0.1234 & 0.1315\end{array}$

Location $=$ ORR; Formation $=$ DISMAL GAP; Site $=43$

$\begin{array}{llrrrrrr}\text { Cs-137 } & \text { PCi/g } & 1.2980 & 0.3329 & 0.0690 & 0.0321 & 0.0300 & 0.0385 \\ K-40 & \text { PCi/g } & 16.9967 & 16.2910 & 15.8280 & 17.6780 & 18.1977 & 22.3797 \\ U-238 & \text { PCi/g } & 4.9464 & 2.6392 & 1.7212 & 2.7298 & 5.6479 & 4.1528 \\ R a-226 & \text { PCi/g } & 0.8646 & 0.9468 & 0.8196 & 0.7193 & 0.6794 & 0.7219 \\ T h-232 & \text { PCi/g } & 1.2621 & 1.1425 & 1.1133 & 1.2199 & 1.1961 & 1.2745 \\ U-235 & \text { PCi/g } & 0.2573 & 0.2029 & N & 0.1681 & 0.1775 & 0.1858\end{array}$

Location $=$ ORR; Formation $=$ NOLJCHUCKY $;$ Site $=3$

$\begin{array}{lllrrrrr}C s-137 & \text { PCi/g } & 1.18164 & 0.3320 & 0.0515 & 0.0236 & 0.0261 & 0.0017 \\ K-40 & \text { PCi/g } & 9.83880 & 11.2675 & 10.9431 & 11.8086 & 15.6450 & 15.6981 \\ U-238 & \text { PCi/g } & 2.51189 & 1.9898 & 1.9450 & 2.2331 & 2.4346 & 0.8476 \\ R a-226 & \text { PCi/g } & 0.76528 & 0.7156 & 0.6469 & 0.7372 & 0.7150 & 0.7377 \\ T h-232 & \text { PCi/g } & 1.00806 & 1.0064 & 0.9741 & 1.1360 & 1.2901 & 1.2811 \\ U-235 & \text { PCi/g } & 0.15702 & 0.1385 & 0.0556 & 0.1362 & 0.1167 & 0.0301\end{array}$

Location $=$ ORR; Formation $=$ NOLICHUCKY $;$ Site $=5$

$\begin{array}{llllrrrr}\text { Cs }-137 & \text { PCi/g } & 1.13769 & 0.42327 & 0.0943 & 0.0288 & 0.0096 & 0.0034 \\ \text { K-40 } & \text { PCi/g } & 8.44980 & 9.81740 & 10.3635 & 11.9426 & 13.3605 & 14.0540 \\ \text { U-238 } & \text { PCi } / 9 & 0.86400 & 2.24251 & 1.9811 & 1.9422 & 2.3860 & 0.8546 \\ \text { Ra-226 } & \text { PCi } / 9 & 0.84649 & 1.03180 & 0.7932 & 0.7644 & 0.7808 & 0.6828 \\ \text { Th-232 } & \text { PSi/g } & 1.09361 & 1.27041 & 1.1657 & 1.1789 & 1.2477 & 1.2289 \\ U-235 & \text { PCi/g } & 0.10365 & 0.16878 & 0.0620 & 0.1338 & 0.1376 & 0.0866\end{array}$


Table B.2 (continued)

\begin{tabular}{|c|c|c|c|c|c|c|c|}
\hline \multirow[b]{2}{*}{ Analysis } & \multirow[b]{2}{*}{ Units } & \multicolumn{6}{|c|}{ Depth (cm) } \\
\hline & & $00-05$ & $05-10$ & $10-15$ & $15-20$ & $20-25$ & $25-30$ \\
\hline \multicolumn{8}{|c|}{ Location $=$ ORR; Formation $=$ NOLICHUCKY; Site $=13$} \\
\hline $\begin{array}{l}\text { Cs }-137 \\
K-40 \\
U-238 \\
R a-226 \\
T h-232 \\
U-235\end{array}$ & $\begin{array}{l}\mathrm{pCi} / \mathrm{g} \\
\mathrm{pCi} / \mathrm{g} \\
\mathrm{pCi} / \mathrm{g} \\
\mathrm{pCi} / \mathrm{g} \\
\mathrm{pCi} / \mathrm{g} \\
\mathrm{pCi} / \mathrm{g}\end{array}$ & $\begin{array}{r}1.2523 \\
15.2972 \\
2.7410 \\
0.8298 \\
1.2134 \\
0.1304\end{array}$ & $\begin{array}{r}0.4773 \\
13.4125 \\
2.1689 \\
0.7163 \\
1.1583 \\
0.1522\end{array}$ & $\begin{array}{r}0.0890 \\
13.4367 \\
2.1121 \\
0.6500 \\
1.1003 \\
0.1367\end{array}$ & $\begin{array}{r}0.0208 \\
12.9746 \\
1.9954 \\
0.6454 \\
1.0305 \\
0.1359\end{array}$ & $\begin{array}{r}0.0035 \\
12.8163 \\
0.7563 \\
0.5691 \\
0.9680 \\
0.1241\end{array}$ & $\begin{array}{r}0.0064 \\
18.2887 \\
1.1959 \\
0.6463 \\
1.2154 \\
0.0759\end{array}$ \\
\hline \multicolumn{8}{|c|}{ Location $=$ ORR; Formation $=$ NOLICHUCKY; Site $=15$} \\
\hline $\begin{array}{l}C s-137 \\
K-40 \\
U-238 \\
R a-226 \\
T h-232 \\
U-235\end{array}$ & $\begin{array}{l}p C i / g \\
p C i / g \\
p C i / g \\
p C i / g \\
p C i / g \\
p C i / g\end{array}$ & $\begin{array}{l}1.31838 \\
7.57668 \\
3.92091 \\
0.75488 \\
1.10444 \\
0.25544\end{array}$ & $\begin{array}{l}0.37165 \\
7.42748 \\
3.55493 \\
0.74257 \\
1.17495 \\
0.19953\end{array}$ & $\begin{array}{l}0.06814 \\
8.37181 \\
3.02203 \\
0.81304 \\
1.21709 \\
0.19982\end{array}$ & $\begin{array}{l}0.03282 \\
9.91225 \\
0.85926 \\
0.68292 \\
1.32450 \\
0.26038\end{array}$ & $\begin{array}{r}0.0439 \\
12.7679 \\
3.2127 \\
0.7380 \\
1.5444 \\
0.2233\end{array}$ & $\begin{array}{r}0.0376 \\
15.2212 \\
3.7710 \\
0.8035 \\
1.6724 \\
0.2312\end{array}$ \\
\hline
\end{tabular}

Location $=$ ORR; Formation $=$ NOLICHUCKX; Site $=16$

$\begin{array}{llcccccc}\text { Cs-137 } & \text { PCi/g } & 1.4118 & 0.3658 & 0.0694 & 0.0235 & N & 0.0261 \\ K-40 & \text { PCi/g } & 18.6789 & 17.0942 & 16.7292 & 16.9414 & 12.1189 & 13.0763 \\ U-238 & \text { PCi/g } & N & 0.7675 & 3.3155 & N & N & 1.0901 \\ R a-226 & \text { PCi/g } & 0.8334 & 0.7990 & 0.7655 & 0.7565 & 0.7995 & 0.7460 \\ T h-232 & \text { PCi/g } & 1.4208 & 1.4286 & 1.3369 & 1.3412 & 1.3938 & 1.3556 \\ U-235 & \text { PCi/g } & N & 0.1074 & N & N & N & 0.0920\end{array}$

Location $=$ ORR; Formation $=$ NOLICHUCKY $;$ Site $=21$

$\begin{array}{llrrrrrr}C s-137 & \text { PCi } / 9 & 3.0932 & 0.1176 & 0.0299 & 0.0294 & 0.0157 & 0.0081 \\ K-40 & \text { PCi/g } & 34.9695 & 30.5231 & 24.6009 & 23.5949 & 27.9409 & 36.0979 \\ U-238 & \text { PCi/g } & 4.6271 & 3.0044 & 2.9325 & 3.2139 & 2.6644 & 1.2930 \\ R a-226 & \text { PCi/g } & 0.7174 & 0.6222 & 0.7803 & 0.5500 & 0.5476 & 0.5080 \\ T h-232 & \text { PCi/g } & 1.7714 & 1.6407 & 1.6096 & 1.7286 & 1.6812 & 1.4985 \\ U-235 & \text { PCi/g } & 0.2569 & 0.0629 & 0.1863 & 0.1076 & 0.2444 & 0.0296\end{array}$

Location $=$ ORR; Formation $=$ NOLICHUCKY; Site $=23$

$\begin{array}{llrrrrrr}C s-137 & \text { PCi/g } & 1.0747 & 0.5820 & 0.0663 & 0.0405 & 0.0283 & 0.0380 \\ K-40 & \text { PCi } / 9 & 10.2992 & 12.4700 & 21.3062 & 27.4506 & 28.2411 & 29.2868 \\ U-238 & \text { PCi / } / 9 & 2.5799 & 2.7668 & 3.8144 & 4.3977 & 4.8436 & 2.5994 \\ R a-226 & \text { PCi } / 9 & 0.7428 & 0.7925 & 0.6529 & 0.5557 & 0.6003 & 0.5588 \\ \text { Th-232 } & \text { PCi/g } & 1.1539 & 1.2869 & 1.5108 & 1.6378 & 1.6599 & 1.6898 \\ U-235 & \text { PCi/g } & 0.2182 & 0.2202 & 0.2275 & 0.2288 & 0.2327 & 0.2311\end{array}$

Location $=$ ORR; Formation $=$ NOLICHUCKY; Site $=24$

$\begin{array}{llrrrrrr}\text { Cs-137 } & \text { PCi/g } & 1.8149 & 0.4331 & 0.1023 & 0.0399 & 0.0273 & 0.0016 \\ K-40 & \text { PCi/g } & 29.1557 & 25.3314 & 27.3521 & 28.6304 & 30.9524 & 31.1790 \\ U-238 & \text { PCi/g } & 4.1051 & 4.5234 & 1.1107 & 2.5580 & 2.8852 & 0.9021 \\ \text { Ra-226 } & \text { PCi/g } & 0.8650 & 0.6682 & 0.6839 & 0.6464 & 0.6724 & 0.6171 \\ \text { Th-232 } & \text { PCi/g } & 1.5330 & 1.3191 & 1.5141 & 1.5585 & 1.6765 & 1.6022 \\ U-235 & \text { PCi/g } & 0.2297 & 0.1567 & 0.1659 & 0.1657 & 0.1667 & 0.0492\end{array}$

Location $=$ ORR; Formation $=$ NOLICHUCKY $;$ Site $=25$

$\begin{array}{llcccccc}\text { Cs }-137 & \text { PCi } / 9 & 1.2195 & 0.0508 & 0.0220 & 0.0459 & 0.0073 & N \\ K-40 & \text { PCi/g } & 22.2143 & 21.5251 & 22.0695 & 28.4921 & 32.4522 & 33.0335 \\ U-238 & \text { PCi } / 9 & N & N & 1.4411 & N & N & N \\ R a-226 & \text { PCi/g } & 0.6716 & 0.6243 & 0.6632 & 0.6693 & 0.6224 & 0.5640 \\ T h-232 & \text { PCi/g } & 1.2591 & 1.3567 & 1.3916 & 1.6653 & 1.6946 & 1.6435 \\ U-235 & \text { PCi } / 9 & 0.2184 & N & N & 0.2563 & N & N\end{array}$


Table B.2 (continued)

\begin{tabular}{|c|c|c|c|c|c|c|c|}
\hline \multirow{2}{*}{ Analysis } & \multirow[b]{2}{*}{ Units } & \multicolumn{6}{|c|}{ Depth (cm) } \\
\hline & & $00-05$ & $05-10$ & $10-15$ & $15-20$ & $20-25$ & $25-30$ \\
\hline \multicolumn{8}{|c|}{ Location $=$ ORR; Fomation $=$ NOLICHUCKY; Site $=28$} \\
\hline $\begin{array}{l}C s-137 \\
K-40 \\
U-238 \\
R a-226 \\
T h-232 \\
U-235\end{array}$ & $\begin{array}{l}p C i / g \\
p C i / g \\
p C i / g \\
p C i / g \\
p C i / g \\
p C i / g\end{array}$ & $\begin{array}{r}1.4521 \\
10.9102 \\
2.6364 \\
0.8626 \\
1.0515 \\
0.1886\end{array}$ & $\begin{array}{r}0.4315 \\
12.0193 \\
2.1114 \\
0.9214 \\
1.1281 \\
0.1687\end{array}$ & $\begin{array}{r}0.1465 \\
12.0245 \\
2.3656 \\
0.8631 \\
1.2079 \\
0.1467\end{array}$ & $\begin{array}{r}0.0288 \\
28.8269 \\
1.5654 \\
0.7461 \\
1.4183 \\
0.3551\end{array}$ & $\begin{array}{r}0.0241 \\
35.8351 \\
3.1094 \\
0.5446 \\
1.4888 \\
0.1632\end{array}$ & $\begin{array}{r}0.0079 \\
35.2583 \\
0.9010 \\
0.6036 \\
1.4185 \\
0.0079\end{array}$ \\
\hline
\end{tabular}

Location $=$ ORR; Fomation $=$ NOLICHUCKY; Site $=31$

$\begin{array}{lllllllr}C s-137 & \text { PCi/g } & 1.81133 & 0.40512 & 0.06764 & 0.01823 & 0.0082 & 0.0093 \\ \text { K-40 } & \text { PCi/g } & 7.58745 & 7.85760 & 7.91839 & 8.72471 & 10.7174 & 13.0808 \\ U-238 & \text { PCi/g } & 3.21282 & 1.08908 & 2.30077 & 1.38541 & 0.8731 & 2.0346 \\ R a-226 & \text { PCi } / 9 & 0.81426 & 0.87903 & 0.84332 & 0.89600 & 0.8555 & 0.7552 \\ \text { Th-232 } & \text { PCi/g } & 1.10946 & 1.08215 & 1.11157 & 1.13255 & 1.1654 & 1.2004 \\ U-235 & \text { PCi/g } & 0.18207 & 0.27899 & 0.14678 & 0.06268 & 0.0492 & 0.0811\end{array}$

Location $=$ ORR; Formation $=$ NOLICHUCKY; Site $=42$

$\begin{array}{llrrrrrr}\text { Cs }-137 & \text { PCi/g } & 1.1265 & 0.1807 & 0.0418 & 0.0249 & 0.0041 & 0.0093 \\ \text { K-40 } & \text { PCi/g } & 12.7187 & 13.3355 & 21.0567 & 23.6743 & 20.8052 & 19.6050 \\ U-238 & \text { pCi/g } & 3.2981 & 2.1334 & 1.1212 & 2.5000 & 1.2277 & 1.6120 \\ \text { Ra-226 } & \text { pCi/g } & 0.7755 & 0.6449 & 0.7492 & 0.5907 & 0.6654 & 0.6581 \\ T h-232 & \text { PCi/g } & 1.1648 & 1.1105 & 1.2794 & 1.3561 & 1.3606 & 1.2759 \\ U-235 & \text { pCi/g } & 0.1788 & 0.1978 & 0.0899 & 0.0443 & 0.0432 & 0.0236\end{array}$

Location $=$ ROA; Formation $=$ DISMAL GAP; Site $=3$

$\begin{array}{llrrrrrr}\text { Cs }-137 & \text { PCi/g } & 0.8547 & 0.1987 & 0.0747 & 0.0327 & 0.0061 & 0.0162 \\ \text { K-40 } & \text { PCi/g } & 11.5364 & 10.8670 & 10.4431 & 10.4249 & 10.9776 & 11.0646 \\ U-238 & \text { PCi/g } & 4.2823 & 1.7353 & 2.7705 & 2.4742 & 2.5445 & 2.4345 \\ \text { Ra-226 } & \text { PCi/g } & 0.7445 & 0.9087 & 0.8165 & 0.7291 & 0.6889 & 0.6538 \\ T h-232 & \text { PCi/g } & 1.1137 & 1.1802 & 1.1179 & 1.1422 & 1.1728 & 1.1765 \\ U-235 & \text { PCi/g } & 0.0547 & 0.1555 & 0.1333 & 0.0442 & 0.1412 & 0.1444\end{array}$

Location $=$ ROA; Formation $=$ DISMAL GAP; Site $=7$

$\begin{array}{lllllllr}\text { Cs }-137 & \text { pCi/g } & 1.00018 & 0.06758 & 0.05618 & 0.0358 & 0.0033 & 0.0022 \\ K-40 & \text { pci/g } & 7.85868 & 9.07075 & 8.85627 & 10.6781 & 15.1970 & 18.7766 \\ \text { U-238 } & \text { pCi/g } & 2.25179 & 1.09497 & 1.61468 & 1.6798 & 1.3074 & 1.1676 \\ R a-226 & \text { pCi/g } & 0.78784 & 0.77877 & 0.80245 & 0.7743 & 0.7757 & 0.7139 \\ \text { Th-232 } & \text { pCi/g } & 0.99946 & 1.15157 & 1.09878 & 1.1931 & 1.3321 & 1.4702 \\ U-235 & \text { pCi/g } & 0.09426 & 0.17547 & 0.32156 & 0.1489 & 0.0679 & 0.0258\end{array}$

Location $=$ ROA; Formation $=$ DISMAL GAP; Site $=8$

$\begin{array}{lllllllr}C s-137 & \text { PCi/g } & 1.80437 & 0.47874 & 0.07930 & 0.02469 & 0.02150 & 0.0078 \\ \mathrm{~K}-40 & \mathrm{PCi} / \mathrm{g} & 6.84697 & 6.31775 & 6.59872 & 7.26735 & 8.17710 & 10.9998 \\ \mathrm{U}-238 & \mathrm{PCi} / \mathrm{g} & 2.17199 & 1.97052 & 2.13584 & 1.33850 & 1.17409 & 1.2732 \\ \mathrm{Ra}-226 & \mathrm{PCi} / \mathrm{g} & 0.73428 & 0.74100 & 0.75145 & 0.79693 & 0.74925 & 0.7442 \\ \mathrm{Th}-232 & \mathrm{PCi} / \mathrm{g} & 1.10390 & 1.07775 & 1.10967 & 1.16853 & 1.29059 & 1.2971 \\ U-235 & \mathrm{PCi} / \mathrm{g} & 0.16511 & \mathrm{~N} & 0.11434 & 0.13874 & 0.14543 & 0.1043\end{array}$

Location $=$ ROA; Formation $=$ DISMAL GAP; Site $=9$

$\begin{array}{llllllll}\mathrm{Cs}-137 & \text { pCi/g } & 2.37360 & 0.33769 & 0.03726 & 0.00893 & 0.00427 & 0.00863 \\ \mathrm{~K}-40 & \text { pCi/g } & 7.97527 & 7.65437 & 7.31478 & 7.00821 & 7.48968 & 7.82609 \\ \mathrm{U}-238 & \text { pCi/g } & 1.68335 & 1.37521 & 0.69534 & 1.51008 & 0.79716 & 1.24066 \\ \mathrm{Ra}-226 & \text { pCi/g } & 0.76762 & 0.79481 & 0.75858 & 0.78649 & 0.78544 & 0.71572 \\ \text { Th-232 } & \text { pCi/g } & 1.07995 & 1.15073 & 1.02602 & 1.03807 & 1.01271 & 0.96568 \\ U-235 & \text { pCi/g } & 0.20495 & 0.16226 & 0.04140 & 0.08682 & 0.08396 & 0.05803\end{array}$


Table B.2 (continued)

\begin{tabular}{|c|c|c|c|c|c|c|c|}
\hline \multirow[b]{2}{*}{ Analysis } & \multirow[b]{2}{*}{ Units } & \multicolumn{6}{|c|}{ Depth (cm) } \\
\hline & & $00-05$ & $05-10$ & $10-15$ & $15-20$ & $20-25$ & $25-30$ \\
\hline \multicolumn{8}{|c|}{ Location $=$ ROA; Formation $=$ DISMAL GAP; Site $=10$} \\
\hline $\begin{array}{l}\text { Cs-137 } \\
K-40 \\
U-238 \\
R a-226 \\
T h-232 \\
U-235\end{array}$ & $\begin{array}{l}p C i / g \\
p C i / g \\
p C i / g \\
p C i / g \\
p C i / g \\
p C i / g\end{array}$ & $\begin{array}{r}0.6479 \\
11.1886 \\
2.2086 \\
0.6988 \\
0.9756 \\
0.1505\end{array}$ & $\begin{array}{r}0.0460 \\
12.2362 \\
0.4811 \\
0.7406 \\
1.1513 \\
0.0913\end{array}$ & $\begin{array}{r}0.0289 \\
13.1197 \\
2.2488 \\
0.7150 \\
1.1846 \\
0.0219\end{array}$ & $\begin{array}{r}0.0182 \\
17.5574 \\
1.1617 \\
0.7095 \\
1.4620 \\
0.0350\end{array}$ & $\begin{array}{r}0.0259 \\
19.4597 \\
1.9573 \\
0.7025 \\
1.5374 \\
0.1580\end{array}$ & $\begin{array}{r}0.0257 \\
21.8072 \\
2.7254 \\
0.7337 \\
1.7462 \\
0.1664\end{array}$ \\
\hline
\end{tabular}

Location $=$ ROA; Formation $=$ DISMAL GAP; Site $=13$

$\begin{array}{llllllrr}\text { Cs-137 } & \text { PCi/g } & 0.20342 & 0.03059 & 0.01112 & 0.0064 & 0.0494 & 0.0253 \\ K-40 & \text { PCi/g } & 6.21173 & 6.58742 & 8.07113 & 11.7095 & 16.5192 & 19.2372 \\ U-238 & \text { PCi/g } & 2.28876 & 1.65555 & 2.15058 & 1.3867 & 1.0939 & 2.4900 \\ R a-226 & \text { PCi/g } & 0.70700 & 0.70586 & 0.71849 & 0.6952 & 0.6415 & 0.6707 \\ T h-232 & \text { PCi/g } & 1.01938 & 0.97115 & 1.01649 & 1.1559 & 1.2880 & 1.4293 \\ U-235 & \text { PCi/g } & 0.10645 & 0.07286 & 0.12643 & 0.1399 & 0.1517 & 0.1553\end{array}$

Location $=$ ROA; Formation $=$ DISMAL GAP; Site $=14$

$\begin{array}{llrrrrrr}\text { Cs-137 } & \text { PCi/g } & 1.5469 & 0.2365 & 0.0486 & 0.0053 & 0.0383 & 0.0366 \\ K-40 & \text { PCi/g } & 15.9916 & 21.2335 & 20.4532 & 17.4199 & 18.2892 & 18.5685 \\ U-238 & \text { PCi/g } & 4.1361 & 3.5846 & 2.4398 & 3.9481 & 4.4876 & 3.7519 \\ R a-226 & \text { PCi/g } & 0.6981 & 0.6281 & 0.6715 & 0.6370 & 0.6924 & 0.5819 \\ T h-232 & \text { PCi/g } & 1.2680 & 1.3550 & 1.4823 & 1.4512 & 1.5986 & 1.5064 \\ U-235 & \text { PCi/g } & 0.1999 & 0.2226 & 0.1907 & 0.2500 & 0.1130 & 0.2129\end{array}$

Location $=$ ROA; Formation $=$ DISMAL GAP; Site $=17$

$\begin{array}{lllllrrr}C s-137 & P C i / g & 1.06811 & 0.41668 & 0.12426 & 0.0491 & 0.0243 & 0.0144 \\ K-40 & P C i / g & 7.86085 & 8.01872 & 8.32302 & 12.3916 & 15.1041 & 17.0407 \\ U-238 & P C i / g & 1.49078 & 0.61736 & 1.98948 & 2.0866 & 2.3101 & 0.7476 \\ R s-226 & P C i / g & 0.74439 & 0.74809 & 0.68905 & 0.6779 & 0.7289 & 0.7326 \\ T h-232 & P C i / g & 1.01544 & 1.02281 & 1.05709 & 1.1309 & 1.2731 & 1.3357 \\ U-235 & P C i / g & 0.08073 & 0.15287 & N^{6} & 0.1279 & N & 0.0391\end{array}$

Location $=$ ROA; Fomation $=$ DISMAL GAP; Site $=19$

$\begin{array}{llllllll}\text { Cs }-137 & \text { PCi } / 9 & 0.77258 & 0.07183 & 0.02488 & 0.02617 & 0.01874 & 0.02448 \\ K-40 & \text { PCi/g } & 7.65583 & 9.00872 & 8.31273 & 9.59149 & 9.03652 & 9.54134 \\ U-238 & \text { PCi/g } & 1.22403 & 2.76961 & 2.52996 & 2.85302 & 2.96084 & 2.53167 \\ R a-226 & \text { PCi/g } & 0.98148 & 1.22142 & 1.13411 & 0.92588 & 1.00616 & 0.94241 \\ T h-232 & \text { PCi/g } & 1.22177 & 1.38829 & 1.21685 & 1.26869 & 1.33524 & 1.25627 \\ U-235 & \text { PCi/g } & 0.10668 & 0.20344 & N & 0.17395 & 0.10917 & 0.06610\end{array}$

Location $=$ ROA; Formation $=$ DISMAL GAP; Site $=20$

$\begin{array}{llllrrrr}C s-137 & \text { PCi/g } & 0.86373 & 0.16857 & 0.0694 & 0.0374 & 0.0177 & 0.0102 \\ K-40 & \text { PCi/g } & 8.37732 & 9.10996 & 15.4059 & 18.4632 & 26.6641 & 29.0528 \\ U-238 & \text { PCi/g } & 2.96989 & 3.16190 & 1.9166 & 3.9993 & 1.1015 & 0.6548 \\ R a-226 & \text { PCi/g } & 0.98084 & 0.64519 & 0.6469 & 0.6153 & 0.5690 & 0.6767 \\ T h-232 & \text { PCi/g } & 0.94018 & 0.97558 & 1.2443 & 1.2996 & 1.4085 & 1.4203 \\ U-235 & \text { PCi/g } & 0.18081 & 0.15951 & 0.1336 & 0.1782 & 0.0445 & 0.0749\end{array}$

Location $=$ ROA; Formation $=$ DISMAL GAP; Site $=2 I$

$\begin{array}{llrrrrrr}\text { Cs }-137 & \text { PCi/g } & 0.9331 & 0.1301 & 0.02048 & 0.03094 & 0.01416 & 0.03293 \\ K-40 & \text { PCi/g } & 12.7528 & 12.7725 & 9.16888 & 8.93252 & 9.76466 & 9.57555 \\ U-238 & \text { PCi/g } & 3.6122 & 1.3212 & 3.17418 & 3.58721 & 2.33844 & 3.60514 \\ R a-226 & \text { PCi/g } & 0.6918 & 0.8860 & 0.79204 & 0.87766 & 1.03810 & 0.89798 \\ T h-232 & \text { PCi/g } & 1.2858 & 1.3053 & 1.11717 & 1.17581 & 1.22282 & 1.26791 \\ U-235 & \text { PCi/g } & 0.1020 & 0.0993 & 0.18391 & 0.20862 & 0.20426 & 0.23618\end{array}$


Table B.2 (continued)

\begin{tabular}{|c|c|c|c|c|c|c|c|}
\hline \multirow[b]{2}{*}{ Analysis } & \multirow[b]{2}{*}{ Units } & \multicolumn{6}{|c|}{ Depth (cm) } \\
\hline & & $00-05$ & $05-10$ & $10-15$ & $15-20$ & $20-25$ & $25-30$ \\
\hline \multicolumn{8}{|c|}{ Location $=$ ROA; Formation $=$ DISMAL GAP; Site $=22$} \\
\hline $\begin{array}{l}C s-137 \\
K-40 \\
U-238 \\
R=-226 \\
T h-232 \\
U-235\end{array}$ & $\begin{array}{l}p C i / g \\
p C i / g \\
p C i / g \\
p C i / g \\
p C i / g \\
p C i / g\end{array}$ & $\begin{array}{l}0.56818 \\
7.22849 \\
3.73728 \\
0.72895 \\
0.92026 \\
0.13483\end{array}$ & $\begin{array}{l}0.11340 \\
8.81645 \\
2.25628 \\
0.65960 \\
0.95387 \\
0.11169\end{array}$ & $\begin{array}{r}0.0243 \\
12.4847 \\
2.3393 \\
0.6180 \\
1.0513 \\
0.1491\end{array}$ & $\begin{array}{r}0.0286 \\
17.6887 \\
3.0799 \\
1.0002 \\
1.3405 \\
0.0546\end{array}$ & $\begin{array}{r}0.0322 \\
21.6703 \\
3.0480 \\
1.0064 \\
1.5092 \\
0.3750\end{array}$ & $\begin{array}{r}0.0077 \\
21.7934 \\
1.1228 \\
0.9894 \\
1.4504 \\
0.0671\end{array}$ \\
\hline
\end{tabular}

${ }^{a} \mathrm{~N}=$ not detected. 
Table B.3. Unweighted gamma screening results for soil samples

\begin{tabular}{|c|c|c|c|c|c|c|c|}
\hline \multirow[b]{2}{*}{ Analysis } & \multirow[b]{2}{*}{ Units } & \multicolumn{6}{|c|}{ Depth (cm) } \\
\hline & & $\overline{00-05}$ & $05-10$ & $10-15$ & $15-20$ & $20-25$ & $25-30$ \\
\hline \multicolumn{8}{|c|}{ Location $=A N D ;$ Formation $=$ DISMAL GAP; Site $=1$} \\
\hline $\begin{array}{l}C s-137 \\
K-40 \\
U-238 \\
R a-226 \\
T h-232 \\
U-235\end{array}$ & $\begin{array}{l}\mathrm{pCi} / \mathrm{cm}^{2} \\
\mathrm{PCi} / \mathrm{cm}^{2} \\
\mathrm{PCi} / \mathrm{cm}^{2} \\
\mathrm{PCi} / \mathrm{cm}^{2} \\
\mathrm{PCi} / \mathrm{cm}^{2} \\
\mathrm{pCi} / \mathrm{cm}^{2}\end{array}$ & $\begin{array}{c}5.245 \\
7.220 \\
N \\
2.977 \\
4.448 \\
N\end{array}$ & $\begin{array}{r}1.112 \\
115.400 \\
10.640 \\
4.256 \\
6.337 \\
N\end{array}$ & $\begin{array}{c}0.216 \\
129.100 \\
N \\
3.549 \\
5.944 \\
N\end{array}$ & $\begin{array}{c}N^{n} \\
161.900 \\
18.050 \\
2.972 \\
6.547 \\
N\end{array}$ & $\begin{array}{c}N \\
154.600 \\
N \\
3.022 \\
6.234 \\
N\end{array}$ & $\begin{array}{r}0.015 \\
162.600 \\
6.472 \\
3.145 \\
6.712 \\
0.333\end{array}$ \\
\hline
\end{tabular}

Location $=A N D ;$ Formation $=$ DISMAL GAP; Site $=3$

$\begin{array}{llrrrrrr}\mathrm{Cs}-137 & \mathrm{PCi} / \mathrm{cm}^{2} & 3.276 & 0.941 & 0.297 & 0.144 & 0.077 & N \\ \mathrm{~K}-40 & \mathrm{PCi} / \mathrm{cm}^{2} & 122.100 & 125.500 & 127.700 & 128.700 & 124.100 & 118.400 \\ \mathrm{U}-238 & \mathrm{PCi} / \mathrm{cm}^{2} & N & 13.240 & N & 9.655 & N & 23.220 \\ \mathrm{Ra}-226 & \mathrm{PCi} / \mathrm{cm}^{2} & 3.227 & 4.262 & 3.743 & 3.456 & 3.448 & 2.850 \\ \mathrm{Th}-232 & \mathrm{PCi} / \mathrm{cm}^{2} & 6.423 & 7.044 & 7.002 & 7.299 & 7.031 & 7.082 \\ \mathrm{U}-235 & \mathrm{PCi} / \mathrm{cm}^{2} & 0.759 & N & 0.863 & 0.306 & N & N\end{array}$

Location $=A N D ;$ Formation $=$ DISMAL GAP; Site $=4$

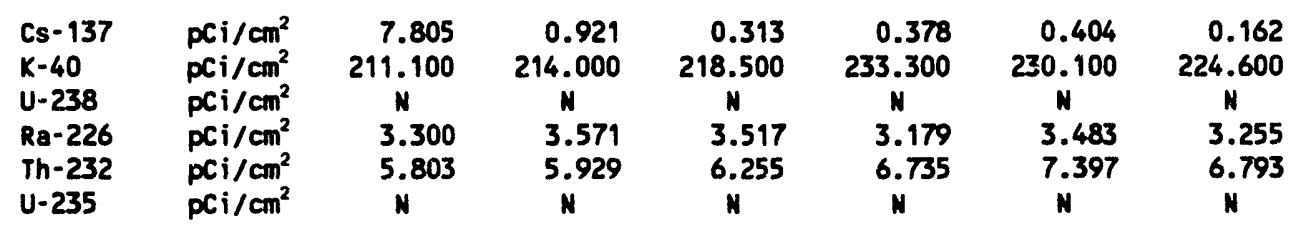

Location $=A N D ;$ Formation $=$ DISMAL GAP; Site $=5$

$\begin{array}{llcrrrrr}\mathrm{Cs}-137 & \mathrm{PCi} / \mathrm{cm}^{2} & 3.946 & 0.560 & 0.062 & N & N & N \\ \mathrm{~K}-40 & \mathrm{PCi} / \mathrm{cm}^{2} & 234.800 & 260.000 & 276.500 & 268.400 & 235.800 & 207.000 \\ \mathrm{U}-238 & \mathrm{PCi} / \mathrm{cm}^{2} & N & N & 22.730 & 22.610 & N & 6.546 \\ \mathrm{Ra}-226 & \mathrm{PCi} / \mathrm{cm}^{2} & 3.146 & 3.499 & 3.47 ! & 3.441 & 3.385 & 3.468 \\ \mathrm{Th}-232 & \mathrm{PCi} / \mathrm{cm}^{2} & 6.167 & 6.967 & 7.672 & 7.487 & 6.929 & 6.241 \\ \mathrm{U}-235 & \mathrm{PCi} / \mathrm{cm}^{2} & \mathrm{~N} & 1.549 & \mathrm{~N} & \mathrm{~N} & 1.606 & 0.450\end{array}$

Location $=A N D ;$ Formation $=$ DISMAL GAP; Site $=9$

$\begin{array}{llrrrrrr}\mathrm{Cs}-137 & \mathrm{PCi} / \mathrm{cm}^{2} & 7.064 & 1.246 & 0.3866 & 0.0465 & 0.190 & 0.0201 \\ \mathrm{~K}-40 & \mathrm{PCi} / \mathrm{cm}^{2} & 93.590 & 95.870 & 92.6000 & 86.5300 & 106.400 & 96.5700 \\ \mathrm{U}-238 & \mathrm{PCi} / \mathrm{cm}^{2} & 6.419 & N & N & 19.9660 & 21.260 & 10.5100 \\ \mathrm{Ra}-226 & \mathrm{PCi} / \mathrm{cm}^{2} & 2.819 & 3.557 & 3.5090 & 3.4620 & 3.541 & 3.4560 \\ \mathrm{~T}-232 & \mathrm{PCi} / \mathrm{cm}^{2} & 4.435 & 6.244 & 6.5830 & 5.7400 & 7.119 & 6.5400 \\ \mathrm{U}-235 & \mathrm{pCi} / \mathrm{cm}^{2} & \mathrm{~N} & 2.502 & \mathrm{~N} & 1.0620 & 1.149 & 0.4574\end{array}$

Location $=A N D ;$ Formation $=$ DISMAL GAP; Site $=10$

$\begin{array}{llrrrrrr}\text { Cs-137 } & \text { PCi } / \mathrm{cm}^{2} & 4.9990 & 2.8280 & 0.5041 & 0.7931 & 0.275 & 0.007 \\ \mathrm{~K}-40 & \mathrm{PCi} / \mathrm{cm}^{2} & 34.0000 & 74.4800 & 78.9000 & 79.3600 & 118.900 & 134.400 \\ \mathrm{U}-238 & \mathrm{PCi} / \mathrm{cm}^{2} & 11.7520 & 16.7600 & 6.9090 & 3.8230 & 9.128 & 7.821 \\ \mathrm{Ra}-226 & \mathrm{PCi} / \mathrm{cm}^{2} & 2.1850 & 4.3820 & 4.8330 & 4.8950 & 5.067 & 5.279 \\ \mathrm{Th}-232 & \mathrm{PCi} / \mathrm{cm}^{2} & 2.7970 & 5.5520 & 5.8990 & 5.8260 & 7.666 & 8.010 \\ \mathrm{U}-235 & \mathrm{PCi} / \mathrm{cm}^{2} & 0.6450 & 0.8568 & 0.9521 & 0.8666 & 0.755 & 0.401\end{array}$


Table B.3 (continued)

\begin{tabular}{|c|c|c|c|c|c|c|c|}
\hline \multirow[b]{2}{*}{ Analysis } & \multirow[b]{2}{*}{ Units } & \multicolumn{6}{|c|}{ Depth (cm) } \\
\hline & & $00-0.5$ & $05-10$ & $10-15$ & $15-20$ & $20-25$ & $25-30$ \\
\hline
\end{tabular}

$$
\text { Location }=\text { AND; Formation }=\text { DISMAL GAP; Site }=11
$$

\begin{tabular}{|c|c|c|c|c|c|c|c|}
\hline $\begin{array}{l}\text { Cs-137 } \\
K-40 \\
U-238 \\
R a-226 \\
T h-232 \\
U-235\end{array}$ & $\begin{array}{l}\mathrm{pCi} / \mathrm{cm}^{2} \\
\mathrm{PCi} / \mathrm{cm}^{2} \\
\mathrm{pci} / \mathrm{cm}^{2} \\
\mathrm{PCi} / \mathrm{cm}^{2} \\
\mathrm{PCi} / \mathrm{cm}^{2} \\
\mathrm{PCi} / \mathrm{cm}^{2}\end{array}$ & $\begin{array}{r}6.389 \\
127.000 \\
13.638 \\
2.596 \\
3.703 \\
1.553\end{array}$ & $\begin{array}{r}2.294 \\
154.900 \\
15.897 \\
2.945 \\
4.666 \\
0.818\end{array}$ & $\begin{array}{r}0.92 i \\
171.200 \\
16.000 \\
2.975 \\
5.410 \\
0.1344\end{array}$ & $\begin{array}{r}0.1121 \\
202.1500 \\
8.982 \\
3.1642 \\
6.1566 \\
0.188\end{array}$ & $\begin{array}{r}0.177 \\
212.400 \\
18.827 \\
3.490 \\
7.296 \\
0.923\end{array}$ & $\begin{array}{r}0.062 \\
202.600 \\
11.190 \\
3.468 \\
7.335 \\
0.296\end{array}$ \\
\hline
\end{tabular}

Lacation $=A$ AND; Formation $=$ DISMAL, GAP; Site $=12$

$\begin{array}{llrrrrrr}\text { Ss-137 } & \text { PCi } / \mathrm{cm}^{2} & 5.909 & 0.815 & 0.139 & 0.1092 & 0.159 & 0.200 \\ \mathrm{~K}-40 & \mathrm{P}-\mathrm{i} / \mathrm{cm}^{2} & 134.800 & 156.130 & 163.400 & 169.700 & 176.300 & 172.700 \\ \mathrm{U}-238 & \mathrm{PCi} / \mathrm{cm}^{2} & N & N & N & 25.1071 & 17.804 & 23.264 \\ \mathrm{Ra}-226 & \mathrm{PCi} / \mathrm{cm}^{2} & 3.058 & 3.829 & 3.739 & 3.725 & 3.744 & 3.479 \\ \text { Th-232 } & \mathrm{PCi} / \mathrm{cm}^{2} & 4.288 & 5.285 & 5.256 & 6.767 & 6.887 & 6.882 \\ U-235 & \mathrm{PCi} / \mathrm{cm}^{2} & 0.573 & N & 1.000 & 1.145 & 0.892 & 1.225\end{array}$

Location $=A N D ;$ Formation $=$ DISMAL GAP; Site $=19$

\begin{tabular}{|c|c|c|c|c|c|c|c|}
\hline $\begin{array}{l}C s-137 \\
K-40 \\
U-238 \\
R a-226 \\
T h-232 \\
U-235\end{array}$ & $\begin{array}{l}\mathrm{PCi} / \mathrm{cm}^{2} \\
\mathrm{PCi} / \mathrm{cm}^{2} \\
\mathrm{pCi} / \mathrm{cm}^{2} \\
\mathrm{PCi} / \mathrm{cm}^{2} \\
\mathrm{PCi} / \mathrm{cm}^{2} \\
\mathrm{pCi} / \mathrm{cm}^{2}\end{array}$ & $\begin{array}{r}11.82500 \\
55.4600 \\
5.19910 \\
2.19940 \\
4.1210 \\
0.5249\end{array}$ & $\begin{array}{r}2.4830 \\
86.8300 \\
5.6140 \\
4.4670 \\
6.3430 \\
C .9140\end{array}$ & $\begin{array}{r}0.4194 \\
97.7600 \\
15.8150 \\
4.4260 \\
7.1320 \\
0.9366\end{array}$ & $\begin{array}{r}0.198 \\
110.100 \\
16.143 \\
3.760 \\
7.275 \\
0.596\end{array}$ & $\begin{array}{r}0.024 \\
108.300 \\
12.070 \\
3.415 \\
6.713 \\
0.831\end{array}$ & $\begin{array}{r}0.050 \\
116.400 \\
7.134 \\
3.382 \\
6.938 \\
0.358\end{array}$ \\
\hline
\end{tabular}

Location = AND; Formation = DISMAL GAP; Site $=20$

$\begin{array}{llcccccc}\mathrm{Cs}-137 & \mathrm{PCi} / \mathrm{cm}^{2} & 6.284 & 0.356 & 0.196 & N & 0.197 & N \\ \mathrm{~K}-40 & \mathrm{PCi} / \mathrm{cm}^{2} & 97.750 & 108.500 & 101.100 & 104.600 & 101.200 & 90.5300 \\ \mathrm{U}-238 & \mathrm{PCi} / \mathrm{cm}^{2} & N & N & N & N & 19.881 & N \\ \mathrm{Ra}-226 & \mathrm{PCi} / \mathrm{cm}^{2} & 3.188 & 4.366 & 3.728 & 3.542 & 3.745 & 2.7410 \\ \text { Th-232 } & \mathrm{PCi} / \mathrm{cm}^{2} & 5.1358 & 6.268 & 6.481 & 6.851 & 6.898 & 6.0540 \\ \mathrm{U}-235 & \mathrm{PCi} / \mathrm{cm}^{2} & \text { N } & \text { N } & \text { N } & \text { N } & 1.102 & 0.4249\end{array}$

Location $=4 N D ;$ Formation $=$ DISMAL GAP; Site $=21$

$\begin{array}{llrrrrrr}\mathrm{Cs}-137 & \mathrm{PCi} / \mathrm{cm}^{2} & 3.965 & 1.209 & 0.471 & 0.1438 & 0.090 & 0.081 \\ \mathrm{~K}-40 & \mathrm{PCi} / \mathrm{cm}^{2} & 99.460 & 136.700 & 164.800 & 156.500 & 139.900 & 151.000 \\ \mathrm{U}-238 & \mathrm{PCi} / \mathrm{cm}^{2} & \mathrm{~N} & N & 31.690 & 20.1803 & 21.349 & 22.795 \\ \mathrm{Ra}-226 & \mathrm{PCi} / \mathrm{cm}^{2} & 2.167 & 3.439 & 3.191 & 2.751 & 2.978 & 2.741 \\ \mathrm{Th}-232 & \mathrm{PCi} / \mathrm{cm}^{2} & 4.082 & 5.474 & 6.3465 & 6.375 & 5.948 & 6.506 \\ \mathrm{U}-235 & \mathrm{PCi} / \mathrm{cm}^{2} & \mathrm{~N} & \mathrm{~N} & \mathrm{~N} & 1.1092 & 1.461 & 1.152\end{array}$

Location $=A N D ;$ Formation $=$ DISMAL GAP; Site $=22$

$\begin{array}{llrrrrrr}\text { Cs-137 } & \text { PCi } / \mathrm{cm}^{2} & 3.019 & 0.325 & 0.144 & 0.144 & 0.139 & 0.045 \\ \mathrm{~K} .-40 & \mathrm{PCi} / \mathrm{cm}^{2} & 167.000 & 162.400 & 157.800 & 136.300 & 158.100 & 176.400 \\ \mathrm{U}-238 & \mathrm{PCi} / \mathrm{cm}^{2} & 17.838 & 16.400 & 18.484 & 17.9110 & 16.82 . & 7.410 \\ \mathrm{Ra}-226 & \mathrm{PCi} / \mathrm{cm}^{2} & 3.532 & 3.781 & 3.603 & 3.243 & 3.484 & 3.378 \\ \mathrm{Th}-232 & \mathrm{PCi} / \mathrm{cm}^{2} & 5.984 & 6.503 & 6.857 & 6.800 & 6.734 & 6.729 \\ \mathrm{U}-235 & \mathrm{PCi} / \mathrm{cm}^{2} & 0.889 & 0.910 & 0.382 & 0.863 & 0.909 & 0.341\end{array}$


Table B.3 (continued)

\begin{tabular}{lllllll}
\hline & \multicolumn{7}{c}{ Depth (cm) } \\
\cline { 2 - 6 } Anatysis Units & $0 j-05$ & $05-10$ & $10-15$ & $15-20$ & $20-25$ & $25-30$ \\
\hline
\end{tabular}

Location $=$ ORR; Formation $=$ DISMAL GAP; Site $=2$

$\begin{array}{llrrrrrr}C s-137 & \text { PCi } / \mathrm{cm}^{2} & 6.188 & 1.2180 & 0.427 & 0.279 & 0.182 & 0.077 \\ K-40 & \text { PCi } / \mathrm{cm}^{2} & 54.710 & 94.3700 & 111.200 & 142.000 & 148.600 & 134.300 \\ U-238 & \text { PCi } / \mathrm{cm}^{2} & 11.796 & 13.7210 & 13.507 & 13.390 & 15.677 & 16.343 \\ R a-226 & \text { PCi } / \mathrm{cm}^{2} & 3.256 & 4.3240 & 3.792 & 4.020 & 4.970 & 3.457 \\ T h-232 & \mathrm{PCi} / \mathrm{cm}^{2} & 3.648 & 5.7230 & 6.086 & 7.001 & 7.637 & 8.072 \\ U-235 & \mathrm{PCi} / \mathrm{cm}^{2} & 0.348 & 0.8346 & 0.836 & 0.918 & 1.223 & 0.943\end{array}$

Location $=$ ORR; Formation $=$ DISMAL GAP; Site $=10$

$\begin{array}{llrrrrrr}\mathrm{Cs}-137 & \mathrm{PCi} / \mathrm{cm}^{2} & 4.7590 & 3.6820 & 1.4140 & 0.8727 & 0.174 & 0.0825 \\ \mathrm{~K}-40 & \mathrm{PCi} / \mathrm{cm}^{2} & 76.6800 & 99.6000 & 95.9900 & 97.5600 & 100.900 & 95.3200 \\ \mathrm{U}-238 & \mathrm{PCi} / \mathrm{cm}^{2} & 12.3210 & 15.5150 & 14.1910 & 14.2840 & 14.644 & 2.2610 \\ \mathrm{Ra}-226 & \mathrm{PCi} / \mathrm{cm}^{2} & 2.5000 & 2.7970 & 2.9260 & 2.9610 & 4.928 & 2.8170 \\ \mathrm{Th}-232 & \mathrm{PCi} / \mathrm{cm}^{2} & 4.0690 & 5.3060 & 5.5650 & 5.8010 & 6.721 & 6.1300 \\ \mathrm{U}-235 & \mathrm{PCi} / \mathrm{cm}^{2} & 0.5908 & 0.7902 & 0.3978 & 0.7672 & 0.872 & 0.1353\end{array}$

Location - ORR; Formation = DISMAL GAP; Site $=11$

$\begin{array}{llrrrrrr}\mathrm{Cs}-137 & \mathrm{PCi} / \mathrm{cm}^{2} & 4.3100 & 1.672 & 0.583 & 0.3982 & 0.200 & 0.086 \\ \mathrm{~K}-40 & \mathrm{PCi} / \mathrm{cm}^{2} & 87.0300 & 106.900 & 113.000 & 97.5500 & 112.200 & 120.800 \\ \mathrm{Ra}-226 & \mathrm{PCi} / \mathrm{cm}^{2} & 2.7680 & 2.947 & 2.979 & 2.9490 & 2.918 & 3.400 \\ \mathrm{Th}-232 & \mathrm{PCi} / \mathrm{cm}^{2} & 4.6310 & 5.391 & 6.231 & 6.0350 & 6.103 & 6.546 \\ \mathrm{U}-235 & \mathrm{PCi} / \mathrm{cm}^{2} & 0.4293 & 0.697 & 0.524 & 0.5058 & 0.476 & 0.530\end{array}$

Location $=$ ORR; Formation $=$ DISMAL GAP; Site $=19$

$\begin{array}{llrrrrrr}\mathrm{Cs}-137 & \mathrm{PCi} / \mathrm{cm}^{2} & 8.0650 & 0.6030 & 0.1264 & 0.0293 & 0.048 & 0.027 \\ \mathrm{~K}-40 & \mathrm{PCi} / \mathrm{cm}^{2} & 68.0500 & 85.7700 & 96.0700 & 93.0800 & 115.000 & 121.400 \\ \mathrm{U}-238 & \mathrm{PCi} / \mathrm{cm}^{2} & 16.3930 & 15.3550 & 4.7170 & 8.4400 & 3.522 & 10.930 \\ \mathrm{Ra}-226 & \mathrm{PCi} / \mathrm{cm}^{2} & 3.8820 & 4.6650 & 4.5660 & 4.2790 & 4.610 & 4.714 \\ \mathrm{Th}-232 & \mathrm{PCi} / \mathrm{cm}^{2} & 4.8300 & 6.4850 & 6.4570 & 6.4280 & 7.356 & 7.756 \\ \mathrm{U}-235 & \mathrm{PCi} / \mathrm{cm}^{2} & 0.9517 & 0.9563 & 0.5841 & 0.3240 & 0.270 & 0.632\end{array}$

Location $=$ ORR; Formation $=$ DISMAL GAP; Site $=22$

$\begin{array}{llrrrrrr}\mathrm{Cs}-137 & \mathrm{PCi} / \mathrm{cm}^{2} & 6.1200 & 2.3930 & 0.568 & 0.156 & 0.142 & 0.055 \\ \mathrm{~K}-40 & \mathrm{PCi} / \mathrm{cm}^{2} & 54.5300 & 99.4500 & 113.400 & 147.200 & 154.600 & 152.500 \\ \mathrm{U}-238 & \mathrm{PCi} / \mathrm{cm}^{2} & 13.0060 & 14.7380 & 15.277 & 15.990 & 15.394 & 7.227 \\ \mathrm{Ra}-226 & \mathrm{PCi} / \mathrm{cm}^{2} & 2.6790 & 4.3340 & 4.723 & 4.330 & 3.743 & 3.081 \\ \mathrm{Th}-232 & \mathrm{PCi} / \mathrm{cm}^{2} & 3.9020 & 6.4800 & 7.290 & 7.805 & 7.806 & 7.730 \\ \mathrm{U}-235 & \mathrm{PCi} / \mathrm{cm}^{2} & 0.7199 & 0.6982 & \mathrm{~N} & 0.926 & \mathrm{~N} & 0.289\end{array}$

Location $=$ ORR; Formation $=$ DISMAL GAP; Site $=26$

$\begin{array}{llrrrrrr}\mathrm{Cs}-137 & \mathrm{PCi} / \mathrm{cm}^{2} & 6.3840 & 1.5310 & 0.519 & 0.136 & 0.056 & 0.063 \\ \mathrm{~K}-40 & \mathrm{PCi} / \mathrm{cm}^{2} & 85.3400 & 93.3400 & 108.600 & 113.700 & 103.800 & 127.600 \\ \mathrm{U}-238 & \mathrm{PCi} / \mathrm{cm}^{2} & 15.5680 & 14.7410 & 15.094 & 16.066 & 12.170 & 7.890 \\ \mathrm{Ra}-226 & \mathrm{PCi} / \mathrm{cm}^{2} & 3.9090 & 2.8950 & 3.338 & 3.495 & 3.129 & 3.269 \\ \mathrm{Th}-232 & \mathrm{PCi} / \mathrm{cm}^{2} & 4.9630 & 5.1140 & 5.928 & 6.511 & 6.291 & 6.814 \\ U-235 & \mathrm{PCi} / \mathrm{cm}^{2} & 0.8203 & 0.5202 & 0.856 & 0.799 & 0.826 & 0.240\end{array}$


Table B.3 (continued)

\begin{tabular}{|c|c|c|c|c|c|c|c|}
\hline \multirow[b]{2}{*}{ Analysis } & \multirow[b]{2}{*}{ Units } & \multicolumn{6}{|c|}{ Depth (cnn) } \\
\hline & & $00-05$ & $05-10$ & $10-15$ & $15-20$ & $20-25$ & $25-30$ \\
\hline
\end{tabular}

Location $=$ ORR; Formation $=$ DISMAL GAP; Site $=27$

$\begin{array}{llrrrrrr}\mathrm{Cs}-137 & \mathrm{PCi} / \mathrm{cm}^{2} & 4.1350 & 1.3330 & 0.8371 & 0.0548 & 0.0427 & 0.0074 \\ \mathrm{X}-40 & \mathrm{PCi} / \mathrm{cm}^{2} & 41.6900 & 59.5400 & 67.1200 & 76.5600 & 86.6300 & 99.0100 \\ \mathrm{Ra-226} & \text { PCi } / \mathrm{cm}^{2} & 2.4840 & 3.4720 & 3.1560 & 3.7550 & 3.9040 & 3.8750 \\ \text { Th-232 } & \text { PCi } / \mathrm{cm}^{2} & 3.2540 & 4.4020 & 4.6760 & 5.1550 & 5.3280 & 5.7990 \\ U-235 & \text { PCi } / \mathrm{cm}^{2} & 0.5355 & 0.4647 & 0.6526 & 0.5576 & 0.5876 & 0.5540\end{array}$

Location $=$ ORR; Formation $=$ DISMAL GAP; Sise $=32$

$\begin{array}{llrrrrrr}\mathrm{Cs}-137 & \mathrm{PCi} / \mathrm{cm}^{2} & 5.2290 & 1.642 & 0.908 & 0.103 & 0.001 & 0.003 \\ \mathrm{~K}-40 & \mathrm{PCi} / \mathrm{cm}^{2} & 76.9900 & 105.300 & 112.200 & 116.100 & 130.400 & 148.800 \\ \mathrm{U}-238 & \mathrm{PCi} / \mathrm{cm}^{2} & 5.1610 & 8.219 & 14.870 & 8.259 & 5.169 & 5.215 \\ \mathrm{Ra}-226 & \mathrm{PCi} / \mathrm{cm}^{2} & 3.2740 & 3.960 & 4.944 & 4.759 & 3.796 & 3.753 \\ \mathrm{Th}-232 & \mathrm{PCi} / \mathrm{cm}^{2} & 5.1200 & 6.369 & 7.043 & 7.172 & 7.508 & 7.493 \\ \mathrm{U}-235 & \mathrm{PCi} / \mathrm{cm}^{2} & 0.3801 & 0.345 & 0.841 & 0.392 & 0.389 & 0.321\end{array}$

Location $=$ ORR; Formation $=$ DISMAL GAP; Site $=33$

$\begin{array}{llrrrrrr}\mathrm{Cs}-137 & \mathrm{PCi} / \mathrm{cm}^{2} & 6.2440 & 2.304 & 0.322 & 0.194 & 0.202 & 0.128 \\ \mathrm{~K}-40 & \mathrm{PCi} / \mathrm{cm}^{2} & 44.9200 & 108.000 & 131.000 & 114.500 & 118.700 & 125.600 \\ \mathrm{U}-238 & \mathrm{PCi} / \mathrm{cm}^{2} & 18.4090 & 21.350 & 24.996 & 19.684 & 15.260 & 15.554 \\ \mathrm{Ra}-22.6 & \mathrm{PCi} / \mathrm{cm}^{2} & 2.8590 & 4.659 & 4.027 & 3.814 & 4.718 & 4.797 \\ \mathrm{Th}-232 & \mathrm{PCi} / \mathrm{cm}^{2} & 3.2060 & 6.592 & 7.584 & 7.203 & 8.378 & 7.648 \\ U-235 & \mathrm{PCi} / \mathrm{cm}^{2} & 0.3353 & 1.100 & 0.827 & 1.053 & N & 0.855\end{array}$

Location $=$ ORR; Formation $=$ DISMAL GAP; Site $=35$

$\begin{array}{llrrrrrr}\mathrm{Cs}-137 & \mathrm{PCi} / \mathrm{cm}^{2} & 4.7840 & 0.6679 & 0.1397 & 0.1281 & 0.0224 & 0.1295 \\ \mathrm{~K}-40 & \mathrm{PCi} / \mathrm{cm}^{2} & 33.8500 & \mathbf{3 8 . 0 7 0 0} & 39.7800 & 46.9500 & 57.5700 & 72.4300 \\ \mathrm{U}-238 & \mathrm{PCi} / \mathrm{cm}^{2} & 16.8100 & 13.7950 & 5.1520 & 27.2000 & 9.4610 & 16.5680 \\ \mathrm{Ra}-226 & \mathrm{PCi} / \mathrm{cm}^{2} & 3.9430 & 5.4660 & 6.0910 & 5.3330 & 5.3370 & 5.5970 \\ T \mathrm{~h}-232 & \mathrm{PCi} / \mathrm{cm}^{2} & 4.3940 & 6.3030 & 6.2200 & 6.3540 & 6.6570 & 7.1480 \\ U-235 & \mathrm{PCi} / \mathrm{cm}^{2} & 0.6461 & 0.7358 & 0.9073 & 0.8943 & 0.1649 & 0.7216\end{array}$

Location $=O R R ;$ Formation $=$ DISMAL GAP; Site $=41$

$\begin{array}{llrrrrrr}\text { Cs }-137 & \mathrm{PCi} / \mathrm{cm}^{2} & 5.8770 & 2.9120 & 1.6000 & 0.3356 & 0.0821 & 0.0866 \\ \mathrm{~K}-40 & \mathrm{PCi} / \mathrm{cm}^{2} & 30.5200 & 48.0500 & 54.2500 & 79.9700 & 73.8200 & 80.4500 \\ \mathrm{Ra}-226 & \mathrm{PCi} / \mathrm{cm}^{2} & 2.1480 & 3.4980 & 3.3720 & 2.6330 & 2.3420 & 3.0050 \\ \mathrm{Th}-232 & \mathrm{PCi} / \mathrm{cm}^{2} & 3.0210 & 4.8850 & 5.1640 & 6.0880 & 6.1560 & 6.4170 \\ \mathrm{U}-235 & \mathrm{PCi} / \mathrm{cm}^{2} & 0.5387 & 0.6378 & 0.5568 & 0.6040 & 0.4883 & 0.5270\end{array}$

Location $=O R R ;$ Formation $=$ DISMAL GAP; Site $=43$

$\begin{array}{llrrrrrr}\mathrm{C}-137 & \mathrm{PCi} / \mathrm{cm}^{2} & 5.5710 & 1.920 & 0.434 & 0.195 & 0.172 & 0.199 \\ \mathrm{~K}-40 & \mathrm{PCi} / \mathrm{cm}^{2} & 72.9500 & 93.950 & 99.590 & 107.500 & 104.200 & 115.300 \\ \mathrm{U}-238 & \mathrm{PCi} / \mathrm{cm}^{2} & 21.2300 & 15.220 & 10.830 & 16.600 & 32.340 & 21.395 \\ \mathrm{Ra}-226 & \mathrm{PCi} / \mathrm{cm}^{2} & 3.7110 & 5.460 & 5.157 & 4.374 & 3.890 & 3.719 \\ \mathrm{Th}-232 & \mathrm{PCi} / \mathrm{cm}^{2} & 5.4170 & 6.589 & 7.005 & 7.418 & 6.849 & 6.566 \\ \mathrm{U}-235 & \mathrm{PCi} / \mathrm{cm}^{2} & 1.1043 & 1.170 & \mathrm{~N} & 1.022 & 1.016 & 0.957\end{array}$


Table B.3 (continued)

\begin{tabular}{|c|c|c|c|c|c|c|c|}
\hline \multirow[b]{2}{*}{ Analysis } & \multirow[b]{2}{*}{ Units } & \multicolumn{6}{|c|}{ Depth (cm) } \\
\hline & & $\overline{00-05}$ & $05-10$ & $10-15$ & $15-20$ & $20-25$ & $25-30$ \\
\hline
\end{tabular}

Location $=$ ORR; Formation $=$ NOLICHUCKY; Site $=3$

$\begin{array}{llrrrrrr}\mathrm{Cs}-137 & \mathrm{PCi} / \mathrm{cm}^{2} & 6.0110 & 2.0850 & 0.3760 & 0.1497 & 0.1431 & 0.0093 \\ \mathrm{~K}-40 & \mathrm{PCi} / \mathrm{cm}^{2} & 50.0500 & 70.7600 & 79.9500 & 74.7600 & 85.7500 & 84.4400 \\ \mathrm{U}-238 & \mathrm{PCi} / \mathrm{cm}^{2} & 12.7780 & 12.4960 & 14.2100 & 14.1380 & 13.3440 & 4.5590 \\ \mathrm{Ra}-226 & \mathrm{PCi} / \mathrm{cm}^{2} & 3.8930 & 4.4940 & 4.7260 & 4.6670 & 3.9190 & 3.9680 \\ \mathrm{Th}-232 & \mathrm{PCi} / \mathrm{cm}^{2} & 5.1280 & 6.3200 & 7.1170 & 7.1920 & 7.0710 & 6.8910 \\ \mathrm{U}-235 & \mathrm{PCi} / \mathrm{cm}^{2} & 0.7988 & 0.8698 & 0.4059 & 0.8622 & 0.6399 & 0.1617\end{array}$

Location $=$ ORR; Formation $=$ NOLICHUCKY; Site $=5$

$\begin{array}{llrrrrrr}\mathrm{Cs}-137 & \mathrm{PCi} / \mathrm{cm}^{2} & 6.0400 & 2.0630 & 0.6278 & 0.2066 & 0.0691 & 0.025 \\ \mathrm{~K}-40 & \mathrm{PCi} / \mathrm{cm}^{2} & 44.8600 & 47.8500 & 69.0000 & 85.7000 & 96.6500 & 103.100 \\ \mathrm{U}-238 & \mathrm{PCi} / \mathrm{cm}^{2} & 4.5870 & 10.9300 & 13.1900 & 13.9370 & 17.2600 & 6.269 \\ \mathrm{Ra}-226 & \mathrm{PCi} / \mathrm{cm}^{2} & 4.4940 & 5.0290 & 5.2810 & 5.4850 & 5.6480 & 5.009 \\ \mathrm{Th}-232 & \mathrm{PCi} / \mathrm{cm}^{2} & 5.8060 & 6.1920 & 7.7610 & 8.4600 & 9.0260 & 9.015 \\ \mathrm{U}-235 & \mathrm{PCi} / \mathrm{cm}^{2} & 0.5503 & 0.8226 & 0.4130 & 0.9602 & 0.9951 & 0.636\end{array}$

Location $=$ ORR; Formation $=$ NOLICHUCKY; Site $=13$

$\begin{array}{llrrrrrr}\mathrm{Cs}-137 & \mathrm{pCi} / \mathrm{cm}^{2} & 5.2460 & 2.8940 & 0.6197 & 0.1523 & 0.028 & 0.045 \\ \mathrm{~K}-40 & \mathrm{PCi} / \mathrm{cm}^{2} & 64.0800 & 81.3200 & 93.6000 & 94.8700 & 101.300 & 127.600 \\ \mathrm{U}-238 & \mathrm{PCi} / \mathrm{cm}^{2} & 11.4820 & 13.1500 & 14.7130 & 14.5900 & 5.978 & 8.344 \\ \mathrm{Ra}-226 & \mathrm{PCi} / \mathrm{cm}^{2} & 3.4760 & 4.3430 & 4.5280 & 4.7190 & 4.498 & 4.509 \\ \mathrm{Th}-232 & \mathrm{PCi} / \mathrm{cm}^{2} & 5.0830 & 7.0230 & 7.6650 & 7.5350 & 7.651 & 8.480 \\ \mathrm{U}-235 & \mathrm{pCi} / \mathrm{cm}^{2} & 0.5462 & 0.9227 & 0.9523 & 0.9934 & 0.981 & 0.530\end{array}$

Location $=$ ORR; Formation $=$ NOLICHUCKY; Site $=15$

$\begin{array}{llrrrrrr}\mathrm{Cs}-137 & \mathrm{PCi} / \mathrm{cm}^{2} & 5.2010 & 2.0500 & 0.3867 & 0.1945 & 0.2486 & 0.2083 \\ \mathrm{~K}-40 & \mathrm{PCi} / \mathrm{cm}^{2} & 29.8900 & 40.9700 & 47.5100 & 58.7400 & 72.3300 & 84.2800 \\ \mathrm{U}-238 & \mathrm{PCi} / \mathrm{cm}^{2} & 15.4680 & 19.6090 & 17.1500 & 5.0920 & 18.2000 & 20.8800 \\ \mathrm{Ra}-226 & \mathrm{PCi} / \mathrm{cm}^{2} & 2.9780 & 4.0960 & 4.6140 & 4.0470 & 4.1810 & 4.4490 \\ \mathrm{Th}-232 & \mathrm{PCi} / \mathrm{cm}^{2} & 4.3570 & 6.4810 & 6.9070 & 7.8490 & 8.7490 & 9.2600 \\ \mathrm{U}-235 & \mathrm{PCi} / \mathrm{cm}^{2} & 1.0077 & 1.1006 & 1.1340 & 1.5430 & 1.2650 & 1.2804\end{array}$

Location $=$ ORR; Formation $=$ NOLICHUCKY; Site $=16$

\begin{tabular}{|c|c|c|c|c|c|c|c|}
\hline $\begin{array}{l}\text { Cs- } 137 \\
K-40 \\
U-238 \\
R a-226 \\
\text { Th-232 } \\
U-235\end{array}$ & $\begin{array}{l}\mathrm{pCi} / \mathrm{cm}^{2} \\
\mathrm{pCi} / \mathrm{cm}^{2} \\
\mathrm{pCi} / \mathrm{cm}^{2} \\
\mathrm{pCi} / \mathrm{cm}^{2} \\
\mathrm{pCi} / \mathrm{cm}^{2} \\
\mathrm{pCi} / \mathrm{cm}^{2}\end{array}$ & $\begin{array}{c}6.754 \\
89.360 \\
N \\
3.987 \\
6.797 \\
N\end{array}$ & $\begin{array}{r}2.168 \\
101.300 \\
4.548 \\
4.735 \\
8.466 \\
0.637\end{array}$ & $\begin{array}{r}0.482 \\
116.000 \\
22.990 \\
5.308 \\
9.270\end{array}$ & $\begin{array}{c}0.158 \\
114.100 \\
N \\
5.095 \\
9.033\end{array}$ & $\begin{array}{c}7^{N} .210 \\
N \\
4.962 \\
8.650\end{array}$ & $\begin{array}{r}0.1715 \\
85.8200 \\
7.1540\end{array}$ \\
\hline
\end{tabular}

Location $=$ ORR; Formation $=$ NOLICHUCKY; Site $=21$

$\begin{array}{llrrrrrr}\mathrm{Cs}-137 & \mathrm{pCi} / \mathrm{cm}^{2} & 10.650 & 0.724 & 0.165 & 0.156 & 0.092 & 0.055 \\ \mathrm{~K}-40 & \mathrm{pCi} / \mathrm{cm}^{2} & 120.400 & 187.900 & 135.600 & 125.100 & 164.600 & 243.300 \\ \mathrm{U}-238 & \mathrm{pCi} / \mathrm{cm}^{2} & 15.931 & 18.495 & 16.164 & 17.040 & 15.696 & 8.715 \\ \mathrm{Ra}-226 & \mathrm{pCi} / \mathrm{cm}^{2} & 2.470 & 3.830 & 4.301 & 2.916 & 3.226 & 3.424 \\ \mathrm{Th}-232 & \mathrm{pCi} / \mathrm{cm}^{2} & 6.099 & 10.100 & 8.872 & 9.165 & 9.904 & 10.100 \\ \mathrm{U}-235 & \mathrm{pCi} / \mathrm{cm}^{2} & 0.885 & 0.388 & 1.027 & 0.570 & 1.440 & 0.200\end{array}$


Table B.3 (continued)

\begin{tabular}{|c|c|c|c|c|c|c|c|}
\hline \multirow[b]{2}{*}{ Analysis } & \multirow[b]{2}{*}{ Units } & \multicolumn{6}{|c|}{ Depth (cm) } \\
\hline & & $00-05$ & $05-10$ & $10-15$ & $15-20$ & $20-25$ & $25-30$ \\
\hline
\end{tabular}

Location $=$ ORR; Formation $=$ NOLICHUCKY $;$ Site $=23$

$\begin{array}{llrrrrrr}\mathrm{Cs}-137 & \mathrm{PCi} / \mathrm{cm}^{2} & 5.4950 & 2.9620 & 0.352 & 0.201 & 0.156 & 0.195 \\ \mathrm{~K}-40 & \mathrm{PCi} / \mathrm{cm}^{2} & 52.6600 & 63.4600 & 113.200 & 136.100 & 155.100 & 150.300 \\ \mathrm{U}-238 & \mathrm{PCi} / \mathrm{cm}^{2} & 13.1910 & 14.0800 & 20.266 & 21.804 & 26.601 & 13.340 \\ \mathrm{Ra}-226 & \mathrm{PCi} / \mathrm{cm}^{2} & 3.7980 & 4.0330 & 3.469 & 2.755 & 3.297 & 2.868 \\ \mathrm{Th}-232 & \mathrm{PCi} / \mathrm{cm}^{2} & 5.9000 & 6.5490 & 8.027 & 8.120 & 9.116 & 8.672 \\ \mathrm{U}-235 & \mathrm{PCi} / \mathrm{cm}^{2} & 1.1156 & 1.1205 & 1.209 & 1.134 & 1.278 & 1.186\end{array}$

Location $=$ ORR $;$ Fomation $=$ NOLICHUCKY $;$ Site $=24$

$\begin{array}{llrrrrrr}\mathrm{Cs}-137 & \mathrm{pCi} / \mathrm{cm}^{2} & 6.922 & 2.744 & 0.586 & 0.251 & 0.166 & 0.009 \\ \mathrm{~K}-40 & \mathrm{pCi} / \mathrm{cm}^{2} & 111.200 & 160.500 & 156.700 & 180.400 & 188.500 & 183.800 \\ \mathrm{U}-238 & \mathrm{pCi} / \mathrm{cm}^{2} & 15.657 & 28.660 & 6.363 & 16.118 & 17.571 & 5.318 \\ \mathrm{Ra}-226 & \mathrm{pCi} / \mathrm{cm}^{2} & 3.299 & 4.234 & 3.918 & 4.073 & 4.095 & 3.638 \\ \text { Th-232 } & \mathrm{pCi} / \mathrm{cm}^{2} & 5.847 & 8.358 & 8.674 & 9.820 & 10.210 & 9.445 \\ \mathrm{U}-235 & \mathrm{pCi} / \mathrm{cm}^{2} & 0.876 & 0.993 & 0.951 & 1.044 & 1.015 & 0.290\end{array}$

Location $=$ ORR; Formation $=$ NOLICHUCKY; Site $=25$

\begin{tabular}{|c|c|c|c|c|c|c|c|}
\hline $\begin{array}{l}C s-137 \\
K-40 \\
U-238 \\
R a-226 \\
T h-232 \\
U-235\end{array}$ & $\begin{array}{l}\mathrm{pCi} / \mathrm{cm}^{2} \\
\mathrm{pCi} / \mathrm{cm}^{2} \\
\mathrm{pCi} / \mathrm{cm}^{2} \\
\mathrm{pCi} / \mathrm{cm}^{2} \\
\mathrm{pCi} / \mathrm{cm}^{2} \\
\mathrm{pCi} / \mathrm{cm}^{2}\end{array}$ & $\begin{array}{c}6.829 \\
124.400 \\
N \\
3.761 \\
7.051 \\
1.223\end{array}$ & $\begin{array}{c}0.357 \\
151.300 \\
\text { N } \\
4.388 \\
9.536 \\
\text { N }\end{array}$ & $\begin{array}{r}0.152 \\
152.500 \\
9.958 \\
4.583 \\
9.616 \\
\text { N }\end{array}$ & $\begin{array}{r}0.265 \\
164.200 \\
N \\
3.857 \\
9.597 \\
1.477\end{array}$ & $\begin{array}{c}0.042 \\
186.600 \\
\text { N } \\
3.579 \\
9.744 \\
\text { N }\end{array}$ & $\begin{array}{c}\text { N } \\
202.000 \\
N \\
3.449 \\
10.050 \\
\text { N }\end{array}$ \\
\hline
\end{tabular}

Location $=$ ORR; Formation $=$ NOLICHUCKY $;$ Site $=28$

$\begin{array}{llrrrrrr}\text { Cs-137 } & \text { pCi } / \mathrm{cm}^{2} & 5.6950 & 2.859 & 0.9707 & 0.179 & 0.156 & 0.053 \\ \mathrm{~K}-40 & \mathrm{PCi} / \mathrm{cm}^{2} & 42.7900 & 79.640 & 79.6500 & 178.900 & 233.000 & 237.500 \\ \mathrm{U}-238 & \mathrm{pCi} / \mathrm{cm}^{2} & 10.3400 & 13.990 & 15.6700 & 9.715 & 20.217 & 6.069 \\ \mathrm{Ra}-226 & \mathrm{pCi} / \mathrm{cm}^{2} & 3.3830 & 6.105 & 5.7170 & 4.630 & 3.541 & 4.066 \\ \mathrm{Th}-232 & \mathrm{pCi} / \mathrm{cm}^{2} & 4.1240 & 7.475 & 8.0010 & 8.802 & 9.680 & 9.555 \\ \mathrm{U}-235 & \mathrm{pCi} / \mathrm{cm}^{2} & 0.7398 & 1.118 & 0.9716 & 2.204 & 1.061 & 0.053\end{array}$

Location $=$ ORR; Fomation $=$ NOLICHUCKY; Site $=31$

\begin{tabular}{|c|c|c|c|c|c|c|c|}
\hline $\begin{array}{l}\text { Cs }-137 \\
K-40 \\
U-238 \\
R a-226 \\
T h-232 \\
U-235\end{array}$ & $\begin{array}{l}\mathrm{pCi} / \mathrm{cm}^{2} \\
\mathrm{pCi} / \mathrm{cm}^{2} \\
\mathrm{pCi} / \mathrm{cm}^{2} \\
\mathrm{pCi} / \mathrm{cm}^{2} \\
\mathrm{pCi} / \mathrm{cm}^{2} \\
\mathrm{pCi} / \mathrm{cm}^{2}\end{array}$ & $\begin{array}{r}8.0260 \\
33.6200 \\
14.2360 \\
3.6080 \\
4.9160 \\
0.8068\end{array}$ & $\begin{array}{r}2.515 \\
48.780 \\
6.761 \\
5.457 \\
6.718 \\
1.732\end{array}$ & $\begin{array}{r}0.4244 \\
49.6800 \\
14.4350 \\
5.2910 \\
6.9740 \\
0.9209\end{array}$ & $\begin{array}{r}0.1162 \\
55.6200 \\
8.8320 \\
5.7120 \\
7.2200 \\
0.3996\end{array}$ & $\begin{array}{r}0.0571 \\
74.2500 \\
6.0490 \\
5.9270 \\
8.0740 \\
0.3406\end{array}$ & $\begin{array}{r}0.0692 \\
97.4000 \\
15.1500 \\
5.6230 \\
8.9380 \\
0.6039\end{array}$ \\
\hline
\end{tabular}

Location $=O R R ;$ Formation $=$ NOLICHUCKY; Site $=42$

$\begin{array}{llrrrrrr}\text { Cs }-137 & \text { PCi } / \mathrm{cm}^{2} & 5.1130 & 1.114 & 0.269 & 0.172 & 0.028 & 0.064 \\ \mathrm{~K}-40 & \mathrm{PCi} / \mathrm{cm}^{2} & 57.7300 & 82.200 & 135.500 & 163.400 & 141.600 & 135.000 \\ \mathrm{U}-238 & \mathrm{PCi} / \mathrm{cm}^{2} & 14.9700 & 13.150 & 7.215 & 17.255 & 8.356 & 11.100 \\ \mathrm{Ra}-226 & \mathrm{PCi} / \mathrm{cm}^{2} & 3.5200 & 3.975 & 4.821 & 4.077 & 4.529 & 4.532 \\ \mathrm{Th}-232 & \mathrm{PCi} / \mathrm{cm}^{2} & 5.2870 & 6.845 & 8.233 & 9.360 & 9.260 & 8.786 \\ \mathrm{U}-235 & \mathrm{PCi} / \mathrm{cm}^{2} & 0.8116 & 1.219 & 0.579 & 0.306 & 0.294 & 0.163\end{array}$


Table B.3 (continued)

\begin{tabular}{|c|c|c|c|c|c|c|c|}
\hline \multirow[b]{2}{*}{ Analysis } & \multirow[b]{2}{*}{ Units } & \multicolumn{6}{|c|}{ Depth (cm) } \\
\hline & & $00-05$ & $05-10$ & $10-15$ & $15-20$ & $20-25$ & $25-30$ \\
\hline
\end{tabular}

$$
\text { Location }=\text { ROA; Formation }=\text { DISMAL GAP; Site }=3
$$

$\begin{array}{llrrrrrr}\mathrm{Cs}-137 & \mathrm{PCi} / \mathrm{cm}^{2} & 3.8940 & 1.0290 & 0.3963 & 0.1884 & 0.0366 & 0.0939 \\ \mathrm{~K}-40 & \mathrm{PCi} / \mathrm{cm}^{2} & 52.5600 & 56.2800 & 55.3800 & 60.1100 & 65.8000 & 64.0200 \\ \mathrm{U}-238 & \mathrm{PCi} / \mathrm{cm}^{2} & 19.5100 & 8.9870 & 14.6920 & 14.2660 & 15.2520 & 14.0860 \\ \mathrm{RB}-226 & \mathrm{PCi} / \mathrm{cm}^{2} & 3.3920 & 4.7060 & 4.3300 & 4.2040 & 4.1290 & 3.7830 \\ \mathrm{Th}-232 & \mathrm{PCi} / \mathrm{cm}^{2} & 5.0740 & 6.1120 & 5.9280 & 6.5860 & 7.0300 & 6.8070 \\ \mathrm{U}-235 & \mathrm{PCi} / \mathrm{cm}^{2} & 0.2494 & 0.8053 & 0.7068 & 0.2551 & 0.8463 & 0.8357\end{array}$

Location $=$ ROA; Formation $=$ DISMAL GAP; Site $=7$

$\begin{array}{llrrrrrr}\mathrm{Cs}-137 & \mathrm{PCi} / \mathrm{cm}^{2} & 5.5770 & 0.4298 & 0.3674 & 0.2311 & 0.0190 & 0.012 \\ \mathrm{~K}-40 & \mathrm{PCi} / \mathrm{cm}^{2} & 43.8200 & 57.6900 & 57.9200 & 68.9700 & 87.9600 & 101.600 \\ \mathrm{U}-238 & \mathrm{PCi} / \mathrm{cm}^{2} & 12.5560 & 6.9640 & 10.5600 & 10.8500 & 7.5670 & 6.318 \\ \mathrm{Ra}-226 & \mathrm{PCi} / \mathrm{cm}^{2} & 4.3930 & 4.9530 & 5.2480 & 5.0010 & 4.4900 & 3.863 \\ \mathrm{Th}-232 & \mathrm{PCi} / \mathrm{cm}^{2} & 5.5730 & 7.3240 & 7.1860 & 7.7060 & 7.7100 & 7.955 \\ \mathrm{U}-235 & \mathrm{PCi} / \mathrm{cm}^{2} & 0.5256 & 1.1160 & 2.1030 & 0.9617 & 0.3932 & 0.140\end{array}$

Location $=$ ROA; Formation $=$ DISMAL GAP; Site $=8$

$\begin{array}{llrrrrrr}\mathrm{Cs}-137 & \mathrm{PCi} / \mathrm{cm}^{2} & 8.0060 & 3.085 & 0.5213 & 0.1544 & 0.1284 & 0.0417 \\ \mathrm{~K}-40 & \mathrm{PCi} / \mathrm{cm}^{2} & 30.3800 & 40.710 & 43.3800 & 45.4500 & 48.8500 & 58.7500 \\ \mathrm{U}-238 & \mathrm{PCi} / \mathrm{cm}^{2} & 9.6371 & 12.698 & 14.0410 & 8.3710 & 7.0140 & 6.8000 \\ \mathrm{Ra}-226 & \mathrm{PCi} / \mathrm{cm}^{2} & 3.2580 & 4.775 & 4.9400 & 4.9840 & 4.4760 & 3.9750 \\ \mathrm{Th}-232 & \mathrm{PCi} / \mathrm{cm}^{2} & 4.8980 & 6.945 & 7.2950 & 7.3080 & 7.7100 & 6.9280 \\ \mathrm{U}-235 & \mathrm{PCi} / \mathrm{cm}^{2} & 0.7326 & \mathrm{~N} & 0.7517 & 0.8677 & 0.8688 & 0.5573\end{array}$

Location $=$ ROA; Formation $=$ DISMAL GAP; Site $=9$

$\begin{array}{llrrrrrr}\mathrm{Cs}-137 & \mathrm{PCi} / \mathrm{cm}^{2} & 8.2530 & 1.5750 & 0.2062 & 0.0478 & 0.0259 & 0.0594 \\ \mathrm{~K}-40 & \mathrm{PCi} / \mathrm{cm}^{2} & 27.7300 & 35.7000 & 40.4800 & 37.5500 & 45.3800 & 53.8200 \\ \mathrm{U}-238 & \mathrm{PCi} / \mathrm{cm}^{2} & 5.8530 & 6.4140 & 3.8480 & 8.0910 & 4.8300 & 8.5320 \\ \mathrm{Ra}-226 & \mathrm{PCi} / \mathrm{cm}^{2} & 2.6690 & 3.7070 & 4.1980 & 4.2140 & 4.7590 & 4.9220 \\ \mathrm{Th}-232 & \mathrm{PCi} / \mathrm{cm}^{2} & 3.7550 & 5.3670 & 5.6780 & 5.5620 & 6.1360 & 6.6410 \\ U-235 & \mathrm{pCi} / \mathrm{cm}^{2} & 0.7126 & 0.7568 & 0.2291 & 0.4652 & 0.5087 & 0.3991\end{array}$

Location $=$ ROA $;$ Formation $=$ DISMAL GAP; Site $=10$

$\begin{array}{llrrrrrr}\mathrm{Cs}-137 & \mathrm{pCi} / \mathrm{cm}^{2} & 3.7990 & 0.3148 & 0.2066 & 0.116 & 0.160 & 0.145 \\ \mathrm{~K}-40 & \mathrm{PCi} / \mathrm{cm}^{2} & 65.6100 & 83.7200 & 93.9500 & 111.700 & 120.300 & 122.600 \\ \mathrm{U}-238 & \mathrm{PCi} / \mathrm{cm}^{2} & 12.9510 & 3.2920 & 16.1040 & 7.391 & 12.100 & 15.322 \\ \mathrm{Ra}-226 & \mathrm{PCi} / \mathrm{cm}^{2} & 4.0980 & 5.0670 & 5.1200 & 4.514 & 4.343 & 4.125 \\ \mathrm{Th}-232 & \mathrm{PCi} / \mathrm{cm}^{2} & 5.7210 & 7.8770 & 8.4830 & 9.301 & 9.504 & 9.817 \\ \mathrm{U}-235 & \mathrm{PCi} / \mathrm{cm}^{2} & 0.8828 & 0.6247 & 0.1569 & 0.223 & 0.977 & 0.936\end{array}$

Location $=$ ROA; Formation $=$ DISMAL GAP; Site $=13$

$\begin{array}{llrrrrrr}\mathrm{Cs}-137 & \mathrm{PCi} / \mathrm{cm}^{2} & 1.2490 & 0.2110 & 0.0775 & 0.0417 & 0.2744 & 0.1310 \\ \mathrm{~K}-40 & \mathrm{PCi} / \mathrm{cm}^{2} & 38.1400 & 45.4400 & 56.2800 & 76.1000 & 91.8300 & 99.6100 \\ \mathrm{U}-238 & \mathrm{PCi} / \mathrm{cm}^{2} & 14.0530 & 11.4200 & 14.9960 & 9.0120 & 6.0810 & 12.8930 \\ \mathrm{Ra}-226 & \mathrm{PCi} / \mathrm{cm}^{2} & 4.3410 & 4.8690 & 5.0100 & 4.5180 & 3.5660 & 3.4730 \\ \mathrm{Th}-232 & \mathrm{PCi} / \mathrm{cm}^{2} & 6.2590 & 6.6990 & 7.0880 & 7.5120 & 7.1600 & 7.4010 \\ \mathrm{U}-235 & \mathrm{pCi} / \mathrm{cm}^{2} & 0.6536 & 0.5026 & 0.8816 & 0.9091 & 0.8434 & 0.8043\end{array}$


Table B.3 (continued)

\begin{tabular}{|c|c|c|c|c|c|c|c|}
\hline \multirow[b]{2}{*}{ Analysis } & \multirow[b]{2}{*}{ Units } & \multicolumn{6}{|c|}{ Depth (cm) } \\
\hline & & $\overline{00-05}$ & $05-10$ & $10-15$ & $15-20$ & $20-25$ & $25-30$ \\
\hline
\end{tabular}

Location $=$ ROA; Formation $=$ DISMAL GAP; Site $=14$

$\begin{array}{llrrrrrr}\mathrm{Cs}-137 & \mathrm{PCi} / \mathrm{cm}^{2} & 6.6610 & 1.273 & 0.240 & 0.0282 & 0.1766 & 0.1812 \\ \mathrm{~K}-40 & \mathrm{PCi} / \mathrm{cm}^{2} & 68.8600 & 114.300 & 101.100 & 91.8900 & 84.2400 & 91.9700 \\ \mathrm{U}-238 & \mathrm{PCi} / \mathrm{cm}^{2} & 17.8100 & 19.296 & 12.060 & 20.8260 & 20.6700 & 18.5830 \\ \mathrm{Ra}-226 & \mathrm{PCi} / \mathrm{cm}^{2} & 3.0060 & 3.381 & 3.319 & 3.3600 & 3.1890 & 2.8820 \\ T h-232 & \mathrm{PCi} / \mathrm{cm}^{2} & 5.4600 & 7.294 & 7.327 & 7.6550 & 7.3630 & 7.4610 \\ U-235 & \mathrm{PCi} / \mathrm{cm}^{2} & 0.8606 & 1.198 & 0.943 & 1.3190 & 0.5204 & 1.0546\end{array}$

Location $=$ ROA; Formation $=$ DISMAL GAP; Site $=17$

$\begin{array}{llrrrrrr}\mathrm{Cs}-137 & \mathrm{PCi} / \mathrm{cm}^{2} & 5.8400 & 2.4480 & 0.8032 & 0.3014 & 0.1427 & 0.0807 \\ \mathrm{~K}-40 & \mathrm{PCi} / \mathrm{cm}^{2} & 42.9800 & 47.1100 & 53.8000 & 76.0100 & 88.5400 & 95.5300 \\ \mathrm{U}-238 & \mathrm{PCi} / \mathrm{cm}^{2} & 8.1510 & 3.6270 & 12.8600 & 12.7990 & 13.5420 & 4.1910 \\ \mathrm{Ra}-226 & \mathrm{PCi} / \mathrm{cm}^{2} & 4.0700 & 4.3950 & 4.4540 & 4.1580 & 4.2730 & 4.1070 \\ \mathrm{Th}-232 & \mathrm{PCi} / \mathrm{cm}^{2} & 5.5520 & 6.0090 & 6.8330 & 6.9370 & 7.4630 & 7.4880 \\ \mathrm{U}-235 & \mathrm{PCi} / \mathrm{cm}^{2} & 0.4414 & 0.8981 & \mathrm{~N} & 0.7843 & \mathrm{~N} & 0.2194\end{array}$

Location $=$ ROA; Formation $=$ DISMAL GAP; Site $=19$

$\begin{array}{llrrrrrr}\mathrm{Cs}-137 & \mathrm{PCi} / \mathrm{cm}^{2} & 3.4210 & 0.2884 & 0.1308 & 0.1243 & 0.0852 & 0.1190 \\ \mathrm{~K}-40 & \mathrm{PCi} / \mathrm{cm}^{2} & 33.9000 & 36.1700 & 43.7000 & 45.5500 & 41.0800 & 46.3900 \\ \mathrm{U}-238 & \mathrm{PCi} / \mathrm{cm}^{2} & 5.4200 & 11.1200 & 13.3000 & 13.5490 & 13.4600 & 12.3090 \\ \mathrm{Ra}-226 & \mathrm{PCi} / \mathrm{cm}^{2} & 4.3460 & 4.9040 & 5.9620 & 4.3970 & 4.5740 & 4.5820 \\ \mathrm{Th}-232 & \mathrm{PCi} / \mathrm{cm}^{2} & 5.4100 & 5.5740 & 6.3970 & 6.0250 & 6.0700 & 6.1080 \\ \mathrm{U}-235 & \mathrm{PCi} / \mathrm{cm}^{2} & 0.4724 & 0.8168 & \mathrm{~N} & 0.8261 & 0.4963 & 0.3214\end{array}$

Location $=$ ROA; Formation $=$ DISMAL GAP; Sire $=20$

$\begin{array}{llrrrrrr}\mathrm{Cs}-137 & \mathrm{PCi} / \mathrm{cm}^{2} & 4.4180 & 0.9735 & 0.3815 & 0.203 & 0.091 & 0.053 \\ \mathrm{~K}-40 & \mathrm{PCi} / \mathrm{cm}^{2} & 42.8500 & 52.6100 & 84.6400 & 100.200 & 137.400 & 150.900 \\ \mathrm{U}-238 & \mathrm{PCi} / \mathrm{cm}^{2} & 15.1910 & 18.2600 & 10.5300 & 21.704 & 5.676 & 3.401 \\ \mathrm{Ra}-226 & \mathrm{PCi} / \mathrm{cm}^{2} & 5.0170 & 3.7260 & 3.5540 & 3.339 & 2.932 & 3.515 \\ \mathrm{Th}-232 & \mathrm{PCi} / \mathrm{cm}^{2} & 4.8090 & 5.6340 & 6.8360 & 7.053 & 7.258 & 7.377 \\ \mathrm{U}-235 & \mathrm{PCi} / \mathrm{cm}^{2} & 0.9249 & 0.9212 & 0.7342 & 0.967 & 0.229 & 0.389\end{array}$

Location $=$ ROA; Formation $=$ DISMAL GAP; Site $=21$

$\begin{array}{llrrrrrr}\mathrm{Cs}-137 & \mathrm{PCi} / \mathrm{cm}^{2} & 4.2540 & 0.6300 & 0.1158 & 0.1568 & 0.0758 & 0.1691 \\ \mathrm{~K}-40 & \mathrm{PCi} / \mathrm{cm}^{2} & 58.1400 & 61.8700 & 51.8500 & 45.2700 & 52.2800 & 49.1800 \\ \mathrm{U}-238 & \mathrm{PCi} / \mathrm{cm}^{2} & 16.4680 & 6.4000 & 17.9500 & 18.1800 & 12.5200 & 18.5160 \\ \mathrm{Ra}-226 & \mathrm{PCi} / \mathrm{cm}^{2} & 3.1540 & 4.2920 & 4.4790 & 4.4480 & 5.5580 & 4.6120 \\ \mathrm{Th}-232 & \mathrm{PCi} / \mathrm{cm}^{2} & 5.8620 & 6.3230 & 6.3210 & 5.9590 & 6.5470 & 6.5120 \\ \mathrm{U}-235 & \mathrm{PCi} / \mathrm{cm}^{2} & 0.4649 & 0.4812 & 1.0400 & 1.0573 & 1.0936 & 1.2130\end{array}$

Location $=$ ROA; Formation $=$ DISMAL GAP; Site $=22$

\begin{tabular}{llrrrrrr}
$\mathrm{Cs}-137$ & $\mathrm{PCi} / \mathrm{cm}^{2}$ & 3.0710 & 0.6686 & 0.1269 & 0.1348 & 0.1314 & 0.0303 \\
$\mathrm{~K}-40$ & $\mathrm{PCi} / \mathrm{cm}^{2}$ & 39.0700 & 51.9700 & 65.2700 & 83.4200 & 88.4800 & 86.2800 \\
$\mathrm{U}-238$ & $\mathrm{PCi} / \mathrm{cm}^{2}$ & 20.2000 & 13.3030 & 12.2300 & 14.5250 & 12.4450 & 4.4450 \\
$\mathrm{Ra}-226$ & $\mathrm{PCi} / \mathrm{cm}^{2}$ & 3.9400 & 3.8890 & 3.2310 & 4.7170 & 4.1090 & 3.9170 \\
$\mathrm{Th}-232$ & $\mathrm{PCi} / \mathrm{cm}^{2}$ & 4.9740 & 5.6240 & 5.4960 & 6.3220 & 6.1620 & 5.7420 \\
$\mathrm{U}-235$ & $\mathrm{PCi} / \mathrm{cm}^{2}$ & 0.7287 & 0.6585 & 0.7796 & 0.2574 & 1.5310 & 0.2656 \\
\hline \multicolumn{2}{c}{$\mathrm{N}=$ not detected. }
\end{tabular}


Appendix C

ORGANIC ANALYSIS DATA 
C-2

NOTE: When available, the validation qualifiers are used in this appendix. When validation qualifiers are not available, the corresponding laboratory data qualifiers are used. 


\section{C-3}

\section{VALIDATOR DATA QUALIFIER DEFINITIONS}

The following definitions provide brief explanations of the qualifiers assigned to the data in this appendix.

U The analyte was analyzed for but was not detected above the reported sample quantitiation limit.

J The analyte was positively identified; the associated numerical value is the approximate concentration of the analyte in the sample.

N The analysis indicates the presence of an analyte for which there is presumptive evidence to make a tentative identification.

JN The analysis indicates the presence of an analyte that has been tentatively identified, and the associated numerical value represents its approximate concentration.

UJ The analyte was not detected above the reported sample quantitation limit. However, the reported quantitation limit is approximate and may or may not represent the actual limit of quantitation necessary to accurately and precisely measure the analyte in the sample.

R The sample results are rejected because of serious deficiencies in the ability to analyze the sample and meet quality control criteria. The presence or absence of the analyte cannot be verified.

UN The laboratory did not register this compound, but there was presumptive evidence of a compound that was within the retention time window but was not reported. No other qualification of the data was made.

UJN The laboratory did not report the compound, but there was presumptive evidence of a compound that was within the retention time window but was not reported. The data were qualified as estimated, J, because of other discrepancies with the data.

RN The laboratory did not report the compound, but there was evidence of a compound that was within the retention time window but was not reported. The data were qualified as unusable, $R$, because of other discrepancies with the data. 


\section{LABORATORY DATA QUALIFIER DEFINITIONS}

The following contract laboratory data qualifiers are used in this project.

\section{Organic}

\begin{tabular}{|c|l||}
\hline Qualifier & Eplanation \\
\hline U & Indicates compound was analyzed for but not detected. \\
J & Indicates an estimated value. \\
N & $\begin{array}{l}\text { Indicates presumptive evidence of a compound [used only for tentatively } \\
\text { identified compounds (TICs)]. }\end{array}$ \\
P & $\begin{array}{l}\text { Used for pesticide/aroclor target analytes when there is greater than 25\% } \\
\text { difference for detected concentrations between the two gas chromatograph (GC) } \\
\text { columns. The lower of the two is reported and flagged. }\end{array}$ \\
C & $\begin{array}{l}\text { Used for pesticide results where the identification has been confirmed by } \\
\text { GC/mass spectrograph (MS). }\end{array}$ \\
B & $\begin{array}{l}\text { Used when the analyte is found in the associated blank as well as in the sample. } \\
\text { D }\end{array}$ \\
A & $\begin{array}{l}\text { Indicates that a TIC is a suspected aldol-condensation product. } \\
\text { X all compounds in an analyte at a secondary dilution factor. }\end{array}$ \\
\hline
\end{tabular}

Inorganic

\begin{tabular}{|c|l||}
\hline Qualifier & Explanation \\
\hline U & Indicates compound was analyzed for but not detected. \\
J & Indicates an estimated value. \\
N & Spiked sample recovery not within control limits. \\
E & Reported value estimated because of the presence of interference. \\
M & $\begin{array}{l}\text { Duplicate injection precision not met. } \\
\text { Beported value was obtained from a reading that was less than the CRDL, but } \\
\text { greater than or equal to the instrument detection limit (IDL). }\end{array}$ \\
S & $\begin{array}{l}\text { Reported value was determined by the method of standard additions (MSA). } \\
\text { Post-digestion spike for furnace atomic absorption is out of control limits, while } \\
\text { W }\end{array}$ \\
$*$ & $\begin{array}{l}\text { Dumple absorbance is less that 50\% of spike absorbance. } \\
\text { Correlation coefficient for the MSA is less than } 0.995 .\end{array}$ \\
\hline
\end{tabular}


Table C.1. Organic analysis results for soil samples

\begin{tabular}{|c|c|c|c|}
\hline Analysis & Units & $\begin{array}{c}\text { A } \\
\text { horizon } \\
\end{array}$ & Qualif \\
\hline \multicolumn{4}{|c|}{ Location $=A N D ;$ Formation $=$ DISMAL } \\
\hline $\begin{array}{l}\text { a-BHC } \\
\text { a-Chlordane } \\
\text {-BHC } \\
\text {-BHC } \\
\gamma \text {-BHC (Lindane) } \\
\gamma \text {-Chlordane } \\
\text { Aldrin } \\
\text { Aroclor } 1016 \\
\text { Aroclor } 1221 \\
\text { Aroclor } 1232 \\
\text { Aroclor } 1242 \\
\text { Aroclor } 1248 \\
\text { Aroclor } 1254 \\
\text { Aroclor } 1260 \\
\text { Acenaphthene } \\
\text { Acenaphthylene } \\
\text { Anthracene } \\
\text { Benzola] anthracene }\end{array}$ & $\begin{array}{l}\mu g / k g \\
\mu g / k g \\
\mu g / k g \\
\mu g / k g \\
\mu g / k g \\
\mu g / k g \\
\mu g / k g \\
\mu g / k g \\
\mu g / k g \\
\mu g / k g \\
\mu g / k g \\
\mu g / k g \\
\mu g / k g \\
\mu g / k g \\
\mu g / k g \\
\mu g / k g \\
\mu g / k g \\
\mu g / k g\end{array}$ & $\begin{array}{r}2.50 \\
2.50 \\
2.50 \\
2.50 \\
2.50 \\
2.50 \\
2.50 \\
49.40 \\
100.30 \\
49.40 \\
49.40 \\
49.40 \\
49.40 \\
49.40 \\
17.00 \\
36.00 \\
1.00 \\
3.80\end{array}$ & $\begin{array}{l}U \\
U \\
U \\
U \\
U J \\
U \\
U J \\
U \\
U \\
U \\
U \\
U \\
U \\
U \\
J N \\
U J \\
U J \\
J N\end{array}$ \\
\hline Benzo $[a]$ pyrene & $\mu \mathrm{g} / \mathrm{kg}$ & 5.90 & $J N$ \\
\hline Benzo $[b]$ f luoranthene & $\mathrm{Mg} / \mathrm{kg}$ & 3.00 & UJ \\
\hline Benzo $[g h, i]$ perylene & $\mathrm{\mu g} / \mathrm{kg}$ & 10.00 & J \\
\hline Benzo [k] fluoranthene & $\mu g / k g$ & 3.00 & UN \\
\hline $\begin{array}{l}\text { Chrysene } \\
\text { Dieldrin } \\
\text { Dibenzo }[a, h] \text { anthracene }\end{array}$ & $\begin{array}{l}\mu g / k g \\
\mu g / k g \\
\mu g / k g\end{array}$ & $\begin{array}{r}22.00 \\
4.90 \\
1.00\end{array}$ & $\begin{array}{l}\text { UJ } \\
\text { UJ } \\
\text { J }\end{array}$ \\
\hline $\begin{array}{l}\text { Endosul fan sul fate } \\
\text { Endosul fan I } \\
\text { Endosul fan II } \\
\text { Endrin } \\
\text { Endrin al dehyde } \\
\text { Endrin ketone } \\
\text { Fluoranthene } \\
\text { Fluorene } \\
\text { Heptachlor } \\
\text { Heptachlor epoxide } \\
\text { Indeno-1,2,3(c,d)-pyrene }\end{array}$ & $\begin{array}{l}\mu g / k g \\
\mu g / k g \\
\mu g / k g \\
\mu g / k g \\
\mu g / k g \\
\mu g / k g \\
\mu g / k g \\
\mu g / k g \\
\mu g / k g \\
\mu g / k g \\
\mu g / k g\end{array}$ & $\begin{array}{r}4.90 \\
2.50 \\
4.90 \\
4.90 \\
4.90 \\
4.90 \\
23.00 \\
6.00 \\
2.50 \\
2.50 \\
5.90\end{array}$ & $\begin{array}{l}U \\
U \\
U \\
U \\
U \\
U \\
J \\
U J \\
U J \\
U \\
J\end{array}$ \\
\hline $\begin{array}{l}\text { Methoxychlor } \\
\text { Naphthalene } \\
\text { Phenanthrene } \\
\text { Pyrene } \\
\text { Toxaphene } \\
4,4^{\prime} \text {-DDD } \\
4,4^{\prime}-\text { DDE } \\
4,4^{\prime}-D D T\end{array}$ & $\begin{array}{l}\mu g / k g \\
\mu g / k g \\
\mu g / k g \\
\mu g / k g \\
\mu g / k g \\
\mu g / k g \\
\mu g / k g \\
\mu g / k g\end{array}$ & $\begin{array}{r}25.40 \\
14.00 \\
4.00 \\
1.00 \\
254.50 \\
4.90 \\
4.90 \\
4.90\end{array}$ & $\begin{array}{l}U \\
J \\
J N \\
U J \\
U \\
U \\
U \\
U J\end{array}$ \\
\hline
\end{tabular}

Location $=A N D ;$ Formation $=$ DISMAL GAP; Site $=3$

$\begin{array}{llll}\text { Q-BHC } & \mu g / \mathbf{k g} & 2.00 & U \\ \text { a-Chlordane } & \mu g / k g & 2.00 & U \\ \beta-B H C & \mu g / k g & 2.00 & U \\ \Delta-B H C & \mu g / k g & 2.00 & U \\ \gamma \text {-BHC (Lindane) } & \mu g / k g & 2.00 & U J \\ \gamma \text {-Chlordane } & \mu g / k g & 2.00 & U \\ \text { Aldrin } & \mu g / k g & 2.00 & U J \\ \text { Aroclor } 1016 & \mu g / k g & 39.50 & U \\ \text { Aroclor } 1221 & \mu g / k g & 80.10 & U \\ \text { Aroclor } 1232 & \mu g / k g & 39.50 & U \\ \text { Aroclor } 1242 & \mu g / k g & 39.50 & U \\ \text { Aroclor } 1248 & \mu g / k g & 39.50 & U \\ \text { Aroclor } 1254 & \mu g / k g & 39.50 & U \\ \text { Aroclor } 1260 & \mu g / k g & 39.50 & U \\ \text { Acenaphthene } & \mu g / k g & 51.00 & J\end{array}$


Table C.1 (continued)

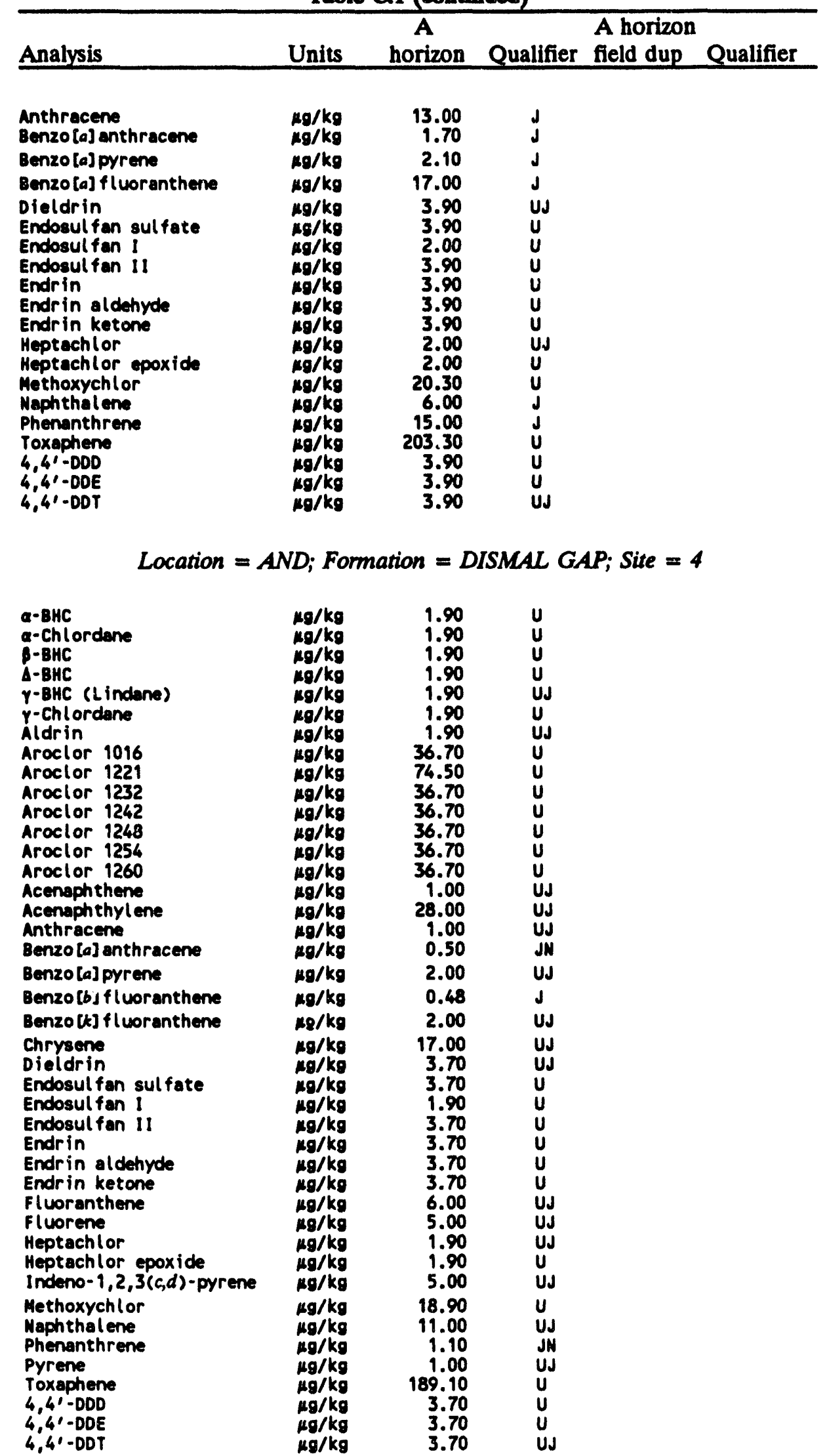


Table C.1 (continued)

\begin{tabular}{lcc}
\hline & A & A horizon \\
Analysis & Units & horizon Qualifier field dup Qualifier \\
\hline
\end{tabular}

$$
\text { Location }=A N D ; \text { Formation }=\text { DISMAL GAP; Site }=5
$$

\begin{tabular}{|c|c|c|}
\hline $\begin{array}{l}\alpha-B H C \\
\alpha-C h l o r d a n e \\
\beta-B H C \\
\text {-BHC } \\
\gamma-B H C \text { (Lindane) } \\
\gamma \text {-Chlordane } \\
\text { Aldrin } \\
\text { Aroclor } 1016 \\
\text { Aroclor } 1221 \\
\text { Aroclor } 1232 \\
\text { Aroclor } 1242 \\
\text { Aroclor } 1248 \\
\text { Aroclor } 1254 \\
\text { Aroclor } 1260 \\
\text { Acenaphthene } \\
\text { Acenaphthylene } \\
\text { Anthracene } \\
\text { Benzo[a] anthracene }\end{array}$ & $\begin{array}{l}\mu g / k g \\
\mu g / k g \\
\mu g / k g \\
\mu g / k g \\
\mu g / k g \\
\mu g / k g \\
\mu g / k g \\
\mu g / k g \\
\mu g / k g \\
\mu g / k g \\
\mu g / k g \\
\mu g / k g \\
\mu g / k g \\
\mu g / k g \\
\mu g / k g \\
\mu g / k g \\
\mu g / k g \\
\mu g / k g\end{array}$ & $\begin{array}{l}1.90 \\
1.90 \\
1.90 \\
1.90 \\
1.90 \\
1.90 \\
1.90 \\
37.80 \\
76.70 \\
37.80 \\
37.80 \\
37.80 \\
37.80 \\
37.80 \\
1.80 \\
21.00 \\
1.00 \\
1.10\end{array}$ \\
\hline Benzo [a] pyrene & $\mu \mathrm{g} / \mathrm{kg}$ & 1.50 \\
\hline Benzo $[b]$ fluoranthene & $\mathrm{Mg} / \mathrm{kg}$ & 2.00 \\
\hline Benzo $[k]$ fluoranthene & $\mu g / k g$ & 2.00 \\
\hline $\begin{array}{l}\text { Chrysene } \\
\text { Dieldrin } \\
\text { Endosul fan sul fate } \\
\text { Endosul fan I } \\
\text { Endosul fan II } \\
\text { Endrin } \\
\text { Endrin aldehyde } \\
\text { Endrin ketone } \\
\text { Fluoranthene } \\
\text { Fluorene } \\
\text { Heptachlor } \\
\text { Heptachlor epoxide } \\
\text { Indeno- } 1,2,3(c, d) \text {-pyrene }\end{array}$ & $\begin{array}{l}\mu g / \mathrm{kg} \\
\mu g / \mathrm{kg} \\
\mu g / \mathrm{kg} \\
\mu g / \mathrm{kg} \\
\mu g / \mathrm{kg} \\
\mu g / \mathrm{kg} \\
\mu g / \mathrm{kg} \\
\mu g / \mathrm{kg} \\
\mu g / \mathrm{kg} \\
\mu g / \mathrm{kg} \\
\mu g / \mathrm{kg} \\
\mu g / \mathrm{kg} \\
\mu g / \mathrm{kg}\end{array}$ & $\begin{array}{r}17.00 \\
3.80 \\
3.80 \\
1.90 \\
3.80 \\
3.80 \\
3.80 \\
3.80 \\
34.00 \\
5.00 \\
1.90 \\
1.90 \\
5.00\end{array}$ \\
\hline $\begin{array}{l}\text { Methoxychlor } \\
\text { Maphthalene } \\
\text { Phenanthrene } \\
\text { Pyrene } \\
\text { Toxaphene } \\
4,4^{\prime}-\text {-DD } \\
4,4^{\prime}-D D E \\
4,4^{\prime}-\text { DD }\end{array}$ & $\begin{array}{l}\mu g / \mathbf{k g} \\
\mu g / \mathbf{k g} \\
\mu g / \mathbf{k g} \\
\mu g / \mathbf{k g} \\
\mu g / \mathbf{k g} \\
\mu g / \mathbf{k g} \\
\mu g / \mathbf{k g} \\
\mu g / \mathbf{k g}\end{array}$ & $\begin{array}{r}19.50 \\
11.00 \\
6.00 \\
1.00 \\
194.60 \\
3.80 \\
3.80 \\
3.80\end{array}$ \\
\hline
\end{tabular}

Location $=A N D ;$ Formation $=$ DISMAL GAP; Site $=9$

\begin{tabular}{|c|c|c|}
\hline $\begin{array}{l}\text { a-BHC } \\
\text { a-Chlordane } \\
\text { p-BHC } \\
\text { A-BHC } \\
y-B H C \text { (Lindane) } \\
\gamma \text {-Chlordane } \\
\text { Aldrin } \\
\text { Aroclor } 1016 \\
\text { Aroclor } 1221 \\
\text { Aroclor } 1232 \\
\text { Aroclor } 1242 \\
\text { Aroclor } 1248 \\
\text { Aroclor } 1254 \\
\text { Aroclor } 1260 \\
\text { Acenaphthene } \\
\text { Benzola] anthracene }\end{array}$ & $\begin{array}{l}\mu g / \mathbf{k g} \\
\mu g / \mathbf{k g} \\
\mu g / \mathbf{k g} \\
\mu g / \mathbf{k g} \\
\mu g / \mathbf{k g} \\
\mu g / \mathbf{k g} \\
\mu g / \mathbf{k g} \\
\mu g / \mathbf{k g} \\
\mu g / \mathbf{k g} \\
\mu g / \mathbf{k g} \\
\mu g / \mathbf{k g} \\
\mu g / \mathbf{k g} \\
\mu g / \mathbf{k g} \\
\mu g / \mathbf{k g} \\
\mu g / \mathbf{k g} \\
\mu g / \mathbf{k g}\end{array}$ & $\begin{array}{r}2.00 \\
2.00 \\
2.00 \\
2.00 \\
2.00 \\
2.00 \\
2.00 \\
38.50 \\
78.10 \\
38.50 \\
38.50 \\
38.50 \\
38.50 \\
38.50 \\
7.60 \\
2.90\end{array}$ \\
\hline
\end{tabular}


Table C.1 (continued)

\begin{tabular}{|c|c|c|c|c|c|}
\hline Analysis & Units & $\begin{array}{c}\text { A } \\
\text { horize }\end{array}$ & Qualifier & $\begin{array}{l}\text { A horizon } \\
\text { field dup }\end{array}$ & Qualifier \\
\hline $\begin{array}{l}\text { Benzo [a] pyrene } \\
\text { Benzo [b] fl luoranthene } \\
\text { Dieldrin } \\
\text { Endosul fan sul fate } \\
\text { Endosul fan I } \\
\text { Endosul fan II } \\
\text { Endrin } \\
\text { Endrin al dehyde } \\
\text { Endr in ketone } \\
\text { Fluoranthene } \\
\text { Fluorene } \\
\text { Heptachlor } \\
\text { Heptachlor epoxide } \\
\text { Methoxychl or } \\
\text { Phenanthrene } \\
\text { Pyrene } \\
\text { Toxaphene } \\
4,4 \text {-DDD } \\
4,41-D D E \\
4,4{ }^{\prime}-D D T\end{array}$ & $\begin{array}{l}\mu g / k g \\
\mu g / k g \\
\mu g / k g \\
\mu g / k g \\
\mu g / k g \\
\mu g / k g \\
\mu g / k g \\
\mu g / k g \\
\mu g / k g \\
\mu g / k g \\
\mu g / k g \\
\mu g / k g \\
\mu g / / g g \\
\mu g / k g \\
\mu g / k g \\
\mu g . k g \\
\mu g / k g \\
\mu g / k g \\
\mu g / k g \\
\mu g / k g\end{array}$ & $\begin{array}{r}4.10 \\
13.00 \\
3.80 \\
3.80 \\
2.00 \\
3.80 \\
3.80 \\
3.80 \\
3.80 \\
3.10 \\
2.30 \\
2.00 \\
2.00 \\
19.80 \\
22.00 \\
2.20 \\
198.20 \\
3.80 \\
3.80 \\
3.80\end{array}$ & $\begin{array}{l}J \\
J N \\
U J \\
U \\
U \\
U \\
U \\
U \\
U \\
U \\
J \\
J \\
U J \\
U \\
U \\
J \\
J \\
U \\
U \\
U \\
U J\end{array}$ & & \\
\hline
\end{tabular}

$$
\text { Location }=A N D ; \text { Formation }=\text { DISMAL GAP; Site }=10
$$

\begin{tabular}{|c|c|}
\hline 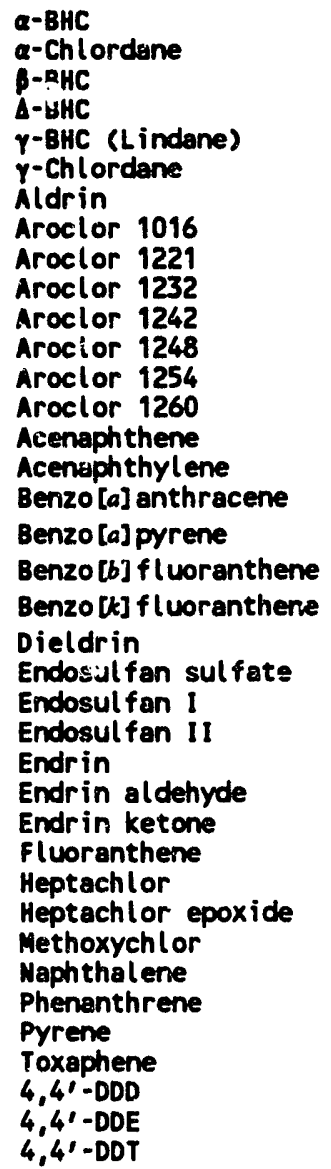 & 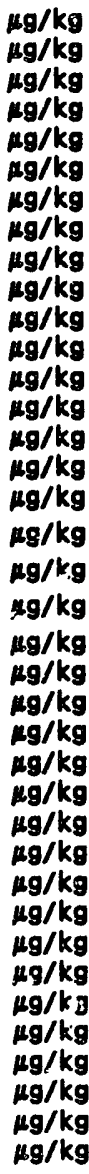 \\
\hline
\end{tabular}

2.50

2.50

2.50

2.50

2.50

2.50

2.50

48.30

98.00

48.30

48.30

48.30

48.30

48.30

38.00

13.00

3.80

5.40

61.00

5.30

4.80

4.80

2.50

4.80

4.80

4.80

4.80

1.80

2.50

2.50

24.90

24.00

19.00

4.20

248.80

4.80

4.80

4.80 
Table C.1 (continued)

\begin{tabular}{lcc}
\hline & A & A horizon \\
Analysis & Units & horizon Qualifier field dup Qualifier \\
\hline
\end{tabular}

$$
\text { Location }=A N D ; \text { Formation }=\text { DISMAL GAP; Site }=11
$$

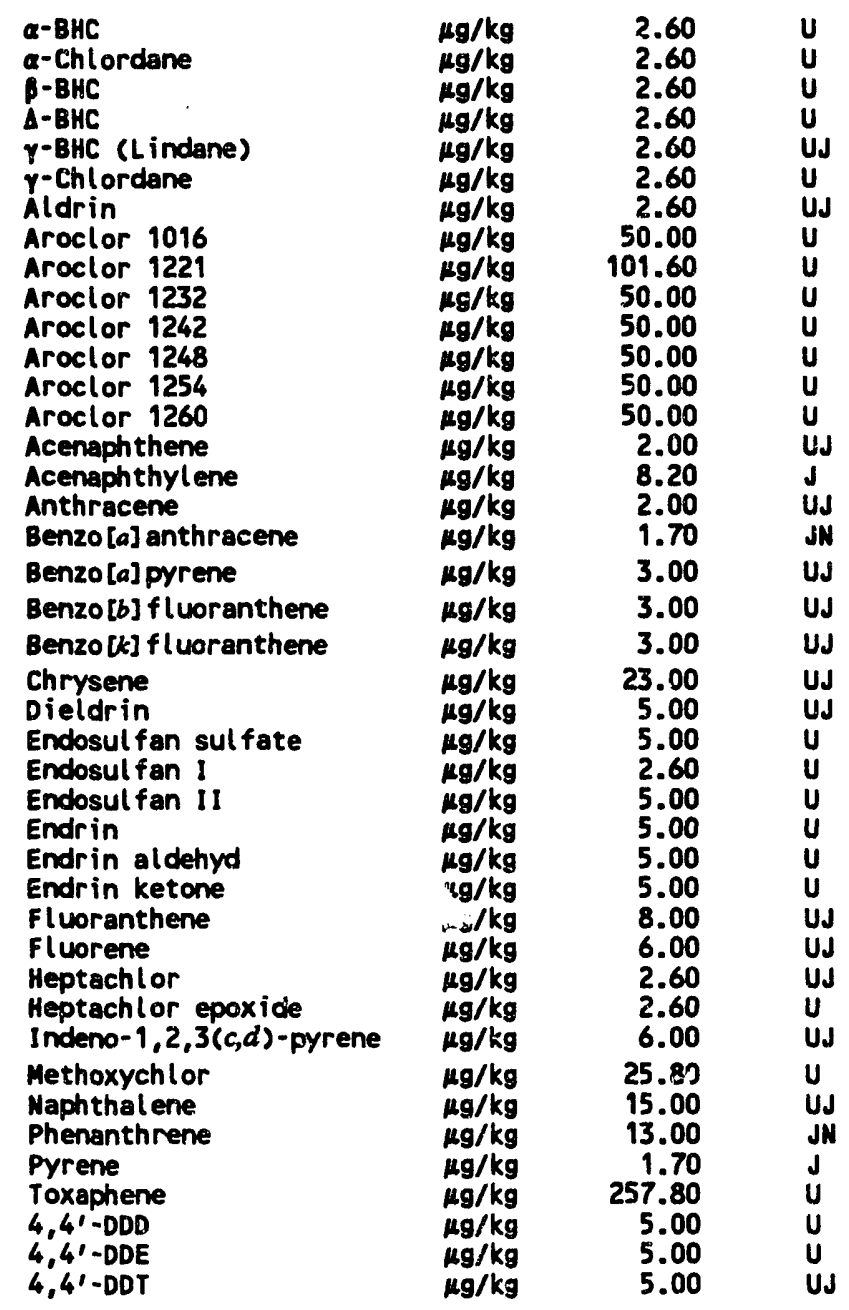

Location $=A N D ;$ Formation $=$ DISMAL GAP; Site $=12$

\begin{tabular}{|c|c|c|}
\hline $\begin{array}{l}\text { a-BHC } \\
\text { a-Chlordane } \\
\text { P-BHC } \\
\text { A-BHC } \\
\gamma \text {-BHC (Limlane) } \\
\gamma \text {-Chlordane } \\
\text { Aldrin } \\
\text { Aroclor } 1016 \\
\text { Aroclor } 1221 \\
\text { Aroclor } 1232 \\
\text { Aroclor } 1242 \\
\text { Aroclor } 1248 \\
\text { Aroclor } 1254 \\
\text { Aroclor } 1260 \\
\text { Acenaphthene } \\
\text { Acenaphthylene } \\
\text { Anthracene }\end{array}$ & $\begin{array}{l}\mu g / k g \\
\mu g / k g \\
\mu g / k g \\
\mu g / k g \\
\mu g / k g \\
\mu g / k g \\
\mu g / k g \\
\mu g / k g \\
\mu g / k g \\
\mu g / k g \\
\mu g / k g \\
\mu g / k g \\
\mu g / k g \\
\mu g / k g \\
\mu g / k g \\
\mu g / k g \\
\mu g / k g\end{array}$ & $\begin{array}{r}2.30 \\
2.30 \\
2.30 \\
2.30 \\
2.30 \\
2.30 \\
2.30 \\
45.30 \\
92.00 \\
45.30 \\
158.70 \\
45.30 \\
45.30 \\
45.30 \\
3.20 \\
34.00 \\
1.00\end{array}$ \\
\hline
\end{tabular}


Table C.1 (continued)

\begin{tabular}{|c|c|c|c|}
\hline Analysis & Units & $\begin{array}{c}\text { A } \\
\text { horizon } \\
\end{array}$ & Qualif \\
\hline $\begin{array}{l}\text { Benzo [a] anthracene } \\
\text { Benzo [a] pyrene } \\
\text { Benzo [b] f l woranthene } \\
\text { Benzo [k] f lworanthene } \\
\text { Chrysene } \\
\text { Dieldrin } \\
\text { Endosul fan sul fate } \\
\text { Endosul fan I } \\
\text { Endosul fan II } \\
\text { Endrin } \\
\text { Endrin al dehyde } \\
\text { Endrin ketone } \\
\text { Fluoranthene } \\
\text { Fluorene } \\
\text { Heptachlor } \\
\text { Heptachlor epoxide } \\
\text { Indeno-1,2,3(c,d)-pyrene }\end{array}$ & $\begin{array}{l}\mu g / k g \\
\mu g / k g \\
\mu g / k g \\
\mu g / k g \\
\mu g / k g \\
\mu g / k g \\
\mu g / k g \\
\mu g / k g \\
\mu g / k g \\
\mu g / k g \\
\mu g / k g \\
\mu g / k g \\
\mu g / k g \\
\mu g / k g \\
\mu g / k g \\
\mu g / k g \\
\mu g / k g\end{array}$ & $\begin{array}{r}2.10 \\
2.60 \\
3.00 \\
2.40 \\
21.00 \\
4.50 \\
4.50 \\
2.30 \\
4.50 \\
4.50 \\
4.50 \\
4.50 \\
7.00 \\
5.00 \\
2.30 \\
2.30 \\
5.00\end{array}$ & $\begin{array}{l}J \\
J \\
\text { UJ } \\
J \\
U J \\
U J \\
U \\
U \\
U \\
U \\
U \\
U \\
U J \\
U J \\
U J \\
U \\
U J\end{array}$ \\
\hline $\begin{array}{l}\text { Methoxychlor } \\
\text { Naphthalene } \\
\text { Phenanthrene } \\
\text { Pyrene } \\
\text { Toxaphene } \\
4,4^{\prime} \text {-DDD } \\
4,4^{\prime} \text {-DDE } \\
4,4^{\prime}-\text { DDT }\end{array}$ & $\begin{array}{l}\mu g / k g \\
\mu g / k g \\
\mu g / k g \\
\mu g / k g \\
\mu g / k g \\
\mu g / k g \\
\mu g / k g \\
\mu g / k g\end{array}$ & $\begin{array}{r}23.30 \\
14.00 \\
18.00 \\
0.49 \\
233.50 \\
4.50 \\
4.50 \\
4.50\end{array}$ & $\begin{array}{l}\text { U } \\
\text { Ud } \\
J N \\
J \\
U \\
U \\
U \\
\text { UJ }\end{array}$ \\
\hline
\end{tabular}

Location $=A N D ;$ Formation $=$ DISMAL GAP; Site $=19$

\begin{tabular}{|c|c|c|c|c|}
\hline $\begin{array}{l}\text { a-BHC } \\
\text { a-Chlordane } \\
\text { p-BHC } \\
\text { A-BHC } \\
\gamma \text {-BHC (Lindane) } \\
\gamma \text {-Chlordane } \\
\text { Aldrin } \\
\text { Aroclor } 1016 \\
\text { Aroclor } 1221 \\
\text { Aroclor } 1232 \\
\text { Aroclor } 1242 \\
\text { Aroclor } 1248 \\
\text { Aroclor } 1254 \\
\text { Aroclor } 1260 \\
\text { Acenaphthene } \\
\text { Acenaphthylene } \\
\text { Anthracene } \\
\text { Benzo[a] anthracene }\end{array}$ & $\begin{array}{l}\mu g / \mathbf{k g} \\
\mu g / \mathbf{k g} \\
\mu g / \mathbf{k g} \\
\mu g / \mathbf{k g} \\
\mu g / k g \\
\mu g / k g \\
\mu g / k g \\
\mu g / k g \\
\mu g / k g \\
\mu g / k g \\
\mu g / k g \\
\mu g / k g \\
\mu g / k g \\
\mu g / k g \\
\mu g / k g \\
\mu g / k g \\
\mu g / k g \\
\mu g / k g\end{array}$ & $\begin{array}{r}2.10 \\
2.10 \\
2.10 \\
2.10 \\
2.10 \\
2.10 \\
2.10 \\
41.00 \\
83.30 \\
41.00 \\
41.00 \\
41.00 \\
41.00 \\
49.00 \\
4.60 \\
13.00 \\
1.00 \\
2.70\end{array}$ & $\begin{array}{l}U \\
U \\
U \\
U \\
U J \\
U \\
U J \\
U \\
U \\
U \\
U \\
U \\
U \\
U \\
J \\
J \\
U J \\
J W\end{array}$ & $\begin{array}{r}2.1 \\
2.1 \\
2.1 \\
2.1 \\
2.1 \\
2.1 \\
2.1 \\
41.1 \\
83.5 \\
41.1 \\
41.1 \\
49.1 \\
41.1 \\
41.1 \\
3.1 \\
32.0 \\
1.0 \\
2.2\end{array}$ \\
\hline Benzo [a] pyrene & $\mathbf{m g} / \mathbf{k g}$ & 3.70 & JN & 2.9 \\
\hline Benzo $[b]$ f luoranthene & $\boldsymbol{\mu g} / \mathbf{k g}$ & 2.00 & UJ & 3.0 \\
\hline Benzo [k] f luoranthene & $\mu g / k g$ & 2.00 & UJ & 3.0 \\
\hline $\begin{array}{l}\text { Chrysene } \\
\text { Dieldrin } \\
\text { Endosulfan sul fate } \\
\text { Endosul fan I } \\
\text { Endosul fan II } \\
\text { Endrin } \\
\text { Endrin al ciehyde } \\
\text { Endrin ketone } \\
\text { Fluoranthene } \\
\text { Fluorene } \\
\text { Heptachlor } \\
\text { Heptachlor epoxide } \\
\text { Indeno-1,2,3(c,d)-pyrene }\end{array}$ & $\begin{array}{l}\mu g / k g \\
\mu g / k g \\
\mu g / k g \\
\mu g / k g \\
\mu g / k g \\
\mu g / k g \\
\mu g / k g \\
\mu g / k g \\
\mu g / k g \\
\mu g / k g \\
\mu g / k g \\
\mu g / k g \\
\mu g / k g\end{array}$ & $\begin{array}{r}19.00 \\
4.10 \\
4.10 \\
2.10 \\
4.10 \\
4.10 \\
4.10 \\
4.10 \\
4.60 \\
3.60 \\
2.10 \\
2.10 \\
5.00\end{array}$ & $\begin{array}{l}U J \\
U J \\
U \\
U \\
U \\
U \\
U \\
U \\
J \\
J \\
U J \\
U \\
U J\end{array}$ & $\begin{array}{r}19.0 \\
4.1 \\
4.1 \\
2.1 \\
4.1 \\
4.1 \\
4.1 \\
4.1 \\
6.0 \\
24.0 \\
2.1 \\
2.1 \\
5.0\end{array}$ \\
\hline $\begin{array}{l}\text { Methoxych lor } \\
\text { Maph thal ene }\end{array}$ & $\begin{array}{l}\mu g / \mathbf{k g} \\
\mu g / \mathbf{k g}\end{array}$ & $\begin{array}{l}21.10 \\
12.00\end{array}$ & $\begin{array}{l}\text { U } \\
\text { UJN }\end{array}$ & $\begin{array}{l}21.2 \\
13.0\end{array}$ \\
\hline
\end{tabular}




\section{C-11}

Table C.1 (continued)

\begin{tabular}{|c|c|c|c|c|c|}
\hline Analysis & Units & $\begin{array}{c}\mathbf{A} \\
\text { horizon }\end{array}$ & Qualifier & $\begin{array}{l}\text { A horizon } \\
\text { field dup }\end{array}$ & Qualifier \\
\hline $\begin{array}{l}\text { Phenanthrene } \\
\text { Pyrene } \\
\text { Toxaphene } \\
4,4 ;-D D D \\
4,4 ;-D D E \\
4,4 ;-D D T\end{array}$ & $\begin{array}{l}\mu g / k g \\
\mu g / k g \\
\mu g / k g \\
\mu g / k g \\
\mu g / k g \\
\mu g / k g\end{array}$ & $\begin{array}{r}6.50 \\
11.00 \\
21.40 \\
4.10 \\
4.10 \\
4.10\end{array}$ & $\begin{array}{l}J_{U} \\
U_{J} \\
U \\
U \\
U \\
U J\end{array}$ & $\begin{array}{r}8.7 \\
1.0 \\
211.8 \\
4.1 \\
4.1 \\
4.1\end{array}$ & $\begin{array}{l}\text { JN } \\
\text { UJ } \\
U \\
U \\
U \\
U \\
U J\end{array}$ \\
\hline
\end{tabular}

Location $=A N D ;$ Formation $=$ DISMAL GAP; Site $=20$

\begin{tabular}{|c|c|c|}
\hline $\begin{array}{l}\text { a-BHC } \\
\text { a-Chlordane } \\
\text { p-BHC } \\
\text { A-BHC } \\
\gamma \text {-BHC (Lindane) } \\
\gamma \text {-Chlordane } \\
\text { Aldrin } \\
\text { Aroclor } 1016 \\
\text { Aroclor } 1221 \\
\text { Aroclor } 1232 \\
\text { Aroclor } 1242 \\
\text { Aroclor } 1248 \\
\text { Aroclor } 1254 \\
\text { Aroclor } 1260 \\
\text { Acenaphthene } \\
\text { Acenaphthylene } \\
\text { Anthracene } \\
\text { Benzo[a] anthracene }\end{array}$ & $\begin{array}{l}\mu g / k g \\
\mu g / k g \\
\mathrm{Mg} / \mathrm{kg} \\
\mu g / k g \\
\mu g / k g \\
\mu g / k g \\
\mu g / k g \\
\mu g / k g \\
\mu g / k g \\
\mu g / k g \\
\mu g / k g \\
\mu g / k g \\
\mu g / k g \\
\mu g / k g \\
\mu g / k g \\
\mu g / k g \\
\mu g / k g \\
\mu g / k g\end{array}$ & $\begin{array}{l}2.10 \\
2.10 \\
2.10 \\
2.10 \\
2.10 \\
2.10 \\
2.10 \\
40.00 \\
81.30 \\
40.00 \\
40.00 \\
40.00 \\
40.00 \\
40.00 \\
1.10 \\
30.00 \\
1.10 \\
1.00\end{array}$ \\
\hline Benzo [a] pyrene & $\mu g / \mathbf{k g}$ & 1.00 \\
\hline Benzo $[b]$ fl woranthene & $\mu \mathrm{g} / \mathrm{kg}$ & 2.00 \\
\hline Benzo [k] f l woranthene & $\mu \mathrm{g} / \mathrm{kg}$ & 2.00 \\
\hline $\begin{array}{l}\text { Chrysene } \\
\text { Dieldrin } \\
\text { Endosul fan sul fate } \\
\text { Endosulfan I } \\
\text { Endosulfan II } \\
\text { Endrin } \\
\text { Endrin aldehyde } \\
\text { Endrin ketone } \\
\text { Fluoranthene } \\
\text { Fluorene } \\
\text { Heptachlor } \\
\text { Heptachlor epoxide } \\
\text { Indeno- } 1,2,3(c, d) \text {-pyrene }\end{array}$ & $\begin{array}{l}\mu g / \mathbf{k g} \\
\mu g / \mathbf{k g} \\
\mu g / \mathbf{k g} \\
\mu g / k g \\
\mu g / k g \\
\mu g / k g \\
\mu g / k g \\
\mu g / k g \\
\mu g / k g \\
\mu g / k g \\
\mu g / k g \\
\mu g / k g \\
\mu g / k g\end{array}$ & $\begin{array}{r}18.00 \\
4.00 \\
4.00 \\
2.10 \\
4.00 \\
4.00 \\
4.00 \\
4.00 \\
6.00 \\
70.00 \\
2.10 \\
2.10 \\
5.00\end{array}$ \\
\hline $\begin{array}{l}\text { Methoxychlor } \\
\text { Maphthalene } \\
\text { Phenanthrene } \\
\text { Pyrene } \\
\text { Toxaphene } \\
4,4^{\prime}-D D D \\
4,4^{\prime}-\text { DDE } \\
4,4^{\prime}-D D T\end{array}$ & $\begin{array}{l}\mu g / \mathbf{k g} \\
\mu g / k g \\
\mu g / k g \\
\mu g / k g \\
\mu g / k g \\
\mu g / k g \\
\mu g / k g \\
\mu g / k g\end{array}$ & $\begin{array}{r}20.60 \\
12.00 \\
3.80 \\
1.00 \\
206.30 \\
4.00 \\
4.00 \\
4.00\end{array}$ \\
\hline
\end{tabular}

Location $=A N D ;$ Formation $=$ DISMAL GAP; Site $=21$

$\begin{array}{llrl}\alpha-B H C & \mu g / k g & 2.30 & U \\ \alpha-C h l o r d a n e & \mu g / k g & 2.30 & U \\ \beta-B H C & \mu g / k g & 2.30 & U \\ \text { A-BHC } & \mu g / k g & 2.30 & U \\ \gamma-B H C \text { (Lindane) } & \mu g / k g & 2.30 & U J \\ \gamma-C h \text { lordane } & \mu g / k g & 2.30 & U \\ \text { Aldrin } & \mu g / k g & 2.30 & U J \\ \text { Aroclor } 1016 & \mu g / k g & 44.50 & U \\ \text { Aroclor } 1221 & \mu g / k g & 90.30 & U\end{array}$




\section{C- 12}

Table C.1 (continued)

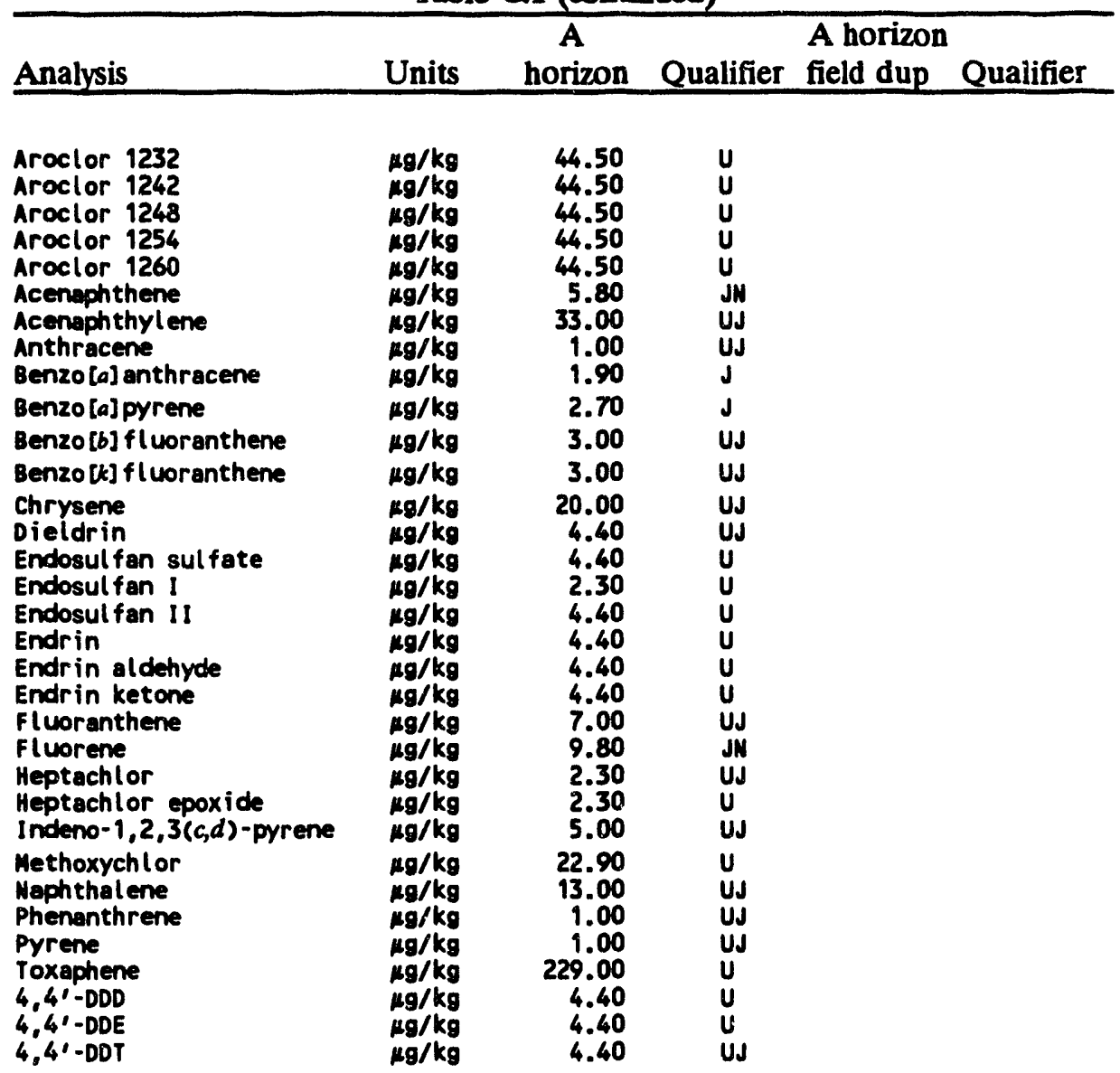

Location $=A N D ;$ Formation $=$ DISMAL GAP; Site $=22$

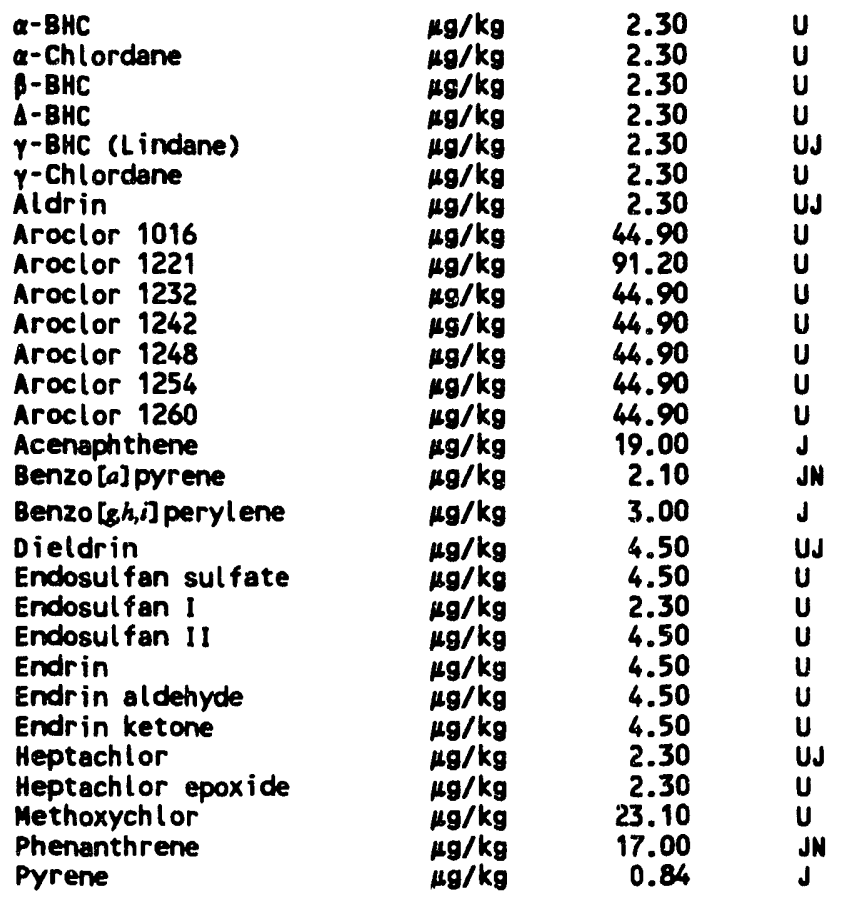


Table C.1 (continued)

\begin{tabular}{|c|c|c|c|c|c|}
\hline Analysis & Units & $\begin{array}{c}A \\
\text { horizon }\end{array}$ & Qualifier & $\begin{array}{l}\text { A horizon } \\
\text { field dup }\end{array}$ & Qualifier \\
\hline $\begin{array}{l}\text { Toxaphene } \\
4,4^{\prime}-D D D \\
4,4^{\prime}-D D E \\
4,4^{\prime}-D D T\end{array}$ & $\begin{array}{l}\mu g / \mathrm{kg} \\
\mu g / k g \\
\mu g / k g \\
\mu g / k g\end{array}$ & $\begin{array}{r}231.30 \\
4.50 \\
4.50 \\
4.50\end{array}$ & $\begin{array}{l}U \\
U \\
U \\
U J\end{array}$ & & \\
\hline
\end{tabular}

$$
\text { Location }=\text { ORR; Formation }=\text { DISMAL GAP; Site }=2
$$

\begin{tabular}{|c|c|c|c|c|c|}
\hline $\begin{array}{l}\text { Q-BHC } \\
\alpha \text {-Chlordane } \\
\beta \text {-BHC } \\
\text { A-BHC } \\
\gamma \text {-BHC (Lindane) } \\
\gamma \text {-Chlordane } \\
\text { Aldrin } \\
\text { Aroclor } 1016 \\
\text { Aroclor } 1221 \\
\text { Aroclor } 1232 \\
\text { Aroclor } 1242 \\
\text { Aroclor } 1248 \\
\text { Aroclor } 1254 \\
\text { Aroclor } 1260 \\
\text { Acenaphthene } \\
\text { Acenaph thylene } \\
\text { Anthracene } \\
\text { Benzo [a] anthracene }\end{array}$ & $\begin{array}{l}\mu g / \mathbf{k g} \\
\mu g / \mathbf{k g} \\
\mu g / \mathbf{k g} \\
\mu g / \mathbf{k g} \\
\mu g / \mathbf{k g} \\
\mu g / \mathbf{k g} \\
\mu g / \mathbf{k g} \\
\mu g / \mathbf{k g} \\
\mu g / \mathbf{k g} \\
\mu g / \mathbf{k g} \\
\mu g / \mathbf{k g} \\
\mu g / \mathbf{k g} \\
\mu g / \mathbf{k g} \\
\mu g / \mathbf{k g} \\
\mu g / \mathbf{k g} \\
\mu g / \mathbf{k g} \\
\mu g / \mathbf{k g} \\
\mu g / \mathbf{k g}\end{array}$ & $\begin{array}{r}4.10 \\
4.10 \\
4.10 \\
4.10 \\
4.10 \\
4.10 \\
4.10 \\
82.00 \\
160.00 \\
82.00 \\
82.00 \\
82.00 \\
82.00 \\
82.00 \\
213.00 \\
272.00 \\
78.00 \\
2.00\end{array}$ & $\begin{array}{l}U J \\
U J \\
U J \\
U J \\
U J \\
U \\
U \\
U \\
U \\
U \\
U \\
U \\
U \\
U \\
U J \\
U J \\
U J \\
U J\end{array}$ & $\begin{array}{r}4.0 \\
4.0 \\
4.0 \\
4.0 \\
4.0 \\
4.0 \\
4.0 \\
80.0 \\
160.0 \\
80.0 \\
80.0 \\
80.0 \\
80.0 \\
80.0 \\
213.0 \\
272.0 \\
78.0 \\
2.0\end{array}$ & $\begin{array}{l}\text { UJ } \\
U J \\
U J \\
U J \\
U J \\
U \\
U \\
U \\
U \\
U \\
U \\
U \\
U \\
U \\
U \\
U J \\
U J \\
U J \\
U J\end{array}$ \\
\hline Benzo [a] pyrene & $\mathrm{Rg} / \mathrm{kg}$ & 3.00 & UJ & 3.0 & UJ \\
\hline Benzo $[b]$ fluoranthene & $\mu g / k g$ & 2.00 & UJ & 2.0 & UJ \\
\hline Benzo $[g h, i]$ perylene & $\mu \mathrm{g} / \mathrm{kg}$ & 9.00 & UJ & 9.0 & UJ \\
\hline Benzo $[k]$ fluoranthene & $\mu \mathrm{g} / \mathrm{kg}$ & 2.00 & UJ & 2.0 & UJ \\
\hline $\begin{array}{l}\text { Chrysene } \\
\text { Dieldrin } \\
\text { Dalapon } \\
\text { Dibenzo }[a, h] \text { anthracene }\end{array}$ & $\begin{array}{l}\mu \mathrm{g} / \mathrm{kg} \\
\mu \mathrm{g} / \mathrm{kg} \\
\mu \mathrm{g} / \mathrm{kg} \\
\mu \mathrm{g} / \mathrm{kg}\end{array}$ & $\begin{array}{r}18.00 \\
4.10 \\
864.00 \\
4.00\end{array}$ & $\begin{array}{l}\text { UJ } \\
\text { UJ } \\
\text { UJ } \\
\text { UJ }\end{array}$ & $\begin{array}{r}18.0 \\
4.0 \\
850.0 \\
4.0\end{array}$ & $\begin{array}{l}U_{J} \\
U_{J} \\
U_{J} \\
U_{J}\end{array}$ \\
\hline $\begin{array}{l}\text { Dicamba } \\
\text { Dichlorprop } \\
\text { Dinoseb } \\
\text { Endosul fan sul fate } \\
\text { Endosul fan I } \\
\text { Endosul fan II } \\
\text { Endrin } \\
\text { Endrin aldehyde } \\
\text { Endrin ketone } \\
\text { Fluorene } \\
\text { Heptachlor } \\
\text { Heptachlor epoxide } \\
\text { Indeno- } 1,2,3(c, d) \text {-pyrene }\end{array}$ & $\begin{array}{l}\mu g / \mathbf{k g} \\
\mu g / k g \\
\mu g / k g \\
\mu g / k g \\
\mu g / k g \\
\mu g / k g \\
\mu g / k g \\
\mu g / k g \\
\mu g / k g \\
\mu g / k g \\
\mu g / k g \\
\mu g / k g \\
\mu g / k g\end{array}$ & $\begin{array}{r}40.00 \\
99.00 \\
21.00 \\
8.20 \\
4.10 \\
8.20 \\
8.20 \\
8.20 \\
8.20 \\
25.00 \\
4.10 \\
4.10 \\
5.00\end{array}$ & $\begin{array}{l}U \\
U \\
U \\
U \\
U J \\
U \\
U \\
U \\
U \\
U J \\
U J \\
U \\
U J\end{array}$ & \begin{tabular}{r}
39.0 \\
98.0 \\
21.0 \\
\hdashline-.0 \\
4.0 \\
8.0 \\
8.0 \\
\hdashline-.0 \\
8.0 \\
25.0 \\
4.0 \\
4.0 \\
5.0
\end{tabular} & 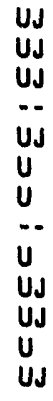 \\
\hline $\begin{array}{l}\text { Methoxychlor } \\
\text { MCPA } \\
\text { MCPP } \\
\text { Naphthalene } \\
\text { Phenanthrene } \\
\text { Pyrene } \\
\text { Silvex } \\
\text { Toxaphene } \\
2,4-0 \\
2,4-D B \\
2,4,5-T \\
4,4^{\prime} \text {-DDD } \\
4,4 \text {-DDE } \\
4,41-\text { DDT }\end{array}$ & $\begin{array}{l}\mu g / \mathbf{k g} \\
\mu g / \mathbf{k g} \\
\mu g / \mathbf{k g} \\
\mu g / k g \\
\mu g / \mathbf{k g} \\
\mu g / \mathbf{k g} \\
\mu g / \mathbf{k g} \\
\mu g / \mathbf{k g} \\
\mu g / \mathbf{k g} \\
\mu g / \mathbf{k g} \\
\mu g / \mathbf{k g} \\
\mu g / \mathbf{k g} \\
\mu g / \mathbf{k g} \\
\mu g / \mathbf{k g}\end{array}$ & $\begin{array}{r}41.00 \\
37230.00 \\
28295.00 \\
213.00 \\
76.00 \\
32.00 \\
25.00 \\
410.00 \\
179.00 \\
134.00 \\
30.00 \\
8.20 \\
8.20 \\
8.20\end{array}$ & $\begin{array}{l}U \\
U \\
U \\
U J \\
U J \\
U J N \\
U J \\
U \\
U \\
U \\
U \\
U J \\
U J \\
U J \\
U\end{array}$ & $\begin{array}{r}40.0 \\
36643.0 \\
27849.0 \\
213.0 \\
76.0 \\
9.1 \\
24.0 \\
400.0 \\
176.0 \\
132.0 \\
29.0 \\
8.0 \\
8.0 \\
8.0\end{array}$ & $\begin{array}{l}U \\
U J \\
U J \\
U J \\
U J \\
J \\
U J \\
U \\
U J \\
U J \\
U J \\
U J \\
U J \\
U\end{array}$ \\
\hline
\end{tabular}


Table C.1 (continued)

\begin{tabular}{lcc}
\hline & A & A horizon \\
Analysis & Units & horizon \\
\hline
\end{tabular}

$$
\text { Location }=O R R ; \text { Formation }=\text { DISMAL GAP; Site }=10
$$

\begin{tabular}{|c|c|c|}
\hline $\begin{array}{l}\text { Q-BHC } \\
\text { Q-Chlordane } \\
\beta-B H C \\
\text { A-BHC } \\
\gamma-\text {-BHC (Lindane) } \\
\gamma \text {-Chlordane } \\
\text { Aldrin } \\
\text { Aroclor } 1016 \\
\text { Aroclor } 1221 \\
\text { Aroclor } 1232 \\
\text { Aroclor } 1242 \\
\text { Aroclor } 1248 \\
\text { Aroclor } 1254 \\
\text { Aroclor } 1260 \\
\text { Acenaphthene } \\
\text { Acenaphthylene } \\
\text { Anthracene } \\
\text { Benzo [a] anthracene }\end{array}$ & 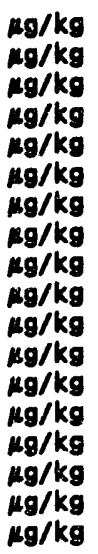 & $\begin{array}{r}4.40 \\
4.40 \\
4.40 \\
4.40 \\
4.40 \\
4.40 \\
4.40 \\
88.00 \\
180.00 \\
88.00 \\
88.00 \\
88.00 \\
88.00 \\
88.00 \\
241.00 \\
307.00 \\
88.00 \\
2.00\end{array}$ \\
\hline Benzo [a] pyrene & $\mathrm{Mg} / \mathrm{kg}$ & 3.00 \\
\hline Benzo $[b]$ f luoranthene & $\mu g / k g$ & 2.00 \\
\hline Benzo [shi] peryl ene & Mg/kg & 10.00 \\
\hline Benzo [k] f l woranthene & $\mathbf{M g} / \mathbf{k g}$ & 2.00 \\
\hline $\begin{array}{l}\text { Chrysene } \\
\text { Dieldr in } \\
\text { Dibenzo }[a, h] \text { anthracene }\end{array}$ & $\begin{array}{l}\mu g / k g \\
\mu g / k g \\
\mu g / k g\end{array}$ & $\begin{array}{r}20.00 \\
8.80 \\
4.00\end{array}$ \\
\hline $\begin{array}{l}\text { Dicanba } \\
\text { Dichlorprop } \\
\text { Dinoseb } \\
\text { Endosul fan sul fate } \\
\text { Endosul fan I } \\
\text { Endosul fan II } \\
\text { Endrin } \\
\text { Endrin al dehyde } \\
\text { Endrin ketone } \\
\text { Fluorene } \\
\text { Heptachlor } \\
\text { Heptachlor epoxide } \\
\text { Indeno- } 1,2,3(c, d) \text {-pyrene }\end{array}$ & $\begin{array}{l}\mu g / k g \\
\mu g / k g \\
\mu g / k g \\
\mu g / k g \\
\mu g / k g \\
\mu g / k g \\
\mu g / k g \\
\mu g / k g \\
\mu g / k g \\
\mu g / k g \\
\mu g / k g \\
\mu g / k g \\
\mu g / k g\end{array}$ & $\begin{array}{r}212.00 \\
531.00 \\
111.00 \\
8.80 \\
4.40 \\
8.80 \\
8.80 \\
8.80 \\
8.80 \\
28.00 \\
4.40 \\
4.40 \\
6.00\end{array}$ \\
\hline $\begin{array}{l}\text { Methoxychlor } \\
\text { MCPA } \\
\text { MCPP } \\
\text { Naph thal ene } \\
\text { Phenanthrene } \\
\text { Pyrene } \\
\text { Silvex } \\
\text { Toxaphene } \\
2,4-D \\
2,4-D B \\
2,4,5-T \\
4,4^{\prime}-\text { DDD } \\
4,4^{\prime}-\text { DDE } \\
4,4^{\prime}-D D T\end{array}$ & $\begin{array}{l}\mu g / \mathbf{k g} \\
\mu g / \mathbf{k g} \\
\mu g / \mathbf{k g} \\
\mu g / \mathbf{k g} \\
\mu g / \mathbf{k g} \\
\mu g / \mathbf{k g} \\
\mu g / \mathbf{k g} \\
\mu g / \mathbf{k g} \\
\mu g / \mathbf{k g} \\
\mu g / \mathbf{k g} \\
\mu g / \mathbf{k g} \\
\mu g / \mathbf{k g} \\
\mu g / \mathbf{k g} \\
\mu g / \mathbf{k g}\end{array}$ & $\begin{array}{r}44.00 \\
199104.00 \\
151319.00 \\
241.00 \\
86.00 \\
36.00 \\
133.00 \\
440.00 \\
956.00 \\
717.00 \\
159.00 \\
8.80 \\
8.80 \\
8.80\end{array}$ \\
\hline
\end{tabular}

Location $=$ ORR; Formation $=$ DISMAL GAP; Site $=11$

\begin{tabular}{|c|c|c|}
\hline $\begin{array}{l}\alpha-B H C \\
\alpha-C h \text { lordane } \\
\beta-B H C \\
A-B H C \\
\gamma-B H C \text { (Lindane) } \\
\gamma-C h \text { lordane }\end{array}$ & $\begin{array}{l}\mu g / \mathbf{k g} \\
\mu g / \mathbf{k g} \\
\mu g / \mathbf{k g} \\
\mu g / \mathbf{k g} \\
\mu g / \mathbf{k g} \\
\mu g / \mathbf{k g}\end{array}$ & $\begin{array}{l}4.30 \\
4.30 \\
4.30 \\
4.30 \\
4.30 \\
4.30\end{array}$ \\
\hline
\end{tabular}




\section{C-15}

Table C.1 (continued)

\begin{tabular}{|c|c|c|c|}
\hline Analysis & Units & $\begin{array}{c}\text { A } \\
\text { horizon } \\
\end{array}$ & Qualifie \\
\hline $\begin{array}{l}\text { Aldrin } \\
\text { Aroclor } 1016 \\
\text { Aroclor } 1221 \\
\text { Aroclor } 1232 \\
\text { Aroclor } 1242 \\
\text { Aroclor } 1248 \\
\text { Aroclor } 1254 \\
\text { Aroclor } 1260 \\
\text { Acenaphthene } \\
\text { Acenaphthylene } \\
\text { Anthracene } \\
\text { Benzo[a] anthracene }\end{array}$ & $\begin{array}{l}\mu g / \mathbf{k g} \\
\mu g / \mathbf{k g} \\
\mu g / \mathbf{k g} \\
\mu g / \mathbf{k g} \\
\mu g / \mathbf{k g} \\
\mu g / k g \\
\mu g / k g \\
\mu g / k g \\
\mu g / k g \\
\mu g / k g \\
\mu g / k g \\
\mu g / k g\end{array}$ & $\begin{array}{r}4.30 \\
86.00 \\
254.00 \\
86.00 \\
86.00 \\
86.00 \\
86.00 \\
86.00 \\
11593.00 \\
14814.00 \\
4254.00 \\
84.00\end{array}$ & 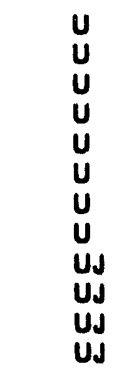 \\
\hline Benzo [a] pyrene & $\mu g / k g$ & 148.00 & UJ \\
\hline Benzo $[b]$ f luoranthene & $\mu g / k g$ & 116.00 & UJ \\
\hline Benzo $[g h, i]$ perylene & $\mu g / k g$ & 490.00 & UJ \\
\hline Benzo [k] fluoranthene & $\mu g / k g$ & 109.00 & UJ \\
\hline $\begin{array}{l}\text { Chrysene } \\
\text { Dieldrin } \\
\text { Dibenzo }[a, h] \text { anthracene }\end{array}$ & $\begin{array}{l}\mu g / k g \\
\mu g / k g \\
\mu g / k g\end{array}$ & $\begin{array}{r}966.00 \\
8.60 \\
193.00\end{array}$ & $\begin{array}{l}\text { UJ } \\
\text { UJ } \\
U_{J}\end{array}$ \\
\hline $\begin{array}{l}\text { Dicamba } \\
\text { Dichlorprop } \\
\text { Dinoseb } \\
\text { Endosul fan sul fate } \\
\text { Endosul fan I } \\
\text { Endosul fan II } \\
\text { Endrin } \\
\text { Endrin al dehyde } \\
\text { Endrin ketone } \\
\text { Fluoranthene } \\
\text { Fluorene } \\
\text { Heptachlor } \\
\text { Heptachlor epoxide } \\
\text { Indeno- } 1,2,3(c, d) \text {-pyrene }\end{array}$ & $\begin{array}{l}\mu g / \mathbf{k g} \\
\mu g / \mathbf{k g} \\
\mu g / \mathbf{k g} \\
\mu g / \mathbf{k g} \\
\mu g / \mathbf{k g} \\
\mu g / \mathbf{k g} \\
\mu g / \mathbf{k g} \\
\mu g / \mathbf{k g} \\
\mu g / \mathbf{k g} \\
\mu g / k g \\
\mu g / k g \\
\mu g / \mathbf{k g} \\
\mu g / \mathbf{k g} \\
\mu g / \mathbf{k g}\end{array}$ & $\begin{array}{r}412.00 \\
1031.00 \\
216.00 \\
8.80 \\
4.30 \\
8.60 \\
8.60 \\
8.60 \\
8.60 \\
348.00 \\
1353.00 \\
4.30 \\
4.30 \\
277.00\end{array}$ & $\begin{array}{l}U J \\
U J \\
U J \\
U \\
U \\
U \\
U \\
U J \\
U \\
U J \\
U J \\
U \\
U \\
U J\end{array}$ \\
\hline $\begin{array}{l}\text { Methoxychlor } \\
\text { MCPA } \\
\text { MCPP } \\
\text { Naphthalene } \\
\text { Phenanthrene } \\
\text { Pyrene } \\
\text { Silvex } \\
\text { Toxaphene } \\
2,4-D \\
2,4-D B \\
2,4,5-T \\
4,4 \text {-DDD } \\
4,4 \text { - DDE }\end{array}$ & $\begin{array}{l}\mu g / \mathbf{k g} \\
\mu g / \mathbf{k g} \\
\mu g / \mathbf{k g} \\
\mu g / \mathbf{k g} \\
\mu g / \mathbf{k g} \\
\mu g / \mathbf{k g} \\
\mu g / \mathbf{k g} \\
\mu g / \mathbf{k g} \\
\mu g / \mathbf{k g} \\
\mu g / k g \\
\mu g / \mathbf{k g} \\
\mu g / \mathbf{k g} \\
\mu g / \mathbf{k g} \\
\mu g / \mathbf{k g}\end{array}$ & $\begin{array}{r}43.00 \\
386449.00 \\
293701.00 \\
11593.00 \\
4122.00 \\
1739.00 \\
258.00 \\
430.00 \\
1855.00 \\
1391.00 \\
309.00 \\
8.60 \\
8.60 \\
8.60\end{array}$ & $\begin{array}{l}\mathbf{U} \\
U J \\
U J \\
U J \\
U J \\
U J \\
U J \\
U J \\
U \\
U J \\
U J \\
U J \\
U \\
U J \\
U \\
U\end{array}$ \\
\hline
\end{tabular}

$$
\text { Location }=\text { ORR; Formation }=\text { DISMAL GAP; Site }=19
$$

\begin{tabular}{|c|c|c|}
\hline $\begin{array}{l}\alpha-B H C \\
\alpha \text {-Chlordane } \\
\beta-B H C \\
\text { A-BHC } \\
y-B H C \text { (Lindane) } \\
\gamma \text {-Chlordane } \\
\text { Aldrin } \\
\text { Aroclor } 1016 \\
\text { Aroclor } 1221 \\
\text { Aroclor } 1232 \\
\text { Aroclor } 1242 \\
\text { Aroclor } 1248 \\
\text { Aroclor } 1254 \\
\text { Aroclor } 1260 \\
\text { Acenaphthene }\end{array}$ & $\begin{array}{l}\mu g / \mathbf{k g} \\
\mu g / \mathbf{k g} \\
\mu g / \mathbf{k g} \\
\mu g / \mathbf{k g} \\
\mu g / k g \\
\mu g / k g \\
\mu g / k g \\
\mu g / k g \\
\mu g / k g \\
\mu g / k g \\
\mu g / k g \\
\mu g / k g \\
\mu g / k g \\
\mu g / k g \\
\mu g / \mathbf{k g}\end{array}$ & $\begin{array}{r}4.30 \\
4.30 \\
4.30 \\
4.30 \\
4.30 \\
4.30 \\
4.30 \\
86.00 \\
254.00 \\
86.00 \\
86.00 \\
86.00 \\
86.00 \\
86.00 \\
2382.00\end{array}$ \\
\hline
\end{tabular}


Table C.1 (continued)

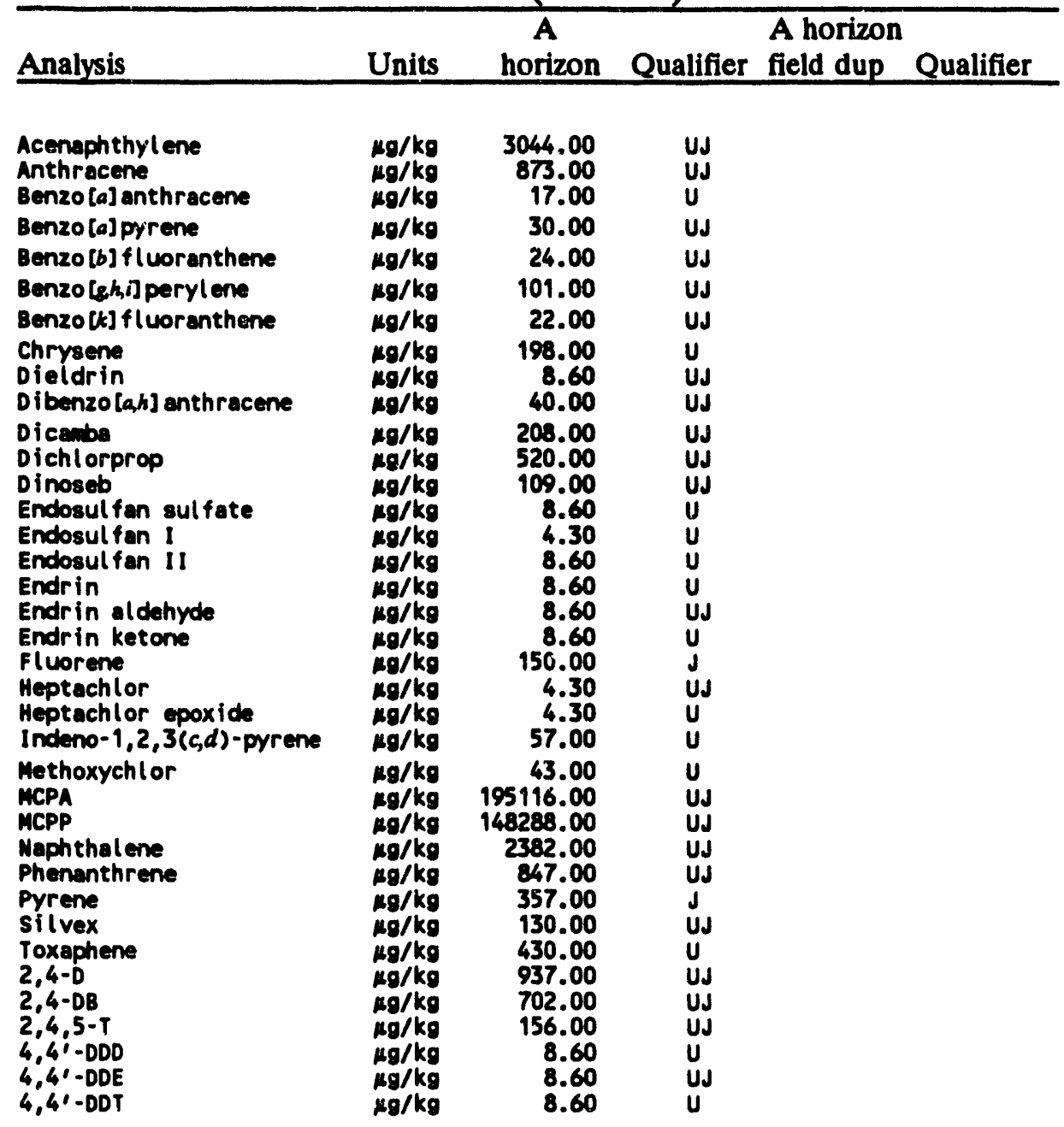

$$
\text { Location }=\text { ORR; Formation }=\text { DISMAL GAP; Site }=22
$$

\begin{tabular}{|c|c|c|}
\hline $\begin{array}{l}a-8 H C \\
\alpha-C h l o r d a n e \\
\text { p-BHC } \\
\text { A-8HC } \\
\gamma-B H C \text { (Lindane) } \\
\gamma-C h l o r d a n e \\
\text { Aldrin } \\
\text { Aroclor } 1016 \\
\text { Aroclor } 1221 \\
\text { Aroclor } 1232 \\
\text { Aroclor } 1242 \\
\text { Aroclor } 1248 \\
\text { Aroclor } 1254 \\
\text { Aroclor } 1260 \\
\text { Acenaphthene } \\
\text { Acenaphthylene } \\
\text { Anthracene } \\
\text { Benzo[a] anthracene }\end{array}$ & $\begin{array}{l}u g / k g \\
u g / k g \\
u g / k g \\
u g / k g \\
u g / k g \\
u g / k g \\
u g / k g \\
u g / k g \\
u g / k g \\
u g / k g \\
u g / k g \\
u g / k g \\
u g / k g \\
u g / k g \\
u g / k g \\
u g / k g \\
u g / k g \\
u g / k g\end{array}$ & $\begin{array}{r}4.30 \\
4.30 \\
4.30 \\
4.30 \\
4.30 \\
4.30 \\
4.30 \\
88.00 \\
176.00 \\
88.00 \\
88.00 \\
88.00 \\
88.00 \\
88.00 \\
2437.00 \\
3114.00 \\
894.00 \\
18.00\end{array}$ \\
\hline Benzo [a] pyrene & $u g / k g$ & 31.00 \\
\hline Benzo $[b]$ f louranthene & $u g / k g$ & 24.00 \\
\hline Benzo [gh,i] peryl erie & $u g / k g$ & 103.00 \\
\hline Benzo [k] f luoranthene & ug/kg & 23.00 \\
\hline $\begin{array}{l}\text { Chrysene } \\
\text { Dieldrin }\end{array}$ & $\begin{array}{l}u g / k g \\
u g / k g\end{array}$ & $\begin{array}{r}203.00 \\
4.30\end{array}$ \\
\hline
\end{tabular}




\section{C-17}

Table C.1 (continued)

\begin{tabular}{|c|c|c|c|c|c|}
\hline Analysis & Units & $\begin{array}{c}\text { A } \\
\text { horizon }\end{array}$ & Qualifier & $\begin{array}{l}\text { A horizon } \\
\text { field dup }\end{array}$ & Qualifier \\
\hline $\begin{array}{l}\text { Dibenzo [a,h] anthracene } \\
\text { Dicamba } \\
\text { Dichlorprop } \\
\text { Dinoseb } \\
\text { Endosul fan sul fate } \\
\text { Endosul fan I } \\
\text { Endosulf fan il } \\
\text { Endrin } \\
\text { Endrin al dehyde } \\
\text { Endrin ketone } \\
\text { Fllworene } \\
\text { Heptachlor } \\
\text { Heptachlor epoxide } \\
\text { Indeno-1,2,3(c,d)-pyrene }\end{array}$ & 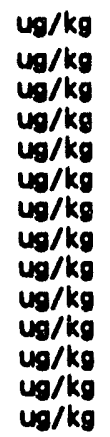 & $\begin{array}{r}41.00 \\
216.00 \\
539.00 \\
113.00 \\
8.80 \\
4.30 \\
8.80 \\
8.80 \\
8.80 \\
8.80 \\
284.00 \\
4.30 \\
4.30 \\
58.00\end{array}$ & 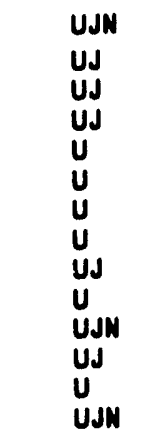 & & \\
\hline $\begin{array}{l}\text { Methoxychlor } \\
\text { MCPA } \\
\text { MCPP } \\
\text { Naphthalene } \\
\text { Phenanthrene } \\
\text { Pyrene } \\
\text { Silvex } \\
\text { Toxaphene } \\
2,4-D \\
2,4-D B \\
2,4,5-T \\
4,4^{\prime}-D D D \\
4,4 ;-D D E \\
4,4,-D D T\end{array}$ & $\begin{array}{l}u g / k g \\
u g / k g \\
u g / k g \\
u g / k g \\
u g / k g \\
u g / k g \\
u g / k g \\
w g / k g \\
u g / k g \\
w g / k g \\
u g / k g \\
u g / k g \\
u g / k g \\
u g / k g\end{array}$ & $\begin{array}{r}43.00 \\
202302.00 \\
153749.00 \\
2437.00 \\
867.00 \\
366.00 \\
135.00 \\
430.00 \\
971.00 \\
728.00 \\
162.00 \\
8.80 \\
8.80 \\
4.30\end{array}$ & 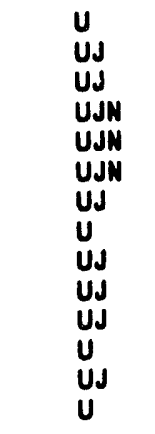 & & \\
\hline
\end{tabular}

$$
\text { Location }=\text { ORR; Formation }=\text { DISMAL GAP; Site }=26
$$

\begin{tabular}{|c|c|c|}
\hline $\begin{array}{l}\text { Q-BHC } \\
a \text {-Chlordane } \\
\text { p-BHC } \\
\text { A-BHC } \\
\gamma-\text {-BC (Lindane) } \\
\gamma \text {-Chlordane } \\
\text { Aldrin } \\
\text { Aroclor } 1016 \\
\text { Aroclor } 1221 \\
\text { Aroclor } 1232 \\
\text { Aroclor } 1242 \\
\text { Aroclor } 1248 \\
\text { Aroclor } 1254 \\
\text { Aroclor } 1260 \\
\text { Dieldrin } \\
\text { Dalapon } \\
\text { Dicamba } \\
\text { Dichlorprop } \\
\text { Dinoseb } \\
\text { Endosulfan sulfate } \\
\text { Endosul fan } 1 \\
\text { Endosulfan il } \\
\text { Endrin } \\
\text { Endrin aldehyde } \\
\text { Endrin ketone } \\
\text { Heptachlor } \\
\text { Heptachlor epoxide } \\
\text { Methoxychlor } \\
\text { MCPA } \\
\text { MCPP } \\
\text { Silvex } \\
\text { Toxaphene } \\
2,4-D\end{array}$ & $\begin{array}{l}\mu g / \mathbf{k g} \\
\mu g / \mathbf{k g} \\
\mu g / \mathbf{k g} \\
\mu g / k g \\
\mu g / k g \\
\mu g / k g \\
\mu g / k g \\
\mu g / k g \\
\mu g / k g \\
\mu g / k g \\
\mu g / k g \\
\mu g / k g \\
\mu g / k g \\
\mu g / k g \\
\mu g / k g \\
\mu g / k g \\
\mu g / k g \\
\mu g / k g \\
\mu g / k g \\
\mu g / k g \\
\mu g / k g \\
\mu g / k g \\
\mu g / k g \\
\mu g / k g \\
\mu g / k g \\
\mu g / k g \\
\mu g / k g \\
\mu g / k g \\
\mu g / k g \\
\mu g / k g \\
\mu g / k g \\
\mu g / k g \\
\mu g / k g\end{array}$ & $\begin{array}{r}4.10 \\
4.10 \\
4.10 \\
4.10 \\
4.10 \\
4.10 \\
4.10 \\
83.00 \\
170.00 \\
83.00 \\
83.00 \\
83.00 \\
83.00 \\
83.00 \\
8.30 \\
877.00 \\
40.00 \\
101.00 \\
21.00 \\
8.30 \\
4.10 \\
8.30 \\
8.30 \\
8.30 \\
8.30 \\
4.10 \\
4.10 \\
41.00 \\
37798.00 \\
28726.00 \\
25.00 \\
410.00 \\
181.00\end{array}$ \\
\hline
\end{tabular}




\section{C-18}

Table C.1 (continued)

\begin{tabular}{|c|c|c|c|c|c|}
\hline Analysis & Units & $\begin{array}{c}\mathbf{A} \\
\text { horizon } \\
\end{array}$ & Qualifier & $\begin{array}{l}\text { A horizon } \\
\text { field dup }\end{array}$ & Qualifier \\
\hline $\begin{array}{l}2,4-D B \\
2,4,5-T \\
4,4 ;-D D D \\
4,4 '-D D E \\
4,41-D D T\end{array}$ & $\begin{array}{l}\mu g / k g \\
\mu g / k g \\
\mu g / k g \\
k g / k g \\
\mu g / k g\end{array}$ & $\begin{array}{r}136.00 \\
30.00 \\
8.30 \\
8.30 \\
8.30\end{array}$ & $\begin{array}{l}U_{U, J} \\
\text { UJ } \\
\text { UJ } \\
\text { UJ } \\
U\end{array}$ & & \\
\hline
\end{tabular}

$$
\text { Location }=O R R ; \text { Formation }=\text { DISMAL GAP; Site }=27
$$

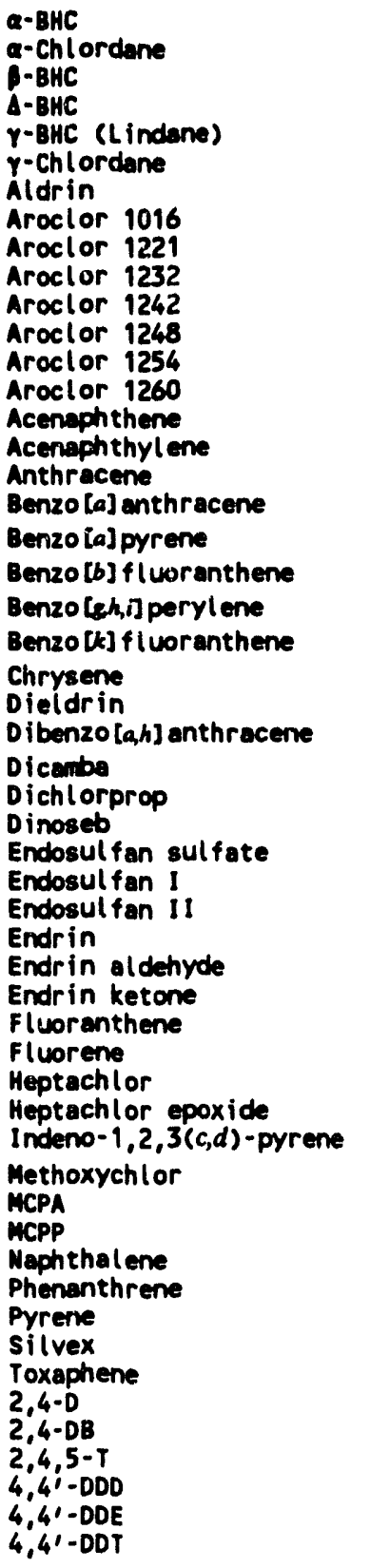

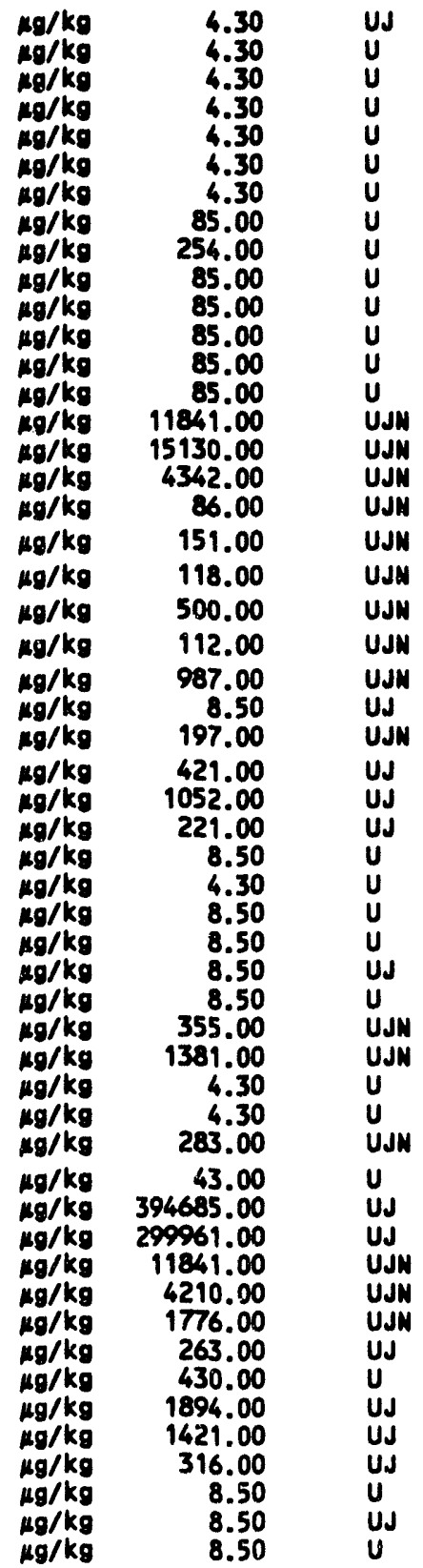


Table C.1 (continued)

\begin{tabular}{lcc}
\hline & A & A horizon \\
Analysis & Units & horizon Qualifier field dup Qualifier \\
\hline
\end{tabular}

Location $=O R R ;$ Formation $=$ DISMAL GAP; Site $=32$

\begin{tabular}{|c|c|c|}
\hline $\begin{array}{l}\text { a-BHC } \\
\text { a-Chlordane } \\
\text {-BHC } \\
\text { A-BHC } \\
\gamma \text {-BHC (Lindane) } \\
\gamma \text {-Chlordane } \\
\text { Aldrin } \\
\text { Aroclor } 1016 \\
\text { Aroclor } 1221 \\
\text { Aroclor } 1232 \\
\text { Aroclor } 1242 \\
\text { Aroclor } 1248 \\
\text { Aroclor } 1254 \\
\text { Aroclor } 1260 \\
\text { Acenaphthene } \\
\text { Acenaphthylene } \\
\text { Anthracene } \\
\text { Benzo } a] \text { anthracene }\end{array}$ & $\begin{array}{l}\mu g / k g \\
\mu g / k g \\
\mu g / k g \\
\mu g / k g \\
n g / k g \\
\mu g / k g \\
\mu g / k g \\
\mu g / k g \\
\mu g / k g \\
\mu g / k g \\
\mu g / k g \\
\mu g / k g \\
\mu g / k g \\
\mu g / k g \\
\mu g / k g \\
M g / k g \\
M g / k g \\
M g / k g\end{array}$ & $\begin{array}{r}4.20 \\
4.20 \\
4.20 \\
4.20 \\
4.20 \\
4.20 \\
4.20 \\
84.00 \\
170.00 \\
84.00 \\
84.00 \\
84.00 \\
84.00 \\
84.00 \\
2291.00 \\
2928.00 \\
840.00 \\
17.00\end{array}$ \\
\hline Benzo [a] pyrene & $\mu g / k g$ & 29.00 \\
\hline Benzo $[b]$ f l woranthene & $\mathrm{mg} / \mathrm{kg}$ & 23.00 \\
\hline Benzo $[k, i]$ perylene & $\mu g / k g$ & 97.00 \\
\hline Benzo $[k]$ f l woranthene & $M g / k g$ & 22.00 \\
\hline $\begin{array}{l}\text { Chrysene } \\
\text { Dieldrin } \\
\text { Dibenzo }[a, h] \text { anthracene }\end{array}$ & $\begin{array}{l}\mu g / k g \\
\mu g / k g \\
\mu g / k g\end{array}$ & $\begin{array}{r}191.00 \\
8.40 \\
38.00\end{array}$ \\
\hline $\begin{array}{l}\text { Dicamba } \\
\text { Dichlorprop } \\
\text { Dinoseb } \\
\text { Endosul fan sulfate } \\
\text { Endosul fan I } \\
\text { Endosul fan II } \\
\text { Endrin } \\
\text { Endrin al dehyde } \\
\text { Endrin ketone } \\
\text { Fluorene } \\
\text { Heptachlor } \\
\text { Heptachlor epoxide } \\
\text { indeno- } 1,2,3(c, d) \text {-pyrene }\end{array}$ & $\begin{array}{l}\mathrm{Mg} / \mathrm{kg} \\
\mathrm{Mg} / \mathrm{kg} \\
\mathrm{Mg} / \mathrm{kg} \\
\mathrm{\mu g} / \mathrm{kg} \\
\mathrm{\mu g} / \mathrm{kg} \\
\mathrm{\mu g} / \mathrm{kg} \\
\mathrm{Mg} / \mathrm{kg} \\
\mathrm{\mu g} / \mathrm{kg} \\
\mathrm{\mu g} / \mathrm{kg} \\
\mathrm{\mu g} / \mathrm{kg} \\
\mathrm{Mg} / \mathrm{kg} \\
\mathrm{Mg} / \mathrm{kg} \\
\mathrm{Mg} / \mathrm{kg}\end{array}$ & $\begin{array}{r}204.00 \\
509.00 \\
107.00 \\
8.40 \\
4.20 \\
8.40 \\
8.40 \\
8.40 \\
8.40 \\
267.00 \\
4.20 \\
4.20 \\
55.00\end{array}$ \\
\hline $\begin{array}{l}\text { Methoxychlor } \\
\text { MCPA } \\
\text { MCPP } \\
\text { Maphthalen: } \\
\text { Phenanthrene } \\
\text { Pyrene } \\
\text { Silvex } \\
\text { Toxaphene } \\
2,4-D \\
2,4-D B \\
2,4,5-T \\
4,4 ;-D D D \\
4,4 ;-D D E \\
4,41-D D T\end{array}$ & $\begin{array}{l}\mu g / k g \\
\mu g / k g \\
\mu g / k g \\
\mu g / k g \\
\mu g / k g \\
\mu g / k g \\
\mu g / k g \\
\mu g / k g \\
\mu g / k g \\
\mu g / k g \\
\mu g / k g \\
\mu g / k g \\
\mu g / k g \\
\mu g / k g\end{array}$ & $\begin{array}{r}42.00 \\
190937.00 \\
145112.00 \\
2291.00 \\
815.00 \\
344.00 \\
127.00 \\
420.00 \\
916.00 \\
687.00 \\
153.00 \\
8.40 \\
8.40 \\
8.40\end{array}$ \\
\hline
\end{tabular}


Table C.1 (continued)

\begin{tabular}{lcc}
\hline & A & A horizon \\
Analysis & Units horizon Qualifier field dup Qualifier \\
\hline
\end{tabular}

$$
\text { Location }=\text { ORR; Fomation }=\text { DISMAL GAP; Site }=33
$$

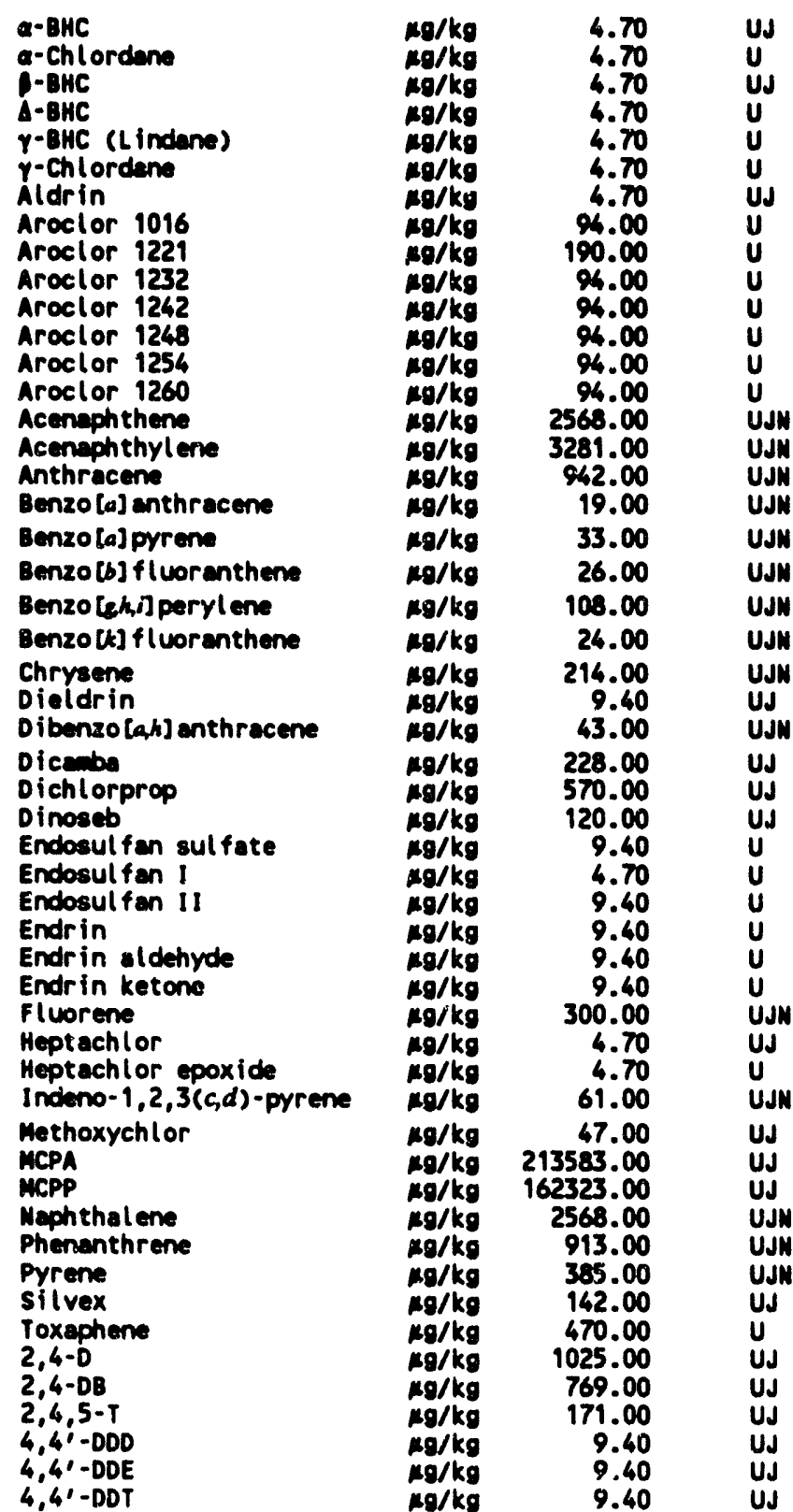

$$
\text { Location }=\text { ORR; Formation }=\text { DISMAL GAP; Site }=35
$$

$\begin{array}{ll}a-B H C & \mu g / k g \\ Q-C h l o r d a n e & \mu g / k g \\ \beta-B H C & \mu g / k g \\ \Delta-B H C & \mu g / k g \\ \gamma-B H C \text { (Lindane) } & \mu g / k g \\ \gamma-C h l o r d a n e & \mu g / k g\end{array}$

$\begin{array}{ll}4.20 & U J \\ 4.20 & U \\ 4.20 & U J \\ 4.20 & U \\ 4.20 & U \\ 4.20 & U\end{array}$


Table C.1 (continued)

\begin{tabular}{|c|c|c|c|c|c|}
\hline Analysis & Units & $\begin{array}{c}\mathbf{A} \\
\text { horizon } \\
\end{array}$ & Qualifier & $\begin{array}{l}\text { A horizon } \\
\text { field dup }\end{array}$ & Qualifier \\
\hline $\begin{array}{l}\text { Aldrin } \\
\text { Aroclor } 1016 \\
\text { Aroclor } 1221 \\
\text { Aroclor } 1232 \\
\text { Aroclor } 1242 \\
\text { Aroclor } 1248 \\
\text { Arocl or } 1254 \\
\text { Aroclor } 1260 \\
\text { Acenaphthene } \\
\text { Acenaphthylene } \\
\text { Anthracene }\end{array}$ & $\begin{array}{l}\mu g / k g \\
\mu g / k g \\
\mu g / k g \\
\mu g / k g \\
\mu g / k g \\
\mu g / k g \\
\mu g / k g \\
\mu g / k g \\
\mu g / k g \\
\mu g / k g \\
\mu g / k g\end{array}$ & $\begin{array}{r}4.20 \\
84.00 \\
170.00 \\
84.00 \\
84.00 \\
84.00 \\
84.00 \\
84.00 \\
2285.00 \\
2920.00 \\
838.00\end{array}$ & 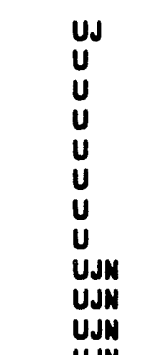 & & \\
\hline $\begin{array}{l}\text { Benzo [a] anthracere } \\
\text { Benzo [a] pyrene }\end{array}$ & $\begin{array}{l}\mathrm{\mu g} / \mathrm{kg} \\
\mathrm{kg} / \mathrm{kg}\end{array}$ & $\begin{array}{l}17.00 \\
29.00\end{array}$ & $\begin{array}{l}\text { UJN } \\
\text { UJN }\end{array}$ & & \\
\hline Benzo $[b]$ fl woranthene & $\mathrm{kg} / \mathrm{kg}$ & 23.00 & UJN & & \\
\hline Benzo $[$ hhi] perylene & Mg/kg & 96.00 & UJM & & \\
\hline Benzo [k] fluoranthene & $\mathbf{g g} / \mathbf{k g}$ & 22.00 & UJN & & \\
\hline $\begin{array}{l}\text { Chrysene } \\
\text { Dieldrin } \\
\text { Dibenzo[a,h] anthracene }\end{array}$ & $\begin{array}{l}\mathrm{kg} / \mathrm{kg} \\
\mathrm{kg} / \mathrm{kg} \\
\mathrm{kg} / \mathrm{kg}\end{array}$ & $\begin{array}{r}190.00 \\
8.40 \\
38.00\end{array}$ & $\begin{array}{l}\text { UJN } \\
\text { UJ } \\
\text { UJN }\end{array}$ & & \\
\hline $\begin{array}{l}\text { Dicembe } \\
\text { Dichlorprop } \\
\text { Dinoseb } \\
\text { Endosulfan sul fate } \\
\text { Endosulfan i } \\
\text { Endosul fan il } \\
\text { Endrin } \\
\text { Endrin al dehyde } \\
\text { Endrin ketone } \\
\text { Fluorene } \\
\text { Heptachlor } \\
\text { Heptachl or epoxide } \\
\text { Indeno-1,2,3(c,d)-pyrene }\end{array}$ & 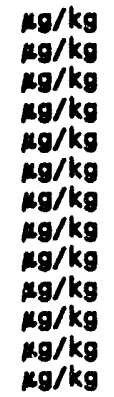 & $\begin{array}{l}202.00 \\
504.00 \\
106.00 \\
8.40 \\
4.20 \\
8.40 \\
8.40 \\
8.40 \\
88.40 \\
267.00 \\
4.20 \\
4.20 \\
55.00\end{array}$ & 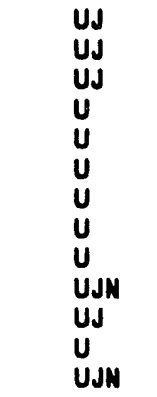 & & \\
\hline $\begin{array}{l}\text { Methoxychlor } \\
\text { MCPA } \\
\text { MCPP } \\
\text { Maphthalene } \\
\text { Phenanthrene } \\
\text { Pyrene } \\
\text { Silvex } \\
\text { Toxaphene } \\
2,4-D \\
2,4-D B \\
2,4,5-T \\
4,4 i-D D D \\
4,41-D D E \\
4,41-D D T\end{array}$ & $\begin{array}{l}\mu g / k g \\
\mu g / k g \\
\mu g / k g \\
\mu g / k g \\
\mu g / k g \\
\mu g / k g \\
\mu g / k g \\
\mu g / k g \\
\mu g / k g \\
\mu g / k g \\
\mu g / k g \\
\mu g / k g \\
\mu g / k g \\
\mu g / k g\end{array}$ & $\begin{array}{r}42.00 \\
189119.00 \\
143730.00 \\
2285.00 \\
813.00 \\
343.00 \\
126.00 \\
420.00 \\
908.00 \\
681.00 \\
151.00 \\
8.40 \\
8.40 \\
8.40\end{array}$ & $\begin{array}{l}\text { UJ } \\
\text { UJ } \\
\text { UJ } \\
\text { UJN } \\
\text { UJN } \\
\text { UJN } \\
\text { UJ } \\
U \text { J } \\
\text { UJ } \\
\text { UJ } \\
\text { UJ } \\
\text { UJ } \\
\text { UJ } \\
\text { UJ }\end{array}$ & & \\
\hline
\end{tabular}

Location $=$ ORR; Formation $=$ DISMAL GAP; Site $=41$

\begin{tabular}{|c|c|c|}
\hline $\begin{array}{l}\alpha \text {-BHC } \\
\alpha \text {-Chlordane } \\
\beta-B H C \\
\Delta-B H C \\
\gamma-B H C \text { (Lindane) } \\
\gamma \text {-Chlordane } \\
\text { Aldrin } \\
\text { Aroclor } 1016 \\
\text { Aroclor } 1221 \\
\text { Aroclor } 1232 \\
\text { Aroclor } 1242 \\
\text { Aroclor } 1248 \\
\text { Aroclor } 1254 \\
\text { Aroclor } 1260 \\
\text { Acenaphthene } \\
\text { Acenaphthylene }\end{array}$ & $\begin{array}{l}\mu g / k g \\
\mu g / k g \\
\mu g / k g \\
\mu g / k g \\
\mu g / k g \\
\mu g / k g \\
\mu g / k g \\
\mu g / k g \\
\mu g / k g \\
\mu g / k g \\
\mu g / k g \\
\mu g / k g \\
\mu g / k g \\
\mu g / k g \\
\mu g / k g \\
\mu g / k g\end{array}$ & $\begin{array}{r}4.20 \\
4.20 \\
4.20 \\
4.20 \\
4.20 \\
4.20 \\
4.20 \\
85.00 \\
169.00 \\
85.00 \\
85.00 \\
85.00 \\
85.00 \\
85.00 \\
11464.00 \\
14648.00\end{array}$ \\
\hline
\end{tabular}


Table C.1 (continued)

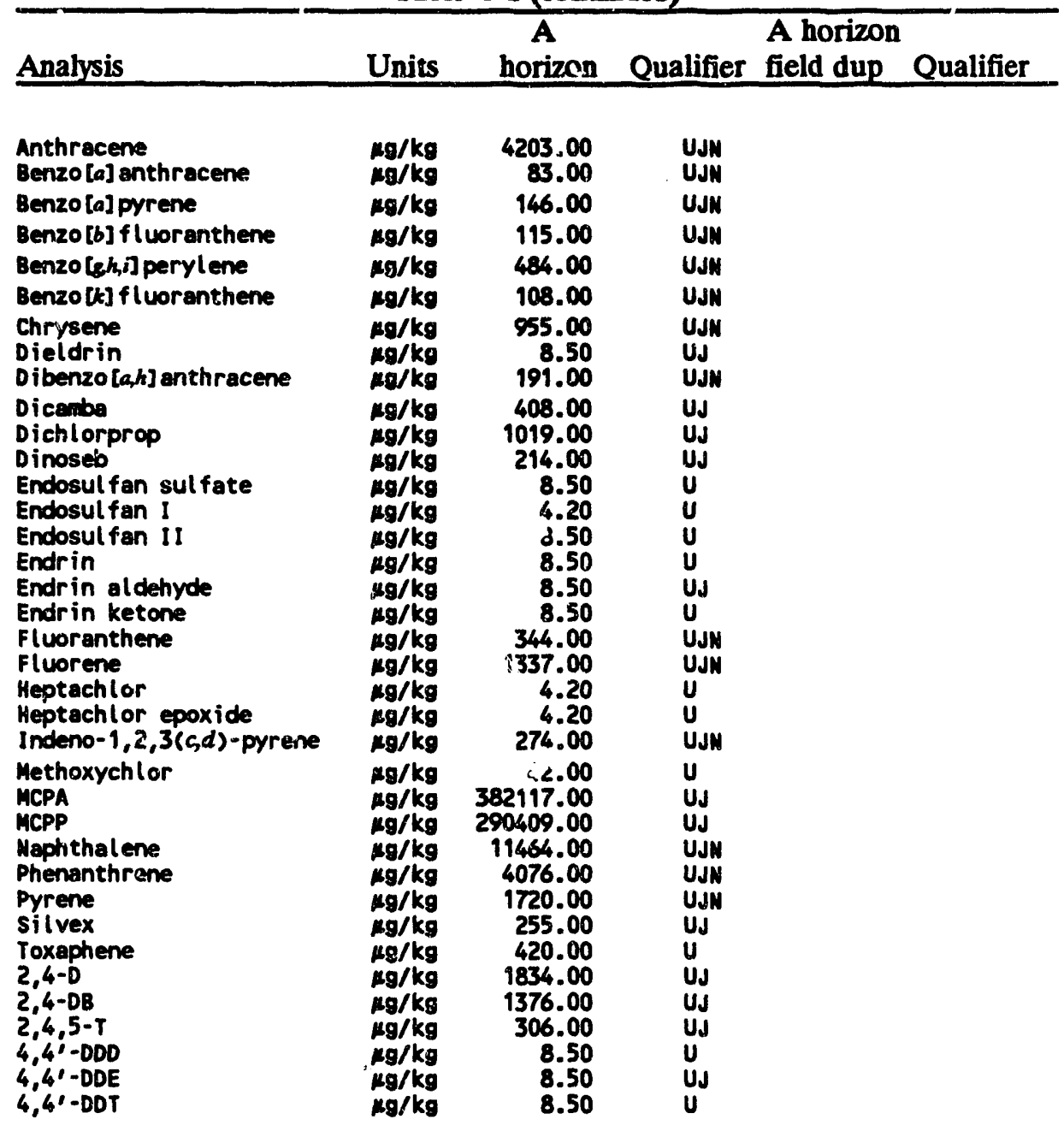

$$
\text { Location }=\text { ORR; Formation }=\text { DISMAL GAP; Site }=43
$$

\begin{tabular}{|c|}
\hline 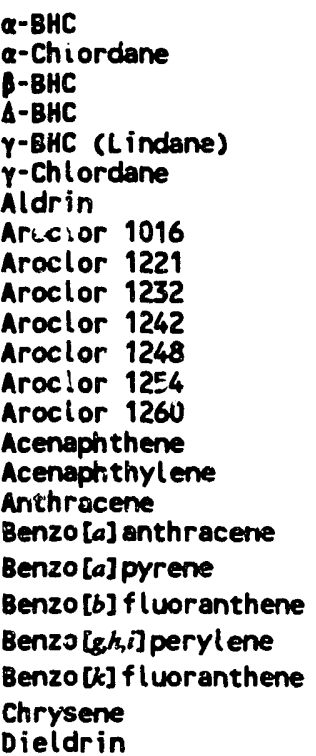 \\
\hline
\end{tabular}

2.10

2.10

2.10

2.10

2.10

2.10

2.10

42.00

85.00

42.00

42.00

42.00

42.00

42.00

233.00

2970.00

850.00

2.00

1.00

2.00

10.00

2.00

19.00

4.20

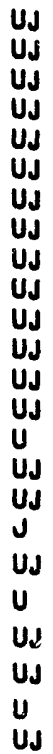


Table C.1 (continued)

\begin{tabular}{|c|c|c|c|c|}
\hline Analysis & Units & $\begin{array}{c}\mathbf{A} \\
\text { horizon }\end{array}$ & $\begin{array}{l}\text { A horizon } \\
\text { Qualifier field dup } \\
\end{array}$ & Qualifier \\
\hline $\begin{array}{l}\text { Dibenzo [a,h] anthracene } \\
\text { Endosul fan sul fate } \\
\text { Endosul fan I } \\
\text { Endosul fan it } \\
\text { Endrin } \\
\text { Endrin al dehyde } \\
\text { Endrin ketone } \\
\text { Fluorene } \\
\text { Heptachlor } \\
\text { Heptachlor epoxide } \\
\text { Indeno-1,2,3(c,d)-pyrene }\end{array}$ & $\begin{array}{l}\mu \mathrm{Mg} / \mathrm{kg} \\
\mathrm{\mu g} / \mathrm{kg} \\
\mu \mathrm{g} / \mathrm{kg} \\
\mathrm{\mu g} / \mathrm{kg} \\
\mu \mathrm{g} / \mathrm{kg} \\
\mathrm{\mu g} / \mathrm{kg} \\
\mu \mathrm{g} / \mathrm{kg} \\
\mu \mathrm{g} / \mathrm{kg} \\
\mu \mathrm{g} / \mathrm{kg} \\
\mu \mathrm{gg} / \mathrm{kg} \\
\mu \mathrm{g} / \mathrm{kg}\end{array}$ & $\begin{array}{r}4.00 \\
4.20 \\
2.10 \\
4.20 \\
4.20 \\
4.20 \\
4.20 \\
270.00 \\
2.10 \\
2.10 \\
60.00\end{array}$ & $\begin{array}{l}\text { U } \\
\text { US } \\
\text { UJ } \\
\text { UJ } \\
\text { US } \\
\text { UJ } \\
\text { UJ } \\
\text { UJ } \\
\text { UJ } \\
\text { UJ } \\
\text { US }\end{array}$ & \\
\hline $\begin{array}{l}\text { Methoxychlor } \\
\text { Naphthalene } \\
\text { Phenanthrene } \\
\text { Pyrene } \\
\text { Toxaphene } \\
4,4^{\prime}-\text {-DD } \\
4,4^{\prime}-D D E \\
4,4^{\prime}-\text {-DT }\end{array}$ & $\begin{array}{l}\mu \mathrm{m} / \mathrm{kg} \\
\mu \mathrm{g} / \mathrm{kg} \\
\mu \mathrm{g} / \mathrm{kg} \\
\mu \mathrm{gg} / \mathrm{kg} \\
\mu \mathrm{g} / \mathrm{kg} \\
\mu \mathrm{g} / \mathrm{kg} \\
\mu \mathrm{g} / \mathrm{kg} \\
\mu \mathrm{g} / \mathrm{kg}\end{array}$ & $\begin{array}{r}22.00 \\
2330.00 \\
830.00 \\
35.00 \\
210.00 \\
4.20 \\
4.20 \\
4.20\end{array}$ & $\begin{array}{l}\text { UJ } \\
\text { UJ } \\
\text { UJ } \\
\text { UJ } \\
\text { UJ } \\
\text { UJ } \\
\text { UJ } \\
\text { UJ } \\
\text { UJ }\end{array}$ & \\
\hline \multicolumn{5}{|c|}{ Location $=$ ORR; Formation $=$ NOLICHUCKY; Site $=3$} \\
\hline $\begin{array}{l}\text { a-BHC } \\
\text { a-Chlordane } \\
\text { p-BHC } \\
\text { A-BHC } \\
\gamma-\text { BHC (Lindane) } \\
\gamma \text {-Chlordane } \\
\text { Aldrin } \\
\text { Aroclor } 1016 \\
\text { Aroclor } 1221 \\
\text { Aroclor } 1232 \\
\text { Aroclor } 1242 \\
\text { Aroclor } 1248 \\
\text { Aroclor } 1254 \\
\text { Aroclor } 1260 \\
\text { Acenaphthene } \\
\text { Acenaphthylene } \\
\text { Anthracene } \\
\text { Benzo[a] pyrene }\end{array}$ & 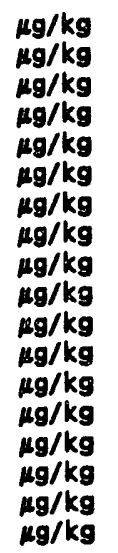 & $\begin{array}{r}2.00 \\
2.00 \\
2.00 \\
2.00 \\
2.00 \\
2.00 \\
2.00 \\
40.00 \\
81.00 \\
40.00 \\
40.00 \\
40.00 \\
40.00 \\
40.00 \\
220.00 \\
2810.00 \\
810.00 \\
3.00\end{array}$ & $\begin{array}{l}: J \\
U \\
U J J \\
U J \\
U J \\
U \\
U \\
U \\
U \\
U \\
U \\
U \\
U \\
U \\
U \\
U \\
U J \\
U J \\
U J \\
U J N\end{array}$ & \\
\hline Benzo $[b]$ fluoranthene & $\mu \mathrm{g} / \mathbf{k g}$ & 2.00 & UJ & \\
\hline Benzo $[g h, i]$ perylene & $\mu g / \mathbf{k g}$ & 9.00 & UJ & \\
\hline Benzo $[k]$ f l woranthene & $\mu \mathrm{g} / \mathrm{kg}$ & 2.00 & UJN & \\
\hline $\begin{array}{l}\text { Dieldrin } \\
\text { Dibenzo }[a, h] \text { anthracene }\end{array}$ & $\begin{array}{l}\mu \mathrm{kg} / \mathrm{kg} \\
\mu \mathrm{g} / \mathrm{kg}\end{array}$ & $\begin{array}{l}4.00 \\
4.00\end{array}$ & $\begin{array}{l}\text { UJ } \\
\text { UJ }\end{array}$ & \\
\hline $\begin{array}{l}\text { Endosul fan sul fate } \\
\text { Endosul fan I } \\
\text { Endosul fan il } \\
\text { Endrin } \\
\text { Endrin al dehyde } \\
\text { ketone } \\
\text { Fluorene } \\
\text { Heptachlor } \\
\text { Heptachlor epoxide } \\
\text { Indeno-1,2,3(c,d)-pyrene }\end{array}$ & $\begin{array}{l}\mu \mathrm{g} / \mathrm{kg} \\
\mu \mathrm{g} / \mathrm{kg} \\
\mu \mathrm{g} / \mathrm{kg} \\
\mu \mathrm{g} / \mathrm{kg} \\
\mu \mathrm{g} / \mathrm{kg} \\
\mu \mathrm{g} / \mathrm{kg} \\
\mu \mathrm{g} / \mathrm{kg} \\
\mu \mathrm{g} / \mathrm{kg} \\
\mu \mathrm{g} / \mathrm{kg} \\
\mu \mathrm{g} / \mathrm{kg}\end{array}$ & $\begin{array}{r}4.00 \\
2.00 \\
4.00 \\
4.00 \\
4.00 \\
4.00 \\
260.00 \\
2.00 \\
2.00 \\
50.00\end{array}$ & $\begin{array}{l}U \\
U \\
U \\
U \\
U \\
U \\
U J \\
U J \\
U \\
U J\end{array}$ & \\
\hline $\begin{array}{l}\text { Methoxychlor } \\
\text { Maphthalene } \\
\text { Phenanthrene } \\
\text { Pyrene } \\
\text { Toxaphene } \\
4,4^{\prime}-\text { DDD } \\
4^{\prime} 4^{\prime}-D D E \\
4,4^{\prime}-D D T\end{array}$ & $\begin{array}{l}\mu \mathrm{g} / \mathrm{kg} \\
\mu \mathrm{g} / \mathrm{kg} \\
\mu \mathrm{g} / \mathrm{kg} \\
\mu \mathrm{g} / \mathrm{kg} \\
\mu \mathrm{g} / \mathrm{kg} \\
\mu \mathrm{g} / \mathrm{kg} \\
\mu \mathrm{g} / \mathrm{kg} \\
\mu \mathrm{g} / \mathrm{kg}\end{array}$ & $\begin{array}{r}20.00 \\
2200.00 \\
780.00 \\
33.00 \\
200.00 \\
4.00 \\
4.00 \\
4.00\end{array}$ & $\begin{array}{l}\text { U } \\
\text { UJ } \\
U J \\
U J N \\
U \\
U J \\
\text { UJ } \\
\text { UJ } \\
\text { UJ }\end{array}$ & \\
\hline
\end{tabular}


Table C.1 (continued)

\begin{tabular}{lcc}
\hline & A & A horizon \\
Analysis & Units & horizon Qualifier field dup Qualifier \\
\hline
\end{tabular}

$$
\text { Location }=\text { ORR; Formation }=\text { NOLICHUCKY; Site }=5
$$

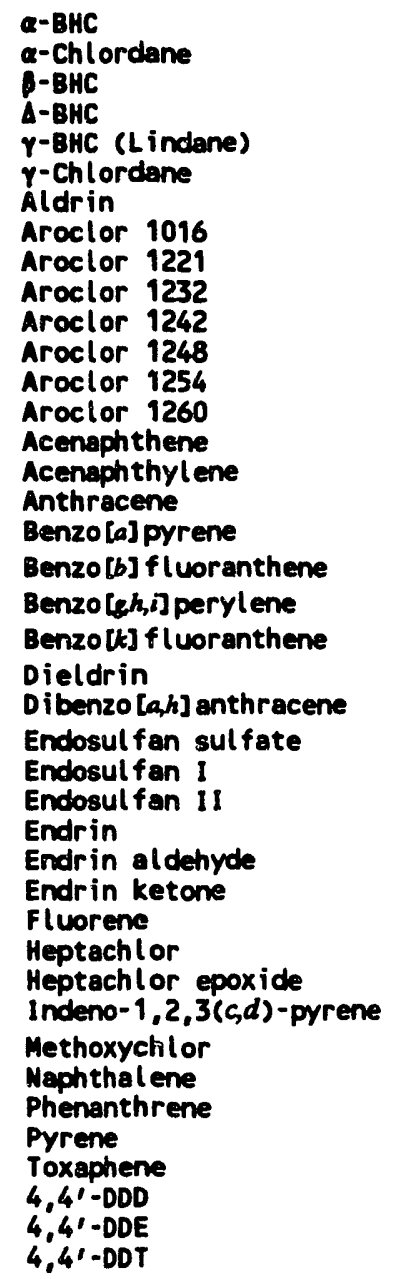

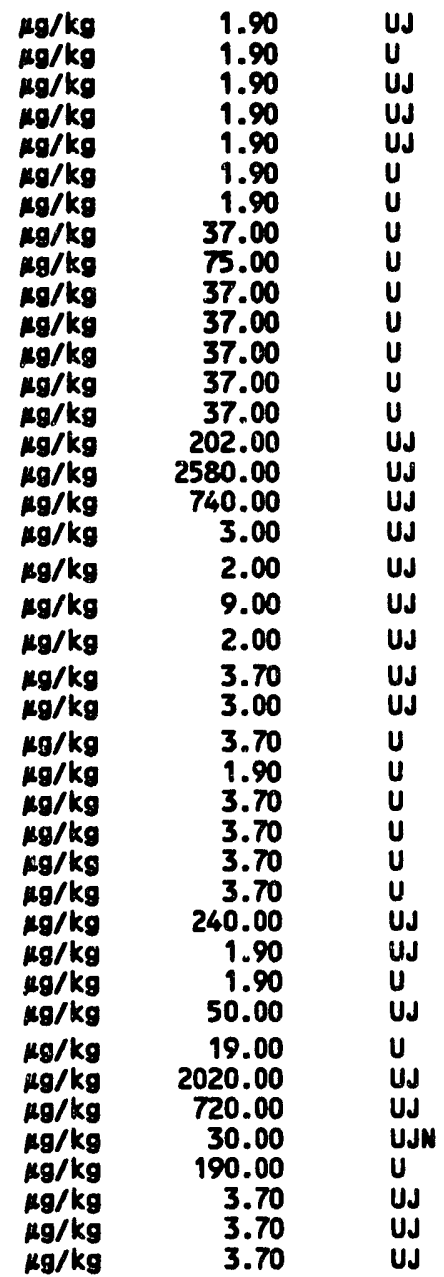

$$
\text { Location }=\text { ORR; Formation }=\text { NOLICHUCKY; Site }=13
$$

\begin{tabular}{|c|c|}
\hline 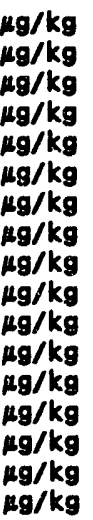 & $\begin{array}{r}2.10 \\
2.10 \\
2.10 \\
2.10 \\
2.10 \\
2.10 \\
2.10 \\
41.00 \\
82.00 \\
41.00 \\
41.00 \\
41.00 \\
41.00 \\
41.00 \\
224.00 \\
286.00 \\
82.00\end{array}$ \\
\hline
\end{tabular}

Q-BHC
a-Chlordare
$\beta$-BHC
A-BHC
$\gamma$-BHC (Lindane)
$\gamma$-Chlordane
Aldrin
Aroclor 1016
Aroclor 1221
Aroclor 1232
Aroclor 1242
Aroclor 1248
Aroclor 1254
Aroclor 1260
Acenaphthene
Acenaph thylene
Anthracene


Table C.1 (continued)

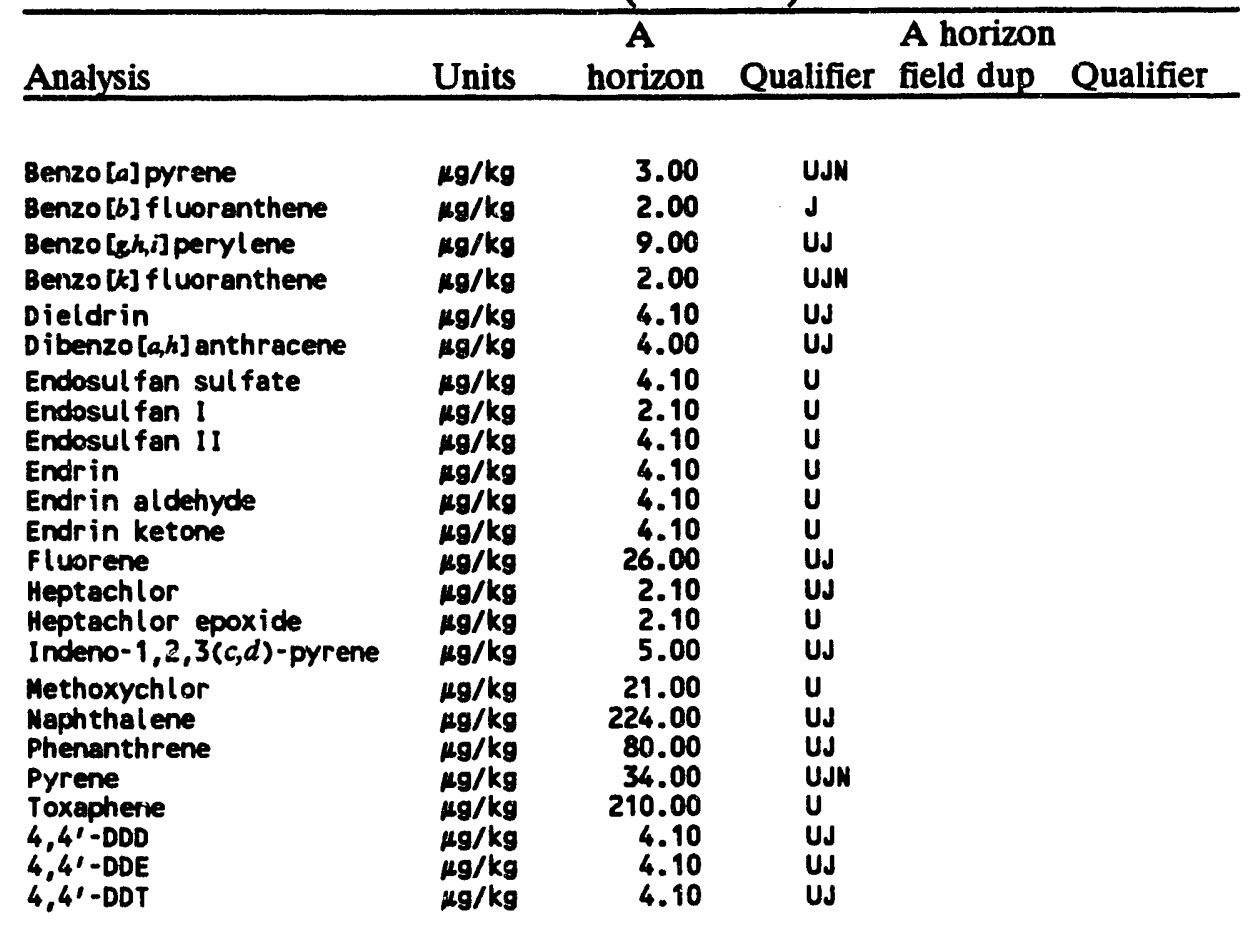

$$
\text { Location }=\text { ORR; Formation }=\text { NOLICHUCKY; Site }=15
$$

\begin{tabular}{|c|c|c|}
\hline $\begin{array}{l}\text { a-BHC } \\
\text { a-Chlordane } \\
\beta \text {-BHC } \\
\text { A-BHC } \\
\gamma \text {-BHC (Lindane) } \\
\gamma \text {-Chlordane } \\
\text { Aldrin } \\
\text { Aroclor } 1016 \\
\text { Aroclor } 1221 \\
\text { Aroclor } 1232 \\
\text { Aroclor } 1242 \\
\text { Aroclor } 1248 \\
\text { Aroclor } 1254 \\
\text { Aroclor } 1260 \\
\text { Acenaphthene } \\
\text { Acenaphthylene } \\
\text { Anthracene } \\
\text { Benzo[a]pyrene }\end{array}$ & $\begin{array}{l}\mu g / \mathbf{k g} \\
\mu g / \mathbf{k g} \\
\mu g / \mathbf{k g} \\
\mu g / \mathbf{k g} \\
\mu g / \mathbf{k g} \\
\mu g / \mathbf{k g} \\
\mu g / \mathbf{k g} \\
\mu g / \mathbf{k g} \\
\mu g / \mathbf{k g} \\
\mu g / \mathbf{k g} \\
\mu g / \mathbf{k g} \\
\mu g / \mathbf{k g} \\
\mu g / \mathbf{k g} \\
\mu g / \mathbf{k g} \\
\mu g / \mathbf{k g} \\
\mu g / \mathbf{k g} \\
\mu g / \mathbf{k g} \\
\mu g / \mathbf{k g}\end{array}$ & $\begin{array}{r}2.00 \\
2.00 \\
2.00 \\
2.00 \\
2.00 \\
2.00 \\
2.00 \\
41.00 \\
82.00 \\
41.00 \\
41.00 \\
41.00 \\
41.00 \\
41.00 \\
224.00 \\
286.00 \\
82.00 \\
3.00\end{array}$ \\
\hline Benzo [b] fluoranthene & $\mu g / k g$ & 2.00 \\
\hline Benzo $[g, h, i]$ peryl ene & $\mu g / k g$ & 9.00 \\
\hline Benzo $[k]$ f luoranthene & $\mu g / \mathrm{kg}$ & 2.00 \\
\hline $\begin{array}{l}\text { Dieldr in } \\
\text { Dibenzo }[a, h] \text { anthracene }\end{array}$ & $\begin{array}{l}\mu g / \mathrm{kg} \\
\mu g / \mathrm{kg}\end{array}$ & $\begin{array}{l}4.10 \\
4.00\end{array}$ \\
\hline $\begin{array}{l}\text { Endosul fan sul fate } \\
\text { Endosul fan I } \\
\text { Endosul fan II } \\
\text { Endrin } \\
\text { Endrin al dehyde } \\
\text { Endrin ketone } \\
\text { Fluorene } \\
\text { Heptachlor } \\
\text { Heptachlor epoxide } \\
\text { Indeno- } 1,2,3(c, d) \text {-pyrene }\end{array}$ & $\begin{array}{l}\mu g / \mathbf{k g} \\
\mu g / \mathbf{k g} \\
\mu g / \mathbf{k g} \\
\mu g / \mathbf{k g} \\
\mu g / \mathbf{k g} \\
\mu g / \mathbf{k g} \\
\mu g / \mathbf{k g} \\
\mu g / \mathbf{k g} \\
\mu g / \mathbf{k g} \\
\mu g / \mathbf{k g}\end{array}$ & $\begin{array}{r}4.10 \\
2.00 \\
4.10 \\
4.10 \\
4.10 \\
4.10 \\
26.00 \\
2.60 \\
2.00 \\
5.00\end{array}$ \\
\hline $\begin{array}{l}\text { Methoxychlor } \\
\text { Maphthalene } \\
\text { Phenanthrene } \\
\text { Pyrene }\end{array}$ & $\begin{array}{l}\mu g / \mathrm{kg} \\
\mu \mathrm{g} / \mathrm{kg} \\
\mu g / \mathrm{kg} \\
\mu g / \mathrm{kg}\end{array}$ & $\begin{array}{r}20.00 \\
224.00 \\
80.00 \\
34.00\end{array}$ \\
\hline
\end{tabular}


Table C.1 (continued)

\begin{tabular}{|c|c|c|c|c|c|}
\hline Analysis & Units & $\begin{array}{c}\mathbf{A} \\
\text { horizon }\end{array}$ & Qualifier & $\begin{array}{l}\text { A horizon } \\
\text { field dup }\end{array}$ & Qualifier \\
\hline $\begin{array}{l}\text { Toxaphene } \\
4,4^{\prime}-D D D \\
4,4^{\prime}-D D E \\
4,4^{\prime}-D D T\end{array}$ & $\begin{array}{l}\mu g / k g \\
\mu g / k g \\
\mu g / k g \\
\mu g / k g\end{array}$ & $\begin{array}{r}200.00 \\
4.10 \\
4.10 \\
4.10\end{array}$ & $\begin{array}{l}U_{U J} \\
\text { UJ } \\
\text { UJ }\end{array}$ & & \\
\hline
\end{tabular}

$$
\text { Location }=\text { ORR; Formation }=\text { NOLICHUCKY; Site }=16
$$

\begin{tabular}{|c|c|c|c|}
\hline $\begin{array}{l}\alpha-B H C \\
a-C h l o r d a n e \\
\beta \text {-BHC } \\
\text { A-BHC } \\
\gamma \text {-BHC (Lindene) } \\
\gamma \text {-Chlordane } \\
\text { Aldrin } \\
\text { Aroclor } 1016 \\
\text { Aroclor } 1221 \\
\text { Aroclor } 1232 \\
\text { Aroclor } 1242 \\
\text { Aroclor } 1248 \\
\text { Aroclor } 1254 \\
\text { Aroclor } 1260 \\
\text { Acenaphthene } \\
\text { Acenaphthylene } \\
\text { Anthracene } \\
\text { Benzo[a]pyrene }\end{array}$ & $\begin{array}{l}\mu g / \mathbf{k g} \\
\mu g / \mathbf{k g} \\
\mu g / \mathbf{k g} \\
\mu g / \mathbf{k g} \\
\mu g / \mathbf{k g} \\
\mu g / \mathbf{k g} \\
\mu g / \mathbf{k g} \\
\mu g / \mathbf{k g} \\
\mu g / \mathbf{k g} \\
\mu g / \mathbf{k g} \\
\mu g / \mathbf{k g} \\
\mu g / \mathbf{k g} \\
\mu g / \mathbf{k g} \\
\mu g / \mathbf{k g} \\
\mu g / \mathbf{k g} \\
\mu g / \mathbf{k g} \\
\mu g / \mathbf{k g} \\
\mu g / \mathbf{k g}\end{array}$ & $\begin{array}{r}2.00 \\
2.00 \\
2.00 \\
2.00 \\
2.00 \\
2.00 \\
2.00 \\
40.00 \\
80.00 \\
40.00 \\
40.00 \\
40.00 \\
40.00 \\
40.00 \\
216.00 \\
276.00 \\
79.00 \\
1.00\end{array}$ & $\begin{array}{l}\mathbf{U J} \\
\mathbf{U} \\
\mathbf{U J} \\
\mathbf{U J} \\
\mathbf{U J} \\
\mathbf{U} \\
\mathbf{U} \\
\mathbf{U} \\
\mathbf{U} \\
\mathbf{U} \\
\mathbf{U} \\
\mathbf{U} \\
\mathbf{U} \\
\mathbf{U} \\
\mathbf{U S} \\
\mathbf{U S} \\
\mathbf{U J} \\
\mathbf{J}\end{array}$ \\
\hline Benzo [b] f l woranthene & $\mathbf{m g} / \mathbf{k g}$ & 1.00 & $J$ \\
\hline Benzo [gh,i] perylene & $\mathbf{M g} / \mathbf{k g}$ & 9.00 & UJ \\
\hline Benzo [k] f luoranthene & $\mathrm{Mg} / \mathrm{kg}$ & 1.00 & $\mathbf{J}$ \\
\hline $\begin{array}{l}\text { Dieldrin } \\
\text { Dibenzo }[a, h] \text { anthracene }\end{array}$ & $\begin{array}{l}\mu g / k g \\
M g / k g\end{array}$ & $\begin{array}{l}4.00 \\
4.00\end{array}$ & $\begin{array}{l}\text { UJ } \\
\text { UJ }\end{array}$ \\
\hline $\begin{array}{l}\text { Endosul fan sulfate } \\
\text { Endosul fan I } \\
\text { Endosul fan II } \\
\text { Endrin } \\
\text { Endrin al dehyde } \\
\text { Endrin ketone } \\
\text { Fluorene } \\
\text { Heptachlor } \\
\text { Heptachlor epoxide } \\
\text { Indeno- } 1,2,3(c, d) \text {-pyrene }\end{array}$ & $\begin{array}{l}\mu g / \mathbf{k g} \\
\mu g / \mathbf{k g} \\
\mu g / k g \\
\mu g / k g \\
\mu g / k g \\
\mu g / k g \\
\mu g / k g \\
\mu g / k g \\
\mu g / k g \\
\mu g / k g\end{array}$ & $\begin{array}{r}4.00 \\
2.00 \\
4.00 \\
4.00 \\
4.00 \\
4.00 \\
25.00 \\
2.00 \\
2.00 \\
5.00\end{array}$ & $\begin{array}{l}\mathbf{U} \\
\mathbf{U} \\
\mathbf{U} \\
\mathbf{U} \\
\mathbf{U} \\
\mathbf{U} \mathbf{J} \\
\mathbf{U J} \\
\mathbf{U} \\
\mathbf{U} \mathbf{J}\end{array}$ \\
\hline $\begin{array}{l}\text { Methoxychlor } \\
\text { Naphthalene } \\
\text { Phenanthrene } \\
\text { Pyrene } \\
\text { Toxaphene } \\
4,4^{\prime} \text {-DDD } \\
4,4^{\prime} \text {-DDE } \\
4,4^{\prime} \text {-DDT }\end{array}$ & 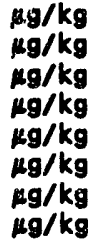 & $\begin{array}{r}21.00 \\
216.00 \\
77.00 \\
32.00 \\
200.00 \\
4.00 \\
4.00 \\
4.00\end{array}$ & $\begin{array}{l}\mathbf{U} \\
\mathbf{U J} \\
\mathbf{U J} \\
\mathbf{U} \mathbf{J N} \\
\mathbf{U} \\
\mathbf{U J} \\
\mathbf{U J} \\
\mathbf{U J}\end{array}$ \\
\hline
\end{tabular}

$$
\text { Location }=\text { ORR; Formation }=\text { NOLICHUCKY; Site }=21
$$

a-BHC

a-Chlordane

p-BHC

$\triangle-B H C$

$\gamma$-BHC (Lindane)

$\gamma$-Chlordane

Aldrin

Aroclor 1016

Aroclor 1221

Aroclor 1232

Aroclor 1242

Aroclor 1248

$\begin{array}{lll}\mu g / \mathrm{kg} & 2.20 & U J \\ \mu g / \mathrm{kg} & 2.20 & U \\ \mu \mathrm{g} / \mathrm{kg} & 2.20 & U J \\ \mu g / \mathrm{kg} & 2.20 & U J \\ \mu g / \mathrm{kg} & 2.20 & U J \\ \mu g / \mathrm{kg} & 2.20 & U \\ \mu g / \mathrm{kg} & 2.20 & U \\ \mu g / \mathrm{kg} & 44.00 & U \\ \mu g / \mathrm{kg} & 88.00 & U \\ \mu g / k g & 44.00 & U \\ \mu g / \mathrm{kg} & 44.00 & U \\ \mu g / \mathrm{kg} & 44.00 & U\end{array}$


Table C.1 (continued)

\begin{tabular}{|c|c|c|c|}
\hline Analysis & Units & $\begin{array}{c}\mathbf{A} \\
\text { horizon }\end{array}$ & Qualifie \\
\hline $\begin{array}{l}\text { Aroclor } 1254 \\
\text { Aroclor } 1260 \\
\text { Acenaphthene } \\
\text { Acenaphthylene } \\
\text { Anthracene } \\
\text { Benzo [a] pyrene }\end{array}$ & $\begin{array}{l}\mu g / k g \\
\mu g / k g \\
\mu g / k g \\
\mu g / k g \\
\mu g / k g \\
\mu g / k g\end{array}$ & $\begin{array}{r}44.00 \\
44.00 \\
240.00 \\
3070.00 \\
880.00 \\
3.00\end{array}$ & $\begin{array}{l}\mathbf{U} \\
\mathbf{U} \\
\mathbf{U J} \\
\mathbf{U J} \\
\mathbf{U J} \\
\mathbf{U J}\end{array}$ \\
\hline Benzo [b] fluoranthene & $\mathrm{kg} / \mathrm{kg}$ & 2.00 & UJ \\
\hline Benzo $[g h, i]$ perylene, & $\mu g / k g$ & 10.00 & UJ \\
\hline Benzo $[k]$ fluoranthene & $\mathrm{ng} / \mathrm{kg}$ & 2.00 & UJ \\
\hline $\begin{array}{l}\text { Dieldr in } \\
\text { Dibenzo }[a, h] \text { anthracene }\end{array}$ & $\begin{array}{l}\mathbf{M g} / \mathbf{k g} \\
\mathbf{M g} / \mathbf{k g}\end{array}$ & $\begin{array}{l}4.40 \\
4.00\end{array}$ & $\begin{array}{l}\text { UJ } \\
\text { UJ }\end{array}$ \\
\hline $\begin{array}{l}\text { Endosul fan sul fate } \\
\text { Endosul fan I } \\
\text { Endosul fan II } \\
\text { Endrin } \\
\text { Endr in al dehyde } \\
\text { Endrin ketone } \\
\text { Fluorene } \\
\text { Heptachlor } \\
\text { Heptachlor epoxide } \\
\text { Indeno-1,2,3(c,d)-pyrene }\end{array}$ & $\begin{array}{l}\mathrm{Mg} / \mathrm{kg} \\
\mathrm{Mg} / \mathrm{kg} \\
\mathrm{Mg} / \mathrm{kg} \\
\mathrm{Mg} / \mathrm{kg} \\
\mathrm{\mu g} / \mathrm{kg} \\
\mathrm{Mg} / \mathrm{kg} \\
\mathrm{\mu g} / \mathrm{kg} \\
\mathrm{Mg} / \mathrm{kg} \\
\mathrm{Mg} / \mathrm{kg} \\
\mathrm{\mu g} / \mathrm{kg}\end{array}$ & $\begin{array}{r}4.40 \\
2.20 \\
4.40 \\
4.40 \\
4.40 \\
4.40 \\
280.00 \\
2.20 \\
2.20 \\
60.00\end{array}$ & $\begin{array}{l}U \\
U \\
U \\
U \\
U \\
U \\
U J \\
U J \\
U \\
U J\end{array}$ \\
\hline $\begin{array}{l}\text { Methoxychlor } \\
\text { Naphthalene } \\
\text { Phenanthrene } \\
\text { Pyrene } \\
\text { Toxaphene } \\
4,4^{\prime}-\text { DDD } \\
4^{\prime} 4^{\prime} \text {-DDE } \\
4,4^{\prime} \text {-DDT }\end{array}$ & $\begin{array}{l}\mathrm{Mg} / \mathrm{kg} \\
\mathrm{\mu g} / \mathrm{kg} \\
\mathrm{\mu g} / \mathrm{kg} \\
\mathrm{Mg} / \mathrm{kg} \\
\mathrm{\mu g} / \mathbf{k g} \\
\mathrm{\mu g} / \mathbf{k g} \\
\mathrm{\mu g} / \mathrm{kg} \\
\mathrm{\mu g} / \mathrm{kg}\end{array}$ & $\begin{array}{r}22.00 \\
2400.00 \\
850.00 \\
36.00 \\
220.00 \\
4.40 \\
4.40 \\
4.40\end{array}$ & $\begin{array}{l}U \\
\text { UJ } \\
\text { UJN } \\
\text { UJN } \\
U \\
\text { UJ } \\
\text { UJ } \\
\text { UJ }\end{array}$ \\
\hline
\end{tabular}

Location $=$ ORR; Formation $=$ NOLICHUCKY; Site $=23$

\begin{tabular}{|c|c|c|}
\hline $\begin{array}{l}\alpha \text {-BHC } \\
\alpha \text {-Chlordane } \\
\beta \text {-BHC } \\
\text { A-BHC } \\
\gamma \text {-BHC (Lindane) } \\
\gamma \text {-Chlordane } \\
\text { Aldrin } \\
\text { Aroclor } 1016 \\
\text { Aroclor } 1221 \\
\text { Aroclor } 1232 \\
\text { Aroclor } 1242 \\
\text { Aroclor } 1248 \\
\text { Aroclor } 1254 \\
\text { Aroclor } 1260 \\
\text { Acenaphthene } \\
\text { Acenaphthylene } \\
\text { Anthracene } \\
\text { Benzo[a] pyrene }\end{array}$ & $\begin{array}{l}\mu g / k g \\
\mu g / \mathbf{k g} \\
\mu g / \mathbf{k g} \\
\mu g / \mathbf{k g} \\
\mu g / \mathbf{k g} \\
\mu g / \mathbf{k g} \\
\mu g / \mathbf{k g} \\
\mu g / \mathbf{k g} \\
\mu g / \mathbf{k g} \\
\mu g / \mathbf{k g} \\
\mu g / \mathbf{k g} \\
\mu g / \mathbf{k g} \\
\mu g / \mathbf{k g} \\
\mu g / \mathbf{k g} \\
\mu g / \mathbf{k g} \\
\mu g / \mathbf{k g} \\
\mu g / \mathbf{k g} \\
\mu g / \mathbf{k g}\end{array}$ & $\begin{array}{r}2.00 \\
2.00 \\
2.00 \\
2.00 \\
2.00 \\
2.00 \\
2.00 \\
40.00 \\
79.00 \\
40.00 \\
40.00 \\
40.00 \\
40.00 \\
40.00 \\
112.00 \\
2750.00 \\
790.00 \\
3.00\end{array}$ \\
\hline $\begin{array}{l}\text { Benzo }[b] \text { f l woranthene } \\
\text { Benzo }[g h, i] \text { perylene }\end{array}$ & $\begin{array}{l}\mu \mathrm{gg} / \mathbf{k g} \\
\mu \mathrm{g} / \mathrm{kg}\end{array}$ & $\begin{array}{l}2.00 \\
9.00\end{array}$ \\
\hline Benzo [k] fluoranthene & $\mu \mathrm{g} / \mathrm{kg}$ & 2.00 \\
\hline $\begin{array}{l}\text { Dieldrin } \\
\text { Dibenzo }[a, h] \text { anthracene }\end{array}$ & $\begin{array}{l}\mu g / k g \\
\mu g / k g\end{array}$ & $\begin{array}{l}4.00 \\
4.00\end{array}$ \\
\hline $\begin{array}{l}\text { Endosul fan sul fate } \\
\text { Endosulfan I } \\
\text { Endosul fan II } \\
\text { Endrin } \\
\text { Endrin aldehyde } \\
\text { Endrin ketone } \\
\text { Fluorene } \\
\text { Heptachlor } \\
\text { Heptachlor epoxide }\end{array}$ & $\begin{array}{l}\mu g / \mathrm{kg} \\
\mu \mathrm{g} / \mathrm{kg} \\
\mu g / \mathrm{kg} \\
\mu g / \mathrm{kg} \\
\mu g / \mathrm{kg} \\
\mu \mathrm{g} / \mathrm{kg} \\
\mu \mathrm{g} / \mathrm{kg} \\
\mu \mathrm{g} / \mathrm{kg} \\
\mu \mathrm{g} / \mathrm{kg}\end{array}$ & $\begin{array}{l}4.00 \\
2.00 \\
4.00 \\
4.00 \\
4.00 \\
4.00 \\
50.00 \\
2.00 \\
2.00\end{array}$ \\
\hline
\end{tabular}




\section{C-28}

Table C.1 (continued)

\begin{tabular}{|c|c|c|c|c|c|}
\hline Analysis & Units & $\begin{array}{c} \\
\text { horizon }\end{array}$ & Qualifier & $\begin{array}{l}\text { A horizon } \\
\text { field dup }\end{array}$ & Qualifier \\
\hline $\begin{array}{l}\text { Indeno-1,2,3(c,d)-pyrene } \\
\text { Methoxychlor } \\
\text { Maphthalene } \\
\text { Phenanthrene } \\
\text { Pyrene } \\
\text { Toxaphene } \\
4,4^{\prime}-D D D \\
4,4^{\prime}-D D E \\
4^{\prime} 4^{\prime}-D D T\end{array}$ & $\begin{array}{l}\mu g / k g \\
\mu g / k g \\
\mu g / k g \\
\mu g / k g \\
\mu g / k g \\
\mu g / k g \\
\mu g / k g \\
\mu g / k g \\
\mu g / k g\end{array}$ & $\begin{array}{r}50.00 \\
20.00 \\
2150.00 \\
760.00 \\
32.00 \\
200.00 \\
4.00 \\
4.00 \\
4.00\end{array}$ & $\begin{array}{l}\text { UJ } \\
U \\
\text { UJ } \\
\text { UJ } \\
\text { UJW } \\
U \\
U_{J} \\
\text { UJ } \\
\text { UJ }\end{array}$ & & \\
\hline
\end{tabular}

$$
\text { Location }=\text { ORR; Formation }=\text { NOLICHUCKY; Site }=24
$$

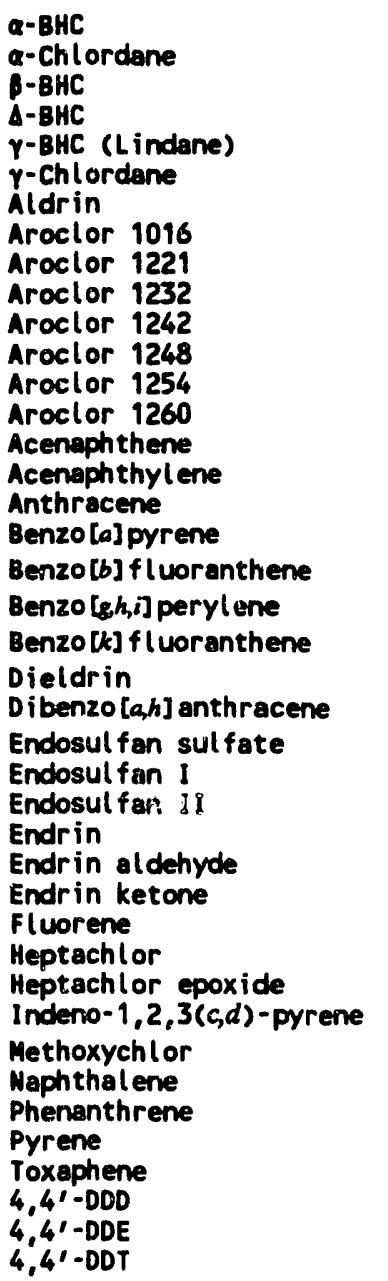

$\begin{array}{lrl}\mu g / k g & 2.10 & U J \\ \mu g / k g & 2.10 & U \\ \mu g / k g & 2.10 & U J \\ \mu g / k g & 2.10 & U J \\ \mu g / k g & 2.10 & U J \\ \mu g / k g & 2.10 & U \\ \mu g / k g & 2.10 & U \\ \mu g / k g & 43.00 & U \\ \mu g / k g & 85.00 & U \\ \mu g / k g & 43.00 & U \\ \mu g / k g & 43.00 & U \\ \mu g / k g & 43.00 & U \\ \mu g / k g & 43.00 & U \\ \mu g / k g & 43.00 & U \\ \mu g / k g & 230.00 & U J \\ \mu g / k g & 2940.00 & U J \\ \mu g / k g & 840.00 & U J \\ \mu g / k g & 3.00 & U J \\ \mu g / k g & 2.00 & U J \\ \mu g / k g & 10.00 & U J \\ \mu g / k g & 2.00 & U J \\ \mu g / k g & 4.30 & U J \\ \mu g / k g & 4.00 & U J \\ \mu g / k g & 4.30 & U \\ \mu g / k g & 2.10 & U \\ \mu g / k g & 4.30 & U \\ \mu g / k g & 4.30 & U \\ \mu g / k g & 4.30 & U \\ \mu g / k g & 4.30 & U \\ \mu g / k g & 270.00 & U J \\ \mu g / k g & 2.10 & U J \\ \mu g / k g & 2.10 & U \\ \mu g / k g & 60.00 & U J \\ \mu g / k g & 21.00 & U \\ \mu g / k g & 2300.00 & U J \\ \mu g / k g & 820.00 & U J N \\ \mu g / k g & 10.00 & J \\ \mu g / k g & 210.00 & U \\ \mu g / k g & 4.30 & U J \\ \mu g / k g & 4.30 & U J \\ \mu g / k g & 4.30 & U J\end{array}$

$$
\text { Location }=\text { ORR; Formation }=\text { NOLICHUCKY } ; \text { Site }=25
$$

a-BHC

a-Chlordane

P-BHC

$\triangle-B H C$

$\gamma$-BHC (Lindane)

$\gamma$-Chlordane

Aldrin ing/kg

$\boldsymbol{\mu g} / \mathbf{k g}$

$\mathrm{mg} / \mathrm{kg}$

$\mathrm{\mu g} / \mathrm{kg}$

$\mu g / k g$

$\mathrm{gg} / \mathrm{kg}$

$\mu \mathrm{g} / \mathrm{kg}$
2.00

2.00

2.00

2.00

2.00

2.00

2.00 
Table C.1 (continued)

\begin{tabular}{|c|c|c|c|}
\hline Analysis & Units & $\begin{array}{c}\mathbf{A} \\
\text { horizon } \\
\end{array}$ & Quailifie \\
\hline $\begin{array}{l}\text { Aroclor } 1016 \\
\text { Aroclor } 1221 \\
\text { Aroclor } 1232 \\
\text { Aroclor } 1242 \\
\text { Aroclor } 1248 \\
\text { Aroclor } 1254 \\
\text { Aroclor } 1260 \\
\text { Acenaphthene } \\
\text { Acenaphthylene } \\
\text { Anthracene } \\
\text { Benzo[a] pyrene }\end{array}$ & $\begin{array}{l}\mathrm{Mg} / \mathrm{kg} \\
\mathrm{Mg} / \mathrm{kg} \\
\mathrm{Mg} / \mathrm{kg} \\
\mathrm{Mg} / \mathrm{kg} \\
\mathrm{Mg} / \mathrm{kg} \\
\mathrm{Mg} / \mathrm{kg} \\
\mathrm{Mg} / \mathrm{kg} \\
\mathrm{Mg} / \mathrm{kg} \\
\mathrm{Mg} / \mathrm{kg} \\
\mathrm{Mg} / \mathrm{kg} \\
\mathrm{Mg} / \mathrm{kg}\end{array}$ & $\begin{array}{r}40.00 \\
80.00 \\
40.00 \\
40.00 \\
40.00 \\
40.00 \\
40.00 \\
220.00 \\
2810.00 \\
810.00 \\
3.00\end{array}$ & 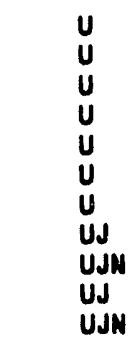 \\
\hline Benzo $[b]$ f l woranthene & mg/kg & 2.00 & UJ \\
\hline Benzo[shi] perylene & Mg/kg & 9.00 & UJN \\
\hline Benzo [k] fluoranthene & $\mathbf{m g} / \mathbf{k g}$ & 2.00 & UJN \\
\hline $\begin{array}{l}\text { Dieldrin } \\
\text { Dibenzo }[a, h] \text { anthracene }\end{array}$ & $\begin{array}{l}\mathrm{Mg} / \mathrm{kg} \\
\mathrm{\mu g} / \mathbf{k g}\end{array}$ & $\begin{array}{l}4.00 \\
4.00\end{array}$ & UJ \\
\hline $\begin{array}{l}\text { Endosul fan sul fate } \\
\text { Endosul fan I } \\
\text { Endosul fan II } \\
\text { Endrin } \\
\text { Endrin al dehyde } \\
\text { Endrin ketone } \\
\text { Fluorene } \\
\text { Heptachlor } \\
\text { Heptachlor epoxide } \\
\text { Indeno- } 1,2,3(c, d) \text {-pyrene }\end{array}$ & $\begin{array}{l}\mu g / \mathbf{k g} \\
\mu g / \mathbf{k g} \\
\mu g / \mathbf{k g} \\
\mu g / \mathbf{k g} \\
\mu g / \mathbf{k g} \\
\mu g / \mathbf{k g} \\
\mu g / \mathbf{k g} \\
\mu g / \mathbf{k g} \\
\mu g / \mathbf{k g} \\
\mu g / \mathbf{k g}\end{array}$ & $\begin{array}{r}4.00 \\
2.00 \\
4.00 \\
4.00 \\
4.00 \\
4.00 \\
260.00 \\
2.60 \\
2.00 \\
50.00\end{array}$ & $\begin{array}{l}U \\
U \\
U \\
U \\
U \\
U \\
U J \\
U J \\
U \\
U S\end{array}$ \\
\hline $\begin{array}{l}\text { Methoxychlor } \\
\text { Maphthalene } \\
\text { Phenanthrene } \\
\text { Pyrene } \\
\text { Toxaphene } \\
4,4^{\prime} \text {-DDD } \\
4,4^{\prime} \text {-DDE } \\
4,4^{\prime}-\text { DDT }\end{array}$ & $\begin{array}{l}\mu g / \mathbf{k g} \\
\mu g / \mathbf{k g} \\
\mu g / \mathbf{k g} \\
\mu g / \mathbf{k g} \\
\mu g / \mathbf{k g} \\
\mu g / \mathbf{k g} \\
\mu g / \mathbf{k g} \\
\mu g / \mathbf{k g}\end{array}$ & $\begin{array}{r}20.00 \\
2200.00 \\
780.00 \\
33.00 \\
200.00 \\
4.00 \\
4.00 \\
4.00\end{array}$ & $\begin{array}{l}\text { U } \\
\text { UJ } \\
\text { UJ } \\
\text { UJN } \\
\mathbf{U} \\
\text { US } \\
\text { UJ } \\
\text { UJ }\end{array}$ \\
\hline
\end{tabular}

Location $=$ ORR; Formation $=$ NOLICHUCKY; Site $=28$

\begin{tabular}{|c|c|c|c|}
\hline $\begin{array}{l}\text { Q-BHC } \\
\alpha \text {-Chlordane } \\
\beta \text {-BHC } \\
\text { A-BHC } \\
\gamma \text {-BHC (Lindane) } \\
\gamma \text {-Chlordane } \\
\text { Aldrin } \\
\text { Aroclor } 1016 \\
\text { Aroclor } 1221 \\
\text { Aroclor } 1232 \\
\text { Aroclor } 1242 \\
\text { Aroclor } 1248 \\
\text { Aroclor } 1254 \\
\text { Aroclor } 1260 \\
\text { Acenaphthene } \\
\text { Acenaphthylene } \\
\text { Anthracene } \\
\text { Benzo [a] pyrene }\end{array}$ & $\begin{array}{l}\mu g / \mathbf{k g} \\
\mu g / \mathbf{k g} \\
\mu g / \mathbf{k g} \\
\mu g / \mathbf{k g} \\
\mu g / \mathbf{k g} \\
\mu g / \mathbf{k g} \\
\mu g / \mathbf{k g} \\
\mu g / \mathbf{k g} \\
\mu g / \mathbf{k g} \\
\mu g / \mathbf{k g} \\
\mu g / \mathbf{k g} \\
\mu g / \mathbf{k g} \\
\mu g / \mathbf{k g} \\
\mu g / \mathbf{k g} \\
\mu g / \mathbf{k g} \\
\mu g / \mathbf{k g} \\
\mu g / \mathbf{k g} \\
\mu g / \mathbf{k g}\end{array}$ & $\begin{array}{r}1.90 \\
1.90 \\
1.90 \\
1.90 \\
1.90 \\
1.90 \\
1.90 \\
38.00 \\
76.00 \\
38.00 \\
38.00 \\
38.00 \\
38.00 \\
38.00 \\
207.00 \\
2650.00 \\
760.00 \\
3.00\end{array}$ & $\begin{array}{l}U J \\
U \\
U J \\
U J \\
U J \\
U \\
U \\
U \\
U \\
U \\
U \\
U \\
U \\
U \\
U J \\
U J N \\
U J \\
U J\end{array}$ \\
\hline Benzo $[b]$ f luoranthene & $\mu \mathrm{g} / \mathrm{kg}$ & 2.00 & Us \\
\hline Benzo $[\mathrm{g} h, i]$ peryl ene & $\mu g / \mathrm{kg}$ & 9.00 & UJ \\
\hline Benzo $[k]$ fluoranthene & $\mu g / \mathrm{kg}$ & 2.00 & UJ \\
\hline $\begin{array}{l}\text { Dieldrin } \\
\text { Dibenzo }[a, h] \text { anthracene }\end{array}$ & $\begin{array}{l}\mu \mathrm{g} / \mathrm{kg} \\
\mu \mathrm{g} / \mathrm{kg}\end{array}$ & $\begin{array}{l}3.80 \\
3.00\end{array}$ & $\begin{array}{l}\text { UJ } \\
\text { UJ }\end{array}$ \\
\hline $\begin{array}{l}\text { Endosulfan sul fate } \\
\text { Endosul fan I } \\
\text { Endosul fan II } \\
\text { Endrin }\end{array}$ & $\begin{array}{l}\mu \mathrm{g} / \mathrm{kg} \\
\mu \mathrm{g} / \mathrm{kg} \\
\mu \mathrm{g} / \mathrm{kg} \\
\mu \mathrm{g} / \mathrm{kg}\end{array}$ & $\begin{array}{l}3.80 \\
1.90 \\
3.80 \\
3.80\end{array}$ & $\begin{array}{l}U \\
U \\
U \\
U\end{array}$ \\
\hline
\end{tabular}


Table C.1 (continued)

\begin{tabular}{|c|c|c|c|c|c|}
\hline Analysis & Units & $\begin{array}{c}\text { A } \\
\text { horizon }\end{array}$ & Qualifier & $\begin{array}{l}\text { A horizon } \\
\text { field dup }\end{array}$ & Qualifier \\
\hline $\begin{array}{l}\text { Endrin al dehyde } \\
\text { Endrin ketone } \\
\text { Fluorene } \\
\text { Heptachlor } \\
\text { Heptachlor epoxide } \\
\text { Indeno- } 1,2,3(c, d) \text {-pyrene }\end{array}$ & $\begin{array}{l}\mathrm{Mg} / \mathrm{kg} \\
\mathrm{Mg} / \mathrm{kg} \\
\mathrm{Mg} / \mathrm{kg} \\
\mathrm{\mu g} / \mathrm{kg} \\
\mathrm{Mg} / \mathrm{kg} \\
\mathrm{\mu g} / \mathrm{kg}\end{array}$ & $\begin{array}{r}3.80 \\
3.80 \\
240.00 \\
1.90 \\
1.90 \\
50.00\end{array}$ & $\begin{array}{l}U \\
U \\
U \\
U J \\
U J \\
U \\
U J\end{array}$ & & \\
\hline $\begin{array}{l}\text { Methoxychlor } \\
\text { Naphthal ene } \\
\text { Phenanthrene } \\
\text { Pyrene } \\
\text { Toxaphene } \\
4,4^{\prime}-\text { DDD } \\
4,4^{\prime}-\text {-DE } \\
4,4^{\prime}-\text { DD }\end{array}$ & $\begin{array}{l}\mu g / k g \\
\mu g / k g \\
\mu g / k g \\
\mu g / k g \\
\mu g / k g \\
\mu g / k g \\
\mu g / k g \\
\mu g / k g\end{array}$ & $\begin{array}{r}19.00 \\
2070.00 \\
740.00 \\
31.00 \\
190.00 \\
3.80 \\
3.80 \\
3.80\end{array}$ & $\begin{array}{l}U \\
U J \\
U J \\
U J W \\
U \\
U J \\
U J \\
U J \\
U J\end{array}$ & & \\
\hline
\end{tabular}

$$
\text { Location }=\text { ORR; Formation }=\text { NOLICHUCKY; Site }=31
$$

\begin{tabular}{|c|c|}
\hline 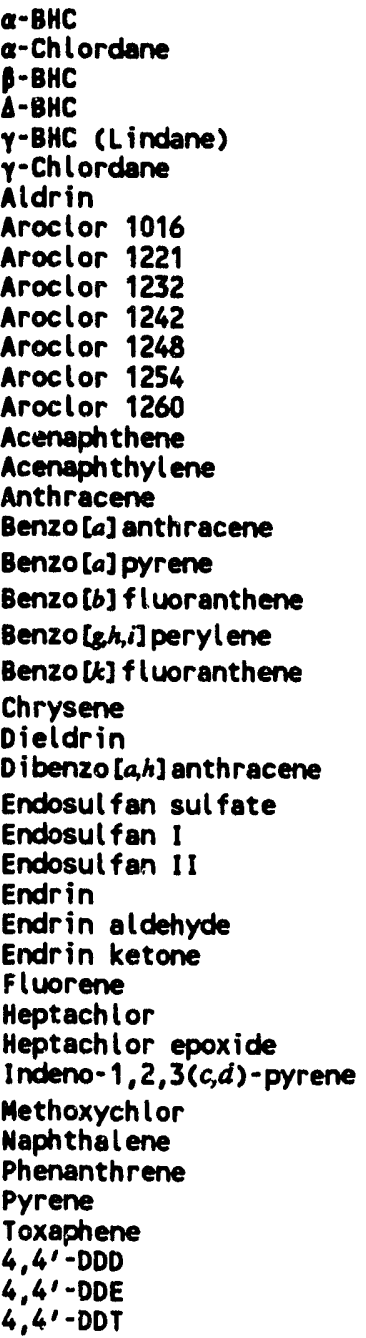 & $\begin{array}{l}\mu g / k g \\
\mu g / k g \\
\mu g / k g \\
\mu g / k g \\
\mu g / k g \\
\mu g / k g \\
\mu g / k g \\
\mu g / k g \\
\mu g / k g \\
\mu g / k g \\
\mu g / k g \\
\mu g / k g \\
\mu g / k g \\
\mu g / k g \\
\mu g / k g \\
\mu g / k g \\
\mu g / k g \\
\mu g / k g \\
\mu g / k g \\
\mu g / k g \\
\mu g / k g \\
\mu g / k g \\
\mu g / k g \\
\mu g / k g \\
\mu g / k g \\
\mu g / k g \\
\mu g / k g \\
\mu g / k g \\
\mu g / k g \\
\mu g / k g \\
\mu g / k g \\
\mu g / k g \\
\mu g / k g \\
\mu g / k g \\
\mu g / k g \\
\mu g / k g \\
\mu g / k g \\
\mu g / k g \\
\mu g / k g \\
\mu g / k g \\
\mu g / k g\end{array}$ \\
\hline
\end{tabular}

$\begin{array}{rl}2.00 & U J \\ 2.00 & U \\ 2.00 & U J \\ 2.00 & U J \\ 2.00 & U J \\ 2.00 & U \\ 2.00 & U \\ 41.00 & U \\ 81.00 & U \\ 41.00 & U \\ 41.00 & U \\ 41.00 & U \\ 41.00 & U \\ 41.00 & U \\ 221.00 & U J \\ 283.00 & U J \\ 81.00 & U J \\ 2.00 & U J \\ 3.00 & U J \\ 1.00 & J \\ 9.00 & U J \\ 2.00 & U J \\ 18.00 & U J \\ 4.10 & U J \\ 4.00 & U J \\ 4.10 & U \\ 2.00 & U \\ 4.10 & U \\ 4.10 & U \\ 4.10 & U \\ 4.10 & U \\ 26.00 & U J N \\ 2.00 & U J \\ 2.00 & U \\ 5.00 & U J \\ 20.00 & U \\ 221.00 & U J \\ 79.00 & U J \\ 33.00 & U J \\ 200.00 & U \\ 4.10 & U J \\ 4.10 & U J \\ 4.10 & U J \\ & \end{array}$


Table C.1 (continued)

\begin{tabular}{lcc}
\hline & A & A horizon \\
Analysis & Units & horizon Qualifier field dup Qualifier \\
\hline
\end{tabular}

$$
\text { Location }=\text { ORR; Formation }=\text { NOLICHUCKY; Site }=42
$$

\begin{tabular}{|c|c|c|}
\hline $\begin{array}{l}\text { a-BHC } \\
\text { a-Chlordane } \\
\text {-BHC } \\
\text { A-BHC } \\
\gamma \text {-BHC (Lindane) } \\
\gamma \text {-Chlordane } \\
\text { Aldrin } \\
\text { Aroclor } 1016 \\
\text { Aroclor } 1221 \\
\text { Aroclor } 1232 \\
\text { Aroclor } 1242 \\
\text { Aroclor } 1248 \\
\text { Aroclor } 1254 \\
\text { Aroclor } 1260 \\
\text { Acenaphthene } \\
\text { Acenaphthylene } \\
\text { Anthracene } \\
\text { Benzo[a] anthracene }\end{array}$ & $\begin{array}{l}\mu g / k g \\
\mu g / k g \\
\mu g / k g \\
\mu g / k g \\
\mu g / k g \\
\mu g / k g \\
\mu g / k g \\
\mu g / k g \\
\mu g / k g \\
\mu g / k g \\
\mu g / k g \\
\mu g / k g \\
\mu g / k g \\
\mu g / k g \\
\mu g / k g \\
\mu g / k g \\
\mu g / k g \\
\mu g / k g\end{array}$ & $\begin{array}{r}2.00 \\
2.00 \\
2.00 \\
2.00 \\
2.00 \\
2.00 \\
2.00 \\
39.00 \\
78.00 \\
39.00 \\
39.00 \\
39.00 \\
39.00 \\
39.00 \\
218.00 \\
2780.00 \\
800.00 \\
2.00\end{array}$ \\
\hline Benzo [a] pyrene & $\mu g / \mathbf{k g}$ & 3.00 \\
\hline Benzo $[b]$ f l woranthene & $\mu g / k g$ & 1.00 \\
\hline Benzo $[s h, i]$ perylene & $\mathrm{Mg} / \mathrm{kg}$ & 9.00 \\
\hline Benzo [k] fluoranthene & $\mu g / k g$ & 2.00 \\
\hline $\begin{array}{l}\text { Chrysene } \\
\text { Dieldrin } \\
\text { Dibenzo }[a, h] \text { anthracene }\end{array}$ & $\begin{array}{l}\mu g / k g \\
\mu g / k g \\
\mu g / k g\end{array}$ & $\begin{array}{r}18.00 \\
3.90 \\
4.00\end{array}$ \\
\hline $\begin{array}{l}\text { Endosulfan sulfate } \\
\text { Endosulfan I } \\
\text { Endosulfan } 11 \\
\text { Endrin } \\
\text { Endrin al dehyde } \\
\text { Endrin ketone } \\
\text { Fluorene } \\
\text { Heptachlor } \\
\text { Heptachlor epoxide } \\
\text { Indeno-1,2,3(c,d)-pyrene }\end{array}$ & $\begin{array}{l}\mu g / \mathbf{k g} \\
\mu g / \mathbf{k g} \\
\mu g / k g \\
\mu g / k g \\
\mu g / k g \\
\mu g / k g \\
\mu g / k g \\
\mu g / k g \\
\mu g / k g \\
\mu g / k g\end{array}$ & $\begin{array}{r}3.90 \\
2.00 \\
3.90 \\
3.90 \\
3.90 \\
3.90 \\
250.00 \\
2.00 \\
2.00 \\
50.00\end{array}$ \\
\hline $\begin{array}{l}\text { Methoxychlor } \\
\text { Maphthalene } \\
\text { Phenanthrene } \\
\text { Pyrene } \\
\text { Toxaphene } \\
4,4^{\prime}-\text { DD } \\
4,4^{\prime}-\text { DDE } \\
4,4^{\prime}-\text { DD }\end{array}$ & $\begin{array}{l}\mu g / \mathbf{k g} \\
\mu g / k g \\
\mu g / k g \\
\mu g / k g \\
\mu g / k g \\
\mu g / k g \\
\mu g / k g \\
\mu g / k g\end{array}$ & $\begin{array}{r}20.00 \\
2180.00 \\
770.00 \\
11.00 \\
200.00 \\
3.90 \\
3.90 \\
3.90\end{array}$ \\
\hline
\end{tabular}

$$
\text { Location }=\text { ROA; Formation }=\text { DISMAL GAP; Site }=3
$$

\begin{tabular}{|c|c|c|}
\hline $\begin{array}{l}\alpha-B H C \\
\alpha-C h l o r d a n e \\
\beta-B H C \\
\Delta-B H C \\
y-B H C \text { (Lindane) } \\
y \text {-Chlordane } \\
\text { Aldrin } \\
\text { Aroclor } 1016 \\
\text { Aroclor } 1221 \\
\text { Aroclor } 1232 \\
\text { Aroclor } 1242 \\
\text { Aroclor } 1248 \\
\text { Aroclor } 1254 \\
\text { Aroclor } 1260 \\
\text { Dieldrin }\end{array}$ & $\begin{array}{l}\mu g / k g \\
\mu g / k g \\
\mu g / k g \\
\mu g / k g \\
\mu g / k g \\
\mu g / k g \\
\mu g / k g \\
\mu g / k g \\
\mu g / k g \\
\mu g / k g \\
\mu g / k g \\
\mu g / k g \\
\mu g / k g \\
\mu g / k g \\
\mu g / k g\end{array}$ & $\begin{array}{r}3.80 \\
3.80 \\
3.80 \\
3.80 \\
3.80 \\
3.80 \\
3.80 \\
76.00 \\
150.00 \\
76.00 \\
76.00 \\
76.00 \\
76.00 \\
76.00 \\
7.60\end{array}$ \\
\hline
\end{tabular}


Table C.1 (continued)

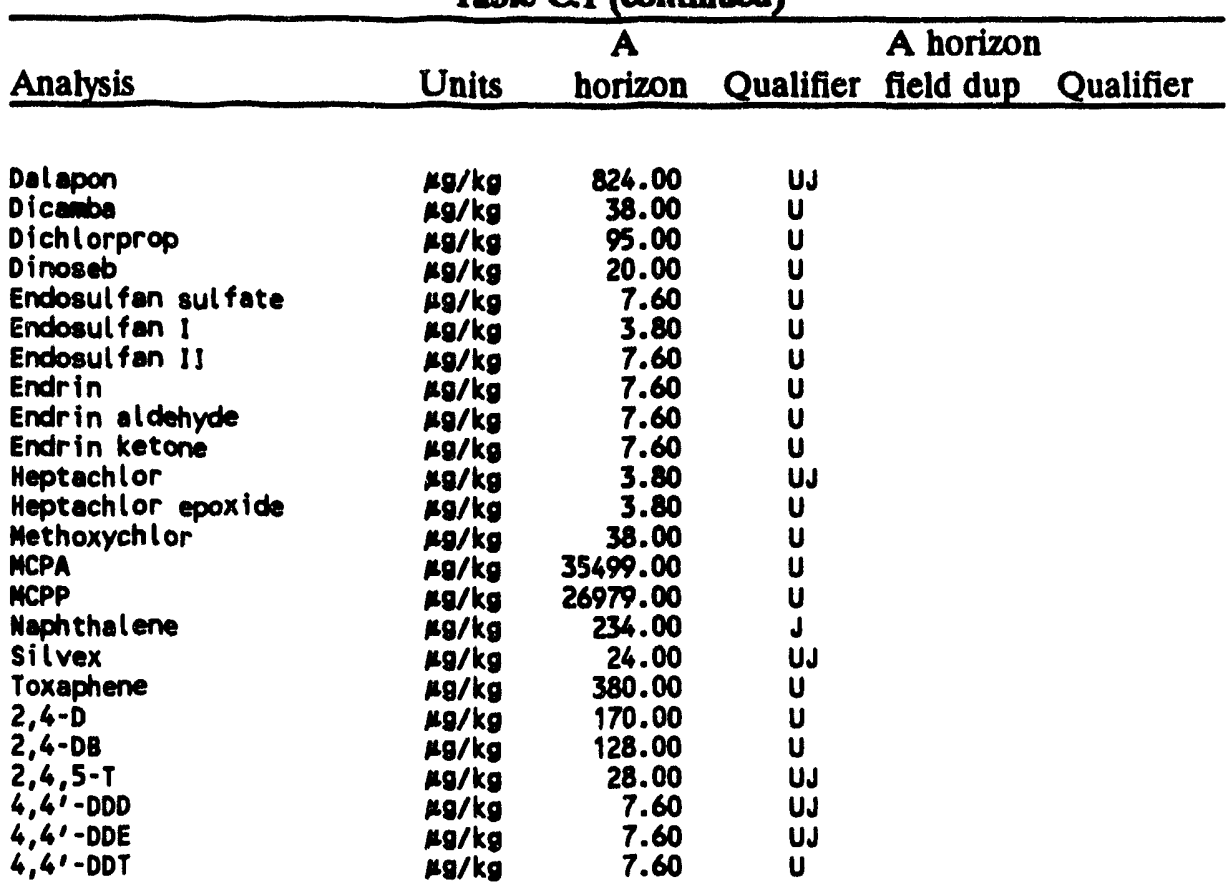

$$
\text { Location }=\text { ROA; Formation }=\text { DISMAL GAP; Site }=7
$$

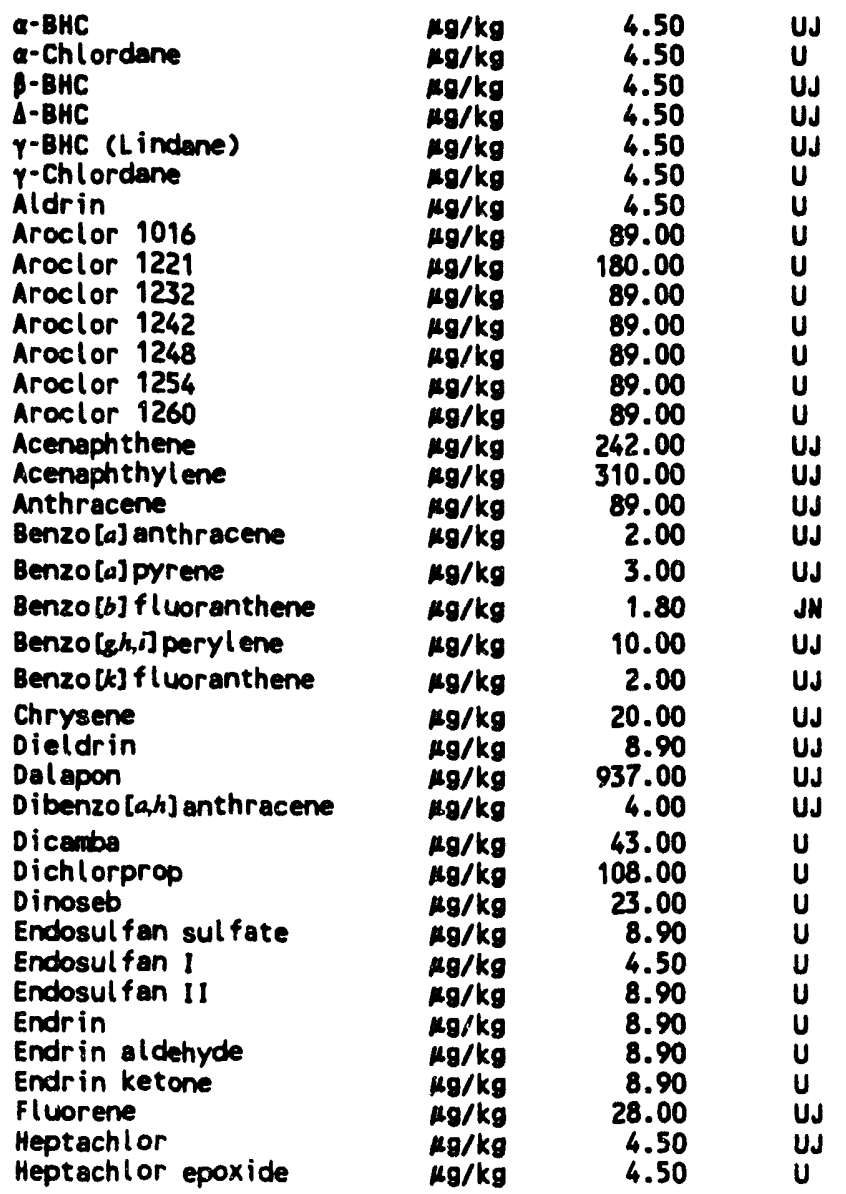


Table C.1 (continued)

\begin{tabular}{|c|c|c|c|c|}
\hline Analysis & Units & $\begin{array}{c}\mathrm{A} \\
\text { horizon }\end{array}$ & $\begin{array}{l}\text { A horizon } \\
\text { Qualifier field dup }\end{array}$ & Qualifier \\
\hline $\begin{array}{l}\text { Indeno-1,2,3(c,d)-pyrene } \\
\text { Methoxychlor } \\
\text { MCPA } \\
\text { MCPP } \\
\text { Maphthal ene } \\
\text { Phenenthrene } \\
\text { Pyrene } \\
\text { Silvex } \\
\text { TOxaphene } \\
2,4-D \\
2,4-D B \\
2,4,5-T \\
4,4 \text {, DDD } \\
4,41-D D E \\
4,4 \text {-DDT }\end{array}$ & $\begin{array}{l}\mu g / k g \\
\mu g / k g \\
\mu g / k g \\
\mu g / k g \\
\mu g / k g \\
\mu g / k g \\
\mu g / k g \\
\mu g / k g \\
\mu g / k g \\
\mu g / k g \\
\mu g / k g \\
\mu g / k g \\
\mu g / k g \\
\mu g / k g \\
\mu g / k g\end{array}$ & $\begin{array}{r}6.00 \\
45.00 \\
40404.00 \\
30707.00 \\
242.00 \\
86.00 \\
36.00 \\
27.00 \\
450.00 \\
194.00 \\
145.00 \\
32.00 \\
8.90 \\
8.90 \\
8.90\end{array}$ & 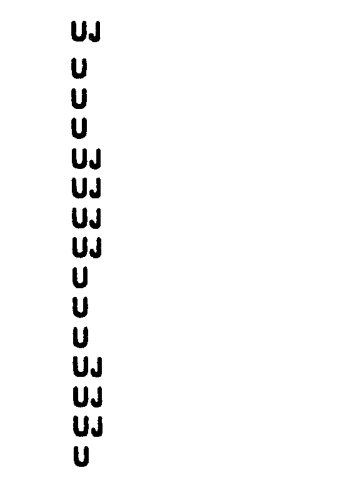 & \\
\hline \multicolumn{5}{|c|}{ Location $=$ ROA; Formation $=$ DISMAL GAP; Site $=8$} \\
\hline 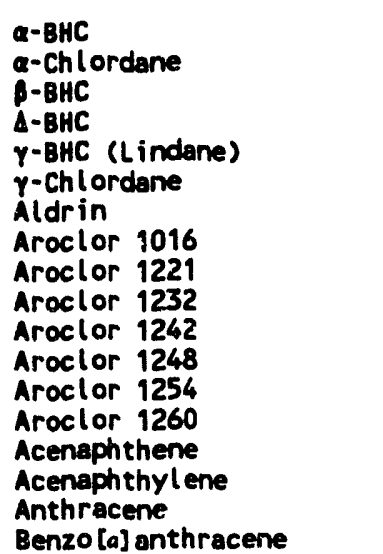 & $\begin{array}{l}\mu g / k g \\
\mu g / k g \\
\mu g / k g \\
\mu g / k g \\
\mu g / k g \\
\mu g / k g \\
\mu g / k g \\
\mu g / k g \\
\mu g / k g \\
\mu g / k g \\
\mu g / k g \\
\mu g / k g \\
\mu g / k g \\
\mu g / k g \\
\mu g / k g \\
\mu g / k g \\
\mu g / k g \\
\mu g / k g\end{array}$ & $\begin{array}{r}4.40 \\
4.40 \\
4.40 \\
4.40 \\
4.40 \\
4.40 \\
4.40 \\
85.00 \\
172.00 \\
85.00 \\
85.00 \\
85.00 \\
85.00 \\
85.00 \\
228.00 \\
291.00 \\
84.00 \\
2.00\end{array}$ & 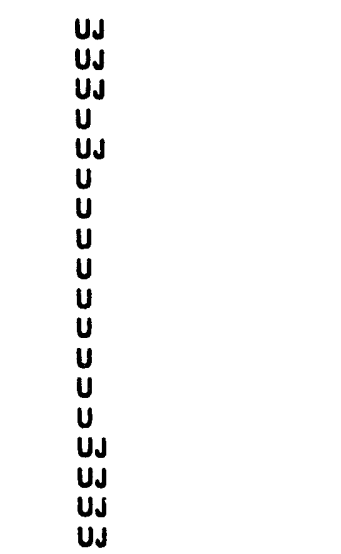 & \\
\hline Benzo [a] pyrene & $\mu \mathrm{g} / \mathrm{kg}$ & 3.00 & UJ & \\
\hline Benzo $[b]$ f l woranthene & $\mu \mathrm{g} / \mathrm{kg}$ & 2.00 & UJ & \\
\hline Benzo[eh,i] perylene & $\mu g / k g$ & 10.00 & UJ & \\
\hline Benzo [k] fluoranthene & $\mu g / k g$ & 2.00 & UJ & \\
\hline $\begin{array}{l}\text { Chrysene } \\
\text { Dieldrin } \\
\text { Dalapon } \\
\text { Dibenzo }[a, h] \text { anthracene }\end{array}$ & $\begin{array}{l}\mu g / k g \\
\mu g / k g \\
\mu g / / g g \\
\mu g / k g\end{array}$ & $\begin{array}{r}19.00 \\
8.50 \\
881.00 \\
4.00\end{array}$ & $\begin{array}{l}\text { UJ } \\
\text { UJ } \\
\text { UJ } \\
\text { UJ }\end{array}$ & \\
\hline $\begin{array}{l}\text { Dicamba } \\
\text { Dichlorprop } \\
\text { Dinoseb } \\
\text { Endosulfan sul fate } \\
\text { Endosul fan } 1 \\
\text { Endosul fan il } \\
\text { Endrin } \\
\text { Endrin al dehyde } \\
\text { Endrin ketone } \\
\text { Fluorene } \\
\text { Heptachlor } \\
\text { Heptachlor epoxide } \\
\text { Indeno-1,2,3(c,d)-pyrene }\end{array}$ & $\begin{array}{l}\mu g / k g \\
\mu g / k g \\
\mu g / k g \\
\mu g / k g \\
\mu g / / k g \\
\mu g / k g \\
\mu g / k g \\
\mu g / k g \\
\mu g / / k g \\
\mu g / k g \\
\mu g / k g \\
\mu g / k g \\
\mu g / k g\end{array}$ & $\begin{array}{r}41.00 \\
101.00 \\
21.00 \\
8.50 \\
4.40 \\
8.50 \\
8.50 \\
8.50 \\
8.50 \\
27.00 \\
4.40 \\
4.40 \\
5.00\end{array}$ & 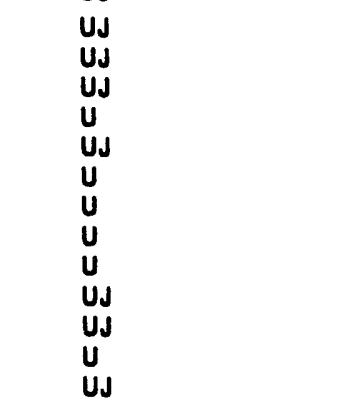 & \\
\hline $\begin{array}{l}\text { Methoxychlor } \\
\text { MCPA } \\
\text { MCPP } \\
\text { Maphthal ene } \\
\text { Phenanthrene } \\
\text { Pyrene } \\
\text { Silvex } \\
\text { Toxaphene }\end{array}$ & $\begin{array}{l}\mu g / k g \\
\mu g / k g \\
\mu g / k g \\
\mu g / k g \\
\mu g / k g \\
\mu g / k g \\
\mu g / k g \\
\mu g / k g\end{array}$ & $\begin{array}{r}44.00 \\
37989.00 \\
28872.00 \\
228.00 \\
203.00 \\
34.00 \\
25.00 \\
436.00\end{array}$ & $\begin{array}{l}\text { UJ } \\
\text { UJ } \\
\text { UJ } \\
U J J \\
J \\
\text { Ju } \\
\text { UJ } \\
U J \\
U\end{array}$ & \\
\hline
\end{tabular}


Table C.1 (continued)

\begin{tabular}{|c|c|c|c|c|c|}
\hline Analysis & Units & $\begin{array}{c}\mathrm{A} \\
\text { horizon }\end{array}$ & Qualifier & $\begin{array}{l}\text { A horizon } \\
\text { field dup }\end{array}$ & Qualifier \\
\hline $\begin{array}{l}2,4-D \\
2,4-D B \\
2,4,5-T \\
4,4 ;-D D D \\
4,4,-D D E \\
4,44^{\prime}-D D T\end{array}$ & $\begin{array}{l}\mathrm{kg} / \mathrm{kg} \\
\mathrm{kg} / \mathrm{kg} \\
\mathrm{kg} / \mathrm{kg} \\
\mathrm{kg} / \mathrm{kg} \\
\mathrm{kg} / \mathrm{kg} \\
\mathrm{kg} / \mathrm{kg}\end{array}$ & $\begin{array}{r}182.00 \\
137.00 \\
30.00 \\
8.50 \\
8.50 \\
14.00\end{array}$ & $\begin{array}{l}\text { UJ } \\
\text { UJ } \\
\text { UJ } \\
\text { UJ } \\
\text { UJ } \\
J\end{array}$ & & \\
\hline
\end{tabular}

$$
\text { Location }=\text { ROA; Formation }=\text { DISMAL GAP; Site }=9
$$

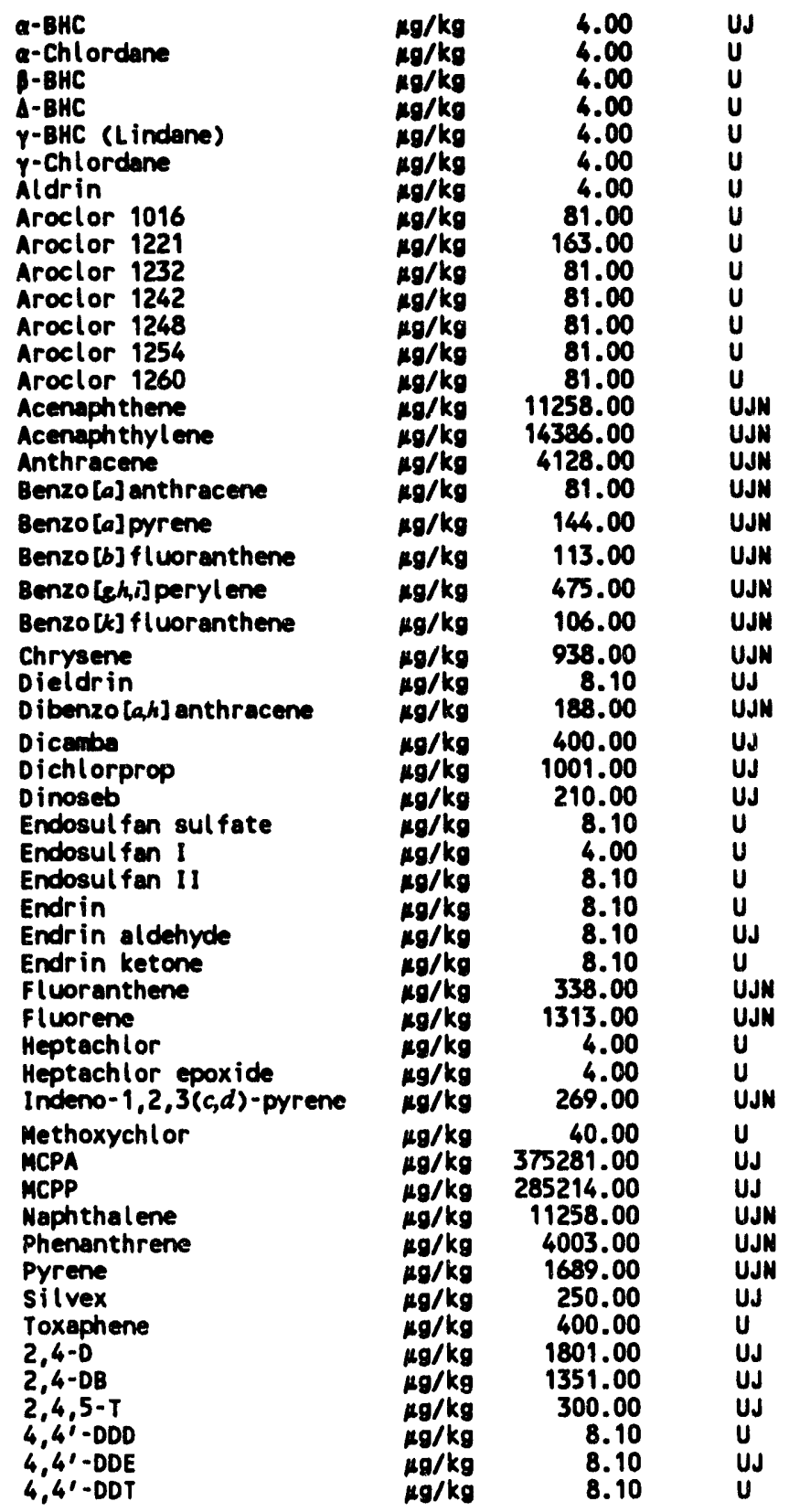




\section{C-35}

Table C.1 (continued)

\begin{tabular}{|c|c|c|c|}
\hline Analysis & Units & $\begin{array}{c}\text { A } \\
\text { horizon }\end{array}$ & Qualifi \\
\hline \multicolumn{4}{|c|}{ Location $=$ ROA; Formation $=$ DISMAL $G$} \\
\hline $\begin{array}{l}\text { a-BHC } \\
\text { a-Chlordane } \\
\text {-BHC } \\
\text { A-BHC } \\
\gamma \text {-BHC (Lindane) } \\
\gamma \text {-Chlordane } \\
\text { Aldrin } \\
\text { Aroclor } 1016 \\
\text { Aroclor } 1221 \\
\text { Aroclor } 1232 \\
\text { Aroclor } 1242 \\
\text { Aroclor } 1248 \\
\text { Aroclor } 1254 \\
\text { Aroclor } 1260 \\
\text { Acenaphthene } \\
\text { Acenaphthylene } \\
\text { Anthracene } \\
\text { Benzo[a] anthracene }\end{array}$ & 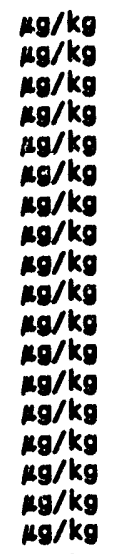 & $\begin{array}{r}6.30 \\
6.30 \\
6.30 \\
6.30 \\
6.30 \\
6.30 \\
6.30 \\
130.00 \\
250.00 \\
130.00 \\
130.00 \\
130.00 \\
130.00 \\
130.00 \\
3407.00 \\
4354.00 \\
1249.00 \\
25.00\end{array}$ & 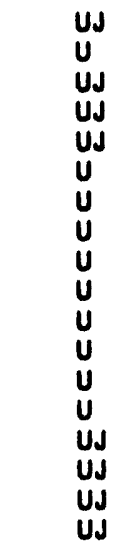 \\
\hline Benzo [a] pyrene & $\mathbf{m g} / \mathbf{k g}$ & 44.00 & UJ \\
\hline Benzo [b] f l woranthene & $\mathrm{mg} / \mathrm{kg}$ & 34.00 & Us \\
\hline Benzo $[g h, i]$ peryl ene & $\mathbf{M g} / \mathbf{k g}$ & 144.00 & UJ \\
\hline Benzo [k] f l woranthene & $\mathrm{mg} / \mathrm{kg}$ & 32.00 & UJ \\
\hline $\begin{array}{l}\text { Chrysene } \\
\text { Dieldrin } \\
\text { Dibenzo }[a, h] \text { anthracene }\end{array}$ & $\begin{array}{l}\mathrm{mg} / \mathrm{kg} \\
\mathrm{mg} / \mathrm{kg} \\
\mathrm{mg} / \mathrm{kg}\end{array}$ & $\begin{array}{r}284.00 \\
13.00 \\
57.00\end{array}$ & $\begin{array}{l}\text { UJ } \\
\text { UJ } \\
\text { UJ }\end{array}$ \\
\hline $\begin{array}{l}\text { Endosulfan sul fate } \\
\text { Endosul fan I } \\
\text { Endosulfan II } \\
\text { Endrin } \\
\text { Endrin aldehyde } \\
\text { Endrin ketone } \\
\text { Fluorene } \\
\text { Heptachlor } \\
\text { Heptachlor epoxide } \\
\text { Indeno-1,2,3(c,d)-pyrene }\end{array}$ & $\begin{array}{l}\mu g / \mathbf{k g} \\
\mu g / k g \\
\mu g / k g \\
\mu g / k g \\
\mu g / k g \\
\mu g / k g \\
\mu g / k g \\
\mu g / k g \\
\mu g / k g \\
\mu g / k g\end{array}$ & $\begin{array}{r}13.00 \\
6.30 \\
13.00 \\
13.00 \\
13.00 \\
13.00 \\
398.00 \\
6.30 \\
6.30 \\
81.00\end{array}$ & $\begin{array}{l}U \\
U \\
U \\
U \\
U \\
U \\
U \\
U J J \\
U \\
U \\
U J\end{array}$ \\
\hline $\begin{array}{l}\text { Methoxychlor } \\
\text { Naphthal ene } \\
\text { Phenanthrene } \\
\text { Pyrene } \\
\text { TOxaphene } \\
4,4^{\prime}-\text {-DDD } \\
4,4^{\prime} \text {-DDE } \\
4,4^{\prime} \text {-DDT }\end{array}$ & $\begin{array}{l}\mu g / k g \\
\mu g / k g \\
\mu g / k g \\
\mu g / k g \\
\mu g / k g \\
\mu g / k g \\
\mu g / k g \\
\mu g / k g\end{array}$ & $\begin{array}{r}63.00 \\
3407.00 \\
1211.00 \\
511.00 \\
630.00 \\
13.00 \\
13.00 \\
13.00\end{array}$ & $\begin{array}{l}U \\
U J J \\
U J \\
U J J \\
U \\
U J J \\
U J \\
U\end{array}$ \\
\hline
\end{tabular}

Location $=$ ROA; Formation $=$ DISMAL GAP; Site $=13$

\begin{tabular}{|c|c|c|c|c|}
\hline $\begin{array}{l}\text { a-BHC } \\
\text { a-Chlordane } \\
\beta-B H C \\
\Delta-8 H C \\
\gamma-8 H C \text { (Lindane) } \\
\gamma \text {-Chlordane } \\
\text { Aldrin } \\
\text { Aroclor } 1016 \\
\text { Aroclor } 1221 \\
\text { Aroclor } 1232 \\
\text { Aroclor } 1242 \\
\text { Aroclor } 1248 \\
\text { Aroclor } 1254 \\
\text { Aroclor } 1260 \\
\text { Acenaphthene } \\
\text { Acenaphthylene }\end{array}$ & $\begin{array}{l}\mu g / k g \\
\mu g / k g \\
\mu g / k g \\
\mu g / k g \\
\mu g / k g \\
\mu g / k g \\
\mu g / k g \\
\mu g / k g \\
\mu g / k g \\
\mu g / k g \\
\mu g / k g \\
\mu g / k g \\
\mu g / k g \\
\mu g / k g \\
\mu g / k g \\
\mu g / k g\end{array}$ & $\begin{array}{r}4.20 \\
4.20 \\
4.20 \\
4.20 \\
4.20 \\
4.20 \\
4.20 \\
84.00 \\
170.00 \\
84.00 \\
84.00 \\
84.00 \\
84.00 \\
84.00 \\
229.00 \\
2930.00\end{array}$ & $\begin{array}{l}U J \\
U \\
U J J \\
U J \\
U J \\
U \\
U \\
U \\
U \\
U \\
U \\
U \\
U \\
U \\
U \\
U \\
U \\
U J\end{array}$ & $\begin{array}{r}4.1 \\
4.1 \\
4.1 \\
4.1 \\
4.1 \\
4.1 \\
4.1 \\
82.0 \\
160.0 \\
82.0 \\
82.0 \\
82.0 \\
82.0 \\
82.0 \\
223.0 \\
2850.0\end{array}$ \\
\hline
\end{tabular}


Table C.1 (continued)

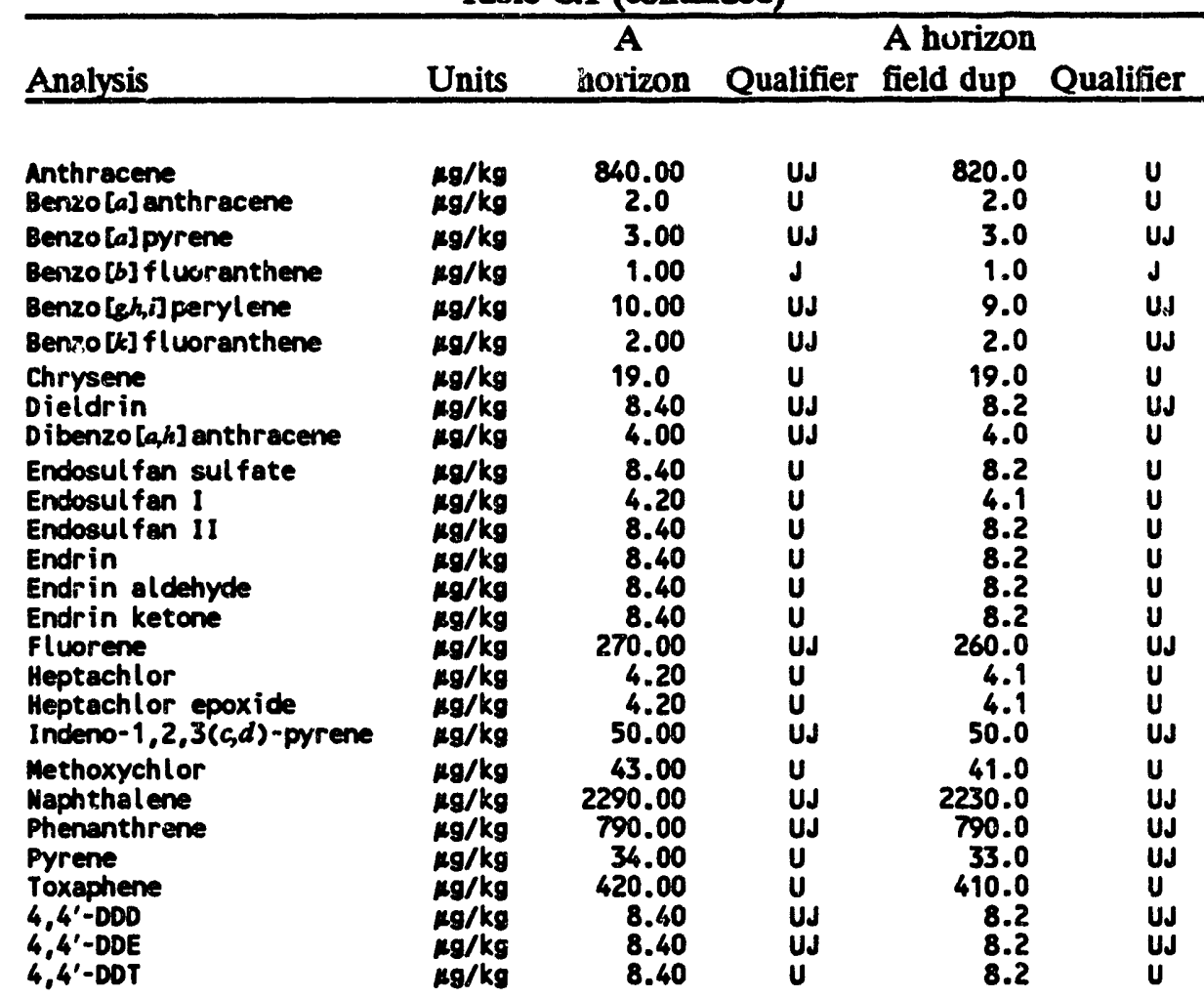

$$
\text { Location }=\text { ROA; Formation }=\text { DISMAL GAP; Site }=14
$$

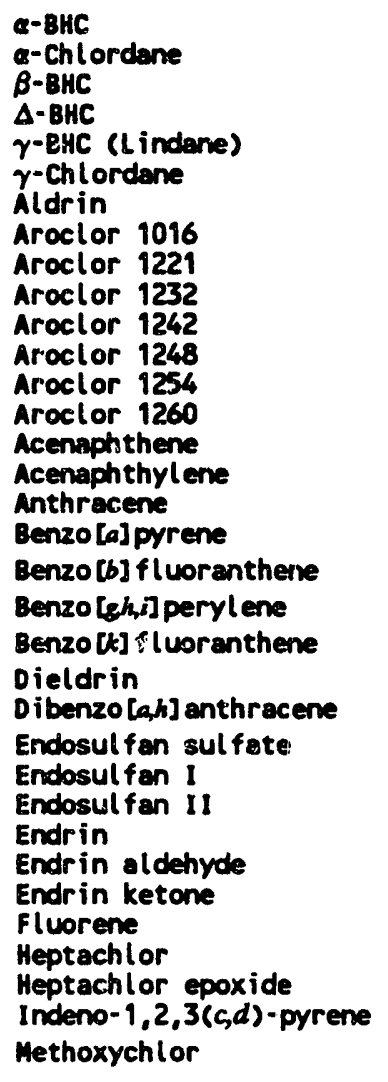

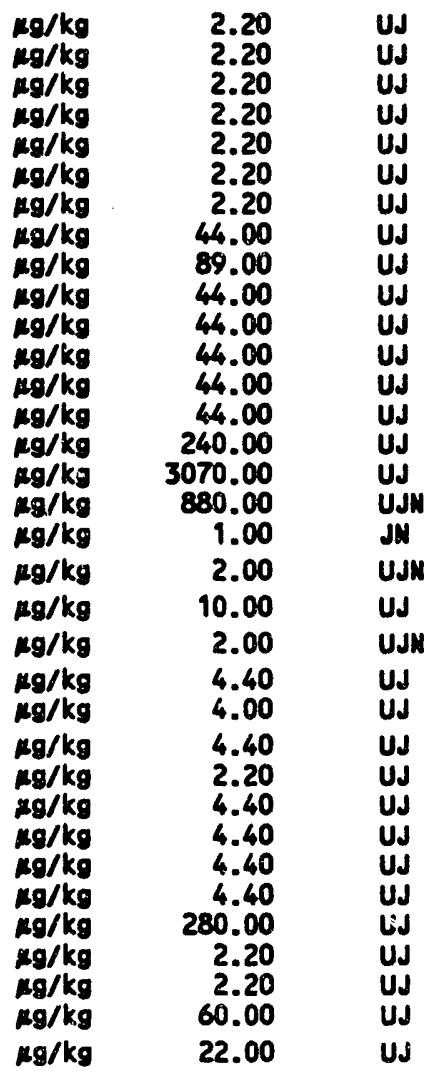


Table C.1 (continued)

\begin{tabular}{|c|c|c|c|c|c|}
\hline Analysis & Units & $\begin{array}{c}\text { A } \\
\text { horizon }\end{array}$ & Qualifier & $\begin{array}{l}\text { A horizon } \\
\text { field dup }\end{array}$ & Qualifier \\
\hline $\begin{array}{l}\text { Maphthalene } \\
\text { Phenanthrene } \\
\text { Pyrene } \\
\text { Toxaphene } \\
4,4^{\prime} \text {-DDD } \\
4,4^{\prime}-\text {-DDE } \\
4,4^{\prime} \text {-DDT }\end{array}$ & $\begin{array}{l}\mathrm{Mg} / \mathrm{kg} \\
\mathrm{kg} / \mathrm{kg} \\
\mathrm{kg} / \mathrm{kg} \\
\mathrm{kg} / \mathrm{kg} \\
\mathrm{kg} / \mathrm{kg} \\
\mathrm{kg} / \mathrm{kg} \\
\mathrm{\mu g} / \mathrm{kg}\end{array}$ & $\begin{array}{r}2400.00 \\
850.00 \\
36.00 \\
220.00 \\
4.40 \\
4.40 \\
4.40\end{array}$ & $\begin{array}{l}\text { UJ } \\
\text { UJ } \\
\text { UJ } \\
\text { UJ } \\
\text { UJ } \\
\text { UJ } \\
\text { UJ }\end{array}$ & & \\
\hline
\end{tabular}

$$
\text { Location }=\text { ROA; Formation }=\text { DISMAL GAP; Site }=17
$$

\begin{tabular}{|c|c|c|c|}
\hline $\begin{array}{l}221 \\
232 \\
242 \\
248 \\
254 \\
260 \\
\text { ene } \\
\text { ylene }\end{array}$ & $\begin{array}{l}\mu g / k g \\
\mu g / k g \\
\mu g / k g \\
\mu g / k g \\
\mu g / k g \\
\mu g / k g \\
\mu g / k g \\
\mu g / k g \\
\mu g / k g \\
\mu g / k g \\
\mu g / k g \\
\mu g / k g \\
\mu g / k g \\
\mu g / k g \\
\mu g / k g \\
\mu g / k g \\
\mu g / k g \\
\mu g / k g\end{array}$ & $\begin{array}{r}3.80 \\
3.80 \\
3.80 \\
3.80 \\
3.80 \\
3.80 \\
3.80 \\
76.00 \\
150.00 \\
76.00 \\
76.00 \\
76.00 \\
76.00 \\
76.00 \\
2091.00 \\
2672.00 \\
767.00 \\
15.00\end{array}$ & $\begin{array}{l}U_{J} \\
U \\
U J \\
U \\
U \\
U \\
U \\
U \\
U \\
U \\
U \\
U \\
U \\
U \\
U J N \\
U J N \\
U J N \\
U N N\end{array}$ \\
\hline Benzo [a] pyrene & $\mu \mathrm{g} / \mathrm{kg}$ & 27.00 & UJN \\
\hline Benzo $[b]$ f luoranthene & $\mu \mathrm{g} / \mathbf{k g}$ & 21.00 & UJN \\
\hline Benzo [gh,i] perylene & $\mu g / k g$ & 88.00 & UJN \\
\hline Benzo $[k]$ fluoranthene & ng/kg & 20.00 & UJN \\
\hline $\begin{array}{l}\text { Chrysene } \\
\text { Dieldrin } \\
\text { Dibenzo }[a, h] \text { anthracene }\end{array}$ & $\begin{array}{l}\mathrm{Mg} / \mathrm{kg} \\
\mathrm{Mg} / \mathrm{kg} \\
\mathrm{Mg} / \mathrm{kg}\end{array}$ & $\begin{array}{r}174.00 \\
7.60 \\
35.00\end{array}$ & $\begin{array}{l}\text { UJN } \\
\text { UJ } \\
\text { UJN }\end{array}$ \\
\hline $\begin{array}{l}\text { Dicanba } \\
\text { Dichlorprop } \\
\text { Dinoseb } \\
\text { Endosulfan sul fate } \\
\text { Endosulfan I } \\
\text { Endosul fan II } \\
\text { Endr in } \\
\text { Endr in al dehyde } \\
\text { Endr in ketone } \\
\text { Fluorene } \\
\text { Heptachlor } \\
\text { Heptachlor epoxide } \\
\text { Indeno-1,2,3(c,d)-pyrene }\end{array}$ & 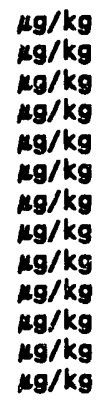 & $\begin{array}{r}185.00 \\
462.00 \\
97.00 \\
7.60 \\
3.80 \\
7.60 \\
7.60 \\
7.60 \\
7.60 \\
244.00 \\
3.80 \\
3.80 \\
50.00\end{array}$ & $\begin{array}{l}U J \\
U J \\
U J \\
U \\
U \\
U \\
U \\
U \\
U \\
U J N \\
U \\
U \\
U J W\end{array}$ \\
\hline $\begin{array}{l}\text { Methoxychlor } \\
\text { MCPA } \\
\text { MCPP } \\
\text { Nephthalene } \\
\text { Pilenanthrene } \\
\text { Pyrune } \\
\text { Silvex } \\
\text { Toxaphene } \\
2,4-0 \\
2,4-D B \\
2,4,5-7 \\
4,4^{\prime}-\text {-DD } \\
4,4^{\prime}-\text { DDE } \\
4,4^{\prime}-\text { DDT }\end{array}$ & 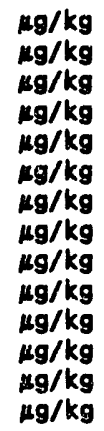 & $\begin{array}{r}38.00 \\
173405.00 \\
131788.00 \\
2091.00 \\
744.00 \\
314.00 \\
116.00 \\
380.00 \\
832.00 \\
624.00 \\
139.00 \\
7.60 \\
7.60 \\
7.60\end{array}$ & $\begin{array}{l}\text { UJ } \\
\text { UJ } \\
\text { US } \\
\text { UJH } \\
\text { UJN } \\
\text { UJN } \\
\text { UJ } \\
\text { U } \\
\text { UJ } \\
\text { UJ } \\
\text { UJ } \\
\text { UJ } \\
\text { UJ } \\
\text { UJ }\end{array}$ \\
\hline
\end{tabular}




\section{C-38}

Table C.1 (continued)

\begin{tabular}{lcc}
\hline & A & A horizon \\
Analysis & Units & horizon Qualifier field dup Qualifier \\
\hline
\end{tabular}

$$
\text { Location }=\text { ROA; Formation }=\text { DISMAL GAP; Site }=19
$$

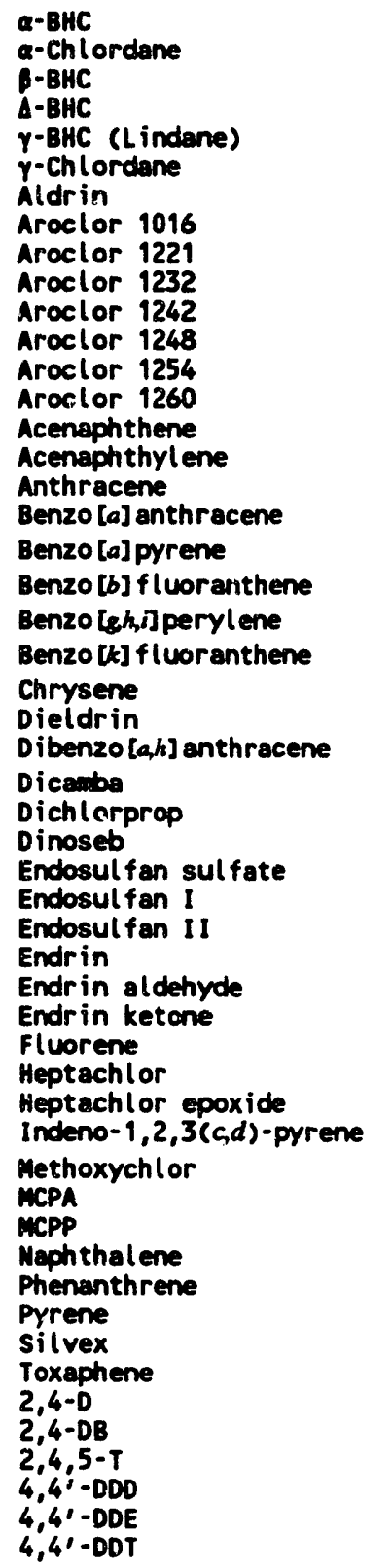

\begin{tabular}{|c|c|}
\hline 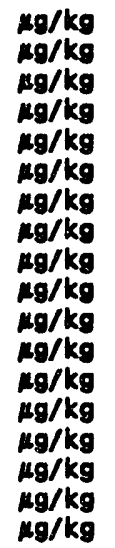 & $\begin{array}{r}4.40 \\
4.30 \\
4.30 \\
4.30 \\
4.30 \\
4.30 \\
4.30 \\
86.00 \\
170.00 \\
86.00 \\
86.00 \\
86.00 \\
86.00 \\
86.00 \\
233.00 \\
298.00 \\
85.00 \\
2.00\end{array}$ \\
\hline$\mu g / k g$ & 3.00 \\
\hline $\mathrm{mg} / \mathrm{kg}$ & 2.00 \\
\hline Rg/kg & 10.00 \\
\hline$\mu g / k g$ & 2.00 \\
\hline $\begin{array}{l}\mathrm{Mg} / \mathrm{kg} \\
\mathrm{Mg} / \mathrm{kg} \\
\mathrm{Mg} / \mathrm{kg}\end{array}$ & $\begin{array}{r}19.00 \\
8.60 \\
4.00\end{array}$ \\
\hline 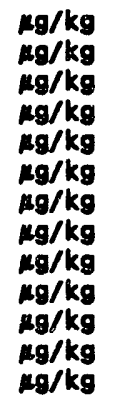 & $\begin{array}{r}204.00 \\
509.00 \\
107.00 \\
8.60 \\
4.30 \\
8.60 \\
8.60 \\
8.60 \\
8.60 \\
27.00 \\
4.30 \\
4.30 \\
6.00\end{array}$ \\
\hline $\begin{array}{l}\mu g / k g \\
\mu g / k g \\
\mu g / k g \\
\mu g / k g \\
\mu g / k g \\
\mu g / k g \\
\mu g / k g \\
\mu g / k g \\
\mu g / k g \\
\mu g / k g \\
\mu g / k g \\
\mu g / k g \\
\mu g / k g \\
\mu g / k g\end{array}$ & $\begin{array}{r}43.00 \\
190824.00 \\
145027.00 \\
233.00 \\
83.00 \\
35.00 \\
127.00 \\
430.00 \\
916.00 \\
687.00 \\
153.00 \\
8.60 \\
8.60 \\
8.60\end{array}$ \\
\hline
\end{tabular}

$$
\text { Location }=\text { ROA; Formation }=\text { DISMAL GAP; Site }=20
$$

a-BHC

a-Chl ordane

Mg/kg

Mg/kg

-BHC

$\triangle-B H C$

Y-BHC (Lindane)

rg/kg

ng/kg

$\mathrm{kg} / \mathrm{kg}$

$\gamma$-Chlordane

ng/kg

Aldrin

Mg/kg

1.90
1.90
1.90
1.90
1.90
1.90
1.90

Us

UJ

US

UJ

UJ

UJ 
Table C.1 (continued)

\begin{tabular}{|c|c|c|c|}
\hline Analysis & Units & $\begin{array}{c}\mathbf{A} \\
\text { horizon }\end{array}$ & Qualifi \\
\hline $\begin{array}{l}\text { Aroclor } 1016 \\
\text { Aroclor } 1221 \\
\text { Aroclor } 1232 \\
\text { Aroclor } 1242 \\
\text { Aroclor } 1248 \\
\text { Aroclor } 1254 \\
\text { Aroclor } 1260 \\
\text { Acenaphthene } \\
\text { Acenaphthylene } \\
\text { Anthracene } \\
\text { Benzo [a] anthracene }\end{array}$ & $\begin{array}{l}\mu g / k g \\
\mu g / k g \\
\mu g / k g \\
\mu g / k g \\
\mu g / k g \\
\mu g / k g \\
\mu g / k g \\
\mu g / k g \\
\mu g / k g \\
\mu g / k g \\
M g / k g\end{array}$ & $\begin{array}{r}39.00 \\
78.00 \\
39.00 \\
39.00 \\
39.00 \\
39.00 \\
39.00 \\
213.00 \\
272.00 \\
78.00 \\
2.00\end{array}$ & $\begin{array}{l}\text { UJ } \\
\text { UJ } \\
\text { UJ } \\
\text { UJ } \\
\text { US } \\
\text { UJ } \\
\text { UJ } \\
\text { UJ } \\
\text { UJ } \\
\text { UJ } \\
\text { UJ }\end{array}$ \\
\hline Benzo [a] pyrene & $\mathrm{mg} / \mathrm{kg}$ & 3.00 & UJ \\
\hline Benzo $[b]$ fluoranthene & $\mathbf{m g} / \mathbf{k g}$ & 2.00 & UJ \\
\hline $\begin{array}{l}\text { Benzo }[\text { gh, }] \text { perylene } \\
\text { Benzo }[k] \text { fluoranthene }\end{array}$ & $\begin{array}{l}\mu g / k g \\
\mu g / k g\end{array}$ & $\begin{array}{l}9.00 \\
2.00\end{array}$ & $\begin{array}{l}\text { UJ } \\
\text { UJ }\end{array}$ \\
\hline $\begin{array}{l}\text { Chrysene } \\
\text { Dieldrin } \\
\text { Dalapon } \\
\text { Dibenzo }[a, h] \text { anthracene }\end{array}$ & $\begin{array}{l}\mu \mathrm{g} / \mathrm{kg} \\
\mathrm{\mu g} / \mathrm{kg} \\
\mathrm{\mu g} / \mathrm{kg} \\
\mu g / \mathrm{kg}\end{array}$ & $\begin{array}{r}18.00 \\
3.90 \\
827.00 \\
4.00\end{array}$ & $\begin{array}{l}\text { UJ } \\
\text { UJ } \\
\text { UJ } \\
\text { UJ }\end{array}$ \\
\hline $\begin{array}{l}\text { Dicamba } \\
\text { Dichlorprop } \\
\text { Dinoseb } \\
\text { Endosulfan sul fate } \\
\text { Endosul fan I } \\
\text { Endosul fan II } \\
\text { Endrin } \\
\text { Endrin aldehyde } \\
\text { Endrin ketone } \\
\text { Fluorene } \\
\text { Heptachlor } \\
\text { Heptachlor epoxide } \\
\text { Indeno-1,2,3(c,d)-pyrene }\end{array}$ & $\begin{array}{l}\mu g / \mathbf{k g} \\
\mu g / \mathbf{k g} \\
\mu g / k g \\
\mu g / k g \\
\mu g / k g \\
\mu g / k g \\
\mu g / k g \\
\mu g / k g \\
\mu g / k g \\
\mu g / k g \\
\mu g / k g \\
\mu g / k g \\
\mu g / k g\end{array}$ & $\begin{array}{r}38.00 \\
95.00 \\
20.00 \\
3.90 \\
1.90 \\
3.90 \\
3.90 \\
3.90 \\
3.90 \\
119.00 \\
1.90 \\
1.90 \\
5.00\end{array}$ & $\begin{array}{l}\text { UJ } \\
\text { UJ } \\
\text { UJ } \\
\text { UJ } \\
\text { UJ } \\
\text { UJ } \\
\text { UJ } \\
\text { UJ } \\
U J \\
J \\
\text { UJ } \\
\text { UJ } \\
U J\end{array}$ \\
\hline $\begin{array}{l}\text { Methoxychlor } \\
\text { MCPA } \\
\text { MCPP } \\
\text { Maphthalene } \\
\text { Phenanthrene } \\
\text { Pyrene } \\
\text { Silvex } \\
\text { Toxaphene } \\
2,4-D \\
2,4-D B \\
2,4,5-T \\
4,4 ;-D D D \\
4,4:-D D E \\
4,4 \text { - DDT }\end{array}$ & $\begin{array}{l}\mu g / \mathbf{k g} \\
\mu g / \mathbf{k g} \\
\mu g / \mathbf{k g} \\
\mu g / k g \\
\mu g / k g \\
\mu g / k g \\
\mu g / k g \\
\mu g / k g \\
\mu g / k g \\
\mu g / k g \\
\mu g / k g \\
\mu g / k g \\
\mu g / k g \\
\mu g / k g\end{array}$ & $\begin{array}{r}19.00 \\
35668.00 \\
27107.00 \\
213.00 \\
76.00 \\
32.00 \\
24.00 \\
190.00 \\
171.00 \\
128.00 \\
29.00 \\
3.90 \\
3.90 \\
3.90\end{array}$ & $\begin{array}{l}\text { UJ } \\
\text { UJ } \\
\text { UJ } \\
\text { US } \\
\text { UJ } \\
\text { UJ } \\
\text { US } \\
\text { UJ } \\
\text { UJ } \\
\text { US } \\
\text { UJ } \\
\text { UJ } \\
\text { UJ } \\
\text { UJ }\end{array}$ \\
\hline
\end{tabular}

$$
\text { Location }=\text { ROA; Formation }=\text { DISMAL GAP; Site }=21
$$

$\begin{array}{ll}\alpha \text {-BHC } & \mu g / k g \\ \alpha-C h l o r d a n e & \mu g / k g \\ \beta-B H C & \mu g / k g \\ \text { A-BHC } & \mu g / k g \\ \gamma \text {-BHC (Lindane) } & \mu g / k g \\ \gamma \text {-Chlordane } & \mu g / k g \\ \text { Aldrin } & \mu g / k g \\ \text { Aroclor } 1016 & \mu g / k g \\ \text { Aroclor } 1221 & \mu g / k g \\ \text { Aroclor } 1232 & \mu g / k g \\ \text { Aroclor } 1242 & \mu g / k g \\ \text { Aroclor } 1248 & \mu g / k g \\ \text { Aroclor } 1254 & \mu g / k g \\ \text { Aroclor } 1260 & \mu g / k g \\ \text { Acenaphthene } & \mu g / k g \\ \text { Acenaphthylene } & \mu g / k g\end{array}$

\subsection{0}

2.10

2.10

2.10

2.10

2.10

2.10

41.00

82.00

41.00

41.00

41.00

41.00

41.00

229.00

2920.00
A horizon

field dup Qualifier 
Table C.1 (continued)

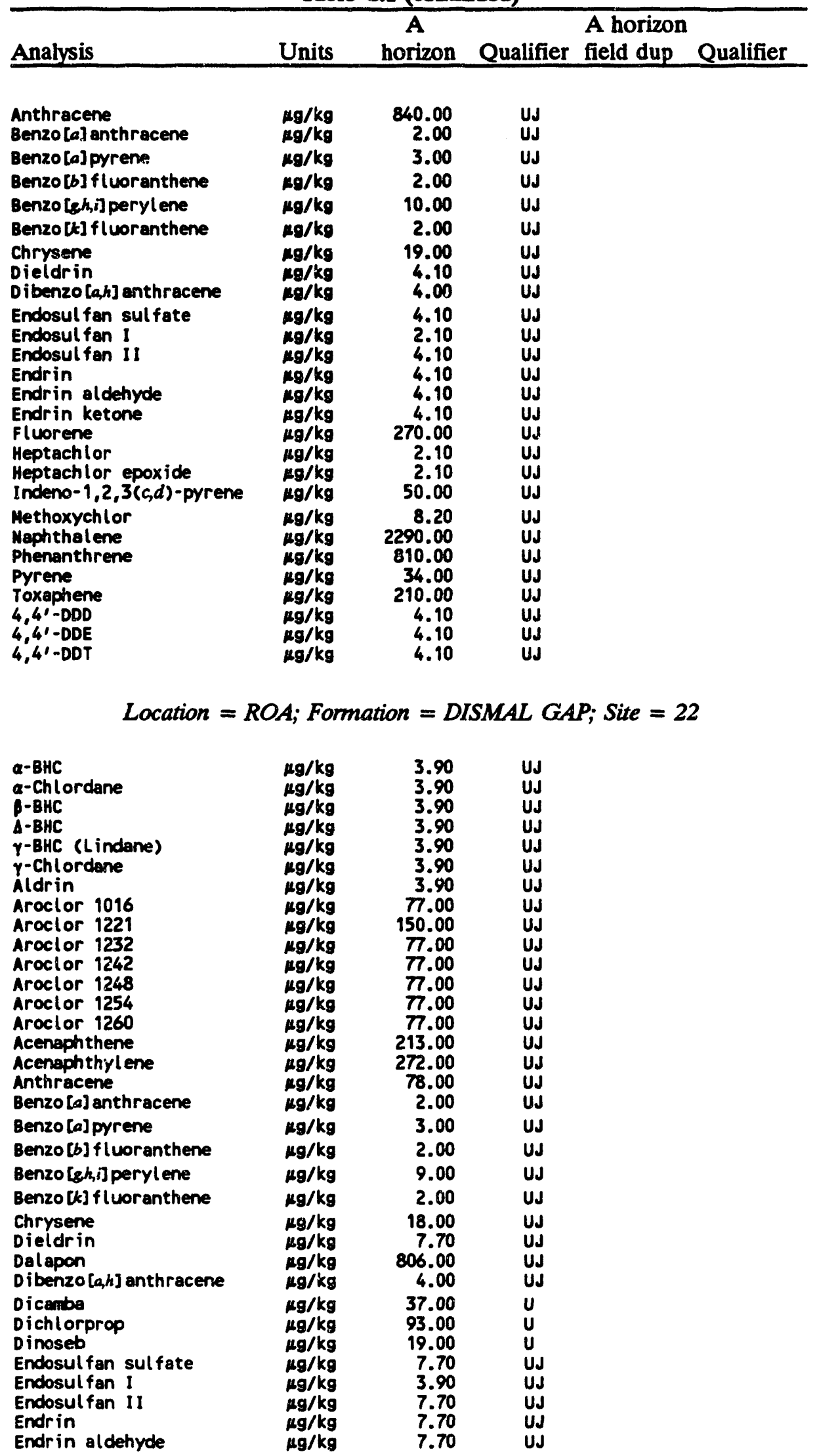




\section{C-41}

Table C.1 (continued)

\begin{tabular}{|c|c|c|c|c|c|}
\hline Analysis & Units & $\begin{array}{c}A \\
\text { horizon }\end{array}$ & Qualifier & $\begin{array}{l}\text { A horizon } \\
\text { field dup }\end{array}$ & Qualifier \\
\hline $\begin{array}{l}\text { Endrin ketone } \\
\text { Fluprene } \\
\text { Heptachlor } \\
\text { Heptachlor epoxide } \\
\text { Indeno- } 1,2,3(c, d) \text {-pyrene }\end{array}$ & $\begin{array}{l}\mu g / \mathrm{kg} \\
\mu g / k g \\
\mu g / k g \\
\mu g / k g \\
\mu g / k g\end{array}$ & $\begin{array}{r}7.70 \\
25.00 \\
3.90 \\
3.90 \\
5.00\end{array}$ & $\begin{array}{l}\text { UJ } \\
\text { US } \\
\text { US } \\
\text { UJ } \\
\text { UJ }\end{array}$ & & \\
\hline $\begin{array}{l}\text { Methoxychlor } \\
\text { MCPA } \\
\text { MCPP } \\
\text { Mephthalene } \\
\text { Phenanthrene } \\
\text { Pyrene } \\
\text { silvex } \\
\text { Toxaphene } \\
2,4-D \\
2,4-D B \\
2,4,5-T \\
4,4 \text {-DDD } \\
4,4 \text {-DDE } \\
4,4 \text { - DDT }\end{array}$ & $\begin{array}{l}\mu g / \mathrm{kg} \\
\mu g / \mathrm{kg} \\
\mu g / \mathrm{kg} \\
\mu g / \mathrm{kg} \\
\mu g / \mathrm{kg} \\
\mu g / \mathrm{kg} \\
\mu g / \mathrm{kg} \\
\mu g / \mathrm{kg} \\
\mu g / \mathrm{kg} \\
\mu g / \mathrm{kg} \\
\mu g / \mathrm{kg} \\
\mu g / \mathrm{kg} \\
\mu g / \mathrm{kg} \\
\mu g / \mathrm{kg}\end{array}$ & $\begin{array}{r}40.00 \\
34722.00 \\
26389.00 \\
496.00 \\
76.00 \\
32.00 \\
23.00 \\
390.00 \\
167.00 \\
125.00 \\
28.00 \\
7.70 \\
7.70 \\
7.70\end{array}$ & $\begin{array}{l}U J \\
U \\
U \\
J \\
U J \\
U J \\
U J \\
U J \\
U \\
U \\
U J \\
U J \\
U J \\
U J\end{array}$ & & \\
\hline
\end{tabular}


Appendix D

INORGANIC ANALYSIS DATA 
D-2

NOTE: When available, the validation qualifiers are used in this appendix. When validation qualifiers are not available, the corresponding laboratory data qualifiers are used. 


\section{VALIDATOR DATA QUALIFIER DEFINITIONS}

The following definitions provide brief explanations of the qualifiers assigned to the data in this appendix.

U The analyte was analyzed for but was not detected above the reported sample quantitiation limit.

J The anaiyte was positively identified; the associated numerical value is the approximate concentration of the analyte in the sample.

N The analysis indicates the presence of an analyte for which there is presumptive evidence to make a tentative identification.

JN The analysis indicates the presence of an analyte that has been tentatively identified, and the associated numerical value represents its approximate concentration.

UJ The analyte was not detected above the reported sample quantitation limit. However, the reported quantitation limit is approximate and may or may not represent the actual limit of quantitation necessary to accurately and precisely measure the analyte in the sample.

R The sample results are rejected because of serious deficiencies in the ability to analyze the sample and meet quality control criteria. The presence or absence of the analyte cannot be verified.

UN The laboratory did not register this compound, but there was presumptive evidence of a compound that was within the retention time window but was not reported. No other qualification of the daia was made.

UJN The laboratory did not report the compound, but there was presumptive evidence of a compound that was within the retention time window but was not reported. The data were qualified as estimated, $\mathrm{J}$, because of other discrepancies with the data.

RN The laboratory did not report the compound, but there was evidence of a compound that was within the retention time window but was not reported. The data were qualified as unusable, $R$, because of other discrepancies with the data. 


\section{LABORATORY DATA QUALIFIER DEFINITIONS}

The following contract laboratory data qualifiers are used in this project.

\section{Organic}

\begin{tabular}{||c|l||}
\hline Qualifier & Explanation \\
\hline U & Indicates compound was analyzed for but not detected. \\
J & Indicates an estimated value. \\
N & $\begin{array}{l}\text { Indicates presumptive evidence of a compound [used only for tentatively } \\
\text { identified compounds (TICs)]. }\end{array}$ \\
P & $\begin{array}{l}\text { Used for pesticide/aroclor target analytes when there is greater than 25\% } \\
\text { difference for detected concentrations between the two gas chromatograph (GC) } \\
\text { columns. The lower of the two is reported and flagged. }\end{array}$ \\
C & $\begin{array}{l}\text { Used for pesticide results where the identification has been confirmed by } \\
\text { GC/mass spectrograph (MS). }\end{array}$ \\
B & $\begin{array}{l}\text { Used when the analyte is found in the associated blank as well as in the sample. } \\
\text { Identifies all compounds in an analyte at a secondary dilution factor. } \\
\text { D }\end{array}$ \\
A & $\begin{array}{l}\text { Indicates that a TIC is a suspected aldol-condensation product. } \\
\text { Xther specific flags may be used to properly define the results. If they are used } \\
\text { they must be fully described and attached to the Sample Data Package. }\end{array}$ \\
\hline
\end{tabular}

Inorganic

\begin{tabular}{|c|c|}
\hline Qualifier & Explanation \\
\hline $\mathrm{U}$ & Indicates compound ras analyzed for but not detected. \\
\hline $\mathbf{J}$ & Indicates an estimated value. \\
\hline $\mathbf{N}$ & Spiked sample recovery not within control limits. \\
\hline $\mathbf{E}$ & Reported value estimated because of the presence of interference. \\
\hline $\mathbf{M}$ & Duplicate injection precision not met. \\
\hline B & $\begin{array}{l}\text { Reported value was obtained from a reading that was less than the CRDL, but } \\
\text { greater than or equal to the instrument detection limit (IDL). }\end{array}$ \\
\hline $\mathbf{S}$ & Reported value was determined by the method of standard additions (MSA). \\
\hline $\mathbf{w}$ & $\begin{array}{l}\text { Post-digestion spike for furnace atomic absorption is out of control limits, while } \\
\text { sample absorbance is less that } 50 \% \text { of spike absorbance. }\end{array}$ \\
\hline * & Duplicate analysis not within control limits. \\
\hline+ & Correlation coefficient for the MSA is less than 0.995 . \\
\hline
\end{tabular}


D.5

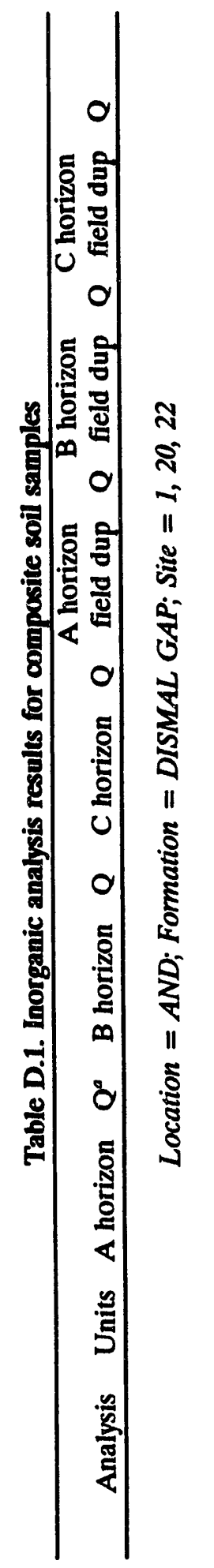

3 J5>0工 $3200733=$

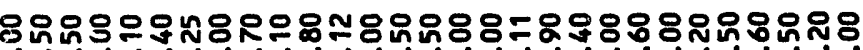

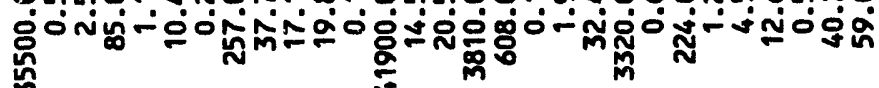

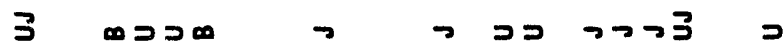

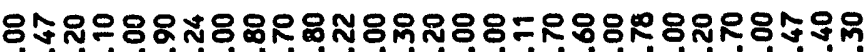

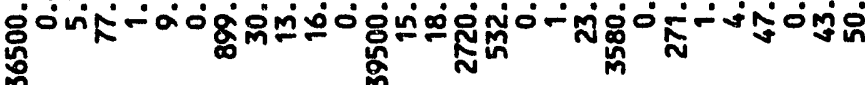

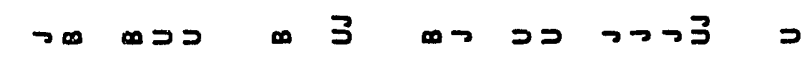

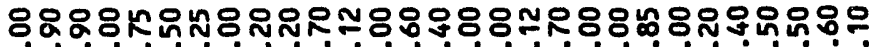

峁

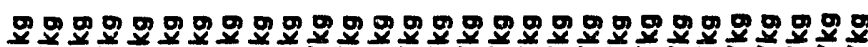

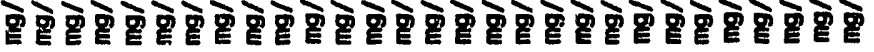

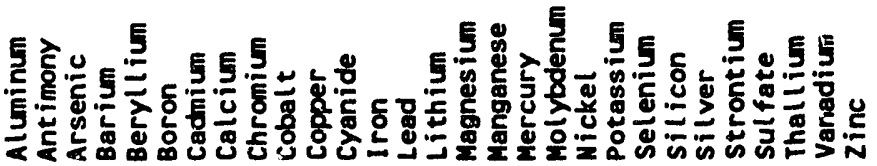




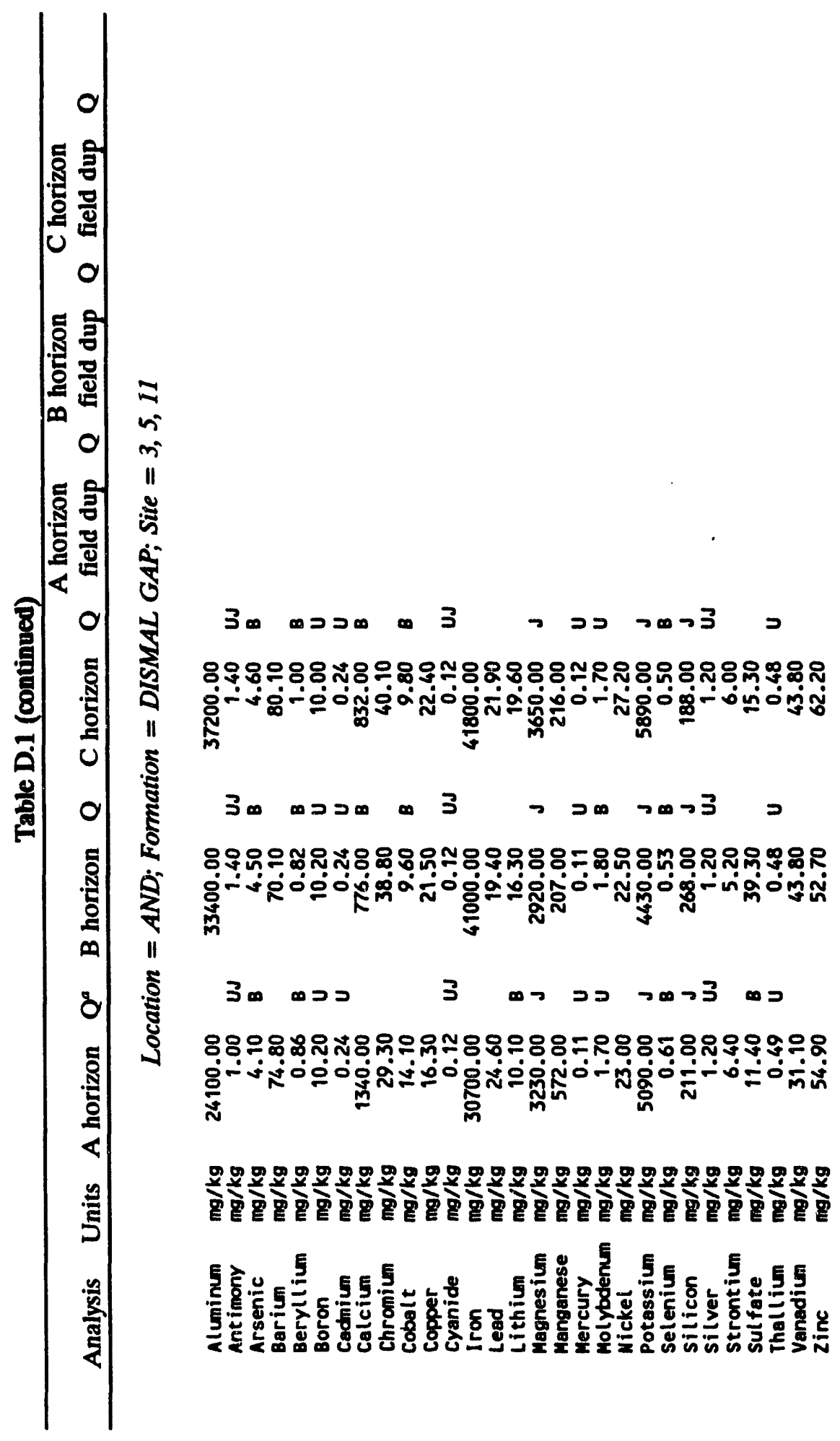


D-7

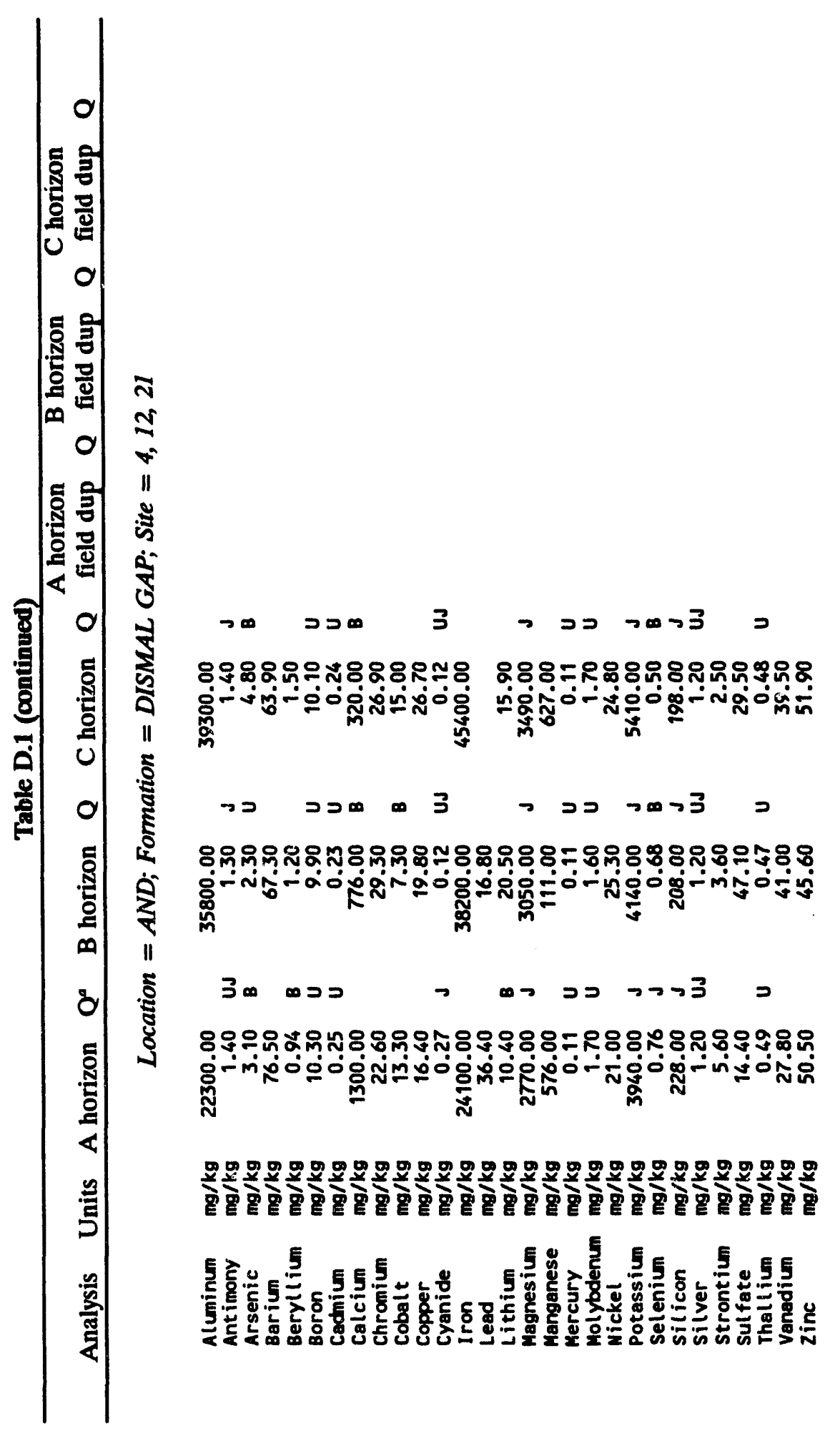




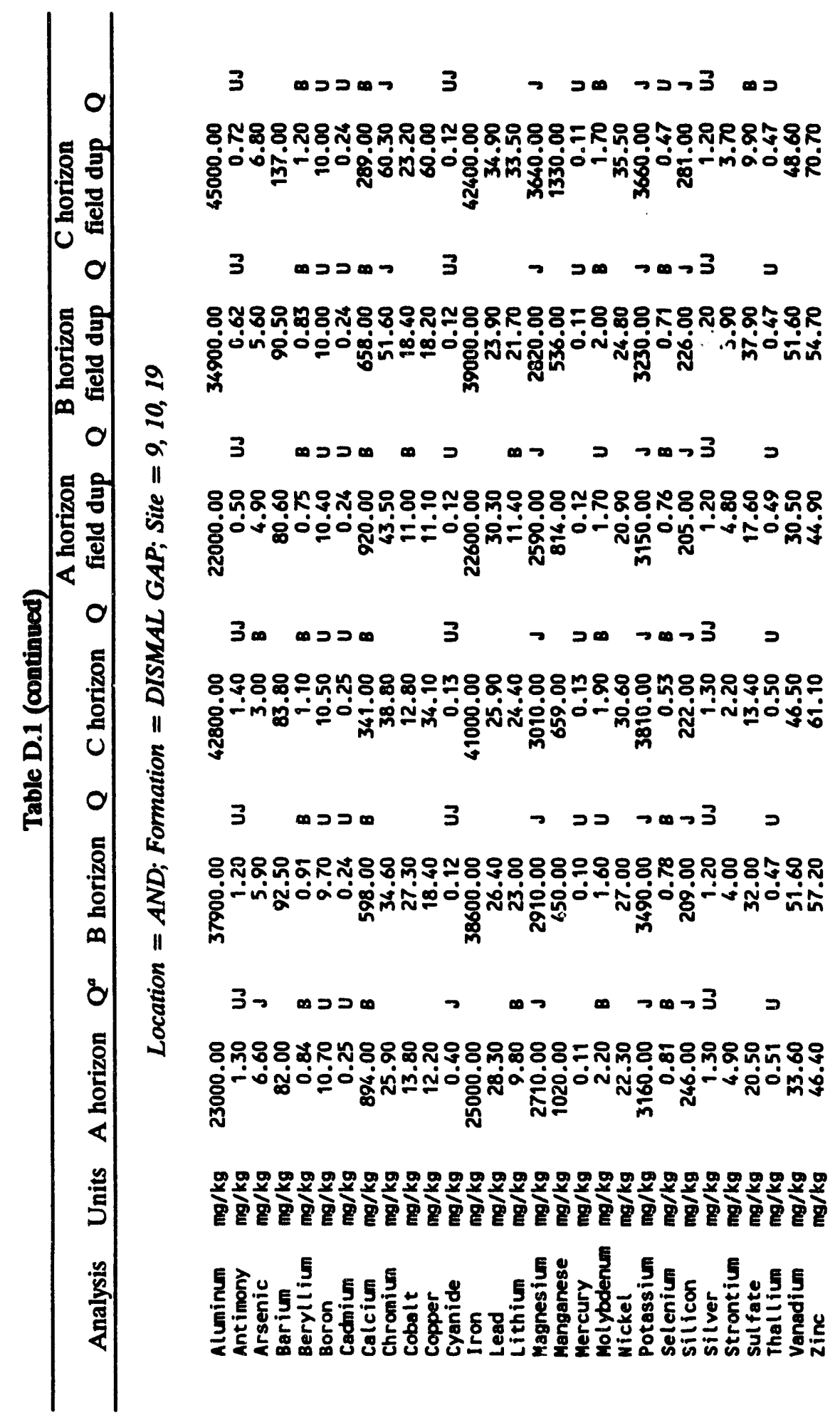


D-9

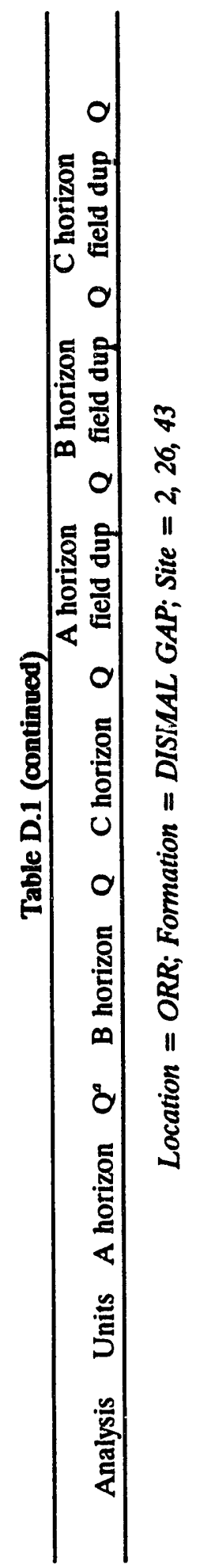

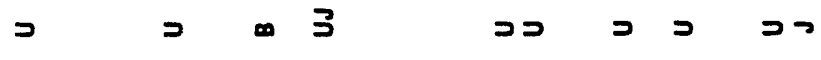

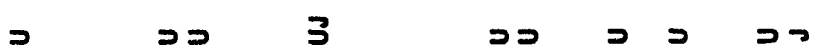

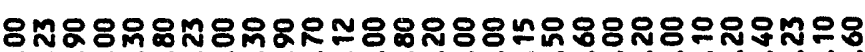
边

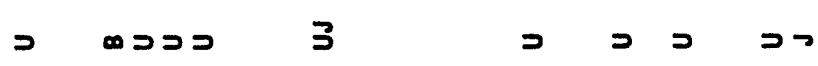

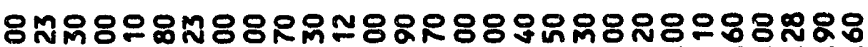

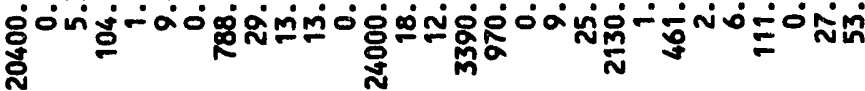
$>$ D 3 D 30,

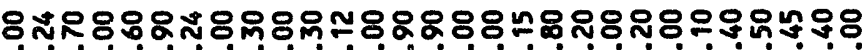
ठ্సু

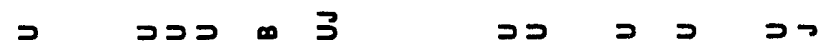

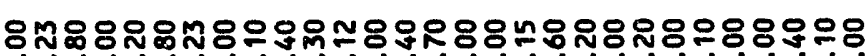

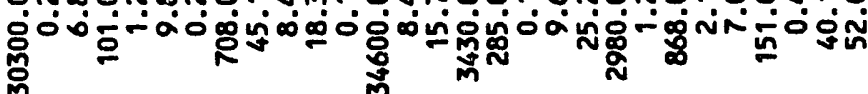

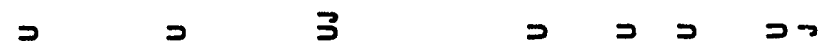

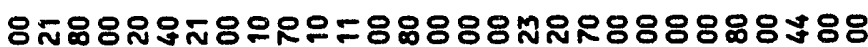

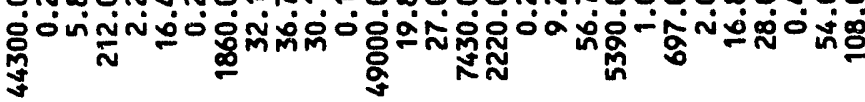

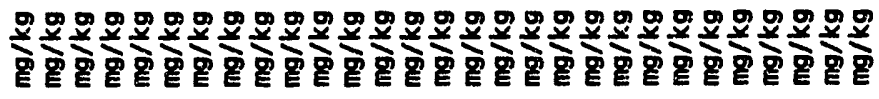

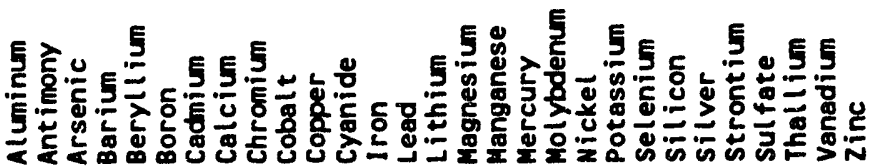


D-10

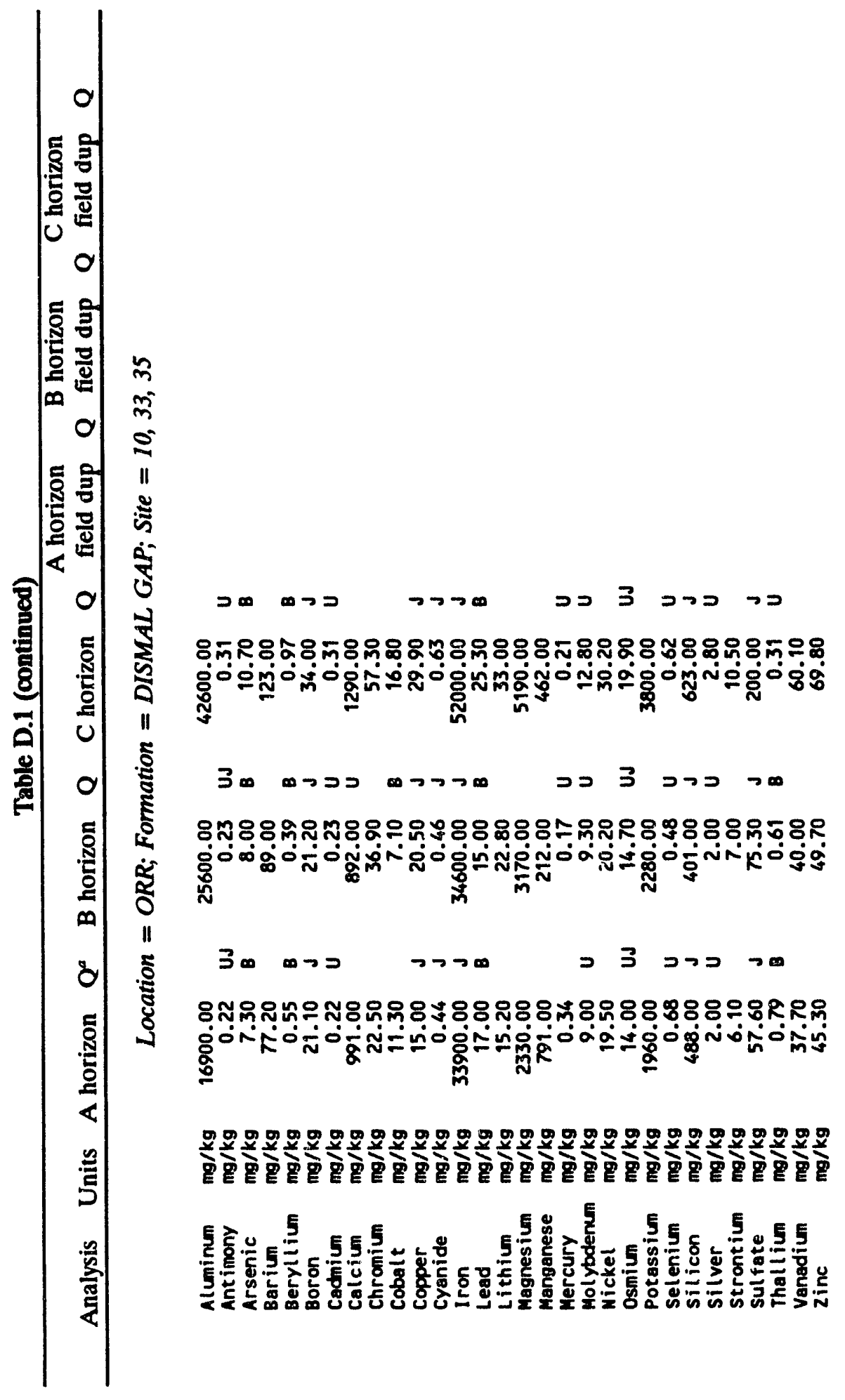


D-11

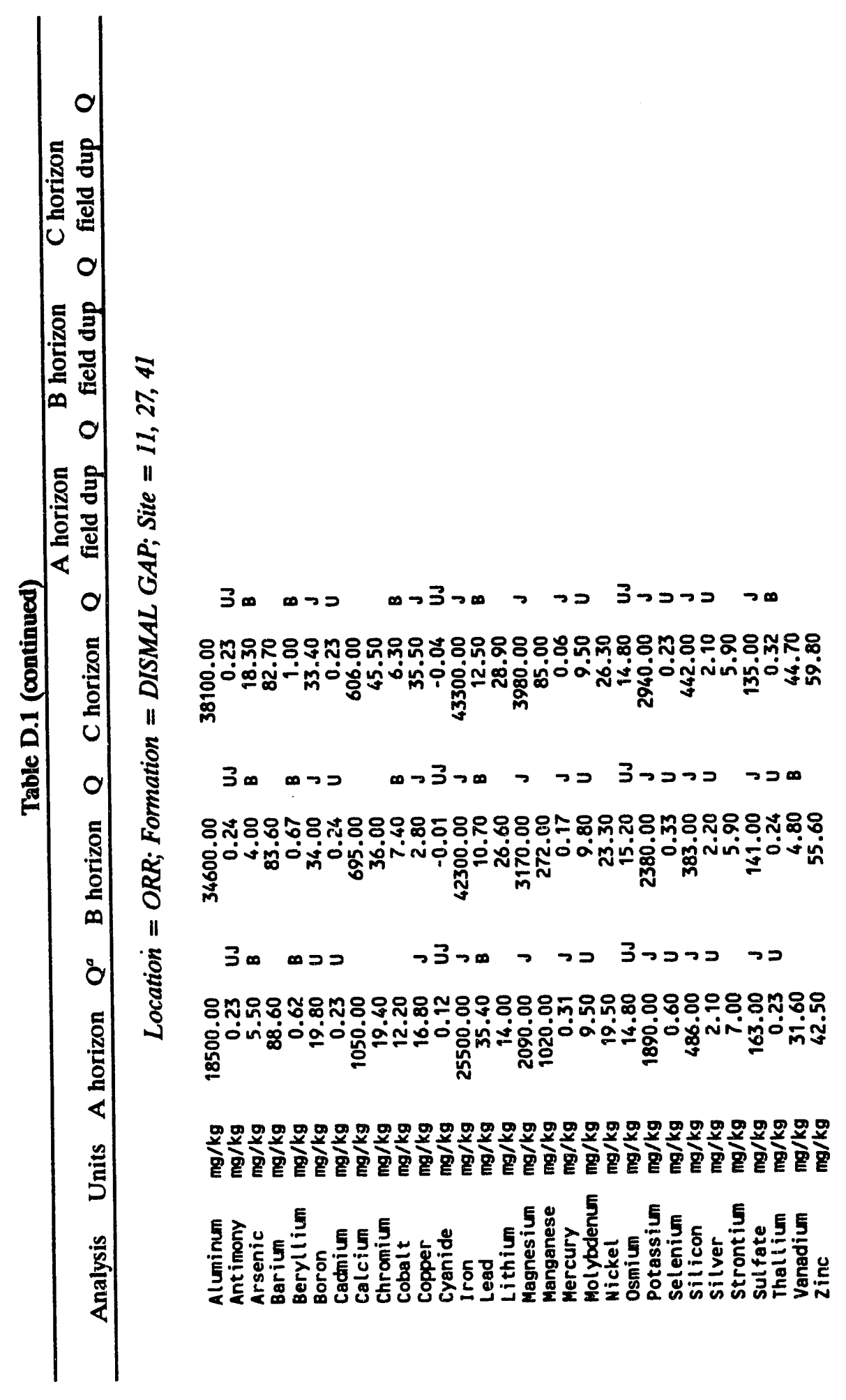


D-12

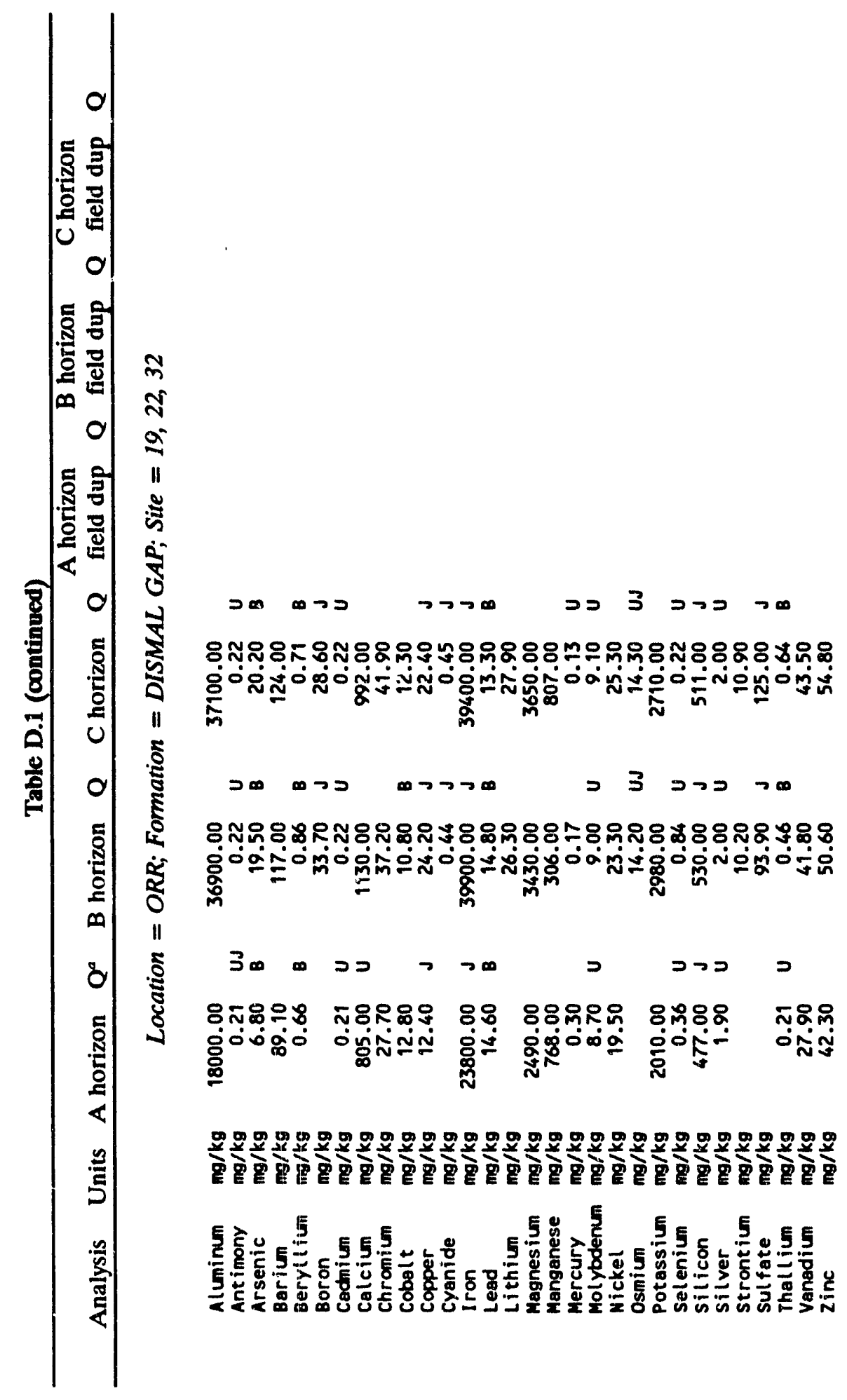


D-13

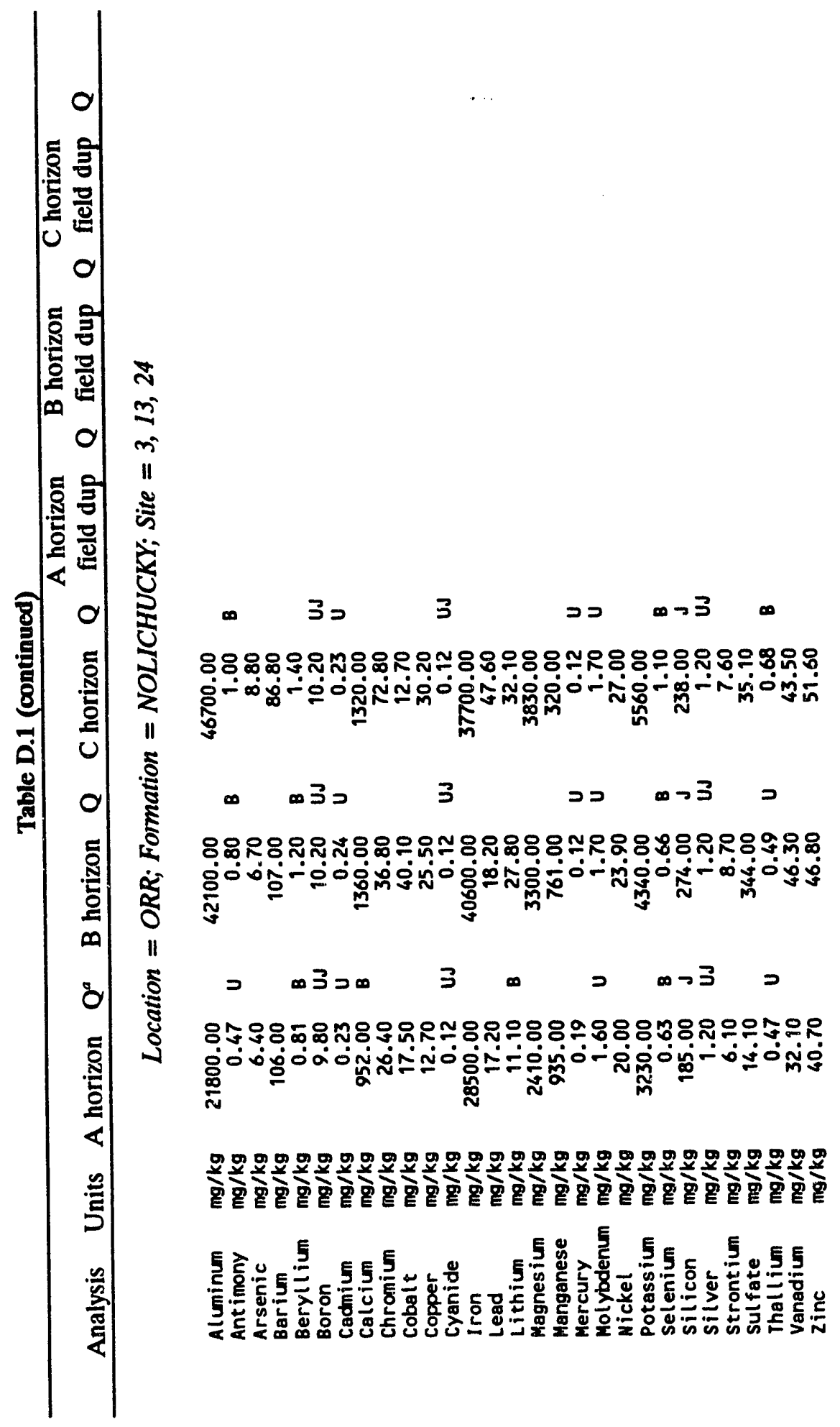


D-14

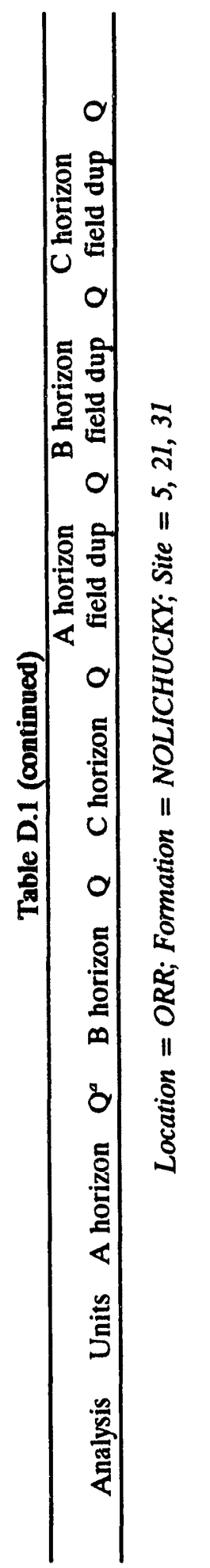

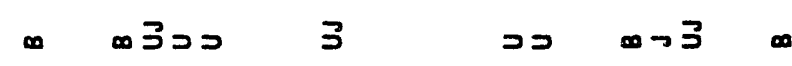

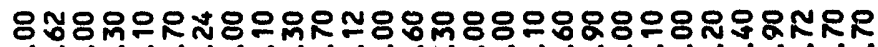

品 =-

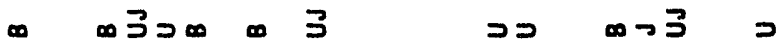

8슨ำ

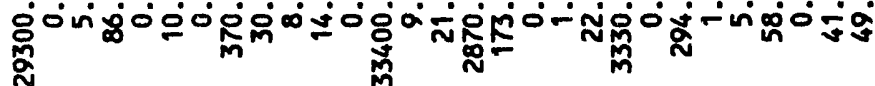

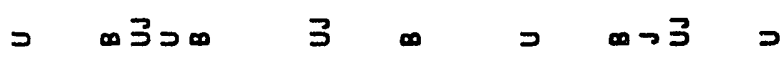

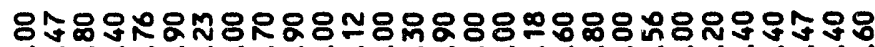

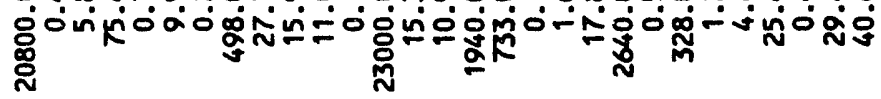

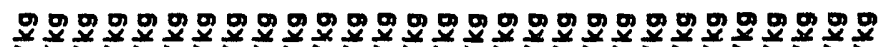

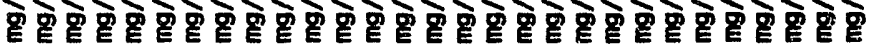

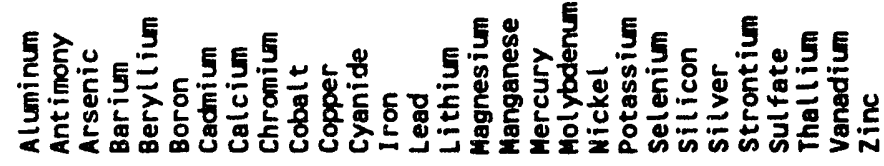


D-15

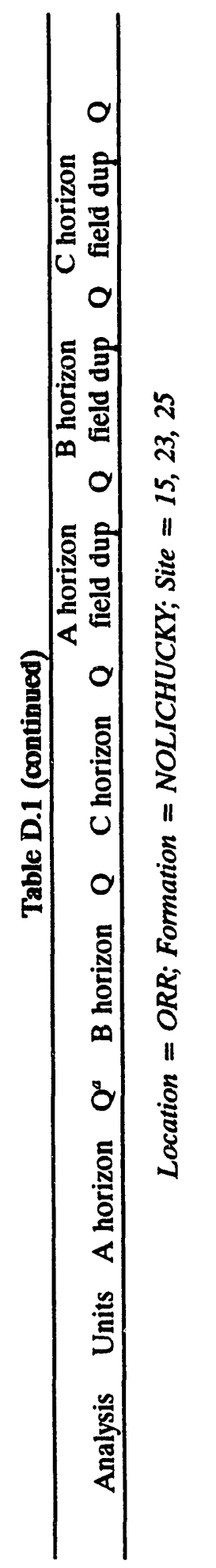

- $\infty 3 ⿻$ - 3 >

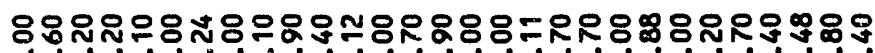

通

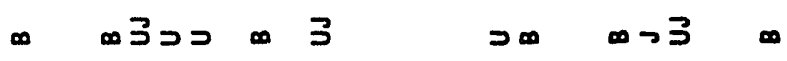

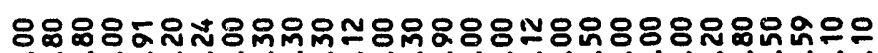

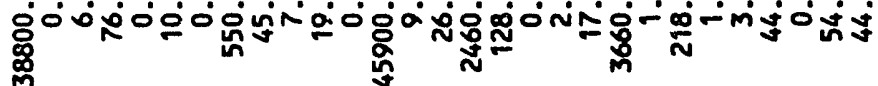

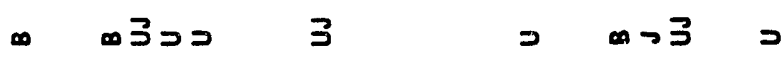

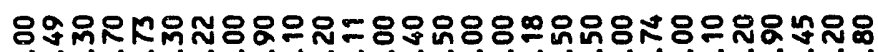

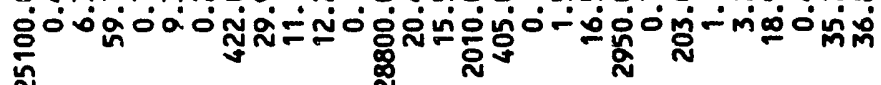

กิ

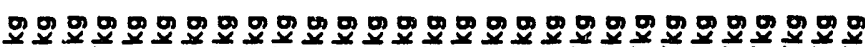

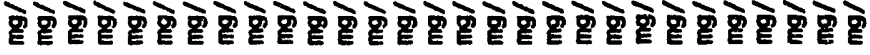

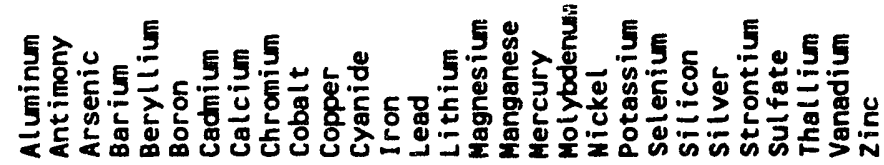


D-16

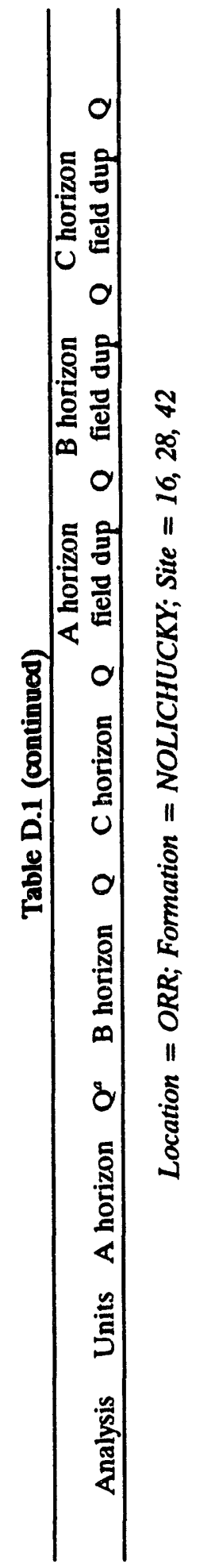

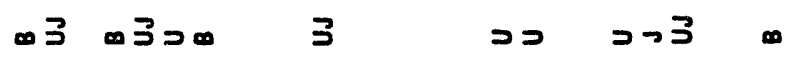

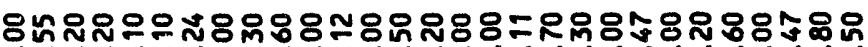

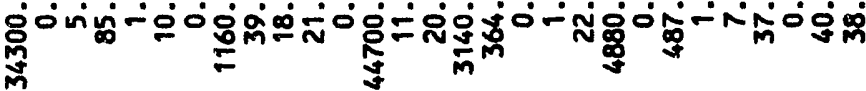

$$
\text { D }
$$

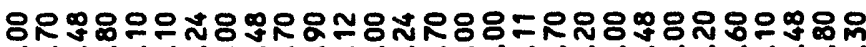

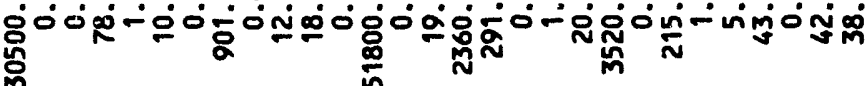

$$
\text { د }
$$

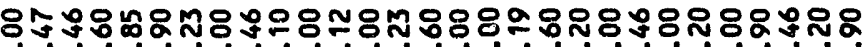
售

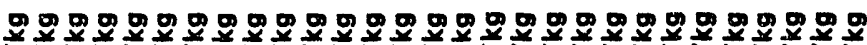

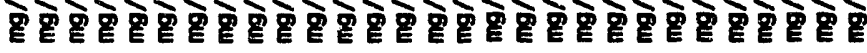

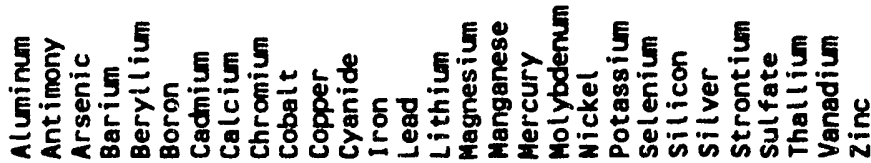


D-17

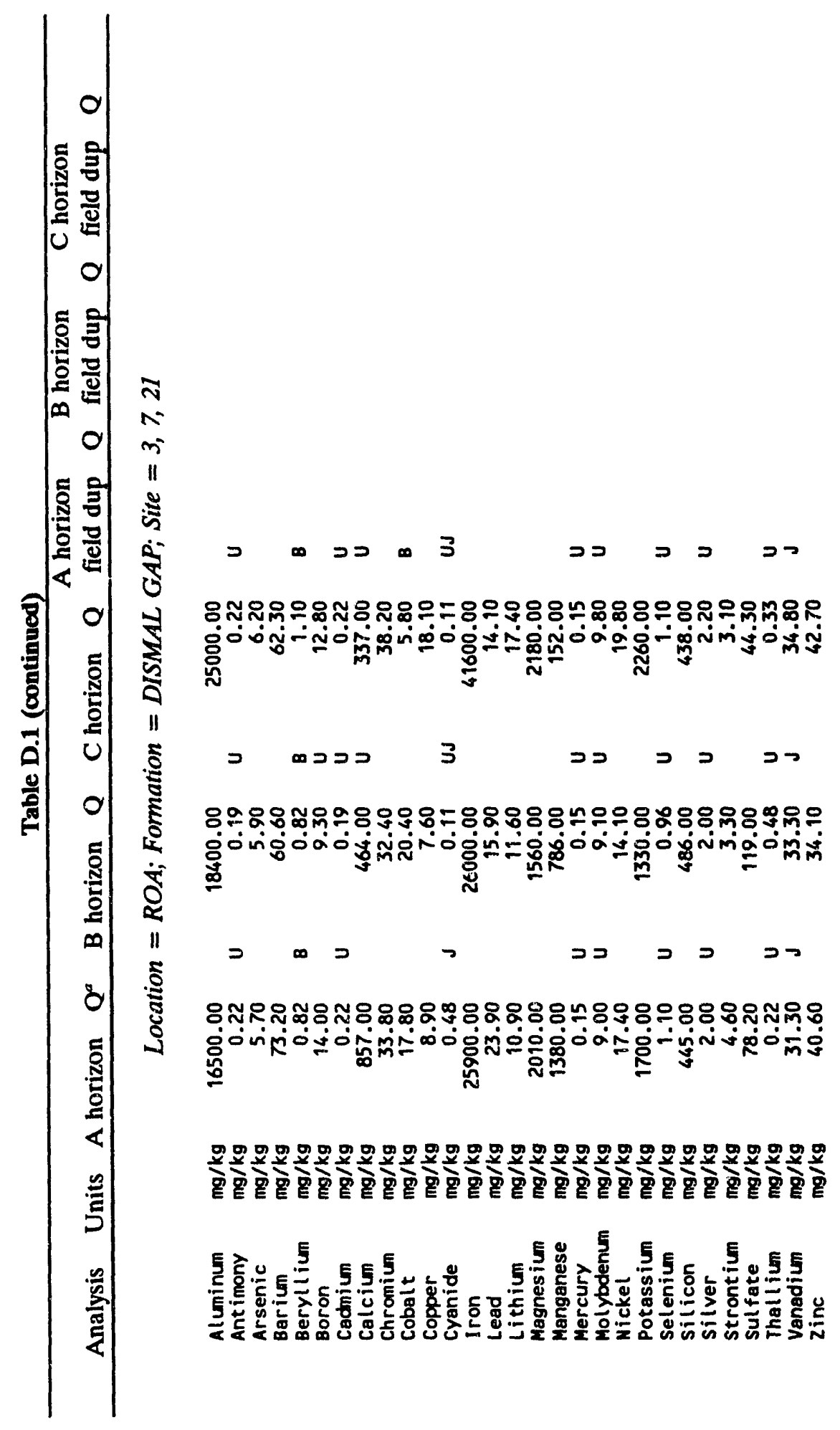


D-18

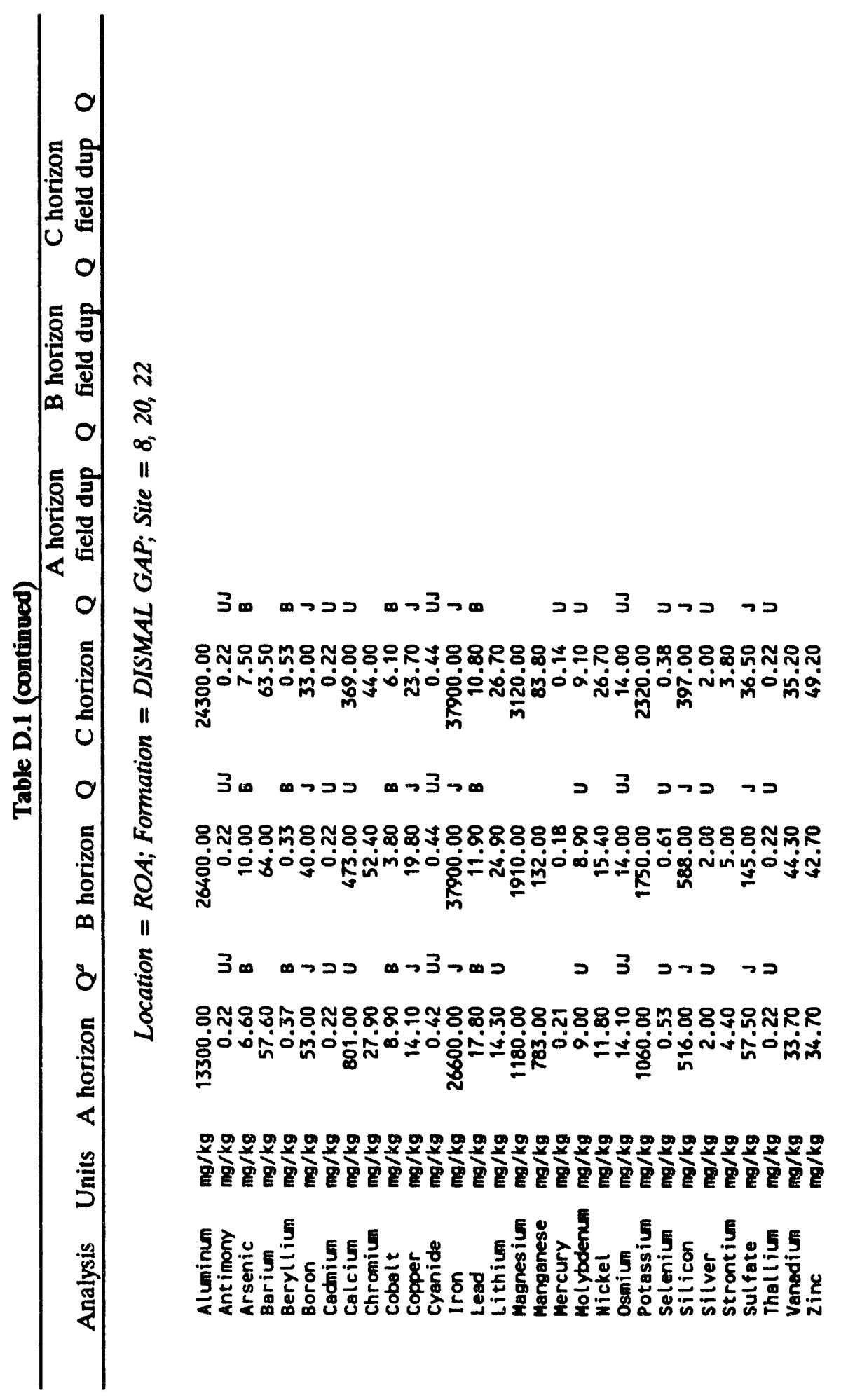


D-19

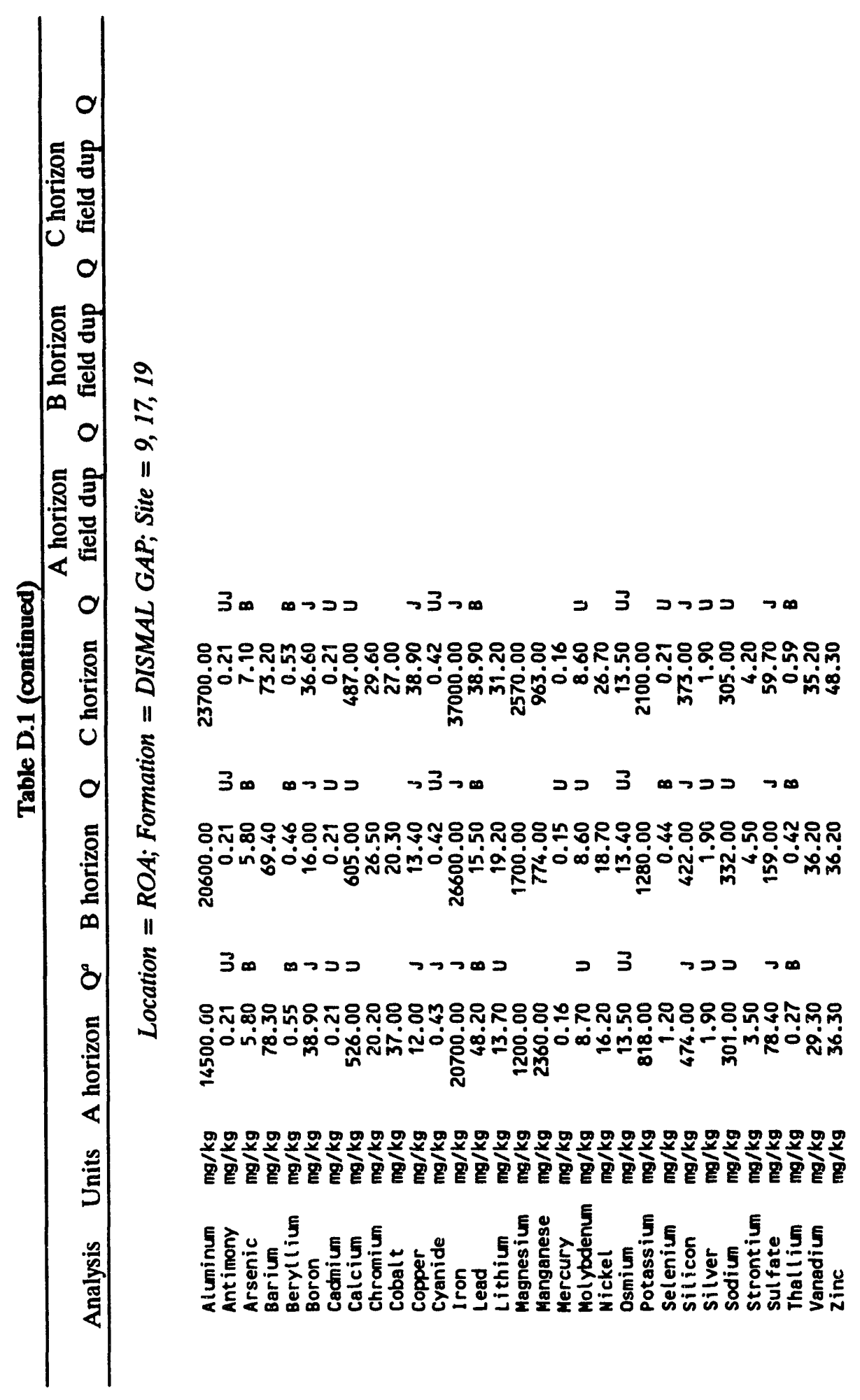




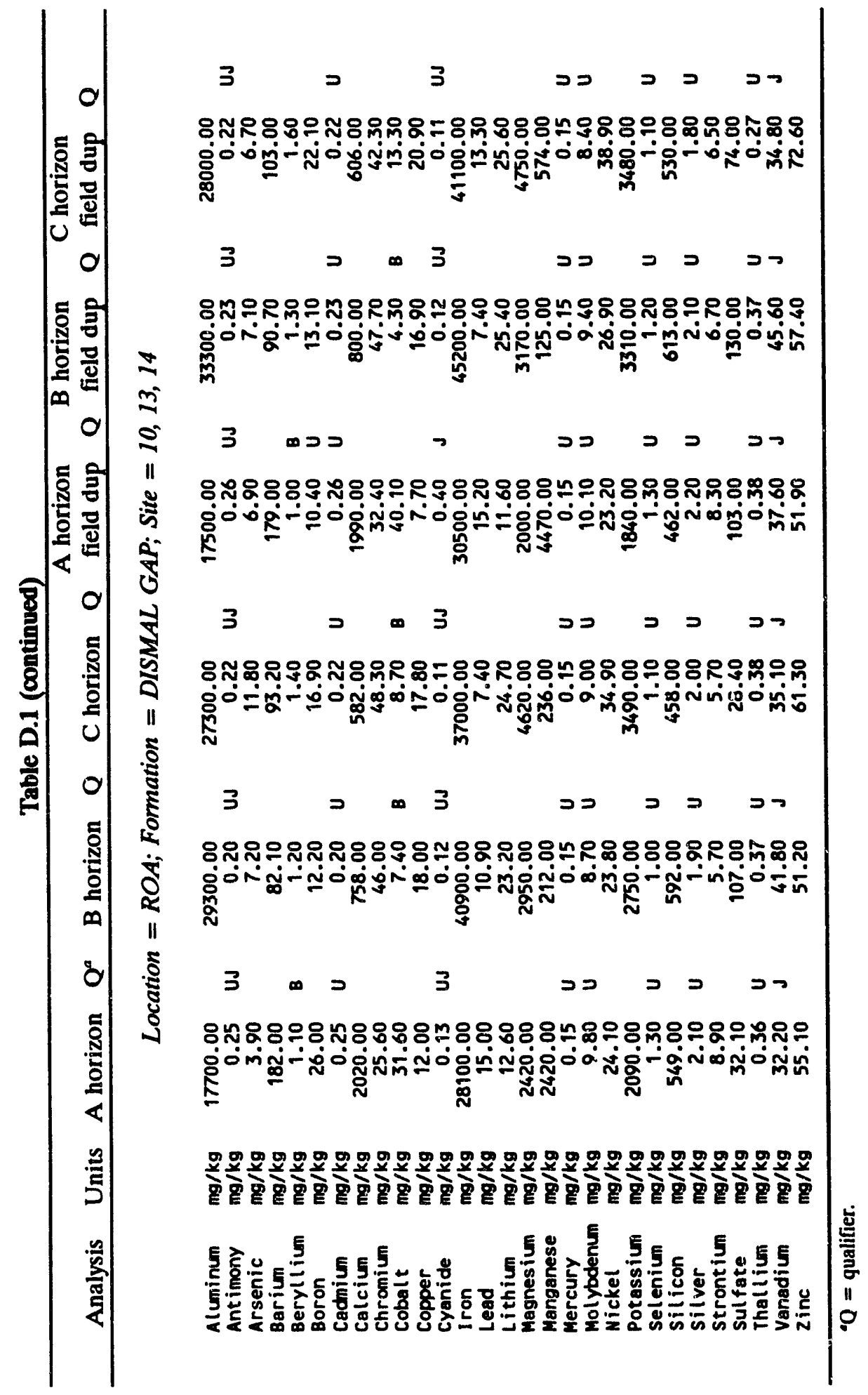


Appendix E

RADIONUCLIDE ANALYSIS DATA 


\section{NOTICE ABOUT UNUSABLE DATA}

The europium-155 data contained in this report have been determined to be unusable, because of possible interference with the gamma spectra of other naturally occurring radionuclides in soils. Although these data were used throughout the analyses discussed in this report, including the evaluation of risk, the effects of eliminating the europium-155 data from risk considerations are minimal, because the risks posed to human health from ingestion of and external exposure to uncontaminated soil on the Oak Ridge Reservation (ORR) containing background levels of europium-155 are relatively small $\left(5.97 \times 10^{-11}\right.$ and $1.49 \times 10^{-7}$, respectively). Furthermore, individual risks are negligible when compared with the total pathway risk from ingestion of and external exposure to background levels of all radionuclides on the ORR (pathway risks are $7.39 \times 10^{-6}$ and $6.38 \times 10^{-4}$, respectively). Hence, elimination of the europium-155 data from this data set would not significantly change the risk values, and similar statements are also true for the europium-155 background risks for Anderson and Roane counties. Revised Phase I results will be presented in the project final report.

NOTE: When available, the validation qualifiers are used in this appendix. When validation qualifiers are not available, the corresponding laboratory data qualifiers are used. 


\section{VALIDATOR DATA QUALIFIER DEFINIIIONS}

The following definitions provide brief explanations of the qualifiers assigned to the data in this appendix.

U The analyte was analyzed for but was not detected above the reported sample quantitiation limit.

J The analyte was positively identified; the associated numerical value is the approximate concentration of the analyte in the sample.

N The analysis indicates the presence of an analyte for which there is presumptive evidence to make a tentative identification.

JN The analysis indicates the presence of an analyte that has been tentatively identified, and the associated numerical value represents its approximate concentration.

UJ The analyte was not detected above the reported sample quantication limit. However, the reported quantitation limit is approximate and may or may not represent the actual limit of quantitation necessary to accurately and precisely measure the analyte in the sample.

R The sample results are rejected because of serious deficiencies in the ability to analyze the sample and meet quality control criteria. The presence or absence of the analyte cannot be verified.

UN The laboratory did not register this compound, but there was presumptive evidence of a compound that was within the retention time window but was not reported. No other qualification of the data was made.

UJN The laboratory did not report the compound, but there was presumptive evidence of a compound that was witbin the retention time window but was not reported. The data were qualified as estimated, $\mathrm{J}$, because of other discrepancies with the data.

RN The laboratory did not report the compound, but there was evidence of a compound that was within the retention time window but was not reported. The data were qualified as unusable, $R$, because of other discrepancies with the data. 


\section{LABORATORY DATA QUALIFIER DEFINITIONS}

The following contract laboratory data qualifiers are used in this project.

Organic

\begin{tabular}{|c|c|}
\hline Qualifier & Explanation \\
\hline $\mathbf{U}$ & Indicates compound was analyzed for but not detected. \\
\hline $\mathbf{J}$ & Indicates an estimated value. \\
\hline $\mathbf{N}$ & $\begin{array}{l}\text { Indicates presumptive evidence of a compound [used only for tentatively } \\
\text { identified compounds (TICs)]. }\end{array}$ \\
\hline $\mathbf{P}$ & $\begin{array}{l}\text { Used for pesticide/aroclor target analytes when there is greater than } 25 \% \\
\text { difference for detected concentrations between the two gas chromatograph (GC) } \\
\text { columns. The lower of the two is reported and flagged. }\end{array}$ \\
\hline C & $\begin{array}{l}\text { Used for pesticide results where the identification has been confirmed by } \\
\text { GC/mass spectrograph (MS). }\end{array}$ \\
\hline B & Used when the analyte is found in the associated blank as well as in the sample. \\
\hline $\mathbf{D}$ & Identifies all compounds in an analyte at a secondary dilution factor. \\
\hline A & Indicates that a TIC is a suspected aldol-condensation product. \\
\hline $\mathbf{X}$ & $\begin{array}{l}\text { Other specific flags may be used to properly define the results. If they are used } \\
\text { they must be fully described and attached to the Sample Data Package. }\end{array}$ \\
\hline
\end{tabular}

Inorganic

\begin{tabular}{|c|l||}
\hline Qualifier & Explanation \\
\hline U & Indicates compound was analyzed for but not detected. \\
J & Indicates an estimated value. \\
N & Spiked sample recovery not within control limits. \\
E & Reported value estimated because of the presence of interference. \\
M & $\begin{array}{l}\text { Duplicate injection precision not met. } \\
\text { B }\end{array}$ \\
S & $\begin{array}{l}\text { Reported value was obtained from a reading that was less than the CRDL, but } \\
\text { Reported value was determined by the method of standard additions (MSA). }\end{array}$ \\
W & $\begin{array}{l}\text { Post-digestion spike for furnace atomic absorption is out of control limits, while } \\
\text { sample absorbance is less that 50\% of spike absorbance. }\end{array}$ \\
$*$ & Duplicate analysis not within control limits. \\
+ & Correlation coefficient for the MSA is less than 0.995. \\
\hline
\end{tabular}




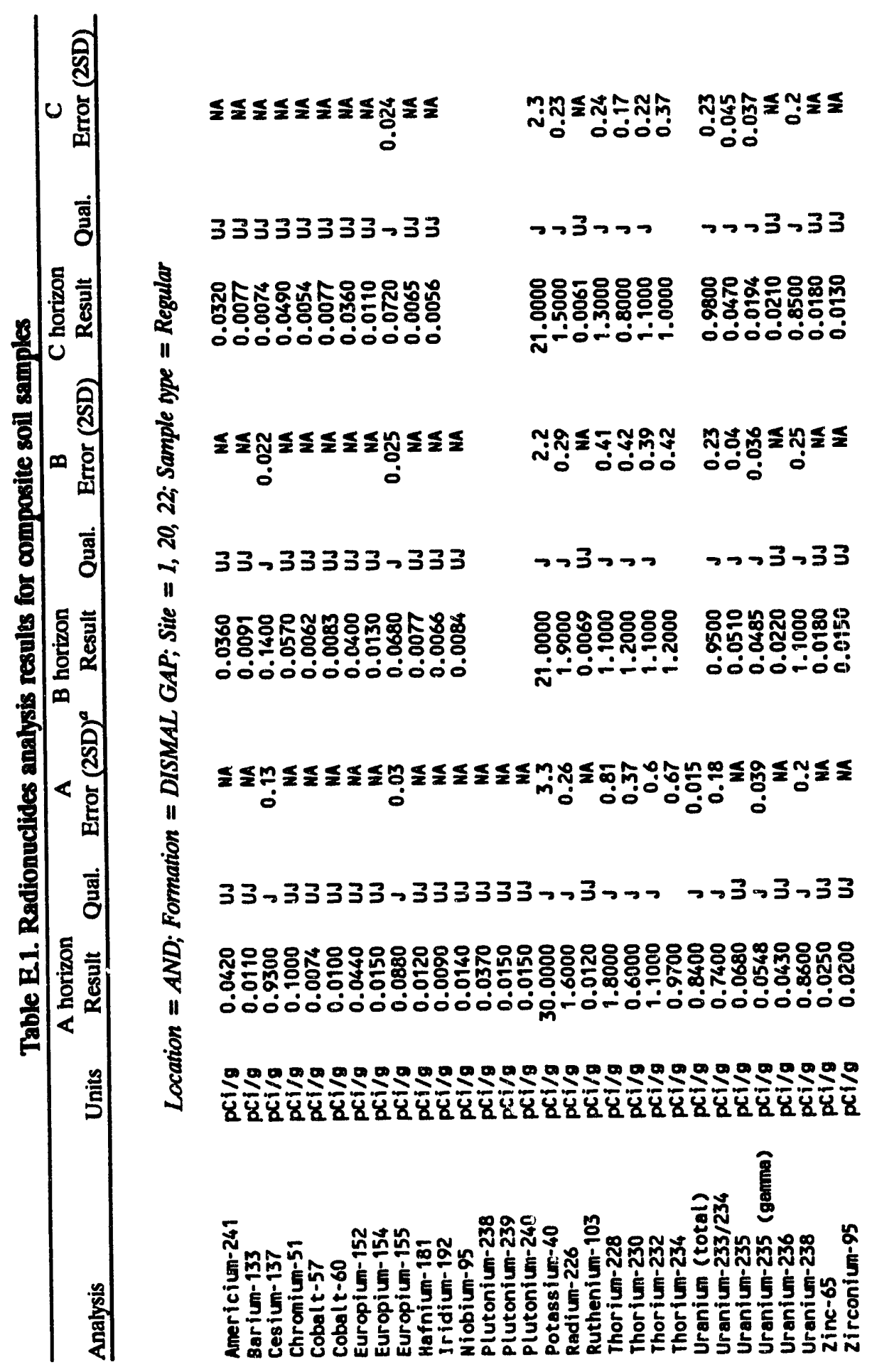




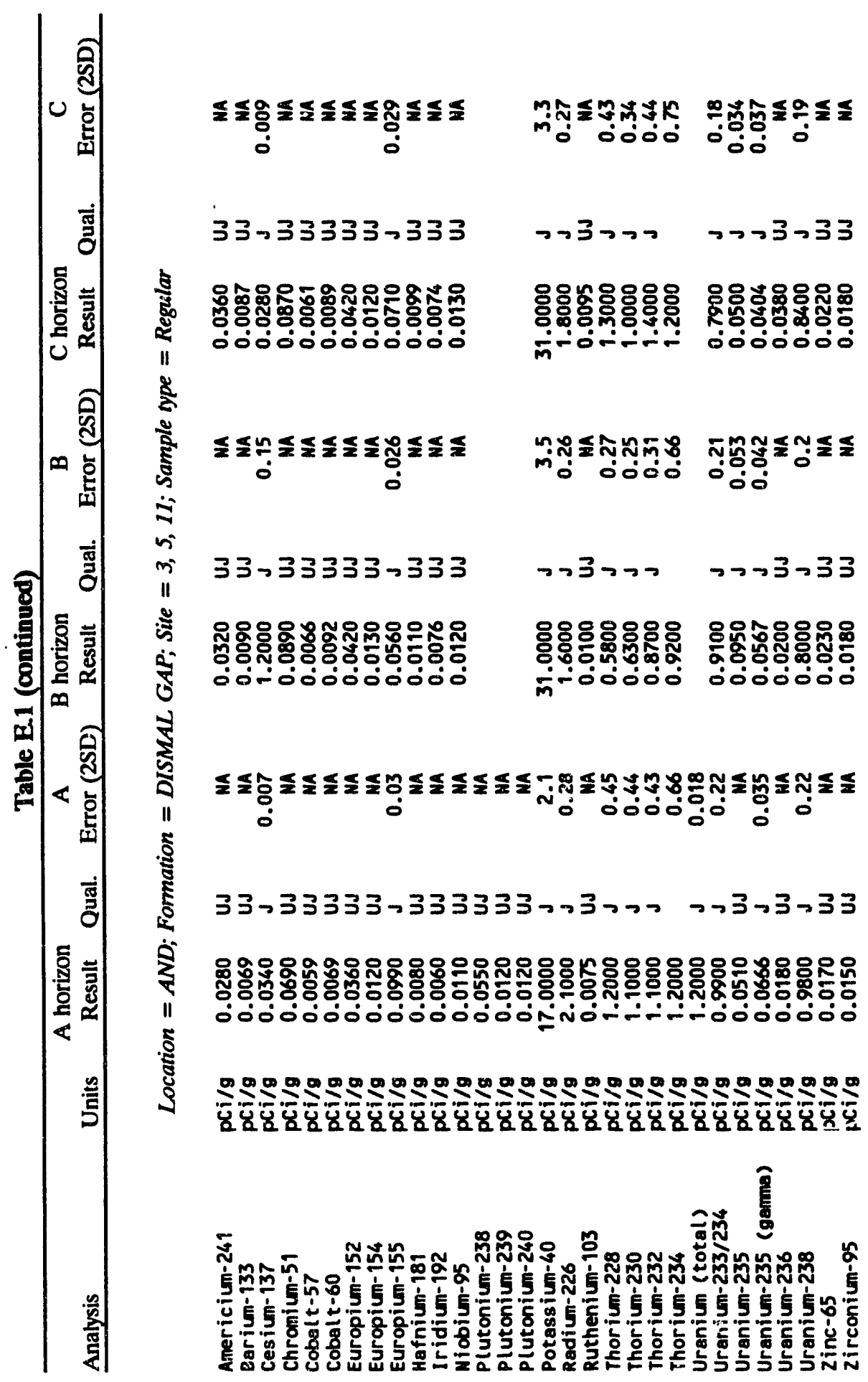


E-7

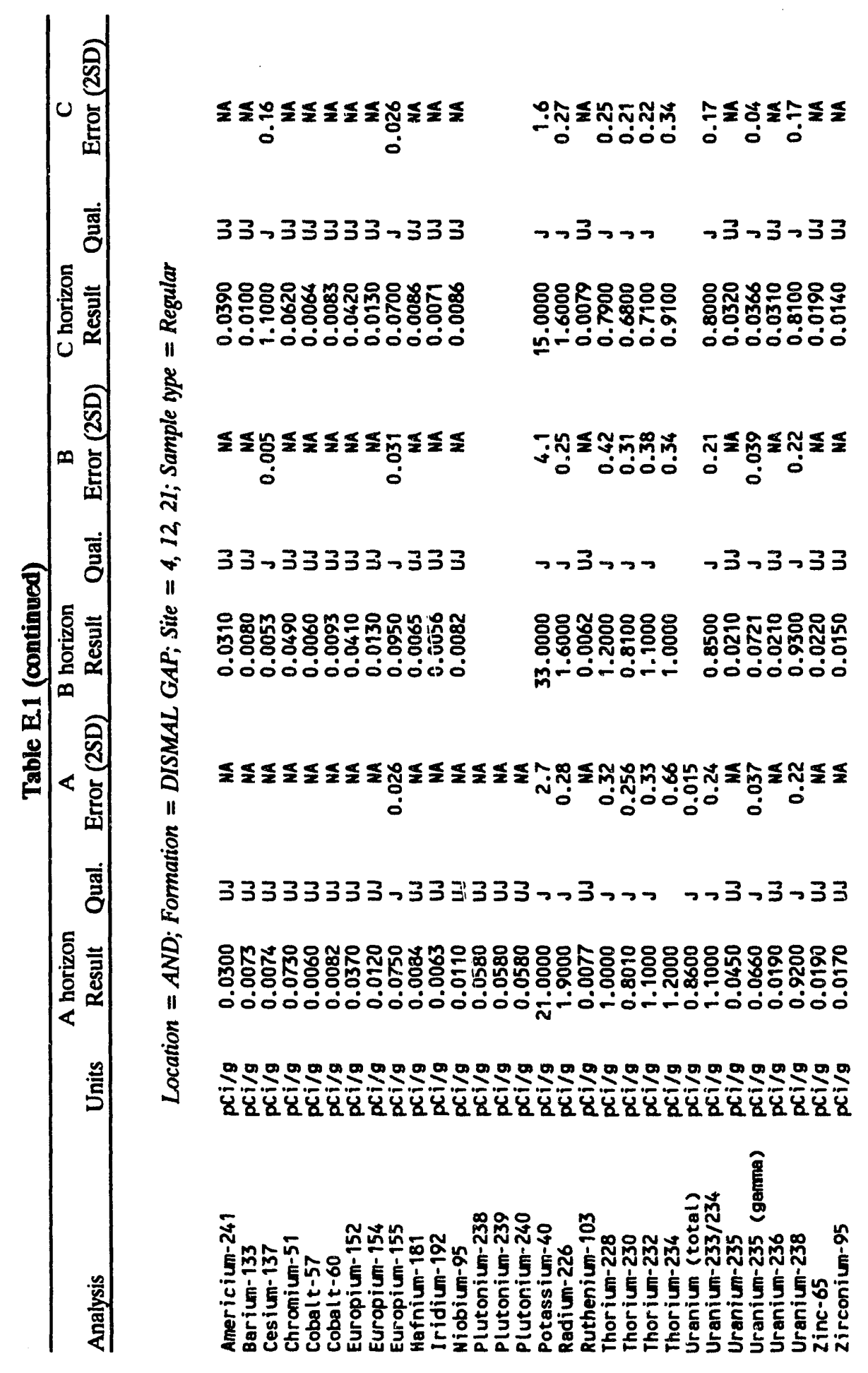




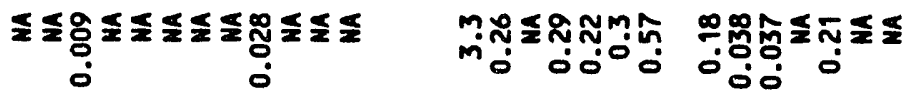

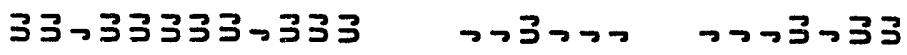

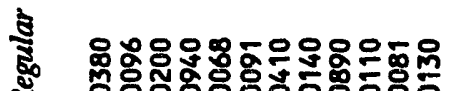

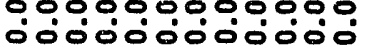

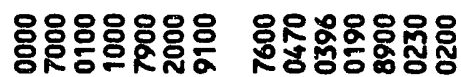

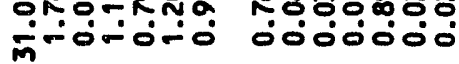
s.

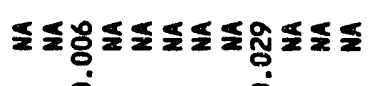

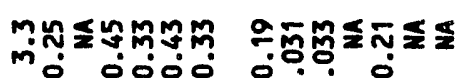
$\dot{a}$ 8

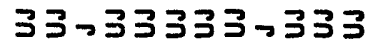

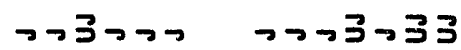

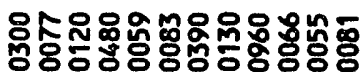

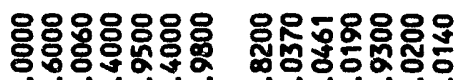

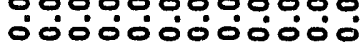

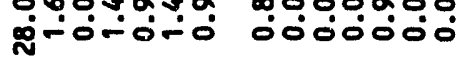

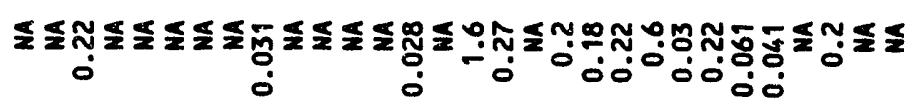

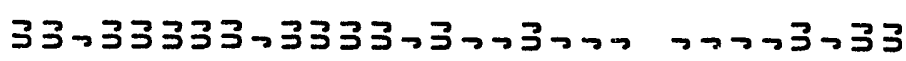

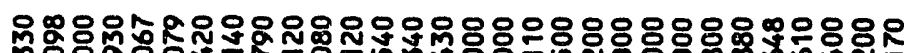

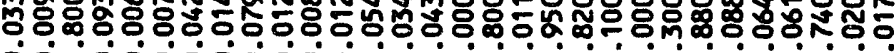

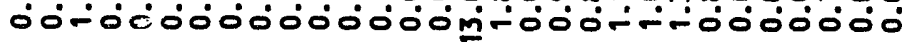

ำㄴำ ব্চব)

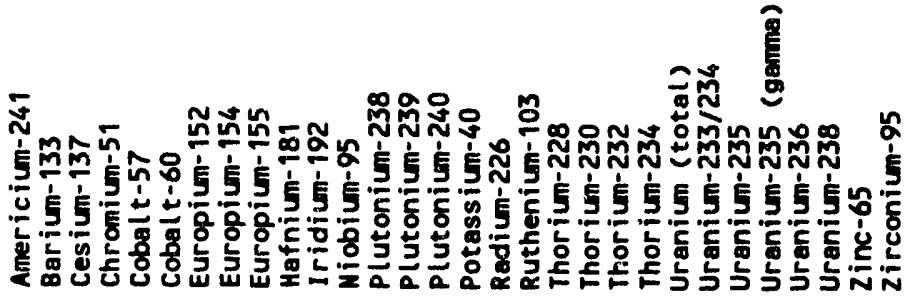


E-9

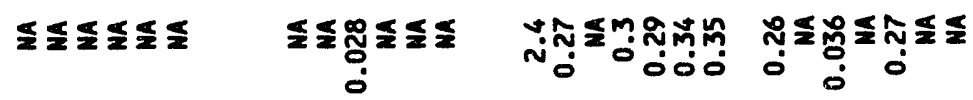

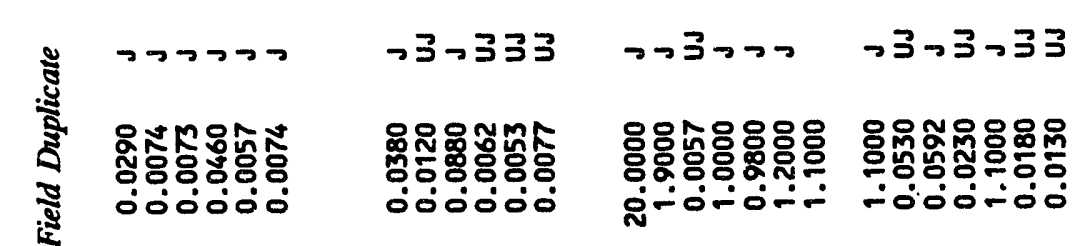

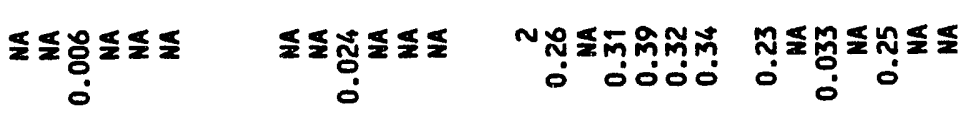

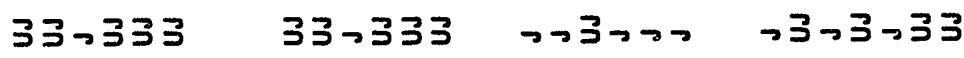

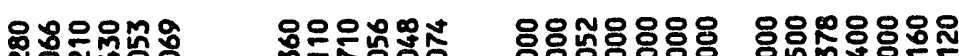

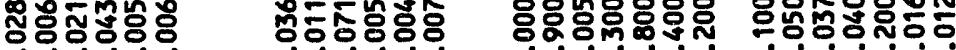

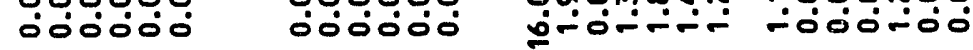

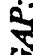

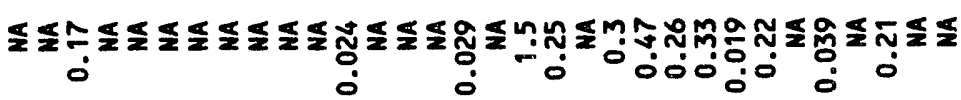

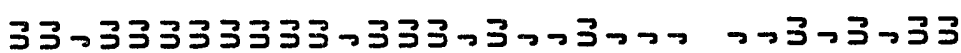

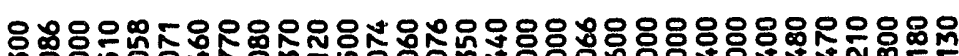

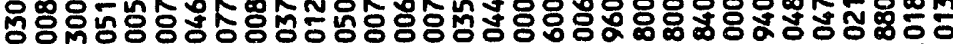

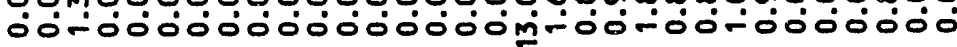

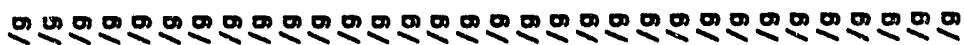
设

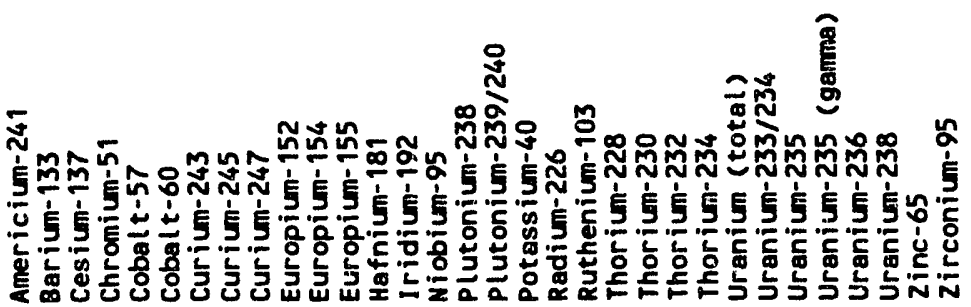




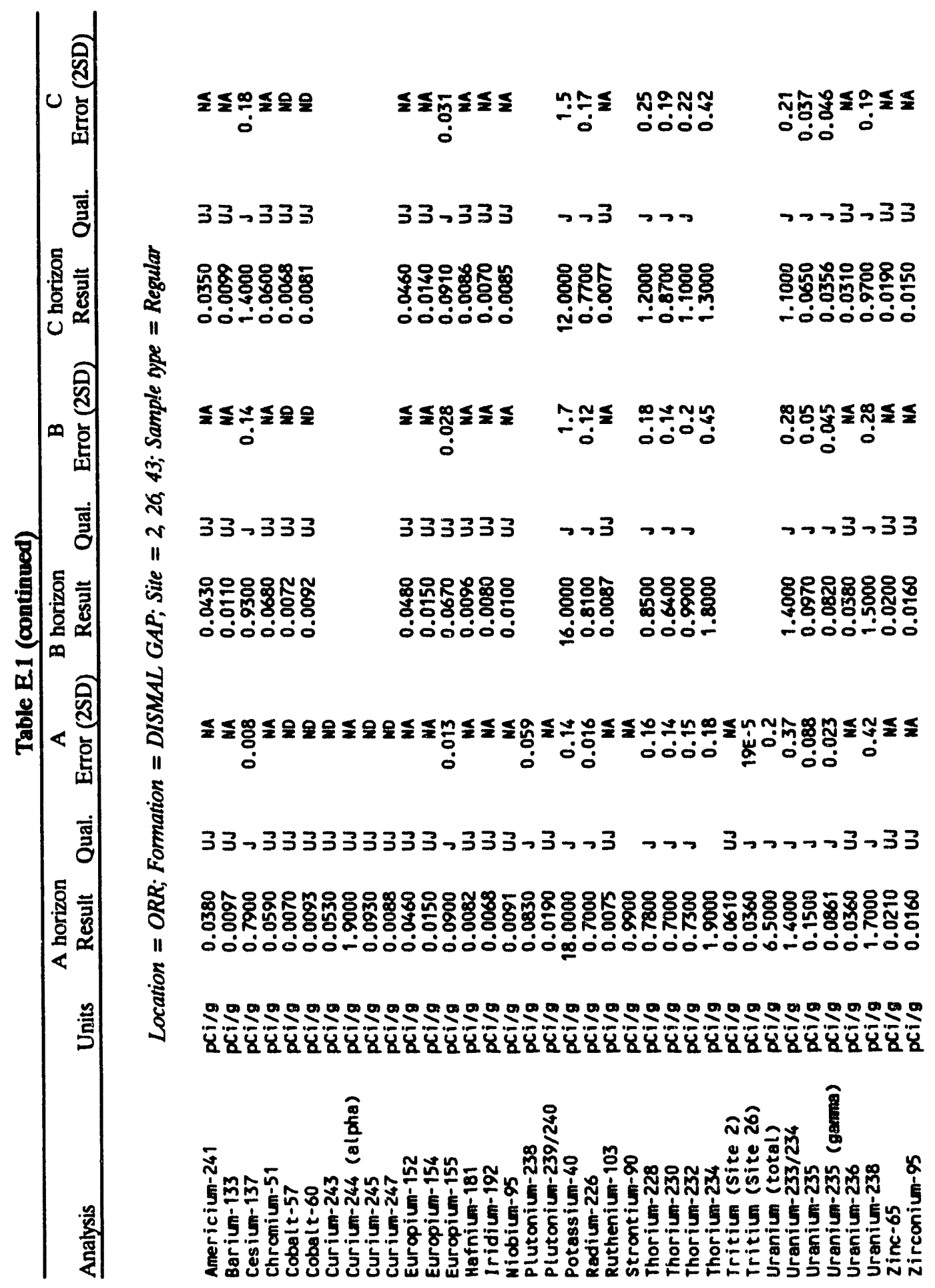


E-11

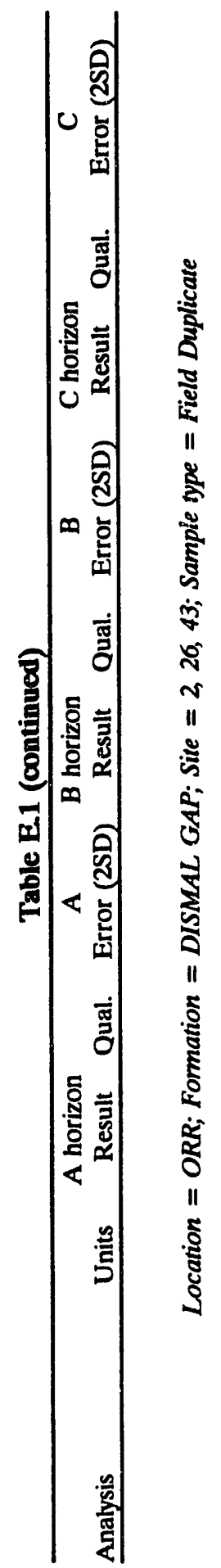

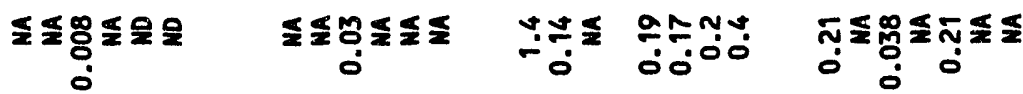

\begin{tabular}{|c|c|c|c|c|}
\hline 33 3ココ & 33ーラ33 & כ כ & כつ & $-3,3-3$ \\
\hline 웅생్ํㅇㅎㅇㅇㅇㅇㅇ & 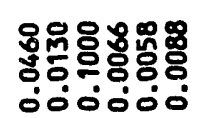 & 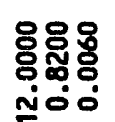 & 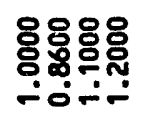 & \\
\hline
\end{tabular}

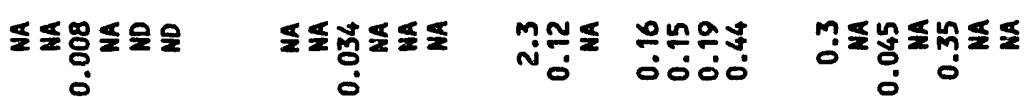

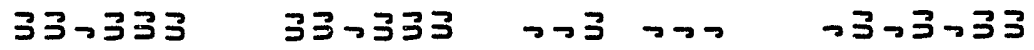

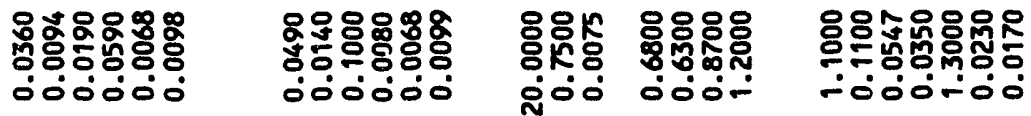

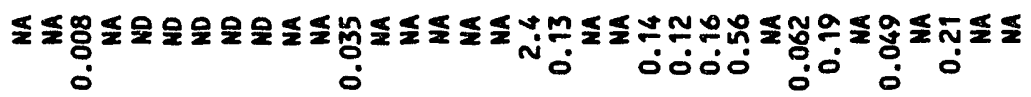

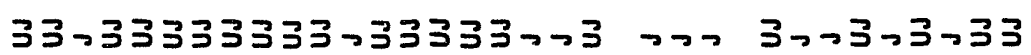

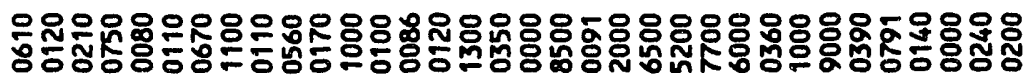

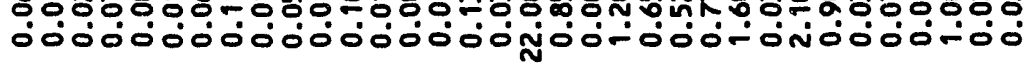

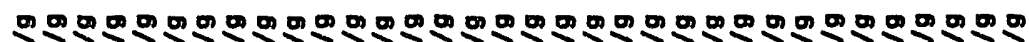

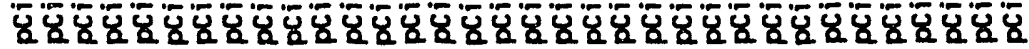

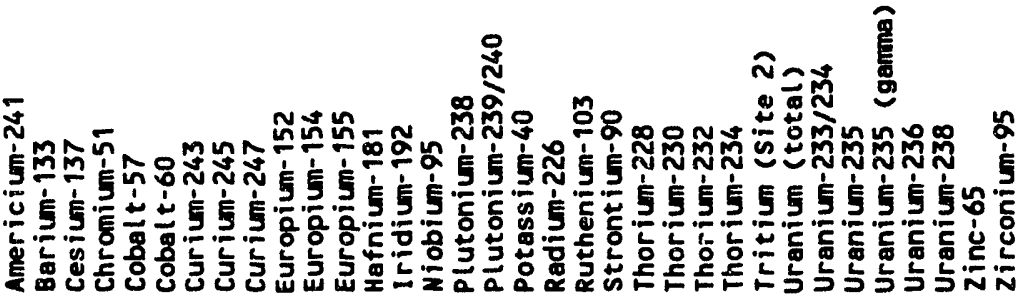


E-12

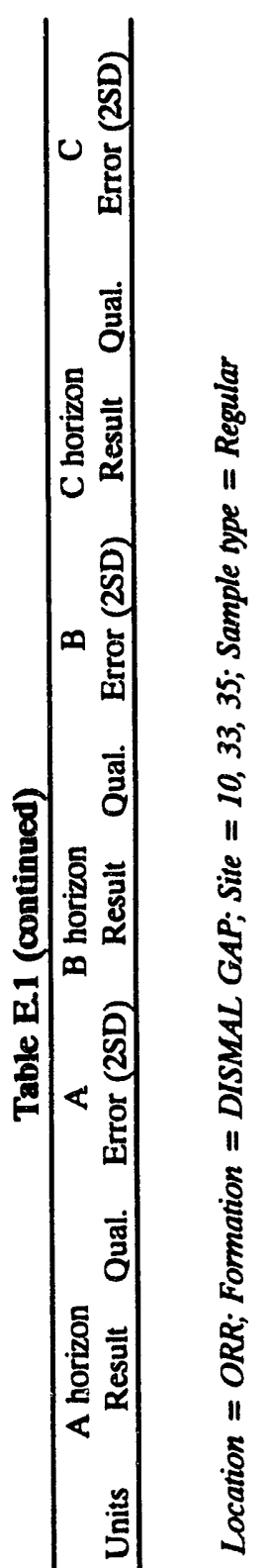

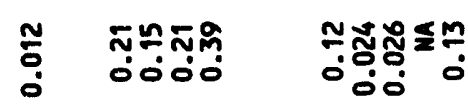

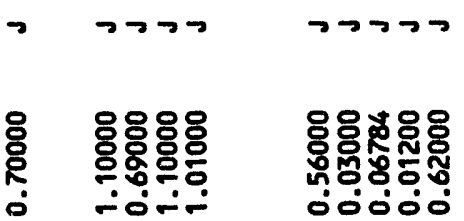

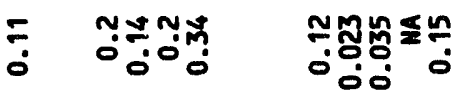

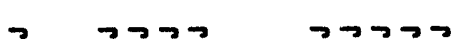

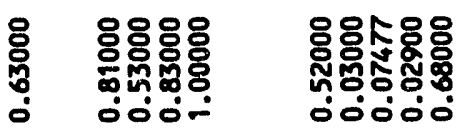

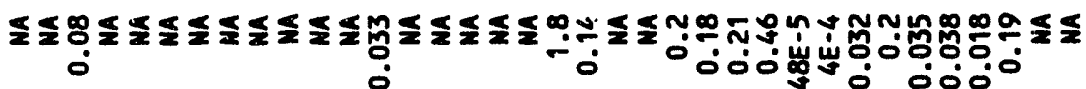

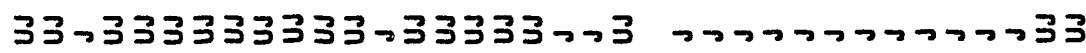

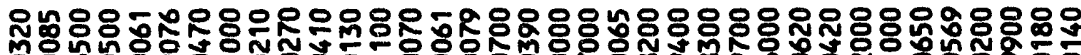

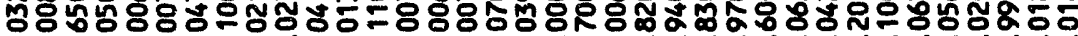

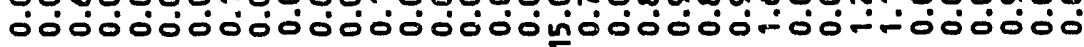

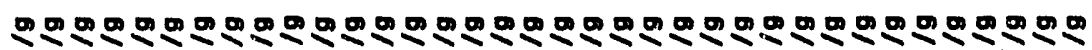

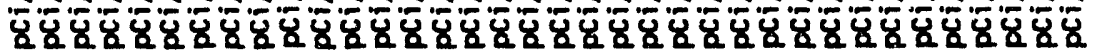

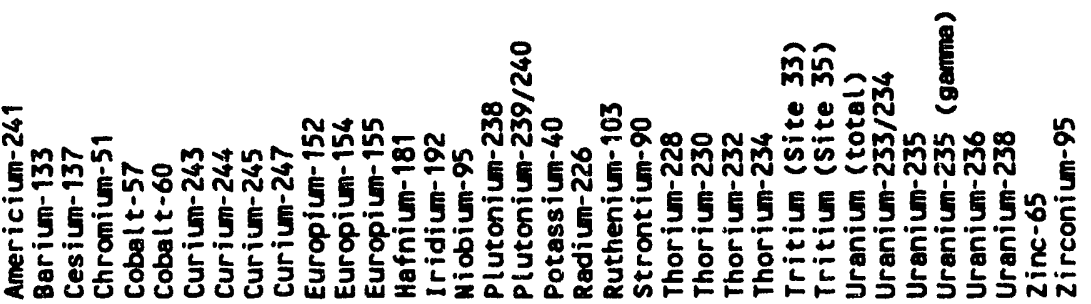




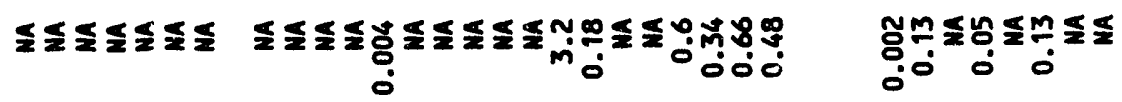

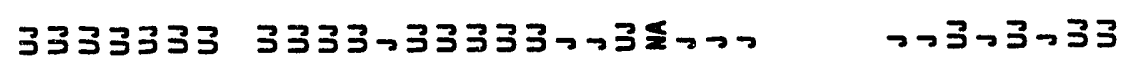

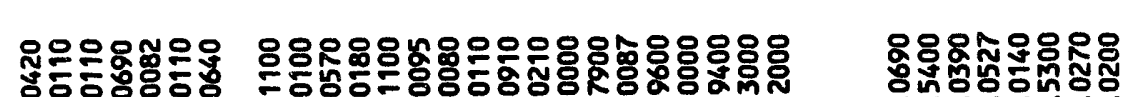

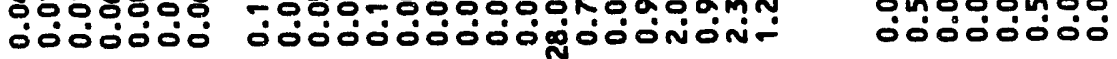
בั้

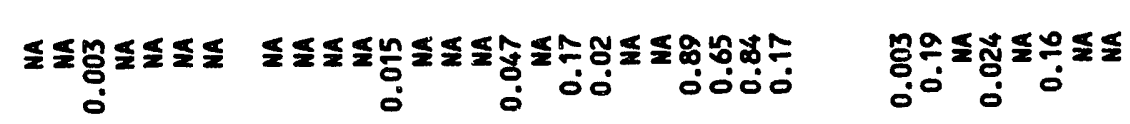

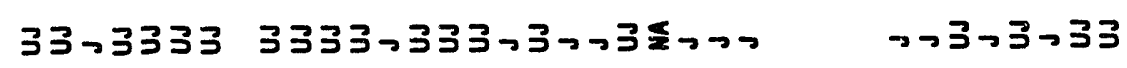

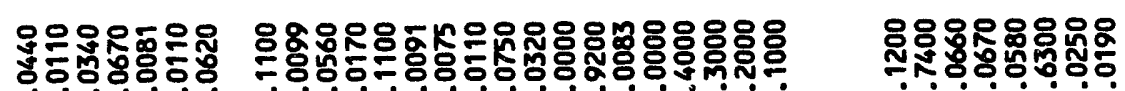

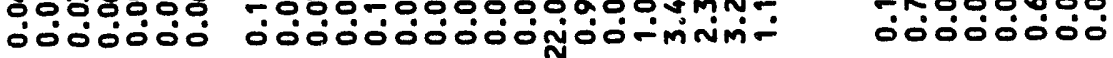

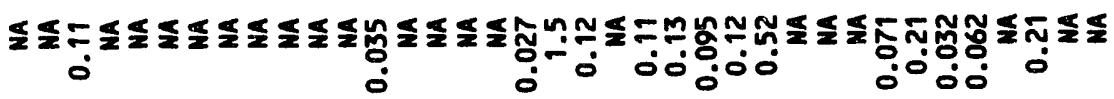

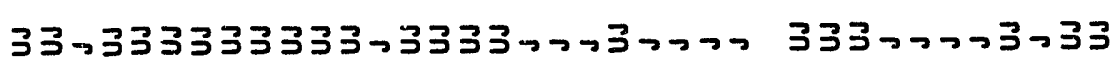

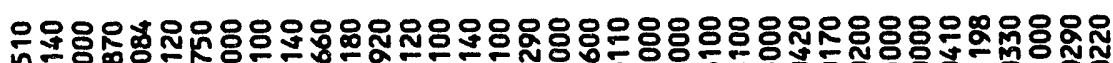

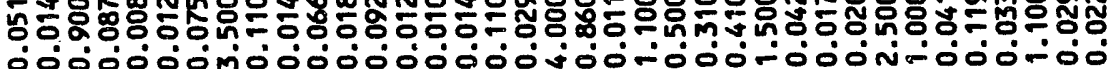

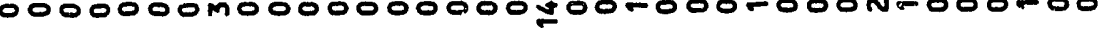

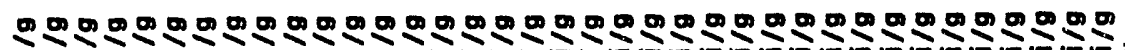

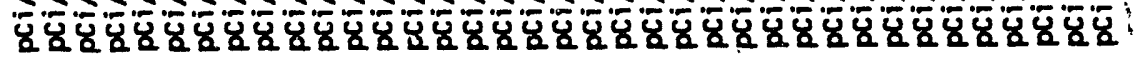

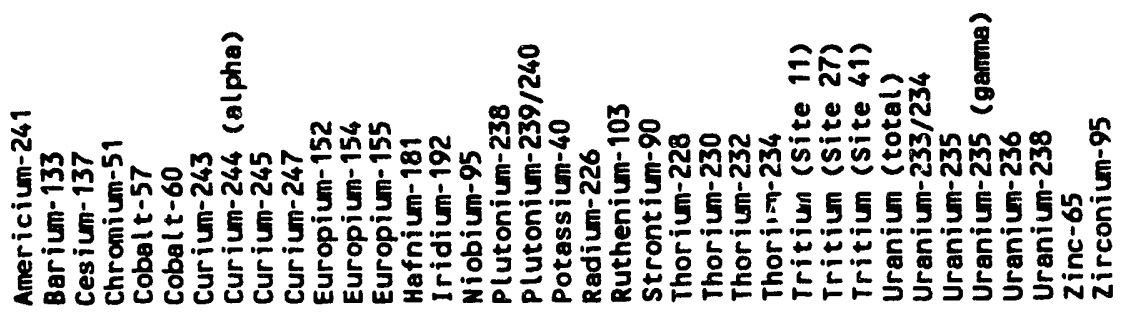


E-14

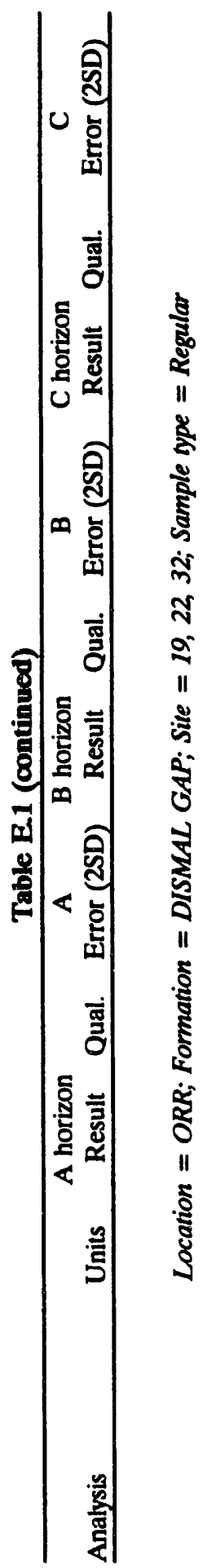

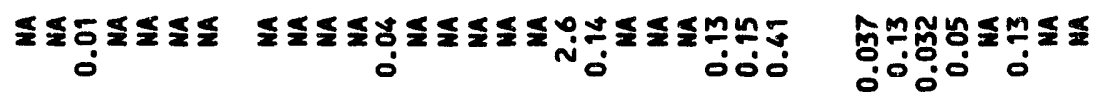

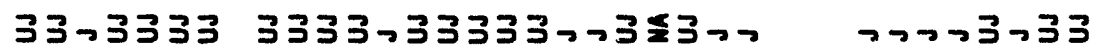

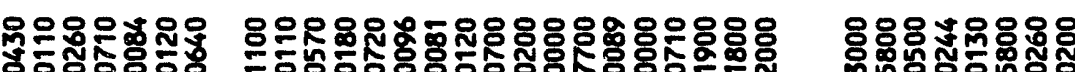

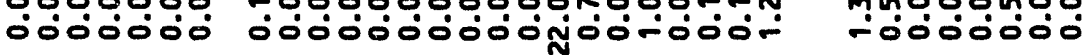

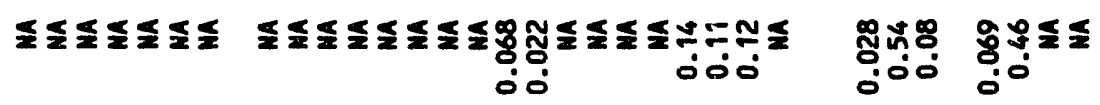

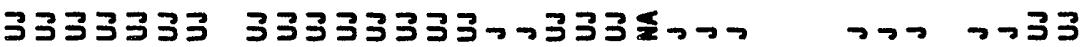

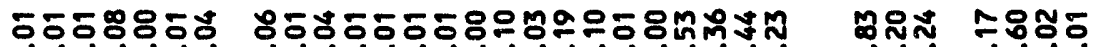

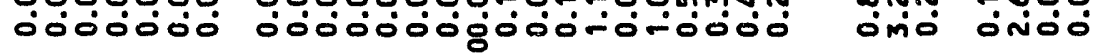
ळั.

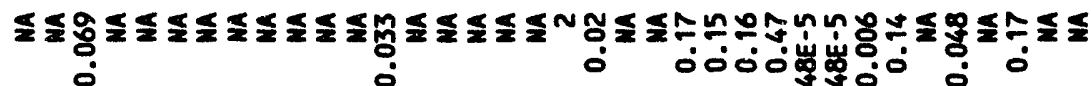

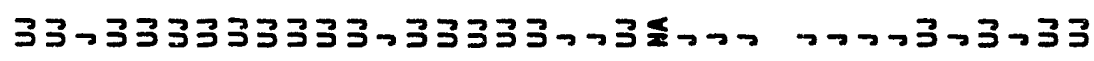

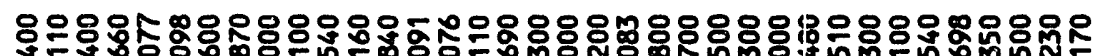
ర大

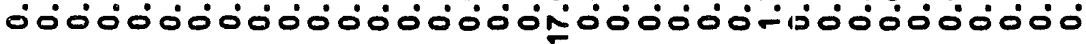

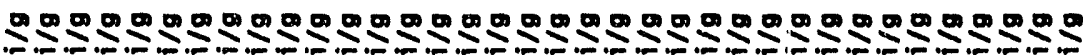

বัه

年 


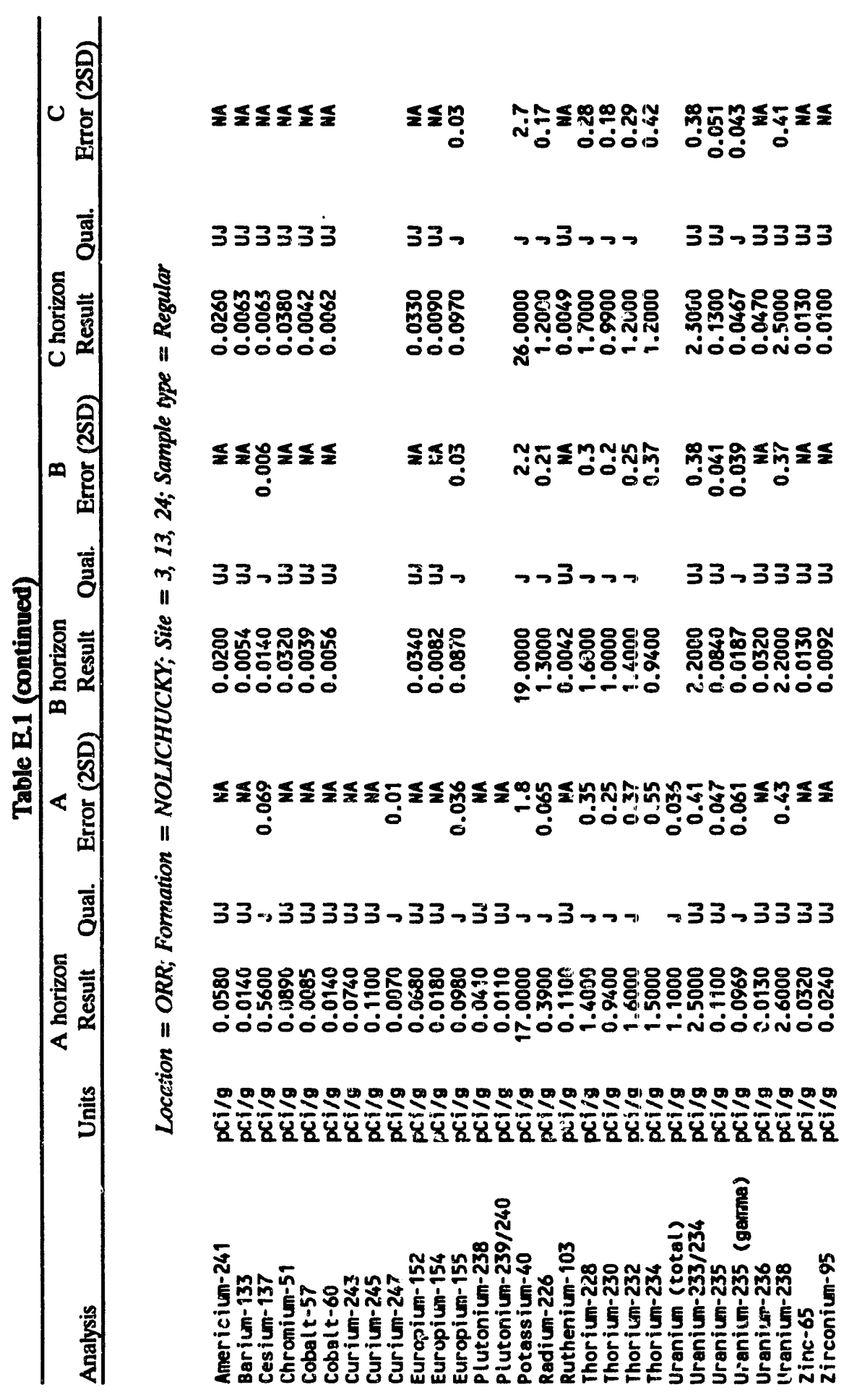




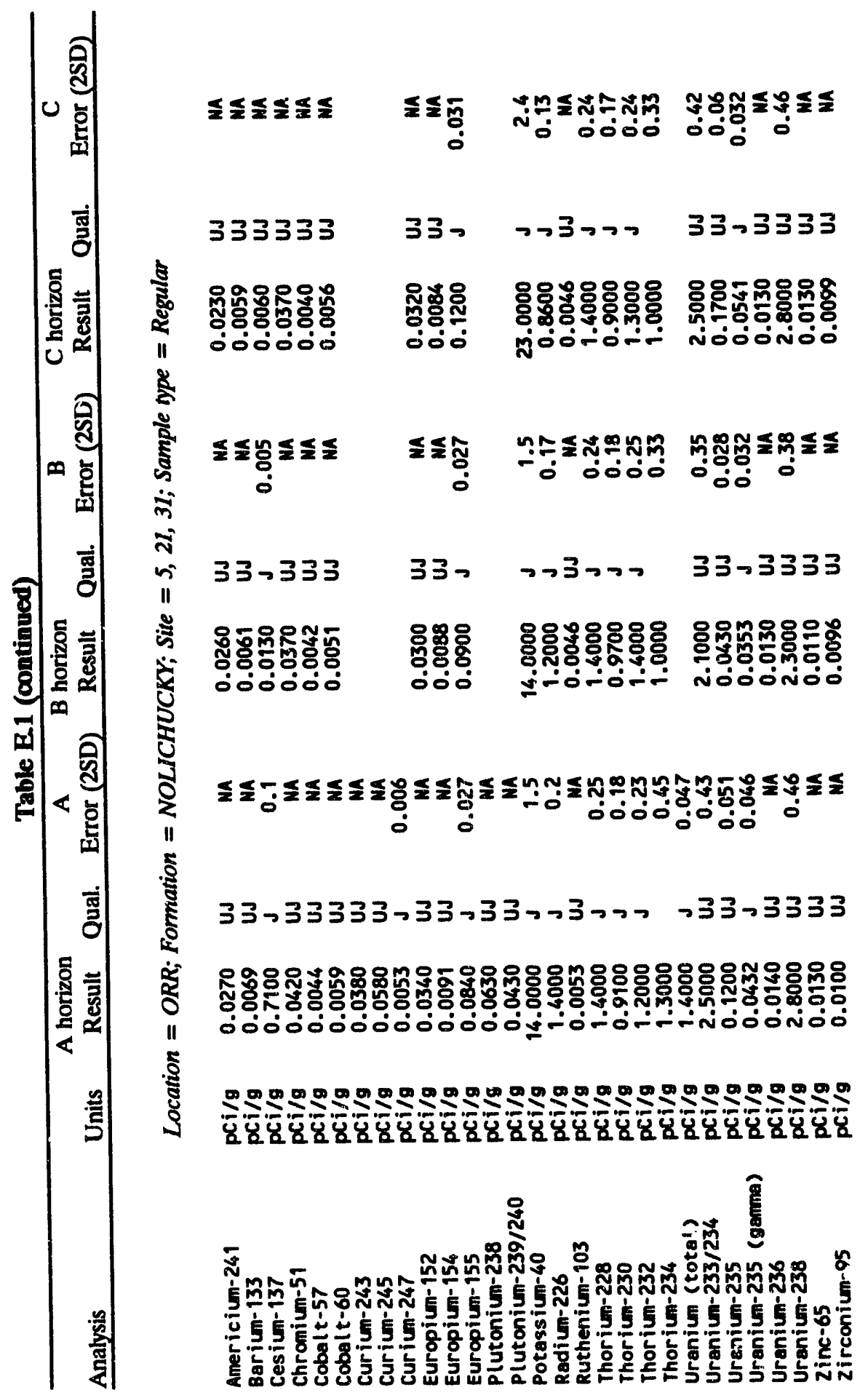




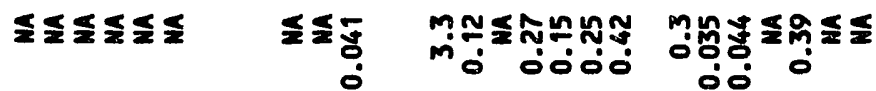

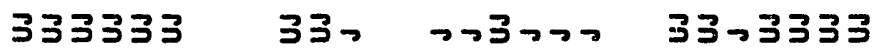

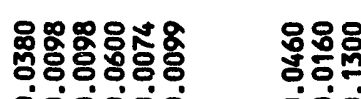

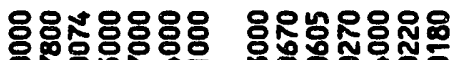

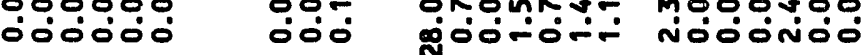

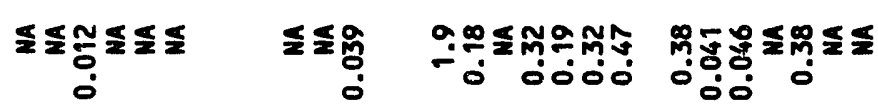

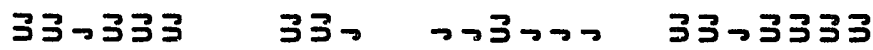

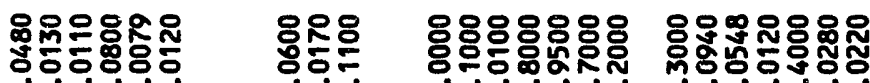

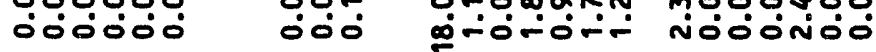

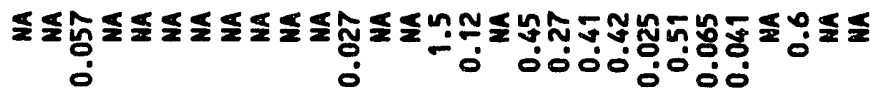

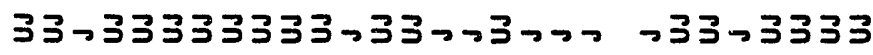

운용ㄷำ

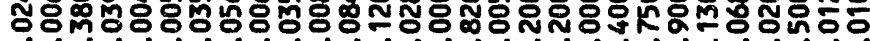

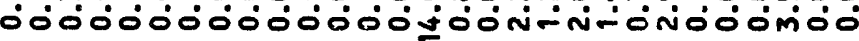

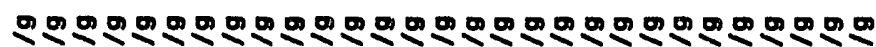

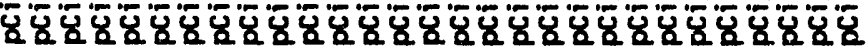

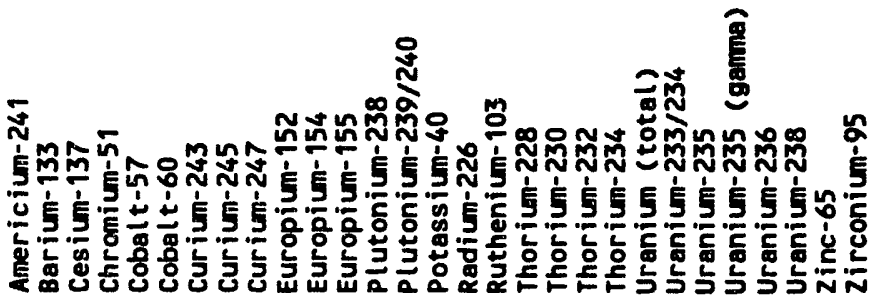




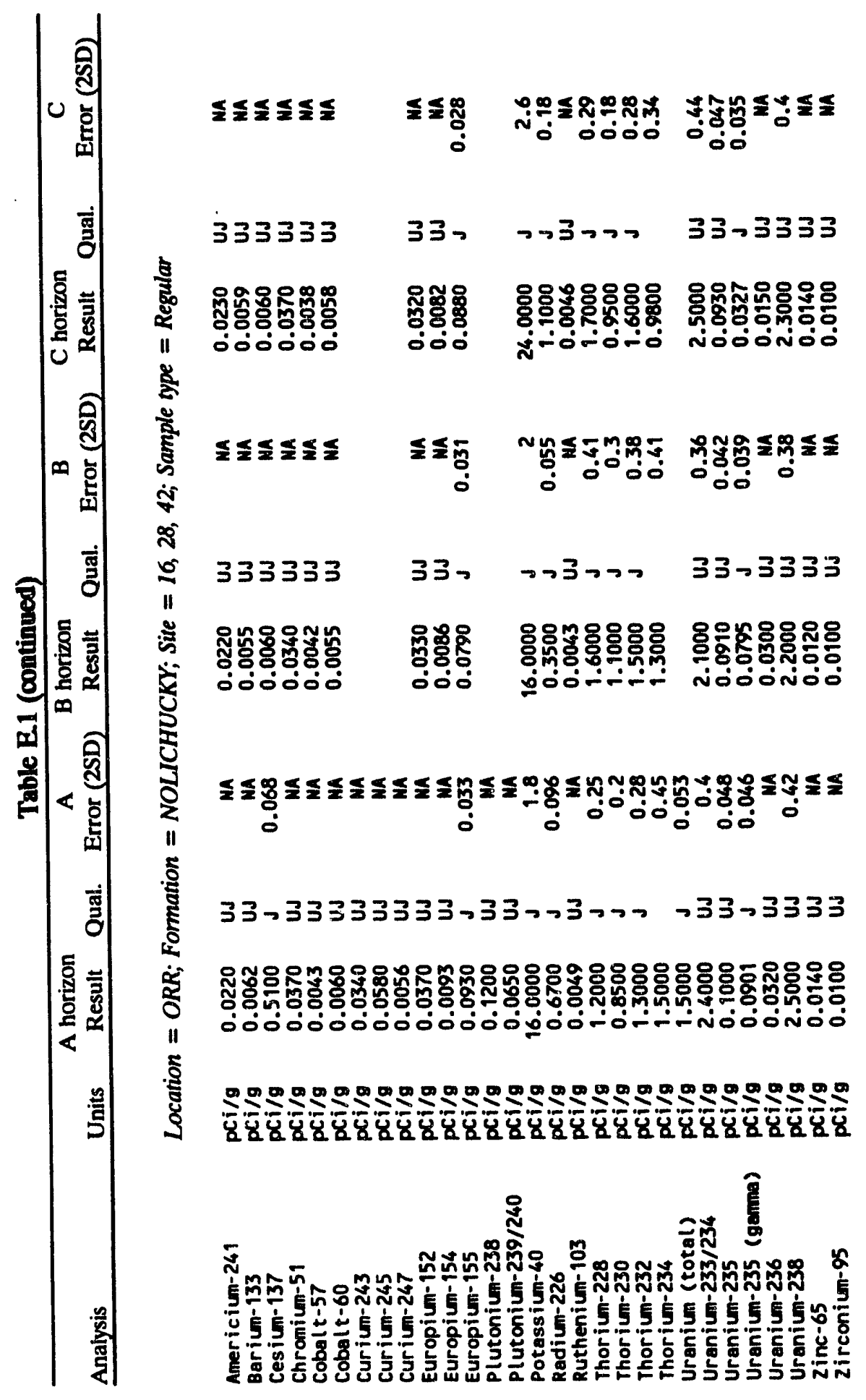


E-19

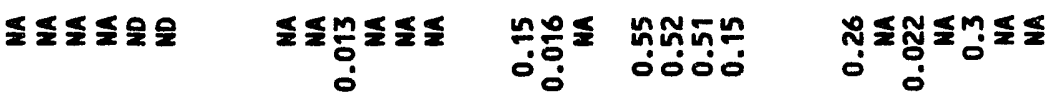

\begin{tabular}{|c|c|c|c|c|}
\hline 33 & アコっふコラ & בת & כ & כמכת מ \\
\hline 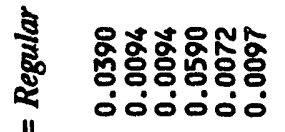 & 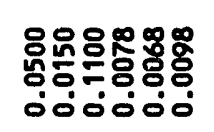 & 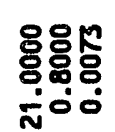 & 융요 & \\
\hline
\end{tabular}

\&

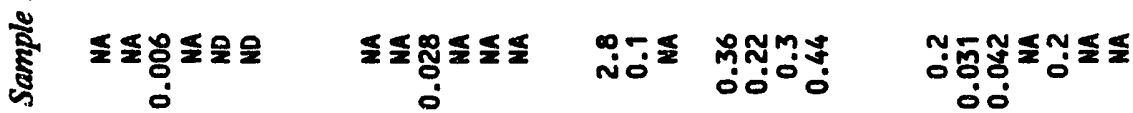
$\ddot{i}$

$\hat{n}$

ア3ープス

$33 \neg 333$

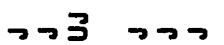

$\neg \neg \neg コ ア コ$

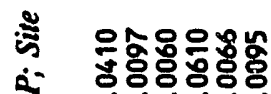

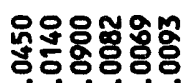

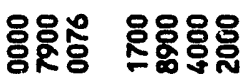

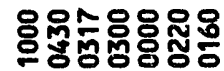

000000

๑0ं00

ío.

ธवं-

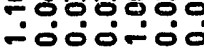

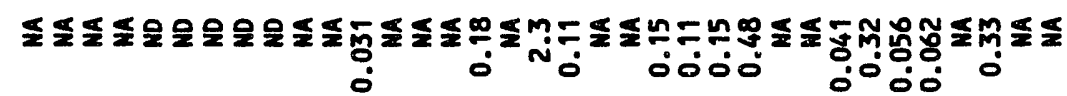

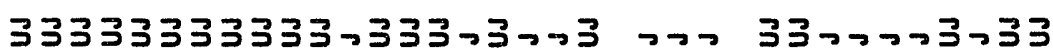

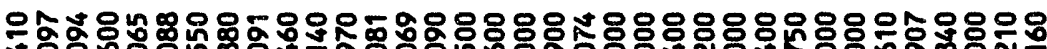
ธั0\%

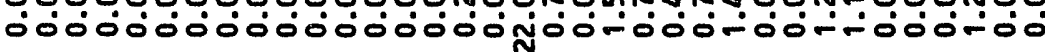

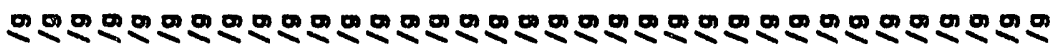
ব্ব)

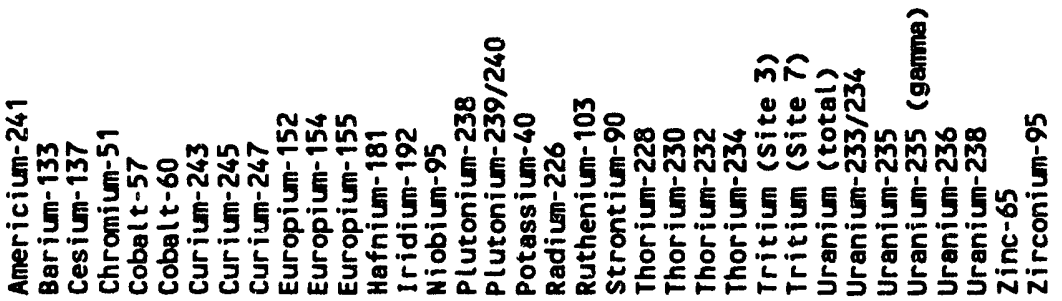




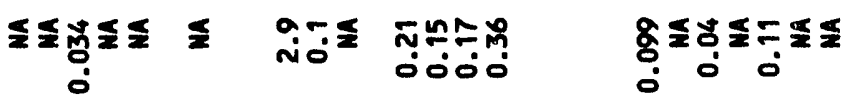

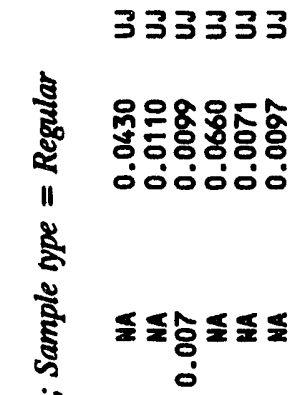

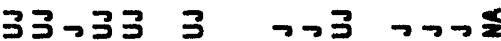

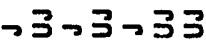

ฟ

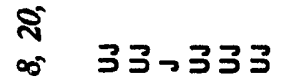

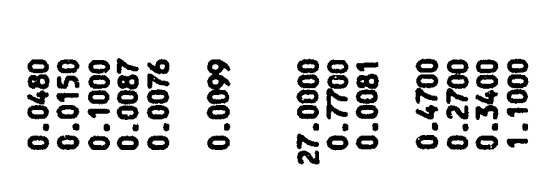

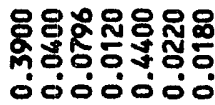

: 윻웡영융영융

iे 000000

00000 సं०๐

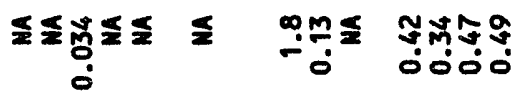

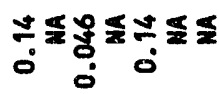

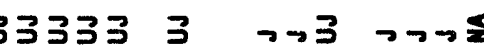

$\neg 3 \neg 3 \neg 33$

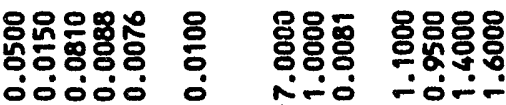

옹강웡ㅇㅇ유요

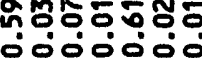

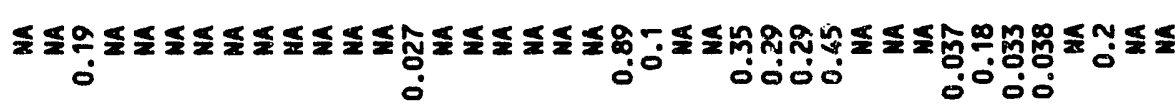

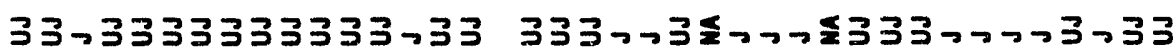

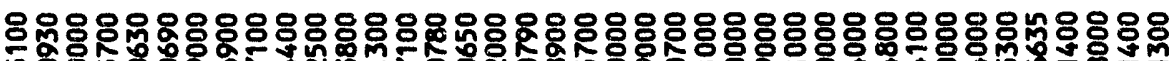

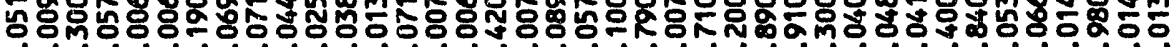

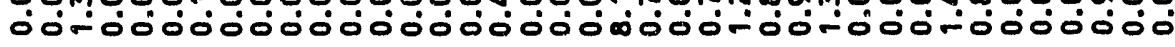

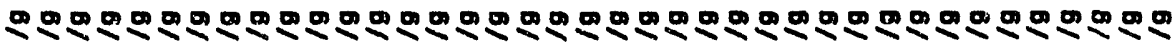

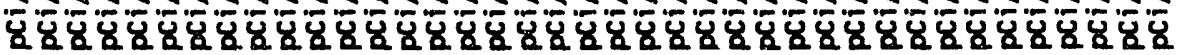

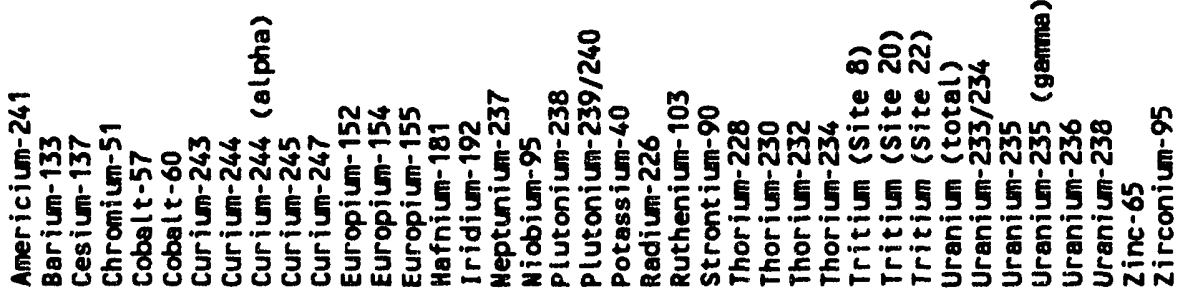


E-21

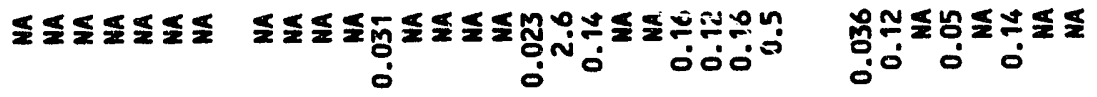

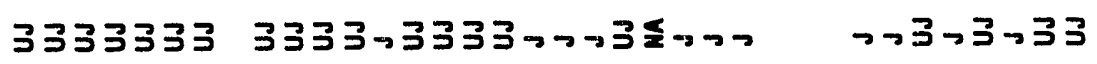

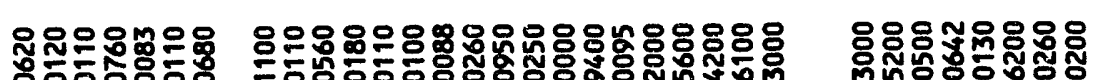

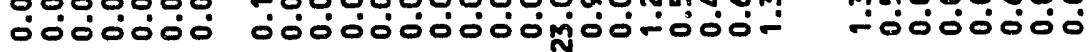

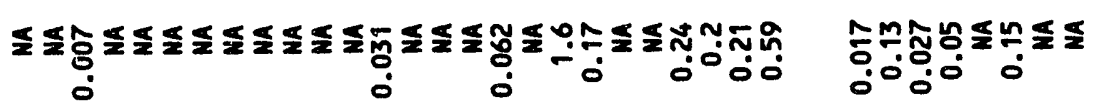

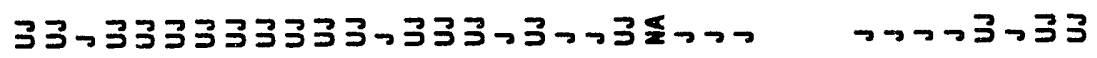

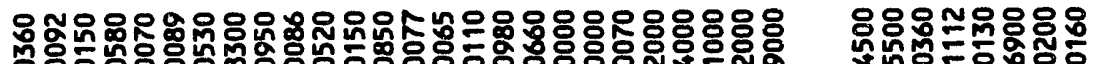

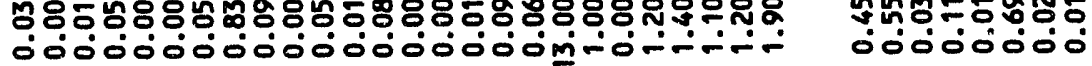

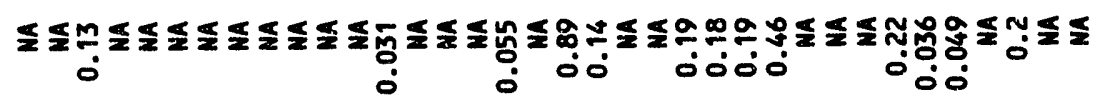

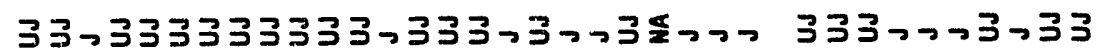

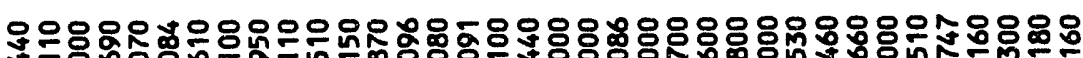

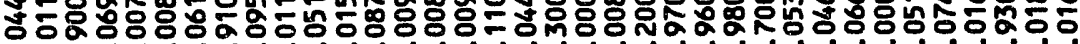

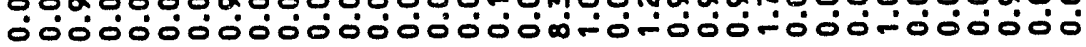

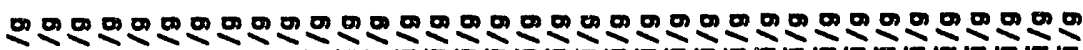

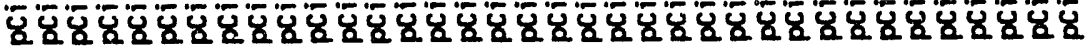

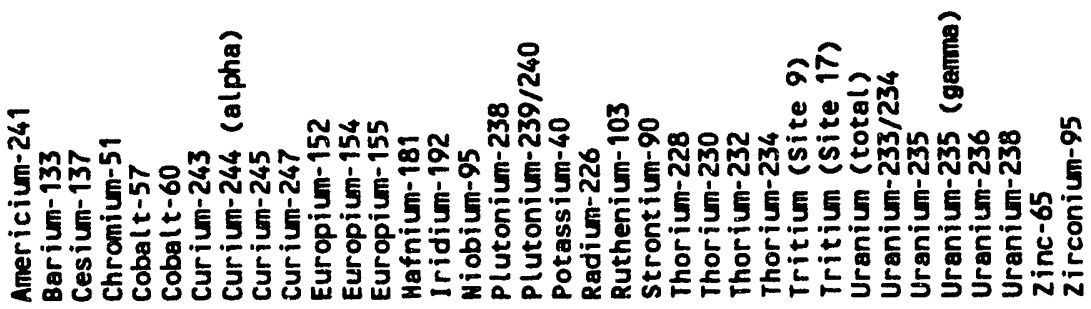




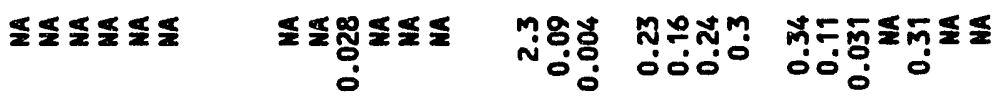

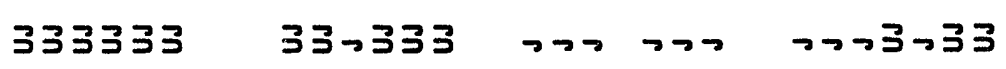

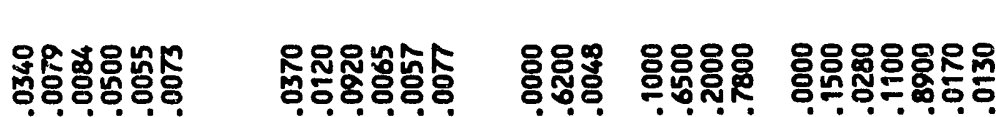

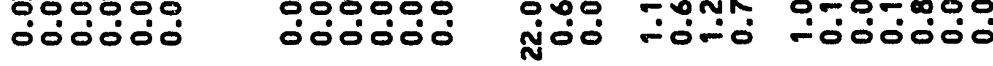

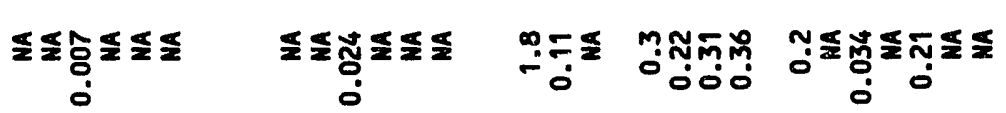

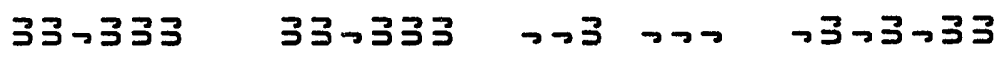

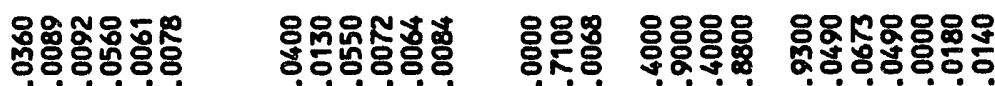

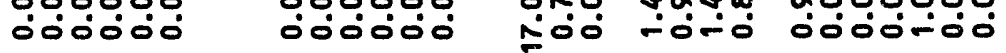

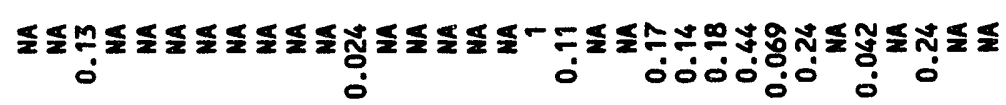

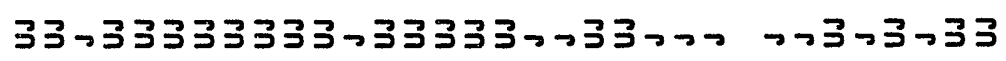

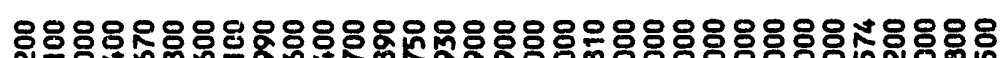

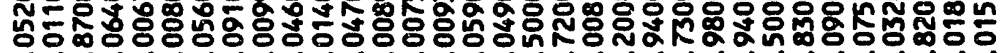

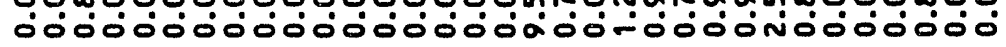

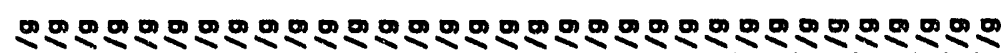

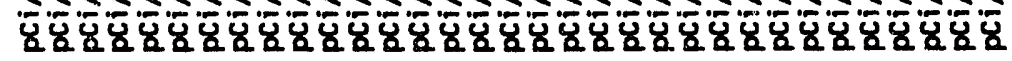

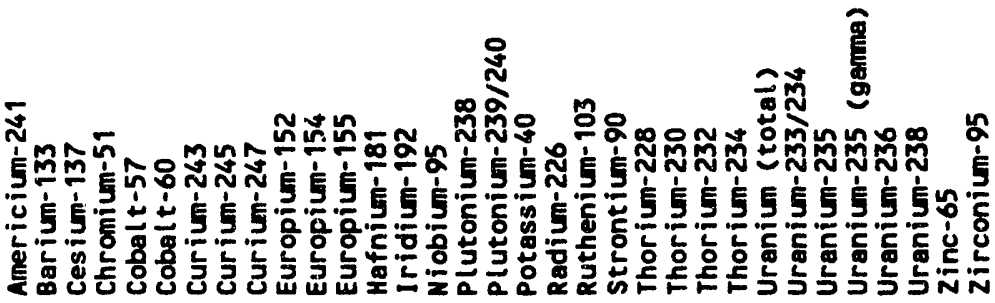




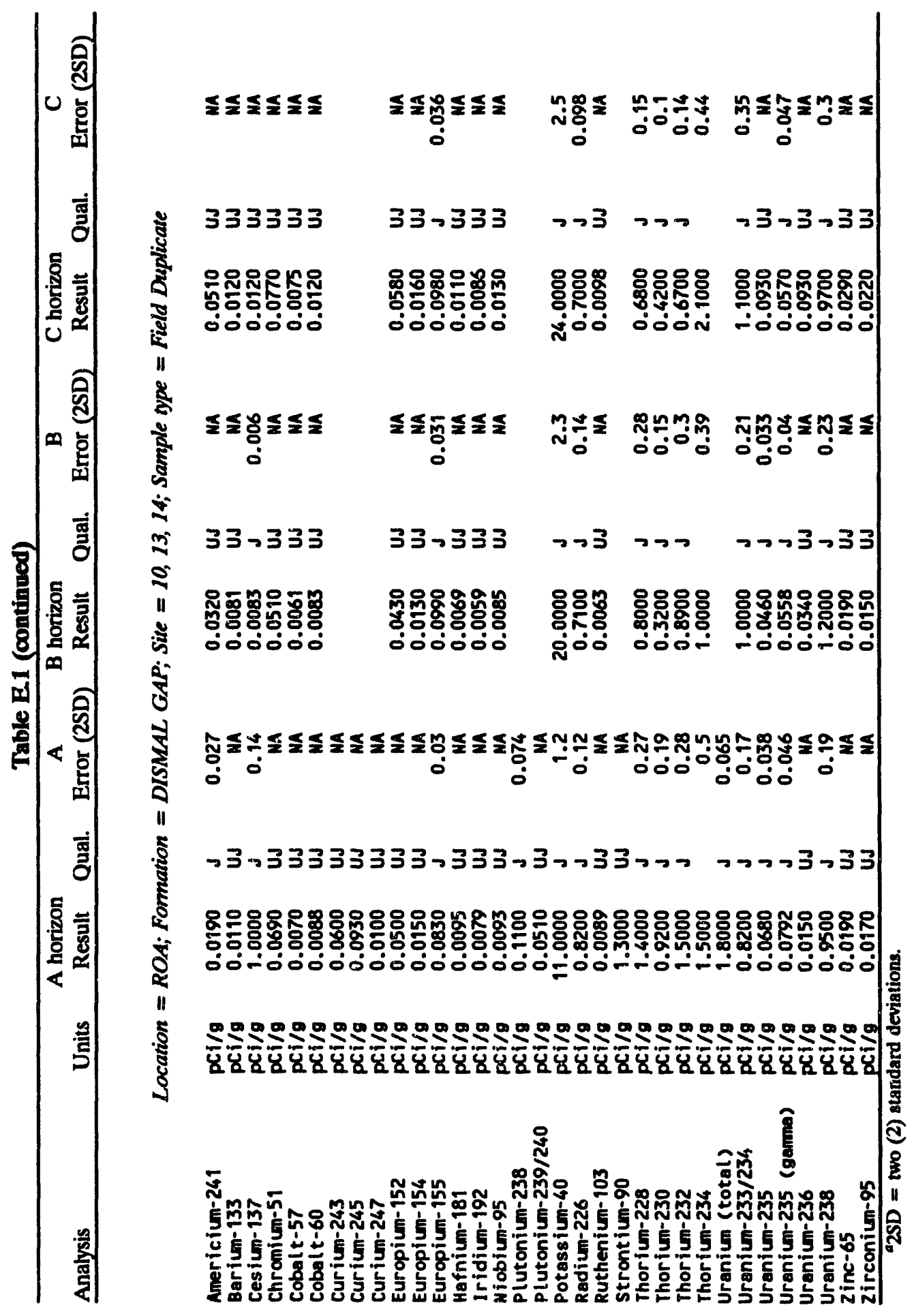


Appendix F

RELATION OF SAMPLE NUMBERS TO LABORATORY

SAMPLE DELIVERY GROUPS (SDGs) 


\section{F-3}

Table F.1. Relatioa of sample numbers to laboratory sample delivery groups (SDGs)

SDG number Sample number

\section{Inorganics}

042260

$5001,5004,5007$

$0422260,0430260 / 0508260 / 05111260$

$5001,5004,5007,5010,5013,5016,5019,5022$, $5025,6001,6004,6007,6010,6013,6016$

0430260

$6001,6004,6007$

0508260

$5010,5013,5016,5019,5022,5025$

0511260

$6010,6013,6016$

0514260

$5028,5031,5034,5037,5040,5043,6019,6022$, 6025

519260

$3144,6028,6031,6034,6037,6040,6043$

0727260/0728260/0729260

$5055,5058,5061,5064,5067,5070,5073,5076$, $5079,5082,5085,5088$

$0722260 / 0723260$

7001, 7001D, 7001, 7004, 7007, 7010, 7013, 7016, 7019, 7022, 7025, 7028, 7031, 7034, 7037FD, ${ }^{a}$

7040FD, 7043FD

0803260

1468

\section{Pesticides/PCBs}

0523260

$1296,1292,1298,1300,1301,1302,1293,1294$, $1297,1299,1303,1295$

0508260

1181, 1190, 1201FD, 1213

0511260

$3058,3099,3072,3085$

042260

$3018,1064,1072,1080,3003$

0424260

1099, 1106, 1107, 1108, 1115

0430260

1127,3032

0514260

3113, 3046, 1231, 3148, 3113RE, 3046RE, 1231RE, 3148RE

0519260

$3127,3139,3168$

0722260

$2070,2059,2157,2090,2101,2120,2116,2080$, $2112,2143,2130,2149,2039$ 


\section{Chlorinated Herbicides}

0508260

0042260

0424260

0430260

0511260

0422260

0430260

0508260

0511260

0514260

0519260

0523260

0722260

$0722260 / 0723260$
1190, 1201FD, 1213

$1072,1080,3003,1064$

$1099,1106,1107,1108,1115,3018$

1127,3032

$3046,3058,3085,3099,3072$

Polynuclear Aromatic Hydrocarbons

$1064,1072,1080,3003,1009,1106,1107,1108$, 1115,3018

1127,3032

1190, 1201FD, 1213

$3046,3058,3099,3072,3085$

1231,3113

$3127,3139,3148,3168$

$1292,1293,1294,1295,1296,1297,1298,1299$, $1300,1301,1302,1303$

2157RE, 2101RE, 2070RE, 2080RE, 2039RE 21'20RE, 2116RE, 2112RE, 2143RE, 2130RE, 2149RE, 2059RE, 2090

$2116,2112,2143,2059,2090,2070,2080,2039$, 2120, 2157, 2149, 2130, 2101

Uranium Isotopes by Alpha Spectroscopy

2684

2633

2391
1469

$7002,7011,7020,7029,7005,7008,7014,7017$, 7023, 7026, 7032, 7035

$5020,5023,5026$ 


\section{F-5}

Table F.1 (continued)

SDG number

Sample number

2419

$5029,5038,6020,5032,5041,6023,5035,5044$, 6026

2423

$6029,6038,6032,6041,6035,6044$

2638

7038FD, 7041FD, 7044FD

2658

$5056,5065,5074,5083,5059,5062,5068,5071$, $5077,5080,5086,5089$

Neptunium-237 by Alpha Spectroscopy

2658

5056, 5065, 5074, 5083

2391

5020

2684

1469

2419

$5029,5038,6020$

2633

7002, 7011, 7020, 7029

2638

7038FD

2423

6029,6038

Plutonium Isotopes by Alpha Spectroscopy

2419

$5029,5038,6020,5032,5041,6023,5035,5044$, 6026

2658

$5056,5065,5074,5083$

2638

7038FD

2391

5020

2633

$7002,7011,7020,7029$

2684

1469

2423

6029,6038 


\section{F-6}

Table F.1 (continued)

SDG number

Sample number

Thorium Isotopes by Alpha Spectroscopy

2684

2638

2633

2658

2419

2423

2419

2423

2633

2638

2658

2684

2638

2684

2658

2423
1469

7038FD, 7041FD, 7044FD

7002, 7011, 7020, 7029, 7005, 7008, 7014, 7017, 7023, 7026, 7032, 7035

$5056,5065,5074,5083,5059,5062,5068,5071$, $5077,5080,5086,5089$

$5029,5038,6020,5032,5041,6023,5035,5044$, 6026

$6029,6038,6032,6041,6035,6044$

Strontium-89/90 by Gas Flow Proportional Counter

$5029,5038,6020$

6029,6038

7002, 7011, 7020, 7029

7038FD

$5056,5065,5074,5083$

1469

Gamma Spectroscopy

7038FD, 7041FD

7044FD

1469

$5056,5065,5074,5083,5059,5062,5068,5071$, $5077,5080,5086,5089$

$6029,6038,6032,6041,6035,6044$ 


\section{F-7}

Table F.1 (continued)

SDG number

2633

2419
Sampie number

$7002,7011,7020,7029,7005,7008,7014,7014$, 7017, 7023, 7026, 7032, 7035

$5029,5038,6020,5032,5041,6023,5035,5044$, 6026

Total Uranium by Pulse Laser Phosphorimetry

2,423

$6029,6038,6020$

2684

1469

2658

$5056,5065,5074,5083$

2638

7938.FD

2633

7002,7011

7020,7029

\section{Technetium-99}

2419

$5029,5038,6020$

2684

1469

$2419 / 2423$

$6029,6038,5029,5038,6020$

$2638 / 2633 / 2658$

7002, 7011, 7020, 7029, 7038FD, 5056, 5065, 5074,5083

Tritium

$1063,1071,1079,1122,1125,3004,3031,1123$,

$1124,1126,1128,3016$

1180, 1189, 1198FD, 1214

3071, 3084, 3098, 3045, 3057, 3082

2399

${ }^{a} \mathrm{FD}=$ field duplicate sample.

${ }^{b} \mathrm{RE}=$ reanalyzed sample. 


\section{Appendix G}

DESCRIPTIONS OF SAMPLES FOR PHASE I ARRANGED NUMERICALLY BY SAMPLE IDENTIFICATION NUMBER 


\section{G-3}

Table G.1. Descriptions of samples for Phase I arranged numerically by sample identification number

\begin{tabular}{|c|c|c|c|c|c|c|c|}
\hline $\begin{array}{c}\text { Sample } \\
\text { ID }\end{array}$ & Location & Formation & Site & Horizon & Matrix & $\begin{array}{c}\text { Field } \\
\text { duplicate }\end{array}$ & $\begin{array}{c}\text { Analysis } \\
\text { type }\end{array}$ \\
\hline $\begin{array}{l}17,09 \\
1010 \\
1011 \\
1012 \\
1013\end{array}$ & $\begin{array}{l}\text { ORR } \\
\text { ORR } \\
\text { ORR } \\
\text { ORR } \\
\text { ORR }\end{array}$ & $\begin{array}{l}\text { DISHAL GAP } \\
\text { DISMAL GAP } \\
\text { DISMAL GAP } \\
\text { DISMAL GAP } \\
\text { DISHAL GAP }\end{array}$ & $\begin{array}{l}27 \\
27 \\
27 \\
27 \\
27\end{array}$ & & $\begin{array}{l}\text { SOIL } \\
\text { SOIL } \\
\text { SOIL } \\
\text { SOIL } \\
\text { SOIL }\end{array}$ & & 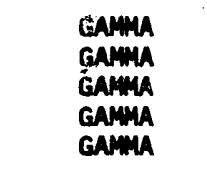 \\
\hline $\begin{array}{l}1014 \\
1028 \\
1029 \\
1030 \\
1031\end{array}$ & $\begin{array}{l}\text { ORR } \\
\text { ORR } \\
\text { ORR } \\
\text { ORR } \\
\text { ORR }\end{array}$ & $\begin{array}{l}\text { DISHAL GAP } \\
\text { DISMAL GAP } \\
\text { DISHAL GAP } \\
\text { DISHAL GAP } \\
\text { DISMAL GAP }\end{array}$ & $\begin{array}{l}27 \\
41 \\
41 \\
41 \\
41\end{array}$ & & $\begin{array}{l}\text { SOIL } \\
\text { SOIL } \\
\text { SOIL } \\
\text { SOIL } \\
\text { SOIL }\end{array}$ & & 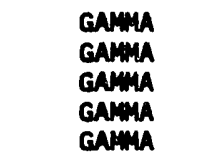 \\
\hline $\begin{array}{l}1032 \\
1033 \\
1040 \\
1041 \\
1042\end{array}$ & $\begin{array}{l}\text { ORR } \\
\text { ORR } \\
\text { ORR } \\
\text { ORR } \\
\text { ORR }\end{array}$ & $\begin{array}{l}\text { DISMAL GAP } \\
\text { DISMAL GAP } \\
\text { DISMAL GAP } \\
\text { DISMAL GAP } \\
\text { DISMAL GAP }\end{array}$ & $\begin{array}{l}41 \\
41 \\
11 \\
11 \\
11\end{array}$ & & $\begin{array}{l}\text { SOIL } \\
\text { SOIL } \\
\text { SOIL } \\
\text { SOIL } \\
\text { SOIL }\end{array}$ & & 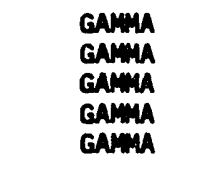 \\
\hline $\begin{array}{l}1043 \\
1044 \\
1045 \\
1063 \\
1064\end{array}$ & $\begin{array}{l}\text { ORR } \\
\text { ORR } \\
\text { ORR } \\
\text { ORR } \\
\text { ORR }\end{array}$ & $\begin{array}{l}\text { DISMAL GAP } \\
\text { DISMAL GAP } \\
\text { DISHAL GAP } \\
\text { DISMAL GAP } \\
\text { DISMAL GAP }\end{array}$ & $\begin{array}{l}11 \\
11 \\
11 \\
41 \\
41\end{array}$ & $\hat{A}$ & $\begin{array}{l}\text { SOIL } \\
\text { SOIL } \\
\text { SOIL } \\
\text { SOIL } \\
\text { SOIL }\end{array}$ & & $\begin{array}{l}\text { GAMmA } \\
\text { GAMHA } \\
\text { GAMHA } \\
\text { H-3 } \\
\text { ORGANICS }\end{array}$ \\
\hline $\begin{array}{l}1071 \\
1072 \\
1079 \\
1080 \\
1087\end{array}$ & $\begin{array}{l}\text { ORR } \\
\text { ORR } \\
\text { ORR } \\
\text { ORR } \\
\text { ORR }\end{array}$ & $\begin{array}{l}\text { DISMAL GAP } \\
\text { DISMAL GAP } \\
\text { DISHAL GAP } \\
\text { DISMAL GAP } \\
\text { DISMAL GAP }\end{array}$ & $\begin{array}{l}27 \\
27 \\
11 \\
19 \\
32\end{array}$ & $\begin{array}{l}\hat{A} \\
\hat{A}\end{array}$ & $\begin{array}{l}\text { SOIL } \\
\text { SOIL } \\
\text { SOIL } \\
\text { SOIL } \\
\text { SOIL }\end{array}$ & & $\begin{array}{l}H-3 \\
\text { ORGANICS } \\
\text { H-3 } \\
\text { ORGANICS } \\
\text { GANA }\end{array}$ \\
\hline $\begin{array}{l}1088 \\
1089 \\
1090 \\
1091 \\
1092\end{array}$ & $\begin{array}{l}\text { ORR } \\
\text { ORR } \\
\text { ORR } \\
\text { ORR } \\
\text { ORR }\end{array}$ & $\begin{array}{l}\text { DISAML GAP } \\
\text { DISHAL GAP } \\
\text { DISHAL GAP } \\
\text { DISHAL GAP } \\
\text { DISMAL GAP }\end{array}$ & $\begin{array}{l}32 \\
32 \\
32 \\
32 \\
32\end{array}$ & & $\begin{array}{l}\text { SOIL } \\
\text { SOIL } \\
\text { SOIL } \\
\text { SOIL } \\
\text { SOIL }\end{array}$ & & 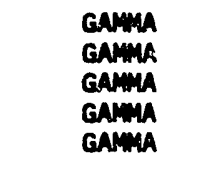 \\
\hline $\begin{array}{l}1093 \\
1094 \\
1095 \\
1096 \\
1097\end{array}$ & $\begin{array}{l}\text { ORR } \\
\text { ORR } \\
\text { ORR } \\
\text { ORR } \\
\text { ORR }\end{array}$ & $\begin{array}{l}\text { DISMAL GAP } \\
\text { DISMAL GAP } \\
\text { DISMAL GAP } \\
\text { DISHAL GAP } \\
\text { DISHAL GAP }\end{array}$ & $\begin{array}{l}19 \\
19 \\
19 \\
19 \\
19\end{array}$ & & $\begin{array}{l}\text { SOIL } \\
\text { SOIL } \\
\text { SOIL } \\
\text { SOIL. } \\
\text { SOIL }\end{array}$ & & 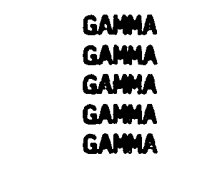 \\
\hline $\begin{array}{l}1098 \\
1099 \\
1100 \\
1101 \\
1102\end{array}$ & $\begin{array}{l}\text { ORR } \\
\text { ORR } \\
\text { ORR } \\
\text { ORR } \\
\text { ORR }\end{array}$ & $\begin{array}{l}\text { DISMAL GAP } \\
\text { I ISMAL GAP } \\
\text { DISHAL GAP } \\
\text { DISMAL GAP } \\
\text { DISMAL GAP }\end{array}$ & $\begin{array}{l}19 \\
19 \\
22 \\
22 \\
22\end{array}$ & $A$ & $\begin{array}{l}\text { SOIL } \\
\text { SOIL } \\
\text { SOIL } \\
\text { SOIL } \\
\text { SOIL }\end{array}$ & & $\begin{array}{l}\text { GAMHA } \\
\text { ORGANICS } \\
\text { GAMIA } \\
\text { GAMNA } \\
\text { GAMHA }\end{array}$ \\
\hline $\begin{array}{l}1103 \\
1104 \\
1105 \\
1106 \\
1107\end{array}$ & $\begin{array}{l}\text { ORR } \\
\text { ORR } \\
\text { ORR } \\
\text { ORR } \\
\text { ORR }\end{array}$ & $\begin{array}{l}\text { DISHAL GAP } \\
\text { DISHAL GAP } \\
\text { DISHAL GAP } \\
\text { DISHAL GAP } \\
\text { DISMAL GAP }\end{array}$ & $\begin{array}{l}22 \\
22 \\
22 \\
22 \\
32\end{array}$ & $\hat{A}$ & $\begin{array}{l}\text { SOIL } \\
\text { SOIL } \\
\text { SOIL } \\
\text { SOIL } \\
\text { SOIL }\end{array}$ & & $\begin{array}{l}\text { GAmIA } \\
\text { GAMIA } \\
\text { GAMUA } \\
\text { ORGANICS } \\
\text { ORGANICS }\end{array}$ \\
\hline $\begin{array}{l}1108 \\
1109 \\
1110 \\
1111 \\
1112\end{array}$ & $\begin{array}{l}\text { ORR } \\
\text { ORR } \\
\text { ORR } \\
\text { ORR } \\
\text { ORR }\end{array}$ & $\begin{array}{l}\text { DISHAL GAP } \\
\text { DISMAL GAP } \\
\text { DISHAL GAP } \\
\text { DISHAL GAP } \\
\text { DISHAL GAP }\end{array}$ & $\begin{array}{l}33 \\
33 \\
33 \\
33 \\
33\end{array}$ & $A$ & $\begin{array}{l}\text { SOIL } \\
\text { SOIL } \\
\text { SOIL } \\
\text { SOIL } \\
\text { SOIL }\end{array}$ & & $\begin{array}{l}\text { ORGANICS } \\
\text { GNMUA } \\
\text { GAMHA } \\
\text { GAmiA } \\
\text { GNmMA }\end{array}$ \\
\hline $\begin{array}{l}1113 \\
1114 \\
1115 \\
1116 \\
1117\end{array}$ & $\begin{array}{l}\text { ORR } \\
\text { ORR } \\
\text { ORR } \\
\text { ORR } \\
\text { ORR }\end{array}$ & $\begin{array}{l}\text { DISMAL GAP } \\
\text { DISHAL GAP } \\
\text { DISHAL GAP } \\
\text { DISMAL GAP } \\
\text { DISMAL GAP }\end{array}$ & $\begin{array}{l}33 \\
33 \\
35 \\
35 \\
35\end{array}$ & A & $\begin{array}{l}\text { SOIL } \\
\text { SOIL } \\
\text { SOIL } \\
\text { SOIL } \\
\text { SOIL }\end{array}$ & & 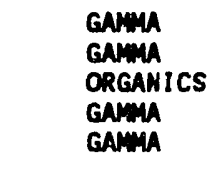 \\
\hline $\begin{array}{l}1118 \\
1119 \\
1120 \\
1121 \\
1122\end{array}$ & $\begin{array}{l}\text { ORR } \\
\text { ORR } \\
\text { ORR } \\
\text { ORR } \\
\text { ORR }\end{array}$ & $\begin{array}{l}\text { DISMAL GAP } \\
\text { DISMAL GAP } \\
\text { DISMAL GAP } \\
\text { DISMAL GAP } \\
\text { DISMAL GAP }\end{array}$ & $\begin{array}{l}35 \\
35 \\
35 \\
35 \\
19\end{array}$ & A & $\begin{array}{l}\text { SOIL } \\
\text { SOIL } \\
\text { SOIL } \\
\text { SOIL } \\
\text { SOIL }\end{array}$ & & $\begin{array}{l}\text { GAmu } \\
\text { GAMMA } \\
\text { GAMmA } \\
\text { GAMHA } \\
H=3\end{array}$ \\
\hline
\end{tabular}


Table G.1 (continued)

\begin{tabular}{|c|c|c|c|c|c|c|c|}
\hline $\begin{array}{l}\text { Sample } \\
\text { ID }\end{array}$ & Location & Formation & Site & Horizon & Matrix & $\begin{array}{c}\text { Field } \\
\text { duplicate }\end{array}$ & $\begin{array}{l}\text { Analysis } \\
\text { type }\end{array}$ \\
\hline $\begin{array}{l}1123 \\
1124 \\
1125 \\
1126 \\
1127\end{array}$ & $\begin{array}{l}\text { ORR } \\
\text { ORR } \\
\text { ORR } \\
\text { ORR } \\
\text { ORR }\end{array}$ & $\begin{array}{l}\text { DI SUAL GAP } \\
\text { DI SWAL GAP } \\
\text { DISMAL GAP } \\
\text { DISMAL GAP } \\
\text { DI SWAL GAP }\end{array}$ & $\begin{array}{l}22 \\
32 \\
33 \\
35 \\
10\end{array}$ & $\begin{array}{l}\hat{A} \\
\hat{A} \\
\hat{A}\end{array}$ & $\begin{array}{l}\text { SOIL } \\
\text { SOIL } \\
\text { SOIL } \\
\text { SOIL } \\
\text { SOIL }\end{array}$ & & $\begin{array}{l}H-3 \\
H-3 \\
H-3 \\
H-3 \\
\text { ORGANICS }\end{array}$ \\
\hline $\begin{array}{l}1128 \\
1129 \\
1130 \\
1131 \\
1132\end{array}$ & $\begin{array}{l}\text { ORR } \\
\text { ORR } \\
\text { ORR } \\
\text { ORR } \\
\text { ORR }\end{array}$ & $\begin{array}{l}\text { DISMAL GAP } \\
\text { DISMAL GAP } \\
\text { DISWAL GAP } \\
\text { DISWAL GAP } \\
\text { DISWML GAP }\end{array}$ & $\begin{array}{l}10 \\
10 \\
10 \\
10 \\
10\end{array}$ & $A$ & $\begin{array}{l}\text { SOIL } \\
\text { SOIL } \\
\text { SOIL } \\
\text { SOIL } \\
\text { SOIL }\end{array}$ & & 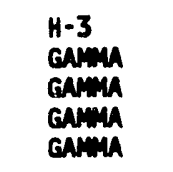 \\
\hline $\begin{array}{l}1133 \\
1134 \\
1180 \\
1181 \\
1183\end{array}$ & $\begin{array}{l}\text { ORR } \\
\text { ORR } \\
\text { ORR } \\
\text { ORR } \\
\text { ORR }\end{array}$ & $\begin{array}{l}\text { DISMAL GAP } \\
\text { DISWAL GAP } \\
\text { DISHAL GAP } \\
\text { DISWAL GAP } \\
\text { DISWAL GAP }\end{array}$ & $\begin{array}{r}10 \\
10 \\
2 \\
2 \\
2\end{array}$ & & $\begin{array}{l}\text { SOIL } \\
\text { SOIL } \\
\text { WATER } \\
\text { WATER } \\
\text { SOIL }\end{array}$ & & $\begin{array}{l}\text { GAmin } \\
\text { GNiMA } \\
\text { H-3 } \\
\text { ORGANICS } \\
\text { GNMMA }\end{array}$ \\
\hline $\begin{array}{l}1184 \\
1185 \\
1186 \\
1187 \\
1188\end{array}$ & $\begin{array}{l}\text { ORR } \\
\text { ORR } \\
\text { ORR } \\
\text { ORR } \\
\text { ORR }\end{array}$ & $\begin{array}{l}\text { DISHAL GAP } \\
\text { DISHAL GAP } \\
\text { DISMAL GAP } \\
\text { DISMAL GAP } \\
\text { DISIAL GAP }\end{array}$ & $\begin{array}{l}2 \\
2 \\
2 \\
2 \\
2\end{array}$ & & $\begin{array}{l}\text { SOIL } \\
\text { SOIL } \\
\text { SOIL } \\
\text { SOIL } \\
\text { SOIL }\end{array}$ & & $\begin{array}{l}\text { GAmun } \\
\text { GAminu } \\
\text { GAmu } \\
\text { GAmA } \\
\text { GAmu }\end{array}$ \\
\hline $\begin{array}{l}1189 \\
1190 \\
1198 \\
1201 \\
1207\end{array}$ & $\begin{array}{l}\text { ORR } \\
\text { ORR } \\
\text { ORR } \\
\text { ORR } \\
\text { ORR }\end{array}$ & $\begin{array}{l}\text { DISMAL GAP } \\
\text { DISMAL GAP } \\
\text { DISMAL GAP } \\
\text { DISMAL GAP } \\
\text { DISMAL GAP }\end{array}$ & $\begin{array}{r}2 \\
2 \\
2 \\
2 \\
26\end{array}$ & $\begin{array}{l}\hat{A} \\
\hat{A}\end{array}$ & $\begin{array}{l}\text { SOIL } \\
\text { SOIL } \\
\text { SOIL } \\
\text { SOIL } \\
\text { SOIL }\end{array}$ & $\begin{array}{l}\text { FIELD DUPLICATE } \\
\text { FIELD DUPLICATE }\end{array}$ & $\begin{array}{l}\text { H-3 } \\
\text { ORGANICS } \\
\text { H-3 } \\
\text { ORGAMICS } \\
\text { GAMAM }\end{array}$ \\
\hline $\begin{array}{l}1208 \\
1209 \\
1210 \\
1211 \\
1212\end{array}$ & $\begin{array}{l}\text { ORR } \\
\text { ORR } \\
\text { ORR } \\
\text { ORR } \\
\text { ORR }\end{array}$ & $\begin{array}{l}\text { DISWAL GAP } \\
\text { DISMAL GAP } \\
\text { DISHAL GAP } \\
\text { DISAAL GAP } \\
\text { DISUAL GAP }\end{array}$ & $\begin{array}{l}26 \\
26 \\
26 \\
26 \\
26\end{array}$ & & $\begin{array}{l}\text { SOIL } \\
\text { SOIL } \\
\text { SOIL } \\
\text { SOIL } \\
\text { SOIL }\end{array}$ & & 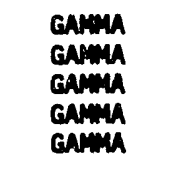 \\
\hline $\begin{array}{l}1213 \\
1214 \\
1231 \\
1240 \\
1241\end{array}$ & $\begin{array}{l}\text { ORR } \\
\text { ORR } \\
\text { ORR } \\
\text { ORR } \\
\text { ORR }\end{array}$ & $\begin{array}{l}\text { DISHAL GAP } \\
\text { DISMAL GAP } \\
\text { DISMAL GAP } \\
\text { DISWAL GAP } \\
\text { DISHAL GAP }\end{array}$ & $\begin{array}{l}26 \\
26 \\
43 \\
43 \\
43\end{array}$ & $\begin{array}{l}A \\
A \\
A\end{array}$ & $\begin{array}{l}\text { SOIL } \\
\text { SOIL } \\
\text { SOIL } \\
\text { SOIL } \\
\text { SOIL }\end{array}$ & & $\begin{array}{l}\text { ORGANICS } \\
\text { H-3 } \\
\text { ORGANICS } \\
\text { GNMA } \\
\text { GNMA }\end{array}$ \\
\hline $\begin{array}{l}1242 \\
1243 \\
1244 \\
1245 \\
1255\end{array}$ & $\begin{array}{l}\text { ORR } \\
\text { ORR } \\
\text { ORR } \\
\text { ORR } \\
\text { ORR }\end{array}$ & $\begin{array}{l}\text { DISMAL GAP } \\
\text { DISUAL GAP } \\
\text { DISMAL GAP } \\
\text { DISMAL GAP } \\
\text { DISMAL GAP }\end{array}$ & $\begin{array}{l}43 \\
43 \\
43 \\
43 \\
.\end{array}$ & & $\begin{array}{l}\text { SOIL } \\
\text { SOIL } \\
\text { SOIL } \\
\text { SOIL } \\
\text { WATER }\end{array}$ & & $\begin{array}{l}\text { GAmA } \\
\text { GAimu } \\
\text { GAmA } \\
\text { GAmA } \\
\text { VOC }\end{array}$ \\
\hline $\begin{array}{l}1257 \\
1258 \\
1259 \\
1260 \\
1261\end{array}$ & $\begin{array}{l}\text { ORR } \\
\text { ORR } \\
\text { ORR } \\
\text { ORR } \\
\text { ORR }\end{array}$ & $\begin{array}{l}\text { DISHAL GAP } \\
\text { DISWAL GAP } \\
\text { DISUAL GAP } \\
\text { DISWAL GAP } \\
\text { DISHAL GAP }\end{array}$ & $\begin{array}{r}2 \\
10 \\
11 \\
19 \\
22\end{array}$ & $\begin{array}{l}\hat{A} \\
\hat{A} \\
\hat{A} \\
\hat{A}\end{array}$ & $\begin{array}{l}\text { SOIL } \\
\text { SOIL } \\
\text { SOIL } \\
\text { SOIL } \\
\text { SOIL }\end{array}$ & & $\begin{array}{l}\text { VOC } \\
\text { VOC } \\
\text { VOC } \\
\text { VOC } \\
\text { VOC }\end{array}$ \\
\hline $\begin{array}{l}1262 \\
1263 \\
1264 \\
1265 \\
1266\end{array}$ & $\begin{array}{l}\text { ORR } \\
\text { ORR } \\
\text { ORR } \\
\text { ORR } \\
\text { ORR }\end{array}$ & $\begin{array}{l}\text { DISMAL GAP } \\
\text { DISMAL GAP } \\
\text { DISMAL GAP } \\
\text { DISMAL GAP } \\
\text { DISMAL GAP }\end{array}$ & $\begin{array}{l}26 \\
27 \\
33 \\
35 \\
41\end{array}$ & $\begin{array}{l}\hat{A} \\
\hat{A} \\
\hat{A} \\
\hat{A}\end{array}$ & $\begin{array}{l}\text { SOIL } \\
\text { SOIL } \\
\text { SOIL } \\
\text { SOIL } \\
\text { SOIL }\end{array}$ & & $\begin{array}{l}\text { VOC } \\
\text { VOC } \\
\text { VOC } \\
\text { VOC } \\
\text { VOC }\end{array}$ \\
\hline $\begin{array}{l}1267 \\
1268 \\
1269 \\
1270 \\
1271\end{array}$ & $\begin{array}{l}\text { ORR } \\
\text { ORR } \\
\text { ORR } \\
\text { ORR } \\
\text { ORR }\end{array}$ & $\begin{array}{l}\text { DISMAL GAP } \\
\text { DISMAL GAP } \\
\text { DISMAL GAP } \\
\text { NOLICHUCKY } \\
\text { NOLICHUCKY }\end{array}$ & $\begin{array}{r}43 \\
32 \\
\dot{3} \\
\dot{3}\end{array}$ & $\begin{array}{l}\hat{A} \\
\hat{A}\end{array}$ & $\begin{array}{l}\text { SOIL } \\
\text { SOIL } \\
\text { WATER } \\
\text { WATER } \\
\text { SOIL }\end{array}$ & & $\begin{array}{l}\text { VOC } \\
\text { VOC } \\
\text { VOC } \\
\text { VOC } \\
\text { VOC }\end{array}$ \\
\hline $\begin{array}{l}1272 \\
1273 \\
1274 \\
1275 \\
1276\end{array}$ & $\begin{array}{l}\text { ORR } \\
\text { ORR } \\
\text { ORR } \\
\text { ORR } \\
\text { ORR }\end{array}$ & $\begin{array}{l}\text { NOL ICHUCKY } \\
\text { NOLICHUCKY } \\
\text { NOLI ICHUCKY } \\
\text { NOL I CHUCKY } \\
\text { NOLI ICHUCKY }\end{array}$ & $\begin{array}{r}5 \\
13 \\
25 \\
15 \\
16\end{array}$ & $\begin{array}{l}\hat{A} \\
\hat{A} \\
\hat{A}\end{array}$ & $\begin{array}{l}\text { SOIL } \\
\text { SOIL } \\
\text { SOIL } \\
\text { SOIL } \\
\text { SOIL }\end{array}$ & & $\begin{array}{l}\text { VOC } \\
\text { VOC } \\
\text { VOC } \\
\text { VOC } \\
\text { VOC }\end{array}$ \\
\hline
\end{tabular}


Table G.1 (continued)

\begin{tabular}{|c|c|c|c|c|c|c|c|}
\hline $\begin{array}{l}\text { Sample } \\
\text { ID }\end{array}$ & Location & Formation & Site & Horizon & Matrix & $\begin{array}{c}\text { Field } \\
\text { duplicate }\end{array}$ & $\begin{array}{l}\text { Analysis } \\
\text { type }\end{array}$ \\
\hline $\begin{array}{l}1277 \\
1278 \\
1279 \\
1280 \\
1281\end{array}$ & $\begin{array}{l}\text { ORR } \\
\text { ORR } \\
\text { ORR } \\
\text { ORR } \\
\text { ORR }\end{array}$ & $\begin{array}{l}\text { NOLI ICHUCKY } \\
\text { NOLI CHUCKY } \\
\text { NOLI I CHUCKY } \\
\text { NOLI ICHUCKY } \\
\text { NOLI CHUCKY }\end{array}$ & $\begin{array}{l}\dot{2} \\
21 \\
23\end{array}$ & $\hat{A}$ & $\begin{array}{l}\text { WATER } \\
\text { WATER } \\
\text { SOIL } \\
\text { SOIL } \\
\text { SOIL }\end{array}$ & FIELD DUPLICATE & $\begin{array}{l}\text { VOC } \\
\text { VOC } \\
\text { VOC } \\
\text { VOC } \\
\text { VOC }\end{array}$ \\
\hline $\begin{array}{l}1282 \\
1283 \\
1284 \\
1285 \\
1286\end{array}$ & $\begin{array}{l}\text { ORR } \\
\text { ORR } \\
\text { ORR } \\
\text { ORR } \\
\text { ORR }\end{array}$ & $\begin{array}{l}\text { NOLI I CHUCKY } \\
\text { NOLI ICHUCKY } \\
\text { NOLI I CHUCKY } \\
\text { NOL I CHUCKY } \\
\text { NOLI I CHUCKY }\end{array}$ & $\begin{array}{l}23 \\
24 \\
24 \\
28\end{array}$ & $\begin{array}{l}\hat{A} \\
\hat{A} \\
\hat{A}\end{array}$ & $\begin{array}{l}\text { SOIL } \\
\text { WATER } \\
\text { SOIL } \\
\text { SOIL } \\
\text { SOIL }\end{array}$ & $\begin{array}{l}\text { FIELD DUPLICATE } \\
\text { FIELD DUPLICATE }\end{array}$ & $\begin{array}{l}\text { VOC } \\
\text { VOC } \\
\text { VOC } \\
\text { VOC } \\
\text { VOC }\end{array}$ \\
\hline $\begin{array}{l}1287 \\
1288 \\
1289 \\
1290 \\
1291\end{array}$ & $\begin{array}{l}\text { ORR } \\
\text { ORR } \\
\text { ORR } \\
\text { ORR } \\
\text { ORR }\end{array}$ & $\begin{array}{l}\text { NOLI ICHUCKY } \\
\text { NOLI ICHUCKY } \\
\text { NOLI I CHUCKY } \\
\text { NOLI CHUCKY } \\
\text { NOLI ICHUCKY }\end{array}$ & $\begin{array}{l}28 \\
31 \\
31 \\
42 \\
42\end{array}$ & $\begin{array}{l}\hat{A} \\
\hat{A} \\
\hat{A}\end{array}$ & $\begin{array}{l}\text { SoIl } \\
\text { SoIL } \\
\text { Soll } \\
\text { SoIL } \\
\text { Soll }\end{array}$ & $\begin{array}{l}\text { FIELD DUPLICATE } \\
\text { FIELD DUPLICATE } \\
\text { FIELD DUPLICATE }\end{array}$ & $\begin{array}{l}\text { VOC } \\
\text { VOC } \\
\text { VOC } \\
\text { VOC } \\
\text { VOC }\end{array}$ \\
\hline $\begin{array}{l}1292 \\
1293 \\
1294 \\
1295 \\
1296\end{array}$ & $\begin{array}{l}\text { ORR } \\
\text { ORR } \\
\text { ORR } \\
\text { ORR } \\
\text { ORR }\end{array}$ & $\begin{array}{l}\text { NOL I CHUCKY } \\
\text { NOLI I CHUCKY } \\
\text { NOLI ICHUCKY } \\
\text { NOLI ICHUCKY } \\
\text { NOLI ICHUCKY }\end{array}$ & $\begin{array}{l}28 \\
25 \\
24 \\
23 \\
21\end{array}$ & $\begin{array}{l}\hat{A} \\
\hat{A} \\
\hat{A}\end{array}$ & $\begin{array}{l}\text { SOIL } \\
\text { SOIL } \\
\text { SOIL } \\
\text { SOIL } \\
\text { SOIL }\end{array}$ & & $\begin{array}{l}\text { ORGANICS } \\
\text { ORGANICS } \\
\text { ORGANICS } \\
\text { ORGANICS } \\
\text { ORGANICS }\end{array}$ \\
\hline $\begin{array}{l}1297 \\
1298 \\
1299 \\
1300 \\
1301\end{array}$ & $\begin{array}{l}\text { ORR } \\
\text { ORR } \\
\text { ORR } \\
\text { ORR } \\
\text { ORR }\end{array}$ & $\begin{array}{l}\text { NOLI I CHUCKY } \\
\text { NOL I CHUCKY } \\
\text { NOL I CHUCKY } \\
\text { NOL I CHUCKY } \\
\text { MOLI I CHUCKY }\end{array}$ & $\begin{array}{r}3 \\
5 \\
13 \\
15 \\
16\end{array}$ & $\begin{array}{l}\hat{A} \\
\hat{A} \\
\hat{A}\end{array}$ & $\begin{array}{l}\text { SOIL } \\
\text { SOIL } \\
\text { SOIL } \\
\text { SOIL } \\
\text { SOIL }\end{array}$ & & $\begin{array}{l}\text { ORGANICS } \\
\text { ORGANICS } \\
\text { ORGANICS } \\
\text { ORGANICS } \\
\text { ORGANICS }\end{array}$ \\
\hline $\begin{array}{l}1302 \\
1303 \\
1307 \\
1308 \\
1309\end{array}$ & $\begin{array}{l}\text { ORR } \\
\text { ORR } \\
\text { ORR } \\
\text { ORR } \\
\text { ORR }\end{array}$ & $\begin{array}{l}\text { MOL I CHUCKY } \\
\text { NOL I CHUCKY } \\
\text { NOL I CHUCKY } \\
\text { NOLI CHUCKY } \\
\text { NOLI I CHUCKY }\end{array}$ & $\begin{array}{l}42 \\
31 \\
15 \\
15 \\
15\end{array}$ & $\hat{A}$ & $\begin{array}{l}\text { SOIL } \\
\text { SOIL } \\
\text { SOIL } \\
\text { SOIL } \\
\text { SOIL }\end{array}$ & & $\begin{array}{l}\text { ORGANICS } \\
\text { ORGANICS } \\
\text { GANAA } \\
\text { GANAA } \\
\text { GANHA }\end{array}$ \\
\hline $\begin{array}{l}1310 \\
1311 \\
1312 \\
1316 \\
1317\end{array}$ & $\begin{array}{l}\text { ORR } \\
\text { ORR } \\
\text { ORR } \\
\text { ORR } \\
\text { ORR }\end{array}$ & $\begin{array}{l}\text { NOL I CHUCKY } \\
\text { NOL I CHUCKY } \\
\text { NOL I CHUCKY } \\
\text { NOL I CHUCKY } \\
\text { NOL I CHUCKY }\end{array}$ & $\begin{array}{l}15 \\
15 \\
15 \\
23 \\
23\end{array}$ & & $\begin{array}{l}\text { SOIL } \\
\text { SOIL } \\
\text { SOIL } \\
\text { SOIL } \\
\text { SOIL }\end{array}$ & & $\begin{array}{l}\text { GANmA } \\
\text { GANmA } \\
\text { GANmA } \\
\text { GANmA } \\
\text { GANmA }\end{array}$ \\
\hline $\begin{array}{l}1318 \\
1319 \\
1320 \\
1321 \\
1325\end{array}$ & $\begin{array}{l}\text { ORR } \\
\text { ORR } \\
\text { ORR } \\
\text { ORR } \\
\text { ORR }\end{array}$ & $\begin{array}{l}\text { NOL I CHUCKY } \\
\text { NOL I CHUCKY } \\
\text { NOL I CHUCKY } \\
\text { NOL I CHUCKY } \\
\text { NOL I CHUCKY }\end{array}$ & $\begin{array}{l}23 \\
23 \\
23 \\
23 \\
25\end{array}$ & & $\begin{array}{l}\text { SOIL } \\
\text { soll } \\
\text { SoIL } \\
\text { soll } \\
\text { SOIL }\end{array}$ & & 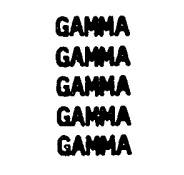 \\
\hline $\begin{array}{l}1326 \\
1327 \\
1328 \\
1329 \\
1330\end{array}$ & $\begin{array}{l}\text { ORR } \\
\text { ORR } \\
\text { ORR } \\
\text { ORR } \\
\text { ORR }\end{array}$ & $\begin{array}{l}\text { NOL I CHUCKY } \\
\text { NOL I CHUCKY } \\
\text { NOL I CHUCKY } \\
\text { NOL I CHUCKY } \\
\text { NOL I CHUCKY }\end{array}$ & $\begin{array}{l}25 \\
25 \\
25 \\
25 \\
25\end{array}$ & & $\begin{array}{l}\text { SOIL } \\
\text { SOIL } \\
\text { SOIL } \\
\text { SOIL } \\
\text { SOIL }\end{array}$ & & $\begin{array}{l}\text { GANmA } \\
\text { GANmA } \\
\text { GAMmA } \\
\text { GAMmA } \\
\text { GANmA }\end{array}$ \\
\hline $\begin{array}{l}1331 \\
1332 \\
1333 \\
1334 \\
1335\end{array}$ & $\begin{array}{l}\text { ORR } \\
\text { ORR } \\
\text { ORR } \\
\text { ORR } \\
\text { ORR }\end{array}$ & $\begin{array}{l}\text { NOL I CHUCKY } \\
\text { NOL I CHUCKY } \\
\text { NOL I CHUCKY } \\
\text { NOL I CHUCKY } \\
\text { NOL I CHUCKY }\end{array}$ & $\begin{array}{l}3 \\
3 \\
3 \\
3 \\
3\end{array}$ & & $\begin{array}{l}\text { SOIL } \\
\text { SOIL } \\
\text { SOIL } \\
\text { SOIL } \\
\text { SOIL }\end{array}$ & & 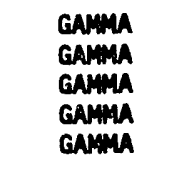 \\
\hline $\begin{array}{l}1336 \\
1337 \\
1338 \\
1339 \\
1340\end{array}$ & $\begin{array}{l}\text { ORR } \\
\text { ORR } \\
\text { ORR } \\
\text { ORR } \\
\text { ORR }\end{array}$ & $\begin{array}{l}\text { NOL I CHUCKY } \\
\text { NOL I CHUCKY } \\
\text { NOL I CHUCKY } \\
\text { NOL I CHUCKY } \\
\text { NOL I CHUCKY }\end{array}$ & $\begin{array}{l}3 \\
28 \\
28 \\
28 \\
28\end{array}$ & & $\begin{array}{l}\text { SOIL } \\
\text { SOIL } \\
\text { SOIL } \\
\text { SOIL } \\
\text { SOIL }\end{array}$ & & $\begin{array}{l}\text { GAMmA } \\
\text { GAMHAA } \\
\text { GAMMA } \\
\text { GAMMA } \\
\text { GAMHA }\end{array}$ \\
\hline $\begin{array}{l}1341 \\
1342 \\
1343 \\
1344 \\
1345\end{array}$ & $\begin{array}{l}\text { ORR } \\
\text { ORR } \\
\text { ORR } \\
\text { ORR } \\
\text { ORR }\end{array}$ & $\begin{array}{l}\text { NOLI I CHUCKY } \\
\text { NOL I CHUCKY } \\
\text { NOL I CHUCKY } \\
\text { NOL I CHUCKY } \\
\text { NOLI I CHUCKY }\end{array}$ & $\begin{array}{l}28 \\
28 \\
16 \\
16 \\
16\end{array}$ & & $\begin{array}{l}\text { SOIL } \\
\text { SOIL } \\
\text { SOIL } \\
\text { SOIL } \\
\text { SOIL }\end{array}$ & & $\begin{array}{l}\text { GAMWA } \\
\text { GAMMA } \\
\text { GAMHA } \\
\text { GAMWA } \\
\text { GAMHA }\end{array}$ \\
\hline
\end{tabular}


Table G.1 (continued)

\begin{tabular}{|c|c|c|c|c|c|c|c|}
\hline $\begin{array}{c}\text { Sample } \\
\text { ID }\end{array}$ & Location & Formation & Site & Horizon & Matrix & $\begin{array}{c}\text { Field } \\
\text { duplicate }\end{array}$ & $\begin{array}{c}\text { Analysis } \\
\text { type }\end{array}$ \\
\hline $\begin{array}{l}1346 \\
1347 \\
1348 \\
1349 \\
1350\end{array}$ & $\begin{array}{l}\text { ORR } \\
\text { ORR } \\
\text { ORR } \\
\text { ORR } \\
\text { ORR }\end{array}$ & $\begin{array}{l}\text { NOL ICHUCKY } \\
\text { NOLI ICHUCKY } \\
\text { MOLI ICHUCKY } \\
\text { MOL ICHUCKY } \\
\text { MOLI ICHUCKY }\end{array}$ & $\begin{array}{r}16 \\
16 \\
16 \\
5 \\
5\end{array}$ & & $\begin{array}{l}\text { Soll } \\
\text { soll } \\
\text { soll } \\
\text { soll } \\
\text { soll }\end{array}$ & & 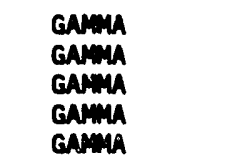 \\
\hline $\begin{array}{l}1351 \\
1352 \\
1353 \\
1354 \\
1355\end{array}$ & $\begin{array}{l}\text { ORR } \\
\text { ORR } \\
\text { ORR } \\
\text { ORR } \\
\text { ORR }\end{array}$ & $\begin{array}{l}\text { NOL I CHUCKY } \\
\text { MOL I CHUCKY } \\
\text { MOL I CHUCKY } \\
\text { NOL I CHUCKY } \\
\text { NOL I CHUCKY }\end{array}$ & $\begin{array}{r}5 \\
5 \\
5 \\
5 \\
13\end{array}$ & & $\begin{array}{l}\text { SoIL } \\
\text { SoIL } \\
\text { SoIL } \\
\text { SoIL } \\
\text { SOIL }\end{array}$ & & $\begin{array}{l}\text { GAmuA } \\
\text { GAimA } \\
\text { GNimA } \\
\text { GAmu } \\
\text { GNimu }\end{array}$ \\
\hline $\begin{array}{l}1356 \\
1357 \\
1358 \\
1359 \\
1360\end{array}$ & $\begin{array}{l}\text { ORR } \\
\text { ORR } \\
\text { ORR } \\
\text { ORR } \\
\text { ORR }\end{array}$ & $\begin{array}{l}\text { NOLICHUCKY } \\
\text { NOLI ICHUCKY } \\
\text { NOLI I CHUCKY } \\
\text { NOLI ICHUCKY } \\
\text { NOLICHUCKY }\end{array}$ & $\begin{array}{l}13 \\
13 \\
13 \\
13 \\
13\end{array}$ & & $\begin{array}{l}\text { SOIL } \\
\text { SOIL } \\
\text { SOIL } \\
\text { SoIL } \\
\text { SOIL }\end{array}$ & & 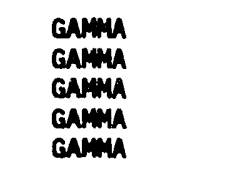 \\
\hline $\begin{array}{l}1361 \\
1362 \\
1363 \\
1364 \\
1365\end{array}$ & $\begin{array}{l}\text { ORR } \\
\text { ORR } \\
\text { ORR } \\
\text { ORR } \\
\text { ORR }\end{array}$ & $\begin{array}{l}\text { NOL ICHUCKY } \\
\text { NOLI ICHUCKY } \\
\text { NOL I CHUCKY } \\
\text { NOL I CHUCKY } \\
\text { NOLICHUCKY }\end{array}$ & $\begin{array}{l}24 \\
24 \\
24 \\
24 \\
24\end{array}$ & & $\begin{array}{l}\text { SOIL } \\
\text { SOIL } \\
\text { SOIL } \\
\text { SOIL } \\
\text { SOIL }\end{array}$ & & $\begin{array}{l}\text { GAmuA } \\
\text { GAminA } \\
\text { GAmina } \\
\text { GAmin } \\
\text { GAma }\end{array}$ \\
\hline $\begin{array}{l}1366 \\
1367 \\
1368 \\
1369 \\
1370\end{array}$ & $\begin{array}{l}\text { ORR } \\
\text { ORR } \\
\text { ORR } \\
\text { ORR } \\
\text { ORR }\end{array}$ & $\begin{array}{l}\text { NOL I CHUCKY } \\
\text { NOL I CHUCKY } \\
\text { NOL I CHUCKY } \\
\text { NOL I CHUCKY } \\
\text { NOL I CHUCKY }\end{array}$ & $\begin{array}{l}24 \\
21 \\
21 \\
21 \\
21\end{array}$ & & $\begin{array}{l}\text { SoIL } \\
\text { SOIL } \\
\text { SoIL } \\
\text { SoIL } \\
\text { SOIL }\end{array}$ & & $\begin{array}{l}\text { GAmA } \\
\text { GAminA } \\
\text { GAmA } \\
\text { GAmuA } \\
\text { GANGA }\end{array}$ \\
\hline $\begin{array}{l}1371 \\
1372 \\
1373 \\
1374 \\
1375\end{array}$ & $\begin{array}{l}\text { ORR } \\
\text { ORR } \\
\text { ORR } \\
\text { ORR } \\
\text { ORR }\end{array}$ & $\begin{array}{l}\text { MOL I CHUCKY } \\
\text { NOL I CHUCKY } \\
\text { NOL I CHUCKY } \\
\text { NOL I CHUCKY } \\
\text { MOL I CHUCKY }\end{array}$ & $\begin{array}{l}21 \\
21 \\
31 \\
31 \\
31\end{array}$ & & $\begin{array}{l}\text { SoIl } \\
\text { SoIl } \\
\text { SoIl } \\
\text { SoIL } \\
\text { SoIl }\end{array}$ & & 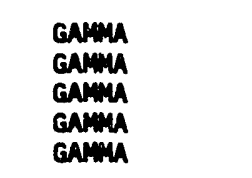 \\
\hline $\begin{array}{l}1376 \\
1377 \\
1378 \\
1379 \\
1380\end{array}$ & $\begin{array}{l}\text { ORR } \\
\text { ORR } \\
\text { ORR } \\
\text { ORR } \\
\text { ORR }\end{array}$ & $\begin{array}{l}\text { NOL I CHUCKY } \\
\text { NOL I CHUCKY } \\
\text { NOL I CHUCKY } \\
\text { NOL I CHUCKY } \\
\text { NOL I CHUCKY }\end{array}$ & $\begin{array}{l}31 \\
31 \\
31 \\
42 \\
42\end{array}$ & & $\begin{array}{l}\text { SoIL } \\
\text { SoIL } \\
\text { SoIL } \\
\text { SoIL } \\
\text { SoIL }\end{array}$ & & 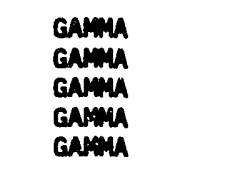 \\
\hline $\begin{array}{l}1381 \\
1382 \\
1383 \\
1384 \\
1456\end{array}$ & $\begin{array}{l}\text { ORR } \\
\text { ORR } \\
\text { ORR } \\
\text { ORR } \\
\text { ORR }\end{array}$ & $\begin{array}{l}\text { NOLI I CHUCKY } \\
\text { NOLI I CHUCKY } \\
\text { NOL I CHUCKY } \\
\text { NOL I CHUCKY } \\
\text { NOLI I CHUCKY }\end{array}$ & $\begin{array}{l}42 \\
42 \\
42 \\
42 \\
.\end{array}$ & & $\begin{array}{l}\text { SOIL } \\
\text { SOIL } \\
\text { SOIL } \\
\text { SOIL } \\
\text { WATER }\end{array}$ & & $\begin{array}{l}\text { GAMUA } \\
\text { GAMHA } \\
\text { GAMHA } \\
\text { GANA } \\
\text { ORGANICS }\end{array}$ \\
\hline $\begin{array}{l}1457 \\
1458 \\
1459 \\
1461 \\
1462\end{array}$ & $\begin{array}{l}\text { ORR } \\
\text { ORR } \\
\text { ORR } \\
\text { ORR } \\
\text { ORR }\end{array}$ & $\begin{array}{l}\text { NOLICHUCKY } \\
\text { NOL I CHUCKY } \\
\text { MOLICHUCKY } \\
\text { NOLICHUCKY } \\
\text { NOLI ICHUCKY }\end{array}$ & $\dot{:}$ & & $\begin{array}{l}\text { WATER } \\
\text { WATER } \\
\text { WATER } \\
\text { WATER } \\
\text { WATER }\end{array}$ & & $\begin{array}{l}\text { ORGANICS } \\
\text { ORGANICS } \\
\text { INORGANICS } \\
\text { RADIONUCLIDES } \\
\text { ORGANICS }\end{array}$ \\
\hline $\begin{array}{l}1463 \\
1464 \\
1465 \\
1467 \\
1468\end{array}$ & $\begin{array}{l}\text { ORR } \\
\text { ORR } \\
\text { ORR } \\
\text { ORR } \\
\text { ORR }\end{array}$ & $\begin{array}{l}\text { NOLICHUCKY } \\
\text { NOLICHUCKY } \\
\text { NOLI CHUCKY } \\
\text { NOLI CHUCKY } \\
\text { NOL I CHUCKY }\end{array}$ & $\dot{:}$ & & $\begin{array}{l}\text { WATER } \\
\text { HATER } \\
\text { WATER } \\
\text { HATER } \\
\text { SOIL }\end{array}$ & & $\begin{array}{l}\text { ORGANICS } \\
\text { ORGANICS } \\
\text { IMCRGANICS } \\
\text { RADIONUCLIDES } \\
\text { INORGANICS }\end{array}$ \\
\hline $\begin{array}{l}1469 \\
2008 \\
2009 \\
2010 \\
2011\end{array}$ & $\begin{array}{l}\text { ORR } \\
\text { AND } \\
\text { AND } \\
\text { AND } \\
\text { AND }\end{array}$ & $\begin{array}{l}\text { NOLICHUCKY } \\
\text { DISMAL GAP } \\
\text { DI SMAL GAP } \\
\text { DISHAL GAP } \\
\text { DI SMAL GAP }\end{array}$ & $\begin{array}{l}11 \\
11 \\
19 \\
11\end{array}$ & & $\begin{array}{l}\text { SOIL } \\
\text { SOIL } \\
\text { SOIL } \\
\text { SOIL } \\
\text { SOIL }\end{array}$ & & $\begin{array}{l}\text { RADIONUCLIDES } \\
\text { GAMA } \\
\text { GAWHA } \\
\text { GAMUA } \\
\text { GAMUA }\end{array}$ \\
\hline $\begin{array}{l}2012 \\
2013 \\
2024 \\
2026 \\
2027\end{array}$ & $\begin{array}{l}\text { AND } \\
\text { AND } \\
\text { AND } \\
\text { AND } \\
\text { AND }\end{array}$ & $\begin{array}{l}\text { DISHAL GAP } \\
\text { DISMAL GAP } \\
\text { DISMAL GAP } \\
\text { DI SMAL GAP } \\
\text { DISHAL GAP }\end{array}$ & $\begin{array}{r}11 \\
11 \\
\dot{9} \\
19\end{array}$ & $\begin{array}{l}A \\
\hat{A}\end{array}$ & $\begin{array}{l}\text { SOIL } \\
\text { SOIL } \\
\text { WATER } \\
\text { SOIL. } \\
\text { SOIL }\end{array}$ & & $\begin{array}{l}\text { GAmu } \\
\text { GAmu } \\
\text { VOC } \\
\text { VOC } \\
\text { VOC }\end{array}$ \\
\hline
\end{tabular}


Table G.1 (continued)

\begin{tabular}{|c|c|c|c|c|c|c|c|}
\hline $\begin{array}{c}\text { Sample } \\
\text { ID }\end{array}$ & Location & Formation & Site & Horizon & Matrix & $\begin{array}{c}\text { Field } \\
\text { duplicate }\end{array}$ & $\begin{array}{l}\text { Analysis } \\
\text { type }\end{array}$ \\
\hline $\begin{array}{l}2028 \\
2029 \\
2030 \\
2031 \\
2032\end{array}$ & $\begin{array}{l}\text { AND } \\
\text { AND } \\
\text { AND } \\
\text { AND } \\
\text { AND }\end{array}$ & $\begin{array}{l}\text { DI SMAL GAP } \\
\text { DISMAL GAP } \\
\text { DI SWHL GAP } \\
\text { DI SMAL GAP } \\
\text { DISHAL GAP }\end{array}$ & $\begin{array}{r}10 \\
1 \\
22 \\
12 \\
3\end{array}$ & $\begin{array}{l}\hat{A} \\
\hat{A} \\
\hat{A}\end{array}$ & $\begin{array}{l}\text { SOIL } \\
\text { SOIL } \\
\text { SOIL } \\
\text { SOIL } \\
\text { SOIL }\end{array}$ & & $\begin{array}{l}\text { Voc } \\
\text { VOC } \\
\text { Voc } \\
\text { Voc } \\
\text { VOC }\end{array}$ \\
\hline $\begin{array}{l}2033 \\
2034 \\
2036 \\
2037 \\
2038\end{array}$ & $\begin{array}{l}\text { AND } \\
\text { AND } \\
\text { AND } \\
\text { AND } \\
\text { AND }\end{array}$ & $\begin{array}{l}\text { DISNAL GAP } \\
\text { DISHAL GAP } \\
\text { DISHAL GAP } \\
\text { DISHAL GAP } \\
\text { DI SNAL GAP }\end{array}$ & $\begin{array}{r}4 \\
5 \\
21 \\
:\end{array}$ & $\begin{array}{l}A \\
A \\
A\end{array}$ & $\begin{array}{l}\text { SOIL } \\
\text { SOIL } \\
\text { SOIL } \\
\text { WATER } \\
\text { WATER }\end{array}$ & & $\begin{array}{l}\text { Voc } \\
\text { VOC } \\
\text { VOC } \\
\text { VOC } \\
\text { VOC }\end{array}$ \\
\hline $\begin{array}{l}2039 \\
2043 \\
2044 \\
2045 \\
2046\end{array}$ & $\begin{array}{l}\text { AND } \\
\text { AND } \\
\text { AND } \\
\text { AND } \\
\text { AND }\end{array}$ & $\begin{array}{l}\text { DISMAL GAP } \\
\text { DISMAL GAP } \\
\text { DISMAL GAP } \\
\text { DISMAL GAP } \\
\text { DISHAL GAP }\end{array}$ & $\begin{array}{l}4 \\
4 \\
4 \\
4 \\
4\end{array}$ & A & $\begin{array}{l}\text { SOIL } \\
\text { SOIL } \\
\text { SOIL } \\
\text { SOIL } \\
\text { SOIL }\end{array}$ & & 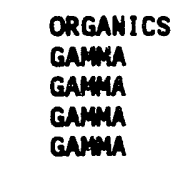 \\
\hline $\begin{array}{l}2047 \\
2048 \\
2053 \\
2054 \\
2055\end{array}$ & $\begin{array}{l}\text { AND } \\
\text { AND } \\
\text { AND } \\
\text { AND } \\
\text { AND }\end{array}$ & $\begin{array}{l}\text { DISHAL GAP } \\
\text { DISHAL GAP } \\
\text { DISMAL GAP } \\
\text { DI SMAL GAP } \\
\text { DISMAL GAP }\end{array}$ & $\begin{array}{l}4 \\
4 \\
5 \\
5 \\
5\end{array}$ & & $\begin{array}{l}\text { SOIL } \\
\text { SOIL } \\
\text { SOIL } \\
\text { SOIL } \\
\text { SOIL }\end{array}$ & & $\begin{array}{l}\text { GAMmA } \\
\text { GAMUA } \\
\text { GANA } \\
\text { GANHA } \\
\text { GAMHA }\end{array}$ \\
\hline $\begin{array}{l}2056 \\
2057 \\
2058 \\
2059 \\
2063\end{array}$ & $\begin{array}{l}\text { AND } \\
\text { AND } \\
\text { AND } \\
\text { AND } \\
\text { AND }\end{array}$ & $\begin{array}{l}\text { DI SMAL GAP } \\
\text { DI SMAL GAP } \\
\text { DI SMAL GAP } \\
\text { DISMAL GAP } \\
\text { DI SMAL GAP }\end{array}$ & $\begin{array}{l}5 \\
5 \\
5 \\
3 \\
3\end{array}$ & A & $\begin{array}{l}\text { SOIL } \\
\text { SOIL } \\
\text { SOIL } \\
\text { SOIL } \\
\text { SOIL }\end{array}$ & & $\begin{array}{l}\text { GAmiA } \\
\text { GAMMA } \\
\text { GAMmA } \\
\text { ORGANICS } \\
\text { GAMUA }\end{array}$ \\
\hline $\begin{array}{l}2064 \\
2065 \\
2066 \\
2067 \\
2068\end{array}$ & $\begin{array}{l}\text { AND } \\
\text { AND } \\
\text { AND } \\
\text { AND } \\
\text { AND }\end{array}$ & $\begin{array}{l}\text { DISMAL GAP } \\
\text { DI SMAL GAP } \\
\text { DISMAL GAP } \\
\text { DISMAL GAP } \\
\text { DISMAL GAP }\end{array}$ & $\begin{array}{l}3 \\
3 \\
3 \\
3 \\
3\end{array}$ & & $\begin{array}{l}\text { SOIL } \\
\text { SOIL } \\
\text { SOIL } \\
\text { SOIL } \\
\text { SOIL }\end{array}$ & & $\begin{array}{l}\text { GAmaA } \\
\text { GAmaA } \\
\text { GAminA } \\
\text { GAmiA } \\
\text { GAmiA }\end{array}$ \\
\hline $\begin{array}{l}2069 \\
2070 \\
2074 \\
2075 \\
2076\end{array}$ & $\begin{array}{l}\text { AND } \\
\text { AND } \\
\text { AND } \\
\text { AND } \\
\text { AND }\end{array}$ & $\begin{array}{l}\text { DISMAL GAP } \\
\text { DISMAL GAP } \\
\text { DISMAL GAP } \\
\text { DISMAL GAP } \\
\text { DISMAL GAP }\end{array}$ & $\begin{array}{l}20 \\
20 \\
20 \\
20 \\
20\end{array}$ & $\hat{A}$ & $\begin{array}{l}\text { SOIL } \\
\text { SOIL } \\
\text { SOIL } \\
\text { SOIL } \\
\text { SOIL }\end{array}$ & & $\begin{array}{l}\text { VOC } \\
\text { ORGANICS } \\
\text { GAMMA } \\
\text { GAMMA } \\
\text { GANMA }\end{array}$ \\
\hline $\begin{array}{l}2077 \\
2078 \\
2079 \\
2080 \\
2084\end{array}$ & $\begin{array}{l}\text { AND } \\
\text { AND } \\
\text { AND } \\
\text { AND } \\
\text { AND }\end{array}$ & $\begin{array}{l}\text { DISMAL GAP } \\
\text { DISMAL GAP } \\
\text { DISMAL GAP } \\
\text { DISMAL GAP } \\
\text { DISMAL GAP }\end{array}$ & $\begin{array}{l}20 \\
20 \\
20 \\
12 \\
12\end{array}$ & $A$ & $\begin{array}{l}\text { SOIL } \\
\text { SOIL } \\
\text { SOIL } \\
\text { SOIL } \\
\text { SOIL }\end{array}$ & & $\begin{array}{l}\text { GAmiA } \\
\text { GANMMA } \\
\text { GAIMMA } \\
\text { ORGANICS } \\
\text { GAMMA }\end{array}$ \\
\hline $\begin{array}{l}2085 \\
2086 \\
2087 \\
2088 \\
2089\end{array}$ & $\begin{array}{l}\text { AND } \\
\text { AND } \\
\text { AND } \\
\text { AND } \\
\text { AND }\end{array}$ & $\begin{array}{l}\text { DISMAL GAP } \\
\text { DISMAL GAP } \\
\text { DISMAL GAP } \\
\text { DISMAL GAP } \\
\text { DISMAL GAP }\end{array}$ & $\begin{array}{l}12 \\
12 \\
12 \\
12 \\
12\end{array}$ & & $\begin{array}{l}\text { SOIL } \\
\text { SOIL } \\
\text { SOIL } \\
\text { SOIL } \\
\text { SOIL }\end{array}$ & $\therefore$ & 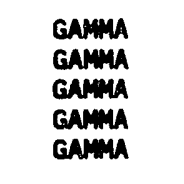 \\
\hline $\begin{array}{l}2090 \\
2094 \\
2095 \\
2096 \\
2097\end{array}$ & $\begin{array}{l}\text { AND } \\
\text { AND } \\
\text { AND } \\
\text { AND } \\
\text { AND }\end{array}$ & $\begin{array}{l}\text { DISMAL GAP } \\
\text { DISMAL GAP } \\
\text { DISMAL GAP } \\
\text { DISMAL GAP } \\
\text { DISMAL GAP }\end{array}$ & $\begin{array}{l}22 \\
22 \\
22 \\
22 \\
22\end{array}$ & $A$ & $\begin{array}{l}\text { SOIL } \\
\text { SOIL } \\
\text { SOIL } \\
\text { SOIL } \\
\text { SOIL }\end{array}$ & & 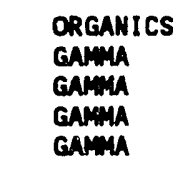 \\
\hline $\begin{array}{l}2098 \\
2099 \\
2101 \\
2105 \\
2106\end{array}$ & $\begin{array}{l}\text { AND } \\
\text { AND } \\
\text { AND } \\
\text { AND } \\
\text { AND }\end{array}$ & $\begin{array}{l}\text { DISMAL GAP } \\
\text { DISMAL GAP } \\
\text { DISMAL GAP } \\
\text { DISMAL GAP } \\
\text { DISMAL GAP }\end{array}$ & $\begin{array}{l}22 \\
22 \\
21 \\
21 \\
21\end{array}$ & A & $\begin{array}{l}\text { SOIL } \\
\text { SOIL } \\
\text { SOIL } \\
\text { SOIL } \\
\text { SOIL }\end{array}$ & & $\begin{array}{l}\text { GAMMA } \\
\text { GAMMA } \\
\text { ORGANICS } \\
\text { GANMA } \\
\text { GAMUA }\end{array}$ \\
\hline $\begin{array}{l}2107 \\
2108 \\
2109 \\
2110 \\
2111\end{array}$ & $\begin{array}{l}\text { AND } \\
\text { AND } \\
\text { AND } \\
\text { AND } \\
\text { AND }\end{array}$ & $\begin{array}{l}\text { DISMAL GAP } \\
\text { DISMAL GAP } \\
\text { DISMAL GAP } \\
\text { DISMAL GAP } \\
\text { DISMAL GAP }\end{array}$ & $\begin{array}{l}21 \\
21 \\
21 \\
21 \\
11\end{array}$ & $A$ & $\begin{array}{l}\text { SOIL } \\
\text { SOIL } \\
\text { SOIL } \\
\text { SOIL } \\
\text { SOIL }\end{array}$ & & $\begin{array}{l}\text { GAMmA } \\
\text { GAMmA } \\
\text { GAMMA } \\
\text { GAMwA } \\
\text { VOC }\end{array}$ \\
\hline
\end{tabular}


Table G.1 (continued)

\begin{tabular}{|c|c|c|c|c|c|c|c|}
\hline $\begin{array}{c}\text { Sample } \\
\text { ID }\end{array}$ & Location & Formation & Site & Horizon & Matrix & $\begin{array}{c}\text { Field } \\
\text { duplicate }\end{array}$ & $\begin{array}{c}\text { Analysis } \\
\text { type }\end{array}$ \\
\hline $\begin{array}{l}2112 \\
2116 \\
2120 \\
2124 \\
2125\end{array}$ & $\begin{array}{l}\text { AND } \\
\text { AND } \\
\text { AND } \\
\text { AND } \\
\text { AND }\end{array}$ & $\begin{array}{l}\text { DI SHAL GAP } \\
\text { DISMAL GAP } \\
\text { DISHAL GAP } \\
\text { DI SMAL GAP } \\
\text { DISHAL GAP }\end{array}$ & $\begin{array}{l}11 \\
19 \\
19 \\
19 \\
19\end{array}$ & $\hat{A}$ & $\begin{array}{l}\text { SOIL } \\
\text { SOIL } \\
\text { SOIL } \\
\text { SOIL } \\
\text { SOIL }\end{array}$ & FIELP DUPLICATE & $\begin{array}{l}\text { ORGANICS } \\
\text { ORGANICS } \\
\text { ORGANICS } \\
\text { GANHA } \\
\text { GANHA }\end{array}$ \\
\hline $\begin{array}{l}2126 \\
2127 \\
2128 \\
2129 \\
2130\end{array}$ & $\begin{array}{l}\text { AND } \\
\text { AND } \\
\text { AND } \\
\text { AND } \\
\text { AND }\end{array}$ & $\begin{array}{l}\text { DISHAL GAP } \\
\text { DI SHAL GAP } \\
\text { DISHAL GAP } \\
\text { DISHAL GAP } \\
\text { DISHAL GAP }\end{array}$ & $\begin{array}{r}19 \\
19 \\
19 \\
19 \\
9\end{array}$ & A & $\begin{array}{l}\text { SOIL } \\
\text { SOIL } \\
\text { SOIL } \\
\text { SOIL } \\
\text { SOIL }\end{array}$ & & $\begin{array}{l}\text { GAmu } \\
\text { CANMA } \\
\text { CANmu } \\
\text { GANMA } \\
\text { ORCANICS }\end{array}$ \\
\hline $\begin{array}{l}2137 \\
2138 \\
2139 \\
2140 \\
2141\end{array}$ & $\begin{array}{l}\text { AND } \\
\text { AND } \\
\text { AND } \\
\text { AND } \\
\text { AND }\end{array}$ & $\begin{array}{l}\text { DISUAL GAP } \\
\text { DISNUL GAP } \\
\text { DISWHL GAP } \\
\text { DISWAL GAP } \\
\text { DISWAL GAP }\end{array}$ & $\begin{array}{l}9 \\
9 \\
9 \\
9 \\
9\end{array}$ & & $\begin{array}{l}\text { SOIL } \\
\text { SOIL } \\
\text { SOIL } \\
\text { SOIL } \\
\text { SOIL }\end{array}$ & & 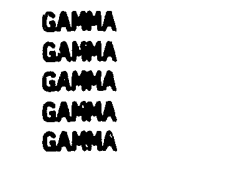 \\
\hline $\begin{array}{l}2142 \\
2143 \\
2149 \\
2151 \\
2152\end{array}$ & $\begin{array}{l}\text { AND } \\
\text { AND } \\
\text { AND } \\
\text { AND } \\
\text { AND }\end{array}$ & $\begin{array}{l}\text { DISMAL GAP } \\
\text { DISSHLL GAP } \\
\text { DISIML GAP } \\
\text { DISNHL GAP } \\
\text { DISUAL GAP }\end{array}$ & $\begin{array}{r}9 \\
10 \\
5 \\
10 \\
10\end{array}$ & $\hat{A}$ & $\begin{array}{l}\text { SOIL } \\
\text { SOIL } \\
\text { SOIL } \\
\text { SOIL } \\
\text { SOIL }\end{array}$ & & $\begin{array}{l}\text { GANAA } \\
\text { ORGANICS } \\
\text { ORGANICS } \\
\text { GANA } \\
\text { GNWA }\end{array}$ \\
\hline $\begin{array}{l}2153 \\
2154 \\
2155 \\
2156 \\
2157\end{array}$ & $\begin{array}{l}\text { AND } \\
\text { AND } \\
\text { AND } \\
\text { AND } \\
\text { AND }\end{array}$ & $\begin{array}{l}\text { DISNAL GAP } \\
\text { DISNALL GAP } \\
\text { DISNAL GAP } \\
\text { DISNULL GAP } \\
\text { DISWAL GAP }\end{array}$ & $\begin{array}{l}10 \\
10 \\
10 \\
10 \\
1\end{array}$ & A & $\begin{array}{l}\text { SOIL } \\
\text { SOIL } \\
\text { SOIL } \\
\text { SOIL } \\
\text { SOIL }\end{array}$ & & 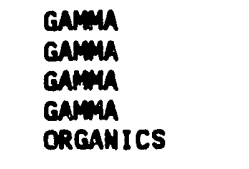 \\
\hline $\begin{array}{l}2161 \\
2162 \\
2163 \\
2164 \\
2165\end{array}$ & $\begin{array}{l}\text { AND } \\
\text { AND } \\
\text { AND } \\
\text { AND } \\
\text { AND }\end{array}$ & $\begin{array}{l}\text { DISWAL GAP } \\
\text { DISSML GAP } \\
\text { DISHAL GAP } \\
\text { DISMAL GAP } \\
\text { DISMAL GAP }\end{array}$ & $\begin{array}{l}1 \\
1 \\
1 \\
1 \\
1\end{array}$ & & $\begin{array}{l}\text { SOIL } \\
\text { SOIL } \\
\text { SOIL } \\
\text { SOIL } \\
\text { SOIL }\end{array}$ & & $\begin{array}{l}\text { GNMA } \\
\text { GANA } \\
\text { GNMA } \\
\text { GANAM } \\
\text { GAMMA }\end{array}$ \\
\hline $\begin{array}{l}2166 \\
2170 \\
2171 \\
2172 \\
2173\end{array}$ & $\begin{array}{l}\text { AND } \\
\text { AND } \\
\text { AND } \\
\text { AND } \\
\text { AND }\end{array}$ & $\begin{array}{l}\text { DISMAL GAP } \\
\text { DISSHLL GAP } \\
\text { DISHLL GAP } \\
\text { DISHAL GAP } \\
\text { DISHAL GAP }\end{array}$ & $\begin{array}{r}1 \\
10 \\
3 \\
3 \\
3\end{array}$ & & $\begin{array}{l}\text { SOIL } \\
\text { HATER } \\
\text { MATER } \\
\text { MATER } \\
\text { WATER }\end{array}$ & & $\begin{array}{l}\text { GAMA } \\
\text { VOC } \\
\text { ORGANICS } \\
\text { ORGANICS } \\
\text { ORGANICS }\end{array}$ \\
\hline $\begin{array}{l}2174 \\
2175 \\
2176 \\
2177 \\
2178\end{array}$ & $\begin{array}{l}\text { AND } \\
\text { AND } \\
\text { AND } \\
\text { AND } \\
\text { AND }\end{array}$ & $\begin{array}{l}\text { DISMAL GAP } \\
\text { DISMAL GAP } \\
\text { DISHAL GAP } \\
\text { DISMAL GAP } \\
\text { DISMAL GAP }\end{array}$ & $\begin{array}{l}3 \\
3 \\
: \\
:\end{array}$ & & $\begin{array}{l}\text { WATER } \\
\text { WATER } \\
\text { WATER } \\
\text { WATER } \\
\text { WATER }\end{array}$ & & $\begin{array}{l}\text { INORGANICS } \\
\text { VOC } \\
\text { ORGANICS } \\
\text { INORGANICS } \\
\text { VOC }\end{array}$ \\
\hline $\begin{array}{l}2179 \\
2180 \\
2181 \\
2182 \\
3003\end{array}$ & $\begin{array}{l}\text { AND } \\
\text { AND } \\
\text { AND } \\
\text { AND } \\
\text { ROA }\end{array}$ & $\begin{array}{l}\text { DISHAL GAP } \\
\text { DISMAL GAP } \\
\text { DISMAL GAP } \\
\text { DISMAL GAP } \\
\text { DI SMAL GAP }\end{array}$ & $\begin{array}{l}\dot{1} \\
\dot{3} \\
3 \\
9\end{array}$ & A & $\begin{array}{l}\text { WATER } \\
\text { WATER } \\
\text { WATER } \\
\text { WATER } \\
\text { SOIL }\end{array}$ & & $\begin{array}{l}\text { ORGANICS } \\
\text { ORGANICS } \\
\text { RADIONUCLIOES } \\
\text { RADIONUCLIDES } \\
\text { ORGANICS }\end{array}$ \\
\hline $\begin{array}{l}3004 \\
3008 \\
3009 \\
3010 \\
3011\end{array}$ & $\begin{array}{l}\text { ROA } \\
\text { ROA } \\
\text { ROA } \\
\text { ROA } \\
\text { ROA }\end{array}$ & $\begin{array}{l}\text { DISHAL GAP } \\
\text { DISHAL GAP } \\
\text { DI SHAL GAP } \\
\text { DI SHAL GAP } \\
\text { DISHAL GAP }\end{array}$ & $\begin{array}{l}9 \\
9 \\
9 \\
9 \\
9\end{array}$ & $A$ & $\begin{array}{l}\text { SOIL } \\
\text { SOIL } \\
\text { SOIL } \\
\text { SOIL } \\
\text { SOIL }\end{array}$ & & $\begin{array}{l}\text { H-3 } \\
\text { GNIMA } \\
\text { GNImA } \\
\text { GNMAM } \\
\text { GANA }\end{array}$ \\
\hline $\begin{array}{l}3012 \\
3013 \\
3014 \\
3015 \\
3016\end{array}$ & $\begin{array}{l}\text { ROA } \\
\text { ROA } \\
\text { ROA } \\
\text { ROA } \\
\text { ROA }\end{array}$ & $\begin{array}{l}\text { DISMAL GAP } \\
\text { DISMAL GAP } \\
\text { DISMAL GAP } \\
\text { DISHAL GAP } \\
\text { DISHAL GAP }\end{array}$ & $\begin{array}{c}9 \\
9 \\
\dot{17}\end{array}$ & A & $\begin{array}{l}\text { SOIL } \\
\text { SOIL } \\
\text { HATER } \\
\text { WATER } \\
\text { SOIL }\end{array}$ & DUPLICATE BLANK & $\begin{array}{l}\text { gavme } \\
\text { GAmA } \\
\text { VO? } \\
\text { VOC: } \\
\text { H-3 }\end{array}$ \\
\hline $\begin{array}{l}3018 \\
3022 \\
3023 \\
3024 \\
3025\end{array}$ & $\begin{array}{l}\text { ROA } \\
\text { ROA } \\
\text { ROA } \\
\text { ROA } \\
\text { ROA }\end{array}$ & $\begin{array}{l}\text { DISMAL GAP } \\
\text { DISMAL GAP } \\
\text { DISMAL GAP } \\
\text { DISMAL GAP } \\
\text { DISMAL GAP }\end{array}$ & $\begin{array}{l}17 \\
17 \\
17 \\
17 \\
17\end{array}$ & A & $\begin{array}{l}\text { SOIL } \\
\text { SOIL } \\
\text { SOIL } \\
\text { SOIL } \\
\text { SOIL }\end{array}$ & & $\begin{array}{l}\text { ORGANICS } \\
\text { GANAMA } \\
\text { GAMHA } \\
\text { GAMMA } \\
\text { GAMMMA }\end{array}$ \\
\hline
\end{tabular}


Table G.1 (continued)

\begin{tabular}{|c|c|c|c|c|c|c|c|}
\hline $\begin{array}{c}\text { Sample } \\
\text { ID }\end{array}$ & Location & Formation & Site & Horizon & Matrix & $\begin{array}{c}\text { Field } \\
\text { duplicate }\end{array}$ & $\begin{array}{c}\text { Analysis } \\
\text { type }\end{array}$ \\
\hline $\begin{array}{l}3026 \\
3027 \\
3031 \\
3032 \\
3036\end{array}$ & $\begin{array}{l}\text { ROA } \\
\text { ROA } \\
\text { ROA } \\
\text { ROA } \\
\text { ROA }\end{array}$ & $\begin{array}{l}\text { DISMAL GAP } \\
\text { DISHAL GAP } \\
\text { DISHAL GAP } \\
\text { DISHAL GAP } \\
\text { DISMAL GAP }\end{array}$ & $\begin{array}{l}17 \\
17 \\
19 \\
19 \\
19\end{array}$ & $\hat{A}$ & $\begin{array}{l}\text { SOIL } \\
\text { SOIL } \\
\text { SOIL } \\
\text { SOIL } \\
\text { SOIL }\end{array}$ & & $\begin{array}{l}\text { GNMMA } \\
\text { GNMAA } \\
\text { H-3 } \\
\text { ORGANICS } \\
\text { GANMA }\end{array}$ \\
\hline $\begin{array}{l}3037 \\
3038 \\
3039 \\
3040 \\
3041\end{array}$ & $\begin{array}{l}\text { ROA } \\
\text { ROA } \\
\text { ROA } \\
\text { ROA } \\
\text { ROA }\end{array}$ & $\begin{array}{l}\text { DISMAL GAP } \\
\text { DISMAL GAP } \\
\text { DISMAL GAP } \\
\text { DI SMAL GAP } \\
\text { DISMAL GAP }\end{array}$ & $\begin{array}{l}19 \\
19 \\
19 \\
19 \\
19\end{array}$ & & $\begin{array}{l}\text { SOIL } \\
\text { SOIL } \\
\text { SOIL } \\
\text { SOIL } \\
\text { SOIL }\end{array}$ & & 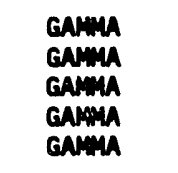 \\
\hline $\begin{array}{l}3045 \\
3046 \\
3050 \\
3051 \\
3052\end{array}$ & $\begin{array}{l}\text { ROA } \\
\text { ROA } \\
\text { ROA } \\
\text { ROA } \\
\text { ROA }\end{array}$ & $\begin{array}{l}\text { DISNAL GAP } \\
\text { DISNAL GAP } \\
\text { DISMAL GAP } \\
\text { DISMAL GAP } \\
\text { DISHAL GAP }\end{array}$ & $\begin{array}{l}20 \\
20 \\
20 \\
20 \\
20\end{array}$ & $\hat{A}$ & $\begin{array}{l}\text { SOIL } \\
\text { SOIL } \\
\text { SOIL } \\
\text { SOIL } \\
\text { SOIL }\end{array}$ & & $\begin{array}{l}\text { H-3 } \\
\text { ORGANICS } \\
\text { GANMA } \\
\text { GANMA } \\
\text { GANMA }\end{array}$ \\
\hline $\begin{array}{l}3053 \\
3054 \\
3055 \\
3057 \\
3058\end{array}$ & $\begin{array}{l}\text { ROA } \\
\text { ROA } \\
\text { ROA } \\
\text { ROA } \\
\text { ROA }\end{array}$ & $\begin{array}{l}\text { DISMAL GAP } \\
\text { DISMAL GAP } \\
\text { DISMAL GAP } \\
\text { DISMAL GAP } \\
\text { DISMAL GAP }\end{array}$ & $\begin{array}{l}20 \\
20 \\
20 \\
22 \\
22\end{array}$ & $\hat{A}$ & $\begin{array}{l}\text { SOIL } \\
\text { SOIL } \\
\text { SOIL } \\
\text { SOIL } \\
\text { SOIL }\end{array}$ & & $\begin{array}{l}\text { GAmiA } \\
\text { GANMA } \\
\text { GAmiA } \\
\text { H-3 } \\
\text { ORGANICS }\end{array}$ \\
\hline $\begin{array}{l}3062 \\
3063 \\
3064 \\
3065 \\
3066\end{array}$ & $\begin{array}{l}\text { ROA } \\
\text { ROA } \\
\text { ROA } \\
\text { ROA } \\
\text { ROA }\end{array}$ & $\begin{array}{l}\text { DISMAL GAP } \\
\text { DISMAL GAP } \\
\text { DI SMAL GAP } \\
\text { DISMAL GAP } \\
\text { DISHAL GAP }\end{array}$ & $\begin{array}{l}22 \\
22 \\
22 \\
22 \\
22\end{array}$ & & $\begin{array}{l}\text { SOIL } \\
\text { SOIL } \\
\text { SOIL } \\
\text { SOIL } \\
\text { SOIL }\end{array}$ & & 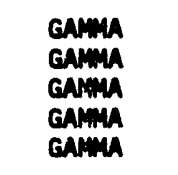 \\
\hline $\begin{array}{l}3067 \\
3071 \\
3072 \\
3076 \\
3077\end{array}$ & $\begin{array}{l}\text { ROA } \\
\text { ROA } \\
\text { ROA } \\
\text { ROA } \\
\text { ROA }\end{array}$ & $\begin{array}{l}\text { DISAAL GAP } \\
\text { DISMAL GAP } \\
\text { DISMAL GAP } \\
\text { DISMAL GAP } \\
\text { DISMAL GAP }\end{array}$ & $\begin{array}{r}22 \\
8 \\
8 \\
8 \\
8\end{array}$ & $\hat{A}$ & $\begin{array}{l}\text { SOIL } \\
\text { SOIL } \\
\text { SOIL } \\
\text { SOIL } \\
\text { SOIL }\end{array}$ & & $\begin{array}{l}\text { GAmA } \\
\text { H-3 } \\
\text { ORGANICS } \\
\text { GAMmA } \\
\text { GNMiA }\end{array}$ \\
\hline $\begin{array}{l}3078 \\
3079 \\
3080 \\
3081 \\
3082\end{array}$ & $\begin{array}{l}\text { ROA } \\
\text { ROA } \\
\text { ROA } \\
\text { ROA } \\
\text { ROA }\end{array}$ & $\begin{array}{l}\text { DISMAL GAP } \\
\text { DISHAL GAP } \\
\text { DISMAL GAP } \\
\text { DISHAL GAP } \\
\text { DISMAL GAP }\end{array}$ & $\begin{array}{l}8 \\
8 \\
8 \\
8 \\
-\end{array}$ & & $\begin{array}{l}\text { SOIL } \\
\text { SOIL } \\
\text { SOIL } \\
\text { SOIL } \\
\text { WATER }\end{array}$ & & 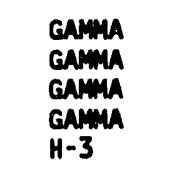 \\
\hline $\begin{array}{l}3084 \\
3085 \\
3089 \\
3090 \\
3091\end{array}$ & $\begin{array}{l}\text { ROA } \\
\text { ROA } \\
\text { ROA } \\
\text { ROA } \\
\text { ROA }\end{array}$ & $\begin{array}{l}\text { DISMAL GAP } \\
\text { DISHAL GAP } \\
\text { DISHAL GAP } \\
\text { DISHAL GAP } \\
\text { DISHAL GAP }\end{array}$ & $\begin{array}{l}7 \\
7 \\
7 \\
7 \\
7\end{array}$ & $\hat{A}$ & $\begin{array}{l}\text { SOIL } \\
\text { SOIL } \\
\text { SOIL } \\
\text { SoIL } \\
\text { SOIL }\end{array}$ & & $\begin{array}{l}H-3 \\
\text { ORGANICS } \\
\text { GAMUA } \\
\text { GANMA } \\
\text { GANMAA }\end{array}$ \\
\hline $\begin{array}{l}3092 \\
3093 \\
3094 \\
3098 \\
3099\end{array}$ & $\begin{array}{l}\text { ROA } \\
\text { ROA } \\
\text { ROA } \\
\text { ROA } \\
\text { ROA }\end{array}$ & $\begin{array}{l}\text { DISMAL GAP } \\
\text { DISMAL GAP } \\
\text { DISMAL GAP } \\
\text { DISMAL GAP } \\
\text { DISMAL GAP }\end{array}$ & $\begin{array}{l}7 \\
7 \\
7 \\
3 \\
3\end{array}$ & $\hat{A}$ & $\begin{array}{l}\text { SoIL } \\
\text { soIL } \\
\text { SoIL } \\
\text { SoIL } \\
\text { SoIL }\end{array}$ & & $\begin{array}{l}\text { GAmiA } \\
\text { GAmMA } \\
\text { GAmMA } \\
H-3 \\
\text { ORGANICS }\end{array}$ \\
\hline $\begin{array}{l}3103 \\
3104 \\
3105 \\
3106 \\
3107\end{array}$ & $\begin{array}{l}\text { ROA } \\
\text { ROA } \\
\text { ROA } \\
\text { ROA } \\
\text { ROA }\end{array}$ & $\begin{array}{l}\text { DISHAL GAP } \\
\text { DISHAL GAP } \\
\text { DISHAL GAP } \\
\text { DISHAL GAP } \\
\text { DISHAL GAP }\end{array}$ & $\begin{array}{l}3 \\
3 \\
3 \\
3 \\
3\end{array}$ & & $\begin{array}{l}\text { SOIL } \\
\text { SOIL } \\
\text { SOIL } \\
\text { SOIL } \\
\text { SOIL }\end{array}$ & & 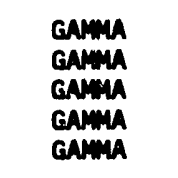 \\
\hline $\begin{array}{l}3108 \\
3113 \\
3117 \\
3118 \\
3119\end{array}$ & $\begin{array}{l}\text { ROA } \\
\text { ROA } \\
\text { ROA } \\
\text { ROA } \\
\text { ROA }\end{array}$ & $\begin{array}{l}\text { DISHAL GAP } \\
\text { DI SMAL GAP } \\
\text { DISMAL GAP } \\
\text { DI SMAL GAP } \\
\text { DISHAL GAP }\end{array}$ & $\begin{array}{l}3 \\
21 \\
21 \\
21 \\
21\end{array}$ & A & $\begin{array}{l}\text { SOIL } \\
\text { SOIL } \\
\text { SOIL } \\
\text { SoIL } \\
\text { SOIL }\end{array}$ & & $\begin{array}{l}\text { GAMNA } \\
\text { ORGANICS } \\
\text { GAMNA } \\
\text { GAMNA } \\
\text { GAMNA }\end{array}$ \\
\hline $\begin{array}{l}3120 \\
3121 \\
3122 \\
3123 \\
3125\end{array}$ & $\begin{array}{l}\text { ROA } \\
\text { ROA } \\
\text { ROA } \\
\text { ROA } \\
\text { ROA }\end{array}$ & $\begin{array}{l}\text { DISHAL GAP } \\
\text { DISMAL GAP } \\
\text { DISHAL GAP } \\
\text { DISMAL GAP } \\
\text { DISHAL GAP }\end{array}$ & $\begin{array}{l}21 \\
21 \\
21 \\
13\end{array}$ & $A$ & $\begin{array}{l}\text { SOIL } \\
\text { SOIL } \\
\text { SOIL } \\
\text { WATER } \\
\text { SOIL }\end{array}$ & & $\begin{array}{l}\text { GAMmA } \\
\text { GAMmAA } \\
\text { GAMmA } \\
\text { VOC } \\
\text { VOC }\end{array}$ \\
\hline
\end{tabular}


G-10

Table G.1 (continued)

\begin{tabular}{|c|c|c|c|c|c|c|c|}
\hline $\begin{array}{l}\text { Sample } \\
\text { ID }\end{array}$ & Location & Formation & Site & zon & Matrix & $\begin{array}{c}\text { Field } \\
\text { duplicate }\end{array}$ & $\begin{array}{l}\text { Analysis } \\
\text { type }\end{array}$ \\
\hline $\begin{array}{l}3127 \\
3131 \\
3132 \\
3133 \\
3134\end{array}$ & $\begin{array}{l}\text { ROA } \\
\text { ROA } \\
\text { ROA } \\
\text { ROA } \\
\text { ROA }\end{array}$ & $\begin{array}{l}\text { DISUAL GAP } \\
\text { DISMAL GAP } \\
\text { DISMAL GAP } \\
\text { DISHAL GAP } \\
\text { DISMAL GAP }\end{array}$ & $\begin{array}{l}13 \\
13 \\
13 \\
13 \\
13\end{array}$ & A & $\begin{array}{l}\text { Soll } \\
\text { SOIL } \\
\text { SOIL } \\
\text { SoIL } \\
\text { SoIL }\end{array}$ & & 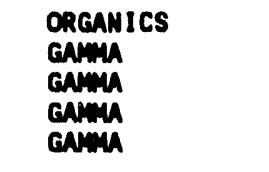 \\
\hline $\begin{array}{l}3135 \\
3136 \\
3137 \\
3139 \\
3143\end{array}$ & $\begin{array}{l}\text { ROA } \\
\text { ROA } \\
\text { ROA } \\
\text { ROA } \\
\text { ROA }\end{array}$ & $\begin{array}{l}\text { DISMAL GAP } \\
\text { DISNAL GAP } \\
\text { DISHAL GAP } \\
\text { DISMAL GAP } \\
\text { DISUAL GAP }\end{array}$ & $\begin{array}{l}13 \\
13 \\
13 \\
13 \\
13\end{array}$ & $\hat{A}$ & $\begin{array}{l}\text { SOIL } \\
\text { SOIL } \\
\text { SOIL } \\
\text { SOIL } \\
\text { WATER }\end{array}$ & $\begin{array}{l}\text { FIELD DUPLICATE } \\
\text { FIELD DUPLICATE }\end{array}$ & $\begin{array}{l}\text { GAmin } \\
\text { GAmin } \\
\text { VOC } \\
\text { ORGANICS } \\
\text { VOC }\end{array}$ \\
\hline $\begin{array}{l}3144 \\
3145 \\
3146 \\
3148 \\
3152\end{array}$ & $\begin{array}{l}\text { ROA } \\
\text { ROA } \\
\text { ROA } \\
\text { ROA } \\
\text { ROA }\end{array}$ & $\begin{array}{l}\text { DISMAL GAP } \\
\text { DISHAL GAP } \\
\text { DISMAL GAP } \\
\text { DISMAL GAP } \\
\text { DISHAL GAP }\end{array}$ & $\begin{array}{l}13 \\
13 \\
14 \\
14 \\
14\end{array}$ & $\hat{A}$ & $\begin{array}{l}\text { MATER } \\
\text { MATER } \\
\text { SOIL } \\
\text { SOIL } \\
\text { SOIL }\end{array}$ & & $\begin{array}{l}\text { INORGANICS } \\
\text { ORGANICS } \\
\text { VOC } \\
\text { ORGANICS } \\
\text { GNMA }\end{array}$ \\
\hline $\begin{array}{l}3153 \\
3154 \\
3155 \\
3156 \\
3157\end{array}$ & $\begin{array}{l}\text { ROA } \\
\text { ROA } \\
\text { ROA } \\
\text { ROA } \\
\text { ROA }\end{array}$ & $\begin{array}{l}\text { DISHAL GAP } \\
\text { DISMAL GAP } \\
\text { DISHAL GAP } \\
\text { DISMAL GAP } \\
\text { DISHAL GAP }\end{array}$ & $\begin{array}{l}14 \\
14 \\
14 \\
14 \\
14\end{array}$ & & $\begin{array}{l}\text { SOIL } \\
\text { SOIL } \\
\text { SOIL } \\
\text { SOIL } \\
\text { SOIL }\end{array}$ & & 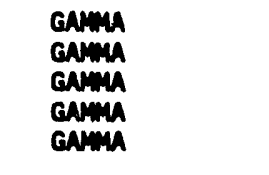 \\
\hline $\begin{array}{l}3163 \\
3164 \\
3166 \\
3168 \\
3176\end{array}$ & $\begin{array}{l}\text { ROA } \\
\text { ROA } \\
\text { ROA } \\
\text { ROA } \\
\text { ROA }\end{array}$ & $\begin{array}{l}\text { DISMAL GAP } \\
\text { DISMAL GAP } \\
\text { DISMAL GAP } \\
\text { DISMAL GAP } \\
\text { DISHAL GAP }\end{array}$ & $\begin{array}{l}14 \\
10 \\
10 \\
10 \\
10\end{array}$ & $\hat{\hat{A}}$ & $\begin{array}{l}\text { WATER } \\
\text { WATER } \\
\text { SOIL } \\
\text { SOIL } \\
\text { WATER }\end{array}$ & & $\begin{array}{l}\text { ORGANICS } \\
\text { VOC } \\
\text { VOC } \\
\text { OREANICS } \\
\text { RADIONUCLIOES }\end{array}$ \\
\hline $\begin{array}{l}3177 \\
3178 \\
3179 \\
3180 \\
3181\end{array}$ & $\begin{array}{l}\text { ROA } \\
\text { ROA } \\
\text { ROA } \\
\text { ROA } \\
\text { ROA }\end{array}$ & $\begin{array}{l}\text { DISMAL GAP } \\
\text { DISNAL GAP } \\
\text { DISMAL GAP } \\
\text { DISMAL GAP } \\
\text { DISMAL GAP }\end{array}$ & $\begin{array}{l}10 \\
10 \\
10 \\
10 \\
10\end{array}$ & & $\begin{array}{l}\text { WATER } \\
\text { SOIL } \\
\text { SOIL } \\
\text { SOIL } \\
\text { SOIL }\end{array}$ & & $\begin{array}{l}\text { ORGANICS } \\
\text { GNmA } \\
\text { GNmA } \\
\text { GNmA } \\
\text { GANAA }\end{array}$ \\
\hline $\begin{array}{l}3182 \\
3183 \\
3184 \\
3185 \\
3186\end{array}$ & $\begin{array}{l}\text { ROA } \\
\text { ROA } \\
\text { ROA } \\
\text { ROA } \\
\text { ROA }\end{array}$ & $\begin{array}{l}\text { DISMAL GAP } \\
\text { DISMAL GAP } \\
\text { DISMAL GAP } \\
\text { DISMAL GAP } \\
\text { DISMAL GAP }\end{array}$ & $\begin{array}{r}10 \\
10 \\
17 \\
7 \\
8\end{array}$ & $\hat{A}$ & $\begin{array}{l}\text { SoIL } \\
\text { SoIL } \\
\text { SoIl } \\
\text { soll } \\
\text { SoIL }\end{array}$ & & $\begin{array}{l}\text { GNwa } \\
\text { Gawn } \\
\text { VOC } \\
\text { VOC } \\
\text { VOC }\end{array}$ \\
\hline $\begin{array}{l}3193 \\
3194 \\
3195 \\
3196 \\
3197\end{array}$ & $\begin{array}{l}\operatorname{ROA} \\
\operatorname{ROA} \\
\operatorname{ROA} \\
\operatorname{ROA} \\
\operatorname{ROA}\end{array}$ & $\begin{array}{l}\text { DISHAL GAP } \\
\text { DISHAL GAP } \\
\text { DISHAL GAP } \\
\text { DISMAL GAP } \\
\text { DISHAL GAP }\end{array}$ & $\begin{array}{r}\dot{ } \\
21 \\
22\end{array}$ & $\hat{A}$ & $\begin{array}{l}\text { WATER } \\
\text { WATER } \\
\text { WATER } \\
\text { SOIL } \\
\text { SOIL }\end{array}$ & & $\begin{array}{l}\text { Voc } \\
\text { Voc } \\
\text { Voc } \\
\text { Voc } \\
\text { Voc }\end{array}$ \\
\hline $\begin{array}{l}3198 \\
3199 \\
3200 \\
3201 \\
5001\end{array}$ & $\begin{array}{l}\text { ROA } \\
\text { ROA } \\
\text { ROA } \\
\text { ROA } \\
\text { ORR }\end{array}$ & $\begin{array}{l}\text { DISMAL GAP } \\
\text { DISMAL GAP } \\
\text { DISMAL GAP } \\
\text { DISMAL GAP } \\
\text { DISMAL GAP }\end{array}$ & $\begin{array}{l}20 \\
19 \\
9 \\
3 \\
11,27,41\end{array}$ & $\begin{array}{l}\hat{A} \\
\hat{A} \\
\hat{A} \\
\hat{A}\end{array}$ & $\begin{array}{l}\text { SOIL } \\
\text { SOIL } \\
\text { SOIL } \\
\text { SoIL } \\
\text { SOIL }\end{array}$ & & $\begin{array}{l}\text { VOC } \\
\text { VOC } \\
\text { VOC } \\
\text { VOC } \\
\text { IMORGANICS }\end{array}$ \\
\hline $\begin{array}{l}5002 \\
5003 \\
5004 \\
5005 \\
5006\end{array}$ & $\begin{array}{l}\text { ORR } \\
\text { ORR } \\
\text { ORR } \\
\text { ORR } \\
\text { ORR }\end{array}$ & $\begin{array}{l}\text { DISMAL GAP } \\
\text { DISMAL GAP } \\
\text { DISMAL GAP } \\
\text { DISMAL GAP } \\
\text { DISMAL GAP }\end{array}$ & $\begin{array}{l}11,27,41 \\
11,27,41 \\
11,27,41 \\
11,27,41 \\
11,27,41\end{array}$ & $\begin{array}{l}\mathbf{A} \\
\hat{A} \\
\mathbf{B} \\
\mathbf{B} \\
\mathbf{B}\end{array}$ & $\begin{array}{l}\text { SOIL } \\
\text { SOIL } \\
\text { SOIL } \\
\text { SOIL } \\
\text { SOIL }\end{array}$ & & $\begin{array}{l}\text { RADIONUCLIDES } \\
\text { EXTRA } \\
\text { INORGANICS } \\
\text { RADIONUCLIDES } \\
\text { EXTRA }\end{array}$ \\
\hline $\begin{array}{l}5007 \\
5008 \\
5009 \\
5010 \\
5011\end{array}$ & $\begin{array}{l}\text { ORR } \\
\text { ORR } \\
\text { ORR } \\
\text { ORR } \\
\text { ORR }\end{array}$ & $\begin{array}{l}\text { DISMAL GAP } \\
\text { DISMAL GAP } \\
\text { DISMAL GAP } \\
\text { DI SMAL GAP } \\
\text { DISMAL GAP }\end{array}$ & $\begin{array}{l}11,27,41 \\
11,27,41 \\
11,27,41 \\
19,22,32 \\
19,22,32\end{array}$ & $\begin{array}{l}c \\
\mathbf{C} \\
\mathbf{C} \\
\hat{A} \\
\mathbf{A}\end{array}$ & $\begin{array}{l}\text { SOIL } \\
\text { SOIL } \\
\text { SOIL } \\
\text { Soll } \\
\text { SOIL }\end{array}$ & & $\begin{array}{l}\text { IMORGANICS } \\
\text { RADIONUCLIDES } \\
\text { EXTRA } \\
\text { INORGANICS } \\
\text { RADIONUCLIDES }\end{array}$ \\
\hline $\begin{array}{l}5012 \\
5013 \\
5014 \\
5015 \\
5016\end{array}$ & $\begin{array}{l}\text { ORR } \\
\text { ORR } \\
\text { ORR } \\
\text { ORR } \\
\text { ORR }\end{array}$ & $\begin{array}{l}\text { DISMAL GAP } \\
\text { DISMAL GAP } \\
\text { DISMAL GAP } \\
\text { DISMAL GAP } \\
\text { DISMAL GAP }\end{array}$ & $\begin{array}{lll}19, & 22, & 32 \\
19, & 22, & 32 \\
19, & 22, & 32 \\
19, & 22, & 32 \\
19, & 22, & 32\end{array}$ & $\begin{array}{l}A \\
B \\
B \\
B \\
C\end{array}$ & $\begin{array}{l}\text { SoIl } \\
\text { soll } \\
\text { soll } \\
\text { Soll } \\
\text { SoIL }\end{array}$ & & $\begin{array}{l}\text { EXTRA } \\
\text { INORGANICS } \\
\text { RADIONUCLIDES } \\
\text { EXTRA } \\
\text { INORGANICS }\end{array}$ \\
\hline
\end{tabular}


Table G.1 (continued)

\begin{tabular}{|c|c|c|c|c|c|c|c|}
\hline $\begin{array}{c}\text { Sample } \\
\text { ID }\end{array}$ & Location & Formation & Site & izon & Matrix & $\begin{array}{c}\text { Field } \\
\text { duplicate }\end{array}$ & $\begin{array}{c}\text { Analysis } \\
\text { type }\end{array}$ \\
\hline $\begin{array}{l}5017 \\
5018 \\
5019 \\
5020 \\
5021\end{array}$ & $\begin{array}{l}\text { ORR } \\
\text { ORR } \\
\text { ORR } \\
\text { ORR } \\
\text { ORR }\end{array}$ & $\begin{array}{l}\text { DISML EAP } \\
\text { DISMAL GAP } \\
\text { DI SNAL GAP } \\
\text { DISMAL GAP } \\
\text { DISMAL GAP }\end{array}$ & $\begin{array}{l}19,22,32 \\
19,22,32 \\
10,33,35 \\
10,33,35 \\
10,33,35\end{array}$ & $\begin{array}{l}\mathbf{C} \\
\mathbf{C} \\
\hat{A} \\
\hat{A}\end{array}$ & $\begin{array}{l}\text { SOIL } \\
\text { SOIL } \\
\text { SOIL } \\
\text { SOIL } \\
\text { SOIL }\end{array}$ & & $\begin{array}{l}\text { RADIONUCLIDES } \\
\text { EXTRA } \\
\text { INORGANICS } \\
\text { RADIONUCLLIDES } \\
\text { EXTRA }\end{array}$ \\
\hline $\begin{array}{l}5022 \\
5023 \\
5024 \\
5025 \\
5026\end{array}$ & $\begin{array}{l}\text { ORR } \\
\text { ORR } \\
\text { ORR } \\
\text { ORR } \\
\text { ORR }\end{array}$ & $\begin{array}{l}\text { DISMAL GAP } \\
\text { DISMAL GAP } \\
\text { DISMAL GAP } \\
\text { DISMAL GAP } \\
\text { DISMAL GAP }\end{array}$ & $\begin{array}{l}10,33,35 \\
10,33,35 \\
10,33,35 \\
10,33, \quad 35 \\
10,33,35\end{array}$ & $\begin{array}{l}\mathbf{B} \\
\mathbf{B} \\
\mathbf{B} \\
\mathbf{C} \\
\mathbf{C}\end{array}$ & $\begin{array}{l}\text { soll } \\
\text { soll } \\
\text { soll } \\
\text { soll } \\
\text { soll }\end{array}$ & & $\begin{array}{l}\text { INORGANICS } \\
\text { RADIONUCLIIDES } \\
\text { EXTRA } \\
\text { INORGANICS } \\
\text { RADIONUCLIDES }\end{array}$ \\
\hline $\begin{array}{l}5027 \\
5028 \\
5029 \\
5030 \\
5031\end{array}$ & $\begin{array}{l}\text { ORR } \\
\text { ORR } \\
\text { ORR } \\
\text { ORR } \\
\text { ORR }\end{array}$ & $\begin{array}{l}\text { DISHAL GAP } \\
\text { DISHL GAP } \\
\text { DISWLL GAP } \\
\text { DISNAL GAP } \\
\text { DISMAL GAP }\end{array}$ & $\begin{array}{r}10,33,35 \\
2,26,43 \\
2,26,43 \\
2,26,43 \\
2,26,43\end{array}$ & $\begin{array}{l}\mathbf{C} \\
\hat{A} \\
\hat{A} \\
\hat{B}\end{array}$ & $\begin{array}{l}\text { Soll } \\
\text { SOIL } \\
\text { SoIL } \\
\text { SoIL } \\
\text { SoIL }\end{array}$ & & $\begin{array}{l}\text { EXTRA } \\
\text { INORGANICS } \\
\text { RADIONUCLLIOES } \\
\text { EXTRA } \\
\text { INORGANICS }\end{array}$ \\
\hline $\begin{array}{l}5032 \\
5033 \\
5034 \\
5035 \\
5036\end{array}$ & $\begin{array}{l}\text { ORR } \\
\text { ORR } \\
\text { ORR } \\
\text { ORR } \\
\text { ORR }\end{array}$ & $\begin{array}{l}\text { DISHAL GAP } \\
\text { DISMAL GAP } \\
\text { DISMAL GAP } \\
\text { DISHAL GAP } \\
\text { DISMAL GAP }\end{array}$ & $\begin{array}{ll}2, & 26,43 \\
2, & 26,43 \\
2, & 26,43 \\
2, & 26,43 \\
2, & 26,43\end{array}$ & $\begin{array}{l}B \\
B \\
C \\
C \\
C\end{array}$ & $\begin{array}{l}\text { SoIL } \\
\text { SoIL } \\
\text { soll } \\
\text { soIL } \\
\text { SoIL }\end{array}$ & & $\begin{array}{l}\text { RADIONUCLIDES } \\
\text { EXTRA } \\
\text { INORGANICS } \\
\text { RADIONUCLIDES } \\
\text { EXTRA }\end{array}$ \\
\hline $\begin{array}{l}5037 \\
5038 \\
5039 \\
5040 \\
5041\end{array}$ & $\begin{array}{l}\text { ORR } \\
\text { ORR } \\
\text { ORR } \\
\text { ORR } \\
\text { ORR }\end{array}$ & $\begin{array}{l}\text { DISMAL GAP } \\
\text { DISMAL GAP } \\
\text { DISMAL GAP } \\
\text { DI SMAL GAP } \\
\text { DISIML GAP }\end{array}$ & $\begin{array}{l}2,26,43 \\
2,26,43 \\
2,26,43 \\
2,26,43 \\
2,26,43\end{array}$ & $\begin{array}{l}\hat{A} \\
\hat{A} \\
\hat{B} \\
B\end{array}$ & $\begin{array}{l}\text { SOIL } \\
\text { SOIL } \\
\text { soIL } \\
\text { SOIL } \\
\text { SOIL }\end{array}$ & $\begin{array}{l}\text { FIELD DUPLICATE } \\
\text { FIELD DUPLICATE } \\
\text { FIELD DUPLICATE } \\
\text { FIELD DUPLICATE } \\
\text { FIELD DUPLICATE }\end{array}$ & $\begin{array}{l}\text { INORGANICS } \\
\text { RADIONUCLIDES } \\
\text { EXTRA } \\
\text { INORGANICS } \\
\text { RADIONUCLIDES }\end{array}$ \\
\hline $\begin{array}{l}5042 \\
5043 \\
5044 \\
5045 \\
5055\end{array}$ & $\begin{array}{l}\text { ORR } \\
\text { ORR } \\
\text { ORR } \\
\text { ORR } \\
\text { ORR }\end{array}$ & $\begin{array}{l}\text { DISMAL GAP } \\
\text { DISHLL GAP } \\
\text { DISML GAP } \\
\text { DISHAL GAP } \\
\text { MOLICHUCKY }\end{array}$ & $\begin{array}{r}2,26,43 \\
2,26,43 \\
2,26,43 \\
2,26,43 \\
15,23,25\end{array}$ & $\begin{array}{l}B \\
C \\
C \\
C \\
A\end{array}$ & $\begin{array}{l}\text { SoIL } \\
\text { SoIL } \\
\text { SoIl } \\
\text { SoIL } \\
\text { SoIL }\end{array}$ & $\begin{array}{l}\text { FIELD DUPLICATE } \\
\text { FIELD DUPLICATE } \\
\text { FIELD DUPLICATE } \\
\text { FIELD DUPLICATE }\end{array}$ & $\begin{array}{l}\text { EXTRA } \\
\text { INORGANICS } \\
\text { RADIONUCLIIDES } \\
\text { EXTRA } \\
\text { INORGANICS }\end{array}$ \\
\hline $\begin{array}{l}5056 \\
5057 \\
5058 \\
5059 \\
5060\end{array}$ & $\begin{array}{l}\text { ORR } \\
\text { ORR } \\
\text { ORR } \\
\text { ORR } \\
\text { ORR }\end{array}$ & $\begin{array}{l}\text { NOL I CHUCKY } \\
\text { NOL I CHUCKY } \\
\text { NOL I CHUCKY } \\
\text { NOL I CHUCKY } \\
\text { NOL I CHUCKY }\end{array}$ & $\begin{array}{l}15,23,25 \\
15,23,25 \\
15,23,25 \\
15,23,25 \\
15,23,25\end{array}$ & $\begin{array}{l}\hat{A} \\
\hat{A} \\
B \\
B \\
B\end{array}$ & $\begin{array}{l}\text { SoIL } \\
\text { SoIL } \\
\text { SoIL } \\
\text { SoIL } \\
\text { SOIL }\end{array}$ & & $\begin{array}{l}\text { RADIONUCL IDES } \\
\text { EXTRA } \\
\text { INORGANICS } \\
\text { RADIONUCL IDES } \\
\text { EXTRA }\end{array}$ \\
\hline $\begin{array}{l}5061 \\
5062 \\
5063 \\
5064 \\
5065\end{array}$ & $\begin{array}{l}\text { ORR } \\
\text { ORR } \\
\text { ORR } \\
\text { ORR } \\
\text { ORR }\end{array}$ & $\begin{array}{l}\text { NOLICHUCKY } \\
\text { NOLI ICHUCKY } \\
\text { NOLI ICHUCKY } \\
\text { NOLICHUCKY } \\
\text { NOLI I CHUCKY }\end{array}$ & $\begin{array}{l}15,23,25 \\
15,23,25 \\
15,23,25 \\
16,28,42 \\
16,28,42\end{array}$ & $\begin{array}{l}c \\
c \\
C \\
A \\
A\end{array}$ & $\begin{array}{l}\text { SoIL } \\
\text { SOIL } \\
\text { SoIL } \\
\text { SoIL } \\
\text { SoIL }\end{array}$ & & $\begin{array}{l}\text { INORGANICS } \\
\text { RADIONUCLIDES } \\
\text { EXTRA } \\
\text { INORGANICS } \\
\text { RADIONUCLIDES }\end{array}$ \\
\hline $\begin{array}{l}5066 \\
5067 \\
5068 \\
5069 \\
5070\end{array}$ & $\begin{array}{l}\text { ORR } \\
\text { ORR } \\
\text { ORR } \\
\text { ORR } \\
\text { ORR }\end{array}$ & $\begin{array}{l}\text { NOLI ICHUCKY } \\
\text { NOL I CHUCKY } \\
\text { NOLI I CHUCKY } \\
\text { NOL I CHUCKY } \\
\text { NOLI ICHUCKY }\end{array}$ & $\begin{array}{l}16,28,42 \\
16,28,42 \\
16,28,42 \\
16,28,42 \\
16,28,42\end{array}$ & $\begin{array}{l}\mathbf{A} \\
\mathbf{B} \\
\mathbf{B} \\
\mathbf{B} \\
\mathbf{C}\end{array}$ & $\begin{array}{l}\text { SOIL } \\
\text { SOIL } \\
\text { SOIL } \\
\text { SOIL } \\
\text { SOIL }\end{array}$ & & $\begin{array}{l}\text { EXTRA } \\
\text { INORGANICS } \\
\text { RADIONUCLIDES } \\
\text { EXTRA } \\
\text { INORGANICS }\end{array}$ \\
\hline $\begin{array}{l}5071 \\
5072 \\
5073 \\
5074 \\
5075\end{array}$ & $\begin{array}{l}\text { ORR } \\
\text { ORR } \\
\text { ORR } \\
\text { ORR } \\
\text { ORR }\end{array}$ & $\begin{array}{l}\text { NOLI I CHUCKY } \\
\text { NOL I CHUCKY } \\
\text { NOL I CHUCKY } \\
\text { NOL I CHUCKY } \\
\text { NOL I CHUCKY }\end{array}$ & $\begin{array}{r}16,28,42 \\
16,28,42 \\
5,21,31 \\
5,21,31 \\
5,21,31\end{array}$ & $\begin{array}{l}C \\
C \\
A \\
A \\
A\end{array}$ & $\begin{array}{l}\text { SoIL } \\
\text { SoIL } \\
\text { SoIL } \\
\text { SoIL } \\
\text { SOIL }\end{array}$ & & $\begin{array}{l}\text { RADIONUCLIDES } \\
\text { EXTRA } \\
\text { INORGANICS } \\
\text { RADIONUCLIDES } \\
\text { EXTRA }\end{array}$ \\
\hline $\begin{array}{l}5076 \\
5077 \\
5078 \\
5079 \\
5080\end{array}$ & $\begin{array}{l}\text { ORR } \\
\text { ORR } \\
\text { ORR } \\
\text { ORR } \\
\text { ORR }\end{array}$ & $\begin{array}{l}\text { NOL I CHUCKY } \\
\text { NOL I CHUCKY } \\
\text { NOL I CHUCKY } \\
\text { NOL I CHUCKY } \\
\text { NOL I CHUCKY }\end{array}$ & $\begin{array}{l}5,21,31 \\
5,21,31 \\
5,21,31 \\
5,21,31 \\
5,21,31\end{array}$ & $\begin{array}{l}\mathrm{B} \\
\mathrm{B} \\
\mathrm{B} \\
\mathrm{C} \\
\mathrm{C}\end{array}$ & $\begin{array}{l}\text { SoIL } \\
\text { SOIL } \\
\text { SOIL } \\
\text { SOIL } \\
\text { SOIL }\end{array}$ & & $\begin{array}{l}\text { INORGANICS } \\
\text { RADIONUCL IDES } \\
\text { EXTRA } \\
\text { INORGANICS } \\
\text { RADIONUCL IDES }\end{array}$ \\
\hline $\begin{array}{l}5081 \\
5082 \\
5083 \\
5084 \\
5085\end{array}$ & $\begin{array}{l}\text { ORR } \\
\text { ORR } \\
\text { ORR } \\
\text { ORR } \\
\text { ORR }\end{array}$ & $\begin{array}{l}\text { NOLICHUCKY } \\
\text { NOLICHUCKY } \\
\text { NOLICHUCKY } \\
\text { NOLICHUCKY } \\
\text { NOLICHUCKY }\end{array}$ & $\begin{array}{l}5,21,31 \\
3,13,24 \\
3,13,24 \\
3,13,24 \\
3,13,24\end{array}$ & $\begin{array}{l}\hat{C} \\
\hat{A} \\
\hat{B}\end{array}$ & $\begin{array}{l}\text { SOIL } \\
\text { SOIL } \\
\text { SOIL } \\
\text { SOIL } \\
\text { SOIL }\end{array}$ & & $\begin{array}{l}\text { EXTRA } \\
\text { INORGANICS } \\
\text { RADIONUCL IDES } \\
\text { EXTRA } \\
\text { INORGANICS }\end{array}$ \\
\hline
\end{tabular}


Table G.1 (continued)

\begin{tabular}{|c|c|c|c|c|c|c|c|}
\hline $\begin{array}{c}\text { Sample } \\
\text { ID }\end{array}$ & Location & Formation & Site & zon & Matrix & $\begin{array}{c}\text { Field } \\
\text { duplicate }\end{array}$ & $\begin{array}{c}\text { Analysis } \\
\text { type }\end{array}$ \\
\hline $\begin{array}{l}\mathbf{5 0 8 6} \\
\mathbf{5 0 8 7} \\
\mathbf{5 0 8 8} \\
\mathbf{5 0 8 9} \\
\mathbf{5 0 9 0}\end{array}$ & $\begin{array}{l}\text { ORR } \\
\text { ORR } \\
\text { ORR } \\
\text { ORR } \\
\text { ORR }\end{array}$ & $\begin{array}{l}\text { NOLI I CHUCKY } \\
\text { NOLI ICHUCKY } \\
\text { NOLI ICHUCKY } \\
\text { NOL I CHUCKY } \\
\text { MOLI ICHUCKY }\end{array}$ & 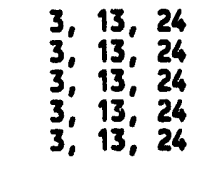 & $\begin{array}{l}B \\
B \\
C \\
C \\
C\end{array}$ & $\begin{array}{l}\text { SOIL } \\
\text { SOIL } \\
\text { SoIL } \\
\text { SoIL } \\
\text { SoIL }\end{array}$ & & $\begin{array}{l}\text { RADIONUCLIDES } \\
\text { EXTRA } \\
\text { INORGANICS } \\
\text { RADIONUCLIDES } \\
\text { EXTRA }\end{array}$ \\
\hline $\begin{array}{l}6001 \\
6002 \\
6003 \\
6004 \\
6005\end{array}$ & $\begin{array}{l}\text { ROA } \\
\text { ROA } \\
\text { ROA } \\
\text { ROA } \\
\text { ROA }\end{array}$ & $\begin{array}{l}\text { DISMAL GAP } \\
\text { DISMAL GAP } \\
\text { DISMAL GAP } \\
\text { DISMAL GAP } \\
\text { DISNAL GAP }\end{array}$ & 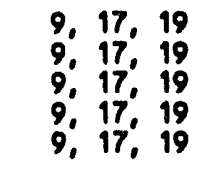 & $\begin{array}{l}0 \\
B \\
B \\
A \\
A\end{array}$ & $\begin{array}{l}\text { SoIL } \\
\text { SOIL } \\
\text { SoIL } \\
\text { SoIL } \\
\text { SOIL }\end{array}$ & & $\begin{array}{l}\text { INORGANICS } \\
\text { RADIONUCLIDES } \\
\text { EXTRA } \\
\text { INORGANICS } \\
\text { RADIONUCLIDES }\end{array}$ \\
\hline $\begin{array}{l}6006 \\
6007 \\
6008 \\
6009 \\
6010\end{array}$ & $\begin{array}{l}\text { ROA } \\
\operatorname{ROA} \\
\operatorname{ROA} \\
\operatorname{ROA} \\
\text { ROA }\end{array}$ & $\begin{array}{l}\text { DISMAL GAP } \\
\text { DISMAL GAP } \\
\text { DISMAL GAP } \\
\text { DISMAL GAP } \\
\text { DISMAL GAP }\end{array}$ & 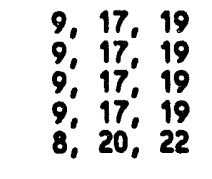 & $\begin{array}{l}A \\
\mathbf{C} \\
\mathbf{C} \\
\mathbf{C} \\
\mathbf{A}\end{array}$ & $\begin{array}{l}\text { SOIL } \\
\text { SOIL } \\
\text { SoIL } \\
\text { SOIL } \\
\text { SOIL }\end{array}$ & & $\begin{array}{l}\text { EXTRA } \\
\text { INORGANICS } \\
\text { RADIONUCLIDES } \\
\text { EXTRA } \\
\text { INORGANICS }\end{array}$ \\
\hline $\begin{array}{l}6011 \\
6012 \\
6013 \\
6014 \\
6015\end{array}$ & $\begin{array}{l}\text { ROA } \\
\text { ROA } \\
\text { ROA } \\
\text { ROA } \\
\text { ROA }\end{array}$ & $\begin{array}{l}\text { DISHAL GAP } \\
\text { DISMAL GAP } \\
\text { DISMAL GAP } \\
\text { DISHAL GAP } \\
\text { DISNAL GAP }\end{array}$ & 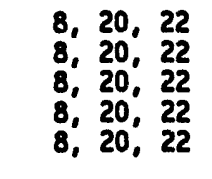 & $\begin{array}{l}\hat{A} \\
\hat{A} \\
\mathbf{B} \\
\mathbf{B} \\
\mathbf{B}\end{array}$ & $\begin{array}{l}\text { SOIL } \\
\text { SOIL } \\
\text { SOIL } \\
\text { SoIL } \\
\text { SOIL }\end{array}$ & & $\begin{array}{l}\text { RADIONUCLIDES } \\
\text { EXTRA } \\
\text { INORGANICS } \\
\text { RADIONUCLIDES } \\
\text { EXTRA }\end{array}$ \\
\hline $\begin{array}{l}6016 \\
6017 \\
6018 \\
6019 \\
6020\end{array}$ & $\begin{array}{l}\text { ROA } \\
\text { ROA } \\
\text { ROA } \\
\text { ROA } \\
\text { ROA }\end{array}$ & $\begin{array}{l}\text { DISNAL GAP } \\
\text { DISMAL GAP } \\
\text { DISNAL GAP } \\
\text { DISMAL GAP } \\
\text { DISNAL GAP }\end{array}$ & 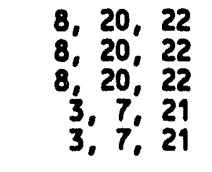 & $\begin{array}{l}\mathbf{C} \\
\mathbf{C} \\
\mathbf{C} \\
\hat{A} \\
\hat{A}\end{array}$ & $\begin{array}{l}\text { SoIL } \\
\text { soll } \\
\text { SoIL } \\
\text { soll } \\
\text { Sit- }\end{array}$ & & $\begin{array}{l}\text { INORGANICS } \\
\text { RADIONUCLIDES } \\
\text { EXTRA } \\
\text { INORGAVICS } \\
\text { RADIONICLIDES }\end{array}$ \\
\hline $\begin{array}{l}6021 \\
6022 \\
6023 \\
6024 \\
6025\end{array}$ & $\begin{array}{l}\text { ROA } \\
\operatorname{ROA} \\
\operatorname{ROA} \\
\operatorname{ROA} \\
\text { ROA }\end{array}$ & $\begin{array}{l}\text { DISUAL GAP } \\
\text { DISHAL GAP } \\
\text { DISNAL GAP } \\
\text { DISHAL GAP } \\
\text { DISNAL GAP }\end{array}$ & $\begin{array}{l}3,7,21 \\
3,7,21 \\
3,7,21 \\
3,7,21 \\
3,7,21\end{array}$ & $\begin{array}{l}\mathbf{A} \\
\mathbf{B} \\
\mathbf{B} \\
\mathbf{B} \\
\mathbf{C}\end{array}$ & $\begin{array}{l}\text { SOIL } \\
\text { SOIL } \\
\text { SOIL } \\
\text { SOIL } \\
\text { SOIL }\end{array}$ & & $\begin{array}{l}\text { EXTRA } \\
\text { INORGANICS } \\
\text { RADIONUCLIDES } \\
\text { EXIRA } \\
\text { IMORGANICS }\end{array}$ \\
\hline $\begin{array}{l}6026 \\
6027 \\
6028 \\
6029 \\
6030\end{array}$ & $\begin{array}{l}\text { ROA } \\
\text { ROA } \\
\text { ROA } \\
\text { ROA } \\
\text { ROA }\end{array}$ & $\begin{array}{l}\text { DISMAL GAP } \\
\text { DISMAL GAP } \\
\text { DISMAL GAP } \\
\text { DISMAL GAP } \\
\text { DISMAL GAP }\end{array}$ & $\begin{array}{r}3,7,21 \\
3,7,21 \\
10,13,14 \\
10,13,14 \\
10,13,14\end{array}$ & $\begin{array}{l}\mathbf{C} \\
\mathbf{C} \\
\hat{A} \\
\hat{A}\end{array}$ & $\begin{array}{l}\text { SoIL } \\
\text { SoIL } \\
\text { SoIL } \\
\text { SoIL } \\
\text { SoIL }\end{array}$ & & $\begin{array}{l}\text { RADIONUCL IDES } \\
\text { EXTRA } \\
\text { INORGANICS } \\
\text { RADIONUCLIDES } \\
\text { EXTRA }\end{array}$ \\
\hline $\begin{array}{l}6031 \\
6032 \\
6033 \\
6034 \\
6035\end{array}$ & $\begin{array}{l}\text { ROA } \\
\operatorname{ROA} \\
\operatorname{ROA} \\
\operatorname{ROA} \\
\text { ROA }\end{array}$ & $\begin{array}{l}\text { DISMAL GAP } \\
\text { DISMAL GAP } \\
\text { DISMAL GAP } \\
\text { DISMAL GAP } \\
\text { DISMAL GAP }\end{array}$ & $\begin{array}{l}10,13,14 \\
10,13,14 \\
10,13,14 \\
10,13,14 \\
10,13,14\end{array}$ & $\begin{array}{l}B \\
B \\
B \\
C \\
C\end{array}$ & $\begin{array}{l}\text { SOIL } \\
\text { SOIL } \\
\text { SoIL } \\
\text { soIL } \\
\text { SoIL }\end{array}$ & & $\begin{array}{l}\text { INORGANICS } \\
\text { RADIONUCLIDES } \\
\text { EXTRA } \\
\text { IMORGANICS } \\
\text { RADIONUCLIDES }\end{array}$ \\
\hline $\begin{array}{l}6036 \\
6037 \\
6038 \\
6039 \\
6040\end{array}$ & $\begin{array}{l}\text { ROA } \\
\text { ROA } \\
\text { ROA } \\
\text { ROA } \\
\text { ROA }\end{array}$ & $\begin{array}{l}\text { DISMAL GAP } \\
\text { DISMAL GAP } \\
\text { DISMAL GAP } \\
\text { DI SMAL GAP } \\
\text { DISMAL GAP }\end{array}$ & $\begin{array}{l}10,13,14 \\
10,13,14 \\
10,13,14 \\
10,13,14 \\
10,13,14\end{array}$ & $\begin{array}{l}\mathbf{C} \\
\hat{A} \\
\hat{A} \\
\hat{B}\end{array}$ & $\begin{array}{l}\text { SoIL } \\
\text { SOIL } \\
\text { SoIL } \\
\text { SoIL } \\
\text { SOIL }\end{array}$ & $\begin{array}{l}\text { FIELD DUPLICATE } \\
\text { FIELD DUPLICATE } \\
\text { FIELD DUPLICATE } \\
\text { FIELD DUPLICATE }\end{array}$ & $\begin{array}{l}\text { EXIRA } \\
\text { INORGANICS } \\
\text { RADIONUCLIDES } \\
\text { EXTRA } \\
\text { IMORGANICS }\end{array}$ \\
\hline $\begin{array}{l}6041 \\
6042 \\
6043 \\
6044 \\
6045\end{array}$ & $\begin{array}{l}\text { ROA } \\
\text { ROA } \\
\text { ROA } \\
\text { ROA } \\
\text { ROA }\end{array}$ & $\begin{array}{l}\text { DISHAL GAP } \\
\text { DISMAL GAP } \\
\text { DISMAL GAP } \\
\text { DISMAL GAP } \\
\text { DISMAL GAP }\end{array}$ & $\begin{array}{ll}10,13, & 14 \\
10, & 13,14 \\
10,13,14 \\
10,13,14 \\
10,13,14\end{array}$ & $\begin{array}{l}B \\
B \\
C \\
C \\
C\end{array}$ & $\begin{array}{l}\text { SOIL } \\
\text { SOIL } \\
\text { SOIL } \\
\text { SOIL } \\
\text { SOIL }\end{array}$ & $\begin{array}{l}\text { FIELD DUPLICATE } \\
\text { FIELD DUPLICATE } \\
\text { FIELD DUPLICATE } \\
\text { FIELD DUPLICATE } \\
\text { FIELD DUPLICATE }\end{array}$ & $\begin{array}{l}\text { RADIONUCLIDES } \\
\text { EXTRA } \\
\text { INORGANICS } \\
\text { RADIONUCLIDES } \\
\text { EXTRA }\end{array}$ \\
\hline $\begin{array}{l}7001 \\
7002 \\
7003 \\
7004 \\
7005\end{array}$ & $\begin{array}{l}\text { AND } \\
\text { AND } \\
\text { AND } \\
\text { AND } \\
\text { AND }\end{array}$ & $\begin{array}{l}\text { DISMAL GAP } \\
\text { DISMAL GAP } \\
\text { DISMAL GAP } \\
\text { DISMAL GAP } \\
\text { DISMAL GAP }\end{array}$ & $\begin{array}{ll}9, & 10,19 \\
9, & 10,19 \\
9, & 10,19 \\
9, & 10,19 \\
9, & 10,19\end{array}$ & $\begin{array}{l}\hat{A} \\
\hat{A} \\
\hat{B} \\
B\end{array}$ & $\begin{array}{l}\text { SoIL } \\
\text { SOIL } \\
\text { SOIL } \\
\text { SOIL } \\
\text { SOIL }\end{array}$ & & $\begin{array}{l}\text { IMORGANICS } \\
\text { RADIONUCLIDES } \\
\text { EXTRA } \\
\text { IMORGANICS } \\
\text { RADIONUCLIDES }\end{array}$ \\
\hline $\begin{array}{l}7006 \\
7007 \\
7008 \\
7009 \\
7010\end{array}$ & $\begin{array}{l}\text { AND } \\
\text { AND } \\
\text { AND } \\
\text { AND } \\
\text { AND }\end{array}$ & $\begin{array}{l}\text { DISMAL GAP } \\
\text { DISMAL GAP } \\
\text { DISMAL GAP } \\
\text { DISMAL GAP } \\
\text { DISWAL GAP }\end{array}$ & $\begin{array}{l}9,10,19 \\
9,10,19 \\
9,10,19 \\
9,10,19 \\
3,5,11\end{array}$ & $\begin{array}{l}B \\
C \\
C \\
C \\
A\end{array}$ & $\begin{array}{l}\text { SOIL } \\
\text { SOIL } \\
\text { SOIL } \\
\text { SOIL } \\
\text { SOIL }\end{array}$ & & $\begin{array}{l}\text { EXTRA } \\
\text { INORGANICS } \\
\text { RADIONUCLIDES } \\
\text { EXTRA } \\
\text { INORGANICS }\end{array}$ \\
\hline
\end{tabular}


G-13

Table G.1 (continued)

\begin{tabular}{|c|c|c|c|c|c|c|c|}
\hline $\begin{array}{c}\text { Sample } \\
\text { ID }\end{array}$ & Location & Formation & Site & inon & Matrix & $\begin{array}{c}\text { Field } \\
\text { duplicate }\end{array}$ & $\begin{array}{c}\text { Analysis } \\
\text { type }\end{array}$ \\
\hline $\begin{array}{l}7011 \\
7012 \\
7013 \\
7014 \\
7015\end{array}$ & $\begin{array}{l}\text { AMD } \\
\text { AMD } \\
A M D \\
A N D \\
A N D\end{array}$ & $\begin{array}{l}\text { DISMAL GAP } \\
\text { DISNAL GAP } \\
\text { DISMAL GAP } \\
\text { DISHAL GAP } \\
\text { DISMLL GAP }\end{array}$ & $\begin{array}{l}3,5,11 \\
3,5,11 \\
3,5,11 \\
3,5,11 \\
3,5,11\end{array}$ & $\begin{array}{l}\mathbf{A} \\
\hat{A} \\
\mathbf{B} \\
\mathbf{B} \\
\mathbf{B}\end{array}$ & $\begin{array}{l}\text { SoIL } \\
\text { SoIL } \\
\text { SoIL } \\
\text { soIL } \\
\text { SoIL }\end{array}$ & & $\begin{array}{l}\text { RADIONUCLIDES } \\
\text { EXTRA } \\
\text { INORGANICS } \\
\text { RADIONUCLIDES } \\
\text { EXTRA }\end{array}$ \\
\hline $\begin{array}{l}7016 \\
7017 \\
7018 \\
7019 \\
7020\end{array}$ & $\begin{array}{l}\text { AND } \\
\text { AND } \\
\text { AND } \\
\text { AND } \\
\text { AND }\end{array}$ & $\begin{array}{l}\text { DISMLL GAP } \\
\text { DISMAL GAP } \\
\text { DISMLL GAP } \\
\text { DISMUL GAP } \\
\text { DISMAL GAP }\end{array}$ & $\begin{array}{l}3,5,11 \\
3,5,11 \\
3,5,11 \\
4,12,21 \\
4,12,21\end{array}$ & $\begin{array}{l}\mathbf{C} \\
\mathbf{C} \\
\mathbf{C} \\
\hat{A} \\
\hat{A}\end{array}$ & $\begin{array}{l}\text { Soll } \\
\text { soll } \\
\text { SoIL } \\
\text { soll } \\
\text { SoIL }\end{array}$ & & $\begin{array}{l}\text { INORGANICS } \\
\text { RADIONUCLIDES } \\
\text { EXTRA } \\
\text { INORGANICS } \\
\text { RADIONUCLIDES }\end{array}$ \\
\hline $\begin{array}{l}7021 \\
7022 \\
7023 \\
7024 \\
7025\end{array}$ & $\begin{array}{l}\text { AND } \\
\text { AND } \\
\text { AND } \\
\text { AND } \\
\text { AND }\end{array}$ & $\begin{array}{l}\text { DISHAL GAP } \\
\text { DISMAL GAP } \\
\text { DISMAL GAP } \\
\text { DISMAL GAP } \\
\text { DISHAL GAP }\end{array}$ & $\begin{array}{l}4,12,21 \\
4,12,21 \\
4,12,21 \\
4,12,21 \\
4,12,21\end{array}$ & $\begin{array}{l}A \\
B \\
B \\
B \\
C\end{array}$ & $\begin{array}{l}\text { SoIL } \\
\text { soll } \\
\text { soll } \\
\text { soll } \\
\text { soll }\end{array}$ & & $\begin{array}{l}\text { EXTRA } \\
\text { INORGANICS } \\
\text { RADIONUCLIDES } \\
\text { EXTRA } \\
\text { INORGANICS }\end{array}$ \\
\hline $\begin{array}{l}7026 \\
7027 \\
7028 \\
7029 \\
7030\end{array}$ & $\begin{array}{l}\text { AND } \\
\text { AND } \\
\text { AND } \\
\text { AND } \\
\text { AND }\end{array}$ & $\begin{array}{l}\text { DISMAL GAP } \\
\text { DISMAL GAP } \\
\text { DISMAL GAP } \\
\text { DISMAL GAP } \\
\text { DISMAL GAP }\end{array}$ & $\begin{array}{l}4,12,21 \\
4,12,21 \\
1: 20,22 \\
1,20,22 \\
1,20,22\end{array}$ & $\begin{array}{l}\mathbf{C} \\
\mathbf{C} \\
\hat{A} \\
\hat{A}\end{array}$ & $\begin{array}{l}\text { SoIL } \\
\text { SOIL } \\
\text { SoIL } \\
\text { soll } \\
\text { SOIL }\end{array}$ & & $\begin{array}{l}\text { RADIONUCLIIDES } \\
\text { EXTRA } \\
\text { INORGANICS } \\
\text { RADIONUCLIDES } \\
\text { EXTRA }\end{array}$ \\
\hline $\begin{array}{l}7031 \\
7032 \\
7033 \\
7034 \\
7035\end{array}$ & $\begin{array}{l}\text { AND } \\
\text { AND } \\
\text { AND } \\
\text { AND } \\
\text { AND }\end{array}$ & $\begin{array}{l}\text { DISMAL GAP } \\
\text { DISHAL GAP } \\
\text { DISMAL GAP } \\
\text { DISMAL GAP } \\
\text { DISHAL GAP }\end{array}$ & $\begin{array}{l}1,20,22 \\
1,20,22 \\
1,20,22 \\
1,20,22 \\
1,20,22\end{array}$ & $\begin{array}{l}\text { B } \\
\mathbf{B} \\
\mathbf{B} \\
\mathbf{C} \\
\mathbf{C}\end{array}$ & $\begin{array}{l}\text { SoIL } \\
\text { SoIL } \\
\text { SoIL } \\
\text { SoIL } \\
\text { SoIL }\end{array}$ & & $\begin{array}{l}\text { INORGANICS } \\
\text { RADIONUCL IDES } \\
\text { EXTRA } \\
\text { INORGANICS } \\
\text { RADIONUCLIDES }\end{array}$ \\
\hline $\begin{array}{l}7036 \\
7037 \\
7038 \\
7039 \\
7040\end{array}$ & $\begin{array}{l}\text { AND } \\
\text { AND } \\
\text { AND } \\
\text { AND } \\
\text { AND }\end{array}$ & $\begin{array}{l}\text { DISMAL GAP } \\
\text { DISMAL GAP } \\
\text { DISHAL GAP } \\
\text { DISMAL GAP } \\
\text { DISMAL GAP }\end{array}$ & $\begin{array}{l}1,20,22 \\
9,10,19 \\
9,10,19 \\
9,10,19 \\
9,10,19\end{array}$ & $\begin{array}{l}\mathbf{C} \\
\hat{A} \\
\hat{A} \\
\mathbf{B}\end{array}$ & $\begin{array}{l}\text { SOIL } \\
\text { SOIL } \\
\text { SOIL } \\
\text { SOIL } \\
\text { SOIL }\end{array}$ & $\begin{array}{l}\text { FIELD DUPLICATE } \\
\text { FIELD DUPLICATE } \\
\text { FIELD DUPLICATE } \\
\text { FIELD DUPLICATE }\end{array}$ & $\begin{array}{l}\text { EXTRA } \\
\text { INORGANICS } \\
\text { RADIONUCLIIDES } \\
\text { EXTRA } \\
\text { INORGANICS }\end{array}$ \\
\hline $\begin{array}{l}7041 \\
7042 \\
7043 \\
7044 \\
7045\end{array}$ & $\begin{array}{l}\text { AND } \\
\text { AND } \\
\text { AND } \\
\text { AND } \\
\text { AND }\end{array}$ & $\begin{array}{l}\text { DISHAL GAP } \\
\text { DISHAL GAP } \\
\text { DISHAL GAP } \\
\text { DISHLL GAP } \\
\text { DISHAL GAP }\end{array}$ & $\begin{array}{l}9,10,19 \\
9,10,19 \\
9,10,19 \\
9,10,19 \\
9,10,19\end{array}$ & $\begin{array}{l}B \\
B \\
C \\
C \\
C\end{array}$ & $\begin{array}{l}\text { SOIL } \\
\text { SOIL } \\
\text { SOIL } \\
\text { soIL } \\
\text { SOIL }\end{array}$ & $\begin{array}{l}\text { FIELD DUPLICATE } \\
\text { FIELD DUPLICATE } \\
\text { FIELD DUPLICATE } \\
\text { FIELD DUPLICATE } \\
\text { FIELD DUPLICATE }\end{array}$ & $\begin{array}{l}\text { RADIONUCLIDES } \\
\text { EXTRA } \\
\text { INORGANICS } \\
\text { RADIONUCLIDES } \\
\text { EXTRA }\end{array}$ \\
\hline
\end{tabular}


Appendix $\mathrm{H}$

OCCURRENCES OF REJECTED DATA 
Table H.1. Occurrences of $R$ validation prefix-Phase I

\begin{tabular}{|c|c|c|c|c|c|c|}
\hline Anatysis Type & Analysis & Location & Formation & Matrix & Horizon & Count \\
\hline \multirow[t]{32}{*}{ Ongunies } & Acenaphthene & AND & Dismal Gap & Soil & $\mathbf{A}$ & 3 \\
\hline & & ORR & Dismal Gap & Soil & $\mathbf{A}$ & 1 \\
\hline & & ROA & Dismal Gap & Soil & $\mathbf{A}$ & $\frac{1}{5}$ \\
\hline & Acenaphthylene & AND & Dismal Gap & Scil & $\mathbf{A}$ & 13 \\
\hline & & ORR & Dismal Gap & Soil & $\mathbf{A}$ & 1 \\
\hline & & ROA & Dismal Gap & Soil & $\mathbf{A}$ & $\frac{1}{15}$ \\
\hline & Anthracene & AND & Dismal Gap & Soil & $\mathbf{A}$ & 11 \\
\hline & & ORR & Dismal Gap & Soil & $\mathbf{A}$ & 1 \\
\hline & & ROA & Dismal Gap & Soil & $\mathbf{A}$ & $\frac{1}{13}$ \\
\hline & Berzo[a]anthracene & ANVD & Dismal Gap & Soil & $\mathbf{A}$ & 13 \\
\hline & & ORR & Dismal Gap & Soil & $\mathbf{A}$ & 1 \\
\hline & & ORR & Nolichucky & Soil & $\mathbf{A}$ & 10 \\
\hline & & ROA & Dismal Gap & Soil & $\mathbf{A}$ & $\frac{3}{27}$ \\
\hline & Benzo[a]pyrene & AND & Dismal Gap & Soil & $\mathbf{A}$ & 2 \\
\hline & & ORR & Dismal Gap & Soil & $\mathbf{A}$ & 1 \\
\hline & & ROA & Dismal Gap & Soil & $\mathbf{A}$ & $-\frac{1}{4}$ \\
\hline & Benzo[b]fl: oranthene & AND & Dismal Gap & Soil & $\mathbf{A}$ & 4 \\
\hline & & ORR & Dismal Gap & Soil & $\mathbf{A}$ & 1 \\
\hline & & ROA & Dismal Gap & Soil & $\mathbf{A}$ & $\frac{1}{6}$ \\
\hline & Benzo $[g, h, i]$ perylene & AND & Dismal Gap & Soil & $\mathbf{A}$ & 23 \\
\hline & & ORR & Dismal Gap & Soil & $\mathbf{A}$ & 1 \\
\hline & & ORP. & Nolichucky & Water & & 2 \\
\hline & & ROA & Dismal Gap & Soil & $\mathbf{A}$ & $\frac{1}{27}$ \\
\hline & Benzo $[k]$ fluoranthene & AND & Dismal Gap & Soil & $\mathbf{A}$ & 12 \\
\hline & & ORR & Dismal Gap & Soil & $\mathbf{A}$ & 1 \\
\hline & & ROA & Dismal Gap & Soil & $\mathbf{A}$ & $\frac{1}{14}$ \\
\hline & Chrysene & AND & Dismal Gap & Soil & $\mathbf{A}$ & 16 \\
\hline & & ORR & Dismal Gap & Soil & $\mathbf{A}$ & 1 \\
\hline & & ORK & Nolichucky & Soil & $\mathbf{A}$ & 10 \\
\hline & & ROA & Dismal Gap & Soil & $\mathbf{A}$ & $\frac{3}{30}$ \\
\hline & Dalapon & $\mathrm{O} / \mathrm{R}$ & Dismai Gap & Soil & $\mathbf{A}$ & 9 \\
\hline & & RUA & Dismal Gap & Soil & $\mathbf{A}$ & $\frac{3}{12}$ \\
\hline
\end{tabular}


H-4

Table H.1 (continued)

\begin{tabular}{|c|c|c|c|c|c|c|}
\hline Analysis Type & Analysis & Location & Formation & Matrix & Horizon & Count \\
\hline & Dibenzo[a,h]anthracene & $\begin{array}{l}\text { AND } \\
\text { ORR } \\
\text { ORR } \\
\text { ROA }\end{array}$ & $\begin{array}{l}\text { Dismal Gap } \\
\text { Dismal Gap } \\
\text { Nolichucky } \\
\text { Dismal Gap }\end{array}$ & $\begin{array}{l}\text { Soil } \\
\text { Soil } \\
\text { Water } \\
\text { Soil }\end{array}$ & $\begin{array}{l}\mathbf{A} \\
\mathbf{A} \\
\mathbf{A}\end{array}$ & $\begin{array}{r}24 \\
1 \\
2 \\
1 \\
28\end{array}$ \\
\hline & Fluoranthene & $\begin{array}{l}\text { AND } \\
\text { ORR } \\
\text { ORR } \\
\text { ROA }\end{array}$ & $\begin{array}{l}\text { Dismal Gap } \\
\text { Dismal Gap } \\
\text { Nolichucky } \\
\text { Dismal Gap }\end{array}$ & $\begin{array}{l}\text { Soil } \\
\text { Soil } \\
\text { Soil } \\
\text { Soil }\end{array}$ & $\begin{array}{l}\mathbf{A} \\
\mathbf{A} \\
\mathbf{A} \\
\mathbf{A}\end{array}$ & $\begin{array}{l}13 \\
10 \\
12 \\
12 \\
47\end{array}$ \\
\hline & Fluorene & $\begin{array}{l}\text { AND } \\
\text { ORR } \\
\text { ROA }\end{array}$ & $\begin{array}{l}\text { Dismal Gap } \\
\text { Dismal Gap } \\
\text { Dismal Gap }\end{array}$ & $\begin{array}{l}\text { Soil } \\
\text { Soil } \\
\text { Soil }\end{array}$ & $\begin{array}{l}\mathbf{A} \\
\mathbf{A} \\
\mathbf{A}\end{array}$ & $\begin{array}{r}14 \\
1 \\
1 \\
16\end{array}$ \\
\hline & Indeno-1,2,3(c,d)-pyrene & $\begin{array}{l}\text { AND } \\
\text { ORR } \\
\text { ROA }\end{array}$ & $\begin{array}{l}\text { Dismal Gap } \\
\text { Dismal Gap } \\
\text { Dismal Gap }\end{array}$ & $\begin{array}{l}\text { Soil } \\
\text { Soil } \\
\text { Soil }\end{array}$ & $\begin{array}{l}\mathbf{A} \\
\mathbf{A} \\
\mathbf{A}\end{array}$ & $\begin{array}{r}15 \\
1 \\
1 \\
17\end{array}$ \\
\hline & Naphthalene & $\begin{array}{l}\text { AND } \\
\text { ORR }\end{array}$ & $\begin{array}{l}\text { Dismal Gap } \\
\text { Dismal Gap }\end{array}$ & $\begin{array}{l}\text { Soil } \\
\text { Soil }\end{array}$ & $\begin{array}{l}\mathbf{A} \\
\mathbf{A}\end{array}$ & $\begin{array}{r}10 \\
1 \\
11\end{array}$ \\
\hline & Phenaibinene & $\begin{array}{l}\text { AND } \\
\text { ORR } \\
\text { ROA }\end{array}$ & $\begin{array}{l}\text { Dismal Gap } \\
\text { Dismal Gap } \\
\text { Dismal Gap }\end{array}$ & $\begin{array}{l}\text { Soil } \\
\text { Soil } \\
\text { Soil }\end{array}$ & $\begin{array}{l}\mathbf{A} \\
\mathbf{A} \\
\mathbf{A}\end{array}$ & $\begin{array}{l}2 \\
1 \\
1 \\
4\end{array}$ \\
\hline & Pyrene & $\begin{array}{l}\text { AND } \\
\text { ORR } \\
\text { ROA }\end{array}$ & $\begin{array}{l}\text { Dismal Gap } \\
\text { Dismal Gap } \\
\text { Dismal Gap }\end{array}$ & $\begin{array}{l}\text { Soil } \\
\text { Soil } \\
\text { Soil }\end{array}$ & $\begin{array}{l}\mathbf{A} \\
\mathbf{A} \\
\mathbf{A}\end{array}$ & $\begin{array}{l}6 \\
1 \\
1 \\
8\end{array}$ \\
\hline Total onganics & & & & & & 284 \\
\hline Inarganics & Osmium & $\begin{array}{l}\text { AND } \\
\text { AND } \\
\text { AND } \\
\text { ORR } \\
\text { ORR } \\
\text { ORR } \\
\text { ORR } \\
\text { ORR } \\
\text { ORR } \\
\text { ORR } \\
\text { ROA } \\
\text { ROA } \\
\text { ROA } \\
\text { ROA }\end{array}$ & $\begin{array}{l}\text { Dismal Gap } \\
\text { Dismal Gap } \\
\text { Dismal Gap } \\
\text { Dismal Gap } \\
\text { Dismal Gap } \\
\text { Dismal Gap } \\
\text { Nolichucky } \\
\text { Nolichucky } \\
\text { Nolichucky } \\
\text { Nolichucky } \\
\text { Dismal Gap } \\
\text { Dismal Gap } \\
\text { Dismal Gap } \\
\text { Dismal Gap }\end{array}$ & $\begin{array}{l}\text { Soil } \\
\text { Soil } \\
\text { Soil } \\
\text { Soil } \\
\text { Soil } \\
\text { Soil } \\
\text { Soil } \\
\text { Soil } \\
\text { Soil } \\
\text { Soil } \\
\text { Soil } \\
\text { Soil } \\
\text { Soil } \\
\text { Water }\end{array}$ & $\begin{array}{l}\text { A } \\
\text { B } \\
\text { C } \\
\text { A } \\
\text { B } \\
\text { C } \\
\\
\text { A } \\
\text { B } \\
\text { C } \\
\text { A } \\
\text { B } \\
\text { C }\end{array}$ & $\begin{array}{l}5 \\
5 \\
5 \\
2 \\
2 \\
2 \\
1 \\
4 \\
4 \\
4 \\
3 \\
3 \\
3 \\
1 \\
\end{array}$ \\
\hline Total inorganies & & & & & & 44. \\
\hline
\end{tabular}


Table H.1 (continued)

\begin{tabular}{|c|c|c|c|c|c|c|}
\hline Analysis Type & Analysis & Location & Formation & Matrix & Horizon & Count \\
\hline \multirow[t]{24}{*}{ Radionuclides } & Neptunium-237 & AND & Dismal Gap & Soil & $\mathbf{A}$ & 5 \\
\hline & & ORR & Dismal Gap & Soil & A & 5 \\
\hline & & ORR & Dismal Gap & Soil & B & 2 \\
\hline & & ORR & Dismal Gap & Soil & C & 2 \\
\hline & & ORR & Nolichucky & Soil & & 1 \\
\hline & & ORR & Nolichucky & Soil & $\mathbf{A}$ & 4 \\
\hline & & ROA & Dismal Gap & Soil & A & 4 \\
\hline & & ROA & Dismal Gap & Soil & B & 1 \\
\hline & & ROA & Dismal Gap & Soil & $\bar{C}$ & $\frac{1}{25}$ \\
\hline & Plutonium-238 & ORR & Nolichucky & Soil & & 1 \\
\hline & Plutonium-239 & ORR & Nolichucky & Soil & & 1 \\
\hline & Plutonium-240 & ORR & Nolichucky & Soil & & 1 \\
\hline & Strontium-90 & AND & Dismal Gap & Soil & $\mathbf{A}$ & 5 \\
\hline & & ORR & Nolichucky & Soil & & 1 \\
\hline & & ORR & Nolichucky & Soil & $\mathbf{A}$ & $\frac{4}{10}$ \\
\hline & Technetium-99 & AND & Dismal Gap & Soil & A & 5 \\
\hline & & ORR & Dismal Gap & Soil & $\mathbf{A}$ & 5 \\
\hline & & ORR & Dismal Gap & Soil & B & 2 \\
\hline & & ORR & Dismal Gap & Soil & C & 2 \\
\hline & & ORR & Nolichucky & Soil & & 1 \\
\hline & & ORR & Nolichucky & Soil & A & 4 \\
\hline & & ROA & Dismal Gap & Soil & $\mathbf{A}$ & 5 \\
\hline & & ROA & Dismal Gap & Soil & B & 1 \\
\hline & & ROA & Dismal Gap & Soil & $\mathrm{C}$ & $\frac{1}{26}$ \\
\hline \multicolumn{2}{|c|}{ Total Radionuclides } & & & & & 64 \\
\hline \multirow[t]{2}{*}{$\boldsymbol{H}-\mathbf{3}$} & Tritium & ORR & Dismal Gap & Soil & $\mathbf{A}$ & 2 \\
\hline & & ROA & Dismal Gap & Soil & $\mathbf{A}$ & $\frac{1}{3}$ \\
\hline Total H-3 & & & & & & 3 \\
\hline \multicolumn{2}{|c|}{ Total data rejected } & & & & & $\underline{395}$ \\
\hline
\end{tabular}




\section{DISTRIBUTION}

1. L. D. Bates

2. F. P. Baxter

3. D. T. Bell

4. H. L. Boston

5. V. J. Brumback

6. J. F. Campbell

7. J. S. Colley

8. K W. Cook

9. R. B. Cook

10. M. F. P. DeLozier

11. D. J. Downing

12. C. W. Francis

13. P. L Goddard

14. C. D. Goins

15. J. T. Grumski

16. P. J. Halsey

17. T. L. Hatmaker

18. J. L. Haymore

19. R. K. Holmes

20. L. A. Hook

21. D. D. Huff

22. B. L Jackson

23. K. G. Kahl

24. R. H. Ketelle

25. C. W. Kimbrough

26. B. L. Kimmel

27. T. M. Koepp

28. A. J. Kuhaida

29. G. R. Larson
30-31. S. Y. Lee

32-34. D. M. Matteo

35. C. W. McGinn

36. B. J. Montgomery

37. M. J. Norris

38. B. D. Nourse

39. F. R. O'Donnell

40-41. P. T. Owen

42. G. E. Rymer

43. A. W. Saulsbury

44. R. L. Schmoyer

45. P. A. Schrandt

46. R. A. Shaw

47. S. H. Stow

48. J. Switek

49. R. I. Van Hook

50. L. D. Voorhees

51. C. S. Walker

52-54. D. R. Watkins

55. S. C. Wells

56. R. K White

57. P. S. Wood

58. J. C. Wright

59. T. F. Zondlo

60. Central Research Library

61-63. ESD Library

64-65. Laboratory Records Department

66-70. ER Document Management Center

71. ORNL Patent Section

72. Office of Assistant Manager for Energy Research and Development, DOE Oak Ridge Operations Office, P.O. Box 2001, Oak Ridge, TN 37831-8600

73-75. J. T. Ammons, J. L. Branson, and B. B. Burgoa, Department of Plant and Soil Science, University of Tennessee, Knoxville, TN 37916

76. D. A. Lietzke, Lietzke Soil Services, Route No. 3, Box 607, Rutledge, TN 37861

77. D. M. Carden, DOE Oak Ridge Operations Office, P.O. Box 2001, Oak Ridge, TN 37831-8541

78. R. N. Farvolden, Department of Earth Sciences, University of Waterloo, Waterloo, Ontario N21 3G1 Canada

79. M. S. Ferre, DOE Oak Ridge Operations Office, P.O. Box 2001, Oak Ridge, TN 37831-8541

80. C. S. Gist, DOE Oak Ridge Operations Office, P.O. Box 2001, Oak Ridge, TN 37831-8541

81-82. R. L. Nace, Branch Chief, Nonenrichment Facilities, Oak Ridge Program Division, Office of Eastern Area Programs, Office of Environmental Restoration, EM-423, Trevion 2, U.S. Department of Energy, Washington, DC 20585

83. S. P. Riddle, DOE Oak Ridge Operations Office, P.O. Box 2001, Oak Ridge, TN 37831-8541

84-85. R. C. Sleeman, DOE Oak Ridge Operations Office, P.O. Box 2001, Oak Ridge, TN 37831-8541 
86-89. J. T. Sweeney, DOE Oak Ridge Operations Office, P.O. Box 2001, Oak Ridge, TN 37831-8541

90. D. W. Swindle, Radian Corporation, 120 S. Jefferson Circle, Oak Ridge, TN 37830

91-92. H. M. Thron, Chief, Enrichment Facilities, Oak Ridge Program Division, Office of Eastern Area Programs, Office of Environmental Restoration, EM-423, Trevion 2, U. S. Department of Energy, Washington, DC 20585

93-94. Office of Scientific and Technical Information, P.O. Box 62, Oak Ridge, TN 37831 

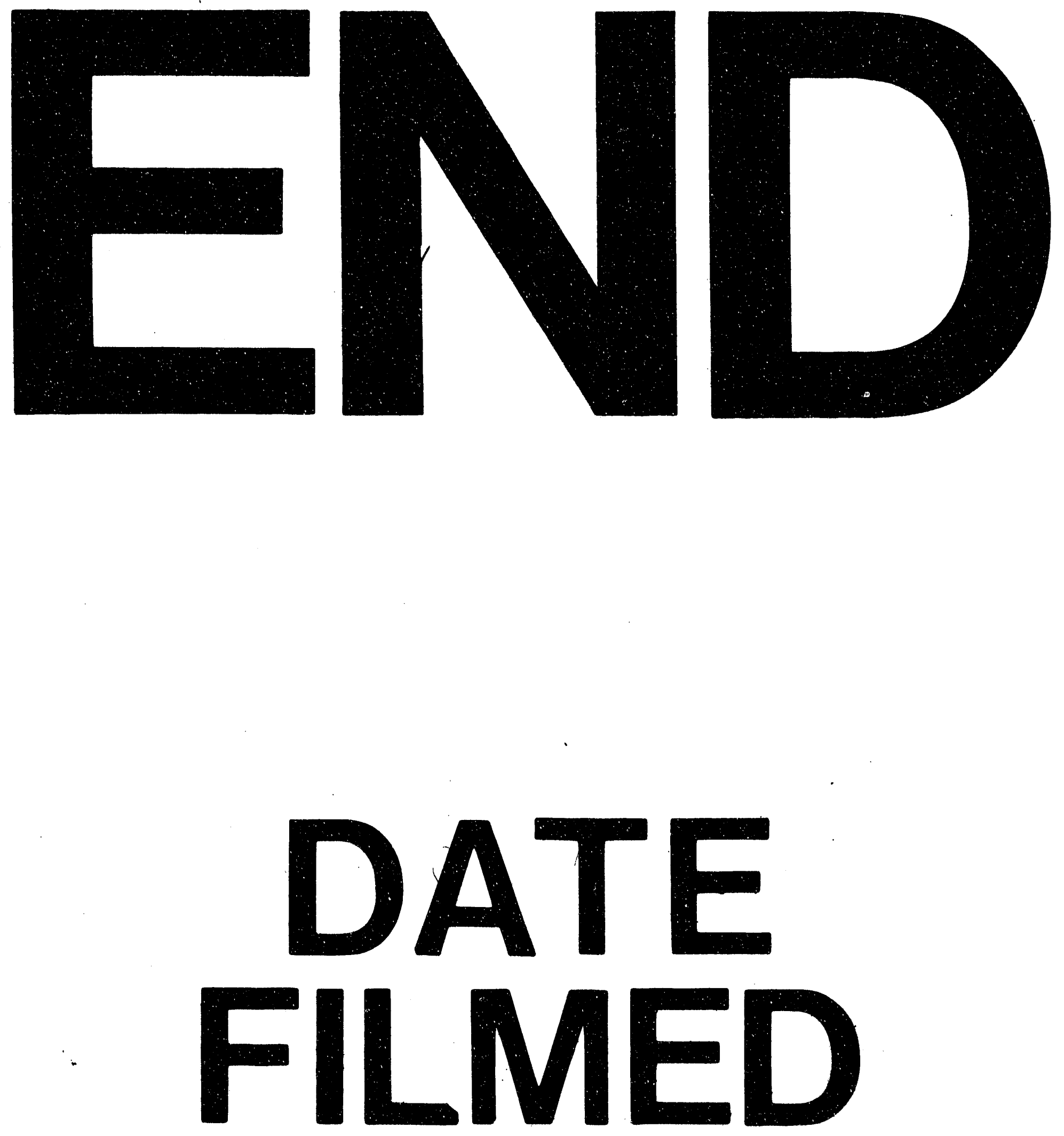

1

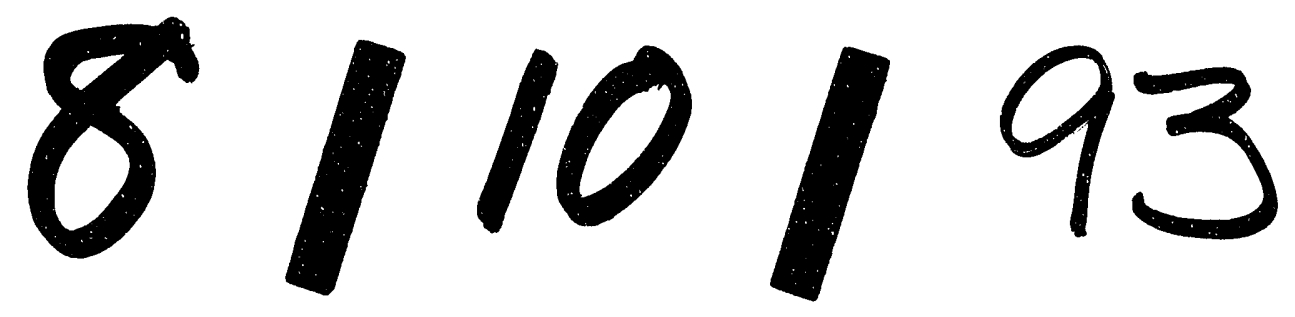


